Luoghi o Pacesiggi

Collana det Dottorato di Ricerca in Progethaione Paesistica
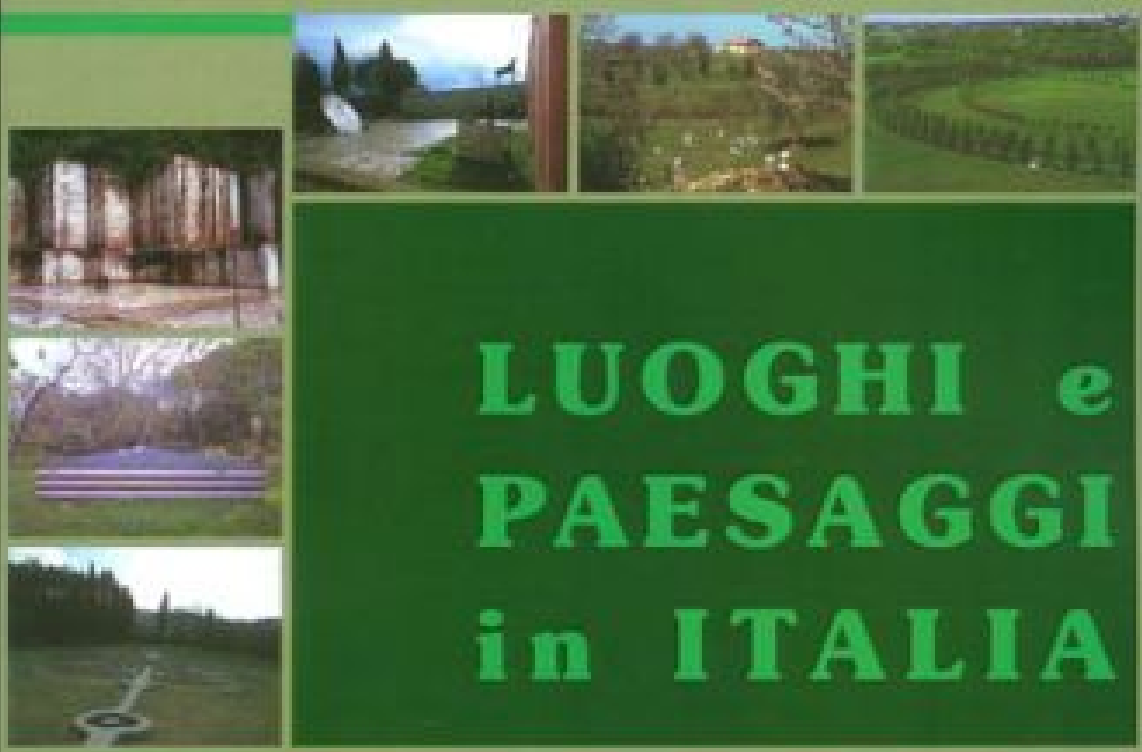

A cura di Giulio G. Rizzo e Antonella Valenmtini 




\section{LUOGHI E PAESAGGI}

Collana del Dottorato di Ricerca in Progettazione Paesistica dell'Università degli Studi di Firenze 



\title{
Luoghi e paesaggi in Italia
}

\author{
a cura di \\ Giulio Gino Rizzo \\ e \\ Antonella Valentini
}

Firenze University Press

2004 
Dottorato di Ricerca in Progettazione Paesistica

Dipartimento di Urbanistica e Progettazione del Territorio

UNIVERSITÀ DEGLI STUDI DI FIRENZE

<http://www.unifi.it/drprogettazionepaesistica>

CoOrdinatore:

Prof. Arch. Giulio G. Rizzo

\section{COLlegio DOCENTI:}

Prof. Arch. Giulio G. Rizzo, Università di Firenze Prof. Arch. Antonello Boatti, Politecnico di Milano

Prof. Arch. Augusto Boggiano, Università di Firenze

Prof. Arch. Carlo Buffa di Perrero, Politecnico di Torino

Prof. Arch. Gabriele Corsani, Università di Firenze

Prof. Arch. Pompeo Fabbri, Politecnico di Torino

Prof. Arch. Guido Ferrara, Università di Firenze

Prof. Carlo Alberto Garzonio, Università di Firenze

Prof. Arch. Attilia Peano, Politecnico di Torino

Prof. Arch. Mariella Zoppi, Università di Firenze

Prof. Arch. Cristina Trev, Politecnico di Milano

Prof. Arch. Danilo Palazzo, Politecnico di Milano

DOTTORI DI RICERCA:

Dott. Arch. Adele Caucci

Dott. Arch. Luigi Latini

Dott. Arch. Gabriele Paolinelli

Dott. Arch. Claudia Cassatella

Dott. Arch. Alessandra Cazzola
Dott. Arch. Enrica Dall'Ara

Dott. Arch. Yuritza Mendoza Garcia

Dott. Arch. Maristella Storti

DOTTORANDI:

Dott. Arch. Giorgio Costa

Dott. Arch. Michele Ercolini

Dott. Arch. Laura Ferrari

Dott. Arch. Francesca Finotto

Dott. Arch. Anna Lambertini

Dott. Arch. Paola Marzorati

Dott. Arch. Tessa Matteini

Dott. Arch. Michela Saragoni

Dott. Arc. Simona Olivieri

Dott. Arch. Silvia Mantovani

Dott. Arch. Andrea Meli

Dott. Arch. Emanuela Morelli

Dott. Arch. Sabrina Tozzini

Dott. Arch. Antonella Valentini

UNIVERSITÀ ASSOCIATE:

Politecnico di Milano

Politecnico di Torino

Contributi presentati in occasione di Configurando Espacios, IV Encuentro Internacional de Arquitectura en Video, Universidad Michoacana de San Nicolás de Hidalgo Morelia (México), 16-18 mayo 2002.

Copertina di Anna Lambertini

Luoghi e paesaggi in Italia / a cura di Giulio Gino Rizzo e Antonella Valentini. - Firenze : Firenze university press, 2004. (Luoghi e paesaggi : collana del Dottorato di Ricerca in Progettazione Paesistica dell'Università degli Studi di Firenze, 1) http://digital.casalini.it/8884531446

Stampa a richiesta disponibile su http://epress.unifi.it

ISBN 88-8453-144-6 (online)

ISBN 88-8453-143-8 (print)

712.0945 (ed. 20)

Architettura del paesaggio - Italia

(C) 2004 Firenze University Press

Università degli Studi di Firenze

Firenze University Press

Borgo Albizi, 28, 50122 Firenze, Italy

http://epress.unifi.it/ 


\section{INDICE}

INTRODUZIONE

Giulio G. Rizzo

PARCHI E PAESAGGIO

Andrea Meli

e paesaggistico della Val di Chiana in Toscana

Michele Ercolini

Il Parco Regionale della Valle del Ticino in Lombardia

Adele G. Caucci

Sistema dei Parchi della Val di Cornia in Toscana

Sabrina Tozzini

Piano per il Parco delle Madonie in Sicilia

Michele Ercolini

Piano del Parco Naturale Regionale

di Migliarino San Rossore Massaciuccoli in Toscana

Andrea Meli

PARCHI METROPOLITANI

La trasformazione "work in progress" di uno spazio aperto

di cintura metropolitana: Parco Nord Milano

Laura Ferrari

Il bosco quale elemento di riordino dello spazio metropolitano:

l'esperienza di "Boscoincittà" a Milano

Laura Ferrari

La bonifica di un sito inquinato quale impulso

alla progettazione dello spazio aperto:

il Bosco delle Querce di Seveso e Meda

Laura Ferrari

SPAZI URBANI

Enrica Dall'Ara 
Da fabbrica a spazio urbano: gli spazi esterni dello stabilimento 163

Fiat Lingotto, Torino

Claudia Cassatella

Il parco diffuso di Tonino Guerra in Romagna

Enrica Dall'Ara

SCHEDE

Il Parco urbano di Volterra

Emanuela Morelli

Parco Amendola a Modena

Michele Ercolini

Il parco Raffaele Viviani a Napoli: il parco delle grotte Antonella Valentini

Riqualificazione paesistico-ambientale di un sito industriale: stabilimento Snam di Panigaglia (La Spezia)

Antonella Valentini

SISTEMI FORTIFICATI

Maristella Storti

Il colle e la fortezza di Osoppo

Maristella Storti

Il sistema dei forti di Genova

Maristella Storti

L'illuminazione delle mura di Verona

Maristella Storti

SCHEDA

La rocca di Ravenna

Maristella Storti

LUOGHI PER L'ARTE

Emanuela Morelli

"Spazi d'arte a Celle: l'invenzione di un collezionista appassionato"

Anna Lambertini

Il Giardino dei Tarocchi di Niki de Saint Phalle a Garavicchio,

Capalbio

Emanuela Morelli

Il Giardino Hic Terminus Haeret di Daniel Spoerri

a Seggiano

Emanuela Morelli

L'Hortus Conclusus a Benevento

Enrica Dall'Ara

SCHEDA

Il recupero ambientale di un attività estrattiva dismessa da luogo di produzione materiale a luogo di produzione culturale:

Cava Borella a Vagli

Sabrina Tozzini 
Antonella Valentini

Il restauro di Villa Garzoni a Collodi

Antonella Valentini

Il Master Plan del giardino di Boboli

Anna Lambertini

Il ripristino dei giardini segreti di Villa Borghese

Anna Lambertini

Il Parco dell'Appia Antica

Alessandra Cazzola

IDEE E PROGETTI

Claudia Cassatella

La scoperta della presenza dei fiumi in città: Torino Città d'acque 391 Claudia Cassatella

La Greenway della Battaglia di Pavia

Michele Ercolini

Il "Programma di Paesaggio Chianti" nel Piano Territoriale

407 di Coordinamento della Provincia di Firenze Alessandra Cazzola 
2 Parco Regionale della Valle del Ticino in Lombardi

3 Sistema dei Parchi della Val di Cornia in Toscana

4 Piano per il Parco delle Madonie in Sicilia

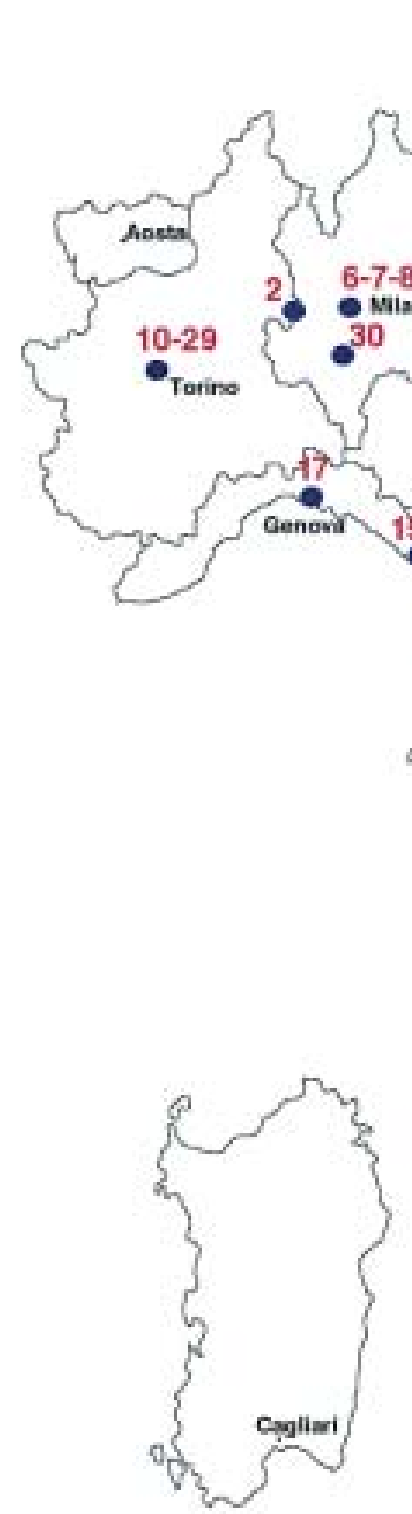

5 Piano del Parco Naturale Regionale di Migliarino San Rossore Massaciuccoli in Toscana

PARCHI METROPOLITANI

6 La trasformazione "work in progress" di uno spazio aperto di cintura metropolitana: Parco Nord Milano

7 Il bosco quale elemento di riordino dello spazio metropo-

litano: l'esperienza di Boscoincitta a Milano

8 La bonifica di un sito inquinato quale impulso alla progettazione dello spazio aperto: il Bosco delle Querce di Seveso e Meda

SPAZI URBANI

9 Il Parco Teodorico a Ravenna

10 Da fabbrica a spazio urbano: gli spazi esterni dello stabi-

limento Fiat Lingotto, Torino

1 ling

nino Guerra in Romag

1211 Parco ubano divolema

4 Parco Raffaele Viviani a Napoli: il parco delle grotte

5 Riqualificazione paesistico-ambientale di un sito ind striale: stabilimento Snam di Panigaglia (La Spezia) SISTEMI FORTIFICATI

16 Il colle e la fortezza di Osoppo (Udine)

17 Il sistema dei forti di Genova

18 L' illuminazione delle mura di Veron

19 La rocca di Ravenna

LUOGHI PER L'ARTE

20 "Spazi d'arte a Celle: linvenzione di un collezionista appassionato"

21 Il Giardino dei Tarocchi di Niki de Saint Phalle a Gar vicchio, Capalbio (Grosseto)

22 II Giardino Hic Terminus Haeret di Daniel Spoerri a Seggiano (Grosseto)

23 L'Hortus Conclusus a Benevento

24 Recupero ambientale di un attivita' estrattiva dismess

da luogo di produzione materiale a luogo di produzione

culturale: Cava Borella a Vagli (Lucca)

PAEsAGGISTORIC

5 Il restaro d Villa Garzoni 2 Collodi (Pistoia)

26 Master Plan di Boboli, Firenze

27 Il ripristino dei giardini segreti di Villa Borghese, Roma

28 Il Parco dell'Appia Antica (Roma)

IDEE E PROGETTI

29 La scoperta della presenza dei fiumi in città: Torino Cit-

tà d'Acque

$30 \mathrm{~L}$ Grenway della batraglia di Pavi

31 Il "Programma di Paesaggio del Chianti" nel Piano Ter-

ritoriale di Coordinamento della Provincia di Firenze 


\title{
INTRODUZIONE
}

\author{
Giulio G. Rizzo
}

Il Dottorato in Progettazione Paesistica è stato istituito in Firenze nel 1997 ed è il solo che si occupa di questo settore disciplinare in Italia. Per certi versi, dunque, ha un compito difficile e oneroso. Il dottorato ha come campo di ricerca la progettazione paesaggistica, in altre parole quel complesso di conoscenze (culturali, storiche, semantiche, metodologiche, teoriche e tecniche) che concorrono alla formazione della cultura del progetto paesistico per gli operatori chiamati a progettare, pianificare, ricuperare e riqualificare nei territori urbani ed extraurbani.

Il Dottorato è articolato in tre anni di studio e di ricerca che prefigurano, alla fine del triennio, due possibili esiti: la formazione di un architetto paesaggista, altamente qualificato, da inserire in organismi di ricerca pubblici (Università, CNR) o privati (Fondazioni operanti nel settore) e il contributo alla formazione di una figura di urbanista particolarmente sensibile ai valori dell'ambiente, delle aree protette e del paesaggio, quindi in grado di coprire ruoli di elevata responsabilità presso Enti pubblici ed Enti locali.

Il curriculum di studio e l'attività di ricerca sono modulati non solo per produrre esiti finalizzati ad un'eventuale carriera universitaria, ma anche per essere valutati e utilizzati nel più vasto campo della sperimentazione applicata al paesaggio.

Il Dottorato si avvale delle strutture del Dipartimento di Urbanistica e Pianificazione del Territorio e della Scuola di Specializzazione in Architettura dei Giardini e Progettazione del Paesaggio entrambi dell'Università di Firenze. In particolare, con la Scuola di Specializzazione in Architettura dei Giardini e Progettazione del Paesaggio (che dispone di un corpo docente multidisciplinare proveniente dalle Facoltà di Agraria, Architettura, Ingegneria, Scienze Forestali e Scienze Politiche), c'è, fin dalla sua istituzione, una fattiva sinergia che si esplica con l'organizzazione di attività svolte congiuntamente: seminari, lezioni integrative, partecipazione a gruppi di lavoro specifici e a viaggi di studio, eccetera. Le sedi aderenti partecipano con strutture analoghe essendo anch'esse, sia Milano, sia Torino, sia Roma, sede di Scuole di Specializzazione con contenuti e finalità simili.

Il Dottorato organizza periodicamente seminari interni e conferenze aperte al pubblico $^{1}$. I seminari e le conferenze hanno consentito ai dottorandi di discutere con i relatori su i temi della pianificazione delle aree protette, delle reti ecologiche, dei piani urbani del verde e del progetto, recupero e restauro degli spazi aperti e dei parchi urbani.

\footnotetext{
${ }^{1}$ Oltre, ovviamente alle normali lezioni previste dall'ordinamento. Ai seminari hanno partecipato personalità di alta qualificazione scientifica come, per esempio, Richard T.T. Forman della Harvard University, che ha inaugurato l'attività seminariale, Petra Potz della Universität Dortmund, Mario Di Fidio del Servizio Tutela ambiente naturale e Parchi della Regione Lombardia, Roberto Gambino del Politecnico di Torino, Bernardino Romano dell'Università de L'Aquila, Lando Bortolotti dello IUAV di Venezia e dell'Università di Trento, Vittoria Calzolari dell'Università di Roma "La Sapienza", Paolo Castelnovi del Politecnico di Torino, Richard Ingersoll della Syracuse University di Firenze.
} 
Attualmente, nei tre anni di Dottorato, sono attivati tre percorsi formativi da intendersi come linee guida per orientare le singole ricerche dei dottorandi, e non come camere stagne:

- aree naturali: piano e progetto;

- verde urbano: piano e progetto;

- le risorse naturali nel paesaggio urbano: l'acqua.

In sintesi, il primo curriculum formativo mira a formare un percorso che abbia l'ambiente e il paesaggio come fattori unificanti per la comprensione (analisi) e la pianificazione del territorio urbano ed extraurbano.

Il tema conduttore e quello della pianificazione del paesaggio e del recupero ambientale delle aree protette, a partire dalla consistenza delle specifiche risorse che queste contengono, entro cui il paesaggio assume un ruolo di sintesi, quale "risorsa delle risorse", e pone dei quesiti le cui risposte non sono per niente scontate.

S'individua, dunque, un percorso formativo che pone in evidenza il ruolo del paesaggio nella trasformazione e gestione del territorio a scala sovracomunale: Piani d'area vasta, Piani territoriali provinciali e regionali, Piani delle Comunità Montane, Piani di Bacino e, infine, in termini più specifici e definiti, i Piani delle varie tipologie di Parco da redigere in base alla vigente legislazione nazionale e regionale. La raccolta, lo studio e il confronto dei vari contributi metodologici sia italiani sia esteri puntano ad evidenziare come il progetto di paesaggio sia il risultato finale di tutto il percorso formativo del piano: partendo dalle analisi, attraverso le valutazioni fino alle proposte progettuali.

Il secondo curriculum formativo mira a formare un dottore di ricerca capace di inserirsi, con autorevolezza, nel recente panorama europeo per la definizione progettuale degli spazi aperti nella città contemporanea.

L'attività nel campo della Progettazione Paesistica, a partire dalla meta' del secolo scorso, ha visto l'affermarsi non solo delle questioni ambientali e paesaggistiche alla grande scala, ma anche il sicuro progresso della riflessione disciplinare e professionale sulle tematiche del progetto alla piccola scala e l'incontro, soprattutto a livello europeo, con una committenza sia pubblica, attenta a non disperdere la domanda sociale sempre più caratterizzata dalla richiesta di maggiore definizione e qualità degli spazi urbani, sia privata che continua a chiedere specializzazioni, spesso rintracciate all'estero per il ritardo formativo accumulato dal nostro paese, capaci di rispondere ad una domanda a volte molto sofisticata, per la progettazione di parchi e giardini per le residenze, di spazi verdi nei luoghi di lavoro, di idonei spazi nei luoghi per la salute e per il tempo libero.

Il quadro di riferimento complessivo di questo curriculum individua alcune costanti nella progettazione: la memoria storica (come termine di confronto e di identità culturale), l'arte (come chiave di ricerca di nuove forme e dimensioni del progetto), la natura (come esperienza diretta e realtà in divenire), la dimensione biologica (come nuova identità di luoghi recuperati), infine, le esigenze sociali e gli stili di vita come generatori di nuove forme del paesaggio urbano o comunque alla piccola scala. Il quadro di riferimento prima descritto sarà integrato con lo studio delle filosofie di intervento e dei metodi progettuali diversi, dal cosiddetto Movimento Moderno, esperienza decisiva per le vicende del paesaggio contemporaneo, alle ultime tendenze riscontrabili nelle recenti realizzazioni.

Lo scopo del terzo percorso di ricerca è quello di delineare il rapporto che intercorre tra le risorse naturali, in particolare l'acqua, ed il paesaggio urbano.

Gli obiettivi sono due: riuscire ad evidenziare quale ruolo possa assumere l'acqua nel progetto del paesaggio urbano per contrastare il degrado che caratterizza la città centrale e le periferie metropolitane; selezionare le conoscenze, gli elementi e gli strumenti necessari affinché l'acqua "penetri" nuovamente nel pae- 
saggio urbano con tutta la sua valenza paesaggistica, attribuendo così nuova identità, qualità e naturalità alle aree urbane.

Le tesi di dottorato, oltre a concentrarsi sulla situazione presente, sono indirizzate a mettere a fuoco il tema di indagine partendo dall'evoluzione storica del ruolo dell'acqua nella vita delle città, anche attraverso analisi comparate di diverse realtà territoriali.

Nel percorso formativo si riconoscono quattro fasi. Nella prima si individua il ruolo della presenza dell'acqua nella creazione e nella trasformazione del paesaggio; nella seconda le informazioni, raccolte principalmente sulla base di bibliografie specialistiche e di cartografie tematiche, sono intrecciate con le visioni narrate o figurate dei paesaggi urbani, dove emergono le "memorie" ed i "segni" che consentono di recuperare le potenzialità dei luoghi. Nella terza fase si indagano a livello europeo e mondiale i casi più interessanti di rivalutazione del sistema acqua nel paesaggio urbano, da risorsa nascosta e negata a risorsa curata, allo scopo di costruire un abaco che renda agevole il confronto su casi studio spesso molto differenti. Nella quarta fase si analizza un caso studio specifico.

Il lavoro che si presenta è il contributo che i dottori e i dottorandi del dottorato in Progettazione Paesistica hanno voluto predisporre per l'occasione del IV Encuentro Internacional de Arquitectura en Video, Configurando Espacios che si è tenuto in Messico nel maggio 2002 al quale siamo stati cortesemente invitati ${ }^{1}$.

$\mathrm{Si}$ è cercato di individuare, spigolando nell'articolato quadro della sperimentazione in tema di progettazione degli spazi aperti in Italia, sei sezioni che, a nostro avviso, ben rappresentano ciò che avviene nel nostro paese:

- Parchi e paesaggio;

- Parchi metropolitani;

- Spazi urbani;

- Sistemi fortificati;

- Luoghi per l'arte;

- Paesaggi storici.

Alle precedenti sezioni è stata aggiunta una settima, inerente le idee e i progetti in itinere, che anticipa, seppure parzialmente, alcuni programmi di intervento.

\footnotetext{
${ }^{1}$ Luoghi e paesaggi in Italia è il risultato dell'impegno e della passione che i dottori e i dottorandi del dottorato di ricerca in Progettazione Paesistica hanno profuso per presentarsi al convegno di Morelia, in Messico, con un contributo collettivo. Pur essendo difficile estrapolare l'mpegno di ciascuno, si ringraziano: Yuritza Mendoza Garcia che ha curato l'organizzazione per la partecipazione al convegno, Gabriele Paolinelli che ha realizzato il video che ha accompagnato l'inervento di Giulio G. Rizzo, Maristella Storti, che con la collaborazione di Alessandra Cazzola, Claudia Cassatela, Enrica Dall'Ara, Michele Ercolini, Anna Lambertini, Laura Ferrari e Sabrina Tozzini, ha coordinato la correzione redazionale, Andrea Melli, Emanuela Morelli e Antonella Valentini che hanno curato l'editing del preprint presentato al convegno in Messico.
} 



\title{
Parchi e Paesaggio
}

\author{
Andrea Meli
}

La tutela e la pianificazione del paesaggio, in Italia, ha origini legislative precoci, nel 1939, con una legge che sottoponeva a vincolo le "bellezze naturali" del nostro paese, assumendo il concetto estetico di Benedetto Croce quale discriminante per ciò che era da tutelare, e introducendo nel successivo Regolamento, del 1940, il Piano Paesistico come possibile strumento da utilizzare per la tutela del paesaggio.

La legge urbanistica ${ }^{1}$ italiana del 1942 , la stessa che introduce il Piano Regolatore Generale a livello comunale, introduce il concetto di Piano Territoriale di Coordinamento, strumento facoltativo, deputato alla gestione strategica dei problemi legati alle aree inedificate, alle infrastrutture ed ai servizi di particolare rilevanza pubblica. Fin da subito si evidenziò come, l'architettura legislativa, restasse fortemente legata ad una precisa gerarchia centralistica, che operava uno stretto controllo su entrambi gli strumenti di pianificazione.

In realtà, il governo del territorio fu praticamente demandato ai P.R.G. comunali, mentre il P.T.C. ed ogni sua possibile attuazione, si dissolsero nel periodo post-bellico, quando l'urgenza della ricostruzione rese la pianificazione ancora più frammentaria, travalicando anche l'autorità dei Piani Regolatori Generali Comunali, a cui si sostituirono piani settoriali più agili, ma slegati tra loro.

Solo con il trasferimento delle competenze urbanistiche dallo Stato alle Regioni, il P.T.C. trova applicazione nella riorganizzazione territoriale regionale.

"Si può parlare fondamentalmente di tre fasi di regionalizzazione dello Stato.

La prima è quella del 1970-72 determinata dalla legge 281/70 ed attuata da un primo trasferimento parziale di funzioni dallo Stato alle Regioni, con il Dpr 8/72 (fra queste, per la prima volta, le funzioni amministrative in materia urbanistica, fino a quel momento esercitate dagli organi centrali periferici dello Stato).

La seconda regionalizzazione è quella del 1975-77, che deriva dalla legge 382/75 di delega al governo per l'attuazione dell'ordinamento regionale, delega che trova sviluppo nel fondamentale Dpr 616/77, che rende realmente operante la riforma istitutiva delle Regioni a statuto ordinario.

La terza regionalizzazione è iniziata con la legge 59/97, di delega al governo per il conferimento di funzioni e compiti agli enti locali per la riforma della pubblica amministrazione, legge delega definita ed implementata a livello legislativo-normativo dal Dlgs 112/98"2.

Appena istituiti, gli Enti Regione trovarono nella normativa, fino ad allora pressoché disattesa, del Piano Territoriale di Coordinamento, lo strumento adatto per la ridefinizione degli assetti urbanistici su scala territoriale, non solo per la pianificazione sovracomunale delle infrastrutture e dei servizi, ma anche per la tutela delle aree di rilevante pregio paesaggistico.

\footnotetext{
${ }^{1}$ Legge Statale 17 agosto 1942, n. 1150.

2 CARLo Alberto BarbierI, Il territorio dello Stato dal Dpr 616/77 al Dlgs 112/98, in INU (a cura di), Rapporto sullo stato della pianificazione del territorio 2000, Ministero dei Lavori Pubblici, Direzione Generale del Coordinamento Territoriale, Roma 2000, vol. 1, pagg. 13-15.
} 
Esemplare in questo senso è il caso del Parco Regionale del Ticino, istituito dalla Regione Lombardia nel 1974, il cui Piano è stato approvato nel 1980. Ci si è avvalsi proprio dello strumento del P.T.C., inserendo tra gli obiettivi la conservazione e la valorizzazione delle risorse ambientali, culturali e storiche, oltre al sostegno alle attività ecocompatibili. L'esperienza del Parco, che vede coinvolti quarantasei comuni, pur con le difficoltà incontrate durante la sua applicazione, dimostra la versatilità che può raggiungere il Piano Territoriale di Coordinamento.

Nel 1985, viene varata la Legge $431^{3}$, che vincola direttamente territori, ambienti e paesaggi ${ }^{4}$ sensibili e fragili, mentre in precedenza, l'iter per istituire un vincolo di tutela sul paesaggio, secondo la legge sulla protezione delle bellezze naturali, del $1939^{5}$, risultava piuttosto complesso, tanto che le aree sottoposte a questo regime, fino al 1985, erano di minima estensione.

Con la nuova legge, alle Regioni viene dato l'obbligo di dotarsi di Piani Paesistici, che la legge del 1939 aveva introdotto come facoltativi oltre quaranta anni prima.

Al contrario del Piano Territoriale di Coordinamento, che trovò applicazione pratica quasi soltanto dopo l'istituzione delle Regioni ${ }^{6}$, il Piano Paesistico cadde in disuso proprio in quell'epoca, la stessa epoca in cui un paese tradizionalmente evoluto da un punto di vista della tutela del territorio come la Germania, si dotava per la prima volta di uno strumento analogo al Piano Paesistico. Tutto ciò avvenne nonostante esperienze di ricerca di rilievo, come quelle nate alla fine degli anni Sessanta, su iniziativa del Ministero della Pubblica Istruzione, che promosse la redazione di Piani Paesistici nel sud Italia.

Liniziativa concorse alla definizione di un metodo di studio che portasse alla comprensione del paesaggio italiano, che si riconosceva come risultato di fattori molteplici, ambientali, storici, culturali, visivi, e, pur nell'esiguità del numero dei lavori terminati, soltanto due, quello della Costiera Amalfitana (M. Coppa, 1972) e quello della Penisola Sorrentina (R. Bonelli, 1975), all'acquisizione di una più completa coscienza del valore culturale dell' intervento umano sulla natura.

\footnotetext{
${ }^{3}$ Legge Statale 08/08/1985 n. 431 (Disposizioni urgenti per la tutela delle zone di particolare interesse ambientale).

${ }^{4}$ L'articolo 1 della legge 8 agosto 1985, n. 431, prevede che siano «sottoposti a vincolo paesaggistico ai sensi della legge 29 giugno 1939, n. 1497:

a) $\mathrm{i}$ territori costieri compresi in una fascia della profondità di 300 metri dalla linea di battigia, anche per i terreni elevati sul mare;

b) i territori contermini ai laghi compresi in una fascia della profondità di 300 metri dalla linea di battigia, anche per i territori elevati sui laghi;

c) $\mathrm{i}$ fiumi, $\mathrm{i}$ torrenti ed $\mathrm{i}$ corsi d'acqua iscritti negli elenchi di cui al testo unico delle disposizioni di legge sulle acque e gli impianti elettrici (...), e le relative sponde o piede degli argini per una fascia di 150 metri ciascuna;

d) le montagne per la parte eccedente 1.600 metri sul livello del mare per la catena alpina e 1.200 metri su livello del mare per la catena appenninica e per le isole;

e) i ghiacciai e i circhi glaciali;

j) i parchi e le riserve nazionali o regionali, nonché i territori di protezione esterna dei parchi;

g) i territori coperti da foreste e da boschi, nonché percorsi o danneggiati dal fuoco, e quelli sottoposti a vincolo di rimboschimento;

h) le aree assegnate alle università agrarie e le zone gravate da usi civici;

i) le zone umide incluse nell'elenco di cui al decreto del Presidente della Repubblica 13 marzo 1976, n. 448;

l) i vulcani;

m) le zone di interesse archeologico».

Nell'articolo successivo ( 1 bis) si prescrive che «con riferimento ai beni e alle aree elencati $(\ldots)$ le Regioni sottopongono a specifica normativa d'uso e di valorizzazione ambientale il relativo territorio mediante la redazione di piani paesistici o di piani urbanistico-territoriali con specifica considerazione dei valori paesistici ed ambientali, da approvarsi entro il 31 dicembre 1986».

${ }^{5}$ Legge Statale 29 giugno 1939, n. 1497 (Norme sulla protezione delle bellezze naturali).

${ }^{6}$ D.P.R. 15 gennaio 1972, n. 8 (Trasferimento alle regioni a statuto ordinario delle funzioni amministrative statali in materia di urbanistica e viabilità, acquedotti e lavori pubblici di interesse regionale e dei relativi personali ed uffici).
} 
I piani di Coppa e Bonelli furono, venti anni dopo, all'attuazione della legge 431/1985, un background culturale indispensabile, per la capacità dimostrata di poter fare dialogare i valori ambientali con quelli della memoria, e per l'aver messo in luce come proprio in quel rapporto biunivoco si possa trovare il concetto stesso di paesaggio.

Con la legge 431/1985 il piano "di area vasta" entra obbligatoriamente nella pratica comune, ponendo il problema del confronto con sistemi territoriali complessi, fatti non solo di parti urbanizzate, ma anche e soprattutto di paesaggi, frutto dei processi di trasformazione del sistema delle risorse naturali, matrici di riferimento, tessuti di relazioni antropiche e naturali, paesaggi insomma come risorsa delle risorse.

Configurare gli spazi a questo livello della pianificazione territoriale e paesistica, significa confrontarsi con temi dal forte carattere di interdisciplinarietà, ed utilizzare gli apporti di scienze diverse per misurare e valutare i fenomeni territoriali; ma da questi si deve anche trascendere, per arrivare ad una lettura dei fattori antropici e delle risorse ambientali, che sono natura stessa del paesaggio, che contiene i valori culturali, simbolici ed estetici delle comunità che quel paesaggio hanno creato.

L'emancipazione dal concetto crociano di "bellezza naturale", non deve portare ad un rifiuto totale della valutazione estetica, "tanto più importante quanto più la bellezza dei luoghi (...) viene percepita come una componente essenziale della qualità della vita e acquista un peso crescente nelle dinamiche, nei comportamenti e nelle attese della società contemporanea"'.

Il bisogno di conservazione dei valori naturali nasce storicamente assieme alla rivoluzione industriale, periodo in cui i profondi cambiamenti socio-economici si accompagnavano alla trasformazione del paesaggio ed all'alienazione di molti dei valori di identificazione che legavano popolazione ed ambiente. Il fenomeno dell'urbanesimo e l'inizio dell'era della mobilità diffusa, spezzavano fisicamente i legami tra uomo e paesaggio, aprendo quel divario in cui hanno trovato collocazione molti dei grandi conflitti caratterizzanti il processo di modernizzazione.

La prima necessità è stata dunque quella di creare occasioni di contatto tra uomo e natura, intesa come fenomeno indipendente dall'azione dell'uomo, secondo un sentimento romantico che faceva stimare il valore dei luoghi in base alla loro wilderness, ma contemporaneamente era necessario offrire anche spazi di ricreazione, per venire incontro alla nuova esigenza, borghese in un primo tempo, poi generalizzata, di impiego del tempo libero.

Nel paesaggio europeo, forgiato da millenni di intensa densità antropica, dopo l'istituzione dei primi parchi per proteggere zone dove l'attività umana era meno intensa, si è fatta strada la coscienza di come anche l'ambiente costruito dall'opera dell'uomo rappresentasse un valore da difendere e conservare, superando il conflitto uomo-natura attraverso il riconoscimento del valore aggiunto che i paesaggi acquisiscono anche nel processo di interazione tra natura, storia ed arte.

In questo contesto si inserisce, ad esempio, l'esperienza dei parchi culturali, o ecomusei, che affondano le proprie radici nei tradizionali musei all'aria aperta diffusi nell'Europa Settentrionale fin dal tardo Ottocento, contesti che, sulla base di studi scientifici ed etnografici, proponevano testimonianze e ricostruzioni di cultura materiale popolare come artigianato ed agricoltura.

Attorno alla metà degli anni Ottanta del secolo scorso, anche in Europa si tentano esperimenti che attraverso "veri e propri strumenti di piano che si prefiggono finalità di riorganizzazione paesistico ambientale: essi, infatti, puntando su uno o più elementi di uno spazio locale, spostano con decisione la loro attenzione dalla salvaguardia/tutela, dalla ricerca scientifica e dalla educazione all'intrattenimento e

${ }^{7}$ Roberto Gambino, Conservare innovare. Paesaggio, ambiente, territorio, UTET, Torino 1997, pag. 29. 
alla valorizzazione turistica (cui si affida il ruolo di motore e collante di uno svi-

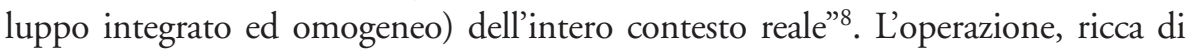
spunti per la promozione economica e culturale del territorio, è stata capace di creare modelli realmente funzionanti quando l'imprenditorialità ha saputo sposarsi con una seria ricerca scientifica ${ }^{9}$, ma reca comunque con sé un forte rischio di retorica e di mitizzazione della storia locale oppure, quando l'interesse economico diventi prevalente, anche di una mercificazione delle risorse, con la sostituzione dei valori culturali originari con altri stereotipati, di largo consumo e facilmente condivisibili dalle masse, slittando dal concetto di ecoparco a quello di parco giochi.

Negli anni Novanta le Regioni italiane pubblicano programmi che si prefiggono lo scopo di perseguire la più ampia possibile fruizione e valorizzazione delle risorse storiche, culturali ed ambientali, prevedendo effetti economici di ordine principalmente turistico, ed allargando le possibilità di investimento e gestione dei parchi anche ai privati.

Moltissime sono state le proposte locali per la creazione di tali parchi culturali e altrettanto varie le tipologie, accomunati di solito da piccole dimensioni, che li esonerano dal confronto con i grandi conflitti territoriali e con la multi gestione tra enti, configurandosi talvolta più che altro come un'operazione di labelling superficiale a dimostrazione dell'ambiguità della definizione "parco culturale", ma tra questi possiamo trovare anche esperienze interessanti che hanno saputo legare reti culturali e qualità ambientale, indagandone le matrici e creando musei, archivi, centri di documentazione strettamente legati alla cultura del paesaggio e capaci di porsi come soggetti interrelazionabili tra loro, di entrare in maniera attiva e culturalmente produttiva in una futura organizzazione sistematica delle aree protette.

Alla pianificazione del territorio e del paesaggio a larga scala, negli ultimi due decenni si è andata affiancando una sempre crescente esperienza legata alla conservazione delle risorse naturali, attraverso la pianificazione e la gestione delle aree protette.

L'Italia vive una breve stagione negli anni Venti-Trenta, che porta all'istituzione dei primi parchi nazionali ${ }^{10}$; dobbiamo arrivare al 1968 per assistere all'istituzione di un altro parco nazionale ${ }^{11}$.

Possiamo considerare come spartiacque fra queste iniziative dell'inizio del secolo scorso, insieme alla stagione vissuta negli anni Settanta-Ottanta con la nascita delle Regioni, l'emanazione della legge n. 394 del 1991, comunemente conosciuta come "legge-quadro sulle aree protette".

Precedentemente a questa, per inquadrare la nascita e l'evoluzione recente del sistema di aree protette nel nostro paese, si deve ricordare il D.P.R n. 616 del 1977, con il quale vengono trasferite alle Regioni "...le funzioni amministrative concernenti gli interventi per la protezione della natura, le riserve ed i parchi naturali...”.

Con questo passaggio di competenze dallo Stato alle Regioni, che copre gli anni Settanta-Ottanta, si assiste ad un notevole incremento sia del numero di aree protette, sia della superficie complessiva di territorio protetto (“...17 regioni su 20 e le due Province Autonome hanno varato un imponente numero di aree pro-

\footnotetext{
${ }^{8}$ LeOnardo Rombai e AnNa Guarducci, Tra natura e cultura : parchi e riserve di Toscana, Centro editoriale Milano, Firenze 1999, pag. 24.

${ }_{9}^{9}$ Vedi gli esempi di Ironbridge e Big Pit di Blanaevon in Gran Bretagna e Le Creusot in Francia. Ibidem.

${ }^{10}$ Il primo Parco Nazionale istituito in Italia risale al 1922, ed è il Parco Nazionale del Gran Paradiso, cui fanno seguito nel corso del tempo, sempre con singole iniziative dello Stato, il Parco Nazionale d’Abruzzo nel 1923, il Parco Nazionale del Circeo nel 1934, il Parco Nazionale dello Stelvio nel 1935.

${ }^{11}$ Il Parco Nazionale della Calabria, che aggiunto agli altri quattro precedentemente istituiti porta il totale della superficie protetta da queste aree a 267.924 ettari (dati riferiti al $3^{\circ}$ Aggiornamento dell'Elenco Ufficiale delle Aree Naturali Protette, a cura del Servizio Conservazione della Natura del Ministero dell'Ambiente). La nascita di queste aree protette è avvenuta in assenza di una normativa generale di riferimento che disciplinasse la procedura istitutiva e concorresse a creare un quadro di riferimento per le politiche di pianificazione e gestione unitario.
} 
tette, per una superficie complessiva di quasi un milione e quattrocentomila ettari, pari al 4,6\% del territorio nazionale, comprendente 81 parchi regionali, 145 riserve naturali e 170 aree protette ad altro titolo"12).

Un'altra fase importante nel processo di formazione di un sistema di aree protette a livello nazionale, è rappresentato dall'azione che lo Stato ha intrapreso con l'istituzione di numerose Riserve Statali (in gran parte negli negli anni Settanta e Ottanta), site generalmente su terreni di proprietà demaniale ${ }^{13}$.

In questa fase, antecedente alla legge quadro 394/91, è possibile riconoscere e delineare due principali modelli nella politica a livello regionale di conservazione della natura:

"1. Regioni che hanno un livello complessivo di macropianificazione regionale ed un livello di micropianificazione delle singole aree; qui l'adozione di macropiani a maglia larga su scala regionale è stata la premessa necessaria per l'adozione dei piani delle singole aree protette...Questo modello, che per primo è stato introdotto dalla Lombardia, è stato poi seguito da altre regioni come l'Emilia Romagna, la Liguria, il Lazio, la Sicilia, la Toscana, la Provincia di Trento, la Valle d'Aosta, il Veneto ed il Piemonte..."14;

"2. Regioni che hanno puntato direttamente al micropiano, e cioè alla istituzione e pianificazione-gestione per singola area protetta, attraverso leggi istitutive, piani e discipline, area per area. Questo modello è stato seguito da regioni come l'Abruzzo, la Basilicata, la Calabria, la Campania, le Marche..."15.

Cercando di riassumere brevemente l'evoluzione storica della vicenda delle aree protette in Italia nel secolo scorso, possiamo individuare alcune come segue ${ }^{16}$ :

1922-1974 - Fase centralistica:

l'attribuzione delle competenze in materia di aree protette era riservata allo Stato; 1974-1985 - Fase regionalistica:

si è affermato prepotentemente il ruolo delle Regioni, unici soggetti pubblici che, a fronte della latitanza dello Stato, hanno avviato più o meno concrete politiche per le aree protette;

1985-1991 - Fase neocentralistica:

si è verificata una forte riconquista di ruolo e competenze da parte dello Stato, in particolare con l'istituzione del Ministero dell'Ambiente (Legge n. 349/86).

Con la legge quadro 394/91, l'Italia avvia un forte spinta alle politiche di tutela dell'ambiente, allineandosi dopo decenni alle molte esperienze ormai consolidate di paesi europei ed extra-europei in materia di sistemi nazionali e regionali di aree protette.

Le principali finalità che la legge si pone possono essere riassunte come segue:

- la conservazione di specie animali e vegetali, di associazioni vegetali o forestali, di singolarità geologiche, di formazioni paleontologiche, di comunità biologiche, di biotipi, di valori scenici e panoramici, di processi naturali, di equilibri idraulici e idrogeologici, di equilibri ecologici;

- l'applicazione di metodi di gestione o di restauro ambientale allo scopo di favorire l'integrazione tra uomo ed ambiente anche mediante la salvaguardia dei valori antropologici, storici e architettonici e dell'attività agro-silvo-pastorale;

- la promozione di attività educative, formative e ricreative compatibili;

- la difesa degli equilibri idraulici;

- la sperimentazione di attività produttive compatibili.

12 Guido Ferrara e Lorenzo Vallerini (a cura di), Pianificazione e gestione della aree protette in Europa, Maggioli Editore, Rimini 1996, pag. 45.

13 "Come per i Parchi Nazionali, per le Riserve Naturali non è esistita, sino all'approvazione della legge-quadro, una disciplina giuridica generale e gli atti di istituzione hanno assunto sempre la forma di singoli decreti ministeriali” in GUIDO FERRARA e LORENZO VALLERINI (a cura di), op. cit., 1996, pag. 38.

${ }^{14}$ Guido Ferrara e Lorenzo Vallerini (a cura di), op cit., 1996, pag. 46.

15 Cfr. Guido Ferrara e Lorenzo Vallerini (a cura di), op. cit., 1996, pag. 46.

${ }^{16}$ Cfr. Franco Migliorini, Gianni Moriani e Lorenzo Vallerini, op. cit., 1999, pag. 18. 
La legge del 1991 si innesta all'interno del quadro di tutela del paesaggio come definito in modo particolare dalla legge 431/85, anche se "la fondamentale novità introdotta con la legge 394/91 consiste (...) nel riconoscimento di un nuovo principio biocentrico, che attribuisce una vera e propria soggettività alla natura in quanto valore in sé, giuridicamente riconosciuto e protetto, non più sottomessa al principio antropocentrico intrinseco alla concezione giuridica propria della pianificazione tradizionale, pur se integrata dai principi della tutela paesaggistica introdotta con la legge 431/85"17.

Con questa forte azione legislativa, si interviene a livello nazionale per dare riferimenti comuni ad un quadro che si presentava quanto mai disomogeneo per tipologie ed esperienze a livello locale, in maniera particolare rispetto a:

1. criteri di classificazione delle aree protette, tra i più svariati ed eterogenei, estremamente differenziati da regione a regione;

2. norme di tutela e promozione, che spaziano dalle più restrittive con divieti estremamente rigidi a quelle più permissive;

3. strumenti di pianificazione e di gestione, assenti o iperstrutturati, operanti spesso in un quadro scarso o totalmente assente di riferimenti comuni, necessari per impostare una moderna politica di gestione delle risorse ambientali e territoriali;

4. organismi di gestione, inesistenti o sottodimensionati rispetto alle necessità di tutela e di sperimentazione di una convivenza compatibile tra uomo-natura.

Nell'ultimo decennio, si assiste in Italia ad un rapido incremento della superficie protetta, che fa raggiungere la soglia del $10 \%$ del territorio nazionale ${ }^{18}$, ponendo il nostro paese in linea con le esperienze estere, e la politica di tutela della natura inizia ad avere un più ampio respiro, anche grazie alle successive leggi di adeguamento regionale agli indirizzi della 394.

Questo processo ha dato origine sia alla riorganizzazione di sistemi di aree protette ormai strutturati e maturi (che fanno riferimento alle vicende precedenti al 1991), sia alla nascita di nuovi sistemi su base regionale, consentendo così la diffusione a livello locale di alcuni principi ed indirizzi relativamente agli aspetti tipologici, alle forme di pianificazione e gestione dei temi che interessano le aree naturali protette (piano del parco, regolamento del parco, piano di sviluppo economico e sociale).

L'introduzione del Piano del Parco ${ }^{19}$ strumento di pianificazione che assume rilievo per quanto attiene non solo la tutela e la conservazione delle risorse naturali, ma ha potere sovraordinato rispetto alla pianificazione urbanistica ordinaria, rappresenta certamente uno degli elementi di maggiore novità e rilievo, assieme alla previsione del Piano Pluriennale di Sviluppo Economico e Sociale, che deve garantire il coordinamento delle azioni di sviluppo compatibile che hanno una incidenza diretta sulle popolazioni locali (allo stato attuale sono rare le esperienze di Piani Pluriennali redatti e/o operanti).

${ }^{17}$ Franco Migliorini, Gianni Moriani e Lorenzo Vallerini, Parchi naturali: guida alla pianifcazione e alla gestione, Franco Muzzio Editore, Padova 1999, pag. 44.

${ }^{18}$ In base al $3^{\circ}$ Aggiornamento dell'Elenco Ufficiale delle Aree Naturali Protette, a cura del Servizio Conservazione della Natura del Ministero dell'Ambiente, 2000, che fornisce i dati ufficiali riferiti a quanto stabilito dalla legge quadro nazionale, in Italia sono attualmente istituite 669 aree protette (suddivise per tipologie di area in 21 parchi nazionali, 143 riserve naturali statali, 15 riserve marine statali, 110 parchi naturali regionali, 252 riserve naturali regionali, 128 altre aree naturali protette), per una superficie totale di 2.752 .952 ettari a terra e 260.992 a mare.

Secondo dati della Federparchi (2001), la superficie totale delle aree protette (terrestri e marine) ammonta a 3.206.215 ettari, per un numero totale di 863 aree, pari al 10,64\% della superficie nazionale. La difformità dei dati è da mettere in relazione al fatto che nell'elenco ufficiale del Ministero dell'Ambiente non vengono prese in considerazione quelle superfici di aree protette dove è consentita l'attività venatoria.

${ }^{19}$ Allo stato attuale, la pianificazione dei parchi nazionali in Italia, dopo la previsione dello strumento del Piano del Parco nella legge quadro del 1991, risulta ad uno stato molto avanzato, con 4 piani in corso di approvazione e 10 piani in fase di redazione (dati Centro Europeo di Documentazione sulla Pianificazione dei Parchi Naturali del Dipartimento Interateneo Territorio del Politecnico di Torino, 2000). 
Accanto al quadro di indirizzo e di politica nazionale sinteticamente sopra delineato, si sono andate affiancando ed integrando negli ultimi dieci anni indirizzi e normative dell'Unione Europea, che hanno definito nuovi concetti di tutela del paesaggio e delle risorse naturali ${ }^{20}$.

Lorigine di queste recenti tendenze dell'Unione Europea, deve essere ricondotta ad una nuova visione dell'azione di tutela e gestione del sistema delle risorse naturali e del paesaggio, non più legato a forme di conservazione "tradizionale", ma dove invece la concezione di habitat abbracci le direttive internazionali in materia di tutela della biodiversità, che trovano nella Conferenza Internazionale delle Nazioni Unite di Rio de Janeiro del 1992 il punto centrale di una nuova considerazione dello sviluppo delle attività umane in termini sostenibili.

Scopo di questa azione congiunta fra Unione Europea e Stati Membri è quello di creare la "...rete Natura 2000, vale a dire un insieme di aree (che prenderanno il nome di Zone Speciali di Conservazione) in grado di mantenere o ripristinare particolari habitat naturali. Per sostenere l'impegno europeo, l'Italia ha avviato il progetto Bioitaly.." ${ }^{21}$, che ha individuato sul territorio nazionale 2.425 aree che, rispondendo ai requisti della direttiva habitat, sono state proposte dall'Italia all'Unione Europea come SIC (Siti di Importanza Comunitaria) ${ }^{22}$.

In questo quadro sintetico, la pianificazione e la gestione della aree naturali protette oggi, sia alla luce delle esperienze pregresse, sia alla luce della necessità di una maggiore integrazione delle politiche di pianificazione e gestione che interessano tali aree, si trova ad affrontare una stagione che si fonda sull'integrazione interdisciplinare, oltre che sullo sviluppo e la sperimentazione di nuove e moderne metodologie di indagine paesistica.

In questa sezione si intende affrontare il tema della pianificazione del paesaggio e delle aree protette, attraverso l'analisi di casi di studio che riguardano un campo oggi ormai vasto ed articolato.

Esperienze che vedono protagonisti parchi regionali storici (Parco Regionale della Valle del Ticino e Parco Regionale di Migliarino San Rossore Massaciuccoli), che hanno rappresentato e rappresentano ancora oggi esempi dell'interazione possibile fra le esigenze di conservazione della natura, proprie dell'istituto dell'area protetta, e la considerazione dei processi umani di trasformazione, che hanno generato sistemi di paesaggi di grande rilievo.

Oppure casi di progetti, alla scala territoriale, che cercano di dare risposta al ripristino di paesaggi fortemente trasformati e sfruttati dall'attività umana (Progetto di riassetto ambientale e paesistico della Val di Chiana), attraverso metodologie di indagine e di intervento sui sistemi naturali ed antropici, anche mediante l'utilizzo di moderne discipline quali l'ecologia del paesaggio (Parco della Madonie).

Ed ancora il caso della pianificazione di sistemi di aree tutelate per il loro insieme di valori naturali, storici, archeologici, insediativi, produttivi (Sistema dei Parchi della Val di Cornia), che sono il segnale della possibile interazione di piani e progetti di recupero e valorizzazione delle risorse paesistiche, in un quadro di integrazione delle diverse componenti che rappresentano l'insieme delle azioni di trasformazione e delle risorse che hanno generato quei paesaggi.

\footnotetext{
${ }^{20}$ Si ricorda qui la principale direttiva CEE che informa queste nuove tendenze, sulle quali ancora non è possibile trarre bilanci sull'effettiva incidenza in termini di pianificazione delle risorse naturali e paesistiche, la 92/43, comunemente conosciuta come direttiva habitat.

${ }^{21}$ Centro Studi Touring Club Italiano (a cura di), La tutela del paesaggio in Italia, Touring Club Italiano, Milano 1998.

${ }^{22} \mathrm{Si}$ pensi che in Italia sono presenti oltre 57.000 specie animali, che rappresentano oltre $1 / 3$ delle specie animali distribuite in Europa. Allo stesso modo il patrimonio floristico italiano è ugualmente importante, ammontando a quasi il 50\% della flora europea su di una superficie di circa $1 / 30$ di quella del continente (fonte: dati Ministero dell'Ambiente).
} 



\title{
Progetto di RIASSETtO AMBIENTALE E PAESAGGISTICO DELLA VAL DI CHIANA IN TOSCANA
}

\author{
Michele Ercolini
}

\section{IL CONTESTO}

La struttura e l'immagine della piana di Arezzo derivano da una serie di dati di fatto territoriali ed ambientali molto specifici, e in particolare sono il prodotto di caratteri fisiografici-geomorfologici, storiciagricoli-insediativi e paesistici.

Da un punto di vista geomorfologico, l'area oggetto d'indagine è identificabile dalla compresenza di una fascia di rilevi collinari e dalla sottostante area pianeggiante, nata come evoluzione del lago pleistocenico e divenuta poi l'attuale Val di Chiana.

Le vicende storiche-agricole riguardanti la piana aretina sono invece un continuo susseguirsi ed alternarsi di opere di impaludamento e di riscatto, con innumerevoli azioni di bonifica iniziate ai tempi degli etruschi e terminate nel secolo XVIII da Vittorio Fossombroni per conto di Pietro Leopoldo di Lorena. Per quanto concerne gli aspetti storico-insediativi, è certo che la Val di Chiana subì fin dall'antichità un forte interesse da parte della comunità urbana, trasformandosi nel tempo in un territorio ricco di memorie legate ad antiche civiltà e di segni testimoni di un'intensa attività agricola, costituendo ancora oggi una delle più importanti zone produttive della Toscana e dell'Italia intera. Anche in questo caso comunque, la collina e la pianura assunsero due ruoli ben distinti.

L'arco collinare infatti, fu fin da subito preferito per fondare gli insediamenti in quanto più salubre e più difendibile, mentre nell'area pianeggiante bonificata ebbe inizio una rilevante attività agricola (in particolare cereali, viti maritate all'acero su seminativo, eccetera). Non a caso, le più significative stratificazioni storiche, come le pievi romaniche e medioevali, il "sistema delle grandi ville Rinascimentali", sono

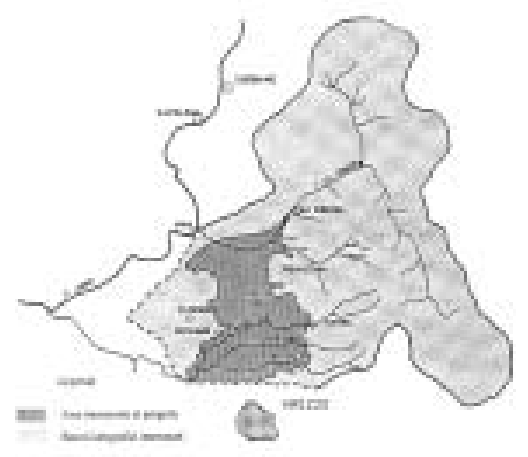

Figura 1 - Localizzazione area di progetto.

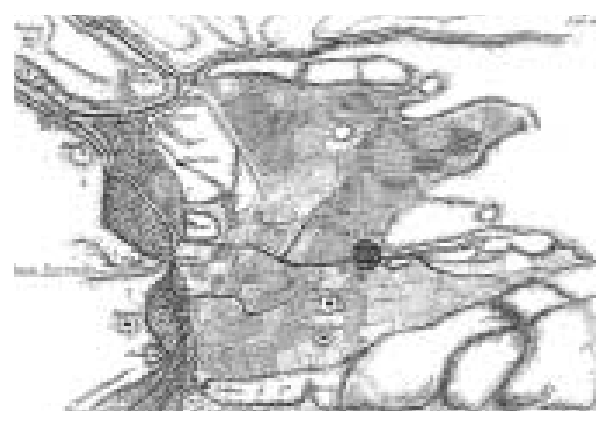

Figura 2 - Mappa delle Memorie idrauliche-storiche. poste sempre a non meno di 200-300 metri d'altitudine.

Questa distinzione tra le due sub-aree è rilevabile non solo da un punto di vista geomorfologico o storico-agricolo-insediativo, ma anche paesaggistico. L'area infatti è inquadrabile in due ambiti paesistici nettamente distinti ma ben integrati:

- l'ambito collinare, ossia il luogo delle stratificazioni storico-culturali, con un carattere spiccatamente conservativo, in cui le trasformazioni tuttora sono rare e si sviluppano in tempi lunghi. Qui, come sottolineato in precedenza, si sono concentrati e consolidati nei secoli i grandi valori conseguenti l'azione dell'uomo, come i borghi storici, le ville, i giardini, e quelli legati allo scenario rurale, come il paesaggio agrario tradizionale, i grandi viali alberati, eccetera.

- l'ambito paesistico della pianura, da sempre contraddistinto da un carattere fortemente innovativo e predisposto alla trasformazione; una tendenza al cambiamento riscontrabile nel sistema idrografico, nella rete viaria e nella destinazione stessa del territorio. Tutto ciò ha comportato, ad esempio, la 


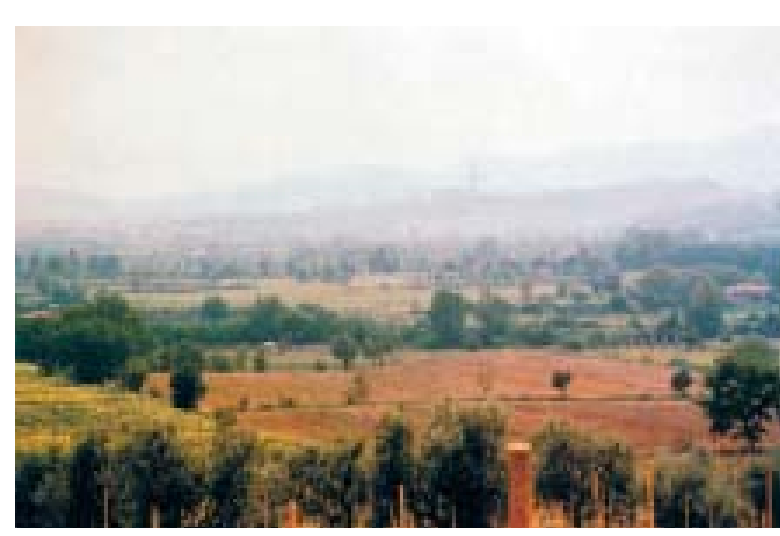

Figura 3 - La piana a nord di Arezzo vista dalle colline.

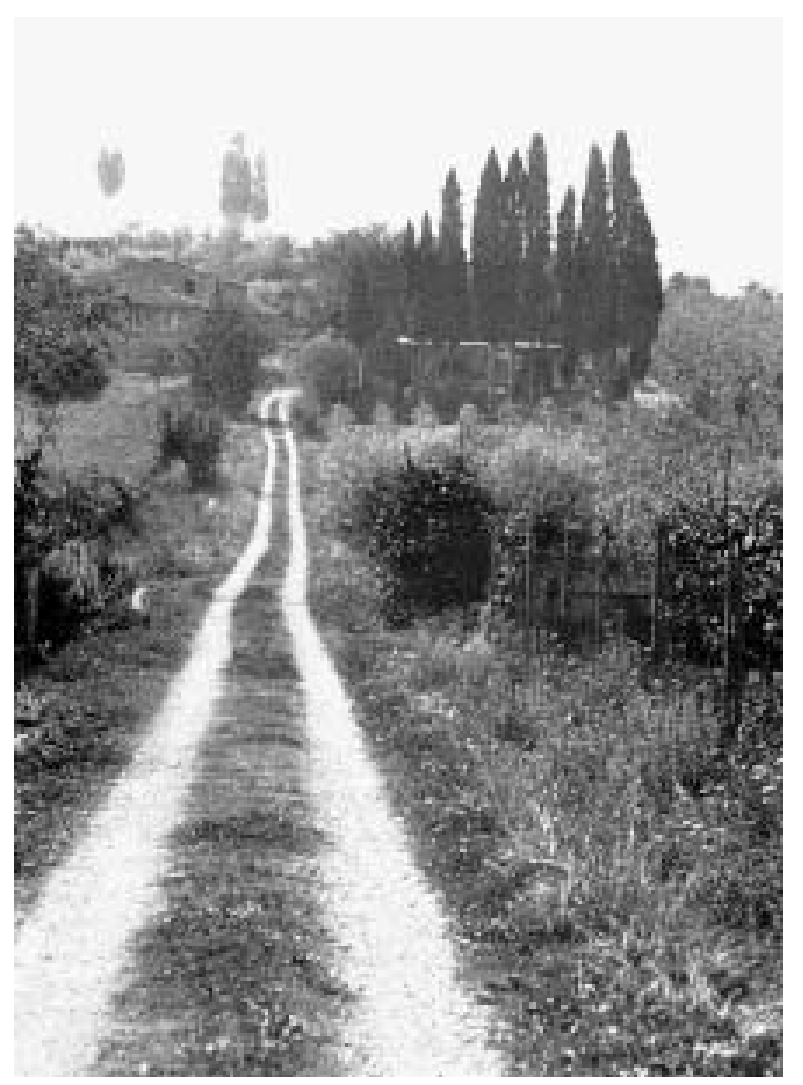

Figura 4 - La viabilità poderale nella piana aretina. scomparsa nell'orditura territoriale della piana aretina delle tracce della centuriazione romana e delle cosiddette "camperie", ossia dei terreni "immediatamente fuori città, dove i mercanti medievali investirono capitali in miglioramenti fondiari, nuove colture, case mezzadrili e case del Signore"1.

Questa attitudine al cambiamento è conseguente a due fattori: la suscettibilità della pianura ad essere da sempre plasmata e disegnata dall'azione antropica e la prossimità del centro urbano di cui ha sempre rappresentato "l'interfaccia".

Ma mentre nel passato la vicinanza alla città era elemento di promozione del territorio agricolo e dell'ambiente rurale, attualmente, con l'assoluta mancanza di interventi di pianificazione urbanistica ed ambientale finalizzati alla conservazione dei delicati equilibri tra città e campagna, questa vicinanza appunto si è tramutata in un fattore di degrado e di scadimento.

Ad oggi dunque, il quadro paesaggistico caratterizzante la pianura non assume più il ruolo complesso di qualità e di alto pregio dell'ambiente rurale, ma piuttosto quello di degradata quanto caotica periferia nord della città, avendo subito, decennio dopo decennio, la diffusione incontrollata di elementi detrattori quali cave, discariche, insediamenti industriali, eccetera.

A tutto ciò ha fatto seguito:

- la scomparsa dell'immagine storica della campagna toscana;

- la nascita di un quadro ambientale definibile "rururbano", derivante cioè da un "uso multiplo dello spazio, che non è più solo agricolo, ma ospita attività di tipo industriale, abitativo e di servizio, secondo una logica causale non legata ad una strutturazione dovuta all'interdipendenza delle funzioni del territorio"2.

Nonostante tutto però, all'interno della sub-area pianeggiante è ancora oggi rilevabile un sistema di microvalori, costituito dalla rete della viabilità poderale e da alcuni biotopi, come la vegetazione riparia, le siepi e i filari, i lembi di bosco, eccetera.

Questi microvalori hanno rappresentato come si vedrà più avanti, il punto di partenza della fase progettuale, in quanto considerati non vincoli o limitazioni, ma veri e propri elementi di stimolo.

\section{GLI OBIETTIVI}

Dall'analisi degli atti relativi alla Conferenza di Coordinamento sulla Pianificazione Territoriale per la Provincia di Arezzo, svoltasi nel febbraio del 1990, si possono desumere innanzitutto, le finalità di carattere generale perseguite in fase progettuale:

${ }^{1}$ Giuliana Campioni, in Guido Ferrara e Roberto Rossi, Valutazione d'impatto ambientale del riordino fondiario e dell'irrigazione della Piana d'Arezzo, Ed. Calosci, Cortona 1991, pag. 134.

${ }^{2}$ Giuliana Campioni, op. cit., 1991, pag. 136. 
- "rispetto della rete idrografica principale;

- rispetto dell'andamento prevalente nella struttura della rete scolante superficiale;

- massima riduzione degli interventi di livellamento, limitandosi prevalentemente ad operazioni di semplice modellamento;

- rispetto delle emergenze storiche e massima attenzione nelle aree indiziate di possibile presenza di siti archeologici;

- rispetto e comunque ripristino della struttura vegetale presente nel territorio;

- mantenimento e raggiungimento di una soglia minima di dotazione di formazioni vegetali lineari non colturali, arboree e/o arbustive;

- controllo della qualità dei deflussi idrici;

- interventi di sistemazione e manutenzione della rete idrica"3.

In parole semplici quindi, l'intervento progettuale si pone come obiettivo prioritario, la costruzione di un paesaggio agrario ex-novo compatibile sì alle richieste e alle necessità della società contemporanea, ma che allo stesso tempo riesca nel suo evolversi sia a mantenere quelle testimonianze, quei segni, quei valori già esistenti, sia ad arricchirsi di nuovi contenuti culturali, ambientali e visuali.

Tutto ciò si è reso possibile grazie al rispetto di determinati "Criteri guida della progettazione e della gestione" ${ }^{4}$, che possono essere così sintetizzati:

- non ritenere il comprensorio irriguo un'isola, ma rafforzare e valorizzare ai vari livelli e nei diversi settori, i legami esistenti tra collina e pianura, considerando quest'ultimi come due ambiti diversificati eppure strettamente compartecipi dello stesso sistema fisiografico e della medesima configurazione paesistica;

- tutelare le risorse culturali e naturali significative sia isolate che in sistema, identificando nei vari casi le destinazioni d'uso più idonee;

- evitare l'effetto desertificante legato all'eliminazione delle microstrutture del paesaggio, cioè di beni culturali ed ambientali definibili come minori, cui è legata la memoria storica e visiva degli abitanti e che rappresentano elementi di riconoscimento ed orientamento del quadro ambientale-ecologico;

- valutare le permanenze come nodi significativi di una maglia territoriale cui agganciare il disegno del nuovo paesaggio, in modo da garantirne la continuità ecologica e storico-territoriale con il passato.

L'intervento ha inoltre cercato di evitare l'adozione dell'ormai superata visione forzatamente ed esclusivamente produttivistica dei progetti di riordino fondiario e irriguo, puntando invece ad una maggiore integrazione tra le risorse naturalistiche, paesistiche ed ambientali esistenti e quelle puramente produttive, sollecitando contemporaneamente, sia un miglioramento delle produzioni agricole che una maggior valorizzazione di tutte le componenti ambientali della piana, con particolare attenzione a quelle paesistiche ed ecologiche.

\section{LA METODOLOGIA}

L'aspetto più interessante del percorso metodologico intrapreso riguarda certamente il punto di partenza: l'intervento nasce infatti come valutazione di impatto ambien-

\footnotetext{
${ }^{3}$ ROBERTO Rossi e Claudio BINI, Criteri e problemi relativi al riordino fondiario e alla conversione irrigua nella particolare situazione ambientale della Toscana, Atti della Conferenza di Pianificazione Territoriale per la Provincia di Arezzo, Arezzo 1990.

${ }^{4}$ Guido Ferrara, Val di Chiana aretina: un progetto per il paesaggio agrario del 2000, "Paesaggio Urbano", 6, 1993, pag. 44.
} 
tale relativa ad un precedente progetto di irrigazione e riordino fondiario, datato 1985 e redatto dai tecnici dell'Assessorato all'Agricoltura della Provincia di Arezzo.

Siamo di fronte però ad un caso "anomalo" ed inedito: per la prima volta una procedura di valutazione di impatto ambientale invece di ridursi al semplice ruolo di copertura e mistificazione del progetto iniziale, ha permesso di controllare pienamente la concezione del nuovo paesaggio agrario derivante dagli interventi di irrigazione e riordino fondiario, "interferendo" fortemente e diventando protagonista attiva dell'intero processo.

La VIA dunque, proponendo opportuni approfondimenti e suggerimenti di tipo progettuale, riguardanti non tanto i valori esistenti quanto la definizione di quelli da realizzare nell'immediato futuro, ha permesso di arrivare alla definizione di un progetto ex novo che, sostituendo completamente quello di partenza, si colloca a pieno titolo entro l'attività della pianificazione del paesaggio.

Prima di iniziare la descrizione dell'iter metodologico caratterizzante il secondo progetto, si ritiene opportuno descrivere brevemente i principali aspetti riguardanti la proposta del 1985, soprattutto per capire le motivazioni che hanno spinto i nuovi progettisti ad abbandonarla completamente.

I maggiori problemi si riscontravano in alcune delle risorse ambientali più significative dell'area di indagine, ovvero in quelle fisiografiche, in particolar modo la morfologia e la pedologia, nella vegetazione e negli aspetti paesaggistici.

Anzitutto, a quanto risulta dall'esame del primo progetto, l'orientamento e la forma geometrica dei nuovi campi nasceva senza minimamente considerare le caratteristiche pedologiche e morfologiche dei suoli. Era inoltre prevista la rimozione di gran parte dei filari che separano gli appezzamenti, ignorando completamente l'importante ruolo che queste formazioni hanno nel limitare, ad esempio, l'erosione dei suoli. Rilevanti conseguenze avrebbero poi colpito anche gli aspetti vegetazionali, sia attraverso la completa distruzione della vegetazione collegata alla rete scolante, sia con la rimozione dell'intera maglia dei filari di vite maritata all'acero campestre.

Ma certamente le conseguenze più gravi erano rilevabili nel settore paesistico. Il progetto infatti, prevedeva la totale semplificazione della maglia tradizionale

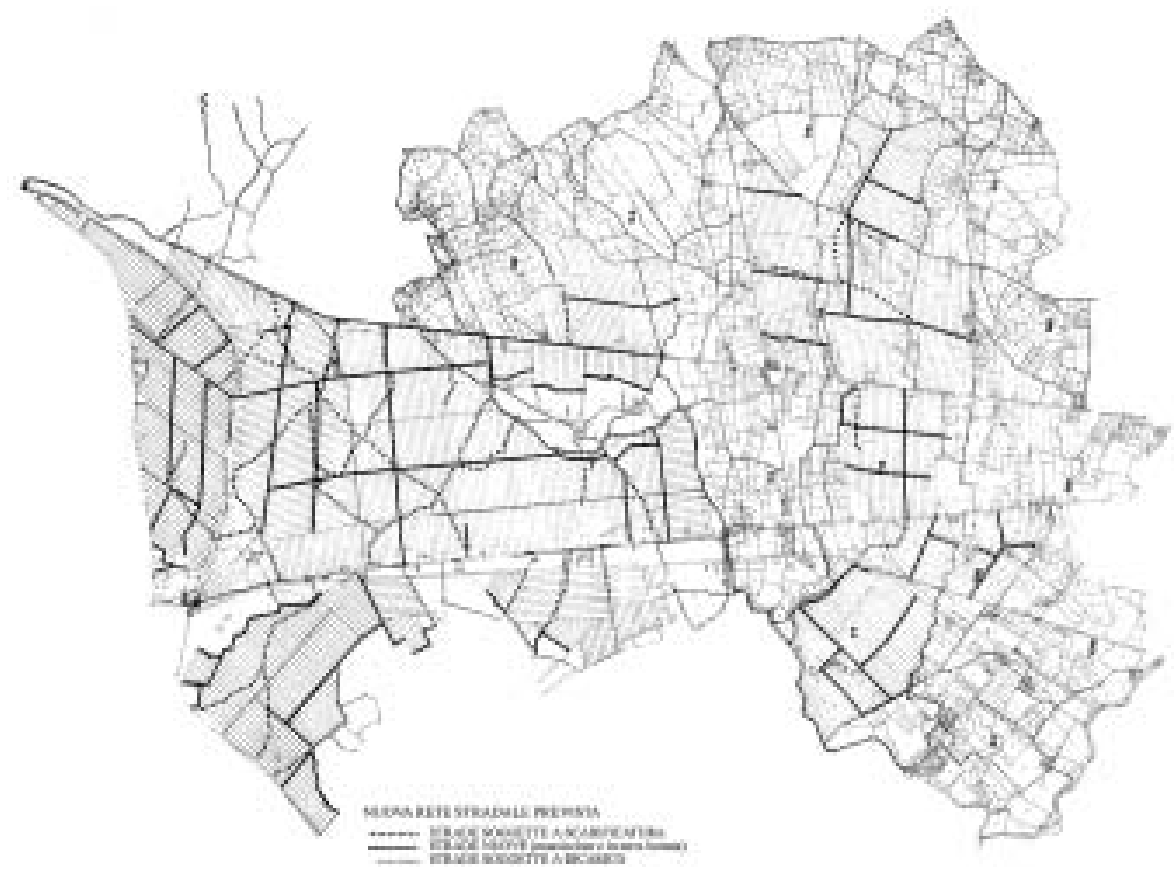

Figura 5 - Progetto 1985: Carta dell'opere stradali. 


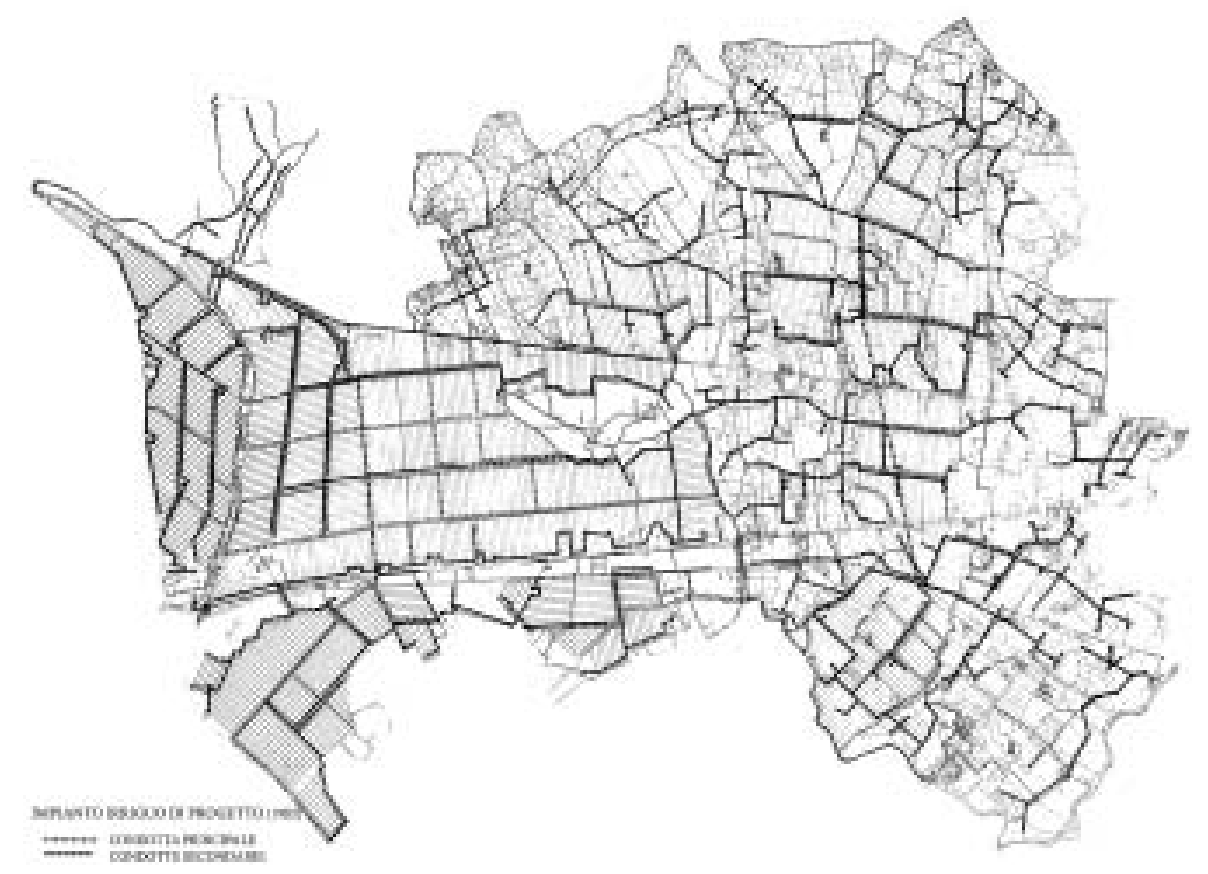

Figura 6 - Progetto 1985: Carta impianto opere irrigue.

degli elementi paesaggistici della campagna aretina. In primis, il paesaggio storico-culturale, con particolare riferimento agli aspetti archeologici, avrebbe subito notevoli danni rischiando persino la completa distruzione, a causa degli interventi di livellamento del terreno e del passaggio delle condotte. Era prevista inoltre la sostituzione dell'intero sistema a rete della viabilità storica con una viabilità progettata esclusivamente sulla base di criteri funzionali alla conduzione agricola.

In poche parole sarebbero andati perduti quei segni e quei valori che ancora oggi evidenziano la logica "fondatrice" del contesto paesistico della piana, ovvero l'orientamento dei campi conseguente alle condizioni idrauliche naturali dei suoli, gli allineamenti prospettici con la corona collinare, il sistema a rete della viabilità interpoderale di servizio agli insediamenti storici, eccetera.

Tutto ciò avrebbe generato un paesaggio agrario uniforme, monotono, e rigido, ben lontano dalle preesistenze d'ordine culturale, ambientale, storico, e derivante solo da considerazioni di carattere economico-funzionale.

In sintesi, il progetto del 1985 prevedeva "la pesante trasformazione del paesaggio, ottenuta mediante livellamenti del terreno, la trasformazione radicale del reticolo dei campi, la definizione della rete irrigua e dei canali scolanti, l'abbattimento di tutti o quasi tutti gli alberi in filare e delle siepi esistenti: in una parola, quanto gli etruschi e i romani prima, e le bonifiche leopoldine poi avevano lasciato sul territorio sarebbe stato definitivamente cancellato" 5 .

Conclusa la prima parte di analisi inerente al progetto del 1985, l'iter metodologico è proseguito sviluppando una dettagliata quanto articolata lettura delle componenti del territorio oggetto di studio. Quest'ultima si è fondata in particolar modo sull'analisi delle pecularietà del paesaggio agrario entro cui "dovevano essere resi intelligibili i meccanismi di continua ricostruzione delle risorse (acque, suoli, flora, fauna, eccetera) sulla base dei cicli naturali e dell'intervento umano"6.

\footnotetext{
${ }^{5}$ Guido Ferrara, op. cit., 1993, pag. 32.

${ }^{6}$ Guido Ferrara, "Valutazione d'impatto ambientale delle opere irrigue e di riordino fondiario sui lavori riferiti al territorio a Nord di Arezzo", intervento alla Conferenza di Pianificazione Territoriale per la Provincia di Arezzo, Arezzo 1990.
} 


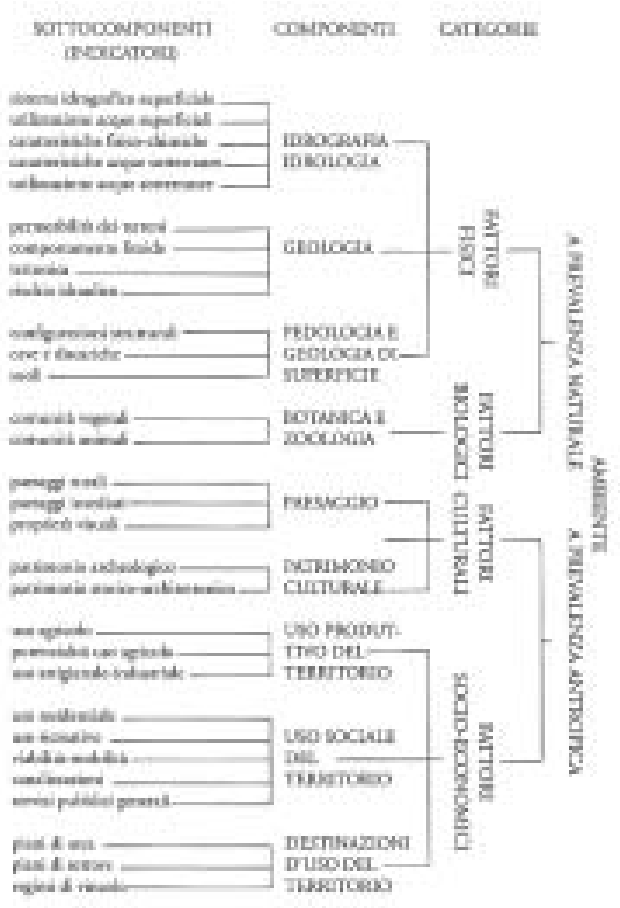

Figura 7 - Schema risorse ambientali.
L'analisi riguardante la lettura delle singole risorse ambientali è stata però condotta inquadrando sempre quest'ultime all'interno di un sistema più ampio e complesso, quale è il territorio oggetto dell'indagine. Tutto ciò ha consentito di valutare sia le problematiche ambientali esistenti, sia di utilizzare le indicazioni sull'ambiente e l'insieme degli ecosistemi in modo utile alla pianificazione e alla progettazione.

L'ambiente risulta dunque il parametro di riferimento dell'intero processo di analisi, ma è anche, come si vedrà di seguito, l'elemento cardine della pianificazione del territorio.

"Le condizioni attuali dell'ambiente sono state analizzate con l'opportuno interscambio di informazioni tra le varie discipline, relativamente ai settori fisiografia, idrologia, agroeconomia, vegetazione, fauna, scenario e paesaggio culturale, che ha compreso anche gli aspetti archeologici"'

In questa sede ci limiteremo ad approfondire esclusivamente la metodologia di analisi inerente i fattori biologici (vegetazione) e i fattori culturali (paesaggio).

Il metodo d'indagine relativo al settore "vegetazione" si è sviluppato attraverso un sopralluogo sommario iniziale, l'analisi delle foto aeree e la descrizione dei vari tipi fisionomici di vegetazione presenti.

All'interno dell'area sono stati individuati due differenti tipi fisionomici: quello delle colture agronomiche, in cui l'azione antropica è costante (oliveti, frutteti, filari di vite maritata all'acero, eccetera), e quello delle formazioni arboree, ove l'azione dell'uomo è presente ma non necessaria per la loro sopravvivenza (zone boscate, zone a bosco-parco, formazioni riparie).

In particolar modo ci si è soffermati su i due soggetti vegetazionali aventi una certa rilevanza vuoi dal punto di vista paesaggistico-storico che nella configurazione dello spazio: le zone a filari di vite maritata all'acero, che rappresentano indubbiamente la tipologia vegetale più significativa, e quello che può essere definito il sistema delle aree "bosco-villa-parco", ovvero quelle permanenze storico-architettoniche in cui sono concentrati e consolidati i grandi valori legati alle componenti antropiche e agli scenari visuali, e qui rappresentate dalle ville cinquecentesche poste sulla corona collinare e pedecollinare e dai loro parchi (Villa Vasari, Villa Ponte alla Chiassa, eccetera).

Per quanto riguarda invece il settore del "paesaggio", nella fase di analisi si sono perseguiti i seguenti obiettivi:

- l'identificazione e misura oggettiva dei connotati e delle caratteristiche complessive del paesaggio della piana, con riferimento anche alle zone contermini costituenti l'interfaccia con cui gli interventi territoriali previsti verranno ad interferire a livello ecologico e visuale;

- la verifica dei possibili effetti negativi indotti sulle componenti del paesaggio dalle trasformazioni connesse alle sistemazioni irrigue e al riordino fondiario;

- l'indicazione dei connettivi e delle strategie giudicate più idonee per la conservazione e valorizzazione delle risorse territoriali esistenti;

- l'individuazione del modello di assetto paesaggistico capace di rendere compatibili le esigenze proprie dell'area di studio di stabilità ecologica, conservazione ambientale e presidio del territorio rurale" ${ }^{\text {. }}$

${ }^{7}$ Guido Ferrara, Valutazione d'impatto ambientale del riordino fondiario e irriguo della Piana d'Arezzo, Ed. Calosci, Cortona 1991, pag. 16.

${ }^{8}$ GiUliana CAmpioni, op. cit., 1991, pag. 121. 


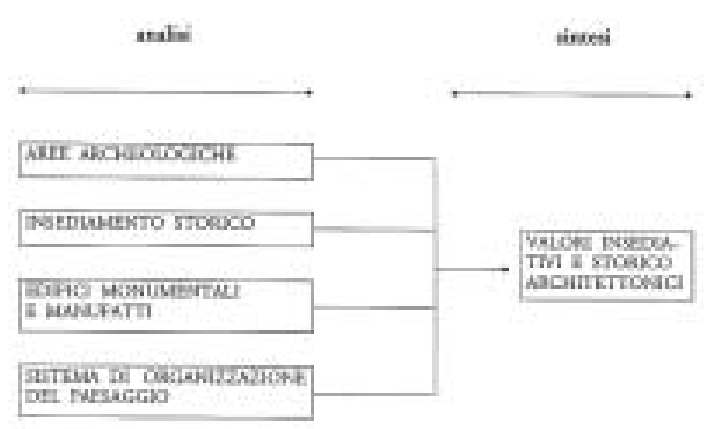

Figura 8 - Analisi del paesaggio: campo della produzione antropica.

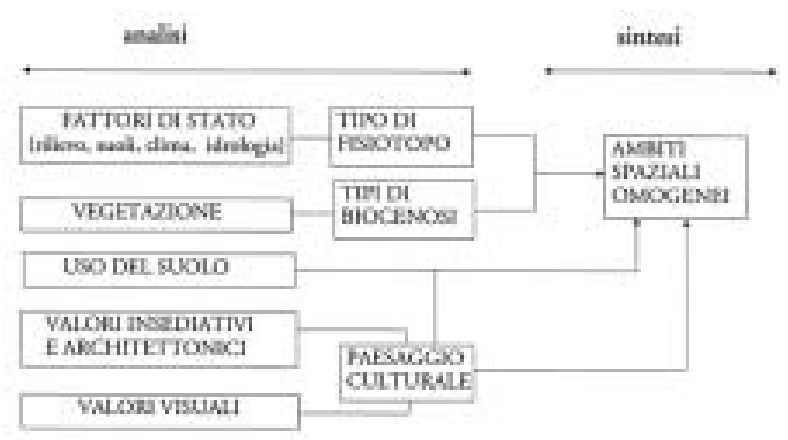

Figura 10 - Analisi del paesaggio: campo dell'ecologia del paesaggio.

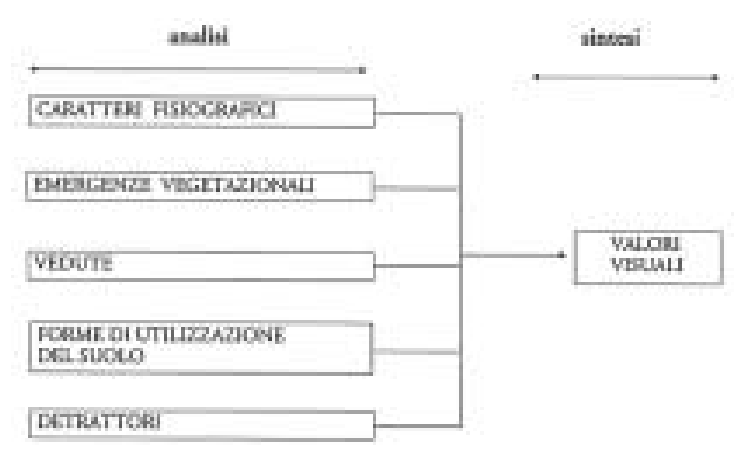

Figura 9 - Analisi del paesaggio: campo dello scenario.

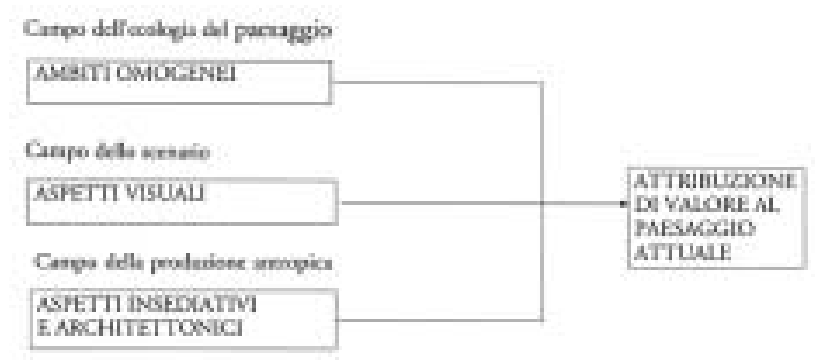

Figura 11 - Schema dell'interazione fra i tre campi di studio.

Il paesaggio è qui inteso quale risultante di elementi abiotici e biotici tra loro correlati; è stato pertanto considerato come "sistema di ecosistemi" secondo i criteri dell'ecologia del paesaggio.

Scendendo nello specifico, l'analisi del paesaggio si è sviluppata distinguendo tre campi di studio: quello della "produzione antropica", inerente i valori storico-architettonici-insediativi, il campo dello "scenario", ovvero i valori visuali, e infine il campo dell'"ecologia del paesaggio". Attraverso l'interazione tra i tre campi, ecologia, scenario e produzione antropica, si è giunti all'attribuzione del valore del paesaggio.

Dall'analisi ecologica paesistica si sono poi ricavate le singole unità di paesaggio, ossia quegli ambiti spaziali aventi caratteristiche abiotiche e biotiche omogenee.

Per l'individuazione di tali ambiti si è resa necessaria la definizione delle unità fisiografiche di base, ovvero dei cosiddetti fisiotopi. I fisiotopi sono da considerare unità spaziali che, rispetto ad una determinata scala cartografica e quindi ad un determinato livello di dettaglio nell'analisi del territorio, risultano caratterizzati da fattori di stato abiotici (litologia, morfologia, pedologia, clivometria, eccetera) relativamente omogenei e che hanno effetti uniformi sulla parte biotica dell'ecosistema, costituendo condizioni specifiche per gli habitat della vegetazione e della fauna.

Per giungere alla successiva definizione delle unità di paesaggio, i fisiotopi sono stati poi integrati con gli altri fattori principali, ovvero la vegetazione e l'uso del suolo. L'individuazione, perimetrazione e successiva descrizione di tali unità è stata attuata mediante il confronto comparato dei seguenti parametri, ricavati dalla cartografia tematica di base disponibile: la carta dell'altimetria, delle pendenze, della vegetazione, della geologia, dell'idrologia, dell'uso del suolo, dei valori storico-architettonici-culturali, dei valori visuali.

Il risultato dei confronti incrociati (tramite la sovrapposizione delle singole carte) ha prodotto l'individuazione di undici zone. Ciascuna di esse rappresenta una unità di paesaggio, ovvero un'entità corrispondente a gruppi di ecosistemi che 


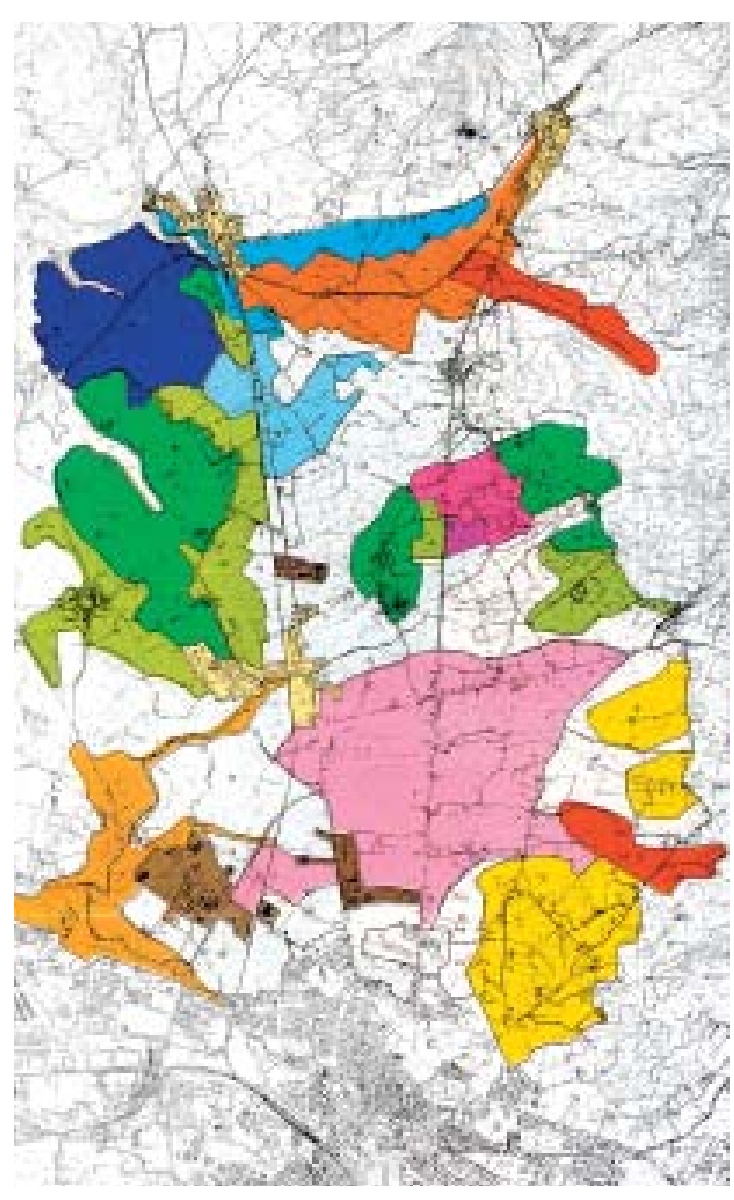

Figura 12 - Tavola delle unità di paesaggio.

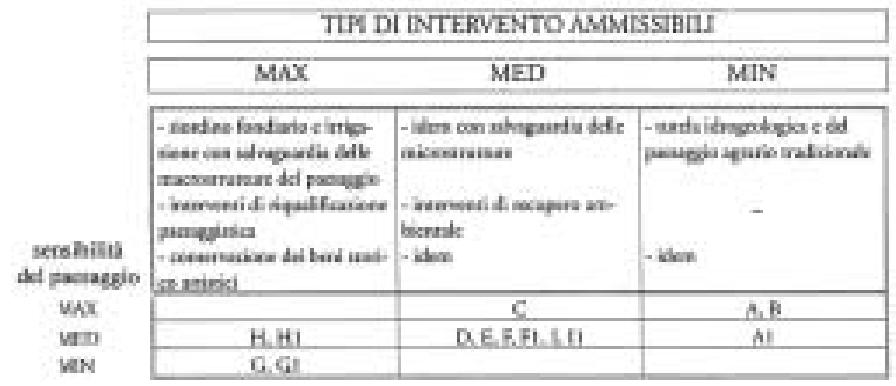

Figura 13 - Matrice del grado di sensibilità del paesaggio.

vi si ripetono spazialmente in maniera simile; pertanto essa risulta al suo interno fondamentalmente omogenea rispetto alle componenti ambientali abiotiche e biotiche considerate, ma diversa o in qualche modo caratterizzata da tutte le altre (e quindi irripetibile). Sono state specificate inoltre, delle sottozone, cioè unità subordinate che presentano una o più componenti diverse da quelle del tipo superiore.

L'individuazione degli undici ambiti spaziali omogenei ha permesso:

- la realizzazione di una sorta di radiografia dell'intero territorio, consentendone una dettagliata lettura;

- l'attuazione di una politica di "conservazione attiva" del paesaggio, legando l'immagine percettiva del contesto paesistico con i contenuti ecologici;

- l'individuazione per ciascuna unità, del "grado di sensibilità del paesaggio" e delle tipologie d'intervento possibili.

Il grado di sensibilità del paesaggio ovvero "la verifica della sua compatibilità o meno alle iniziative di intervento fondiario e irrigazione previste" ${ }^{\text {, }}$, risulta massimo nelle zone collinari (unità $\mathrm{A}$ e B) e nell'ambito spaziale della piana del fiume Castro (unità $\mathrm{C}$ ), ove si è riscontrata proprio la più elevata concentrazione di risorse culturali-storico-paesistiche.

Conseguentemente dette zone più sensibili alle trasformazioni del paesaggio hanno richiesto livelli minimi di intervento.

Viceversa nelle aree con un grado di sensibilità medio o basso (esempio unità $\mathrm{D}, \mathrm{E}$ ), i livelli d'intervento concessi sono stati massimi: addirittura in alcuni casi, il progetto di riordino fondiario e irriguo ha rappresentato una vera e propria occasione di recupero ambientale e di riqualificazione paesistica dell'intero territorio.

L'analisi ambientale compiuta ha inoltre consentito di evidenziare le tendenze in atto consolidate e ben radicate nell'area della piana aretina. Come si può constatare dalle foto aeree allegate, una prima linea di tendenza ancora oggi pesantemente avvertibile, è quella orientata verso un'espansione dei processi di urbanizzazione a partire da Arezzo.

Una seconda è invece rappresentata da un rilevante aumento del grado di frammentazione degli appezzamenti, conseguente all'apertura di nuove vie, alla diffusione di colture ortive, eccetera.

Tutto ciò ha comportato una forte semplificazione del paesaggio agrario, semplificazione accentuata anche dalla sensibile diminuzione delle superfici a vigneto e dalla scomparsa delle alberature e di altri elementi di suddivisione dei campi.

${ }^{9}$ GiUliana CAmpioni, op. cit., 1991, pag. 142. 


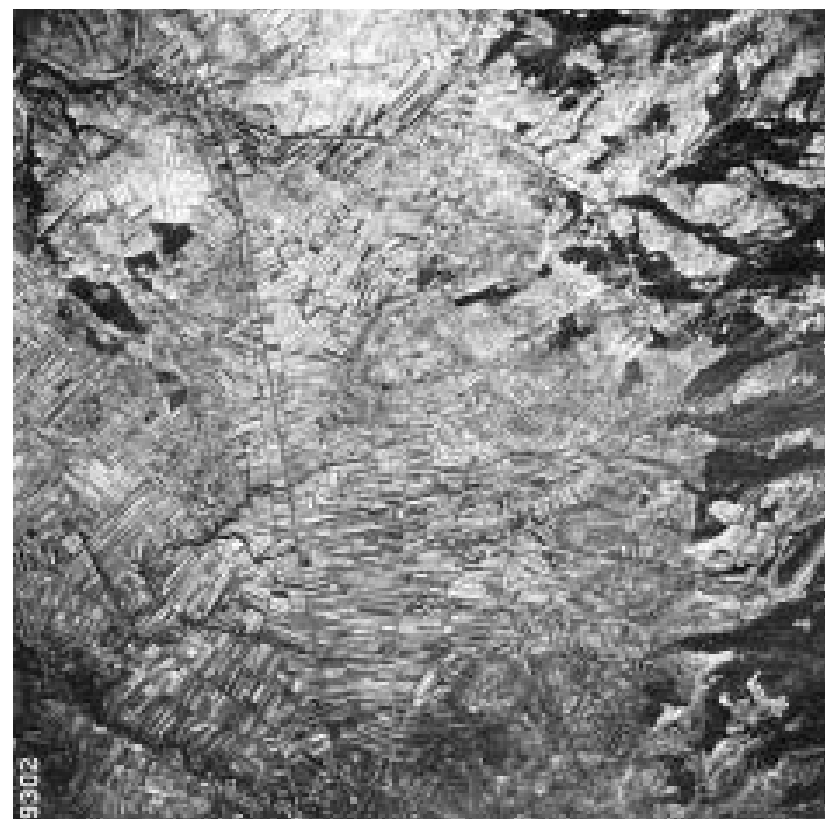

Figura 14 - Foto aerea zona di progetto (1955).

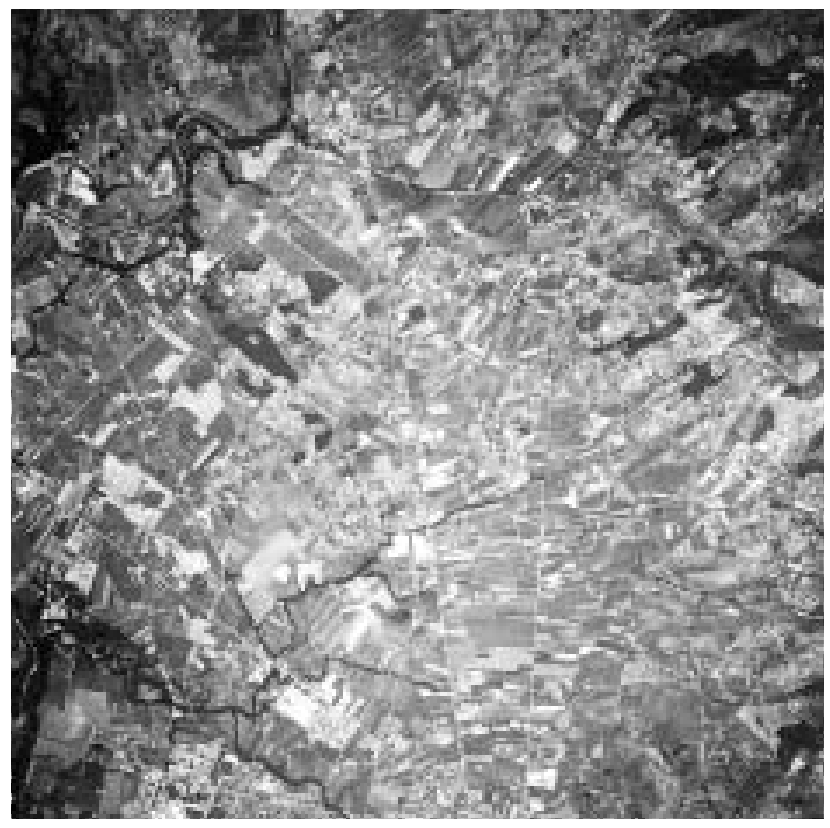

Figura 15 - Foto aerea zona di progetto (1987).

\section{GLI ESITI}

Il sistema di microvalori costituito dalla rete della viabilità poderale e da alcuni biotopi, come la vegetazione riparia, le siepi, i filari, i lembi di bosco, eccetera, rappresentano, come ricordato in apertura, il punto di partenza della fase progettuale. Questi microvalori sono stati qui considerati non vincoli o limitazioni, ma elementi di stimolo, "nodi di una maglia da ricostruire su basi diverse, per una società diversa, con un paesaggio condotto ad un nuovo equilibrio, senza per questo dover negare o cancellare quello passato" 10 .

Ci troviamo di fronte ad un classico esempio di paesaggio culturale costruito. A causa però di una marcata fragilità delle componenti fisiografiche, il paesaggio in questione è stato oggetto, fin dall'antichità, di rilevanti interventi antropici: sistemazioni di tipo idraulico, impianti arborei, costruzioni di arginature, nascita di nuovi agglomerati urbani, eccetera.

"In termini storici quindi una trasformazione del paesaggio anche profonda, come quella spontaneamente in corso nell'area, non è un fatto nuovo. Ciò che non è accettabile nella situazione attuale però non è il mutamento in se stesso, quanto la casualità dei modi e degli scopi per cui esso avviene, la sua rispondenza a leggi diverse e talvolta in conflitto tra loro, cosa che produce l'effetto negativo di un quadro ambientale caotico, di qualità decisamente più scadente di quanto non sia mai stata nel passato"11.

E stata pertanto esclusa a priori la possibilità di avviare una politica progettuale fondata sull'idea, ancor oggi piuttosto diffusa, che lo stato di fatto in quanto tale debba essere considerato sempre e comunque una condizione preferibile ad un qualsiasi tipo d'intervento.

Una politica di questo tipo avrebbe infatti generato nel caso della piana aretina, pesanti ripercussioni su tutte le principali risorse ambientali (vegetazione, fauna, scenari visivi, idrologia, pedologia, eccetera).

Il piano di irrigazione e riordino fondiario è stato qui al contrario conside-

\footnotetext{
${ }^{10}$ GiUliana Campioni, op. cit., 1991, pag. 135.

${ }^{11}$ Giuliana Campioni, op. cit., 1991, pag. 233.
} 


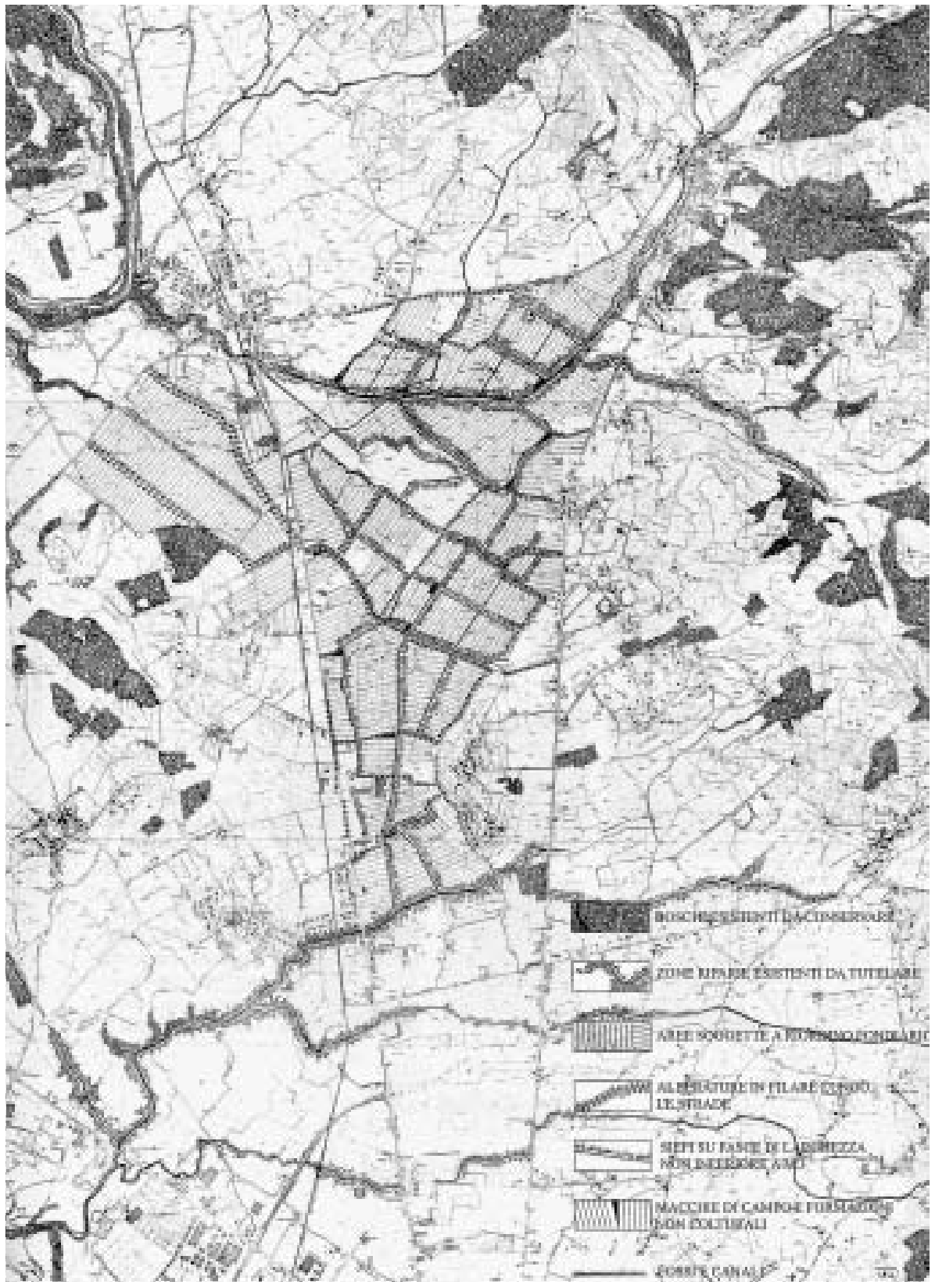

Figura 16 - Planimetria generale del progetto definitivo (riduzione da scala 1:10.000).

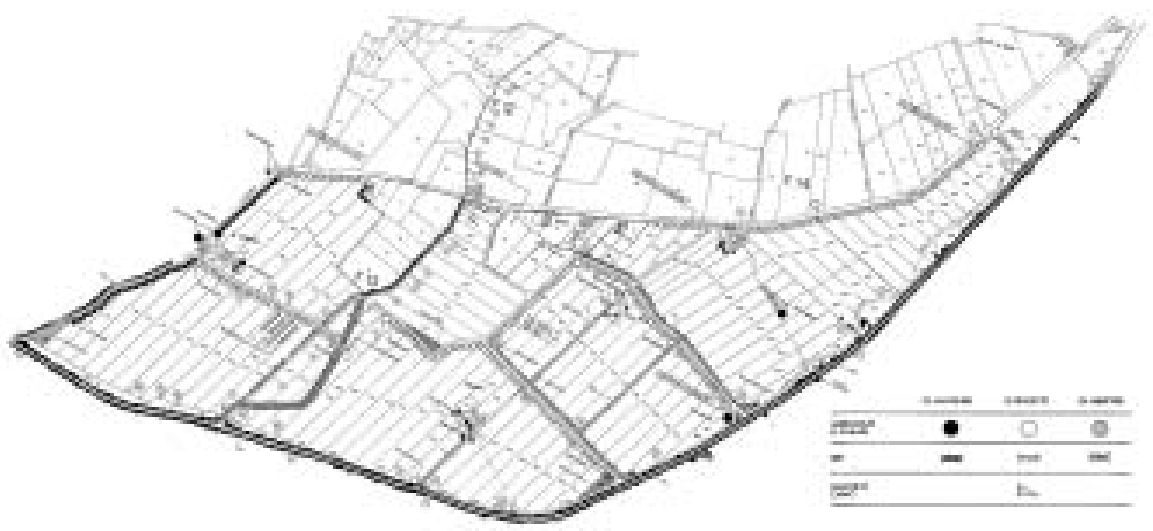

Figura 17 - Planimetria progetto definitivo (riduzione dalla scala 1:2.000). 

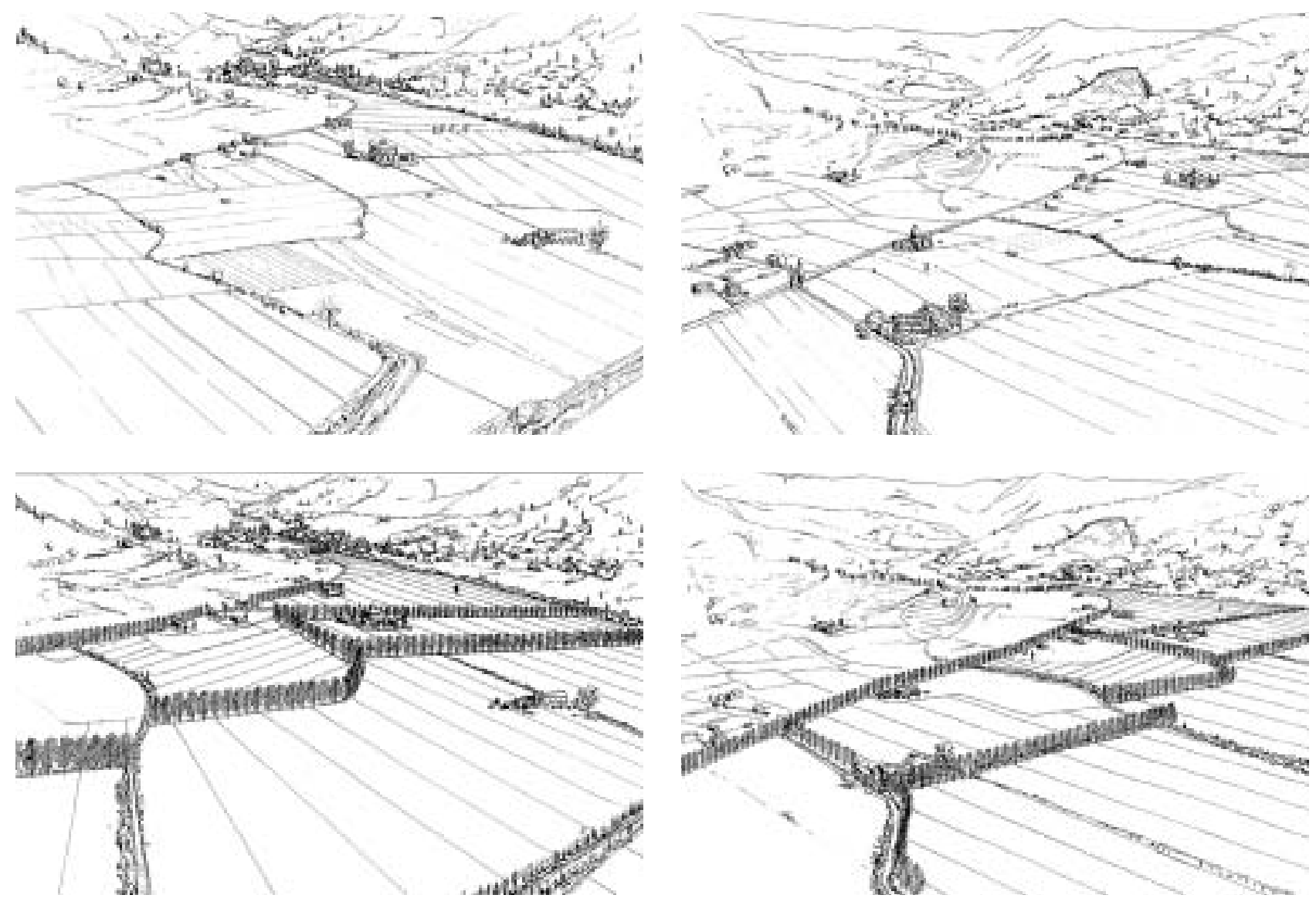

Figura 18 - Confronto tra lo stato di fatto e le sistemazioni proposte e di seguito realizzate (loc. Capanne).

Figura 19 - Confronto tra lo stato di fatto e le sistemazioni proposte e di seguito realizzate (loc. S. Salvatore).

rato "un importante quanto insostituibile strumento di guida delle modificazioni nell'uso del suolo e un efficiente fattore di incentivazione di uno sviluppo durevole"12.

Gli esiti derivanti da questo tipo di logica progettuale sono emersi con una certa rilevanza in tutte le componenti ambientali del territorio oggetto di studio.

$\mathrm{Nel}$ settore fisiografico, ad esempio, gli interventi, riducendo tra l'altro la superficie complessiva soggetta al riordino fondiario, hanno comportato tre significative trasformazioni: una modifica all'orientamento dei campi; la rete scolante segue maggiormente la morfologia naturale del suolo; i livellamenti del terreno sono stati sostituiti da azioni di rimodellamento, finalizzate all'eliminazione delle possibili cause di ristagno delle acque dei terreni ed alla riduzione del rischio di erosione superficiale.

Nel settore della vegetazione il progetto ha portato alla costruzione di un nuovo paesaggio rurale con la messa a coltura di nuovi alberi ed arbusti, riuscendo a definire una geometria degli appezzamenti più rispettosa della preesistente orditura dei campi e conservando completamente la vegetazione riparia. In questo modo si è ulteriormente sottolineata l'importanza del reticolo delle piantagioni arboree ed arbustive, responsabile diretto della configurazione paesistica alla scala di area vasta.

Ma sicuramente gli effetti positivi più evidenti si riscontrano nel settore del paesaggio. In primis va sottolineato come l'intervento di riordino fondiario ed irriguo non interessando, se non in minima parte, la corona collinare ne ha sal-

${ }^{12}$ Guido Ferrara, Valutazione impatto ambientale delle opere irrigue e di riordino fondiario sui lavori riferiti al territorio a Nord di Arezzo, intervento alla Conferenza di Pianificazione Territoriale per la Provincia di Arezzo, Arezzo 1990. 
vaguardato la trama del tessuto paesaggistico. Allo stesso modo nell'area della piana si è garantita la piena tutela delle risorse culturali-storiche più significative e maggiormente vulnerabili, come le aree archeologiche.

Nello specifico, le zone archeologiche sono state classificate in base al loro livello d'importanza, in tre categorie: le risorse archeologiche vere e proprie, escluse ovviamente da qualsiasi tipo di intervento; i siti di "preminente interesse archeologico", in cui allo scavo hanno fatto seguito una serie di provvedimenti specifici di tutela dei reperti rinvenuti; e infine, i siti che non hanno comportato alcuna azione di tutela.

Lintervento progettuale ha altresì tenuto conto delle microstrutture del paesaggio, come le siepi e le alberature, necessarie sia nello spezzare l'uniformità e la monotonia dello scenario paesistico che a svolgere una funzione di carattere produttivo e di difesa del suolo, attraverso la ricostruzione di una rete collegata alla vegetazione riparia fluviale e alle realtà boschive contigue. Per quanto concerne la scelta delle piante, nelle alberature si sono utilizzate undici diverse specie: Quercus cerris, Quercus ilex, Quercus pubescens, Quercus robur, Cupressus sempervirens pyr, Populus nigra italica, Prunus avium, Ulmus pumila, Sorbus aucuparia, Acer campestre e Morus nigra.

Gli alberi sono stati collocati ad una distanza variabile, compresa tra i 6-7 metri per il Morus nigra e i 15-18 metri della Quercus cerris, con una densità lineare minima pari a $46 \mathrm{~m} / \mathrm{ha}$. Nell'impianto delle siepi, realizzato su fasce di larghezza di almeno 3 metri, si sono impiegate 8 specie differenti: Asparagus acutifolius, Cornus sanguinea, Crataegus monogyna, Ligustrum vulgare, Prunus spinosa, Pyracantha coccinea, Rosa canina e Ulmus campestris, con una densità minima lineare pari a $40 \mathrm{~m} / \mathrm{ha}$.

Il sistema lineare delle siepi e delle alberature è stato poi integrato da una struttura vegetazionale puntiforme, rappresentata dalle macchie di campo, ovvero formazioni non colturali areali arboree ed arbustive. Le macchie di campo, collocate agli incroci di una rete aventi maglie con lati di lunghezza massima pari a 500 metri formati da siepi, coprono una superficie variabile da 500 a $1500 \mathrm{mq}$, con una densità lineare minima di $60 \mathrm{mq} / \mathrm{ha}$. Sono state utilizzate le seguenti specie: Morus nigra, Prunus avium, Quercus spp., Sorbus aucuparia, Corylus avellana, Pyrus communis, Malus domestica, Taxus baccata, Juniperus communis e Juiniperus oxycedrus. Per le macchie si è rispettata inoltre una larghezza minima di dieci metri, fondamentale per garantire un'adeguata possibilità di rifugio e alimentazione alla selvaggina.

In questo modo, si è cercato di riproporre l'immagine storicamente consolidata del paesaggio della piana all'interno di uno schema compatibile con le preesistenze culturali-ambientali, ma anche adeguato alle necessità e finalità di un intervento di riordino fondiario e irriguo. Grazie inoltre alle nuove sistemazioni infrastrutturali e vegetali si sono poi ulteriormente rafforzati e consolidati gli organismi portanti dell'intero sistema ambientale, ovvero la natura dei terreni, la rete idrologica, l'orientamento dei campi, gli allineamenti prospettici, eccetera.

\section{RIFERIMENTI BIBLIOGRAFICI}

Di Fidio Mario, Architettura del Paesaggio, Ed. Pirola, Milano 1993 (quarta edizione).

FERraRa Guido, Val di Chiana aretina: un progetto per il paesaggio agrario del 2000, "Paesaggio Urbano", 6, 1993, pagg. 30-44.

FERRARA GUIDO e Rossi RoberTO, Valutazione d'impatto ambientale del riordino fondiario e dellirrigazione della Piana di Arezzo, Ed. Calosci, Cortona 1991.

FERrara Guido, Progetto irriguo in Val di Chiana: tra continuità e innovazione in Recupero del paesaggio e riflessioni sul trend occupazionale, Atti del Seminario Internazionale - Regione Liguria, Goethe Institut Genua, Genova 1998. 
Ferrara Guido, Verso uno studio d'impatto ambientale dell'invaso di Montedoglio, relazione alla Giornata studio Complesso Montedoglio, Sansepolcro 1988.

FERraRA GUIDO, Valutazione d'impatto ambientale delle opere irrigue e di riordino fondiario sui lavori riferiti al territorio a Nord di Arezzo, intervento alla Conferenza di Pianificazione Territoriale per la Provincia di Arezzo, Arezzo 1990.

Focardi Andrea e TANi Piergiorgio, Lavori di irrigazione e riordino fondiario nel comprensorio alimentato da Montedoglio, serbatoio di compenso n. 2, nel comune di Arezzo, - Relazione tecnica, edizione dattiloscritta, Amministrazione provinciale di Arezzo, 1985.

Fossombroni VitTorio, Memorie idraulico-storiche sopra la Val di Chiana, Ed. Atesa, Bologna 1978.

ROSSI ROBERTO e BINI CARLO, Criteri e problemi relativi al riordino fondiario e alla conversione irrigua nella particolare situazione ambientale della Toscana, Atti della Conferenza di pianificazione territoriale per la Provincia di Arezzo, Arezzo 1990.

\section{RIFERIMENTI ICONOGRAFICI}

Le immagini sono tratte da: FERRARA GUIDO e Rossi RoberTO, Valutazione d'impatto ambientale del riordino fondiario e dellirrigazione della Piana di Arezzo, Provincia di Arezzo, Ed. Calosci, Cortona 1991. 
SCHEDA DI PROGETTO

Progetto: $\quad$ intervento di riassetto fondiario e irriguo

Luogo: l'area oggetto dell'intervento è collocata fra l'immediata periferia a Nord della città di Arezzo, l'abitato di Ponte alla Chiassa e le colline poste ad Est e Ovest

Committente: $\quad$ Provincia di Arezzo

Superficie: $\quad 2,5$ ettari di territorio, primo lotto di un'opera che interesserà l'intera Val di Chiana, per una superficie complessiva pari a 60.000 ettari

Progettista: $\quad$ Prof. Arch. Guido Ferrara

Collaboratori:

COMMISSIONE TECNICO-SCIENTIFICA INTERDISCIPLINARE:

Vittoria Calzolari Ghio - Urbanistica - Università di Roma;

Adriano Ciani - Economia agraria - Università di Perugia;

Carla Corsi Miraglia - Sovrintendente ai Beni architettonici e Ambientali di Arezzo;

Giorgio Federici - Ingegneria idraulica - Università di Firenze;

Adriano Gradi - Selvicoltura - Università di Padova;

Luigi Omodei Zorini - Economia agraria - Università di Firenze;

Bernardino Ragni - Scienze naturali - Università di Perugia;

Roberto Rossi - Pedologia e geografia fisica - Dipartimento Agricoltura e Foreste della Regione

Toscana;

Mario Torelli - Archeologia - Università di Perugia;

GRUPPO DI STUDIO:

Giuliana Campioni - Paesaggio e beni culturali;

Paola Savini - Beni naturali;

Alessandro Cocchi, Franco Gabrielli, Paolo Gandi - Economia agraria;

Fabrizio Cassi, Alessandro Fonseca, Claudio Marchisio, Daniele Gallorini, Edafos s.p.a. - Idraulica e pedologia;

Stefanie Risse - Pubbliche relazioni.

ALTRI CONTRIBUTI TECNICO-SCIENTIFICI:

Claudio Lubello, Federico Preti - Idraulica - Università di Firenze;

Guido Tellini - Fauna - Istituto Difesa del Suolo;

Laura Bigi, Enrico Favi, Serena Maiani, Luca Rustici, Andrea Vinci - Pedologia - Regione

Toscana.

Anno di progettazione: 1989

Anno di realizzazione: 1990-1993 


\title{
IL PARCO REGIONALE DELLA VALLE DEL TICINO IN LOMBARDIA
}

\author{
Adele G. Caucci
}

\section{IL CONTESTO}

Il Parco Regionale Lombardo della Valle del Ticino, istituito nel 1974, si estende su una superficie di 23.989 ettari $^{1}$ nelle province di Varese, Milano e Pavia, e comprende tutta la fascia fluviale del corso inferiore del fiume Ticino nel versante lombardo che, unitamente a quello piemontese, tutela l'intera valle fluviale, per una lunghezza complessiva del corso di circa 115 chilometri fino alla confluenza con il fiume Po.

Il Parco Regionale del Ticino rappresenta un importante tassello del sistema delle aree protette lombarde e identifica la prima istituzione (L.R. 2/74) conseguente alla legge quadro (L.R. 58/73) per l'istituzione delle aree protette della Regione Lombardia; vengono istituite successivamente altre aree protette sui fiumi di particolare rilevanza naturale e ambientale: Adda, Mincio, Lambro, Serio. Il sistema regionale dei parchi naturali viene completato con l'inserimento di altre due tipologie di aree protette: i parchi

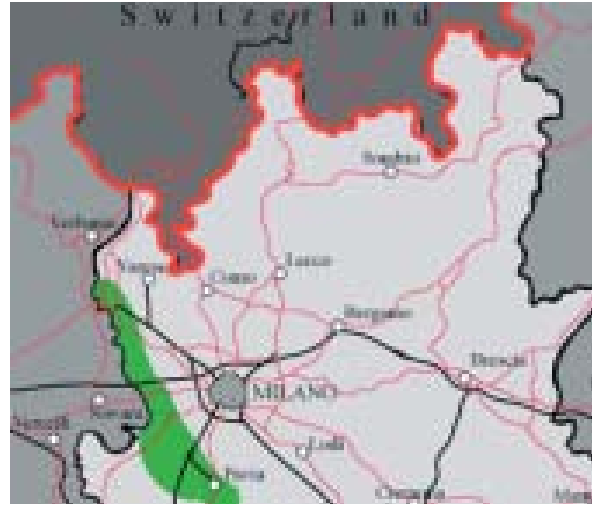

Figura 1 - Localizzazione area di progetto. subalpini ed i parchi periurbani. Listituzione dei parchi naturali ha creato in Lombardia un sistema di aree protette che, insieme al Piemonte, rappresenta una realtà consolidata nel quadro nazionale della conservazione della natura.

Nell'evoluzione dei sistemi insediativi dell'area metropolitana milanese, le aree protette hanno progressivamente assunto il ruolo di polmoni verdi, e sono meta del turismo ricreativo dei fine settimana per milioni di persone.

Nell'ottica di recupero della sostenibilità ambientale dello sviluppo locale, la Provincia di Milano ha istituzionalmente consolidato l'importanza delle aree protette quali nodi di connessione della rete ecologica provinciale, recentemente costituita.

Il Parco del Ticino svolge un ruolo fondamentale in questo sistema, per posizione e dimensione dell'area, proprio per la sua natura di sistema fluviale, e quindi di importante corridoio di connettività ecologica.

\section{GLI OBIETTIVI}

L'istituzione dell'area protetta, voluta per conservare ambienti naturali di particolare valore nel contesto metropolitano circostante, oltre alla tutela di gran parte degli ecosistemi presenti, ha assunto quale obiettivo primario la gestione partecipata dell'agricoltura e della forestazione, come mezzo fondamentale per la conservazione di molti paesaggi tipici dell'area del Ticino.

Il territorio del parco si sviluppa da nord-ovest a sud-est seguendo il corso del fiume, attraverso paesaggi che variano sostanzialmente al variare della morfologia e soprattutto delle funzioni antropiche storicamente insediate.

\footnotetext{
${ }^{1}$ L'estensione di 23.989 ettari, indicata nell'Elenco Ufficiale della Aree Naturali Protette del Ministero dell'Ambiente nella G.U. n.14 del 19/06/1997, risulta effettivamente pari a 90.640 ettari, poiché comprende l'intero territorio dei comuni interessati dal parco.
} 


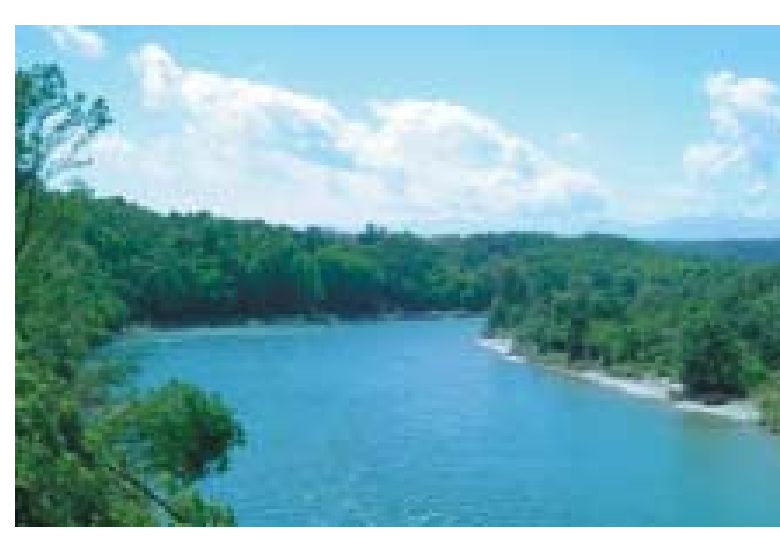

Figura 2 - Il fiume Ticino a Castelnovate.
La parte alta del parco, situata nelle province di Varese e Milano, è caratterizzata da brughiere alle quali si alternano colline moreniche fittamente coperte da boschi, con la costante presenza di acque, che attraverso corsi secondari, a volte paralleli, alimentano il Ticino; è questa la zona dove la pressione dell'area metropolitana milanese si fa più forte, tanto da estendere le sue propaggini all'interno dell'area protetta, con la presenza dell'aeroporto intercontinentale della Malpensa.

Nell'area centrale dei terrazzi alluvionali il fiume assume un andamento meandriforme, che crea ambienti naturali di grande rilevanza con boschi di ripa che rappresentano gli ultimi lembi di foresta planiziale; in alcuni di questi tratti, il suo alveo raggiunge l'ampiezza di 2,5 chilometri, dove l'interazione tra fenomeni naturali ed attività umana ha generato ecosistemi e paesaggi di grande rilievo nell'ambito dell'intero territorio circostante.

La parte bassa del parco si estende da Pavia fino alla confluenza del Ticino con il Po ed è la zona tipicamente di pianura, con piccole aree boscate ed una conduzione agraria fortemente industrializzata, dove le colture irrigue rappresentano la matrice principale del paesaggio.

In ognuna delle macrozone morfologicamente individuate, si identificano gli ambiti che strutturano il parco in tutta la sua estensione, fortemente differenziati ma strettamente correlati fra loro:

- il corpo d'acqua con le ampie fasce boscate che rappresenta il nucleo centrale dell'area protetta;

- le aree agricole che caratterizzano il corso del fiume fino alla confluenza con il fiume Po;

- gli insediamenti che costellano l'intero territorio, dai piccolissimi centri storici alle aree propriamente urbane - Pavia - fino alle forti presenze industrali ed infrastrutturali.

La zona propriamente fluviale comprende l'alveo e le fasce boscate che ne completano la struttura, è indubbiamente la componente del parco col più alto grado di naturalità: qui sono insediate la vegetazione e la fauna che non trovano spazi di sopravvivenza nei territori contermini. Il microclima, determinato dalle condizioni del sistema delle acque e dalla vegetazione, ha creato ecosistemi di gran valore naturalistico, che necessitano di un controllo attento affinché sopravvivano agli effetti del sistema che li racchiude.

Le foreste all'interno del parco costituiscono il $17 \%$ dell'intera area protetta, e coprono un estensione pari a circa 25.000 ettari, quasi totalmente in zona perifluviale e storicamente utilizzate come riserve, oggi necessitano di una gestione unitaria, che superi le differenziazioni generate dai diversi usi pubblici o privati.

Il fiume però non è solamente questo, ma rappresenta anche una importante risorsa idrica, con portate piuttosto rilevanti. Il Canale Villoresi ed il Naviglio Grande costituiscono le due principali opere di derivazione del Ticino: il primo fu realizzato per la produzione di energia elettrica e per l'alimentazione delle industrie, il secondo sostanzialmente per servire la città di Milano con una via di navigazione fluviale.

Questi due canali principali, insieme agli altri canali storici ${ }^{2}$ ed una rete minore di derivazioni ad uso irriguo, fanno sì che la portata d'acqua del Ticino, mediamente stimata in $900 \mathrm{mc} / \mathrm{sec}$, raggiunga anche minimi di $30-40 \mathrm{mc} / \mathrm{sec}$; oscillazioni così forti sono determinanti per la funzionalità degli ecosistemi fluviali, che

${ }^{2}$ Canale Sforzesco e Naviglio di Bereguardo. 
possono subire trasformazioni sostanziali, fino ad arrivare alla scomparsa.

Appena al di sotto dell'aeroporto della Malpensa, ha inizio l'ambito di riferimento per la moderna agricoltura industrializzata, dove la parte più alta é caratterizzata da pianura asciutta coltivata a seminativo semplice, a prevalenza di cereali, che variano in relazione ai contributi accordati dalla PAC (Politica Agricola Comunitaria).

Le risorgive segnano il confine con la pianura irrigua posta più a sud, che invece è costituita da monocolture ad alta redditività, quali ad esempio la risicoltura e la pioppicoltura.

Sempre in queste zone, l'allevamento dei bovini rappresenta una realtà economica consolidata. La moderna

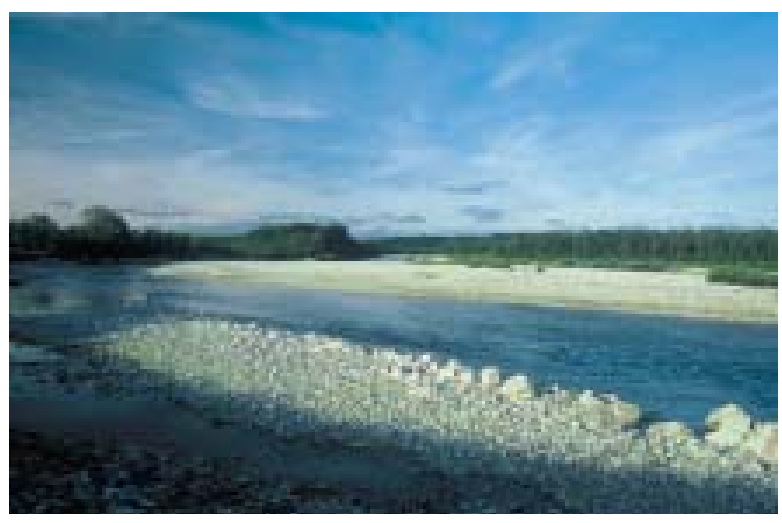

Figura 3 - Il fiume e gli ontaneti. conduzione delle aziende agricole ha completamente modificato il tradizionale insediamento rurale, fondato sulla cascina e sulle piantate che caratterizzavano il sistema insediativo storico; le attuali aziende, che si configurano sempre come piccoli nuclei industriali, rappresentano i nodi di aree coltivate molto estese, e qui le dimensioni delle aziende agricole variano da 80 a 100-200 ettari.

Il parco include interamente entro i suoi confini quarantasei comuni: questa scelta è stata operata per poter attuare uniformemente le politiche di gestione e deriva anche dal fatto che la maggior parte dei comuni interessati ha una estensione molto limitata (anche $5 \mathrm{kmq}$ ), con un numero di residenti che raggiunge appena, in alcuni casi, le 500 unità.

Nell'intero territorio dell'area protetta gravitano circa 420.000 residenti, con le massime punte a Pavia e Vigevano: in corrispondenza di questi due poli urbani, sono localizzate le aree di maggiore produttività economica, rispettivamente agricola ed industriale.

Il sistema insediativo nell'area del parco annovera la più ampia casistica di testimonianze storiche e contemporanee di manufatti e complessi, che identificano e caratterizzano il paesaggio fluviale ed agrario.

I centri storici scandiscono la trama viaria che affianca il corso fluviale per l'intero tratto; essi rappresentano, insieme alle cascine e gli altri manufatti isolati (opifici, torri, ville), il patrimonio storico-architettonico del parco che, coniugato al sistema degli spazi aperti, definisce l'identità culturale del parco stesso.

È in questa realtà sociale che il parco ha fondato la propria esistenza ed ha delineato i propri obiettivi, tanto da eleggere a propria sede istituzionale uno dei centri minori che si trovano nel cuore dell'area protetta, la frazione di Pontevecchio, nel comune di Magenta.

La posizione del parco, marginale rispetto all'area metropolitana più estesa della penisola, ha determinato la presenza diffusa dei segni della civiltà industriale.

Oltre ad insediamenti industriali di varie dimensioni, diffusi un pò ovunque, sono presenti cave di inerti, infrastrutture viarie quali l'attraversamento dell'autostrada A4 Milano-Torino e della parallela S.S. n. 11 all'altezza di Magenta, dell'A7 Genova-Milano all'altezza di Bereguardo ed il relativo tratto di collegamento con Pavia che si sviluppa parallelo al fiume per alcuni chilometri. Infine, non ultimo per importanza, troviamo l'aeroporto della Malpensa, che é situato in prossimità del fiume, nel tratto di maggior interesse naturalistico.

La volontà di attribuire all'area del Ticino la valenza di ambito di particolare rilevanza per valori naturalistici, culturali e paesaggistici con l'istituzione del parco, ha messo in moto un processo di regolamentazione delle attività umane che è tuttora in fase di evoluzione.

Il processo di sviluppo della zona, che precedentemente rispondeva esclusivamente ad interessi di settore, generando anche situazioni di incompatibilità, 

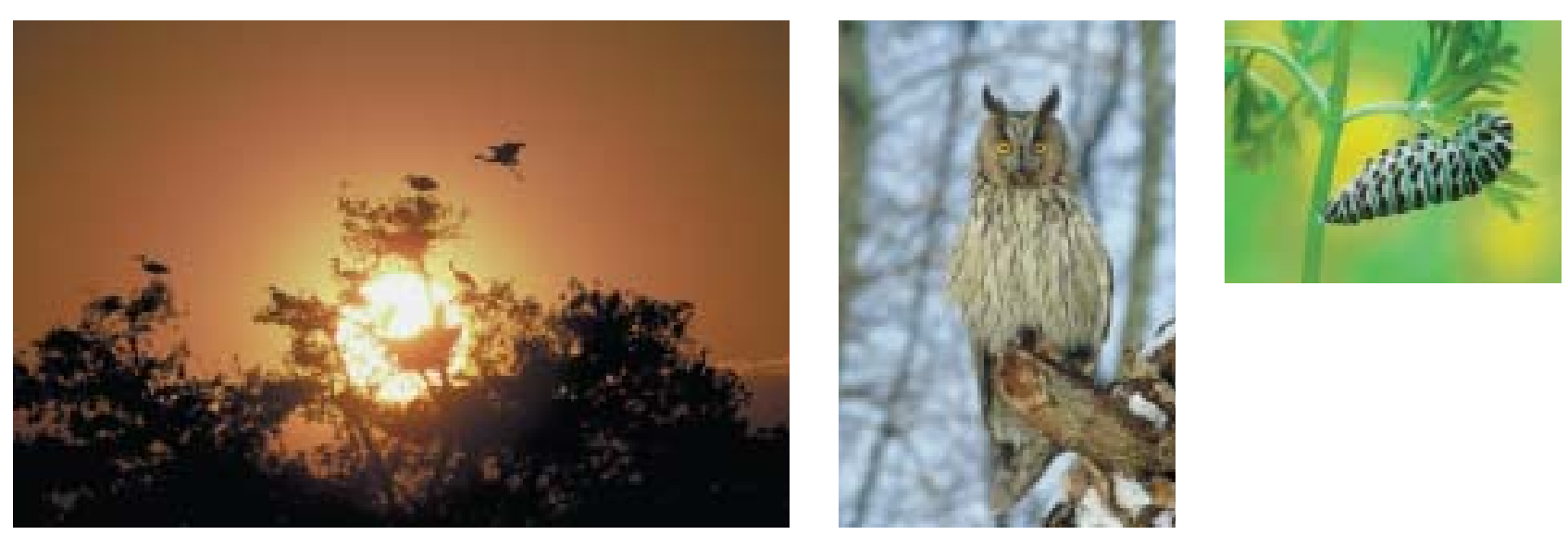

Figure 4,5 e 6 - Il volo degli aironi al tramonto, il gufo, il bruco di macaona.

deve ora concertare le diverse finalità che integrano gli interessi economici con quelli di tutela e valorizzazione delle risorse ambientali.

Le ampie zone naturali del corso d'acqua sono strettamente correlate ad un sistema insediativo agrario fortemente specializzato, oltre ad essere in stretto rapporto con l'ambito metropolitano milanese ed il sistema degli insediamenti connessi a questo (industriali, infrastrutturali, eccetera).

Le aree che hanno maggiormente conservato i caratteri di naturalità, sono soggette alla forte pressione turistica legata agli spostamenti dei fine settimana da parte dei residenti dell'intera area metropolitana, che gravita sull'area protetta; il flusso, che ormai è divenuto una consuetudine, genera fenomeni di pressione sull'area con effetti derivati quali:

- il sovraccarico della viabilità locale, che si manifesta con punte massime in due giorni alla settimana di alcuni mesi dell'anno;

- l'utilizzo di ampie zone a parcheggio, con conseguente degrado delle superfici a suolo;

- la fruizione incontrollata degli ambiti naturali a scopo ricreativo e la pressione su ambienti fragili;

- la proliferazione di "seconde case" ed insediamenti abusivi lungo le sponde fluviali.

Le vaste aree boscate, un tempo utilizzate come riserve, hanno assunto il ruolo di ecosistemi di interesse botanico-vegetazionale o faunistico, oppure di aree interessate dalla fruizione turistica e ricreativa, generando conflitti con la proprietà privata delle aree e con usi delle risorse naturali, quali l'attività selvicolturale e l'attività venatoria.

L'uso della risorsa idrica, storicamente consolidato, deve confrontarsi oggi con la riduzione della portata del fiume e con gli effetti indotti dalle trasformazioni territoriali ed insediative (si pensi, ad esempio, all'impatto provocato dagli agenti inquinanti prodotti dagli insediamenti civili, industriali e dalle attività agricole meccanizzate).

L'agricoltura è comunque l'attività umana che in misura maggiore entra in conflitto con le finalità dell'area protetta, sia per la sua estensione sul territorio ${ }^{3}$, sia per la sua incidenza diretta sugli ecosistemi, ed infine per il rilievo economico che riveste nell'intera area.

Gli impatti principali derivano dall'uso monocolturale dei terreni, che impoverisce la fertilità del suolo; lo sfruttamento intensivo richiede un apporto sempre maggiore di elementi nutritivi, che si traduce nell'uso massiccio di concimi 
chimici e fertilizzanti, sostanze che, filtrando negli acquiferi sotterranei, si ritrovano sotto forma di agenti inquinanti le acque di falda e fluviali.

Il paesaggio agrario, dove l'organizzazione territoriale e produttiva è legata alla cascina lombarda, rappresenta la matrice dell'intero sistema rurale, e rischia quotidianamente la manomissione anche nei complessi edilizi, a causa degli adeguamenti funzionali delle aziende agricole. Con la trasformazione dei metodi colturali, il progressivo degrado delle strutture storiche e l'introduzione di manufatti pseudoindustriali (silos, capannoni, rimesse), si inseriscono nel paesaggio rurale elementi di disturbo visivo, che spesso assumono rilievo come elementi detrattori del paesaggio.

I sistemi insediativi (civile ed infrastrutturale) generano, insieme al sistema agrario, i maggiori conflitti con la gestione del parco.

Il controllo delle trasformazioni si é evoluto secondo le logiche di mercato; sono molti $\mathrm{i}$ casi in cui la strumentazione urbanistica dei comuni non può esprimersi coerentemente con le finalità dell'area protetta, a causa delle limitate dimensioni territoriali.

\section{LA METODOLOGIA}

L'istituzione dell'area protetta, legata ad una volontà popolare diffusa, ha tratto spunto dall'esigenza di tutelare un sistema di risorse naturali dall'impatto negativo delle attività umane, quali l'inquinamento delle acque, i rischi di edificazione sulle rive e soprattutto dai fenomeni di fruizione di massa della risorsa naturale ${ }^{4}$.

Il parco, dalla sua istituzione, ha costantemente assolto alla funzione ricreativa a scala metropolitana; con la progressiva istituzione degli altri parchi della cintura metropolitana, il Ticino si è trovato all'interno di un più vasto sistema di aree protette, dove comunque continua a svolgere un ruolo centrale.

A quattro anni dalla sua istituzione, l'area protetta si è dotata di un piano territoriale di coordinamento, approvato nel 1980: il P.T.C., nella fase preliminare di redazione ha prodotto un ricco repertorio di conoscenze sulle risorse ambientali e territoriali dell'area, attraverso indagini sul sistema fisico-morfologico, sulla vegetazione, la fauna, sui sistemi idraulici ed i processi di urbanizzazione, sul sistema storico, ambientale e sociale, sulle infrastrutture, le cave e la fruizione turistico-ricreativa.

Il Piano Territoriale di Coordinamento pone, alla base delle scelte di pianificazione, la tutela e la valorizzazione delle caratteristiche storiche, ambientali e
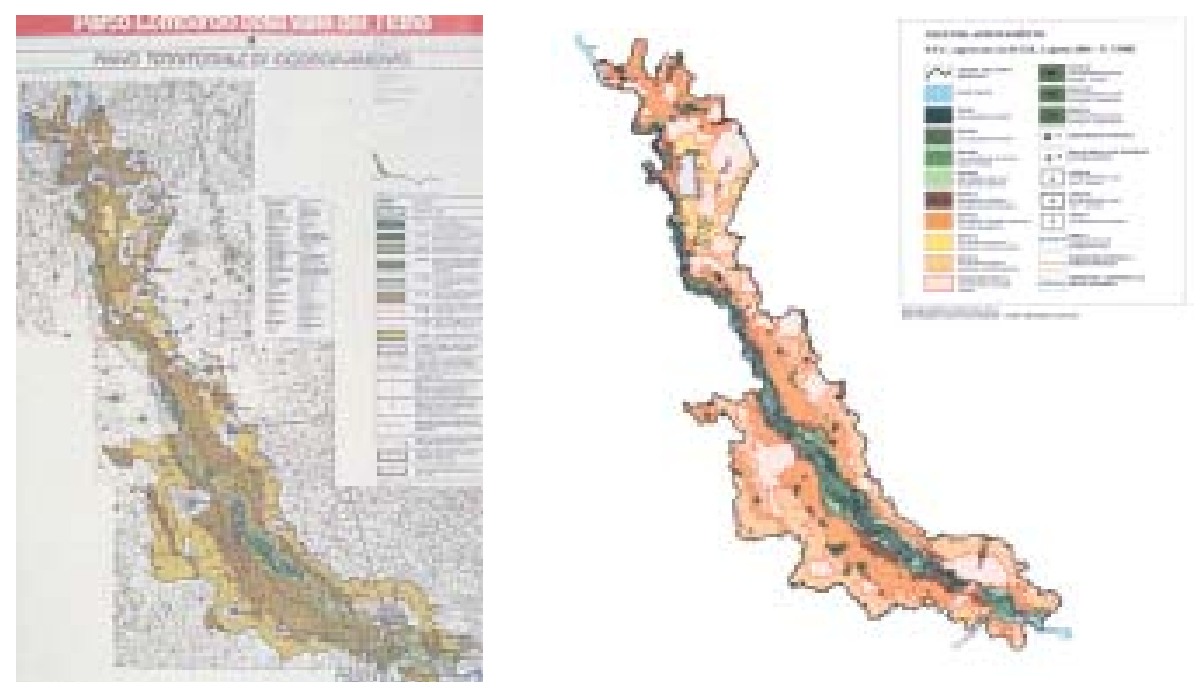

Figura 7 - Il PTC del Parco - 1984.

${ }^{4}$ Cfr. Alberto Tenconi, I parchi fluviali della Regione Lombardia, allegato di "Parchi”, 13, ottobre 1994. 
naturalistiche dell'area, in funzione dell'uso sociale e dei suoi valori ${ }^{5}$, rivestendo anche la funzione di strumento urbanistico, che si articola in zone territoriali che disciplinano l'uso del suolo e le attività che vi sono consentite.

Il piano individua cinque ambiti, che corrispondono a differenti gradi di tutela: Zona A - riserva integrale; zona $\mathrm{B}$ - riserva orientata; zona $\mathrm{C}$ - parco agricolo e forestale; zona $\mathrm{G}$ - zona agricola; zona IC - di rinvio all'iniziativa comunale orientata.

Il piano, dalla sua prima approvazione, ha avuto successivi adeguamenti: l'ultima versione approvata in via definitiva dalla Regione nel 2001.

Il parco, costituitosi istituzionalmente come consorzio di enti locali, affida la gestione dell'area all'assemblea del consorzio che, attraverso un Consiglio Direttivo, si organizza operativamente per mezzo di commissioni consultive e servizi tecnici.

Lattività di gestione dell'area protetta è ormai consolidata ed ha avuto il compito primario di attenuare e risolvere i conflitti e le incompatibilità esistenti nell'area.

La tutela delle aree più fragili e la valorizzazione del sistema socio-economico interno al parco sono perseguiti mediante piani di settore: questi piani attuativi, oltre al territorio direttamente vincolato e tutelato, coinvolgono anche le aree contermini, innescando così meccanismi di pianificazione integrata.

\section{GLI ESITI}

Il parco ha svolto e sta svolgendo un ruolo fondamentale nel coordinamento degli strumenti urbanistici comunali e nella loro attuazione.

Ha infatti definito ambiti di azione totale, mediata e di "non interferenza", lasciando in quest'ultimo caso totale libertà di azione agli strumenti comunali; sono questi gli ambiti di riferimento dei centri abitati, in cui ogni comune ha totale giurisdizione.

Gli ambiti di azione mediata coinvolgono gran parte delle zone ad uso agricolo, dove le normative di piano hanno valenza di indirizzo programmatico; negli ambiti di totale giurisdizione del piano, la normativa diventa vincolante e rappresenta la disciplina del territorio a cui attenersi.

La maggiore difficoltà incontrata dagli organi di gestione è stata quella di far convivere gli obiettivi di tutela con gli interessi economici degli operatori che gravitano nell'area del parco, soprattutto in campo agricolo, dove le possibilità di invertire tendenze di sviluppo consolidate sono piuttosto complesse.

Recentemente il Piano Territoriale di Coordinamento è stato sottoposto ad una revisione generale, a cura dei tecnici dell'ente parco. Lo strumento di pianificazione rimane legato all'azzonamento per caratteri e destinazioni d'uso, anche se comunque esiste una maggiore attenzione ad alcuni fattori legati alla valoriz-

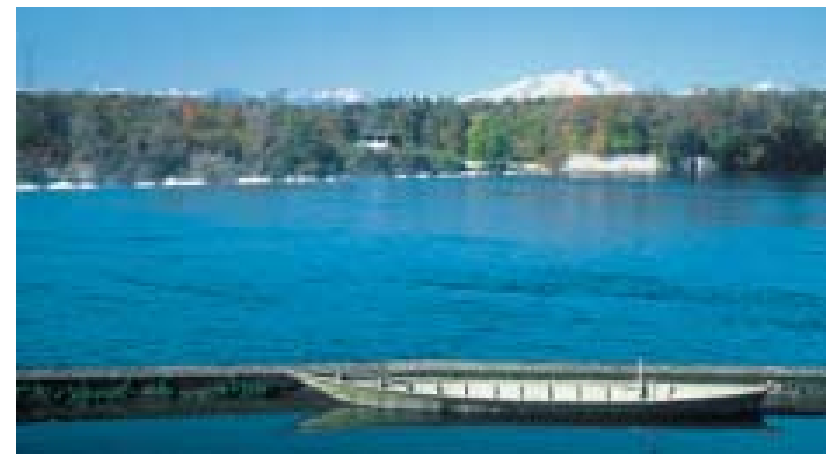

Figura 8 - Un tratto del fiume navigabile.

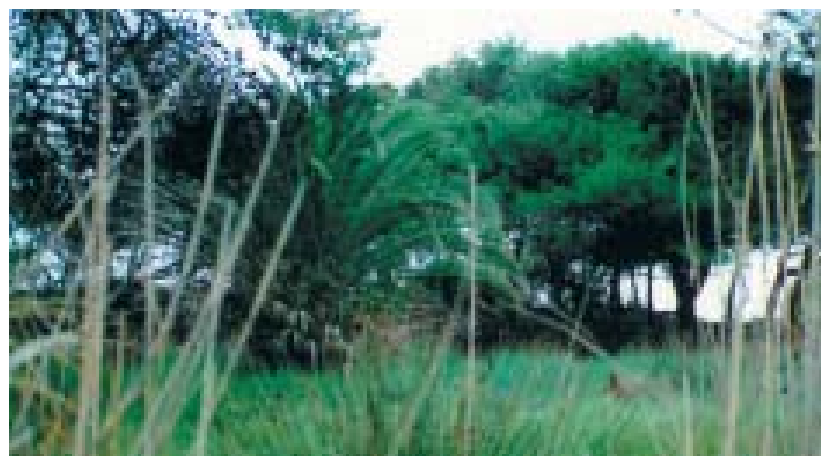

Figura 9 - La palude Pollini. 
zazione paesaggistica, che contribuiscono nella definizione di scelte progettuali tali da poter valutare e controllare gli interventi programmati.

Anche se in modo non coordinato, le componenti paesaggistiche entrano a far parte dei progetti e delle strategie, sotto forma di ambiti visuali, di compatibilità ecologica, di coesione dei sistemi culturali, eccetera. Le scelta di fondo effettuata dal parco consiste nella partecipazione attiva all'economia locale, cercando di invertire le tendenze in atto che risultano perdenti a lungo termine; l'impegno maggiore profuso dagli organi di gestione è rivolto al settore agricolo ed alla fruizione sociale del parco. La strategia adottata nei confronti delle aziende agricole ha identificato come obiettivo il miglioramento ambientale e paesaggistico, attraverso una migliore qualificazione delle produzioni a scapito delle quantità, attualmente perseguita poiché quest'ultima è sempre meno competitiva nei mercati internazionali, cui l'agricoltura partecipa a pieno titolo.

La partecipazione del parco si attua con strumenti tecnici di supporto, che agevolano le aziende nel proporre progetti finalizzati alla trasformazione dei sistemi colturali, contribuendo al miglioramento ambientale e paesaggistico, essi vengono finanziati con contributi pubblici ${ }^{6}$, oppure attraverso interventi diretti dell'ente parco.

Dei 25.000 ettari di colture ad alto reddito, circa il $50 \%$ ha instaurato con l'area protetta un rapporto di cooperazione, che ha permesso la reintroduzione della rotazione colturale, il reimpianto di filari alberati, la costituzione di piccoli nuclei boscati in aree non coltivate, oppure la conservazione delle marcite (circa 200 ettari) e di alcune zone di brughiera.

Sono state operate anche scelte fondamentali per la fruizione controllata del fiume, ma soprattutto è iniziata una politica di miglioramento degli sistemi insediativi, che permette agli abitanti del parco di poter trarre vantaggi dalla presenza dell'area protetta.

Gli interventi attuati dall'ente parco hanno la capacità di produrre effetti sostanziali e programmati sul paesaggio, ed è proprio questa la finalità ultima di tutto il meccanismo di gestione che, attraverso operazioni specifiche in ognuno dei settori coinvolti, riesce a condizionare ed indirizzare i processi di trasformazione che generano paesaggio all'interno dell'area protetta. Attualmente il Parco è fortemente impegnato a mitigare gli interventi infrastrutturali programmati che interessano l'area protetta (tracciato del treno ad alta velocità BolognaMilano, ampliamento dell'autostrada Milano-Torino, potenziamento dell'aeroporto intercontinentale della Malpensa).

Questi interventi, oltre a creare forti impatti sull'ambiente naturale e sul paesaggio, pongono serie ipoteche sulla qualità della vita della popolazione insediata nell'area. I forti interessi economici che gravitano in questo contesto metropolitano rendono particolarmente difficile il raggiungimento dell'equilibrio fra processi di trasformazione e tutela delle risorse naturali.

\section{RIFERIMENTI BIBLIOGRAFICI}

Brenga Monica, Piste ciclabili: un modello compatibile, relazione al convegno Paradeisos, Monza 1999. Bocca Umberto e Magna CARlo (a cura di), Ticino: natura, arte, storia, agricoltura, itinerari del Parco Lombardo, IEVVE edizioni, 2002.

FurlanetTo Dario, Acque agitate nel Parco del Ticino, "Parchi”, 15, giugno 1995.

Parco Ticino, rivista del Consorzio Parco regionale Lombardo della Valle del Ticino, 1998.

FURLANETTO DARIO, Reti ecologiche: azioni locali di gestione territoriale per la conservazione dell'ambiente, relazione al convegno internazionale di Gargnano, 12/13 ottobre 2000.

Malcevschi Sergio, La rete ecologica della Provincia di Milano a supporto della pianificazione di area vasta, in DimagGio C. e GHIRINGHELLI R. (a cura di), Reti ecologiche in aree urbanizzate, Franco Angeli Editore, Milano 1999.

\footnotetext{
${ }^{6} \mathrm{Nel}$ settore agricolo l'Unione Europea arriva a finanziare interventi fino al $49 \%$ delle spese sostenute.
} 
Regione Lombardia, Le aree protette della Regione Lombardia, cd rom, 2000.

REGIONE LOMBARDia, Le reti ecologiche dell'alta pianura lombarda, cd rom, 2000.

SAINo LuCiano, Relazione tenuta al seminario del Dottorato di Ricerca in Progettazione Paesistica (Firenze) il 26/11/1997.

siti web: <www.parcoticino.it>

\section{RIFERIMENTI ICONOGRAFICI}

Figura 1: sito internet <www.parcoticino.it>.

Figure 2-6 e 8-10: per gentile concessione del Consorzio Parco Lombardo della Valle del Ticino (archivio foto del parco).

Figura 7: Regione Lombardia, Parchi Naturali Parchi Urbani, catalogo mostra.

\section{SCHEDA DI PROGETTO}

Tipologia di area protetta:

Istituzione:

Dati dimensionali dell'area protetta:

Residenti all'interno dell'area:

Enti locali interessati:

Strumento di pianificazione:

Progettisti del Piano:

Ufficio di Piano:

Consulente giuridico:

Consulenti di settore:

Revisione del PTC:
Parco Regionale

Legge Regionale Lombarda n. 2 del 9 gennaio 1974 (su proposta di iniziativa popolare)

90.000 ettari, di cui 16\% di aree boscate fluviali, $72 \%$ di aree agricole, $11 \%$ di aree urbanizzate

420.000 abitanti

Province di Varese, Milano e Pavia; 46 Comuni compresi nell'intero territorio amministrato

Piano Territoriale di Coordinamento, approvato con Legge Regionale n. 33 del 22 marzo 1980. Successivamente revisionato, l'ultima variante è in fase di attesa dell'approvazione in Regione Lombardia

Arch. Maurice Cerasi, Arch. Paolo Favole, Arch. Empio Malara, Arch. Roberto Rizzini

Arch. Maurizio Colombo, Arch. Antonio De Leo, Arch. Chiara De Rosa, Arch. Marco Engei, Arch. Vito Pasi, Arch. Giampiero Spinelli

Avv. Achille Cutrera

Università di Pavia - Istituto di Botanica - Istituto di Zoologia, Dott. Giovanni Braga (geologia-geomorfologia), Dott. Luigi Chilò (agricoltura), Ing. Ugo Raffa (idraulica fluviale)

a cura degli uffici del Parco 


\title{
Sistema del Parchi della Val di Cornia in Toscana
}

\author{
Sabrina Tozzini
}

\section{IL CONTESTO}

La Val di Cornia occupa la parte più settentrionale della Maremma Toscana, quella gestita dalla provincia di Livorno e, partendo dal massiccio del monte Calvi, nelle colline Metallifere, si protrae da est a ovest verso il rilievo di Piombino. Nell'ultimo tratto attraversa una vasta e fertile pianura, consolidata dalle bonifiche leopoldine nella prima metà dell'Ottocento, dove il fiume Cornia si articola in una serie di fossi e canali che scandiscono geometricamente le divisioni fondiarie tra coltivazioni orticole, seminativi e residui incolti acquitrinosi, sfociando infine a sud, nella zona della Sterpaia, dove il paesaggio, a testimonianza del proprio passato, conserva ancora quasi integralmente $i$ caratteri della foresta umida maremmana con farnie, frassini, lecci, sughere,

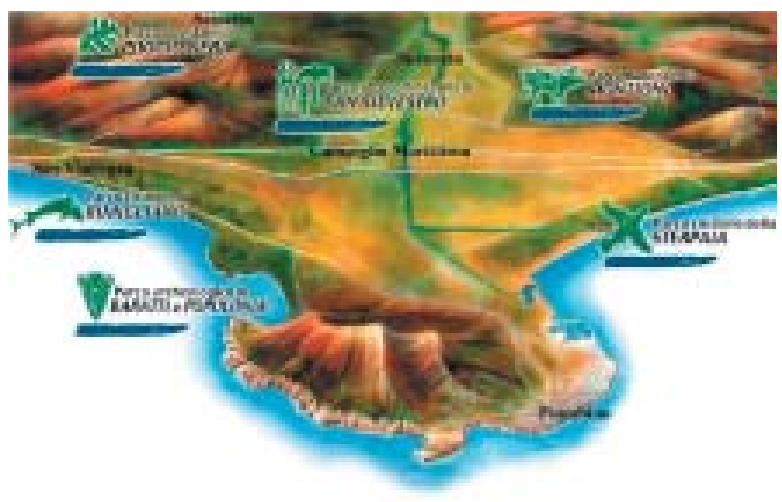

Figura 1 - La Val di Cornia e l'insieme dei parchi. mentre a nord, tra San Vincenzo e Baratti il confine tra terra e mare resta definito dalle dune di Rimigliano, ricoperte di pineta e macchia mediterranea.

Motivo caratteristico e costante della costa Tirrenica da La Spezia ai Campi Flegrei è l'alternanza di promontori rocciosi a picco sul mare e di lunghe spiagge sabbiose che li collegano tra loro con andamento concavo. In Toscana tali promontori sono strettamente imparentati con le isole dell'arcipelago toscano e non solo da un punto di vista geologico, ma anche culturale: spesso essi sono stati isole, ed alcuni, come Piombino ed Argentario, solo in tempi storici sono stati saldati definitivamente alla terra ferma.

Questi rilievi costieri sono stati nei secoli luoghi deputati all'insediamento umano per la loro altitudine, che permetteva di sfuggire dall'aria malsana delle paludi e, al contempo, di godere delle ricchezze naturali che il territorio offriva, e le tracce archeologiche ci mostrano come questi fossero frequentati già dalle popolazioni nomadi preistoriche.

Il promontorio di Piombino fu scelto dagli Etruschi per la fondazione della loro unica città costiera: Populonia. Affacciato sul Mar Tirreno e l'isola d'Elba l'insediamento godeva di una visuale completa anche sulla pianura retrostante fino alle colline Metallifere e poteva controllare la costa a perdita d'occhio, mentre il piccolo golfo a nord, quello di Baratti dove si trovava anche la loro necropoli più importante, offriva riparo alle imbarcazioni ed era sede della fiorente industria metallurgica etrusca. Già allora infatti il territorio della Val di Cornia aveva mostrato la sua vocazione metallifera, non solo per il materiale ferroso importato dall'Elba, ma anche per quello estratto e lavorato in loco, come nelle miniere di Rocca S. Silvestro che fruttavano metalli preziosi come rame ed argento, ma anche piombo, stagno e pregiati calcari da costruzione.

Era l'inizio di una sintonia tra uomo e topos destinata a durare nei secoli, di un equilibrio che, pur con alterne vicende, ha portato alla attuale consapevolezza 
delle comunità locali riguardo all'unitarietà del paesaggio Val di Cornia anche al di là delle divisioni territoriali.

\section{GLI OBIETTIVI}

In un recente seminario tenuto all'Università di Firenze, riprendendo le raccomandazioni espresse dalla conferenza di Rio del 1992 per una territorializzazione delle politiche ambientali, il Prof. Roberto Gambino ${ }^{1}$ ha sottolineato come l'unica possibilità di far funzionare un sistema di aree protette sia quella di inserirle in una rete costituita non tanto e non solo dai parchi stessi, ma da tutta una serie di funzioni e di infrastrutture che li valorizzino, accompagnando l'istanza di conservazione con quella di "comunicazione sociale", che pone i parchi come "metafora vivente" della domanda diffusa di senso di identità e di appartenenza.

La Val di Cornia, pur nella propria ridotta dimensione, sta rappresentando un interessante laboratorio per la declinazione del paradigma paesistico ambientale integrato in senso locale. Le sei aree protette che ne fanno parte si caratterizzano per i differenti valori al loro interno, antropologici e naturalistici, e possono concretamente mirare a diventare punti di eccellenza per la comprensione dei rapporti uomo-territorio, testimoni fondamentali di un equilibrio paesaggistico che non è solo quello che essi contengono all'interno, ma è soprattutto quello del sistema in cui si inseriscono.

Se infatti in passato è sembrato sufficiente demandare alle aree protette la conservazione dei valori paesaggistici e al di fuori di esse ogni devastazione diventava lecita, oggi la comunità scientifica attribuisce ai parchi principalmente una funzione educativa, quasi didascalica, per mettere il visitatore in condizione di apprezzare quegli stessi valori anche all'esterno, anche in paesaggi che hanno subito maggiori o più gravi trasformazioni, mentre l'attenzione alla gestione delle risorse e l'analisi approfondita delle argomentazioni del processo di sviluppo devono diventare pratica comune in ogni politica territoriale, senza distinzione tra dentro e fuori dell'area protetta.

L'esperienza dalla Val di Cornia mira al conseguimento di un'integrazione tra parchi e con il territorio, abbinando insieme riserve naturalistiche aperte gratuitamente al pubblico ed ecomusei capaci di generare risorse economiche che costituiscono un fondo comune per la protezione anche degli ambienti a minor richiamo turistico. Il complesso dei parchi, la cui completa realizzazione

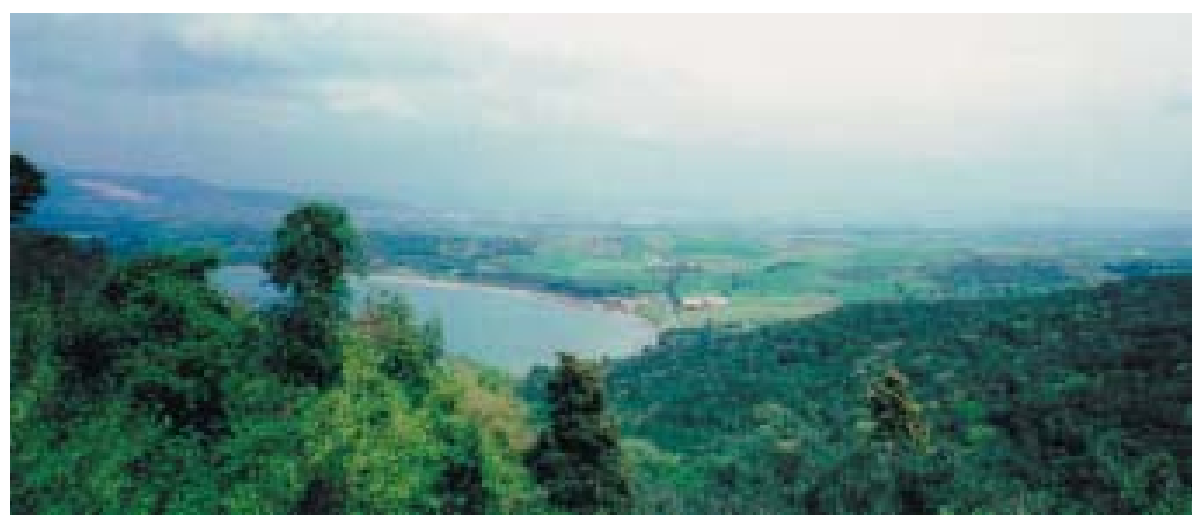

Figura 2 - I boschi e la piana di Baratti.

${ }^{1}$ Roberto Gambino, "Politiche dei Parchi e Pianificazione del territorio", Seminario del 10 aprile 2002 presso il Corso di Dottorato in Urbanistica dell'Università di Firenze. 


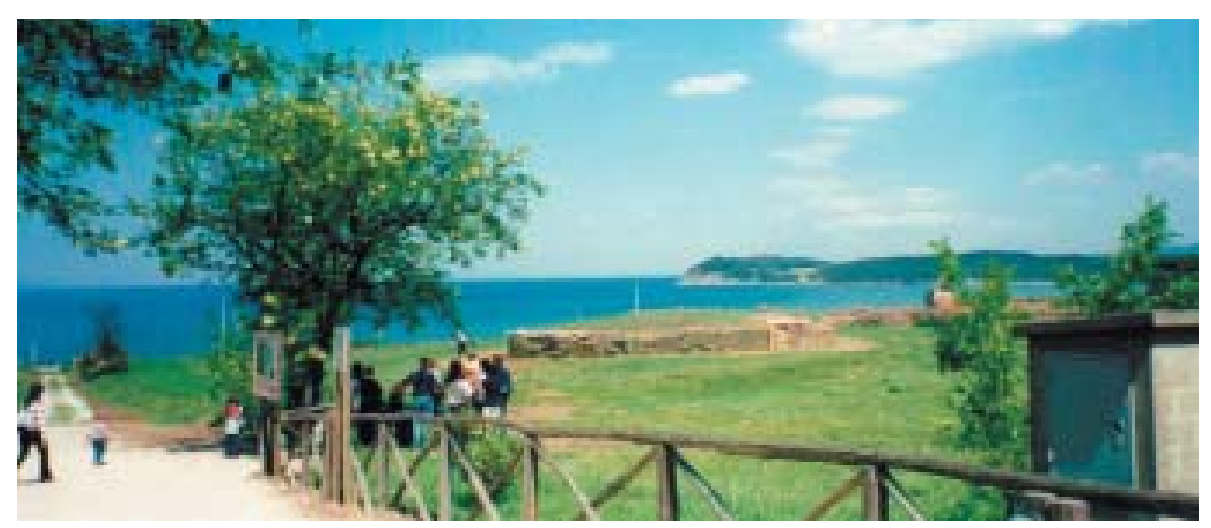

Figura 3 - Parco Archeologico di Baratti e Populonia: ingresso alla necropoli di San Cerbone.

è ancora in corso, si prefigge dunque lo scopo dell'autosostentamento, ma anche di una forte correlazione con il proprio territorio, attraverso da una parte convenzioni con le strutture ricettive locali esterne ai parchi e dall'altra accordando, anche nei casi dei parchi con accesso regolato da biglietteria, alla fruizione pubblica gratuita l'uso di almeno parte delle aree di ristoro, sosta, documentazione.

\section{LA METODOLOGIA}

A partire dagli anni Sessanta il territorio della Maremma settentrionale è stato oggetto di interessanti esperienze di pianificazione coordinata: i comuni della fascia costiera compresa tra i due centri industriali di Rosignano Solvay e di Piombino (Bibbona, Castagneto Carducci, Cecina, San Vincenzo e, alle spalle di questi, senza sbocco al mare ma forte di un notevole patrimonio naturale, Sassetta) sentirono la necessità di definire insieme i lineamenti di uno sviluppo futuro che li vedeva stretti tra i due poli, chimico e metallurgico, e pressati da aspettative turistiche non facilmente controllabili ${ }^{2}$. L'operazione ${ }^{3}$ scelse la strada di cinque Piani Regolatori Generali indipendenti, ma coordinati tra loro e disegnò un sistema integrato mare collina che privilegiava i rapporti tra costa ed entroterra e salvaguardava le risorse boschive, posizionando gli insediamenti turistici alle spalle delle pinete costiere oppure a mezza collina. Furono destinati a parco naturale le aree di dune, macchia mediterranea e pinete tra Donoratico e Marina di Bibbona e la parte sud del comune di San Vincenzo composta sia dalla formazione boschiva costiera di Rimigliano sia dalle aree agricole retrostanti. L'esperienza ebbe a confrontarsi con le pressioni degli interessi imprenditoriali che avevano portato all'occupazione di molte pinete con strutture di campeggi e villaggi turistici, che avrebbero portato alle edificazioni al Forte di Bibbona e nella parte nord di Rimigliano entro la zona classificata parco, ma fu comunque significativa ed incisiva, anche considerato gli strumenti legislativi che i progettisti ebbero a disposizione.

\footnotetext{
${ }^{2}$ Apparteneva al decennio precedente l'esperienza dell'urbanizzazione della pineta di Donoratico, promossa dal proprietario, il conte Gaddo della Gherardesca, in modo assolutamente indipendente dalla volontà del Comune di Castagneto Carducci, progettata da Giancarlo De Carlo e realizzata dopo alterne vicende in modi completamente difformi dal progetto iniziale, tanto da indurre il progettista alla famosa auto critica durante il I Seminario di Italia Nostra nel 1963 con la denuncia del "fenomeno della copertura professionale delle grandi operazioni di trasformazione che si compiono sul territorio più pregiato".

${ }^{3}$ Affidata ai progettisti L. Benevolo, L. Bortolotti, L. Gazzola (entrato a far parte del gruppo in un secondo tempo), V. Giorgini (dimessosi prima del termine del lavoro), T. Giuralongo, I. Insolera, C. Melograni.
} 
Successivamente nel 1975, sulla scia di tali vicende, i comuni di Campiglia, Piombino, San Vincenzo e Suvereto incaricarono un analogo gruppo di tecnici ${ }^{4} \mathrm{di}$ revisionare e aggiornare in modo coordinato i propri Piani Regolatori Generali.

Erano gli anni in cui per Piombino sembrava profilarsi un futuro di polo siderurgico d'importanza nazionale, e le comunità del luogo sentivano fortemente la necessità di un'organizzazione comune che potesse gestire i futuri sviluppi. Forse le aspettative economiche che si prospettavano furono la molla che spinse il consenso popolare verso la protezione del patrimonio ambientale e culturale ${ }^{5}$ con un processo non frequentemente riscontrabile durante gli anni del boom edilizio e che appare ancora più evidente confrontando lo spregiudicato uso delle risorse naturali operato da comuni limitrofi; proprio l'illusoria prospettiva di un'economia stabile di tipo industriale ha paradossalmente permesso la riconversione, certo ancora in itinere, verso un turismo ambientale e culturale di qualità.

Il lavoro di pianificazione coordinato aveva l'innegabile vantaggio di poter cogliere l'insieme territoriale da un punto di vista privilegiato, svincolato da confini comunali troppo ristretti, per poter poi progettare in modo razionale, individuando così un sistema integrato di parchi naturali ed archeologici. La logica dell'intervento si inseriva comunque nei desiderata locali, come dimostrano le successive riconferme di quelle scelte.

\section{GLI ESITI}

Nel 1993 riunitisi in conferenza, i comuni istituirono la Parchi Val di Cornia S.p.a., società mista pubblico e privato con prevalenza di capitale pubblico ${ }^{6}$. Il progetto si è avvalso dei finanziamenti dell'Unione Europea, dei Comuni, di privati e della collaborazione di diverse Università, Istituti di Ricerca, Soprintendenze e Ministeri ed è coordinata dal presidente Massimo Zucconi.

I parchi che le furono affidati furono sei, taluni già esistenti, altri ancora da organizzare:

- Il Parco archeominerario di San Silvestro nel comune di Campiglia Marittima

- Il Parco archeologico di Baratti-Populonia nel comune di Piombino

- Il Parco naturalistico costiero della Sterpaia nel comune di Piombino

- Il Parco naturalistico costiero di Rimigliano nel comune di San Vincenzo

- Il Parco naturalistico di Montoni, prevalentemente nel comune di Suvereto

- Il Parco naturalistico forestale di Poggio Neri nel comune di Sassetta

La politica che aveva portato a questa organizzazione perseguiva una diversificazione economica che tenesse conto dei valori ambientali e culturali presenti prevedendo di strutturare e rendere operativo ogni parco dando la precedenza ai

${ }^{4}$ G. Benucci, L. Gazzola, G. Menichetti, D. Parducci con C. Melograni coordinatore.

${ }^{5}$ Già il Comune di Piombino, dopo l'iniziale inserimento nel 1967 di un milione ottocentomila metri cubi di insediamenti turistici e residenziali ed il loro successivo stralcio, aveva dovuto controllare le mire della società "Populonia Italica" che premeva per ripetere nel golfo di Baratti (ed esattamente sopra l'area archeologica che comunque era in massima parte già portata alla luce) la spregiudicata impresa che trenta chilometri più a sud aveva visto nascere l'insediamento turistico di Punta Ala. Ancora nel Comune di Piombino il piano Amati-Codellia, approvato in via definitiva dalle Regione Toscana nel 1973, classificava come "verde arborato di rispetto" il bosco litoraneo della Sterpaia.

Erano state inoltre, se non respinte almeno limitate dal Comune di Campiglia le richieste per aprire nella zona di Rocca San Silvestro miniere a cielo aperto, che avrebbero reso di nuovo conveniente l'escavazione dei minerali presenti, ma che con la loro invasiva presenza avrebbero ulteriormente distrutto il paesaggio del luogo e cancellato le tracce archeologiche che il sito ancora conserva.

${ }^{6}$ Il vincolo di "prevalente capitale pubblico" viene inserito nel 1998, per consentire l'affidamento diretto alla società delle aree archeologiche di Baratti-Populonia, di proprietà del Ministero per i Beni Culturali ed Ambientali. 
due casi di maggior richiamo, San Silvestro e Populonia, che stanno permettendo anche, grazie al biglietto d'ingresso, di far fronte non solo alle loro stesse necessità, ma anche a quelle degli altri parchi aperti gratuitamente al pubblico.

Si sta attuando una gestione strategica coordinata con i comuni stessi per la valutazione dei problemi a contorno dei parchi, i quali, soprattutto in periodo estivo, sono soggetti a flussi di visitatori controllabili solo attraverso la collaborazione dei servizi comunali. Il legame tra i parchi della Val di Cornia ed il fenomeno turismo è sicuramente molto profondo, e proprio su questo si basa la possibilità di autofinanziamento dell'Ente. Il tentativo di "ridistribuire" nell'arco dell'anno le presenze non è sempre facilmente attuabile, specialmente nei casi di Rimigliano e della Sterpaia che si affacciano direttamente sul mare e sono meta di balneazione molto apprezzata. In quest'ottica si collocano molti degli interventi esterni, come i collegamenti con navetta da varie località, strutture di parcheggio, punti informativi. Esiste inoltre una politica educativa e formativa a vari livelli: dalla collaborazione con le scuole locali all'archeologia sperimentale negli spazi attrezzati, ai musei interni ed esterni dedicati a vari tematismi.

\section{Il Parco archeominerario di San Silvestro}

Il primo episodio ad essere inaugurato è stato nel 1996 il Parco Archeominerario di San Silvestro, dove fin dal 1983 erano iniziati gli scavi ad opera del Prof. Riccardo Francovich del dipartimento di archeologia medioevale dell'Università di Siena.

Posta in una zona tanto ricca di minerali da essere sfruttata fin dai tempi preistorici prima, ed etruschi e romani poi, la Rocca di San Silvestro venne fondata nell'alto medioevo per iniziativa baronale e fu dotata di un'organizzazione completamente diversa da altri esempi di città di fondazione coeve: non era, come spesso accade nella Toscana del tempo, basata su un'economia agricola, ma sull'estrazione e lavorazione dei metalli. I capitali, mossi dalle famiglie Della Gherardesca e Della Rocca che facevano parte del ceto dirigente pisano, erano stati precisamente indirizzati alla produzione di metalli da conio come rame, argento, piombo e, caso praticamente unico in Italia, addirittura stagno, mentre il ferro occupava una posizione marginale nell'economia produttiva e, come dimostrano i ritrovamenti archeologici, veniva principalmente impiegato per la costruzione di attrezzi destinati ad un uso locale. Estremamente interessante è l'organizzazione urbanistica, che mostra ancora chiaramente il controllo operato dal potere feudale, ponendo le lavorazioni dei metalli da conio all'interno delle mura del castrum, mentre quella del ferro, pur sempre sotto stretto controllo, al di fuori di esse 7 . È stato sottolineato dal Prof. Francovich che, non solo nel caso specifico, ma nella maggior parte dei siti analoghi, per i primi destinati alla produzione di moneta, pregiatissimi, non esista un locale destinato alla forgia,: probabilmente, appunto per l'alto valore di tali metalli il signore del castello riservava questo compito alla propria città di residenza, dove il suo controllo poteva essere ancora più stretto.

\footnotetext{
7 Il forno da ferro utilizzato a Rocca San Silvestro era di tipo molto semplice: un incavo nel terreno di forma ovale, con una sovrastruttura di protezione in pietre ed argilla ed una apertura frontale, senza alcuna coibentazione termica. Pur essendo così semplice rientra nella comune tipologia dei Fabri pisani: operai specializzati che con una migrazione stagionale verso le coste di Piombino e Populonia collocavano le loro lavorazioni dove era possibile avere a disposizione una sufficiente quantità di legname e operavano la prima estrazione del ferro elbano prima di inviarlo a Pisa per la forgia finale. Il pressoché completo disboscamento dell'isola d'Elba aveva infatti reso necessaria quest'organizzazione, di cui restano numerose tracce archeologiche sul promontorio di Piombino.
}

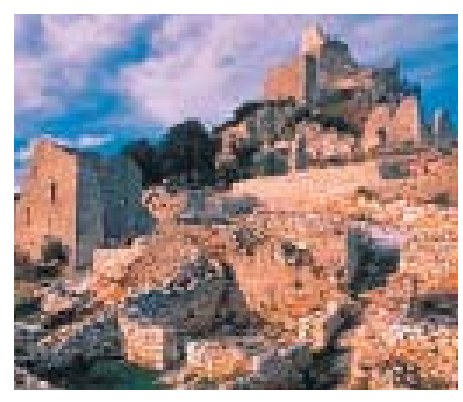

Figura 4 - Il sito archeologico di Rocca San Silvestro. 
La crisi dell'insediamento cominciò con l'avvento delle nuove tecnologie che utilizzavano la forza idrica per la produzione e che resero molto più redditizie le lavorazioni in prossimità dei corsi d'acqua: per Rocca San Silvestro, priva di risorse idriche in loco, fu l'inizio di un lento declino che nel XVI secolo la vide completamente abbandonata, mentre probabilmente tra i suoi boschi si svolgeva ancora qualche lavorazione marginale, destinata all'uso della comunità di Campiglia Marittima.

Dopo più di duecento anni dall'abbandono dell'abitato, sull'inizio del XIX secolo, Rocca San Silvestro attirò di nuovo l'attenzione di geologi ed ingegneri inviati sul posto da società minerarie francesi prima e inglesi poi, lasciando, nelle loro relazioni tecniche, preziosa testimonianza sulla mineralizzazione e sulle lavorazioni antiche, in seguito largamente cancellate dai loro stessi saggi ed escavazioni, mentre le strutture e le gallerie di questo periodo sono ancora visitabili all'interno del parco.

L'impronta più interessante da un punto di vista archeoindustriale è quella lasciata dalla società inglese "Etruscan Copper Estate Mines": furono aperti cinque pozzi di estrazione collegati tra loro e con le strutture di lavorazione da una ferrovia a scartamento ridotto, costruiti imponenti edifici industriali, recuperate e restaurate strutture antiche, ma, nonostante il cospicuo investimento effettuato, tutto fu abbandonato dopo pochi anni.

Il progetto paesaggistico del parco, approntato dal gruppo formato da J. Buchanan (paesaggista), R. Francovich (archeologo) e L. Greppi (architetto che si è occupato della ristrutturazione e del riuso degli edifici presenti) nel 1993, ha concorso principalmente a valorizzare gli aspetti storici e scientifici del parco, individuando percorsi a tema integrati tra natura e reperti archeologici e percorribili da diversi tipi di utenza.

"La prima considerazione che abbiamo fatto, pensando alla progettazione di un parco, è stata quella di affermare la necessità di superare i limiti spaziali e cronologici della ricerca specifica sul castello di Rocca San Silvestro e di tener conto viceversa del vasto potenziale presente nell'intero territorio campigliese, valorizzandolo nel suo complesso e sottolineando in particolare quegli aspetti caratterizzati in termini di lunga durata delle attività estrattíve e metallurgiche. Si è deciso quindi di passare dalla scala puntiforme della musealizzazione di uno scavo alla dimensione di un parco comprensoriale (...) i piani dei parchi della Valle della Cornia, che Italo Insolera, con grande sensibilità ed efficacia, aveva predisposto negli anni precedenti per conto delle amministrazioni locali, erano assolutamente compatibili con il nostro progetto e, per certi aspetti, ne prevedevano gli sviluppi. La seconda considerazione che ci ha guidato è stata quella relativa all'opportunità di «importare» in Italia, che ancora nella seconda metà degli anni Ottanta ne era priva, l'esperienza dei parchi archeo-minerari che molti paesi europei avevano realizzato coniugando con successo le esigenze dello sfruttamento delle risorse e la salvaguardia dei valori ambientali, storici e naturalistici. In questi tipi di strutture infatti i centri d'interesse non rispondono più a meri principi classíficatori delle forme del paesaggio o degli oggetti e delle tecniche, ma si tende piuttosto a, attraverso varie forme di organizzazione espositiva, la complessità delle pratiche scientifiche o tecnologiche, con gli assetti sociali e naturali, con le logiche imprenditoriali e le culture tradizionali, con le «vocazioni» ambientali e le scelte operate nella lunga durata" ${ }^{8}$.

La fase preparatoria del lavoro ha operato una vasta ricognizione su analoghe esperienze europee ${ }^{9}$ individuando in alcune di esse il limite di un troppo stretto

\footnotetext{
${ }^{8}$ RicCARDO FrANCOVICH, Le ragioni di un parco alle radici dell'archeologia mineraria: le miniere di Campiglia Marittima nelle pagine dei naturalisti e dei geologi dell'Ottocento, Marsilio, Venezia 1994, pag. 15.

${ }^{9}$ I centri museali e i parchi minerari visitati sono stati: gli austriaci Hallstatt e Hallaim, Bex, in Svizzera, Ironbridge Museum in Inghilterra, il Deutsches Bergbau Museum in Germania, Le Creusot in
} 
legame tra tecnica mineraria e conservazione, che aveva portato a trascurare gli aspetti di continuità storica con il territorio. L'analisi storico archivistica ed archeologica hanno evidenziato la presenza sul posto di lavorazioni minerarie con una prima inimmaginata continuità nel tempo, dalla preistoria agli anni Settanta del secolo scorso, fatto in realtà obliato dalla comunità locale. Scrive Italo Insolera "le miniere nel patrimonio culturale di Campiglia sono sempre state qualcosa di esterno, di straniero: hanno sempre avuto dei padroni lontani (...) a Campiglia hanno sempre chiesto di fornire la forza lavoro di base. È logico quindi che la memoria delle miniere sia una memoria di lotte contro i padroni, un patrimonio storico eccezionale, ma ben diversa da quella che ha con la terra" ${ }^{10}$.

Obbiettivo del parco è proteggere e conservare quest'area d'importanza storica contro le minacciose attività

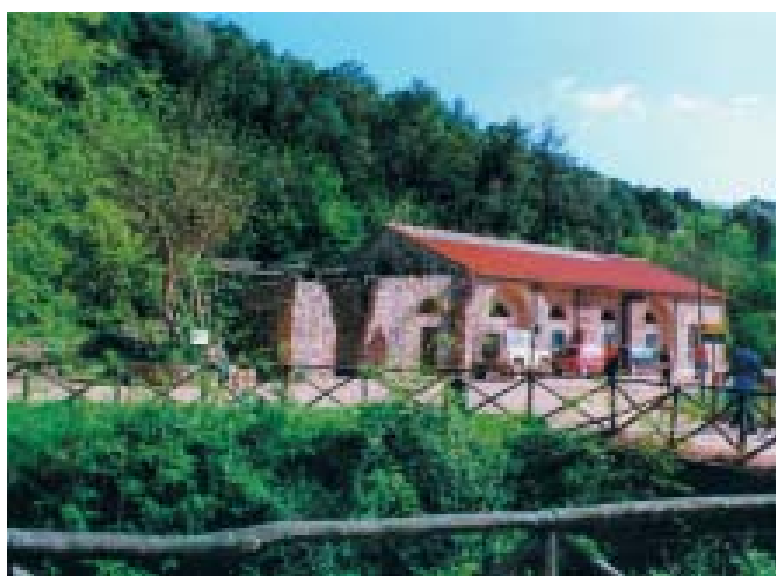

Figura 5 - Il ristoro del Parco di Rocca San Silvestro, l'edificio industriale è stato restaurato dall'architetto L. Greppi. estrattive limitrofe, ed anche interne, come nel caso della cava di calcare di Monte Calvi, ancora in attività nel cuore del parco ${ }^{11}$, che con le loro produzioni di basso valore (il calcare estratto viene in massima parte impiegato per ricavarne calce) hanno provocato distruzioni e danni irreversibili. La considerazione motrice del progetto reputa necessario rendere questo patrimonio da proteggere una fonte di occupazione e di ricchezza per il territorio, in modo da generare un conflitto virtuoso che suggerisca come preferibile l'investimento nel turismo ecocompatibile.

Il piano del parco e stato molto attento alla conservazione di quelle testimonianze che ancora si celano sotto la coltre verde dei boschi, per permettere future indagini ed approfondimenti. Il filo conduttore del progetto di ricreare un rapporto organico tra le scoperte operate dall'archeologia e con quanto conosciuto attraverso le ricerche storiche e bibliografiche tradizionali ed il paesaggio, con l'individuazione di quei parametri che, reinseriti in contesti compatibili di uso moderno, portassero valorizzazione dell'identità del luogo.

Messe in sicurezza, alcune delle gallerie delle miniere sono diventate un'interessante excursus didattico dalle coltivazioni minerarie etrusche all'archeologia industriale delle società inglesi.

Di grande pregio ed interesse è anche la vegetazione che nel parco vede rappresentate le tre fasi di sviluppo della Macchia Mediterranea: dalla gariga, alla macchia, alla foresta. La forte attività di deforestazione a vantaggio delle lavorazioni ha lasciato tracce anche sull'aspetto morfologico del territorio, con ampie zone che hanno subito forti dilavamenti, mentre i saggi minerari, gli scarti, le trincee sono ancora chiaramente leggibili nella distribuzione della gariga e dei suoli nudi. I camminamenti naturalistici permettono ed agevolano una lettura del paesaggio come risultato dell'interazione uomo natura, ma è possibile anche usufruire del servizio navetta verso la Rocca oppure della recuperata ferrovia a scartamento ridotto. Negli edifici paleoindustriali sono state alloggiate le strutture di accoglienza del parco, musei, ristori, laboratori per l'archeologia sperimentale. La parte di progetto fin qui realizzata è solo una minima quota di quanto previsto nel Masterplan, ma, seguendo un condivisibile disegno strategico, si è preferito destinare i primi investimenti europei alla gestione e conserva-

Francia, Schwaz in Tirolo, Huttemberg, Lolling e Bleiberg in Carinzia, Eísenerz in Stiria. Ancora più importante la cooperazione con l'analogo parco di Brandes sull'Alpes d'Huez presso Grenoble.

${ }^{10}$ Italo Insolera, Il parco minerario della Val di Cornia Firenze in RoberTo Mancini (a cura di), Campiglia Marittima. Percorsi Storici e turistici, Tosca, San Giovanni Valdarno 1990, pag 24.

11 L'estrazione del calcare dovrebbe concludersi ormai prossimamente, con l'esaurimento della licenza di coltivazione. 
zione dei beni culturali ed ambientali, lasciando le pur interessanti previsioni accessorie ad un momento successivo. Tra gli interventi futuri sono stati previsti percorsi trekking di interesse anche specialistico, come quello per rocciatori sulla cava di calcare microcristallino del monte Calvi, ma anche servizi di trasporto diversi, tra cui funicolare e seggiovia, che l'architetto Buchanan progetta di recuperare dalle preesistenti vie di trasporto industriali, ricostruzioni storiche educative, porte d'accesso al parco più comode e consone.

\section{Il Parco archeologico di Baratti}

Fu la ripresa delle attività minerarie attorno agli anni Venti a cambiare il volto di Populonia. Chi visita adesso il Golfo di Baratti e la sua Necropoli, forse non immagina neppure l'aspetto che aveva meno di un secolo fa. La millenaria attività metallurgica etrusca aveva prodotto una tale quantità di scorie ferrose da cancellare quasi ogni altra traccia della loro civiltà. Solo quando la tecnologia permise di recuperare il ferro scartato e i noduli furono portati indietro sull'isola d'Elba, ripercorrendo a ritroso il cammino intrapreso tremila anni fa, riemerse la necropoli.

Invano i viaggiatori dell'antichità avevano cercato tra quelle nere colline le vestigia dei suoi antichi abitanti. Strabone si meravigliò del deserto del golfo di Baratti, dove pochi metallurghi si ostinavano a far lavorare i forni

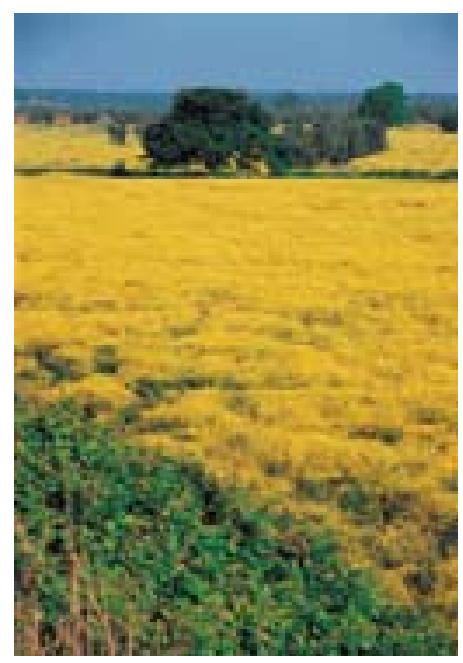

Figura 6 - Fioritura primaverile nella piana di Baratti. fusori, e quattro secoli più tardi, nel 417 d.C. arrivato a Populonia Namaziano dichiarò che questa è la prova che "anche le città possono morire". Solo nel XIX secolo cominciarono ad affiorare i reperti e nel 1832 tra le reti dei pescherecci restò impigliata una statua bronzea di circa un metro di altezza: era l'Apollo di Piombino, ora conservata al Louvre. Altri reperti vennero alla luce 50 anni dopo durante la costruzione del podere di San Cerbone, oggi centro di accoglienza del parco, e cominciarono i primi scavi clandestini dei contadini che trafugarono oggetti preziosi per rivenderli per poche lire agli antiquari. Gli scavi ufficiali cominciarono all'inizio del XX secolo e subirono un'accelerazione forzata all'arrivo delle escavatrici meccaniche delle ditte minerarie nel 1920 per contrastare i loro effetti distruttivi.

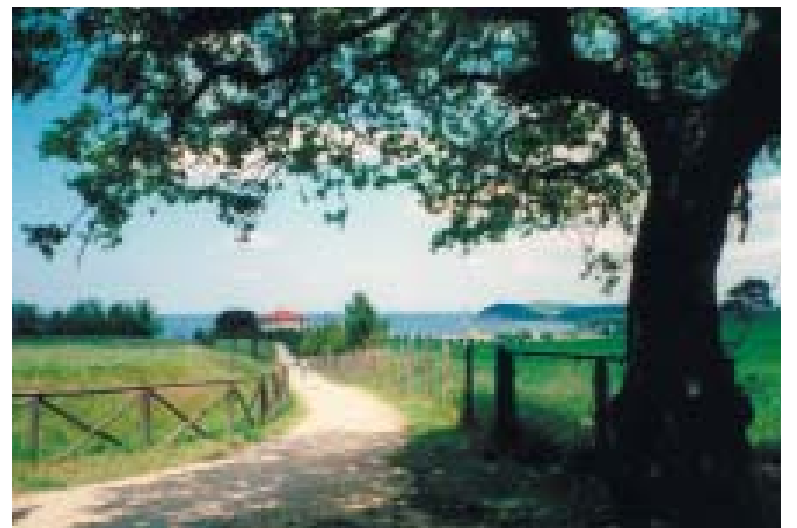

Figura 7 - Parco Archeologico di Baratti: il centro visite.
Per decenni il patrimonio archeologico di Baratti è stato protetto soltanto da un recinto attorno alle tombe di maggiore importanza e, nonostante le interessanti proposte per un ampliamento ed organizzazione dell'area parco, solo nel 1997 la Regione Toscana inserisce l'area nel programma regionale. Nel 1998 il parco viene considerevolmente ampliato, valorizzando non più soltanto gli episodi più eclatanti e famosi come la necropoli di San Cerbone, ma tutta una serie di manufatti e di strutture storiche che rappresentano ed illustrano le dinamiche territoriali che hanno portato alla creazione del paesaggio, non solo del promontorio piombinese, ma di tutta la Maremma settentrionale.

Il Centro Visita del parco ha riutilizzato l'edificio del Podere di san Cerbone, rappresentativo, con la sua solida 
forma a pianta quadrata, dell'architettura spontanea dell'appoderamento maremmano ottocentesco, a cui si giunge dopo un sentiero d'ingresso che costeggia la necropoli più antica, detta appunto di San Cerbone, lasciando che l'occhio del visitatore colga subito l'eccezionalità della posizione dei reperti, direttamente sul mare. Questa zona costituisce il nucleo antico del parco archeologico, non solo per la datazione delle tombe, ma anche per la sua precoce escavazione e si caratterizza per la notevole concentrazione di strutture imponenti in uno spazio relativamente piccolo. La delimitazione della stessa verso nord e verso est, con confini così netti dettati da strade moderne, lascia intuire subito come ancora il parco abbia un'estensione dettata più dalla burocrazia e da problemi gestionali che da un'organica riflessione scientifica, tradendo per adesso le aspettative che

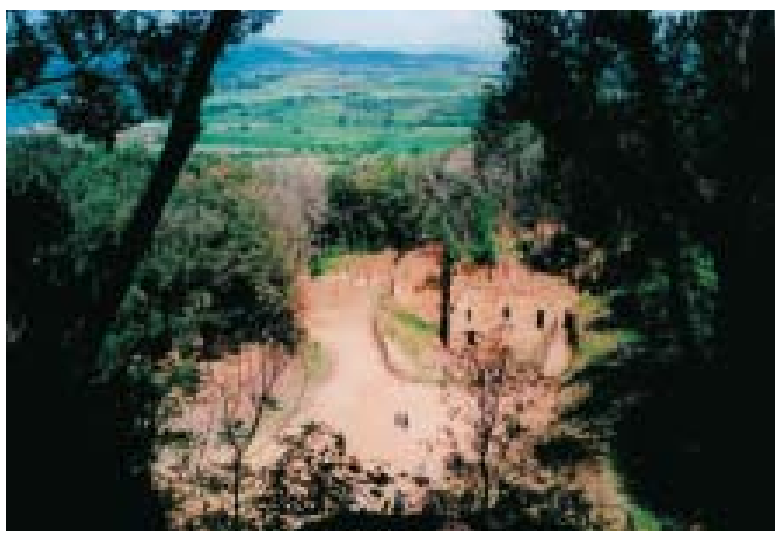

Figura 8 - Parco Archeologico di Baratti: la Necropoli delle Cave vista dal Belvedere. Italo Insolera e Antonella Romualdi ${ }^{12}$ esprimevano nel 1988, per un parco che abbracciasse il territorio fino a lambire il parco di Rimigliano a nord e la città di Piombino a sud.

Il resto del parco si presenta invece con una sistemazione più organica, ruotando attorno alla distribuzione di tre diversi itinerari, che contemplano sia valori archeologici, che storici e naturalistici.

La Via del Ferro è il sentiero che conduce attraverso gli antichi quartieri industriali della città di Populonia, qui gli operai tenevano accesi giorno e notte i forni che resero la città fiorente. Lungo il cammino incontriamo sepolcri costruiti nel VII secolo a.C. quando ancora la città era scalo per il ferro lavorato direttamente sull'Elba, mentre in seguito, a causa del totale disboscamento che per l'attività delle fucine l'isola aveva patito, le lavorazioni si trasferirono sulle coste peninsulari, coprendo lentamente anche le aree di sepoltura. Se in un primo momento gli operai dovevano scendere dall'insediamento cittadino verso i luoghi di lavoro, attorno al 540-530 a.C. il poggio della Porcareccia divenne anche quartiere di abitazione per i metallurghi, mentre Populonia, con la sua tipica disposizione su due colli, uno per i templi ed uno per le residenze, rimaneva appannaggio dei nobili.

I quartieri della Porcareccia furono a più mandate distrutti e ricostruiti, come ha dimostrato l'indagine stratigrafica, fino alla distruzione definitiva nel III secolo a.C. Anche qui i lavori di recupero delle scorie ferrose hanno profondamente inciso l'aspetto dei luoghi, nel Campo Sei, toponimo evocativo della nostra recente storia industriale, molti danni sono stati arrecati alle strutture archeologiche, interi tratti di mura della città bassa sono crollati sotto le spinte delle escavatrici, vittime di un senso storico distorto, che valutava degno di conservazione solo l'edificio eccezionale, o il manufatto utilizzabile in una esposizione museale.

La Via del Ferro è collegata attraverso un sentiero di raccordo alla Via delle Cave, che conduce attraverso la fitta macchia mediterranea all'esplorazione delle antiche coltivazioni della Panchina ${ }^{13}$, la pietra da costruzione impiegata per i sepolcri, per le mura e per gli edifici della Populonia antica e medioevale. Qui nel periodo ellenistico (IV-III secolo a.C.) gli etruschi scavarono le loro tombe ipogee, creando strette aperture nelle lisce pareti di pietra, che intimorirono con la loro aura di mistero la cultura popolare posteriore, che ebbe a chiamarle Buche

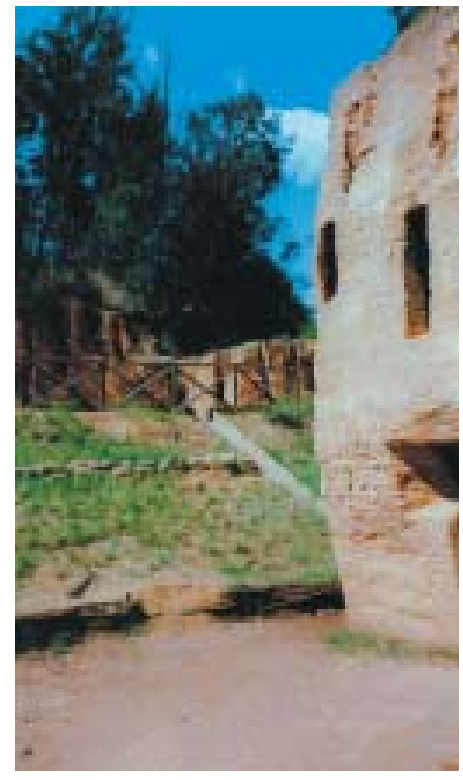

Figura 9 - La necropoli delle Cave.

\footnotetext{
${ }^{12}$ Italo Insolera e Antonella Romualdi, Il parco archeologico di Populonia in Bruna AmendoLEA, Rosanna Cazzella e LaURa Indio, I siti archeologici un problema di musealizzazione all'aperto, Multigrafica Editrice, Roma 1988, Primo seminario di studi, Roma, febbraio 1988, pagg. 76-83.

${ }^{13}$ Calcarenite: roccia calcareo arenacea.
} 
delle Fate. Ma camminando tra le ombre del bosco compatto, che caratterizza il percorso di Via delle Cave, ecco all'improvviso aprirsi la terrazza del Belvedere, ricavata sul fronte più alto del cratere della Cava delle Grotte. Da qui lo sguardo può spaziare sull'intero sistema del parco, sulla linea di costa verso nord e verso le verdi colline metallifere dove le cave aprono ancora ferite bianche, creando un momento di riflessione su come tutto ciò si sia creato in secoli di interazione tra uomo e natura, di come un paesaggio che talvolta ci sembra l'unico possibile per il proprio luogo, non sia in realtà altro che uno dei molti possibili arrivato a noi tra tanti, per una serie di piccole scelte puntiformi, compiute nel tempo da chi ha vissuto quel territorio. Il belvedere è la più bella invenzione paesaggistica del parco, per il senso di serendipity che riesce a creare nel visitatore, improvvisamente proiettato nel vivo del processo di estrazione della panchina: la roccia, protetta dalle intemperie da decine di metri di detriti del bosco e della lavorazione accumulatesi nel tempo, conserva ancora i segni dello scalpello che la lavorò e i pezzi ancora incompiuti che vi si ritrovano raccontano una tecnologia basata sul rispetto delle risorse naturali. Ė il caso del "dado", scelto come logo per le indicazioni del meeting point del parco, si tratta di un tentativo interrotto di aprire una cavità da destinare a tomba nella parete nord della cava, non secondo il concetto moderno della frantumazione, ma dell'accurato taglio della pietra da asportare per poterla poi utilizzare altrove. Sulla Cava delle Grotte si affaccia anche una terrazza destinata alla sosta, più bassa rispetto al Belvedere e quasi invisibile da questo, protetta com'è dall'ombra del bosco.

Il patrimonio archeologico, costituito dai reperti mobili ritrovati durante gli scavi sul promontorio, oppure restituiti dal mare, si trova sparso per i musei di tutto il mondo, anche ad opera degli antiquari e dei tombaroli, instancabili soprattutto nel secolo scorso. Il materiale proveniente dalla necropoli di San Cerbone si conserva in massima parte al Museo Archeologico di Firenze, mentre quello di più recente raccolta (necropoli delle Cave, quartieri metallurgici della Porcareccia, edificio romano sull'Acropoli di Populonia, ma anche acquisizioni da collezioni private) è esposto nei musei di Piombino e Populonia, recentemente istituiti e pensati anche come poli espositivi per un collegamento tra parco e città.

\section{Il Parco costiero della Sterpaia}

Localizzato lungo la costa ad est del promontorio di Piombino, il Parco della Sterpaia rappresenta una significativa testimonianza di una Maremma antica, ormai sempre meno riconoscibile a causa delle trasformazioni che ha subito negli ultimi due secoli. Questo lembo di foresta umida si è conservata nonostante le profonde metamorfosi paesaggistiche comportate nell'Ottocento dalle bonifiche di Pietro Leopoldo, il quale, capendo le potenzialità produttive sia in senso agricolo che in senso minerario della zona, intendeva, attraverso interventi di risanamento e forti investimenti, capitalizzare le risorse locali. Quella che era stata una vasta palude utilizzata pro indiviso dalle comunità limitrofe per la pesca e la raccolta di vegetazione spontanea, ma anche fonte di miasmi e malaria, divenne una redditizia pianura appoderata. La foresta della Sterpaia fu lasciata intatta e non fu mai governata a ceduo, perché essa garantiva riparo dai venti marini, proteggendo le coltivazioni. Fu invece adibita a pascolo boschivo del Podere del Vignale prima, e a riserva di caccia poi. Dopo la seconda Guerra Mondiale fu sottoposta ad un rimboschimento ad opera del Corpo Forestale dello Stato, per proteggere ulteriormente la campagna retrostante. Soggetta a periodici allagamenti la Sterpaia conserva ancora al suo interno aree umide come quella di Orti di Bottegone, riserva naturale affidata in gestione al WWF, oasi sicura per l'avifauna migratoria e stanziale. 
Nel 1962 la Sterpaia venne inclusa come bellezza naturale tra le aree vincolate ai sensi della legge del '39 n. $1497^{14}$ come "tipico esempio di foresta retrodunale, ormai raro residuo in quel settore costiero".

Negli anni Settanta si verificò sul posto un caso particolare di abuso edilizio, a cui il comune assistette quasi impotente per la mancanza di legislazione ${ }^{15}$ in materia: il proprietario operò un violento frazionamento catastale della tenuta, vendendo poi i micro lotti così ottenuti e ribattezzando la zona "Riva Verde", quasi uno slogan pubblicitario, banale ed insieme ricco di capacità evocative ed accattivanti, secondo quel tipico inquinamento toponomastico che spesso accompagna la speculazione.

Pubblicizzata come un'operazione democratica che permetteva di avere un proprio luogo di villeggiatura a

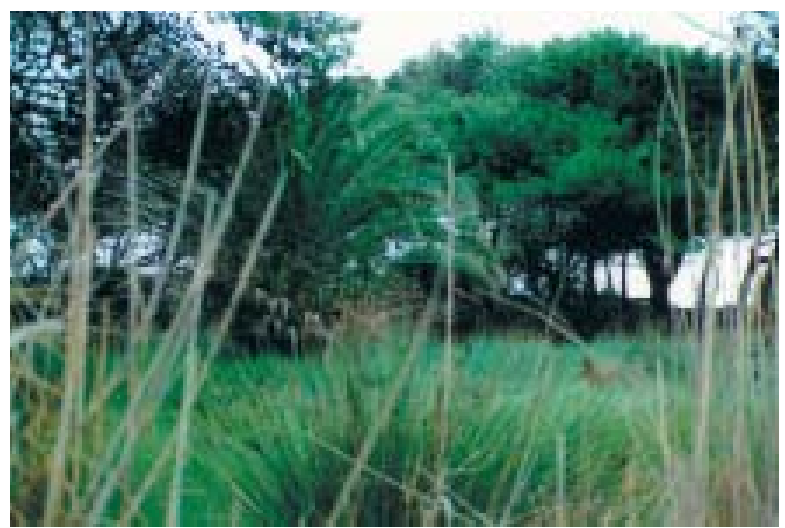

Figura 10 - Commistione di vegetazione autoctona ed alloctona nel Parco della Sterpaia. contatto con la natura anche alle famiglie con reddito medio - basso, Riva Verde generò una forte attesa edificatoria da parte dei piccoli proprietari, ed i lotti che rientravano sul mercato riscuotevano quotazioni via via crescenti, mentre le speranze venivano incoraggiate dal malcostume del condono edilizio che lo Stato Italiano varava nel frattempo per regolarizzare anche le costruzioni abusive e riannetterle ad un regime di legalità, ma che al contrario agli occhi degli abusanti pareva la scappatoia per cui avrebbero potuto legittimare in seguito il proprio operato. Fu l'inizio di un lungo contenzioso: la foresta della Sterpaia aveva ormai perso buona parte del suo sottobosco, con la costruzione di migliaia di manufatti con materiali e tecniche tra le più svariate, che nel periodo estivo ospitavano in massa circa 10.000 persone.

La lotta del Comune di Piombino contro l'abusivismo si protrasse per decenni, tra offerte di trasferimenti in altro luogo dell'insediamento, ricorsi in tribunale, pressioni di vario genere.

La prima vera vittoria del Parco della Sterpaia è stata la sua affermazione di esistenza: a metà degli anni Ottanta il Comune di Piombino aveva già avuto i ver-

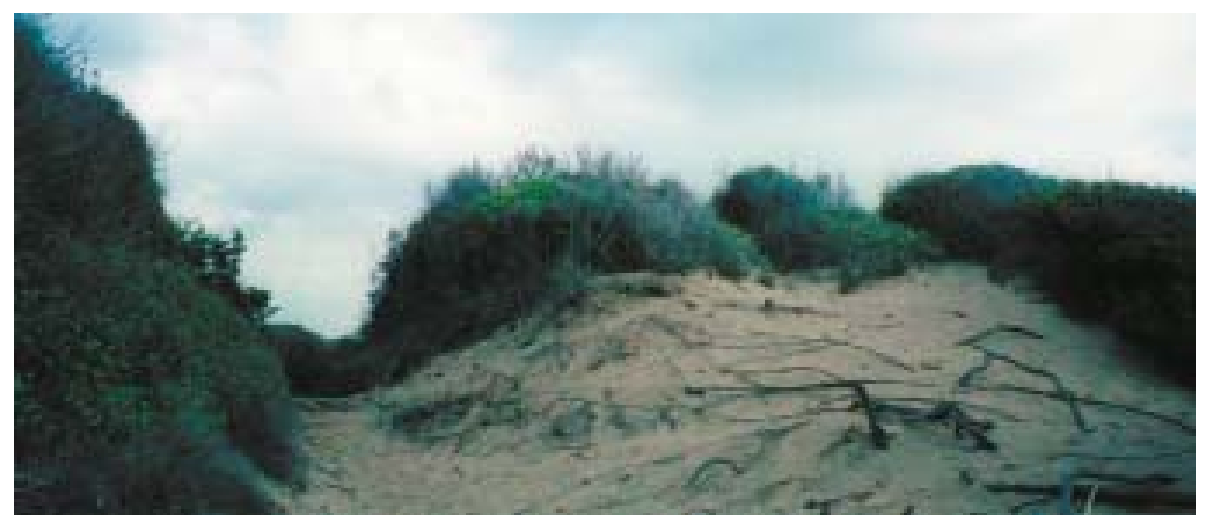

Figura 11 - Dune sabbiose nel Parco di Rimigliano.

\footnotetext{
${ }^{14}$ Legge Statale 29/06/1939 n. 1497 (Norme sulla protezione delle bellezze naturali).

${ }^{15}$ Solo con la legge Statale 29/02/1985 n. 47 (Norme in materia di controllo dell'attività urbanistico-edilizia (...)) viene previsto questo tipo di abuso. Recita l'articolo 18:

"Si ha lottizzazione abusiva di terreni a scopo edificatorio quando vengono iniziate opere che comportino trasformazione urbanistica od edilizia dei terreni stessi in violazione delle prescrizioni degli strumenti urbanistici, (...) quando tale trasformazione venga predisposta attraverso il frazionamento e la vendita, o atti equivalenti, del terreno in lotti che, per le loro caratteristiche quali la dimensione in relazione alla natura del terreno e alla sua destinazione secondo gli strumenti urbanistici, il numero, l'ubicazione o la eventuale previsione di opere di urbanizzazione ed in rapporto ad elementi riferiti agli acquirenti, denuncino in modo non equivoco la destinazione a scopo edificatorio".
} 
detti a lui favorevoli del T.A.R. ${ }^{16}$ della Toscana, intraprendendo così le prime demolizioni, nel 1993 la IV Sezione del Consiglio di Stato conferma i tali verdetti del T.A.R., alla metà degli anni Novanta sono state demolite oltre 2000 costruzioni abusive ed operati espropri al prezzo di mercato del terreno boschivo ${ }^{17}$.

Affidato alla gestione della società Val di Cornia S.p.a. il parco è stato bonificato dai manufatti edilizi abusivi, dotato di servizi igienici, parcheggi esterni, navette dai centri limitrofi, regolamentazioni per la tutela dal massiccio uso per la balneazione che lo interessa in estate. L'accesso è libero e gratuito, ma è possibile prenotare visite guidate alla scoperta del pregevole ambiente naturale, che, pur gravemente compromesso dalla pesante 'colonizzazione', mantiene ancora forti valenze paesaggistiche e scientifiche.

Il parco attende ora un accurato restauro naturalistico, che lo liberi dalle banali presenze floristiche impiantate di recente, prima che esse assumano carattere infestante compromettendo per sempre un patrimonio unico nel suo genere sulla costa toscana.

\section{Il Parco costiero di Rimigliano}

Situato sul litorale sud del territorio del comune di San Vincenzo, confina con i comuni di Piombino e di Campiglia Marittima, era già stato inserito nell'elenco delle aree protette ai sensi della legge 1939/n. 1497. Si tratta di un tombolo con dune recenti, sabbiose, ed antiche, costituite da calcareniti rossastre, ricoperto da una fitta associazione vegetale fatta di essenze tipiche della macchia mediterranea, (lecci, pini marittimi, pino d'Aleppo, sughera, erica, ginepro, mirto, lentisco, eccetera ma anche roverella e orniello) e pini domestici impiantati tra fine Ottocento e inizi Novecento perché l'attività di produzione del carbone che vi si svolgeva non depauperasse la materia prima di cui si serviva.

Sono ancora identificabili i sentieri, gli spiazzi, le fosse di combustione aperte nel bosco dai carbonai, ma anche dalle aree agricole retrostanti, nate dalle bonifica leopoldina ${ }^{18}$, separate dal bosco dalla Via della Principessa, strada vicinale agreste consolidata dall'uso veicolare recente.

La prima parte del parco è stata aperta al pubblico nel maggio del $1973 \mathrm{e}$ costituisce uno dei più vecchi esempi di area protetta comunale.

Nel piano di sistemazione erano previsti interventi per limitare la forte erosione causata dai venti marini, che, a causa delle aperture nella vegetazione normali alla linea di costa, minacciavano le essenze arboree interne al bosco. La sentieristica del parco, dedicata esclusivamente alla percorrenza pedonale, fu così in massima parte recuperata tra i percorsi storici al suo interno, ma in prossimità dell'accesso al mare deviati in modo da ottenere un'incidenza obliqua verso l'arenile e non offrire mai uno sbocco di penetrazione alle brezze salmastre. Alla base delle dune verso il mare fu messa in opera in diversi punti una palificazione in legno inclinata verso le dune stesse, perché fungesse da raccolta dei detriti di accumulo evitando che l'erosione aggredisse il tombolo alla base delle dune. Si trattava in realtà di un tentativo di rallentamento del moto dunale, anche perché

\footnotetext{
${ }^{16}$ Tribunale Amministrativo Regionale, competente in controversie di ordine amministrativo.

${ }^{17}$ Negli ultimi anni della lottizzazione il prezzo dei lotti superava le 20.000 lire al metro quadrato, il prezzo di esproprio del comune, legittimato da una sentenza della corte d'appello del Tribunale di Firenze nel 1996, fu di 650 lire per metro quadrato.

${ }^{18} \mathrm{Nel} 1842$ il governo granducale acquista dalla famiglia Alliata il Lago di Rimigliano e, dopo i lavori di bonifica che andranno avanti dal 1842 al 1860, lo vende di nuovo agli Alliata che lo organizza in 74 poderi.

MAURO PARIGI, Rimigliano, patrimonio della cultura e governo del territorio, "Urbanistica Informazioni”, 165, 1999, pagg. 69-70.
} 
la retrostante strada della Principessa costituisce ormai un limite invalicabile, sbarrando la via ad un movimento naturale della formazione. Furono individuate zone attrezzata per la sosta e per i servizi igienici, ma anche accessi motorizzati esclusivamente per scopi specialistici o di emergenza.

\section{Parco Naturale inTerprovinCiale Di Montioni}

La proposta di una costituzione in forma di parco interprovinciale della formazione boschiva di Montioni fu avanzata nel 1994 per la creazione di un parco culturale. Il piano regionale per il biennio 1997-99 appoggiò l'ipotesi e nel 1998 le province di Grosseto e Livorno deliberarono l'istituzione dell'area protetta. Con i suoi 6.495 ettari è il parco più esteso tra quelli gestiti dalla società Val di Cornia, e contiene al suo interno la Riserva Statale di Popolamento animale della Marsiliana (443 ettari) e la Riserva Naturale Integrale Statale di Poggio dei Tre Cancelli (99 ettari).

Il ricco ambiente naturale, prevalentemente sempreverde, soprattutto lecci, sughere e pini, ricco di fauna selvatica anche rara, è scandito da significative memorie della presenza umana, come castelli, case poderali, opifici per la lavorazione dell'allume, sentieri storici e si presta ad un recupero naturalistico e culturale. Sono già in funzione percorsi segnalati che, a partire dal centro visitatori di Pratini di Valle, conducono alla scoperta dei resti della ferrovia lorenese per il trasporto della lignite, delle sorgenti solforose, delle installazioni termali volute da Elisa Baciocchi, sorella di Napoleone, a cui lei stessa volle dedicare la stele che è diventata logo del parco. Già attrezzato in molti punti il parco, procede nella sua organizzazione in maniera puntuale, fruendo dei finanziamenti europei. Tra questi il recupero di due edifici rurali abbandonati da adibire a "Casa per Ferie ed Ostello per la Gioventù" e a "Casa per Vacanze" su progetti dell'architetto Italo Insolera.

\section{Parco forestale di Poggio Neri}

Ubicato nel comune di Sassetta, il parco forestale di Poggio Neri è costituito da 200 ettari di bosco, divisi tra quello ceduo, composto da lecci, querce e castagni, e quello coltivato a castagneto da frutto.

Per adesso non sono previsti progetti per quest'area, che è stata considerata relativamente al sicuro da trasformazioni violente.

L'ingresso è libero e gratuito ed il visitatore può fruire di percorsi trekking percorribili a piedi, a cavallo o in mountain bike, ma l'accessibilità è garantita anche da sentieri adatti a disabili.

I sentieri si snodano nel bosco seguendo la linea delle sorgenti, mentre le aree di sosta ed i servizi sfruttano, quando possibile, gli antichi metati, i tradizionali seccatoi per le castagne.

\section{RIFERIMENTI BIBLIOGRAFICI}

AMENDOlEa BRUna (a cura di), I siti archeologici: un problema di musealizzazione all'aperto: secondo seminario di studi, Gruppo editoriale internazionale, Roma 1995, Secondo seminario di studi, Roma gennaio 1994.

AmEndolea Bruna, Cazzella Rosanna e Indio LaURa, I siti archeologici un problema di musealizzazione all'aperto, Multigrafica Editrice, Roma 1988, Primo seminario di studi, Roma febbraio 1988.

Carlini Giovanni e Sandra Bellandi, I parchi della Val di Cornia, "Architettura del Paesaggio", 5, 2000, pagg. 55-60.

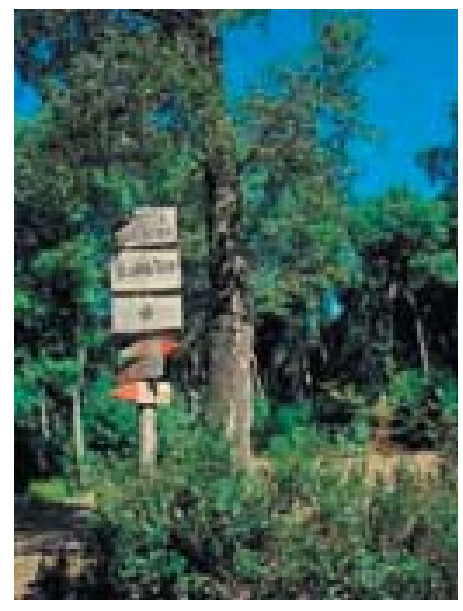

Figura 13 - Poggio Neri. 
Benedettini Gianfranco, Benesperi Paolo, Benucci Giorgio, Bianconi Umberto, Gazzola Luigi e Melograni Carlo, Una Pianificazione coordinata. Urbanistica ed architettura a Piombino e nella Val di Cornia, Be-Ma, Milano 1984.

Campos Venuti Giuseppe e Oliva Federico (a cura di), Cinquantanni di Urbanistica in Italia 1942-1992, Laterza, Bari 1993.

Coppa Mario, Paesaggio ed Ambiente, UTET, Torino 1990 in Coppa MARIo, Piccola storia dell'urbanistica, UTET, Torino 1990.

Cortese Maria Elena e Francovich Riccardo, La lavorazione del ferro in Toscana nel medio evo, "Ricerche Storiche", XXV, 2, 1995, pagg. 435-457.

Dallai Luigi, Opifici metallurgici sul promontorio di Piombino. Primi dati topografici in Atti del II Congresso di Archeologia Medievale, All'insegna del giglio, Firenze 2000, pagg. 186-201.

FrANCOVICH RICCARDO, Archeologia delle attività estrattive e metallurgiche, All'insegna del giglio, Firenze 1993.

Francovich Riccardo e Buchanan Jamie, Il progetto del Parco Archeominerario di Rocca San Silvestro (Campiglia Marittima) in AMENDolea Bruna (a cura di), I siti archeologici: un problema di musealizzazione all'aperto: secondo seminario di studi, gruppo editoriale internazionale, Roma 1995, Secondo seminario di studi, Roma gennaio 1994, pagg. 176-196.

FRANCOVICH RICCARDO, Le ragioni di un parco alle radici dell'archeologia mineraria: le miniere di Campiglia Marittima nelle pagine dei naturalisti e dei geologi dell'Ottocento, Marsilio, Venezia 1994.

Francovich Riccardo e Farinelli Roberto, Potere e attività minerarie nella Toscana altomedievale, in La storia dell'alto medioevo italiano alla luce dell'archeologia, secoli VI-X. Atti del convegno internazionale tenutosi a Siena il 2-6 dicembre 1992, École Française de Rome, Università degli Studi di Siena, All'insegna del giglio, Firenze 1994.

GAMBIno RoberTo, Conservare innovare, UTET, Torino 1997.

Gambino Roberto, I parchi naturali europei, Nis, Roma 1994.

Gazzola Luigi e Insolera Italo, Parchi Naturali. L'esperienza di Rimigliano, Edizioni delle autonomie, Roma 1982.

Giacomini Valerio e Romani Valerio, Uomini e parchi, Franco Angeli, Milano 1986.

InSOlera ItAlo, Il parco minerario della Val di Cornia in MANCINi Roberto (a cura di), Campiglia Marittima. Percorsi Storici e turistici, Tosca, San Giovanni Valdarno 1990.

Insolera Italo e Romuldi Antonella, Il parco archeologico di Populonia in Amendolea Bruna, Cazzella Rosanna e Indio Laura, I siti archeologici un problema di musealizzazione all'aperto, Multigrafica Editrice, Roma 1988, Primo seminario di studi, Roma febbraio 1988.

Mancini Roberto (a cura di), Campiglia Marittima. Percorsi Storici e turistici, Tosca, San Giovanni Valdarno 1990.

Minto Antonio, L'antica industria mineraria in Etruria ed il porto di Populonia 1954, "Studi Etruschi”, 33, 1954, pagg. 43-49.

PARIGI MAURO, Rimigliano, patrimonio della cultura e governo del territorio, "Urbanistica Informazioni”, 165, 1999, pagg. 69-70.

Preite Massimo, I parchi-museo minerari in Toscana: un primo bilancio attuativo, "Urbanistica Informazioni", 157, 1998, pagg. 21-22.

Rombai LEONARDO e GUARDUCCI ANnA, Tra natura e cultura: parchi e riserve di Toscana, Centro editoriale toscano, Firenze 1999.

Semplici Andrea, Parco archeologico di Baratti e Populonia, Edizioni Polistampa, Firenze 2000.

Zanchini Edoardo, Dall'Abusivismo al Parco. Storia del bosco della Sterpaia a Piombino, Franco Angeli, Piombino 2000.

Zucconi Massimo, Un'esperienza di coordinamento: il P.R.G. di Piombino, "Urbanistica Informazioni”, 141, 1995, pagg. 31-34.

\section{RIFERIMENTI ICONOGRAFICI}

Figure 1, 4, 6, 12 e 13: immagini tratte dal sito Internet della società Parchi Val di Cornia S.p.a. $<$ www.parchivaldicornia.it>.

Figure 2, 3, 5, 7, 8, 9, 10 e 11: fotografie di Sabrina Tozzini. 


\section{SCHEDA DI PROGETTO}

\section{Progetto:}

- Area protetta di interesse locale

- Area protetta di interesse locale

- Area protetta di interesse locale

- Area protetta comunale

- Parco naturale Interprovinciale

- Area protetta comunale

Luogo:

Committente:

Tipologia di parco:

Superficie:

Anno di progettazione:

Anno di realizzazione:
Sistema dei parchi della Val di Cornia

Parco archeominerario di San Silvestro nel comune di Campiglia Marittima

Parco archeologico di Baratti-Populonia nel comune di Piombino

Parco naturalistico costiero della Sterpaia nel comune di Piombino

Parco naturalistico costiero di Rimigliano nel comune di San Vincenzo

Parco naturalistico di Montoni, province di Livorno e di Grosseto, prevalentemente nel comune di Suvereto.

Parco naturalistico forestale di Poggio Neri nel comune di Sassetta

bacino del Fiume Cornia, al confine tra le province di Livorno e di Grosseto, comuni di Campiglia Marittima, Piombino, San Vicenzo, Sassetta, Suvereto

Società Parchi Val di Cornia S.p.a.

coordinamento di Parchi, a prevalente valenza museale, ma anche ambientale e faunistica

8.217 ettari

1994

dal 1996 



\title{
PIANO PER IL PARCO \\ DELLE MADONIE IN SICILIA
}

\author{
Michele Ercolini
}

\section{IL CONTESTO}

Le Madonie costituiscono un territorio molto variegato sotto il profilo delle componenti ambientali, con condizioni variabili, e in alcuni casi alternative, anche entro poche centinaia di metri. Il tema del "contesto" quindi, non può che essere affrontato attraverso la lettura delle ricche e straordinarie risorse su cui il medesimo Parco si fonda. Risorse ambientali, naturali e storiche, numerose, di alta qualità e, ciò che più conta, distribuite su tutto l'entroterra in un sistema diffuso, complesso e interrelato.

Ma l'aspetto ancor più interessante è dato dal fatto che non esiste in questo particolare ambito, né un'unica cultura né tanto meno un unico ambiente madonita, ma sussistono diverse entità spaziali, fra loro correlate, ben definite a livello paesaggistico, storico e produttivo.

Appare dunque utile in questa sede illustrare, ovviamente in sintesi, i risultati derivanti dalla lettura delle principali risorse territoriali presenti nelle Madonie sviluppata nella fase analitico-diagnostica del Piano Territoriale.
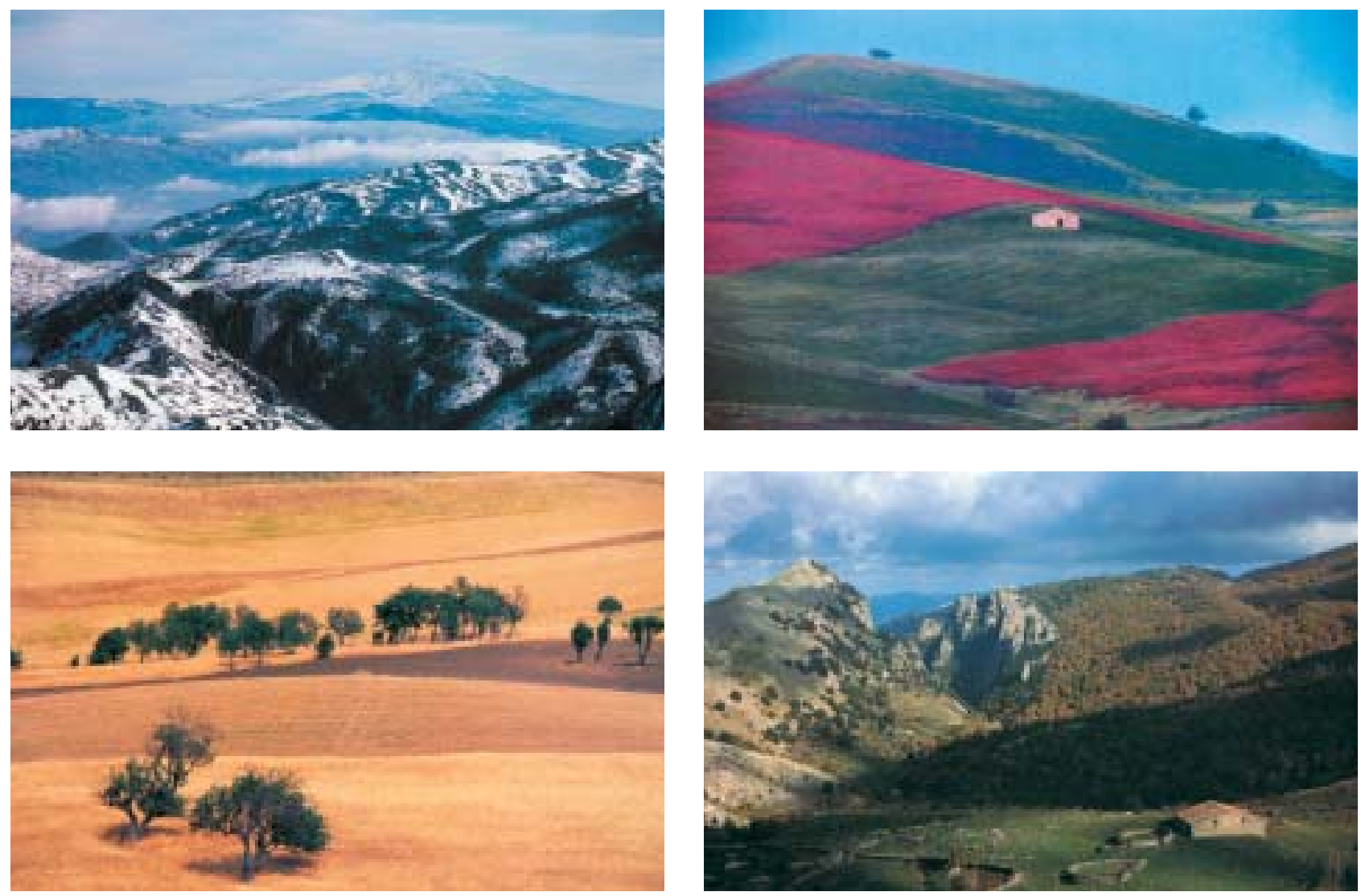

Figure 1, 2, 3, 4 - La forte eterogeneità del paesaggio che contraddistingue il Parco delle Madonie. 


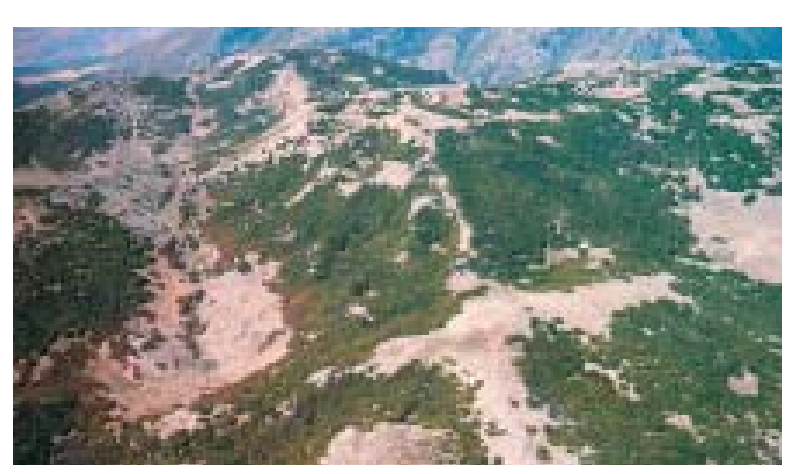

Figura 5 - Esempio di paesaggio carsico.

Iniziamo con il descrivere le principali peculiarità geologiche e geomorfologiche che fanno del territorio oggetto d'indagine, una delle zone di maggior interesse scientifico ed attrattiva naturalistica della Sicilia.

Si possono così scoprire località caratterizzate dalla presenza di morfologie rilevanti dal punto di vista scientifico e/o paesaggistico, come le gole, i fenomeni franosi complessi, le scarpate di faglia, mentre valori eccezionali di assoluto rilievo vengono assunti da fenomeni come gli affioramenti di rocce, le stazioni di interesse paleontologico, le forme dovute all'erosione accelerata, il carsismo, eccetera.

$\mathrm{Nel}$ corso della fase analitica sono state così individuate-cartografate-descritte ben 21 emergenze geologico-ambientali e 27 emergenze di carattere geomorfologico da sottoporre ad un regime congiunto di tutela e valorizzazione.

$\mathrm{Si}$ è rivolta inoltre una particolare attenzione ai processi carsici, che sui massicci madoniti risultano ancora attivi, in virtù delle condizioni climatiche caratterizzate da un'elevata piovosità e da una notevole persistenza del manto nevoso.

La ricca gamma di morfologie carsiche, creatasi nei millenni in funzione delle diverse condizioni delle rocce e delle variazioni climatico-ambientali, origina infatti uno dei paesaggi più caratteristici delle Alte Madonie. Il tutto assume una certa importanza anche per le implicazioni di natura idrologica, essendo i territori calcarei ricchi contenitori di acque in grado di originare un fitto sistema di circolazione idrica sotterranea che alimenta le grandi sorgenti.

Anche su questa particolare risorsa è stata sviluppata un'azione conoscitiva, consistente nell'individuazione, classificazione e "cartografazione" delle fenomenologie carsiche, suddivise poi in categorie in relazione alla specifica localizzazione, al livello di interesse e al grado di vulnerabilità.

Si è poi passati all'analisi delle risorse idriche: nei territori dei comuni del Parco complessivamente sono presenti oltre 400 punti d'acqua raggruppati lungo i margini dei rilievi. A seguito però della diminuzione degli apporti meteorici dell'ultimo venticinquennio (ed in particolare negli anni Ottanta) la portata di numerose sorgenti, anche di una certa entità, ha subito una notevole flessione, tanto che alcune manifestazioni si sono addirittura azzerate.

Questa fondamentale risorsa naturale, definita "l'oro bianco delle Madonie", si è andata dunque in questi decenni impoverendo con conseguente alterazione

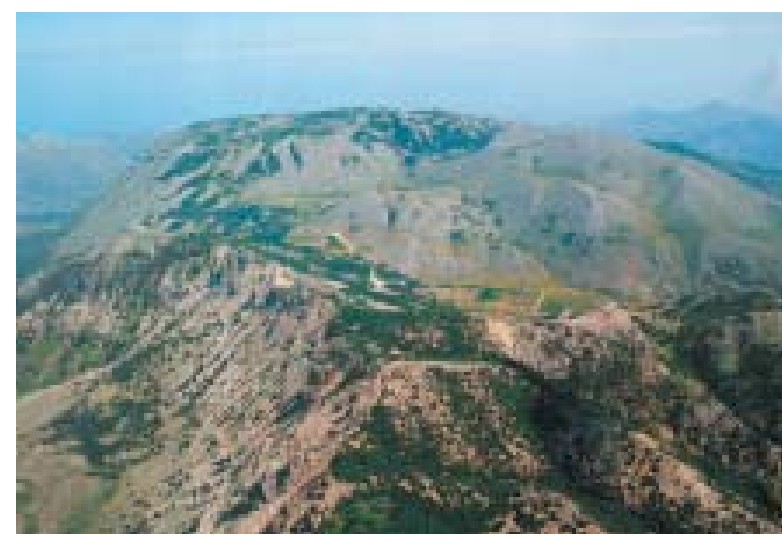

Figura 6 - "L'anfiteatro" delle rocce dolomitiche. degli equilibri naturali, vuoi per motivi direttamente legati ad eventi meteorologici, e vuoi, principalmente, per il suo sfruttamento per usi esterni all'area. "Tutto questo costituisce un'indubbia testimonianza di espropriazione culturale, prima ancora che economica, del valore delle risorse ecologiche"

È seguita poi l'analisi dell'immenso e straordinario patrimonio vegetazionale, contraddistinto da un elevato grado di diversità biologica e dalle peculiarità degli elementi che lo caratterizzano. Questo territorio, estremamente vario dal punto di vista geologico e climatico, costituisce infatti un crocevia internazionale dove convivono piante caratteristiche di decine di regioni geografiche dai climi molto differenti (mediterraneo, continen-

${ }^{1}$ Guido Ferrara e Giuliana Campioni, Piano Territoriale del Parco delle Madonie - Relazione di sintesi, Palermo 1999, pag. 26 (versione su Cd-rom gentilmente concessa dagli autori del Piano). 
tale, eccetera). Un areale capace di ospitare fino a 1.500 specie di piante vascolari complesse, tra cui circa 120 locali ed in parte esclusive di quest'ambiente, e molte altre di notevole interesse, perché rare o ai limiti della loro distribuzione geografica. Tra le specie "esclusive" presenti, quella più significativa, tanto da poter essere considerata l'emblema del Parco, è l'Abete delle Madonie (Abies nebrodensis).

Oltre a questa, oggi seriamente minacciata di estinzione, varie altre (Viola nebrodensis, Senecio candidus, Rhamnus lojaconoi, Peucedanum nebrodense, Genista cupanii, Bupleurum elatum, Astracantha nebrodensis, eccetera) risultano presenti esclusivamente nelle Madonie. Altre ancora testimoniano collegamenti fra il territorio siciliano e regioni più o meno lontane, come la Calabria (Armeria nebrodensis), la Sardegna (Orchis brancifortii), fino alla Tunisia (Alyssum nebrodense). Da ricordare inoltre, entità più comuni e diffuse in territori ampi, quali il faggio (Fagus sylvatica) e la rovere (Quercus petraea).

Relativamente alla distribuzione territoriale, le specie locali sono presenti in tutta l'area ma con maggiore frequenza nelle parti più elevate, secondo un rapporto crescente a partire dal mare fino alle sommità.

I caratteri speciali della flora hanno riscontro in quelli della vegetazione e costituiscono altrettante straordinarie espressioni del paesaggio vegetale delle Madonie.

Ricordiamo ad esempio la faggeta, qui considerata come un vero e proprio relitto geografico che raggiunge il suo estremo limite meridionale in Italia, entrando in contatto diretto addirittura con una formazione tipicamente mediterranea quale il leccio. Di non meno rilevante significato è la foresta mista relittuale di rovere e agrifoglio che insiste sui versanti di Monte Catarineci e in contrada Pomieri.

L'agrifoglio in taluni contesti, è presente con popolamenti monumentali costituiti da piante di dimensioni inconsuete capaci di raggiungere fino a 14 metri di altezza.

Non si possono poi dimenticare i terreni a pascolo, insediatesi a seguito della distruzione del manto forestale. All'interno dell'area protetta essi coprono complessivamente una superficie stimata attorno ai 12.367 ettari, il cui utilizzo risulta vitale per l'allevamento zootecnico che costituisce una delle principali attività connesse all'agricoltura.

I prati-pascolo e le praterie d'altitudine sono interrotti nella loro continuità $\mathrm{da}$ areali residuali di faggeto o da arbusti spinosi di rilevanza naturalistica, come l'astragalo dei Nebrodi, il ginepro emisferico, la genista di cubani. L'alternarsi di tali aspetti con i prati, con la vegetazione delle aree umide, delle depressioni dei pianori, e con i complessi boscati, contribuisce in maniera determinante all'unicità del paesaggio montano madonita. "In questo ambiente inoltre, un antico equilibrio si era storicamente realizzato tra risorse naturali ed attività antropiche e l'importanza dell'allevamento bovino ed ovino aveva portato ad una montagna intensamente vissuta e presidiata dall'uomo, particolarmente in estate, quando al bestiame locale si aggiungeva una transumanza molto attiva proveniente dalla marina tirrenica" ${ }^{2}$.

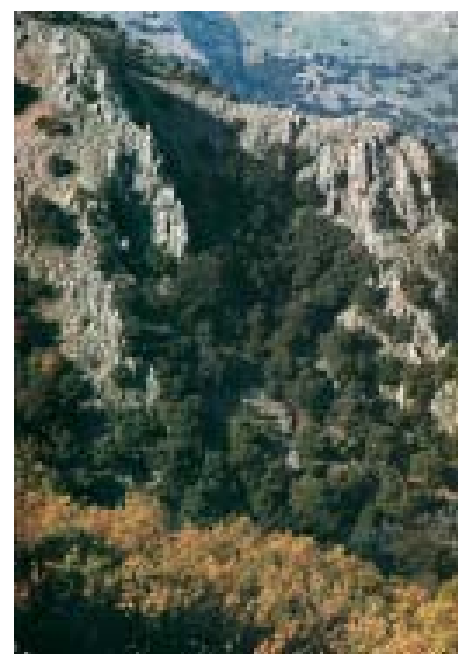

Figura 7 - Nucleo di Lecci frammisti all'Acero.

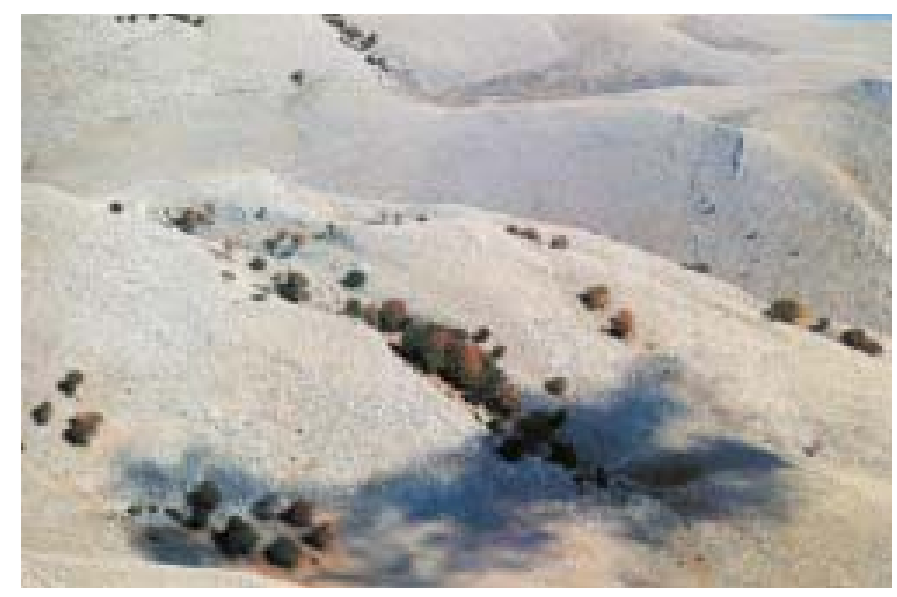

Figura 8 - Versante montuoso reso calvo dall'azione congiunta del pascolo e dell'erosione subentrata a massicce deforestazioni. 


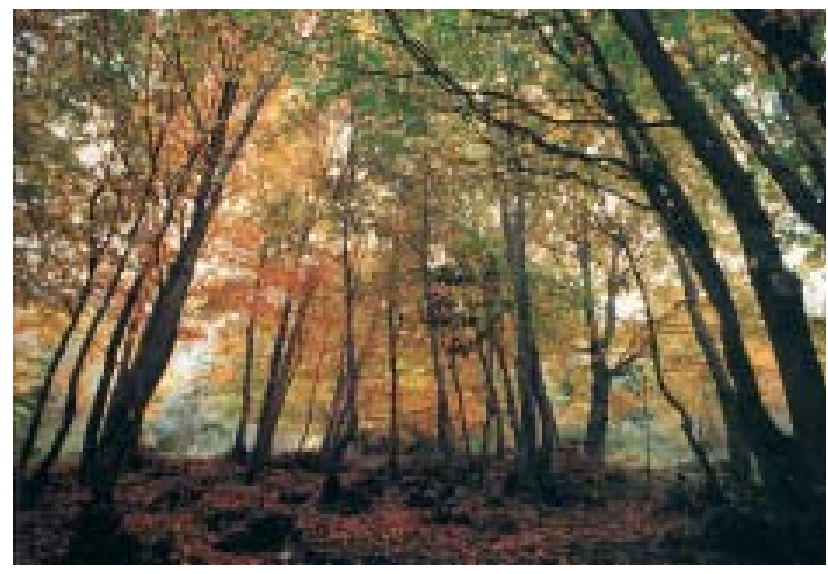

Figura 9 - Il giallo della faggeta preannuncia i primi freddi.

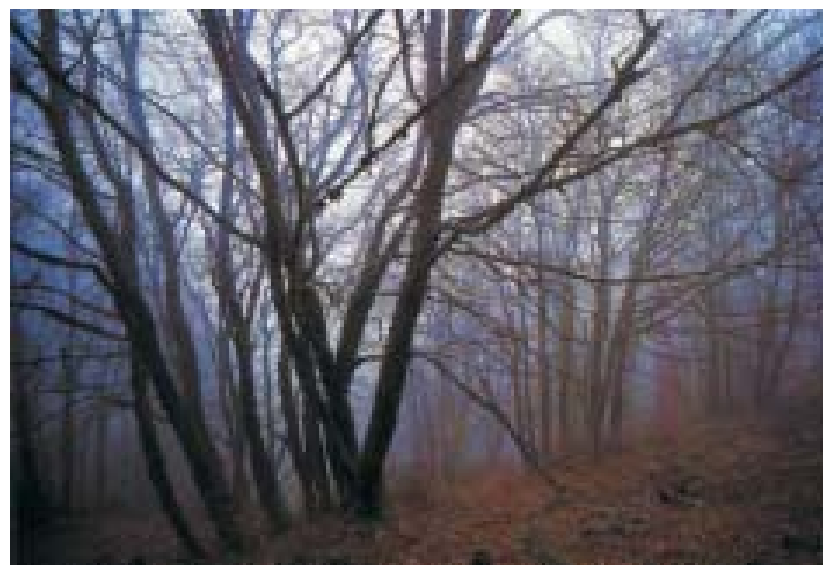

Figura 10 - La nebbia determina inconsueti scenari nel bosco.

Il paesaggio madonita, dal livello del mare sino ai circa 1.700 metri di quota è poi per larghi tratti dominato dai complessi boschivi che presentano una vegetazione e una struttura molto varia. Nella fascia costiera, il paesaggio forestale è caratterizzato da sclerofille sempreverdi-latifoglie con un'elevata varietà di specie comprendente, oltre ad individui sporadici di leccio e sughera, tutti gli arbusti tipici della macchia mediterranea. Tale formazione è particolarmente soggetta ad azioni di disturbo da parte del fuoco. Le sugherete sono presenti nei complessi boscati dei comuni della fascia settentrionale e nord orientale, mentre ad altitudini maggiori, a volte sino alle quote del faggio, in formazioni miste con la sughera e la roverella, insistono i boschi a prevalenza di leccio.

Nei querceti a foglia caduca la specie più diffusa è la roverella, presente negli ambienti caldo-aridi a quote inferiori a quelle del leccio, in formazioni miste intensamente pascolate per la presenza della ghianda. Il castagno, coltivato da molto tempo anche se in misura non molto estesa ed oggi per di più danneggiato dal cancro, si colloca nella fascia intermedia del leccio e della roverella, arrivando ad avere contatti con la sughera al limite inferiore e con il faggio al limite superiore. Nei pianori della zona montana centrale, dove trovano la migliore espressività le formazioni naturali, è presente il faggio in posizione marginale rispetto all'areale della specie.

In totale la copertura forestale si estende su 12.313 ettari all'interno dei quali le specie di gran lunga più rappresentate sono: il leccio, che copre il $23,4 \%$ della superficie forestale totale, la sughera $(16,5 \%)$, il faggio $(16,5 \%)$, la roverella $(10,2 \%)$. In merito al tipo di bosco si hanno, per il 64\% boschi misti e per il 36\% boschi puri soggetti alle seguenti forme di governo: $57,5 \%$ a fustaia, il $27,5 \%$ a ceduo semplice, il $13,3 \%$ a ceduo composto e il restante $1,7 \%$ a boscaglia e formazioni irregolari.

Un ruolo a parte infine, è svolto dai numerosi rimboschimenti effettuati nel tempo con specie autoctone e alloctone; oggi in particolare va affermandosi la tendenza ad effettuare rimboschimenti con acero, frassino, olmo campestre, castagno , noce, eccetera, allo scopo di ripristinare, sia pure nella consapevolezza dei lunghi processi indispensabili alla ricostituzione della fase di climax e della necessità di cure colturali, l'originaria foresta di latifoglie eliofile un tempo presente sul territorio e scomparsa con la messa a coltura dei terreni o per l'eccessivo sfruttamento a ceduo.

Così come quelle naturali anche le risorse antropiche sono state attentamente valutate. Nelle Madonie infatti esiste un rilevante patrimonio insediativo storico e tradizionale rappresentato da numerosi insediamenti creati per lo svolgimento delle attività agricole o pastorali, come i màrcati, spesso incuneati ad altitudini che sfiorano i 1.500 metri fin entro il cuore dei rilievi montani, dove hanno costituito per secoli, veri e propri avamposti della presenza e dell'azione antropica. In buona parte abbandonati da quando l'uomo ha allentato il presidio della 
montagna, essi rivestono notevoli aspetti di peculiarità e rarità e motivi molteplici di interesse, sia come manufatti fortemente esemplificativi delle tipologie e delle tecniche costruttive dell'architettura rurale locale (muratura in pietra a pezzatura irregolare reperita sul posto, scarso legante, copertura a travi lignee e coppi siciliani, focolare centrale, eccetera), sia quale luoghi di conservazione e propagazione dei valori etnici-antropologici e della cultura più strettamente legata alla matrice agropastorale del mondo madonita.

A queste preesistenze ben si adatta la definizione di bene culturale ambientale. E ciò non solo per il rapporto diretto che intrattengono con il contesto naturale, cui si legano sia nei materiali che nelle forme, tanto che spesso è difficile capire dove inizia l'opera dell'uomo e ove finisce quella della natura, ma anche e soprattutto per i particolari aspetti di conservazione degli equilibri naturali legati ai noti effetti del pascolamento.

Da tempo l'abbandono di queste sedi, avanza a ritmi accelerati non solo e non tanto per la scarsa redditività dell'attività pastorale in se stessa, quanto per i vincoli e i divieti che le sono stati imposti, per la riforestazione dei prati-pascoli, per la difficoltà di approvvigionamento d'acqua e, non ultimo, per il presunto decadimento della figura e del ruolo sociale del pastore a fronte, ad esempio, di quella dello stipendiato. "La prospettiva di una progressiva perdita dei màrcati, con il conseguente compimento della desertificazione e il calo di diversità della montagna, rappresenta nondimeno uno spreco immenso sia dal punto di vista culturale e naturalistico che da quello produttivo ed economico che rende improrogabile e urgente un'incisiva azione di tutela attiva"'3.

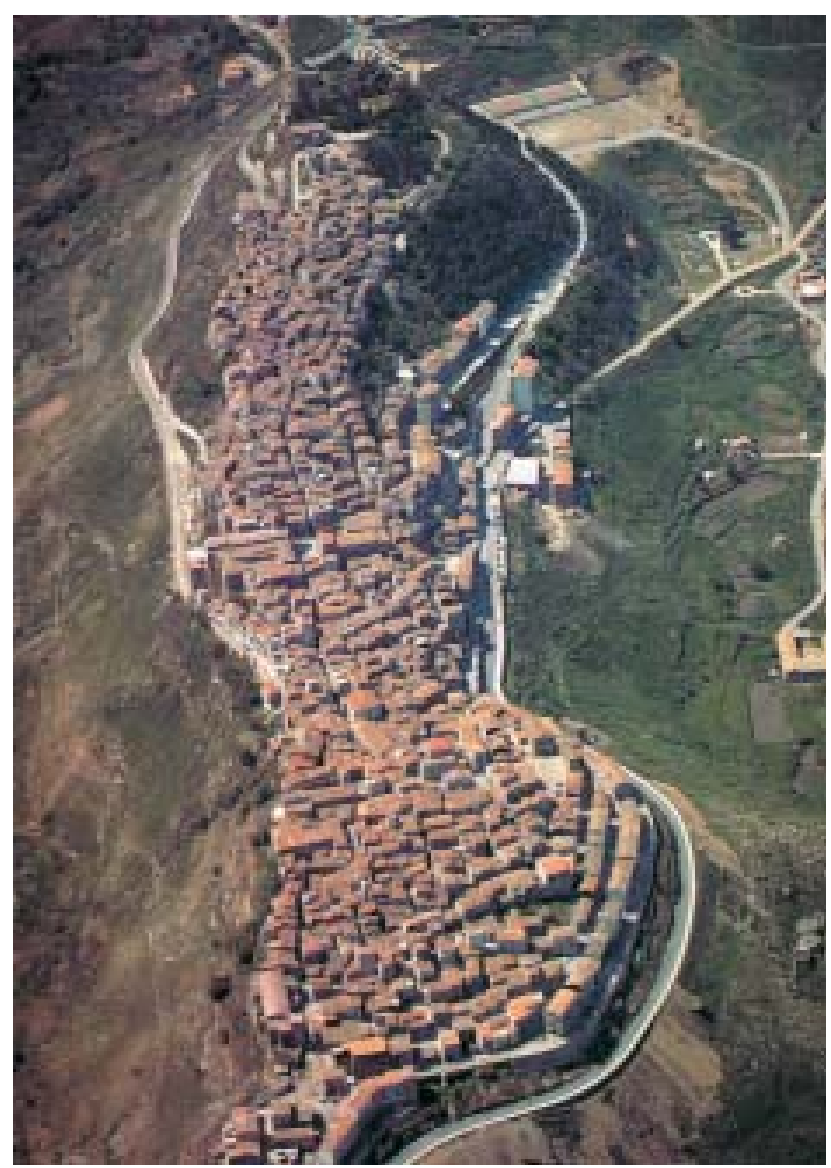

Figura 11 - Veduta aerea dell'abitato di Castelbuono.

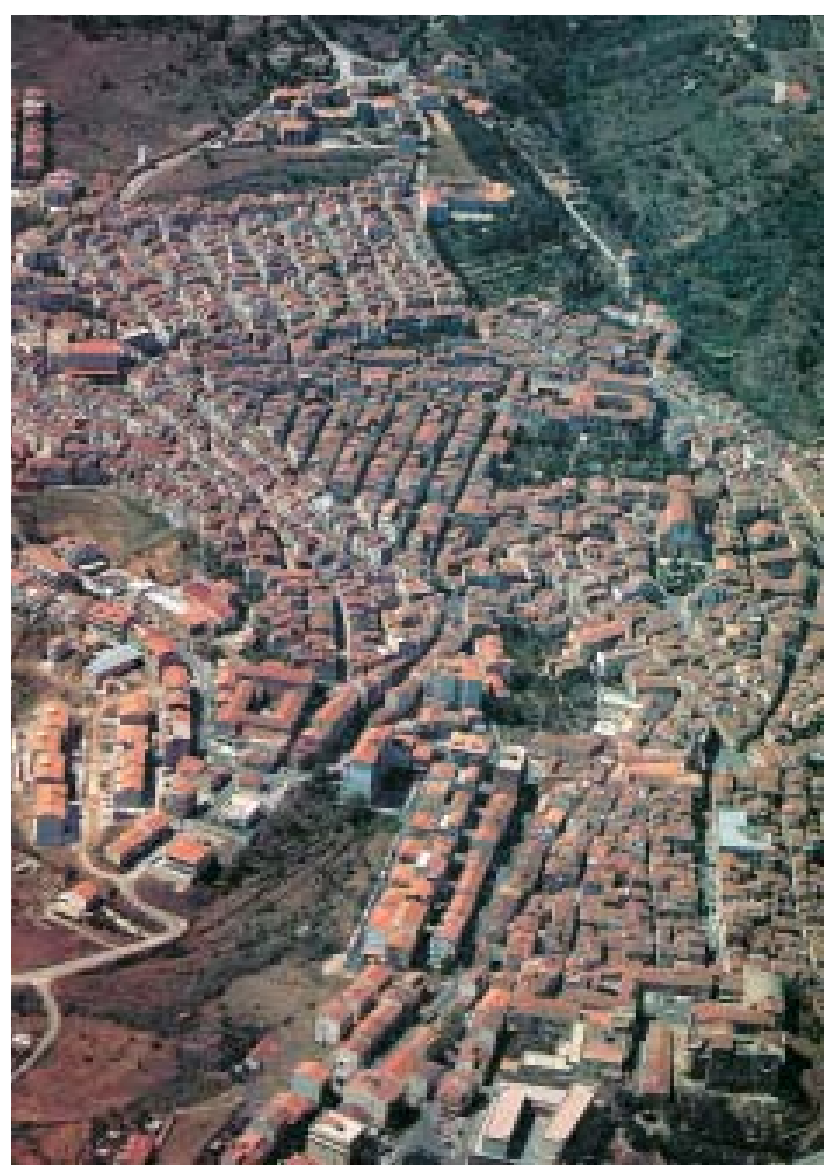

Figura 12 - Veduta aerea dell'abitato di Gerici. 


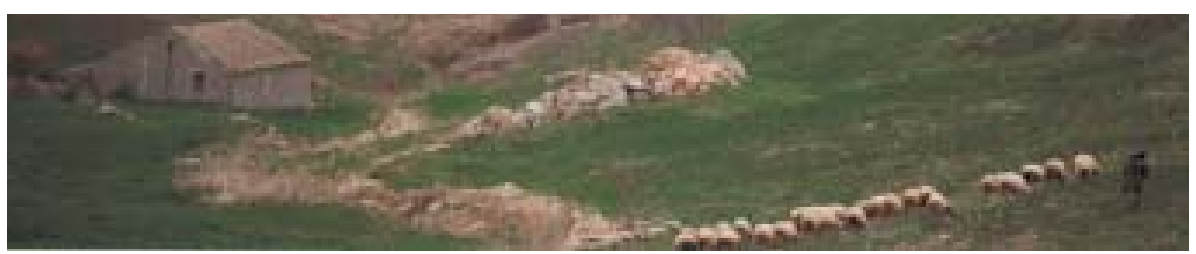

Figura 13 - Prati e pascoli sui versanti madoniti.

Gli esempi più significativi di architettura spontanea rurale, in aggiunta a quelli rilevanti di edilizia religiosa e di impianti di servizio all'agricoltura, costituiscono dunque, nel loro complesso, uno dei maggiori elementi di caratterizzazione dell'ambiente antropizzato delle Madonie, coprendo il paesaggio urbano e rurale del Parco con una maglia continua che, in alcune situazioni si addensa a costituire complessi di straordinario interesse. Si può parlare a ragione di veri e propri capisaldi dell'assetto territoriale storico, luoghi dove si sono espresse al meglio le valenze della spiritualità, dell'architettura e dell'artigianato colto.

Analoghe considerazioni valgono anche per i manufatti connessi alla residenza tradizionale rurale sparsa, stabile o temporanea, e, in particolare, alla produzione agricola ed all'industria domestica. Nel territorio madonita questi esempi di "archeologia rurale", trovano la massima espressività nella tipologia dei mulini, vere e proprie macchine ad acqua capaci di testimoniare l'affermata tradizione cerealicola del territorio. La loro presenza risulta stimolante e carica di implicazioni, vuoi quando essi segnalano l'esistenza di attività antropiche in ambiti impervi, sia quando si addensano in complessi, o addirittura in veri e propri sistemi territoriali in prossimità dei centri abitati.

La fase relativa alla lettura delle principali risorse storico-ambientali, si è conclusa analizzando il patrimonio archeologico. In questo territorio di intensa ed antichissima antropizzazione, il panorama archeologico risulta infatti particolarmente significativo. Esso comprende un vasto repertorio di materiali e di segni che testimoniano la vita e il lavoro degli uomini e le presenze animali-vegetali, in un arco temporale lunghissimo che va dall'Eneolitico alla piena età romana, per proseguire, seppure con documenti più rari e meno certi, in età bizantina ed altomedievale.

Tra i siti archeologici più significativi e suggestivi, dove si celebra in modo ineguagliabile la sintesi tra natura e cultura, si annoverano le numerose grotte, spesso ubicate oltre i mille metri di quota alle falde delle formazioni calcaree, dove le frequentazioni avvenute coprono una sequenza che va dal Neolitico

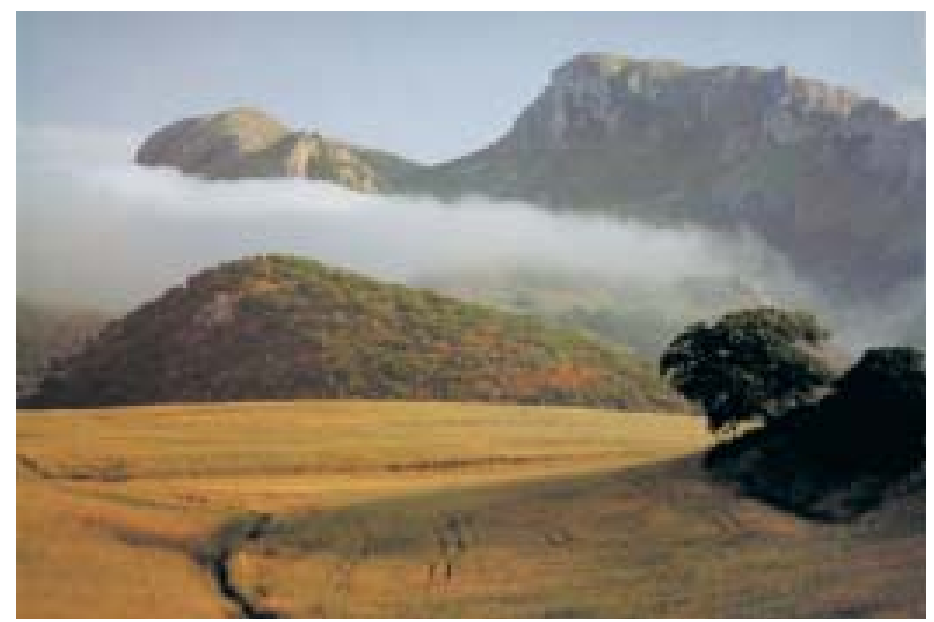

Figura 14 - Il paesaggio madonita tra prati e versanti montuosi. all'Età del Bronzo. Infine, particolarmente numerose risultano le presenze archeologiche d'età romana riferibili a manufatti (come ponti, resti di acquedotti, ville rustiche) e a necropoli.

In ragion di ciò il territorio madonita, contraddistinto da vette montane, da vedute, sorgenti, fiumi e torrenti, forme e grotte carsiche, grandi boschi, elementi floristici, prati e pascoli, ma anche frassineti e noccioleti, oliveti, mulattiere, santuari rurali, edilizia spontanea e infine feste locali e tradizioni, può certamente rappresentare la vera risorsa delle risorse, il vero petrolio di questi luoghi, ossia il punto di partenza "obbligato" per l'azione di tutela e valorizzazione attuata dal medesimo piano. 


\section{GLI OBIETTIVI}

Nel caso del Piano Territoriale delle Madonie si può fare una distinzione tra gli obiettivi di carattere generale, gli obiettivi strategici e gli obiettivi "dell'evitare", ossia quelli utili per non ripetere gli errori "tipici" della pianificazione delle aree protette.

Tra gli obiettivi di carattere generale ricordiamo:

- la volontà di attuare una politica di conservazione e tutela degli ecosistemi attraverso l'intervento antropico permanente, purché fondato su un'analisi diagnostica e comparata di tutte le componenti del territorio;

- compiere una ricerca scientifica continua multidisciplinare e interdisciplinare;

- impostare una didattica educativa e formativa, dal momento che il Parco costituisce di per sé già un laboratorio dedicato, fra l'altro, alla formazione di esperti in problemi ambientali e di gestione del territorio;

- sperimentare nuove tecniche di agricoltura montana e collinare, di zootecnia, di selvicoltura naturalistica, di insediamenti produttivi non inquinanti, di turismo estensivo, mediante l'utilizzazione equilibrata delle risorse territoriali disponibili;

- coinvolgere le popolazioni dell'area di influenza del Parco in attività compatibili con un ambiente da cui scaturiscono le principali opportunità culturali, sociali ed economiche locali;

- promuovere in particolar modo la funzione ricreativa del Parco, perché esso rappresenta, complessivamente, un servizio sociale qualificato e qualificante indispensabile per stabilire un contatto proficuo tra cultura urbana e cultura rurale;

- infine, dare vita ad una politica di conservazione dinamica dei valori antropologici autoctoni, in quanto il Parco tutela le comunità locali riservando però loro il diritto di proposta alle scelte di governo del territorio e alla difesa e la gestione delle tradizioni culturali.

In altre parole il Piano si è posto i seguenti obiettivi strategici, ognuno dei quali entra nel merito:

- "delle modalità con cui la tutela dei beni protetti si esplica, dando giustificazione dei limiti entro cui porre l'interferenza umana, giustificazione basata sulla conoscenza reale dei fattori che si intendono controllare;

- degli aspetti patologici presenti sul territorio (dalle cave abbandonate, agli svincoli delle autostrade, dalla crisi delle attività tradizionali, allo spreco delle risorse), diagnosticandoli e proponendo di volta in volta le cure più adatte. Non sempre, infatti, il Parco significa impedire di fare, dal momento che i nostri ecosistemi sono dinamici, sottoposti prima agli interventi sporadici e poi all'azione costante dell'uomo che, in più di un caso, ne ha specificato e caratterizzato le peculiarità originarie;

- dell'importanza relativa alle diversità che il Parco offre, per poter articolare in conseguenza le differenti modalità d'azione conservazionali; tutto ciò conferma ancora una volta, che le limitazioni d'uso non costituiscono nel nostro caso l'unica ricetta praticabile" .

\footnotetext{
${ }^{4}$ Guido Ferrara e Giuliana Campioni, Il Piano del Parco delle Madonie in Sicilia, "Architettura del Paesaggio", 4, 2000, pag. 34.
} 
Il Piano Territoriale ha cercato infine di perseguire gli obiettivi "dell'evitare", tentando cioè di non commettere i seguenti possibili errori:

- ignorare che dentro il Parco vivono intere collettività insediate, portatrici di tradizioni e di cultura ambientale oltreché di aspirazioni, che meritano di essere ascoltate, confrontate e discusse;

- escludere a priori dal perimetro del Parco le attività produttive, con particolare riferimento a quelle più direttamente e storicamente legate alla dinamica della società rurale-montana e alla riproduzione dinamica degli ecosistemi, anche se effettuate in zone di particolare pregio ambientale o naturale;

- credere di poter mantenere ogni cosa esattamente com'è su un areale amministrativo che conta ben 15 comuni, sapendo che alcuni cambiamenti possono risultare opportuni e a volte assolutamente necessari;

- impedire la fruizione allargata dei beni ambientali, naturali, dei monumenti e dei siti, invece di garantire una gestione equilibrata che assicuri gli accessi e i prelievi insieme alla conservazione delle risorse;

- attuare la politica della cosiddetta "campana di vetro", ossia operando con un'impostazione esclusivamente di tipo vincolistico.

\section{LA METODOLOGIA}

La stesura del Piano Territoriale presenta interessanti novità, che potremmo quasi definire delle "anomalie positive", relative in particolare, al tipo di approccio metodologico che gli stessi progettisti hanno voluto dare fin dal principio.

Le finalità elencate in precedenza innanzitutto, sono state perseguite impostando un criterio metodologico rigorosamente interdisciplinare. In particolare, nel corso della fase analitica lo stato delle conoscenze esistenti sul territorio madonita, già particolarmente approfondito, è stato "completato, sistematizzato, unificato, e soprattutto orientato ad una strategia per decidere, grazie al compimento di numerose indagini specifiche mirate, realizzate entro l'universo del massiccio racchiuso tra le valli fluviali del Pollina e dell'Imera Settentrionale"'. Le analisi ambientali, infatti, ed ecco la prima interessante novità, sono state svolte su una superficie territoriale molto più ampia (praticamente doppia) rispetto ai 39.679 ettari compresi entro la perimetrazione cui fa riferimento il Decreto Assessorile del 9 novembre 1989 Istitutivo del Parco, al fine di poter ottenere una documentazione adeguata non solo sul sistema di ecosistemi costituente l'universo madonita, ma anche sull'area di interfaccia con il territorio limitrofo. Ciò ha così reso possibile, nel corso della successiva fase propositiva (illustrata nel paragrafo a seguire), la rilettura ponderata dei confini del Parco e la gerarchizzazione dell'azzonamento interno.

È stato adottato inoltre, un particolare metodo di lavoro fondato principalmente sulla presa di contatto diretto con la realtà ambientale e con le popolazioni. I colloqui e le indagini sul campo sono stati infatti ritenuti il complemento più opportuno alle analisi tematiche redatte in sede tecnico-scientifica. In questo tipo di approccio al territorio si sono presi in considerazione, allo stesso tempo, sia gli aspetti specificatamente connessi alla natura ed alla consistenza delle risorse territoriali sia quelli legati alla struttura culturale, sociale ed economica delle comunità residenti del Parco, cui è in larga parte delegata la diretta gestione dell'ambiente antropizzato, sia pure in termini di conservazione attiva.

Ma sicuramente l'elemento che più di ogni altro contraddistingue il Piano Territoriale delle Madonie è rappresentato dalla chiara volontà di superare una

\footnotetext{
${ }^{5}$ Guido Ferrara, e Giuliana Campioni, op. cit., Palermo 1999, pag. 11.
} 


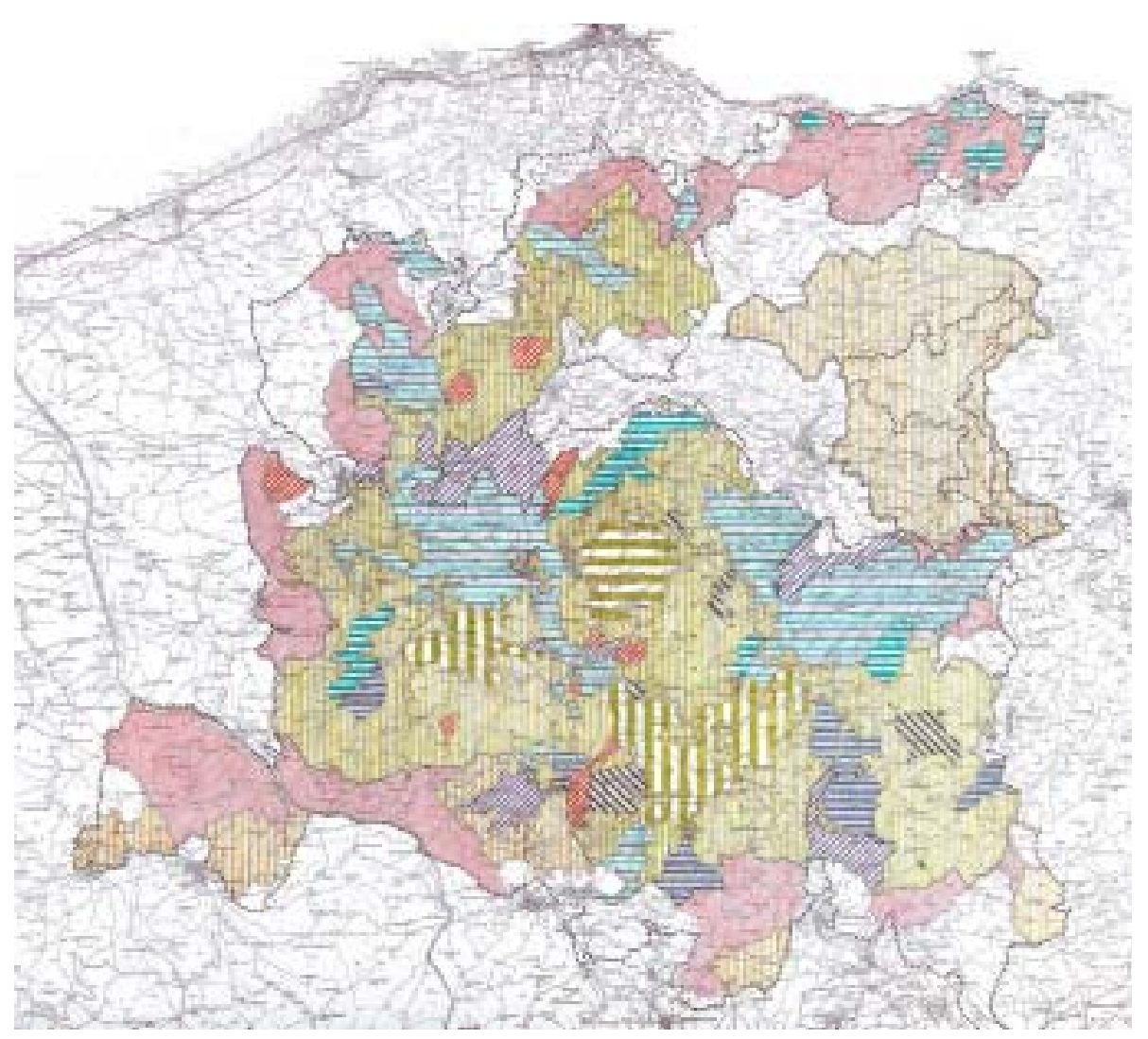

Figura 15 - Carta degli Interventi di conservazione attiva rispetto alla gerarchizzazione delle aree protette sulla base delle categorie I.U.C.N.

volta per tutte la logica vincolistica, andare cioè oltre "la politica della zonizzazione", dei Piani costruiti a "tavolino". Si è cercato in poche parole, di riconsiderare sotto nuova luce l'articolazione zonale (A, B, C, D) imposta dall' istituzione del Parco, facendo riferimento alle conclusioni del IV Congresso Internazionale sui Parchi e le Aree Protette tenutosi nel 1992 a Caracas, ove si era rilevata la necessità che per il futuro "le aree protette fossero strettamente legate alle strategie proposte a livello nazionale e regionale per lo sviluppo riproducibile ed il cor-

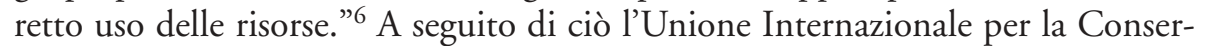
vazione della Natura (IUCN), cercò di formulare delle linee guida utili per l'individuazione delle categorie di gestione delle aree protette.

Il massimo organismo conservazionale pose l'accento in particolare sul fatto che le categorie individuate non potevano essere rigide e vincolanti, ma solo costituire uno strumento atto a facilitare la comunicazione, l'informazione e l'adeguamento delle aree protette agli obiettivi gestionali unitariamente riconosciuti.

Questo tipo di approccio metodologico ha dato luogo ad un interessante elaborato grafico definito dai progettisti "Interventi di conservazione attiva rispetto alla gerarchizzazione delle aree protette sulla base delle categorie I.U.C.N."

Una tavola che, anticipando quella finale, si è posta il principale obiettivo di "ricondurre le diverse condizioni di stato analizzate a livello interdisciplinare, alle categorie di aree protette valide in campo internazionale, stabilendo in termini corretti le diverse azioni conservazionali rispetto alle risorse disponibili"'

\footnotetext{
${ }^{6}$ Guido Ferrara e Giuliana Campioni, op. cit., 2000, pag. 34.

7 Guido Ferrara, Il Piano Territoriale del Parco delle Madonie, intervento al Convegno di Genova "Pianificazione e sviluppo locale in ambienti sensibili: esperienze di progettazione e gestione in alcuni paesi mediterranei”, Genova, 6-8 novembre 1997, pag. 4.
} 


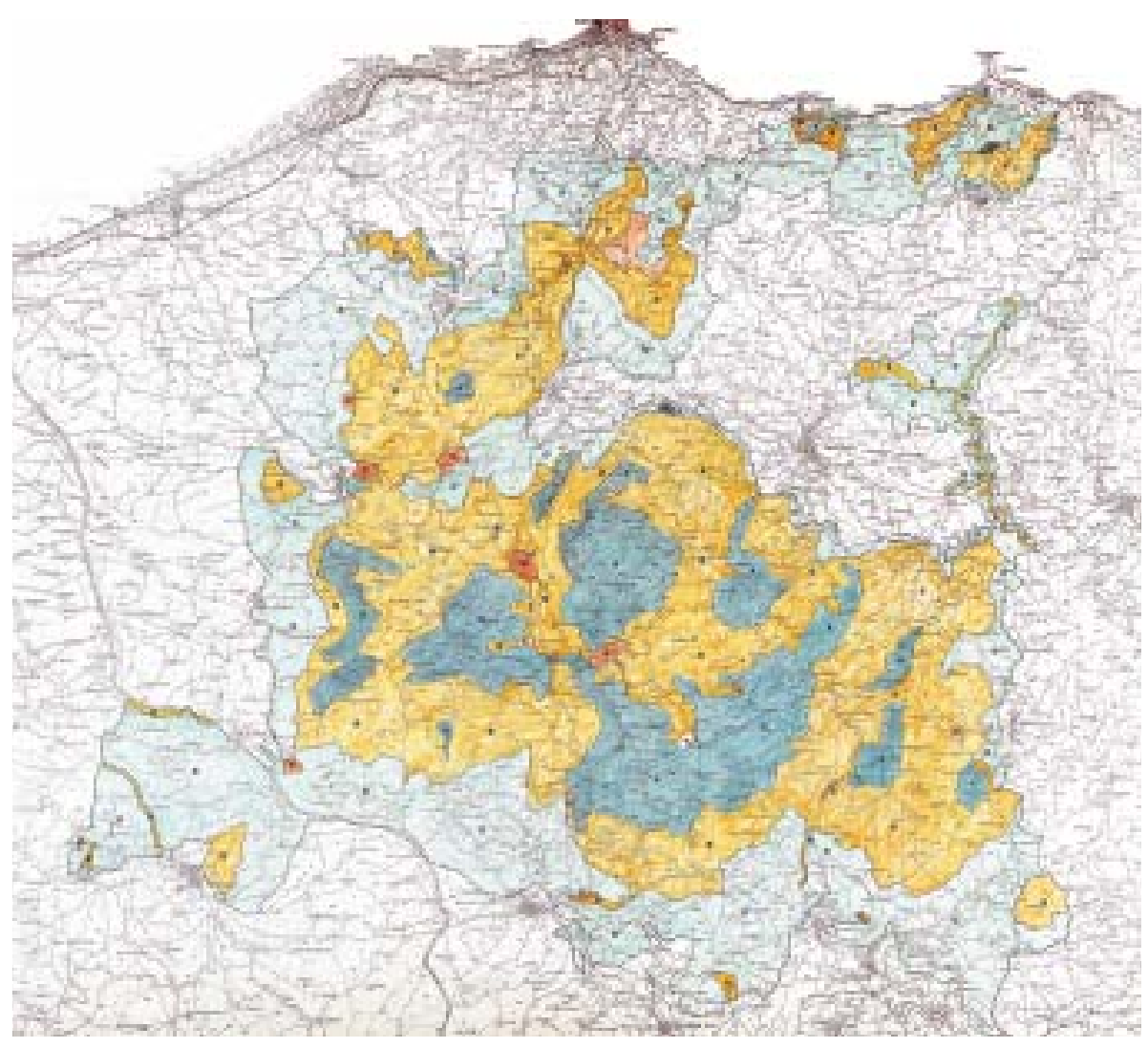

Figura 16 - Carta della zonizzazione derivante dall'applicazione del Decreto Istitutivo del Parco.

Si sottolinea, peraltro, ed ecco un ulteriore elemento di novità, come detto elaborato non precostituisca un "azzonamento" dotato di valore giuridico, come è invece compito della tavola finale (illustrata nel paragrafo successivo), ma rappresenti una sintesi delle letture diagnostiche suggerite nella fase analitica, ovvero la trama concettuale su cui si sono potute fondare le destinazioni d'uso del suolo, nella prospettiva della comparazione del Parco delle Madonie ai criteri di pianificazione ed agli obiettivi conservazionali stabiliti a livello internazionale.

Tutto ciò ha consentito, sia di mettere in discussione per la prima volta la politica fondata sull'articolazione zonale classica, "figlia" della Legge Quadro Nazionale e della Legge Regionale istitutiva del Parco medesimo, sia di superare finalmente la logica dei confini ideologici che non hanno cioè nessuna corrispondenza con la realtà territoriale. Non a caso, dall'analisi della carta con la perimetrazione esterna ed interna dell'area protetta, derivante dall'applicazione del Decreto Istitutivo del Parco, detti confini apparivano completamente sfalsati rispetto alla vera natura degli ecosistemi protetti. Il livello di "lontananza" tra questi confini ideologici e la realtà territoriale era talmente grande che, ad esempio, un paesaggio agrario fortemente antropizzato e con interessi conservativi circoscritti esclusivamente ad aspetti geologici, era stato inserito nella zona di Riserva Integrale (zona A) con conseguente obbligo di interruzione delle pratiche agricole ed esproprio, mentre paradossalmente, entro un contesto storicamente pastorale e strettamente legato ad un biotopo (segnalato perfino dalla Società Botanica Italiana), veniva proposta la localizzazione di un insediamento residenziale e di servizi (Zona $\mathrm{C}$ ).

I confini del Parco sono stati quindi messi in discussione e completamente riconsiderati, sulla base delle condizioni di stato analizzate a livello interdisciplinare, in modo da poter stabilire correttamente le diverse azioni conservazionali rispetto alle risorse disponibili. Il tutto però sempre nel pieno rispetto 
della perimetrazione di Riserva integrale e di Riserva generale fissata dal Decreto Istitutivo.

Per una miglior comprensione, si riporta di seguito l'elenco delle quattro principali categorie di aree protette di cui si è riscontrata la contemporanea presenza all'interno del Parco, a loro volta suddivise in sottozone, in virtù della nota e più volte sottolineata complessità ambientale e stratificazione antropica tipica del territorio madonita:

\section{"CATEGORIA I: Riserva integrale}

CATEGORIA Ia: Riserva integrale di prevalente interesse scientifico

Definizione: area caratterizzata da ecosistemi di rilevante interesse o rappresentativi, da emergenze geologiche, geomorfologiche e/o da specie rare, la cui gestione deve essere finalizzata in primo luogo alla ricerca scientifica e/o al monitoraggio ambientale.

CATEGORIA Ib: Riserva integrale di protezione di aree remote e dei santuari di natura

Definizione: vasta porzione di territorio intatto o scarsamente interessato da processi di modificazione, privo di insediamenti stabili significativi, che conserva le proprie caratteristiche naturali e la propria influenza, sottoposto a protezione e gestione al fine di preservarne le condizioni naturali.

CATEGORIA IV: Riserva orientata (area protetta gestita principalmente per la conservazione attuata mediante interventi gestionali):

Definizione: area gestita con interventi di conservazione attiva finalizzati ad assicurare il mantenimento degli habitat e/o soddisfare le necessità di determinate specie.

CATEGORIA IVa: Biotopo, riserva floristica o vegetazionale

CATEGORIA IVb: Riserva forestale

CATEGORIA IVc: Riserva geologico ambientale

CATEGORIA IVd: Riserva pastorale

CATEGORIA IVe: Bosco ad evoluzione naturale

CATEGORIA V: Paesaggio protetto (area protetta gestita principalmente per la conservazione del paesaggio e per la ricreazione)

Definizione: area dove l'interazione tra uomo e natura ha prodotto nel tempo un paesaggio ragguardevole di notevole valore estetico, ecologico e/o culturale, spesso ad alta diversità biologica. Salvaguardare l'integrità di questa tradizionale interazione risulta di importanza vitale per la protezione, il mantenimento e l'evoluzione di questo tipo di area protetta.

CATEgoria Va: Paesaggio agrario di preminente interesse culturale ed ambientale

CATEGORIA Vb: Area di tutela paesaggistica generale

CATEGORIA VI: Area di gestione delle risorse (area protetta gestita principalmente per un uso degli ecosistemi naturali capace di garantirne la durata):

Definizione: area contenente prevalentemente sistemi naturali non modificati, gestiti al fine di assicurarne la protezione a lungo termine ed il mantenimento della diversità biologica, fornendo contemporaneamente un flusso durevole di prodotti naturali e di servizi capaci di soddisfare i bisogni della collettività.

CATEGORIA VIa: Forestazione produttiva

CATEGORIA VIb: Aree in equilibrio instabile (da programmare)" 8 . 
Questo tipo di approccio metodologico ha permesso altresì di "considerare l'aspetto che nella prassi conservazionale italiana la definizione di Parco Nazionale o Regionale, in quanto area protetta contraddistinta da una specifica Authority e da un piano strategico, è in realtà un mosaico complesso di molte situazioni diverse, ognuna delle quali richiede una specifica politica di tutela ed utilizzazione. E poiché le definizioni internazionali sono relazionate specificatamente agli obiettivi di gestione, il riferimento appare quanto mai opportuno per indirizzare in termini concreti l'azione di governo del Parco in quanto Ente, coincidendo la conservazione del paesaggio (inteso come sistema di ecosistemi) in buona misura con la gestione del Parco"'.

Il paesaggio delle Madonie è stato dunque qui inteso quale "sistema di ecosistemi", conseguente cioè alle interazioni dinamiche tra processi geomorfologici, e vicissitudini geologiche e climatiche, popolamenti ed azioni antropiche, e connotato da un organizzazione spaziale caratteristica degli ecosistemi territoriali (unità di paesaggio), ognuna delle quali possiede aspetti ambientali specifici e meccanismi di riproduzione molto particolari.

$\mathrm{Si}$ è proceduto quindi alla stesura di una carta di sintesi relativa alla individuazione delle unità di paesaggio. Ciascuna unità individuata rappresenta un'entità corrispondente a gruppi di ecosistemi che vi si ripetono spazialmente in maniera simile; pertanto essa risulta al suo interno sostanzialmente omogenea rispetto alle componenti ambientali abiotiche e biotiche considerate, ma allo stesso modo diversa o in qualche modo caratterizzata da tutte le altre (e per tanto irripetibile).
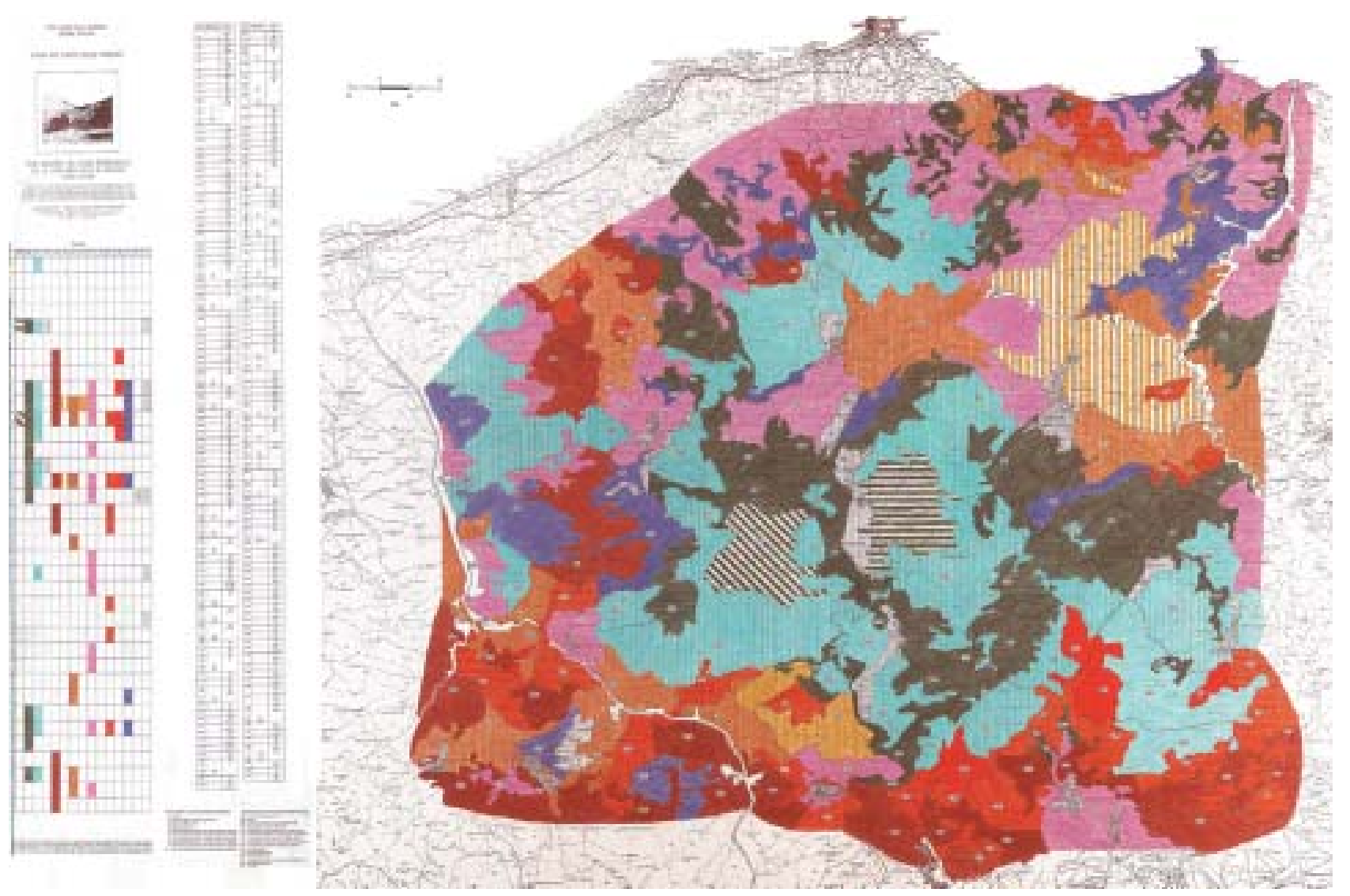

Figura 17 - Carta dell'unità di paesaggio: ecomosaico delle Madonie. 
Elemento primario per l'individuazione di tali unità sono le unità fisiografiche di base definite nell'ambito della disciplina dell'ecologia del paesaggio, quali "fisiotopi". I fisiotopi sono da considerare come unità spaziali che, rispetto ad una determinata scala cartografica e quindi ad un determinato livello di dettaglio nell'analisi del territorio, risultano caratterizzati da fattori di stato abiotici (litologia, morfologia, pedologia, idrologia, eccetera) omogenei, aventi effetti relativamente uniformi sulla parte biotica dell'ecosistema e costituendo condizioni specifiche per gli habitat della vegetazione e della fauna.

In un contesto prevalentemente montano come quello oggetto di studio, i fattori fisiografici determinanti risultano essere quelli legati essenzialmente alla morfologia (ad esempio i processi geomorfologici, di erosione, del carsismo, eccetera). Per la successiva definizione delle unità di paesaggio, i fisiotopi sono stati poi integrati con altri fattori principali, ossia la vegetazione e l'uso del suolo.

Nello specifico dunque, la definizione, individuazione e successiva descrizione delle unità di paesaggio è stata attuata mediante il confronto comparato dei seguenti parametri, ricavati dalla cartografia di base disponibile: altimetria, clivometria, litologia, idrografia, fisiografia, uso del suolo, vegetazione.

Il risultato dei confronti incrociati tra le varie carte tematiche, ha prodotto l'individuazione di 159 aree. Ogni unità di paesaggio è stata così numerata e contrassegnata da una sigla riassuntiva e schematica. Sono state specificate inoltre delle sottozone, cioè unità subordinate che presentano una o più componenti diverse da quelle del tipo superiore.

Le aree infine, sono state perimetrate e campite in un elaborato (Carta delle Unità di paesaggio), utilizzando una matrice-legenda che, tenuto conto del ripetersi di situazioni, ha consentito di visualizzare le 159 unità riducendole a sua volta in 18 macro unità, indispensabili per suggerire indirizzi di Piano.

\section{GLI ESITI}

Il quadro delle proposte progettuali avanzate dal Piano deve necessariamente essere individuato a due differenti scale: quella "generale", in cui si sono definite le politiche strategiche (di conservazione attiva, analizzata nel paragrafo precedente, di gestione ambientale e di promozione turistica-ricreativa), e la scala più dettagliata, finalizzata cioè ad indicare in concreto, le azioni opportune a raggiungere gli obiettivi di tutela attiva per ogni specifica tipologia di risorsa, bene o valore culturale e naturale presente o potenzialmente attivabile entro il Parco delle Madonie.

In questa sede appare più opportuno concentrarsi sulla lettura del Piano rimanendo alla scala generale, vuoi per ovvi motivi di sintesi, sia perché, proprio in essa, sono rintracciabili i principali aspetti innovativi, quasi "provocatori", caratterizzanti nel bene e nel male, l'iter progettuale del Piano del Parco.

Come prima cosa, è stata compiuta un'attenta verifica dei confini interni ed esterni del Parco naturale, in quanto la dettagliata fase analitico-diagnostica (illustrata in precedenza) ha messo a disposizione materiali assolutamente inediti (per esempio la carta dell'uso del suolo, quella delle unità di paesaggio, eccetera) che, uniti alle carte tematiche già disponibili (ma ulteriormente aggiornate e dettagliate in scala maggiore) hanno consentito di non ripetere gli errori materiali e concettuali precedentemente segnalati.

Inoltre, il Piano Territoriale ha potuto disporre per la prima volta nella storia della pianificazione di quest'area, di uno strumento conoscitivo particolarmente dettagliato, qual'è la Carta Tecnica Regionale in scala 1:10.000. Pertanto, pur rispettando in modo rigoroso l'attuale configurazione e localizzazione dei con- 
fini esterni ed interni, ha costituito l'occasione per una loro "interpretazione autentica", condotta cioè, sulla scorta delle ulteriori conoscenze, apportando le seguenti modifiche:

- "la confinazione è sempre stata specificata e resa certa rispetto alle principali caratteristiche insediative e geografiche dei luoghi, in modo da rendere chiaramente identificabile sul terreno, la soluzione di continuità tra area protetta e il suo esterno e fra le stesse sottozone interne al Parco. Dette confinazioni si sono interpretate come segue: nel caso di un crinale, il confine è identificato dalla linea di massima pendenza; nel caso di una soluzione di continuità nell'uso del suolo (per esempio bosco/prato-pascolo), il confine è identificato dallo stato reale dei luoghi; nel caso in cui il confine segue l'alveo di un corso d'acqua, deve intendersi che entrambe le sponde o ripe ricadono nella zona a maggior tutela; nel caso in cui il confine segue il tracciato di una strada o trazzera o sentiero esistente, deve intendersi che l'intero corpo stradale ricade nella zona a minor tutela;

- sono stati deliberatamente esclusi dal perimetro del Parco, sulla scorta delle numerose segnalazioni pervenute da Enti e privati, i manufatti ed i complessi edilizi che non sono assimilabili alle condizioni di stato delle diverse tipologie di aree a tutela naturalistica;

- sono stati classificati come parte integrante delle aree a minor tutela i manufatti edilizi e gli insediamenti non assimilabili alle aree a maggior protezione;

- sono state individuate e delimitate aree di modesta estensione su cui insistono manufatti o infrastrutture da considerare servizi di interesse collettivo, al fine di specificare nel Piano un'apposita normativa d'uso, sostanzialmente diversa da quella della sottozona circostante" 10 .

Il quadro delle proposte avanzate dal Piano in merito alle Politiche di Promozione Ricreativa, Turistica e Culturale ha invece mirato ad un fondamentale obiettivo: definire cioè "un modello di sviluppo personalizzato ed originale per questa montagna, legato al soddisfacimento delle aspettative dei visitatori del Parco, ma soprattutto congruente da un lato, con le esigenze conservazionali legate all'esistenza di una grande area protetta, e dall'altro con le attese delle comunità insediate che dovranno trovare nel Parco un'occasione di crescita economica, di rottura di isolamento, di conferimento di ruolo sociale al pari con i tempi"11.

Nell'attuazione delle strategie nel delicato settore turistico, si è cercato anzitutto di evitare l'adozione sia di politiche eccessivamente consumistiche, distruttici delle risorse, sia la promozione di atteggiamenti "ottusamente conservazionistici" che pretendono cioè di porre il territorio sotto una campana di vetro. Si è pertanto operato in modo che la cultura definita "del ritardo" potesse esprimere invece un'originale "cultura dello sviluppo", per consentire alle Madonie di trasformarsi in breve tempo, da "luogo di consumo di reddito, a luogo di produzione di reddito". Tuttora infatti, ad ogni ritorno della stagione estiva, ad ogni caduta della neve, si verifica che ondate di turisti provenienti dai centri urbani vicini e non solo, invadono caoticamente la montagna madonita, senza interessare minimamente o quasi i paesi, causando problemi di impatto anzichè prospettive per lo sviluppo.

\footnotetext{
${ }^{10}$ Guido Ferrara, op. cit,. 6-8 novembre 1997, pag. 2.

${ }^{11}$ Guido Ferrara e Giuliana Campioni, op. cit., 2000, pag. 35.
} 
Vediamo dunque sinteticamente, le soluzioni strategiche avanzate dal Piano Territoriale per ovviare a tale problema. È stata proposta in particolare:

- la progettazione di una rete capillare di servizi ricreativi e culturali basati sulla rivalorizzazione delle risorse ambientali, in grado di svolgere un fondamentale ruolo di informazione per residenti e turisti. Una vera e propria maglia di percorsi per le escursioni, il trekking e lo sci di fondo usufruibile sia in estate che in inverno, in alternativa all'attuale sistema sentieristico, caotico oltre che privo di riferimenti certi;

- la costruzione di un sistema integrato di trasporti e di aree di sosta e la possibile messa in opera di alcuni impianti di trasferimento del tipo "a fune" per poter, con il minimo livello d'impatto ambientale, consentire gli spostamenti nelle zone idonee allo sci di fondo;

- la progettazione di un nuovo sistema di attrezzature ricettive (accantonamenti, bivacchi, rifugi, campeggi, alberghi montani, centri di accoglienza, centri studi, eccetera) realizzato sia attraverso il recupero del patrimonio edilizio esistente, sia rafforzando (in termini di qualità e di quantità) l'attuale struttura di alloggi e ristoro.

L'offerta si è poi completata con due interessanti proposte: l'individuazione innanzitutto, di mete di importanza essenziale sia da un punto di vista naturalistico-ambientale che architettonico-culturale (con particolare riferimento alle presenze archeologiche); ed infine, la creazione attraverso un apposito Progetto, di una rete museale coordinata dal Museo del Territorio da sviluppare attorno a quella corona di centri abitati che costituiscono nei fatti i veri "biglietti da visita", le "porte d'ingresso", i punti di partenza ed arrivo di ogni escursione del Parco.

Le Politiche di Gestione Ambientale sono state invece descritte nell'elaborato conclusivo, "Destinazioni d'uso del suolo", realizzato attraverso l'incrocio tra le categorie di aree protette, come classificate e gerarchizzate nella carta descritta nel paragrafo precedente (vedi Fig.15), e l'azzonamento derivante dal Decreto Istitutivo del Parco (vedi fig.16), che il Piano Territoriale ha rispettato puntigliosamente, limitandosi a modestissimi cambiamenti di confine riferiti esclusivamente alla correzione di errori materiali.

In sostanza, sulla base della lettura delle reali condizioni di stato, si è potuto individuare, all'interno delle quattro grandi categorie di aree protette stabilite dalla Legge Regionale Istitutiva dei Parchi e delle Riserve in Sicilia (L.R. n. 98/1981) - Riserva integrale (zona A), Riserva generale (zona B), Zone di protezione (zona C), Zona di controllo (zona D) - delle sottozone "che specificano e dettagliano nei loro contenuti reali le caratteristiche delle tipologie di aree protette, consentendo di mettere in dettaglio le norme relative alla disciplina urbanistica e le modalità di gestione ambientale più appropriate. E ciò, con particolare riguardo ai grandi areali definiti dalla Legge n.98/1981 come Riserva integrale e Riserva generale, nei quali i vincoli destinati alla protezione della natura e dell'ambiente sono stati gerarchizzati con valore decrescente, non però in astratto, ma a seconda delle peculiari caratteristiche scientifiche ed ambientali delle sottozone stesse, indipendentemente se esse ricadano in zona A o B del Parco"12.

Sulla base delle premesse valutazioni, il grande areale contenente le emergenze geomorfologiche e ambientali, le sorgenti, le aree di ricarica degli acquiferi, i prati-pascoli d'altitudine, le faggete, i popolamenti forestali a rovere e agrifoglio, i biotopi, ovvero le più eclatanti e vulnerabili risorse presenti nel Parco, è stato localizzato come vera e propria "Area di preminente interesse naturalistico", cioè come sito ove le attività produttive e la stessa presenza umana sono da considerarsi in sottordine rispetto ai caratteri naturali e ai loro meccanismi di riproducibilità. È da notare inoltre che alcune zone "D" (Zona di controllo) restano

\footnotetext{
${ }^{12}$ Guido Ferrara e GiUliana Campioni, op. cit., 1999, pag. 93.
} 
incluse in modo organico in questa nuova perimetrazione, con l'importante conseguenza di venire sottratte alla normativa urbanistica comunale.

Dalla lettura dell'elaborato finale si può in pratica individuare l'articolazione della zonizzazione delle quattro categorie e delle loro relative sottozone. Si inizia con la categoria A "Riserva Integrale" così composta: A1 - riserva integrale di prevalente interesse scientifico, $A 2$ - riserva integrale di protezione di aree remote e dei santuari di natura, A3/B1 - biotopo, riserva floristica o vegetazionale, A4/B2 - riserva forestale, A5/B3 - riserva geologico ambientale, A6/B4/D1 - pascoli montani, A7/B5/D2 - bosco ad evoluzione naturale, A10 - foresta giardinata.

All'interno delle Riserve integrali, il Piano Territoriale è stato in grado di specificare ben 9 differenti tipologie, in quanto le condizioni risultanti di ciascuna di esse risultano spiccatamente caratterizzate e, pertanto, suggeriscono modalità di gestione conservazionale altrettanto diverse. Non si tratta però di una semplice differenziazione nominalistica, ma di vere e proprie politiche di tutela differenziate: in effetti, accanto alle zone da classificare quali Riserve integrali di prevalente interesse scientifico, dove la normativa prescrive non solo il divieto dello svolgimento di qualunque attività, ma perfino l'abbandono dei sentieri da parte del visitatore, si collocano le Riserve integrali di protezione di aree remote e dei santuari di natura, dove tale divieto non ha ragione d'essere, pur rimanendo le aree stesse assolutamente interdette a qualunque tipo di insediamento, attività produttiva o raccolta di prodotti. Le zone definite Biotopo e Riserva floristico vegetazionale, identificano invece areali caratterizzati da forti diversità naturalistiche, la cui presenza sulle Madonie non solo non interferisce con la prosecuzione degli usi pastorali, ma trae addirittura dalla presenza di questi, alcuni fattori di supporto; pertanto sono stati vietati prelievi ma vi è consentito il mantenimento del pascolo.

Non così nella Riserva forestale, dove la presenza di soprassuoli e di componenti vegetazionali particolarmente significative, pur consentendo la raccolta dei prodotti del sottobosco, non ammette la pratica del pascolo, che sicuramente ha danneggiato in passato la rinnovazione naturale della vegetazione arborea ed arbustiva.

Ancora un diverso scenario è proposto dalle Riserve geologico ambientali, che devono le loro particolari esigenze conservative alla presenza di emergenze geologiche o geomorfologiche, e che pertanto nulla hanno da temere né dagli usi agro-silvo-pastorali di tipo tradizionale, né tanto meno dai prelievi delle risorse riproducibili, né infine dalle attività escursionistiche.

Il Piano Territoriale ha individuato altresì una vasta superficie caratterizzata da forme diverse di soprassuoli, ma sempre riconducibile al prato-pascolo, definita Pascoli Montani. In questo caso è la forestazione, attuata a mezzo di cantieri forestali, ad aver prodotto negli anni passati rilevanti e non sempre desiderabili trasformazioni. Il Piano Territoriale ha assunto per tanto la difesa del paesaggio pastorale proponendo la conservazione per le sue peculiari caratteristiche.

Sempre nelle Riserve integrali si è rilevata la presenza di ampie fasce boscate che, diversamente dalle Riserve forestali, hanno meritato una forma di governo finalizzata alla gestione del bosco in termini di presidio ambientale, di tutela del paesaggio, e di supporto alle attività ricreazionali, oltrechè a fini produttivi di tipo selvicolturale.

E stato infine perimetrato un areale di contenute dimensioni connotato dal carattere monumentale delle formazioni forestali e denominato "Foresta giardinata". In questo specifico settore, le strategie si sono indirizzate ad un tipo di governo e di trattamento del sistema forestale finalizzato all'esaltazione della biodiversità e della funzione culturale del bosco.

Infine, insiste nella zona di Riserva integrale (zona A) un solo caso di paesaggio agrario, storicamente insediato, dove la finalità conservazionale è stata individuata soltanto nella tutela degli emergenti aspetti geomorfologici.

Le Riserve generali hanno subito praticamente lo stesso tipo di gerarchizzazione interna e pertanto finiscono per afferire, dal punto di vista delle destina- 
zioni d'uso del suolo, alle precedenti categorie, ricomponendo così caratteri geomorfologici, soprassuoli e paesaggi assolutamente identici e dando vita ad un'unica forma di gestione conservazionale.

Dalle zone a Riserva integrale e generale, si è passati alle Zone C (Zone di Protezione).

Il Piano in questo caso, ha dovuto riconoscere sostanziali differenze sia all'interno delle aree $\mathrm{C}$ cosiddette "estese", che all'interno delle C "puntuali". Si è pertanto attuata una gerarchizzazione che ha permesso di distinguere 7 differenti tipi di zone estese e 8 diverse tipologie di $\mathrm{C}$ puntuali, secondo il seguente elenco: (Aree Estese) C1 - Aree altomontane di tipo pluriuso; C2 - Alberghi e rifugi montani con servizi; C3 - Campeggi con aree attrezzate per attività ricreative e sportive; C4 - Stabilimenti termali e relative aree attrezzate; C5 - Ricettività organizzata di tipo sociale con servizi; C6 - Aree di picnic; C7 - Attrezzature sportive speciali; (Aree Puntuali) Porta del Parco, meta, ricettività organizzata di tipo sociale, centro didattico-informativo, rifugio, centro di accoglienza, accantonamento, bivacco e servizio di soccorso alpino.

In particolare, le zone $\mathrm{C}$ estese, di cui il Piano Territoriale ha predisposto gli indirizzi normativi quadro e le destinazioni d'uso, sono state specificate in termini urbanistici tramite: Piani di recupero ambientale, edilizio e funzionale; Concessioni edilizie per gli interventi di razionalizzazione e riqualificazione delle strutture alberghiere delle zone altomontane, (con particolare riferimento all'adeguamento della rete fognante e dei sistemi di depurazione); Piani particolareggiati, per la creazione di aree di campeggio e di attrezzature ricreative e sportive o per il completamento e la riqualificazione di quelle esistenti; Piani particolareggiati per gli stabilimenti termali e le relative aree attrezzate.

Si sono poi individuate le Aree di Tutela e di Intervento Speciale, fondamentali per stabilire ulteriori importanti precisazioni localizzative in merito alle aree ad inedificabilità assoluta, alle aree destinate ad interventi di restauro ambientale ed alle aree da assoggettare a Piani di recupero del paesaggio.

In tutte le Zone A e B è previsto il divieto assoluto di edificabilità (salvo le attività di recupero e gestione del patrimonio esistente); altresì molte delle Zone $\mathrm{C}$, ricadenti all'interno dei confini dell'area a preminente interesse naturalistico, sono state praticamente interdette all'edificazione; infine, per la rimanente Zona $\mathrm{D}$ si è effettuata una perimetrazione degli unici casi in cui l'inedificabilità si rendeva necessaria.

Le aree destinate ad interventi di restauro ambientale sono state invece individuate e cartografate sulla base delle indicazioni della fase analitico-diagnostica del Piano Territoriale e comprendono discariche dismesse, cave di pietra inattive $o$ in esercizio, aree sottoposte a scavi, sbancamenti e riporti per la realizzazione di opere di captazione idrica.

Per quanto riguarda infine le aree da assoggettare a Piano di recupero del paesaggio, "sono stati segnalati alcuni areali cerniera, di contatto fra il Parco Naturale e le zone $\mathrm{D}$ di paesaggio agrario, che soffrono forme di degrado e di mancanza di identità tipiche delle zone di frangia. Per queste aree si è reso pertanto necessario un intervento attivo da parte del Parco, capace di pilotare l'auspicabile ritorno a forme di equilibrio, con particolare riferimento all'assunzione di nuove stabili configurazioni dei soprassuoli”'13.

Il Piano Territoriale ha inoltre rilevato alcune situazioni obiettive di "paesaggio protetto" anche nelle zone A e B, proprio laddove apparirebbe del tutto inconsueta la definizione di riserva integrale o orientata, come nel caso dei rimboschimenti o del paesaggio agrario di tutela geologico-ambientale. Rientrano in

\footnotetext{
${ }^{13}$ Guido Ferrara, op. cit., 6-8 novembre 1997, pag. 13.
} 
questa categoria "i contesti ambientali di antica e permanente antropizzazione, la cui conservazione e manutenzione è affidata soprattutto alla continuità nel tempo delle pratiche culturali e alla correttezza e compatibilità degli stessi fenomeni insediativi." 14 Pertanto alla categoria di "paesaggio protetto" afferiscono le seguenti situazioni ambientali: A8/B6 - Impianti artificiali; A9/B7 - Paesaggio agrario di tutela geologico-ambientale, B8/B9/D3/D4/D5/D7 - Paesaggio agrario di preminente interesse ambientale; D6 - Area protetta a tutela ordinaria.

Siamo di fronte dunque ad una strategia pianificatoria decisamente articolata, innovativa e graduale, entro cui la variabile tempo, più di qualunque altra, è considerata elemento cardine per la maturazione di situazioni di governo dell'ambiente e del territorio certamente più avanzate delle attuali.

In particolar modo si è tenuto conto dell'esistenza, anche nel caso del comprensorio madonita, come del resto in tutti quelli inerenti la pianificazione di aree protette, del timore delle comunità locali di vedersi progressivamente espropriate dei diritti d'uso del proprio ambiente e territorio, in analogia ad altri casi italiani in cui proprio la presenza di un Parco naturale ha determinato ed esasperato i conflitti, anziché risolverli. Questo però, come più volte sottolineato dai progettisti, non deve essere assolutamente paragonato ad un tentativo di promozione della politica dei compromessi, ma alla volontà di attuare una trasformazione consapevole dei comportamenti rispetto alla gestione delle risorse stesse. Ciò anche tenendo conto della sostanziale validità di alcune forme di autogoverno preesistenti che, storicamente e culturalmente, hanno dimostrato notevole efficacia conservazionale.

Rispetto a quanto esposto, appare utile in conclusione proporre un quadro riepilogativo della strategia pianificatoria avanzata. In particolare, dalla lettura del prospetto di seguito allegato risulta come:

- il "parco naturale" vero e proprio, cioè quella parte del territorio madonita che risponde ai requisiti di tale istituto di tutela, da tempo consolidati su base internazionale, copre esattamente 21.500 ettari, pari al 53\% dell'area protetta, e purchè si comprenda in esso la totalità della "riserva pastorale" che con i suoi 12.784 ettari interessa quasi il 60\% della superficie utile;

- le aree di riserva integrale (nel senso stretto del termine di istituti conservazionali a doc, e non intese quindi come zone vincolate di tipo A), appaiono alquanto modeste, essendo pari solo al 5,3\% del totale;

- alle aree più interessate da attività antropiche permanenti, (coltivazioni, insediamenti, rimboschimenti, degradi, eccetera) deve essere imputata invece una superficie territoriale relativamente ampia, essendo questa pari a quasi 19.000 ettari, ovvero al $47 \%$ del totale.

\footnotetext{
14 Guido Ferrara e Giuliana Campioni, op. cit., 2000, pag. 39.

15 Guido Ferrara e Giuliana Campioni, op. cit., 1999, pag. 103.
} 
PROSPETTO DI SINTESI ${ }^{15}$

\begin{tabular}{|c|c|c|c|c|}
\hline $\begin{array}{l}\text { CAT. } \\
\text { I.U.C.N. }\end{array}$ & $\begin{array}{c}\text { Tipologie Delle AREE } \\
\text { PROTETTE }\end{array}$ & $\begin{array}{l}\text { Area di preminente } \\
\text { interesse naturalistico } \\
\text { perimetrata dal Piano } \\
\text { Territoriale (cifre in ettari) }\end{array}$ & $\begin{array}{l}\text { Restante area protetta } \\
\text { come definita dal } \\
\text { Decreto Assessorile, } \\
9 \text { novembre } 1989\end{array}$ & $\begin{array}{c}\text { Area contigua } \\
\text { e ulteriore } \\
\text { area protetta } \\
\text { come proposta } \\
\text { dal Piano Territoriale }\end{array}$ \\
\hline I & $\begin{array}{c}\text { A1 - RISERVA INTEGRALE DI } \\
\text { PREVALENTE INTERESSE } \\
\text { DI SCIENTIFICO }\end{array}$ & 575,84 & & \\
\hline I & $\begin{array}{c}\text { A2 - RISERVA INTEGRALE DI } \\
\text { PROTEZIONE DI AREE } \\
\text { REMOTE E DEI SANTUARI } \\
\text { DI NATURA }\end{array}$ & $1.589,66$ & & \\
\hline IV & $\begin{array}{l}\text { A3/B1 - BIOTOPO, RISERVA } \\
\text { FLORISTICA O VEGETAZIONALE }\end{array}$ & 488,02 & & \\
\hline IV & A4/B2 - RISERVA FORESTALE & 757,32 & 122,27 & \\
\hline IV & $\begin{array}{c}\text { A5/B3 - RISERVA GEOLOGICO } \\
\text { AMBIENTALE }\end{array}$ & 444,95 & 104,12 & \\
\hline IV & A6/B4/D1 - PASCOLI MONTANI & $12.784,50$ & & $\begin{array}{c}22,96 \\
\text { (interna al parco) }\end{array}$ \\
\hline IV & $\begin{array}{c}\text { A7/B5/D2 - BosCO } \\
\text { AD EVOLUZIONE NATURALE }\end{array}$ & $4.859,85$ & 364,20 & \\
\hline VI & $\begin{array}{l}\text { A8/B6 - IMPIANTI ARTIFICIALI } \\
\text { E A10 - FORESTA GIARDINATA }\end{array}$ & & 985,42 & \\
\hline V & $\begin{array}{c}\text { A9/B7/D3/D4 - PAESAGGIO } \\
\text { AGRARIO DI TUTELA } \\
\text { GEOLOGICO-AMBIENTALE } \\
\text { E PAESAGGIO AGRARIO } \\
\text { DI PREMINENTE INTERESSE } \\
\text { AMBIENTALE }\end{array}$ & & $3.231,09$ & $\begin{array}{c}3.463,64 \\
\text { (area contigua) }\end{array}$ \\
\hline V & $\begin{array}{l}\text { B8/D5 - AREA DI TUTELA } \\
\text { PAESAGGISTICA GENERALE }\end{array}$ & & $7.181,36$ & \\
\hline $\mathrm{V}$ & $\begin{array}{l}\text { D6 - AREA PROTETTA } \\
\text { A TUTELA ORDINARIA }\end{array}$ & & $5.542,33$ & \\
\hline VI & $\begin{array}{c}\text { C/B9/D7 - AREE } \\
\text { DA PROGRAMMARE E DA } \\
\text { ASSOGGETTARE A PIANI DI } \\
\text { RECUPERO DEL PAESAGGIO }\end{array}$ & 95,49 & $1.391,11$ & \\
\hline (ETTARI) & $\begin{array}{l}\text { TOTALI PARZIALI } \\
\text { TOTALI COMPLESSIVI }\end{array}$ & $21.595,63$ & $\begin{array}{l}18.921,90 \\
40.517,53\end{array}$ & $\begin{array}{l}3.486,60 \\
44.004,13\end{array}$ \\
\hline
\end{tabular}

\section{RIFERIMENTI BIBLIOGRAFICI}

Raimondo Francesco Maria (a cura di), Il Parco delle Madonie, un crocevia dove convivono le piante di tre continenti, Ed. Arbor, Palermo 1993.

Ferrara Guido (a cura di), I.U.C.N., Parchi naturali e cultura dell'uomo, Ed. Maggioli, Rimini 1994.

Ferrara Guido, Il Piano Territoriale del Parco delle Madonie, intervento al Convegno di Genova "Pianificazione e sviluppo locale in ambienti sensibili: esperienze di progettazione e gestione in alcuni paesi mediterranei”, Genova, 6-8 novembre 1997. 
Ferrara Guido e Campioni Giuliana, Piano Territoriale del Parco delle Madonie - Relazione di sintesi, Palermo 1999 (versione su Cd-rom gentilmente concessa dagli autori del Piano).

Ferrara Guido e Campioni Giuliana, Il Piano del Parco delle Madonie in Sicilia, "Architettura del Paesaggio", 4, 2000, pagg. 34-39.

InGEGNOLI VITTORIO, Fondamenti di ecologia del paesaggio, Ed. Cittàstudi, Milano 1993.

\section{RIFERIMENTI ICONOGRAFICI}

Le immagini di seguito riportate sono rielaborazioni di Michele Ercolini da RAIMONDO FRANCEsCo Maria (a cura di), Il Parco delle Madonie, un crocevia dove convivono le piante di tre continenti, Ed. Arbor, Palermo 1993.

Figure 1, 2, 3, 4, 7, 9, 11,12: Franco Barbagallo, op. cit., 1993, pagg. 26, 128, 129, 76, 32, 41, 203, 202.

Figure 5, 6, 8: Benedetto Abate, op. cit., 1993, pagg. 43, 36, 45.

Figura 10: Nello Gaudioso, op. cit., 1993, pag. 69.

Figg.13-18: immagini gentilmente concesse dall'autori del Piano, Prof. Arch. Guido Ferrara, Arch. Giuliana Campioni. 
Progetto:

Luogo:

Committente:

Superficie:

Progettisti:

Collaboratori:

ConsulenZE TECNICO-SCIENTIFICHE

Centro Interdipartimentale di Ricerca sulle Interazioni Tecnologia e Ambiente dell'Università di Palermo (CIRITA)

Idrogeologia e risorse idriche: Prof. Gioacchino Cusimano, con la collaborazione del Dr. Antonio Contino

Geomorfologia: Prof. Valerio Agnesi, con la collaborazione del Dr. Cipriano di Maggio e del Dr. Calogero Gagliano

Geolitologia: Prof. Benedetto Abate, con la collaborazione del Prof. Antonino di Cara, Dr. Giuseppe Greco e Dr. Fabrizio Nigro

Pedologia: Prof. Giovanni Fierotti, con la collaborazione del Prof. Carmelo Dazzi e della Dr.ssa Donatella Marchiafava

Botanica e fitogeografia: Prof. Piero Mazzola, con la collaborazione del Dr. Lorenzo Gianguzzi Selvicoltura e assestamento forestale: Prof. Giuseppe Asciuto

Economia agraria: Prof. Maria Crescimanno

Agronomia e coltivazioni: Prof. Giuseppe Barbera, Prof. Giovanni Fatta del Bosco;

Zootecnia e produzioni animali: Prof. Giovanni Leto

Zoologia e faunistica: Prof. Bruno Massa, con la collaborazione del Dr. Maurizio Sarà e Dr. Fabio Lo Valvo

Coordinamento CIRITA: Prof. Raimondo Catalano

\section{ESPERTI E CONSULENTI DI SETTORE}

Antropologia: Prof. Mario Giacomarra, Università di Palermo

Rilevamento servizi, itinerari, sentieri e infrastrutture: Cooperativa Agritur, Polizzi Generosa Schedatura beni architettonici comuni di Polizzi Generosa e Petralia Soprana: Cooperativa Agritur, Polizzi Generosa

Schedatura beni architettonici altri comuni del parco: Arch. Antonio Michele Dino, Castellana Sicula Rilevamento vincoli e proprietà demaniali: dr. Antonio Sferruzza, Castelbuono

Anno di progettazione: l'incarico di redazione del Piano Territoriale del Parco delle Madonie è stato affidato con delibera del Comitato Esecutivo del Parco, il 15 giugno 1993

Anno di realizzazione (tempi di esecuzione): la fase analitica è stata completata in data 27 novembre 1994. La fase propositiva è stata presentata in bozza in data 27 Maggio 1995. Il Piano Territoriale è stato infine adottato con delibera del Consiglio del Parco, il 29 ottobre 1999 



\title{
IL PIANO del PARCo NatURALE REGIONALE \\ DI MIGLIARINO SAN ROSSORE \\ MASSACIUCCOLI IN TOSCANA
}

\author{
Andrea Meli
}

\section{IL CONTESTO}

L'area pianeggiante della costa settentrionale toscana rappresenta l'ultima fase della formazione della bassa valle del fiume Arno.

Il Parco Naturale Regionale di Migliarino San Rossore Massaciuccoli si estende su quello che, in epoca storica, è stato l'ulteriore ampliamento dell'area di pianura, in larga parte dovuto all'apporto solido dei fiumi Arno e Serchio.

Fino ai primi decenni del Novecento, questa zona risultava occupata da ampie ed estese aree umide, interessate dalle piene periodiche dei due fiumi, con le conseguenti modificazioni alla morfologia superficiale del terreno ed un dinamismo di trasformazione piuttosto marcato, e risultava caratterizzata da un sistema di cordoni dunali ben riconoscibili.

Fra il 1920 ed il 1940 vennero attuati estesi interventi di bonifica, che interessarono sia l'intera zona, sia la regimazione dei corsi dell'Arno e del Serchio, determinando quindi un rapido processo di stabilizzazione della morfologia e delle caratteristiche naturali dell'area nel suo complesso ${ }^{1}$.

Le principali trasformazioni territoriali ed ambientali della zona attualmente interessata dall'area protetta risalgono ai secoli XV e XVI, indotte dai primi processi di antropizzazione diffusa, che hanno inciso su quella che era la struttura morfologica ed ambientale prevalente, e cioè estese formazioni forestali con associazioni vegetali proprie delle aree umide, a prevalenza di specie igrofile (Acer campestre, Alnus glutinosa, Fraxinus angustifolia, Populus alba, Quercus robur, Ulmus minor).

Con il passaggio della Repubblica di Pisa sotto l'influenza di Firenze, nel 1406, ha inizio un lungo periodo nel quale si assiste alla nascita di grandi tenute e fattorie, legate alle più influenti famiglie fiorentine: la tenuta Salviati a Migliarino, le tenute medicee di San Rossore, Col-

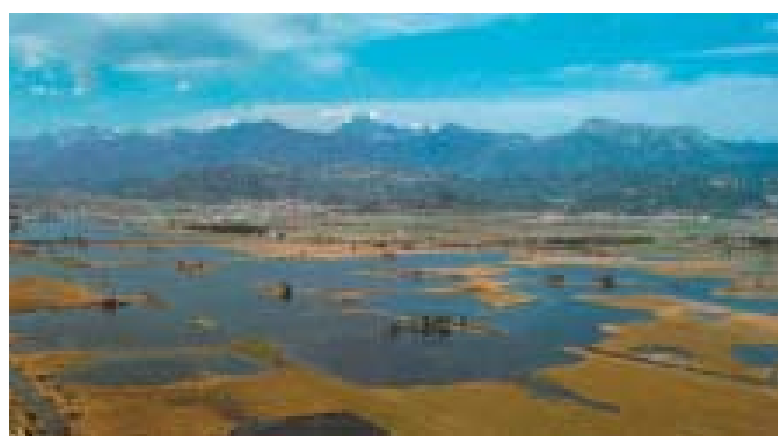

Figura 1 - Una vista aerea del Padule di Massaciuccoli: sullo sfondo la catena montuosa delle Alpi Apuane. tano e castagno lo. le tenute infine di Vecchiano, Casablanca e Collesalvetti ${ }^{2}$.

Con la nascita e l'evoluzione delle tenute, inizia un lento ma costante fenomeno di inurbamento e di diffusione degli insediamenti nelle zone più interne, mentre le aree costiere rimarranno a lungo spopolate.

Nella seconda metà del XVIII secolo, sotto il governo del Granduca Pietro Leopoldo, le tenute vengono progressivamente trasformate in riserve di caccia,

${ }^{1}$ Una residua variabilità morfologica naturale è ancora presente alla foce del fiume Serchio, mentre le attuali forti modificazioni alla linea di costa nell'area interessata dal fiume Arno, in ragione della loro dipendenza da azioni antropiche (escavazioni in alveo, pennelli, dighe a mare, eccetera), non si possono considerare come variazioni morfologiche naturali.

${ }^{2}$ Attualmente il sistema delle Tenute, seppure modificato nel corso dei secoli, è ancora percepibile e rappresenta una delle strutture portanti dell'assetto territoriale ed ambientale del Parco. Le Tenute attuali sono: Coltano, Macchia Lucchese, Migliarino, San Rossore, Tombolo, molto diversificate al loro interno per numero e struttura delle aziende agricole operanti. 


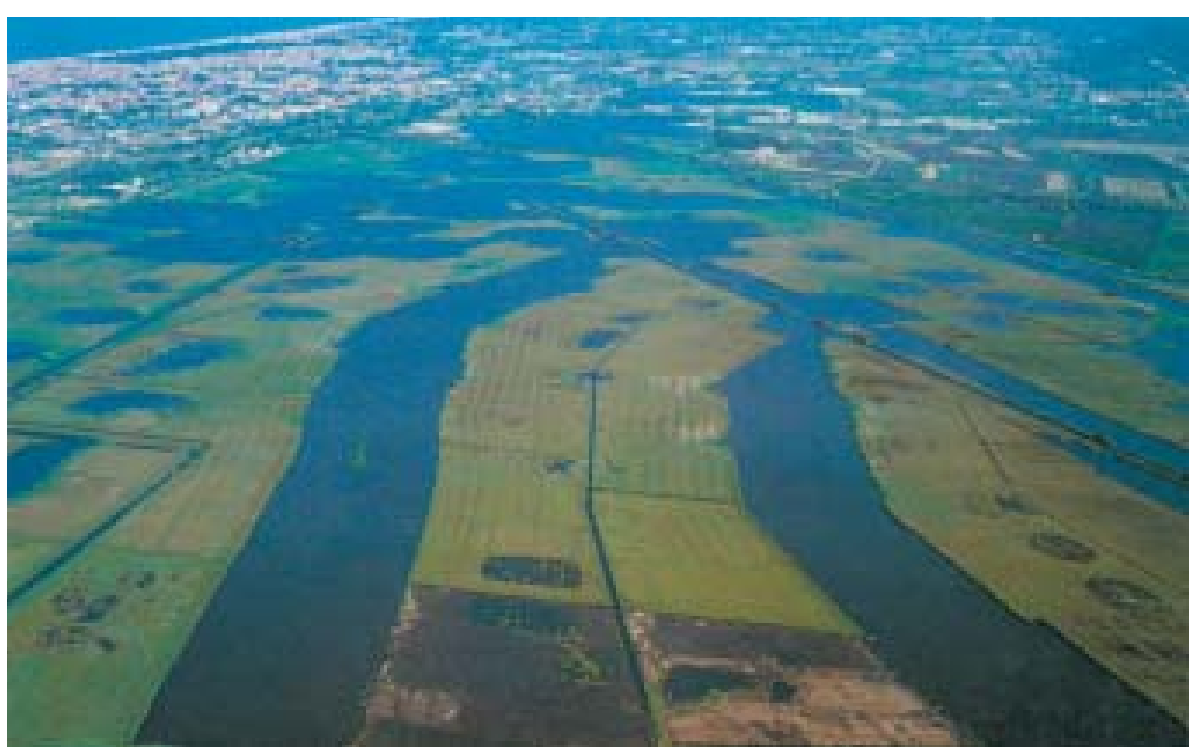

Figura 3 - Veduta a volo d'uccello del Padule di Massaciuccoli in direzione del litorale costiero della Versilia.

sostituendo in gran parte al ruolo produttivo una valenza ricreativa, costituendo di fatto un esteso sistema di "aree naturali", che si sono conservate in gran parte inalterate fino alla prima metà del XX secolo.

Quello che oggi vediamo è possibile definirlo come un sistema di ambienti naturali, interessato da forti elementi di caratterizzazione antropica, dovuti anche agli sviluppi urbanistici che hanno interessato i margini ed i confini dell'area specialmente nel corso del XX secolo ${ }^{3}$.

Attualmente il territorio del Parco è interessato da un complesso mosaico ambientale, che per grandi classi ambientali si articola in aree boscate, aree agricole ed aree umide, all'interno delle quali la variabilità ecosistemica raggiunge anche livelli di notevole interesse e rarità (a solo scopo esemplificativo si ricorda, ad esempio, il sistema delle zone umide delle Lame di San Rossore, caratterizzate da un alternarsi di tomboli ${ }^{4}$ e lame ${ }^{5}$, che formano habitat di grande interesse dal punto floristico e faunistico).

\section{GLI OBIETTIVI}

Nel 1979, a seguito di una forte campagna di opinione pubblica e non senza forti contrasti, e facendo seguito ad una lunga vicenda che ha inizio a partire dagli anni Cinquanta, la Regione Toscana istituisce il Parco Naturale Regionale ${ }^{6}$, con lo scopo di tutelare e conservare le caratteristiche naturali, ambientali e storiche del litorale pisano e lucchese, in funzione anche dell'uso sociale di questi valori e promuovendo le attività di ricerca scientifica e la didattica ambientale.

\footnotetext{
${ }^{3}$ Basti pensare che l'area del parco ha delle strette relazioni con le città di Pisa, Livorno e Viareggio, oltre che con una serie di centri balneari di notevole importanza quali Torre del Lago, Marina di Pisa, Tirrenia. L'area è inoltre interessata direttamente ed indirettamente da infrastrutture lineari (autostrada A12, strada statale Aurelia, ferrovia) e da infrastrutture puntuali (aereoporto di Pisa, porti di Livorno e Viareggio), che contribuiscono, assieme al sistema di attività produttive legate ai centri urbani, a definire un quadro di forte urbanizzazione e carico antropico nel quale il parco si colloca.

${ }^{4}$ I tomboli sono antichi cordoni dunali interessati da vegetazione mista a Pinus pinaster, Pinus pinea, Quercus ilex e Quercus pubescens.

${ }^{5}$ Le lame sono paludi retrodunali, in genere originate in prossimità delle foci dei fiumi, di carattere spesso permanente ed interessate da vegetazione igrofila di acque dolci e/o salmastre.

${ }^{6}$ Legge Regionale Toscana n. 61 del 13 dicembre 1979 "Istituzione del Parco Naturale di Migliarino San Rossore Massaciuccoli”.
} 


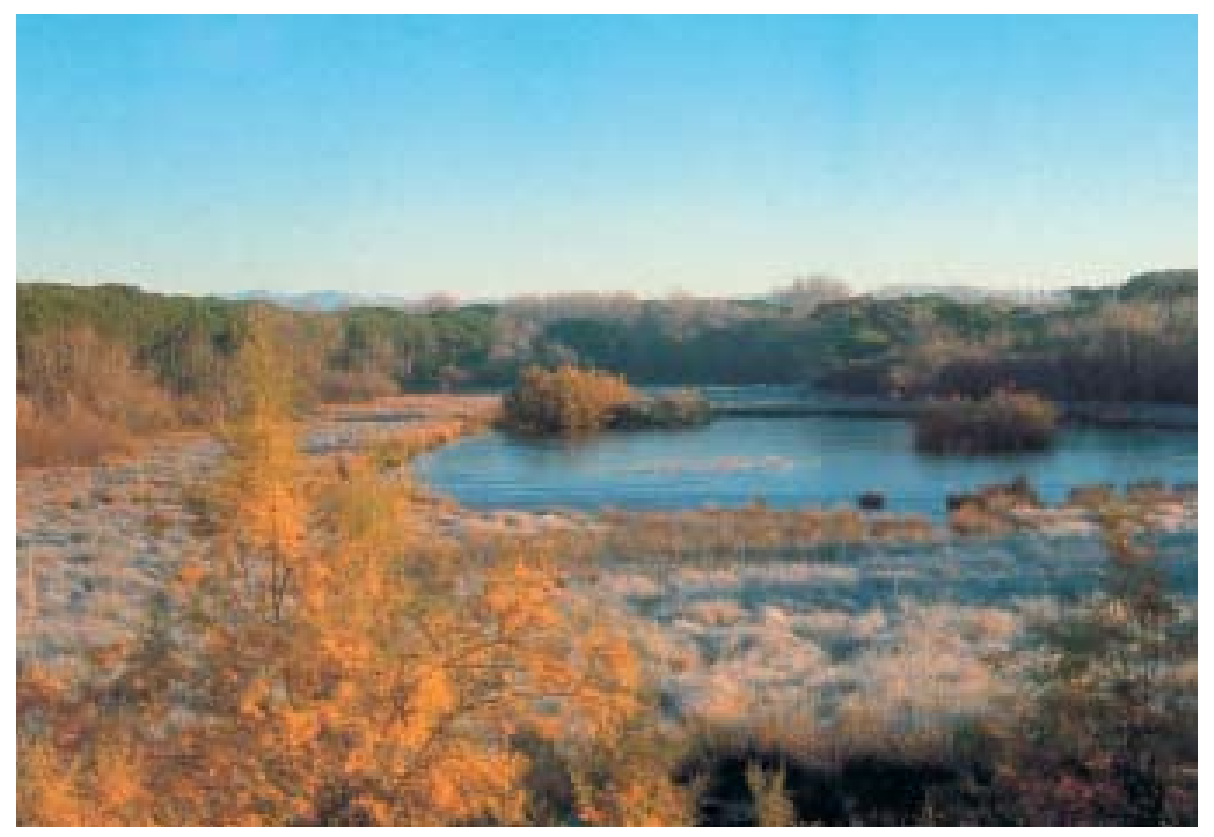

Figura 4 - Le caratteristiche aree umide retrodunali (lame) presenti nella Tenuta di San Rossore.

Attualmente il territorio del Parco interessa un'area di circa 23.000 ettari, ed il Piano li disciplina sia attraverso le previsioni in esso contenute, sia attraverso il rimando a specifici Piani di Gestione.

Con il Piano del Parco, si trattava di dare risposta al destino di un'area costiera di grande importanza dal punto di vista della ricchezza delle risorse ambientali, oltre che prefigurare un nuovo destino ad un territorio interessato da forti pressioni antropiche, strettamente legato ad importanti città (Pisa, Livorno) ed a famosi e frequentati centri balneari (Viareggio, Torre del Lago, Tirrenia, Marina di Pisa), tanto da poter considerare l'area protetta a tutti gli effetti inserita all'interno di una complessa area urbanizzata ed antropizzata.

Certamente la ricchezza e la diversificazione degli ambienti naturali ha rappresentato, insieme alla presenza umana diffusa, motivo di contrasto/arricchimento del Piano che, fin dall'inizio, ha cercato uno stretto legame con le vicende storiche che hanno portato alla definizione della attuale struttura territoriale.

\section{LA METODOLOGIA}

Dall'istituzione dell'area protetta, al momento in cui questa si è dotato di uno strumento di pianificazione, sono passati circa dieci anni: è del 1989 l'approvazione del Piano Territoriale, elaborato da un gruppo di lavoro coordinato dall'architetto Pier Luigi Cervellati .

Fin dall'inizio della suo processo, il Piano ha posto due questioni preliminari che hanno accompagnato la sua redazione: "la prima questione riguardava lo stato della pianificazione dell'ampio territorio, una vera e propria area sub-regionale, in cui è inserito il Parco; l'altra concerneva gli obiettivi - culturali ed economici che si intendevano perseguire con la realizzazione del progetto di Parco" ${ }^{8}$.

\footnotetext{
${ }^{7}$ Oltre al coordinatore, il gruppo era formato dall'Arch. Enza Citterio, dall'Ing. Roberto Corlaita, dall'Arch. Giovanni Maffei Cardellini, dall'Arch. Enrico Ghiselli, dall'Ing. Stefano Paglialunga.

${ }^{8}$ Pier Luigi Cervellati e Giovanni Maffei Cardellini, (a cura di), Il Parco di Migliarino San Rossore Massaciuccoli: la storia e il progetto, Giunta Regionale Toscana-Marsilio Editori, Venezia 1988, pag. 163.
} 


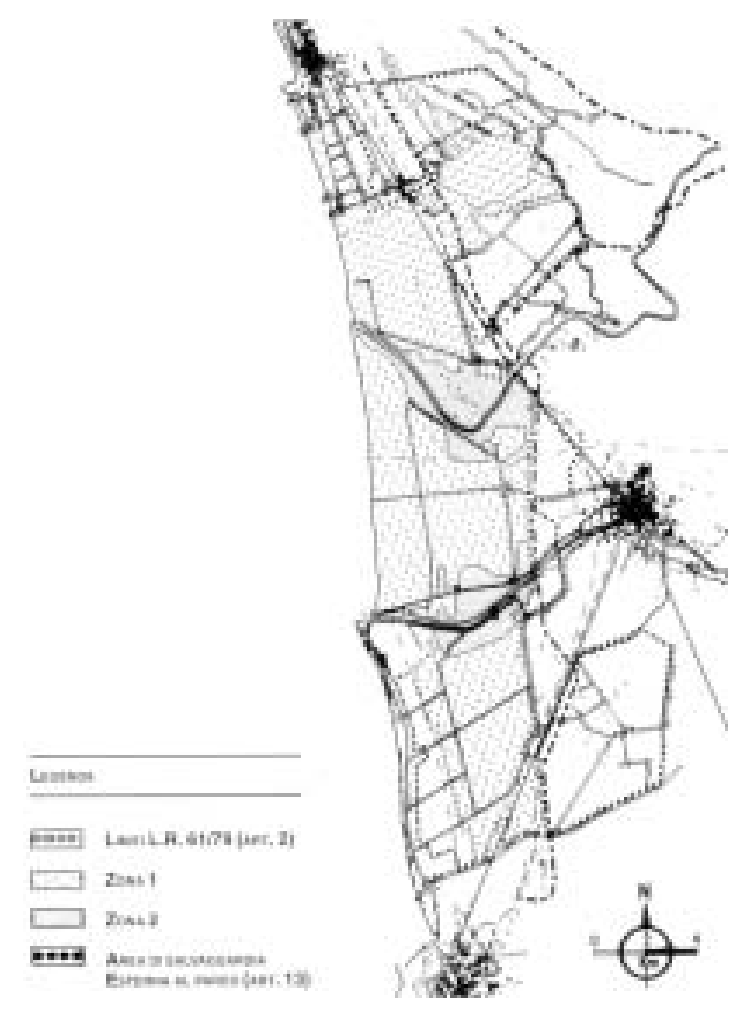

Figura 5 - La zonizzazione conseguente alla legge di istituzione dell'area protetta.

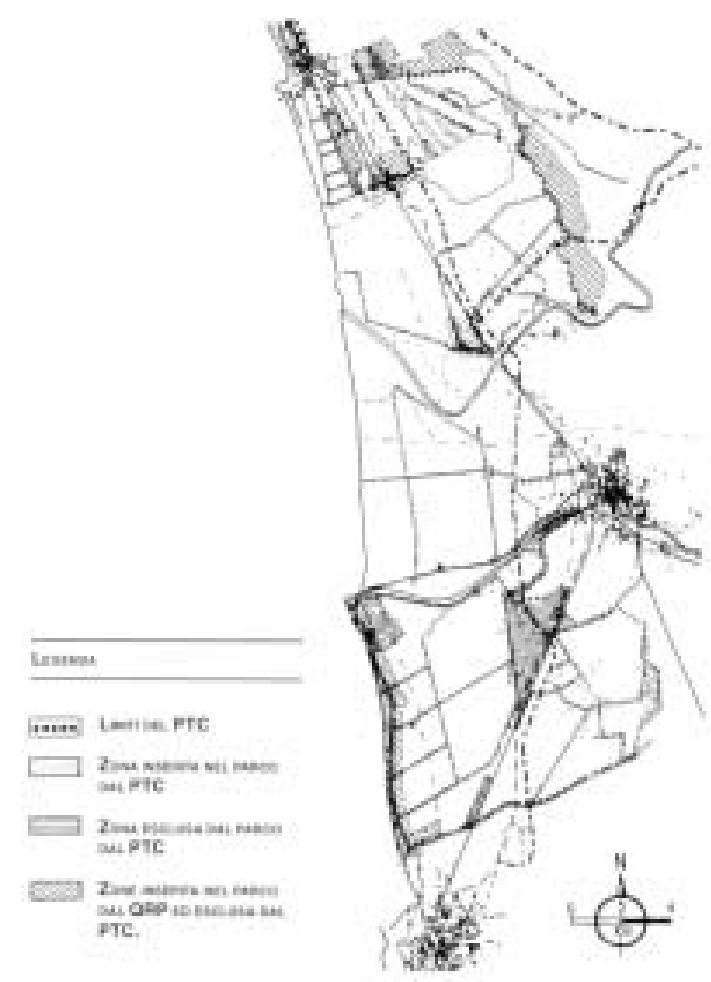

Figura 6 - I limiti definiti dal Piano Territoriale di Coordinamento del Parco.

Questa era una esigenza sentita in gran parte per l'assenza di quadri di riferimento di pianificazione sovracomunale, e per la limitatezza metodologica, culturale e programmatoria dei singoli strumenti urbanistici di livello comunale, che informavano le linee di pianificazione e gestione territoriale.

Inoltre, vi è da ricordare che il Piano nasceva in un periodo nel quale la realtà italiana in materia di pianificazione e gestione delle risorse naturali non disponeva di molti riferimenti metodologici ed esperienze consolidate, tali da delineare uno specifico filone culturale della progettazione e pianificazione delle aree protette in Italia.

Certamente, dal punto di vista metodologico, nel processo di formazione del Piano ha assunto grande rilievo la storia del territorio interessato dall'area protetta, come indicato dagli stessi autori del Piano secondo i quali "alcuni principi sono comunque irrinunciabili e consequenziali (...). Il primo di questi principi è relativo all'equivalenza della conoscenza con la prassi pianificatoria. Non si può progettare senza conoscere, senza sapere, senza capire il perché, il quando e il come avvengono sul territorio le trasformazioni antropiche, oltre a quelle vegetazionali, faunistiche, geomorfologiche. E poi, è fondamentale, si deve saper tradurre questa conoscenza in scelte progettuali che sappiano evidenziare, e dunque mantenere/rinforzare, i caratteri, la peculiarità del territorio interessato alla progettazione"'.

Certamente questa impostazione preliminare alla redazione del piano trova una sua ragione di essere nella particolare condizione territoriale ed ambientale nella quale si colloca l'area interessata, in un insieme di storia e natura, di presenza umana e di ambienti naturali, di zone interessate da forti processi di urbanizzazione e intensi carichi di presenze estive, di luoghi importanti per la conservazione del sistema delle risorse naturali. 
Quindi "Migliarino San Rossore Massaciuccoli, in termini generali, quale Parco non solo istituzionale, bensì quale luogo in cui stupire e istruire, può rappresentare l'alternativa economica - e dunque culturale - a quell'area metropolitana ora inquinante e schizofrenica nel suo volere essere tutto: porto/industria/litorale/vacanza/residenza/agricoltura/territorio (...)"10.

L'esigenza di un confronto preliminare con gli enti locali interessati, insieme quindi alla necessità di creare un quadro di riferimento programmatico e culturale che informasse la redazione del Piano, ha delineato un momento intermedio $^{11}$, che ha definito alcuni riferimenti progettuali relativi a:

- l'individuazione delle strutture storiche di gestione del territorio quali elementi di organizzazione del Parco, attraverso l'individuazione di sette aree definite, autonome, ma interconnesse, che sono state il presupposto progettuale per l'individuazione del parco delle tenute quale struttura portante dell'assetto territoriale ed ambientale dell'area protetta;

- il ripristino/restauro dell'ambiente naturale, attraverso il riallagamento di vaste aree occupate, in tempi recenti, da stagni e paludi, poi bonificate, assieme ad interventi di riforestazione in aree tradizionalmente interessate da vaste zone boscate a prevalenza di latifoglie decidue;

- l'organizzazione del Parco, facente riferimento alla struttura delle Tenute, ognuna interessata da un sistema integrato di accessi, percorsi e strutture per la visita e la conoscenza del territorio dal punto di vista storico ed ambientale.

Questa impostazione programmatica generale, fa riferimento ad una cultura progettuale che vede il Parco non quale "(...) perimetro invalicabile, ma al contrario quale assetto di un territorio naturale fortemente antropizzato. La presenza

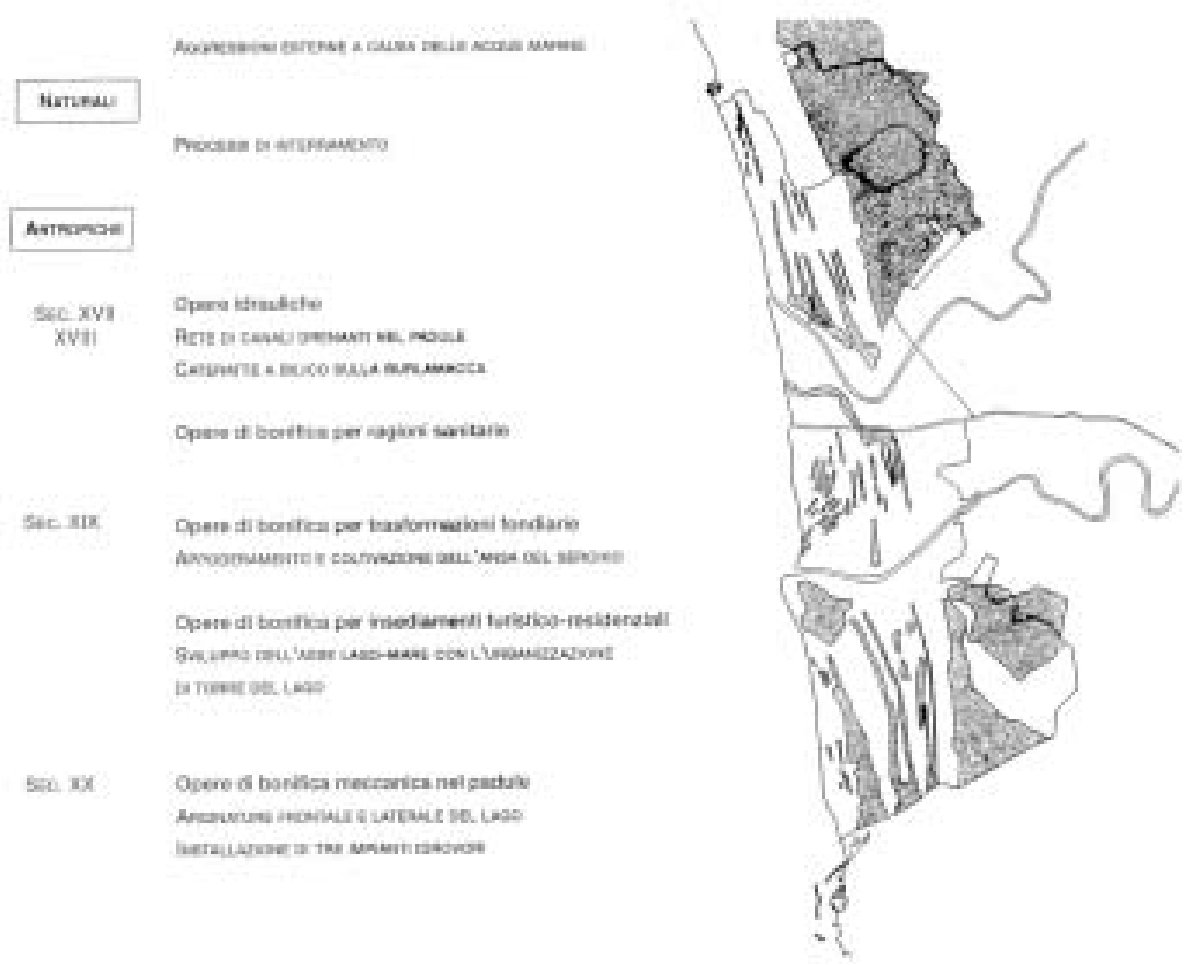

Figura 2 - Le trasformazioni storiche dell'assetto morfologico del Parco.

\footnotetext{
${ }^{10}$ Pier Luigi Cervellati e Giovanni Maffei Cardellini, (a cura di), op. cit., 1988, pag. 164.

${ }^{11}$ Questo step è stato definito "Quadro di Riferimento Progettuale", ed è servito quale momento di orientamento comune con gli altri enti territoriali interessati dall'iter di redazione del Piano del Parco.
} 
di zone abitate deve indurre, da un lato al recupero funzionale ed urbanistico di queste stesse zone (...), e dall'altro deve avviare quel processo di integrazione del Parco con il suo intorno, che rappresenta l'utenza principale del Parco"12.

Se vogliamo, questo rappresenta anche un primo tentativo di rendere evidenti anticipatamente gli obiettivi generali che col Piano ci si poneva, in un quadro di discussione/confronto con gli enti locali interessati, precorrendo una logica oggi ormai diffusa ed accettata in Italia, che prevede la concertazione preventiva in materia di istituzione, pianificazione e gestione di nuove aree protette, per favorire forme di consenso sociale, assolutamente necessarie per rendere percorribili le azioni di conservazione della natura e promozione compatibile delle attività economiche delle popolazioni locali.
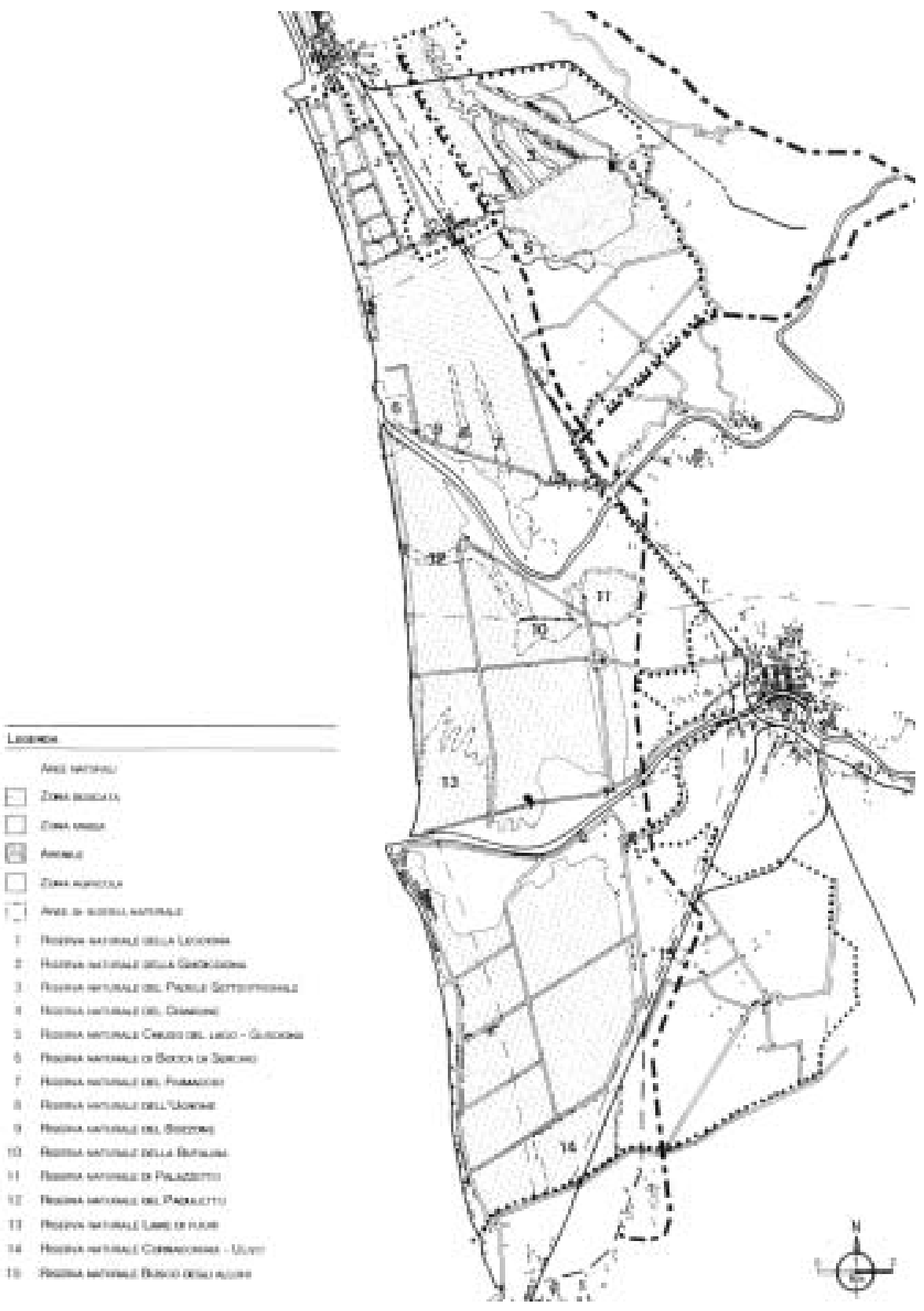

Figura 7 - Il Piano del Parco: aree naturali e di riserva. 
I principali contenuti del Piano possono essere riassunti rispetto ad uno zoning, che definisce settori di intervento diversificati per uso e tutela delle risorse naturali e per sistema infrastrutturale:

- le aree di restauro o ripristino ambientale, nelle quali si definisce un processo di riconduzione alla morfologia originaria dei luoghi attraverso il rimboschimento (per complessivi 903 ettari di aree agricole), le zone umide di ripristino (320 ettari), i riallagamenti (1.200 ettari) di aree oggi agricole, per ricreare vaste aree umide quali habitat di grande importanza, oggi rari e minacciati ${ }^{13}$, il ripristino delle sponde dei canali come segni storici importanti di un disegno territoriale fortemente legato alla presenza dell'elemento acqua;

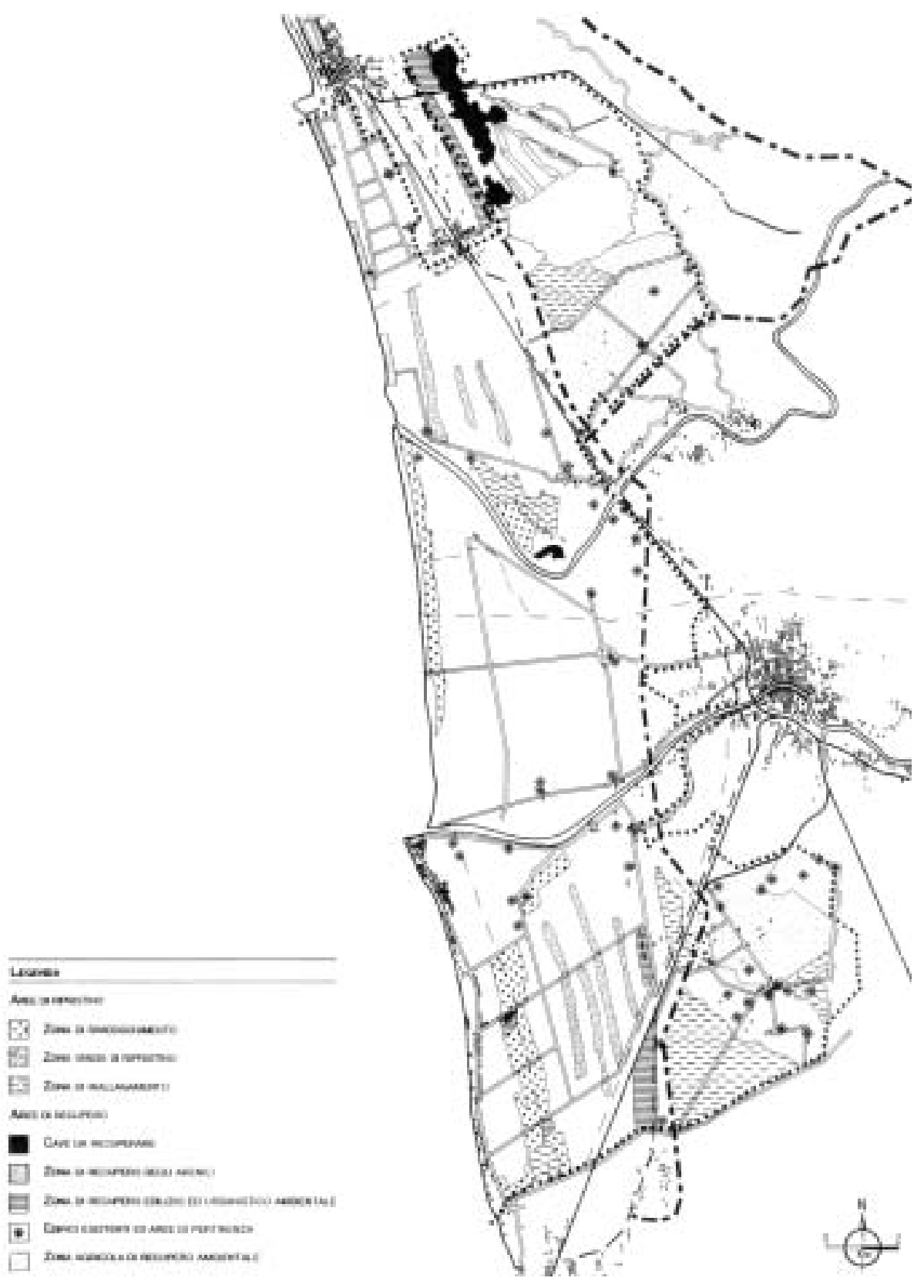

Figura 8 - Il Piano del Parco: aree di ripristino e di recupero.

${ }^{13}$ Questa è la proposta più originale ed innovativa del Piano del Parco, ed anche quella che ha determinato forti contrasti con gli enti locali e parte della popolazione del parco. Ad oggi, questa previsione 
- le zone di recupero, principalmente riferite a cave, arenili, edifici esistenti e relative aree di pertinenza, zone di recupero edilizio ed urbanistico, nelle quali l'azione umana e progressivi processi di abbandono hanno determinato delle modificazioni, che necessitano di azioni recupero e rifunzionalizzazione, in armonia con i principi e le finalità dell'area protetta;

- le aree naturali, che sono vaste zone per le quali il Piano prevede il mantenimento delle destinazioni e degli usi attuali, e la gestione del sistema di risorse rispetto alle aree boscate, le zone umide, gli arenili e le zone agricole;

- le riserve naturali, individuate in quindici aree (per complessivi 2.250 ettari), che rappresentano quelle più significative per la presenza di valori faunistici e floristici rari e di pregio, e nelle quali è consentita la ricerca scientifica ed il mantenimento delle caratteristiche naturali, senza introdurre modificazioni;

- le infrastrutture, con una ipotesi di riduzione del carico veicolare della strada statale Aurelia e la definizione di una rete di percorrenza interna all'area protetta;

- le attrezzature, che facendo riferimento alla struttura territoriale principale, riferita al sistema delle Tenute, individua l'insieme dei servizi offerti al visitatore, quali porte del parco, centri visite, foresterie, eccetera.

Il quadro generale delle previsioni del Piano del Parco, trova una sua specifica attuativa nei Piani di Gestione, che devono interessare almeno il territorio di una singola Tenuta, definendo nel dettaglio gli interventi ammessi sul patrimonio edilizio, sulle attività produttive (agricola, forestale, eccetera) e sulla natura degli interventi legati alle aree precedentemente descritte.

"La Tenuta è individuata come un tassello del Parco, con percorsi e circuiti storico-naturalistici organizzati, e con le attrazioni e le strutture ricettive, luogo autonomo e (...) interdipendente per la gestione unitaria delle altre Tenute"14.

Allo stato attuale sono stati redatti i primi Piani di Gestione per alcune Tenute (San Rossore, Coltano), i cui esiti, ad oggi, sono ancora difficilmente valutabili.

Certamente però, l'organizzazione generale del Piano, con la suddivisione in Tenute, permette il raggiungimento di obiettivi specifici anche per parti, ovviando in questo modo alle difficoltà ed ai conflitti che sempre emergono nella gestione di un'area protetta.

\section{GLI ESITI}

Il Piano del Parco di Migliarino San Rossore Massaciuccoli, a distanza di oltre tredici anni dalla sua entrata in vigore, si può considerare ancora come un piano attuale e innovativo, sia per la sua impostazione generale, che ha portato alla integrazione degli elementi di stratificazione storica con quelli di importanza naturalistica, sia perché ha cercato di affrontare il difficile rapporto con un contesto urbanizzato fortemente dinamico e piuttosto articolato.

Certamente, le intuizioni iniziali del parco delle tenute e del parco della acque, hanno rappresentato delle idee forti attorno alle quali aggregare un ricco e complesso sistema di previsioni che sanciscono, accanto alla conservazione del patrimonio naturale già esistente, un suo miglioramento/incremento/ripristino, anche attraverso previsioni coraggiose quali il riallagamento di vaste aree agricole per ricostituire aree umide estese.

del piano è rimasta del tutto inattuata, ma conserva ancora un forte carattere di innovazione culturale in un paese come l'Italia dove la riduzione di questi importanti habitat è stata drastica nel corso del XX secolo.

14 Sergio Paglialunga, Il Piano del Parco Naturale di Migliarino San Rossore Massaciuccoli, in Rosini Rino e VECCHIETTI SANDRA (a cura di), La pianificazione dei parchi regionali, Alinea Editrice, Firenze 1994, pag. 186. 


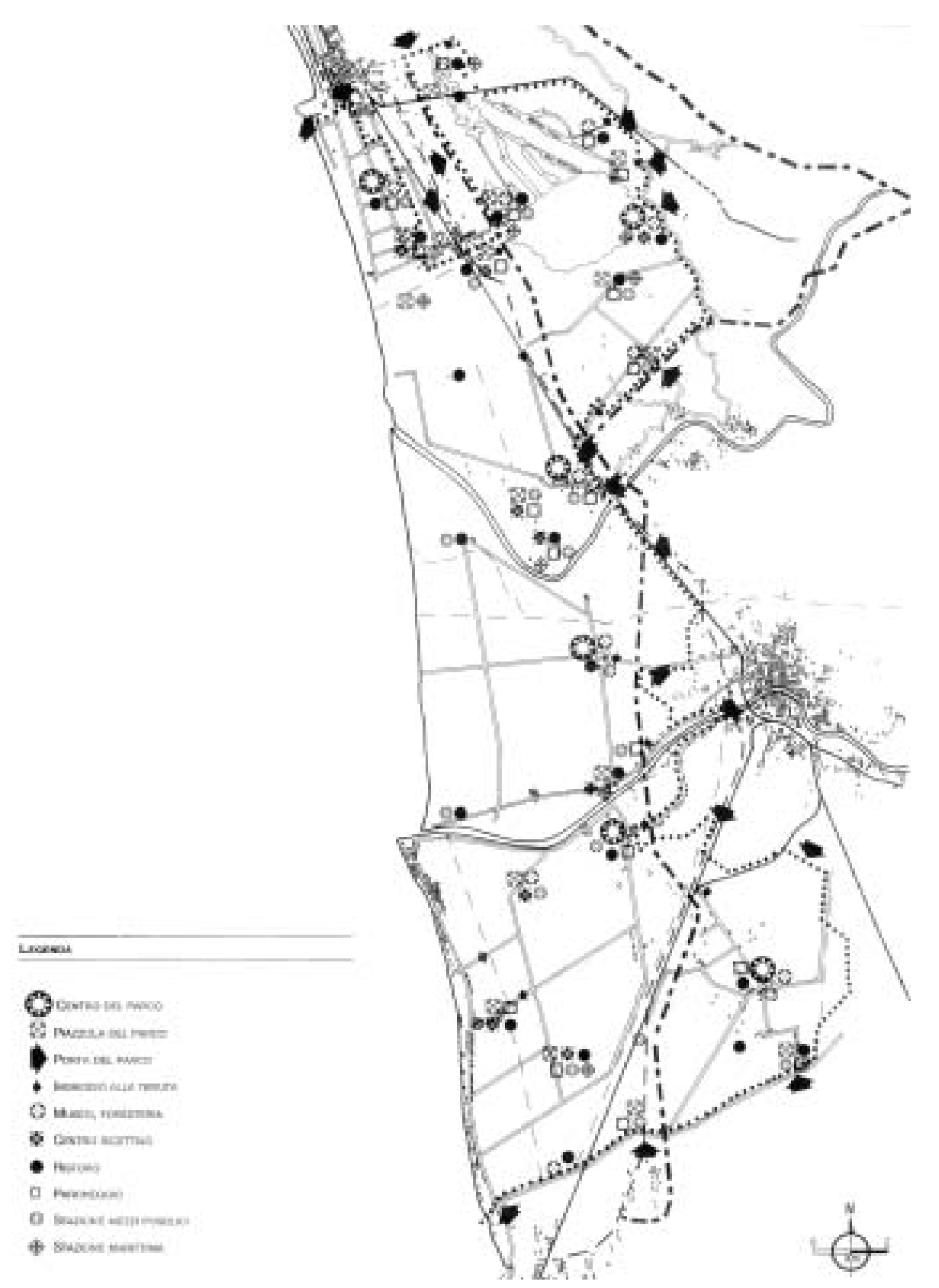

Figura 9 - Il Piano del Parco: attrezzature ed infrastrutture.

Ciò non toglie che "il piano territoriale, più che come strumento naturalistico in senso stretto, è uno strumento di gestione e governo del territorio con forte valenza naturalistica" 15 .

Certamente il Piano è nato anche come mediazione di necessità portate avanti dagli enti locali, sulla base di considerazioni che hanno comportato l'esclusione dei centri abitati dal Parco, ma comunque l'inclusione di molte aree fortemente antropizzate, determinando un ripensamento verso una filosofia che vede, nell'area protetta, uno strumento esclusivamente votato alla pianificazione e gestione delle risorse naturali.

Se di limiti si può parlare, questi derivano dal "(...) rimando attuativo delle previsioni a strumenti successivi, e la scarsa chiarezza normativa con cui è indivi- 
duata la priorità degli interventi territoriali, finalizzata soprattutto alla tutela ed alla conservazione della natura, con gradazioni nella modalità dell'uso del suolo"16; si fa chiaramente riferimento ai Piani di Gestione, che nell'idea di Piano sono i veri strumenti attuativi ed operativi rispetto al complesso di azioni previste.

Invece, per quanto riguarda il regime d'uso dei suoli, seppure con una certa rigidità, il Piano ha rappresentato fino ad oggi uno strumento efficace per il controllo e l'indirizzo delle azioni sul territorio (si ricorda, ad esempio, il blocco dell'attività estrattiva nel padule di Massaciuccoli, che generava forti impatti ed era assolutamente incompatibile con gli obiettivi e le finalità dell'area protetta).

Rimane da riconoscere, quale merito finale al Piano, anche se processi di questo genere sono lunghi e faticosi ed allo stato attuale solo in fase iniziale, quello di avere perseguito una filosofia progettuale non di chiusura nei confronti dell'esterno, ma di integrazione con un intorno territoriale ricco di condizionamenti ed opportunità, anche in questo caso operando secondo modalità ormai generalmente accettata nel panorama italiano, e non solo, della pianificazione e gestione delle aree protette.

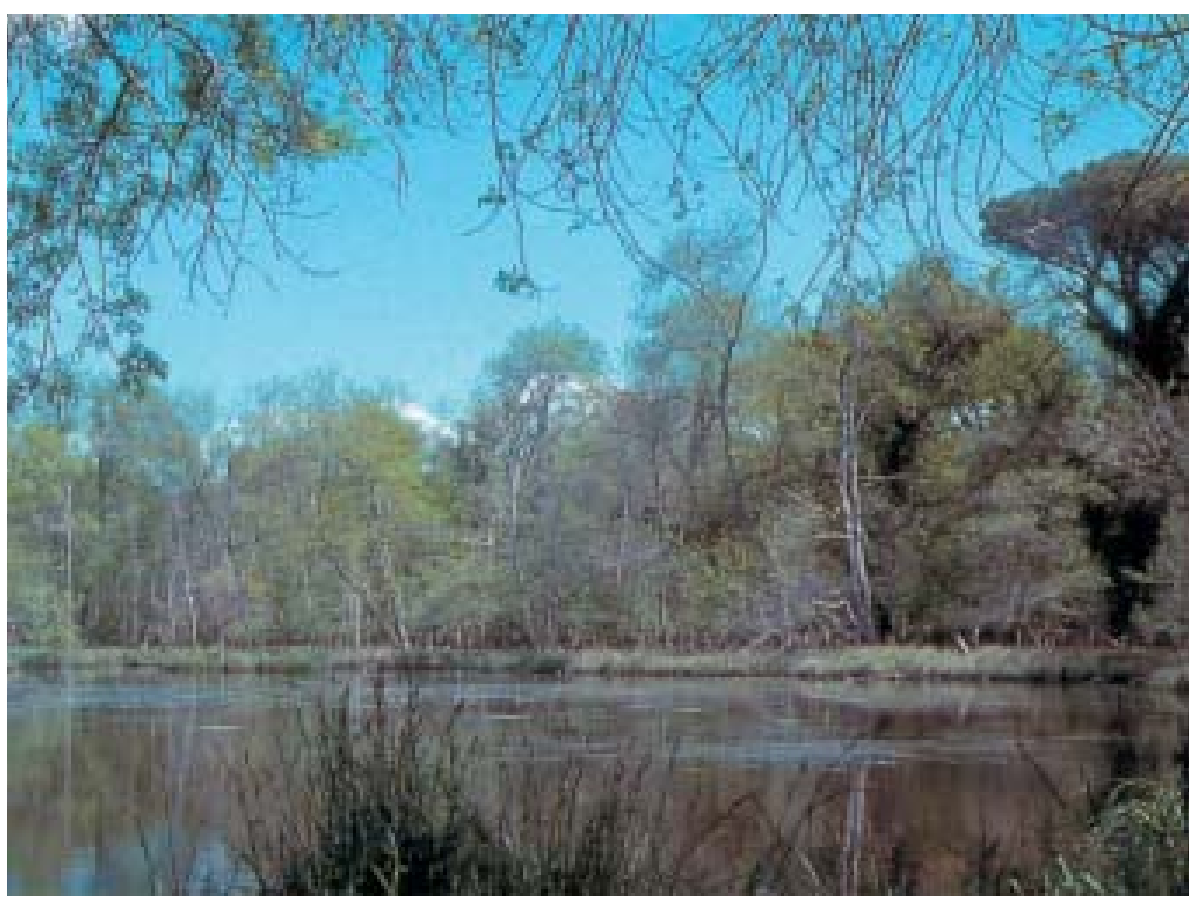

Figura 10 - I boschi igrofili della Riserva Naturale del Paduletto.

\section{RIFERIMENTI BIBLIOGRAFICI}

Cavalli Stefano e Lambertini Marco, Il Parco Naturale di Migliarino San Rossore Massaciuccoli, Pacini Editore, Pisa 1990.

Cervellati Pier Luigi e Maffei Cardellini Giovanni (a cura di), Il Parco di Migliarino San Rossore Massaciuccoli: la storia e il progetto, Giunta Regionale Toscana-Marsilio Editori, Venezia 1988.

Paglialunga Sergio, Il Piano del Parco Naturale di Migliarino San Rossore Massaciuccoli, in Rosini Rino e VeCCHIETt SANDra (a cura di), La pianificazione dei parchi regionali, Alinea Editrice, Firenze 1994. 
TORnati DANiela, Parco Naturale Regionale Migliarino San Rossore Massaciuccoli (Italia-Toscana) e Parque Nacional de Donana (Spagna), in Ferrara Guido e Vallerini Lorenzo (a cura di), Pianificazione e gestione delle aree protette in Europa, Maggioli Editore, Rimini 1996.

\section{RIFERIMENTI ICONOGRAFICI}

Figure 1 e 3: rielaborazione di Andrea Meli da CENNI MARIO (a cura di), Lago di Massaciuccoli: 13 ricerche finalizzate al risanamento $-2^{\circ}$ contributo, Editrice Universitaria Litografia Felici, Pisa 1997, ultima di copertina e prima di copertina.

Figure 2 e 5-9: rielaborazione di Andrea Meli da ToRnati Daniela, Parco Naturale Regionale Migliarino San Rossore Massaciuccoli (Italia-Toscana) e Parque Nacional de Donana (Spagna), in Ferrara Guido e Vallerini Lorenzo (a cura di), Pianificazione e gestione delle aree protette in Europa, Maggioli Editore, Rimini 1996, pagg. 130, 137, 137, 139, 138, 140.

Figure 4 e 10: rielaborazione di Andrea Meli da Cavalli Stefano e Lambertini Marco, Il Parco Naturale di Migliarino San Rossore Massaciuccoli, Pacini Editore, Pisa 1990, pag. 165 e 40.

SCHEDA DI PROGETTO

Tipologia di area protetta:

Istituzione:

Dati dimensionali dell'area protetta:

Enti locali interessati:

Organo di gestione:

Caratteristiche ambientali:

Strumento di pianificazione:

Progettisti del Piano:
Parco Naturale Regionale

Legge Regionale Toscana n. 61 del 13 dicembre 1979

23.000 ettari, su una fascia litorale di circa $32 \mathrm{~km}$ ed una profondità variabile dai 3 ai $6 \mathrm{~km}$

Province di Lucca e Pisa, Comuni di Massarosa, Viareggio, Vecchiano, San Giuliano Terme, Pisa: i primi due ricadono nella provincia di Lucca, i rimanenti tre in provincia di Pisa

Ente Parco Regionale

situato su una fascia litoranea che si affaccia sul Mare Tirreno, il territorio dell'area protetta è caratterizzato da un complesso di ecosistemi riferiti a boschi di latifoglie decidue, pinete, zone umide, ambienti dunali e retrodunali, ampie zone agricole

Piano Territoriale di Coordinamento, approvato con Delibera di Consiglio Regionale n. 515 del 12 dicembre 1989. Il Piano rimanda poi a successivi Piani di Gestione, riferiti al sistema delle Tenute

coordinatore del gruppo di lavoro, Arch. Pierluigi Cervellati, gruppo di lavoro formato da: Arch. Enza Citterio, Ing. Roberto Corlaita, Arch. Giovanni Maffei Cardellini, Arch. Enrico Ghiselli, Ing. Stefano Paglialunga 



\title{
PARCHI METROPOLITANI
}

\author{
Laura Ferrari
}

La ridefinizione e la progettazione degli spazi aperti metropolitani costituisce senza dubbio una delle tematiche più rilevanti che tanto la pianificazione territoriale quanto la progettazione paesistica si trovano oggi ad affrontare.

L'urbanistica d'altronde si è sempre occupata della città e della sua crescita, trascurando le caratteristiche e i problemi di ciò che stava al di là, della "non città"; dal canto suo l'architettura si è sempre più preoccupata del singolo manufatto edilizio e della sua funzionalità piuttosto che del suo rapporto con il contesto urbano, un contesto astratto e sovente idealizzato.

Nel processo di urbanizzazione il "non urbano", lo spazio aperto, è sempre stato considerato luogo di attesa, luogo delle discariche e dell'esportazione degli scarti urbani, "territorio da abbandonare od occupare volta per volta per i propri comodi" .

A questi fattori si deve senz'altro aggiungere l'incontrollato processo di crescita e di concentrazione delle funzioni economiche, abitative e di servizio nelle aree urbane $e^{2}$. Processo che, manifestatosi in tutti i paesi industrializzati, ha determinato profonde trasformazioni sociali, funzionali e morfologiche nella città e nel più vasto contesto paesistico ed ambientale.

Dal punto di vista geografico queste trasformazioni hanno cancellato i confini delle città dando luogo al fenomeno della conurbazione continua, della progressiva saldatura dei diversi nuclei urbani, inizialmente lungo alcune direttrici principali e, successivamente, lungo ramificazioni secondarie. Le agglomerazioni si sono gradualmente dilatate e la "città compatta" si è trasformata progressivamente in "area metropolitana".

Gli spazi liberi, ridotti ad isole, sono stati spesso abbandonati al degrado in attesa di ulteriore urbanizzazione; aree sottratte anche per decenni a qualsiasi utilizzazione, abbandonate al gioco delle speculazioni immobiliari, dei cambi di destinazione, delle lottizzazioni auspicate o manovrate, squallide "steppe" trasformate ben presto in discariche abusive o depositi provvisori. Anche dove ciò non è avvenuto il territorio aperto è diventato una sorta di "patchwork", dove terreni ancora coltivati, capannoni industriali, edifici residenziali, opere di grande viabilità, centri commerciali, incolti, cascinali, orti, rogge, strade vicinali si accalcano in una sequenza fitta, disordinata e casuale, illeggibile e spesso fisicamente impenetrabile.

In tempi recenti, l'accelerazione di questi fenomeni e il progressivo estendersi dei processi che hanno caratterizzato l'esperienza americana negli anni passati ${ }^{3}$ hanno progressivamente messo in crisi il grande "recinto" dell'agglomerazione

\footnotetext{
${ }^{1}$ Edoardo Salzano, La montagna vista dalla città, "Unione delle Comunità Montane", Venezia 1999.

${ }^{2} \mathrm{Si}$ è trattato di un processo sospinto da potenti leggi economiche verificatosi in assenza di adeguati strumenti di pianificazione e controllo dello sviluppo urbano.

${ }^{3}$ Processi di diffusione delle residenze nelle aree verdi periurbane, con tipologie edilizie a bassa densità e conseguentemente ad elevato consumo di suolo; importazione di modelli localizzativi ed organizzativi e di tipologie edilizie del suburbano americano, come i grandi centri commerciali e ricreativi con vasta area di gravitazione ed estesa mobilità indotta.
} 
metropolitana. Un "recinto" che, se già aveva perso la sua specificità di grande spazio aperto, rischia ora di perdere ulteriormente la propria, pur precaria, "salute" per via di uno snaturamento operato per pezzi, per singoli frammenti che, andando ad incollarsi alla realtà urbana circostante, costituiscono ulteriori tessere di un mosaico non concluso, di un "puzzle" a cui è mancato e a cui manca un disegno di riferimento complessivo.

A causa di questi fenomeni, il dissesto ambientale del territorio urbano e metropolitano è giunto dunque alla soglia dell'invivibilità, spesso superandola: inquinamento atmosferico, corsi d'acqua canalizzati e inquinati, agricoltura periurbana abbandonata, spazi aperti degradati, luoghi urbani ostili, patrimonio storico in decadimento, sono le più appariscenti manifestazioni di questa alterazione ambientale.

Nella maggior parte dei paesi industrializzati lo stato di alterazione è talmente avanzato che si sta cercando di invertire la tendenza e definire un "progetto-processo" per risalire a livelli di sicurezza rispetto alla soglia di rischio ormai largamente superata; si sta cercando di definire, ma soprattutto di realizzare, un progetto del territorio che assuma quale elemento principale la definizione degli spazi aperti, vero tessuto connettivo delle agglomerazioni urbane in grado di coniugare funzioni ricreative e di servizio con ben più nobili e forse più necessarie funzioni di natura ecologico-ambientale.

La questione degli spazi aperti in realtà non è un tema nuovo, è una questione antica quanto la città industriale; da sempre, infatti, questi spazi sono stati insufficienti per quantità e qualità, da sempre vengono minacciati da ogni nuovo progetto di espansione urbana.

Nella storia dell'urbanistica diversi, in ogni epoca e in ogni paese, sono stati i tentativi operati dall'uomo per circondare e limitare l'espansione della città. I "boulevard" di Parigi, la "Green Belt" di Londra, il "Ring" di Vienna, la "Randstad" olandese, la "Ceinture Verte" di Parigi, il "GrünGürtel" di Francoforte sono solo alcuni di questi tentativi.

Se tuttavia il contesto internazionale ha saputo essere terreno fertile per il proliferare di cinture verdi e di nuovi progetti per gli spazi aperti sin da tempi antichi, altrettanto non si può affermare per quanto riguarda la situazione italiana, dove l'interesse verso il modello della cintura verde e verso una più corretta progettazione degli spazi aperti si è manifestato solo in tempi relativamente recenti.

In Italia, infatti, la forma di pianificazione più conosciuta e consolidata per il governo degli spazi aperti si è da sempre riferita fondamentalmente a tre tipologie di uso e gestione del territorio: le zone agricole che, per definizione, hanno quasi sempre rappresentato "aree bianche" destinate ad ospitare l'espansione degli aggregati urbani e, ancora peggio, le funzioni che la città espelle; le aree verdi distribuite "a pelle di leopardo" all'interno del tessuto urbano solo per soddisfare il bilancio della contabilità degli standard urbanistici imposti dalla legislazione nazionale; ; parchi e le aree protette con i quali si sono sottoposti al vincolo della conservazione solo le porzioni di territorio più rilevanti dal punto di vista naturalistico e paesistico.

Si è trattato evidentemente di una pianificazione incapace di comporre in un disegno unitario e funzionale zone agricole, verde urbano e aree protette; l'impo-

\footnotetext{
${ }^{4} \mathrm{E}$ interessante vedere come all'evoluzione del modello di cintura verde abbia fatto seguito in questi esempi anche una evoluzione concettuale del modo di concepire lo spazio verde: da elemento di decoro, a strumento di controllo dell'espansione urbana, a elemento di riequilibrio ecologico dei 'disordini' metropolitani.

${ }^{5}$ Con il Decreto Ministeriale 2 aprile 1968, n. 1444, lo Stato introduce, infatti, le quantità minime di spazi pubblici o riservati alle attività collettive, a verde pubblico o a parcheggi da osservare in rapporto agli insediamenti residenziali, produttivi e terziari.
} 
sizione di un vincolo di inedificabilità e di tutela ha avuto solo il "merito" (se così lo si può definire) di aver preservato, anche se in maniera del tutto passiva, una parte delle aree libere ancora rimaste all'interno del grande "recinto" metropolitano. Aree che tuttavia, in assenza di un disegno d'insieme e di obiettivi chiari, sono rimaste per anni spazi "vuoti", senza nome e senza volto, in attesa che lo scorrere del tempo e le utilizzazioni provvisorie, più o meno abusive, cancellassero progressivamente quei segni che la natura e la storia imprimono ad ogni paesaggio.

In Italia è solo a partire dagli anni Settanta che il continuo proliferare degli episodi urbani, l'inadeguatezza delle forme di tutela e pianificazione fino ad allora sperimentate e l'emergere di una nuova coscienza/sensibilità rispetto ai problemi ambientali e di scarsità delle risorse, spostano l'attenzione di studiosi, amministratori e associazioni ambientaliste, verso i temi della pianificazione e della progettazione degli spazi aperti.

Il confronto con altre discipline che si occupano di territorio induce a riflettere su una ridefinizione del linguaggio disciplinare e sulla individuazione di nuove ipotesi di ricerca e di intervento. Ad ipotesi strutturate sul sistema del verde pubblico e dei percorsi tematici, si affiancano processi di riqualificazione basati sulla ridefinizione del sistema agricolo e in generale sul significato e sull'identità del territorio extraurbano.

Nascono all'interno di questo dibattito le prime idee per la realizzazione di una cintura verde intorno a Milano; è negli anni Settanta che l'attenzione verso l'ambito meridionale dell'area metropolitana porta alla proposta, ormai da tempo presente nel dibattito culturale, di un "parco diffuso" che, integrandosi alle aree agricole, garantisca il mantenimento e la valorizzazione degli spazi aperti e delle attività agricole ancora largamente presenti ${ }^{6}$. È di questi stessi anni l'idea di realizzare un "grande polmone verde" nel cuore della zona più densamente edificata e a maggior rischio di saturazione del nord Milano; uno spazio di cintura metropolitana da riqualificare e a cui riconferire l'identità perduta attraverso la realizzazione di un grande parco metropolitano denominato "Parco Nord Milano".

Nel tempo, l'esempio di Milano è seguito da altre città italiane.

Bologna, già alla fine degli anni Sessanta, prevede di contrastare la crescita urbana "a macchia d'olio" proponendo uno schema di sviluppo appoggiato ad un forte sistema verde e al principio della salvaguardia delle aree agricole. Un sistema ipotizzato come insieme di parchi fluviali e di corona dell'urbanizzato in cui assume particolare rilievo il ruolo della Collina, che per le sue caratteristiche fisiche, socioeconomiche e storiche si impone quale elemento territoriale di grande valore.

Ferrara nel 1975 avvia una politica di recupero del territorio agricolo di cintura, perimetrando l'area che si estende dalle mura fino al Po e denominandola "Parco Campagna"; un'area tutelata come "Parco del Barco" anche negli strumenti urbanistici più recenti, nei quali se ne prefigurano addirittura gli esiti formali e funzionali.

Firenze nello Schema strutturale per l'area metropolitana Firenze-PratoPistoia dei primi anni Novanta, propone la creazione del parco metropolitano dell'area fiorentina e il rimodellamento dei bordi frammentati e dispersi della città e degli spazi aperti interclusi nelle larghe maglie del sistema insediativo.

Ravenna con il Piano Regolatore del 1993 propone la realizzazione di una cintura verde intorno all'urbanizzato, intesa come fascia di moderazione tra città e campagna. Una previsione che nel Piano del Verde acquista addirittura una

\footnotetext{
${ }^{6}$ Una proposta che riuscirà finalmente ad avere concretezza solo negli anni Novanta con l'istituzione del Parco Agricolo Sud Milano.
} 
dimensione progettuale ${ }^{7}$, tanto da contenere il disegno dello spazio, le indicazioni dei percorsi, delle aree di sosta e di parcheggio, delle sistemazioni generali, delle attrezzature per la pratica sportiva, del quadro delle specie vegetali da utilizzare e delle colture agrarie da praticare.

Palermo alla fine degli anni Novanta per ritrovare la bellezza della città del passato e costruire quella della città del futuro, assume le aree periurbane quali "nuovi e intangibili baluardi" della città, quali elementi strutturali in grado di conferire alla città una nuova "forma urbis" e propone la realizzazione del Parco Agricolo di Ciaculli ${ }^{8}$.

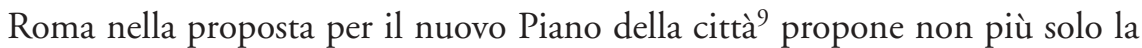
realizzazione della "ruota verde" 10 ma la costruzione di un disegno più complesso e minuto, di una vera e propria "rete" di spazi aperti e di aree verdi che tocca e attraversa tutti i tessuti urbani e le nuove trasformazioni urbanistiche.

Torino con i recenti progetti "Corona Verde" e "Torino Città d'acque" porta a compimento l'idea avanzata già negli anni Settanta di ricomporre in un disegno, unitario e funzionale, verde urbano, fasce fluviali e parchi di interesse regionale. Un vero e proprio sistema del verde che, pur con caratteristiche differenti a seconda della potenzialità degli spazi aperti, assume la comune peculiarità di essere a disposizione dei cittadini per le varie finalità a cui è preposto in genere ogni sistema di verde urbano.

Nonostante il modello delle cinture verdi sia stato oggetto di un grande interesse da parte di diverse città italiane (e gli esempi citati lo dimostrano) occorre sottolineare tuttavia che il quadro delle effettive realizzazioni risulta poco confortante. Se si è assistito infatti ad un proliferare di idee e di progetti non altrettanto si può dire per le realizzazioni; molti sono ancora i progetti "sulla carta" e pochissimi sono invece gli esiti fisici di questo nuovo modo di intendere gli spazi aperti di cintura metropolitana.

Certo qualcosa si è fatto ma si tratta ancora di singoli "episodi verdi”, assimilabili per la logica fortemente interna che li struttura più alla tipologia dei parchi che non a dei veri spazi aperti di cintura metropolitana; molto spesso è solo la loro collocazione, ai margini delle grandi conurbazioni metropolitane, che gli conferisce il valore e le funzioni degli spazi di cintura.

Nel nostro Paese, infatti, la particolare predisposizione del legislatore e delle istituzioni ad identificare e tutelare le porzioni di territorio dotate di alto valore paesistico-ambientale attraverso la formazione dei "parchi", ovvero mediante il ricorso all'istituto del "vincolo", ha fatto sì che la stessa metodologia di intervento venisse adottata anche negli spazi aperti collocati ai margini dei grandi insediamenti. Proprio per questi spazi è stata addirittura introdotta una specifica tipologia di parco, il "parco metropolitano" o "parco di cintura metropolitana", attraverso la quale si sottopongono a tutela e valorizzazione le "zone di importanza strategica per l'equilibrio ecologico delle aree metropolitane, per la tutela e il recupero paesistico e ambientale delle fasce di collegamento tra città e campagna, per la connessione delle aree esterne, dei sistemi verdi urbani, per la ricreazione e il tempo libero dei cittadini”"11.

Se dunque l'avvio dei dibattiti e delle prime esperienze di progettazione degli spazi aperti non ha condotto, al pari di altre realtà internazionali, ad esiti rile-

\footnotetext{
${ }^{7}$ Progetto "Cintura del verde urbano".

${ }^{8}$ Un parco la cui realizzazione è stata resa possibile grazie al programma Life '94 dell'Unione Europea.

${ }^{9}$ Approvata dalla Giunta Comunale nell'ottobre del 2000.

${ }^{10}$ La presenza sul territorio di vaste aree e corridoi liberi che si incuneano nell'area urbana fino nel centro antico della città ha conferito una specifica connotazione territoriale al sistema delle aree libere, connotazione che nel Piano delle Certezze del '97 ha fatto parlare di "green wheel", ruota verde, come declinazione locale del concetto di "green belt".

${ }^{11}$ Legge Regione Lombardia 23 aprile 1985, n. 41 “Integrazioni e modifiche alla L.R. 86/83”.
} 
vanti, esso ha comunque avuto il merito di aver stimolato in quasi tutte le città italiane un'inversione di tendenza nella pianificazione e progettazione degli spazi aperti $^{12}$. Ad un approccio "difensivo" per parti isolate ${ }^{13}$, è andato sostituendosi un approccio di tipo "propositivo" e progettuale, orientato cioè alla costruzione di sistemi di spazi non edificati: non più aree residuali o di riserva, ma elementi costitutivi e strutturanti lo sviluppo urbano ${ }^{14}$. Non più spazi da tutelare passivamente, ma luoghi di progetto, luoghi a cui attribuire un valore storico, culturale, ambientale, spazi in cui "costruire quel senso "ora fattosi assente"

$\mathrm{Si}$ è trattato di atteggiamenti stimolati senza dubbio anche dal progressivo affermarsi di un approccio ecologico alla pianificazione degli spazi verdi della città che ha condotto sempre più verso una visione ecosistemica di questi spazi. Un sistema la cui funzione non sembra più essere solo quella di "sistema di verde pubblico" ma, in linea con gli obiettivi e le politiche di sviluppo sostenibile, anche di "sistema di rigenerazione e riequilibrio ecologico, (...) fattore di compensazione ambientale, (...) struttura per l'incremento della potenzialità biotica dell'ecosistema urbano"16.

In quest'ottica dunque il sistema degli spazi aperti metropolitani diventa un grande insieme a funzioni multiple, integrato e complementare al sistema insediativo, capace di riorganizzare l'insieme dei beni ambientali attraverso politiche di valorizzazione e di sviluppo e non semplicemente di vincolo e conservazione. La sua funzione diviene quella di orientare la riorganizzazione della struttura urbana e territoriale, di mettere a disposizione dei cittadini luoghi e spazi per l'attività ricreativa e di preservare quelle risorse fisiche, naturali e paesistiche indispensabili per l'equilibrio dell'ecosistema metropolitano.

A partire da queste considerazioni e tenuto conto della scarsità dei progetti compiuti, si è scelto di affrontare il tema degli spazi aperti di cintura metropolitana attraverso la lettura di tre esperienze che, per obiettivi, tecniche di realizzazione ed esiti conseguiti, possono rappresentare importanti riferimenti metodologici per la progettazione di altri spazi aperti.

In questa direzione dunque Parco Nord Milano, Bosco delle Querce di Seveso e Meda, Boscoincittà a Milano sono stati assunti quali casi studio (progetti-pilota) dai quali far emergere indicazioni, criteri-guida e suggerimenti metodologici per le esperienze future.

Tre esperienze che per la durata della loro realizzazione, quasi trent'anni, permettono di ripercorrere l'evoluzione del tema degli spazi aperti; progetti che segnano il passaggio da una considerazione del verde come "standard" ad una lettura e progettazione del territorio a partire dai "vuoti", dalle aree libere, dalle relazioni che avvengono, o sono prevedibili, tra spazi liberi e luoghi abitati.

Tre realizzazioni accomunate non solo dalla collocazione geografica, l'area metropolitana milanese, ma anche dalla tecnica di costruzione dello spazio verde; una tecnica, quella della forestazione urbana, che lega la configurazione

\footnotetext{
12 La sola 'conservazione' del paesaggio aperto non poteva più rappresentare la soluzione alle contraddizioni e ai fenomeni di compromissione territoriale; l'esperienza aveva dimostrato, infatti, che essa non contribuiva né alla totale preservazione delle aree strategiche per il sistema metropolitano, né a dare senso finito, con nuova qualità, ad una conurbazione cresciuta per frammenti.

${ }^{13}$ Approccio che troppo spesso ha visto la provvisorietà dei risultati raggiunti, con un ripensamento, ad ogni salto demografico e ad ogni cambiamento di 'colore' politico, del destino delle aree fino a quel momento preservate all'edificazione.

${ }^{14}$ Non più "aree in attesa di edificazione", ma "aree in attesa di diventare parco", evocando in questo modo l'immagine che, a medio e lungo termine, questi spazi dovranno assumere nel contesto urbano e metropolitano in cui si collocano.

15 Aimaro Isola, Necessità di architettura, in Antonio De Rossi, Giovanni Durbiano, FranceSCA Governa, LuCa ReInerio e MATTEO Robiglio, Linee nel paesaggio. Esplorazioni nei territori della trasformazione, UTET, Torino 1999, pag. 15.

${ }^{16}$ Lorenzo Vallerini e Sergio Pelligra, Spazi aperti e città sostenibile. Le greenways di Vancouver e Victoria, "Architettura del paesaggio", 7, 2001, pag. 38.
} 
dello spazio allo scorrere del tempo, un tempo che a sua volta dipende in larga misura dai "materiali vegetali" impiegati e dalla loro evoluzione naturale.

È una strategia di realizzazione "work in progress" quella che sta alla base di tutti e tre i progetti, della rinuncia al progetto "finito" a favore di una sequenza di azioni e di interventi inquadrati da un disegno di grande scala destinato continuamente a definirsi e ad integrarsi ${ }^{17}$.

La lettura dei tre casi studio mette in evidenza come il futuro delle nostre città e il nostro stesso "abitare" si debba giocare sul terreno del limite urbano (Boscoincittà), degli spazi aperti pieni di rifiuti (Parco Nord Milano), o ancora sul terreno degli spazi inquinati da bonificare (Bosco delle Querce).

Spazi il cui limite, il cui confine si presenta sempre più incerto e indecifrabile; spazi in cui il progettista deve "oggi, assumere la responsabilità di trovare di volta in volta il limite (...), di imporlo, di comporre la misura, di individuare nei luoghi (nel silenzio) "ciò di cui ancora si può parlare", i segni, le tracce, e a partire da queste costruire quel senso "ora fattosi assente". Ma per comporre luoghi dotati di senso bisogna che questi limiti, anziché chiudere, aprano spazi"18.

In questo senso dobbiamo dunque leggere gli esempi analizzati; essi infatti, attraverso la lettura dei segni e delle tracce del passato, attraverso l'attingere alle proprie radici, naturali e culturali, cercano di costruire il "senso" mancante, di conferire nuova identità. E mano a mano che acquistano senso, questi spazi non si chiudono in loro stessi ma all'interno dei loro confini aprono a nuovi spazi e allo spazio della città in una tensione continua tra natura e cultura.

Il porsi rispetto al tema degli spazi aperti che emerge dai tre esempi è proprio quello della ricerca di integrazione tra natura e cultura ${ }^{19}$, tra natura e città. Una ricerca che induce a creare negli spazi aperti nuove condizioni di naturalità, ad introdurre nel disegno degli spazi gli elementi costitutivi del paesaggio naturale; elementi che, oltre a svolgere funzioni ecologiche, possono contribuire anche ad offrire identità ai quartieri urbani e ad articolare visivamente gli spazi aperti. Gli elementi costitutivi del paesaggio naturale (boschi, filari di alberi, cespugli) diventano quindi gli elementi chiave del progetto attraverso i quali ecosistemi e spazi fruibili si integrano e si sovrappongono.

Gli esiti formali delle singole esperienze, ma soprattutto il ruolo che ciascuno spazio assume nei confronti di un ambito di riferimento ben più esteso, rappresentano una conferma che "quel che resta dello spazio extraurbano, non è più lo sfondo immobile ed inerte della città-cantiere come nelle rappresentazioni rinascimentali, ma il teatro principale del cambiamento" 20 .

\footnotetext{
${ }^{17}$ Un disegno degli spazi che si costruisce gradualmente, in tempi lunghi e diversificati: a parti del territorio che raggiungono una loro configurazione ottimale in tempi abbastanza brevi si affiancano infatti spazi la cui configurazione è 'provvisoria' anche per lunghi periodi di tempo.

${ }_{18}$ Aimaro Isola, Necessità di architettura, in Antonio De Rossi, Giovanni Durbiano, FranceSCA Governa, LuCa ReInerio e MatTeo Robiglio, Linee nel paesaggio. Esplorazioni nei territori della trasformazione, UTET, Torino 1999, pagg. 14-15.

19 "Quale è la relazione tra città e natura? Per alcuni nessuna. Gli amanti della natura affermano che natura significa stare fuori dalla città: nella città la natura non esiste. Anche per molti planners e progettisti la risposta è la medesima: la città è luogo di cultura, di strade, di piazze, di edifici. Eppure, l'aver dimenticato che anche nella città, "suo malgrado", avvengono processi ecologici, ha contribuito a rendere progressivamente invivibile anche per l'uomo il "proprio prodotto". La scoperta della natura nella città assume allora un significato più vasto del solo tutelare piante e animali o prestare attenzione per le relazioni tra azioni umane e ambiente. Significa capire che senza natura non c'è cultura" (SYBRAND P. TJallingil e E. Koning, Ecologie van de stad. En verkenning, Platform Stadecologie, Wageningen 1991).

${ }^{20}$ Roberto Gambino, Il paesaggio tra conservazione e innovazione, in AnTONio De Rossi, Giovanni Durbiano, Francesca Governa, LuCa Reinerio e Matteo Robiglio, Linee nel paesaggio. Esplorazioni nei territori della trasformazione, UTET, Torino 1999, pag. 24.
} 


\title{
LA TRASFORMAZIONE "WORK IN PROGRESS" DI UNO SPAZIO APERTO DI CINTURA METROPOLITANA: PARCO NORD MILANO
}

\author{
Laura Ferrari
}

\section{IL CONTESTO}

L'opportunità di salvaguardare le aree libere rimaste alla periferia della città, unitamente all'esigenza di prevedere utilizzazioni alternative per le porzioni di territorio che via via vengono dismesse dalle grandi industrie, rappresenta un obiettivo e, al tempo stesso, una occasione molto importante per la pianificazione territoriale e la progettazione paesistica di molte città italiane. Nel caso della metropoli milanese lo storico, o meglio, il 'cronico' disinteresse per i servizi e gli spazi collettivi che ha caratterizzato le pratiche urbanistiche e, parallelamente, l'assenza di un progetto coordinato di sviluppo, hanno reso sempre più difficile il raggiungimento di un simile obiettivo e, in particolar modo, il soddisfacimento del fabbisogno pregresso di aree verdi.

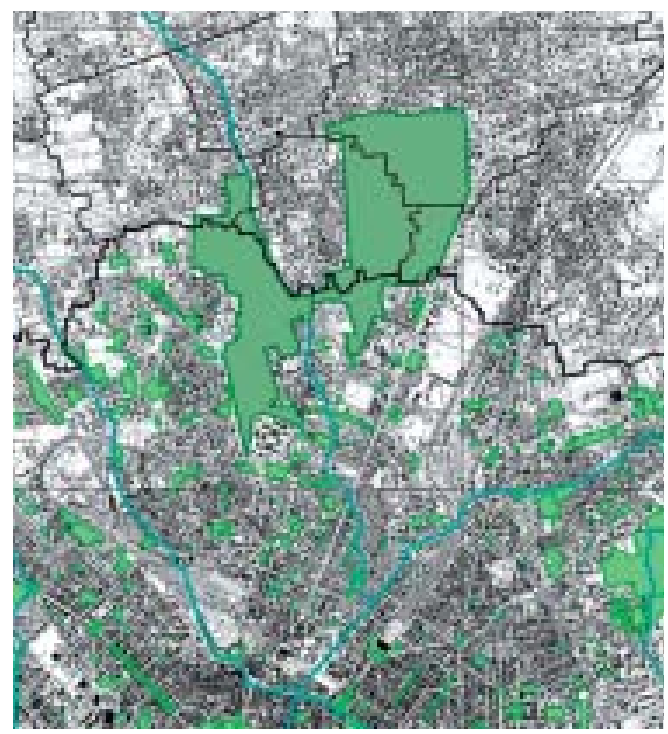

Figura 1 - Inquadramento territoriale.

In una simile situazione l'idea, elaborata alla fine degli anni Sessanta, di realizzare un "grande polmone verde" nel cuore della zona più densamente edificata e a maggior rischio di saturazione del nord Milano, rappresenta senza dubbio un fatto di grande 'rottura' nei confronti degli interessi speculativi che avevano caratterizzato la politica di sviluppo milanese di quegli anni.

Il parco, scaturito da questa idea e denominato "Parco Nord Milano" per la sua collocazione, è un'area verde di 600 ettari situata all'interno della più importante asta di conurbazione della metropoli lombarda sulla quale si affacciano i comuni di Milano, Bresso, Cinisello Balsamo, Cormano, Cusano Milanino e Sesto San Giovanni. Un parco particolare, per l'oggettiva dimensione e collocazione geografica, per la complessità amministrativa e istituzionale della vicenda, per la strategia che è sottesa alla sua definizione progettuale e per il disegno degli spazi che ne è conseguito. Un parco che "è riuscito a trasformare il territorio in cui è nato "colorando" di verde quello che era grigio: aree industriali dismesse, terreni incolti, capannoni, baracche abusive, avviando un processo di riqualificazione di tutte le aree della periferia a nord di Milano"1. 
Il Parco sorge in un contesto tra i più densamente urbanizzati, caratterizzato dalla presenza di fabbriche storiche ${ }^{2}$, oggi quasi del tutto scomparse a seguito della de-industrializzazione, e grandi quartieri edilizi che, nel tempo, hanno saldato la periferia nord di Milano al suo hinterland quasi senza soluzione di continuità.

Prima della sua realizzazione il territorio si presentava con l'immagine tipica "dell'estrema periferia urbana, fatta di aree agricole residuali in attesa di trasformazione e disseminate di discariche abusive, di depositi all'aperto, di sfasciacarrozze, di orti spontanei, di baraccopoli più o meno mimetizzate tra gli orti e, da ultimo, di aree industriali dismesse e in stato di abbandono"3.

Situato nell'alta pianura asciutta e racchiuso all'interno di un triangolo costituito da tre grandi arterie stradali ${ }^{4}$, questo territorio non si è mai distinto per la presenza di aree significative dal punto di vista naturalistico; questo sia per le sue dimensioni, ma soprattutto per l'enorme influenza esercitata dall'uomo sul territorio. Il passato utilizzo a fini agricoli e successivamente il progressivo sviluppo insediativo ed industriale hanno fatto sì che le zone rifugio per le specie vegetali ed animali di un certo interesse si concentrassero principalmente lungo i corsi d'acqua limitrofi al parco ${ }^{5}$, dai quali però sono oggi quasi completamente scomparse per via del forte impatto urbano.

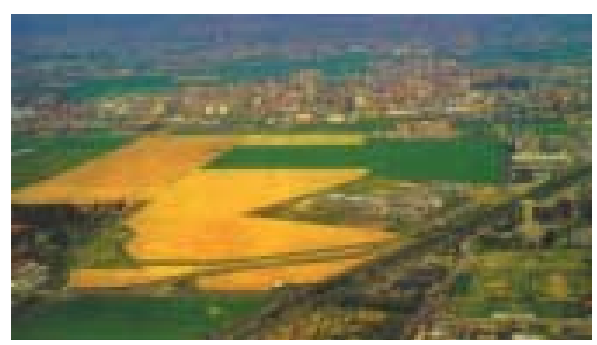

Figura 2 - L'area del Parco prima dell'intervento.

Il primo documento ufficiale in cui si riconosce la necessità di utilizzare come verde pubblico l'area 'miracolosamente' risparmiata alla crescita insediativa è la proposta del Piano Intercomunale Milanese ${ }^{6}$, elaborato proprio dal Centro studi del P.I.M. ${ }^{7}$ nella seconda metà degli anni Sessanta ${ }^{8}$.

L'istituzione del parco risale tuttavia al 1975, anche se la definizione legislativa più consona all'ambito tutelato è del 1985, anno in cui la Regione Lombardia affianca alle altre ormai consolidate tipologie di aree protette un nuovo tipo di parco, il "parco regionale di cintura metropolitana", con il quale vengono identificate le "zone di importanza strategica per l'equilibrio ecologico delle aree metropolitane, per la tutela e il recupero paesistico e ambientale delle fasce di collegamento tra città e campagna, per la connessione delle aree esterne, dei sistemi verdi urbani, per la ricreazione e il tempo libero dei cittadini, mediante la più efficace gestione del paesaggio con particolare riguardo alla continuazione $\mathrm{e}$ al potenziamento delle attività agro-silvo-colturali” ${ }^{9}$.

${ }^{2}$ Breda, Falck, Pirelli, Agusta.

${ }^{3}$ Francesco Borella, Il Parco Nord Milano, oggi, "Folia di Acer", 2, 1995, pag. 36.

${ }^{4}$ A nord, l'autostrada Milano-Venezia; ad est, viale Fulvio Testi e ad ovest, la superstrada Milano-Meda.

${ }^{5}$ Il Seveso e i derivatori del canale Villoresi.

${ }^{6}$ In realtà il primo atto significativo nella direzione del parco è stato senza dubbio il coinvolgimento della cittadinanza. La presenza, all'interno di questa densa conurbazione, di una vasta area edificabile ed eccezionalmente non edificata, infatti, risvegliò dapprima l'interesse dei cittadini che, provati da una situazione sempre più insostenibile, decisero di rivendicare la salvaguardia delle vaste aree comprese tra le zone di margine dei comuni di Milano, Sesto San Giovanni, Cinisello Balsamo, Bresso e Cormano. In particolar modo furono i cittadini di Bresso, forse più sensibili vista la presenza dell'aeroporto e la pressante urbanizzazione, ad organizzarsi in movimento spontaneo con l'obiettivo primario di creare su quest'area un esteso "polmone verde". Le rivendicazioni del movimento, a causa della loro eccessiva spontaneità, non trovarono tuttavia un'immediata reazione negli ambienti politici. Successivamente le spinte dei partiti politici, che interpretavano le esigenze della cittadinanza locale, spinsero l'assessore all'urbanistica di Milano, nonché presidente del Piano Intercomunale Milanese (P.I.M.), a promuovere la realizzazione su questo territorio di un grande parco ad uso collettivo.

${ }^{7}$ Istituito con l'obiettivo di elaborare un piano urbanistico di livello sovracomunale per l'area metropolitana milanese.

${ }^{8}$ Approvato dall'assemblea dei Sindaci del P.I.M. il 23 novembre del 1967.

${ }^{9}$ La vicenda normativa del parco è abbastanza complessa. Il primo atto amministrativo per la 


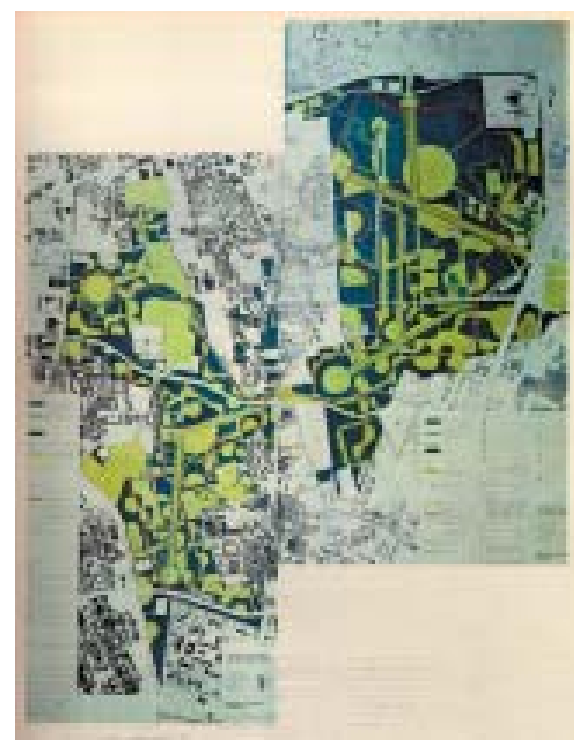

Figura 3 - Il Progetto di massima del 1979.

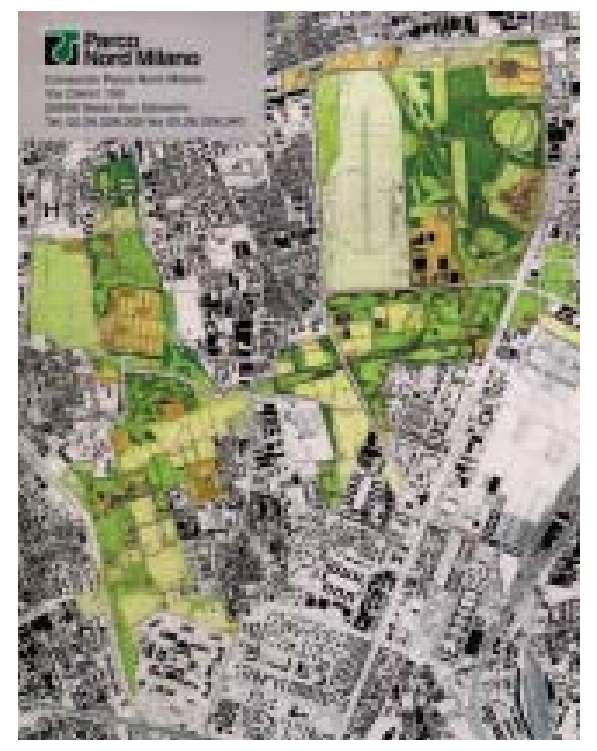

Figura 4 - Il Progetto di massima del 1995.

Dopo la proposta di Piano Intercomunale Milanese, l'idea comincia ad assumere concretezza nel 1970 quando il Consorzio Parco Nord Milano viene ufficialmente istituito con decreto prefettizio.

I primi due anni di attività furono impegnati principalmente nella difesa del territorio dalle proposte di utilizzazione non a verde che i privati, proprietari delle aree, ma anche le Amministrazioni partecipanti all'iniziativa, avanzavano di continuo. Negli stessi anni viene avviata la ricerca delle aree da acquisire per estendere e dare maggiore compattezza ai confini del parco; in questa direzione devono essere viste le attenzioni rivolte alle aree di proprietà della società finanziaria Breda collocate nella fascia nord est del parco, lungo viale Fulvio Testi.

Gli anni successivi furono dedicati invece all'attività di pianificazione del territorio e di inizio della progettazione vera e propria del parco. Nel 1973 viene affidato allo stesso Centro studi P.I.M. l'incarico di redigere il primo pre-progetto del parco, ma è solo nel 1975 che il Consorzio decide di elaborare il Piano Territoriale di Coordinamento.

Nel 1977, per cercare di tradurre in un disegno architettonico e paesaggistico gli obiettivi e le scelte individuate dal piano, viene affidata allo stesso gruppo di progettisti l'elaborazione di un progetto di massima che, consegnato nel 1979, chiude la

nascita del parco è la legge regionale 11 giugno 1975, n. 78 "Istituzione del Parco di interesse regionale Nord-Milano" che, oltre a fornire al Consorzio (ente che si occupa della gestione del parco sin dalla sua istituzione) i poteri necessari per vincolare i terreni e per procedere agli eventuali espropri, obbliga i comuni interessati ad adeguare i propri strumenti urbanistici e apre la strada alla concessione dei contributi finanziari da parte della Regione Lombardia.

Nei primi anni Ottanta, al mutare dell'idea di parco da realizzare diviene essenziale modificare anche l'atto legislativo che l'aveva istituito; la legge 78/1975 ispirata, infatti, ad una filosofia di conservazione delle bellezze naturali, della flora e della fauna, mal si adattava al caso in questione, considerato che nel parco erano del tutto assenti episodi significativi dal punto di vista naturalistico e faunistico.

Un primo passo per superare lo 'scollamento' tra la realtà del parco e il suo riconoscimento legislativo avviene con la L.R. 86/1983 "Piano generale delle aree protette regionali. Norme per l'istituzione e la gestione delle riserve, dei parchi e dei monumenti naturali nonché delle aree di particolare rilevanza naturale e ambientale". Con tale legge infatti la Regione introduce la tipologia del "parco locale di interesse sovracomunale" con la quale qualifica Parco Nord sottraendolo almeno in parte alla disciplina restrittiva prevista per i parchi e le riserve naturali. Nell'arco di breve tempo, tuttavia, anche quest' ultima definizione legislativa appare troppo rigida e induce il Consorzio a richiederne una revisione che avviene, in tempi brevi, con l'approvazione della legge regionale 41/1985 "Integrazioni e modifiche alla L.R. 86/83". Con questa legge la Regione introduce finalmente una nuova tipologia di parco, più consona alla tutela degli spazi aperti che ancora permangono ai margini delle grandi aree urbanizzate e, in particolare, al caso in questione. 
prima lunga fase di pianificazione del parco. Ad essa seguiranno poi a metà degli anni Ottanta una revisione del Piano Territoriale conclusasi con l'elaborazione di una nuova Variante ${ }^{10}$, l'avvio delle opere di costruzione del parco e la predisposizione nel 1995 di un nuovo progetto di massima che, integrando in un disegno complessivo l'esistente e il progettato, è stato definitivamente approvato solo nel maggio del 2000, ovvero contemporaneamente alla costruzione del parco stesso.

Dopo una lunga fase di pianificazione e di progettazione rimasta senza esiti visibili si era scelto dunque di intraprendere la strada della 'costruzione sul campo'. A partire dalle indicazioni emerse dai disegni dei primi anni Ottanta, assunti come impianto strategico di base, si è proceduto a disegnare direttamente sul territorio gli spazi aperti, le masse arboree, le quinte prospettiche, i percorsi attraverso la giustapposizione di tutti gli strumenti e gli elementi necessari per 'costruire' un paesaggio necessariamente 'artificiale', in grado tuttavia di riconferire un'identità forte ad un ambito urbano fortemente degradato e privo di qualità. Un disegno, un parco che si è costruito nel tempo attraverso progressivi aggiustamenti, ripensamenti, modifiche, adattandosi alle risorse economiche, amministrative, umane e tecnologiche di cui si poteva disporre e che solo ora, quasi al completamento della sua realizzazione, trova restituzione sulla carta.

\section{GLI OBIETTIVI}

L'obiettivo prioritario dei promotori di questa singolare esperienza è stato senza dubbio duplice: da un lato risparmiare all'espansione urbana un ambito strategico per la sua collocazione, dall'altro dotare la periferia milanese di un spazio verde ben disegnato con funzioni ricreative ed ecologiche. Finalità che sono state assunte evidentemente dai piani e progetti che si sono susseguiti, i quali tuttavia si sono dati anche obiettivi concreti di configurazione degli spazi attraverso il disegno dei margini, delle aree da destinare a prato o alle piantumazioni ed infine dei percorsi verdi e delle aree di connessione tra i diversi spazi.

Singolare appare anche il modello di parco da 'far sorgere' su questo territorio; al "parco-giardino" tradizionale subentra il modello del "bosco urbano", caratterizzato da costi molto più contenuti, da una quasi immediata figurabilità e già realizzato in alcune città europee ${ }^{11}$.

Il parco si presentava d'altronde come un grande spazio 'vuoto', privo di qualità, da disegnare e 'modellare' attraverso la giustapposizione degli elementi tradizionali di costruzione del verde: i filari, le masse alberate, le grandi superfici a prato dai contorni geometrici o naturali, l'intreccio delle visuali.

Il piano del $1975^{12}$, elaborato in stretto concerto con il P.I.M. seguendo le linee orientative emerse negli anni precedenti, non contiene un disegno vero e proprio del parco; esso definisce semplicemente lo schema di accessibilità, le direttrici e gli obiettivi prioritari per la stesura del progetto di massima, cioè per l'organizzazione funzionale e spaziale del parco $^{13}$.

Concependo l'area come un grande spazio verde in contrapposizione al tessuto edificato della periferia milanese, il piano cerca innanzitutto di accentuare

\footnotetext{
${ }^{10}$ Approvata solo nel 1990.

${ }^{11}$ Oltre che nella stessa metropoli milanese in un'area gestita dall'associazione Italia Nostra e denominata in maniera abbastanza provocatoria "Boscoincittà".

${ }^{12}$ Affidato agli architetti Mario Brunati, Demetrio Costantino, Raffaele Selleri e Virgilio Vercelloni viene approvato dal Consiglio regionale nel 1977.

${ }^{13}$ A differenza della maggior parte degli altri parchi regionali italiani, dunque, il piano non prevede una zonizzazione con specifiche norme di tutela e di uso. Ė evidente che una simile concezione deriva dalla natura stessa del parco: un grande spazio 'vuoto' dove non si pongono tanto problemi di tutela, quanto di ricostruzione dell'ambiente naturale e del paesaggio.
} 
questa visione distribuendo i nuovi edifici per le attrezzature di quartiere ai margini del parco, nelle aree di frangia, considerandoli quali 'strumenti' di integrazione morfologica e funzionale con la realtà urbana e come 'veicoli' di penetrazione dell'utenza all'interno del parco stesso.

Il piano formula inoltre tre scelte di grande rilievo: l'acquisizione delle proprietà private incluse nel perimetro del parco ${ }^{14}$; l'acquisizione e l'utilizzazione a fini sociali del patrimonio storico monumentale ${ }^{15}$ ed infine la realizzazione sul territorio del parco di un vero e proprio bosco urbano. A questo riguardo il piano prevede di sistemare a bosco o a piantumazioni d'alto fusto circa il $50 \%$ dell'intera superficie, mentre per la rimanente parte, ad esclusione delle aree edificate o lastricate in funzione delle attrezzature insediate, è prevista la formazione di radure prative calpestabili che si alternino in un gioco di pieni e vuoti quasi a riproporre il composito disegno della città fatto di lotti edificati, di superfici pertinenziali più o meno permeabili, di spazi vuoti destinati alla mobilità ed infine di aree libere vincolate ad uso pubblico o in attesa di edificazione.

È solo quattro anni più tardi che gli obiettivi del piano vengono tradotti in termini architettonici e paesaggistici attraverso il disegno e l'identificazione degli spazi e delle alberature che avrebbero dovuto caratterizzare questo grande spazio verde.

Nel progetto di massima l'organizzazione del parco viene definita da un disegno a grande scala, costituito da un sistema di grandi assi prospettici a piantumazioni regolari e non necessariamente coincidenti con i percorsi pedonali e ciclabili; da radure circolari circondate da aree boscate, di cui una grande al centro del quadrilatero orientale; da due sistemi di canali e specchi d'acqua di notevoli dimensioni e da una ciclopista perimetrale coincidente, per la gran parte del suo tracciato, con il confine tra le aree interne e le aree di frangia.

Una differente configurazione spaziale connota le aree interne e le aree di frangia del parco. Se per le prime vengono previste consistenti sistemazioni a bosco che definiscono i limiti e le quinte di una serie di spazi aperti dalle forme più o meno regolari, per le aree di frangia il progetto prospetta invece soluzioni

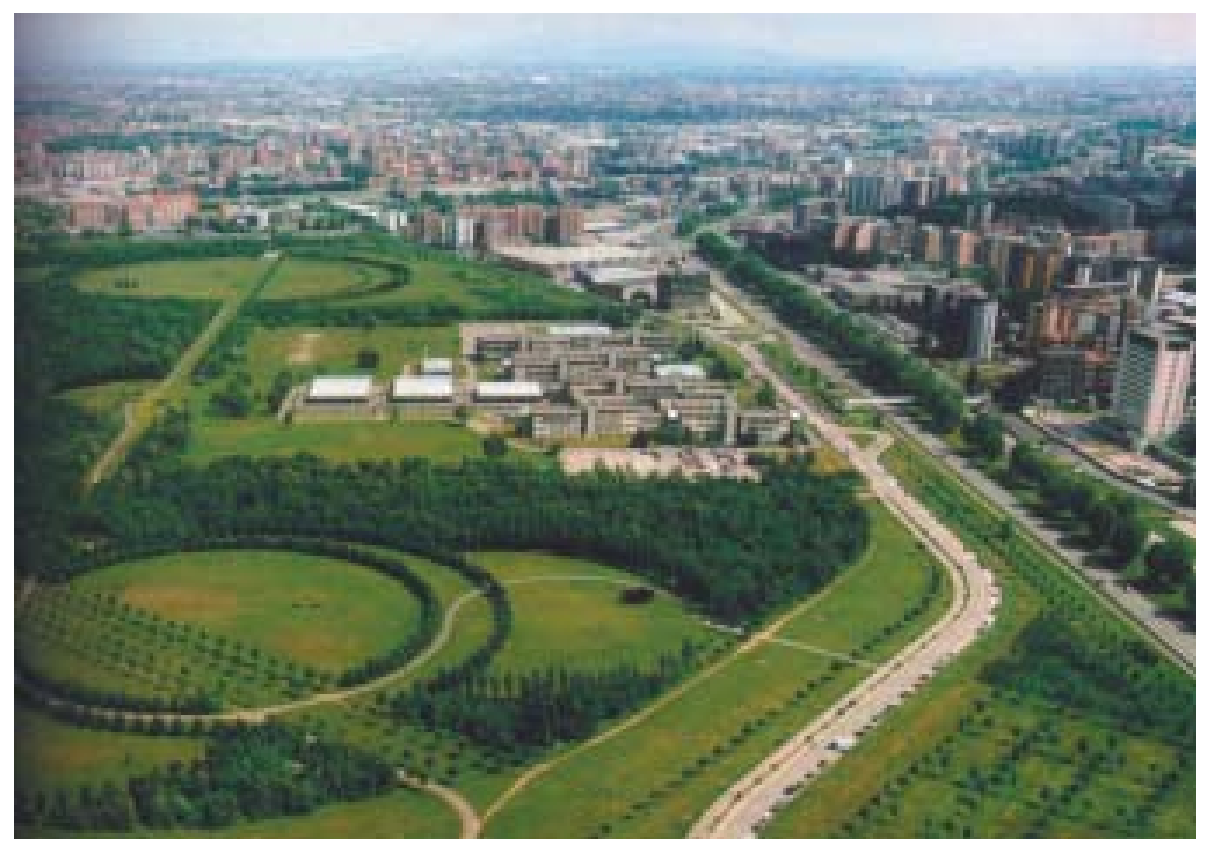

Figura 5 - Veduta aerea della porzione nord est del Parco.

\footnotetext{
${ }^{14} \mathrm{Si}$ tratta in particolare di 6 milioni di metri quadrati nei quali sono comprese l'area dell'aeroporto di Bresso e le aree sulle quali già insistono edifici privati.

${ }^{15}$ Villa Torretta a Sesto San Giovanni e villa Manzoni a Cormano.
} 
simili a quelle sperimentate nei parchi urbani veri e propri, con la creazione di superfici a prato variamente attrezzato con elementi di arredo e strutture per il tempo libero ${ }^{16}$; insomma degli spazi altamente fruibili dai cittadini che costituiscano una sorta di 'filtro' tra l'uniformità e la rigidità della maglia urbana e la varietà e la ricchezza degli spazi seminaturali da realizzare all'interno del parco.

Lungo le aree di frangia il progetto colloca anche le limitate possibilità edificatorie di attrezzature previste dal Piano Territoriale connettendole con gli ambienti del parco e tra di loro attraverso il percorso ciclopedonale che, snodandosi tra le radure e i boschi, percorre tutto il perimetro del parco.

$\mathrm{Al}$ progetto di massima fece seguito nel 1981 l'acquisto di 120 ettari di proprietà della Breda Finanziaria ${ }^{17}$ e la definizione del primo stralcio del progetto esecutivo dal costo di attuazione stimato in circa 18 miliardi di lire sui 90 miliardi del costo complessivo previsto per la realizzazione del parco secondo il disegno elaborato nel progetto di massima.

Nonostante la quantità di studi, piani e progetti elaborati negli anni precedenti, al 1983 non esisteva ancora una sola area ove la costruzione del parco potesse dirsi avviata. I fattori a cui è possibile imputare le difficoltà di avvio della fase realizzativa vera e propria sono principalmente quattro: la rigidità del piano territoriale del parco; la diffusa situazione di compromissione delle aree; l'impostazione del progetto esecutivo stralcio ereditato dalla precedente amministrazione; le difficoltà economiche conseguenti all'acquisto delle aree Breda.

Innanzitutto "le scelte del piano furono drastiche: tutte le aree dovevano essere recuperate a verde attrezzato metropolitano, ad attrezzature di quartiere potevano essere destinate alcune aree marginali, le aree private dovevano essere tutte acquisite alla disponibilità pubblica, le attività insediate dovevano essere tutte trasferite e nel frattempo gli occupanti avrebbero potuto unicamente compiere sugli immobili interventi di manutenzione ordinaria.

È evidente che un disegno così ambizioso (...) si sarebbe potuto attuare solo ad opera di un consorzio forte, soprattutto capace di eccezionale rapidità di intervento esecutivo.

(...) Nelle aree esterne (...) in assenza di una capacità di intervento realizzativo rapido quale il piano ipotizzava (...) la situazione sembrava sfuggire poco a poco di mano.

(...) Si era pensato di poter congelare per molti anni una realtà (...) in attesa di disporre del progetto definitivo e delle risorse per attuarlo "pronto effetto", e invece la realtà non si era lasciata congelare e aveva preso a muoversi, nell'unica direzione possibile per le aree periferiche "sotto vincolo di piano", quella del provvisorio, del marginale, dell'abusivo, verso cioè il degrado e lo squallore delle popolate terre di nessuno delle nostre periferie urbane.

(...) $\mathrm{Ma}$ ciò che ha soprattutto dato la misura dell'impraticabilità della "maniera grande" ipotizzata dal piano e perseguita dal consorzio nella sua prima fase (...) è stato il quadro finanziario venutosi a determinare. Il progetto esecutivo stralcio (...) nel contesto del debito Breda e delle terribili difficoltà a farvi fronte, apparve impraticabile, fuori dalla realtà" 18 .

$\grave{E}$ in seguito a queste considerazioni che il Consorzio decide di "mutare filosofia, nella convinzione che il parco non si sarebbe costruito se non gradual-

${ }^{16}$ Secondo il progetto il parco doveva essere tutto recintato ad esclusione degli spazi di margine in cui la recinzione doveva arretrare per lasciare libera una 'fascia' di parco sempre aperta al pubblico.

${ }^{17}$ Le prime trattative concrete iniziano nel 1980. Si tratta di terreni localizzati nel settore nord est del parco e comprendenti alcuni campi sportivi e la Torretta, una sontuosa villa di campagna dei nobili milanesi con affreschi di notevole valore artistico. Il pesante onere finanziario conseguente all'acquisto delle aree Breda pone il Consorzio in notevoli difficoltà economiche rendendo di difficile attuazione il primo stralcio del progetto esecutivo.

${ }^{18}$ FrANCESCO BORELlA, I parchi metropolitani come strumenti di riqualificazione: il Parco Nord nell'area milanese, "Edilizia Popolare", 205, 1988. 
mente, in tempi lunghi, recuperando il degrado e riqualificando area dopo area, riconquistando poco a poco, sul campo, la credibilità in parte perduta" ${ }^{19}$.

"La scelta era dunque quella del "work in progress", della rinuncia al progetto rigido e predefinito, nella consapevolezza che un parco territoriale di queste dimensioni e di questa collocazione non può che essere un'opera corale, cui molte persone, nell'arco probabilmente di alcuni decenni, sono e saranno chiamate a portare il loro contributo: un progetto generale "aperto", definito nella grande maglia, nel disegno di grande scala e che poi, all'interno delle singole "stanze verdi", avrebbe consentito di operare per successive addizioni, proposte o reinterpretazioni, oggi ma anche domani e dopodomani, da parte di chi fosse chiamato a progettare quel tassello; progetto destinato quindi a continuamente integrarsi e definirsi e ridefinirsi, per successivi contributi e approfondimenti, anche tornando talvolta a modificare e integrare il già realizzato" 20 .

Una scelta che ha consentito di uscire pressoché subito dall'immobilismo degli anni precedenti e di avviare la realizzazione vera e propria del parco con i primi rimboschimenti, le prime bonifiche ambientali, i primi recuperi e le prime acquisizioni di nuove aree.

Mantenendo pressoché inalterato il disegno di massima del parco $^{21}$, il Consorzio si pose l'obiettivo di una realizzazione graduale, per successive addizioni, affinché anche l'attuazione di un solo lotto del parco potesse soddisfare i fabbisogni dei cittadini, convincere le Amministrazioni dell'effettiva necessità di uno spazio verde così configurato ed infine costituire 'volano' per il completamento del parco e per la riqualificazione complessiva di tutto l'ambito periferico.

$\bar{E}$ con riferimento alla mutata filosofia di base che nel 1984 il Consorzio avvia una fase di revisione del Piano Territoriale conclusasi solo nel 1990 con l'approvazione della Variante generale.

I criteri guida e gli obiettivi del nuovo strumento possono essere così sintetizzati 22: "individuazione delle aree di primo intervento, destinate al verde metropolitano o, secondo la collocazione e la vocazione, al verde urbano; tutela delle aree agricole compatte, intese come aree di intervento di seconda fase; individuazione delle incompatibilità ambientali più rilevanti, da rimuovere, e definizione degli interventi per il recupero o il provvisorio inglobamento delle incompatibilità meno gravi o delle realtà più difficili da trasformare o da rimuovere; approntamento degli strumenti giuridici per la salvaguardia delle aree nella fase intermedia, fino all'attuazione del piano, pur senza ricorrere a vincoli e divieti intollerabili per gli attuali fruitori degli immobili; apertura di nuovi canali di partecipazione e di collaborazione alla

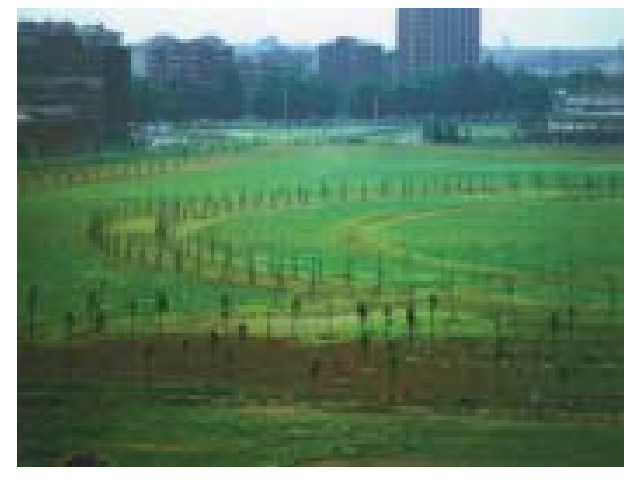

Figura 6 - I primi interventi di rimboschimento.

${ }^{19}$ Francesco Borella, op. cit., 1988.

${ }^{20}$ Francesco Borella, Il Parco Nord Milano, 1983-1998: riflessioni sopra un'esperienza, "Parchi”, 25, 1998, pag. 33.

${ }^{21}$ Furono apportate solo alcune modifiche conseguenti all'indisponibilità delle aree dell'aeroporto di Bresso incluse nel disegno del progetto di massima.

${ }^{22} \mathrm{Dal}$ punto di vista territoriale, il Piano individua all'interno del parco cinque ambiti aventi carattere, modalità e tempi di realizzazione differenti: la "zona a parco naturale attrezzato di livello metropolitano" comprende tutte le aree destinate prevalentemente al recupero naturalistico e ambientale attraverso il potenziamento delle aree boscate esistenti, i nuovi rimboschimenti e la salvaguardia e la realizzazione di prati, oltre alla fruizione per il tempo libero e la ricreazione; la "zona a parco metropolitano agricolo" comprende le aree per ora destinate alla prosecuzione dell'attività agricola ma che, nei progetti di lungo periodo, saranno destinate in linea di massima a parco metropolitano attrezzato; la "zona a parco attrezzato urbano" raccoglie invece tutte le aree di frangia e le aree connotate da una minore uniformità e compattezza, dove dovrebbe delinearsi un disegno di verde più "urbano" ed integrato ai tessuti edificati esistenti; la "zona monumentale", di modeste dimensioni ma di prioritaria importanza, investe le aree e le costruzioni di villa Torretta, sita nel comune di Sesto San Giovanni, e di villa Manzoni in comune di Cormano; la "zona edificata", ovvero tutti gli edifici che, con le relative pertinenze, insistono sulla superficie del parco, per i quali sono previsti interventi di recupero se i relativi usi sono compatibili con il parco o l'acquisto da parte del Consorzio e lasuccessiva successiva rimozione seritenuti ritenuti incompatibili. 
costruzione del parco, soprattutto riguardo alle attrezzature per lo sport e il tempo libero e al recupero della Villa Torretta, anche per operatori privati”"23.

"L'immagine che la variante esprime è (...) quella di un abito confezionato su misura per "vestire" ogni parte anatomica del parco, dopo una serie di prove o stadi intermedi, necessari per ottenere una visione finale perfetta" ${ }^{24}$.

Contestualmente alla revisione del Piano vengono approvati una serie di progetti a breve scadenza che mirano essenzialmente a rendere fruibile il territorio da parte della cittadinanza. Vengono così iniziati i primi interventi di forestazione urbana a cura dell'Azienda regionale delle Foreste; anche se un primo modesto intervento di impianto arboreo aveva avuto luogo già nel 1981, il vero programma di rimboschimento del parco viene realmente avviato solo nel 1983. Da quell'anno gli interventi sono proseguiti senza sosta dando luogo ad un processo di sistematica e graduale formazione del "sistema vegetale" 25 attraverso il quale si sono configurati gli spazi aperti, le quinte scenografiche, gli assi prospettici e gli spazi della fruizione tenendo quale struttura di fondo il disegno emergente dal progetto di massima della fine degli anni Settanta.

\section{LA METODOLOGIA}

Quanto detto finora rende senza dubbio evidente come l'originalità e l'unicità di questa esperienza ${ }^{26}$ siano dovute principalmente alla gradualità con cui è stata pensata la realizzazione del parco; una scelta, quella della gradualità, che costituisce obiettivo e, al tempo stesso, scelta metodologica per la costruzione di un nuovo 'brano' di paesaggio. L'impossibilità di ottenere 'tutto e subito' legata a problemi urbanistici, burocratici, economici hanno fatto prediligere la strada del "work in progress sotto gli occhi di tutti i cittadini che con le loro azioni di appropriazione possono indurre a modifiche progettuali senza tuttavia sconvolgere l'assetto generale del parco"27. Un parco che "non si realizza sul tavolo da disegno, non si realizza in pochi anni mirando ad un pronto effetto, si realizza (invece) mediante un impegno continuo che sappia distribuire il giusto peso ad ogni azione"28.

L'esigenza di costruire un paesaggio più vivibile dove gli elementi naturali costituiscono la struttura portante del nuovo 'tessuto' formato da prati, boschi, siepi, filari e specchi d'acqua ha indotto il Consorzio a sperimentare sul proprio territorio i metodi della "forestazione urbana", una innovazione teorica e metodologica che, nata negli Stati Uniti negli anni Sessanta, trova applicazione in Italia solo agli inizi degli anni Settanta grazie ad una associazione non governativa che introduce, pubblicizza ed applica questa metodologia per realizzare un parco urbano di soli trentacinque ettari all'estrema periferia di Milano ${ }^{29}$.

Una disciplina e una metodologia avente per obiettivo principale la coltivazione e la gestione degli alberi, ma che nel suo significato più ampio include anche la cura dei corsi d'acqua urbani, il ripristino degli habitat faunistici, lo studio di opportunità ricreative fuori città, la progettazione del paesaggio, il recupero di aree degradate, la cura degli alberi in genere e la loro gestione come fonti di materia prima.

${ }^{23}$ Francesco Borella, L'esperienza del Parco Nord Milano, "Folia di Acer", 2, 1995, pag. 42.

${ }^{24}$ Roberta Peverelli, Parco Nord Milano: parco metropolitano in corso di realizzazione, "Acer", 5, 1988, pag. 12.

${ }^{25}$ Un sistema che all'anno 2000 interessa circa 250 ettari di aree verdi.

${ }^{26}$ Almeno nel panorama italiano.

27 Andreas Kipar, Il Parco Nord Milano nel panorama europeo, "Folia di Acer", 2, 1995, pag. 35.

${ }^{28}$ ANDREAS KIPAR, op. cit., 1995, pag. 35.

29 Si fa riferimento all'esperienza di Boscoincittà. 
Una tecnica operativa che prevede la messa a dimora di un gran numero di piante al minor costo possibile, con formazione in tempi medio lunghi di ecosistemi equilibrati con ridotto e costante apporto di energia e materiali dall'esterno. Una tecnica che consente di configurare piccoli e grandi spazi aperti attraverso l'uso equilibrato degli elementi essenziali del paesaggio in un disegno in grado di coniugare esigenze ricreative ed estetiche con esigenze ecologiche e produttive tipiche del bosco.

Come si è detto la fase di realizzazione del parco viene concretamente avviata a partire dal 1983 con l'attuazione del primo lotto di rimboschimento effettuato nell'area più compatta del parco e relativa ai terreni acquistati dalla Breda. Da quell'anno il Consorzio inizia infatti la trasformazione a verde di tutte le aree industriali dismesse di cui era divenuto proprietario, dando priorità alle operazioni di pulizia e sgombero da attività abusive e agli interventi di forestazione urbana.

L'impianto del primo lotto su un'area di dieci ettari fu realizzato secondo uno schema semplice, con piante messe a dimora in file allineate, ortogonali tra loro e distanti 2 metri l'una dall'altra con una densità di 1.100 piante per ettaro ${ }^{30}$.

Nel secondo lotto di intervento, realizzato nel 1984 su un'area di circa dodici ettari posta nel settore orientale del parco tra il comune di Cinisello Balsamo e quello di Sesto San Giovanni, venne abbandonata la tecnica usata precedentemente a favore di un sesto di impianto a quadrato con distanza tra le piante di 2,50 metri e una densità pari a 1.600 piante per ettaro. Tale schema permetteva la minore ramificazione dei fusti, la più rapida chiusura delle chiome e lo sviluppo in tempi relativamente brevi dello strato organico del suolo.

Per i lotti di rimboschimento successivi si ritenne opportuno aumentare ulteriormente la densità degli impianti arborei e abbandonare lo schema di lavorazione incrociata ${ }^{31}$ a favore di un tracciato ad andamento curvilineo in grado di

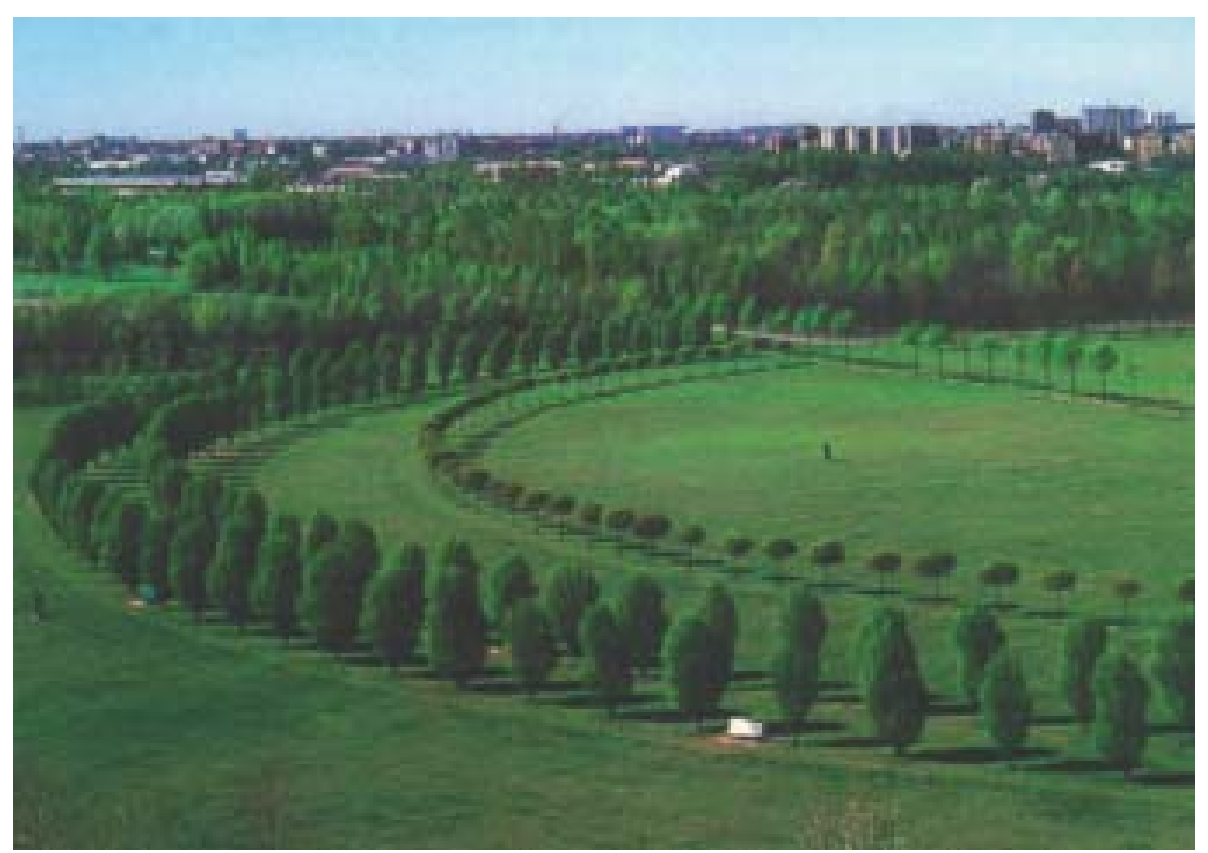

Figura 7 - Lo sviluppo dei filari che disegnano le grandi rotonde.

\footnotetext{
${ }^{30} \mathrm{Si}$ trattava di una disposizione in grado di garantire un'ottima possibilità di lavorazione incrociata del terreno con mezzi meccanici e, in genere, facilità di manutenzione.

${ }^{31}$ La densità venne infatti portata a 2.500 piante per ettaro con una distanza sulla fila di 1,60 metri e una distanza tra le file di 2,60 metri. Le ragioni dell'abbandono della lavorazione incrociata andavano ricercate nei danni che progressivamente erano stati causati al piede delle alberature dai mezzi meccanici di lavorazione del suolo.
} 
rompere la monotonia, la rigidità e la persistenza nel tempo di un impianto di tipo ortogonale.

Se con il primo e il secondo lotto erano stati impiantati pochi filari di alberi adulti, è a partire dal terzo lotto, realizzato nel 1985 ed esteso ad una superficie di circa 18.000 metri quadrati, che viene avviata la realizzazione del disegno del parco, con la creazione dei grandi assi visivi a filari e radure erbose circondate da alberi d'alto fusto. Con il quarto lotto di intervento, riguardante il rimboschimento forestale di 2,8 ettari confinanti con i lotti già rimboschiti, continuò la costituzione delle prime grandi direttive d'impianto del disegno del parco, con la creazione di filari per circa 9.000 metri lineari, la formazione di quattro gruppi ornamentali, l'impianto di specie arbustive su 0,25 ettari ed alcuni interventi di risarcimento forestale sui lotti precedenti.

Inizialmente per il rimboschimento furono impiegate pianticelle di 1-2 metri di altezza appartenenti alla tipica associazione vegetale padana: quercia, frassino, ornello, acero montano, olmo campestre, pino silvestre, pioppo e betulla; mentre per quanto riguarda la formazione di filari furono utilizzati esemplari più adulti di 4-5 metri di altezza e di specie diverse: pioppo cipressino, ippocastano, carpino bianco, farnia, quercia rossa, acero, tiglio e bagolaro. Sono stati inoltre creati quattro gruppi di piante ornamentali scelti tra le varietà che meglio si prestano a questo scopo: cedri, magnolie, liriodendri, sofore, paulownie, gleditsie.

Ai classici interventi di forestazione, tesi per lo più a dare vita al 'cuore' verde del parco, sono seguiti inserimenti di specie arbustive e di essenze ornamentali, finalizzati a creare un ambiente più gradevole dal punto di vista paesaggistico in grado di offrire sul piano estetico notevoli variazioni nella dimensione, nelle fioriture stagionali e nei colori.

All'inizio degli anni Novanta, a sette anni di distanza dai primi rimboschimenti, venne ritenuto indispensabile effettuare uno studio approfondito e interdisciplinare sullo stato del patrimonio vegetale del parco e sulle sue linee evolutive; questo appariva necessario non solo per comprendere come si stesse evolvendo l'ecosistema avviato artificialmente, ma anche per capire quali linee di intervento e quali tecniche adottare al fine di favorirne l'evoluzione verso la maggiore stabilità e complessità ambientale e paesistica.

Gli interventi che ne seguirono si ispirarono agli orientamenti operativi emersi dai diversi studi sia per quanto concerne l'assestamento e la riqualificazione delle aree già rimboschite ed affermate, sia per quanto riguarda i nuovi impianti.

Relativamente alla riqualificazione forestale e naturalistica degli impianti esistenti, la scelta delle specie da inserire ${ }^{32}$ e il loro posizionamento seguì modalità differenti ai margini o all'interno del rimboschimento. La necessità di cancellare la rigidità dell'impianto era particolarmente avvertita ai margini dei lotti boscati e nelle radure aperte in occasione della formazione di percorsi stabiliti, per cui il posizionamento divenne il fattore determinante per la riuscita dell'intervento.

Tenendo dunque come base la maglia quadrata dell'impianto esistente, tutti i nuovi inserimenti arbustivi furono collocati nel modo più casuale possibile per evitare qualsiasi schema ripetitivo che non avrebbe fatto altro se non evidenziare le preesistenti linee di impianto. Tutti questi interventi di riqualificazione non furono estesi ovviamente a tutte le superfici, ma solo ai punti strategici individuati dagli incroci delle linee visuali e dove l'effetto indesiderato era evidenziato anche dalla presenza massiccia di una sola specie arborea.

Per quanto riguarda invece i nuovi rimboschimenti gli interventi si orientarono verso la creazione di composizioni arboree ed arbustive, con predominanza

\footnotetext{
32 Gli studi misero in evidenza la necessità di inserire negli impianti esistenti essenze arboree ed arbustive, mancanti o insufficienti, e ritenute essenziali per il raggiungimento della migliore complessità ambientale e paesistica del sistema vegetale.
} 
di alcune essenze ritenute fondamentali per la vegetazione autoctona. A queste venivano comunque associati alcuni elementi arborei a più rapido accrescimento, così da permettere alla vegetazione di crescita più lenta, uno sviluppo in condizioni migliori. Il tracciamento dei nuovi rimboschimenti venne effettuato con un sesto d'impianto di 2,60 metri per 1,50 metri circa su una fila ad andamento curvilineo per rendere più naturale l'impianto.

Nel 2000 è stato realizzato il diciassettesimo lotto di rimboschimento che ha elevato a quota 200.000 circa il numero di alberi ed arbusti messi a dimora nei diciassette anni di realizzazione del parco.

Se l'attività di forestazione è stata prevalente nei primi anni di realizzazione del parco, successivamente l'opera di riqualificazione e di attuazione ha incluso anche tutta una serie di interventi complementari ai rimboschimenti Tra questi vanno senza dubbio ricordati: la bonifica ambientale e gli sgomberi; la realizzazione dei percorsi ciclopedonali e delle passerelle di collegamento; il recupero e la ristrutturazione degli edifici storici inclusi nel parco; la realizzazione delle attrezzature; la costruzione degli impianti per l'irrigazione e la distribuzione dell'acqua all'interno del parco e la creazione di alcuni specchi d'acqua artificiali.

\section{GLI ESITI}

L'impiego delle tecniche di forestazione, la varietà delle specie impiegate e la loro disposizione sul territorio delimitato dal parco hanno consentito di ottenere esiti spaziali e visivi di notevole interesse.

Nonostante la scelta iniziale fosse stata quella di favorire le necessità funzionali, ecologiche e sociali del parco rinunciando almeno in parte alla cura degli aspetti puramente estetici, in realtà osservando una fotografia aerea o, più semplicemente, percorrendo uno dei numerosi sentieri del parco si rimane colpiti dalla calibrata ricerca formale delle composizioni arboree ed arbustive impiegate. In particolare colpiscono i grandi cerchi concentrici di querce rosse che, attraversati da un doppio filare di ippocastani e sottolineati dalle spalliere di carpini fastigiati, disegnano gli spazi a prato del quadrilatero orientale; o ancora i grandi assi visivi disegnati dai filari di tiglio o di ippocastano che guidano il visitatore alla scoperta dei luoghi più suggestivi del parco e lo inducono ad immergersi nei numerosi sentieri che corrono tra $\mathrm{i}$ boschi per ammirare i colori del parco e i giochi di luce e ombra creati dalla disposizione degli elementi naturali.

Allo stesso modo del disegno del paesaggio forestale anche altri interventi hanno assunto un ruolo rilevante nella configurazione degli spazi e nella connotazione paesaggistica del parco. Tra questi certamente considerevoli sono gli esiti spaziali scaturiti dal recupero ambientale della cosiddetta "montagnetta" e dalla realizzazione dell'importante sistema di canali e specchi d'acqua ancora in corso di completamento.

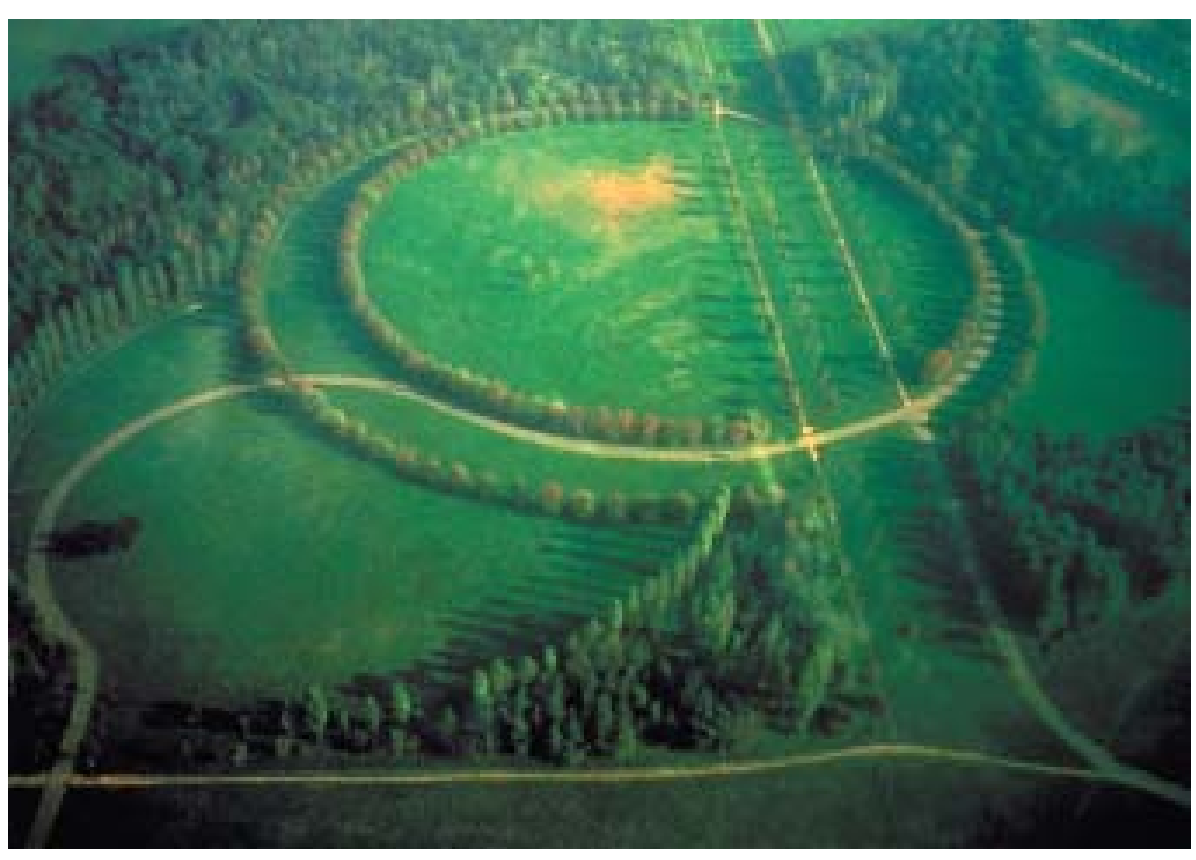

Figura 8 - I grandi cerchi concentrici di querce rosse. 
Nella sua opera di riqualificazione ambientale il parco si era trovato a gestire pesanti situazioni di degrado dovute alla presenza di manufatti o di residui industriali incompatibili con le sue finalità; con l'acquisto delle aree Breda erano state annesse al parco tutta una serie di strutture industriali in stato di abbandono e con esse anche una vera e propria discarica di inerti provenienti dagli altiforni delle fabbriche siderurgiche allora operanti su questo territorio ${ }^{33}$.

La presenza di quest'area così fortemente degradata aveva indotto il Consorzio ad inserire il progetto di sistemazione nel quadro del programma triennale di interventi 1984-1986; la sistemazione a verde di quest'area rappresentava, nell'ambito del paesaggio pianeggiante del parco, una grande occasione di valorizzazione dell'ambiente.

L'operazione di ripristino comportò un'attenta progettazione e alcuni anni di lavoro; movimento di terra per coprire la discarica, rimodellazione della superficie, riporto di terreno fertile furono le operazioni principali per il recupero della montagnetta.

Il progetto di massima, elaborato nel 1985 a cura dell'architetto Andreas Kipar, si basò su un'attenta analisi della situazione esistente nella convinzione che il recupero ambientale di un'area dovesse basarsi innanzitutto sullo stato di fatto della stessa e ad esso ispirarsi.

A partire da questi presupposti l'idea formale del progetto venne subito orientata dall'elemento verticale della montagnetta; l'accentuazione della verticalità e l'inserimento della montagnetta come struttura armonica, consona al paesaggio circostante rappresentavano, infatti, le scelte principali e vincolanti del progetto.

La "verticalità, che è un fattore isolato in un ambiente complessivamente pianeggiante, è stata ripresa nel progetto per creare nuovi punti di vista panoramica sulla pianura" 34 . Reso dinamico il profilo del rilievo, l'intervento ha teso a raccordare dolcemente le pendici all'area pianeggiante circostante.

"Gli ampi spazi pianeggianti all'interno sono stati destinati alle più varie funzioni (...), come ad esempio l'esposizione floreale e l'orto botanico, mentre la sommità è (stata) interpretata quale luogo di tranquillità su cui potersi isolare ed osservare lo scenario sottostante" 35 .

L'elemento vegetale ha assunto in quest'area un'importanza determinante; circa l' $80 \%$ dell'area totale è stata, infatti, destinata a verde e in particolare il $30 \%$ a piantagioni e il $50 \%$ a prato, distinto tra manto erboso delle scarpate e quello delle aree pianeggianti che rendono leggibile il disegno morfologico della montagnetta e lasciano spazio alle più varie funzioni ricreative.

L'attuazione del progetto, avviata nel 1986, avvenne gradualmente tenendo conto delle disponibilità finanziarie ed organizzative del Consorzio e, soprattutto, di quella strategia del "work in progress" che animava qualsiasi scelta progettuale da attuare nel parco.

Gli esiti ottenuti sono decisamente notevoli. Da lontano la montagnetta si presenta all'orizzonte come una 'grande' collina verde, come un 'segno' indelebile della storia che ha segnato questo territorio. Una collina a tutt'oggi fruibile dalla popolazione; un luogo suggestivo per il disegno morfologico del terreno, per la varietà delle piante ornamentali con i colori delle fioriture che si alternano nell'avvicendarsi dei mesi, per la varietà degli scorci e dei paesaggi che si possono osservare percorrendo la vasta rete di percorsi sinuosi che circondano e attraversano questo sorprendente rilievo artificiale.

\footnotetext{
${ }^{33}$ Nata come cumulo di scorie prodotte dagli altiforni, la montagnetta era poi 'cresciuta' negli anni adempiendo al ruolo di discarica.

${ }^{34}$ ANDREAS Kipar, Recupero a verde: la montagnetta nel Parco Nord, "Acer", 5, 1988, pag. 19.

${ }^{35}$ ANDREAS KiPAR, op. cit., 1988, pag. 19.
} 


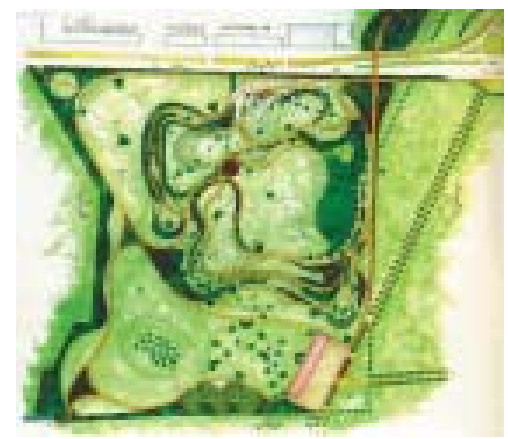

Figura 9 - Progetto di recupero della montagnetta (1985).

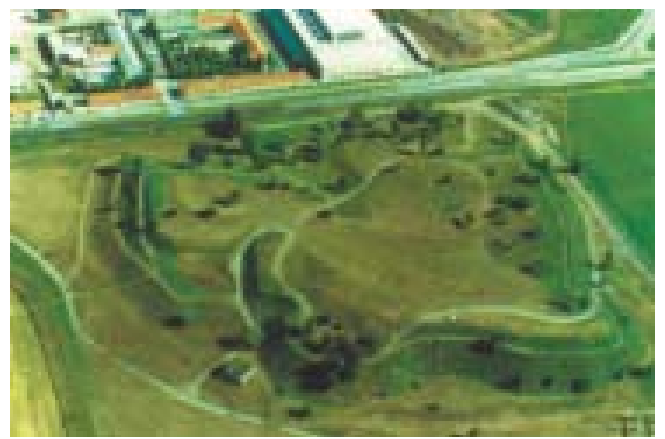

Figura 10 - Veduta aerea della montagnetta poco dopo gli interventi di rinverdimento.

Relativamente all'inserimento dell'elemento acqua all'interno del paesaggio del parco occorre innanzitutto premettere che il progetto si era venuto via via definendo e concretizzando sulla base di una ricerca relativa al ruolo e alle funzioni dell'acqua nei parchi metropolitani ${ }^{36}$.

La ricerca che aveva messo in evidenza come "l'acqua (...) (fosse) sempre stata un elemento base per la formazione e l'abbellimento del verde di ogni tipo sia che in esso prevalga l'architettura, la natura o l'agricoltura" ${ }^{37}$, era scaturita in una proposta di utilizzo a scopi irrigui delle acque del Canale Villoresi; proposta che andava ad affiancarsi alla decisione già assunta dal Consorzio di realizzare un condotto sotterraneo in pressione per l'irrigazione degli impianti vegetali della cosiddetta "montagnetta".

Il progetto prevedeva di distribuire l'acqua proveniente dal Canale Villoresi nell'area principale del parco sotto forma di un sistema di laghetti e canali che potevano garantire al tempo stesso il mantenimento di un discreto sistema di acque superficiali ${ }^{38}$.

Il sistema di laghetti e canali, alimentato a regime dal condotto di adduzione del Canale Villoresi, è stato attuato per la parte a sud di via Clerici: il primo gruppo di stagni è stato costruito nel 1992 tra la montagnetta e viale Fulvio Testi; altri due laghetti sono stati realizzati nel 1999 nella vasta area che si estende ad ovest della montagnetta, tra Bresso e Niguarda. A quest'ultimi l'acqua proviene dal laghetto già esistente e posto ad est attraverso un canale in parte sotterraneo che sfocia poi nella sede del vecchio "canale Breda" ora intubato e diretto al depuratore.

Si tratta di un sistema di acque artificiali fortemente caratterizzante il parco; il disegno libero e sinuoso degli specchi d'acqua e la presenza di una

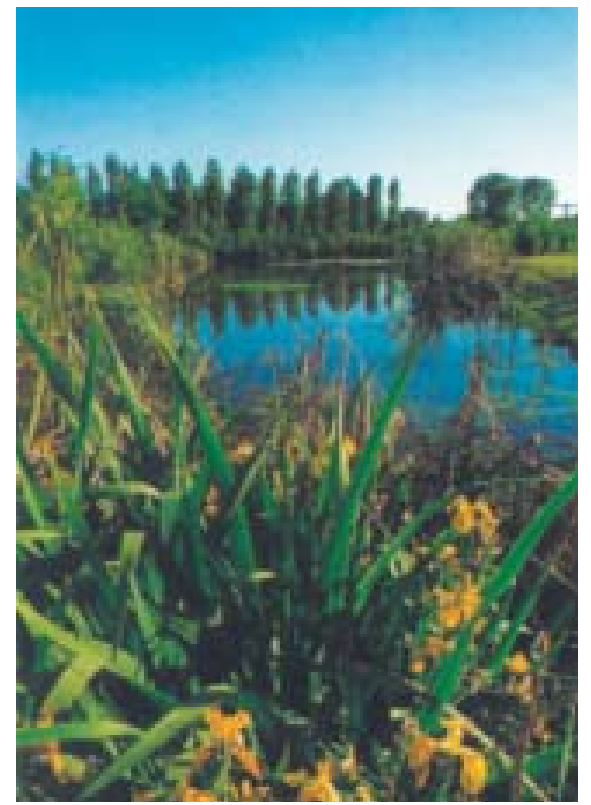

Figura 11 - Uno scorcio sul primo laghetto realizzato.

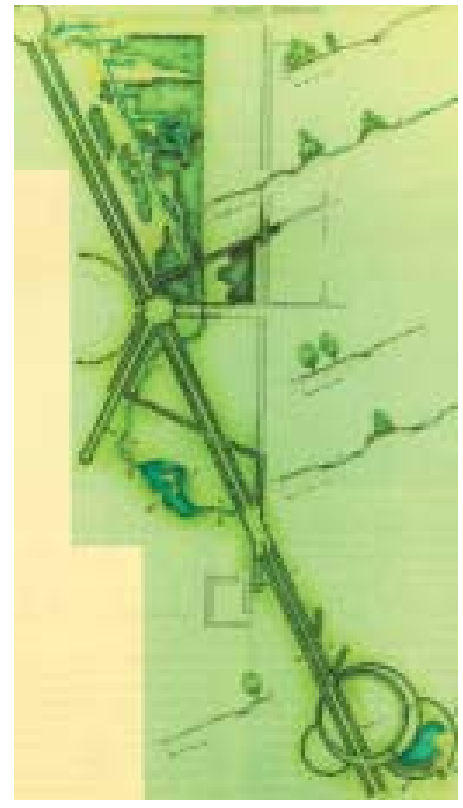

Figura 12 - Il disegno del "progetto acqua”.

${ }^{36}$ Alla ricerca, dal titolo "Ruolo e funzione delle acque nei parchi metropolitani europei, storici e recenti, con riferimento alla possibilità di utilizzazione a scopo irriguo di acque addotte dal Canale Villoresi nel Parco Nord”, partecipò anche il Dott. Luigi Brambilla, profondo conoscitore della campagna irrigua lombarda.

${ }^{37}$ PaOlo Lassini, Progetto di irrigazione del Parco Nord Milano mediante le acque addotte dal Canale Villoresi, "Acer", 3, 1991, pag. 17.

38 Il disegno paesaggistico delle acque è stato realizzato dagli architetti Francesco Borella e Andreas Kipar. 


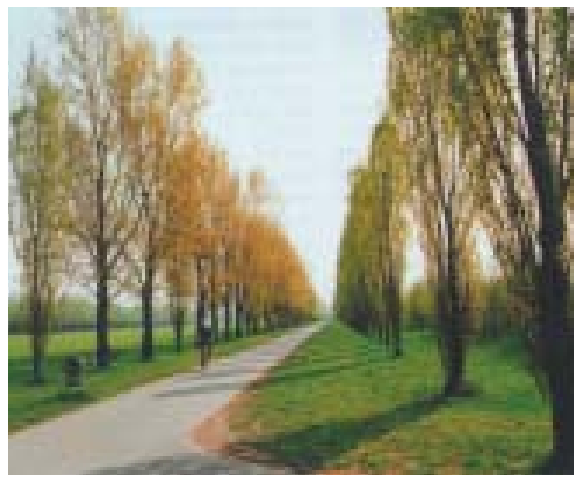

Figura 13 - Il grande viale alberato.

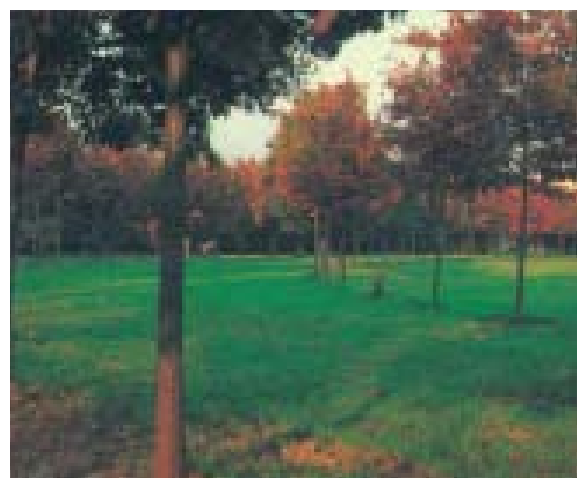

Figura 14 - La grande rotonda delle querce rosse.

ricca vegetazione acquatica lungo le sponde hanno contribuito a creare degli ambiti naturalistici e paesaggistici di grande interesse e suggestione.

La presenza all'interno di un contesto così densamente urbanizzato degli elementi tipici del paesaggio naturale, quali le modeste ondulazioni del terreno e i piccoli stagni, contribuisce a rendere reale e pienamente vivibile un paesaggio altrimenti presente solo nell'immaginario collettivo.

Con il definitivo interramento dell'ultimo tratto rimasto a cielo aperto del "canale Breda" e la costruzione delle opere idrauliche necessarie, nei prossimi anni l'elemento acqua è destinato a divenire via via sempre più importante anche all'interno del settore nord del parco. Dal 1999 è infatti iniziata la realizzazione di un nuovo sistema di canali e laghetti nella zona nord, un sistema che partendo da Cinisello Balsamo e correndo con un disegno libero e a tratti sinuoso verso sud, fino alla via Clerici e oltre, si ricollega al sistema di laghetti già esistenti.

Il disegno di questo nuovo sistema di acque si articola secondo tre ambiti differenti collegati tra loro mediante il grande asse a quadruplice filare che costituisce l'elemento centrale del settore orientale del parco.

Nel primo tratto "a nord emerge una sistemazione "dichiaratamente libera" seppure impostata su uno schema che ricalca le strutture primarie del parco"39; il progetto prevede per questo primo lago, lo sviluppo di ambienti acquatici di una certa rilevanza naturalistica, grazie ad un disegno che rende inaccessibili in molti punti le sponde dello specchio d'acqua, favorendo la colonizzazione di flora e fauna selvatica specifica.

Il tratto centrale che pure è caratterizzato dalla presenza di un lago naturalistico circondato dai prati e dalle aree di sosta, "si inserisce in un contesto che già per sua natura si presenta con caratteristiche meno formalizzate e più mosse; la presenza del laghetto intende sottolineare il carattere di questo spazio, unico nel paesaggio del parco" ${ }^{40}$.

L'ultima parte, più formalizzata ed orientata ad assecondare il disegno dell'esistente struttura vegetale, è invece caratterizzata da un lungo tratto di canale rettilineo che, correndo tra un doppio filare di ippocastani, si va a collegare all'ultimo laghetto attraverso un tracciato con andamento semicircolare sottolineato dal filare di carpini fastigiati.

L'immagine del parco che è derivata da questi e da altri interventi è dunque "quella di una grande area verde, di un vero parco metropolitano, tuttora in corso di realizzazione e di completamento, ma già ben strutturato e definito" ${ }^{41}$.

\footnotetext{
${ }^{39}$ Francesco Borella, L'esperienza del Parco Nord Milano, "Folia di Acer", 2, 1995, pag. 44.

${ }^{40}$ Francesco Borella, op. cit., 1995, pag. 44.

41 Francesco Borella, Parco Nord Milano, 1983-1998: riflessioni sopra un'esperienza, in Consorzio Parco Nord Milano (a cura di), Parco Nord Milano. Notizie 1998, Milano 1998, pagg. 3-4.
} 
Un parco che alterna zone di ripristino ambientale in cui sono stati realizzati interventi di forestazione urbana ${ }^{42}$ a zone a parco urbano attrezzato in un disegno naturaliforme del bosco caratterizzato dall'intervallarsi di spazi poligonali, rettilinei e mistilinei all'interno dei quali si aprono i tanti e diversi ambienti del parco: la grande rotonda, la montagnetta, il pratone, i laghetti, i grandi viali, i campi agricoli, la rotonda delle sophore, il cerchio delle ginestre, la scacchiera dei ciliegi, il teatrino, il bosco, i percorsi pedonali e ciclabili, gli orti.

Lultimo elemento che si vuole considerare trattando degli esiti del parco riguarda l'aspetto più propriamente gestionale dell'area. Elemento di successo e fattore determinante per il conseguimento degli esiti descritti è stato senza dubbio l'assetto istituzionale, operativo e cooperativo che ha caratterizzato il parco sin dai primi anni della sua istituzione. Innanzitutto la costituzione di un Consorzio di comuni, un ente pubblico che, pur dovendo rispettare la volontà politica delle Amministrazioni comunali consorziate, ha mantenuto nel tempo una certa autonomia, agilità e snellezza burocratica; fattori che hanno consentito l'assunzione diretta delle scelte progettuali e delle priorità di intervento. In secondo luogo la possibilità di disporre di una struttura tecnico-operativa propria, cresciuta in parallelo con il parco, che ha permesso di realizzarne il disegno in "economia diretta", ricorrendo solo in casi particolari all'appalto a ditte esterne ${ }^{43}$. Infine la convinzione che dall'"ascolto strutturato", dal coinvolgimento e dalla cooperazione con i cittadini e le associazioni locali potesse scaturire l'immagine del parco e la forza necessaria alla sua realizzazione.

Con il 2000 e in particolare con la sostituzione del direttore ${ }^{44}$ e l'approvazione quasi contestuale del progetto di massima e del nuovo statuto è stata chiusa un'epoca molto importante per l'esistenza del parco; la fase pionieristica della sua costruzione ha lasciato il posto alla fase, forse più complessa, di governo di una realtà ben definita e strutturata, ma in costante evoluzione poiché "non tutto è finito, non tutto è perfetto; ancora molto resta da fare e, in qualche caso, da correggere, da riqualificare, da "reinventare" 45 .

\section{RIFERIMENTI BIBLIOGRAFICI}

Borella FranCESCO, I parchi metropolitani come strumenti di riqualificazione: il Parco Nord nell'area milanese, "Edilizia Popolare", 205, 1988, pagg. 25-36.

Borella FranCESCO, L'elemento acqua nel Parco Nord: stato attuale e previsioni future, "Acer", 3 , 1991, pag. 19.

Borella FranCesco, Il Parco Nord Milano, oggi, "Folia di Acer", 2, 1995, pagg. 36-37.

Borella Francesco, L'esperienza del Parco Nord Milano, "Folia di Acer", 2, 1995, pagg. 39-43.

Borella Francesco, "Progetto acqua", "Folia di Acer", 2, 1995, pag. 44.

Borella Francesco, Il Parco Nord Milano, 1983-1998: riflessioni sopra un'esperienza, "Parchi", 25, 1998, pagg. 33-38.

Borella FranCesCo, Parco Nord Milano, 1983-1998: riflessioni sopra un'esperienza, in Consorzio Parco Nord Milano (a cura di), Parco Nord Milano. Notizie 1998, Milano 1998, pagg. 2-45.

Carra Mauro, Il bosco urbano del Parco Nord Milano, "Folia di Acer", 2, 1995, pag. 45.

Colombo Tomaso e Gini Riccardo, Parco Nord Milano. Perla d'Europa, "Parchi e Riserve naturali", 1, 2001, pagg. 4-11.

Consorzio Parco Nord Milano, Parco Nord Milano. Venticinque anni in un soffio..., Edireport, Milano 2001.

\footnotetext{
${ }^{42} \mathrm{Al} 2001$ il bosco occupa complessivamente una superficie di 70 ettari.

${ }^{43}$ Un'impostazione decisamente controcorrente con la tendenza sempre più diffusa tra le amministrazioni pubbliche dell'area milanese a trasferire a società esterne la realizzazione e la cura delle opere a verde, con il risultato di ridurre progressivamente, fino quasi a cancellarle, le proprie strutture operative.

${ }^{44}$ A Francesco Borella che ha seguito le vicende di Parco Nord sin dalla nascita dell'idea stessa di parco succede in qualità di nuovo direttore del parco Riccardo Gini.

${ }^{45}$ Francesco Borella, op. cit., 1998, pag. 45.
} 
Dente Bruno, Il Parco Nord Milano, in IReR-Progetto Milano (a cura di), Istituzioni e nuovi modelli di governo urbano, Franco Angeli, Milano 1989, pagg. 72-82.

Engel Marco, Parco Nord: ricucire le periferie metropolitane, "Folia di Acer", 2, 1995, pagg. 46-47.

ENGEL MARCO, I traguardi di un direttore, "Acer", 4, 2000, pagg. 64-67.

Guccione Biagio, Parchi e giardini contemporanei. Cenni sullo specifico paesaggistico, Alinea, Firenze 2001, pagg. 57-60.

KIPAR ANDREAS, Recupero a verde: la montagnetta nel Parco Nord, "Acer", 5, 1988, pagg. 17-21.

Kipar Andreas, Il Parco Nord Milano nel panorama europeo, "Folia di Acer", 2, 1995, pagg. 34-35.

LASSINI PAOLO, Progetto di irrigazione del Parco Nord Milano mediante le acque addotte dal Canale Villoresi, "Acer", 3, 1991, pagg. 17-22.

Peverelli Roberta, Parco Nord Milano: parco metropolitano in corso di realizzazione, "Acer", 5, 1988, pagg. 11-13.

Regione Lombardia, In/Arch Lombardia, Parchi naturali e urbani, Regione Lombardia, Milano 1979.

Urso Massimo, Aspetti naturalistici ed educazione ambientale, "Folia di Acer”, 2, 1995, pagg. 46-47.

\section{RIFERIMENTI ICONOGRAFICI}

Figura 1: elaborazione di Laura Ferrari. Base dati Laboratorio Metodi e Tecniche di Pianificazione Urbanistica, Dipartimento di Architettura e Pianificazione, Politecnico di Milano.

Figure 2, 4, 6, 7, 13: Consorzio Parco Nord Milano (a cura di), Parco Nord Milano. Notizie 1998, Milano 1998, pagg. 3, 49, 23, 22, 16.

Figura 3: Regione Lombardia, In/Arch Lombardia, Parchi naturali e urbani, Regione Lombardia, Milano 1979.

Figura 5: ENGEL MARCO, I traguardi di un direttore, "Acer", 4, 2000, pag. 65.

Figura 8: GuCCIOne Biagio, Parchi e giardini contemporanei. Cenni sullo specifico paesaggistico, Alinea, Firenze 2001, pag. 60.

Figure 9, 10: KIPAR ANDREAS, Recupero a verde: la montagnetta nel Parco Nord, "Acer", 5, 1988, pagg. 18, 19.

Figura 11: Colombo Tomaso e Gini Riccardo, Parco Nord Milano. Perla d'Europa, "Parchi e Riserve naturali”, 1, 2001, pag. 11.

Figura 12: Borella FranCeSCO, "Progetto acqua", "Folia di Acer", 2, 1995, pag. 44.

Figura 13: Consorzio Parco Nord Milano (a cura di), Parco Nord Milano. Notizie 1998, Milano 1998, pag. 16.

Figura 14: Borella Francesco, L'esperienza del Parco Nord Milano, "Folia di Acer", 2, 1995, pag. 40. 
SCHEDA DI PROGETTO

Progetto:

Comuni interessati:

Ente gestore:

Tipologia:

Superficie:

Progettisti:

Ditte esecutrici:

Anno di istituzione:

Anno di progettazione:

Specie vegetali principali:

Anno di realizzazione:

Costo di intervento: riqualificazione e progettazione degli spazi aperti di cintura metropolitana

Milano, Sesto San Giovanni, Cinisello Balsamo, Bresso, Cormano, Cusano Milanino

Consorzio Parco Nord Milano (costituito nel 1973)

parco regionale di cintura metropolitana (Regione Lombardia, Legge 41/85)

6.000.000 mq di cui $2.800 .000 \mathrm{mq}$ già consolidati nelle opere a verde, nei percorsi e nelle attrezzature

Dott. Arch. Francesco Borella, in qualità di Direttore del parco dal 1983 al 2000,

Dott. Arch. Andreas Kipar, in qualità di consulente paesaggista dal 1985 al 2000

Dott. Agr. Paolo Lassini, in qualità di consulente per il "progetto acqua"

Dott. Arch. Mario Brunati, Dott. Arch. Demetrio Costatino, Dott. Arch. Raffaele Selleri

Dott. Arch. Virgilio Vercelloni (Piano Territoriale di Coordinamento del Parco 1975, Progetto di massima 1977)

Azienda Regionale delle Foreste, Milano (Progetto forestale 1983-1990)

Dott. Arch. Davide Papa, Dott. Arch. Marco Fiori, Dott. Arch. Silvia Calabroni, Dott. Arch. Alessandro Ferrari, in qualità di componenti dell'Unità operativa demanio, progetti e lavori, Consorzio Parco Nord Milano

Azienda Regionale delle Foreste, Milano (1983-1990)

Unità operativa gestione, impianti forestali, manutenzione e pulizia, Consorzio Parco Nord Milano (1990-2002)

1975 (Legge regionale 11 giungo 1975, n. 78)

1973 - primo pre-progetto del parco

1975 - elaborazione del Piano Territoriale di Coordinamento del Parco (approvato nel 1977)

1977 - elaborazione del primo Progetto di massima del Parco

1984 - elaborazione della Variante generale al Piano Territoriale di Coordinamento del Parco (approvata nel 1990)

1995 - elaborazione del secondo Progetto di massima del Parco (approvato nel 2000)

Acer campestre, Acer pseudoplatanus, Acer platanoides, Celtis australis, Populus hybrida, Ulmus campestre, Crataegus cru-agali, Pinus sylvestris, Tilia parvifolia, Fraxinus excelsior, Betula alba, Carpinus betulus, Quercus robur, Prunus avium, Corpus sanguinea, Corylus avellana, Ligustrum vulgare, Rosa rugosa, Sambucus racemosa, Viburnum lantana, Viburnum rhytidophyllum

1980 - acquisto delle aree di proprietà della Breda Finanziaria

1983 - primo lotto di rimboschimento

$1985 / 1988$ - interventi di recupero della "montagnetta"

1992/2000 - realizzazione del primo sistema di laghetti e canali

2000 - diciassettesimo lotto di rimboschimento

35 miliardi di lire non rivalutate di cui:

25 miliardi per l'acquisto delle aree necessarie e per la ristrutturazione della sede del parco e degli altri immobili consortili; 10 miliardi per la sistemazione a verde 



\title{
IL BOSCO QUALE ELEMENTO DI RIORDINO DELLO SPAZIO METROPOLITANO: L'ESPERIENZA DI "BOSCOINCITÀ" A MILANO
}

\author{
Laura Ferrari
}

\section{IL CONTESTO}

"Boscoincittà" nasce a Milano nei primi anni Settanta grazie ad una iniziativa della sezione milanese di Italia Nostra ${ }^{1}$, con l'intento di sperimentare nuove forme di creazione e di gestione del verde pubblico nelle aree di cintura urbana e metropolitana, troppo spesso trascurate dalla progettazione urbanistica tradizionale. L'idea legata alla creazione del Bosco era infatti quella di sperimentare un modello di 'parco metropolitano' caratterizzato dall'essere operativamente semplice e facilmente riproducibile.

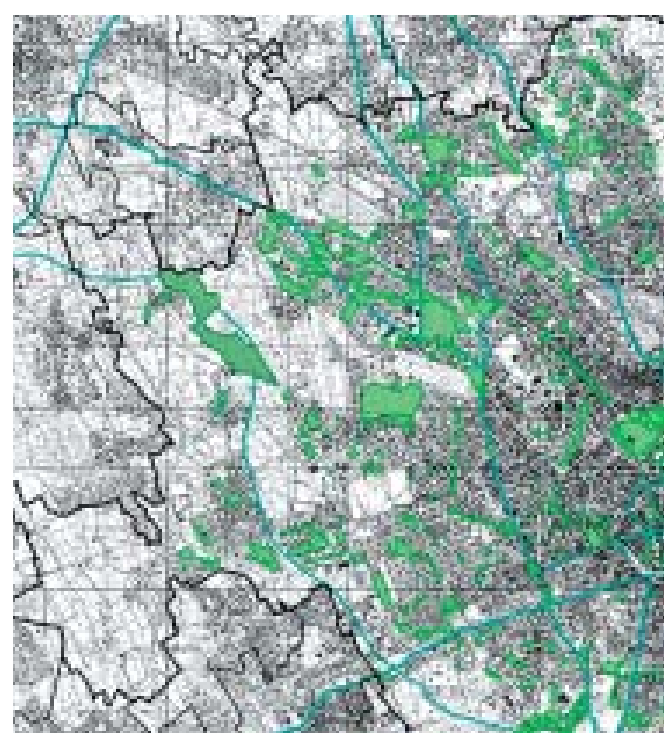

Figura 1 - Inquadramento territoriale.

Situato all'estrema periferia ovest della città di Milano ai margini del territorio edificato, il Bosco, con un'estensione di ottantatre ettari ${ }^{2}$, si colloca in un contesto territoriale ancora caratterizzato dalla presenza di un sistema eterogeneo di spazi aperti. Aree agricole dismesse, aree di risulta delle attività edilizie, aree infrastrutturali e aree fortemente compromesse da usi impropri ${ }^{3}$ rappresentano infatti gli elementi strutturali di questo spazio, solo in minima parte caratterizzato dalla presenza di boschi lineari in corrispondenza delle teste di fontanile e dei canali costruiti per utilizzare ai fini agricoli le risorgive.

Le aree su cui sorge il Bosco sono di proprietà del Comune di Milano che sin dai primi anni Settanta le ha offerte in concessione gratuita demandando l'attuazione, la gestione e la manutenzione del Bosco ad Italia Nostra attraverso la stipula di convenzioni novennalí.

L'area è destinata dal Piano Regolatore del 1953 e dalle successive varianti a verde agricolo e comunale; in particolare la Variante del 1973, raccogliendo le

\footnotetext{
${ }^{1}$ L'associazione Italia Nostra, fondata nel 1955 con lo scopo di concorrere alla tutela e alla valorizzazione del patrimonio storico, artistico e ambientale della nazione, è un'Organizzazione Non Lucrativa di Utilità Sociale (O.N.L.U.S.) i cui proventi sono destinati esclusivamente al funzionamento dell'associazione e alla realizzazione dei suoi fini istituzionali.

${ }^{2}$ Prima degli ampliamenti la superficie iniziale del Bosco era di trentacinque ettari.

${ }^{3}$ Tra questi si segnalano la presenza di discariche, orti abusivi, sfasciacarrozze, baraccopoli e depositi all'aperto.

${ }^{4}$ Convezioni rinnovate di volta in volta sia in termini di forma che di contenuti per via della costante evoluzione dell'iniziativa.
} 
indicazioni del Piano Intercomunale Milanese della fine degli anni Sessanta ${ }^{5}$, prevedeva che i territori del Bosco fossero destinati alla creazione di un parco comprensoriale finalizzato alla salvaguardia delle ampie zone a verde agricolo ancora presenti.

Purtroppo negli anni successivi all'approvazione della Variante il Comune, trovandosi ad affrontare forti vincoli di bilancio, dovette ridurre gli investimenti e rimandare l'attuazione del parco. Fu proprio in questa situazione di incertezza che Italia Nostra ottenne dal Comune l'assegnazione dell'area per la creazione del bosco urbano; una tipologia di intervento fortemente voluta dall'associazione e in linea con le indicazioni che il Piano Intercomunale Milanese aveva fornito per il previsto Parco Ovest: un parco all'interno del quale realizzare grandi recuperi boschivi che, tenendo conto della presenza di numerosi terreni agricoli, doveva realizzarsi lungo la linea dei fontanili, oltre la tangenziale e verso Cusago.

Se negli anni a seguire, nonostante il superamento delle difficoltà economiche, le indicazioni della Variante sono state ugualmente disattese, Boscoincittà è diventato invece una realtà in crescita e in costante trasformazione, un'esperienza che nata come sperimentazione quasi utopica, ha raggiunto alti livelli qualitativi sia dal punto di vista progettuale, sia da quello gestionale.

In un periodo in cui si iniziavano a ricercare forme di gestione del verde più convenienti che permettessero di 'tagliare' i costi ma fossero al tempo stesso qualitativamente e quantitativamente soddisfacenti, l'esperimento del Boscoincittà dimostrò infatti la possibilità di realizzare un bosco urbano anche in casi di limitata estensione territoriale e in situazioni caratterizzate da scarsità di mezzi economici, assenza di contributi finanziari pubblici e di mano d'opera specializzata da impiegare nei lavori di piantagione. Se in Europa e negli Stati Uniti le nuove sperimentazioni in atto venivano condotte grazie alla collaborazione degli organismi statali, nel caso di Boscoincittà la situazione è stata totalmente differente ${ }^{6}$. Nei primi anni di attività gli accordi tra il Comune di Milano e Italia Nostra non includevano infatti alcun tipo di contributo economico a sostegno della creazione del Bosco tanto che l'associazione dovette provvedere ad autofinanziare il progetto e ad avviare i lavori in economia forzata.

\section{GLI OBIETTIVI}

Negli anni Settanta il mutamento nei modelli insediativi, l'espansione incontrollata delle periferie e la progressiva degradazione degli spazi aperti portarono alla necessità di trovare un elemento unificante che, identificato nella tipologia del bosco o del parco, fosse in grado di rispondere in modo soddisfacente sia alle problematiche ambientali, sia a quelle del disegno paesaggistico. A queste esigenze andavano aggiunte anche una crescente domanda di spazi verdi e una tendenza sempre più diffusa della popolazione ad aprirsi alla natura e a rifugiarsi in essa.

La scelta di realizzare un bosco sembrava soddisfare tutte queste necessità. Il bosco risponde, infatti, ad esigenze ambientali, quali la depurazione chimica e batteriologica dell'atmosfera, la fissazione di gas tossici e polveri, l'azione di ter-

${ }^{5}$ Il Piano Intercomunale proponeva l'istituzione di una serie di parchi atti a creare una 'cintura verde' intorno alla città. Nelle previsioni la cintura doveva essere costituita da: Parco Nord, con aree di pertinenza dei comuni di Milano, Bresso, Cinisello, Sesto San Giovanni; Parco Ovest (nord) comprendente San Siro, il Parco di Trenno, Cormano e Settimo Milanese; Parco Ovest (sud) con Baggio, Cesano Boscone, Cusago e Gaggiano; Parco Sud cui afferivano i territori di Chiaravalle, Assago, Corsico e Opera; Parco Est con la zona Forlanini e Peschiera Borromeo.

${ }^{6}$ Va ricordato infatti che la prima convenzione stipulata con il Comune di Milano non prevedeva che pochi punti fermi: la durata della concessione, la collaborazione da attuarsi con l'ufficio tecnico, la gratuità della concessione e nessuna forma di finanziamento da parte dell'Amministrazione. 


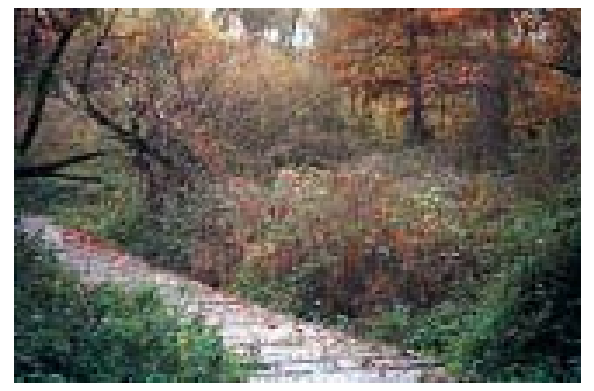

Figura 2 - I colori del Bosco.

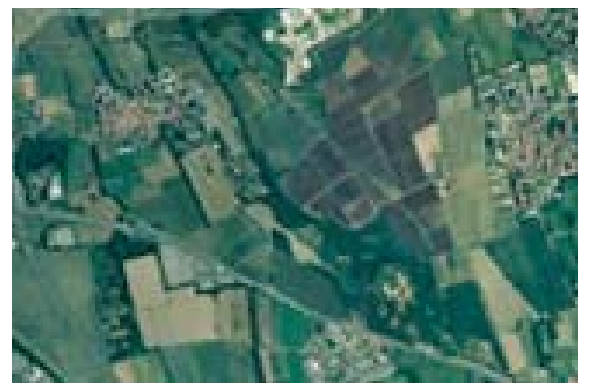

Figura 3 - Vista aerea dell'ambito del Bosco

moregolazione dovuta all'emissione di vapore acqueo, la funzione di barriera antirumore. Allo stesso modo la natura dinamica del bosco, unitamente allo sviluppo spontaneo di alcune specie floristiche tipiche dell'area di intervento, favoriscono lo sviluppo di un disegno paesaggistico di notevole suggestione, un disegno sempre più naturale e 'spontaneo' in cui la rigidità dello schema iniziale impresso dall'uomo perde via via consistenza per lasciare spazio ad un ecosistema sempre più 'reale', dall'equilibrio ecologico sempre più perfetto.

Infine la creazione di un bosco risponde ad esigenze ricreative e di fruizione; una fruizione però che non è di tipo intensivo come nel caso del parco urbano, ma che permette al visitatore di entrare in contatto con ambienti naturali integri e di favorire l'incontro tra l'uomo e la natura nella forma più diretta possibile.

Queste considerazioni, unitamente alla presa di coscienza dell'esiguità del patrimonio boschivo milanese $\mathrm{e}^{7}$, portarono dunque alla decisione di realizzare su questo territorio il primo di una serie di 'boschi urbani' che, sull'esempio di molti altri paesi del nord Europa ${ }^{8}$, dovevano andare a colmare il grande fabbisogno di aree verdi.

La scelta di creare un bosco urbano rispondeva inoltre anche ad alcune ragioni pratiche specifiche: si doveva tenere conto, infatti, della scarsità dei mezzi economici a disposizione e della mancanza di mano d'opera specializzata? .

Sulla base di tutte queste considerazioni la realizzazione del Bosco prese avvio nel 1974 con l'affidamento dell'incarico all'architetto Giulio Crespi, il quale predispose per il Bosco un progetto 'aperto', un progetto fatto semplicemente di 'idee' e di 'intenti' e non accompagnato dagli elaborati cartacei e dalle planimetrie che solitamente corredano qualunque progetto architettonico, urbanistico o paesistico.

Per descrivere le idee e gli intenti del progetto sembra allora utile riferirsi a quanto lo stesso Crespi scrive nella relazione allegata alla domanda di concessione dell'area: "La progettazione del Bosco ha avuto come obiettivo quello di creare degli spazi naturali che nella moltiplicazione del fronte del bosco mediante numerose radure, riuscisse a ricreare un ambiente vario e facilmente connettibile al futuro Parco Ovest previsto nelle zone circostanti. Questo indirizzo progettuale nasce dalla impossibilità di sfruttare a fini paesaggistici dislivelli

\footnotetext{
${ }^{7}$ Si pensi che solo il 4,5\% circa delle pianure lombarde può essere considerato area boschiva e che tale percentuale nell'area metropolitana milanese diminuisce fino al $3 \%$ circa.

${ }^{8} \mathrm{Si}$ pensi ad esempio all'Amsterdam Bos della fine degli anni Trenta o ancora allo Spaarnwoude Park (2.750 ettari) che la città di Amsterdam realizzò negli anni Sessanta su un'area pianeggiante, all'Oslo's Oslomarka, al Vestkoven a Copenhagen, al Thames Chase di Londra, alle antiche riserve di caccia del Bois de Boulogne e del Bois de Vincennes a Parigi, o ancora a Berlino che vanta un'estensione boschiva pari a 7.803 ettari di cui il $98 \%$ aperti al pubblico.

${ }^{9}$ La possibilità di realizzare un giardino urbano era stata esclusa a priori. La sua realizzazione infatti oltre a prevedere un'intensa fruizione di tipo ricreativo rendeva necessaria la sistemazione di manufatti di diverso genere che avrebbero inciso in modo rilevante sui costi complessivi. Il ricorso poi a tecniche di giardinaggio che tendono ad ottenere il massimo risultato produttivo ed alla realizzazione estetica nel minor tempo possibile avrebbe richiesto un illimitato apporto di energia, di materiali e di continue cure dall'esterno con notevole aumento dei costi.
} 
e punti di vista particolarmente significativi. La presenza della Cascina San Romano suggeriva di creare attorno uno spazio sufficientemente ampio che mantenesse l'originario rapporto spaziale con i campi coltivati. Il grande emiciclo a nord della cascina dovrebbe concludere questo spazio a prato: le chiome cresciute delle piante, viste dalla cascina, formeranno un orizzonte. Lo sviluppo delle piante oggi messe a dimora raggiungerà un effetto significativo tra 10-15 anni, mentre l'uso degli spazi a prato potrà essere visto in tempi più brevi.

$\mathrm{Si}$ è quindi pensato di formare uno specchio d'acqua che crei subito un polo di interesse oltre la cascina, che pure dovrà ospitare attività confacenti alla destinazione dell'area. La presenza dell'acqua, ampliata in seguito verso ovest, migliora il microclima favorevole al rapido sviluppo delle piante, oltre a presentare vantaggi per i mesi più asciutti.

A tal fine si cercherà di alimentare lo stagno con le acque del Villoresi anche a scopi irrigui attraverso i vecchi canali. Lo stagno, benché artificiale, sarà eseguito mediante tecniche sperimentate in Inghilterra per la formazione dei bacini di riserva d'acqua con strati di argilla ed insediando sulle rive particolari specie di piante. Lo stagno avrà così contorni naturali e potrà ospitare numerose specie di animali, che come in tutta l'area del Bosco (sono previste piante con bacche) saranno protette.

La piantagione è eseguita con metodo che garantisca la naturalità del Bosco. All'interno delle radure verranno piantati gruppi di piante particolarmente significative per il loro portamento o colorazione, sempre però rispettose di una naturalità che prescinde dall'artificio. Unico inserimento formalizzato saranno grandi gruppi di arbusti fioriferi che saranno disposti nelle radure o ai margini di queste. Il più rapido sviluppo di questi arbusti permetterà di percepire prima dello sviluppo delle grandi essenze forestali gli spazi, e la vivacità della fioritura e della colorazione, che saranno elemento significativo per gli spazi così creati.

La crescita del Bosco e la formazione di un sottobosco spontaneo portato dagli uccelli e da un rinnovamento spontaneo ricondurrà alla naturalità più libera. Solo a posteriori, quando le masse arboree saranno formate, si tracceranno pochi percorsi ed aree per le soste formalizzate.

Il Bosco, comunque, prima di essere una realtà volumetrica di grandi piante e di prati regolati è un'area di incontro tra uomini e natura in un rapporto non predatorio, ma creativo, con nuove piante seminate e cresciute grazie all'aiuto di molti nel vivaio che alimenterà la realtà del Bosco"10.

Dalla descrizione del progetto fatta dall'architetto Crespi emerge dunque l'originalità della concezione del Bosco rispetto alla tradizionale impostazione delle aree verdi e dei parchi pubblici ${ }^{11}$, non solo nel panorama italiano notoriamente poco innovativo in questo senso, ma anche nel più ampio contesto internazionale dove le sperimentazioni per la ricerca di formule innovative erano già in atto da tempo ${ }^{12}$.

Sono state proprio queste sperimentazioni ad ispirare, almeno in parte la realizzazione di Boscoincittà; una realizzazione che ha dovuto tuttavia fare i conti con

${ }^{10}$ Giulio Crespi, Relazione di progetto, Milano 1974.

${ }^{11}$ Agli inizi degli anni Settanta infatti la progettazione del verde risultava essere ancora legata a canoni molto formali, gli elementi fondamentali del disegno erano rigidi e la dotazione irrinunciabile del parco pubblico era costituita dalle strade, dai vialetti, dalle panchine lungo i percorsi, dall'illuminazione e dai giochi per i bambini; tutti elementi che contribuivano a fare del parco un ambiente immutabile, quasi museale, le cui spese di mantenimento risultavano inevitabilmente molto alte.

${ }^{12} \mathrm{Nel}$ panorama internazionale le sperimentazioni in atto, pur rivolte in direzioni differenti, erano tutte ugualmente tese alla ricerca di forme progettuali maggiormente fruibili dal pubblico e dai costi contenuti nelle fasi di realizzazione e di gestione: in Inghilterra si sviluppò la teoria dei grandi parchi metropolitani e delle new towns, fondate sulle basi della tradizione inglese del parco-paesaggio; in America, negli anni Quaranta, all'interno dei dibattiti sul paesaggio si iniziò a criticare la simmetria; in Germania si svolgevano esperimenti proprio nella creazione di aree boschive. 
una serie di vincoli tipicamente locali quali ad esempio le ridotte possibilità economiche e le particolari condizioni ambientali, prima fra tutte la limitata estensione territoriale, trentacinque ettari, non certo ideale per la realizzazione di un bosco. Anche la scelta di utilizzare come mano d'opera personale volontario non qualificato risultava vincolare fortemente il disegno del Bosco, un disegno che doveva essere necessariamente semplice e flessibile, così da renderne più agevole l'esecuzione materiale. La disponibilità stessa del materiale vegetale imponeva forti vincoli al progetto; nei primi anni, infatti, tutte le piante messe a dimora erano state regalate ad Italia Nostra da altri enti e in tempi diversi; questo aveva costretto a lavorare con essenze non previste a priori dal progetto e a volte inadatte all'ambiente del Bosco.

Lultimo vincolo era infine rappresentato dalla presenza di alcuni manufatti edilizi con i quali il progettista era tenuto a confrontarsi ${ }^{13}$ : la cascina, il cui uso era stato inizialmente contemplato dalla convenzione con il Comune di Milano ed in seguito divenuta parte fondamentale del progetto; le rogge e i piccoli canali irrigui; i fontanili e la loro vegetazione.

Ẽ evidente che questa serie di fattori ha determinato nel caso del Boscoincittà precise conseguenze pratiche sul progetto, sulle successive realizzazioni e sulla gestione stessa dell'area ${ }^{14}$.

\section{LA METODOLOGIA}

Se come già è stato sottolineato l'idea di realizzare un bosco ai margini dell'agglomerazione milanese ha rappresentato un'innovazione radicale nel campo della progettazione del paesaggio e delle aree verdi sia in ambito italiano, sia nel più ampio panorama internazionale, altrettanto innovative si manifestano anche le metodologie adottate per la realizzazione del Bosco.

Innanzitutto il progetto che, non contando né di elaborati cartacei, né di planimetrie, consisteva semplicemente di una serie di indicazioni di massima, una soluzione in grado dunque di 'tollerare' eventuali modifiche e migliorie in corso d'opera.

Il Bosco prese infatti forma pionieristicamente, senza la guida di un disegno preordinato; i disegni e le planimetrie furono predisposti solo a piantumazioni avvenute come rendiconto delle operazioni svolte ${ }^{15}$.

L'approccio progettuale adottato a Boscoincittà trova riscontro nell'"Urban Forestry", una disciplina che, nata negli Stati Uniti negli anni Sessanta ${ }^{16}$, non si

\footnotetext{
${ }^{13}$ La decisione di confrontarsi con i vincoli preesistenti avvicinava Boscoincittà alle esperienze allora in corso in Olanda, Germania e America, dove veniva privilegiato il confronto con il paesaggio esistente, anche se marginale o parzialmente compromesso; in Italia la tendenza in atto era invece quella di una protezione integrale del territorio che veniva dunque messo in contrapposizione all'ambiente costruito più che in dialogo con esso.

${ }^{14}$ La gestione del Bosco, i progetti di ampliamento nonché l'organizzazione delle attività di promozione per la fruizione del Bosco e di tutte le iniziative ad esso legate, vengono coordinate direttamente dal Centro di Forestazione Urbana, un ente istituito nel 1981 quale distaccamento operativo all'interno della sezione milanese di Italia Nostra al fine di poter operare al meglio le scelte riguardanti il Parco.

${ }^{15} \mathrm{La}$ tipologia di verde da realizzare e il metodo di progettazione e realizzazione adottato hanno sempre privilegiato gli aspetti gestionali rispetto alle decisioni estetiche; è proprio per queste ragioni che l'esperienza di Boscoincittà per il suo carattere puntuale e progressivo può essere paragonabile a quella di un'azienda agricola. I problemi di carattere eccezionale, quali ad esempio i rapporti con gli agricoltori della zona, la prevenzione degli incendi, i conflitti con i cacciatori, sono infatti stati affrontati via via che si sono presentati, cercando ogni volta il compromesso più vantaggioso per tutti gli attori coinvolti.

${ }^{16}$ In un momento in cui lo sviluppo edilizio ed urbanistico cresceva in modo frenetico, incontrollato e quanto mai caotico, parte della popolazione dei quartieri più poveri, generalmente i più danneggiati dalle nuove espansioni, diede vita a movimenti di opposizione caratterizzati dalla propria decisa partecipazione alla costruzione e gestione dei quartieri stessi.
} 
interessa solo degli alberi delle città o della manutenzione delle singole piante, ma di tutti i territori influenzati ed utilizzati dalla popolazione urbana ${ }^{17}$.

I principi che ispirarono l'Urban Forestry hanno avuto diffusione, seppur con alcune differenze, anche in Italia dove la disciplina ha assunto il nome di "Forestazione Urbana"18.

Seguendo dunque i principi della "Forestazione Urbana"19, le piantumazione del Bosco sono state realizzate progressivamente tenendo conto dell'uso agricolo preesistente e mediandolo con la nuova destinazione a bosco urbano.

Inizialmente sono state adottate tecniche di piantagione tipiche della forestazione, tecniche che hanno come obiettivo la messa a dimora di un gran numero di piante al minor costo possibile. Sono stati utilizzati semenziali di dimensioni ridotte $^{20}$, se non direttamente la semina a dimora, e sono stati previsti diradamenti selettivi e altri interventi di manutenzione minimi.

Successivamente tenuto conto che l'applicazione fedele delle tecniche forestali avrebbe comportato tempi di realizzazione e dunque di fruizione decisamente troppo lunghi, si è optato per l'utilizzo di tecniche ispirate da criteri di tipo più agronomico-forestale ${ }^{21}$, in grado di consentire la realizzazione del Bosco in tempi relativamente brevi e a costi contenuti ${ }^{22}$.

Le 15.000 piantine impiegate per la piantagione dei primi trentacinque ettari di terreno furono selezionate tra semenziali o trapianti di 1,2 o 3 annii $^{23}$ allevate in vivaio e messe definitivamente a dimora quando giudicate capaci di emergere tra le infestanti. L'impianto di esemplari di tale sviluppo comporta infatti una buona riduzione dei tempi di realizzazione rispetto alla messa a dimora di semenziali, anche se il loro impiego comporta una particolare cura e prepara-

${ }^{17}$ Il termine "Urban Forestry" venne utilizzato per la prima volta all'Università di Toronto nel 1965; successivamente nel 1968 all'interno del rapporto sullo stato dell'ambiente che il Citizen Advisory Committee on Recreation and Natural Beauty pubblicò e presentò al Presidente degli Stati Uniti, il tema delle alberature negli ambienti urbanizzati assunse grande rilievo tanto che, all'interno dello stesso rapporto, veniva richiesta la creazione di un Programma di Urban Forestry a cura del Servizio Forestale dello Stato. Un programma rivolto ai governi locali, alle organizzazioni e ai singoli cittadini in grado di fornire supporto tecnico e teorico alla cura e alla gestione di parchi pubblici, spazi aperti, alberature stradali, 'green belt' e proprietà private. Fu con l'accoglimento di questa richiesta che la Urban Forestry divenne una disciplina ufficialmente riconosciuta.

${ }^{18}$ La prima differenza tra le due discipline è riconducibile ai movimenti popolari che l'hanno ispirata: se i movimenti popolari statunitensi miravano a 'ritagliarsi', a conquistarsi delle aree verdi in quartieri così densamente edificati da risultare invivibili, in Italia il 'verde' veniva considerato un elemento di integrazione dei diversi, non organici, movimenti edificatori. Questa considerazione permette di focalizzare l'attenzione anche su un'altra differenza: nel nostro paese il movimento che promuove la creazione del 'verde' all'interno delle città non è di tipo spontaneo, ma frutto di elaborazioni teoriche; negli Stati Uniti al contrario erano stati i cittadini esasperati a muoversi per primi e solo in seguito il movimento spontaneo era stato ufficializzato. A differenziare ulteriormente le due discipline è anche il diverso accoglimento trovato presso i rispettivi governi: negli Stati Uniti le istanze provenienti dal movimento popolare sfociarono nella istituzione di appositi organismi e programmi statali; in Italia invece fu un associazione non governativa ad introdurre, pubblicizzare ed applicare le nuove metodologie e, a distanza di più di trent'anni da questa prima esperienza, non esiste ancora nel nostro paese una cultura e una politica organica di tutela del verde e dell'ambiente.

${ }_{19}$ Secondo questa tecnica le piante devono essere disposte secondo griglie modulari il cui disegno assume forma definitiva solo dopo il diradamento delle pianticelle, ovvero quando vengono eliminati gli esemplari malati o deboli. Le specie da utilizzare sono prevalentemente indigene o di provata adattabilità all'ambiente in cui vengono inserite. La successiva cura del bosco non deve riguardare le singole piante, ma il loro insieme.

${ }^{20}$ Piante nate da seme che non hanno mai subito trapianti.

${ }^{21}$ Frutto di un giusto compromesso tra le tecniche forestali vere e proprie e le più tradizionali tecniche del giardinaggio. La necessità di sperimentare queste nuove tecniche stimolò l'avvio di un rapporto di collaborazione tra l'Istituto di Coltivazioni Arboree dell'Università degli Studi di Milano e lo staff tecnico di Boscoincittà, collaborazione tuttora in atto.

${ }^{22}$ Era stato scartato a priori il ricorso alle tecniche di giardinaggio tradizionali, le quali avrebbero sì consentito una più rapida realizzazione del Bosco a scapito tuttavia di un notevole aumento dei costi di impianto e del materiale vegetale che mal si adattavano alla situazione specifica.

${ }^{23}$ Con sviluppo medio di 1,5-2 metri fino a 2,5 m di altezza. 
zione dell'apparato radicale che, mediante zollature e trapianti annuali, si deve presentare ben rinnovato ed irrobustito, ovvero in grado di superare con relativa facilità la crisi di trapianto.

I sesti di impianto adottati nella realizzazione del Bosco sono stati di $4,5 \mathrm{~m} \mathrm{x}$ $4,5 \mathrm{~m}$ e quindi larghi; ciò ha consentito una minor spesa di lavorazione e un minor impiego di materiale vegetale rispetto alle tecniche prettamente forestali.

La scelta di tale sesto d'impianto $\left(1\right.$ pianta $\left./ \mathrm{m}^{2}\right)$ scaturiva dalla situazione aziendale cui i promotori del Bosco si erano trovati di fronte; la mancanza di macchine agricole e l'impiego di personale volontario per la messa a dimora delle piantine aveva reso necessario l'avvio di una stretta collaborazione con gli agricoltori presenti intorno al $\mathrm{Bosco}^{24}$.

I sesti più larghi, determinando uno sviluppo della chioma molto esteso, avevano anche l'importante funzione di favorire il ricovero di piccoli animali e la nidificazione ${ }^{25}$.

Terminati i lavori di impianto, iniziarono i lavori necessari per la manutenzione del Bosco e la cura delle prime piante. Venne posta particolare attenzione all'irrigazione dei terreni, garantita dalla riapertura di diversi canali irrigui preesistenti ${ }^{26}$; attraverso una convenzione tra il Bosco e il Consorzio Villoresi, fu infatti possibile recuperare e riattivare una parte di queste canalizzazioni.

La successiva gestione del Bosco ha fatto riferimento all'evoluzione naturale dell'ecosistema; se i primi tre quattro anni furono dedicati alla cura e alla manutenzione degli impianti attraverso diradamenti o reimpianti, a partire dal quinto anno il Bosco è stato lasciato crescere spontaneamente, limitando al minimo gli interventi. Diventava essenziale non modificare artificialmente l'equilibrio naturale che si andava raggiungendo in seguito alle variazioni del microclima determinate dalla crescita delle piante introdotte artificialmente.

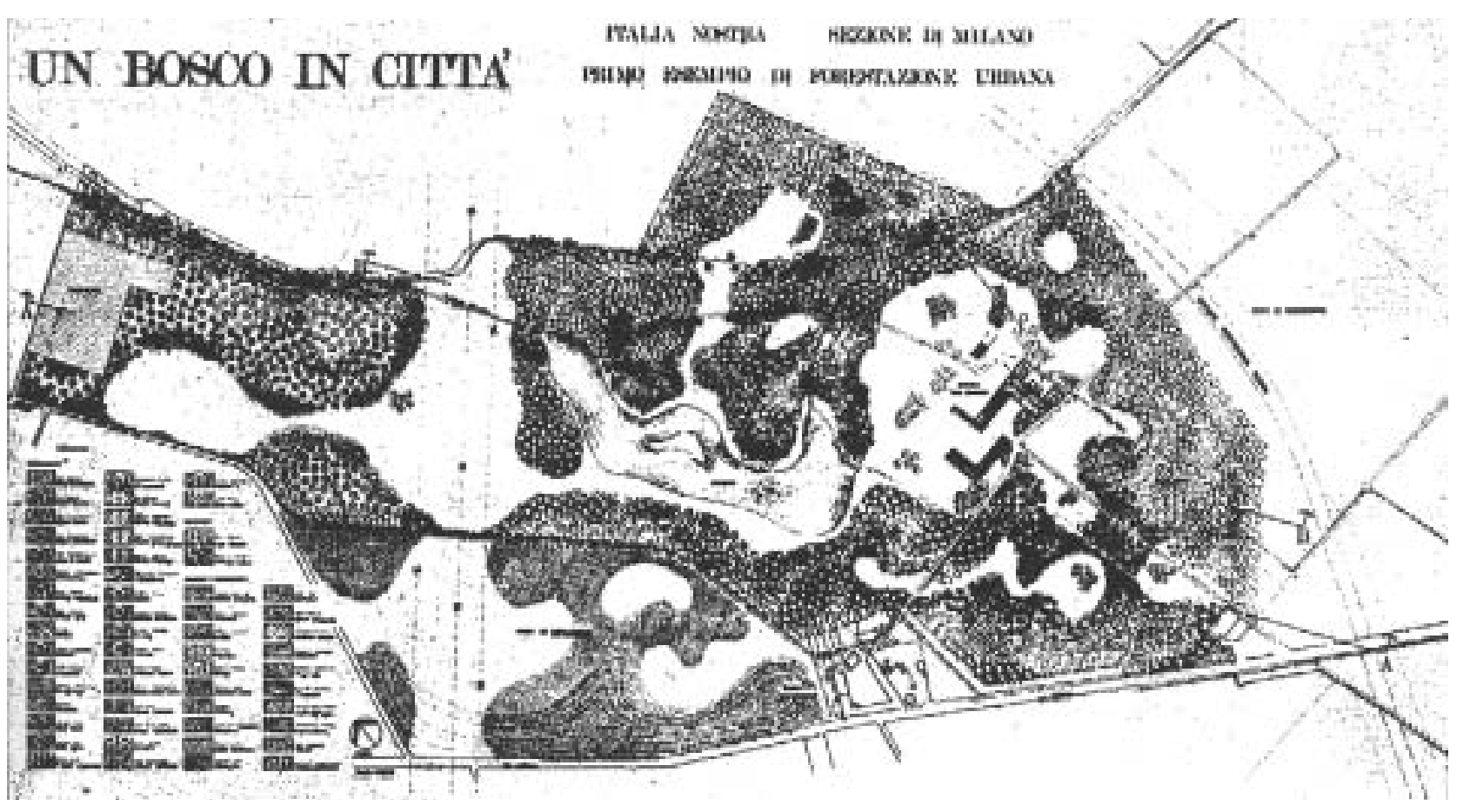

Figura 5 - La struttura finale del Bosco con l'indicazione del bacino lacustre.

${ }^{24}$ Luso delle macchine agricole consentiva infatti di attuare tutte le operazioni necessarie allo sviluppo delle giovani piante, altrimenti soffocate dalle erbe infestanti.

${ }^{25}$ Il ricorso a sesti di impianto più stretti avrebbe garantito una più vistosa copertura del terreno fin dalle prime fasi, e quindi una fruizione più immediata, ma rendendosi necessario un forte diradamento successivo, il raggiungimento dell'equilibrio biologico del bosco sarebbe stato ritardato. 
Per quanto riguarda le specie introdotte occorre innanzitutto sottolineare l'esigenza di impiegare essenze particolarmente significative per velocità di accrescimento e adattabilità all'ambiente ${ }^{27}$. Proprio per rispondere a questa esigenza accanto alle specie tipiche della pianura lombarda (Acer campestre, Quercus robur, Fraxinus excelsior, Populus nigra, Salix alba) sono state affiancate alcune essenze pioniere, compatibili con il clima lombardo (Acer negundo, Quercus rubra, Ulmus pumila).

Non va dimenticato inoltre che il Bosco è stato progettato e realizzato nel rispetto di un paesaggio formato da gruppi di piante preesistenti tipiche della realtà agricola lombarda quali: Populus nigra, Robinia pseudoacacia, Alnus glutinosa.

La piantagione è avvenuta in modo più o meno fitto, così da alternare aree più densamente boscate con radure più direttamente destinate alla ricreazione. Proprio ad ornamento delle radure sono state introdotte, nella seconda fase di realizzazione del Bosco, alcune essenze arbustive scelte tra quelle tipiche dell'ambiente naturale (Sambucus nigra, Cornus sanguineus, Prunus spinosa, Crataegus monogyna) e quelle più propriamente da giardino (Liriodendron tulipifera, Acer negundo, Ginkgo biloba). Sia per le specie di seconda grandezza, sia per le essenze arbustive, è stata prestata particolare attenzione alla capacità di fornire vivaci fioriture, alla loro scalarità nel tempo e alla possibilità di offrire rifugio ai piccoli animali.

Nella messa a dimora delle piante altrettanta attenzione è stata rivolta al rapporto essenza-ambiente; il salice e l'ontano sono stati ad esempio utilizzati lungo i corsi d'acqua per la creazione di fasce riparie, mentre sono stati utilizzati arbusti sciafili per la creazione di un fitto sottobosco.

Accanto alle tecniche di impianto, altrettanto innovative sono state le modalità di realizzazione dei lavori di piantagione. Uno dei presupposti dell'iniziativa era infatti quello di utilizzare per la realizzazione del Bosco il lavoro offerto spontaneamente e volontariamente dai cittadini; questo sia perché rappresentava una delle scelte fondamentali per la realizzazione di un progetto che prevedeva spese contenute, sia perché il gruppo che guidava l'iniziativa "credeva fortemente nell'impegno individuale e nella risoluzione collettiva dei problemi”. Il ricorso al volontariato se da un lato non dava le maggiori garanzie ${ }^{28}$, dall'altro consentiva importanti risultati economici e sociali: i costi di impianto sono stati minimi, ma soprattutto l'intervento ha permesso di conseguire anche altre utilità quali, ad esempio, l'educazione ambientale e la sensibilizzazione della popolazione, il recupero degli anziani e lo sviluppo di forme di lavoro associate allo studio.

\section{GLI ESITI}

I lavori di realizzazione del Bosco iniziarono nel 1974 quando l'Azienda Forestale di Stato regalò ad Italia Nostra le 30.000 piantine allo stato semenziale che costituirono il nucleo del primo vivaio ${ }^{29}$.

Una volta risolto il problema dell'impianto delle essenze arboree, gli operatori si dedicarono al ripristino delle canalizzazioni esistenti nell'area e cadute in disuso da tempo. Successivamente vennero affrontati i problemi relativi al recupero e all'arricchimento dei terreni, operazione resasi necessaria a causa dell'impoverimento che tanti anni di sfruttamenti abusivi avevano determinato.

\footnotetext{
${ }^{26}$ Si trattava per lo più di canali ormai asciutti e abbandonati a causa dell'abbassamento della falda per prelievi idrici urbani.

${ }^{27}$ La scelta delle essenze da impiegare nelle piantagioni è stata fatta anche in funzione dell'insediamento delle specie animali tipiche della pianura lombarda (coniglio, lepre, passeracei, corvo, allocco, eccetera).

${ }^{28}$ Proprio per queste ragioni si era dovuti ricorrere ad un disegno semplice e flessibile nella realizzazione del Bosco.

${ }^{29}$ Il vivaio venne creato appositamente per le piantine ricevute dalla Forestale in quanto le stesse non potevano essere messe a dimora immediatamente.
} 


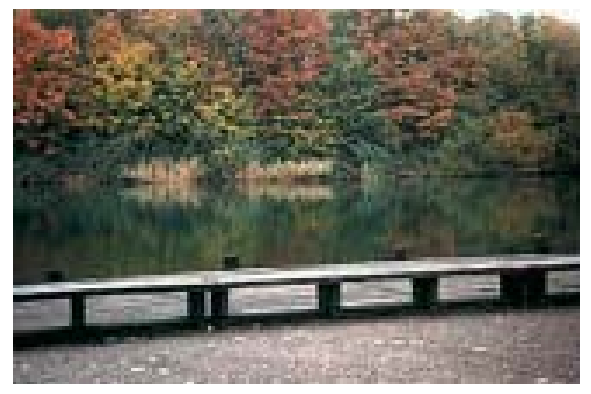

Figura 6 - L'orizzonte del lago formato dall'accostamento degli elementi vegetali.

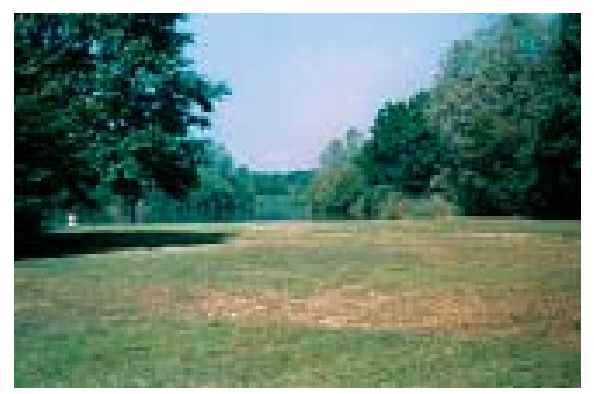

Figura 8 - L'alternanza di boschi, radure e acqua.

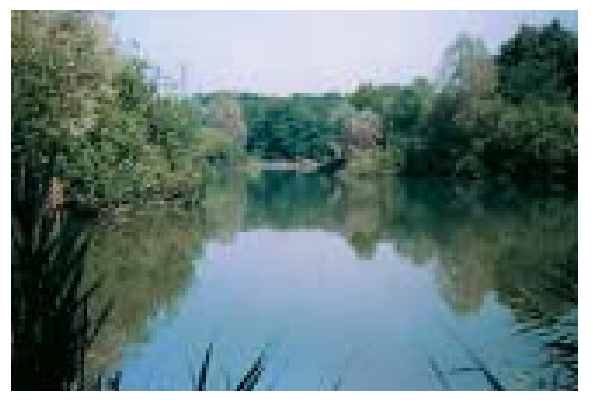

Figura 7 - L'ambiente naturale del lago.

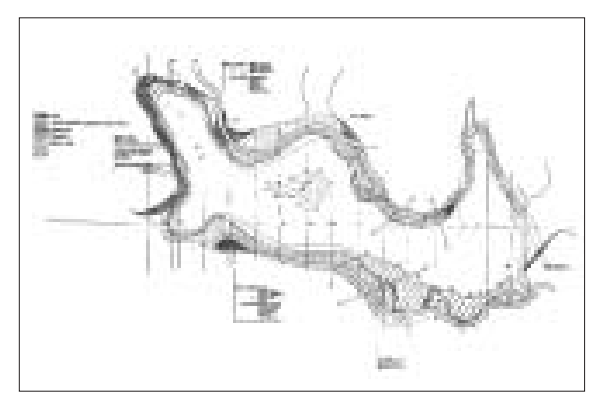

Figura 9 - Il disegno dei contorni del lago.

Durante il 1975 le attività furono più numerose: vennero costruite le passerelle in legno sui canali, si realizzarono un semenzaio ed un sentiero di collegamento fra il bosco ed il vivaio, si proseguirono le operazioni di impianto.

Al termine del 1976 il Bosco contava tre vivai e le attività di impianto e di manutenzione delle piantagioni proseguivano senza sosta. All'impianto iniziale si procedette ad un perfezionamento e ad un arricchimento del patrimonio vegetale secondo criteri più 'liberi': le macchie di sottobosco e quelle fiorifere vennero infatti affidate ai gruppi autogestiti di volontari che, nel frattempo avevano acquisito una maggiore dimestichezza ed una maggiore conoscenza dei problemi colturali.

Nei primi anni Ottanta con il rinnovo della concessione, il Comune affidava ad Italia Nostra altri quindici ettari di terreno per attuare il primo ampliamento del Bosco. Considerata la limitata superficie del lotto di ampliamento, la realizzazione del Bosco su questi nuovi terreni seguì gli stessi metodi adottati negli anni Settanta: non si elaborò nessun piano particolareggiato dell'intervento e si ricorse ancora una volta al lavoro dei volontari per l'impianto delle essenze secondo griglie modulari.

Le uniche differenze rispetto al vecchio impianto erano date da: l'esperienza ormai consolidata; la presenza di due vivai in grado di fornire le piante necessarie evitando così il ricorso alle donazioni; il finanziamento concesso dal Comune che permetteva evidentemente maggiori margini di spesa.

Al conseguimento di pregevoli esiti visivi e spaziali contribuì anche la presenza dell'acqua che, in quanto elemento fondamentale della natura nei suoi processi di regolazione, si rendeva innanzitutto necessaria all'interno del Bosco per l'irrigazione delle piante e la difesa dagli incendi. A questo scopo fin dal 1974 era stato proposto di realizzare un lago all'interno del Bosco e più precisamente nei pressi della Cascina San Romano, nel cuore dell'area, dove presumibilmente si sarebbe verificata la maggiore concentrazione di utenti.

Secondo il progetto iniziale la superficie occupata dallo specchio d'acqua avrebbe dovuto essere di 20.000 metri quadrati ed assolvere a molteplici funzioni: irrigue, ecologico-biologiche e paesaggistiche. 
Dal punto di vista paesaggistico il parco era stato studiato come composizione di boschi, radure e acqua, nella quale il lago diventava una sorta di specchio in grado di riflettere i colori e le luci dell'ambiente circostante; la sua presenza era poi fondamentale per la creazione della diversità ambientale in quanto avrebbe consentito l'insediamento di forme di vita vegetale ed animale proprie delle zone umide. L'utilità infine di una così estesa superficie d'acqua era quella di costituire un importante serbatoio per l'irrigazione del Bosco e per eventuali interventi antincendio.

Se il progetto era già presente all'avvio delle attività di piantumazione, fu però solo alla fine degli anni Ottanta che si decise di dare inizio ai lavori di realizzazione del lago. Nel rispetto delle linee progettuali indicate dall'architetto Crespi, si studiarono più dettagliatamente i diversi aspetti della sua costruzione. In fase preliminare, lavorarono a questi studi: un paesaggista americano, per lo studio dei margini; un paesaggista tedesco, per la realizzazione dei percorsi; un professionista italiano per la stesura degli elaborati da presentare per la concessione edilizia.

Dagli elaborati che approfondivano prevalentemente gli aspetti del progetto più legati al profilo altimetrico e planimetrico del lago, emergeva chiaramente la scelta di realizzare un invaso dalla forma particolarmente sinuosa, scelta determinata sia da ragioni di tipo paesaggistico sia da ragioni di tipo più naturalistico. Il profilo sinuoso del lago avrebbe favorito la creazione di configurazioni spaziali più suggestive per lo sviluppo e l'accostamento di elementi vegetali dai profili e dalle coloriture differenti e per le maggiori variazioni luminose che potevano riflettersi sulla superficie del lago. Dal punto di vista naturalistico invece lo sviluppo sinuoso del lago poteva favorire la creazione di ambienti e di habitat naturali adatti all'insediamento della fauna acquatica.

Ottenuta la licenza edilizia, il Centro di Forestazione Urbana avviò il progetto esecutivo e la realizzazione vera e propria del lago. In questa fase, al fine di diversificare per profondità il perimetro del lago assunse grande importanza lo studio dei profili verticali; lo scopo era quello di ottenere un profilo di scavo consono allo sviluppo della vegetazione igrofila, che permettesse anche il controllo dell'aspetto paesaggistico finale. Considerato che lo sviluppo della vegetazione igrofila è strettamente correlato alla profondità dell'acqua, fu possibile

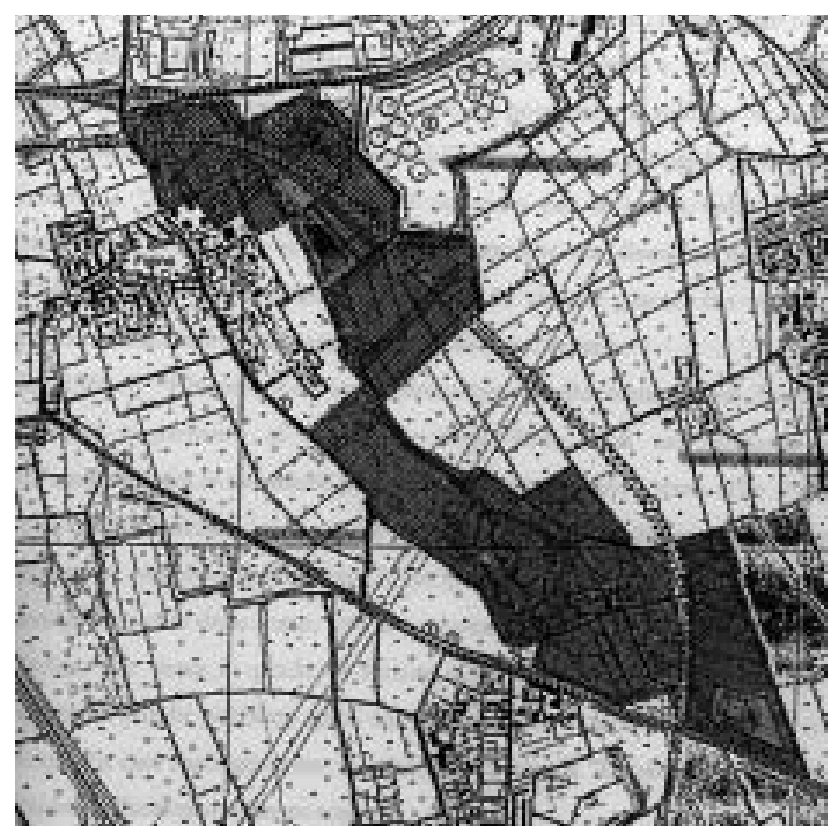

Figura 10 - Le fasi di ampliamento del Bosco. determinare il rapporto tra acque 'libere' e acque 'occupate' da detta vegetazione, con conseguente creazione di zone marginali a basso ricambio d'acqua e dunque adatte all'insediamento ed alla riproduzione di particolari specie animali.

La realizzazione dell'opera, iniziata nel 1989, fu articolata in fasi differenti: lo scavo del bacino, l'allontanamento del materiale di scavo, l'impermeabilizzazione del fondo e delle sponde tramite materiali argillosi compattati, la ricarica di terre miste e vegetali a protezione delle zone impermeabilizzate, la sistemazione degli impianti di approvvigionamento dell'acqua, la formazione e la sistemazione dei percorsi pedonali e di servizio.

Purtroppo per difficoltà legate alla collocazione dei materiali di risulta, le operazioni di scavo, impermeabilizzazione e ricarico furono terminate solo nel 1992. Da allora il lago si è arricchito di vegetazione lungo le sponde; la quale contribuisce a creare una sorta di quinta scenografica e di orizzonte intorno al lago, nelle cui acque si riflettono i profili delle chiome cresciute delle piante, la vivacità delle fioriture e della colorazione delle diver- 
se essenze, i giochi di luce e ombra creati dall'accostamento delle essenze forestali e dei grandi gruppi di arbusti fioriferi.

Nel 1988 venne programmato il secondo ampliamento del bosco che, per un totale di trenta ettari, estendeva la superficie complessiva di Boscoincittà a circa ottantatre ettari.

Il progetto di ampliamento rappresenta la sintesi teorica che traduce la fase sperimentale in un modello esportabile e riproducibile, la cui formulazione nasce dall'esperienza maturata in un ventennio di attività.

Con il secondo ampliamento fu riproposto il tema dell'integrazione tra verde estensivo e verde intensivo con una stretta correlazione all'edificato ${ }^{30}$. La nuova porzione di Bosco venne infatti studiata in relazione alle nuove necessità della città ${ }^{31}$ : la richiesta di verde da parte dei cittadini era notevolmente aumentata nel corso degli anni incrementando, di conseguenza, l'affluenza e il tipo di attività svolte e ricercate nei parchi; le aree libere nei pressi degli agglomerati urbani non erano più aree marginali destinate all'espansione edilizia, ma al contrario rappresentavano la sola possibilità di miglioramento delle città. Nel caso specifico dell'area metropolitana milanese, l'istituzione del Parco Agricolo Sud Milano aveva introdotto un nuovo soggetto istituzionale nella gestione del territorio ma soprattutto aveva fatto mutare radicalmente la concezione del

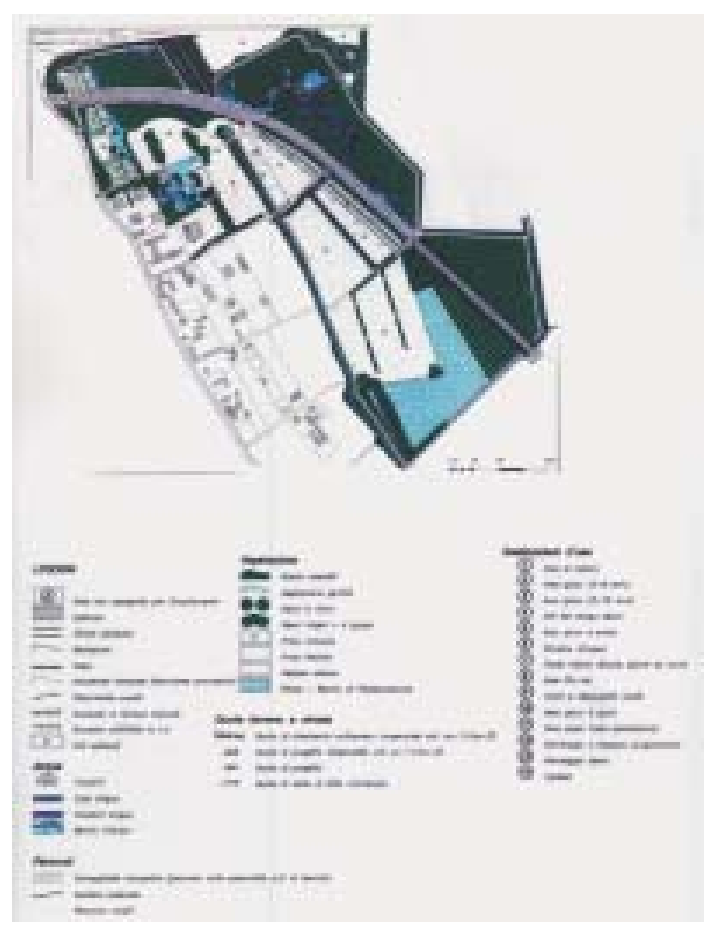

Figura 11 - Planimetria del progetto. parco urbano e in generale del verde pubblico inteso come insieme organico atto ad armonizzare edificato e spazi aperti.

Alla luce di queste nuove esigenze, il nuovo progetto di ampliamento assunse quale obiettivo principale la realizzazione di un parco destinato a soddisfare le necessità dei residenti nelle zone ad esso contigue, ma in grado al contempo di 'servire' la città nel suo complesso. In questa direzione furono attentamente valutati i bisogni dei fruitori così come emergevano dalle esperienze condotte nel primo nucleo del Bosco. Le passate sperimentazione avevano infatti permesso ai progettisti di giungere ad alcune conclusioni in merito: l'osservazione naturalistica necessita di ambienti diversi, di cui sia possibile osservare componenti e interazioni; la differenziazione estetica degli ambienti è funzionale allo sviluppo degli aspetti emotivi e sensoriali legati all'esplorazione dell'ambiente; il gioco è lo strumento attraverso cui si costruisce il rapporto con gli spazi apertii3 ${ }^{32}$; sono necessari spazi dedicati alla sosta ed all'isolamento ma anche zone attrezzate da adibire alla socializzazione; il lavoro pratico è fondamentale per l'educazione del cittadino e necessita di spazi adeguati; la comprensione del territorio è favorita dall'assenza di confini rigidi che impediscono di cogliere l'interazione fra parco, agricoltura ed infrastrutture; il confronto tra i soggetti che agiscono all'interno del parco trova buona collocazione nelle aree dove questi operano.

Sulla base di queste considerazioni i progettisti hanno dunque pensato di strutturare il Bosco come unità organica all'interno della quale far convivere zone atte allo svolgimento di attività specifiche ma non esclusive.

L'intero disegno che propone la realizzazione di un paesaggio semplice ed essenziale è basato sull'interazione di quattro elementi: l'acqua dei laghetti e dei canaletti irrigui, i percorsi, le radure e il bosco; su questa trama fondamentale

\footnotetext{
${ }^{30}$ Le aree del secondo ampliamento si collocano, infatti, adiacenti al borgo di Figino.

${ }^{31}$ Necessità mutate rispetto agli anni di avvio del progetto Boscoincittà.

32 E dunque necessario approntare aree protette per i bambini più piccoli ed aree di dimensioni maggiori dove gli adulti possono dedicarsi ad attività dinamiche.
} 


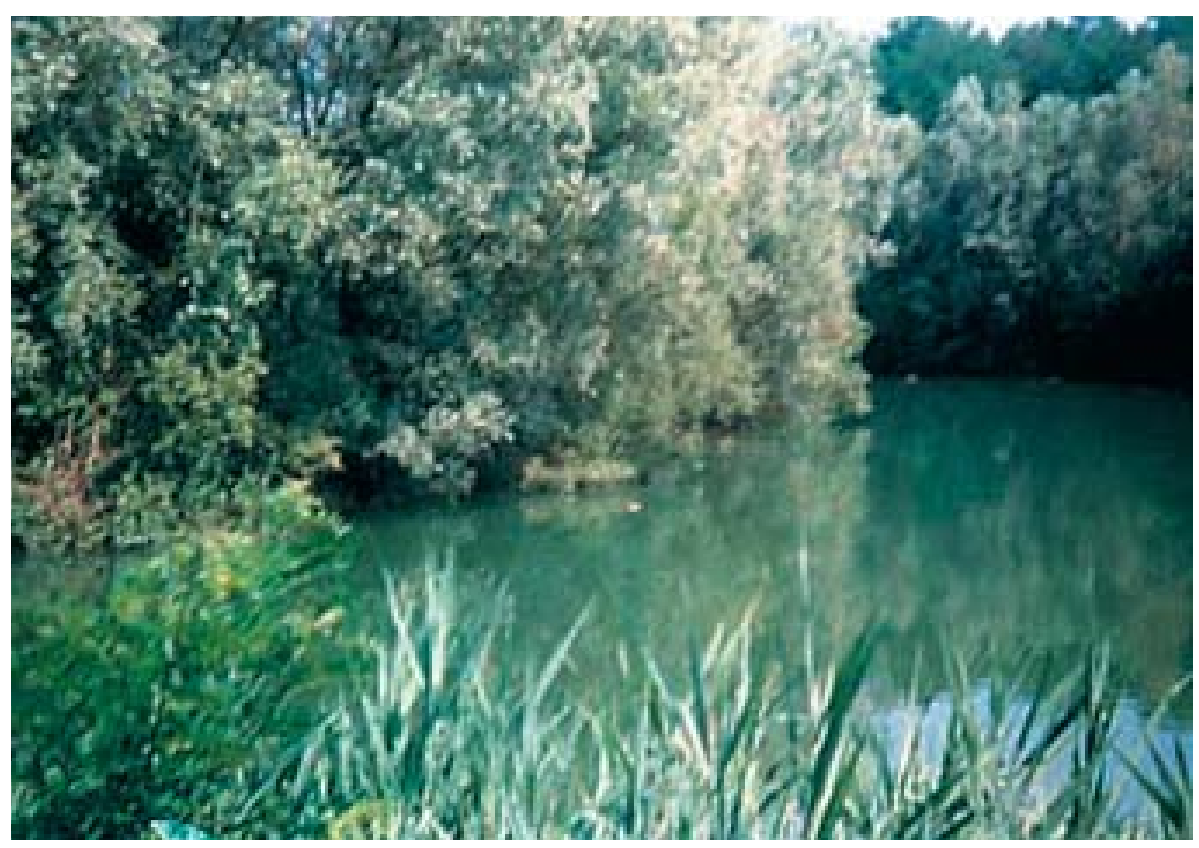

Figura 12 - Lo sviluppo del bosco ai margini del lago.

sono state inserite alcune funzioni specializzate: gli orti del tempo libero, le aree gioco per i bambini, un "giardino d'acqua" e una piazza quale punto di ristoro.

Insieme alla cornice boschiva le acque della nuova zona settentrionale del Bosco hanno assunto un ruolo molto importante nella configurazione dello spazio e nella connotazione paesaggistica e naturalistica del luogo. La realizzazione di tre laghetti che delimitano un'ampia radura ha permesso infatti di ottenere notevoli effetti paesaggistici; la sinuosità dei profili dei laghi, la vegetazione lungo le sponde, i riflessi colorati che si specchiano sull'acqua, il contrasto con la radura circostante consentono di creare spazi fortemente caratterizzati che si alternano e si susseguono in un continuo mutare di forme e colori.

Il bacino più meridionale dei tre laghi delimita un "giardino d'acqua" dove è stata pensata la successiva realizzazione di una collezione di specie vegetali igrofile.

I laghetti sono collegati tra loro con canalette di superficie ed utilizzati per irrigare gli orti e l'abbeverata prevista all'interno del percorso equestre; all'interno dell'area boscata è stata realizzata invece una sequenza di zone umide costituite da una serie di piccoli bacini fra loro collegati, il cui insieme tende a ricreare ambienti periodicamente invasi dall'acqua, ove si alternano spazi ampi e luminosi a spazi contenuti ed ombrosi.

Il progetto ha tenuto conto anche dei dislivelli esistenti fra i campi e li ha utilizzati come stacco tra gli stagni e le tratte degli alvei dei fontanili, a loro volta inglobate nel bosco. Verso l'esterno, in particolare lungo il tracciato di collegamento autostradale, lo spazio è occupato da un bosco compatto confinante con l'analogo bosco creato attorno al Depuratore Nord Milano e funge, dunque, da barriera antirumore.

Lo schema dei percorsi ha confermato invece nelle linee essenziali gli antichi tracciati agricoli permettendo la realizzazione di numerosi collegamenti nel Bosco, percorribili su vie differenziate a piedi, in bicicletta, a caval-

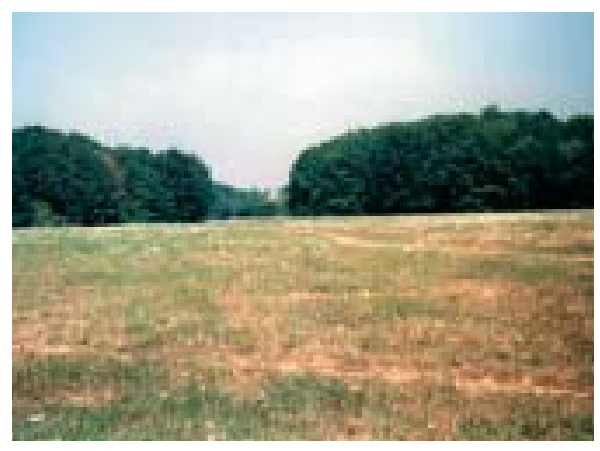

Figura 13 - I boschi delimitano lo spazio delle radure. 


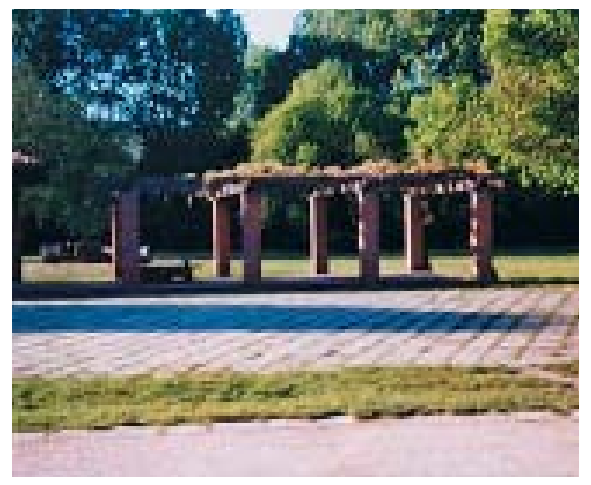

Figura 14 - Il disegno essenziale dei manufatti architettonici del Bosco.

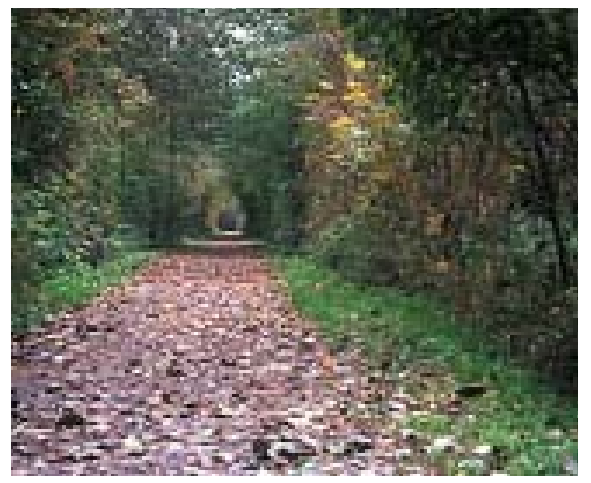

Figura 15 - I sentieri si sviluppano all'interno del Bosco quasi 'avvolti' dalla natura.

lo, secondo due tipologie di percorsi: le strade sterrate, di tipo campestre, caratterizzate da tracciati rettilinei ed ortogonali con il sedime in terra mista cilindrata e con margini che si inseriscono a raso nel suolo circostante; i sentieri che seguono invece percorsi informali con un andamento che suggerisce la formazione spontanea.

Le radure, spazi aperti di grande dimensione destinati alla fruizione più intensa, coprono circa il $23 \%$ della superficie totale; in particolare la superficie è condotta a tappeto erboso per le aree più sfruttate, a prato per le fruizioni meno intense e a "prato naturale" nelle zone naturalistiche.

Il bosco occupa invece il 53\% della superficie dell'ampliamento ed assolve a funzioni paesaggistiche e naturalistiche. Nel bosco, composto da latifoglie e destinato ad assumere la forma della fustaia, sono stati ridotti al minimo gli attraversamenti dei percorsi principali ad eccezione di alcuni percorsi pedonali necessari all'osservazione naturalistica.

La progettazione e la realizzazione hanno riguardato anche la struttura da conferire a quelle poche attrezzature e manufatti da collocare nel Bosco. Nella logica di integrazione con la natura pochi sono gli elementi metallici che compongono barriere, cancelli o cartelli segnaletici, e le forme degli oggetti non si scontrano con la linearità del disegno della natura, in modo che tutto sia quasi 'invisibile' in esso. In particolare le attrezzature e i manufatti sono caratterizzati dall'uso di materiali semplici e da un disegno essenziale, senza compromettere la sensazione di naturalità che si vuole trasmettere all'utilizzatore' del Bosco. A questo fine sono stati progettati i ponti, le chiuse di derivazione delle acque irrigue, i margini dei laghetti, le pergole, le recinzioni, utilizzando calcestruzzo a vista, legno squadrato e tondame.

Gli esiti formali, ma anche sociali di questa esperienza dimostrano che se il primo nucleo di Boscoincittà nasce come realtà isolata e sperimentale, ricalcando l'idea corrente di parco quale episodio a sé stante, gli ampliamenti successivi hanno permesso di arrivare a formulare un'idea di parco come elemento complesso e articolato, che non può e non deve essere considerato uno spazio chiuso, un episodio fortuito di sottrazione d'aree alla città. La visione del parco quale elemento di riordino dell'ambiente urbano, era già presente nelle teorizzazioni della Forestazione urbana, ma il progetto studiato e realizzato dal Centro di Forestazione Urbana ne rappresenta la concretizzazione.

Boscoincittà infine induce ad una riflessione circa il fattore tempo nella costruzione del verde; la costruzione di un parco non può essere istantanea, richiede al contrario una differenziazione temporale degli interventi studiata in fase di progetto, che preveda la possibilità dell'adeguamento e delle migliorie in corso d'opera dello stesso. Il parco è infatti un'opera in crescita, in costante trasformazione; per la sua progettazione non è sufficiente un unico disegno iniziale ma occorre un costante apporto progettuale. 


\section{RIFERIMENTI BIBLIOGRAFICI}

Italia Nostra (a cura di), Un Bosco in città, "Italia Nostra", 172/173, 1979.

Centro di Forestazione Urbana, Italia Nostra (a cura di), Boscoincittà, il parco pubblico nato dalla collaborazione tra Italia Nostra e il Comune di Milano. Sintesi 1983-1992, Milano 1992.

Italia Nostra (a cura di), Il Bosco diventa più grande, "Italia Nostra", 311, 1994.

Centro di Forestazione Urbana, Italia Nostra (a cura di), Boschi per la città, Milano 1994.

Centro di Forestazione Urbana, Italia Nostra (a cura di), Dossier: il Lago del Boscoincittà, Milano 1996.

Centro di Forestazione Urbana, Italia Nostra (a cura di), Ampliamento Boscoincittà, Milano 1997.

ATZORI GIORGIA, Innovazioni gestionali e progettuali del parco milanese "un Boscoincittà", Tesi di Laurea, Facoltà di Architettura, Politecnico di Milano, Relatore: Prof. M. V. Erba, Correlatore: Arch. Laura Ferrari, Milano 1998.

CARRA LuCA, Il Bosco cresce!, "Sentieri in città", Italia Nostra, 2, 2000.

Della Rovere Piero, Tecniche estensive di impianto per Boscoincittà, "Acer", 3, 1996, pagg. 16-19. Masera Carlo e Pellizzoni Sergio, Il Parco dei Sentieri Interrotti, "Acer", 2, 1992, pagg. 5-9.

Regione lombardia, Azienda Regionale delle Foreste, Forestazione urbana per la Lombardia, Regione Lombardia, Milano 1998.

Saluzzo Alberto Maria, Milano: un "Boscoincittà", "Acer", 6, 1986, pagg. 32-33.

SAlUZzO AlberTo Maria, Tecniche impiegate per la realizzazione del "Boscoincittà": problemi e proposte, "Acer", 1, 1987, pagg. 33-35.

SAlUZzo Alberto Maria, "Boscoincittà": un bosco per imparare, "Acer”, 5, 1987, pagg. 26-27.

Toeschi Luigi, Un Boscoincittà, Franco Angeli, Milano 1984.

\section{RIFERIMENTI ICONOGRAFICI}

Figura 1: Elaborazione di Laura Ferrari. Base dati Laboratorio Metodi e Tecniche di Pianificazione Urbanistica, Dipartimento di Architettura e Pianificazione, Politecnico di Milano.

Figure 2, 6, 7, 8, 12, 13, 14, 15: http://web.tiscali.it/diverimento/index/home.html

Figura 3: http://www.milanoin.it//parchi_giardini/boscoincitta.htm

Figure 4, 5, 9, 10, 11: ATZORI GIORGIA, Innovazioni gestionali e progettuali del parco milanese "un Boscoincittà”, Tesi di Laurea, Facoltà di Architettura, Politecnico di Milano, Relatore: Prof. M.V. Erba, Correlatore: Arch. Laura Ferrari, Milano, 1998, pagg. 111, 114, 125, 115, 146. 


\section{SCHEDA DI PROGETTO}

Progetto:

Luogo:

Committente:

Tipologia:

Superficie:

Progettisti:

Ditte esecutrici:

Anno di progettazione:

Anno di realizzazione:

Specie vegetali principali: realizzazione di un bosco urbano

comune di Milano

Italia Nostra, Comune di Milano

parchi metropolitani

$830.000 \mathrm{mq}$

Dott. Arch. Giulio Crespi, Milano (progetto generale - 1974)

Centro di Forestazione Urbana (progetto primo e secondo ampliamento - 1984, 1988)

Centro di Forestazione Urbana, Milano

Allievi della Scuola Agraria di Minoprio (Co)

Volontari

1974 - progetto generale

1984 - progetto primo ampliamento

1988 - progetto secondo ampliamento

1974/1978 - lavori di impianto e prima manutenzione

1989/1992 - realizzazione del lago artificiale

1984 - lavori di impianto nell'area del primo ampliamento

1988/2000 - lavori di impianto e realizzazione del secondo ampliamento

Acer campestre, Quercus robur, Fraxinus excelsior, Populus nigra, Salix alba, Quercus rubra, Ulmus pumila, Sambucus nigra, Corpus sanguineus, Prunus spinosa, Crataegus monogyna, Liriodendron tulipifera, Acer negundo, Ginkgo biloba 



\title{
LA BONIFICA DI UN SITO INQUINATO QUALE IMPULSO
}

\author{
ALLA PROGETTAZIONE DELLO SPAZIO APERTO: \\ "Bosco delle Querce" di SeVeso e Meda
}

\author{
Laura Ferrari
}

\section{IL CONTESTO}

"10 luglio 1976. È una giornata caldissima (...). Una squadra di operai sta facendo lavori di manutenzione in una fabbrica chimica (...) che sorge a ridosso della superstrada Milano-Meda, in territorio di Meda, ai confini con Seveso. Accanto passa un torrente del solito colore delle nostre acque indefinibili. Si chiama Certosa e fa rima con il nome della fabbrica: Icmesa. Ad un tratto come un rombo. È scoppiato qualcosa ..."1.

Così ha inizio la storia del Bosco delle Querce, un parco che, con una superficie territoriale di circa quarantatre ettari, si colloca al confine settentrionale della provincia di Milano, a circa venti chilometri dal capoluogo lombardo, in una delle zone più densamente urbanizzate ed industrializzate del territorio milanese, in cui le aree a verde agricolo e forestale che ancora permangono rappresentano veri e propri spazi di risulta 'risparmiati' all'espansione urbana.

Il Bosco, che sorge a cavaliere del territorio di Meda e di Seveso, è un grande triangolo rettangolo situato tra il Parco Regionale delle Groane e il Parco della Brughiera Briantea; una superstrada, la Milano-Meda, delimita il confine orientale e un torrente, il Certosa, definisce in parte il confine occidentale.

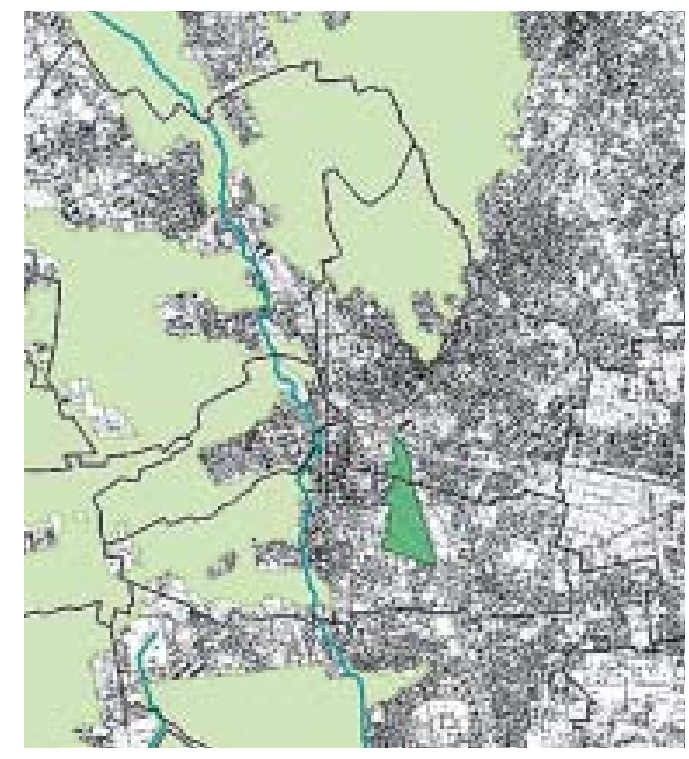

Figura 1 - Inquadramento territoriale.

Il contesto territoriale del Bosco è sicuramente tra quelli più radicalmente trasformati dall'opera dell'uomo che via via ha cancellato i caratteri morfologici originari; la presenza di un articolato sistema di infrastrutture di trasporto, grandi e piccole, ha determinato infatti sia la formazione dei principali nuclei storici, tra cui quelli di Seveso e Meda, sia l'edificazione più recente, a carattere misto residenziale e artigianale, che sviluppandosi tutto intorno al Bosco sembra volerne oscurare la presenza.

Un territorio, dunque, in cui gli antichi terrazzi fluviali ${ }^{2}$ sono stati rimodellati dall'agricoltura o 'sepolti' dall'urbanizzazione e dove i corsi d'acqua ${ }^{3}$, per lunghi tratti canalizzati e inquinati, hanno perso il loro corredo vegetale originario $^{4}$. La vegetazione naturale è infatti quasi completamente scomparsa e laddove

\footnotetext{
${ }^{1}$ Renato Farina, Seveso diossina e scuola, Gribaudi, Torino 1978.

2 Dal punto di vista geomorfologico, il Bosco è situato nell'alta pianura diluviale presso il margine tra l'area collinosa degli archi morenici a nord e le spianate terrazzate dei depositi fluvio-glaciali, dovuti allo smantellamento erosivo degli accumuli morenici, a sud. Il terreno di origine alluvionale è permeabile, con una composizione prevalentemente ghiaio-sabbiosa. Tutti i terrazzi però sono ricoperti da un orizzonte pedologico rossastro, costituito da argille di alterazione (ferretto), impermeabili o poco permeabili.

${ }^{3}$ Sotto il profilo idrografico la zona a ovest del parco è sicuramente molto più ricca di corsi d'acqua; tuttavia il Bosco è interessato dal torrente Certosa o Terrò, che ne lambisce per un tratto il perimetro, dopo aver drenato un vasto territorio a monte fino al lago di Montorfano, per entrare poco più a valle del Bosco nel torrente Seveso.

${ }^{4}$ Le associazioni vegetali che un tempo coprivano questo territorio sono individuabili nell'ambito delle latifoglie mesofile e in particolare del Querco-Betuletum insubricum, Querco carpinetum, Querco ostrieto, con buona variabilità a seconda delle freschezza e composizione del terreno profondamente alterato.
} 
permane in alcune 'oasi' superstiti è stata quasi completamente infiltrata dalla robinia e da altre specie esotiche.

Il significato e l'importanza che l'esperienza del Bosco delle Querce assume è sicuramente duplice: da un lato la sua realizzazione dimostra la possibilità di un riscatto della natura dopo una catastrofe ecologica e ambientale di notevole dimensioni; dall'altro l'interesse che questo bosco di nuovo impianto riveste nei confronti della popolazione e del territorio che lo circonda dimostra il ruolo ecologico e ricreativo che gli spazi aperti di cintura metropolitana possono svolgere all'interno di un paesaggio densamente urbanizzato quale quello dell'area metropolitana milanese.

\section{GLI OBIETTIVI}

La storia del Bosco delle Querce ha inizio dunque il 10 luglio 1976, giorno in cui dalla ciminiera dell'industria chimica ICMESA $^{5}$ si alzò una nuvola bianca di diossina prodotta da una reazione chimica imprevista $^{6}$. La nube tossica venne subito dispersa dal vento che, contrariamente alle condizioni climatiche stagionali, spirava in direzione sud-est depositando il carico inquinante lungo un percorso lineare di circa sei chilometri.

"In assenza di vento, i siti contaminati si sarebbero concentrati su una

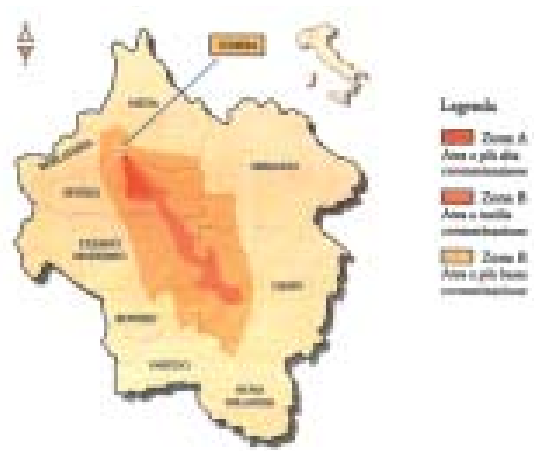

Figura 2 - La delimitazione delle zone contaminate dalla diossina.

superficie molto più limitata e la storia sarebbe stata diversa. (...) i confini del Bosco delle Querce, corrispondenti a quelli dell'area più inquinata nel primo tratto della nube tossica, sono stati disegnati dal vento"7.

In seguito all'incidente l'ambito interessato dalla nube tossica venne delimitato e suddiviso in tre zone in modo tale da differenziare le misure di tutela e di bonifica in base al grado di contaminazione dell' $\mathrm{area}^{8}$.

La rilevanza e la gravità dell'incidente resero evidentemente necessari numerosi provvedimenti da parte dello Stato e della Regione Lombardia per risolvere i molteplici problemi giuridici, amministrativi, organizzativi, tecnico-scientifici e finanziari che si sarebbero presentati nei mesi immediatamente seguenti e soprattutto negli anni a venire?

\footnotetext{
${ }^{5}$ Industrie Chimiche Meda Società Azionaria, di proprietà della Società Givaudan di Ginevra.

${ }^{6}$ In un reattore che da qualche tempo produceva triclorofenolo, composto chimico usato per defoglianti ed erbicidi, l'aumento improvviso e incontrollato della temperatura provocò complesse reazioni chimiche che portarono alla formazione di composti altamente tossici, in primo luogo il tetraclorodibenzodiossina, più conosciuto come diossina.

${ }^{7}$ Mario Di Fidio, Il "Bosco delle Querce" di Seveso e Meda, Regione Lombardia, Milano 2000, pag. 25.

${ }^{8}$ La prima e più inquinata prese il nome di zona "A"; la seconda, con un grado di contaminazione medio, fu denominata zona " $B$ "; la terza ed ultima, denomina zona " $R$ ", fu invece istituita per creare una sorta di 'filtro' tra le aree inquinate e quelle non ritenute tali.

${ }^{9}$ Tra questi i più importanti furono: il D.L. 10 agosto 1976, n. 542, convertito nella legge 8 ottobre 1976, n. 688 ("Interventi urgenti per le popolazioni della zona colpita dall'inquinamento da sostanze tossiche verificatosi in Provincia di Milano il 10 luglio 1976") con cui lo Stato assegnava alla Regione un contributo speciale di 40 miliardi di lire per fronteggiare l'emergenza e nel quale prevedeva che le modalità e i criteri degli interventi venissero determinati con leggi regionali; il Decreto del Presidente del Consiglio dei Ministri 4 agosto 1976 con cui fu istituita una Commissione speciale tecnico-scientifica, con il compito di studiare e proporre misure di decontaminazione. A livello regionale fu emanata la legge 17 gennaio 1977, n. 2 ("Interventi per le popolazioni della zona colpita dall'inquinamento da sostanze tossiche verificatosi in Provincia di Milano il 10 luglio 1976") che, in attuazione della legge $688 / 76$, definiva i programmi operativi degli interventi di decontaminazione e di bonifica. Con la legge
} 
Nel 1977 venne approvato il piano di bonifica con la definizione degli interventi da attuarsi, dei tempi di attuazione, dei soggetti responsabili e delle risorse economiche necessarie alla loro realizzazione. Il piano, articolato in due distinti intervent $\mathrm{i}^{10}$, seguì fasi cronologiche differenti: vennero innanzitutto realizzati gli interventi nelle zone meno contaminate nelle quali era più semplice intervenire ma anche più urgente vista l'estensione e la rilevanza sociale ed economica; relativamente invece alla zona più inquinata dovettero passare alcuni anni di incertezze e discussioni prima che venissero identificati i metodi di smaltimento dei materiali inquinanti provenienti dalla bonifica ed individuato l'assetto definitivo da conferire all'area.

Lo spunto per la sistemazione dell'area inquinata derivò da una ricerca avviata nel 1977 a cura dell'Università degli Studi di Milano ${ }^{11}$ avente quale obiettivo la verifica della possibilità di impiego di vegetali per la bonifica del suolo inquinato; i risultati della ricerca, infatti, mettevano in evidenza un parziale processo di eliminazione della diossina attribuibile a traspirazione e fotodegradazione o metabolizzazione della stessa da parte delle piante. Occorre tuttavia sottolineare che se i metodi naturali o seminaturali apparivano adatti alle zone meno contaminate, gli stessi non offrivano prospettive pratiche per la decontaminazione del rimanente territorio in tempi ragionevoli; per abbreviare i tempi della bonifica occorreva necessariamente fare ricorso ad alcuni interventi tecnici di decontaminazione.

Se dunque non fu del tutto possibile condividere l'ipotesi avanzata da varie associazioni locali, consistente nel trasformare l'area inquinata in bosco ed attendere il degrado naturale della diossina ad opera degli agenti naturali, gli uffici competenti decisero tuttavia di realizzare ugualmente un bosco di nuovo impianto facendolo però precedere da alcuni importanti interventi tecnici di bonifica. In particolare, una Commissione internazionale approvò quale metodo di bonifica la scarificazione dell'area sino agli strati non inquinati ${ }^{12}$ e l'apertura di due discariche speciali nelle quali depositare tutti i materiali contaminati provenienti dalle operazioni di bonifica ${ }^{13}$.

regionale 17 giugno 1977, n. 27 ("Norme per l'attuazione dei programmi operativi di cui alla L.R. 2/27 nella zona della Provincia di Milano inquinata da sostanze tossiche") la Regione istituì un Ufficio Speciale decentrato che, con sede a Seveso, doveva assicurare la maggiore tempestività e il coordinato svolgimento degli interventi e dei servizi delle pubbliche amministrazioni e dei privati. Questo Ufficio che per un decennio fu il protagonista delle decisioni e delle azioni intraprese sull'area inquinata, si caratterizzò sin dall'inizio per l'impostazione interdisciplinare della sua attività; impostazione che consentì una corretta sintesi dei diversi problemi in campo e l'esperienza di un nuovo metodo di lavoro, più adeguato alla pluralità e complessità delle situazioni da affrontare.

${ }^{10}$ Il primo riguardava la bonifica della zona B e della parte di zona A socialmente rilevante attraverso interventi di diserbo e defoliazione, accatastamento della vegetazione in attesa del successivo incenerimento, decorticazione del terreno agricolo e bonifica delle abitazioni e delle relative pertinenze; il secondo riguardava invece la bonifica del territorio più contaminato, ossia la zona $\mathrm{A}$, attraverso demolizione degli edifici, decorticazione e accatastamento del terreno in condizioni di sicurezza a ridosso dello stabilimento chimico.

${ }^{11}$ Istituti di Agronomia, Chimica agraria e Scienze botaniche.

${ }^{12} \mathrm{Si}$ tratta di un intervento di asporto del terreno per uno spessore rapportato al tasso di inquinamento rilevato nell'area. Complessivamente la superficie interessata da queste operazioni fu pari a 43 ettari circa (l'intero sedime del Bosco), la profondità media di scarificatura di 46 centimetri e il volume di terreno asportato e messo in sicurezza pari a 200.000 metri cubi circa.

${ }^{13}$ La decisione di conservare sul posto i residui della bonifica portò ad identificare due aree di confinamento: la prima, più piccola, in comune di Meda tra il torrente Certosa e lo svincolo della superstrada; la seconda, più grande, collocata in territorio di Seveso a nord del cimitero. Entrambe le aree furono preventivamente sottoposte ad accurate indagini geologiche, geotecniche, idrologiche e idrogeologiche per stabilire la compatibilità delle due discariche con le caratteristiche del territorio interessato dal loro collocamento. I progetti e le realizzazioni che seguirono assunsero inevitabilmente come principio fondamentale la necessità di garantire sicurezza ad un territorio già intensamente e drammaticamente colpito. I materiali provenienti dalle operazioni di bonifica comprendevano: il terreno scarificato, il materiale di scasso delle strade, le macerie degli edifici civili, il materiale della demolizione dello stabilimento ICMESA, i fanghi, il legname, la vegetazione e i detriti vari. Per la messa in sicurezza del materiale contaminato venne adottato 
La prima fase di bonifica, iniziata nel 1977, si concluse nel 1984 con la realizzazione dei due depositi tossici e con la messa a punto di un complesso sistema di monitoraggio per entrambe le vasche di discarica.

La scelta del metodo di bonifica, ma soprattutto l'identificazione delle due aree di confinamento all'interno dell'area più inquinata, portarono inevitabilmente alla decisione di trasformare tutta questa zona in un'area da mantenere a verde con la creazione di un prato-bosco formato da soprassuoli vegetali originali della Brianza; una decisione che teneva conto delle istanze della popolazione e delle autorità locali e soprattutto della necessità di garantire sicurezza attorno alle due discariche.

La realizzazione del bosco, che non solo difende l'ambiente, ma produce anche il legno lavorato dall'uomo, si legava simbolicamente anche all'attività di lavorazione del legno che si era via via affermata su questo territorio a partire dall'Ottocento, dapprima con la produzione tradizionale di suppellettili, attrezzi agricoli e macchine, e successivamente con la produzione di legname per uso cantieristico e di arredi finiti.

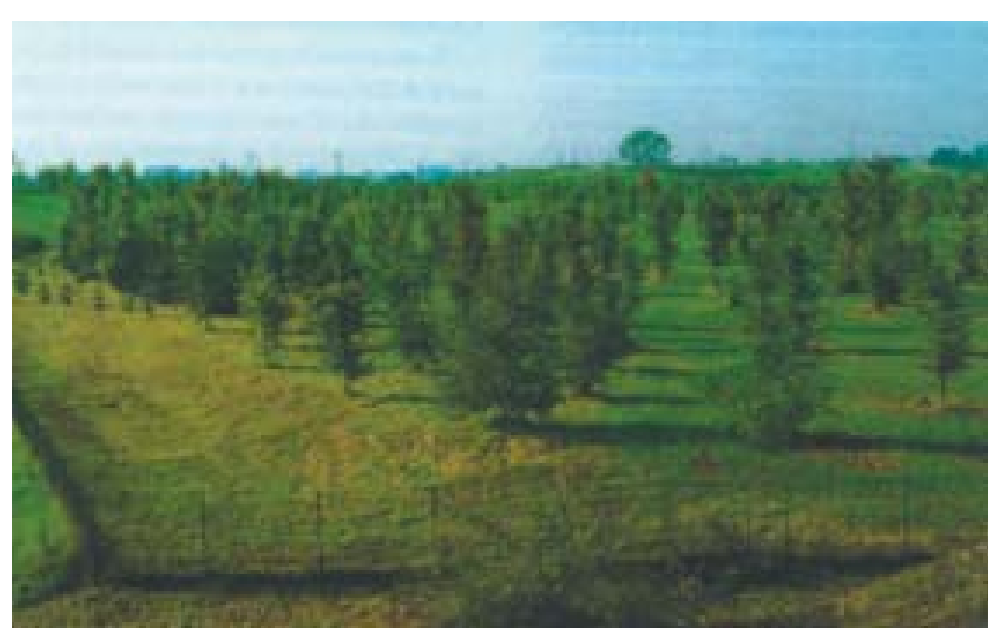

"Il ritorno al bosco dopo una catastrofe come quella della diossina non è quindi da vedersi in termini restrittivi, come un mero fatto di forza maggiore o - peggio - una fuga dalla modernità ed una regressione, bensì come la ricerca di un nuovo equilibrio, attingendo alla forza che sale come una linfa dalle proprie radici, naturali e culturali, per riprendere l'avventura della vita" 14 .

Emerge chiaramente come il Bosco delle Querce non sia nato come parco disegnato e progettato nei minimi dettagli ma come spazio in continuo movimento e mutamento. Questo principalmente per due ragioni: la prima è che l'obiettivo da perseFigura 3 - Gli impianti arborei nel 1989. guire su quest'area era innanzitutto legato alla bonifica delle zone inquinate e non alla creazione di un parco fruibile; la seconda deriva invece dalle tecniche agroforestali adottate per il recupero delle aree bonificate, tecniche che affidano all'evoluzione naturale e seminaturale degli impianti vegetali il disegno e l'esito spaziale del parco.

Se in origine dunque ben altre erano le preoccupazioni e non vi erano le condizioni per un progetto articolato del parco, con il passare del tempo e parallelamente al crescere degli impianti vegetali, prendeva forma una nuova idea di assetto che modificava l'intento originario.

Una volta terminate le operazioni di bonifica e la messa a dimora della prima generazione di piante, infatti, l'obiettivo primario dell'ente gestore divenne il parco stesso, tanto che la gestione del Bosco non si limitò più ad assicurare soltanto lo sviluppo e la manutenzione dell'ecosistema venutosi a creare, ma si aprì anche alla dimensione estetico-paesaggistica nella consapevolezza che fosse necessario attribuire al Bosco un carattere strutturale più specifico.

In questa direzione si inquadrano gli incarichi dei primi anni Novanta per la redazione dei progetti di sistemazione paesaggistica del parco, i quali se inizialmente operano in una logica puramente interna al Bosco, in un secondo tempo

in fase costruttiva un sistema di quattro barriere successive tra l'inquinante e l'ambiente esterno simile a quello messo a punto dall'OCDE per i materiali radioattivi. La capacità delle due discariche, entrambe collocate in zona A, era nell'ordine di 500.000 metri cubi circa.

${ }^{14}$ Mario Di Fidio, op. cit., 2000, pag. 17. 
aprono lo sguardo anche verso l'orizzonte esterno nel tentativo di estendere i confini del parco attraverso la prosecuzione del disegno vegetale.

Verso l'obiettivo della valorizzazione e del completamento del Bosco si collocano anche molti dei progetti futuri per il parco; tra questi vale la pena di richiamarne i principali: il progetto di sviluppo dell'ecosistema ${ }^{15}$; i progetti di disciplina e lo sviluppo della fruizione ${ }^{16}$; il progetto di ampliamento e di connessione con l'ambiente esterno; i progetti di valorizzazione culturale ${ }^{17}$.

$\mathrm{E}$ evidente che non tutti questi progetti potranno essere realizzati in tempi brevi; alcuni infatti riguardano direttamente il territorio e la gestione del Bosco, altri richiedono più complesse verifiche di carattere giuridico, amministrativo, territoriale e politico. Tuttavia quello che sembra emergere da una simile esperienza e dalla consistente quantità di progetti e attività in essere è sicuramente l'estrema vitalità di un piccolo parco, solo 43 ha, che ha "nel suo DNA una storia unica al mondo"18.

\section{LA METODOLOGIA}

Occorre innanzitutto sottolineare che il progetto e i lavori di realizzazione del bosco si trovarono di fronte ad una situazione di partenza del tutto assimilabile a quella di un deserto; la scarificazione dell'area con l'eliminazione della parte fertile, infatti, aveva profondamente alterato il profilo morfologico del terreno distruggendo tutto il soprassuolo vegetale e il terreno di coltura ad eccezione di un grande pioppo collocato al centro dell'area.

Il progetto originario del parco, datato $1983^{19}$, fu realizzato a partire dall'ultimazione dei lavori di bonifica dall'Ufficio Speciale di Seveso che affidò la direzione dei lavori all'Azienda regionale delle Foreste.

La metodologia adottata fu quella della "forestazione urbana" con la quale si potevano ottenere interessanti risultati sia dal punto di vista naturalistico sia sotto il profilo del disegno morfologico e paesistico del parco. Questa tecnica comportava, infatti, costi molto contenuti a fronte della varietà delle specie da impiantare, della possibilità di una loro selezione prima della messa a dimora e, soprattutto, della possibilità di realizzare il Bosco per gradi, per piccole e progressive aggiunte in grado di migliorare o di modificare il disegno originario.

Allo scopo di rendere visibile ed apprezzabile in tempi brevi il disegno del nuovo parco, il progetto propose l'impiego di piante sviluppate a pronto effetto, con sesto medio d'impianto piuttosto largo $(7 \mathrm{~m} \times 8 \mathrm{~m})$ e la formazione generalizzata di un prato, ossia in sostanza non un vero e proprio bosco, ma piuttosto

\footnotetext{
${ }^{15}$ La ricchezza di conoscenze acquisite tramite gli studi floristici e faunistici ha consentito non soltanto di valutare lo stadio evolutivo del Bosco, ma anche di elaborare un complesso piano di sviluppo dell'ecosistema con ricadute sulla sua struttura e sulla sua gestione. Si tratta complessivamente di una trentina di interventi diversi da realizzare gradualmente nel tempo in funzione delle priorità e delle risorse economiche disponibili.

${ }^{16}$ Con l'apertura al pubblico del parco e con il continuo incremento di visitatori si rendono sempre più necessari una serie di interventi che consentano la fruizione del patrimonio verde ma che ne garantiscano la conservazione. Il regolamento per l'accesso del pubblico, la realizzazione di un Centro visitatori, il potenziamento della segnaletica e delle infrastrutture sul territorio ed infine la realizzazione di una serie di attrezzature ludiche per l'intrattenimento dei bambini, sono solo alcuni degli interventi che il parco si propone di realizzare in tempi relativamente brevi.

${ }^{17}$ Sin dall'inizio si intuì che il Bosco poteva rappresentare qualcosa di più dell'esito finale di un evento contingente, potendo svilupparsi al contrario come centro vitale, capace di unire e coordinare nuove iniziative non solo ambientali, ma anche sociali e culturali. Negli anni Novanta quando ormai il parco aveva assunto una struttura verde sufficientemente caratterizzata ed efficiente si iniziò ad avviare la discussione su una serie di progetti per la valorizzazione culturale del parco; tra questi i principali riguardano: l'archivio storico dell'Ufficio Speciale di Seveso, il Museo del Legno e la Biblioteca dell'Ambiente.

${ }^{18}$ MARIO Di Fidio, op. cit., 2000, pag. 142.

${ }^{19}$ Firmato dall'Arch. Silvano Barbarani e dall'Ing. Nino Noseda con la consulenza di un agronomo.
} 


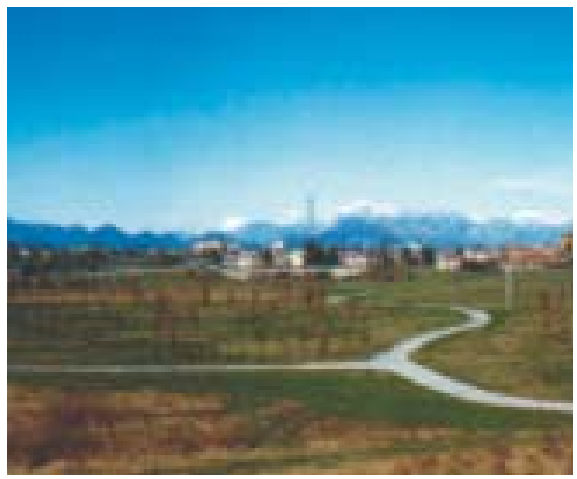

Figura 4 - Il Bosco delle Querce visto dalla collina di Seveso nel 1987.

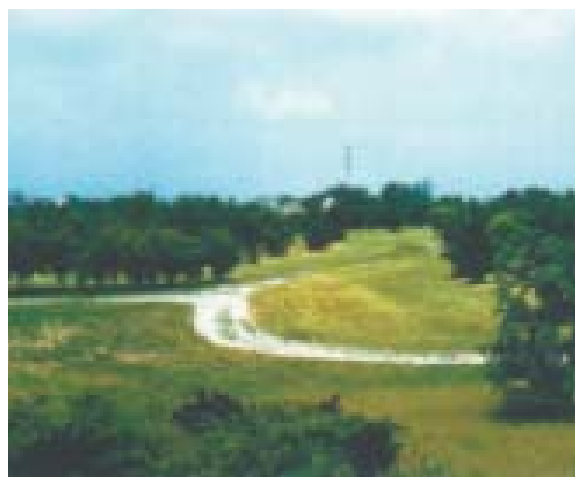

Figura 5 - Il Bosco delle Querce visto dalla collina di Seveso nel 1994.

un prato alberato, che tenesse conto dei limiti tecnici, legati alla presenza delle due vasche di rifiuti tossici, di un metanodotto e di due elettrodotti ${ }^{20}$.

Le specie arboree previste comprendevano: querce indigene, aceri, carpino, pino silvestre, tigli, salici, betulla, pioppo nero e bianco, frassino, orniello; tra quelle arbustive si selezionarono il biancospino, la ginestra, il viburno, il ginepro e la rosa canina.

Nel progetto le piante delle diverse specie erano distribuite piuttosto uniformemente sull'intera superficie del parco, ad esclusione delle colline sopra le vasche, dove la limitata profondità del terreno di coltura ${ }^{21}$ consentiva lo sviluppo delle sole essenze arbustive.

I primi lavori per la formazione del parco vennero attuati tra 1984 e 1986 a cura di una ditta florovivaistica privata che, tramite studi approfonditi e adeguate tecniche di piantagione e di irrigazione, riuscì ad assicurare uniformità ai trentasette ettari su cui inizialmente venne a crearsi il nuovo parco.

Sull'intera superficie del parco vennero riportati 15-20 centimetri di terra di coltura proveniente da zone distanti almeno 10 chilometri dal luogo dell'incidente, alla quale furono aggiunte speciali sostanze organiche atte a favorire lo sviluppo di una flora batterica adatta alla successiva messa a dimora di alberi ed arbusti.

Vennero successivamente messi a dimora 5.789 piante arbustive, prevalentemente autoctone, e 4.960 piante arboree, di 3-4 metri di altezza e circonferenza di 10-16 centimetri, precedentemente ambientate in un vivaio provvisorio e selezionate in base alla loro resistenza.

Nel 1986, quando i lavori in appalto vennero terminati, il risultato, anche se visivamente scarso, poteva essere giudicato abbastanza soddisfacente considerate le condizioni estreme di partenza, le oggettive difficoltà di un cantiere complesso, la necessità del rigoroso rispetto delle normative esistenti e il costo contenuto delle diverse operazioni di formazione e manutenzione del bosco e del verde ${ }^{22}$.

Dal 1987, dopo il primo rinverdimento della zona più inquinata, i lavori di completamento, gestione e manutenzione del Bosco furono affidati all'Azienda regionale delle Foreste $^{23}$ ed in particolare all'Ufficio Operativo di Milano ${ }^{24}$.

${ }^{20} \mathrm{Nel}$ progetto le strutture e le infrastrutture da collocare nel parco erano ridotte al minimo e riguardavano principalmente: la recinzione, la palazzina di servizio, i percorsi, l'impianto di irrigazione e l'impianto per il trattamento del percolato delle due discariche di materiali tossici.

${ }^{21} \mathrm{La}$ copertura delle vasche era stata infatti completata con soli $70 \mathrm{~cm}$ di terra di coltura rinverdita.

${ }^{22}$ Il costo delle operazioni sostenute nel triennio 1984-1986 è stato dell'ordine di circa 30 milioni di $£ /$ ha, escluso il costo degli impianti e delle infrastrutture.

${ }^{23}$ La gestione e lo sviluppo del Bosco fu oggetto di una convenzione stipulata dall'Ufficio Speciale di Seveso al termine del suo mandato (1986); convenzione che venne rinnovata periodicamente dall'Assessorato all'Ambiente-Ecologia della Regione Lombardia (subentrato all'Ufficio Speciale) con la definizione di anno in anni degli interventi di manutenzione ordinaria e straordinaria che l'Azienda avrebbe dovuto eseguire e con lo stanziamento delle risorse finanziarie necessarie alla realizzazione delle stesse. 
Quando il 1 gennaio del 1987 l'Azienda regionale delle Foreste prese in consegna il Bosco, i primi lavori di rinverdimento già eseguiti costituivano solo la base di partenza per lo sviluppo di un ecosistema complesso, che risultava ancora molto povero e poteva evolvere solo con continui interventi.

Nel primo biennio (1987-1988) l'Azienda si preoccupò di assicurare la sopravvivenza degli impianti arborei iniziali e di completare le varie rifiniture indispensabili. Accanto agli interventi di manutenzione ordinaria vennero tuttavia realizzati anche significativi lavori straordinari quali: l'ampliamento della rete di irrigazione e l'avviamento in forma sparsa di una seconda generazione di piante alte 1,5 metri con diversificazione del soprassuolo e incremento del numero delle specie. Inoltre, poiché il parco

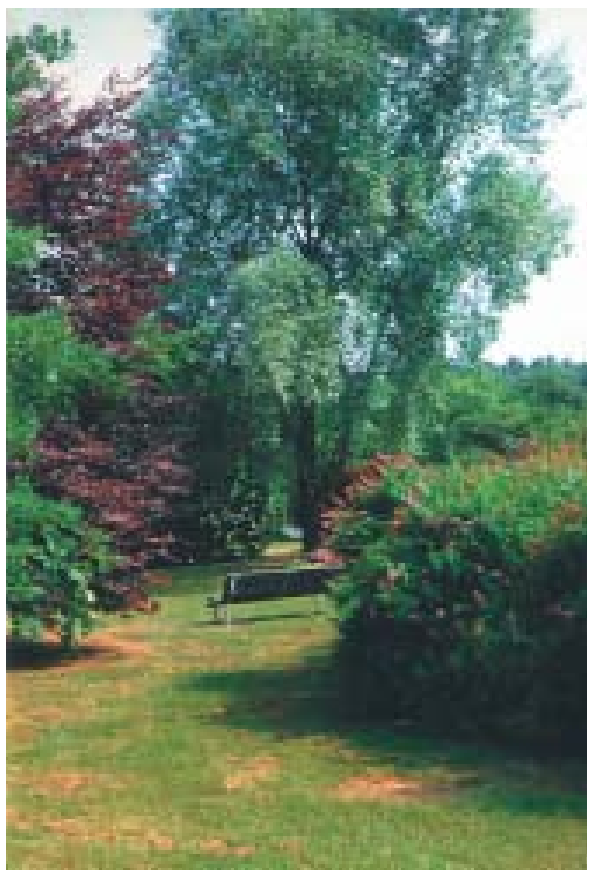

Figura 6 - La varietà delle specie all'interno del Bosco presentava ancora un impianto troppo artificiale poco consono all'idea di bosco, si decise di avviare la formazione di alcune aree rinaturalizzate intensivamente che, definite aree 'sporche', avevano lo scopo di promuovere lo sviluppo dell'avifauna e della microfauna all'interno del Bosco.

Nel triennio successivo (1989-1991) l'Azienda si occupò del miglioramento del parco, del completamento dell'impianto arboreo ${ }^{25}$ e della sua finalizzazione.

$\hat{E}$ in questa direzione che nel 1990 venne commissionato anche il primo progetto di sistemazione paesaggistica del parco $^{26}$, le cui indicazione furono messe subito in atto.

Il progetto interessò dapprima il riassetto della viabilità interna con la creazione di una serie di percorsi che, articolandosi sinuosi tra i prati e gli ambiti boscati, venivano a formare interessanti e suggestivi itinerari di fruizione degli spazi e degli ambienti naturali esistenti all'interno del parco.

Vennero successivamente individuate anche le zone dove effettuare uno sfoltimento delle alberature già esistenti, oppure dove realizzare nuovi impianti arborei, con accrescimento del numero di piante già presenti o la messa a dimora di esemplari nuovi, più caratterizzanti sia per la maggiore dimensione, sia per il profilo delle chiome.

L'attenzione per gli aspetti estetici e paesaggistici, unitamente a quelli ecologici, portò ad attribuire sempre maggiore importanza anche all'inserimento di numerose specie arbustive di pronto effetto aventi funzioni ornamentali; la fioritura, la coloritura persistente o stagionale del fogliame o ancora il profilo delle chiome, diventarono i requisiti fondamentali per la scelta delle specie da adottare. La varietà delle specie poteva consentire infatti di creare particolari configu-

\footnotetext{
${ }^{24}$ Diretto dal Dott. Paolo Lassini, che già dal 1984 aveva svolto le funzioni di direttore dei lavori di costruzione del parco.

${ }^{25}$ In relazione all'aumento delle superfici di rimboschimento e delle aree 'sporche' sottratte al taglio per favorire lo sviluppo naturale dell'ecosistema, venne ridotta anche la superficie totale interessata dai tagli: dai 28 ettari del 1989 si passò ai 16 ettari del 1991.

${ }^{26}$ I professionisti incaricati furono il Prof. Pier Fausto Bagatti Valsecchi e l'Arch. Paola Froncillo di Milano.
} 
razioni spaziali differenziando la fisionomia e la percezione dei luoghi attraverso molteplici accostamenti di colore, fioriture e profili differenti.

Il progetto prestò particolare attenzione anche alla percezione e all'aspetto visivo del parco; a questo scopo si impiantarono grandi macchie arbustive ai piedi e sulle scarpate delle due colline e si cercò di migliorare notevolmente l'aspetto delle aree circostanti il centro operativo del parco che costituivano il punto di accesso principale al Bosco da parte dei visitatori. A tal fine, vennero sistemati gruppi di arbusti a fioritura stagionale nelle superfici al contorno del grande piazzale di accesso, dell'edificio direzionale e dell'attiguo laghetto, che con la sua fauna acquatica veniva ad assumere sempre più un prevalente significato estetico-ricreativo accanto alla più concreta funzione di serbatoio per il fabbisogno idrico nei periodi di crisi.

Lattenzione che i consulenti prestarono agli aspetti paesaggistici e formali del parco non si concretizzò solamente nella configurazione degli spazi interni al parco; a questi si affiancò, anche se in misura minore, l'obiettivo di un migliore rapporto con l'ambiente circostante principalmente attraverso due interventi sulle aree di margine.

Venne innanzitutto posta attenzione ad alcuni episodi edilizi che, posti ai margini del parco, disturbavano la percezione del Bosco; per contrastarne l'impatto si realizzò una quinta prospettica, una sorta di zona filtro, con la messa a dimora di alberi ad alto fusto e a rapido accrescimento.

Sempre ai margini del parco si prestò attenzione alla sistemazione della scarpata lungo la superstrada Milano-Meda, una barriera molto forte per la percezione del parco dai territori più orientali e un elemento fortemente negativo per le condizioni di degrado che inevitabilmente un'arteria di grande traffico porta con $\mathrm{sec}^{27}$.

Accanto alle operazioni di rinforzo della recinzione perimetrale già esistente, gli interventi più consistenti riguardarono la preparazione del suolo con fresatura, bonifica da sassi, macerie e rifiuti, a cui fece seguito la messa a dimora di 2.340 arbusti in gruppi e con fioritura a scalare nelle varie stagioni dell'anno che, intervallati a spazi a prato, dovevano conseguire un notevole effetto paesaggistico sia per chi si trovava all'interno del parco, sia per chi si trovava a percorrere la superstrada. Occorre sottolineare che una simile sistemazione era stata concepita tenendo conto della possibilità di trasformare la superstrada in un'"autostrada-parco" con l'impianto di arbusti su entrambe le scarpate e nell'ottica di un possibile ampliamento del Bosco negli spazi agricoli al di là della superstrada.

Al fine di perseguire la strategia del progressivo arricchimento del parco, l'Azienda delle Foreste, sostenuta anche dai finanziamenti regionali, continuò gli interventi straordinari di impianto di nuovi alberi e arbusti ${ }^{28}$, realizzando in questo modo anche le barriere arbustive di protezione delle due discariche, la barriera acustica con specie arboree sull'intero fronte della superstrada Milano-Meda e il primo e secondo

\footnotetext{
${ }^{27}$ Principalmente per tre ragioni: l'impatto acustico dovuto al flusso di traffico molto intenso; la frequente distruzione dell'impianto boschivo a causa degli incendi più o meno vasti che venivano alimentati nei tratti di Bosco confinanti con la superstrada e nei pressi dello svincolo di Meda; ed infine la scarsa manutenzione e pulizia delle aree di pertinenza della superstrada ridotte, come nella maggior parte di questi spazi di risulta, a discarica a cielo aperto dei più svariati materiali. Proprio con il fine di contrastare questi tre riflessi negativi, venne stipulato un accordo di collaborazione tra l'Agenzia regionale delle Foreste e la Provincia di Milano, alla quale subentrò la Società Autostrade Serravalle a cui venne trasferita la competenza della superstrada.

${ }^{28}$ La continua necessità di disporre di piante per nuovi impianti fece optare per la realizzazione in loco di un piccolo vivaio e di un piantonaio.
} 


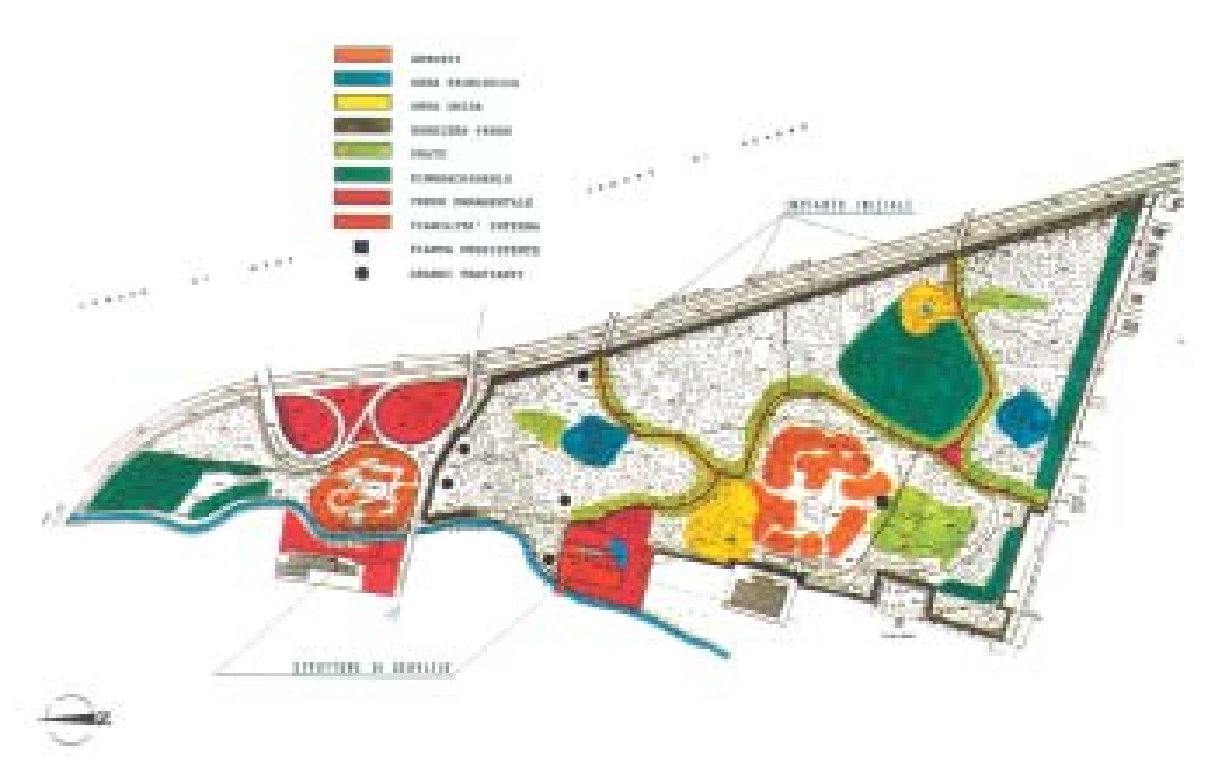

Figura 8 - Lo sviluppo differenziato del Bosco nel 1992.

lotto di rimboschimento dell'area naturalistica.

$\mathrm{Al}$ termine del primo quinquennio di gestione il parco nel 1992 si presentava sostanzialmente suddiviso in due parti. A nord la parte in comune di Meda che comprendeva, oltre al sistema di svincoli della superstrada, la collina della discarica più piccola disegnata con arbusti e a nord della stessa un rimboschimento di latifoglie ben affermato. A sud la parte in comune di Seveso che si presentava come una grande distesa di piante e cespugli interrotta da radure, prati ornamentali ed aree faunistiche e comprendente la collina della discarica più grande sistemata a prati ed arbusti. In questa parte si era inoltre provveduto a realizzare una barriera acustica verde lungo il confine esterno, in particolare lungo la superstrada; un settore ornamentale con laghetto attorno alla sede operativa dell'Azienda regionale ed infine a sud della stessa una grande area umida.

Complessivamente nel 1992 circa il 77\% dell'area era coperto da alberi ed arbusti, un delicato ecosistema destinato a trasformarsi in bosco, pur molto diversificato in funzione delle tecniche iniziali di impianto ${ }^{29}$.

Nel 1992 a seguito del graduale assestamento dell'ecosistema, della forma sempre più precisa assunta dal parco e della prevista apertura al pubblico, l'Azienda regionale delle Foreste si impegnò nella redazione di un vero e proprio azzonamento in modo da poter meglio finalizzare e ottimizzare gli investimenti e gli interventi di gestione degli anni successivi. A seguito di un attento studio vocazionale furono così individuate cinque tipologie di aree con destinazione funzionale differente ma anche con caratterizzazione spaziale e paesaggistica diversa: le aree paesaggistiche che svolgono essenzialmente funzioni di barriera verde, per schermare il parco nei confronti degli effetti nocivi provenienti dall'esterno, in termini sia di visuale - schermo ottico - che di immissioni - schermo fonoassorbente - ma anche per abbellire strutture ed infrastrutture $^{30}$; le aree naturalistiche aventi la funzione di garantire un buon inseri-

${ }^{29} \mathrm{Al} 1992$ sull'area erano presenti 8.723 piante arboree e 15.133 piante arbustive, con una consistenza più che doppia rispetto a quella dell'impianto iniziale.

${ }^{30}$ Con una superficie complessiva di 5 ettari queste aree comprendono gli svincoli della superstrada Milano-Meda e la barriera verde che circonda quasi interamente la parte del parco ricadente nel comune di Meda.

${ }^{31} \mathrm{~A}$ tal fine è stata prevista una superficie compatta ed estesa che, dislocata prevalentemente nel settore più appartato del parco, vicino alla superstrada, occupa circa $1 / 3$ della superficie totale del Bosco (16,5 ettari). Completamente rimboschite, queste aree sono state tutte recintate per ridurre al minimo i fattori di disturbo per la fauna naturale e la manutenzione dell'erba è stata ridotta all'indispensabile. 


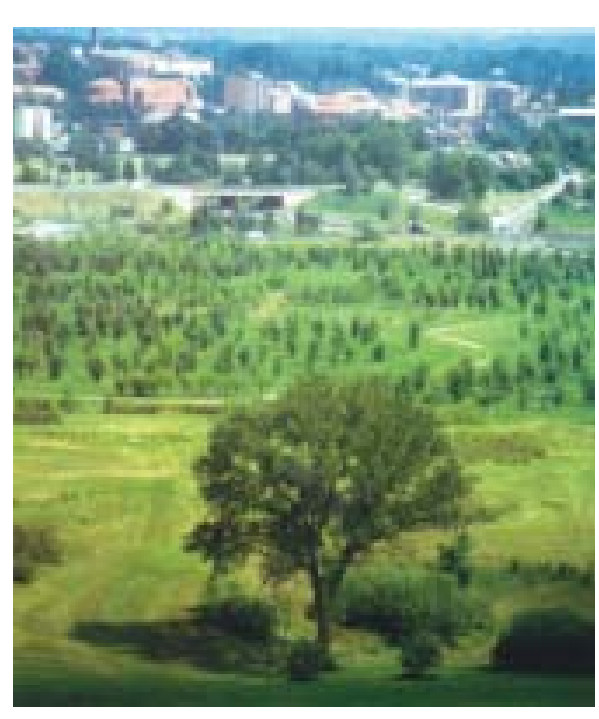

Figura 9 - Il grande pioppo al centro del Bosco rappresenta l'unico albero risparmiato nelle operazioni di bonifica. mento della micro e della macrofauna tipica dei boschi ${ }^{31}$; le aree ricreative intensive localizzate in punti periferici di particolare interesse per l'accesso al pubblico ${ }^{32}$; le aree ricreative estensive prevalentemente destinate ad una fruizione di tipo escursionistico e contemplativo $^{33}$; infine le aree di rispetto che comprendono innanzitutto gli impianti tecnologici ${ }^{34}$.

La dimensione naturalistica e ricreativa che veniva sempre più attribuita al Bosco, unitamente alla sua dimensione estetica e paesaggistica, richiesero un'attenzione sempre maggiore verso i territori esterni; le aree naturalistiche dovevano essere inserite in un sistema a rete di più ampio respiro, le aree ricreative collegate tra loro attraverso percorsi pedonali e ciclabili e le sistemazioni paesaggistiche dovevano trovare una naturale prosecuzione nella qualità dell'ambiente esterno.

Proprio a partire da questi obiettivi nel 1993 venne affidata l'elaborazione di un progetto paesaggistico di più ampio respiro ${ }^{35}$, le cui indicazioni tuttavia non sono ancora state portate a compimento ${ }^{36}$.

Il progetto considerava essenziale ricondurre l'area verso un nuovo e più adeguato equilibrio, ottenibile non solo attraverso il ridisegno della forma urbana e il riordino delle grandi infrastrutture di trasporto ma anche e soprattutto attraverso la realizzazione di un tessuto connettivo di aree verdi e percorsi pedonali e ciclabili che consentisse di dare forma ad un sistema integrato di spazi naturali e paesistici altamente fruibile.

Muovendosi in questa direzione, il progetto prese innanzitutto in considerazione la trama preesistente dei tracciati ancora presenti e di quelli cancellati dall'incidente del 1976 e, interpretandoli in un disegno d'insieme rispettoso delle diverse tracce e segni storici dei luoghi ${ }^{37}$, cercò di dare vita ad un insieme articolato di proposte organizzate fondamentalmente in due gruppi: gli interventi lungo il perimetro del parco e gli interventi nelle aree adiacenti.

Con riferimento ai primi il progetto cercò innanzitutto di valorizzare l'importante ruolo di cerniera di quest'area nel sistema verde circostante. A questo scopo il progetto disegnava una serie di connessioni e di accessi da collocare su tutto il perimetro del parco per una adeguata ricucitura del territorio. Se prima

${ }^{32}$ Comprendono tre aree per un totale di 7,2 ettari: a nord lungo il torrente Certosa, il complesso edilizio collocato di fronte all'area dell'ex ICMESA, oggi trasformata in un centro sportivo; al centro, l'area perimetrale con annesso laghetto in corrispondenza dell'ingresso principale del parco; infine a sud, l'area ornamentale esistente in corrispondenza dell'accesso secondario di fronte alla piscina comunale di Seveso.

${ }^{33} \mathrm{Su}$ queste aree che occupano 8 ettari non è prevista la sistemazione di attrezzature impattanti.

${ }^{34}$ Ovvero le colline che ospitano le vasche con i residui della bonifica delimitate da una spessa barriera arbustiva e l'area compresa tra la superstrada, lo svincolo e l'area di fruizione intensiva a nord in cui è stato per alcuni anni realizzato il compostaggio dei residui verdi del parco, poi spostato vicino al cimitero. La superficie complessiva di queste aree è di circa 6 ettari.

35 Il progetto, elaborato dagli architetti Paola Froncillo e Carlo Masera di Milano, prese innanzitutto avvio da un'analisi abbastanza approfondita dell'ambiente immediatamente circostante il parco; l'immagine che venne messa in luce fu quella tipica di un'area in cui una crescita tumultuosa e disordinata aveva portato da uno sviluppo graduale per piccoli centri ad una loro rapida 'dilatazione' ed accorpamento in una unica grande conurbazione.

${ }^{36}$ Nonostante le difficoltà e i ritardi nella realizzazione si trattò di una proposta di notevole interesse non solo progettuale ma anche politico e metodologico in quanto consentì di avviare un dialogo concreto con le Amministrazioni comunali di Seveso e Meda. La possibilità di discutere su un progetto di riassetto complessivo dell'area gravitante sul Bosco delle Querce portò infatti dapprima all'avvio della necessaria e contestuale revisione degli strumenti urbanistici dei due comuni confinanti e successivamente ad una riflessione sul ruolo che questo parco poteva assumere all'interno della grande area metropolitana milanese. Come ben esprimono le parole di Mario Di Fidio, "nel processo di sviluppo metropolitano degli anni Duemila, il piccolo Bosco delle Querce di Seveso e Meda ha due carte da giocare: la centralità geografica e la potenza evocativa del suo nome; entrambe fanno sì che esso non sia un'area verde tra le tante" (MARIO Di FIDIO, op. cit., 2000, pag. 140).

${ }^{37}$ In particolare, si fece riferimento alla topografia del luogo, alle preesistenze architettoniche, alle antiche trame del territorio agricolo e alle presenze infrastrutturali. 


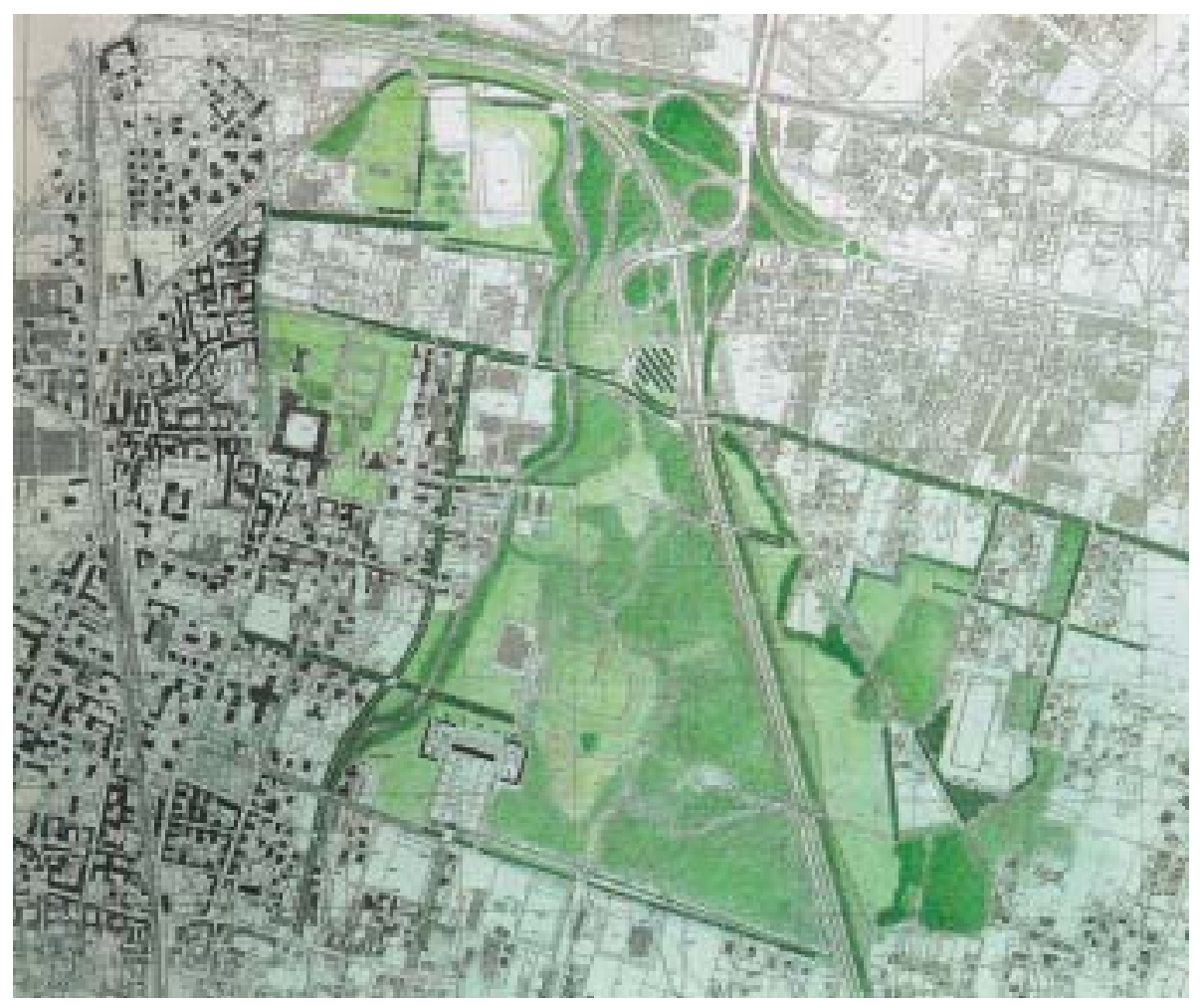

Figura 10 - Progetto di sistemazione paesaggistica collegata al territorio circostante (1993).

dell'incidente, le aree occidentali del territorio di Seveso erano collegate a quelle orientali attraverso una serie di percorsi paralleli alle vicinali dei Boschi che si interrompevano al confine del parco, con il nuovo progetto questi stessi percorsi venivano potenziati attraverso il disegno di tracciati sinuosi che, sovrapposti agli antichi percorsi rettilinei, terminavano nei medesimi punti di sbocco, ossia i due cavalcavia che oltrepassano la superstrada per collegarsi con la viabilità delle aree più orientali.

Il progetto, oltre a ridisegnare gli ingressi, cercava di mettere a sistema il Bosco con gli spazi pubblici esistenti attraverso un insieme morfologicamente ben strutturato di strade alberate a filari. Nel progetto il sistema dei filari veniva rafforzato e proseguito sin dall'interno del Bosco, in direzione dell'unico grande pioppo 'risparmiato' alla bonifica e superstite dell'antica vicinale dei Boschi.

Il progetto riservava particolare attenzione al corso del torrente Certosa che, in considerazione del suo valore naturale e paesistico, rappresenta un importante asse di collegamento tra aree verdi di interesse sovracomunale. Per recuperare pienamente il suo potenziale venivano proposti lavori di sistemazione dell'alveo e delle sponde con criteri di ingegneria naturalistica, al fine di realizzare lungo tutto il corso una pista pedonale e ciclabile piantumata.

Per quanto concerne le proposte relative alle aree adiacenti il Bosco, un primo gruppo di interventi riguardava l'area costituita dagli svincoli a nord est della superstrada. Traendo spunto dalla già attuata sistemazione paesaggistica della superstrada e delle sue pertinenze che mirava a trasformare tutto il tracciato contiguo al Bosco in un'"autostrada-parco" 38 e tenuto conto della fascia boschiva già esistente tra la superstrada e la ferrovia Milano-Como, il progetto intendeva estendere l'impianto arboreo anche alle altre aree comprese nel nodo infrastrutturale attribuendo così a tutta quest'area una finalità essenzialmente paesistica.

\footnotetext{
${ }^{38} \mathrm{Ci}$ si riferisce alle sistemazioni paesaggistiche iniziate negli anni precedenti sulla base delle indicazioni del Prof. Bagatti Valsecchi e dell'Arch. Froncillo.
} 


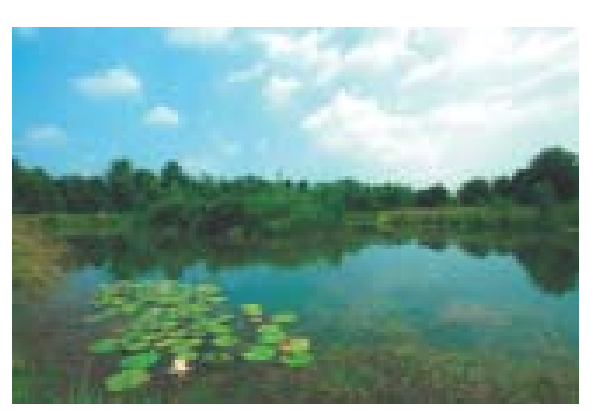

Figura 11 - Il laghetto artificiale tra la collina di Seveso e la superstrada MilanoMeda realizzato nel 1996.

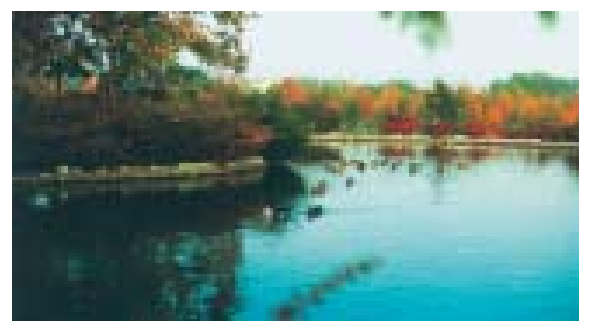

Figura 12 - Il secondo laghetto artificiale realizzato nei pressi della sede operativa.

Ancora una volta era stata posta particolare attenzione alla percezione dello spazio 'vissuta' dall'automobilista transitante lungo la superstrada: dalla chiusura della vegetazione compatta proposta per l'area degli svincoli all'apertura su un sistema più articolato di spazi verdi, visibile dalla superstrada all'alzarsi del rilevato.

Altri interventi riguardarono infine la vasta area agricola situata ad est della superstrada e collegata al parco attraverso la prevista riapertura dei due cavalcavia già esistenti. La proposta, considerata la localizzazione dell'area prossima alla parte più naturalistica del Bosco, ipotizzava una sistemazione a prato alberato con funzioni ricreative, isolato acusticamente dalla superstrada mediante la costruzione di una serie di terrapieni alberati. Il progetto prevedeva un completamento ed un potenziamento della vegetazione anche sul lato opposto alla superstrada, dove le fasce arboree avrebbero lasciato spazio alle sole aree di accesso alla vasta radura. Oltre questa fascia arborea, gli spazi aperti residuali erano interessati da un sistema di piantagioni a bosco avente lo scopo di rimodellare i margini frammentati dell'abitato e di schermare qualsiasi impatto provenisse dall'area urbanizzata.

Dal 1993 al 1998, mentre la struttura fondamentale del Bosco era ormai consolidata, proseguirono solo le operazioni di rimboschimento tanto che alla fine del 1998 il parco comprendeva 21.753 piante arboree e 23.898 piante arbustive, ossia un patrimonio quadruplo rispetto a quello dell'impianto iniziale ereditato dall'Ufficio Speciale di Seveso.

Le specie utilizzate risultavano più numerose di quelle diffuse nei boschi naturali vicini, poiché si era seguito un indirizzo naturalistico che aveva portato a ricercare una maggiore diversificazione dell'ecosistema, associando al bosco zone umide e fasce di transizione (ecotoni), ma anche un indirizzo esteticoricreativo, legato alla crescente fruizione del parco da parte della popolazione ${ }^{39}$.

\section{GLI ESITI}

Grazie ai costanti interventi di piantumazione, manutenzione e gestione degli impianti vegetali la struttura del parco può sicuramente considerarsi ormai consolidata; le grandi macchie arbustive, i prati, le aree naturalistiche, gli specchi d'acqua e i percorsi di fruizione costituiscono un insieme armonioso di spazi, forme e colori. L'attenzione per gli aspetti estetici e paesaggistici, unitamente a quelli ecologici, ha portato alla creazione di differenziate configurazioni spaziali.

Se è vero tuttavia che la struttura complessiva del parco può dirsi consolidata, non altrettanto si può dire per il sistema vegetale che lo caratterizza. La continua evoluzione naturale degli impianti arborei ed arbustivi, la coloritura e la fioritura stagionale contribuiscono infatti a conferire al Bosco una fisionomia in continuo mutamento ed evoluzione.

\footnotetext{
${ }^{39}$ La crescente fruizione del parco da parte degli istituti scolastici locali è culminata con la definitiva apertura al pubblico del Bosco in occasione del ventennale dell'incidente ICMESA. Il Bosco infatti non era stato subito accessibile alla popolazione in osservanza ad una disposizione della Commissione tecnico-scientifica governativa del 1976, con la quale si stabiliva che il parco non venisse aperto al pubblico per un periodo di almeno 6-7 anni per evitare di compromettere le essenze arboree ed arbustive appena impiantate. Nonostante il divieto di accesso l'ente gestore cercò comunque di stabilire un rapporto tra la popolazione e il Bosco in maniera tale da abituare i cittadini a frequentare un ambiente che nel giro di pochi anni sarebbe diventato un parco fruibile a tutti gli effetti, seppur non omologabile a tanti altri parchi aperti al pubblico. A questo scopo l'Azienda regionale delle Foreste cercò la collaborazione delle associazioni locali e delle scuole per cercare di concordare le specifiche esigenze di fruizione culturale e ricreativa; in questa direzione è iniziata, a partire dal 1998, una collaborazione con la Lega Italiana per la Protezione degli Uccelli finalizzata alla gestione di un calendario di eventi a sfondo naturalistico e di una serie di visite guidate destinate alle scolaresche locali.
} 
Il Bosco dunque deve ancora crescere, svilupparsi, evolversi verso la sua configurazione definitiva; una configurazione finale che non è stata scritta a priori ma che sarà il frutto delle azioni iniziali, delle aggiunte successive, delle proposte e delle reinterpretazioni che seguiranno negli anni a venire.

Quello che però preme qui sottolineare non è tanto l'esito formale raggiunto quanto il significato intrinseco di una simile esperienza; significato che si collega innanzitutto alla dimostrazione di un avvenuto riscatto nei riguardi della natura dopo un incidente come quello della diossina. La realizzazione del Bosco infatti dimostra che, se è stato possibile bonificare e costruire ex novo un ecosistema boscato su un'area come questa, a maggior ragione sarà possibile bonificare e riutilizzare le decine o le centinaia di siti contaminati ancora presenti nel territorio italiano.

Il Bosco delle Querce ha permesso di sperimentare una metodologia idonea al graduale recupero dell'ambiente e al reinserimento nel paesaggio di aree fortemente degradate. L'esperienza dunque deve essere di stimolo per un concreto recupero e per una efficace progettazione degli spazi aperti di cintura metropolitana.

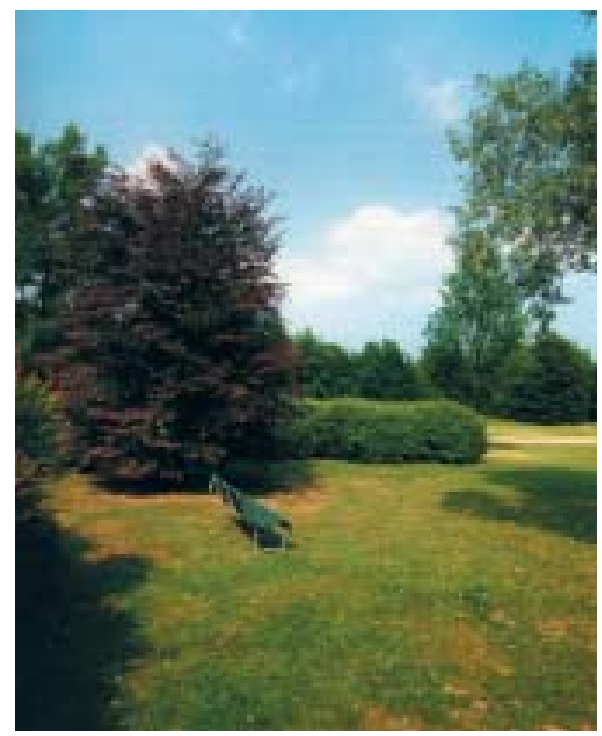

Figura 13 - Uno degli ambienti caratterizzanti il Bosco.

\section{RIFERIMENTI BIBLIOGRAFICI}

Ballardini PaOlo e Lassini PaOlo, Le tecniche agroforestali per il recupero ambientale delle discariche e delle aree degradate, "Acer", 6, 1990, pagg. 83-88.

Ballardini Paolo, Lassini Paolo, Mambriani Antonio e Monzani Francesco, il Bosco delle Querce di Seveso, "Acer", 4, 1993, pagg. 54-58.

Di Fidio Mario, Il "Bosco delle Querce" di Seveso e Meda, Regione Lombardia, Milano 2000.

Farina Renato, Seveso diossina e scuola, Gribaudi, Torino 1978.

Testori Carla, Seveso: un progetto per il Bosco delle Querce, Fondazione Lombardia per l'Ambiente, Milano 1996.

Zaini GraZIELLA, Seveso: il Bosco delle Querce, “Acer”, 6, 1996, pagg. 50-51.

\section{RIFERIMENTI ICONOGRAFICI}

Figura 1: Elaborazione di Laura Ferrari. Base dati Laboratorio Metodi e Tecniche di Pianificazione Urbanistica, Dipartimento di Architettura e Pianificazione, Politecnico di Milano.

Figure 2, 4, 5, 6, 7, 9, 10, 11, 12, 13: Di Fidio Mario, Il "Bosco delle Querce" di Seveso e Meda, Regione Lombardia, Milano 2000, pagg. 25, 52, 7, 62, 43, 134, 60, 83.

Figura 3: BALLARDini PAOLO e LASSINI PAOLO, Le tecniche agroforestali per il recupero ambientale delle discariche e delle aree degradate, "Acer", 6, 1990, pag. 85.

Figura 8: Ballardini Paolo, Lassini Paolo, Mambriani Antonio e Monzani Francesco, $I l$ Bosco delle Querce di Seveso, “Acer”, 4, 1993, pag. 55. 
SCHEDA DI PROGETTO

Progetto:

Luogo:

Committente:

Tipologia:

Superficie:

Progettisti:

Direttore dei lavori:

Ditte esecutrici:

Anno di progettazione:

Anno di realizzazione:

Specie arboree principali:

Costo intervento: intervento di bonifica e di recupero ambientale di un sito inquinato

comuni di Seveso e Meda (provincia di Milano)

Regione Lombardia, Ufficio speciale di Seveso

parchi metropolitani

$427.600 \mathrm{mq}$

Dott. Arch. Silvano Barbarani, Milano e Dott. Ing. Nino Noseda, Milano (progetto generale - 1983)

Dott. Arch. Pier Fausto Bagatti Valsecchi, Milano e Dott. Arch. Paola Froncillo, Milano (progetto di sistemazione paesistica - 1990)

Dott. Arch. Paola Froncillo, Milano e Dott. Arch. Carlo Masera, Milano (proposta di ampliamento e di sistemazione paesaggistica - 1993)

Dott. Agr. Paolo Lassini, Azienda Regionale delle Foreste, Milano (1984-1997)

Dott. Agr. Antonio Mambriani, Azienda Regionale delle Foreste, Milano (1998-2000)

Scarpellini s.p.a., Alzano Lombardo (1984-1986)

Azienda Regionale delle Foreste, Milano (1987-2000)

1983 - progetto generale

1990 - progetto di sistemazione paesistica

1993 - proposta di ampliamento e di sistemazione paesaggistica

1977/1983 - bonifica precedente l'impianto di rimboschimento

1984/1986 - lavori di impianto e prima manutenzione

1987/2000 - lavori di completamento, manutenzione e gestione

Acer campestre, Acer pseudoplatanus, Fraxinus excelsior, Carpinus betulus, Populus spp., Quercus spp.

1984/1986 - opere a verde e cure colturali, 1.001.000.000 di lire

$1987 / 2000$ - complessivo degli interventi di investimento, manutenzione straordinaria e gestione, 5.479.000.000 di lire 


\title{
SPAZI URBANI
}

\author{
Enrica Dall'Ara
}

Parlare di spazio urbano implica un ragionamento sull'immagine stessa di città, a cui riferirsi nel discorso. Nel tempo contemporaneo della famosissima globalizzazione la riflessione muove intorno al rapporto fra la Generic City quale iconografata da Rem Koolhas ${ }^{1}$ - che si configura come un'istallazione effimera e policentrica montata su un suolo occupato da vegetazione ipertrofica completamente dimentica di qualsiasi regione d'origine o di processi di acclimatazione e una città governata localmente dal proprio genius loci.

La città Cyburbia, presentata da Michael Sorkin nell'introduzione a Variations on a theme park ${ }^{2}$, indagando la realtà americana, ha aspetti analoghi a Generic City.

Si costruisce secondo le stesse logiche che regolano il sistema televisivo, per cui ogni elemento appartiene ad una rete ed ha al suo interno un uguale valore. È la connessione ad attribuire un senso allo spazio. Alla città storica, fisicamente spaziale e fenomenologia della relazione sociale, "in cui l'ordine è una funzione della prossimità" , si contrappone uno spazio invisibile, solo concettuale, scevro sia dalla geografia fisica sia da quella culturale: "La nuova città sostituisce l'anomalia e il diletto di tali luoghi ( le città tradizionali ) con un particolare universale (...). Ossessionata dal principio della produzione e del prezzo, la nuova città è poco più che uno sciame di bit urbani, buttando a mare la visione fisica del complesso, sacrificando l'idea di città come posto della comunità e delle relazioni umane" ${ }^{\prime 4}$.

Allo stesso proposito Richard Ingersoll evidenzia una modalità di percezione dello spazio urbano, che porta a quello che definisce Jumpcut urbanism ${ }^{5}$, dovuta alla visione dall'automobile in movimento, fatta di "imprecisioni ed interruzioni", che "ricompone frammenti temporali attraverso un processo di montaggio" nel quale viene annullata l'unità di tempo e di spazio del teatro classico. "L'unità spazio-temporale condivisa dal palcoscenico prospettico e dallo spazio pubblico figurato è stata definitivamente sconvolta dall'introduzione dell'automobile (...). Insomma, i film e le automobili si occupano di quel progetto delusorio che è la libertà individuale" 6 .

Contemporaneamente soprattutto in Italia - l'affermazione è una semplificazione - il genius loci ha preso storicamente forme di tale valore, quasi ingombranti a volte, che sembra impossibile non andarlo a scoprire, nell'esplorazione

\footnotetext{
${ }^{1}$ Rem Koolhas e Brus Mau, La città generica, in S,M,L,XL, Taschen, Germany 1997.

${ }^{2}$ Michael SORKIN (a cura di), Variations on a theme park. The new American city and the end of public space, Hill and Wang, New York 1999.

${ }^{3}$ Michael Sorking (a cura di), op. cit., 1999, pag. xii, trad. it. nostra.

${ }^{4}$ Michael SORKING (a cura di), op. cit., 1999, pag. xiii, trad. it. nostra.

5 RichARD InGERSOlL, Jumpcut urbanism, in "Casabella", 597-598, Il disegno degli spazi aperti, Electa, Milano 1993, pagg. 52-57.

In nota Richard Ingersoll spiega: "Jumpcut" è termine di origine cinematografica: indica un improvviso stacco da un fotogramma all'altro, diversi fra loro per soggetto, per profondità o per senso".

${ }^{6}$ RiCHARD INGERSOLL, op. cit., 1993.
} 
come nell'azione progettuale, garante di un senso di appartenenza e suggeritore benevolo delle possibili trasformazioni. Rimane ancora istanza della città essere luogo del riconoscimento personale correlato al sentimento di una similitudine, per cui Umberto Saba poteva dire di Trieste: "La mia città che in ogni sua parte è viva / ha il cantuccio a me fatto, alla mia vita / pensosa e schiva.", rivelandola allo stesso tempo, in Città vecchia ${ }^{7}$, spazio del contatto con gli altri, abitanti, stranieri, di passaggio.

Alla comprensione della realtà d'esistenza precisa, particolare, consegue la condivisione di umanità, che è condizione di tutti.

I progetti che si presenteranno di seguito sono, ognuno con espressione differente, soluzioni di questo interrogarsi sulle relazioni fra lo spazio, la sua storia e l'attribuzione di un nuovo valore condivisibile. Su come l'intervento si pone nei confronti dell'identità.

Al progetto di Renzo Piano per il Lingotto a Torino e al progetto del Parco di Teodorico si giunge dopo una fase di concorso. I contesti sono completamente differenti ma in entrambi gli oggetti della trasformazione consistono in immagini emblema: nella Torino, città associata all'industria, lo stabilimento FIAT costruito dopo la prima guerra mondiale, a Ravenna, città d'arte, lo spazio aperto circostante il Mausoleo di Teodorico (VI secolo d.C.), dichiarato recentemente patrimonio universale dell'UNESCO. In entrambe le situazioni emergono vincitrici le proposte che esplicitano un'interpretazione del luogo tesa all'equilibrio: la valorizzazione parte dal considerare il patrimonio in riferimento alle istanze che l'hanno costruito e contemporaneamente ai significati odierni, liberi da nostalgie - l'ambiente naturale costiero perduto del Mausoleo - o, all'opposto, da sensi di colpa - la tecnologia come peccato originale.

Le realizzazioni di Tonino Guerra nei territori della Romagna sono un'affermazione della necessità, ancora viva, di empatia con un luogo specifico. Allo spazio viene affidato un racconto fatto di memoria "o meglio dell'ECO prolungata del passato nella coscienza, che determina anche l'incertezza delle frontiere tra la memoria e il SOGNO"8, quindi anche di fantasia. Esse dilatano il tempo e lo spazio dal vicino al lontano assorbendo la presenza di altre terre e civiltà per cui l'bic et nunc si affaccia sull'universale.

\footnotetext{
7 “Spesso, per ritornare alla mia casa / prendo un'oscura via di città vecchia. / Giallo in qualche pozzanghera si specchia / qualche fanale, e affollata è la strada./

Qui tra la gente che viene che va / dall'osteria alla casa o la lupanare, / dove son merci ed uomini il detrito / di un gran porto di mare, / io ritrovo, passando, l 'infinito / nell'umiltà./

Qui prostituta e marinaio, il vecchio / che bestemmia, la femmina che bega, / il dragone che siede alla bottega / del friggitore, / la tumultuante giovane impazzita / d'amore, / sono tutte creature della vita / e del dolore; / s'agita in esse, come in me, il Signore./

Qui degli umili sento un compagnia / il mio pensiero farsi/ più puro dove più turpe è la via."

8 JaCQUes Le Goff, presentazione in Tonino Guerra, Dizionario fantastico, Pietroneno Capitani Editore, Rimini 2000, pag. 10.
} 


\title{
IL PARCO DI TEODORICO A RAVENNA
}

\author{
Enrica Dall'Ara
}

Il valore del Mausoleo del re dei goti Teodorico a Ravenna (VI sec. D.C.) rende singolare l'esperienza di dare una configurazione a un parco circostante. La presenza monumentale richiede allo spazio esterno di essere scena discreta al servizio di un fuoco centrale, rapportandosi, nell'ideazione, sia con l'eloquenza dell'arte, iterata nel tempo, sia con l'assenza della compagine originaria: il mausoleo fu eretto nelle vicinanze della linea di costa e della foce del fiume Badareno o Podoreno che collegava il Po al porto di Classe, in un'area ai margini della città, adibita a necropoli già dai romani e mantenuta dai goti come luogo di sepoltura.

È semplice immaginare un paesaggio costituito, nella dimensione vasta, dalle forme dendritiche della foce verso la superficie omogenea del mare, entro il quale si pone, puntuale,

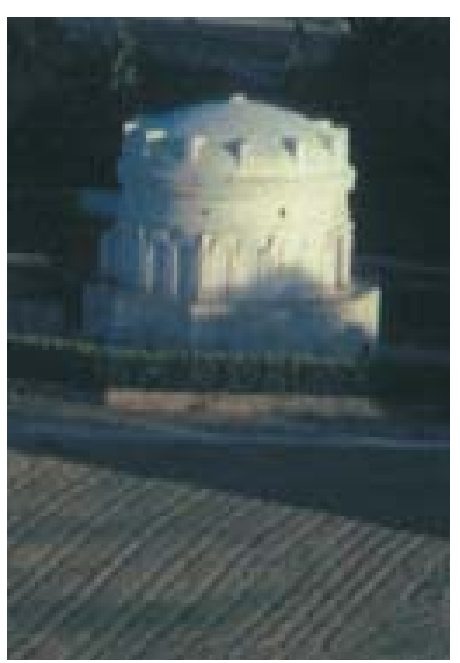

Figura 1 - Mausoleo di Teodorico.

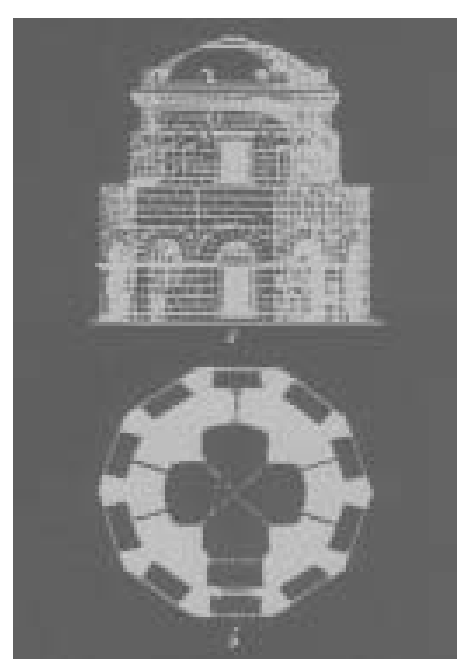

Figura 2 - Pianta e prospetto. 1 architettura a pianta centrale del mausoleo, in grandi conci di pietra bianca, severa ${ }^{1}$.

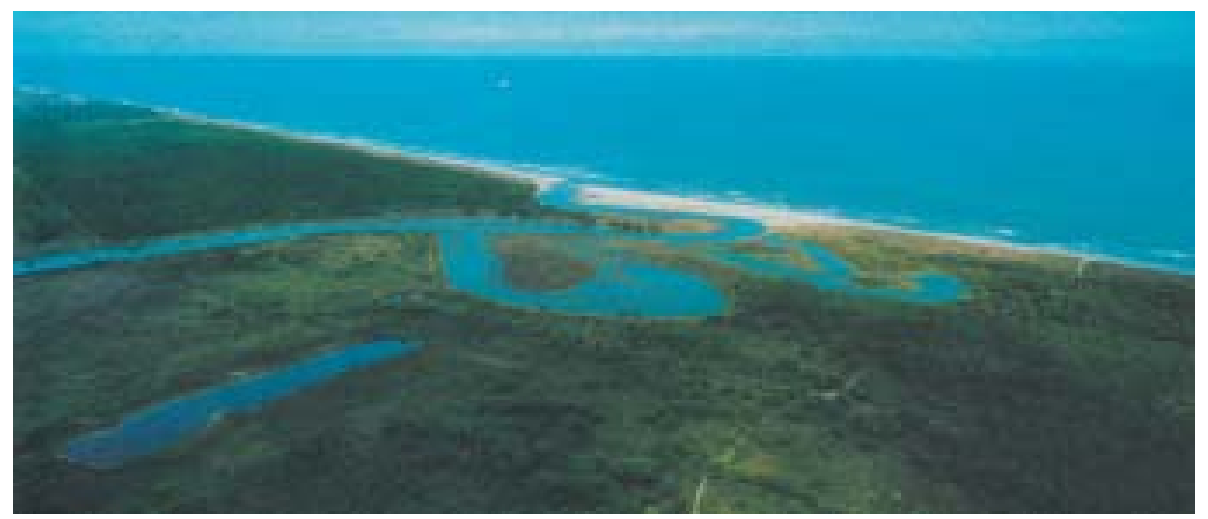

Figura 3 - Paesaggio costiero nel territorio di Ravenna: foci del fiume Bevano nel Parco Regionale del Delta del Po.

${ }^{1}$ Fatto eccezionale in quanto le coeve architetture di Ravenna erano costruite in mattoni: "Sorprende, come affermazione di una forza brutale, l'enorme monolito che copre il mausoleo, poiché gli esperti costruttori ravennati avrebbero facilmente potuto sostituirvi una cupola; esso rammenta i monumenti eretti tra il secolo IV e il VI nel Hauran in Siria nei quali le coperture non sono formate di volte ma di lastroni enormi, chè i costruttori vi dimostrarono grande perizia nell'uso dei conci di pietra, come nel mausoleo." in Pietro Toesca, Storia dell'Arte Italiana I, Il Medioevo, vol. I, Unione TipograficoEditrice Torinese, Torino 1965. 
$\mathrm{Al}$ tema di ridelineare un paesaggio-rappresentazione si affianca la volontà di costruire un parco, un luogo quindi disponibile ad essere vissuto, con modalità proprie della contemporaneità, in relazione alle funzioni della città odierna.

Larea del mausoleo si trova in posizione nodale nel territorio di Ravenna: il progetto del parco è occasione per la soluzione meditata del rapporto fra città e paesaggio rurale, per il completamento di un percorso monumentale che colleghi il centro storico, il mausoleo di Teodorico e la Darsena di città, per la realizzazione di una continuità fra il sistema degli spazi verdi delle mura storiche e il sistema del "verde di cintura", a sua volta connesso con il Parco Regionale del Delta del Po, come individuato dagli strumenti della pianificazione comunale ${ }^{2}$. L'anello verde intende dichiarare compiuta la forma urbis mediante il contenimento dell'espansione, nel disegno di un parco lineare a margine della periferia urbana che intercetti i parchi significativi di Ravenna: il Parco Baronio ad Ovest, il parco Cesarea a Sud ed il Parco di Teodorico a Nord, il quale diviene, in occasione dei finanziamenti del Ministero dei Lavori pubblici per I Programmi di Riqualificazione (1998), uno dei tasselli della riqualificazione del comparto Darsena, un tempo area di insediamento di numerose attività produttive e portuali, attualmente per ampia parte interessate da processi di dismissione.

La progettazione è nei termini pertanto di ricerca d'equilibrio fra proposizioni attuali e memoria.

\section{LE METODOLOGIE}

Nello schema guida del concorso bandito nel 1996 per la progettazione del Parco di Teodorico, era già suggerita una strategia: l'area risultava frazionata in modo tale che il contatto interno-esterno avvenisse con omogeneità di carattere, per cui il confronto fra aspetti e funzioni diverse era "posticipato" dentro al parco, la sua risoluzione affidata alle modalità con cui i progetti avrebbero definito le relazioni fra le parti e il loro disegno.

$\mathrm{Ne}$ conseguiva la tematizzazione degli spazi: in un parco monumentale a contatto con il mausoleo e il parco esistente delle Rimembranze, realizzato negli anni Venti a ricordo dei Caduti durante la I Guerra Mondiale, in un parco agricolo verso la campagna, in spazi-filtro contro la ferrovia e sul lato prospiciente il quartiere residenziale ad est, in un parco attrezzato al centro. Doveva inoltre essere recuperata la memoria dell'antico tracciato del Canale Badareno.

In questo modo veniva guidata la presa in considerazione delle trasformazioni avvenute nel tempo per cui il paesaggio contemporaneo restituisce la presenza del mausoleo in un teatro realizzato da azioni diverse da quella del dispiegare forza nella solitudine della necropoli gota sulla costa. Allo stesso tempo, comunque, emergeva la consapevolezza che: "Il vero problema è posto (...) dal vasto pubblico degli individui per i quali la visita dei monumenti non è un fine in sé, per coloro che individualmente, attendono dal patrimonio storico qualcosa di diverso dalla distrazione, si aspettano una iniziazione alla felicità del sapere storico e dei piaceri dell'arte"3.

I progetti del concorso muovono - costituisce un'eccezione la proposta del gruppo, risultato vincitore, dell'Arch. Boris Podrecca - dall'attribuire allo spazio attuale una carenza di capacità evocativa dell'ambiente supposto coevo al mausoleo. Più genericamente il paesaggio è apparso tacere il racconto delle pinete e delle dune proprio del territorio di Ravenna, troppo solcato dalla linea ferrovia-

\footnotetext{
${ }^{2}$ Piano di Settore del verde urbano - Piano Regolatore Generale 1993.

${ }^{3}$ Françoise Choay, Lallegoria del patrimonio, in ERnesto D’Alfonso e Ilaria Valente (a cura di), Officina Edizioni, Roma 1995, pag. 154.
} 

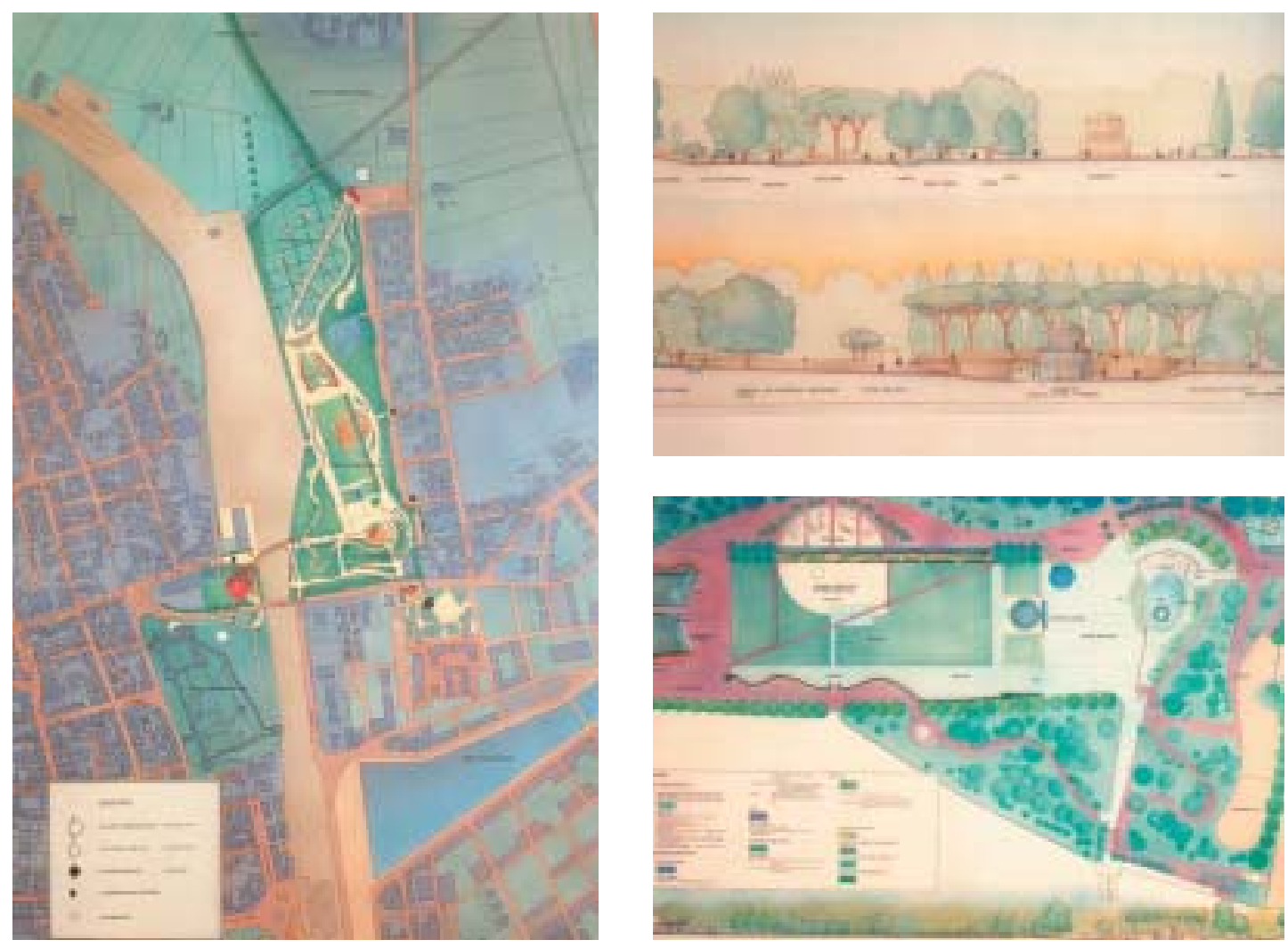

Figura 4 - Elaborati progettuali del gruppo del Prof. Arch. Nigro.

ria e chiuso contro il quartiere residenziale sul lato est, a spogliare l'arte del proprio contesto, considerato fondamentale per la piena ricezione del suo valore.

L'inserzione di elementi dal passato avviene con modalità differenti.

Il progetto del gruppo del Prof. Arch. Nigro ripropone l'ecosistema litoraneo, la presenza della duna, le formazioni vegetali del Pinus, in un sistema le cui componenti si dispongono con segni incisi: accentuano la definizione della geometria agricola, allontanano dietro il bosco la lottizzazione recente, si densificano al centro: lo spazio con le attrezzature sovraimpone forme eterogenee identificative di concetti. Significano acqua, gioco, parterre, sedute, collina panoramica - che ricopre bar e servizi - canale, piazza del mausoleo. La sintassi è fornita da un percorso in conglomerato, fascia definita nei margini dal canale del parco agricolo e dai boschi. La connessione rimane labile e lascia che all'interno gli oggetti restino singoli. Il rapporto con la storia consiste nella reinterpretazione delle idee, presentate mediante un'iconografia da essa autonoma, in cui l'arte è affiancata da istanze che assumono quasi valore paritetico.

La duna che si stende lunga, nel progetto del gruppo del Prof. Arch. Melograni, collegando una pineta a nord e la pineta prevista attorno al mausoleo, ricostruisce un paesaggio costiero assente: "il disegno generale rievoca alcuni caratteri della situazione ambientale quale si suppone che fosse quando il mausoleo fu costruito: una laguna dove i fiumi che vi confluivano scendevano fra cordoni litorali" ${ }^{\prime}$. Inoltre si attinge alle forme in senso analogico, non filologicamente, in maniera che possano dire mare, porto...: "Lungo la ferrovia corre un percorso pedonale e ciclabile sopraelevato da cui, come moli, si diramano raccordi a pettine. Ai piedi del percorso, i moli definiscono una serie di campi attrezzati" ${ }^{\prime}$. La sensazione di ambiente vasto viene ricostruita, sembra, lasciando 

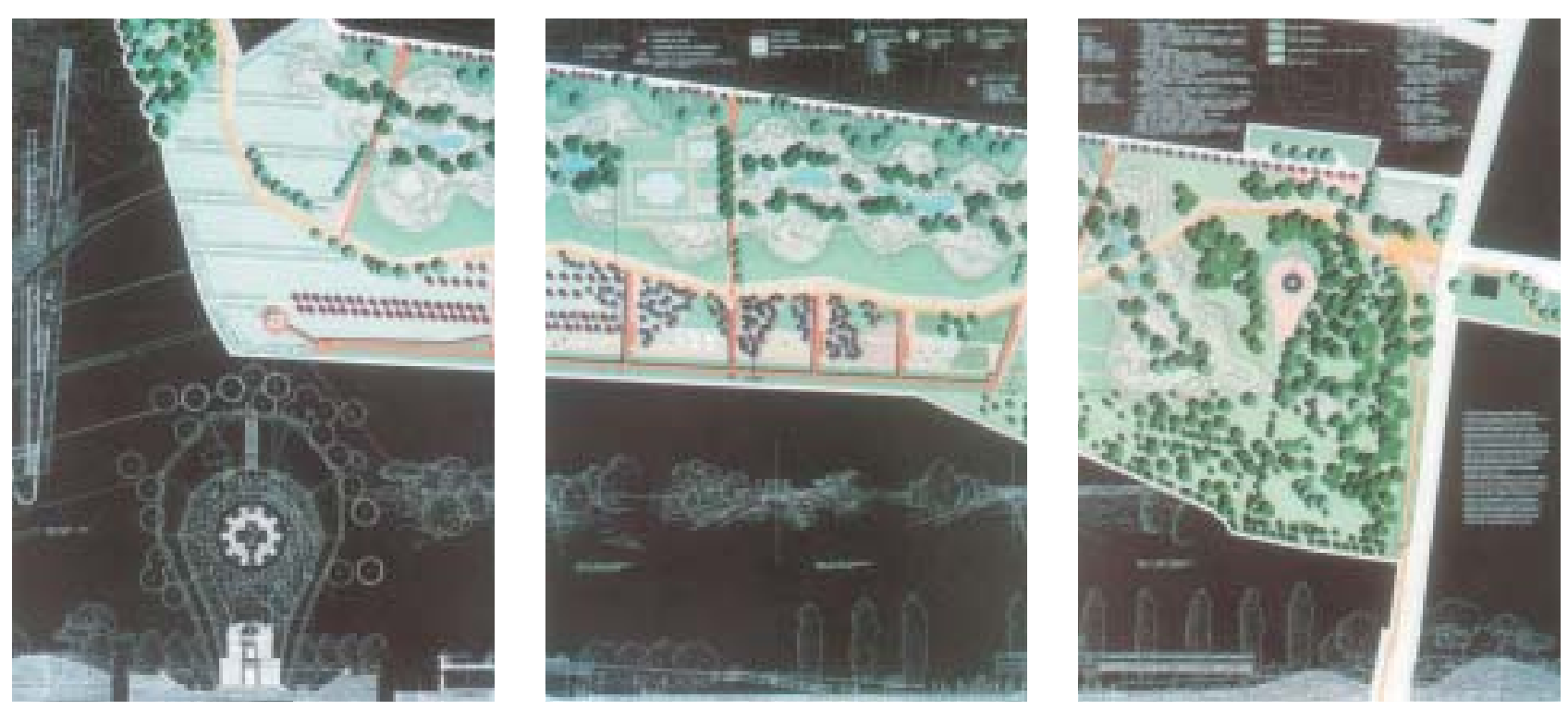

Figura 5 - Elaborati progettuali del gruppo del Prof. Arch. Melograni.

che da ogni parte la cosa desiderata sia tenuta distante: immaginando di percorrere il sentiero che muove dall'ingresso nord fino al mausoleo questo appare lontano e nascosto recuperando un'idea, forse, di solitudine. La sacralità del monumento è potenziata nel lasciarlo indisturbato. Il quartiere residenziale è separato, mediante le dune, dai campi "dei bambini", "dell'incontro", "degli anziani", "della didattica", "del pic-nic". La soluzione vuole probabilmente conservare il silenzio anche nella moltiplicazione delle attività. Le cose appaiono però più isolate che aggregate da un desiderio fatto attendere.

L'ambiente della duna compare anche nel progetto del gruppo dell'Arch. Rosini inserita nel disegno in continuità con le partizioni agricole della campagna adiacente. All'interno, quindi, di binari appartenenti ad una geometria estranea alla sua genesi naturale. L'operazione è disinvolta. Così è dichiarato che alla memoria si ricorre al servizio della composizione, la quale riesce ad essere nitida e sottile allo stesso tempo. Traccia una maglia regolare in cui il mausoleo è l'unico elemento puntiforme. Ogni partitura del tessuto, identica alle altre per ampiezza, è lavorata con materiale diverso - dune, bosco, pioppeto, acqua, piani inclinati ... - in funzione della variazione di ruolo e della creazione di prospettive calibrate: "L'asse del parco è costituito da un lungo percorso arcuato pedonale ciclabile che lo attraversa tutto in senso diagonale (...). Da questo asse si snodano i sentieri di penetrazione nel parco e lungo di esso si succedono i punti di vista e i cannocchiali ottici che permettono al visitatore di osservare il Mausoleo di Teodorico con rappresentazioni, alcune attuali, altre ormai perdute ..."6.

Il gruppo del Prof. Arch. Tintori intende "offrire una sezione storica del territorio, della sua struttura e antropizzazione in modo da accendere nell'utente-fruitore il ricordo, la memoria, l'identità che lo fanno diventare soggetto (...), allontanare - metaforicamente e anche concretamente - il contesto alieno che assedia il mausoleo (...), generare habitat vegetazionali intesi per ridare spazio a elementi e organismi - la vegetazione tardo-antica, l'inselvaticamento alto-medievale, l'apparire di un'agricoltura intensiva agli albori del nostro millennio" 7 . Il disegno fa apparire due fasce a bosco che delimitano prima un impianto agricolo, poi una piana vuota che diviene un bacino d'acqua, vicino al mausoleo, giungendo dal

\footnotetext{
${ }^{6}$ Dalla relazione di progetto.

${ }^{7}$ Dalla relazione di progetto.
} 

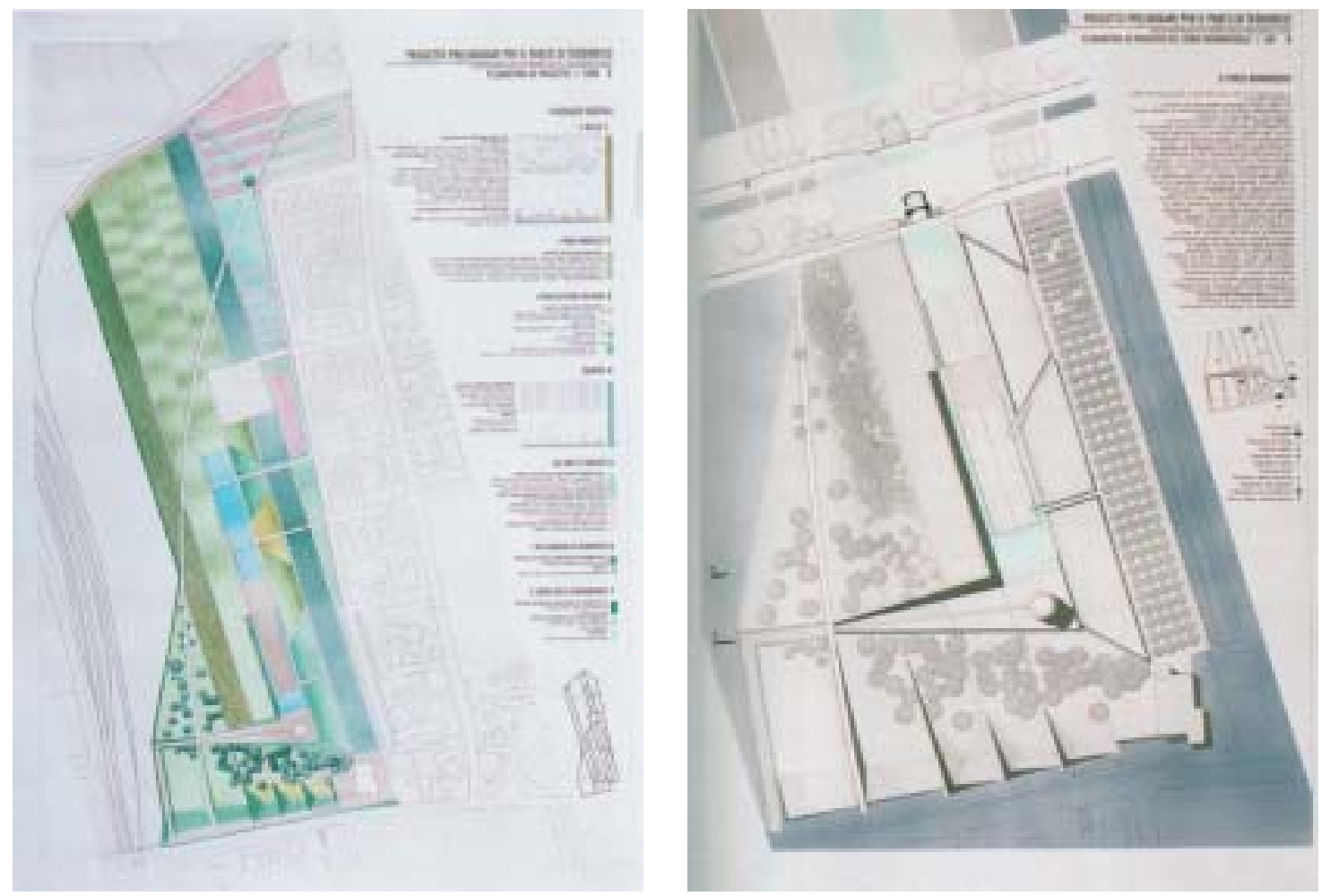

Figura 6 - Concorso 1996 - elaborati progettuali del gruppo dell'Arch. Rosini.
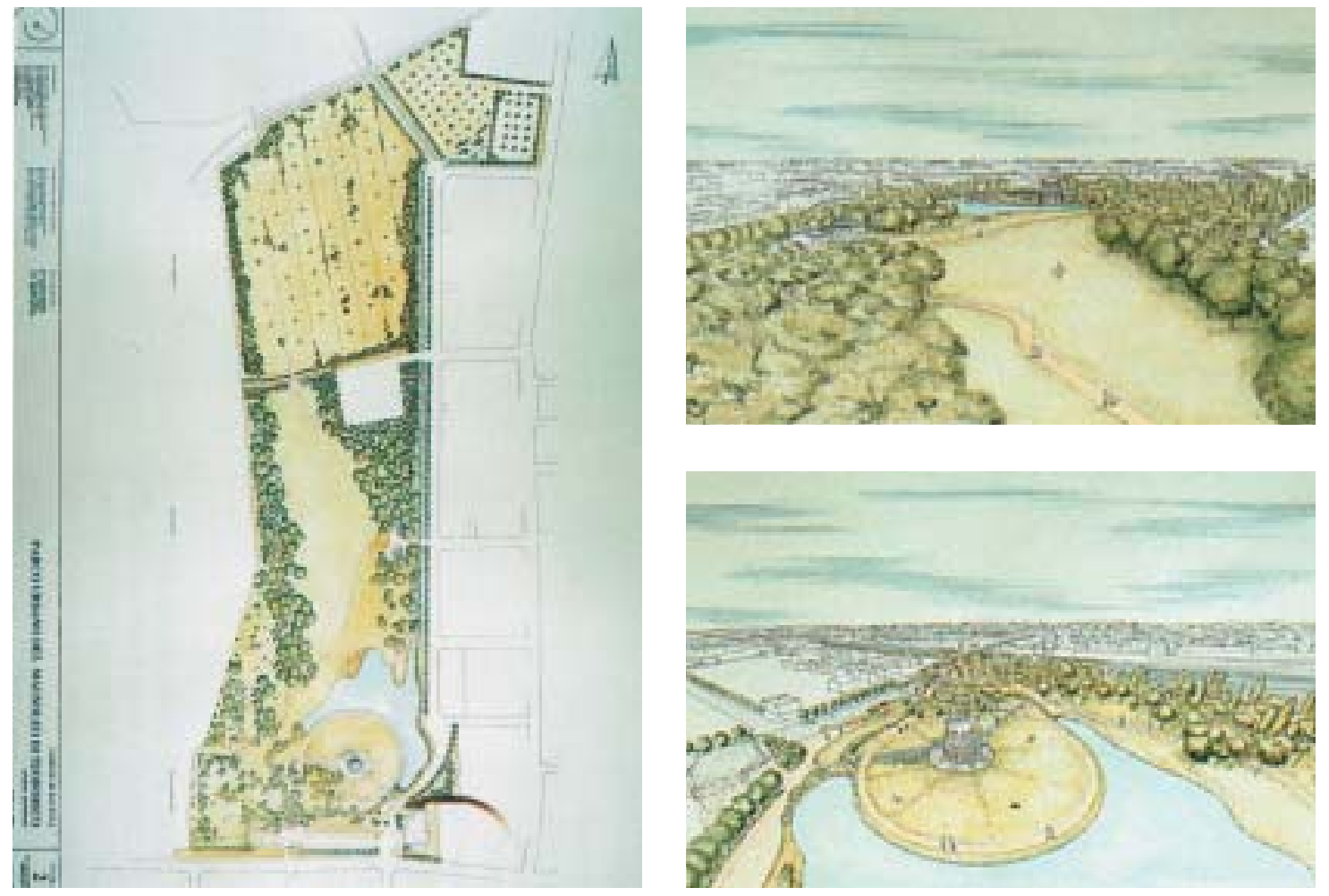

Figura 7 - Concorso 1996 - elaborati progettuali del gruppo del Prof. Arch. Tintori. 
recuperato corso del fiume Badareno. "L'avvicinamento al mausoleo avviene lungo un tracciato che lo inviluppa come in un ornato tardo-antico ...”.

Il progetto del gruppo del Prof. Ing. Ghio presenta punti di similitudine con la proposta di Melograni. La storia struttura la planimetria nel riproporre, muovendo da ovest verso est, il bosco, la pianura e l'ambiente delle dune - sono onde di sabbia, cemento bianco e "erbe azzurrine". Quest'ultimo diviene a sua volta luogo in cui esporre la storia delle trasformazioni subite dal territorio nel tempo.

Il riferimento è di secondo grado e lo spazio si configura come citazione. Un'altra citazione è concepire, presso il mausoleo, "segni da realizzare con erbe e pietre, evocanti opere scomparse perché demolite o perché inghiottite". La trasformazione del materiale culturale in elementi di disegno avviene per mezzo di un tratto elegante. Sull'aspetto di "naturalità" diffuso degli impianti boschivi ad isolare dalle presenze del paesaggio contemporaneo - lottizzazione e area ferroviaria - si sovrappongono pochi segni geometrici che esplicitano l'artificialità dell'azione. Sono il quadrato che stabilisce le pertinenze del mausoleo, i percorsi paralleli interni al parco monumentale esistente, i terrapieni tagliati come bastioni - quello centrale proteso, con i pini affacciati, come molo verso la tomba del re goto.

Il progetto dell'arch. Podrecca e di Teprin-Associati esplicita la necessità di non inserire nuovi elementi di discorso ma di realizzare "Non un parco nella tradizione ricca e meditata della nostra cultura mitteleuropea, non un Parco che ricorre al linguaggio di una nostalgia al confine del romantico e non un Parco impregnato dall'idea di un'architettura più concettuale che reale. Semplicemente un parco che non si impone, ma che mette nella giusta relazione quello che c'è, prendendo in prestito quello che il contesto offre: l'apertura della campagna, le prospettive del monumento, le geometrie dell'edificato, il disegno del suolo ed il movimento della terra. Tutto ciò senza chiudersi al proprio interno, senza entrare nell'inutile competizione con il mausoleo e senza cadere nella tentazione di riempire laddove lo spazio aperto è la massima espressione di qualità dei nostri tempi pieni più che mai". 8
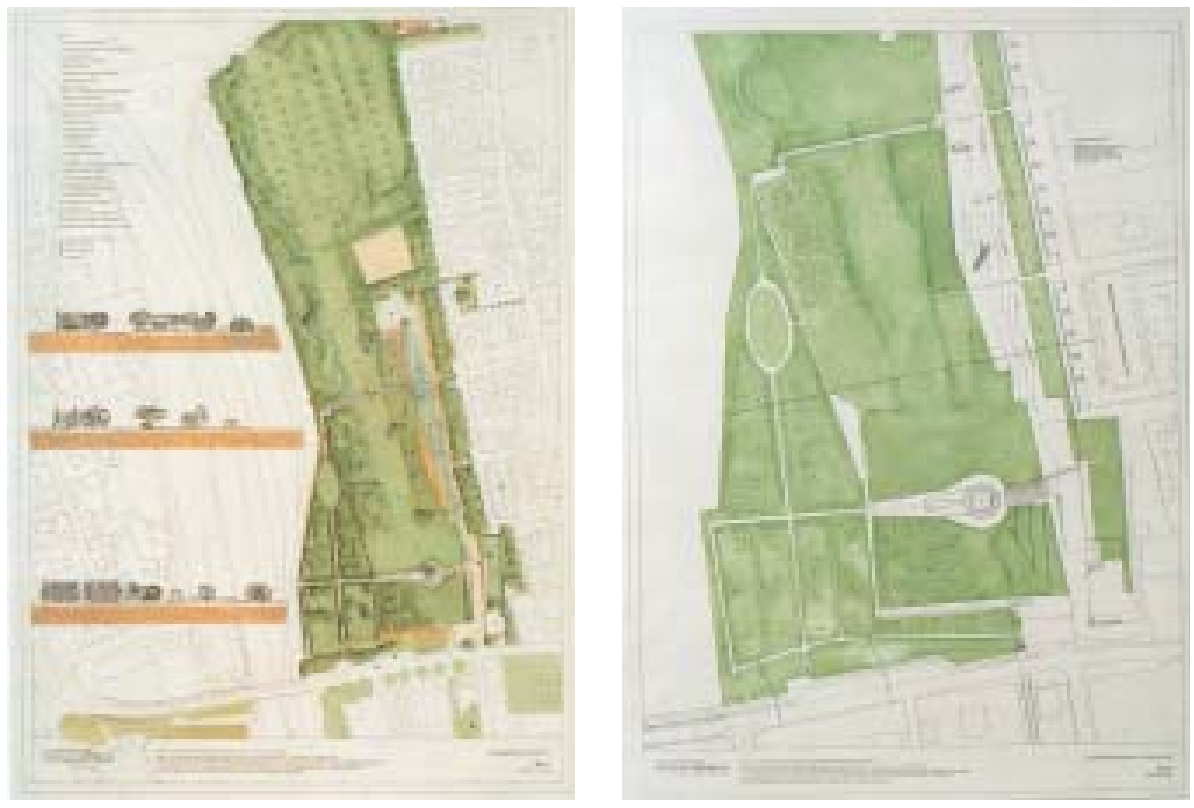

Figura 8 - Concorso 1996 - elaborati progettuali del gruppo del Prof. Arch. Ghio.

${ }^{8}$ Andreas KIPAR, Il parco nel paesaggio, in Parco Teodorico - I sei progetti del Concorso, Danilo Montanari Editore, Ravenna 1998, pag. 7. 


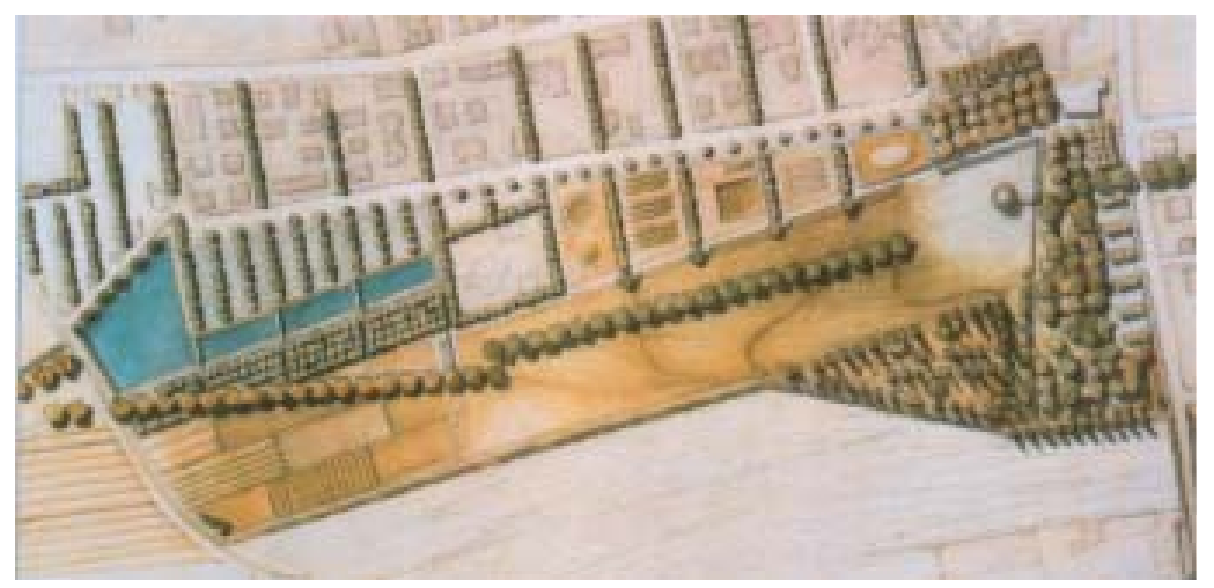

Figura 9 - Concorso 1996 - elaborati progettuali del gruppo del Prof. Arch. Podrecca.

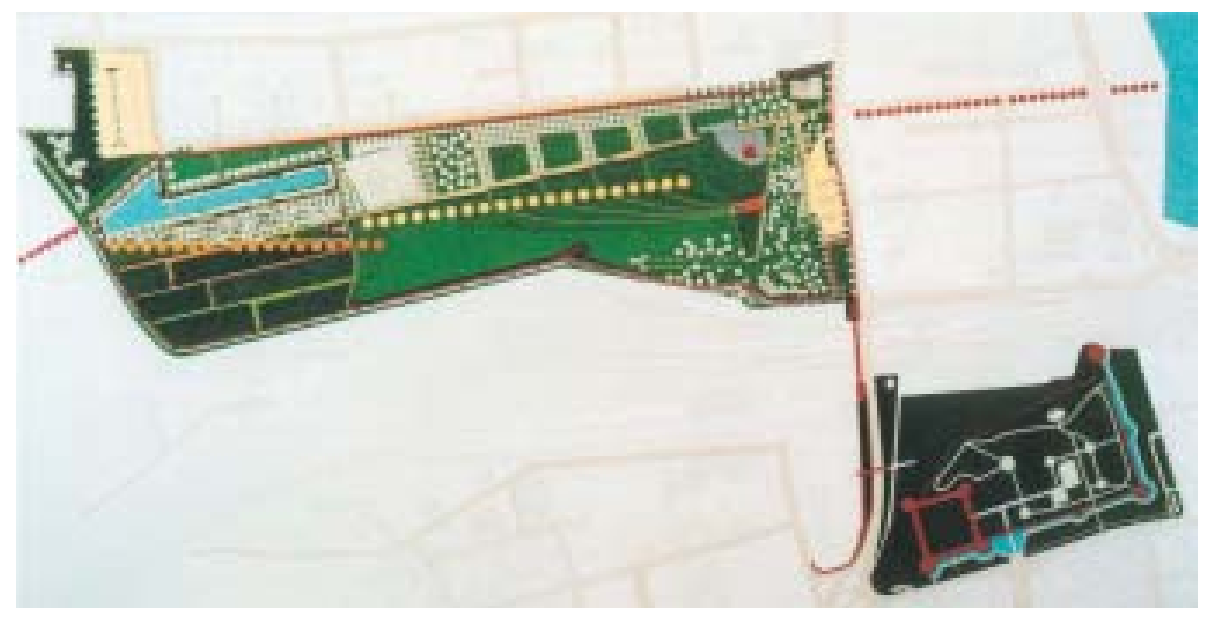

Figura 10 - Prof. Arch. Podrecca - Teprin Associati: progetto definitivo-esecutivo - planimetria generale.

Il rapporto fra i diversi temi/spazi avviene con un meccanismo abile, come un rimbalzo, ovvero tramite la relazione significativa che intercorre fra essi e il mausoleo - in questo modo l'unico a raccontare la storia antica - il quale assume funzione di medio proporzionale nel sistema percettivo: i confini fra le parti eterogenee vengono fatti coincidere con punti e percorsi da cui si ha un prospettiva particolare del monumento.

\section{GLI ESITI}

Arrivando dalla strada statale Romea, si accede al parco attraversando su un ponte di legno un macero, progettato seguendo il tracciato del vecchio canale Badareno. Insieme ad un filare di farnie e ad una serie di orti agricoli intende costituire una continuità, nel momento d'ingresso da Nord, con il paesaggio rurale. In corrispondenza di un nuovo fabbricato a servizi si incrociano l'asse del parco agricolo con l'asse monumentale costituito di un filare di Pinus pinea riferimento esplicito, ma minimo, alle pinete del territorio ravennate. In questo punto origina la prospettiva "monumentale" verso il mausoleo, accompagnata dal digradare lieve del terreno fino alla quota del basamento del mausoleo: "prospettiva lontana, tranquilla, leggermente monumentale, che ci fa rivedere in tutta la sua interezza l'architettura del monolite"'. 

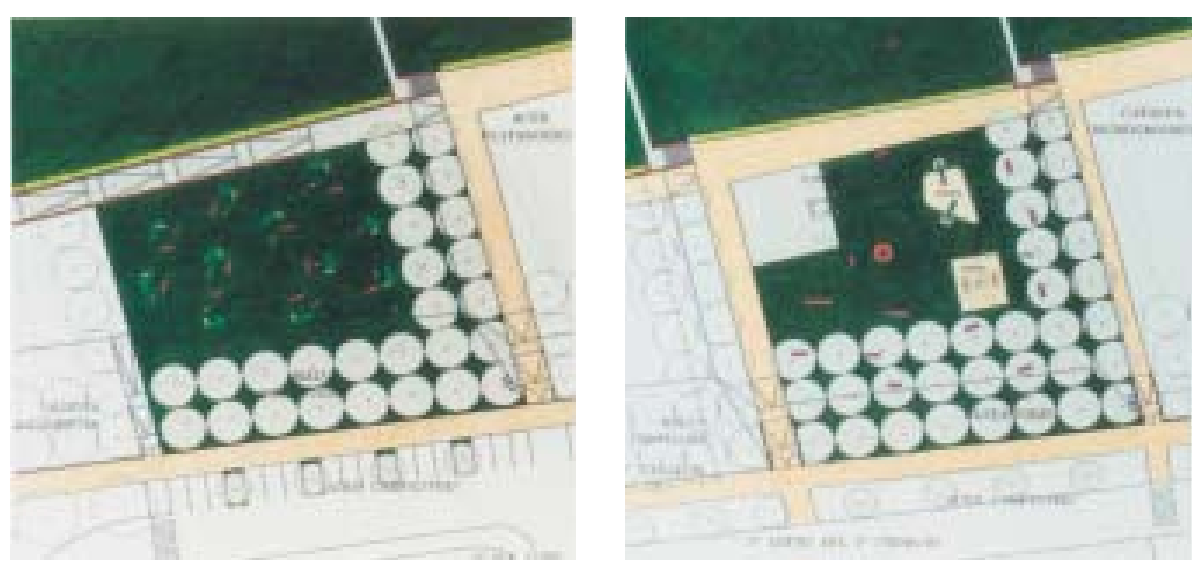

Figura 11 - Progetto definitivo-esecutivo - Il giardino dei colori e Il giardino dei giochi: planimetria.

Dall'edificio dei servizi il percorso prosegue ad est lungo orti conclusi tematici, per il riposo e il gioco, che si organizzano secondo la struttura del quartiere residenziale adiacente integrandolo nelle sue funzioni. Gli orti conclusi si sono caratterizzati in fase di progettazione esecutiva come: "Il giardino delle ombre", semplicemente un bosco," il giardino dei fuochi" in cui muri alti 2,70 metri e lunghi 4 definiranno l'area in cui sarà possibile cucinare e mangiare, "il giardino del vento", spazio per far volare gli aquiloni, giocare con lo skate-board...., "il giardino dei giochi" dedicato ai bambini, "il giardino dei colori", con piccoli monoliti in cemento armato e rivestiti a mosaico, "la gradinata degli spettacoli".

Da ogni orto concluso è possibile scendere nel prato del sistema monumentale mediante scale da cui nascono sentieri in pietra che definiscono le gradinate. Questo movimento in sezione, dato dai diversi livelli del terreno e dei percorsi, struttura il passaggio dallo spazio consueto degli orti conclusi allo spazio d'eccezione in cui si ammira la tomba di Teodorico. La prospettiva monumentale ha come linee guida il filare di pini e il percorso pedonale, a quota più alta, che fiancheggiando gli orti conclusi avvicina al mausoleo.

A ovest, dal parco agricolo e abbandonando il cono visuale della prospettiva monumentale, si allontana un pista ciclabile, proseguendo lungo il confine dell'area ferroviaria che assume il volume di un terrapieno, segnato da una siepe mista di Crataegus monogyna, Ligustrum sinensis, Cornus sanguinea, Euonymus europeans. Percorrendolo ci si sposta su un crinale di margine, ai piedi del quale esiste solo la pianura semplice a prato e, in lontananza, il mausoleo: prospettiva "più sfumata e lontana". Il passaggio fra il carattere remoto del percorso lungo la ferrovia e quello storico del Parco delle Rimembranze e del mausoleo a sud avviene lungo il viale, già esistente, di accesso al mausoleo, in cui si apre la prospettiva "domestica", "piano piano (...) riportando agli occhi degli spettatori, lucidamente incorniciato, il bianco della pietra aurisina"10.

Esiste poi un modo diretto di relazionarsi, appena si entra dall'ingresso a sud, dalla zona del Centro informazioni, con il mausoleo. Il progetto prevede un breve percorso nascosto fra gli alberi fino ad una scala in discesa che proietta inaspettatamente - è la prospettiva "improvvisa" - davanti al mausoleo, alla quota originaria del suo basamento: l'area circostante il monumento viene resa libera da due muri ad angolo, rivestiti in bronzo, inclinati come pareti di un bastione, che tagliano con segno netto il terreno, confinano il bosco e contengono i percorsi di collegamento fra la quota attuale e quella prevista.

Attualmente è stato realizzato il primo stralcio del progetto, quello che ne definisce la struttura generale (e non coinvolge l'area strettamente a contatto con

${ }^{10}$ Dalla relazione di progetto del Concorso. 


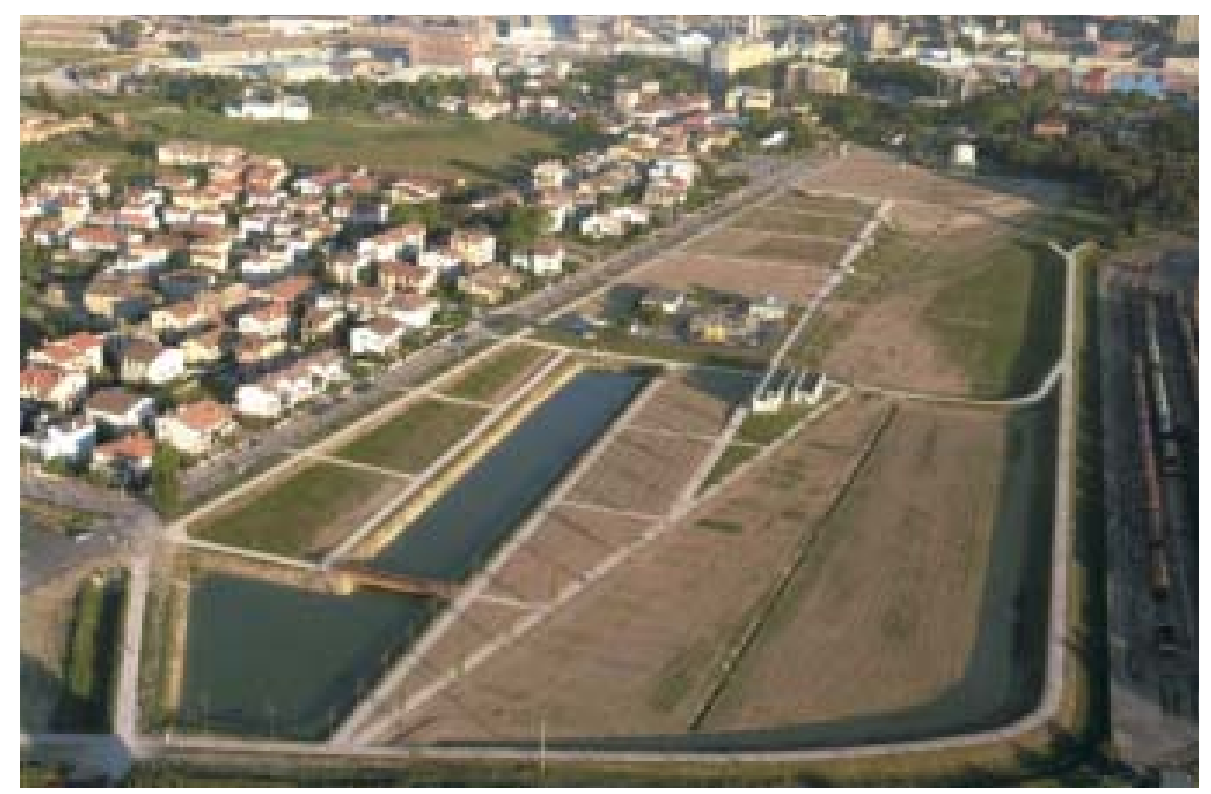

Figura 12 - Il Parco di Teodorico (a realizzazione ultimata del primo stralcio).

il mausoleo): ne risulta un luogo umile, che assume aspetti quotidiani - nel disegno degli orti conclusi, nella tranquillità della ciclabile di crinale lungo la ferrovia, nella semplicità dei materiali dei percorsi, ghiaia e pietra di Luserna - e capace di silenzio nel realizzare una conca libera, costituita solo di sentieri che, nel prato, attraversano la linea dei Pinus Pinea deviando, con forma curva, dalla prospettiva monumentale. Sembra che l'unico loro fine sia quello di invitare a passeggiare, con la calma del tempo a disposizione. Le attività avvengono tutte negli orti conclusi: spazi che si pensano brulicanti, affacciati su un vuoto che accoglie la densità dei significati della presenza dell'arte.

\section{RIFERIMENTI BIBLIOGRAFICI}

Amministrazione Comunale di Ravenna (a cura di), Parco Teodorico, dal progetto all'attuazione, Danilo Montanari Editore, Ravenna 1998.

Antoniacci Raffaella, Sulla via dei Romei - Il Parco di Teodorico a Ravenna, "Paesaggio Urbano", 4, Maggioli Editore, Perugia 1999, pagg. 28-35.

Baldiserri Claudio, Prospettiva sul mausoleo, "Folia", supplemento ad "Acer",4, Il Verde Editoriale, Milano 2001, pagg. 8-9.

ChoAy Françoise, L'allegoria del patrimonio, a cura di Ernesto d'Alfonso e Ilaria Valente, Officina Edizioni, Roma 1995.

Mercatali Vidmer, Kipar Andreas, Stringa Franco e Proni Francesca, Parco Teodorico, i sei progetti di Concorso, Danilo Montanari Editore, Ravenna 1998.

Toesca Pietro, Storia dell'Arte Italiana I, Il Medioevo, vol. I, Unione Tipografico-Editrice Torinese, Torino 1965.

\section{RIFERIMENTI ICONOGRAFICI}

Figure 1, 12: rielaborazione di Enrica Dall'Ara di foto Biserni (RA), per gentile concessione del Comune di Ravenna.

Figura 2: rielaborazione di Enrica Dall'Ara di immagine di copertina in MERCATALI Vidmer, Kipar Andreas, Stringa Franco e Proni Francesca, Parco Teodorico, i sei progetti di Concorso, Danilo Montanari Editore, Ravenna 1998.

Figura 3: rielaborazione di Enrica Dall'Ara di foto Biserni (RA) in "Folia", 1, Speciale Ravenna, Il Verde Editoriale, Milano 1998, pag. 48.

Figure 4, 5, 6, 7, 8, 9, 10, 11: Kipar Andreas, Stringa Franco e Proni Francesca, Parco Teodorico, i sei progetti di Concorso, Danilo Montanari Editore, Ravenna 1998. 
SCHEDA DI PROGETTO

Tipo di intervento:

Committenti:

Iter del progetto:

Progetto vincitore del concorso:

Consulenti:

\section{Collaboratori:}

Progetto preliminare adeguato:

Consulenti:

Collaboratori:

Progetto definitivo-esecutivo:

Consulenti:

\section{Collaboratori:}

Direzione dei lavori del I stralcio:

Coordinamento Comune di Ravenna:

Superficie complessiva:

Superficie $1^{\circ}$ stralcio:

Superficie $2^{\circ}$ stralcio: progettazione di parco urbano

comune di Ravenna

bando di concorso ad curricula: luglio 1996

progetto preliminare: gennaio/febbraio 1997

nomina vincitore: marzo 1997

progetto preliminare adeguato: luglio 1997

progetto definitivo esecutivo: febbraio 1998

appalto: giugno 1998

Arch. Boris Podrecca (capogruppo)

Teprin Associati*: Arch. Aldo Aymonino*, Arch. Claudio Baldisserri*, Ing. Lorenzo Sarti*, Arch. Emilio Rambelli*

Dott.ssa Alessandra Carretta

Prof. Ferdinando Rebecchi (consulente storico archeologico), Dott. Edoardo Vaccari (consulente per la progettazione e gestione del verde), Prof. Ing. Sandro Artina (consulente idraulico), Dott. Massimiliano Costa (consulente naturalista) Arch. Roberto Eleuteri, Arch. Irwin Miller

Arch. Boris Podrecca (capogruppo)

Teprin Associati*: Arch. Aldo Aymonino*, Arch. Claudio Baldisserri*, Ing. Lorenzo Sarti*, Arch. Emilio Rambelli*

Dott.ssa Alessandra Carretta

Prof. Ing. Sandro Artina (consulente idraulico), Dott. Massimiliano Costa (consulente naturalista)

Ing. Gianluca Bonini

Arch. Boris Podrecca (capogruppo)

Teprin Associati*: Arch. Aldo Aymonino*, Arch. Claudio Baldisserri*, Ing. Gianluca Bonini*, Arch. Francesco Muti*, Ing. Lorenzo Sarti*, Arch. Emilio Rambelli*, Dott.ssa Alessandra Carretta

per le strutture - Studio Lenzi: Ing. Francesco Ricci, Ing. Mario de Lorenzi, Ing. Sergio Marchetti

per la sistemazione idraulica: Prof. Ing. Sandro Artina

per la sistemazione geotecnica - Studio EN SER: Prof. Ing. Maurizio Merli, Prof. Ing. Gianfranco Marchi, Ing. Andrea Beccati

Ing. Paola Campana

Impianti elettrici: Ing. Franco Errani

Impianti termosanitari: Ing. Gianni Minori

Rilievi topografici: Geom. Leo Brugnara

Prove geotecniche: Dott. Angelo Angeli

Dott. Messino Generini, Luigi Agi, Arch. Marco Bottoni, Giuseppe Catania, Silvio D'Amore, Alessandra De Gregorio, Arch. Roberto Eleuteri, Arch. Adriana Feo, Arch. Irwin Miller, Geom. Erik Sassi

Ing. Lorenzo Sarti di Teprin Associati

Arch. Franco Stringa

$140.000 \mathrm{mq}$

$120.500 \mathrm{mq}$

$15.000 \mathrm{mq}$ 


\section{SEGUE SCHEDA}

Costo complessivo:

Costo $1^{\circ}$ stralcio:

Costo $2^{\circ}$ stralcio:

Tempi di realizzazione:

Finanziamenti:

Specie arboree impiegate:

Orti conclusi:
10 miliardi e 170 milioni di lire

6 miliardi e 470 milioni di lire

3 miliardi e 700 milioni di lire

$1^{\circ}$ stralcio: ottobre 1999-dicembre 2000

$2^{\circ}$ stralcio: dicembre 2002

Fondi per il Giubileo: $€ 1.700 .000,00$

P.R.U.: $€ 1.300 .000,00$

Comune di Ravenna: $€ 242.000,00$

Programma speciale d'area per il porto: $€ 1.400 .000,00$

filare dell'asse monumentale: Pinus pinea

filare del Parco agricolo: Quercus robur

integrazioni del Parco Monumentale esistente in corrispondenza del Mausoleo: Quercus ilex, Pinus pinea, Gynko biloba, Tilia platiphillos, Cupressus sempervirens.

siepe lungo il percorso adiacente la ferrovia - in forma libera: Viburnum tinus, Ligustrum vulgare, Pittosporo tobira; tratto in quota: Crataegus monogyna, Ligustrum sinensis, Cornus sanguinea, Euonymus europeans Ripristino viale esistente del Cippo del Caduti: Cupressus sempervirens

bosco a sesto d'impianto regolare: Albizzia julibrissin, Robinia pseudoacacia umbraculifera, Catalpa bignioides

perimetro idrovora: Quercus ilex, Laurus nobilis

siepe del percorso pedonale lungo gli orti - formale: Buxus sempervirens. 



\title{
DA FABBRICA A SPAZIO URBANO: GLI SPAZI ESTERNI DELLO STABILIMENTO FIAT LINGOTTO, TORINO
}

\author{
Claudia Cassatella
}

\section{IL CONTESTO}

I cambiamenti dei sistemi produttivi e la crisi del settore industriale che ha colpito molte città europee all'inizio degli anni Ottanta hanno reso obsoleti molti stabilimenti industriali, cosicchè il problema del loro riuso con destinazione a funzioni diverse ha spesso significato il cambiamento del volto di intere parti di città. Il caso dello stabilimento FIAT Lingotto a Torino è fra questi, ed ha costituito una palestra per il dibattito sulle aree dismesse, sull'archeologia industriale, sul marketing urbano. Una vastissima area recintata ed impenetrabile è diventata un pezzo di città, e, senza sensibili trasformazioni volumetriche, gli stessi spazi un tempo usati come piazzali per le automobili sono diventati spazio pubblico urbano.

\section{Il Lingotto: origini ed architettura}

Dopo la prima guerra mondiale la FIAT decise la costruzione di un nuovo grande stabilimento, che ebbe subito il primato europeo per la produzione di automobili di serie e per l'integrazione del ciclo di produzione. Il progetto è dell'Ing. Giacomo Matté Trucco, ufficio progetti FIAT (già progettista dello stabilimento FIAT Grandi Motori), e la costruzione avvenne tra il 1915 ed il 1923. Grande opera di ingegneria, l'edificio è lungo 500 metri e largo 80, ha 29.000 metri quadrati coperti per 5 piani, ed è basato su di un'anticipatrice struttura in cemento armato, prefabbricata e modulare (pilastri-travi-solaio), che ne connota l'architettura. All'e-

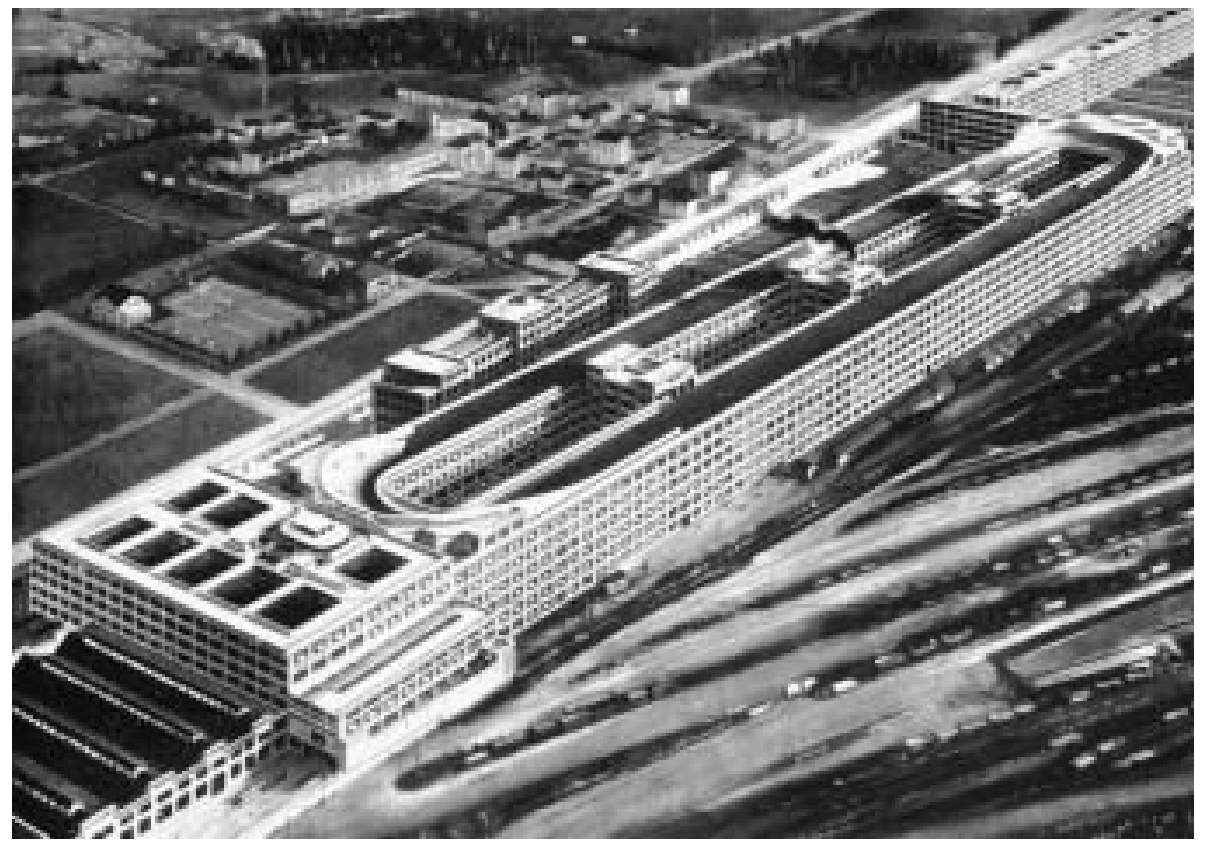

Figura 1 - Veduta delle officine Fiat appena costruite (acquerello di L. Francesetti, 1929). Lo stabilimento sorgeva fuori città; è ben visibile l'ampio campo binari. 
poca il progetto fu ampiamente pubblicato e commentato come esempio razionalista, ricevendo gli elogi di Persico e Le Corbusier.

La catena di montaggio (sistema Taylor) si sviluppava in senso verticale, culminando nella fase del collaudo in una pista sul tetto della lunghezza di un chilometro, che fu oggetto di grande ammirazione. Due torri costitute da rampe elicoidali furono aggiunte in seguito per permettere l'accesso diretto dal piazzale alla pista sul tetto.

Lo stabilimento sorgeva fuori città, collegato alla ferrovia e dotato di un ampio campo binari; il quartiere Lingotto si sviluppò solo in seguito, stretto tra ferrovia e fiume, quartiere operaio e denso. Tuttora per chi arriva in treno da sud lo stabilimento si impone alla vista come un enorme segnale di ingresso alla città.

Lo stabilimento cessò la produzione alla fine degli anni Settanta, non potendo

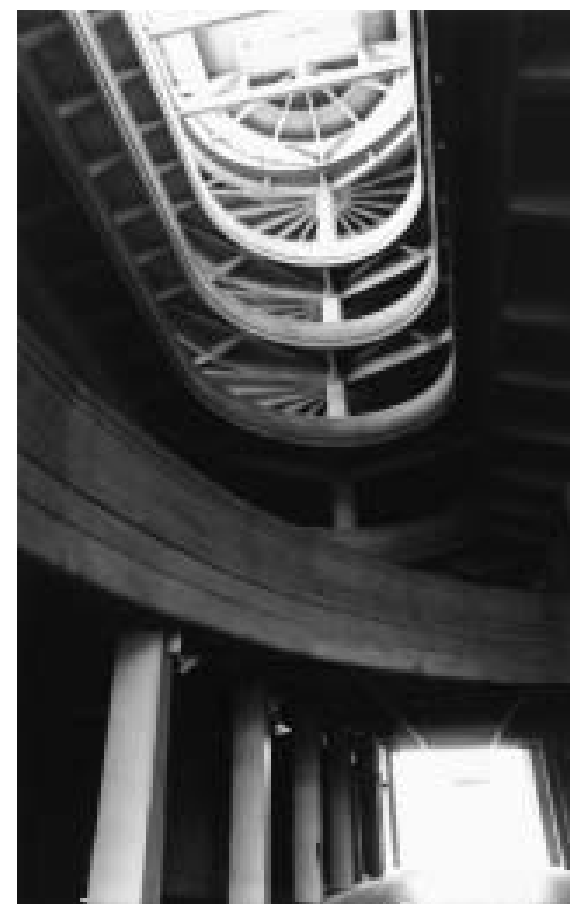

Figura 2 - La rampa elicoidale. ospitare le catene di montaggio robotizzate, ed attese decisioni sul suo utilizzo fino alla fine degli anni Ottanta. La chiusura dello stabilimento si inseriva in una fase di crisi dell' industria che per Torino, "città operaia", rappresentò anche una crisi di vocazione ed immagine: la città promosse studi sociologici ed economici per approfondire le proprie risorse umane e materiali, puntando infine su di un rilancio come città dell' innovazione scientifico-tecnologica.

\section{GLI OBIETTIVI}

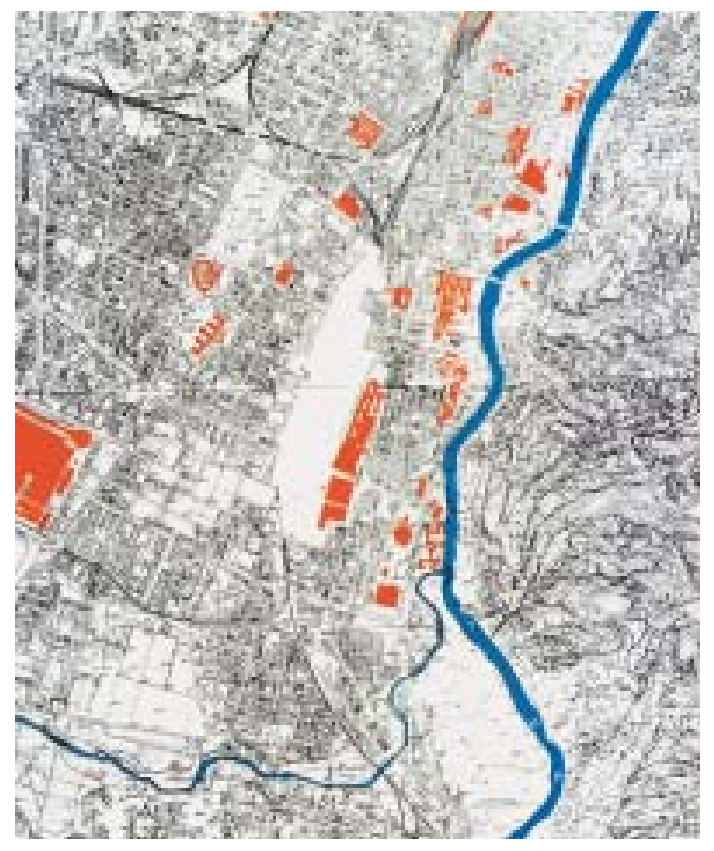

Figura 3 - Localizzazione delle attrezzature di interesse pubblico nell'area circostante il Lingotto.
Il concorso di idee per il riuso ed il progetto vincitore di Renzo Piano

"Tutti gli interventi di recupero di ex-fabbriche si scontrano con il fatto che non esiste oggi una funzione che richieda tanto spazio quanto la produzione industriale. Ma il problema del Lingotto non si fermava a questo, era a monte dell'architettura e dell'urbanistica. Prima di decidere che cosa fare dei muri, bisognava ricreare un'identità, un rapporto, un'immagine all'altezza delle aspettative della città.

Della portata del problema erano consapevoli sia le autorità pubbliche, sia la dirigenza FIAT. Perciò, fin da subito, la consultazione sulle sorti del Lingotto avvenne al massimo livello. Nel 1984 si tenne una mostra internazionale, cui erano stati chiamati a contribuire con i propri progetti venti architetti ${ }^{1}$. Tra le proposte per il riuso dell'edificio fu scelta la mia, che si basava sulla trasformazione in centro polivalente dedicato al terziario e all'innovazione: un pezzo di città, con tutte le sue complessità e sfumature.

(...) In un contesto di cambiamento profondo dell'economia cittadina occorreva reinventare non solo l'uso, ma anche

${ }^{1}$ Tra gli altri Aulenti, Gabetti \& Isola, Gregotti, Halprin, Hollein, Meier, Pelli, Pesce, Roche, Stirling (N.d.R.). 
il ruolo della ex-fabbrica; non solo la sua funzione urbana, ma anche la sua funzione simbolica": così Renzo Piano ricorda il momento del concorso ${ }^{2}$.

Le soluzioni proposte dai partecipanti colpiscono gli osservatori per il carattere generale di divertissement: la scatola modulare viene trattata quasi come un gioco di costruzioni tipo Lego, camuffata o truccata, fino a esiti parodistici. Renzo Piano riconosce invece la dignità dell'architettura di Matté Trucco, il carattere ormai identitario dell'edificio per la città, e sceglie di rispettarlo e conservarlo, pur cercando di collocare nei 796.000 metri cubi (!) tutte le diverse funzioni urbane immaginabili: imprese e servizi alle imprese, gallerie commerciali, residenze, università e laboratori, hotel, un grande centro fieristico, un centro convegni ed un auditorium dall'acustica all'avanguardia. "Il Lingotto: un pezzo di città" è lo slogan vincitore. Il progetto cerca anche di ricucire le parti di città che lo stabilimento ed il campo binari hanno separato, proponendosi come cuneo verde verso il centro città.

\section{LA METODOLOGIA}

Quando vinse il concorso per il Lingotto, Piano aveva appena terminato la ristrutturazione della fabbrica Schlunberger a Mountrouge (Francia), ma, come ammette egli stesso, essa non metteva in gioco l'immagine della città, né
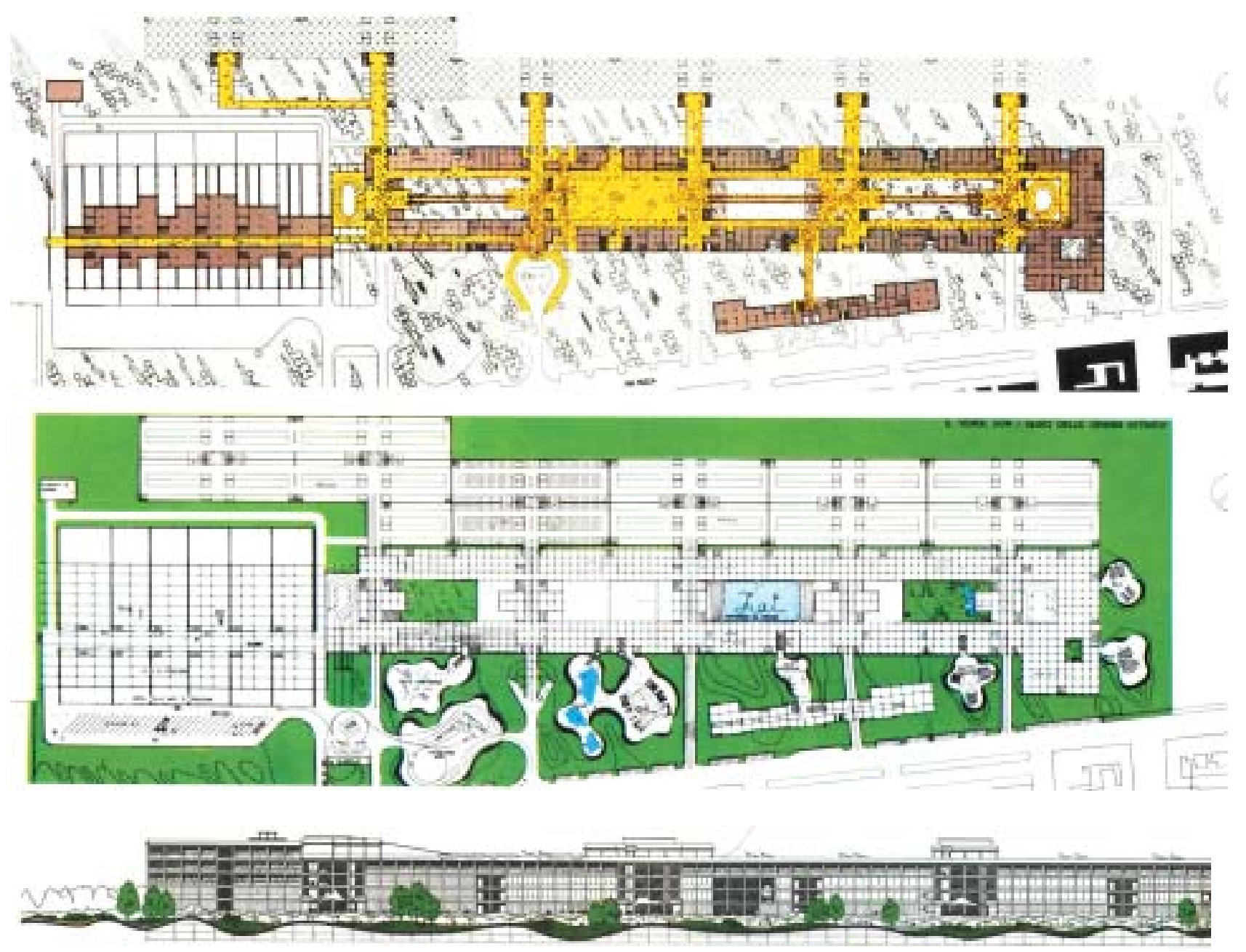

Figure 4, 5 - Renzo Piano Building Workshop, planimetria e sezioni del progetto di concorso.

2 Renzo Piano, Giornale di bordo, Passigli Editore, Firenze 1997, pag. 91. 

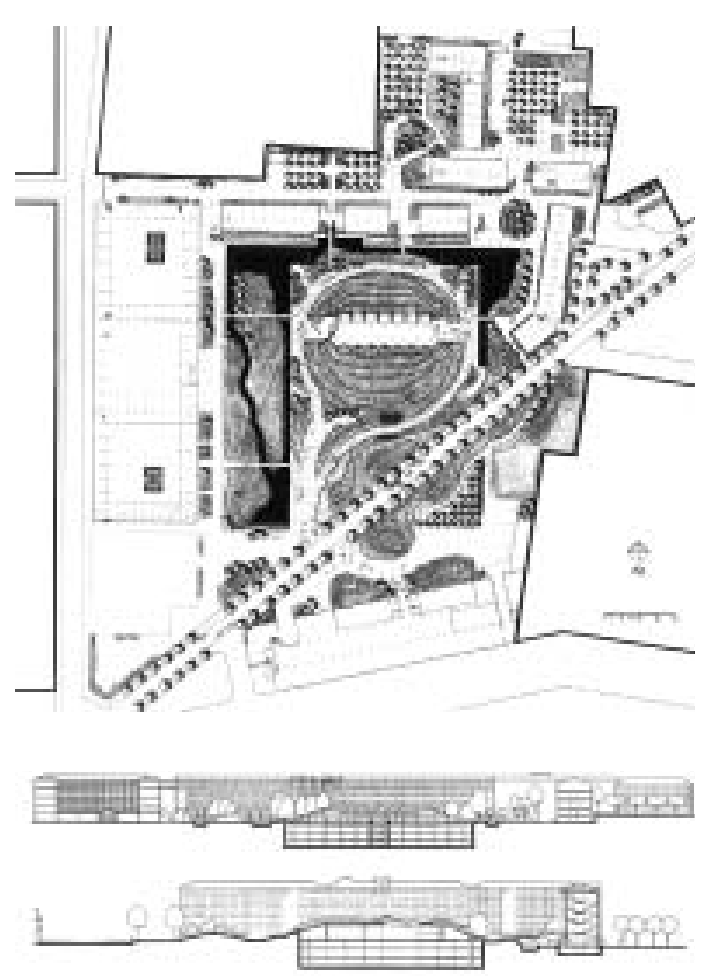

Figure 6, 7 - Renzo Piano Building Workshop, ristrutturazione Schlunberger, planimetria e sezioni. così vasti spazi pubblici: rimaneva infatti uno spazio privato. Alcune analogie possono essere trovate nella concezione del rapporto tra edificio e natura: all'hi-tech del primo si contrappone uno spazio verde movimentato da colline e arbusti, decisamente "all'inglese". Per Lingotto pensò inizialmente a qualcosa di simile: "Di fronte a questa prevalenza della tecnica, inizialmente cademmo nella tentazione di proporre, come per Schlumberger, la rivincita della natura. (...) Poi prevalse un'altra linea progettuale: la scelta di assecondare, invece di contrastare, la potenza con cui il Lingotto si impone al paesaggio urbano. Oggi di fronte all'edificio c'è un grande spiazzo, l'equivalente del sagrato delle cattedrali: un sagrato molto forte e molto minerale." ${ }^{3}$

\section{Gli spazi urbani ed il rapporto con la città}

Renzo Piano confessa: "Lingotto è stato il primo progetto in cui ho affrontato in modo organico il tema dello spazio urbano"4. Gli spazi esterni oggetto d'intervento raggiungono i 98.000 metri quadrati.

Il progetto finale abbandona l'idea dei movimenti del terreno e degli specchi d'acqua, estendendo invece la geometria dell'edificio anche agli spazi aperti, adottando una griglia di base che ricalca la maglia strutturale. La soluzione è più dura (anche nei materiali, poiché la griglia è in cemento grigio) ed economica, ma forse anche più consona al sito naturalmente pianeggiante ed alla città di Torino, la cui tradizione, prima barocca e poi neoclassica, è di spazi urbani "misurati” (dove la misura è spesso data dai portici e dalla scansione ritmica delle facciate).

\section{GLI ESITI}

Tre sono gli spazi aperti principali: il piazzale di ingresso, le corti interne, il parco pubblico sul lato ferrovia. È stato oggetto di riqualificazione anche il viale prospiciente, la via Nizza (uno degli assi di accesso alla città), dove una doppia alberatura sempreverde di magnolie maschera il fronte del quartiere popolare.

Il piazzale di accesso è un'enorme e monumentale spazio vuoto e "minerale", che subisce diverse sistemazioni in occasione di ogni fiera o manifestazione grazie ad alcuni arredi mobili - piante in vaso e dissuasori, che delimitano le aree

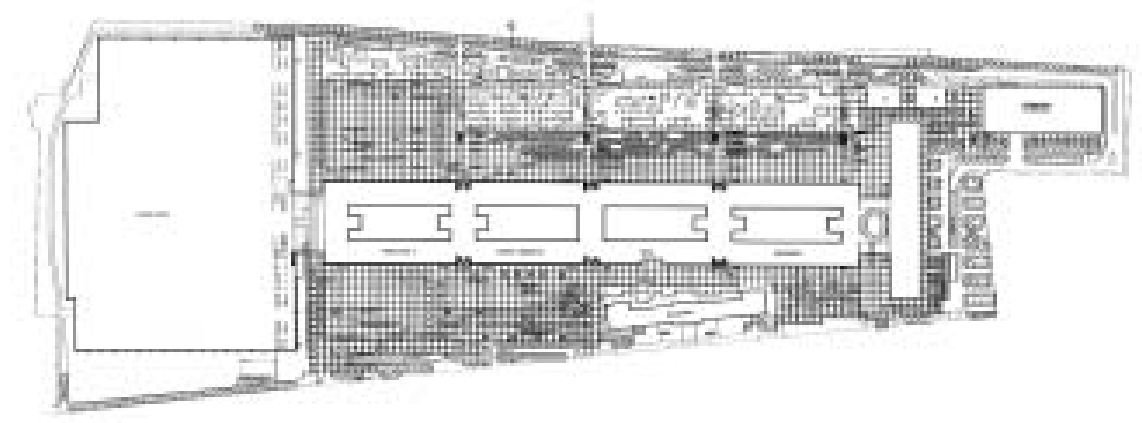

Figura 8 - Renzo Piano Building Workshop, il progetto finale per il Lingotto, planimetria.

\footnotetext{
${ }^{3}$ Renzo Piano, op. cit., 1997, pag. 92.

${ }^{4}$ RenZO Piano, op. cit., 1997, pag. 91.
} 

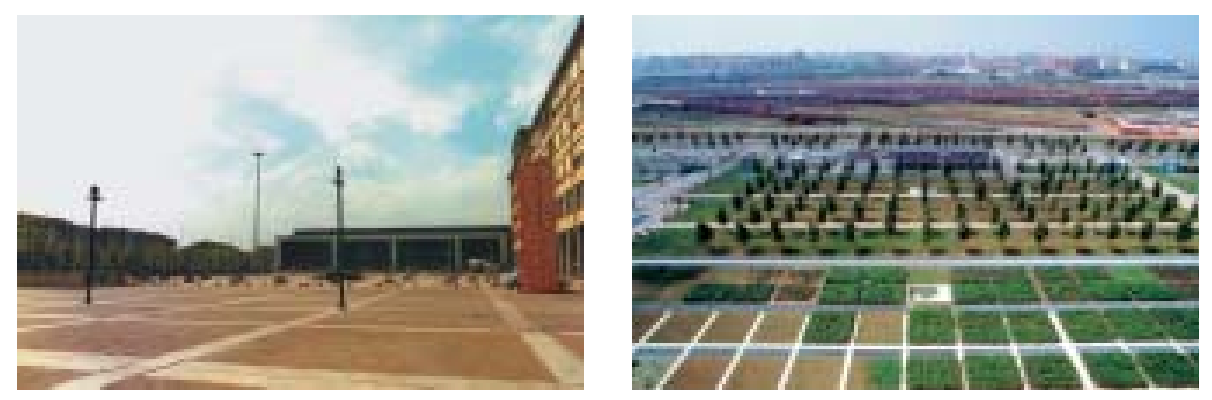

Figure 9, 10 - Il piazzale d'ingresso; il parco al momento dell'impianto delle alberature.

per taxi, i parcheggi riservati, i box office del Centro Fiere. Stenta insomma ad essere una piazza, anche perché non vi si affacciano direttamente le attività che invece hanno spazi pubblici coperti, come le gallerie commerciali del primo piano, raggiungibili dai grandi blocchi di distribuzione passanti.

Il parco è sul retro dell'edificio, stretto tra i 500 metri del fronte costruito e la ferrovia, ed è assai poco frequentato: scelte urbanistiche generali e di utilizzo del piano terreno dell'edificio in particolare ne fanno un margine anziché un tratto di "spina verde".

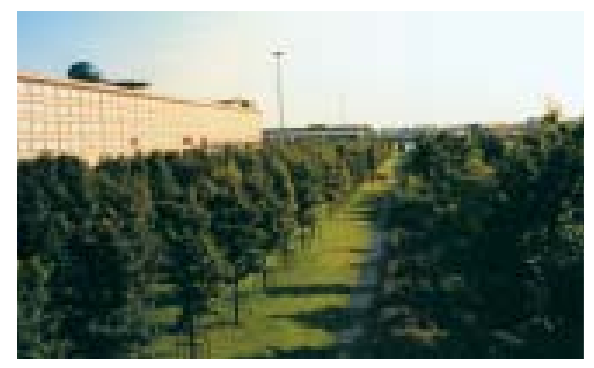

Figura 11 - Veduta del fronte posteriore (in evidenza l'eliporto)

\section{L'edificio}

Esteriormente l'edificio non ha mutato aspetto, tranne per l'inserimento di due "ospiti inattesi", come li definisce Piano, che quasi ammette di aver rivaleggiato con le invenzioni di Matté Trucco: sul tetto infatti ha collocato un eliporto ed una bolla di vetro, che è diventata il nuovo simbolo dell'edificio, stupefacente oggetto visibile anche da lontano nel panorama urbano, una sala riunioni dalla quale si gode della visione dell'arco alpino5. Buchanan osserva che, per il loro carattere elitario, questi oggetti contraddicono il resto dell'edificio (non è insolito in effetti veder planare Giovanni Agnelli ed i suoi ospiti, che frequentano solo la cima dell'edificio e la palazzina per uffici).

Interrati si trovano invece l'auditorium ed il centro congressi. L'interno del nuovo auditorium, interamente in legno di ciliegio, rispecchia l'architettura del Lingotto ripetendone la struttura.

\section{Il parco}

Dal lato della ferrovia una parte del campo binari è diventata parco. I lunghi filari paralleli costringono il fruitore a muoversi lungo passeggiate rettilinee, quasi ricalcando i vecchi binari (di cui in realtà non hanno l'orientamento, essendo legati alla griglia dell'edificio). Il disegno modulare è apprezzabile soprattutto dall'alto, ossia dall'interno dell'edificio e dagli ingressi sopraelevati. Quasi a conferma dell'impraticabilità, e del fatto che è un parco "da guardare", sono assenti punti di sosta. Sono impiegati esclusivamente tigli e viburni, ad alcuni vasi di aceri rossi e di arbusti a fioritura bianca.

\footnotetext{
${ }^{5} \mathrm{Nel} 2002$ è stato aggiunto anche il cosiddetto "Scrigno", che ospita una collezione d'arte moderna donata alla Città dalla famiglia Agnelli. Anch'esso si presenta come un volume sospeso al di sopra del fabbricato, dominando il panorama, ma, a differenza della bolla di vetro, è completamente opaco e privo di aperture, come un forziere.
} 
Rispetto al piazzale "minerale", vuol essere lo spazio dedicato alla natura e alla vegetazione, che in realtà è assai poca. Del resto, trattandosi della copertura del parcheggio interrato, la terra ha una profondità variabile tra i 40 e i $120 \mathrm{~cm}$.

Per la concezione generale del parco Piano non cita consulenti, ma riconosce di essere stato aiutato da conversazioni con Larry Halprin.

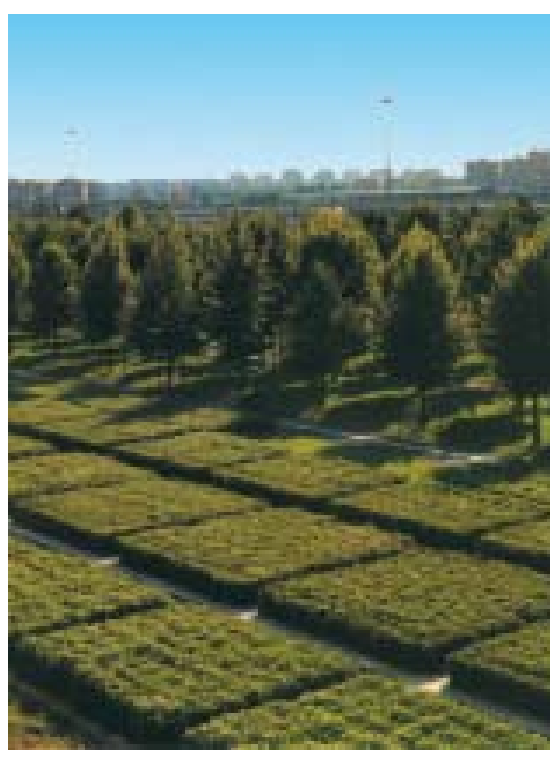

Figura 12 - Veduta del parco (sullo sfondo la stazione ferroviaria).

\section{Le corti interne}

Le corti interne sono diventate inaspettati giardini. La corte della fontana era un giardino formale di conifere nane ed azalee, arricchito da una fontana mobile di Susumu Shingu; la "corte delle feste" aveva arredi mobili (fioriere) per consentire la massima libertà di utilizzo: entrambe sono state recentemente coperte per essere utilizzate come gallerie commerciali. La terza corte è concepita come un "giardino delle meraviglie" ricco di esotismi, che Piano descrive come "un piccolo orto botanico" di piante comunque familiari per i torinesi frequentatori della Riviera: palme, magnolie, banani, ulivi, yucche (sono state impiegate solo piante grandi e formate, alcune secolari). Esso è utilizzato in particolar modo dall'hotel di prima categoria e dal Centro Congressi. Per la concezione di questo giardino, l'unico libero dalla geometria, Piano dichiara le collaborazione del nipote Daniele Piano (direttore dei lavori per tutte le sistemazioni esterne).
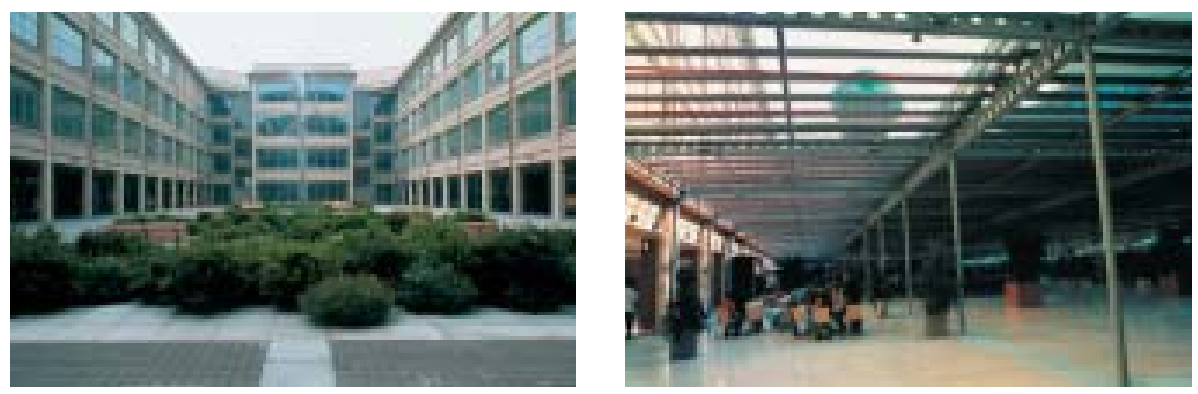

Figure 13, 14 - La "corte della fontana” prima che venisse coperta e la "corte delle feste" dopo la copertura.

\section{I dettagli}

I dettagli delle sistemazioni esterne mantengono una forte unitarietà con l'architettura dell'edificio, e sono risolti con il ricorso ad una gamma molto ristretta di forme e materiali: come già detto, la pavimentazione ricalca la maglia strutturale $(6 \mathrm{x}$ $6 \mathrm{~m}$ ), tramite elementi di cemento prefabbricati di 60 centimetri di spessore; i quadrati di base sono di volta in volta riempiti con piastrelle in cemento, green-block o siepi, e dettano il passo dell'alberatura; gli alberi sono esclusivamente tigli, le siepi viburni; in vasi quadrati troviamo bambù, aceri, ed arbusti dalla fioritura bianca.

L'alberatura lungo il viale prospiciente è di magnolie, contro il cui fogliame lucido e scuro contrastano i pali bianchi, che accendono il panorama notturno: multifunzionali, portano stendardi, lampade o diffusori. Tutti gli elementi di arredo sono stati appositamente disegnati da Meccano. 


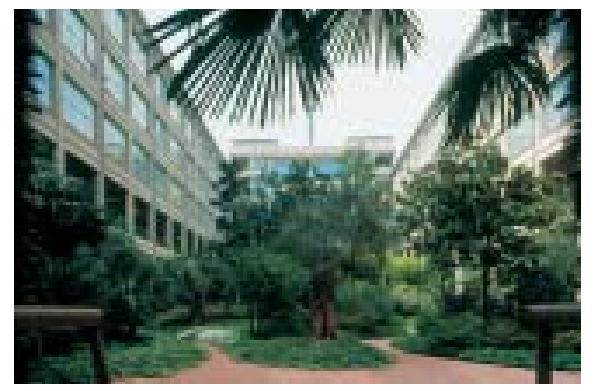

Figura 15 - Il "giardino delle meraviglie".

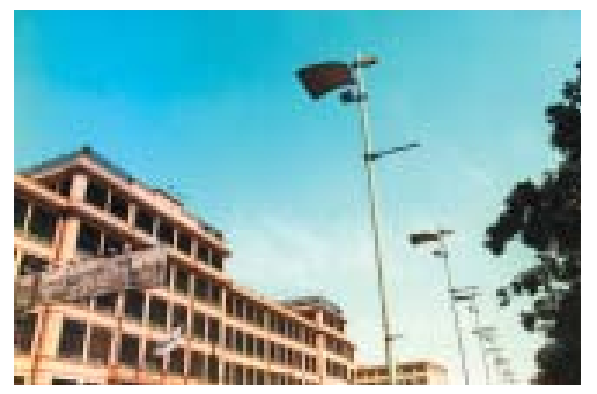

Figura 16 - I pali porta lampade e stendardi.

\section{I cambiamenti in corso: il "cantiere permanente"}

Fin dall'inizio il motto del Lingotto è stato "il cantiere permanente". Vista l'enormità dell'impresa, si è scelto di completare e aprire man mano le varie parti (per primo il centro fiere, le parti esterne, l'auditorium e il centro congressi, la galleria commerciale). All'interno dell'edificio, al posto dell'artigianato e delle imprese, è cresciuta la superficie di terziario, si è aggiunto un cinema da undici sale, ed è nato un nuovo edificio per ospitare un hotel di prima categoria; anche vari servizi legati alle Olimpiadi invernali del 2006 verranno ospitati qui. Il parcheggio interrato non è più sufficiente: una superficie di parcheggi a raso circa doppia rispetto a quella del parco ha già occupato un'altra porzione del campo binari, e dai blocchi di distribuzione della galleria commerciale si protendono le passerelle aeree per raggiungerli.

Queste non raggiungeranno invece la stazione ferroviaria e, con essa, l'altro lato della città - altra rinuncia alle connessioni urbanistiche possibili.

Il progetto con cui Piano vinse il concorso metteva giustamente in luce il ruolo dell'area rispetto alla città ad alle previsioni del Piano Regolatore Generale Comunale, enfatizzando il possibile cuneo verde verso il centro storico (fino a proporre l'interramento della Stazione Centrale di Porta Nuova) e di ricucitura tra due parti di città separate dalla ferrovia attraverso il parco e lo spostamento della stazione passeggeri Lingotto. In realtà nulla di tutto ciò si è ancora realizzato: gli unici interventi attuati ad oggi sono quelli legati alle iniziative commerciali, mentre l'area ex FFSS finora non ha assunto alcuna centralità. Nei primi mesi del 2001 questa ipotesi è tornata di attualità legandosi strategicamente ad altri progetti di trasformazione della città di Torino in occasione delle Olimpiadi Invernali del 2006, tanto che la Città ha siglato un protocollo d'intesa per l'arretramento della stazione centrale al Lingotto e l'utilizzo della fascia ferroviaria a fini edificatori.

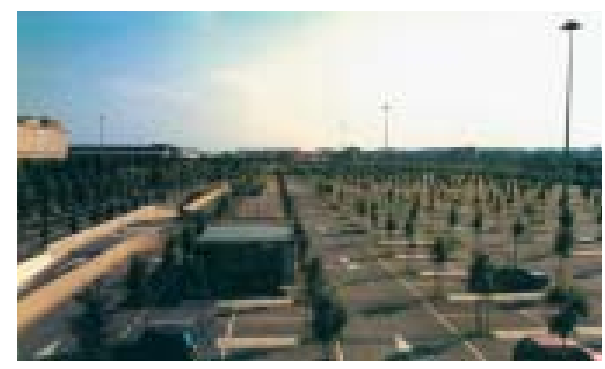

Figura 17 - L'ampliamento del parcheggio in superficie verso ferrovia.

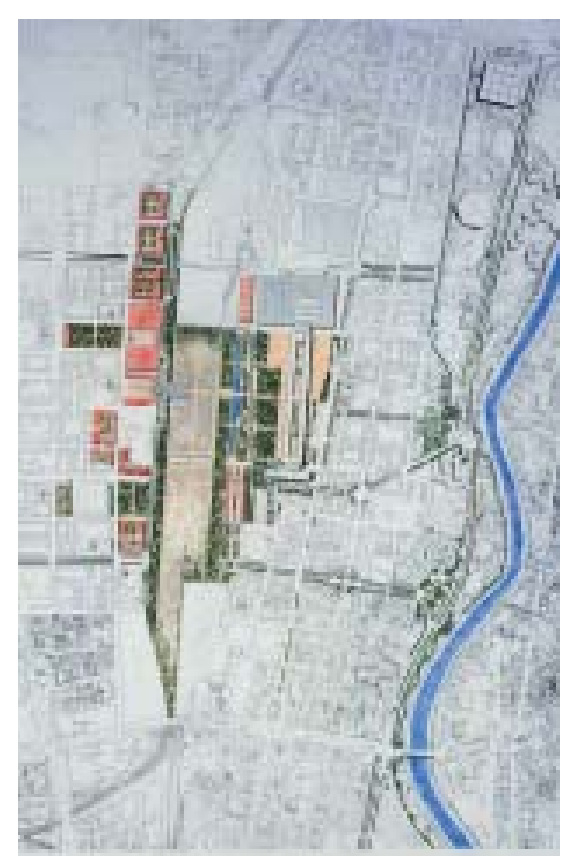

Figura 18 - Il sistema del verde e della "spina" nel progetto di concorso. 


\section{RIFERIMENTI BIBLIOGRAFICI}

BIAMINO BRUNA, L'architettura del moderno a Torino, Lindau s.r.l., Torino 1993.

Buchanan Paul, Renzo Piano. L'opera completa del Renzo Piano Building Workshop, Umberto Allemandi \& C., Torino 1993 (vol. I, in part. pagg. 90-109 Ristrutturazione Schlunberger) e 1997 (vol. III, in part. pagg. 40-53 Ristrutturazione della fabbrica del Lingotto).

Camerana Benedetto, Tecnica versus natura? Tre progetti di Renzo Piano: Aeroporto di Kansai, Lingotto a Torino, Centro Culturale Kanak a Nouméa, "Eden. Rivista dell'Architettura nel paesaggio", 3, Automobilia, Milano 1996, pagg. 26-33.

Carbonara Giovanna, L'altro Lingotto, "Acer", 1, Il Verde editoriale, Milano 1998, pagg. 61-64.

CITTÀ DI TORINO, Spazio Torino. Idee e progetti per la riqualificazione urbana, Torino, Assessorato all'Ambiente e allo Sviluppo Sostenibile 1996.

Yukio FutAgaWa (a cura di), Renzo Piano Building Workshop, numero monografico, "GA (Global Architecture) Architect", 14, A.D.A. EDITA, Tokyo 1997, pagg. 90-99.

Gruppo FIAT (a cura di), Venti progetti per il futuro del Lingotto, Etas Libri, Milano 1984, pagg. 154-163.

Magnaghi Agostino, Monge Mariolino e Re Luciano (a cura di), Guida dell'architettura moderna a Torino, Lindau s.r.l., Torino 1982.

Piano Renzo, Giornale di bordo, Passigli Editore, Firenze 1997 (in part. pagg. 90-99).

Olmo Carlo (a cura di), Il Lingotto 1915-1939. L'architettura, l'immagine, il lavoro, Umberto Allemandi \& C, Torino 1994.

Touring Club Italiano, Piemonte. Attraverso l'Italia, Illustrazione delle regioni italiane, vol. I, T.C.I., Milano 1930.

\section{RIFERIMENTI ICONOGRAFICI}

Figura 1: rielaborazione di Claudia Cassatella di immagine dell'Archivio Storico Fiat. Figure 2, 9, 11, 12, 14, 16, 17: fotografie di Claudia Cassatella.

Figure 3, 18: rielaborazioni di Claudia Cassatella di immagini tratte da GRUPPO FIAT (a cura di), Venti progetti per il futuro del Lingotto, Etas Libri, Milano 1984, pagg. 30 e 156.

Figure 4-8: Renzo Piano Building Workshop, per gentile concessione.

Figure 10, 15: fotografie di Gianni Berengo Gardin, per gentile concessione RPBW.

Figura 13: fotografia di Michel Denancé, per gentile concessione RPBW. 


\section{Progetto:}

Luogo:

Committente:

Tipologia di intervento:

Superficie:

Progettista:

Iter del progetto:

Anno di progettazione:

Anno di realizzazione:

Architetto responsabile:

Imprese esecutrici:

Costo intervento:

Costo manutenzione annuale:

Materiali impiegati per la pavimentazione:

Specie vegetali impiegate:

Arredo urbano: ristrutturazione dello stabilimento FIAT Lingotto, realizzazione di nuovi spazi pubblici urbani: viale, piazza, parco, corti interne

Torino

Gruppo FIAT, poi Lingotto S.r.l.

spazi pubblici urbani

$98.000 \mathrm{mq}$

Renzo Piano Building Workshop (RPBW), con la collaborazione di Meccano per l'arredo urbano

concorso internazionale ad inviti 1983, vincitore Renzo Piano Building Workshop; affidamento del progetto al RPBW

$1992 / 1993$

1994-96 sistemazioni esterne

1988-1995 ristrutturazione dell'edificio $1^{\circ}$ fase

$19962^{\circ}$ fase ed ampliamento

Dott. Arch. Daniele Piano (spazi aperti)

pavimentazioni: Tecneco, Torino

opere a verde:Vivai Piante Mari, Roma

4 miliardi e 400 milioni di lire

200 milioni di lire circa $(103.291,00)$

cordoli prefabbricati in cemento armato, atuobloccanti in cemento, green blocks

Tilia cordata "argentea", Viburnum tinus per il parco; aceri nani, conifere nane, azalee e bamboo per le fioriere; olivi, palme, magnolie e banani per il giardino delle meraviglie; Magnolia grandiflora per l'alberatura di via Nizza. Nota: piantagione su soletta con $40-120 \mathrm{~cm}$ di terreno di riporto

progettato e brevettato per il Lingotto da Meccano, in particolare pali multifunzionali che portano elementi illuminanti a riflessione e stendardi 



\title{
IL PARCO DIFFUSO DI TONINO GUERRA IN ROMAGNA
}

\author{
Enrica Dall'Ara ${ }^{1}$
}

\section{IL CONTESTO}

Il territorio su cui si estende il parco diffuso realizzato da Tonino Guerra, scenografo e poeta originario di Santarcangelo (Rimini), va dalla costa adriatica romagnola (Cervia) fino all'estremità della Valmarecchia, in provincia di Pesaro, nel cuore della regione storica del Montefeltro.

$\mathrm{Si}$ tratta di una terra carica di contraddizioni che nel tempo hanno generato una dialettica di situazioni oggi coesistenti in un improbabile equilibrio.

Da un lato ci sono i paesaggi storici delle vallate, con le loro rocche e pievi antichissime (la Madonna di Saiano, San Leo, Verucchio, Maioletto, Torriana e Montebello, Pennabilli...), le fughe d'occhio del Rinascimento con la luce di Piero della Francesca celebrata, tra gli altri, da Ezra Pound, dall'Alta Valmarecchia fino al tempio malatestiano di Rimini, gli ambienti naturali caratteristici, i massi erratici di calcare su cui sono sorte San Marino e le altre cittadelle fortificate che dai crinali vicini appaiono come un esercito con le lance puntate verso il mare, i boschi di querce e il letto enorme di ghiaia bianca della "Marecchia argentina", come la vedeva Giovanni Pascoli.

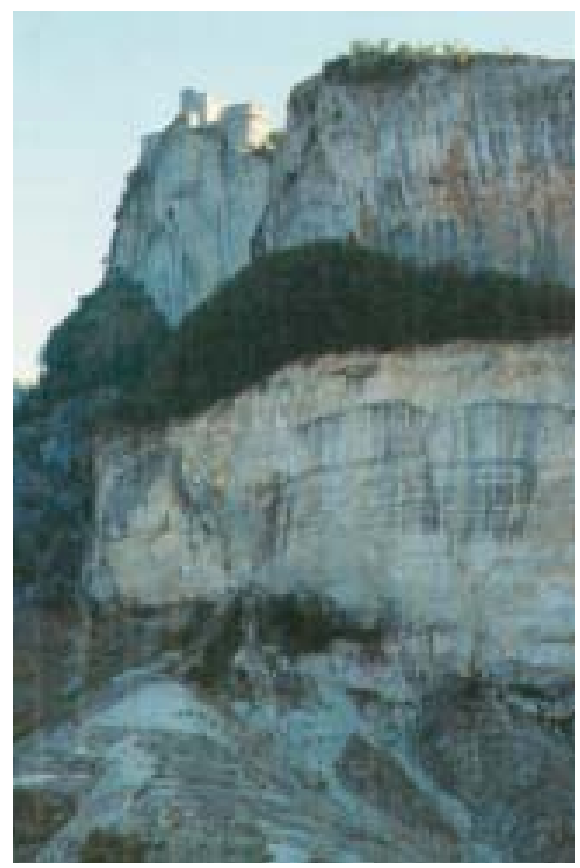

Figura 1 - Paesaggio della Valmarecchia: San Leo.

Dall'altro queste valli rimaste al margine della storia, con i loro personaggi straniti da cui Tonino Guerra ha tratto ispirazione, sfociano su una costa che ha fatto del divertimento e dello spettacolo la struttura delle sue città, mangiandosi le dune costiere e sostituendole con una conurbazione metropolitana tentacolare.

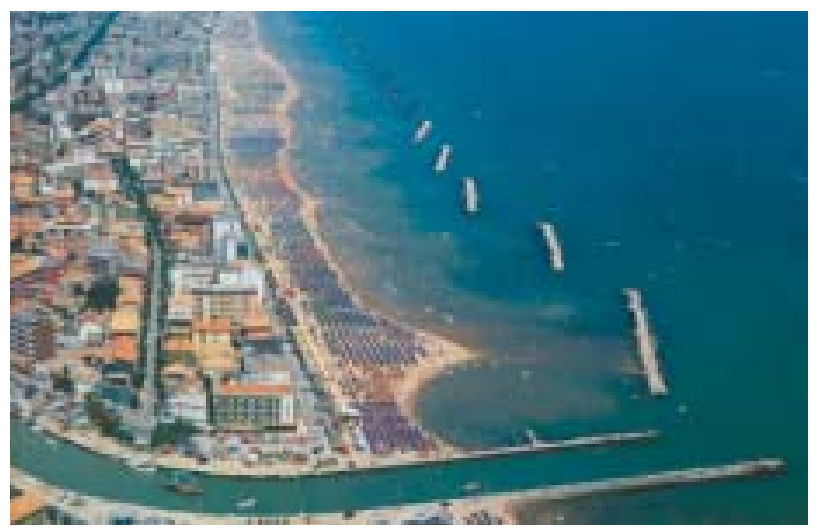

Figura 2 - Costa romagnola nei pressi di Bellaria.

${ }^{1}$ Le presenti considerazioni sono un ampliamento dell'articolo I giardini Narranti pubblicato nei 'Quaderni' n. 1 della "Ri-Vista", rivista on line del Dottorato in Progettazione Paesistica dell'Università degli Studi di Firenze. 


\section{GLI OBIETTIVI}

Le realizzazioni recenti di Tonino Guerra, sparse in questo territorio, vogliono richiamare interesse su "luoghi minimi, con il fascino della memoria e dell'abbandono" ${ }^{2}$. Il valore di muoversi nelle brevi distanze consiste nella possibilità di incontrare la propria storia: nei termini di presa di coscienza del radicamento dovuto alla famigliarità con un paesaggio singolare e contemporaneamente dell'appartenenza all'universale per cui "Vicino a casa improvvisamente vi potete perdere perché il viaggio non si ferma dove arrivate ma continua dentro la vostra memoria." È possibile trovare i "depositi di immagini che ci ha consegnato l'umanità. Possiamo imbatterci in apparizioni fissate da quando eravamo primitivi."

\section{LA METODOLOGIA}

Nelle opere letterarie di Tonino Guerra, apparizione, interesse, concentrazione sono associati e costruiscono i momenti in cui l'aspetto fantastico avvicina ad una verità.

Ricorrono spesso situazioni in cui improvvisamente un luogo è invaso da un'unica cosa. Essa fa scomparire, oppure trasfigura, gli elementi che lo identificano, perché ne impedisce la vista o anche perché il suo avvento è straordinario e attira tutta l'attenzione.

L'immagine è coperta da una sensazione esclusiva:

“...Vedo solo bianco. Neve, nevica, nevicando questo pulviscolo nell'aria e io che cammino, felicità più completa" ${ }^{3}$.

"Poi sono arrivate tutte quelle farfalle bianche / che venivano dal fiume / e si sono posate su tutto, e la roba lì attorno ha preso il colore del latte: porte, / finestre, muri e noi che eravamo a sedere/ sembravamo dei bambocci di gesso" ${ }^{3}$.

Analogamente la fioritura eccezionale del racconto L'inverno fiorito 5 è presenza continua in fondo alle strade. Dopo una notte in cui è piovuto e soffiato il vento, "lei si affaccia alla finestra e vede che tutta la campagna fino al mare è bianca come se avesse nevicato".

Il film Amarcord $^{6}$ di Federico Fellini, il cui soggetto è ideato da Tonino Guerra, inizia con una danza nell'aria delle "manine", i pollini dei pioppi, che viaggiano sui tetti delle case, scendono per le strade del Borgo - arriveranno al mare - mentre le persone, nella meraviglia, si scompongono tentando di afferrarle. Successivamente una nebbia "che ha cancellato tutto attorno a lui" disorienta il nonno, fra i protagonisti, davanti alla propria casa e alimenta un monologo nello smarrimento crescente: "Non sono più da nessuna parte". "Ma dove sono? Se la morte è così non è un bel lavoro ${ }^{7}$...finito tutto! (...)".

Queste visioni totalizzanti stravolgono l'ordinarietà mediante uno svago o un sentimento intensi, dovuti al mutamento subito dallo spazio intorno. La descrizione è in bilico fra assolutezza dell'immagine e chiarezza del dettaglio proponendo comunque una ricomposizione forte attorno all'idea centrale: "Ci sono dei momenti in cui il corpo fa attenzione a tutto: gli occhi vedono un fiore rosso, le mani sentono il calore di una stoffa, la bocca ritrova un vecchio sapore. E così uno si sente frantumato. Si potrebbe ricomporre con un grosso dolore, concen-

${ }^{2}$ Tonino Guerra, cit. in Rita Giannini, Tonino Guerra e la sua valle, Raffaelli Editore, Rimini 1998.

${ }^{3}$ Tonino Guerra, Dizionario fantastico, Pietroneno Capitani, Rimini 2000, pag. 68.

${ }^{4}$ Tonino Guerra, Il viaggio, Maggioli Editore, Rimini 1986, pagg. 25-26. L'immagine dello sciame di farfalle si ritrova anche in TONINO GUERRA, Dizionario fantastico, Pietroneno Capitani, Rimini 2000, pag. 66.

${ }^{5}$ Tonino Guerra, Il Polverone, Maggioli Editore, Rimini 1992, pagg. 9-11.

${ }^{6}$ Il titolo è in dialetto romagnolo e significa "Mi ricordo".

7 "Non è un bel lavoro" è un'altra espressione dialettale romagnola, per significare "non è una bella cosa". 
trarsi insomma attorno ad un interesse unico. Quando mi capita, io guardo il sole e così raduno il mio corpo attorno ad un dolore accecante"s.

Negli allestimenti sul territorio la ricerca dell'assolutezza si affievolisce. La sottolineatura è operata inserendo forme decorative che informano i luoghi di figuratività, offrendo una realtà ricreata, ridata, tramite la sovrapposizione, da parte dell'artista, del suo stile - per questo è possibile una loro lettura come episodi di un parco unico sotto il "timbro" di Tonino Guerra.

\section{GLI ESITI}

Ci sono tanti oggetti parlanti. La natura è scelta a raccontare se stessa esponendosi: nelle mostre museografiche spesso convivono gli oggetti reali e la loro rappresentazione. Così a Cervia, nel giardino Il Tappeto sospe$s o$, un canneto di ceramica e rame è cresciuto in un minuscolo "lago" ed un altro, naturale, nel prato accanto. Se ci si avvicina a questo si scopre anche qui, fra le foglie, qualche fusto di ferro, sparso.

A Pennabilli nell' Orto dei Frutti dimenticati - realizzato dove era un orto conventuale - una lumaca di bronzo della dimensione di un gatto abita Il bosco incantato, un labirinto di steli di pietra serena, mentre dopo la pioggia sicuramente qualche lumaca viva si potrà vedere lungo i muretti e altre sono in rilievo sulla Porticciola delle lumache realizzata da Aldo Rontini, ceramista faentino.

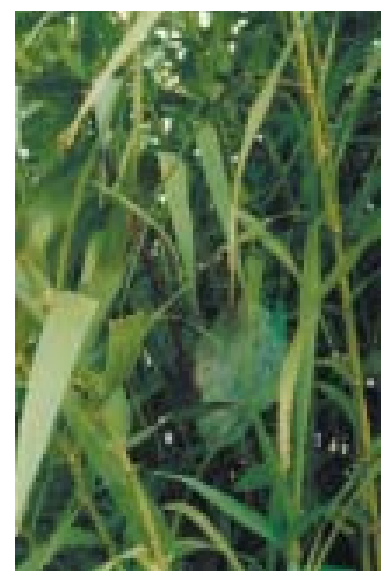

Figura 4 - Foglia di rame nel canneto.

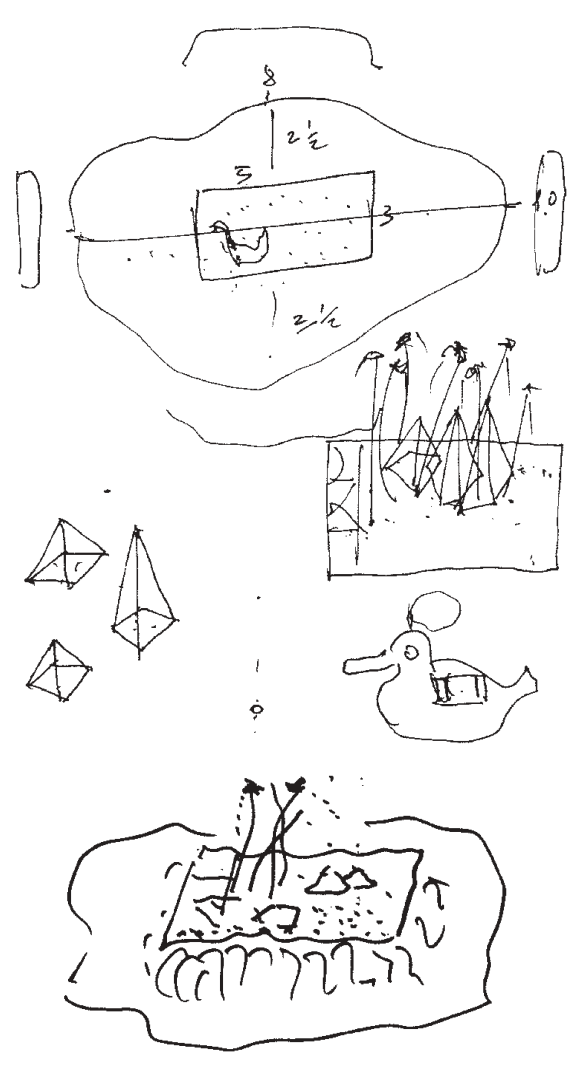

Figura 3 - Schizzi di Tonino Guerra per la fontana Il Tappeto sospeso a Cervia.

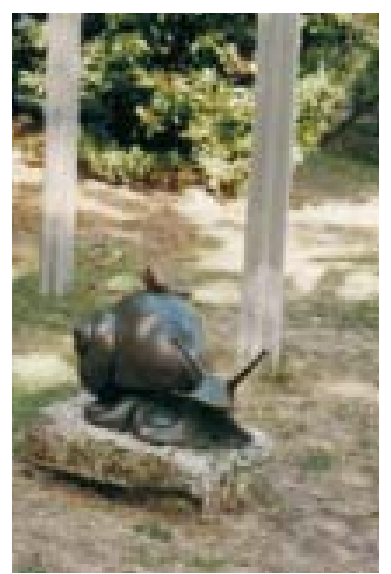

Figura 5 - Lumaca di bronzo.

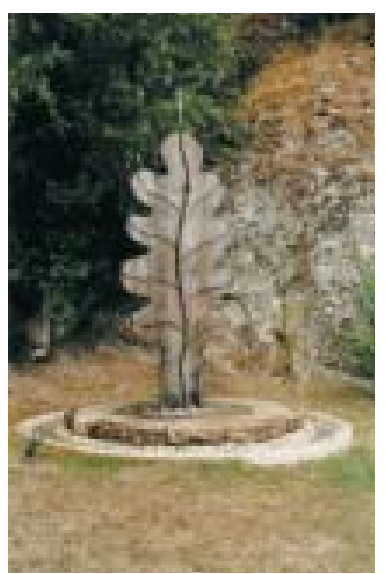

Figura 6 - Fontana La Voce della foglia.

8 Tonino Guerra, Dizionario fantastico, Pietroneno Capitani, Rimini 2000, pagg. 83-84.

${ }^{9}$ Dalla dichiarazione di poetica dell'autore in occasione di un'intervista di Giancarlo Papi per l'apertura dell'esposizione Con la poesia alle spalle, Rimini 15 dicembre 2001-19 gennaio 2002, Galleria Fabjbasaglia. 


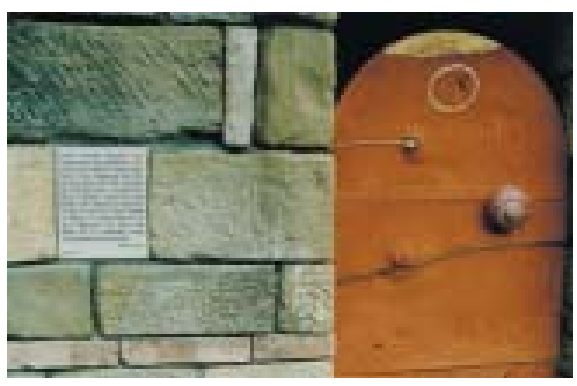

Figura 7 - Porticciola delle lumache.

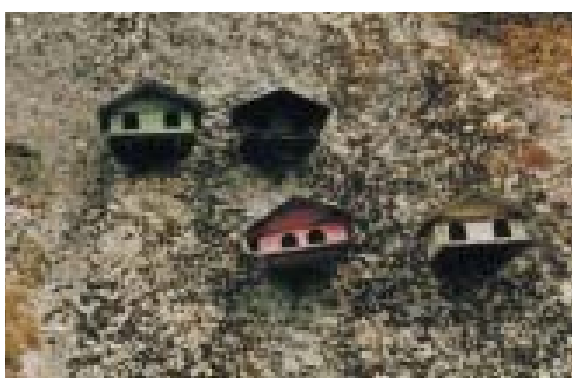

Figura 8 - Nidi a casetta di legno colorato.

Così nello stesso orto vivono le foglie dei tanti alberi da frutto esposti, una foglia di legno alta tre metri che versa acqua sulla pietra di un antico mulino - la fontana La Voce della foglia - una sagoma di foglia di dimensioni analoghe, realizzata a fil di ferro e mimetizzata contro i muschi della parete rocciosa, e le foglie scritte del mese di Ottobre su una delle targhe in ceramica in un vecchio lavatoio - Le parole dei mesi: OTTOBRE $i$ tappeti di foglie secche sotto $i$ piedi. Alla roccia che delimita l'orto stanno appesi nidi a casetta di legno colorati, per gli uccelli, mentre due colombi di bronzo proiettano la loro ombra su un disco bianco, a terra, disegnando i volti di Federico Fellini e Giulietta Masina - La Meridiana dell'incontro.

Una griglia di ferro tridimensionale a forma di elefante struttura una pianta rampicante: è un prototipo per i successivo allestimento, a Perticara (Pesaro), di uno Zoo verde di animali giganteschi. Ci sono un altro elefante, una giraffa, una tartaruga, un rinoceronte.

A Torriana la Fontana dell'Albero dell'acqua è un albero con il tronco di legno ed i rami effimeri disegnati da getti d'acqua.

Il risultato è un senso ambiguo fra reale e surreale per cui le cose possono acquistare tutte le dimensioni ed essere fatte di tutti i materiali senza che muti la loro sostanza, ma amplificando i rimandi fantastici contenuti nel loro nome. In questo modo anche il racconto si amplifica, trasforma la natura in personaggio recitante simultaneamente molte eco, a chi, in particolare, conosca l'opera poetica dell'autore.

Le piante di rosmarino a Cervia: “(...) Petrèlla Guidi par léu / l'era un udòur ad smaroin / ch u t butéva la tèsta indrì." 10

ed i ciottoli ordinati a bordo d'acqua: " $U$ i era te fiom dal béusi ' $\mathrm{d}$ sabia/ in dò ch'u s'avdéva in qua e in là / di sas e alòura Rico u i amucéva s'una cana/ te mèz, che i féva péna si stéva smanèd." 11

Gli esemplari di alberi da frutto "dimenticati" a Pennabilli, costituiscono un orto botanico di piccole dimensioni che presenta le piante tipiche di un'agricoltura pre-industriale.

Eppure non sono solo materia didattica o una possibilità di ricordo, ma soprattutto materia poetica:

"In tutta la valle del Marecchia ne parlavano. E cioè da San Leo a Gradara, da Verucchio a Montebello e a tutta la zona che qualcuno vorrebbe chiamare il trapezio Medievale. I vecchi dicevano che una fioritura d'inverno così totale e completa l'avevano avuta soltanto alla fine dell'altro secolo. Eravamo soltanto a metà gennaio e il sole sbriciolava la terra. Ciliegi meli e tutti gli alberi da frutto erano

10 "Petrella Guidi per lui / era un odore di rosmarino / così forte che ti buttava la testa indietro." da Tonino Guerra, Il Viaggio, Maggioli Editore, Rimini 1986, pagg. 30-31.

11 "C'erano nel fiume delle buche di sabbia / dove si vedevano qua e là / dei sassi e allora Rico li ammucchiava nel mezzo / con una canna perché gli facevano pena se stavano isolati." da TONINO GuERrA, Il Viaggio, Maggioli Editore, Rimini 1986, pagg. 28-29. 


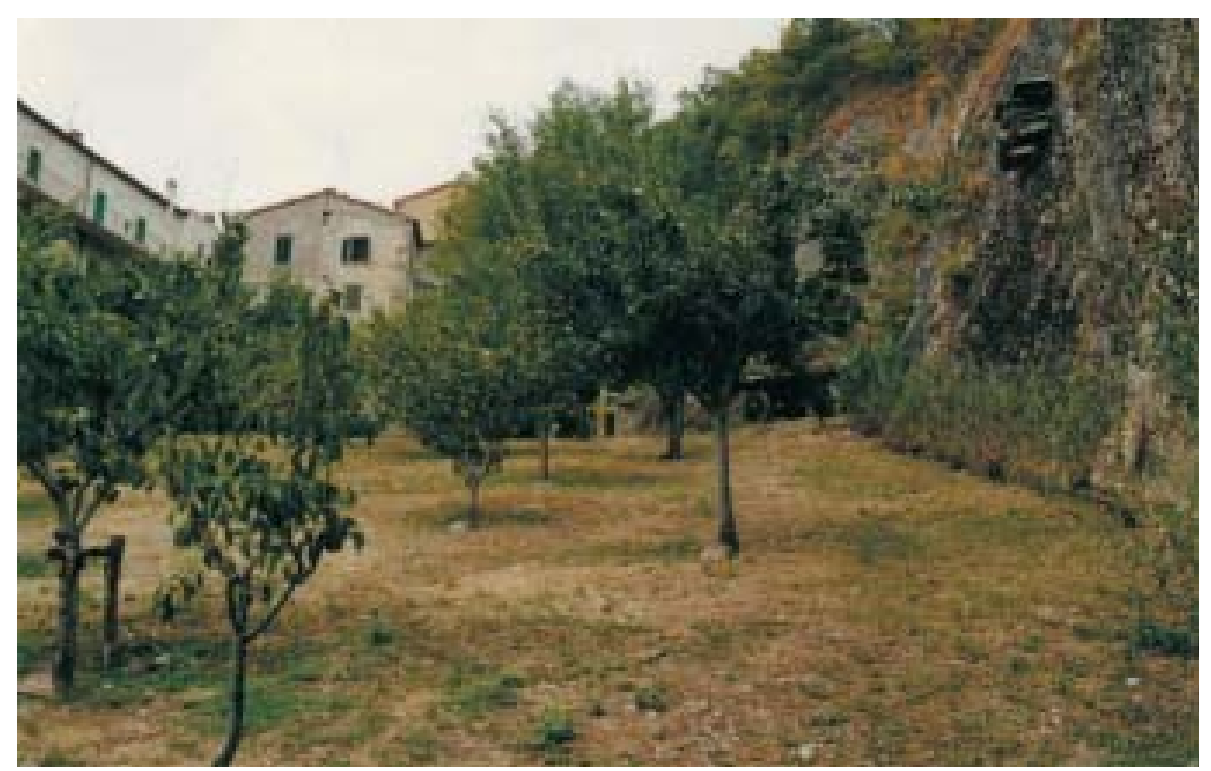

Figura 9 - Alberi da frutto ne L'orto dei frutti dimenticati a Pennabilli.

carichi di fiori e di api golose. Al paese si vedevano gli alberi fioriti in fondo a tutte le strade e la gente parlava d'affari ma ogni tanto accennava a questa primavera precoce..." ${ }^{\prime 2}$.

"...Così ho cominciato a capire che stavo andando al paese. / Infatti c'erano gli ulivi e anche molti mandorli nei campi. / Ulivi e mandorli grossi come cominciano a vedersi solo a un centinaio di chilometri dal mio paese. (...) Probabilmente quindi andavo verso il mio paese. / Siccome non ho la certezza di essere in nessun posto..."13.

Sono anche gli oggetti di Il ciliegio in fiore ${ }^{14}$, Al foi de zris - Le foglie del ciliegio $^{15}$, L'amòur - Il gelso ${ }^{16}$.

Materia letteraria e materia del giardino coincidono: naturale/meraviglioso, memoria/presenza, esotico-orientale/locale. Sintetico è il tappeto volante della fontana a Cervia, realizzato a mosaico - come un oggetto d'arte bizantina della vicina Ravenna e dell'antico oriente - sul quale si sollevano dall'acqua due cumuli di sale a ricordare la Cervia delle saline e la funzione originaria di magazzini del sale degli edifici che delimitano la piazza.

Lassonanza fra i giardini di Tonino Guerra e i paesaggi scritti delle sue opere esiste non solo perché gli elementi della narrazione sono gli stessi, ma anche per il fatto che in entrambi il timbro è simultaneamente di gravità e leggerezza:

"(...) Ci sono dei giorni che questi alberi si caricano di tanti pezzetti di stoffa così da sembrare fioriti specialmente negli anni delle guerre e delle pestilenze.

Lalbero più amato è un grande caragac. Senza foglie tutto l'anno perché strappate dalle mani avide di grazie per far posto ai pezzi di stoffa, l'albero si alza in una gola di pochi metri in mezzo a due montagne che dividono il deserto dai giardini di albicocchi. Una è rossa e l'altra è bianca. Tutte le sere nasce un vento risucchiato oltre la gola delle foglie ancora calde degli albicocchi e dai frutti gialli di sole.

I pellegrini che arrivano a chiedere la grazia, scrivono il loro desiderio sul pezzetto di stoffa che appendono ai rami del grande caragac. Così quando di notte il

\footnotetext{
${ }^{12}$ Tonino Guerra, Il ciliegio fiorito, in Il polverone, Maggioli Editore, Rimini 1992, pag. 9.

13 Tonino Guerra, Treno, in Dizionario fantastico, Pietroneno Capitani, Rimini 2000, pag. 163.

${ }_{14}$ Tonino Guerra, Il polverone, Maggioli Editore, Rimini 1992, pagg. 9-11.

${ }^{15}$ Tonino Guerra, Il libro delle chiese abbandonate, Maggioli Editore, Rimini, 1988, pagg. 83-85.

16 Tonino Guerra, op. cit., Maggioli Editore, Rimini 1988, pagg. 109-111.
} 


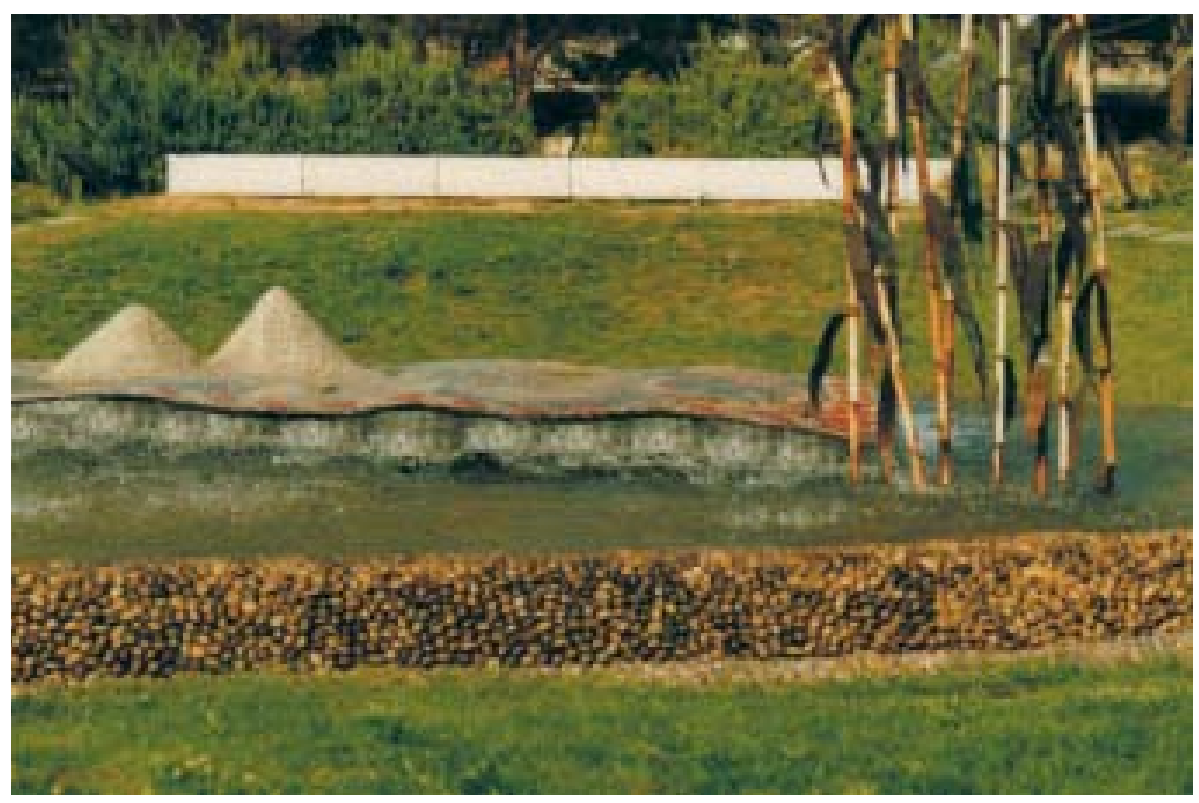

Figura 10 - Il Tappeto sospeso a Cervia.

vento scuote le fettucce, le parole scritte si tramutano magicamente in suoni che si ascoltano a chilometri di distanza: se le sente anche chi le ha scritte questo vuol dire che il desiderio è accolto. (...) Per due notti si alzò appena un vento leggero e a valle arrivarono delle parole dilatate nell'aria. Erano parole sciolte che appartenevano un po' a tutte le scritte. Quindi un groviglio di pensieri confusi. Ma poi una notte si alzò vento grosso che scosse violentemente i rami del caragac agitando le banderuole di panno che schioccarono come fruste protese verso i giardini" ${ }^{17}$.

"In una delle tante favole raccontate in un villaggio ai confini con il Turkmenistan, si parlava sempre di un giardino sonoro che incantava i cammellieri e deviava il corso delle carovane cariche di seta. (...) Un giovane si mise in cerca di questo giardino. L'unica cosa che trovò fu una foresta pietrificata. Cento alberi di duro marmo rugoso e verdastro con solchi come fessure gelate. (...) Battendo i tronchi il giovane si accorse che mandavano un suono duro senza nessuna vibrazione. Con un coltello spinse a lungo in una fessura che era chiusa da un materiale friabile e polveroso. D'improvviso fu investito da un gettito d'acqua. Il tronco era pieno di acqua limpidissima e fresca. (...) Ma un giorno l'acqua finì e la piccola foresta pietrificata rimase con gli alberi vuoti d'acqua. Così una notte di vento gli alberi suonarono e tutti capirono che quel bosco era il famoso giardino sonoro di cui parlavano le favole"18.

Nel giardino di Cervia la gravità è nella collina con i blocchi di pietra squadrata che costituiscono la lunga panca, nei due massi lungo il sentiero d'assi di legno e nella semisfera di pietra con inciso il messaggio-premessa dell'autore, nella forma chiusa della fontana e del sentiero. La leggerezza è negli spruzzi d'acqua dal canneto di ceramica e rame, nell'acqua che apparentemente solleva il tappeto volante, nell'alternarsi d'erba e legno a terra, nella presenza delle cavolaie attorno ai cespugli di lavanda: le stesse farfalle, probabilmente, del prodigio raccontato Alla voce farfalla in Dizionario Fantastico e del ricordo di Rico in Il Viaggio.

Nell' Orto dei frutti dimenticati a Pennabilli, la gravità del bronzo, del legno e della pietra delle sculture è negata dalla composizione degli elementi nello spazio, che muove per aggregazione di punti a costituire nebulose: l'insieme delle steli de Il bosco incantato, la distribuzione seriale degli alberi da frutto, l'ammuc-

${ }^{17}$ Tonino GuerRA, L'albero nero, in Il polverone, Maggioli Editore, Rimini 1992, pagg. 12-14.

${ }^{18}$ Tonino Guerra, Il giardino sonoro, in Il polverone, Maggioli Editore, Rimini 1992, pagg. 70-71. 


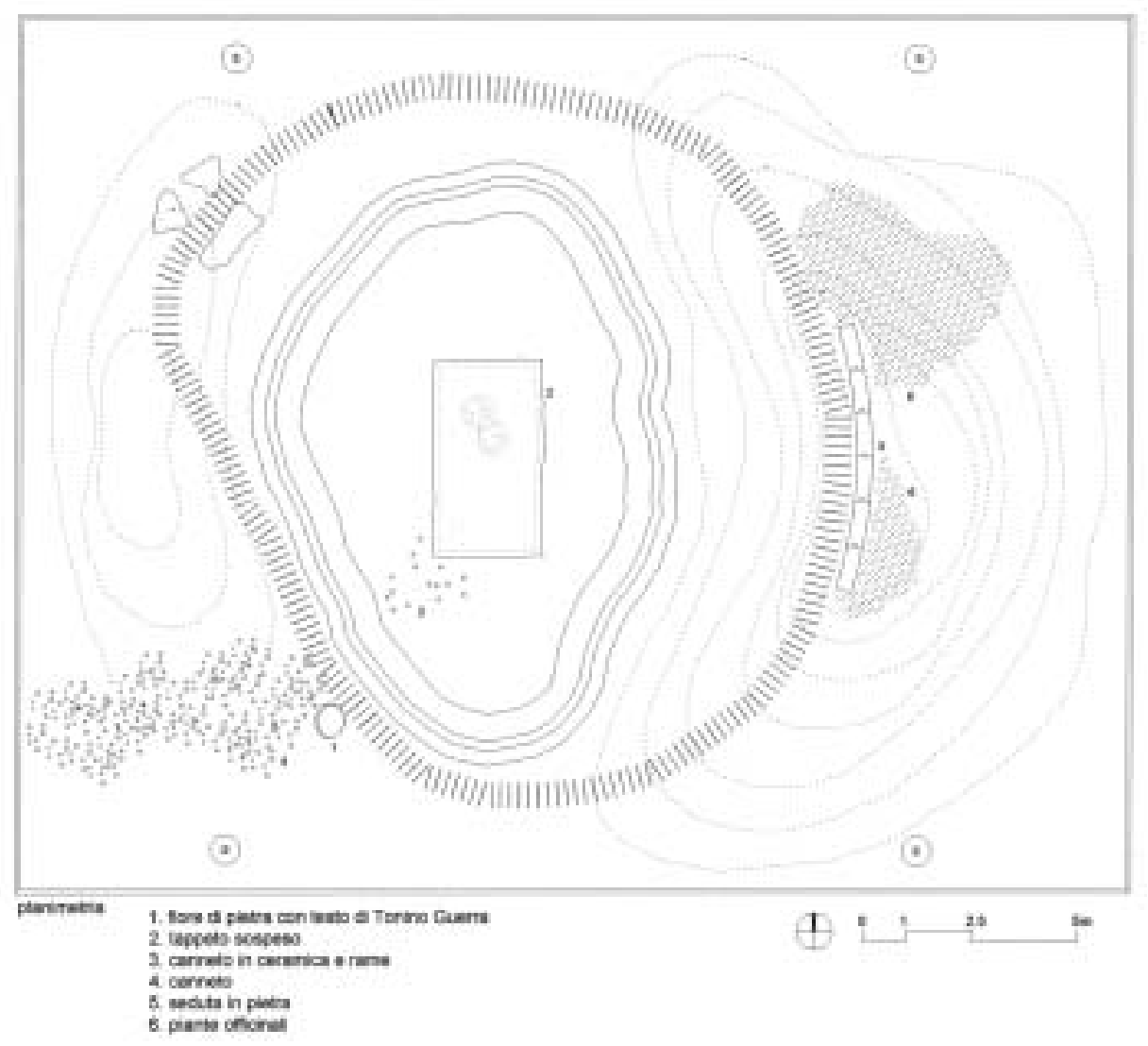

Figura 11 - "Il Tappeto sospeso" a Cervia (RA) - planimetria: 1. "Fiore di pietra” con iscrizione di Tonino Guerra, 2. tappeto a mosaico, 3. canneto di ceramica e rame, 4. canneto, 5. panca in pietra, 6. piante officinali.

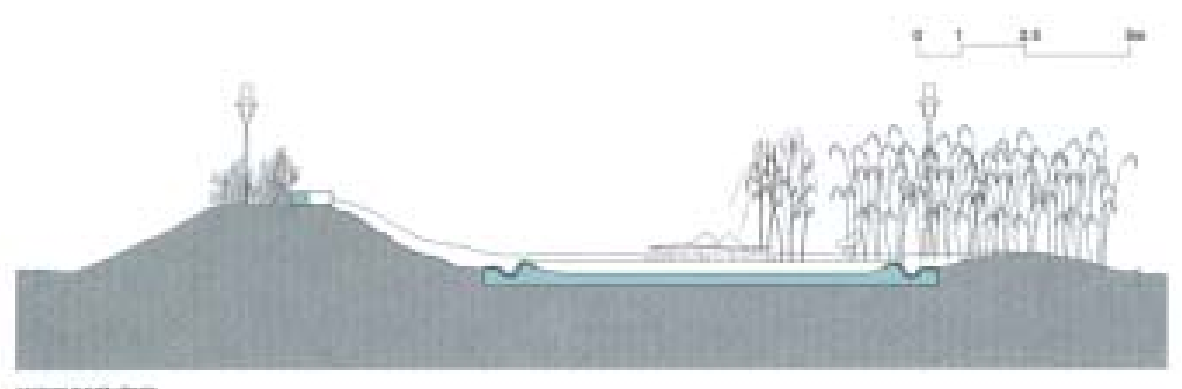

Figura 12 - "Il Tappeto sospeso" a Cervia - sezione.

chiarsi delle formelle appese a un muro con le madonne "raccolte" dai cippi abbandonati. Le raffigurazioni dell' Arco delle favole per gli occhi dell'infanzia che immette nel bosco incantato, delle formelle sacre, delle istallazioni ed il loro decorativismo orientale-popolare, costituiscono un ulteriore elemento smaterializzante, riducendo la percezione del volume degli oggetti che rivestono.

Lieve è il carattere fantastico di tutti gli eventi/presenze dell'Orto, che sono addirittura a volte - è il caso dei volti di Fellini e della Masina e della meridiana in cui a segnare l'ora è il corpo dello stesso visitatore - semplici ombre. Inoltre leggerezza e gravità stanno in un rapporto ambiguo nella volontà di memoria che rappresenta l'intento esplicito dell'Orto dei frutti dimenticati e nell'inserimento delle due meridiane, per cui il tempo diviene componente del giardino ai pesi diversi del passato e del presente.

Avviene qualcosa di simile, nonostante siano diverse le culture di riferimento - da un lato la cultura classica, dall'altro il rapporto con la tradizione popolare - 


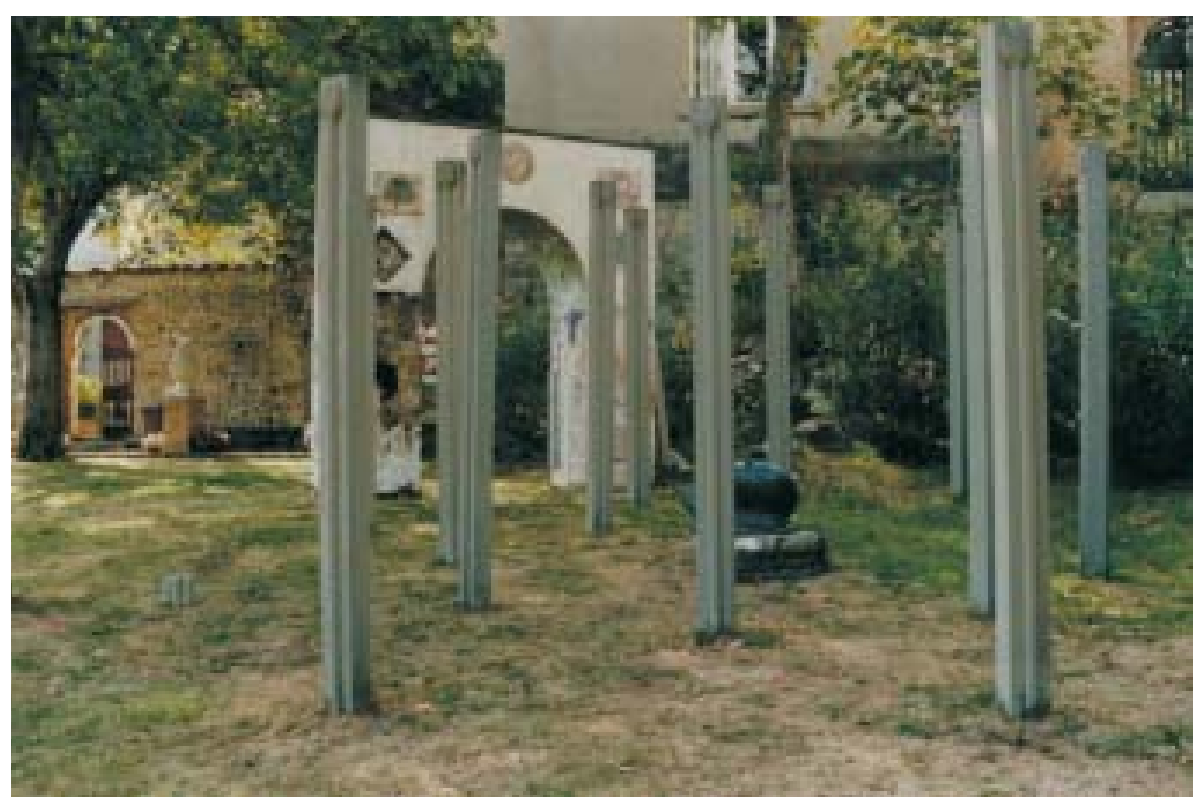

Figura 13 - L'orto dei frutti dimenticati a Pennabilli: labirinto di steli e Arco delle favole per gli occhi dell'infanzia.

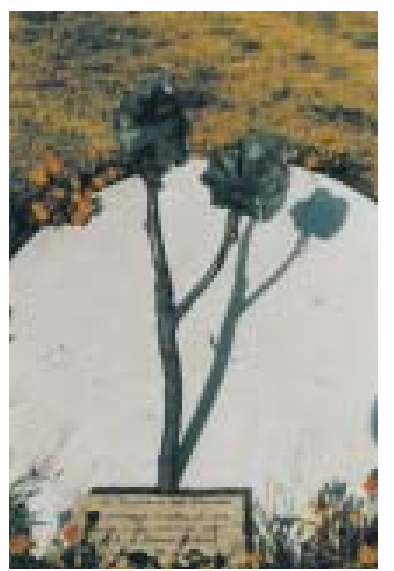

Figura 14 - La meridiana dellincontro.

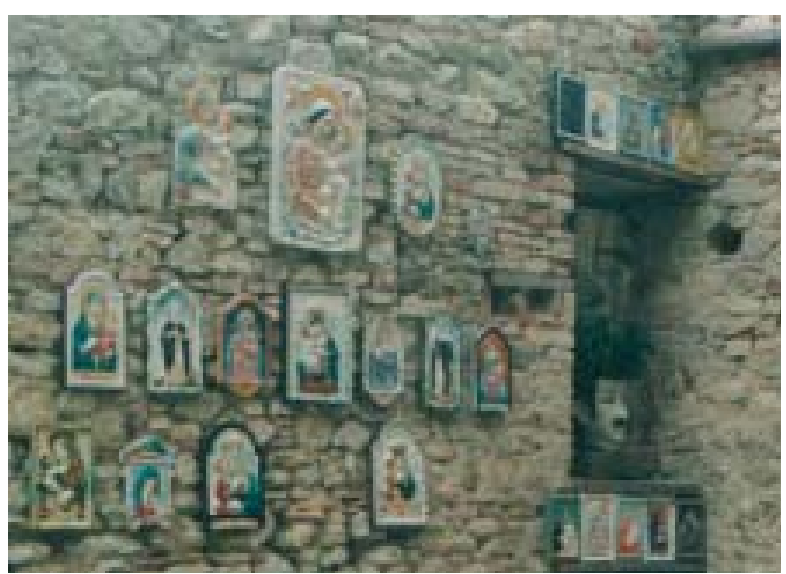

Figura 15 - Formelle in ceramica Le madonne abbandonate.

al legame fra reale e simbolico, fra natura ed evocazione letteraria, che Ian Hamilton Finlay istaura con le sue opere. Tonino Guerra firma messaggi scritti su pietra e ceramica: non sono solo semplici chiarimenti dell'autore a prologo della visione, ma operano un trasferimento dalle cose alla cultura dietro le cose. Impediscono la distrazione, guidano l'assegnazione di significati diminuendo i gradi di libertà dell'immaginazione. Eppure se per Finlay - in Selence per la foresta di Dean, A Remembrance of Annette a Monaco, paesaggio firmato Hodler a Furka Pass in Svizzera, I sing for the Muses And for Myself a Stockwood Park a Luton in Inghilterra - l'iscrizione ha potere di trasformazione sul paesaggio mutandone l'estetica e il contenuto, per cui il rapporto fra lettere, materie e ambiente circostante è quello proprio della poesia concreta, nei giardini di Tonino Guerra la parola scritta non diviene paesaggio e dichiara più esplicitamente il ruolo di filtro: il giardino viene annunciato e immediatamente interpretato. L'autore racconta il giardino che a sua volta manifesta l'autore e ha la sua voce.

L'Orto dei frutti dimenticati di Pennabilli fa parte di un itinerario tematico più ampio I luoghi dell'anima, in cui il discorso prosegue, con un linguaggio maggiormente popolare, fino a Il Santuario dei pensieri. 


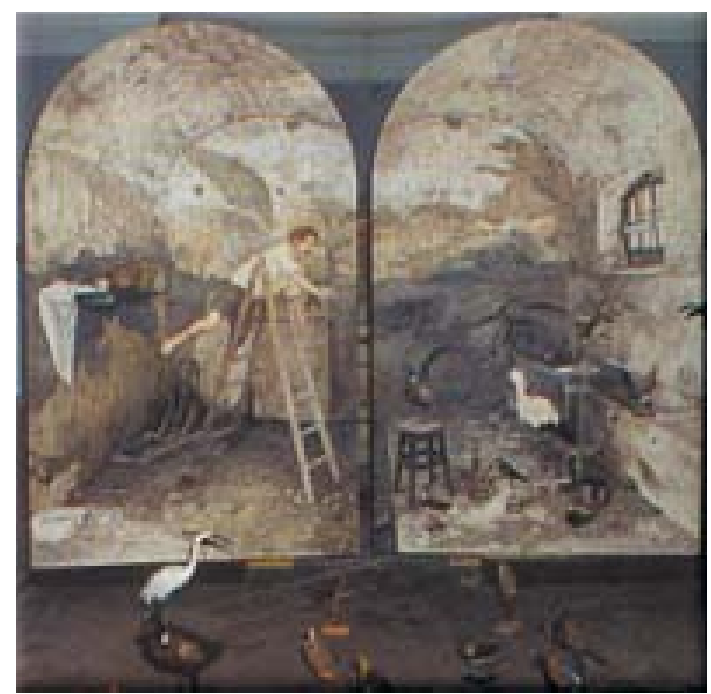

Figura 16 - L'Angelo coi baffi.

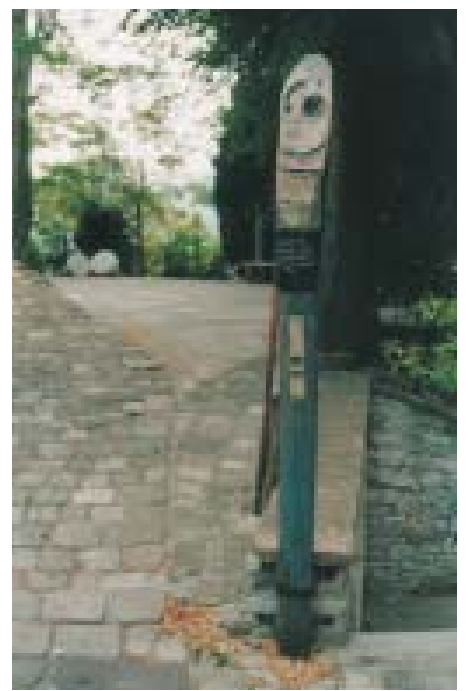

Figura 17 - Totem.

Si incontrano La strada delle meridiane in cui sono esposte altre sei meridiane artistiche appese agli edifici del borgo, l'Angelo coi baffi all'interno di una piccola chiesa - un dittico di Luigi Poiaghi affiancato dalla poesia omonima di Tonino Guerra, con coreografia di uccelli imbalsamati e riproduzione dei cinguettii - e totem lungo il percorso.

Il Santuario dei pensieri trasforma, mediante un restauro ideato dall'autore, i muri di un'antica abitazione del guasto malatestiano in un giardino raccolto.

Nel prato sono disposte sette pietre implicitamente fitomorfe, disposte secondo linee "magiche" spiegate in un'iscrizione. Il loro rapporto con la natura è nascosto ed esse assomigliano solo vagamente a cose già viste: un tulipano, una foglia. Lo spazio ospita poche cose e nessuna decorazione.

Le parole di Tonino Guerra invitano, prima di entrare, altre parole - quelle del visitatore - da rivelare ai "sette confessori muti". Poi l'autore esce di scena aspettandosi il silenzio, ma rimanendo comunque, nel rito, un celebrante:

"Mi nascosi dietro ad un muretto e cercai di raccogliere il silenzio come si prende un uccello in trappola. Una volta registrato il silenzio, sapevo che in quel silenzio poteva inserirsi un grido o la risata o la sillaba mistica di un Dio purchessia che fa vibrare il nulla e ripete l'atto della creazione, con la miseria degli uomini e il gelo e il fuoco e l'acqua e tutte le cose piene di sostanza sonora"19.

Il timbro si alleggerisce al fine di una narrazione aperta il cui strumento sono i vuoti fra le pietre.

A Bascio il silenzio è costruito diversamente, nel Giardino pietrificato: ai piedi della torre malatestiana sono disposti i tappeti del ceramista Giovanni Urbinati, fra le pieghe del prato, a memoria dei grandi personaggi della storia e dell'arte che sono passati nel territorio vicino, Giotto, Dante, Buonconte da Montefeltro, Uguccione della Faggiola, Matteo da Bascio, Ezra Pound, Fanina dei Borboni.

Tonino Guerra esprime il ricordo come fosse una favola semplice, comunicabile a tutti, su cui si potrebbe anche chiaccherare. Non si crea però nessun rumore perché il racconto avviene a livello del terreno, fra piramidi e chiese minuscole, negli interstizi, lungo segni orizzontali che non costituiscono né massa né volume da consentirgli di entrare in vibrazione contrappuntistica con la torre eretta contro la vallata. 


\section{RIFERIMENTI BIBLIOGRAFICI}

BAnn Stephan, Giardino e arti visive. Arcadia, postclassico e "land art", in Mosser MONIQUe e TeYssot Georges, L'architettura dei giardini d'Occidente, Electa, Milano 1990, pagg. 491-502.

Bann Stephen, I giardini di Ian Hamilton Finlay, in Mosser Monique, Teyssot Georges, L'architettura dei giardini d'Occidente, Electa, Milano 1990, pagg. 518-520.

BANN STEPHen, Vivi ignorato: l'arte dei giardini di ian Hamilton Finlay, "Lotus", 88, Electa, Milano 1996, pagg. 94-131.

Giannini Rita, Guerra Tonino e Urbinati Giovanni, Il giardino pietrificato, Ramberti Arti Grafiche, Rimini.

GIANNINI RITA, Tonino Guerra e la sua valle, Raffaelli Editore, Rimini 1998.

Guerra Tonino, Con la poesia alle spalle, Edizioni Tipertì - Galleria Fabjbasaglia, Rimini 2001.

Guerra Tonino, Dizionario fantastico, Pietroneno Capitani, Rimini 2000.

Guerra Tonino, Il libro delle chiese abbandonate, Maggioli Editore, Rimini 1988.

Guerra Tonino, Il Polverone, Maggioli Editore, Rimini 1992.

GUERRA Tonino, Il Viaggio, Maggioli Editore, Rimini 1986.

Il tappeto sospeso - fontana monumentale, catalogo, Maurizio Montanari, Ravenna 1997.

LocATElli VitTORIO, Le architetture parlanti di Tonino Guerra, "ANAGKH" , 4, Alinea Editrice, Firenze 1993.

Panzini Franco (a cura di), Giardini di Tonino Guerra - Pennabilli (Pesaro) in Giardini delle Marche, Federico Motta, Milano 1998.

Sleiter Rossella, L'orto di Tonino, "Il Venerdì di Repubblica" del 14 luglio 1989.

Siti web: I luoghi dell'anima <http://www.montefeltro.net/pennabilli.htm>

\section{RIFERIMENTI ICONOGRAFICI}

Figura 1: rielaborazione di Enrica Dall'Ara di foto Pubbliaerfoto in TURRI EUGENIO (a cura di), Adriatico mare d'Europa - La geografia e la storia, Arti grafiche Amilcare Pizzi S.p.A., Cinisello Balsamo (Milano) 1999, pag. 129.

Figura 2: rielaborazione di Enrica Dall'Ara di fotografia in SANTOLINI RICCARDO (a cura di), La Valle del Marecchia, Regione Emilia Romagna, Grafiche Zanini, Bologna 1988, pag. 68.

Figura 3: rielaborazione di Enrica Dall'Ara da Il tappeto sospeso - fontana monumentale, catalogo, Maurizio Montanari, Ravenna 1997.

Figure 4-10: fotografie di Enrica Dall'Ara.

Figure 11-12: rielaborazione degli elaborati grafici di progetto a cura di Enrica Dall'Ara.

Figure 13-15: fotografie di Enrica Dall'Ara.

Figura 16: da <http://www.montefeltro.net/pennabilli.htm>.

Figure 17-19: fotografie di Enrica Dall'Ara.

Figure 20-21: foto Luciano Liuzzi, in Giannini Rita, GuerRa Tonino e Urbinati Giovanni Il giardino pietrificato, Ramberti Arti Grafiche, Rimini. 
IL TAPPETO SOSPESO A CERVIA (RA)

$\begin{array}{ll}\text { Tipo di intervento: } & \text { giardino pubblico con fontana } \\ \text { Committente: } & \text { Comune di Cervia (RA) } \\ \text { Ideazione: } & \text { Tonino Guerra } \\ \text { Progetto e direzione tecnico-artistica: } & \text { Rita Ronconi , Claudio Lazzarini, Marco Bravura } \\ \text { Realizzazione musiva: } & \text { Marco Bravura } \\ \text { Realizzazione operativa: } & \text { Settore Lavori Pubblici - Servizio edilizia Pubblica - Comune di Cervia; } \\ & \text { Ufficio tecnico Romagna Acque S.p.A. } \\ \text { Imprese: } & \text { C.M.C.-Ravenna, Moviter Strade- Cervia, SATA Tecnologie per l'acqua- } \\ & \text { Riccione } \\ \text { Superficie: } & 47 \mathrm{mq} \\ \text { Realizzazione: } & 1997\end{array}$

L'ORTO DEI FRUTTI DIMENTICATI A PENNABILLI (PS)

Tipo di intervento:

Committente:

Ideazione:

Collezione botanica:

Realizzazioni artistiche:
Superficie:

Realizzazione:

Specie arboree antiche esposte: giardino pubblico, botanico ed espositivo

Comune di Cervia (RA)

Tonino Guerra

Carlo Pagani - Flora 2000 vivai - Budrio (BO)

Meridiana dell'Incontro (1996)

Scultore polacco

Meridiana umana-orologio solare orizzontale analemmatico (1990)

calcoli gnomonici e direzione lavori: Giovanni Paltrinieri

formelle in ceramica: Giovanni Urbinati

Tempietto dedicato a Tarkowski (1993)

architettura del tempietto: Celio Francioni

porta in ceramica Porticciola delle Lumache: Aldo Rontini

Bosco incantato (1995)

steli in pietra: Fr.lli Catani-Alfero (FC), su disegno di Tonino Guerra

lumaca in bronzo: Scultore marchigiano (donazione di Tonino Guerra)

Arco delle favole per gli occhi dell'infanzia (1995)

decorazioni in ceramica: Giovanni Urbinati

La Voce della foglia - fontana (1999)

scultura in legno: Gruppo di Ferro - Pennabilli

progettazione idraulica: Luigi Bernardi

Le Parole dei mesi (1998)

formelle in ceramica: Vaiolet Muki

ruota equinoziale in ceramica: Luigi Serafini (donazione di Tonino Guerra) Il Rifugio delle Madonne abbandonate (1994)

formelle in ceramica: Artisti vari

Promozione e realizzazione operativa: associazioni: Mostra Mercato Nazionale dell'Antiquariato - Pennabilli, Amici della Val Marecchia, Pro Loco - Pennabilli, in collaborazione con Comune di Pennabilli, Provincia di Pesaro-Urbino, Comunità Montana Alta Val Marecchia

$2015 \mathrm{mq}$

1988-1998

Meli: "Verdone”, "Limoncella", "Cotogno Gigante di Vranya”, "Rosa romana", "Renetta grigia di Torriana", "Luigi", "Lavina bianca", "Decio verde", "Durello di Forli", "Scudellino", "Puppino ferrarese", "Annurca", "Campanino", "Commercio", "Winter Winesap", "Renetta del Canadà", "Durello giallo", "Ruggine", "Renetta Doree”, "Delbard” - varietà moderna Peri: "S. Pietro", "Martin Pescatore", "Mora di Faenza", "Cannella", "Moscatello Gambolungo", "Garofanino”, "San Giovanni”, "Cotogno Periforme", "Zugnin", "Volpino", "Martin Secc", "Spadona Estiva", 
"Scipiona”, "Butirra Hardy", "La Praza”, "La Madernassa”, "Moscatello", "Curato"

Susini: "Agostiniana”, "Luglienga", "Biricoccolo", "Rusticano"

Ciliegi: "Durone della Marca", "Visciola nera" , "Durone di Vignola"

Peschi: "Buco Incavato", "S. Anna Calducci”, "Giambela”, "J. Hale"

Fichi: "Verdino", "Brogiotto bianco", "Brogiotto nero"

Frutti minori: mora, lampone, mirtillo, melograno "Gigante del

Convento", nespolo del giappone, nespolo goccia, giuggiolo, uva spina bianca, uva spina rossa, uva spina verde, ribes bianco, ribes rosso, ribes nero, azzaruolo rosso, azzaruolo giallo, mandorlo, "Zaccarella", sorbo domestico, noce nostrano, azzaruolo bianco, kaki "Vaniglia", castagno , "Castel del Rio", sambuco

Nel Giardino delle farfalle: Buddleia davidii

Piante officinali: Santolina spp., Lavandula spp., Salvia spp.

dono del Dalai Lama del Tibet: Morus nigra (gelso)

IL SANTUARIO DEI PENSIERI A PENNABILLI ( PS)

Tipo di intervento:

Ideazione:

Sculture in pietra:

Contributi di:

Promozione e realizzazione operativa:

Superficie:

Realizzazione: giardino privato aperto al pubblico

Tonino Guerra

Pietre rinvenute sul luogo e nuove realizzate su disegno di Tonino Guerra da Camillo e Lorenzo Catani - Riofreddo e dai fratelli Ballerini- Torriana Giovanni Urbinati, Giordano Geri, Luigi Piaghi, Aldo Canducci associazioni: Mostra Mercato Nazionale dell'Antiquariato-Pennabilli, Amici della Val Marecchia

$70 \mathrm{mq}$

1993

IL GIARDINO PIETRIFICATO A BASCIO DI PENNABILLI ( PS)

Tipo di intervento:

Finanziamento:

Ideazione:

Progetto dell'allestimento:

Tappeti in ceramica:

Promozione: associazioni:

Realizzazione: allestimento artistico

"Il Messaggero", giornale quotidiano, Roma

Tonino Guerra

Rita Ronconi

Giovanni Urbinati

Mostra Mercato Nazionale dell'Antiquariato-Pennabilli, Amici della Val Marecchia

1992 


\title{
IL PARCO URBANO DI VOLTERRA
}

\author{
Emanuela Morelli
}

\section{IL CONTESTO}

Quando si realizza un parco urbano dentro una grande città, gli elementi che guidano il processo progettuale sono riferiti principalmente all'intorno urbano e alla sua maglia compositiva, ai fruitori e all'ambiente sociale che lo avvolge, alle tradizioni degli spazi aperti che hanno contribuito alla formazione della città stessa.

$\mathrm{Nel}$ caso in cui si operi in un piccolo centro urbano, gli elementi precedenti restano comunque validi, ma a questi se ne associa un altro di grande importanza, che è il rapporto che nasce tra lo spazio urbano e lo spazio aperto circostante, ovvero con il paesaggio agrario e naturale.

Il parco - giardino viene quindi ad instaurare uno stretto legame, quasi come un elemento cardine, tra il nucleo storico dell'abitato e la campagna ed il bosco.

$\grave{E}$ qui che il progettista deve cogliere ed interpretare le componenti paesaggistiche dei due mondi, "affinché il progetto sia in sintonia con esse"1.

Volterra è una piccola città che sorge a coronamento di un elevato dosso collinare (531 m sul livello del mare), e riassume l'intera storia toscana: lucumonia (città-stato) durante il periodo etrusco, divenne municipio romano ed infine libero Comune nel periodo medievale. Il suo centro storico è ancor oggi ben

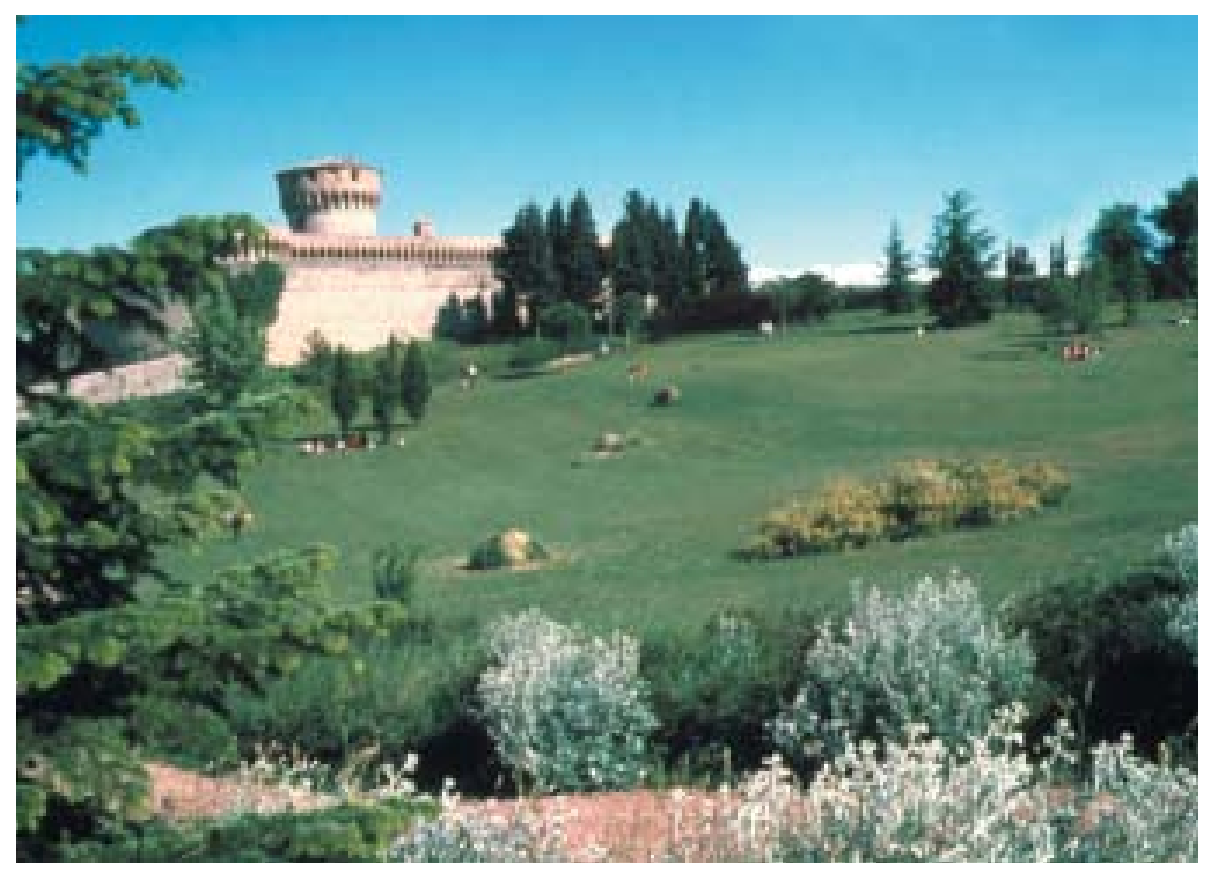

Figura 1 - Vista della Fortezza Medicea e dell'ampio prato centrale del parco urbano.

${ }^{1}$ Biagio Guccione, Paesaggio Parchi Giardini, unintroduzione all'architettura del paesaggio, Aquarius editore, Sesto Fiorentino (Fi) 1990, pag. 26. 


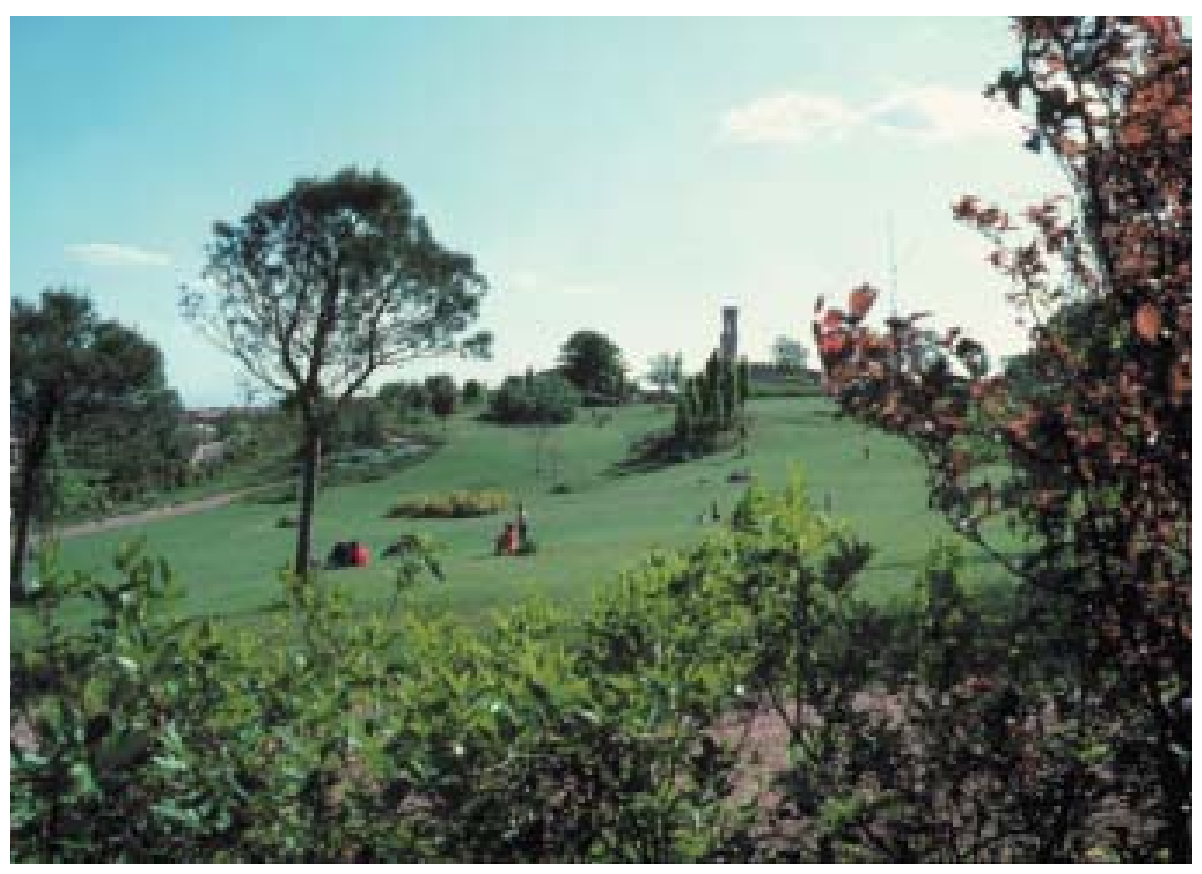

Figura 2 - Composizione della vegetazione nel parco.

conservato ed intatto, caratterizzato da pietra grigia locale che conferisce all'insieme un aspetto severo. Esso è delimitato dalla grande Fortezza medicea, utilizzata per molti anni come carcere, formata da due nuclei principali e dai cui bastioni si può osservare l'intero tessuto urbanistico e le ondulazioni collinari del paesaggio agricolo circostante che discendono verso il mare.

Il parco nasce tra la Fortezza e gli scavi archeologici delle terme romane, in un'area incolta ed abbandonata, dove sono presenti deboli tracce, per lo più tratti di filari di viti, del suo passato agricolo.

\section{GLI OBIETTIVI}

L'idea di recuperare quest'area incolta, in posizione strategica per il centro urbano di Volterra, parte dall'Amministrazione Comunale. L'intenzione è quella di creare un parco pubblico a corredo del centro urbano che deve fungere da raccordo tra questo e l'imponente Fortezza, gli scavi archeologici e la campagna circostante. Lintera operazione trova anche un importante sostenitore nella Cassa di Risparmio di Volterra, che ha finanziato la realizzazione del parco.

\section{LA METODOLOGIA}

Nel caso di piccoli centri urbani, anche quando vi sia un finanziatore privato come in questo caso, in genere le risorse economiche sono molto esigue. Ci si trova quindi davanti al problema di realizzare, e in seguito a mantenere, uno spazio che abbia una propria dignità nonostante i pochi mezzi a disposizione. Il paesaggista, oltre a fare i conti con il suolo presente che non sempre è di buona qualità, deve quindi trovare soluzioni formali che limitino i movimenti di terra $\mathrm{e}$ ad utilizzare specie botaniche e elementi di arredo il più possibile economici e che non creino problemi di manutenzione.

Nel 1972 l'architetto paesaggista Marco Pozzoli viene incaricato dall'Amministrazione Comunale di realizzare un progetto per il parco di Volterra. Già pre- 
cedentemente l'area era stata oggetto di studio da parte dell'arch. Salghetti che aveva elaborato un primo progetto. Così il paesaggista si trova a coordinare tre aspetti fondamentali per la realizzazione del parco: la rielaborazione del vecchio progetto, le esigue disponibilità economiche e instaurare un rapporto coerente tra la Fortezza medicea, oggetto di grande imponenza, la campagna circostante e gli scavi archeologici.

Si pone quindi l'obiettivo di limitare l'intervento alla sottolineatura dei caratteri del paesaggio esistente

\section{GLI ESITI}

Il risultato che ne scaturisce è un delicato e controllato impianto a verde, che nel rispetto del contesto storico e paesistico, tende ad esaltare i monumenti presenti.

Il parco si compone di un grande spazio centrale a prato a forma di anfiteatro, da cui si può ammirare l'immagine complessiva del centro urbano di Volterra. Questo nucleo viene poi definito da un viale, che si svolge perimetralmente e accompagnato solo in alcuni punti strategici da gruppi di arbusti accuratamente scelti nelle loro specie botaniche. Il percorso viene individuato in modo da poter accedere con facilità a luoghi che offrono punti panoramici di particolare valore.

La scelta del corredo vegetale segue l'obiettivo di assecondare il carattere monumentale della Fortezza e anche l'utilizzo delle specie arboree è molto limitato: le specie sono quasi tutte autoctone o comunque naturalizzate, come il leccio, il cipresso e il cedro dell'Atlante. La loro ubicazione segue motivi per lo più funzionali, quali quello di mitigare e nascondere brutte attrezzature, come i serbatoi d'acqua, o delimitare spazi particolari, come i campi gioco, o ancora guidare lo sguardo di chi usufruisce di questo ampio spazio.

L'insieme dei leggeri movimenti di terra, che digradano verso il muro della Fortezza, degli spazi aperti e dei gruppi di vegetazione, compongono un gioco di prospettive che collega percettivamente il visitatore a tutto l'intorno paesistico.

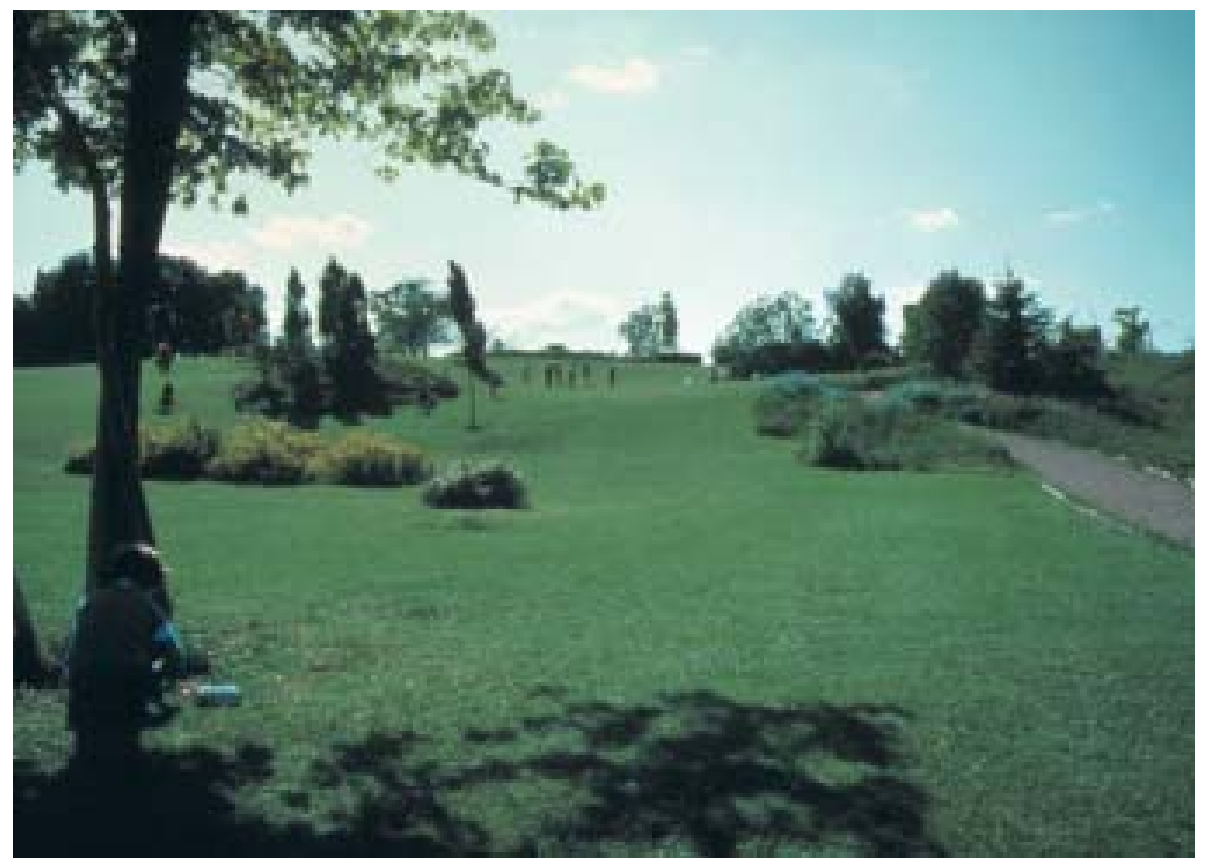

Figura 3 - Il sentiero che delimita l'ampio prato. 


\section{RIFERIMENTI BIBLIOGRAFICI}

Guccione Biagio, Paesaggio Parchi Giardini, unintroduzione all'architettura del paesaggio, Aquarius editrice, Sesto Fiorentino (Fi) 1990.

GuCCIONE BIAGIO, Il parco di Volterra, "Ville e Giardini", giugno 1983.

RIFERIMENTI ICONOGRAFICI

Le fotografie sono di Biagio Guccione.

\section{SCHEDA DI PROGETTO}

Tipo di intervento:

Località:

Committente:

Proprietà:

Progettista:

Anno progetto:

Anno di realizzazione:

Superficie complessiva:

\section{Finanziamento:}

Specie arboree impiegate: parco urbano

comune di Volterra, provincia di Pisa

Amministrazione Comunale e Cassa di Risparmio di Volterra

Amministrazione Comunale

rielaborazione di un vecchio progetto dell'Arch. Salghetti, da parte dell'architetto paesaggista Marco Pozzoli.

$1972-1973$

1974

circa 5-6 ettari

Cassa di Risparmio di Volterra

vegetazione autoctona o naturalizzata (cedro dell'atlante) 


\title{
IL PARCO AMENDOLA A MODENA
}

\author{
Michele Ercolini
}

\section{IL CONTESTO}

Il parco Amendola nasce in una (allora) disordinata e quanto mai desolata periferia residenziale della città di Modena. Un contesto del tutto privo di identità e di riconoscibilità tipico dei quartieri periferici italiani di inizio anni Settanta.

I progettisti, fin dalla prima versione del parco, hanno cercato di compensare a questa mancanza proponendo un segno forte (un riferimento lineare nordsud) in grado di dare un significato all'intera composizione architettonica.

\section{GLI OBIETTIVI}

Nel progetto del parco ritroviamo alcuni tra gli obiettivi più ricorrenti nel lavoro dei due progettisti:

- voler concepire il sistema dei percorsi "come fattore dinamico, linee e figure geometriche elementari di cemento grezzo che stabiliscono tensioni cariche di energia;

- voler analizzare gli alberi, nelle loro fisionomie di individui e quindi nel loro mutamento continuo nel tempo di forme, densità e colori;

- voler imporre l'idea iper-realistica del parco pubblico concepito come attrezzatura elementare hardcore, dura e indistruttibile quanto sicura, aperta e antigerarchica" ${ }^{1}$.

- voler compensare la mancanza di carattere e di identità del luogo, proponendo la creazione di un asse nordsud completamente indipendente dall'ambiente, ma in grado di dare un significato all'intero progetto.

\section{LA METODOLOGIA}

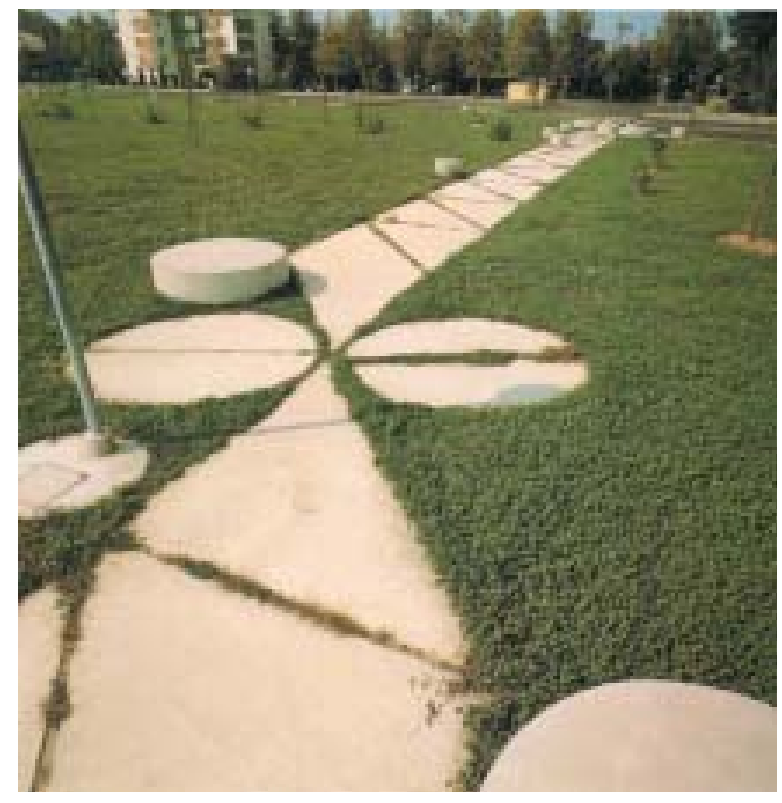

Figura 1 - Percorsi e sedute del parco (dettaglio).

La metodologia progettuale ruota attorno a due parametri visivi principali entrambi riferibili al concetto del tempo.

In primo luogo, la distribuzione della gamma di colori delle piante utilizzate si è basata sul loro aspetto di giorno, ossia alla luce del sole, ma anche di notte. Secondariamente, nella scelta dei luoghi destinati alle piante e alle specie selezionate, si sono considerati i mutamenti stagionali della vegetazione non solo però nella scelta degli alberi e degli arbusti sempre verdi oppure caduchi, ma anche in re- 
lazione al cromatismo delle loro foglie e dei loro fiori. "L'aspetto temporale del parco acquisisce così il significato dei riti stagionali e delle cerimonie, riconducibili all'epoca Neolitica in Europa"2.

\section{GLI ESITI}

Tutti gli elementi costitutivi del progetto si collocano lungo un asse nord-sud. Si comincia con un planetarium posto in testa all'asse, seguito da un anfiteatro in stile greco e da un lago di 7000 metri quadrati. A chiudere il semicerchio dell'anfiteatro, un'area gioco per bambini, una torre alta 50 metri dotata di sei riflettori e concepita come una sorta di meridiana notturna, principale fonte luminosa del parco medesimo, una zona ristoro comprendente un bar, una terrazza, i servizi. Si procede poi con un secondo lago più piccolo $(5000 \mathrm{mq})$, ed una serie di colline artificiali dotate di percorsi e realizzate utilizzando la terra estratta dal fondale dei laghi. Questi insoliti e curiosi terrapieni ricoperti di erba rievocano secondo Francisco Asensio Server, "i tumuli sacrali di sepoltura baltici"3.

Il parco Amendola è inoltre dotato di 500 parcheggi auto, di un ristorante, di un punto di incontro realizzato in un edificio preesistente e di aree gioco che fiancheggiano l'asse nord-sud. La struttura che ospita il ristorante è stata ampliata con un'interessante pergola radiale, una pista da ballo ed un teatro all'aperto.

L'arredo consiste molto semplicemente di sei fontane, cestini per i rifiuti ed un numero rilevante di sedute, tutte disegnate in esclusiva dall'Arch. Cesare

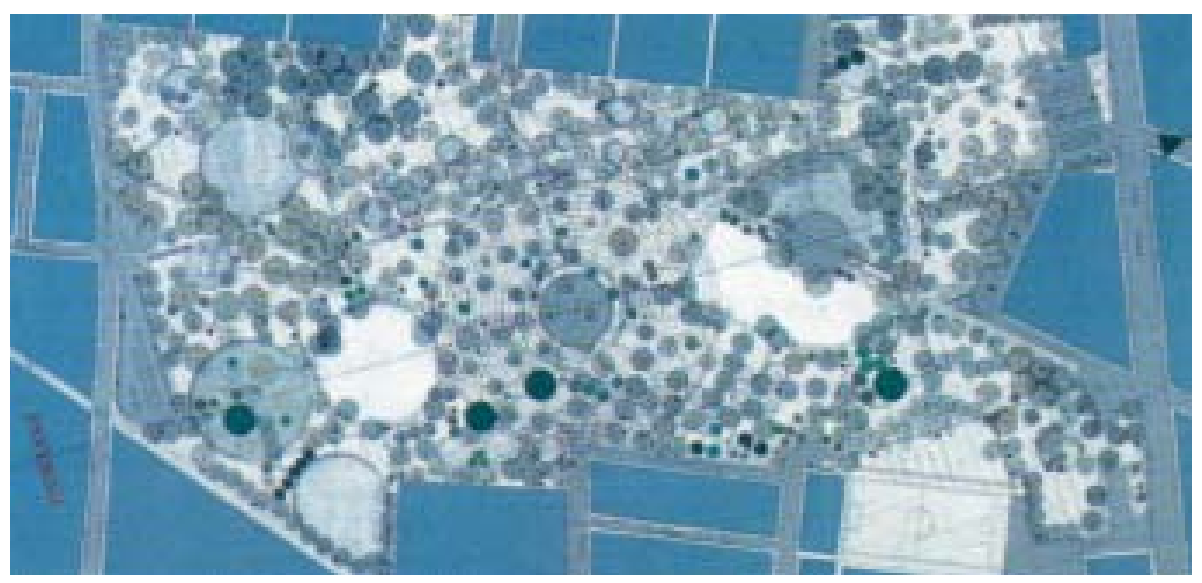

Figura 2 - Planimetria del progetto.
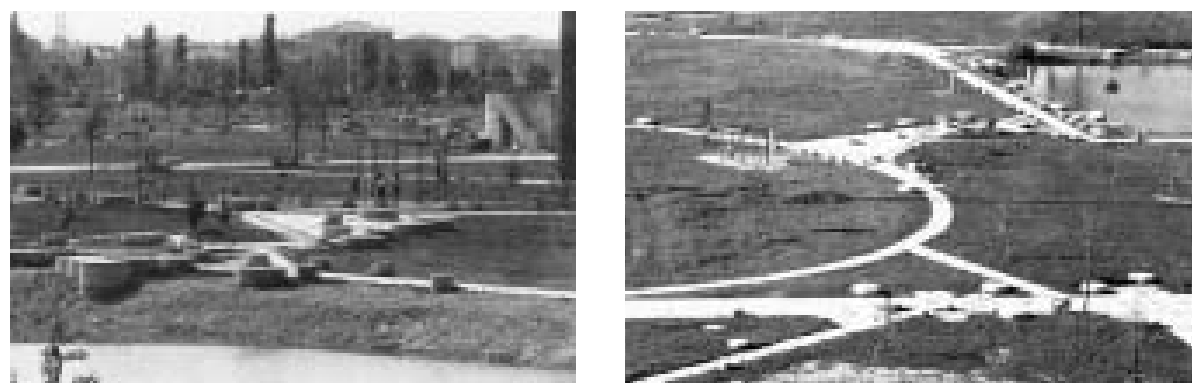

Figure 3, 4 - Vedute generali del parco a pochi mesi dall'inaugurazione.

${ }^{2}$ Francisco Asensio Cerver, Collana World of Environmental Design - Urban Space II (Urban Parks), Ed. Arco, Barcellona 1994, pagg. 100-101.

${ }^{3}$ Francisco Asensio Cerver, op. cit., 1994, pag. 100. 
Leonardi. In particolare le sedute colpiscono il visitatore per il loro design astratto di cilindri con diametro variabile che rileva un'insospettabile funzione ricreativa. A sua volta le pavimentazioni sperimentano un design giocoso di forme circolari, triangolari e trapezoidali quasi plasmate sul manto erboso capaci di risvegliare l'immaginazione e lo spirito creativo dei passanti.

Da un'attenta lettura del parco emergono soprattutto tre interessanti aspetti.

Il primo è rappresentato da questa forte geometria e da una perfetta regolarità caratterizzante tutti gli elementi del progetto: le panche circolari, le colline a forma conoidale, le pavimentazioni triangolari e trapezoidali, eccetera

In secondo luogo, il parco risulta non concepito come entità fisica immutata ma al contrario "vive" e cambia continuamente. "Il progetto stesso risponde a questo cambiamento in modo appropriato e contemporaneamente dimostra chiaramente la sintesi dialettica di elementi naturali ed artificiali" 4 .

$\mathrm{Ma}$ indubbiamente l'aspetto più significativo da sottolineare è il forte carattere di "identità" che il parco è riuscito ad imprimere ad una allora vuota e desolata periferia della città di Modena. Il parco Amendola infatti, in questi quasi trenta anni di vita ha assunto la funzione di vero e proprio "catalizzatore sociale" per tutti i quartieri limitrofi, ospitando anno dopo anno interessanti e seguitissime iniziative di carattere ricreativo e culturale.

Nel parco infine, ritroviamo un inedito e curioso particolare: un tentativo cioè di trasposizione della visione dell'albero, sempre concepito dai progettisti come una sorta di meridiana che, attraverso la sua ombra scandisce l'evoluzione del tempo, in un contesto notturno attraverso la realizzazione di una meridiana-faro costituita da un proiettore rotante la quale, segnando il passare delle ore della notte, propone uno scenario paesistico-visivo diverso ed insolito per un parco pubblico.

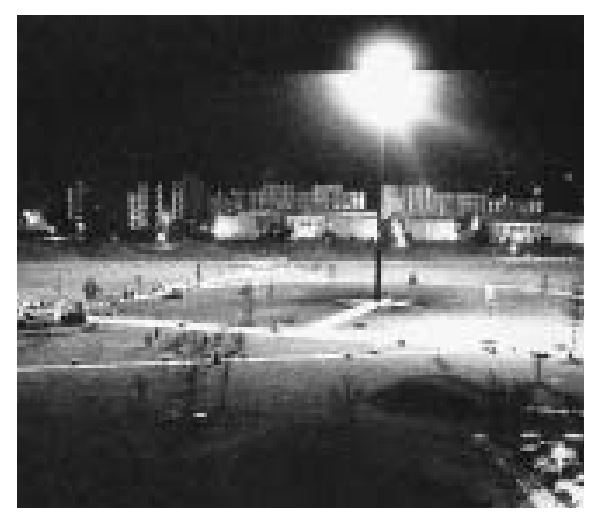

Figura 5 - Il parco illuminato dalla meridiana notturna.

\section{RIFERIMENTI BIBLIOGRAFICI}

Cerver Francisco Asensio, Collana World of Environmental Design - Urban Space II (Urban Parks), Ed. Arco, Barcellona 1994.

Guccione Biagio, Parchi e giardini contemporanei. Cenni sullo specifico paesaggistico, Ed. Alinea, Firenze 2001.

ZaGARI FranCO, L'architettura del giardino contemporaneo, Ed. Mondadori, Milano 1988.

Siti web: <www.comune.modena.it>

\section{RIFERIMENTI ICONOGRAFICI}

Figure 1, 3, 4, 5: rielaborazione di Michele Ercolini da ZAGARI FRANCO, L'architettura del giardino contemporaneo, Ed. Mondadori, Milano 1988, pag. 48.

Figura 2: rielaborazione di Michele Ercolini da Cerver Francisco AsEnsio, Collana World of Environmental Design - Urban Space II (Urban Parks), Ed. Arco, Barcellona 1994, pag. 100. 


\section{SCHEDA DI PROGETTO}

Progetto:

Luogo:

Committente:

Superficie:

Progettista:

Strutture presenti:

Anno di progettazione:

Anno di realizzazione: realizzazione di un parco urbano

periferia residenziale della città di Modena

Comune di Modena

24 ettari, suddivisi in 2 parti:

Parco Nord (Circoscrizione 4): superficie di 7 ettari, di cui 6 a verde

Parco Sud (Circoscrizione 3): superficie 17 ettari, di cui 16 a verde

Arch. Cesare Leonardi, in collaborazione con l'Arch. Franca Stagi

2 piste ciclabili;

aree gioco per bambini;

2 laghi;

bar e ristorante;

centro conferenze;

planetario;

anfiteatro all'aperto;

campo da calcio e pallavolo

1972

1979-1986 


\title{
Parco Raffaele Viviani a Napoll: IL PARCO DELLE GROTTE
}

\author{
Antonella Valentini
}

\section{IL CONTESTO}

Il parco si trova su di un versante della collina del Vomero, a Napoli, non distante dal complesso di S. Martino e S. Elmo. Nelle intenzioni dei suoi progettisti $^{1}$, esso rappresenta la "concretizzazione" di una ricerca che ruota attorno al tema del rapporto tra "siti, luoghi e paesaggi" come presupposto indispensabile per la creazione di nuovi spazi. Francesco Ghio precisa cosa intende con questi termini: sito è una parte di territorio ben delimitata, forse più mentalmente che fisicamente; i luoghi derivano dalla trasformazione dei siti attraverso l'opera continua dell'uomo che vi abita; il paesaggio "è legato al fenomeno mentale, intellettuale ed emotivo attraverso il quale i luoghi sono guardati, descritti, giudicati e interpretati, appunto come paesaggi"' ${ }^{2}$. Presupposto fondamentale per creare nuovi paesaggi è conoscere le qualità di un sito, la sua identità, le sue potenzialità, non solo quelle palesi ma anche quelle nascoste, e re-interpretarle progettualmente; il cosiddetto genius loci, di cui parla Christian Norberg Schulz ${ }^{3}$ deve essere, per Ghio, elaborato attraverso l'azione progettuale. La nostra storia è fatta di esperienze di questo tipo $^{4}$, che hanno portato alla realizzazione di paesaggi memorabili.

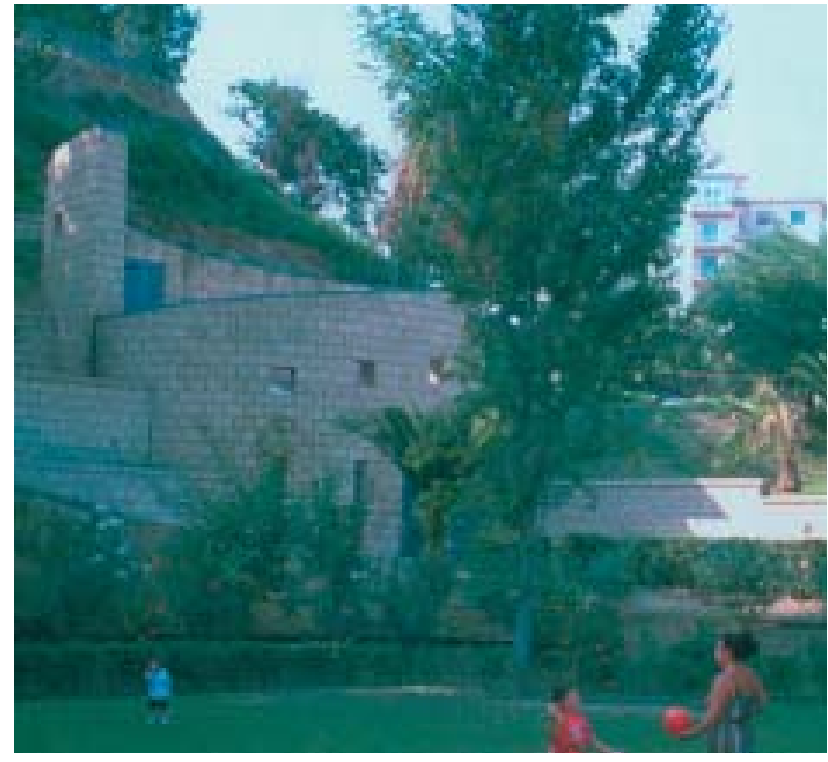

Figura 1 - Il pianoro per il gioco con la scala elicoidale sullo sfondo.

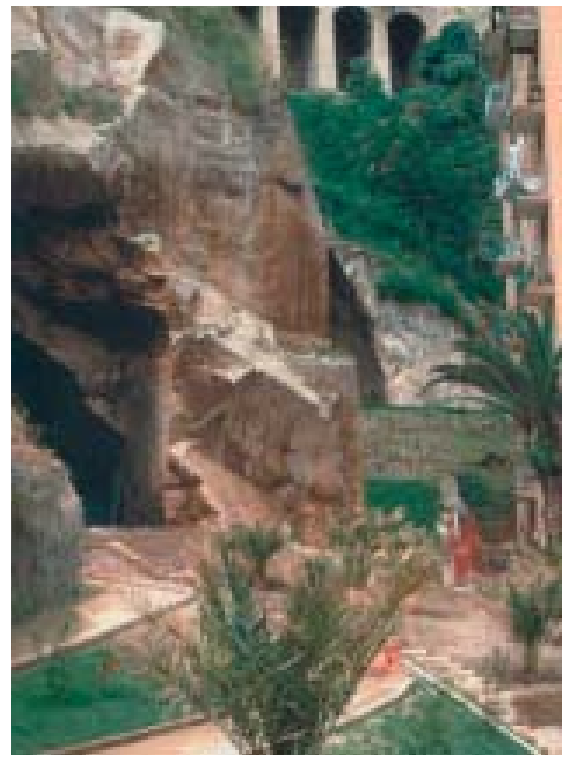

Figura 2 - Ingresso al parco al livello inferiore.

\footnotetext{
${ }^{1}$ Francesco Ghio e Vittoria Calzolari lo hanno progettato negli anni 1984-1990. Il parco è stato realizzato nel 1990-1993.

${ }^{2}$ FranCesCo GHIO, Siti e paesaggi, Alinea, Firenze 1999, pagg. 5-6.

${ }^{3}$ Christian Norberg Schultz, Genius Loci, Electa, Milano 1979.

${ }^{4}$ Francesco Ghio, nel suo libro, fa due esempi completamente diversi tra loro ma emblematici: Villa Farnese a Caprarola in Italia ed i giardini di Versailles in Francia (Cfr. FrANCESCO GHIO, op. cit., 1999, pagg. 6-9).
} 


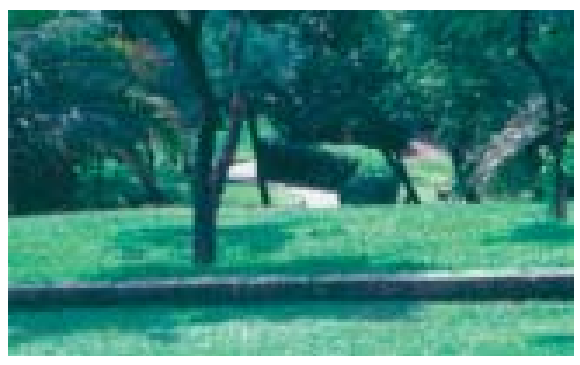

Figura 3 - Passeggiata degli agrumi.

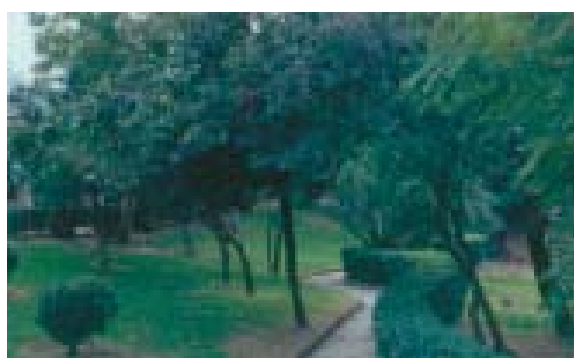

Figura 4 - Percorso lungo il pianoro di gioco.

\section{GLI OBIETTIVI}

Il progetto interpreta tre temi, distinti ma interconnessi: quello del rapporto tra il parco ed il sito su cui sorge, con i suoi caratteri morfologici e geologici; la relazione con il paesaggio naturale ed agrario che lo circonda; il rapporto, infine, con la città che lo ospita.

Il sito è caratterizzato da due costoni in tufo alti più di venticinque metri ed intervallati da un ampio pianoro. Alla base del costone più basso si può accedere ad una serie di grotte (ex cave di materiale da costruzione, poi sede di una serie di attività come carrozzerie, depositi e laboratori) che sono diventate elementi fondamentali del parco. Da qui parte una scala elicoidale ricavata nel pozzo che serviva a trasportare il materiale di cava ed emerge, con la sua struttura in tufo, a livello del pianoro intermedio. Anche a questo livello si trovano delle piccole grotte che possono ospitare mostre, spettacoli ed altre attività di quartiere.

Il contesto in cui è inserito il parco è caratterizzato da aree residuali di paesaggio agrario collinare costituito da orti, vigneti, frutteti ed agrumeti, di cui la sistemazione del parco evoca la presenza: una trama di alberi di palma è l'elemento ordinatore che richiama ed ingloba la trama residua del frutteto-agrumeto che anche qui era presente. Inoltre, questo spazio può costituire, insieme a tutti gli altri che ancora esistono sulla collina del Vomero, un "tassello" di un percorso verde che colleghi il parco Sant'Antonio ai Monti - nome con cui è conosciuto dai suoi abitanti il parco Raffaele Viviani - con S. Martino e S. Elmo.

Il terzo tema, il rapporto tra la città ed il parco, è risolto attraverso il progetto differenziato dei tre diversi accessi allo spazio pubblico legati ai tre livelli di quota che corrispondono però a differenti tipologie di fruitori e diverso carattere sociale dei quartieri sui quali si affacciano. L'ingresso al parco al livello più basso è preceduto da una piccola piazza che si apre nel quartiere densamente popolato del centro storico; l'accesso a quota intermedia è quasi la continuazione di una piccola strada che collega Corso Vittorio Emanuele con la sommità della collina; la terza porta di accesso al livello superiore è costituita da una piazzola belvedere dalla quale si gode della vista sul golfo.

\section{LA METODOLOGIA}

La scala elicoidale, su cui si aprono piccole feritoie che, lasciando filtrare la luce nel vano, ne consentono l'illuminazione e permettono molteplici viste sul parco, costituisce il fulcro del parco stesso, dove convergono tutti i percorsi del livello intermedio. Il tufo che riveste la scala è il materiale che caratterizza tutta la sistemazione,

${ }^{5}$ Il Parco Scampia nel quartiere di Secondigliano è stato progettato da un gruppo di tecnici coordinato da Giulio Fioravanti con la consulenza per gli aspetti paesaggistici degli architetti Carlo Bruschi, Valeria de Folly d'Auris e Ippolito Pizzetti. 


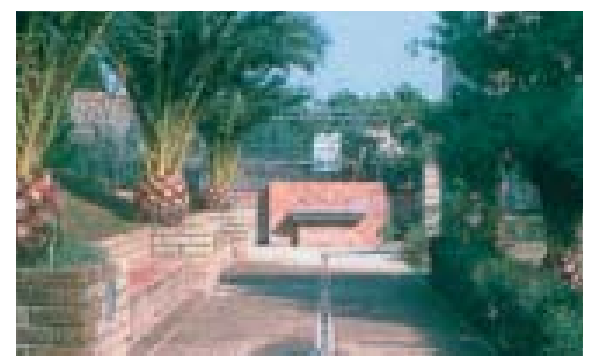

Figura 5 - Percorso dalla scala elicoidale lungo il pianoro.

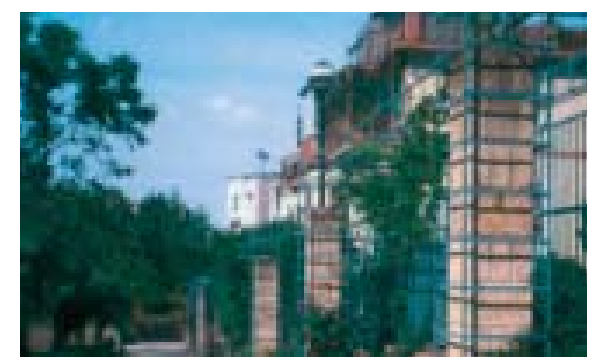

Figura 6 - Passeggiata pergolata.

uniformandosi all'esistente (gli speroni, le grotte, i resti di costruzioni preesistenti) ed è impiegato, con lavorazioni diverse, sia per i rivestimenti dei muri di sostegno e delle costruzioni, che per le pavimentazioni dei percorsi e scalinate. Nella parte centrale del parco è riproposta l'antica trama del frutteto ed agrumeto impiantato dai frati del convento che un tempo coltivavano il terreno. Le piante residue di questa coltivazione (albicocchi, peri, nespoli, noci, limoni, meli) sono state mantenute ed inserite in una nuova trama a grandi maglie quadrate, costituita da palme. Accanto agli alberi da frutto e fiore, variamente colorati, si contrappone una macchia verde di lecci, corbezzoli ed allori che ricopre la sommità degli speroni di tufo, ed una macchia costituita da vegetazione di diverse tonalità di verde e piccole fioriture primaverili (mirto, lavanda, rosmarino, cisto, eccetera) nelle scarpate meno ripide, in cui ci si immerge percorrendo i sentieri e le scalinate.

\section{GLI ESITI}

Il parco, che alla sua quota più bassa si affaccia sul quartiere popolare dell'Avvocata Monte Calvario, è molto frequentato e vissuto dalla popolazione locale, la quale ha costituito un gruppo di volontari che ne curano la gestione, organizzando le attività che si svolgono nelle grotte. Questo presidio fa sì che le grotte non siano diventate ricettacolo di usi e comportamenti deviati, ma siano effettivamente utilizzate per una serie di attività culturali e di svago, in collegamento con le scuole ed i comitati di quartiere.

Per fortuna, dunque, il parco Viviani ha avuto un diverso destino rispetto un altro parco napoletano, il parco Scampia ${ }^{5}$, inaugurato nello stesso anno 1994. Nato in un contesto degradato, non solo per essere stato in passato una discarica che l'intervento dei paesaggisti era riuscito a nobilitare con un progetto di grande sensibilità (che qui ha stemperato la forte connotazione architettonica iniziale), il parco di Secondigliano non è riuscito a vincere il degrado ambientale e sociale del quartiere ed a coinvolgere gli abitanti, presentandosi a pochi anni di distanza dalla sua nascita già in pessimo stato di conservazione.

\section{RIFERIMENTI BIBLIOGRAFICI}

GHio FranCesco, Siti e paesaggi, Alinea, Firenze 1999.

GHio Francesco, Il parco delle grotte al Vomero, "Architettura del Paesaggio", 4, 2000, pagg. 20-24. Guccione Biagio, Parchi e giardini contemporanei. Cenni sullo specifico paesaggistico, Alinea, Firenze 2001.

\section{RIFERIMENTI ICONOGRAFICI}

Le fotografie sono tratte da: GHIO FrAnCESCO, Siti e paesaggi, Alinea, Firenze 1999. 
SCHEDA DI PROGETTO

Progetto:

parco pubblico

Luogo:

collina del Vomero, Napoli

Committente:

Commissariato Straordinario di Napoli, Comune di Napoli

Progettisti:

Dott. Arch. Vittoria Calzolari, Dott. Arch. Francesco Ghio, Roma

Collaboratori:

L. Cannella, M.G. Cianci, I. Lutri, E. Von Normann

Anno di progettazione:

$1984-90$

Anno di realizzazione:

1990-93, inaugurazione 1994 


\title{
RIQUALIFICAZIONE PAESISTICO-AMBIENTALE \\ DI UN SITO INDUSTRIALE: \\ STABILIMENTO SNAM DI PANIGAGLIA
}

\author{
Antonella Valentini
}

\section{IL CONTESTO}

Lo stabilimento della SNAM ${ }^{1}$ di Panigaglia è collocato in un contesto di notevole valore ambientale e paesaggistico: all'interno del golfo di La Spezia, non lontano da Portovenere, in un ambiente ricco di formazioni vegetali appartenenti alla macchia mediterranea. Il complesso industriale si trova proprio sul mare ed alle sue spalle si eleva un fitto bosco di lecci. Quest'area, però, non fu scelta negli anni Sessanta per impiantarvi lo stabilimento di rigassificazione del gas naturale liquefatto $^{2}$ per i suoi caratteri di pregio, ma per la sua posizione strategica, lontano dai centri abitati e facilmente accessibile sia dalla terraferma che dal mare. Il golfo di La Spezia ospita diversi impianti industriali legati alla cantieristica navale, al settore energetico (centrale ENEL, area IP, centrale SNAM) ed alla Marina Militare. Di fatto gli impianti nascono su un'area artificiale di riporto, realizzata per ospitare dapprima una polveriera della Marina Militare e poi lo stabilimento SNAM, che ha realizzato anche un pontile di attracco per le navi metaniere.

La proposta di redigere un progetto di inserimento paesaggistico dello stabilimento industriale avviene molti anni più tardi, quando la legislazione e la sensibilità per questo genere di argomenti è notevolmente mutata. Infatti, anche in Italia iniziano ad essere realizzati vari interventi volti al recupero ambientale di aree di grande valenza paesistica come questa ma degradate dalla presenza di atti-

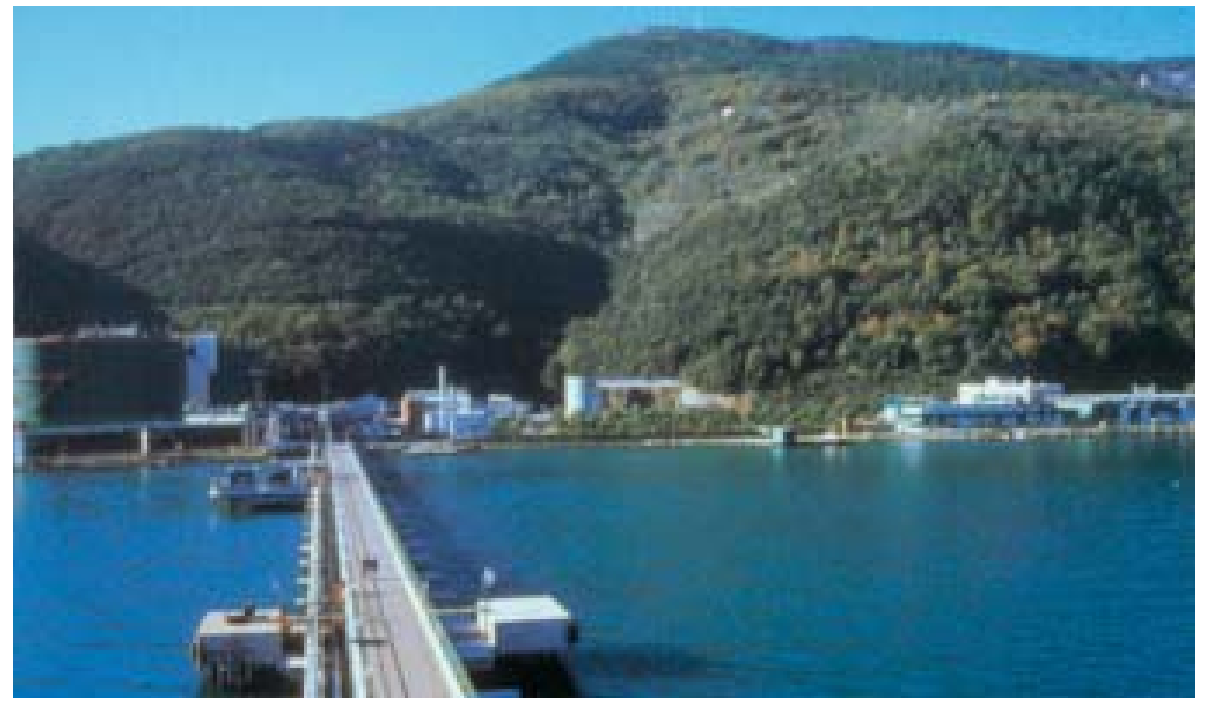

Figura 1 - Veduta dello stabilimento dal mare.

\footnotetext{
${ }^{1}$ Società Nazionale Approvvigionamento Metano, facente parte dal 1953 del Gruppo Eni (Ente Nazionale Idrocarburi).

${ }^{2}$ Lo stabilimento di gas naturale liquefatto (Gnl) comprende impianti di vaporizzazione, serbatoi ed un pontile di attracco lungo 500 metri.
} 


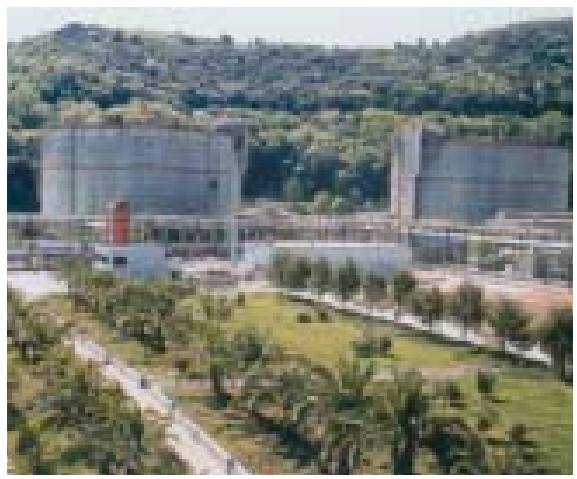

Figura 2 - Veduta verso i silos.

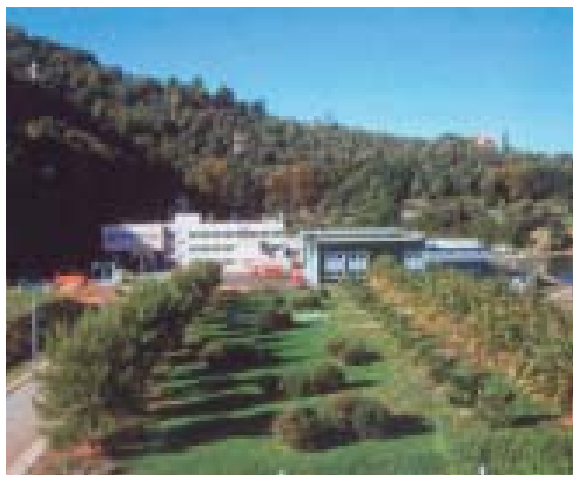

Figura 3 - Veduta verso gli uffici.

vità antropiche. In questi ultimi anni si assiste inoltre al diffondersi di grandi complessi di edifici per affari (siano essi industrie, uffici o centri commerciali) per i quali viene previsto un vero e proprio progetto di inserimento paesaggistico. I "business park" stanno diventando un campo di attività sempre più esplorato dai paesaggisti italiani.

Tra questi progetti possiamo citare il Centro Cavagnari a Parma ${ }^{3}$ o lo stabilimento Loro Piana a Roccapietra ${ }^{4}$, che hanno un minimo comune denominatore oltre i progettisti: il superamento della natura prettamente "industriale" del luogo per inserirsi nel paesaggio circostante, che nel primo caso è la pianura padana, con le sue orditure geometriche della centuriazione romana e nel secondo caso il paesaggio montano, con i suoi torrenti ed affioramenti rocciosi.

\section{GLI OBIETTIVI}

Alla scadenza ventennale per il rinnovo della concessione industriale, il Ministero dei Lavori Pubblici ordinò la redazione di un progetto di riqualificazione paesistico-ambientale, peraltro previsto dal Piano Territoriale di Coordinamento Paesaggistico Regionale, come condizione indispensabile per procedere alla ristrutturazione degli impianti produttivi che dovevano essere adeguati alle nuove tecniche di trasformazione. Alla fine degli anni Ottanta fu affidato alla Scuola di Specializzazione in Architettura del Paesaggio di Genova ${ }^{5}$ il progetto preliminare per la riqualificazione dell'area industriale ed a questo seguirono, nella prima metà degli anni Novanta, il progetto definitivo ed esecutivo ${ }^{6}$.

Il progetto prevedeva la ristrutturazione e l'adeguamento tecnologico dell' impianto industriale, con una conseguente compattazione degli edifici; in tal modo venivano liberate circa il $40 \%$ delle aree, che potevano essere quindi destinate alla riqualificazione architettonica dello stabilimento ed il suo inserimento ambientale e paesistico allo scopo di "ridisegnare" il rapporto tra l'area industriale ed il

\footnotetext{
${ }^{3}$ Centro destinato ad uffici, mensa, auditorium, magazzini e centrali tecnologiche della Cassa di Risparmio di Parma a Parma. Progetto architettonico di Vico Magistretti, progetto paesaggistico degli architetti Elena Balsari Berrone e Chiara Curami Balsari; anno di realizzazione 1985-88.

${ }^{4}$ Lanificio ing. Loro Piana \& C. a Roccapietra, in provincia di Vercelli. Progetto architettonico Arch. Dario Montagni, progetto paesaggistico Arch.tti Elena Balsari Berrone, Chiara Curami Balsari, Marco Bay; anno di realizzazione 1994-95.

${ }^{5}$ L'incarico di redigere il progetto preliminare fu affidato nel 1989 alla direttrice della Scuola di Specializzazione, Annalisa Maniglio Calcagno e ai suoi collaboratori, Francesca Mazzino e Giampiero Buffoni.

${ }^{6}$ Il progetto definitivo e quello esecutivo sono stati redatti nel 1992 dall'architetto Giampiero Buffoni per la parte architettonica e dalla dottoressa Francesca Neonato per la parte paesaggistica. Il progetto ha partecipato al Premio Mediterraneo del Paesaggio 2000 promosso dalla Junta de Andalucía, Région Languedoc-Rousillon e Regione Toscana.
} 
contesto naturale. Il principale elemento che caratterizza il progetto consiste proprio in questo: non si tratta di riconvertire un'area industriale dismessa, ma di progettare uno spazio di pertinenza di un'industria in piena attività, pensandolo però con caratteri legati al leisure che consentano anche una sua possibile futura riconversione ad altri usi.

\section{LA METODOLOGIA}

Il progetto si basa su una accurata analisi visiva dell'intero ambito paesistico in cui è inserito il complesso industriale, al fine di individuare i caratteri peculiari del luogo e definire gli elementi fondamentali attraverso i quali ottenere un miglioramento dell'immagine paesistica della baia nella sua complessità. La vista dello stabilimento, con i suoi serbatoi alti trenta metri, risultava particolarmente critica dal mare e dal golfo di La Spezia, mentre dalla strada provinciale l'impatto visivo era stato già ridotto con il rimboschimento delle pendici collinari. L'intervento di riqualificazione coinvolge sia le architetture sia, soprattutto, la vegetazione. La mitigazione dell'impatto visuale è ottenuta proprio attraverso l'utilizzo della vegetazione, la scelta dei materiali di finitura ed il trattamento cromatico dei manufatti architettonici con tonalità consone al paesaggio circostante ${ }^{7}$, oltre che attraverso il gioco altimetrico dei corpi di fabbrica. Due sono le operazioni che permettono di realizzare questo sistema del verde attorno agli edifici industriali: la dismissione degli impianti nell'area prospiciente il mare $(4.500 \mathrm{mq})$ e la dismissione di un'altra area a monte $(5.500 \mathrm{mq})$ in modo da creare una continuità con il paesaggio collinare retrostante.

\section{GLI ESITI}

La sistemazione dell'area prospiciente il mare consente di realizzare una "passeggiata a mare" che richiama molte situazioni della riviera ligure, lunga centoventi metri con un doppio filare di palme delle Canarie (Phoenix canariensis), che segue la linea artificiale della costa, ed un doppio filare di palmette di S. Pietro (Chamaerops humilis) e di palme delle Canarie lungo il percorso interno. Questa area, una "collinetta verde" di altezza massima centottanta centimetri, delimitata da muretti di contenimento e da piccole scarpate verso i percorsi pedonali e viari, viene attraversata da

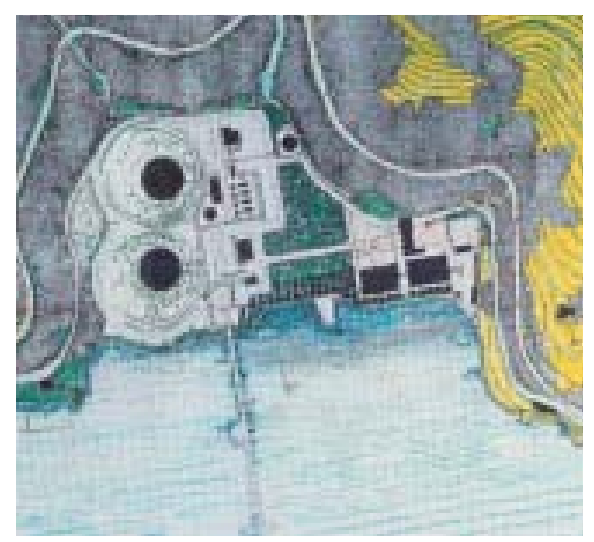

Figura 4 - Studio di inquadramento urbanistico e d'impatto paesistico. due percorsi tra loro perpendicolari pavimentati con masselli autobloccanti di colore grigio e beige. Accanto alla messa a dimora di alberi, i due doppi filari di palme ed il filare di pini d'Aleppo (Pinus halepensis) lungo la strada principale di accesso allo stabilimento, sono utilizzate piante tappezzanti (come timo, rosmarino, lavanda) scelte sia per l'effetto decorativo del loro fogliame che in funzione del loro diverso periodo di fioritura, e gruppi di arbusti (sempre caratteristici della macchia mediterranea come corbezzolo, lentisco, oleandro) posti in un'ampia zona a prato.

\footnotetext{
${ }^{7}$ Gli edifici civili hanno colori grigio e rosa che richiamano la tradizione ligure, gli edifici industriali varie tonalità di verde che corrispondono alle diverse sfumature della vegetazione.
} 


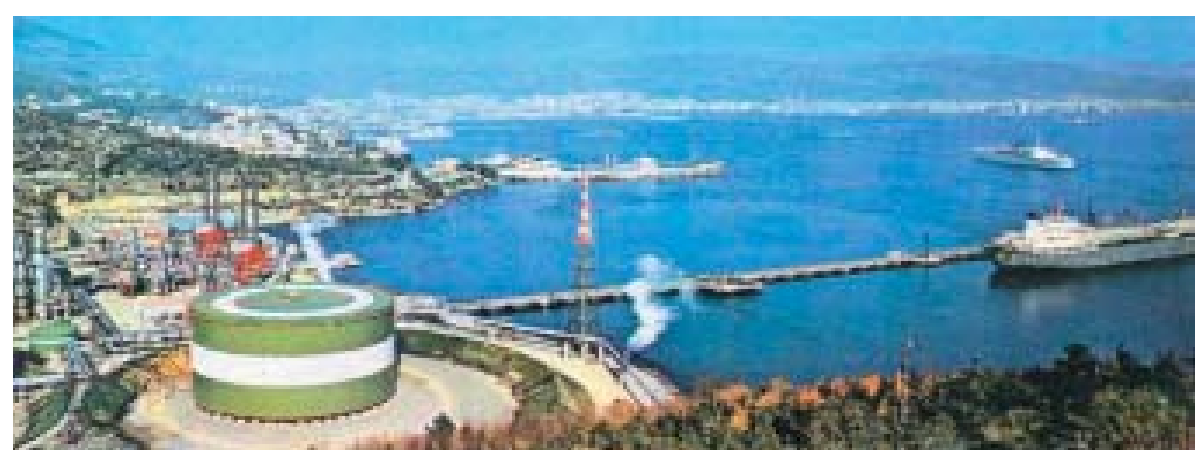

Figura 5 - L'area dello stabilimento Snam nel 1971.

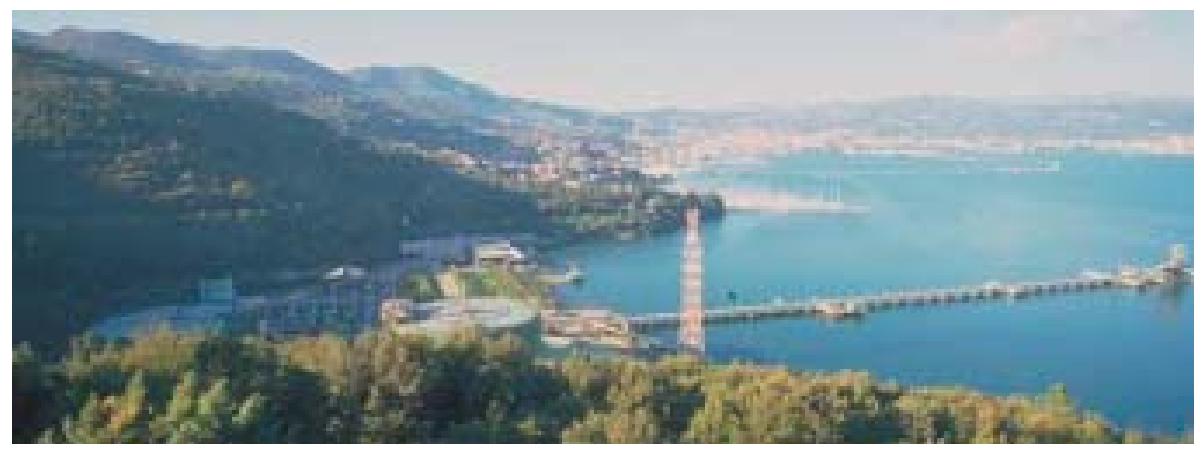

Figura 6 - Lo stabilimento nel 1998 dopo l'intervento di riqualificazione paesistico-ambientale.

La sistemazione dell'area a monte permette, invece, di realizzare l'integrazione con il paesaggio circostante attraverso la messa a dimora di specie arboree ed arbustive caratteristiche della macchia mediterranea (leccio, olivo, carrubo, mirto, fillirea, eccetera) ed il rimodellamento altimetrico delle pendici. Il progetto si caratterizza per questo graduale passaggio, dalla linea di costa alle colline, da elementi fortemente costruiti ed artificiali - la passeggiata alberata - ad elementi naturali e mimetici - la ricomposizione delle pendici boscate. La continuità paesaggistica tra l'area verso il mare e quella più interna è ottenuta attraverso il modellamento morfologico del terreno a creare delle piccole "onde" con cui realizzare anche la mitigazione visiva degli impianti.

\section{RIFERIMENTI BIBLIOGRAFICI}

Buffoni Giampiero e NeOnato Francesca, Un possibile equilibrio tra paesaggio e industria, "Folia di Acer", 5, 1997, pagg. 54-55.

Guccione Biagio, Limpianto GNL della Snam a Panigaglia, "Linea Verde", 9, 1997, pagg. 26-28. Guccione Biagio, Parchi e giardini contemporanei. Cenni sullo specifico paesaggistico, Alinea,

Firenze 2001.

Junta de Andalucía, Région languedoc-Rousillon, Regione Toscana, Premio Mediterraneo del paesaggio PMP 2000, Artes Gráficas, Sevilla 2001.

NeOnato Francesca, La convivenza possibile: lintervento, "Ecos", 3, 1997.

Rapporto Salute Sicurezza Ambiente 1998, Immobiliare Metanopoli Spa., 1998.

\section{RIFERIMENTI ICONOGRAFICI}

Figura 1: Junta de Andalucía, Région languedoc-Rousillon, Regione Toscana, Premio Mediterraneo del paesaggio PMP 2000, Artes Gráficas, Sevilla 2001, pag 33. 
Figure 2-3: GuCCIONe Biagio, Parchi e giardini contemporanei. Cenni sullo specifico paesaggistico, Alinea, Firenze 2001, pagg. 97-98.

Figura 4: BUfFONI GIAMPIERO e NEONATO FRANCESCA, Un possibile equilibrio tra paesaggio e industria, "Folia di Acer", 5, 1997, pag. 55.

Figure 5-6: fotografie di Francesca Neonato.

\section{SCHEDA DI PROGETTO}

Progetto:

Luogo:

Committente:

Tipologia di giardino:

Superficie:

Progettisti:

Direttore dei lavori:

Ditte esecutrici:

Anno di progettazione:

Anno di realizzazione:

Costo intervento: intervento di riqualificazione paesistico-ambientale di un sito industriale

Panigaglia, Comune di Portovenere (Provincia di La Spezia)

Snam S.p.a., San Donato Milanese (MI)

spazi aperti di stabilimento industriale

$135.000 \mathrm{mq}$ di cui $10.000 \mathrm{mq}$ di verde $80.000 \mathrm{mq}$ di bosco ed oliveto

$45.000 \mathrm{mq}$ stabilimenti

Dott. Arch. Annalisa Maniglio Calcagno, in qualità di Direttrice della Scuola di Specializzazione in Architettura del Paesaggio di Genova (Progetto preliminare) Dott. Arch. Giampiero Buffoni, Genova - opere architettoniche, Dott. Agr. Francesca Neonato, Milano - opere a verde (Progetto definitivo ed esecutivo)

Dott. Agr. Francesca Neonato, Milano

COFAR, Castelnuovo ne' Monti (RE)

COAF, Villafranca Lunigiana (MS)

1989 (progetto preliminare)

1992 (progetto definitivo ed esecutivo)

1994-1998

Area a verde suddivisa in tre fasi di attuazione:

$4.500 \mathrm{mq}-1^{\circ}$ fase

$5.500 \mathrm{mq}-2^{\circ}$ fase

$1.200 \mathrm{mq}-3^{\circ}$ fase

(di questa terza fase non è stato realizzato, per scelta della committenza, l'impianto di cogenerazione previsto)

3 miliardi di lire (per le opere a verde) 



\title{
SISTEMI FORTIFICATI
}

\author{
Maristella Storti
}

\section{DALla CONOSCENZA ALL'IDEA DEL PROGETTO “INTEGRATO”: ALCUNI ESEMPI DI INTERVENTO SUL PATRIMONIO FORTIFICATO ITALIANO}

La ri-progettazione degli spazi aperti "monumentali” è un tema ormai all'ordine del giorno e costituisce una corrente innovativa sotto molti punti di vista che implica l'acquisizione e l'integrazione di tutta una serie di considerazioni sul recupero, la tutela, la messa in valore e la gestione del patrimonio storico-artistico e ambientale nazionale.

L'interesse è rivolto alla valorizzazione di ciò che sta attorno ai monumenti piuttosto che agli immobili in sé in quanto architetture di un certo pregio, dando risalto alla dimensione e all'articolazione dei loro spazi aperti, così spesso trascurati e sottovalutati.

Solo di recente nella nostra cultura progettuale ha cominciato ad avere un certo rilievo lo spazio esterno al costruito; sono sotto agli occhi di tutti gli esiti degli ultimi cinquant'anni di urbanizzazione delle nostre città, sia sul versante della nuova progettazione urbana, sia su quello della tutela e del recupero dell'esistente. Infatti, se da un lato le lottizzazioni cresciute a macchia d'olio negli ultimi trent'anni hanno praticamente ignorato anche la realizzazione di quel minimo di disegno degli spazi aperti imposto dagli standard urbanistici di legge (D.M. n. 1444/68), permettendo la proliferazione di non luoghi, di aree-deposito abbandonate ai margini dell'urbanizzato, dall'altro l'imposizione del vincolo di tutela su gran parte del nostro patrimonio storico-artistico e ambientale, spesso non ha interessato (o, almeno, non è riuscita a "proteggere") i parchi, i giardini e le pertinenze di ville e palazzi storici, consentendo la perdita di questo enorme patrimonio, talvolta in favore di nuove arterie stradali oppure di nuove edificazioni che hanno cancellato grandi e piccoli sistemi monumentali di alto valore dal punto di vista ambientale e paesistico.

$\mathrm{Ci}$ troviamo di fronte a una quantità enorme di emergenze storico-artistiche, ambientali e paesaggistiche presenti sul territorio nazionale del cui recupero o stato di degrado il nostro tempo è responsabile: questo pensiero è in oggi alla base di molte azioni di intervento e di sensibilizzazione verso il problema ${ }^{1}$. Già da una decina d'anni o forse più, infatti, soprattutto per opera di associazioni ambientaliste e di studiosi illuminati, si è sentita l'esigenza di dare avvio a un movimento di opinione pubblica in favore dei beni culturali, cercando di promuovere la conoscenza come base indispensabile per qualsiasi azione sul nostro patrimonio, di cui l'impiego dei monumenti è uno degli aspetti più complessi.

Intervenire sul patrimonio "monumentale" per portarlo a nuova vita, riconoscere la sua portata culturale, comporta sia una presa di coscienza sulla totale

\footnotetext{
${ }^{1}$ Anna Barra, "Presentazione Archeoclub d'Italia", in Giuliano Bosco (a cura di), Progetti integrati per le antiche fortificazioni costiere, Atti del Convegno (La Spezia-Portovenere 16-17 maggio 1998) organizzato da "Archeoclub d'Italia" (Associazione nazionale per la tutela e la valorizzazione del patrimonio storico-artistico e ambientale), Agorà Edizioni, La Spezia 1999, pagg. XI-XII.
} 
entità delle valenze storico-artistiche, ambientali e paesistiche nazionali, sia una notevole integrazione di forze pubbliche e private capaci di condurre all'attuazione di scelte davvero implementabili e sostenibili.

È solo partendo dalla lettura storica del territorio che si individuano, tutelano e valorizzano i caratteri identitari e le risorse rilevanti, soprattutto in vista della loro futura progettazione "sostenibile" che può scaturire solo dalla conoscenza approfondita del nostro territorio in quanto "palinsesto" e "archivio". Occorre tutelare, conservare, mantenere le "tracce" della storia impresse sul territorio, preservando i monumenti dal loro degrado e, se possibile, intervenendo con il restauro solo laddove non se ne possa fare più a meno.

Viviamo in una fase della vita sociale ed economica del nostro paese in cui non si può più fare affidamento su determinate risorse che nel passato hanno poderosamente contribuito a sostenere lo sviluppo di certe zone. Lo sforzo dovrà andare nella direzione dell'individuazione di altre risorse, la cui valorizzazione possa consentire uno sviluppo dell'economia, del lavoro, della vita sociale, prefigurando l'uso alternativo di manufatti non più utilizzabili allo stato attuale. Fra le risorse disponibili per queste finalità, in molte parti d'Italia - come già si è fatto in parte per il recupero dei centri storici e delle opere d'arte - si può mettere in gioco un volume poderoso ed esplosivo: dal paesaggio dei terrazzamenti storico-agrari costieri e collinari alle valli e ai pascoli alti con i loro insediamenti, dai numerosi nuclei rurali alle testimonianze archeologiche, dalle rarità naturalistiche e geologiche ai castelli, ai forti, alle chiese, ai conventi ${ }^{2}$.

Si pone così il problema di riutilizzare una gigantesca risorsa partendo dal presupposto che le azioni di diffusione della conoscenza e di conservazione, in conseguenza del valore storico e documentario dei luoghi, devono condurre alla costruzione di un nuovo progetto d'uso degli stessi, necessariamente diverso da quello originario ma ugualmente caratterizzante.

In altri termini, la tutela, la manutenzione, la conservazione e il restauro, non sono sufficienti da soli a preservare il patrimonio culturale italiano; queste azioni di recupero devono essere supportate da un programma complesso che finalizzi i sistemi di beni culturali a nuove esigenze di vita, facendo propri anche gli strumenti della pianificazione urbanistica e territoriale, con conseguente pluralità di attori, fattori ed interessi.

Nel processo di costruzione di questa "rete" è indispensabile l'apporto di soggetti economici privati, in cui le scelte sulle finalità generali e specifiche degli interventi, l'uso e le modalità di azione e di fruizione dovrebbero però derivare dalle strategie delle amministrazioni locali e dalle loro politiche urbanistiche.

È necessario a questo punto almeno accennare a due questioni molto importanti relative al tema trattato: la prima, è che l'imposizione del vincolo monumentale (su sistemi fortificati, ville, palazzi, chiese, complessi monastici eccetera, e relativi spazi aperti), a cui è poi stata spesso sovrapposta anche una tutela ambientale e paesaggisti$\mathrm{ca}$, ha preservato le forme e le strutture originarie dei monumenti italiani, privilegiando però una posizione "passiva" di taglio conservativo portando frequentemente alla rovina se non alla perdita del bene tutelato. "Troppo spesso nelle nostre città si trovano i fondi per restaurare un monumento, un'opera d'arte, ma è molto difficile che si riesca a creare un progetto complessivo all'interno del quale i diversi restauri, pur dilazionati nel tempo, vengono ad avere un loro significato. Eppure, il restauro di un monumento non ha molto senso se questo non viene di nuovo integrato nella vita quotidiana, non viene di nuovo riportato ad un uso che sia adatto alla sua caratteristica originaria e che, allo stesso tempo, lo reinserisca nella vita della cittadinanza. Un monumento restaurato e non usato è un monumento destinato a morire di nuovo" ${ }^{3}$.

\footnotetext{
2 ANNA BARRA, Ibidem.

${ }^{3}$ ANNA BARRA, Ibidem.
} 
Una seconda questione importante, per entrare nel tema, è che tra i beni monumentali, quelli che appartengono al Demanio Pubblico (le servitù militari) hanno avuto un percorso di vita un po' diverso, riguardando aree off limits sino a quando lo Stato italiano, a partire dagli anni Ottanta circa, ne propose la loro dismissione attraverso apposite disposizioni legislative emanate caso per caso sul territorio nazionale. Lo scopo di questa operazione è stato, ed è tuttora, quello di portare denaro alle casse dello Stato e di rendere meno pesante la spesa riferibile al dicastero della Difesa. Gli Enti locali però, in genere, non hanno disponibilità finanziarie adeguate per l'acquisto dei monumenti e ancor meno potrebbero investire per il loro recupero. Oltre alla pesante questione del prezzo di vendita, la presenza dei vincoli ambientali è recepita come un ostacolo all'iniziativa del pubblico ma anche, e soprattutto, del privato. In un disegno di recupero misto pubblico-privato, le istituzioni hanno il compito di svolgere una funzione di ricomposizione organica di quello che fu un sistema monumentale e delle singole opere in quanto beni ambientali e paesaggistici, ma anche beni architettonici, talvolta esempi di archeologia militare, quindi meritevoli di essere oggetto della tutela culturale nazionale ${ }^{4}$.

La dismissione dei beni demaniali, tema attualissimo che vede sullo stesso campo Enti locali, Soprintendenze, Associazioni di volontariato, Fondazioni bancarie, Università, ai fini dell'attuazione di progetti di recupero sui beni monumentali italiani, deve essere finalizzata all'obiettivo della riutilizzazione dei beni stessi in funzione di una fruizione e di un interesse generali.

$\mathrm{Si}$ intende affrontare, quindi, la questione del "progetto integrato" 5 nel riuso di grandi spazi aperti monumentali, ancora oggi carichi di storia, di cultura e di memoria, rispettandone dignità e funzione primaria. "Un progetto complessivo che, muovendo dalle ragioni della tutela, sappia essere progetto finanziario, riorganizzato e ripensato in funzione turistica e culturale in grado di offrire: godimento estetico per l'oggetto in sé e per la posizione panoramica, supportata da gratificanti informazioni culturali; forme di turismo culturale giovanile e scolastico; forme per il passeggio e per lo sport; luoghi per convegni, seminari, incontri universitari e quant'altro, così da contribuire al rilancio dell'economia complessiva, piuttosto che immaginare questi beni come museo di se stessi" ${ }^{6}$.

Si prendono in considerazione grandi sistemi monumentali (quando per dimensione spaziale, quando per la loro importanza nel contesto nazionale), privilegiando esempi forse meno conosciuti rispetto ad altri, ma selezionati per il tipo di approccio intrapreso nella ri-progettazione di questi spazi e perché costituiscono sistemi di relazioni fra elementi naturali, architettonici e storici di una certa rilevanza nel contesto nazionale.

Sistemi considerati "monumentali" sia dalla legislazione italiana in materia di tutela, sia dalla nostra tradizione storico-culturale dato che come tali sono nelle nostre coscienze, almeno le più sensibili, per il loro alto valore storico, documentario, ambientale e paesaggistico.

Il sistema delle fortificazioni è una particolare categoria dei monumenti nazionali: un patrimonio sconosciuto, immenso e anche se oggi sono ormai numerosi gli studi settoriali, non abbiamo ancora un'idea esatta della quantità di fortificazioni che esistono in Italia fra castelli, torri costiere, cinte fortificate, forti di varie epoche, castellari anche preistorici.

Si pensa che una valutazione attendibile si aggiri sul dato dei 20.000-40.000 manufatti presenti in tutta Italia ${ }^{7}$.

\footnotetext{
${ }^{4}$ Per un approfondimento del tema specifico, si confronti GiULiano BosCo (a cura di), op. cit., 1999.

${ }^{5}$ Giuliano Bosco, Ibidem.

${ }^{6}$ Giuliano Bosco (a cura di), op. cit., 1999, pagg. XV-XVII.

${ }^{7}$ Giuliano Bosco, Ibidem.
} 
Tutte queste fortificazioni, importanti architetture e testimonianze storiche, stanno subendo un degrado continuo e progressivo pur essendo un patrimonio enorme, importantissimo sia per la storia dell'architettura, sia per la nostra storia civile. Ai fini del loro recupero è necessaria perciò un'azione che non riguardi soltanto il tema dell'architettura militare e difensiva, ma che si rivolga alla complessa economia dei luoghi.

A maggior ragione in questi contesti, la straordinaria fusione tra risorse naturali, ambientali ed antropiche necessita di progetti unitari, perché le fortificazioni costituiscono una parte molto singolare delle categorie dei beni appartenenti al patrimonio nazionale, in quanto "emergenze" talvolta di una tale imponenza da non poterne circoscrivere localmente il raggio d'azione rispetto all'ambito di appartenenza.

Il tema delle fortificazioni storiche di crinale, in particolare, è legato a quello della fruizione dei panorami; il giro d'orizzonte era tipico della loro funzione originaria che è convertibile in oggi proprio nella nuova fruizione ed utilità di queste emergenze paesistiche: ad esempio nuove "porte" di città o "rifugi" e "punti di arrivo" delle nostre aree collinari.

Si tratta di un grande patrimonio, questo, di beni architettonici spesso dismessi, eppure le fortificazioni storiche (medioevali, napoleoniche, post-unitarie) rappresentano segni forti di riconoscibilità dei luoghi, tali da poter prefigurare nuove opportunità per l'instaurazione di un rapporto strategico con il territorio nel quale sono inserite. E ciò in quanto queste architetture, viste come nuove dimensioni spaziali soprattutto nei sistemi collinare e costiero, costituiscono delle presenze e delle tracce che esprimono relazioni e significati tali da doverli necessariamente ripensare nel contesto della città attuale.

Progettare la reintegrazione di queste emergenze storico-architettoniche nella società contemporanea, significa intervenire sui manufatti e i relativi sistemi di spazi aperti in modo da consentirne, non solo il mantenimento delle strutture murarie ma, soprattutto, un uso consono alla loro importanza nel territorio, rispettandone le caratteristiche originarie ed i "limiti prestazionali".

Gli esempi riportati in questo contesto sono relativi al recupero culturale, ambientale e paesistico di fortificazioni urbane, di "sistemi di fortificazioni", con quel grande potenziale di conoscenza, di cultura, di ricreazione che il sistema nel suo insieme costituisce.

Ogni situazione di insieme richiede però una mirata e diversificata azione d'intervento coerente con il quadro strategico complessivo. Si tratta quindi di coniugare le scelte di riequilibrio ambientale con lo sviluppo di iniziative tese ad un effettivo recupero e ad una gestione innovativa della realtà esistente, sia essa di tipo monumentale singola, sia in quanto inserita in un sistema urbano.

Si presentano quattro casi-studio, scelti tra i molti, esemplificativi di quattro diversi aspetti del tema della progettazione "integrata" (dal punto di vista ambientale, paesistico, economico e gestionale) di grandi spazi aperti monumentali: il colle e la fortezza di Osoppo a Udine, il sistema dei forti di Genova, l'illuminazione delle mura di Verona e infine la rocca di Ravenna.

\section{Il colle e la fortezza di Osoppo (Udine)}

Nel 1984, quando è stato avviato il piano di recupero del colle e della fortezza di Osoppo, il luogo appariva dominato dalla vegetazione e punteggiato di ruderi. La natura si stava riappropriando del sito, cancellando i segni della storia.

Una prima ripulitura della vegetazione, il rilievo e un'ampia ricerca sulle fonti storiche hanno consentito di scoprire una realtà insediativa più complessa, fatta di segni e tracce, in parte ancora coperte da macerie o interrate, e di coglierne il "significato" nell'ambito della storia del colle di Osoppo. Qui gli uomini si sono cimentati, dall'antichità fino all'epoca moderna, in un'imponente trasforma- 
zione del rilievo naturale, per farne una macchina da guerra, continuamente adeguata al modificarsi delle strategie militari, ridotta però, dopo i bombardamenti del 1945 e il sisma del 1976, a un coacervo di rovine.

Assumendo il "progetto" e il "parco" come strumenti rispettivamente di indirizzo e di valorizzazione di questo intreccio esemplare tra le componenti del paesaggio e gli elementi della storia del colle e della fortezza, per consentirne la fruizione e per immettervi nuove funzioni volute dalla comunità locale, l'Amministrazione Comunale di Osoppo ha promosso nei primi anni Ottanta il coinvolgimento di enti e operatori pubblici, avviando una serie di interventi di recupero in maniera autonoma ma all'interno di un disegno unitario, la maggior parte dei quali sono stati realizzati entro il $1995^{\circ}$.

\section{Il sistema dei forti di Genova}

"La città è mirabilmente situata ad anfiteatro sul mare (...). Fra la montagna (...) e il mare (...) la parte di Genova occupata dalle case e dai giardini è alta trecentoquattro volte Montmartre, ma la montagna nuda senza piante e case è molto più alta. Nel punto più alto della montagna è costruito un forte (...); i forti di Genova (...) dominano e la loro imponenza dà sicurezza”. Così Stendhal in "Memoire d'un touriste" nel 1837 descrive la città ammirandola a bordo del "Sully" al suo arrivo nel porto di Genova. Tuttora a chi giunge dal mare Genova offre lo spettacolo del suo anfiteatro naturale coronato da Mura e Forti.

Certo, il paesaggio è sempre più congestionato da costruzioni; il limite superiore della città si è alzato e la conseguente fascia verde di rispetto con le Mura che seguono il crinale si è assottigliata, ma la caratteristica conformazione del territorio della città di Genova è da sempre uno dei maggiori fattori della sua riconoscibilità, della sua identità urbana.

L'imponente complesso delle mura, costruito negli anni 1630-1633, note come le mura Nuove, e dei Forti edificati per la maggior parte intorno agli anni 1800-1833, alcuni situati lungo il tracciato della suddetta cinta ed altri isolati sulle alture circostanti, rappresentano sicuramente una delle componenti fondamentali dell'immagine della città, sia storica che contemporanea.

Si è citato un viaggiatore illustre quale Stendhal e la sua percezione della città portuale; oggi i turisti, nella stragrande maggioranza dei casi, giungono a Genova in auto o in treno e l'impatto visivo con la città avviene lungo le direttrici autostradali e ferroviarie costiere e della Val Polcevera.

Ebbene i forti, alcuni isolati, altri a formare un tutt'uno con le mura, sono gli alfieri, i portavoce, i rivelatori della città e rappresentano i primi segnali a scala monumentale che si colgono della "Superba".

Alla stessa stregua, arrivando dal mare, la loro silhouette inconfondibile è la prima che si staglia contro il cielo emergendo dalla linea blu dell'orizzonte. I forti spuntano all'improvviso, maestosi, dietro un palazzo, una curva, il profilo di un colle, a dare inequivocabilmente la posizione e l'orientamento di chi sta guardando.

Il Progetto Preliminare per il recupero, il riuso e la valorizzazione del sistema dei forti di Genova, intende esaltare le valenze ambientali, paesistiche e architettoniche di questi manufatti, per riportarli a nuova vita e a nuove funzioni per la collettività.

Il progetto di illuminazione di tutto il sistema, in particolare, visti i buoni risultati ottenuti di recente su alcuni forti, si pone come strumento indispensabile per aumentare l'impatto scenografico della città. Lo scopo è quello di rilanciare Genova come città turistica ed invogliare a valorizzare, nella giusta misura,

\footnotetext{
${ }^{8}$ Pierluigi Grandinetti, Il progetto di recupero della fortezza e del colle di Osoppo (Udine), "Casabella", 634, 1996, pagg. 12-23.
} 
i tesori d'arte e di architettura che così gelosamente custodisce, offrendoli quindi alla fruizione di un turismo maggiormente sensibile a questi aspetti.

Rispetto ad altre città, Genova "versione notturna" è una città piuttosto buia e tale particolarità colpisce il forestiero. Illuminare le fortificazioni sulle alture della città avrebbe quindi, per contrasto, un risultato più spettacolare; la cinta muraria illuminata costituirebbe il naturale alter ego del fronte portuale, definendo lo skyline dell'anfiteatro naturale in quel rapporto mare/monte, porto/mura così fondamentale per la città; segno inconfondibile di riconoscimento per il cittadino e di attrattiva per il turista.

Una cortina difensiva ininterrotta di circa dodici chilometri e di diciotto complessi fortificati immersi in un incomparabile scenario in parte urbano e in parte collinare, rappresentano un patrimonio culturale di architettura e storia che non ha eguali in Europa'.

\section{L'illuminazione delle mura di Verona}

La gestione sostenibile di una comunità locale e del suo territorio implica un'opera di valorizzazione globale delle risorse economiche, socioculturali e ambientali.

In città come Verona, dove le attività produttive si realizzano a livelli molto elevati, è indispensabile incrementare le opportunità di sviluppo garantendo allo stesso tempo che quantità di ricchezza e qualità della vita non diventino contrastanti.

La storia e le vicende economiche di Verona sono sempre state favorite dalla sua posizione geografica privilegiata: l'eredità lasciata nei secoli è quindi così varia e ricca da fare della città un luogo unico. Come spesso accade però, lo sviluppo urbano nel dopoguerra e l'abitudine di sfruttare disorganicamente il patrimonio monumentale, hanno fatto sì che parti significative dei beni della città non abbiano avuto negli anni il dovuto risalto.

È il caso dell'importante sistema di architettura militare e il recupero ambientale, il restauro e la valorizzazione di questi mirabili monumenti si prospettano oggi come interventi capaci di attivare investimenti e di produrre forme di occupazione.

Mura, castelli, torri, forti, bastioni: molte sono le opere fortificatorie diffuse sul territorio di Verona e provincia; in esse arte e tecnica si fondono in armoniose strutture. Si tratta di un patrimonio architettonico storico per lo più dismesso e dimenticato che più di altri beni è esposto al rischio del degrado, essendo venuta meno nel tempo la loro funzione originaria. Da qui la necessità di approfondire i temi del recupero e della valorizzazione prima di tutto attraverso la conoscenza accurata delle opere monumentali. Questo è un tema di grande attualità per le difficoltà che spesso incontrano le amministrazioni nell'individuare destinazioni d'uso appropriate, volte ad impedire la perdita di monumenti che costituiscono una straordinaria testimonianza di civiltà.

Il tema dell'illuminazione dei giardini e delle aree verdi, in particolare, impegna sempre più i paesaggisti e l'intervento, da tecnologico, diventa indispensabile progetto di paesaggio a maggior ragione in tutte le città fortificate (Verona, Lucca, Ferrara), dove le mura sono sopravvissute alla dismissione della funzione difensiva e militare e dove ci si pone il problema del loro riuso. Superato l'infelice periodo in cui con grande disinvoltura queste pregevoli strutture architettoniche venivano abbattute, oggi ci troviamo davanti straordinari manufatti di notevole pregio, nella maggior parte dei casi circondati da ampi spazi aperti. Pertanto, una volta che la loro fruizione viene aperta al pubblico, è indispensabile la sistemazione degli spazi verdi limitrofi con il duplice scopo, da una parte di ren-

\footnotetext{
${ }^{9}$ Comune di Genova, "Progetto Preliminare di recupero, riuso e valorizzazione del sistema dei forti di Genova", Settore Progetti Speciali, gennaio 2002.
} 
derli facilmente accessibili e gradevoli alla visita e dall'altra, di recuperare un quadro paesaggistico di solito pregevole, che nel corso del tempo per incuria o disattenzione ha perso l'originario carattere.

Il Progetto guida per l'illuminazione delle mura di Verona va in questo senso; integra l'intero programma di valorizzazione delle opere monumentali e interessa lo sviluppo della Cinta magistrale a destra del fiume Adige, che si estende per circa cinque chilometri.

Verona è città fortificata per eccellenza; la sua cinta muraria è un sistema tra i più importanti ed estesi d'Europa, uno dei più interessanti per la sua collocazione a cavallo d'Adige, per il paesaggio vario di collina e di pianura, per le stratificazioni di interventi di duemila anni.

Le Mura, infatti, documentano dal vivo l'origine e l'ultima evoluzione di un'arte oggi estinta come quella dell'architettura militare.

Dopo un periodo di eclissi e di distacco, l'opinione pubblica, gli studiosi, l'Amministrazione Comunale, oggi riscoprono per molteplici aspetti questa preziosa eredità storica, architettonica ed urbanistica.

$\mathrm{Si}$ riconosce che questo grandioso insieme di edifici e spazi, nonché di ambiente naturale, un tempo generato dalle ferree leggi di guerra, possa essere di ragguardevole vantaggio per la vita civile e per l'intera collettività; è un monumento che si arricchisce di nuovi significati. Questo ha fatto sì che l'Unesco dichiarasse il centro storico di Verona "Patrimonio storico e culturale dell'umanità" in quanto "esempio di eccezionale bellezza dell'evoluzione della città fortificata attraverso i secoli".

I criteri informatori della proposta per il recupero delle mura di Verona partono appunto dalla consapevolezza che la Cinta magistrale ha cambiato destinazione nella città con il passare del tempo ed è divenuta in epoca moderna anche un parco urbano ${ }^{10}$.

\section{La rocca di Ravenna}

La rocca di Ravenna è sostanzialmente un punto focale della città: un luogo ridotto ad un'enclave, una zona avulsa dal territorio circostante che solo nel corso degli ultimi anni è stato parzialmente aperto alla fruizione cittadina, prima con la sistemazione della "Cittadella" a giardino e poi con la sistemazione della rocca "Brancaleone" ad arena estiva, in occasione del Festival internazionale di Musica Classica ed Operistica, che nel corso degli anni ha attirato un sempre maggior numero di fruitori.

Il progetto è fondamentalmente una ricontestualizzazione dell'esistente, non avulsa dal reale, ma inserita in esso. Il leit-motiv portante dell'intero intervento è quello di riuscire ad imprimere una dinamica a tutto il progetto: un progetto in continua evoluzione.

Il monumento della rocca di Ravenna necessitava di un contesto che lo rendesse indipendente e tuttavia profondamente integrato in tutte le trasformazioni territoriali proposte. Non un episodio meramente aggregato ad altri spazi verdi della città, ma monumento versatile e flessibile polo di sviluppo ${ }^{11}$.

I quattro casi-studio esaminati si riferiscono a processi di recupero, riuso e valorizzazione degli spazi aperti monumentali che nascono talvolta perché incentivati dal lungo operato delle associazioni ambientaliste o storico-culturali che hanno a cuore il patrimonio nazionale; iniziative poi promosse e portate avanti

\footnotetext{
${ }^{10}$ Comune di Verona, "Progetto guida per l'illuminazione della Cinta magistrale a destra d'Adige (Verona)", 1999 e Biagio GuCCIONE, Illuminazione: mura di Verona, un esempio da seguire, "Lineaverde", nov./dic. 2001, pagg. 26-28.

${ }^{11}$ DaVide Brandoli e STEFANo BaldisSerRi, La rocca, il teatro, il giardino: un progetto per Ravenna, "Paesaggio urbano", sett./ott. 1993.
} 
soprattutto dagli Enti locali che si fanno promotori dei processi, pilotando l'insieme delle forze pubbliche e private che intervengono nel corso dell'iter procedurale delle azioni di intervento.

Questo è il caso di Osoppo, Genova e Verona: sono esempi di processi che essendo già avviati, anche se esperienze recentissime (l'approvazione del progetto genovese risale ai primi mesi del 2003), rappresentano l'inizio di un cammino verso la valorizzazione delle opere monumentali.

Ravenna rappresenta un caso a sé, in quanto il progetto che viene presentato non è in itinere ma è il prodotto di una tesi di laurea in "Arte dei Giardini e Architettura del Paesaggio", discussa nel 1991 al Politecnico di Milano. Viene inserita in questo contesto perché ritenuta meritevole di attenzione per obiettivi, metodologia e criteri adottati per l'esecuzione del progetto alla rocca di Ravenna.

Nel loro insieme, per opera di studiosi illuminati, di amministratori, di ambientalisti, di soprintendenti, di fondazioni bancarie o di studenti di architettura, questi casi fanno capire che è iniziata ormai da qualche anno un'azione di sensibilizzazione verso il recupero del nostro patrimonio, in particolare riferendoci a quello fortificatorio.

Questi quattro esempi permettono inoltre di percorrere, dalla scala territoriale a quella prettamente urbana, il tema della valorizzazione degli spazi monumentali partendo dal presupposto che è solo sulla base di un progetto integrato (con le risorse disponibili pubbliche e private, con gli operatori locali, con le nuove esigenze della collettività nel rispetto delle strutture originarie) che è possibile, oggi, procedere a programmi veramente implementabili e sostenibili. 


\title{
IL COLLE E LA FORTEZZA DI OSOPPO
}

\author{
Maristella Storti
}

\section{IL CONTESTO}

Il colle di Osoppo è un singolare rilievo friulano posto a nord del centro abitato omonimo, che emerge come baluardo naturale nel punto in cui il fiume Tagliamento incide le Prealpi.

Si tratta di un complesso di rilevante interesse storico e ambientale, caratterizzato dall'intreccio tra aspetti insediativi, geomorfologici e vegetazionali: infatti il colle di Osoppo rappresenta un insediamento fortificato assolutamente singolare nella compresenza tra manufatti di diversi periodi e nella sovrapposizione di più sistemi difensivi, nonché nella varietà e ricchezza delle specie vegetali presenti.

Il colle (dal 1923 monumento nazionale e dal 1978 parco di interesse regionale) ha avuto, proprio grazie alla sua posizione strategica, una lunghissima storia insediativa come luogo fortificato, almeno dal periodo romano fino alla seconda guerra mondiale.

La fortezza è stata oggetto di continue trasformazioni e distruzioni per episodi bellici o naturali o, ancora, per il modificarsi delle esigenze difensive.

Cessato l'uso militare, i bombardamenti del 1945 ed in seguito il terremoto del 1976 hanno provocato ulteriori crolli e danni alla struttura, per cui essa è diventata un coacervo di ruderi e di frammenti della sua lunga storia.

Nel 1984, all'avvio del piano di recupero della fortezza e del colle di Osoppo, su incarico dell'Amministrazione Comunale, il colle appariva dominato dalla

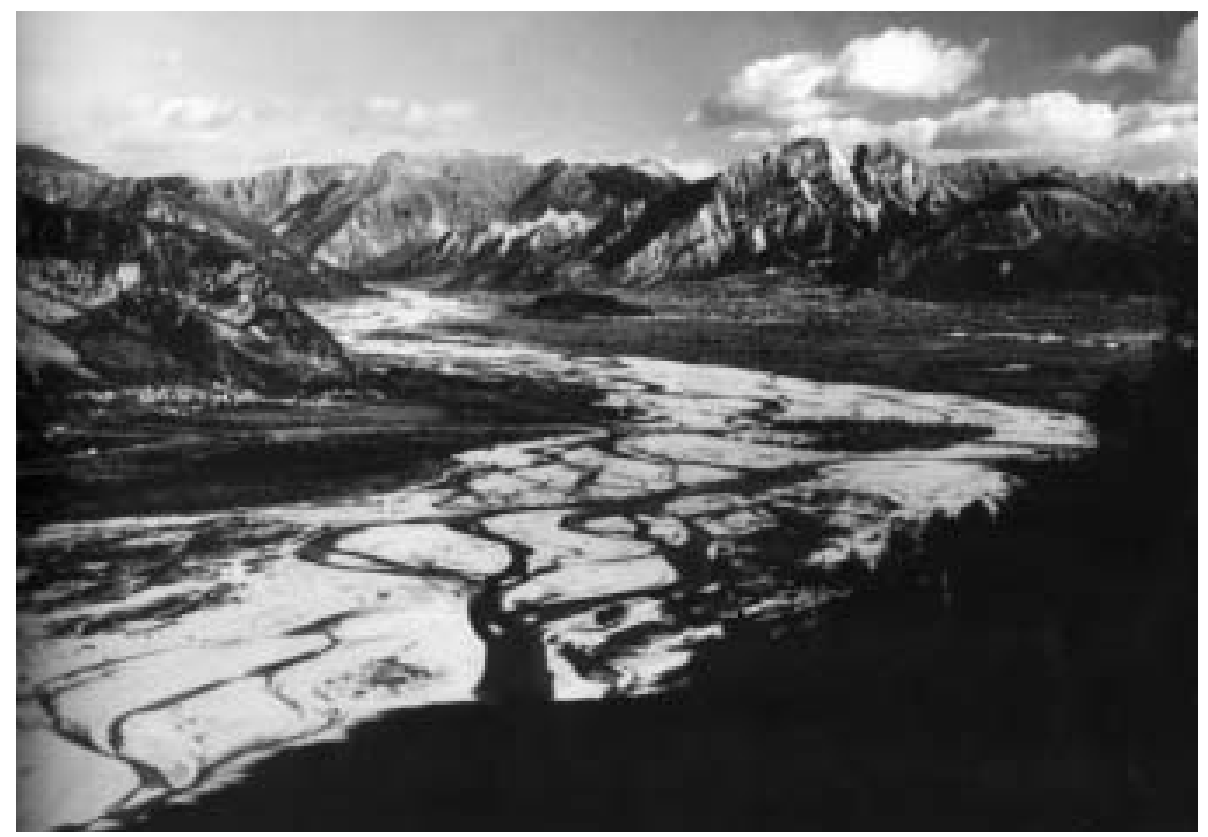

Figura 1 - Il colle di Osoppo al centro della valle del fiume Tagliamento (Udine). 


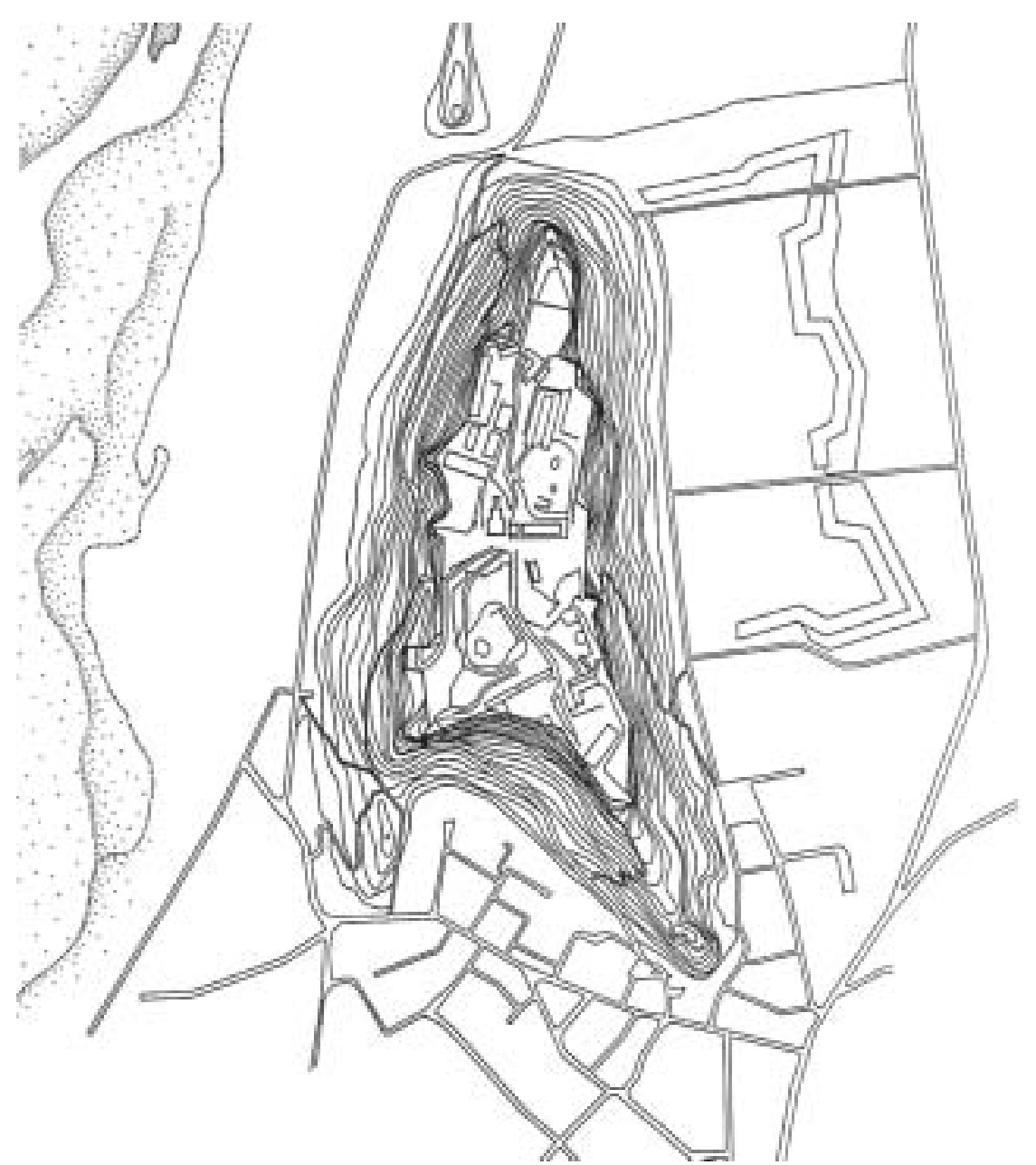

Figura 2 - Planimetria generale del colle e della fortezza di Osoppo e di parte del centro abitato.

vegetazione e punteggiato di ruderi, in quanto la natura si stava riappropriando del luogo, ricoprendo i segni della storia.

Nell'ambito del progetto generale (un "piano particolareggiato"), attraverso il rilievo del sito e delle strutture esistenti, è emersa - dopo un intervento consistente di pulizia della vegetazione - una realtà complessa, fatta di tracce murarie, di manufatti impraticabili, coperti da vegetazione e macerie o addirittura interrati.

Le conoscenze storiche disponibili per spiegare queste tracce, in gran parte indecifrabili, erano però scarse e frammentarie, per cui è stata avviata un'opera sistematica di raccolta e di interpretazione delle fonti storiche, attraverso la quale si sono potuti riscoprire e documentare i segni fisici del passato tuttora presenti, per tentare di coglierne il ruolo, il "significato" nell'ambito della storia della fortezza. Essa ha cominciato così a svelare la sua singolarità insediativa, la sua struttura "stratificata", per essere stata utilizzata per un lunghissimo arco di tempo come insediamento e struttura di difesa.

Qui gli uomini si sono cimentati in un'imponente trasformazione e costruzione del rilievo naturale, per farne una "macchina da guerra", continuamente adeguata al modificarsi delle strategie e delle tecniche militari.

Il colle, inoltre, è caratterizzato da un mosaico di situazioni microclimatiche che hanno favorito lo sviluppo di numerose formazioni vegetali, in alcuni casi (come il leccio o il pino nero) non comuni nel paesaggio collinare.

A ciò si è aggiunta l'azione dell'uomo attraverso il rimboschimento parziale delle pendici e la piantagione di specie ornamentali ed esotiche sull'altopiano. 


\section{GLI OBIETTIVI}

Se l'analisi ha messo in luce i caratteri di "unità" (implicita nei concetti stessi di colle e di fortezza) e insieme di "complessità" (nella compresenza di tanti elementi storici sovrapposti) costitutivi dei caratteri fondativi della "natura storica" del colle, il piano si è posto l'obiettivo di far riemergere e conservare i segni della storia della fortezza, nella loro stratificazione, anche attraverso la ricerca di un nuovo equilibrio tra natura e insediamento.

Questo non solo negli aspetti più propriamente insediativi (che sono quelli dominanti), ma anche nel loro combinarsi con la componente geomorfologica (il rilievo appare come un "monte" che emerge dalla piana, come lo chiamava Gerolamo Savorgnan) e con quella vegetazionale, per la ricchezza delle specie presenti.

Per scomporre e sciogliere la complessità data dall'intreccio delle diverse componenti, il progetto ha posto la conoscenza a capo di qualsiasi intervento, attraverso l'individuazione degli obiettivi e la messa a punto di tipi e categorie di intervento, giungendo, proprio attraverso il progetto, a una nuova "sintesi" di questa complessità.

Un tema, in particolare, è anche un obiettivo fondamentale del progetto: quello della "conservazione". La conservazione richiede, per esplicarsi, "giudizi di valore". Su che cosa basare tali giudizi? È giusto conservare la vegetazione cresciuta sulle macerie dei ruderi (che progressivamente li sta distruggendo) o eliminarla riportandoli alla luce?

È giusto conservare i riporti di terra "italiani" o rimuoverli per far riemergere i manufatti più antichi interrati? Non c'è una risposta univoca a tali domande.

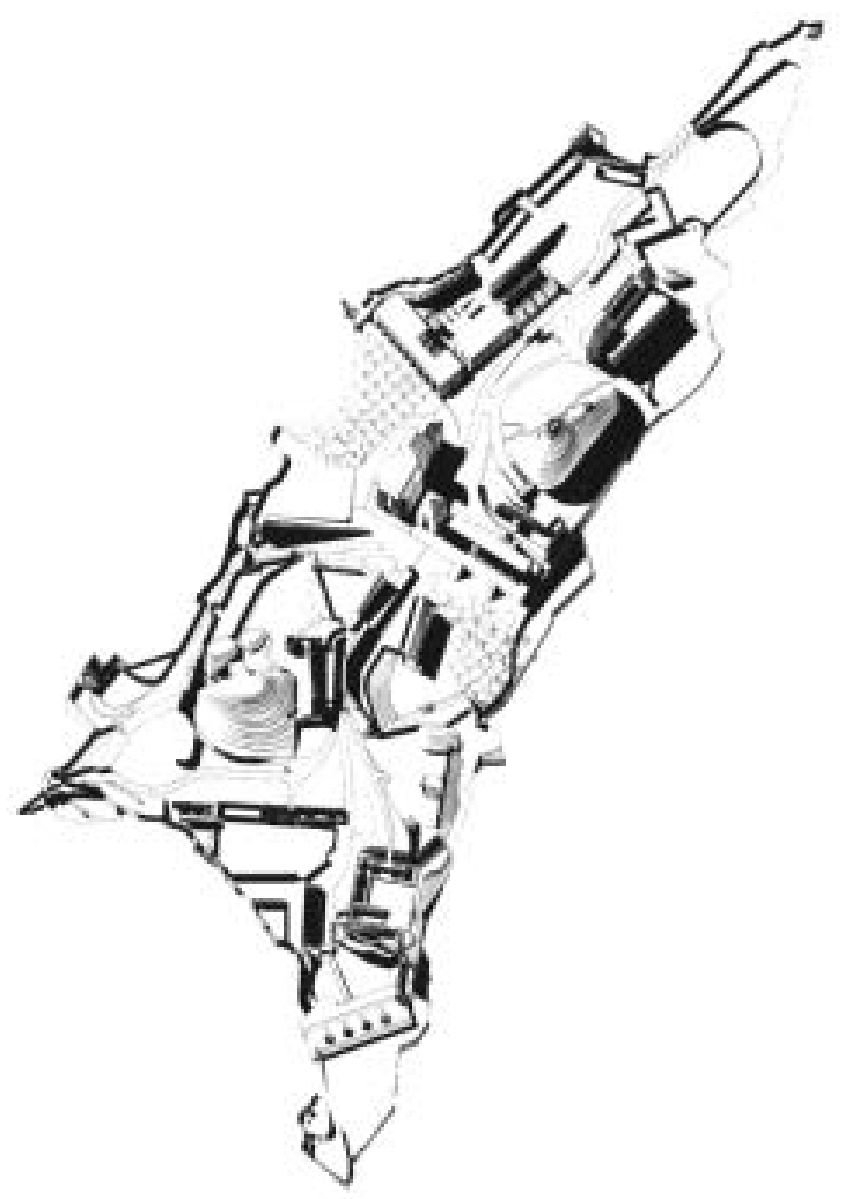

Figura 3 - Piano di recupero della fortezza di Osoppo: visione assonometrica. 


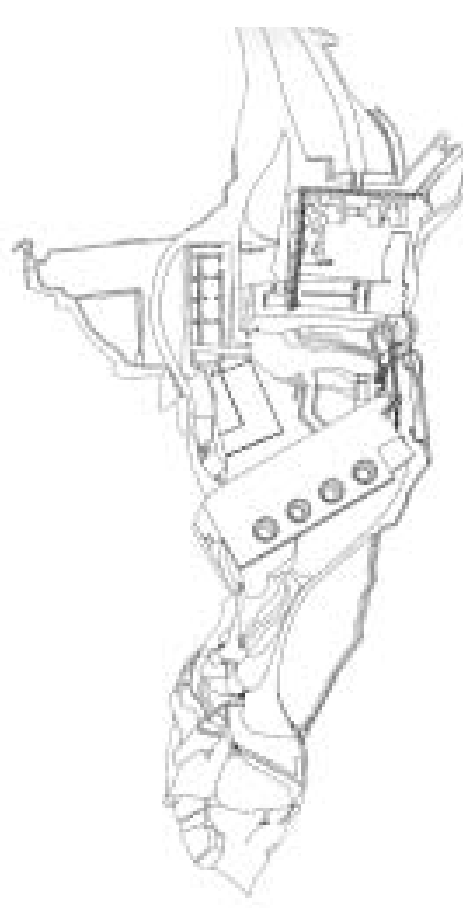

Figura 4 - La punta meridionale dell'altopiano: assonometria del progetto di piano.
I giudizi di valore vanno articolati e motivati. Ciò avviene, nel progetto, attraverso l'individuazione, per ogni sito, delle regole specifiche di costruzione insediativa dello spazio, nella sua storicità, assumendo i segni della storia come prodotto, spesso incompiuto, di tale costruzione: "storicità" che viene letta rispetto al singolo manufatto, ai sistemi di manufatti, alla struttura dell'insediamento, facendo emergere le tracce della storia e mostrandone il più possibile le stratificazioni e le discordanze. Nel caso della fortezza questo lavoro è reso più complesso dal fatto che il concetto di "conservazione" va applicato alla natura oltre che alla storia e dal fatto che all'obiettivo della conservazione si sovrappone quello della "rimessa in funzione" di alcuni elementi del sistema insediativo, per consentire la localizzazione di nuove attività, come chiede la comunità locale, che vede in prospettiva la fortezza non solo come "monumento", come luogo della memoria, come museo di se stessa, ma anche come risorsa, come luogo ricco di nuovi fermenti vitali.

Il recupero e la valorizzazione della fortezza, a partire dall'individuazione delle tracce materiali che di essa sono rimaste, costituisce il motivo conduttore del piano, il quale, riportando in luce e conservando tali segni, tende a far sì che la fortezza sia recuperata nel suo valore di "monumento" e di luogo della memoria.

\section{LA METODOLOGIA}

Il metodo adottato è fondato su due cardini fondamentali per l'impostazione progettuale:

- il riconoscimento del ruolo centrale del progetto, inteso non solo come momento di controllo degli aspetti fisico-formali e funzionali, ma anche e soprattutto come strumento di programmazione degli interventi, attraverso il quale il Comune di Osoppo ha coinvolto enti e operatori pubblici diversi, che hanno agito, pur nella loro autonomia, all'interno di un disegno unitario, di un programma integrato. Ciò ha consentito di realizzare compiutamente il recupero di una parte della fortezza e di aprirla al pubblico;

- l'utilizzazione dell'idea di parco come strumento in grado di dare risposte efficaci ai problemi di recupero e valorizzazione di situazioni insediative e ambientali complesse, nelle quali assume significato il rapporto tra elementi della natura ed elementi della storia.

La valorizzazione dell'identità delle parti e dei luoghi che compongono il colle e la fortezza di Osoppo deve inoltre tener conto della combinazione, ogni volta diversa, tra i segni della storia, la forma del sito, i caratteri della vegetazione, la configurazione del paesaggio: dalla fortezza come "monumento" alla fortezza come "parco".

A partire dal recupero del colle come bene culturale e ambientale, il piano ne prevede infine la valorizzazione come specifica risorsa territoriale, con l'immissione in essa di nuove funzioni connesse alla cultura, alla ricreazione, al turismo. Il piano si propone di organizzare e mostrare queste diverse possibilità d'uso: facendo emergere i segni della storia incorporati nell'attuale assetto del colle (organizzati nella forma del "parco archeologico"), rimettendo in funzione alcuni elementi del suo sistema insediativo, individuando tipi di attività congruenti con i caratteri specifici delle strutture da recuperare, selezionando e valorizzando la vegetazione (organizzata nella forma del "parco-arboreo"), al fine di consentire - in questo intreccio tra elementi artificiali e naturali - forme differenziate di fruizione.

Su questi criteri si fonda la progettazione delle parti e dei luoghi del colle, nella specificità delle regole di costruzione insediativa, storicamente determinate, 
che ciascuno di essi esprime. Ad essi viene anche riferita la progettazione degli elementi "nuovi".

Il "nuovo" può essere infatti, di volta in volta, strumento per valorizzare l'antico, momento di riconfigurazione dello spazio per consentire l'immissione di nuove funzioni all'interno dell'ex fortezza, operazione di completamento di manufatti in condizione di rudere (qualora sia richiesto il loro riuso), che agisce nello "scarto" tra ciò che oggi rimane dell'opera e la sua configurazione preesistente, assunta come prodotto delle trasformazioni subite nel corso della vita dell'edificio.

Il passaggio dall'analisi storica al progetto ha consentito l'individuazione del "significato" delle tracce ancora presenti, del ruolo cioè che i manufatti ad esse corrispondenti svolgevano all'interno della fortezza. Ad esempio, ha permesso di individuare diversi "sistemi difensivi", intesi come insiemi di opere (edifici e infrastrutture) realizzati in funzione appunto difensiva, corrispondenti a momenti storici significativi di funzionamento della fortezza, i quali diventano - una volta recuperati attraverso il progetto - il supporto a itinerari di fruizione turistico-culturale.

\section{GLI ESITI}

Assumendo il piano come strumento di direzione e indirizzo, l'Amministrazione Comunale ha promosso il coinvolgimento di enti e operatori pubblici, che hanno avviato una serie di interventi di recupero e valorizzazione in maniera autonoma ma all'interno di un disegno unitario. Infatti, l'analisi delle relazioni tra natura e insediamento, il riconoscimento delle tracce della storia nel loro organizzarsi in "sistemi difensivi" e l'individuazione degli obiettivi di progetto hanno consentito di articolare l'altopiano in tre "parti": settentrionale, meridionale e centrale, alle quali corrispondono modi diversi di recupero, di utilizzazione e specificazione progettuale del piano insieme con i successivi progetti esecutivi e i corrispondenti lavori, i più significativi realizzati tra il 1987 e il 1995.

\section{La "parte settentrionale"}

Anche se è l'ultima in ordine di priorità di realizzazione e al 1999 non ancora interessata dagli interventi, è storicamente la più recente.

Il piano si propone di far emergere e valorizzare quei segni di costruzione del sito (realizzati soprattutto nell'ultimo periodo italiano), che rappresentano oramai elementi costitutivi del paesaggio, organizzato per piani incassati e terrazzi, e individuando contemporaneamente le azioni necessarie a rimetterne in funzione alcune parti da destinare a strutture per attività culturali, sociali, ricreative, di servizio: le tracce murarie delle scuderie a ovest, l'ipotesi di una struttura polifunzionale sul terrapieno del magazzino italiano a est, il complesso delle caserme italiane (da utilizzare come "museo dei sistemi difensivi italiani") a sudovest, un nuovo parcheggio a nord.

\section{La "parte meridionale"}

A questa parte corrispondono i lavori più significativi effettuati dalla Soprintendenza, con il restauro delle mura venete dalla porta di accesso fino alla chiesa settecentesca di San Pietro (nella quale sono stati eseguiti gli scavi archeologici e il recupero della sagrestia a fini museali) e dalla Provincia di Udine, che ha sistemato la punta meridionale (con il castello cinquecentesco e il forte italiano) e ha avviato il restauro e il completamento della chiesa di San Pietro. La "parte meridionale " è infatti quella a più alto interesse storico, perché di più antico insediamento e perché presenta la maggior parte dei resti delle mura, delle strade e degli edifici veneti, i quali si intrecciano qui con le vie, le gallerie e gli edifici sotterranei italiani.

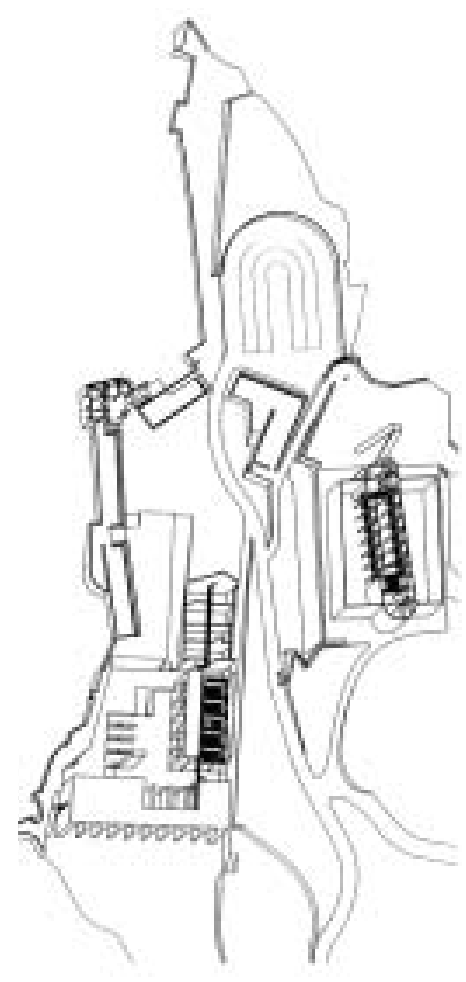

Figura 5 - La parte settentrionale dell'altopiano: assonometria del progetto di piano. 
Si tratta di un patrimonio storico di rilevante interesse, di cui il piano propone il recupero nella forma del "parco archeologico", prevedendone la visita secondo percorsi organizzati per "sistemi difensivi".

La punta sud-orientale della parte meridionale è stata oggetto di un progetto esecutivo della Provincia di Udine, già realizzato. Essa corrisponde alla zona più complessa del parco archeologico, per la singolare compresenza dei ruderi del Castel novo (eretto nel 1525 da Gerolamo Savorgnan, di cui rimane la cortina muraria del fossato e del piano sotterraneo, utilizzato dagli Italiani come "ridotto delle polveri") e dal forte corazzato (realizzato dagli Italiani ai primi del Novecento fuori terra nella depressione a sud del castello e ad esso collegato attraverso rampe e gallerie, poi completamente ricoperto a costituire l'attuale piazzale in calcestruzzo).

Ma tutta la punta meridionale è ricca di elementi di interesse storico e archeologico (mura, bastioni, pusterle, batterie, eccetera), appartenenti soprattutto al periodo veneto, ma oggi non più visibili a causa dei riporti di terra, inerti e macerie, realizzati dagli Italiani nell'ultimo secolo a partire dalla costruzione del forte sotterraneo, e per una fitta boscaglia cresciuta negli ultimi decenni. Il progetto propone, dunque, di far riemergere i resti delle strutture storiche e di valorizzarne le connessioni attraverso i percorsi, sia per creare nuovi rapporti di percezione e fruizione tra il castello e il forte che esaltino la presenza, nello stesso sito, di manufatti difensivi così lontani tra loro nel tempo e nella concezione, sia per sottolineare la funzione di cerniera che il castello svolge tra il sistema difensivo "Savorgnan" (costituito dagli edifici del periodo veneto, in condizione di rudere, posti a nord e a ovest del castello e dal recinto perimetrale delle mura) e quello "italiano sotterraneo" (costituito da alcune polveriere e dal forte, collegate da strade in galleria e in trincea). Gli interventi di progetto hanno riguardato un imponente lavoro di asportazione dei materiali di riporto, il restauro degli elementi archeologici e storici rimessi in luce, la riconfigurazione del piano superiore del forte sotterraneo che diventa il nuovo "piazzale delle cupole", la sistemazione ambientale degli spazi scavati, la realizzazione infine di un nuovo sistema di percorsi: un percorso pedonale sopraelevato lungo le mura del castello fino alla torre circolare ripristinata; un ponte in acciaio; il suo appoggio triangolare, che costituisce il nuovo monumento ai caduti in sostituzione di quello preesistente; una scala in acciaio che scende al piazzale.
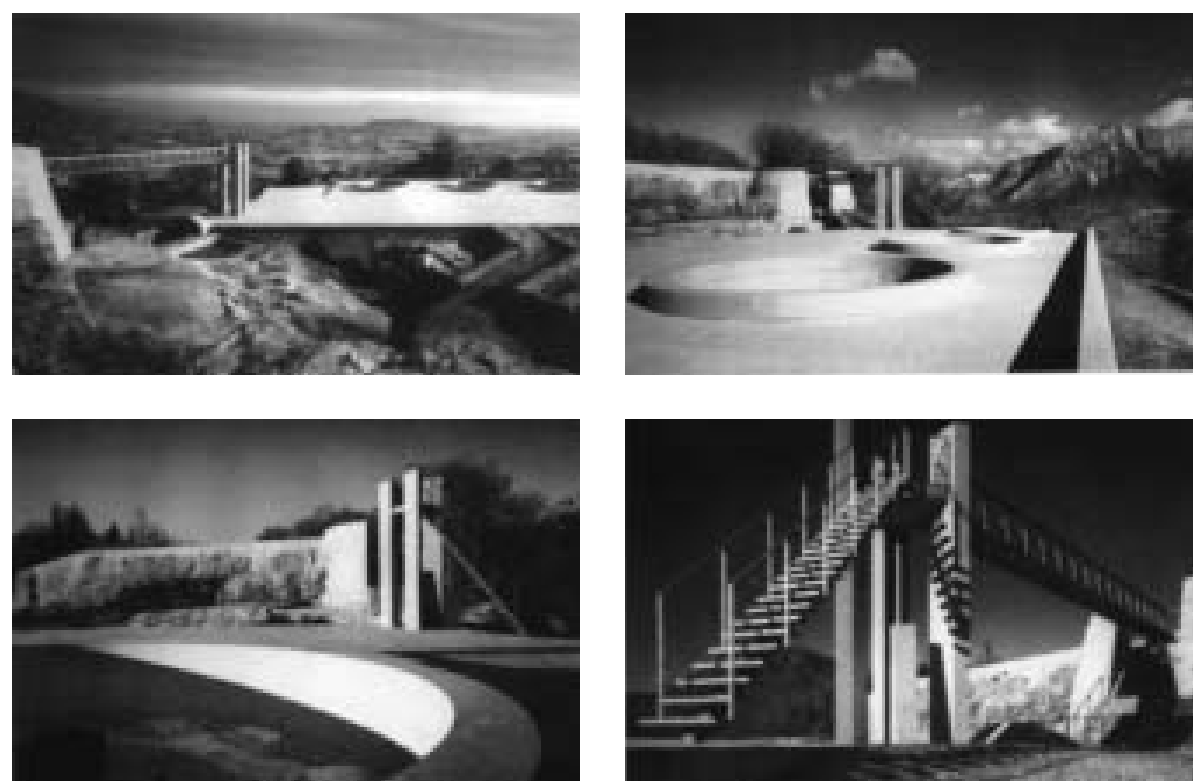

Figura 6 - Il Castel novo, il ponte con la scala, il monumento e il piazzale delle cupole. 
Il ponte, il monumento, la scala e gli altri percorsi sono concepiti, nella forma e nei materiali, come oggetti autonomi, in quanto diventano elementi di raccordo tra "episodi" storici profondamente diversi: il castello rinascimentale, i resti archeologici, le mura medievali e il forte moderno.

\section{La "parte centrale"}

Il Comune di Osoppo, infine, con il contributo finanziario della Regione, ha recuperato e valorizzato la "parte centrale" dell'altopiano come area di servizi del

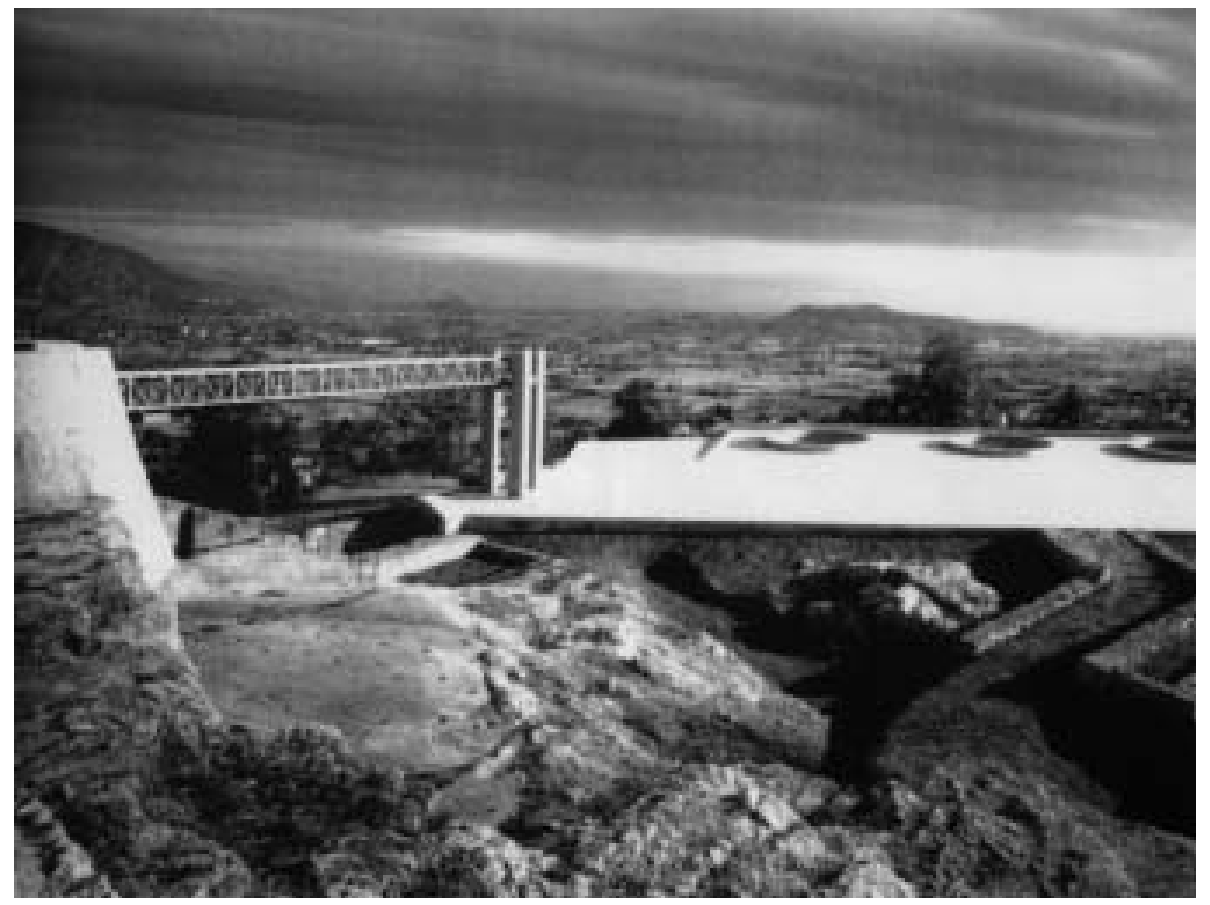

Figura 7 - Il ponte, il monumento e il piazzale delle cupole dal percorso sopraelevato del Castel novo.

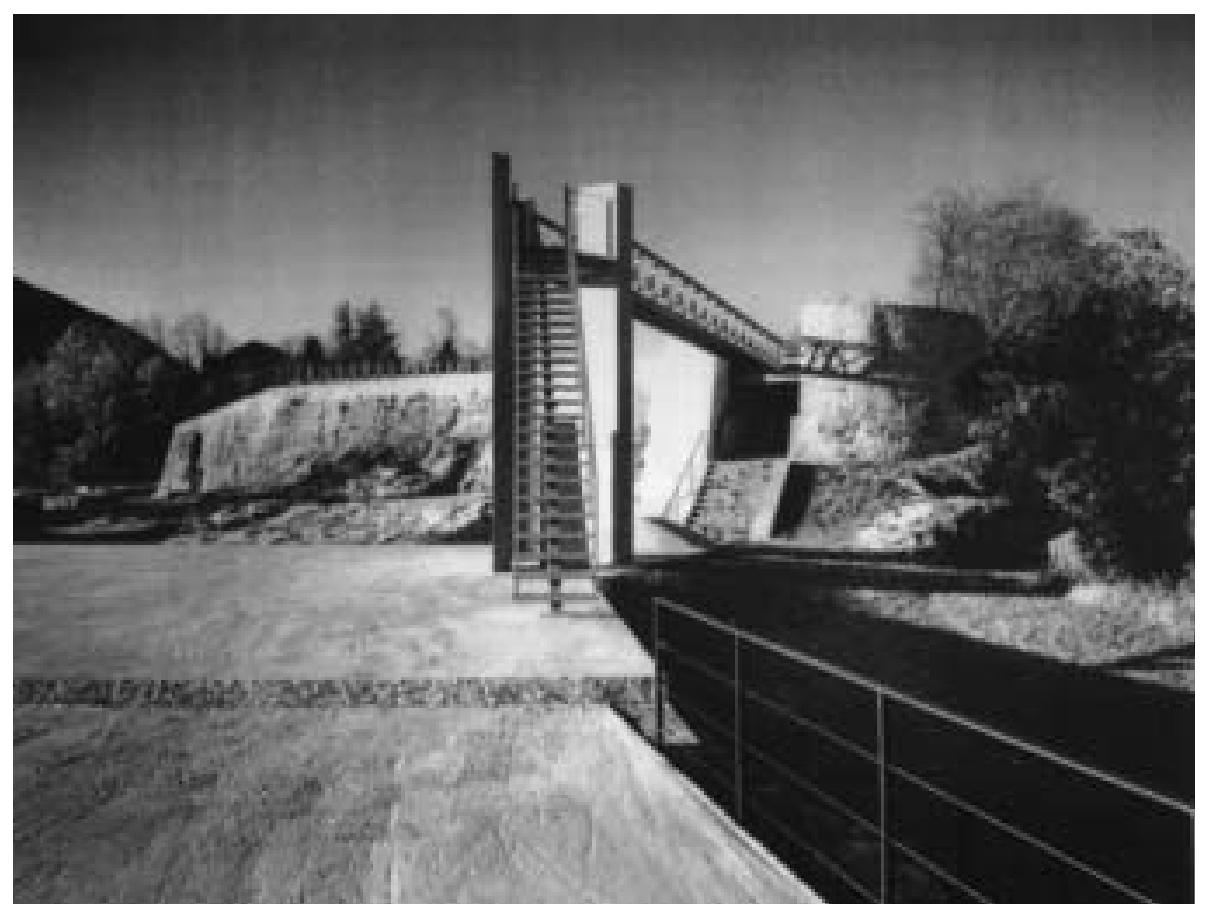

Figura 8 - Il monumento, il ponte e la scala da sud. 


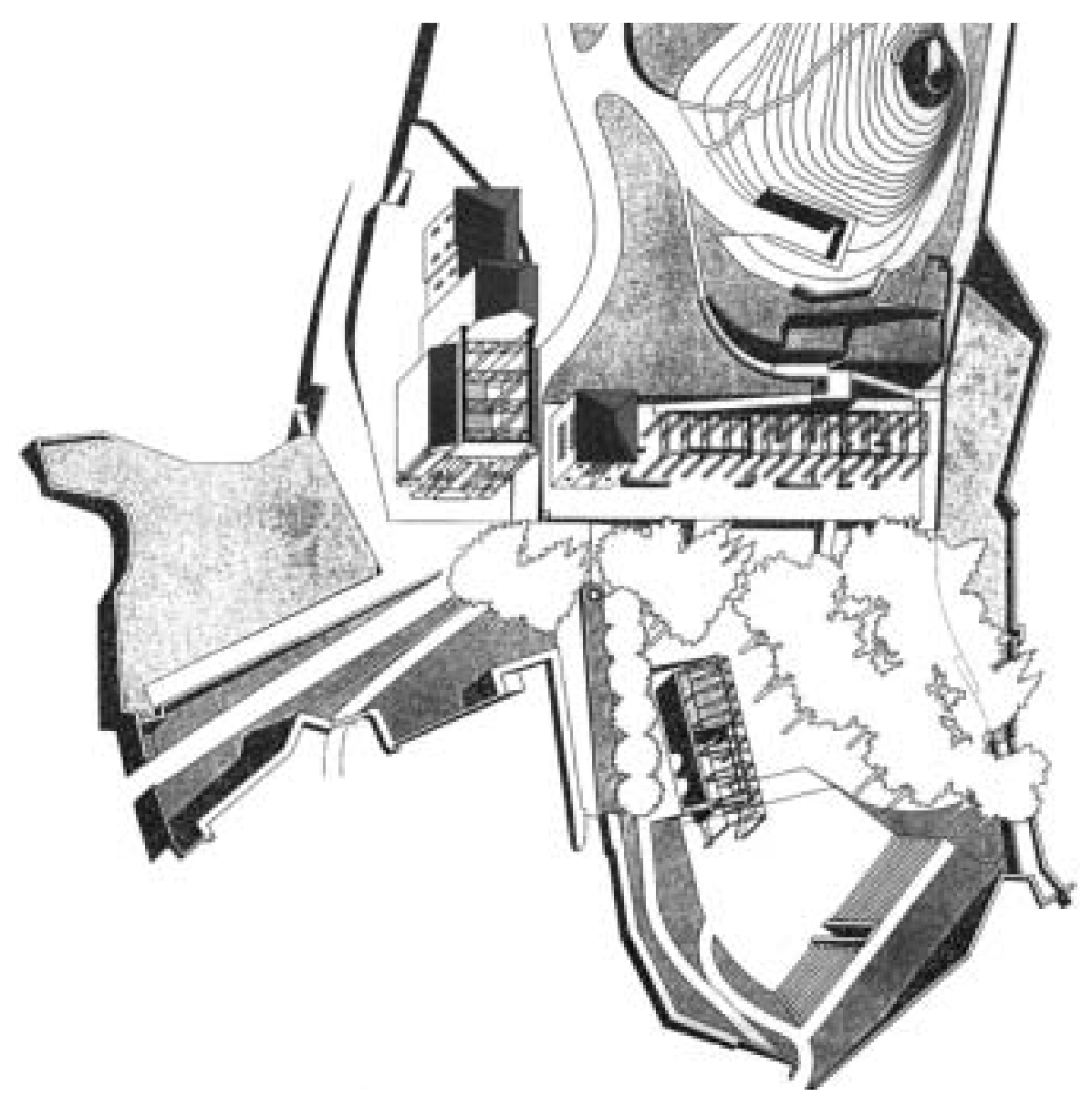

Figura 9 - La "parte centrale" dell'altopiano come area dei servizi del parco: assonometria del progetto di piano.

parco, con la ristrutturazione della Casa del Custode a centro visite e il completamento della Casa del Tamburo adibita a punto di ristoro.

Il progetto di attuazione è stato avviato per primo dall'Amministrazione Comunale, non solo per recuperare una parte storica significativa della fortezza, ma soprattutto per realizzare i servizi necessari al funzionamento del parco. L'area si configura come un invaso, chiuso a nord dal colle Napoleone, delimitato sugli altri lati dal recinto delle mura venete, caratterizzato da una struttura insediativa complessa, ricca di elementi storici, quasi totalmente occultati però dai riporti di terra realizzati in periodo italiano, dalle macerie provocate dai bombardamenti e dal terremoto, nonché da una vegetazione rigogliosa cresciuta negli ultimi anni. Tali condizioni hanno posto l'esigenza, all'interno di un recupero attento degli elementi storici superstiti nella loro integrità, di una ricomposizione di questo sito, anche attraverso interventi di ristrutturazione e di completamento che, coniugandosi con l'antico, ne valorizzassero le permanenze e le regole di costruzione insediativa, consentendo il riuso dei manufatti esistenti.

Il progetto propone, da una parte la ricomposizione del complesso formato dalla chiesa di San Pietro e dal terrapieno della Casa del Comandante, dall'altra dell'ambito compreso tra la Casa del Tamburo e le pendici delle mura. Pur nell'autonomia dei singoli edifici, i due insiemi tendono a configurarsi come "sistemi architettonici": il primo connesso alla geometria della parte settentrionale, il secondo a quella della parte meridionale. Attraverso la loro valorizzazione, si esalta la funzione di "fulcro" - come luogo dei servizi - che l'area centrale ha svolto storicamente e potrà svolgere in futuro, in funzione del parco archeologico e del parco-arboreto previsti nelle parti restanti dell'altopiano. 
In particolare, l'area centrale diventa quindi un luogo a servizio dell'intera fortezza, sia come punto di partenza e di arrivo dei percorsi di interesse storico e botanico, che si snodano lungo le mura e all'interno dell'altopiano, sia come area attrezzata per manifestazioni, sia come luogo di sosta e di visita, per le sue peculiarità storiche e paesaggistiche: la presenza di piante d'alto fusto nella sua parte centrale a formare una gradevole zona ombreggiata; i terrazzamenti e il profilo del colle Napoleone sullo sfondo a nord; l'anfiteatro delle mura venete a sud; i resti degli edifici veneti e italiani; il belvedere a est sulla piana di Gemona e quello sul colle Napoleone a spaziare sull'intero panorama dalle montagne alla pianura.

Il progetto di recupero di quest'area è stato articolato in lotti d'intervento, corrispondenti a progetti autonomi per impostazione e realizzazione.

Gli interventi, in particolare, hanno riguardato: la sistemazione ambientale del colle Napoleone a nord, dell'invaso centrale e del pendio delle mura a sud, la liberazione dalla vegetazione e dalle macerie e il recupero dei terrazzamenti del colle Napoleone e dei resti murari del terrapieno della Casa del Comandante, la ricostruzione del muro di sostegno del terrapieno, la predisposizione delle reti tecnologiche, la realizzazione di un parcheggio alberato a ovest, nel piano incassato antistante le Caserme italiane, in modo da consentire la pedonalizzazione dell'altopiano. Particolare attenzione è stata posta alle tecniche del restauro, per conservare i manufatti nella loro identità, segnata da stratificazioni e discontinuità per le continue trasformazioni subite nel corso dei secoli, e a quelle del ripristino, in modo da ricostituire le parti crollate o demolite nella loro conformazione preesistente.

Nella parte occidentale del terrapieno della Casa del Comandante, è stata ristrutturata l'ex Casa del Custode, realizzata negli anni Cinquanta, per destinarla a "centro visite" del parco.

L'intervento ha mantenuto il volume esistente, modificando le aperture e gli accessi e riorganizzando gli spazi interni.

Sul pendio che chiude a ovest l'invaso centrale, la Casa del tamburo, cioè un piccolo edificio veneto ridotto a rudere, è stato restaurato negli elementi superstiti e completato per essere utilizzato come punto di ristoro.

Una nuova struttura, composta da pilastri, travi e capriate in acciaio, è stata inserita all'interno del rudere. Attraverso l'articolazione dei profili metallici, essa
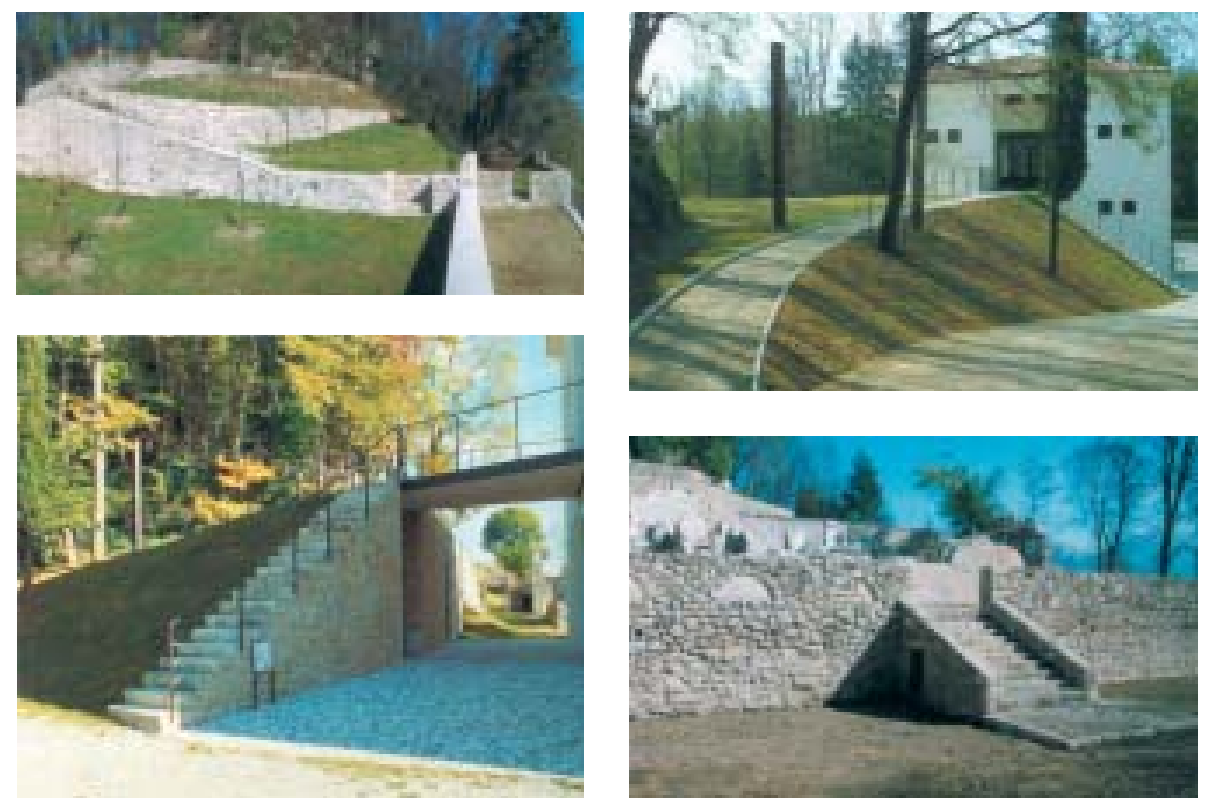

Figura 10 - I terrazzamenti del colle di Napoleone, il terrapieno della Casa del Comandante e il centro visite dopo gli interventi di recupero. 


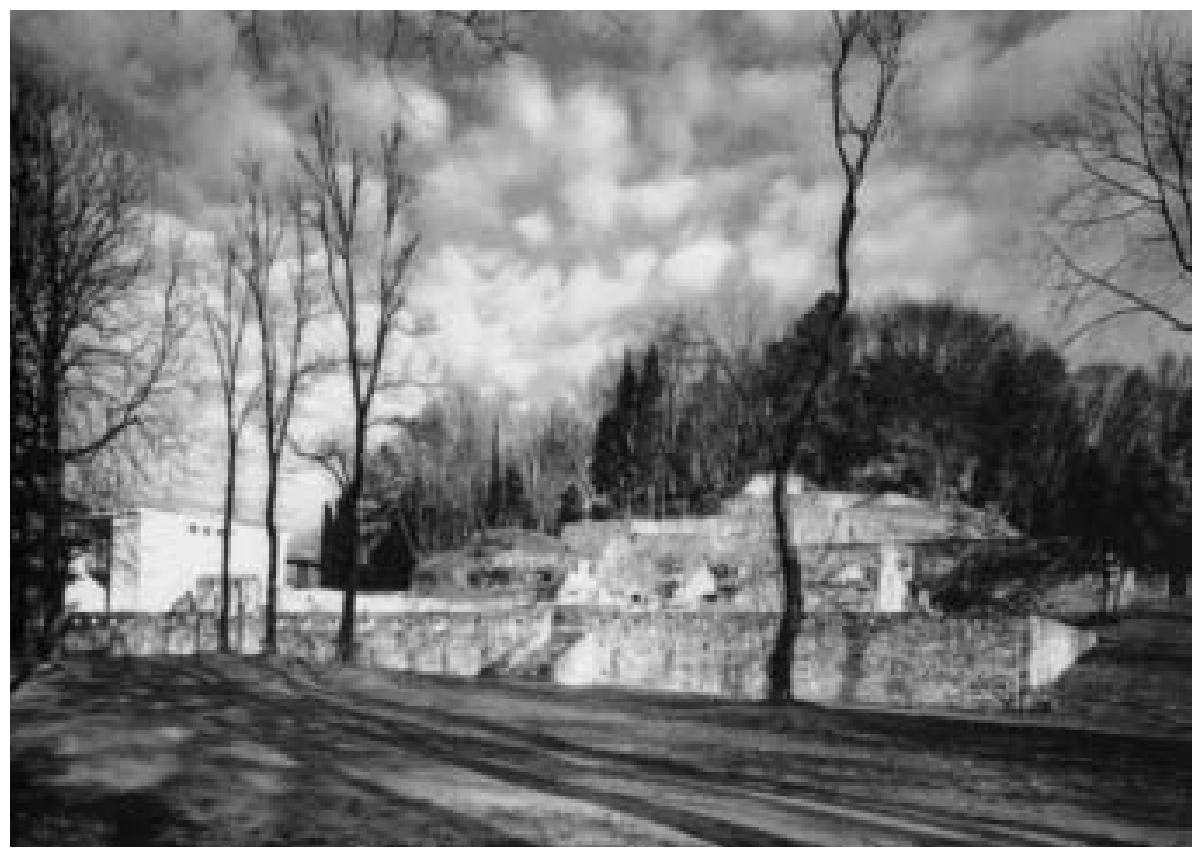

Figura 11 - Il centro visite e i muri del terrapieno della Casa del Comandante e dei terrazzamenti del colle Napoleone, a conclusione degli interventi.

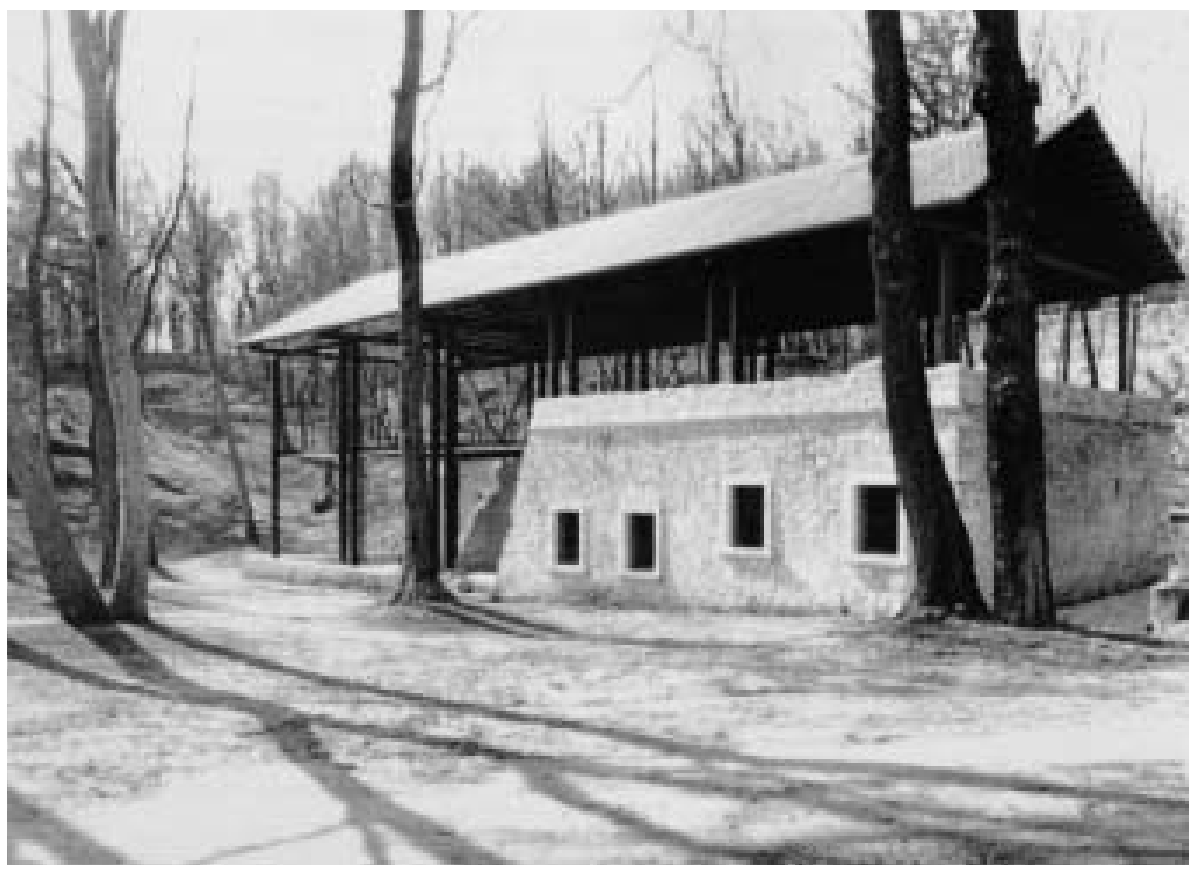

Figura 12 - La casa del Tamburo.

ripropone nelle sue linee essenziali, quasi come uno "scheletro", la figura storica dell'edificio preesistente.

Negli interventi di completamento della Casa del Tamburo e della chiesa di San Pietro, si sono rifiutate sia una operazione mimetica, sia una concezione del nuovo in contrasto con l'antico, tentando piuttosto di stabilire tra essi relazioni di natura "strutturale", immettendo cioè il "nuovo" all'interno delle regole di costruzione storica del manufatto ${ }^{1}$. L’operazione di completamento agisce così nello "scarto" tra

${ }^{1}$ Forma, posizione e modi di connessione dei profili metallici nascono da un'idea di "articolazione", che sottende una ricerca sulla essenzialità della forma, come risultato di un percorso progettuale complesso. 


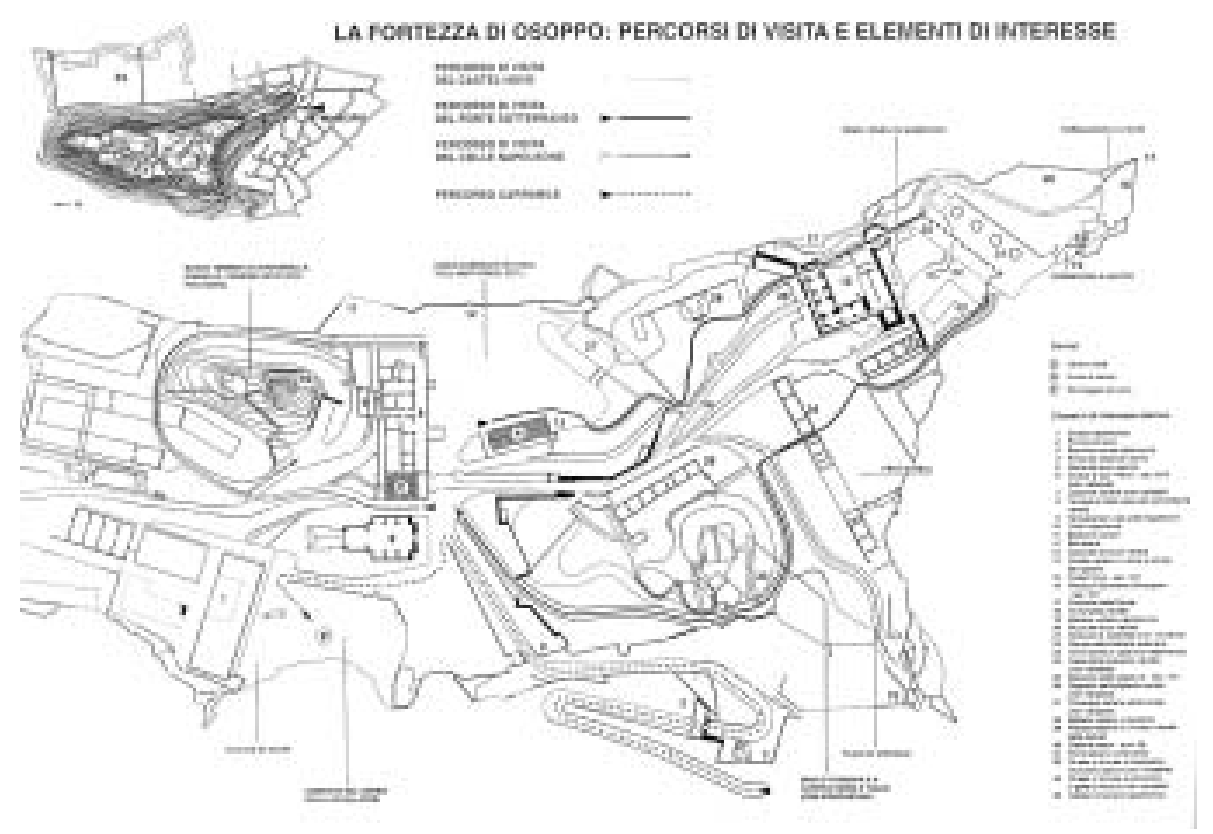

Figura 13 - Il parco della fortezza di Osoppo: mappa per i visitatori, con i percorsi di visita e gli elementi di interesse.

ciò che rimane dell'opera oggi e la sua configurazione preesistente, al fine di ricostituire l'unità formale e d'uso dell'opera, attraverso la definizione degli elementi di "analogia" e di "differenza" tra le sue parti esistenti e quelle di nuova costruzione.

Anche se permangono alcuni problemi (tra cui la sicurezza geologica della strada di accesso e la gestione delle opere), l'insieme dei lavori realizzati ha consentito di aprire al pubblico, nel 1995, un primo ambito del parco, corrispondente alle parti centrale e meridionale della fortezza, secondo tre percorsi di visita (quello del periodo veneto, quello delle opere militari italiane e quello del colle Napoleone), che collegano i diversi elementi, di interesse storico, botanico e paesaggistico, in una sorta di museo all'aperto.

\section{RIFERIMENTI BILIOGRAFICI}

Grandinetti Pierluigi, Un progetto integrato per la fortezza di Osoppo, in Bosco Giuliano (a cura di), Progetti integrati per le antiche fortificazioni costiere, Atti del Convegno (La SpeziaPortovenere 16-17 maggio 1998) organizzato da "Archeoclub d'Italia" (Associazione nazionale per la tutela e la valorizzazione del patrimonio storico-artistico e ambientale), Agorà Edizioni, La Spezia 1999, pagg. 67-86.

Grandinetti Pierluigi, Il progetto di recupero della fortezza e del colle di Osoppo (Udine), “Casabella”, 634, 1996, pagg. 12-23.

GRANDINETTI PIERLUIGI (a cura di), La geometria in funzione nell'architettura e nella costruzione della città, Cluva, Venezia 1985.

Grandinetti Pierluigi, Die Casa del Tamburo auf dem Festungshugel von Osoppo, "Detail”, 4, 1995.

Ciascuna parte ha una sua autonomia, formale e costruttiva, a partire dalla quale essa dialoga con le altre parti della nuova struttura e con il manufatto antico, in modo che, rispetto all'incompletezza del rudere, l'unità architettonica dell'opera sia costituita dall'insieme delle parti, nuove e antiche, dell'edificio. I criteri compositivi utilizzati nella Casa del Tamburo si ritrovano nel progetto per la chiesa di San Pietro. La chiesa, posta al centro dell'area, realizzata nel XVIII sec., mai completata secondo il progetto originario, dopo una storia complessa di trasformazioni, distruzioni e ricostruzioni, è oggi in condizione di rudere. Al suo interno sono visibili, in seguito a una campagna di scavi archeologici, i resti delle chiese precedenti. Il progetto propone il restauro, il parziale ripristino e il completamento della chiesa, con il recupero di tutti gli elementi archeologici, architettonici e stilistici in essa presenti, al fine di consentire la loro conservazione e protezione, di attrezzare l'edificio alla visita e alla fruizione, di ricostituire l'immagine della chiesa nella valorizzazione della sua monumentalità ma anche della sua storica incompiutezza. 


\section{RIFERIMENTI ICONOGRAFICI}

Figure 1, 6, 10: rielaborazioni di Maristella Storti da GRANDINETTI PiERLuIGI, Il progetto di recupero della fortezza e del colle di Osoppo (Udine), "Casabella", 634, 1996, pagg. 13, 20, 16.

Figure 2-5, 7-9, 11-13: rielaborazioni di Maristella Storti da GRANDINETTI PIERLUIGI, Un progetto integrato per la fortezza di Osoppo, in Bosco Giuliano (a cura di), Progetti integrati per le antiche fortificazioni costiere, Atti del Convegno (La Spezia-Portovenere 16-17 maggio 1998) organizzato da "Archeoclub d'Italia" (Associazione nazionale per la tutela e la valorizzazione del patrimonio storico-artistico e ambientale), Agorà Edizioni, La Spezia 1999, pagg. 69, 74, 76, $77,79,80,81,83,84,86$.

SCHEDA DI PROGETTO

Progetto unitario:

Luogo:

Committente:

Progetto e direzione lavori:

Anno di progettazione:

Realizzazione:

Tipi di intervento relativi alle singole parti del progetto

Progetto 1:

Committente:

Collaboratori:

Consulenti:

Realizzazione:

Progetto 2:

\author{
Committente: \\ Collaboratori: \\ Consulenti:
}

Realizzazione:

Progetto 3:

\section{Committente:}

Collaboratori:

Ditte esecutrici:

G. Dri, D. Mucin 1984-1986 servizi del parco

Comune di Osoppo

D. Mucin centrale

Comune di Osoppo progetto di recupero della fortezza e del colle di Osoppo

Udine

Comune di Osoppo - Provincia di Udine

Pierluigi Grandinetti

1984

$1987-1995$
Piano particolareggiato della fortezza di Osoppo

Comune di Osoppo - Provincia di Udine

G. Menchini, R. Meriggi (aspetti geologici), E. Screm, A. Tomè (aspetti vegetazionali), M. Asquini, L. Cargnelutti, F. Micelli, P. Toldo (aspetti storici)

Recupero e valorizzazione della parte centrale della fortezza di Osoppo come area di

strutture, Conti associati, O. Molinari, P. Mazzanti, M. Del Piccolo, G. Tavano; geologia, G. Menchini;

impianti, M. Bortolotti, S. Massarino

1986-89 recupero dei terrazzamenti del colle Napoleone

1987-90 restauro dei ruderi della Casa del tamburo e dei muri dell'area centrale

1987-95 ristrutturazione della Casa del custode a centro visite

1990-95 completamento della Casa del Tamburo a punto di ristoro

1991-95 sistemazione ambientale e realizzazione delle reti infrastrutturali nell'area

Restauro e completamento della Casa del tamburo nella fortezza di Osoppo

D. Mucin, S. Ronco;

strutture, M. Del Piccolo, G. Tavano

opere edili, Impresa Lizzi Giobattista;

strutture metalliche, Moro Lodovico e figli 


\section{SEGUE SCHEDA}

Realizzazione:

Progetto 4:

Committente:

Collaboratori:

Consulenti:

Ditte esecutrici:

Realizzazione:

Progetto 5:

Committente:

Collaboratori:

Consulenti:

Ditte esecutrici:

Realizzazione:
1987-94

Restauro conservativo e sistemazione ambientale del complesso Castel novo-forte corazzato nella fortezza di Osoppo

Provincia di Udine

G. Dri, D. Mucin

strutture, S. Urbano, M. Del Piccolo;

geologia, G. Menchini, R. Meriggi;

vegetazione, A. Tomè;

archeologia, F. Piuzzi

opere edili, Impresa Midolini;

strutture metalliche, Moro Lodovico e figli

1989-1995

Restauro e completamento della chiesa di San Pietro nella fortezza di Osoppo

Provincia di Udine

D. Mucin, E. Ariis, R. Bertossi, K. Ambrosini, K. Miniutti, F. Narduzzi strutture, M. Del Piccolo;

geologia, G. Menchini;

archeologia, F. Piuzzi;

impianto elettrico, S. Massarino

opere edili, Impresa Midolini;

strutture metalliche, Moro Lodovico e figli

1995 



\title{
IL SISTEMA DEI FORTI DI GENOVA
}

\author{
Maristella Storti
}

\section{IL CONTESTO}

Cenni sui caratteri delle architetture fortificate liguri

Sono degli inizi degli anni Settanta le prime ricognizioni attendibili e complete sulla consistenza dell'architettura fortificata in Liguria che riguardano, in particolare, sia il rilievo sistematico di questo tipo di patrimonio, sia l'approfondimento di una specifica tipologia nel repertorio delle architetture fortificate costiere liguri e ciò̀ quella delle torri di avvistamento. Da queste ricerche sistematiche, effettuate da diversi docenti della Facoltà di Architettura dell'Università di Genova, le circa cinquecento architetture fortificate regionali risultano più numerose nella Liguria di Ponente rispetto a quella di Levante, anche se nelle province di Genova e La Spezia le fortificazioni a contorno delle due città portuali sembrano riguardare quasi la metà dell'intero patrimonio fortificato provinciale $^{1}$.

Nei primi anni Ottanta, le ricerche preliminari per la formazione del piano paesistico regionale ligure ${ }^{2}$ comprendono uno specifico capitolo sulla classificazione sistematica del patrimonio architettonico fortificato relativo a circa duecento localizzazioni, sparse nell'intera regione e caratterizzate da preponderanti valori paesistico-ambientali.

In questa indagine, $\mathrm{i}$ valori proporzionali di ripartizione tra le varie province corrispondono quasi a quelli tratti dal rilevamento delle ricerche condotte dai docenti dell'Università di Genova e questo perché i dati si riferiscono solo alle emergenze rilevanti sul piano della visibilità, con l'esclusione delle tracce storiche e dei ruderi ancora percettibili.

Da tutti questi studi emerge, già negli anni Ottanta, che a caratterizzare questo tipo di patrimonio non è tanto la quantità o la dislocazione nelle quattro province liguri, quanto la qualità e le tipologie relative alle modalità di impianto

\footnotetext{
${ }^{1}$ Leone Carlo ForTi, Le fortificazioni di Genova, Stringa Editore, Genova 1971; PaOlo STRINGA, Guide di Genova (nn. 24-25-26), Sagep Editrice, Genova 1976. In particolare, ci si riferisce al lavoro svolto dal Prof. Perogalli negli anni Settanta, con la collaborazione di quattro docenti della Facoltà di Architettura dell'Università di Genova, comprendente il rilievo sistematico del patrimonio dell'architettura fortificata suddiviso nelle quattro province della Regione Liguria. Dalle schede di studio risultava che l'architettura fortificata in Liguria consta di circa 500 siti distribuiti in maniera disomogenea nelle varie province e ben diversificati secondo la tipologia di localizzazione sul territorio. In generale, da quegli studi si notava una maggiore densità di architetture fortificate a Ponente ed una minore a Levante; infatti, ne risultavano censite circa 100 in provincia di Imperia, 170 in provincia di Savona, 100 in provincia di Genova ed altrettante in quella della Spezia. Il Prof. Riccardo De Maestri, sempre negli anni Settanta, rilevava invece una tipologia specifica nel repertorio delle architetture fortificate e cioè quella delle fortificazioni costiere, con particolare riferimento alle torri di avvistamento. Gli studi sul sistema fortificatorio di avvistamento della regione si riferivano tuttavia solo alla Riviera di Ponente. Cfr. PAOLO STRINGA, Prospettive di recupero delle architetture fortificate in Liguria nel contesto territoriale ed ambientale regionale, in GiUliano BOSCO (a cura di), Progetti integrati per le antiche fortificazioni costiere, Atti del Convegno (La Spezia-Portovenere 16-17 maggio 1998) organizzato da "Archeoclub d'Italia" (Associazione nazionale per la tutela e la valorizzazione del patrimonio storico-artistico e ambientale), Agorà Edizioni, La Spezia 1999, pagg. 33-37.

${ }^{2}$ Piano Territoriale di Coordinamento Paesistico della Regione Liguria, approvato nel 1990.
} 
sul territorio, alla collocazione nel paesaggio e alle motivazioni storico-strategiche che lo giustificano.

Nel complesso, si tratta di ricerche che si sono occupate dell'aspetto storicostrategico-tipologico dei singoli manufatti, legato più alle loro caratteristiche intrinseche, piuttosto che alla specifica localizzazione e quantificazione.

Dagli anni Ottanta ad oggi, le relazioni intercorrenti tra le problematiche del recupero delle fortificazioni regionali, intese nella loro consistenza storico-architettonica, nonché i problemi, per tanti aspetti forse anche più complessi, della loro riqualificazione funzionale alla scala territoriale, con uno specifico interesse dedicato ai valori ambientali connessi all'intero sistema di tipologie cui appartengono, sono state evidenziate, in particolare, dal lungo lavoro condotto dal Prof. Paolo Stringa. Come rileva l'autore “... l'approccio alla conoscenza del sistema fortificato della Liguria è ancora abbastanza incompleto; la valutazione complessiva e sistematica di questo patrimonio è infatti ancora tutta da fare, ci si riferisce in prima battuta a valori numerici che comunque dovrebbero superare le 400/500 unità per gli elementi ancora visibili e tangibili sul territorio comprendenti torri, castelli, mura, bastioni, forti, ridotte, trinceramenti, eccetera (...). La conoscenza sistematica del fenomeno potrebbe consentire di arrivare ad una strategia generale regionale di recupero e riqualificazione che evidenzi le caratteristiche sistemiche di ogni singola tipologia e di ogni singolo insieme"3.

Si riconoscono sul territorio della Liguria almeno quattro importanti sistemi omogeneamente diffusi, con una caratterizzazione di impianto che certamente suggerisce un'altrettanto specifica caratterizzazione per le prospettive di intervento:

1 - un insieme di fortificazioni liguri relative al sistema strategico limitaneo dello spartiacque alpino ed appenninico, dove si riconoscono diversi complessi fortificati per lo più di epoca recente (XIX-XX secolo), in gran parte collegati alla progettazione napoleonica delle direttrici rotabili di valico, generalmente di realizzazione Sabauda, e integrate alle più recenti opere militari di valico e di spartiacque eseguite tra le due guerre mondiali;

2 - un sistema abbastanza omogeneo fa riferimento a tutte le fortificazioni del Golfo della Spezia: tale insieme rappresenta sul piano territoriale un'unità ben definibile e ben individuabile anche dal punto di vista delle prospettive di recupero $^{4}$;

3 - un altro sistema dotato più o meno delle medesime caratteristiche, di cui si tratterà, è quello che fa riferimento a Genova, cioè il sistema a corona della città, esteso dalle fortificazioni più antiche fino alle nuove mura, e quello dei forti del settore orientale con caratteristiche strettamente collegate alla storia urbana della città;

4 - un ultimo sistema riguarda le fortificazioni costiere che caratterizzano la lunga durata del dominio della Repubblica di Genova a partire dai secoli XII-XIII. Le testimonianze fisiche più importanti e diffuse, costituenti questo grande insieme, sono comunque databili intorno al XVI secolo e corrispondono all'intendimento di creare un sistema di avvistamento per garantire (con dei metodi abbastanza rapidi quali quelli visivi) la protezione dell'intero litorale. Sono ricomprese in questo sistema torri quadrate, torri rotonde, castelli, forti, fortini e fortificazioni varie realizzate con la specifica finalità, da un lato di proteggere

${ }^{3}$ PaOlo STRINGa, Prospettive di recupero delle architetture fortificate in Liguria nel contesto territoriale ed ambientale regionale, in GiUliano BosCo (a cura di), op. cit. (1999), pagg. 33-37; PAOlo STRINGA, Un itinerario turistico-culturale, Sagep, Genova 1985. Inoltre si veda: RENATO FINOCCHIO, Le fortificazioni di Genova, Valenti Editore, Genova 1983; ANDrEA PARODI, I monti di Genova, Andrea Parodi Editore, Genova 1999.

${ }^{4} \mathrm{Si}$ ricorda il Progetto "Saxa Ligustica” promosso dall'Archeoclub d'Italia in tempi recentissimi. Cfr. AnNa Barra, "Presentazione Archeoclub d'Italia”, in Giuliano BosCo (a cura di), op. cit., 1999, pagg. XI-XII. 
le popolazioni costiere e, dall'altro, di inviare segnalazioni di pericolo in modo da consentire alla flotta della Repubblica, che si muoveva principalmente da Genova, di intervenire. $\mathrm{Ci}$ possiamo rendere conto dell'antichità di questo patrimonio fortificatorio anche dalla tipologia planimetrica dei manufatti; per esempio, le torri di avvistamento quadrate corrispondono al sistema fortificato più antico, che è anche il più diffuso nella regione, invece le torri rotonde caratterizzano il Ponente ligure e sono più recenti. La tipologia della torre di protezione e di avvistamento a pianta rotonda corrisponde a quel sistema fortificato pianificato dalla Repubblica di Genova anche oltre confine, come in Corsica dove ben oltre novanta torri contornano il perimetro dell'intera isola.

Questi quattro sistemi principali, ben identificabili sul territorio ligure, comprendono al loro interno circa quaranta localizzazioni relative alle fortificazioni che fanno riferimento allo spartiacque; altrettante si contano sia nel Golfo della Spezia che in quello di Genova, mentre quelle dell'arco costiero sono più di cento 5 .

A questi grandi sistemi di fortificazioni vanno poi aggiunte le realtà fortificate delle aree interne, in generale meno strutturabili in insiemi ben definiti come quelli a cui si è fatto cenno. Ad esempio, un sistema di fortificazioni liguri altrettanto interessante sul piano ambientale è quello relativo alla testata terminale dell'asta del fiume Vara nell'estrema Liguria di Levante: sono sia le fortificazioni dei borghi-castello che rappresentano uno degli elementi più caratteristici dell'intera regione, sia le architetture fortificate di derivazione feudale concentrate all'interno dei borghi e degli insediamenti sottomessi. Appartengono, per esempio, alla tipologia feudale i borghi-fortezza di Ameglia, Arcola e soprattutto Varese Ligure, come caso esemplare di una fortezza feudale inserita all'interno di un tessuto.

Una categoria a sé è quella che attiene alle fortificazioni di derivazione comunale dove la fortezza è parte di un sistema fortificato con mura, torri e castelli posti a contorno del borgo e della comunità che proteggono. $\mathrm{Al}$ sistema comunale più diffuso nella Liguria di Ponente fanno capo per esempio le fortificazioni di Pieve di Teco, Albenga e Noli ma anche la stessa Genova è modello esemplare del genere comunale.

Il problema della conoscenza, della classificazione e della comprensione dei valori di questo notevole insieme di architetture fortificate liguri è reso poi ancora più complesso per effetto delle relazioni di stile e di cultura architettonica che, soprattutto nella diaspora genovese nel Mediterraneo, arricchiscono e complicano la diffusione dei modelli tra le architetture fortificate di terraferma e quelle di oltremare. Si tratta quindi in totale di un patrimonio di architettura fortificata ligure e mediterranea di quasi mille localizzazioni ancora ben emergenti sul paesaggio e fortemente connotate sotto il profilo paesistico ${ }^{6}$.

In sintesi, si può affermare che dalle prime esperienze di fortificazione ligure dei castellari pre-romani fino alle più recenti fortificazioni disposte sul fronte francese nella seconda guerra mondiale e agli esempi oltremare, si evidenzia un patrimonio complessivo che forse nessuna regione d'Italia possiede.

Per valorizzare e far conoscere questo tipo di patrimonio esemplare, occorre pensare ad un progetto strategico complessivo di scala regionale ${ }^{7}$; questo perché il recupero dell'architettura fortificata non può essere attuato operando esclusivamente sull'oggetto architettonico, sia per le relazioni che lo legano alla complessità

${ }^{5}$ Paolo STRINGa, Prospettive di recupero delle architetture fortificate in Liguria nel contesto territoriale ed ambientale regionale, in GIULIANO BOSCO (a cura di), op. cit. (1999), pagg. 33-37.

${ }^{6}$ A partire dagli anni Ottanta il Prof. Paolo Stringa ha anche avviato, con diverse pubblicazioni, una classificazione sistematica del patrimonio storico-insediativo ligure nel Mediterraneo. In quell'occasione si è potuto osservare come nel bacino mediterraneo vi siano altrettante fortificazioni di tradizione ligure quanto quelle presenti in Liguria. Tra Corsica, Sardegna, Sicilia, Tunisia, Anatolia, Mar Nero si possono contare 300-400 unità fortificate direttamente riconducibili alla storia della presenza genovese nel Mediterraneo. Cfr. GiUliano BosCo (a cura di), op. cit. (1999), pagg. 33-37.

${ }^{7}$ Alcune regioni hanno inserito questo tipo di iniziative all'interno del proprio piano paesistico, come nel caso della Valle d'Aosta. 
più vasta, sia perché la soluzione del problema non è solo di tipo architettonico ma anche storico, tipologico, paesistico-ambientale e soprattutto funzionale.

"Là dove non è possibile un intervento coordinato ad una prospettiva funzionale nella scala urbana, nelle relazioni che l'oggetto ha con il suo territorio, è forse meglio attendere occasioni di recupero e tempi di intervento migliori" ${ }^{8}$.

Fra le suindicate categorie di fortificazioni, in questa scheda si prende in considerazione il sistema del punto 3 , cioè quello caratterizzato dai forti a corona del centro antico genovese.

I forti di Genova sono tutti localizzati dall'interno verso la costa del capoluogo ligure, lungo il perimetro delle mura nuove, elementi di fortificazione e di protezione del porto e della città e non elementi di controllo del suo tessuto urbano e della popolazione, come invece avviene nelle fortificazioni di derivazione feudale.

I forti genovesi rappresentano un patrimonio che per decenni è rimasto in abbandono e il progetto che qui si intende presentare è rivolto a frenare la loro graduale distruzione recuperandoli, per quanto possibile, all'uso culturale e funzionale, attraverso una presa di coscienza generale e una serie di azioni mirate ai singoli complessi architettonici e ambientali.

\section{I forti di Genova: principali notizie storiche}

La città di Genova è contornata verso monte da un esteso sistema di fortificazioni, edificate e potenziate nel corso del XVIII e del XIX secolo, a volte su precedenti costruzioni.

I forti genovesi, integrati in una imponente cinta muraria quelli più vicini alla città, oppure a coronamento delle ripide colline quelli verso l'interno, sono stati costruiti e si trovano collocati territorialmente secondo due ampi sistemi: il "Sistema Centrale", posto sui crinali dei monti che separano le due vallate dei torrenti Polcevera e Bisagno a ridosso della città antica, e il "Sistema Orientale" posto sui crinali dei monti che separano la valle del Bisagno dalla Valle Sturla a protezione della città moderna del Levante. Oltre alle mura e ai sedici grandi complessi che coronano la città di Genova, esiste poi una serie di torri e di edifici minori9.

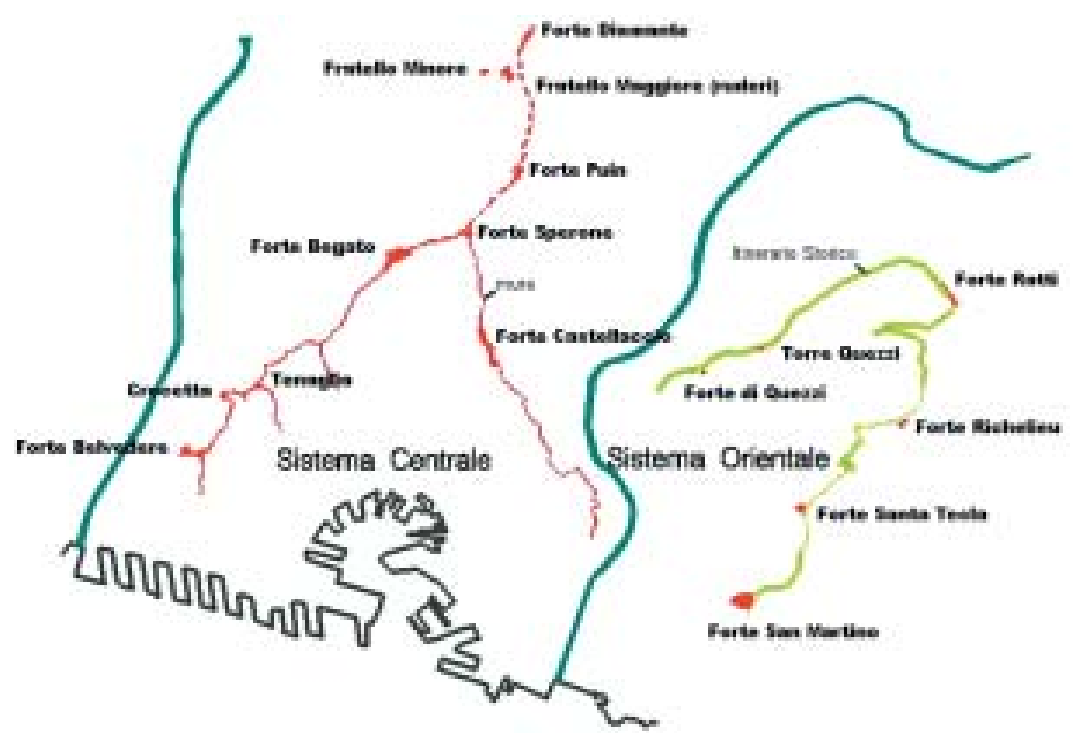

Figura 1 - Schema generale del sistema dei forti di Genova.

${ }^{8}$ PaOlo STRINGa, Prospettive di recupero delle architetture fortificate in Liguria nel contesto territoriale ed ambientale regionale, in GiUliano BosCo (a cura di), op. cit. (1999), pagg. 33-37.

${ }^{9}$ Partendo dal lato occidentale, salendo da Sampierdarena, sino alla collina di Granarolo, si incontrano i forti Belvedere, Crocetta, Tenaglia, per poi proseguire con i forti Begato, Sperone, Puin, Fratello 
Le mura nuove di Genova, cioè quelle che vengono concepite a protezione della città alla fine del Cinquecento e realizzate nel Seicento, sono conseguenza di esigenze difensive diverse da quelle medioevali e del periodo rinascimentale, perché rapportate allo sviluppo degli armamenti bellici, alle artiglierie e alle più lunghe gittate dei cannoni. Vengono quindi costruite non più in stretta aderenza con l'agglomerato urbano, come avveniva per quelle medievali, ma come baluardo posto a ragionevole distanza dall'urbanizzato, sul crinale dei monti circostanti, a sovrastare le vallate dei torrenti Polcevera e Bisagno e alla confluenza dei crinali stessi, nei punti più elevati, con funzione di avvistamento e preventiva offensiva.

L'idea di costruire la linea difensiva che dalla città sale fino al Forte Diamante, con riferimento particolare al "Sistema Centrale", venne suggerita dal Padre Domenicano Gaspare Vannoni fin dal 1568, come prosecuzione del baluardo del Castellaccio, già costruito negli anni precedenti dal lato del torrente Bisagno, e del Forte del Promontorio, corrispondente agli attuali Forti Tenaglia e Crocetta dal lato del torrente Polcevera. Anche sul Colle del Peralto, a ridosso del Castelletto, esisteva già una bastia di difesa costruita nel primo periodo rinascimentale, a forma di punta, nel luogo dove sorse il Forte Sperone. Pertanto, tra la seconda metà del XVI e l'inizio del XVII secolo, viene confermato e sviluppato architettonicamente un complesso sistema difensivo di particolare significato strategico, nel periodo in cui la Repubblica genovese vede il suo massimo splendore e crescono parallelamente le sue esigenze difensive nei confronti dei grandi Stati nemici e concorrenti (Spagna e Francia) e dei loro alleati (Ducato del Piemonte).

Le mura nuove, lungo i crinali tra le due valli del Polcevera e del Bisagno ed il centro città, ebbero inizio nel 1625 per l'imminente esigenza di potersi difendere tempestivamente dagli attacchi non preventivati e da possibili assedi ma, nell'anno successivo, divennero il "tracciato" su cui si attestarono le costruzioni difensive più imponenti relative al sistema dei forti di Genova.

Negli stessi anni, venne istituito il "Magistrato delle Mura" e si reperirono le necessarie risorse finanziarie per assicurare il nuovo programma difensivo; diversi illustri progettisti si avvicendarono nella direzione degli interventi, che videro periodi di sospensione e di modifiche fino al 1633, anno in cui venne conclusa la realizzazione delle Mura.

Con l'ulteriore evoluzione della tecnica militare, dalla metà dell'Ottocento in poi, la semplice disposizione difensiva dei piccoli fortilizi addossati alle mura non risulta più sufficiente alla difesa della città dai bombardamenti; vengono perciò pensate e progettate delle fortificazioni vere e proprie con corpi edilizi possenti e coperture piane, a loro volta ricoperte da uno spesso strato di terrapieno per attutire e smorzare la caduta delle bombe.

L'Ottocento è il secolo in cui, grazie al Regno Sabaudo Piemontese, vennero costruiti i forti di Genova (tra gli anni 1815-1830) e adattati, in seguito, taluni come forti difensivi, altri come batterie antiaeree, prigioni o caserme, altri ancora come forti per l'approvvigionamento, l'avvistamento o caserme-campo prigionieri.

\section{GLI OBIETTIVI}

\section{Dalla conoscenza allidea del progetto}

La configurazione dei forti genovesi a corona della città è relativa a un "sistema" in quanto i singoli elementi (forti, mura, porte, scalinate, torri, trincee, 
eccetera), ancorché di notevole valore storico ed architettonico ma anche naturalistico e mineralogico, per la loro collocazione nel territorio comunale, per le possibili funzioni, per la tipologia costruttiva che li accomuna, per le potenzialità d'uso e soprattutto per gli aspetti gestionali conseguenti, possono essere studiati, progettati ed inquadrati solo in un insieme sistematico.

Si tratta di un unicum architettonico di eccezionale valore che connota la città di Genova alla stessa stregua dell'insieme ambientale della costa e del porto o dell'agglomerato edilizio urbano del centro storico.

Allo stato attuale i forti, fatta eccezione per quelli vicini al centro città, in ragione del loro abbandono, presentano una difficile accessibilità e non sono serviti da mezzi pubblici di trasporto, essendo le strade esistenti anguste, tortuose e ripide. Attraverso le vie carrabili esistenti, i turisti, i cittadini e gli escursionisti accedono ai forti con autoveicoli privati che, specialmente nelle giornate estive e di maggior affluenza, intasano in modo improprio e disordinato le percorrenze veicolari, interferendo in modo pesante con i pedoni e con la godibilità del sito, anche dal punto di vista ambientale.

Una prima condizione per realizzare il recupero funzionale di questo estesissimo complesso monumentale è quindi quella di consentire una adeguata accessibilità, con relativi parcheggi e spazi per la sosta, permettendo la fruizione da parte di una ampia fascia di persone.

La condizione attuale di abbandono e di degrado in cui versano i manufatti edilizi delle fortificazioni, in generale, è dovuta anche al fatto che essi non sono stati oggetto da tempo di sistematici interventi manutentivi e che, non essendo inseriti nelle priorità d'intervento pubblico, ne è stata trascurata a lungo la fruibilità d'uso con assegnazioni in concessione di tipo frammentario e a soggetti diversi che, tuttavia, spesso li hanno preservati da occupazioni abusive ed hanno contribuito indirettamente alla loro conservazione ${ }^{10}$.

Ancora oggi i beni sono in uso ai suddetti soggetti assegnatari, tra cui lo stesso Comune di Genova, per iniziative temporalmente limitate, diverse da forte a forte, con notevoli aggravi manutentivi sui concessionari. Tale situazione di carattere provvisorio, in attesa del più generale intervento, può essere considerata, nella sua relatività, un dato di partenza verso il raggiungimento dell'obiettivo di recupero, data la fortissima valenza culturale e le potenzialità d'uso, e di conseguenza la necessità del loro recupero e relativa valorizzazione.

In quest'ottica lo Stato, attraverso i suoi organi centrali e decentrati (Soprintendenza e Agenzia del Demanio), negli ultimi anni ha posto particolare attenzione alla possibilità e all'opportunità di una nuova scelta gestionale, concordata con gli Enti locali che possono farsi garanti, nel presupposto di una concreta operatività d'intervento sui beni stessi, di una fattiva restituzione alla fruibilità cittadina.

I manufatti architettonici hanno caratteristiche tali da poter essere oggetto di svariate utilizzazioni, ma è indispensabile che il loro recupero e la loro riqualificazione si muovano entro linee strategiche e disciplinari chiare e la scelta d'uso non venga disgiunta da una reale prospettiva gestionale che valorizzi i beni e offra qualità funzionale ed ambientale all'utenza.

L'opportunità che vi sia una scelta globale strategica di recupero del "Sistema dei Forti” è data dalla ormai sentita esigenza a livello cittadino della piena godibilità dei manufatti, degli spazi, dei percorsi ludico-sportivi e di tutte le attrattività

\footnotetext{
${ }^{10}$ Oggi i forti genovesi e i relativi spazi aperti sono sostanzialmente utilizzati per svariate funzioni che vanno, ad esempio, dal campo da gioco del calcio e relativi spogliatoi, all'area attrezzata oasi del cane, ai depositi per mobili, all'allevamento cani, agli impianti tecnologici, ai depositi militari, alla biblioteca della Marina Militare, ai locali di tipo ricreativo, all'uso estivo per manifestazioni teatrali, ai cantieri di recupero, ai ricoveri di animali e abitazioni private, agli spazi esterni in concessione a privati, agli spazi in uso alla Protezione Civile. Sui sedici forti, inoltre, cinque risultano attualmente inutilizzati (ex abitazioni private) e uno è allo stato di rudere.
} 
ambientali relative al sistema. Puntare alla riqualificazione dell'esistente ed al recupero degli elementi urbani consolidati è un assunto delle strategie del Piano Urbanistico Comunale (PUC) della città di Genova ${ }^{11}$. Lo strumento urbanistico, infatti, vincola sia i forti militari che circondano il territorio genovese quali immobili di pregio storico-architettonico e ambientale soggetti a salvaguardia, conservazione e valorizzazione, sia le aree circostanti i forti stessi come sistemi di parchi urbani pubblici, anch'essi soggetti a tutela per la valenza ambientale: in particolare, il "Parco delle Mura" è relativo al sistema più attinente alla città; il "Parco dei Forti”, invece, si riferisce al sistema più interno.

Nel maggio del 1999, la Civica Amministrazione, tramite la Conferenza Strategica "Genova: le vie del Mediterraneo all'Europa" indetta a livello cittadino, ha definito le linee strategiche di sviluppo della città di Genova nelle quali si è inserito anche il progetto di riqualificazione del sistema dei forti. Inoltre, da qualche anno a questa parte, la città ha avviato un certo numero di interventi e sta elaborando strategie di valorizzazione di tutte le funzioni culturali nell'ambito del programma Genova Capitale europea della Cultura $2004^{12}$.

Il Comune di Genova, in particolare, è risultato il promotore dell'iniziativa di recupero di questo immenso patrimonio, riuscendo ad avviare le procedure per l'attuazione di un progetto complessivo sui due sistemi di manufatti monumentali e relativi spazi aperti ${ }^{13}$.

Il progetto di recupero del "Sistema dei Forti" non può che collocarsi, dunque, all'interno di questa strategia complessiva e sintonizzarsi all'insieme d'iniziative che vedranno la città sede delle attività culturali più importanti e significative in ambito europeo.

Tra tutti i forti emerge, non solo per importanza storica e per posizione strategica, ma anche perché oggetto d'interventi pubblici particolarmente significativi, il complesso di Forte Begato che è stato considerato il punto di forza del "Sistema Centrale" intorno al quale si possono sostenere programmi e progetti di recupero urbano e di valorizzazione consistenti, che vedono il coinvolgimento logico e conseguente dell'altrettanto importante e limitrofo Forte Sperone. Caratterizzano questo sistema anche il Forte Puin e l'imponente Forte Diamante.

Nel "Sistema Orientale", invece, si segnalano il Forte Quezzi, la Torre Quezzi e il Forte Ratti che è il più imponente ma anche il più lontano dall'abitato e pertanto di più arduo intervento (progettuale e d'uso).

Gli immobili, quale proprietà del Demanio dello Stato, sono patrimonio architettonico monumentale di pregio e di "specificità", vincolati ai sensi della legge n. 1089/39 così come modificata dal D.Lgs. 490/99, "Testo Unico delle disposizioni legislative in materia di Beni culturali e ambientali".

La costruzione del quadro delle conoscenze del progetto preliminare, inerenti le problematiche, le potenzialità ambientali e gli spazi di pertinenza delle fortifi-

${ }^{11}$ Il Piano Urbanistico Comunale (PUC) del Comune di Genova, ai sensi della Legge Urbanistica Regionale n. 36/1997, approvato con DPGR n. 44 del 10/03/2000, di cui alla delibera di C.C. n. 90/00, indica per tutti i Forti zona "AS Struttura Urbana Storica", disciplinata dalle norme di conservazione, salvaguardia e valorizzazione di cui agli artt. 60 e segg. delle NTA del PUC.

${ }^{12}$ In data 28 maggio 1998 la città di Genova è stata designata dai Governi degli Stati membri dell'Unione Europea "Capitale Europea della Cultura" per l'anno 2004, unitamente alla città di Lille.

13 Con Decreto del Ministero delle Finanze, di concerto con il Ministero del Tesoro, D.M. 27/03/2000, i cinque complessi fortificati del "Sistema Centrale" sono stati inseriti in elenchi finalizzati alla alienazione ai fini di assicurare un consistente introito al Demanio Statale. Avverso tale Decreto il Comune di Genova, sostenuto dalla Soprintendenza per i Beni Architettonici e per il Paesaggio della Liguria, ha opposto ricorso al T.A.R., motivato dal grande interesse, affinché i beni demaniali fossero trasferiti in capo al Comune ai fini di assicurarne la fruibilità pubblica nell'ambito di un progetto di recupero urbano. Il tribunale ha emesso sentenza favorevole al Comune e pertanto, effettuate le necessarie verifiche degli aspetti giuridico-patrimoniali sui beni medesimi, con successivo Decreto Ministeriale, i forti sono stati stralciati dall'elenco dei beni alienabili dando così possibilità all'Amministrazione Comunale, in accordo con la locale Soprintendenza, di avviare il progetto complessivo del loro recupero. 


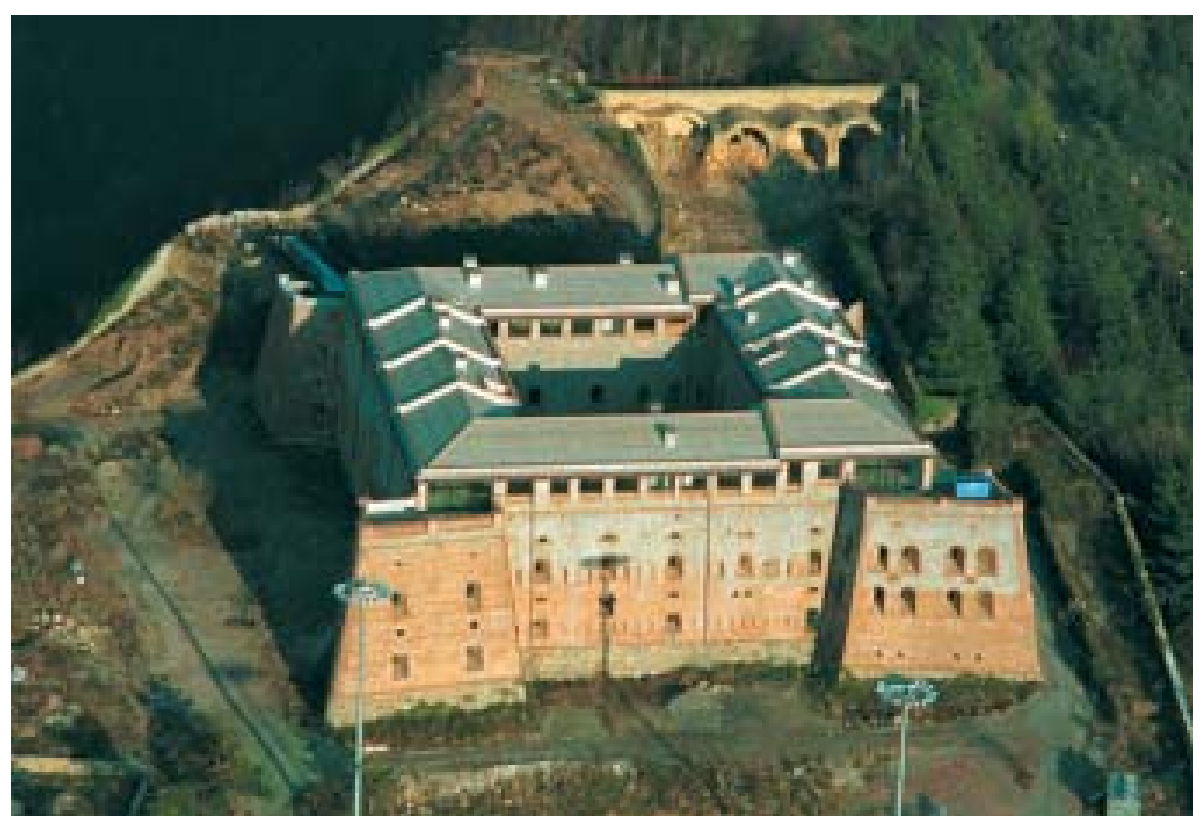

Figura 2 - Forte Begato.

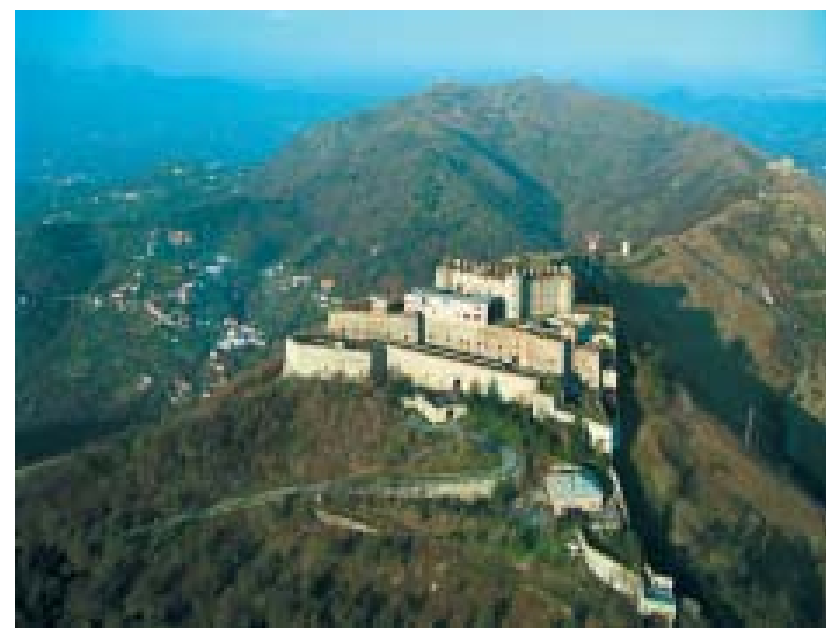

Figura 3 - Forte Sperone: veduta aerea.

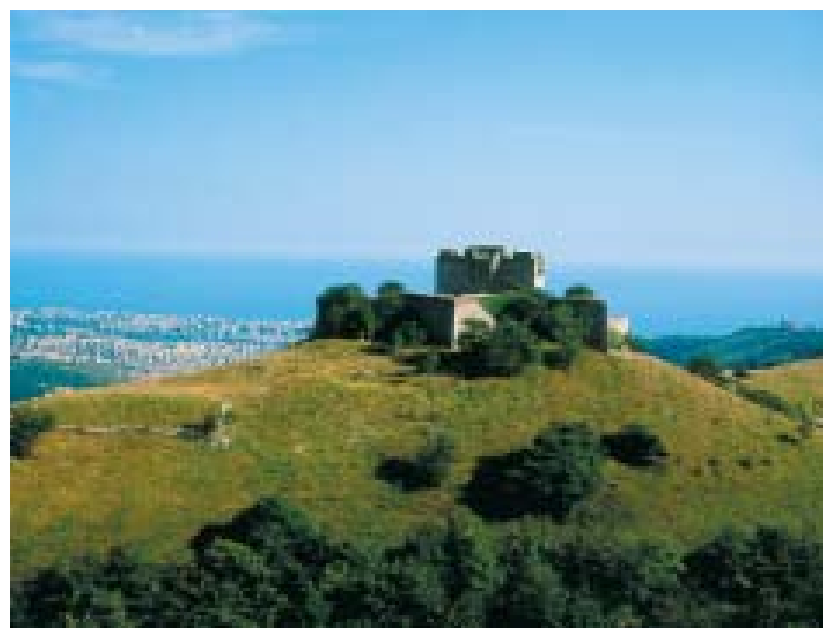

Figura 4 - Forte Puin: veduta aerea.

cazioni genovesi, si è basata su diverse operazioni: analisi delle fonti bibliografiche e degli strumenti urbanistici vigenti, contatti con Associazioni Ambientaliste, disamina visiva attraverso sopralluoghi diretti e riunioni presso l'Amministrazione Comunale.

Nell'aprile 2001 veniva presentato all'Agenzia del Demanio, Sede Centrale, da parte dell'Assessore delegato al Progetto dei Forti del Comune di Genova, il primo studio ricognitivo ${ }^{14}$ delle possibilità d'uso e dei vincoli urbanistici sui forti dei due sistemi centrale ed orientale.

Faceva seguito a tale presentazione un analogo incontro con la Soprintendenza per i Beni Architettonici e per il Paesaggio della Liguria alla presenza dell'Agenzia

${ }^{14}$ È stata fatta una prima ricognizione dello stato dei beni oggetto della progettazione, l'assemblaggio e la restituzione informatizzata dei rilievi esistenti, nonché una campagna fotografica rivolta a mettere in evidenza le condizioni generali dei beni sotto il profilo della consistenza materica delle condizioni di degrado delle murature in vista, dell'assetto complessivo dell'accessibilità e della presenza di condizioni igieniche particolari. Le condizioni di staticità dei forti e delle mura genovesi, alla prima rilevazione, sono risultate discrete. 

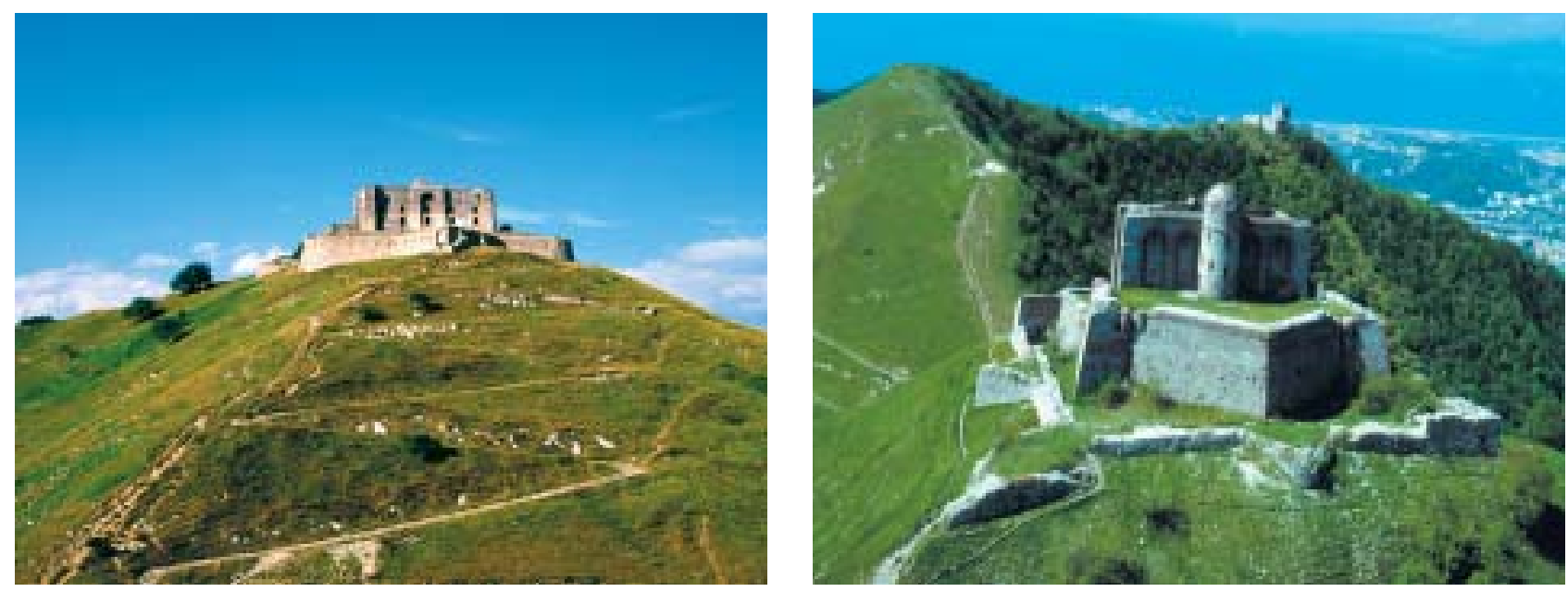

Figure 5 e 6 - Forte Diamante.
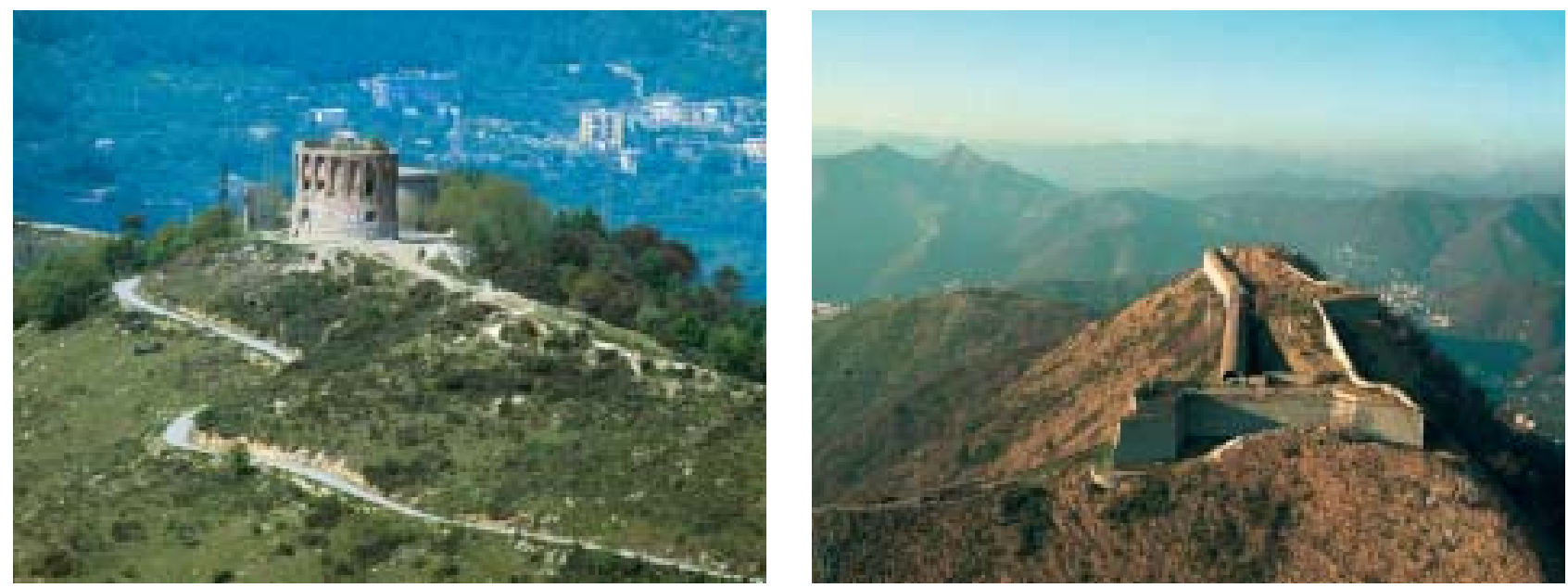

Figura 7 - Torre Quezzi: veduta aerea.

Figura 8 - Forte Ratti: veduta aerea.

del Demanio, Filiale di Genova; entrambi gli uffici hanno espresso il loro parere favorevole sulla previsione di un progetto di recupero del sistema dei forti, così come elaborato dal Comune, offrendo altresì, anche in vista della scadenza del 2004, la loro fattiva disponibilità ${ }^{15}$.

Infine, in un incontro presso l'Agenzia del Demanio, nel mese di settembre 2001, sono state concordate le linee procedurali relative al programma sui passaggi formali per l'attuazione dell'intervento, di cui la recente approvazione del Progetto preliminare da parte del Consiglio Comunale di Genova, costituisce la prima tappa significativa di questo iter ${ }^{16}$.

${ }^{15}$ L'11 maggio 2001 il Comune di Genova ha firmato con l'Agenzia del Demanio e con il Ministero dei Beni Culturali un Protocollo d'Intesa generale per la messa a punto degli obiettivi d'intervento riguardanti Genova Capitale europea della Cultura 2004; protocollo nel quale è previsto un consistente contributo pubblico sia per la messa a punto della progettazione, sia per i conseguenti interventi da realizzare nell'ambito del programma complessivo di recupero dei forti genovesi.

16 "Progetto preliminare del recupero, del riuso e della valorizzazione dei sistemi centrale ed orientale dei forti genovesi”, approvato nella seduta della Giunta Comunale del 25/01/2002.

L'intero iter procedurale di attuazione del processo relativo al recupero del sistema dei forti genovesi, si può riassumere nei passaggi principali: Elaborazione da parte del Comune del Progetto preliminare e sua approvazione in Consiglio Comunale; Approvazione di un Protocollo d'Intesa con l'Agenzia del Demanio e la Soprintendenza locale per l'attuazione del progetto complessivo; Indizione di gara, da parte dell'Agenzia del Demanio, per l'individuazione dei soggetti proponenti la progettazione e gestione degli interventi; Accordo di Programma per l'attuazione degli interventi, per la messa a disposizione delle risorse pubbliche, per la specificazione dei diversi ruoli tra gli Enti pubblici competenti e per assicurare la regia complessiva dell'intervento. 
Mentre, quindi, sono stati avviati i presupposti per concretizzare il più generale progetto di recupero e valorizzazione dei forti, con separato Atto stipulato in data 17 maggio 2001, il Comune otteneva in Concessione triennale da parte dell'Agenzia del Demanio cinque forti per le prime azioni di intervento e per l'uso temporaneo per manifestazioni e attività culturali varie in attesa dell'attuazione del programma complessivo.

Successivamente, in data 28 maggio 2001, veniva firmato un Protocollo d'Intesa con l'Istituto Nazionale per la ricerca sul Cancro, per la richiesta alla Presidenza del Consiglio dei Ministri di un finanziamento per il Forte Santa Tecla, per l'attuazione di un intervento di riqualificazione e recupero avente finalità concordate con gli Istituti scientifici presenti sul territorio vincolato dal PUC a servizi sanitari dell'Ospedale di livello regionale di S. Martino.

L'insieme dei forti dei due sistemi può altresì costituire un Parco Tematico d'eccezionale interesse dal punto di vista funzionale, ambientale e gestionale: pertanto, lo sviluppo delle tematiche progettuali ed operative connesse al parco può essere pensato come un unico filo conduttore, ovvero essere oggetto di strategie operative che lo investano nella sua globalità e non solo come somma di singole emergenze architettoniche e paesistiche.

Il "Sistema Centrale", in particolare, è a diretto contatto della città storica, forma un tutt' uno con le mura, le porte, le scalinate, i forti, le trincee, i bastioni; è quasi totalmente conservato ed in parte già recuperato. L'intero sistema, in tutte le sue parti, può essere considerato un museo all'aperto delle opere edilizie per strategie militari e dei beni architettonici ed archeologici presenti, quali reperti vivibili e fruibili del percorso museale di valenza storico-culturale.

\section{LA METODOLOGIA}

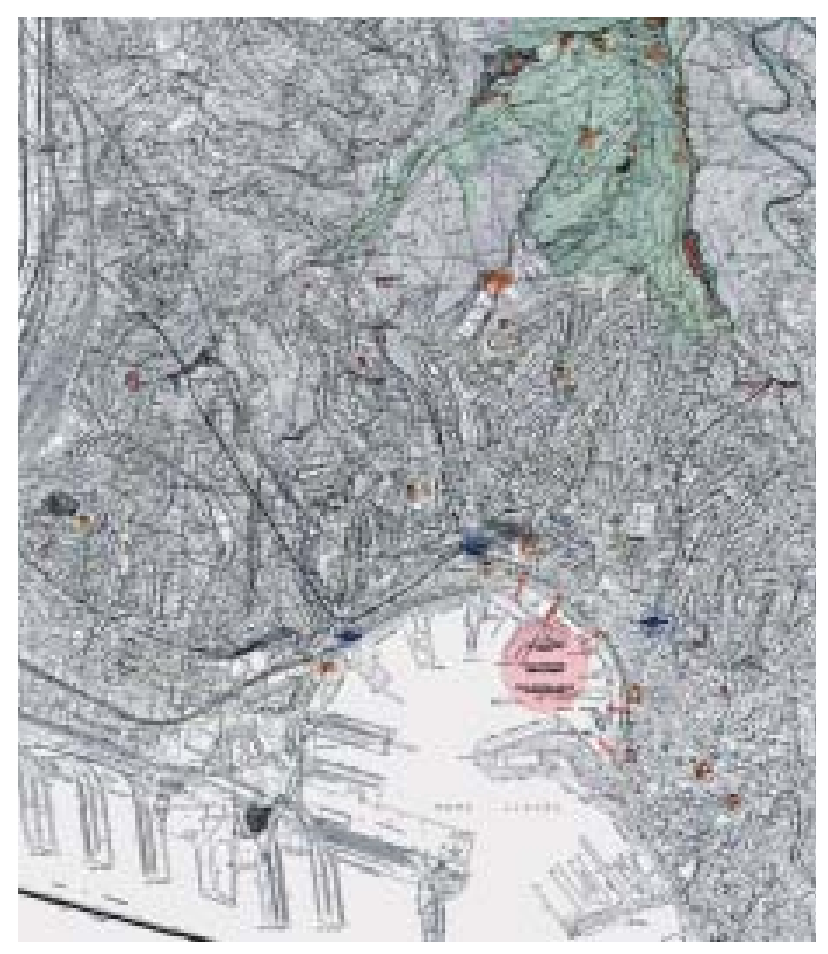

Figura 9 - Progetto preliminare di recupero, riuso e valorizzazione dei forti di Genova: il "Sistema Centrale" (particolare).
Opportunità delle scelte di recupero e riqualificazione urbana alla base di ogni strategia di intervento: principali funzioni individuate nel progetto preliminare per la compatibilità con $i$ diversi contesti architettonici e urbani

Gli studi relativi alla redazione del Progetto preliminare interessano 14 fortificazioni a corona della città di Genova con spazi, tratti di mura o percorsi connessi:

- "Sistema Centrale":

Forte Belvedere, Forte Tenaglia, Forte Crocetta, Forte Diamante, Forte Fratello Maggiore,

Forte Fratello Minore, Forte Puin, Forte Castellaccio, Forte Sperone, Forte Begato.

- "Sistema Orientale": Forte San Martino, Forte Santa Tecla, Forte Richelieu, Forte Ratti, Forte Quezzi, Torre Quezzi.

I due sistemi dei forti, i volumi, gli spazi circostanti, il verde, i percorsi di collegamento e le mura si collocano prevalentemente nell'ambito di quei manufatti ed ambienti che offrono grande potenzialità di sviluppo delle funzioni ludico-sportive e turistico-culturali.

Secondo gli indirizzi della Civica Amministrazione, messi a punto anche in vista delle già citate manifestazioni di Genova Capitale europea della Cultura 2004, ma soprattutto per dare al turismo genovese un assetto stabile e qualitativamente elevato, il preliminare ritiene indispensabile formulare proposte di utilizzo che si con- 


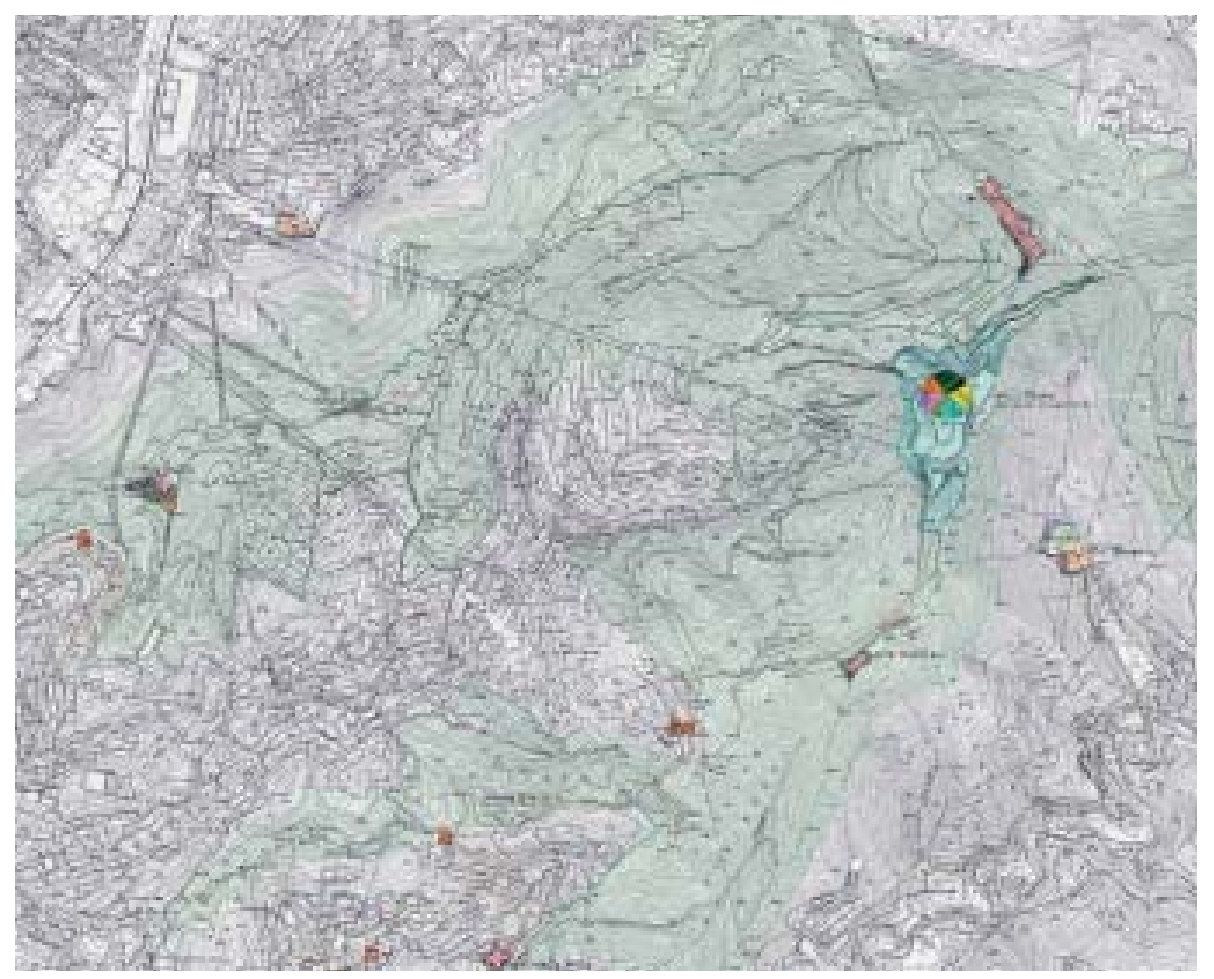

Figura 10 - Progetto preliminare di recupero, riuso e valorizzazione dei forti di Genova: il "Sistema Orientale" (particolare).

frontino con quanto, a livello europeo e mondiale, viene realizzato e gestito per una attrattività a scala internazionale duratura nel tempo. Il progetto intende anche far emergere le peculiarità cittadine derivanti, in questo contesto, dalla sua storia e dal suo essere proiettata verso l'esterno, nonché dalla sua connotazione ambientale, per puntare su funzioni strategiche che possano essere sorrette da importanti interessi culturali, scientifici ed imprenditoriali, considerando la cultura quale campo strategico e innovativo per una nuova imprenditorialità.

La città offre, per la sua particolare posizione orografica e climatica, spazi straordinariamente fruibili per la godibilità del clima e delle sue risorse ambientali, garantendo un buon livello di integrazione con la natura circostante: da un lato il mare e dall'altro le montagne, ne fanno senz'altro un sito appetibile per investimenti duraturi nel campo delle attrattività sportive, del divertimento individuale e collettivo, delle attività musicali, teatrali, culturali e di ricerca scientifica.

La previsione delle funzioni, per il progetto, non può che collocarsi dentro queste linee generali ed essere disponibile ad accogliere, nelle grandi famiglie funzionali, le proposte che maggiormente possano garantire la loro sostenibilità economica ed ambientale. Le due famiglie di funzioni ludico-sportivo-culturali e della musica sono quelle che possono richiamare la maggior frequentazione del pubblico, con tutto quello che ne consegue in termini di attrezzature per l'accessibilità veicolare pubblica e privata, nonché per i servizi di trasporto pubblico. Ma occorre altresì potenziare la godibilità degli spazi per le frequentazioni pedonali, per l'attività escursionistica, per lo sviluppo delle connessioni tra i forti e i vasti spazi a parco urbano, già in parte attrezzati, nonché per tutte quelle azioni concertate tra l'ente pubblico e le istituzioni scolastiche che da tempo hanno individuato nei forti e negli spazi circostanti obiettivi per la didattica sul tema dell'ambiente lungo i percorsi naturalistici di notevole interesse. Queste sono senz'altro le funzioni che maggiormente interessano anche l'utenza cittadina che potrebbe finalmente godere, sulle colline circostanti il territorio urbanizzato, di spazi analoghi per attrattiva a quelli delle aree del porto storico recuperato. 
È proposto quindi uno stretto collegamento con il polo a mare sia con le funzioni del Porto Storico (Acquario, Centro Congressi, Città dei Bambini, eccetera) e del Porticciolo turistico, della Darsena recuperata a funzioni urbane e del nuovo insediamento pubblico di Ponte Parodi, sia di quelle che si collocano presso la Stazione Marittima, il Terminal Traghetti e navi crociere, questi ultimi oggi in piena funzionalità.

Per il progetto non si dovrebbe trattare solo di un collegamento fisico tra queste centralità, attraverso sistemi misti di trasporto, bensì di collegamenti funzionali che risolvano la richiesta di circuitazione in ambito urbano, di prolungamento dello stazionamento turistico in città, di offerta programmata per grandi eventi, nonché di sistematica attività promozionale per la durabilità e sostenibilità delle scelte produttive e offerte di servizio. L'offerta culturale genovese dovrebbe pertanto collocarsi in reti internazionali di "pacchetti turistici" qualitativamente elevati, per essere inserita a livelli di diffusione dell'immagine della città e del suo sistema produttivo.

Tra i due sistemi, centrale ed orientale, vi sono sostanziali differenze dal punto di vista urbanistico e di collocazione, tuttavia in entrambi i casi si possono raggiungere, quanto meno per una parte delle fortificazioni, utilizzazioni in sintonia tra loro secondo un disegno strategico complessivo, dove i progetti risultano realizzabili solo attraverso la disponibilità di finanziamenti misti pubblici e privati ${ }^{17}$.

Le linee guida per la sistemazione ambientale degli spazi aperti di pertinenza dei forti

Il preliminare si fonda, oltre che su tutta una serie di temi progettuali che esulano dagli interessi di questa scheda, su alcune linee-guida fondamentali relative, in particolare, alla configurazione futura degli spazi aperti adiacenti i forti genovesi che qui si riportano in sintesi:

- il problema dell'accessibilità: in previsione di un incremento notevole delle attività derivanti dall'uso dell'intero sistema, diventa indispensabile assicurare l'accessibilità ai forti per garantire il raggiungimento dell'obiettivo del recupero complessivo dell'insieme urbano costituito dai manufatti e dai parchi urbani. Il sistema dell'accessibilità si presenta necessariamente come un insieme di azioni di governo della mobilità e di offerta di infrastrutture e servizi di tipo misto, connessi tra loro, che vedranno il coinvolgimento, all'atto della realizzazione, degli Enti pubblici e dei soggetti privati concorrenti, finalizzato a garantire la fattibilità degli interventi e la gestione delle funzioni insediate ed insediabili. Il tema dell'accessibilità riguarda quindi: il sistema viario, il trasporto pubblico, il reperimento di spazi per parcheggi e le connessioni d'interscambio con il sistema della mobilità esistente. La progettazione delle infrastrutture di servizio di trasporto pubblico di collegamento tra i forti e la città è stata sviluppata in collaborazione con l'Azienda Mobilità e Trasporti genovese ${ }^{18}$. Per la sistemazione delle aree a parcheggi di stretta pertinenza dei forti, gli interventi saranno studiati per una

${ }^{17}$ Il Progetto preliminare ha già impostato tutta una serie di previsioni che vanno dai servizi pubblici di quartiere, d'interesse comune e impianti sportivi, alle funzioni di limitata entità connesse al servizio e compatibili con il recupero architettonico del manufatto, ad esempio: centri sociali e per attività artigianali, piccole biblioteche, sale per la musica, agriturismi, soggiorni, stanze attrezzate, godimento punti panoramici pubblici, servizi culturali, teatrali, didattici, d'interesse scientifico e turistico, cittadella dello sport, mostre e musei interattivi dello sport, scuola superiore per le attività sportive, spazi all'aperto attrezzati per allenamento professionale, foresteria e soggiorni temporanei, Planetario, ristorazione e soggiorno per cure, punti di avvistamento per la Protezione Civile antincendio boschivo eccetera.

${ }^{18}$ Su richiesta dell'Assessorato ai Forti l'Azienda di Mobilità e Trasporti S.p.a. ha sviluppato, tramite i suoi uffici ed anche avvalendosi di progettisti esterni, un progetto preliminare e un programma di fattibilità per la realizzazione di un sistema di trasporto pubblico al servizio del Parco dei Forti del "Sistema Orientale" e del Parco delle Mura del "Sistema Centrale" (progettazione in parte in attuazione delle previsioni del PUC). Si tratta dello Studio di fattibilità per il sistema del trasporto pubblico al servizio del parco dei forti centrale (Aprile 2001), nonché delle Indicazioni per l'accessibilità al sistema orientale dei forti di Genova (Dicembre 2001). 
corretta compatibilità ambientale. Si individuano altresì aree private e per campers e roulottes, opportunamente attrezzate sotto il profilo igienico e impiantistico e oggetto di inserimento di segnaletica particolare finalizzata alla loro gestione rispetto alla rete cittadina;

- forti, mura, scalinate, porte, ponti, elementi architettonici in pietra: oltre a verifiche e operazioni puntuali di risanamento conservativo e restauro (effettuate con la locale Soprintendenza) per quanto riguarda l'inserimento di elementi architettonici nuovi, sono stati individuati i più appropriati materiali tradizionali (legno, pietra, laterizi, ferro);

- percorsi sugli spalti, percorsi d'accesso ai forti e percorsi all'interno delle aree fortificate: le percorrenze pedonali d'accessibilità ai forti e tutte quelle lungo gli spalti delle mura, con riferimento particolare alle zone panoramiche, dovranno essere trattati con gli stessi criteri conservativi, di salvaguardia e valorizzazione che caratterizzeranno le parti storiche di particolare pregio architettonico;

- sistemazioni a verde degli spazi esterni: le aree verdi in stretta adiacenza o pertinenza dei forti e delle mura saranno oggetto, per il preliminare, di uno specifico progetto di sistemazione del verde da sottoporre a verifica dei competenti uffici per quanto riguarda le tipologie delle specie vegetali, della struttura del verde e gli accorgimenti tecnici di impianto. Di massima, il preliminare ritiene compatibili le sistemazioni a copertura erbacea nell'intorno dei forti che non precludano la vista dei manufatti storici che sono oggetto dell'intervento di restauro conservativo, mentre l'impianto di nuove alberature, ovvero la conservazione di quelle di pregio esistenti, sarà possibilmente sviluppata per gruppi o macchie compositivamente distribuite negli spazi liberi oggetto di progettazione, secondo le tipologie che verranno individuate nella successiva progettazione esecutiva. In tutte le aree verdi circostanti le mura e i forti andrà effettuato un generale intervento di bonifica, di decespugliamento e di scerbatura della vegetazione; tali interventi, nel caso in cui la vegetazione ostruisca la visibilità dei manufatti o produca lesioni o deterioramenti delle parti murarie da conservare, dovranno essere improntati all'eliminazione delle specie vegetali non previste. Non si esclude, su taluni percorsi o su manufatti di tipo leggero accessori alle fortificazioni, l'impianto di nuovo verde con funzione di arredo o schermatura. La progettazione del verde, la scelta della specie e la loro collocazione più appropriata dovrà altresì essere mirata alla razionalizzazione delle spese manutentive. Per quanto riguarda i contenuti minimi che la progettazione del verde dovrà comprendere, è stata allegata al progetto preliminare una scheda con le specifiche tecniche per parti di progettazione definitiva ed esecutiva del verde e delle linee guida per la progettazione.

Fanno parte del Progetto preliminare tutte le schede specifiche dei forti che riportano i dati relativi allo stato di fatto e i vincoli urbanistico-edilizi, nonché le proposte progettuali per ogni singola situazione.

Particolarmente significativo sarà lo sviluppo della progettazione alla scala edilizia per la quale nelle schede vengono tracciate le linee e gli indirizzi princi-

Gli interventi sarebbero tali da non interferire troppo da vicino con gli edifici monumentali, stimolando invece un minimo di percorso a piedi su facili strade e sentieri. Sarebbe possibile anche escludere completamente l'accesso ai veicoli a motore, che per la verità già adesso trovano un serio impedimento nel forte degrado e nell'incuria del fondo delle antiche strade militari. Il progetto prevede un insieme di opere infrastrutturali delle quali una parte possono essere considerate in attuazione delle previsioni del PUC ed una parte sono totalmente innovative; per quest'ultime, ai fini dell'approvazione, sarà necessaria una variante al PUC con l'attivazione di apposita Conferenza dei Servizi nell'ambito di procedura di Accordo di Programma (LUR 36/97). 


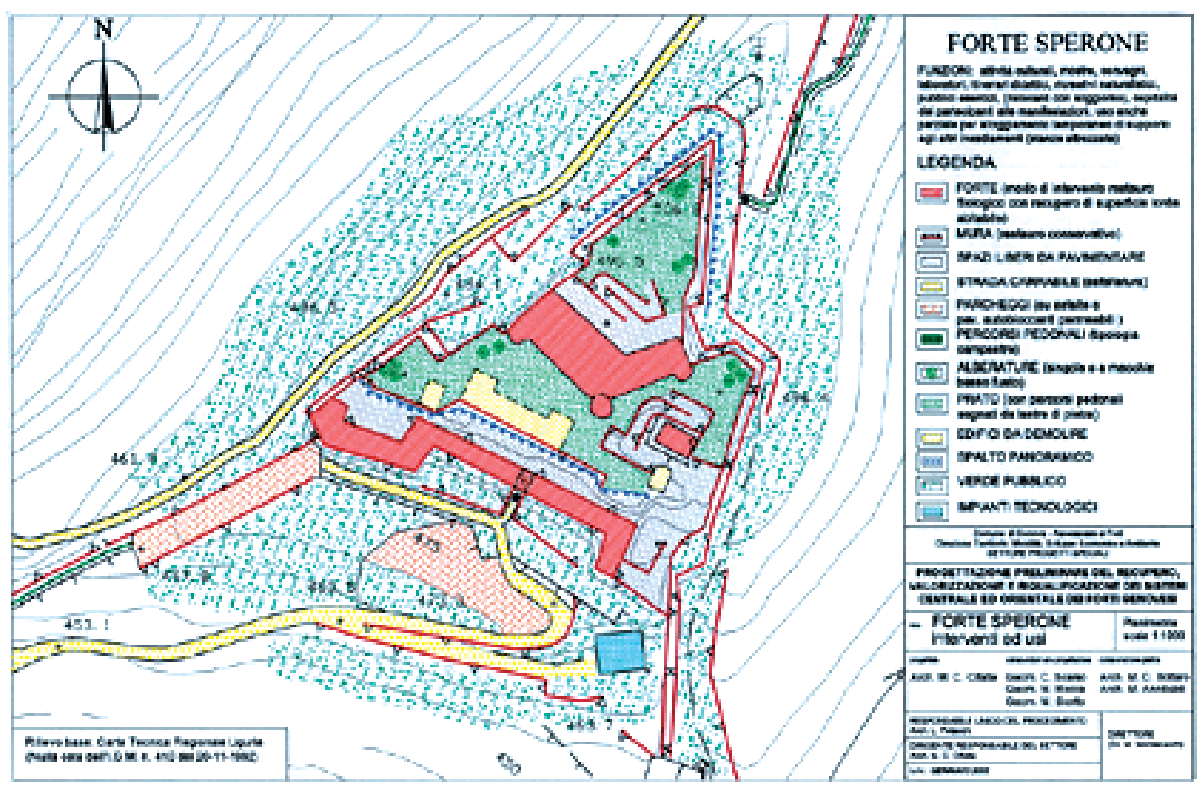

Figura 11 - Progetto preliminare di recupero, riuso e valorizzazione dei forti di Genova: scheda di Forte Sperone.

pali caratterizzanti il livello preliminare. Ogni progetto dovrà essere proceduto da un'accurata rilevazione degli elementi architettonici e strutturali esistenti, da appropriate indagini diagnostiche di tipo archeologico ed architettonico, nonché da sondaggi del sottosuolo tali da consentire la scelta progettuale più appropriata, sia per gli interventi sui manufatti edilizi esistenti, sia sugli spazi ed aree libere o trattate a verde, sia sui percorsi pedonali.

L'individuazione delle tipologie di intervento sugli spazi esterni destinati a verde pubblico è stata ottenuta attraverso differenti linee guida, intese come parti di un unicum strettamente integrato.

Le sistemazioni ambientali individuate ed il potenziamento del verde esistente rispondono alle seguenti esigenze:

- paesistiche ed estetiche: rispondenza all'unicum paesaggistico del singolo forte nel contesto circostante e rispetto all'orizzonte visivo che lo unisce con le altre emergenze fortificate; sottolineatura di percorsi e porte di accesso alle fortificazioni; valorizzazione estetica degli elementi di pregio e caratterizzanti il singolo forte; richiamo dell'antica peculiarità di funzione; individuazione di specie di verde che fossero già in uso comune nell'epoca storica di costruzione del forte;

- ambientali: necessità di riduzione del rischio incendio, sia rispetto alla situazione attuale, sia in previsione dell'insediamento delle nuove funzioni indicate dal progetto; necessità di messa in sicurezza del suolo rispetto alle problematiche geomorfologiche dovute al tipo di assetto, agli interventi antropici effettuati, agli interventi previsti dal progetto; individuazione dell'ingegneria naturalistica quale metodo di intervento in grado di garantire, oltre al risultato tecnico, anche un effetto ecologico, economico ed estetico; inoltre, la particolare collocazione dei singoli forti non permette certo l'insediamento di vaste aree di cantiere anche in riferimento all'approvvigionamento dei materiali ed alle loro lavorazioni, ossia è preferibile potersi approvvigionare di materiali già pronti per la messa in opera che non necessitino di particolari condizioni di lavorazione sia prima che durante l'intervento; considerazione della fauna presente quale elemento facente parte della progettualità, effettuando scelte che ne favoriscano lo sviluppo o la limitazione a seconda delle esigenze; 
- funzionali: individuazione delle specie vegetali a seconda delle aree di intervento in relazione all'utilizzo previsto (esempio zona di sosta) nella stagione di riferimento (esempio zona di sosta utilizzata soprattutto d'estate); individuazione di specie che siano tipiche dei luoghi, reperibili sul mercato e già adattate alla situazione climatica; individuazione di specie di verde che non necessitino di costante manutenzione sia dal punto di vista della crescita e sviluppo, sia degli effetti indotti dalla pianta stessa.

Proposta di restauro e risanamento conservativo del Forte Begato nel Parco Urbano delle Mura (Assessorato ai Lavori Pubblici - Assessorato all'Ambiente del Comune di Genova)

Forte Begato è parte integrante della cinta muraria più estesa e meglio conservata di Genova, quella risalente al sec. XIX, che cingeva l'antica città dalle alture sino al mare.

La costruzione del forte ebbe inizio nel 1819 ad opera del Genio Militare del Regno Sardo quale ampliamento della cinta delle nuove mura in corrispondenza del terrapieno della batteria settecentesca di Begato, in posizione particolarmente dominante sulla città, ad una altitudine di 475 metri s.l.m. Fra le prime opere realizzate vi fu la caserma, ultimata nel 1823 , dalla forma architettonica caratteristica a pianta quadrata con la corte interna ed i quattro baluardi angolari; edificio che risulta ancor oggi particolarmente imponente e rappresentativo con i suoi 33.000 metri cubi di volume.

La definitiva recinzione del forte mediante cortina protetta da fronti bastionate, avvenuta tra il 1832 ed il 1836, si presenta ben conservata e racchiude un'area verde sulla quale insistono cinque edifici minori, ex casermette o munizionieri, anch'essi oggetto dell'intervento. Prima dell'attuale restauro, iniziato nell'agosto del 1993, lo stato di conservazione dell'intero complesso era discreto, nonostante molti anni di completo abbandono.

La realizzazione dell'intervento, a fronte di una progettazione unitaria, è stata articolata, per la sua estensione e complessità (non ultimi i problemi delle bonifiche belliche), in tre lotti temporalmente distinti.

Su un'area di circa 3,5 ettari di un altopiano completamente cintato da mura ed a tratti bastionato, i lavori di restauro del complesso hanno interessato (e parzialmente interessano tuttora) la riconversione funzionale di edifici per circa 5.000 metri quadrati di spazi coperti e per la restante parte la sistemazione a verde attrezzato, sport e percorsi.

Oltre a divenire sede della centrale per il monitoraggio del territorio del Parco Urbano delle Mura, del quale costituirà il fulcro operativo, il Forte Begato ospiterà al suo interno una serie di attività a carattere culturale, ludico e sportivo, tali da garantirne la frequentazione e l'uso attivo a vari livelli durante l'intero arco dell'anno, con punte di frequentazione durante la stagione climaticamente favorevole. Infatti, si prevedono attività a frequenza quotidiana di operatori legati agli spazi per lo sport e per la musica e a frequenza legata al carattere turistico stagionale del museo, del teatro all'aperto e, ancora, delle attività sportive quali gli spazi per l'ippica e per le attività di ricezione e ristoro costituite da due ristoranti (di cui uno panoramico, posto all'ultimo piano del forte) e da un bar attrezzato con spazi all'aperto.

Le nuove destinazioni d'uso sono state scelte dal preliminare in compatibilità ed in armonia con il carattere del luogo; il loro insediamento all'interno dell'edificio principale del forte e degli edifici minori, pur garantendo la più completa accessibilità, contempla infatti interventi tesi al massimo rispetto dell'esistente.

L'intero complesso militare è di proprietà del Demanio dello Stato, ottenuto in concessione dal Comune di Genova che ne sta trattando l'acquisizione definitiva. Il restauro realizzato dal Comune come ente attuatore è stato finanziato, oltre che con stanziamenti propri della stessa Amministrazione civica, anche gra- 


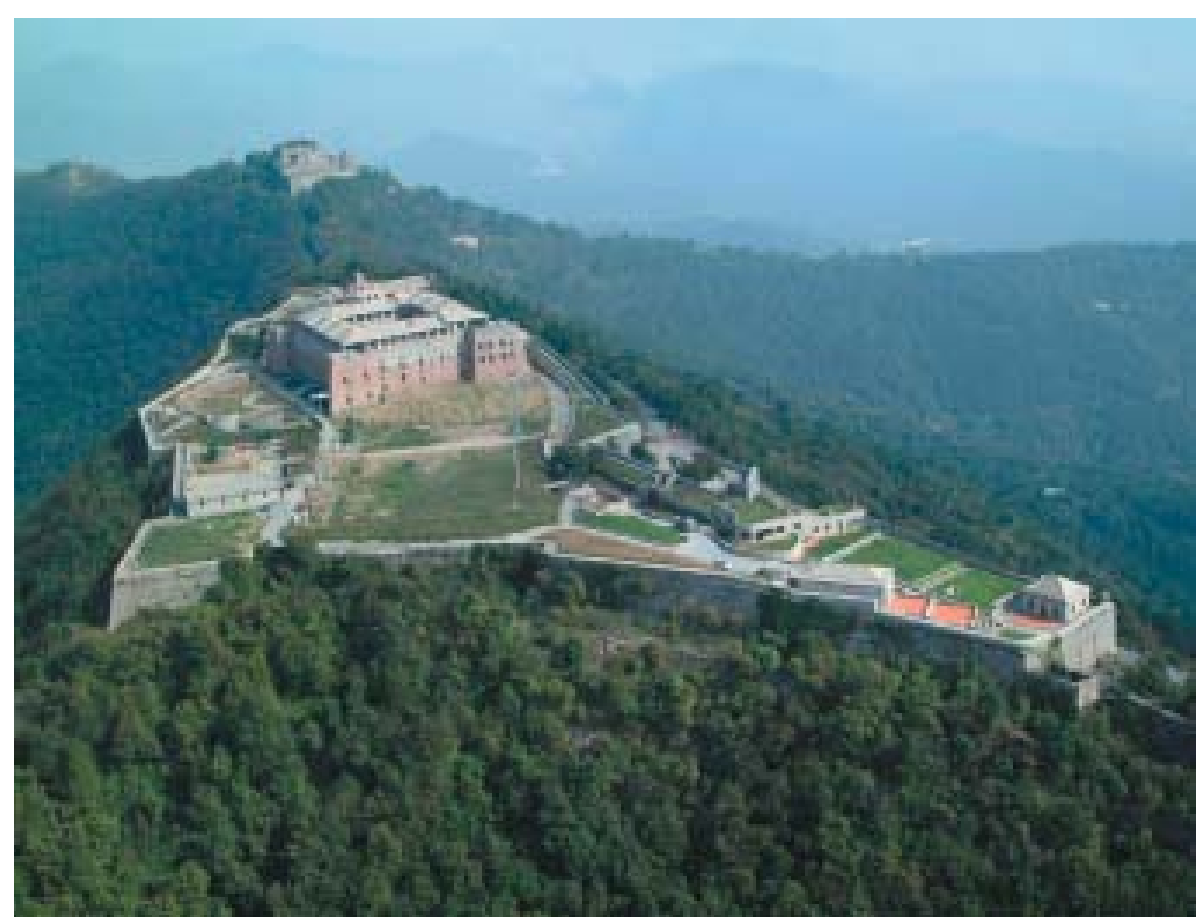

Figura 12 - Forte Begato con la sistemazione degli spazi aperti: veduta aerea.

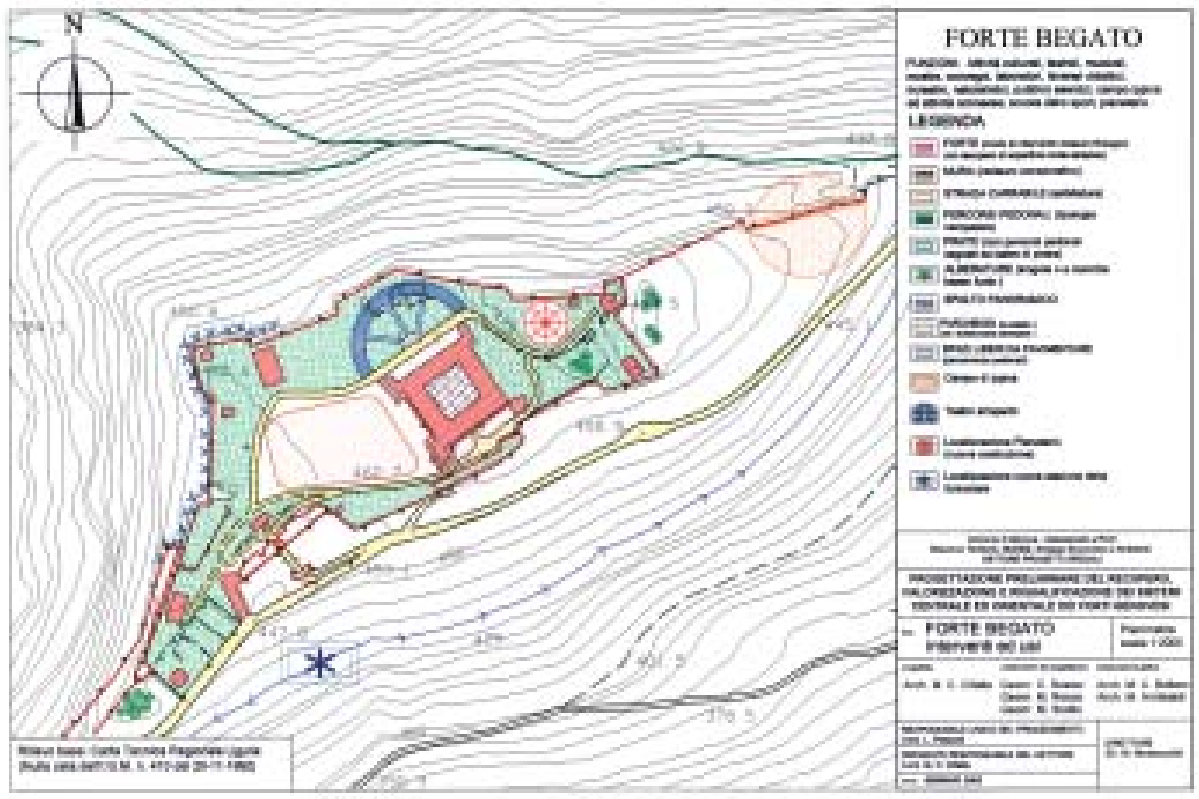

Figura 13 - Progetto preliminare di recupero, riuso e valorizzazione dei forti di Genova: scheda di Forte Begato.

zie ai fondi comunitari inseriti nel Programma Operativo Pluriennale del Fondo Europeo di Sviluppo Regionale - Obiettivo 2.

La realizzazione dei lavori e del completamento delle opere in corso, sia di quelle ancora in fase di studio, porrà le basi per la concreta individuazione (procedura già attualmente avviata) delle metodologie e dei soggetti a cui affidare la gestione dell'intero complesso e delle relative attività. Sono allo studio due ulteriori e successivi lotti: uno riguardante i sistemi di accessibilità e l'altro l'inserimento di una struttura a forte componente attrattiva, peraltro già prevista nel preliminare, costituita dal "planetario": un nuovo edificio di forma emisferica, simile allo geod del parco della Villette a Parigi. 
Il progetto di un sistema illuminotecnico esterno per la valorizzazione dei forti genovesi

L'inserimento degli impianti tecnologici, come quelli idrico-sanitari, di riscaldamento, di condizionamento e ventilazione, elettrici e del gas, rappresenta un ulteriore fase di progettazione esecutiva molto delicata. Tra questi impianti tecnologici, già nel preliminare è presente un progetto di un sistema illuminotecnico esterno allargato all'intero complesso dei forti genovesi ${ }^{19}$.

Tutto il progetto di recupero e riqualificazione tende ad una valorizzazione dei beni, soprattutto dal punto di vista economico: tali beni si prestano ad essere un forte richiamo turistico, pertanto l'aspetto economico della valorizzazione non può essere disgiunto da un'esaltazione della specificità, del valore estetico, dell'importanza architettonica, paesaggistica e d'immagine che i forti, le mura e i percorsi nel loro insieme presentano. Di conseguenza, nel progetto illuminotecnico una delle componenti più importanti risulta la cura dell'immagine dei singoli elementi collegati a sistema e la loro visibilità nel paesaggio urbano dalle diverse angolature visuali, dai punti panoramici e dalle percorrenze d'accesso alla città.

Dato che oggi sono fortemente presenti nel contesto panoramico cittadino per la loro imponenza, pertanto caratterizzanti lo stesso paesaggio nelle ore diurne, il progetto ritiene che i forti possano essere adeguatamente segnalati come richiamo paesaggistico anche nelle ore serali e notturne.

Il preliminare ritiene che solo l'attuazione di un intervento sull'intero sistema dei forti possa costituire, coerentemente con il progetto complessivo di tipo funzionale e

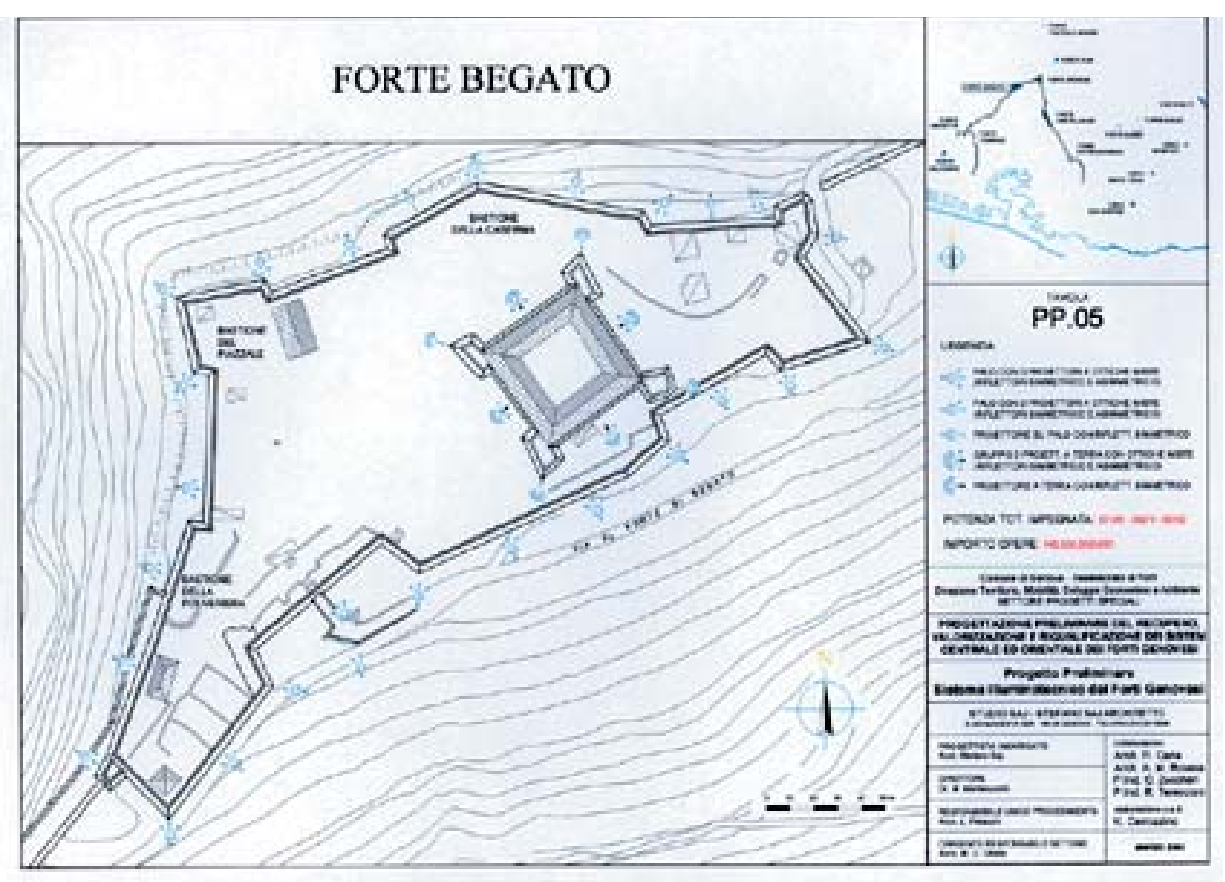

Figura 14 - Progetto preliminare del sistema illuminotecnico dei forti di Genova: scheda di Forte Begato.

\footnotetext{
${ }^{19}$ Un altro interessante progetto è quello relativo al riordino delle servitù e dei rapporti con i gestori di impianti (antenne): l'eccezionale posizione delle fortificazioni, poste sui crinali dei monti più alti intorno al tessuto urbano, si è prestata nel tempo ad accogliere in modo del tutto casuale e disordinato il posizionamento delle più disparate antenne per radio, telefonia, televisioni, forniture di energia eccetera Molti di questi insediamenti sono stati realizzati in modo abusivo ed oggetto di autorizzazione in sanatoria. Tali impianti deturpano in modo evidente l'aspetto architettonico e paesaggistico delle fortificazioni e delle mura; si rende pertanto necessario progettare adeguatamente un riordino delle concessioni demaniali per tali impianti e verificare prima di tutto essenzialità, necessità ed uso degli stessi. Gran parte delle antenne esistenti oggi inutilizzate, ovvero collocate in luogo inadatto o incongruente con l'ambiente architettonico di pregio, dovranno (in sede di progetto definitivo ed esecutivo), essere oggetto di un sistematico progetto di demolizione con ripristino dei siti inquinati.
} 


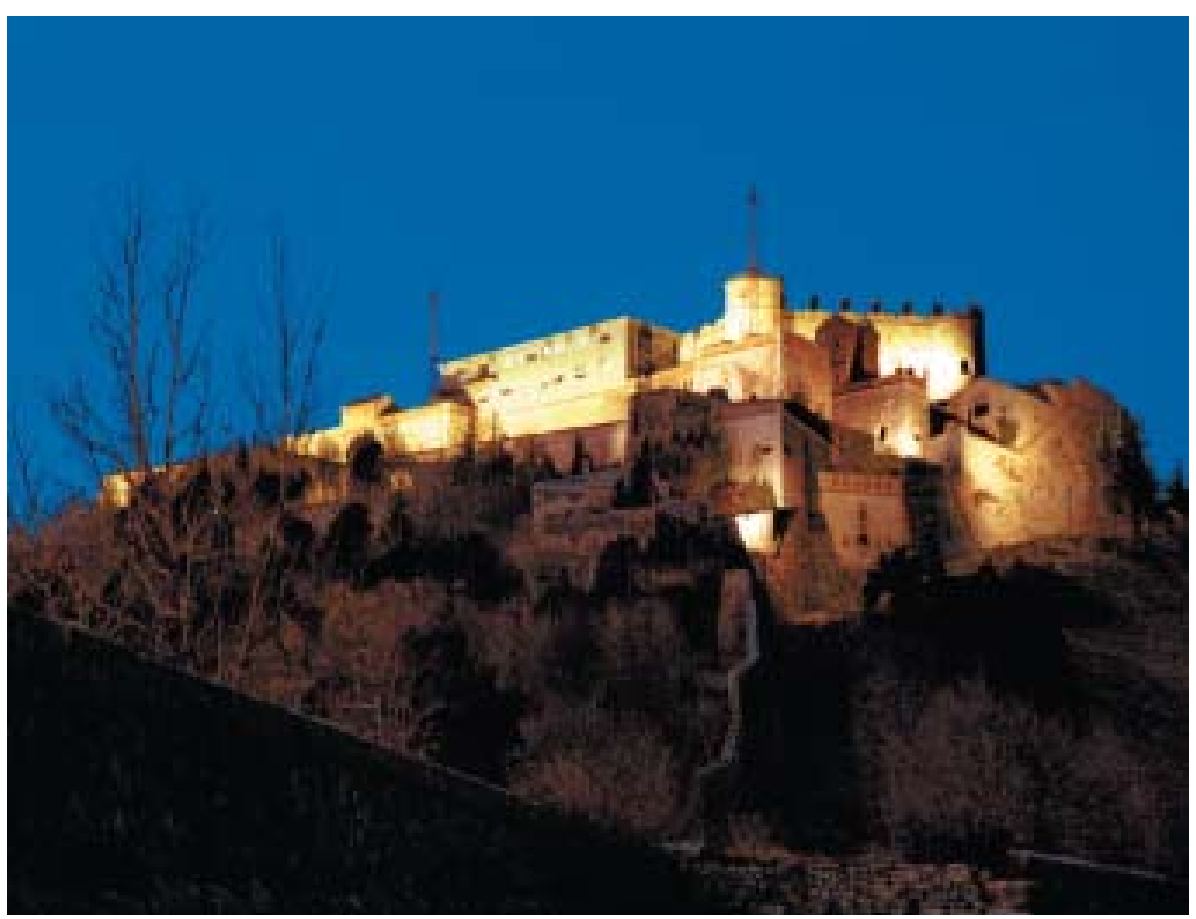

Figura 15 - Visione notturna del Forte Sperone.

urbanistico-architettonico, un elemento d'attrattività e di richiamo: gli impianti già installati al Forte Puin e al Forte Sperone hanno determinato una significativa valorizzazione paesaggistica notturna.

I criteri progettuali che sottendono il progetto illuminotecnico sono riassumibili in:

- esaltazione delle valenze ambientali dei manufatti e dell'intorno murario, con funzione di comunicazione dell'entità degli elementi architettonici: facciate, speroni, spigoli vivi, portali, presenza di torri, eccetera;

- gioco di luce "sparata dal basso verso l'alto" per rimarcare l'imponenza dei muri e della plasticità volumetrica dei corpi edilizi;

- disposizione delle fonti di luce a cornice dell'intero manufatto, con almeno un punto luce su ogni fronte prospettico, al fine di assicurare il completo coinvolgimento della luce e la visibilità da tutti i punti panoramici;

- estensione dell'illuminazione scenografica alle mura nei punti di maggiore evidenza e imponenza e in prossimità dei forti;

- ogni forte, così come ogni tratto di mura preso in esame, ha la sua scheda illuminotecnica e può essere oggetto di specifico progetto realizzato in autonomia, in riferimento alle esigenze funzionali legate agli eventi e alle possibilità di carattere finanziario.

Questo progetto, risultando fondamentale per la presentazione e la valorizzazione paesaggistica del sistema dei forti, costituisce uno degli obiettivi prioritari dell'Amministrazione civica, raggiungibile in prima istanza come effetto promozionale ma ritenuto di particolare importanza strategica all'interno dell'intero progetto di recupero del sistema dei forti genovesi.

\section{RIFERIMENTI BIBLIOGRAFICI}

Bosco Givliano (a cura di), Progetti integrati per le antiche fortificazioni costiere, Atti del Convegno (La Spezia-Portovenere 16-17 maggio 1998) organizzato da "Archeoclub d'Italia" (Asso- 
ciazione nazionale per la tutela e la valorizzazione del patrimonio storico-artistico e ambientale), Agorà Edizioni, La Spezia 1999.

Comando $19^{\circ}$ Zona Militare della Liguria, Forti di idee - Proposte per il recupero delle fortificazioni di Genova, Genova, gen. 1991.

Comune di Genova, Il parco urbano delle Mura, Sagep Editrice, Genova 1994.

Finocchio Renato, Le fortificazioni di Genova, Valenti Editore, Genova 1983.

Forti LeOne Carlo, Le fortificazioni di Genova, Stringa Editore, Genova 1971.

Parodi Andrea, I monti di Genova, Andrea Parodi Editore, Genova 1999.

Stringa PaOlo, Guide di Genova (nn. 24-25-26), Sagep Editrice, Genova 1976.

STRINGA PAOLO, I forti di Genova - Un itinerario turistico-culturale, Sagep, Genova 1985.

\section{RIFERIMENTI ICONOGRAFICI}

Le schede di progetto e i materiali illustrativi e fotografici sono stati tratti dai documenti relativi al "Progetto preliminare per il recupero, il riuso e la valorizzazione dei sistemi centrale e orientale dei forti genovesi", reperiti presso il Comune di Genova, Settore Progetti Speciali. In particolare, si intende ringraziare l'Arch. Marina D'Onofrio Caviglione del Settore Pianificazione Urbanistica e il Geom. Mario Rocca del Settore Progetti Speciali per l'estrema disponibilità, nonché l'Arch. Anna Maddaluno, senza la cui collaborazione questa scheda non sarebbe mai stata compiuta.

\section{SCHEDA DI PROGETTO}

Progetto:

Committente:

Superficie:

Gruppo di lavoro:

Progettista:

Collaborazione alla progettazione:

Assistenza alla redazione progettuale:

Progettazione impiantistica:

Schedatura urbanistica:

Progettazione accessibilità mezzi di trasporto pubblico:

Schede specifiche progettuali del verde:

Progetto preliminare geologico:

Supporti cartografici: progettazione preliminare del recupero, riuso e valorizzazione dei sistemi centrale ed orientale dei forti genovesi

Comune di Genova

$20.480 \mathrm{mq}$ (comprendono solo l'assetto costruito del sistema di forti di Genova)

Comune di Genova

Direzione Territorio, Mobilità, Sviluppo Economico, Ambiente SETTORE PROGETTI SPECIALI

Direttore: Dr. Massimo Montecucchi

Responsabile unico del procedimento: Arch. Laura Petacchi

Dirigente del Settore Progetti Speciali: Arch. Maria Caterina Cifatte

Arch. Maria Caterina Cifatte

Geom. Carlo Scarso, Geom. Mario Rocca, Geom. Maurizio Scotto

Arch. Carla Bottaro, Arch. Monica Annibaldi

Ing. Paolo Marrè-Brunenghi, Per. Giuseppe Vella

Arch. Marina D’Onofrio Caviglione, Arch. Ivana Sciutto, Geom. Piero Luciani

AMT Ing. Michele Montanari

Dr. Giorgio Costa, Arch. Stefano Ortale

Dr. Michele Bonfante, Dr.ssa Sabrina Razzore, Dr. Diego Bruzzo

SIT Ing. Danilo Bertini 
SEGUE SCHEDA

Agenzia del Demanio - Filiale di Genova: Ing. Giuseppe Izzo e Geom. Giovanni Coiana

Soprintendenza per i Beni Architettonici e per il Paesaggio della Liguria:

Ing. Rita Pizzone, Geom. Gianni Gianardi

Progettazione:

approvazione con deliberazione adottata dalla Giunta Comunale nella seduta del 25/01/2002

Realizzazione:

in corso

Gli uffici comunali competenti, in collaborazione con gli uffici della locale Soprintendenza per i Beni Architettonici e per il Paesaggio della Liguria e dell'Agenzia del Demanio, Filiale di Genova, hanno elaborato una progettazione preliminare approvata, ai fini della delibera del Consiglio Comunale, che si compone di due elaborati: Relazione Tecnica e Protocollo d'Intesa.

\section{Allegato 1:}

- "Relazione Tecnica" con gli indirizzi ed i criteri di restauro e suoi allegati descrittivi;

- "Dossier progettuale" con schede tecniche per ogni forte, comprensivo delle tavole relative ai vincoli urbanistici, alle destinazioni d'uso, ai modi d'intervento, alla documentazione fotografica, alla toponomastica e agli estratti catastali;

- Planimetrie di ricognizione dello stato delle mura;

- Planimetrie dell'Accessibilità dei due Sistemi Centrale ed Orientale;

- Planimetrie con l'individuazione dei parcheggi per auto e campers;

- Progetto preliminare dell'assetto geologico ed idrogeologico;

- Progetto per impianto illuminotecnico speciale;

- Linee guida per la sistemazione ambientale degli spazi di pertinenza;

- Preventivo di massima delle spese da sostenersi per il restauro dei forti e mura comprensive degli impianti e dei sistemi di trasporto di tipo innovativo;

- Capitolato Speciale d'Appalto per l'attività di progettazione esecutiva e definitiva;

- Studio preliminare per la sicurezza nei cantieri;

- Stato d'occupazione del suolo degli immobili demaniali.

L’Agenzia del Demanio e la Soprintendenza per i Beni Architettonici e per il Paesaggio della Liguria hanno espresso parere favorevole su tale progettazione convenendo sull'opportunità di sottoscrizione di apposito Protocollo d'intesa la cui bozza, concordata tra i diversi soggetti, viene allegata alla deliberazione quale parte integrante e sostanziale del documento, ai fini dell'approvazione del progetto. 


\title{
L'ILLUMINAZIONE DELLE MURA DI VERONA
}

\author{
Maristella Storti
}

\section{IL CONTESTO}

\section{Le mura di Verona: monumento esemplare all'architettura militare italiana}

La città di Verona conserva, da duemila anni, come nessun'altra città italiana, un sistema fortificatorio relativo alla sua cinta muraria ritenuto tra i più importanti, interessanti ed estesi d'Europa, frutto di un intreccio singolare tra aspetti morfologici, storico-culturali e paesistici e testimonianza suprema di un'arte oggi estinta: l'architettura militare.

Tra la collina e la pianura, tra la terraferma e il fiume Adige, la Cinta delle mura di Verona è il prodotto di diverse stratificazioni che via via ne hanno modificato il disegno per dimensioni, tipologie e materiali.

Verona è, infatti, città fortificata per eccellenza; monumento di assoluto interesse storico e di impareggiabile qualità architettonica ed ambientale in cui in diverse epoche (comunale, scaligera, sanmicheliana e asburgica) sono stati realizzati manufatti, seppur diversi tra loro, di grande sapienza tecnica ed artistica. In particolare, tra il 1530 e il 1548 il sommo architetto veronese Michele Sanmicheli diede alla sua città capolavori assoluti della moderna fortificazione italiana: terrapieni, bastioni, cortine, cavalieri e porte urbane, di cui oggi rimangono alcune opere esemplari.

Il Sanmicheli è architetto militare, ma la sua importanza risiede non tanto nella progettazione di singole opere difensive, quanto nello studio urbanistico della cinta muraria in relazione allo sviluppo della città. Le mura infatti, considerate di importanza strategica, nel tempo hanno condizionato la vita stessa della città, ponendosi come limite all'edificazione verso la spianata esterna.

Nei primi anni dell'Ottocento, i francesi di Napoleone demolirono gran parte della Cinta a destra d'Adige che venne in seguito ricostruita dagli austriaci con criteri più evoluti di difesa militare e con tecniche costruttive raffinate che stupiscono ancora oggi. In particolare, il progetto dei bastioni di Franz von Scholl (1833-36) integra e rafforza le opere superstiti del Sanmicheli.

Nel corso del Novecento, questo grandioso insieme di edifici e spazi, nonché di ambiente naturale, un tempo generato dalle ferree leggi di guerra, viene abbandonato e convertito ad altre funzioni spesso incongrue.

Molteplici sono stati i destini urbanistici delle cinte magistrali nelle città del Novecento:

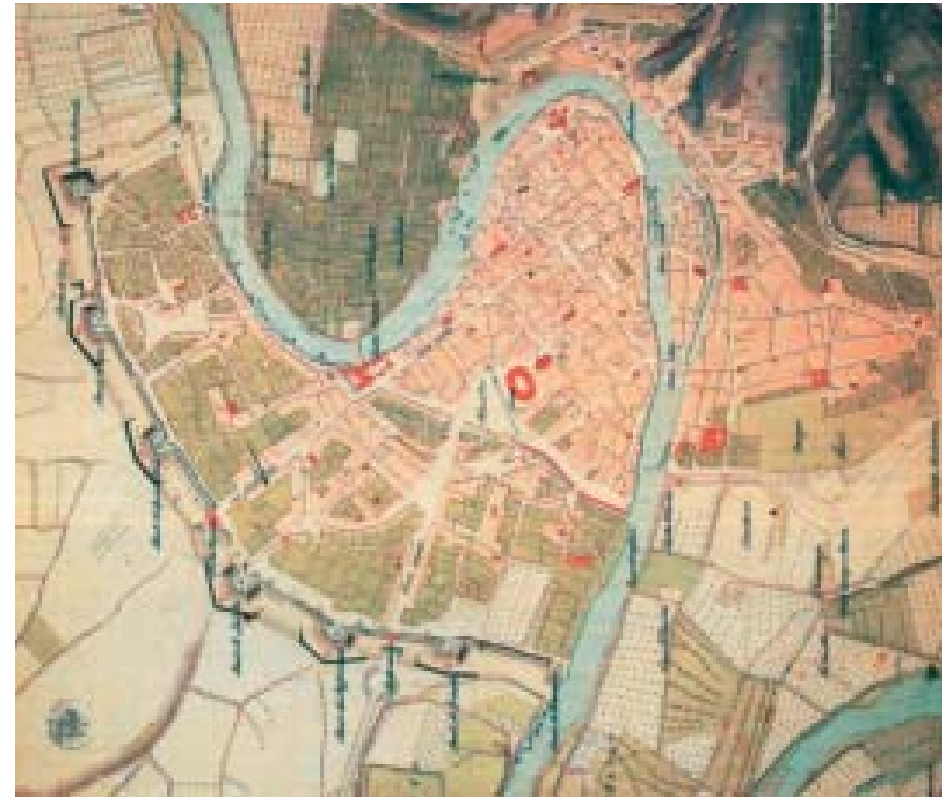

Figura 1 - Plan der Stadt und Umgebung von Verona (1835) - Grande rilevamento topografico in scala 1:6.914, eseguito sotto la direzione del maggiore Johann von Hlavaty dell'Ingenieur Corps asburgico. 


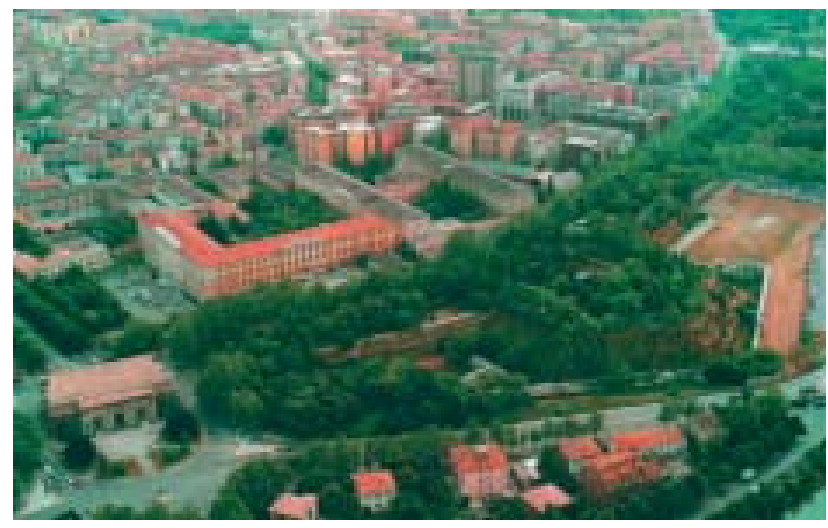

Figura 2 - Il bastione asburgico di S. Spirito (1836) e il grande ospedale militare d'armata (1852): veduta aerea.

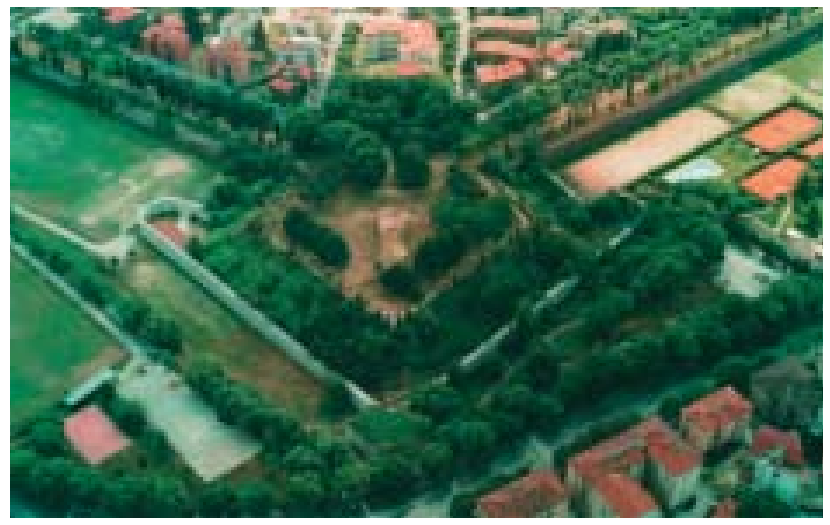

Figura 3 - Il bastione asburgico di S. Procrolo (1836-38): veduta aerea.

spesso vennero smantellate, in modo parziale o totale; a volte erose progressivamente, incorporate nella crescita urbana; altre volte mantenute in uso dall'esercito o convertite agli usi civili. Quasi sempre l'originaria unità dell'organismo fortificato è stata compromessa.

Oggi questi grandi esempi di spazi aperti e di architetture monumentali possono tornare ad essere riscoperti a Verona come altrove: monumenti che si arricchiscono di nuovi significati a distanza di secoli.

Come un tempo vi appartenevano in quanto opere destinate alla difesa nazionale, anche oggi, per il loro eminente interesse storico e artistico, le mura di Verona fanno ancora parte del Demanio dello Stato nonostante l'imminente passaggio alla gestione comunale. Inoltre, nel 2000 l'UNESCO dichiara il centro storico di Verona "Patrimonio storico e culturale dell'umanità", in quanto "esempio di eccezionale bellezza dell'evoluzione della città fortificata attraverso i secoli".

\section{Verona abbandona le sue mura}

Dopo quest'estrema sintesi sulla costituzione della grande opera monumentale che sono le mura di Verona, seguita dal riconoscimento a livello mondiale della loro importanza nel campo dell'architettura militare italiana e la riscoperta del loro valore da parte della città, si ripercorrono più nel dettaglio le tappe principali dell'abbandono e della rinascita di questo patrimonio fortificatorio ripartendo dalle vicende del XX secolo, così importanti per le diverse trasformazioni e destinazioni d'uso che hanno interessato la cinta muraria e il suo immediato intorno.

Dopo l'intervento austriaco, la città di Verona non ha più instaurato un buon rapporto con le sue mura e dall'inizio del secolo scorso in poi, esse sono state considerate nel migliore dei casi spazio da sfruttare, intaccate soprattutto nelle opere di terra (rampari, cortine, fosso magistrale) per realizzarvi le più disparate funzioni: dai giochi allo zoo, dai campi sportivi (calcio, tennis...) ai parcheggi, dalle piscine alle balere.

Di questi interventi forse i più critici sono stati, da un lato, intorno agli anni Trenta, l'apertura delle brecce, che hanno interrotto la continuità della fortificazione e relegato in isole spartitraffico le porte sanmicheliane; dall'altro, nel secondo dopoguerra, la scelta dei valli esterni - ossia, il fosso magistrale, "vuoto" prospettico essenziale alle fortificazioni - come discarica per le macerie della città bombardata. Eppure, durante le innumerevoli incursioni aeree, le mura avevano protetto $\mathrm{i}$ veronesi nei rifugi approntati nelle poterne, nelle caponiere, nelle gallerie sanmicheliane di contromina, quindi le loro misteriose architetture sotterranee avevano garantito spesso la vita. In tempo di pace, però, l'urgenza della ricostruzione imponeva alle mura un sacrificio che adombrava il loro 
futuro destino di opere ritenute prive di qualità; altri interramenti, oltre a quello del fosso magistrale, riducevano la visibilità e la bellezza delle fortificazioni. Intanto, veniva proposto addirittura l'abbattimento delle mura a destra d'Adige e si costruivano abitazioni popolari nella zona di S. Bernardino, cancellando gli impianti originari delle pertinenze del bastione omonimo.

Infranta la delicata resistenza del "vuoto" esterno (il vallo), soprattutto tra gli anni 1960-85, si andavano edificando imponenti edifici sportivi pubblici a ridosso delle mura e per l'avvenimento dei mondiali di calcio del 1990 si costruirono anche nuovi parcheggi e sottopassi, sottraendo sempre più spazi al fosso magistrale. Ad ogni piè sospinto si insediavano campi sportivi di varia vocazione con i loro corollari di lampioni e di "torri faro", creando così luoghi accessibili solo a poche persone. Strade e parcheggi facevano la loro parte: invadevano, intersecavano e riducevano gli spazi esterni della Cinta magistrale.

Nuove edificazioni private seguirono l'esempio invadendo spontaneamente lo spazio di pertinenza delle mura, ormai destituito a terra di nessuno. Nelle aree rimaste di uso pubblico, la frequentazione si è man mano degradata fino alla creazione di veri e propri villaggi di senzatetto. Non solo, per abbellire le mura di destra d'Adige, che nel 1960 erano solo parzialmente e ragionevolmente piantumate, si intraprese un'intensa, quanto improvvisata, eterogenea e fittissima sistemazione a verde pubblico (con piante sempreverdi) di terreni storicamente assai poveri di vegetazione d'alto fusto, mentre i terrapieni vennero recintati con reti metalliche di bassa qualità. Perdute le consuetudini della manutenzione, una coltre vegetale spontanea con le più disparate specie arboree ha poi omogeneizzato il tutto e, lentamente e inavvertitamente, le mura di entrambe le rive dell'Adige sono state cancellate dal paesaggio urbano. Immagini fotografiche antecedenti alla "boscaglia" restituiscono visioni sorprendenti; originariamente le mura non avevano vegetazione né arborea né arbustiva ma solo un continuo tappeto erboso. I massicci impianti arborei a cavallo degli anni Sessanta sono stati eseguiti senza tener conto delle caratteristiche morfologiche del manufatto e senza avere un qualsiasi criterio di impianto, con il risultato che è stata per gran parte persa la percezione delle verdi geometrie.

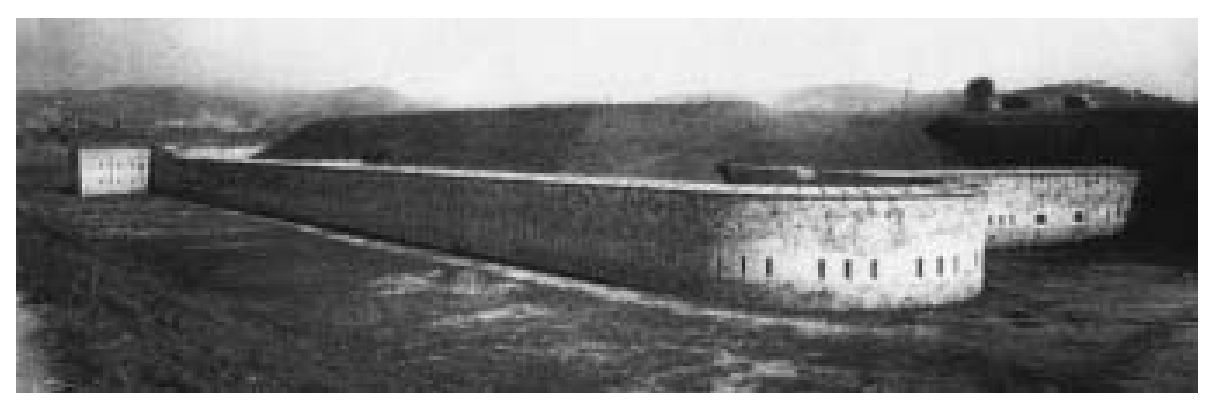

Figura 4 - Il bastione asburgico di San Procrolo in una fotografia dell'anno 1940.

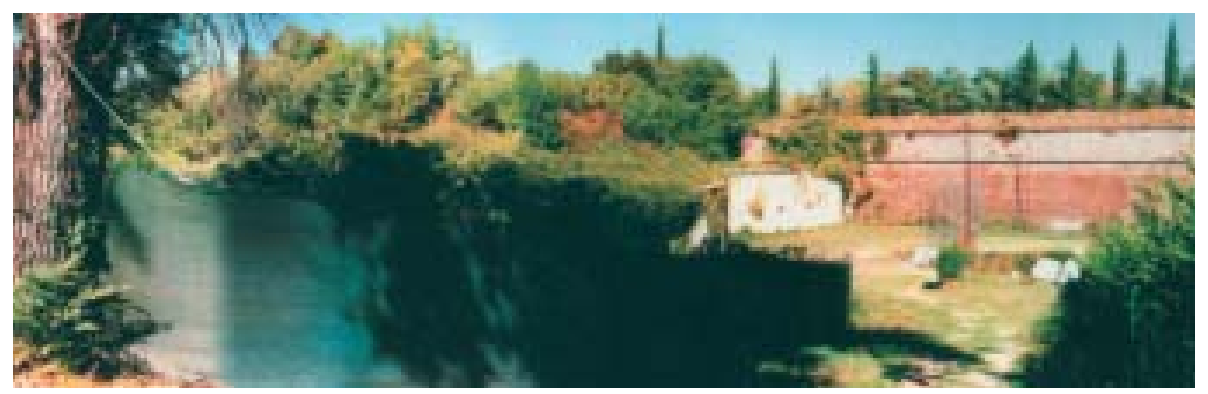

Figura 5 - Il bastione asburgico di San Procrolo assediato dalla vegetazione. Sono iniziati i lavori di diradamento. 


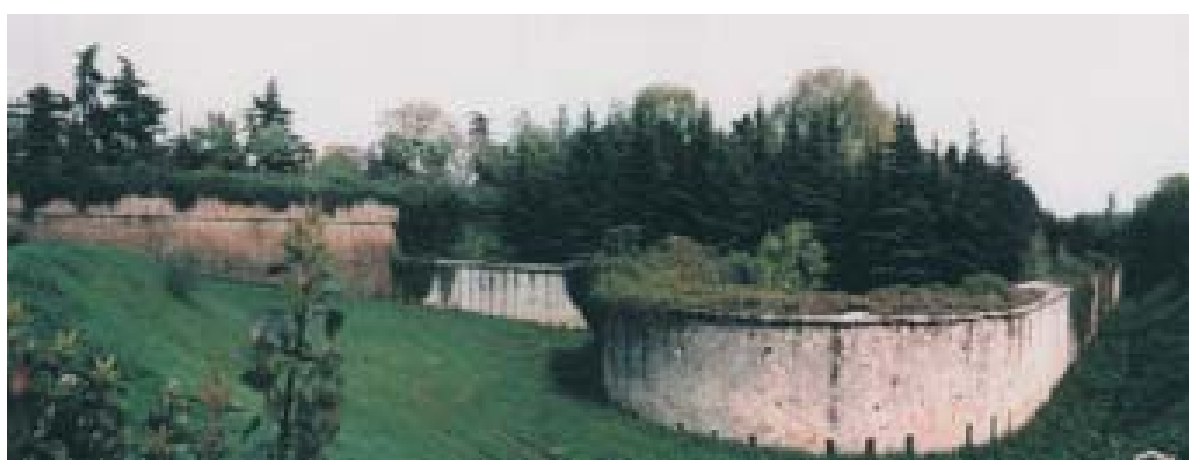

Figura 6 - Il muro veneziano, l'orecchione austriaco e il vallo del bastione dei Riformati. La vegetazione invade il bastione negando l'opera.

Le mura, abbandonate sempre più dai cittadini, divennero il luogo prediletto di malintenzionati, di vite marginali e disperate in un contesto ambientale pessimo, trascurato, le reti metalliche sfondate in diversi punti, la sporcizia e l'incuria crescenti, sino all'eclissi totale degli anni Novanta. Nonostante ciò è da sottolineare il fatto che, nel secolo scorso, il vero danno subito dalle mura di Verona non furono tanto le trasformazioni materiali, fisiche, l'assedio urbanistico, quanto la perdita di coscienza e di significato delle mura per l'identità artistica e civile della città come se, con dissennata demagogia, taluni ritenessero di condannarle all'oblio perché testimonianza dell'odiato nemico.

Verona riscopre il suo patrimonio fortificatorio: le tappe significative di questo percorso

Dopo un periodo di eclissi e di distacco l'opinione pubblica, gli studiosi, l'Amministrazione civica, riscoprono per molteplici ragioni questa preziosa eredità storica, architettonica ed urbanistica. La rinascita è promossa soprattutto da associazioni di volontariato mentre con spirito conservativo la Soprintendenza documenta, rileva le fortificazioni, ne raccoglie la schedatura per l'Istituto Centrale del Catalogo. All'Università, soprattutto gli studenti di architettura, si cimentano in tesi di laurea storiche, urbanistiche e progettuali sulle fortificazioni veronesi. Tra gli anni Ottanta e Novanta, quindi, la bibliografia sull'architettura militare veronese si arricchisce di fondamentali pubblicazioni.

Nel 1989 l'opinione pubblica, gli studiosi, i cittadini veronesi, accolsero con soddisfazione la legge, approvata dal Parlamento della Repubblica, con la quale si autorizzava il trasferimento della Cinta magistrale di Verona dallo Stato all'Amministrazione Comunale (legge n. 225 del 30 maggio 1989). Oggi, dopo più di dieci anni, il trasferimento ufficiale non si è ancora compiuto, anche se gli atti conclusivi sono stati recentemente perfezionati e sono al vaglio dell'Avvocatura dello Stato per il parere di conformità, viste le notevoli difficoltà interpretative della legge per quanto riguarda gli oneri relativi al passaggio e all'individuazione del giusto perimetro della totalità delle opere fortificatorie stesse.

Le mura sono una parte importante di Verona, connotano la forma urbana dell'edificato storico e ne hanno determinato vincoli e direttrici di espansione; costituiscono un patrimonio architettonico di grande rilevanza ma anche, una volta abbandonata la loro pura funzione militare e difensiva, un cospicuo polmone verde. Malgrado l'abbandono, hanno conservato gran parte della loro "potenza" architettonica e monumentale.

Queste considerazioni hanno spinto l'associazione di volontariato "Legambiente" e alcuni professionisti a credere che fosse possibile intervenire, con limitate risorse finanziarie, su uno dei più estesi e abbandonati tratti di mura della parte in destra d'Adige: i bastioni di S. Bernardino e di S. Zeno. 
Prima del 1995 questo era un luogo che i veronesi evitavano assolutamente, le condizioni sociali e igieniche erano disastrose, la vegetazione spontanea aveva invaso le scarpate esterne e tutta la parte bassa dei bastioni, nascondendo completamente le opere monumentali.

Nel 1995 il Comune di Verona, con la partecipazione della Prefettura e della Fondazione Cassa di Risparmio, affida a "Legambiente" la manutenzione delle aree a verde pubblico di questa zona (per una superficie complessiva di circa 100.000 metri quadrati) mediante una convenzione poi rinnovata per altri cinque anni.

Nel frattempo, prendendo atto che la manutenzione dell'opera non era sufficiente per avviare un vero recupero e per la valorizzazione sia dei manufatti monumentali che dell'area verde, viene redatto il progetto per il recupero dell'area, a cura dello studio di architettura Lino Vittorio Bozzetto e dello studio di paesaggistica Ballestriero-Muscari coadiuvati dall'arch. Alessandra Morsiani. Il progetto viene finanziato in parte dalla Prefettura di Verona con fondi sequestrati alla criminalità e in parte dalla Fondazione della Cassa di Risparmio.

Su indicazione dei progettisti, "Legambiente" elabora un proprio programma di massima, redatto e approvato nella stesura esecutiva nel 1999, per l'esecuzione di lavori urgenti: è il primo intervento sulle mura volto al loro recupero.

Lesperienza di "Legambiente", che ha permesso il coinvolgimento di numerose istituzioni e di iniziative collaterali, si pone come "intervento pilota" in quanto, oltre all'esecuzione dei lavori di recupero, viene poi garantita la gestione degli spazi fortificati riconvertiti, facendo leva sull'importanza del "pianificare la manutenzione".

Per la prima volta viene eseguito un diradamento mirato della vegetazione, che ha restituito sorprendenti scorci di spazialità prospettica ormai dimenticati da tutti i veronesi.

Intanto il Comune, con la consulenza dell'arch. Bozzetto, per la prima volta inserisce la Cinta magistrale nel Nuovo Piano Regolatore Generale (PRG), prevedendone la tutela, il recupero e la valorizzazione anche dal punto di vista ambientale, proponendo un "Parco per la Cinta magistrale".

Lo stesso gruppo di progettisti, su incarico del Comune, redige anche il "Progetto guida per l'illuminazione della Cinta magistrale a destra d'Adige".

Nel 2000 "Legambiente" ottiene dalla Fondazione della Cassa di Risparmio un nuovo finanziamento di centocinquanta milioni di lire per completare le opere iniziate, i cui lavori urgenti terminano l'anno successivo.

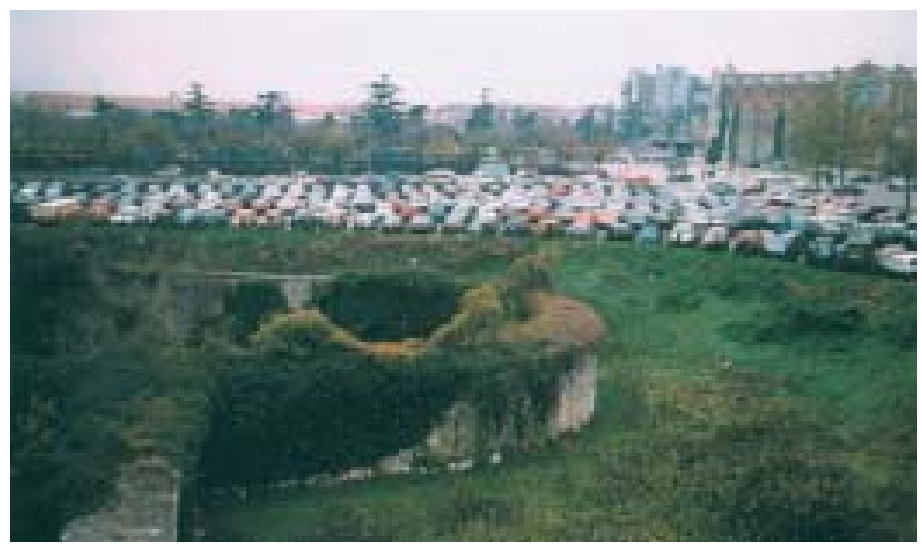

Figura 7 - Parcheggi sul riempimento del vallo del bastione dei Riformati, a due passi dalla Stazione di Verona-Porta Nuova

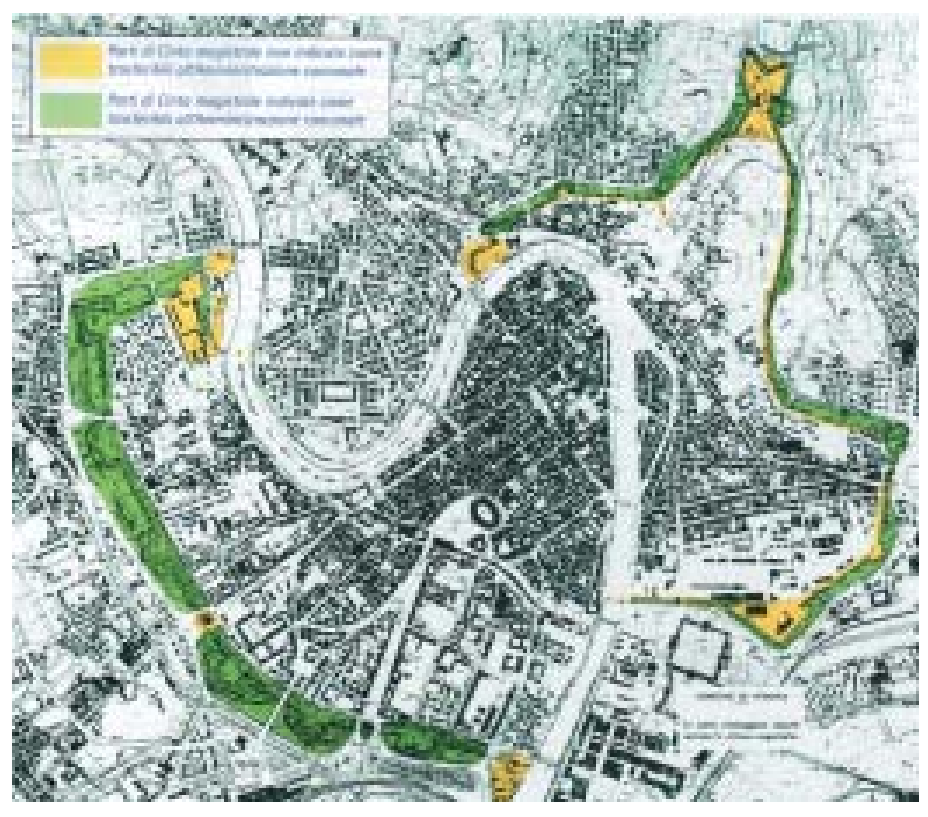

Figura 8 - Tavola allegata alla Legge 30 Maggio 1989, n. 225 - Trasferimento della "Cinta magistrale" della città di Verona all'Amministrazione Comunale.
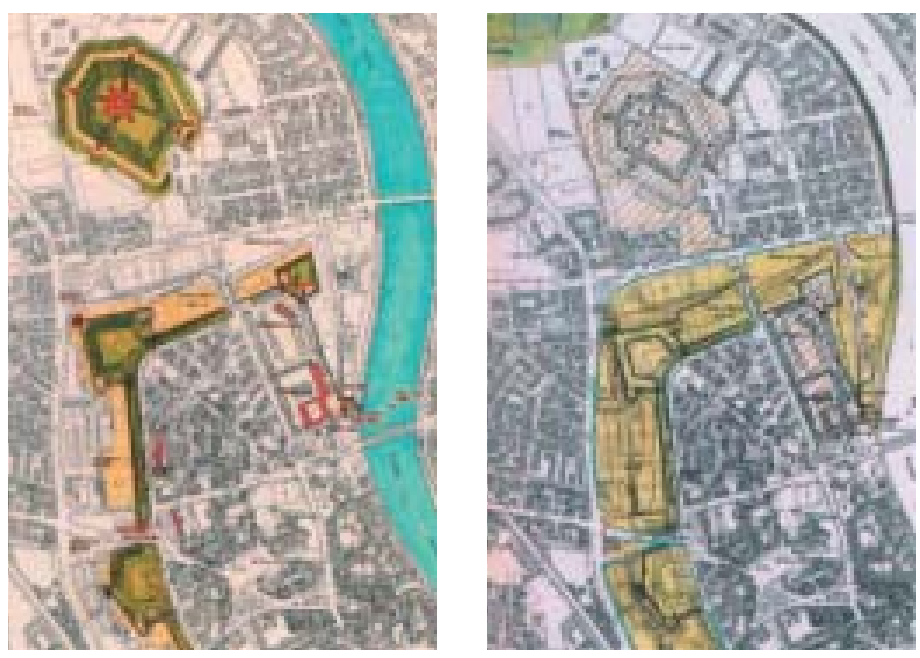

Figura 9 - Dettagli delle tavole allegate al Piano per il Parco della Cinta magistrale (Variante Generale al Piano Regolatore Generale, 1999). 


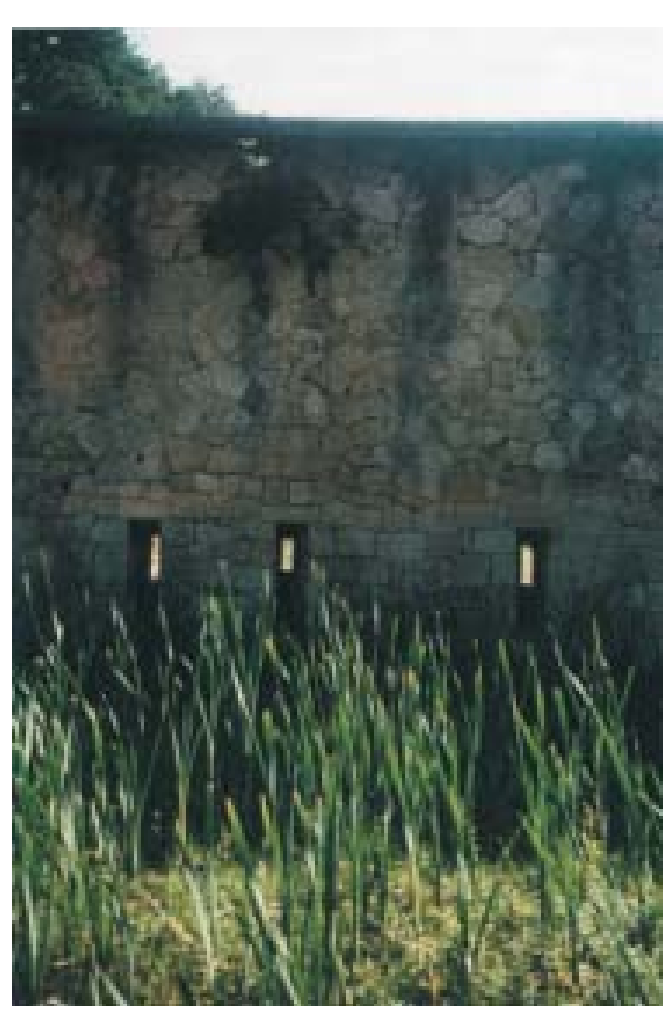

Figura 10 - Fessure di luce nel bastione di San Bernardino. Il tufo, con paramenti ad opus poligonale, di accuratissimo taglio, messo in opera nella complessa sequenza dei muri alla Carnot, è il contrassegno stilistico della fortificazione asburgica, che risalta per il bellissimo colore dorato, sui fondali erbosi.
La direzione lavori viene svolta dall'Arch. Bozzetto e dal paesaggista Ballestriero che coordinano anche gli interventi del Comune sul bastione di S. Bernardino e per la posa in opera di panchine e cestini portarifiuti.

Nel frattempo nel bastione di S. Procrolo viene eseguito, a cura dell'Azienda dei Servizi Municipalizzati di Verona, un primo lotto di lavori per l'illuminazione del piazzale del bastione e delle cortine, sulla scorta del Progetto guida sopra menzionato, redatto nel 1999.

Il percorso di progressiva conoscenza e riappropriazione del patrimonio militare di Verona fu intrapreso, quindi, a partire dal 1989 con il passaggio dal Demanio Militare al Comune ed è continuato nel 1999, in particolare, con la redazione di due strumenti cognitivi che costituiscono una tappa importante di questo percorso e che, nello stesso anno, sono stati allegati al PRG di Verona: la "Relazione del Parco della Cinta Magistrale" e il "Progetto guida per l'illuminazione della Cinta magistrale a destra d'Adige".

Le prime azioni di intervento sulle mura e sulle aree verdi pubbliche

Intervenire in un contesto oramai consolidato non è compito facile, richiedendo interventi graduali, complessi e delicati, nonché molteplici contributi finanziari e materiali.

I lavori eseguiti a S. Bernardino e a S. Zeno sono stati poi allargati ad altre zone e hanno riguardato principalmente la pulizia e il disboscamento dei valli, l'eliminazione delle piante infestanti, il riordino della vegetazione arborea e la risemina di erba nelle zone manomesse dai lavori, al fine di ritornare alla perfetta leggibilità dei paramenti murari della Cinta e dell'imponenza della sua concezione.

Sono state realizzate inoltre delle rampette e delle scale rustiche in legno e terra per il collegamento tra le banchine del ramparo, al fine di dare continuità ai percorsi e di facilitare le operazioni di manutenzione. Sono state anche installate tre bacheche in legno con cartelli esplicativi sulla storia delle mura.

Togliere alberi in città è sempre un'operazione traumatica tuttavia in questo caso, sia perché rientrava in un intervento più ampio di recupero, sia perché condotta da un'associazione ambientalista, l'operazione non ha suscitato l'ostilità che si temeva da parte della cittadinanza.

Anche la decisione di togliere definitivamente la maggior parte delle fatiscenti e inutili recinzioni sul perimetro dell'area è stata difficoltosa e preceduta da qualche dubbio circa la sicurezza da parte dei frequentatori. Tuttavia, la nuova percezione delle mura liberate dall'ingombrante presenza e la risposta della cittadinanza, che sempre più numerosa frequenta questo luogo, conferma che le scelte operate nel passato erano corrette, mentre si attende che presto venga ampliata la realizzazione delle opere indicate nel Progetto guida per l'illuminazione delle mura, al fine di procedere nel recupero e nella valorizzazione di altre aree di questo grande monumento esemplare dell'architettura militare della città di Verona.

Per i lavori di manutenzione ordinaria della cinta muraria "Legambiente", in collaborazione con l'Azienda Municipale di Igiene Ambientale (AMIA), in oggi aderisce a un progetto finanziato dalla Comunità europea e impiega giovani provenienti da tutta Europa. L'associazione ha iniziato anche una collaborazione con il Museo di Storia Naturale di Verona per effettuare una ricerca sull'ambiente naturale delle mura (insetti, pipistrelli, uccelli, flora) per ora rivolta ai bastioni di S. Bernardino e S. Zeno.

Inoltre, le associazioni "Legambiente", "Italia Nostra" e "WWF" si sono fatte promotrici di una nuova proposta per la tutela e valorizzazione di questo grande 
complesso monumentale: una "Fondazione Parco delle Mura di Verona", un ente no-profit che, sulla base della legge n.368/98, consenta ad enti pubblici e privati di collaborare alla rinascita della Cinta magistrale di Verona.

Lintervento sui bastioni di S. Bernardino e S. Zeno può essere considerato un "modello" che è possibile applicare agli altri bastioni in destra d'Adige. È anche un esempio di coordinamento tra $\mathrm{i}$ vari soggetti interessati (Associazioni di volontariato, Progettisti, Cittadini, imprese esecutrici, Comune, Soprintendenza, Aziende municipalizzate) che testimonia come, con ridotte risorse finanziarie e interventi mirati, si possa e si debba intervenire su tutta la cinta muraria perché costituisce, oltre che un anello di verde preziosissimo per la qualità della vita urbana, un patrimonio culturale di straordinario interesse.

Col patrocinio della Regione si è costituita anche l'Associazione delle Città Murate del Veneto, le cui molteplici finalità si fondano sul duplice significato delle fortificazioni quali beni culturali e beni economici. Il Comune di Verona ha istituito l'Ufficio per la Valorizzazione dell'Architettura Militare (UVAM) per nuove idee per il recupero culturale delle mura e per operazioni di ampia portata che si avvalgono di partner europei. Infine, il Parco delle Mura, predisposto con la Variante Generale del PRG, nel momento della sua adozione, segnalerà l'inizio di una nuova vita per questo grande spazio aperto monumentale.

\section{GLI OBIETTIVI}

Un Progetto guida in sintonia con il nuovo Piano Regolatore Generale e con il Parco delle Mura di Verona

In sintonia con le previsioni di recupero formulate nel nuovo piano urbanistico comunale, il "Progetto guida per l'illuminazione della Cinta magistrale a destra d'Adige", redatto nel 1999 e allegato al PRG, accoglie i medesimi principi di riconversione ambientale e valorizzazione del sistema delle fortificazioni urbane.

Studiato con queste finalità, ma applicato allo stato attuale dei luoghi, un progetto guida delle opere di fortificazione e della vegetazione sarebbe privo di significato, in quanto condizionato da situazioni in totale contrasto con $\mathrm{i}$ principi di rispetto, conservazione e valorizzazione della Cinta magistrale medesima. Il Progetto guida prevede, invece, condizioni dei

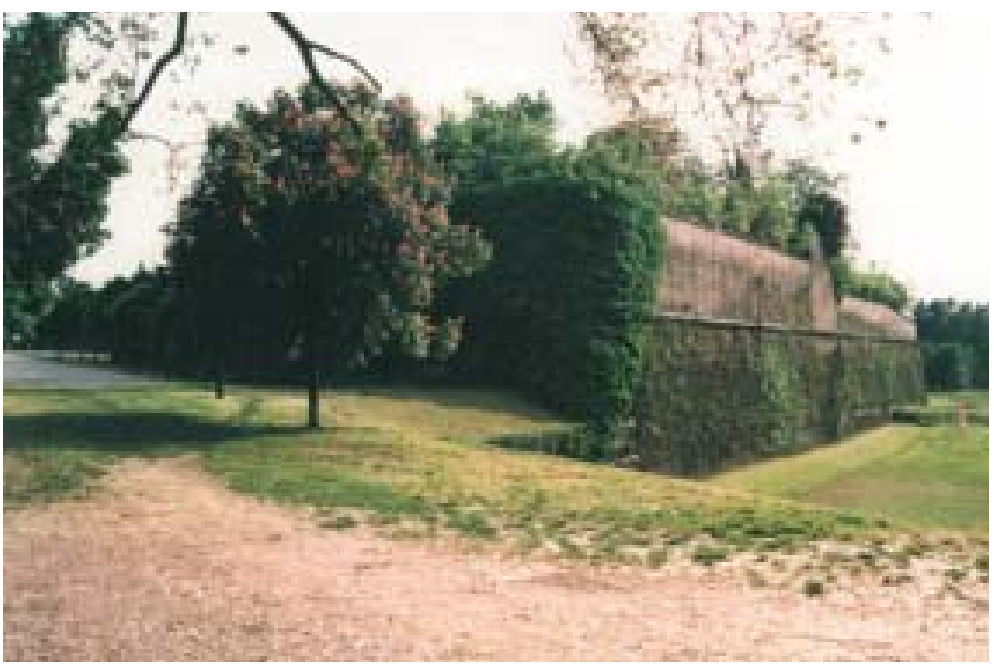

Figura 11 - Le Mura veneziane: la cortina di S. Procrolo. Fosso magistrale, controscarpa del fosso, spalto, conformano il vuoto prospettico all'esterno della cortina. luoghi, delle opere e della vegetazione, ragionevolmente riordinate e ripristinate, prive di tutti gli interventi incongrui operati nel tempo, gravemente offensivi dell'eminente carattere storico e artistico del monumento fortificatorio veronese.

Secondo il progetto, il recupero di quest'area a destra d'Adige potrà avvenire per gradi, come si è già iniziato a fare in parte in altri contesti come quelli di $S$. Bernardino e $\mathrm{S}$. Zeno, con le seguenti priorità:

- recupero della percezione visiva mediante pulizia delle scarpate, dei cammini di ronda, degli orecchioni e del fosso magistrale con eliminazione dell'eccessiva vegetazione; 
- eliminazione delle strutture che schermano o alterano la percezione visiva delle mura (piscine, campi da tennis, baracche, spogliatoi, impianti tecnologici, recinzioni);

- ricostituzione del fosso magistrale con eliminazione o riduzione degli interramenti (parcheggi) e arretramento della controscarpa (salvo eventuali parcheggi inerbiti a livello del fosso e a ridosso della controscarpa);

- eliminazione dei corpi illuminanti infissi sui manufatti storici per illuminare spazi ed attività estranei ed in contrasto con la percezione delle mura.

È un progetto rispettoso dell'alto valore artistico delle opere e di quello che saranno secondo la previsione del nuovo PRG: un parco storico-monumentale a servizio della città e del turismo culturale. La Cinta, infatti, con il passare del tempo, ha cambiato destinazione nella città, ed in epoca moderna è divenuta anche parco: il "Parco urbano delle Mura".

Il tema dell'illuminazione delle mura è perciò anche il tema dell'illuminazione di un parco e si inserisce nel contesto di quell'apparente contraddizione che è la "fruizione" di un monumento. Attualmente il parco, inteso come area verde con vegetazione, è in gran parte disordinato e inselvatichito. Sulla cinta muraria ogni intervento deve rispondere a coerenti criteri di restauro e di manutenzione, limitandosi talvolta a rimuovere le superfetazioni e le occupazioni abusive ed a riportare in luce i manufatti sepolti dai detriti, dalla vegetazione e dai rifiuti, con lo scopo soprattutto di rendere fruibile ai cittadini di tutte le età e ai turisti il Parco urbano delle Mura. In destra d'Adige, esso rappresenta il corridoio verde che incontra e collega i due futuri Parchi dell'Adige Nord, a Ponte Catena, e dell'Adige Sud, a Ponte S. Francesco, come previsto anche dal progetto di Variante Generale al PRG vigente e che delimita un'area che è ora sotto la tutela dell'Unesco.

Il Parco delle Mura sarà, dopo quella scaligera, veneziana e asburgica, la quarta cinta urbana sul perimetro più esterno della città $o$, se si preferisce, la terza ricostruzione delle mura di Verona. Come le altre, questa cerchia di monumenti e di aree verdi servirà a difendere la città, la sua forma, la sua storia e la qualità della vita dei cittadini, salvaguardando gli ultimi spazi liberi rimasti e la possibilità stessa per Verona di avere un parco.

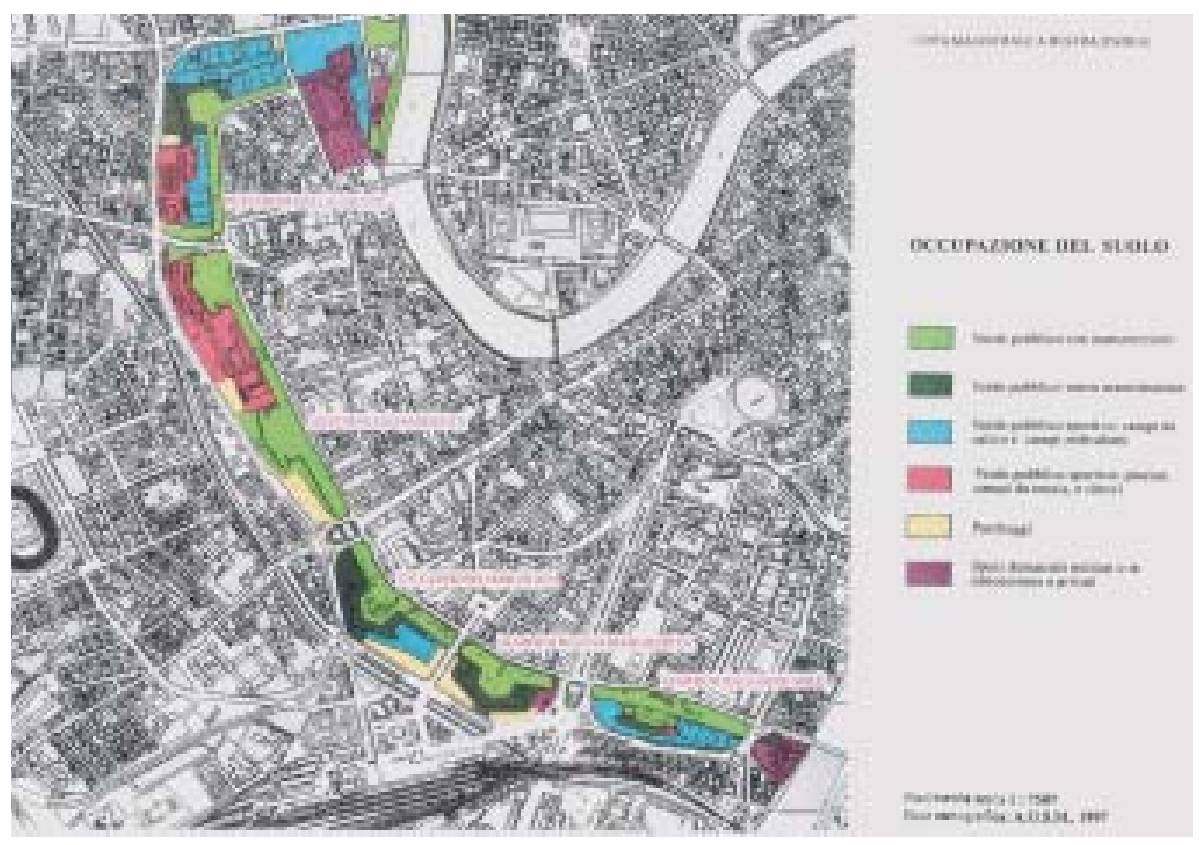

Figura 12 - Progetto guida per l'illuminazione della Cinta magistrale a destra d'Adige: l'occupazione del suolo, scala 1:7500 (1999). 
In anni non lontani, l'impegno di "Italia Nostra" fu all'origine del mitico restauro delle mura di Ferrara; a Verona, "Legambiente" rilancia il ruolo di "apripista" svolto dall'associazionismo, andando aldilà della mera proposta culturale ed impegnandosi direttamente nelle realizzazioni delle opere.

I principi guida progettuali sono già nell'immediato applicabili per buona parte al lato interno della Cinta magistrale stessa, ossia per le opere e gli spazi che si estendono dalla strada di circonvallazione interna al perimetro dei muri distaccati alla Carnot e dei muri veneziani, nel fosso magistrale.

Risulta invece parziale l'applicabilità immediata dei medesimi principi guida all'esterno della Cinta, ossia negli spazi del fosso magistrale fino al limite dei viali di circonvallazione esterna. Questa parte del compendio fortificato urbano, invasa da costruzioni incongrue, richiederà, infatti, una pianificazione più graduale, procedendo, ove possibile, per interventi campione.

La finalità più immediata della nuova illuminazione è di rendere riconoscibili le mura, identificabili anche di notte, facendone sicura la frequentazione al pubblico, sia ai cittadini, sia ai turisti.

Verona deve recuperare l'immagine e la percezione delle sue mura quale opera d'arte di scala urbanistica, unificante e caratterizzante la forma urbana: esse sono, infatti, monumento, spazio verde, percorso urbano ma, se valorizzate e reintegrate nella vita della città, saranno anche luogo originale di aggregazione per manifestazioni, attività e oggetto di attrazione turistica.

Il Progetto guida fornisce indicazioni generali, tipologiche e sistematiche per illuminare la Cinta magistrale, non solo per renderla visibile e sicura, ma anche per evidenziarne adeguatamente il complesso pregio monumentale e le cangianti suggestioni ambientali, tenendo conto di opere e luoghi con destinazioni ed usi diversi.

\section{Lilluminazione e l'esperienza di altre città bastionate}

L'illuminazione di un monumento è una lente di ingrandimento che permette di scoprire aspetti che di giorno non si colgono.

"Si dice che la luce è uno strumento urbanistico e anche un materiale da costruzione. Attraverso la luce artificiale si crea un'immagine dell' architettura e della città e si pone in essere una gerarchia negli elementi compositivi, accentuando certi elementi sopra gli altri. La luce ha molte funzioni, innanzitutto di ordine pratico: deve rendere visibili gli ostacoli, in modo da procurare un sentimento di sicurezza, rischiarare l'intorno, in modo da rendere riconoscibile lo spazio e facilitare l'orientamento. Inoltre, sul piano degli effetti estetici, si può utilizzare la luce per concretizzare idee, per conferire identità a una situazione o evocare una reazione emozionale in chi osserva. Di un'opera, di un luogo, la luce mette in evidenza il carattere, per identificare, comunicare un messaggio, creare un'atmosfera e un ambiente, ed anche divertire"1.

Per l'elaborazione del Progetto guida, i professionisti incaricati hanno studiato l'esperienza di altre città bastionate. Sono state acquisite le conoscenze maturate sul campo da progettisti, tecnici e amministratori che si sono occupati di questo tema ed hanno realizzato l'illuminazione di altri sistemi fortificati, come le cinte magistrali di Lucca e Ferrara, di Portoferraio e di Vicenza. È stata inoltre presa in esame la nuova illuminazione del centro monumentale di Cremona, dei Fori imperiali a Roma e di alcuni siti analoghi in Verona.

\section{Percezione visiva delle mura e illuminazione esistente}

Il tema dell'illuminazione delle mura è innanzi tutto strettamente collegato alla "percezione" delle mura stesse: si illumina ciò che si può percepire.

Le mura di Verona sono un monumento complesso e unico: mura di pianura e di collina, con stratificazioni di interventi di varie epoche, in stretta relazione con il fiume Adige.

\footnotetext{
1 "Progetto guida per l'illuminazione della Cinta magistrale a destra d'Adige", "Relazione".
} 
Tuttavia, attualmente, le mura a destra d'Adige quasi non si vedono, o meglio la percezione che se ne ha è molto frammentaria e risulta difficile, se non impossibile, coglierne l'unitarietà, la complessità e la varietà formale e spaziale.

$\mathrm{Al}$ contrario di altre città fortificate, dove spesso si ha un precario stato di conservazione dei manufatti, in un contesto ambientale abbastanza integro, a Verona la cinta muraria è complessivamente in buone condizioni, ma in un contesto ambientale pessimo.

Si potrebbe avere una buona percezione visiva delle mura da diversi punti di vista e angolazioni: dall'interno della città, dall'esterno, dal ciglio della controscarpa, camminando sulla sommità dei bastioni stessi, da punti panoramici lontani e infine dall'aereo. Attualmente, provenendo dal centro della città, si percepiscono solo le masse vegetali che ricoprono i rilevati di terra e alcune coperture delle poterne e delle polveriere. Dall'alto dei terrapieni stessi si può avere ancor oggi la visione delle opere fortificate, ma spesso ciò si limita al perimetro interno dei muri alla Carnot, mentre al loro esterno si presenta un paesaggio desolante.

Inoltre, la visione che si ha dall'esterno e dagli assi di penetrazione in città, intensamente motorizzati, è limitata a brevi tratti delle mura, che sono occultate dagli svariati impianti sportivi e dai terrapieni per i parcheggi. Il riempimento del fosso magistrale (o vallo), attuato dopo l'ultima guerra, fa sì che nella maggior parte delle situazioni la sommità della scarpata si trovi a ridosso delle opere murarie, alterandone l'intorno prospettico. Le opere murarie ottocentesche si elevano dal fondo del fosso magistrale senza superare il piano medio urbano di viabilità.

La percezione, già limitata ${ }^{2}$, è ulteriormente compromessa dalla folta vegetazione, sia d'impianto sia spontanea, che ostacola anche la visione delle opere di terra, soprattutto in primavera ed estate. In bicicletta o a piedi la visibilità migliora un poco, ma anche in senso negativo, in quanto si percepiscono ulteriori elementi di disturbo e di degrado: rifiuti, recinzioni, vegetazione infestante, superfetazioni.

Lilluminazione stradale della zona, delle circonvallazioni interna ed esterna, non interessa le mura, le lascia in penombra. Le opere murarie stesse sono impiegate come sostegni di corpi illuminanti a beneficio di campi sportivi e piscine, creando fastidiosi effetti di controluce per chi percorre i terrapieni interni. Anche i marciapiedi delle vie di circonvallazione interna, che sono i principali accessi alle mura, rimangono al buio, perché l'illuminazione esistente è volta a rendere sicura principalmente la sede stradale. Tuttavia, in alcune situazioni la vegetazione arborea scherma e rende insufficiente anche l'illuminazione stradale.

Il tracciato urbanistico della circonvallazione interna ha origine da ragioni di pura funzionalità militare: la sua definizione completa è contemporanea alla ricostruzione asburgica della Cinta magistrale. Oggi è uno dei percorsi di maggiore scorrimento e di attraversamento veicolare, in contrasto con il carattere urbano e civile dei luoghi che esso connette, soprattutto ove si consideri la contiguità e il contatto con il Parco storico e monumentale delle Mura. È infatti incongruente il contatto tra la strada di grande traffico e di attraversamento, interna al centro storico, ed il maggiore parco urbano. Inoltre, lungo la strada di circonvallazione interna si succedono le porte sanmicheliane, tra i più illustri monumenti del Rinascimento europeo, e si dispone prospetticamente la grandiosa architettura dei rampari, complessa e geometrica, con le poterne, le riservette, le polveriere, a formare uno dei più suggestivi viali del centro storico di Verona.

Nonostante il traffico e il movimento frenetico che animano le circonvallazioni, la Cinta magistrale dà una sensazione di vuoto, di isolamento; un aspetto

${ }^{2}$ Lo sviluppo complessivo della Cinta a destra d'Adige, misurato lungo l'andamento delle murature, è di 3650 metri. Di questi, si è potuto calcolare che di giorno la percezione attuale dalla circonvallazione esterna è del $25 \%$, la percezione a piedi o in bicicletta dalla controscarpa è del $50 \%$ e la percezione dall'alto dei terrapieni è del $30 \%$. Di notte, addirittura, le mura spariscono nell'oscurità. "Progetto guida per l'illuminazione della Cinta magistrale a destra d'Adige", "Relazione", op. cit. 
simile all'abbandono, all'emarginazione di certe zone periferiche, totale nelle ore serali e notturne. Manca ogni luce atta non solo a valorizzare le opere, ma anche a renderle accessibili. La città è lì accanto, ma di notte, tranne i tratti delle Porte, le mura non si vedono ed è come se non esistessero.

"Verona, la città fortificata per eccellenza, non si identifica più nelle sue grandiose fortificazioni. È necessario avviare il recupero figurativo delle mura, rendendole accoglienti e visibili, utili alla vita della città. Fare luce sulle mura significa ricomporre l'immagine di Verona"3.

Il tema dell'illuminazione dunque non conclude, ma inizia il processo di recupero di questo straordinario monumento.

\section{LA METODOLOGIA}

\section{Perché illuminare le mura: il metodo e le prescrizioni del progetto}

Il Progetto guida persegue un'idea unitaria ed omogenea della riqualificazione della Cinta magistrale e per far ciò deve necessariamente tener conto delle diverse caratteristiche costruttive, architettoniche e spaziali delle parti e degli elementi che compongono la Cinta stessa. Per analogia di forme e di costituzione costruttiva e spaziale, si possono distinguere nella Cinta magistrale a destra d'Adige sia elementi architettonici ripetuti, ricorrenti, sia elementi singoli.

Gli elementi architettonici ripetuti sono i seguenti:

(1) il bastione cinquecentesco sanmicheliano;

(2) la cortina cinquecentesca con il cavaliere;

(3) la porta urbana cinquecentesca sanmicheliana;

(4) il bastione ottocentesco con il ramparo;

(5) la cortina ottocentesca;

(6) il terrapieno-ramparo cinque-ottocentesco;

(7) la strada di circonvallazione interna con il sistema delle poterne.

Gli elementi architettonici singolari sono le testimonianze dell'epoca scaligera.

Sulla scorta dei temi progettuali di illuminazione, delle caratteristiche e delle prescrizioni generali sulle modalità d'illuminazione, è stato composto un "Repertorio tipologico" relativo a schede di progetto e ad abachi dei corpi illuminanti utilizzabili per le finalità e le modalità individuate.

Il "Repertorio tipologico" individua i sette distinti insiemi di opere ricorrenti o singole suindicati ed è assunto per altrettanti modelli di progetto adattabili ed applicabili ai singoli elementi della Cinta magistrale; vi è perciò una corrispondenza diretta e univoca tra repertorio di elementi architettonici e modelli di progetto. In questo modo, per ogni insieme di elementi simili, per forma e costituzione, ripetuti nello sviluppo della Cinta, si dispone di un modello di progetto

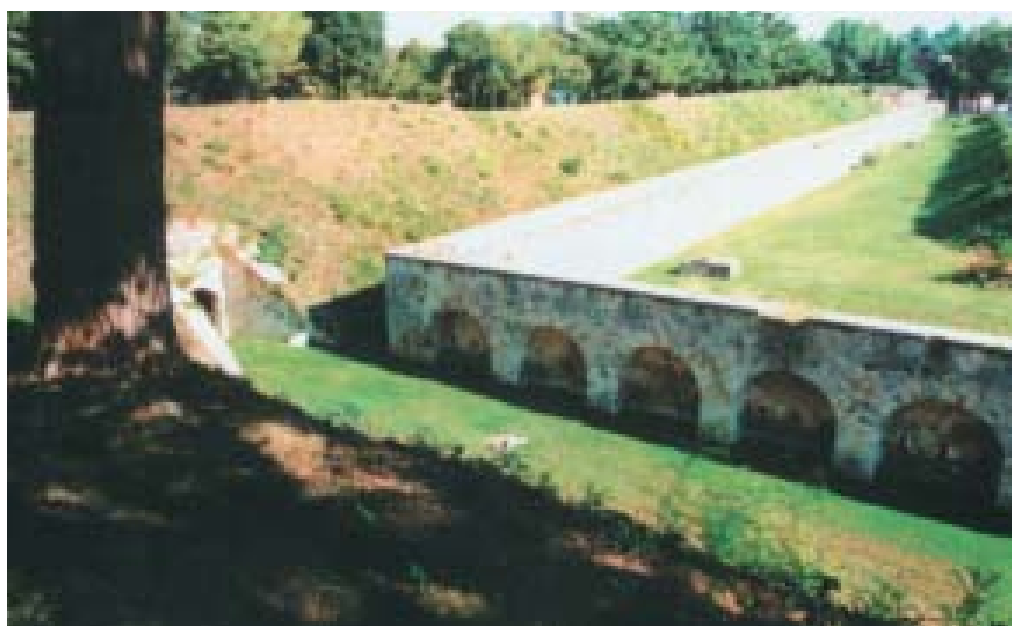

Figura 13 - La cortina verso Porta Palio con il muro alla Carnot, vista dal fianco del bastione di San Bernardino, dopo i lavori di sistemazione del verde e di recupero ambientale. ripetibile per elemento dell'insieme. Per gli elementi o parti singolari, invece, viene presentato un modello di progetto specifico. 


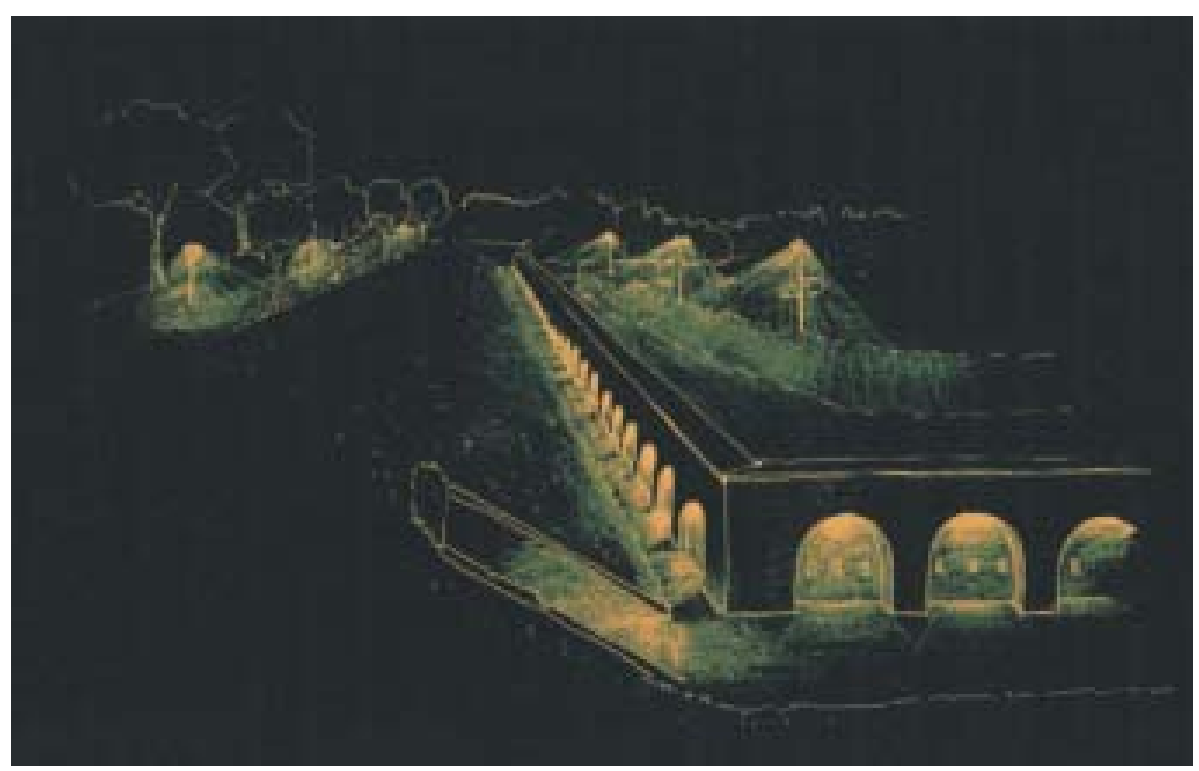

Figura 15 - Progetto guida per l'illuminazione della Cinta magistrale a destra d'Adige: disegno relativo al bastione di San Bernardino.

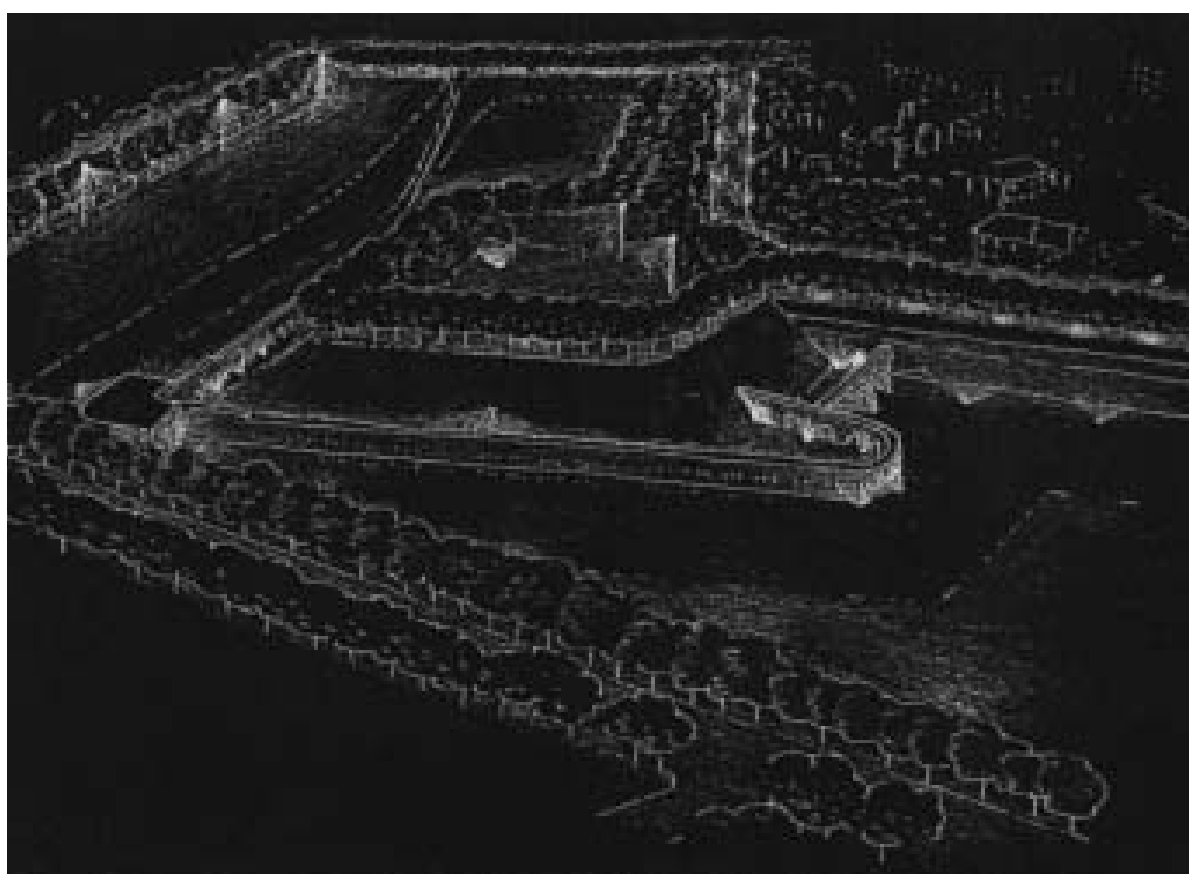

Figura 16 - Progetto guida per l'illuminazione della Cinta magistrale a destra d'Adige: disegno relativo al bastione di S. Procrolo. Secondo il Progetto Guida dell'illuminazione, la prospettiva notturna della Cinta, al piano del fosso magistrale, sarà costituita visivamente dall'illuminazione generale, monumentale e di dettaglio architettonico, ritmata e dosata in intensità sulla complessa e dinamica successione delle opere fortificate.

In ogni caso sia l'elemento luce, sia le caratteristiche morfologiche e ambientali di ciascun bastione, presentano una complessità di situazioni e variabili tali che le soluzioni proposte vanno sperimentate caso per caso in sede di progetto esecutivo; in alcune situazioni sono ipotizzabili più soluzioni egualmente proponibili.

La Relazione tecnica del progetto riporta l'analisi dei singoli elementi della Cinta a destra d'Adige con relative schede dello stato di fatto; per meglio comprendere, ad esempio, l'entità effettiva della percezione dei bastioni, sono stati attribuiti alla visibilità valori numerici percentuali. 
Le prescrizioni di carattere generale, inerenti l'intero sistema di illuminazione, prevedono che:

- l'illuminazione sarà di intensità moderata, a carattere diffuso, per valorizzare le opere e leggerne le qualità di forma, spazio e costruzione;

- le tonalità di colore delle luci emesse saranno in perfetto accordo con le caratteristiche cromatiche dei materiali (pietra, tufo, laterizio), nonché con la vegetazione ed i manti erbosi;

- gli angoli di emissione e di incidenza delle fonti di illuminazione dovranno evitare ombre portate, in contrasto figurativo con le strutture e gli apparati architettonici;

- posizioni, distanze, angoli di emissione e di incidenza saranno studiati per evitare gli effetti di ombre in movimento (ombre cinesi) dei visitatori, proiettate sulle pareti delle opere illuminate;

- l'illuminazione di servizio dei percorsi, lungo le opere di terra asseconderà le geometrie delle opere medesime con l'andamento dei punti luce;

- posizioni, angoli di emissione e di incidenza dei corpi illuminati saranno studiati per evitare, da vicino e da lontano, effetti di abbagliamento e di controluce verso i pedoni, i ciclisti ed il traffico veicolare;

- i corpi illuminanti, i loro sostegni, le loro proiezioni a terra, saranno scelti tenendo conto della minima incidenza nell'insieme figurativo e ambientale delle opere;

- la posizione dei medesimi corpi illuminanti dovrà produrre la minima interferenza con le opere; sono da evitare fissaggi diretti dei medesimi corpi illuminanti sulle pareti delle opere di fortificazione;

- nella scelta dei corpi illuminanti da impiegare si terrà conto della normativa regionale per la prevenzione dell'inquinamento luminoso. Si terrà inoltre conto degli aspetti di gestione, manutenzione e consumo energetico, nonché della prevenzione contro il vandalismo ${ }^{4}$;

- ogni disposizione di illuminazione prefigurata nel Progetto Guida, all'atto dei progetti esecutivi conseguenti, verrà sperimentata in loco per verificare e mettere a punto, nel dettaglio, posizioni, cadenze, angolazioni, portata ed intensità, ottiche, tonalità di colore della luce.

In particolare, il sistema di illuminazione ideato per la Cinta magistrale a destra d'Adige si sviluppa per classi tematiche di progetto, ossia:

1. illuminazione generale e monumentale: di intensità moderata, variabile $\mathrm{e}$ dosata, per evitare sia sulle opere murarie, sia sulle opere di terra, effetti di accentuazione drammatica e di contrasto, in relazione alle caratteristiche morfologiche e spaziali delle opere, per ottenere una lettura corretta dell'insieme nonché una equilibrata relazione prospettica tra le parti e gli elementi;

2. illuminazione di dettaglio architettonico: di intensità media, per quanto necessario a mettere in risalto elementi singolari o parti di speciale configurazione delle opere, o loro qualità architettoniche come: motivi di serialità, orizzontalità, imponenza e geometria delle masse, loro dinamismo, corrispondenze prospettiche tra elementi non prossimi; originarie continuità fisiche tra elementi

\footnotetext{
${ }^{4}$ Il problema del vandalismo è sempre tenuto presente nelle tematiche dell'illuminazione pubblica e monumentale e variamente (mai definitivamente) risolto. Alcune amministrazioni (Lucca, Portoferraio) hanno studiato apposite gabbie metalliche più o meno resistenti, per contenere e proteggere i riflettori. Anche a Verona, in alcune situazioni, dove i proiettori sono a rischio, è stata attuata la stessa soluzione. Il Comune di Ferrara ha invece progettato appositi contenitori, interamente chiusi, con vetri anti-sfondamento e, nel caso dei lampioni, ha escluso i pali in alluminio e scelto corpi illuminanti particolarmente robusti e collaudati. In ogni caso, saranno la frequentazione e il controllo, assieme all'illuminazione, a dare sicurezza e valorizzazione all'area. Se uno di questi elementi manca, qualsiasi soluzione tecnica risulta vana.
}

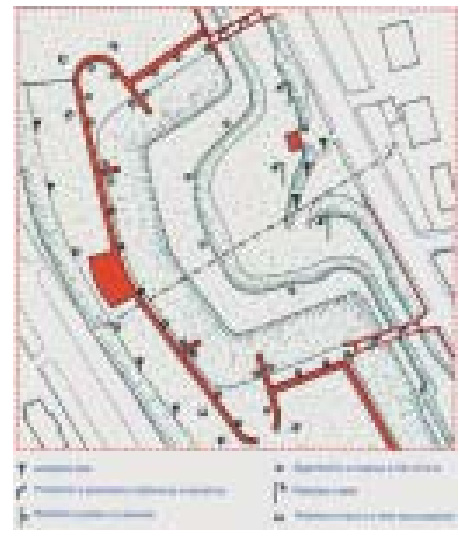

Figura 14 - Progetto guida per l'illuminazione della Cinta magistrale a destra d'Adige: il bastione di San Bernardino. 
della Cinta, oggi separati dall'organismo di appartenenza. Uno degli obiettivi del progetto è quello di rendere percepibili dall'esterno gli interni architettonici di poterne, polveriere e caponiere evitando effetti di accentuazione artificiosa, di connotazione drammatica o eccessivamente scenografica e ombre portate in contrasto con i motivi compositivi delle stesse architetture;

3. illuminazione di servizio: riguarda i piazzali dei bastioni, i percorsi sui terrapieni, gli accessi ai bastioni, gli ingressi alle poterne, il sistema dei percorsi poterna-sortita, il sistema dei cammini di ronda, i muri alla Carnot, gli orecchioni, i percorsi lungo il fosso magistrale e il viale di circonvallazione interna. I piazzali dei bastioni, per la loro principale importanza come luoghi d'incontro, di sosta e di relazione, oltre che di osservazione delle opere fortificate esterne, saranno illuminati con la maggiore intensità rispetto alle altre parti della Cinta. Saranno adottate soluzioni che valorizzano adeguatamente, sia lo spazio aperto del piazzale, sia i filari di alberi che lo contornano.

Per i percorsi, l'illuminazione si integrerà con gli apparati arborei d'impianto esistenti sulle mura e con la loro disposizione planimetrica provvedendo, ove necessario, a selezionare una eccessiva presenza arborea e ad innalzare o ridurre l'impalcatura inferiore dei rami.

Gli ingressi dei singoli bastioni riceveranno una maggior intensità di illuminazione per essere identificabili e per produrre un effetto di attrazione verso il pubblico. Sia per i piazzali sui bastioni, sia per i percorsi sui terrapieni-rampari delle cortine, vale il principio di ottenere un'intensità di illuminazione diffusa, adeguata anche per le attività collaterali, ad impianto fisso, quali i percorsi della salute o i punti di sosta. In questo modo non si dovrà disporre una distinta serie di apparecchi illuminanti, ad esclusivo servizio di singole attività o funzioni.

Per i percorsi lungo il fosso magistrale, nella pianificazione urbanistica il recupero della Cinta magistrale prevede il ripristino della continuità spaziale sull'intero arco della Cinta di destra d'Adige anche al piano del vallo. La prospettiva notturna della Cinta, a questo piano, sarà costituita visivamente dall'illuminazione generale, monumentale e di dettaglio architettonico, ritmata e dosata in intensità sulla complessa e dinamica successione delle opere fortificate. Il quadro sarà completato da irradiazioni di luce secondaria sui terrapieni retrostanti e dalla linea guida luminosa, con cadenza distesa e regolare, a demarcare il percorso di collegamento generale, attrezzato con punti di sosta.

Nella sua costituzione spaziale originaria la Cinta magistrale era organicamente integrata alla circonvallazione interna, spazio di relazione di pura funzionalità militare a scala urbanistica. L'architettura delle fortificazioni, sul lato interno, rivolto alla città, è definita, anche in termini prospettici, dallo spazio aperto, a sviluppo lineare, della strada di circonvallazione. Oggi, invece, la circonvallazione interna determina una frattura tra la città ed il suo sistema fortificato, non solo per il flusso di traffico che la percorre, ma anche per la sua configurazione ambientale. Di giorno presenta un aspetto periferico, trascurato, con i marciapiedi sacrificati e disagevoli, la preponderanza del traffico veicolare, le recinzioni al piede dei terrapieni, la vegetazione spesso conformata a barriera visuale. Di notte, la Cinta magistrale e gli esigui marciapiedi scompaiono, spinti nelle tenebre dal sistema di illuminazione rivolto esclusivamente al percorso stradale. Si tratta, allora, di riconvertire l'immagine d'insieme della circonvallazione: da strada periferica di traffico, a viale urbano ricco di incontri, di storia e di architettura, non solo fortificata. Il nuovo sistema di illuminazione, necessariamente pensato assieme ad un migliore dimensionamento dei marciapiedi collaterali ed al diradamento delle barriere arboree, sarà l'intervento decisivo per riconfigurare il viale di circonvallazione, secondo i modelli civili della città ottocentesca. Pur adeguata anche alla funzionalità del percorso veicolare, la nuova 
disposizione a lampioni bilaterali, diffonderà luce sui marciapiedi e sulle adiacenti scarpate della Cinta 5 .

Terrapieni, rampate, poterne, polveriere, porte urbane, con le loro specifiche illuminazioni, daranno forma alla sequenza continua lungo i viali di circonvallazione. In questo modo, nel paesaggio urbano notturno sarà ricomposta l'unità prospettica e spaziale tra le fortificazioni e la città.

Valorizzazione scenografica ed effetti speciali: questa, infine, è studiata per dare effetti di tridimensionalità a struttura prospettica alle varie opere architettoniche monumentali.

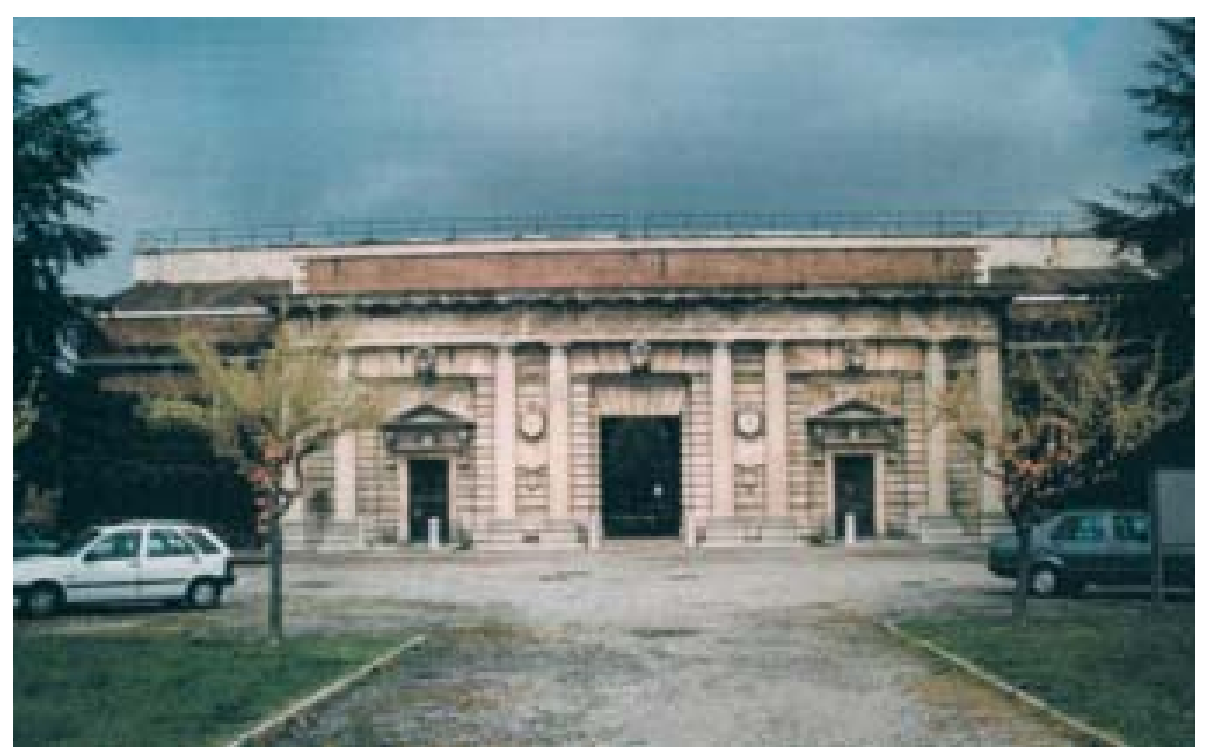

Figura 17 - Porta Palio (1547-1557) del sommo architetto veronese Michele Sanmicheli. Le Porte spiccavano incastonate nelle cortine rettilinee, del tutto spoglie; oggi, isolate dalle brecce e circondate dal traffico, hanno completamente perduto il carattere del loro legame con la Cinta. I materiali impiegati sono i più nobili della tradizione veronese: laterizio combinato con la pietra da taglio e il tufo.

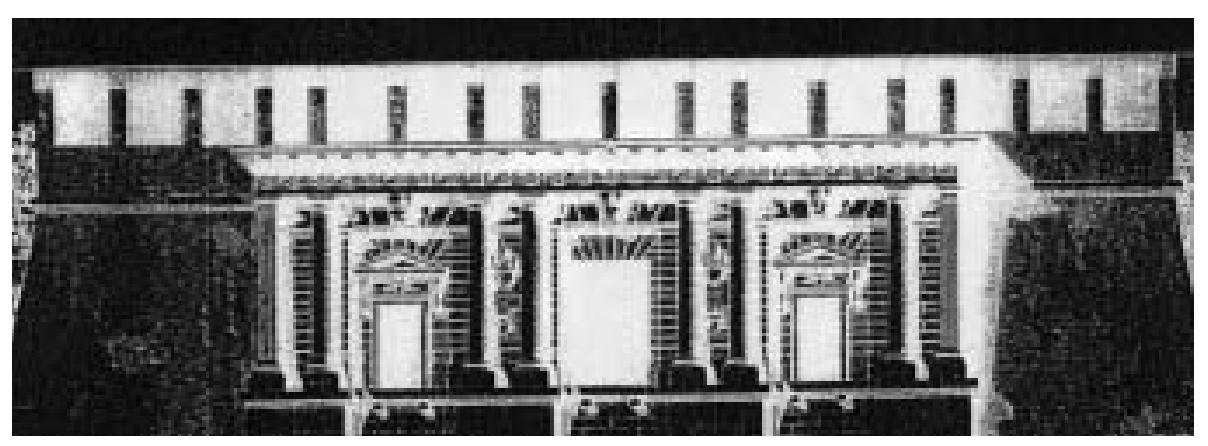

Figura 18 - Porta Palio. Ė una delle tre Porte cinquecentesche di Verona, veri e propri edifici monumentali d'ingresso alla città che saranno illuminati anche all'interno, sia per valorizzare elementi architettonici altrimenti non percepibili, sia per "interpretare" lo spazio di attraversamento delle porte.

${ }^{5}$ Lilluminazione esistente della circonvallazione interna, volta a rendere sicura la sede stradale, lascia in ombra i marciapiedi che, quindi, non sono adatti per i pedoni. In realtà, un progetto del Comune prevedeva, già nel 1926, "un viale (...), da costruirsi nella cinta interna delle mura, ombreggiato da otto fila di alberi di alto fusto, (...) fiancheggiato a sua volta da tre viali pedonali (...)". Oggi le prospettive del nuovo Piano Regolatore Generale, che spostano a sud le arterie importanti di attraversamento e le limitazioni già esistenti al traffico automobilistico, restituiscono di fatto questa strada alla sua vocazione di viale cittadino. Le linee del progetto guida proseguono in questa prospettiva, prevedendo una nuova illuminazione dei marciapiedi. Inoltre, dal marciapiede della circonvallazione interna si possono sottopassare i bastioni attraverso le poterne, che diventano così elemento di collegamento tra la città antica e i quartieri periferici; di notte, la luce che filtrerà attraverso i loro cancelli contribuirà a dare il senso di sicurezza. 
Per ogni elemento o parte della Cinta, individuato nel "Repertorio tipologico", saranno risolte le quattro distinte tematiche di illuminazione secondo combinazioni variabili, dipendenti dal carattere spaziale e funzionale della specifica parte in esame, in modo da ottenere sempre un insieme armonico dal punto di vista estetico e della pratica utilità. Perciò potrà anche essere che in particolari situazioni due o più tematiche di illuminazione siano risolte con lo stesso dispositivo, o che i corpi illuminanti previsti per una specifica tematica, concorrano secondariamente a risolverne un'altra di illuminazione.

Tutti i bastioni sistemati a verde pubblico hanno la stessa struttura: una vasta area centrale corrispondente al piazzale e lunghi percorsi sulle cortine e sulle balze intermedie dei terrapieni. Nel progetto guida le cortine, usate soprattutto per le passeggiate, sono illuminate da lampioni a palo, posti sulla sommità della scarpata intermedia, con un'unica fila di lampioni ed un palo ogni 20 metri circa, che garantisce un buon illuminamento, senza interferire sostanzialmente con la percezione delle

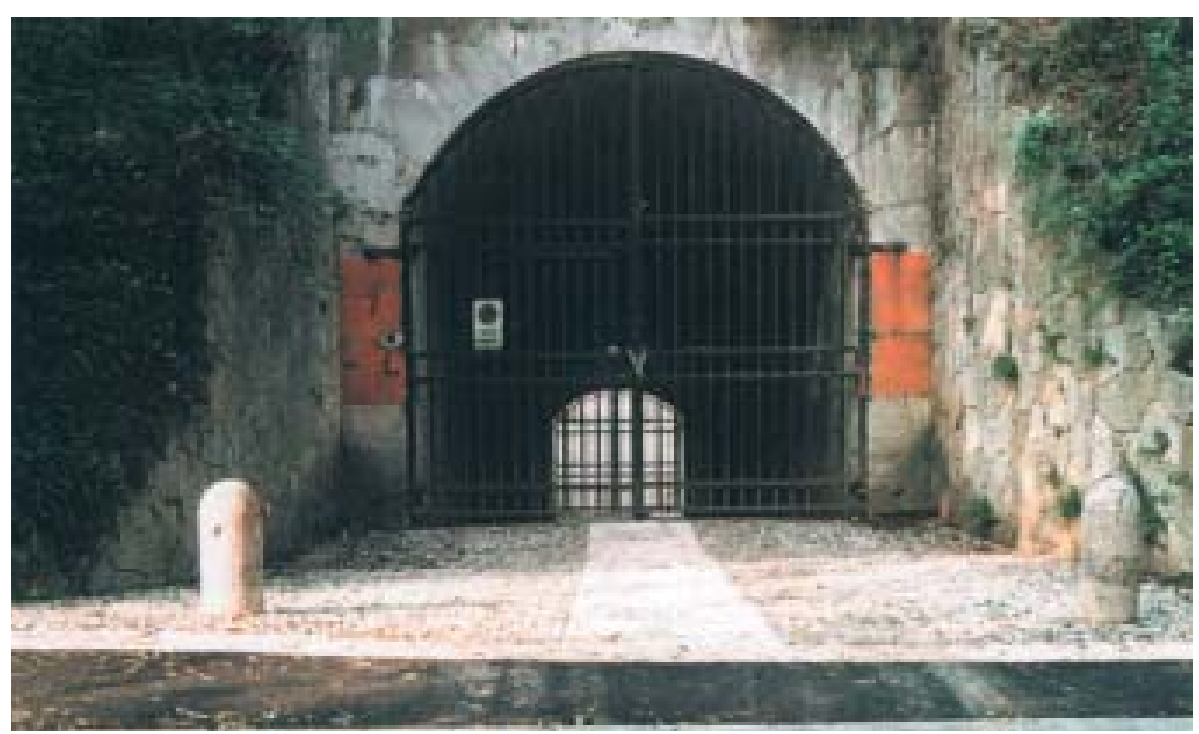

Figura 19 - La "poterna” restaurata del Bastione di San Bernardino. Le "poterne" saranno illuminate al loro interno da proiettori a piantana.

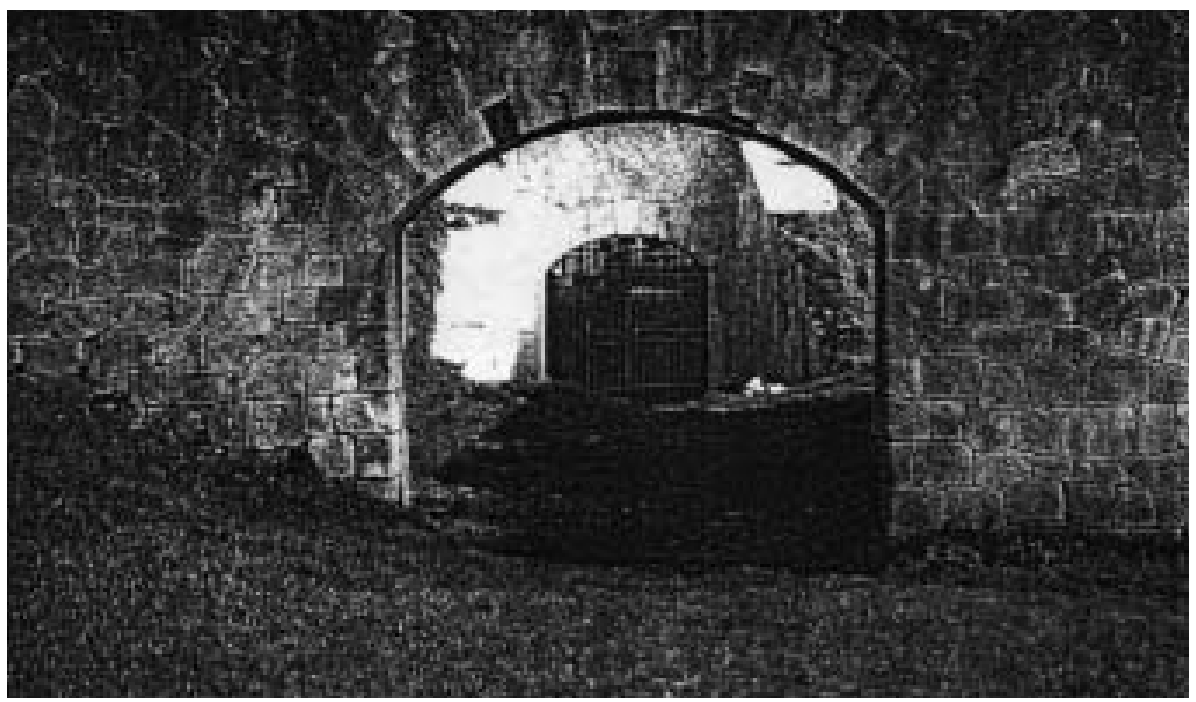

Figura 20 - Nell'illuminazione notturna, i muri distaccati alla Carnot, con la sequenza delle nicchie archeggiate sull'interno perimetro dei bastioni, risalteranno per la loro bellezza architettonica. Saranno inoltre l'elemento conduttore del percorso interno, dalle poterne, lungo il cammino di ronda. 
mura dall'esterno. Gli ampi piazzali, oggi liberati dalla vegetazione superflua ed infestante, sono il luogo più idoneo alla sosta ed allo svolgimento di giochi ed attività di incontro che possono protrarsi anche di sera. Qui la sorgente luminosa è concentrata su proiettori a pali alti 10-12 metri: si tratta di un'illuminazione intensa che consente di mantenere la percezione d'insieme di tutto lo spazio disponibile. Inoltre, alcuni piccoli proiettori incassati nel terreno illumineranno dal basso le chiome di gruppi di alberi esistenti. Dall'esterno, invece, l'ottica è differente: l'obiettivo è quello di evidenziare, anche di notte, la presenza del monumento e il fosso magistrale ha un'illuminazione di fondo di tipo indiretto che scaturisce dalla luce di proiettori a terra, riflessa sui muri. Per gli spalti, larghi spazi a verde esterni alle mura che si estendono tra la controscarpa e i viali di circonvallazione esterna, oggi riservati ad un utilizzo vario di gioco e attività all'aperto, l'illuminazione più idonea, di non forte intensità per non interferire con la percezione visiva delle mura, è quella con proiettori su palo al limite delle alberature della circonvallazione.

Ovviamente, il nodo principale che il progetto doveva sciogliere era quello dell'illuminazione delle mura vere e proprie. Un problema non facile poiché le mura sino a noi pervenute non sono omogenee ma di diverse epoche. La scelta del Progetto guida è stata quella di dare un senso di continuità alla Cinta e, contestualmente, valorizzare tipologie architettoniche e materiali diversi. Lilluminazione dall'esterno dei muri di rivestimento veneziani, in mattoni, proviene da proiettori da terra. Le caponiere, gli orecchioni e i muri delle cortine austriache, in tufo, avranno la stessa tipologia di illuminazione dei muri veneziani, mentre i muri distaccati rettilinei delle cortine austriache, detti alla Carnot, saranno illuminati con proiettori posti sulla scarpata.

\section{GLI ESITI}

Il Progetto guida prende avvio dal presupposto che vengano eseguiti lavori preliminari di ricomposizione dei luoghi, attraverso opere di diradamento della vegetazione, pulizia, manutenzione e restauro; ma non solo, esso testimonia dell'importanza della figura del paesaggista in operazioni delicate come queste.

In alcune situazioni, dato il buon livello di ripristino, le opere possono già partire. La stima del loro costo complessivo e il preventivo preliminare di spesa (calcolato in base a una media dei prezzi dei corpi illuminanti e relativa posa in opera), indicano la possibilità di iniziare i lavori per stralci significativi con costi limitati e con grossi risultati.

Il Progetto è stato consegnato nell'agosto del 1999; fino all'aprile 2002 è stato realizzato, a cura dell'Azienda Generale dei Servizi Municipalizzati di Verona, solo un primo intervento, nel bastione di San Procrolo, dove sono state parzialmente recepite le direttive del Progetto guida.

La "messa in luce" del "primo bastione" potrebbe essere di stimolo a una nuova conoscenza e frequentazione, in una parola a una riappropriazione da parte di Verona della sua Cinta magistrale.

\section{RIFERIMENTI BIBLIOGRAFICI}

Bozzetto Lino Vittorio (a cura di), Eclissi e rivelazione delle mura di Verona, "Architetti Verona”, 53, marzo/aprile 2001.

Bozzetto Lino Vittorio, Verona, la cinta magistrale asburgica, Cassa di Risparmio di Verona, Vicenza, Belluno e Ancona, Verona 1993.

Perbellini Gianni e Bozzetto Lino VitTorio, Verona. La Piazzaforte ottocentesca nella cultura europea, "Architetti Verona", Verona 1990. 
Ballestriero Alberto, Sintesi storica delle mura di Verona e Intervento sulla Cinta muraria di Verona. Il recupero dei Bastioni di S. Bernardino e S. Zeno, Studio di paesaggistica BallestrieroMuscari, Verona, 01/10/01.

Conforti Calcagni Annamaria, Le mura di Verona, Cierre edizioni, Verona 1999.

FARA AMELIO, La città da guerra, Einaudi, Torino 1993.

GuCCIOne Biagio, Illuminazione: mura di Verona, un esempio da seguire, "Lineaverde", nov./dic. 2001, pagg. 26-28.

\section{RIFERIMENTI ICONOGRAFICI}

Le schede di progetto e i materiali illustrativi e fotografici sono stati tratti dai documenti relativi al "Progetto guida per l'illuminazione della Cinta magistrale a destra d'Adige", reperiti presso lo Studio di paesaggistica Ballestriero-Muscari di Verona che si intende ringraziare, assieme al Prof. Biagio Guccione (Università di Firenze) e all'Arch. Anna Maddaluno, per l'estrema disponibilità e per la mole di informazioni e materiali forniti.

Il "Progetto guida per l'illuminazione della Cinta magistrale a destra d'Adige" è composto principalmente dai seguenti materiali:

- "Abaco dei corpi illuminanti"

- "Repertorio tipologico degli elementi architettonici e dei tipi di illuminazione applicati”

- "Relazione"

- "Simulazione delle cortine e mura austriache La Carneaux ottocentesche"

\section{SCHEDA DI PROGETTO}

Progetto:

Luogo:

Committente:

Superficie:

Progetto e direzione lavori:

Assistenza:

Anno di progettazione:

Realizzazione:

Costo previsto:
Progetto guida per l'illuminazione della Cinta magistrale a destra d'Adige

\section{Verona}

\section{Comune di Verona}

lo sviluppo complessivo della Cinta a destra d'Adige, misurato lungo l'andamento delle murature, è di 3.650 metri di lunghezza sui 9.350 complessivi delle Mura

Studio di architettura Lino Vittorio Bozzetto (VR)

Studio di paesaggistica Ballestriero-Muscari (VR)

assistenza illuminotecnica della ditta Philips

1999

in corso

importo dell'opera 2 milioni di Euro 


\title{
LA ROCCA DI RAVENNA
}

\author{
Maristella Storti
}

\section{IL CONTESTO}

\section{La storia e le caratteristiche della rocca Brancaleone}

La Romagna annovera nel suo territorio un notevole numero di fortezze che risalgono a epoche assai antiche e che nel corso dei secoli hanno subito numerose modifiche e varianti, adeguandosi alle mutate esigenze belliche connesse all'evoluzione delle armi da urto in armi da fuoco. L'introduzione di nuovi elementi di difesa ha quindi modificato l'aspetto delle fortificazioni stesse e la rocca Brancaleone della città di Ravenna si inserisce proprio all'interno di questo quadro. Essa si colloca a pieno titolo nella transizione fra l'architettura militare medievale e quella rinascimentale, racchiudendo e fondendo le caratteristiche di entrambi i periodi.

Ciò che differenzia la fortezza ravennate rispetto ad altre città romagnole, quali Imola, Forlì, Forlimpopoli, Faenza e Riolo, è che nelle seconde l'adeguamento difensivo moderno è stato ottenuto attraverso la lenta trasformazione di un organismo medievale ed il processo di rinnovamento si è sviluppato dando origine a differenti tipologie; nella rocca di Ravenna, invece, il collegamento fra le due epoche si fa molto più ravvicinato in quanto ad una pianificazione obsoleta è seguita una decadenza immediata.

La produzione architettonica a Ravenna, fino al XV secolo, ricalca ancora nei propri edifici civili e religiosi le forme bizantine che l'avevano resa famosa nel V-VI secolo d.C. e che la renderanno riconoscibile poi nel mondo intero fino ai giorni nostri.

È con l'inizio della dominazione veneziana all'interno del territorio romagnolo (XI secolo) che la vita culturale e architettonica della città si risveglia dalla stasi millenaria tramite lo sviluppo di un "programma" edilizio che incide profondamente sul volto urbano della città.

Da questo momento in poi, per lo stato veneziano Ravenna diventa un polo fondamentale legato soprattutto ai traffici mercantili.

Fra gli edifici realizzati nel periodo della rinascita e del rinnovamento civile e religioso, si trova la rocca Brancaleone che venne costruita inizialmente come rinforzo per la difesa della città in appoggio alla cinta muraria già esistente, anche se questa funzione primaria venne rapidamente decadendo poiché la sua struttura non era stata pensata per poter resistere e rispondere ad attacchi condotti con armi da fuoco. Sarà quindi solo settant'anni dopo, al termine della sua costruzione, che il fortilizio risulterà completamente inutilizzabile ed adibito in più occasioni a svariati impieghi come il ricovero di profughi provenienti dall'Albania e dalle isole dalmate, fuggiti dall'incalzare delle truppe ottomane.

Da allora, la decadenza del monumento diviene progressiva sino al culmine del Settecento quando la parte superiore e le opere di difesa esterna vengono addirittura demolite per impiegare il materiale di risulta in opere di salvaguardia idraulica del territorio. A questo si deve aggiungere che l'interno della Cittadella veniva usato già da tempo come orto e tale uso prosegue fino ai giorni nostri quando, con l'acquisizione da parte del Comune, viene attuata tutta una serie di trasformazioni. Gli spazi interni della Cittadella e della rocca, infatti, risultano 


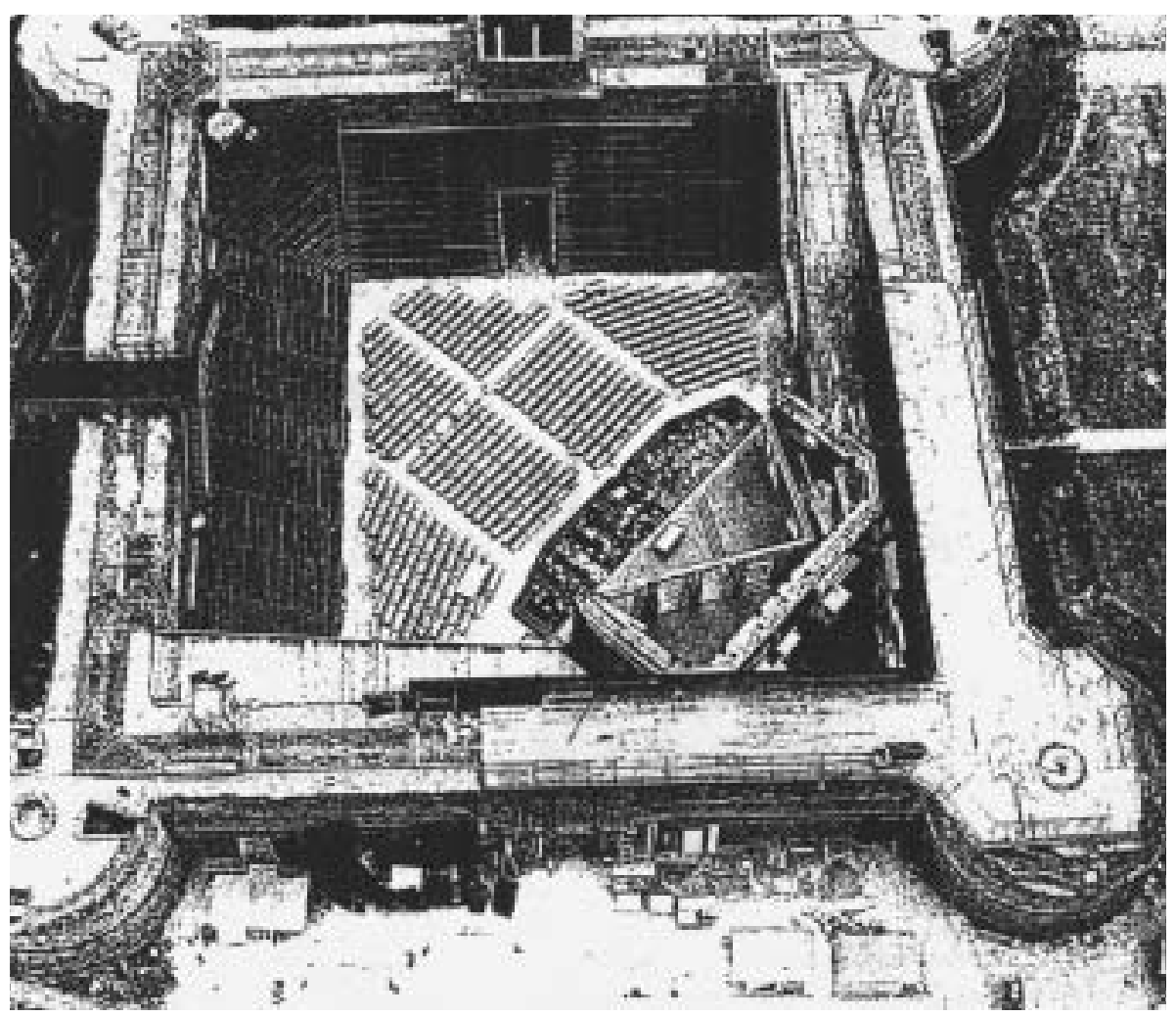

Figura 1 - Vista aerea della Rocca Brancaleone di Ravenna in occasione del Ravenna Festival 1987. Scenografie: Arch. Gae Aulenti.

oggi rispettivamente adibiti a giardino e a teatro, così caratterizzanti per l'odierna vita sociale e culturale della città.

L'aumento d'importanza delle manifestazioni artistiche, con il passare degli anni, hanno collocato Ravenna all'interno di un circuito culturale internazionale. Eppure Ravenna appare come una città che non riesce a decollare completamente per le proprie caratteristiche di città chiusa, dove negli ultimi anni il centro storico è stato degradato, svuotato per scelta politica di tutte le sue prerogative e peculiarità, favorendo il decentramento delle funzioni in un nuovo polo di sviluppo esterno alla città antica. A ciò si deve aggiungere la carenza sia di parcheggi pubblici in occasione di manifestazioni, sia di spazi idonei per il normale godimento del centro antico della città.

La rocca di Ravenna rappresenta, data la notevole estensione planimetrica, una parte di città racchiusa tra la selvaggia lottizzazione attuata negli anni Sessanta (che non ha risparmiato nemmeno le antiche mura), la ferrovia (che aveva già dal 1860 bloccato lo sviluppo urbano verso est) e una serie di strade che ne limitano la visuale quando addirittura non la sovrastino, facendole perdere quasi completamente quella monumentalità che aveva conservato nel corso dei secoli.

Negli ultimi anni, la rocca è stata parzialmente aperta alla fruizione pubblica con la sistemazione ad arena estiva in occasione del Festival internazionale di Musica Classica e Operistica, che nel corso degli anni ha visto crescere il proprio peso culturale nelle rassegne nazionali ed internazionali.

Nonostante questa meritoria rivitalizzazione culturale e sociale avvenuta nelle ultime decine d'anni, lo storico manufatto non è stato inserito all'interno della vita cittadina e ciò ha portato ad un degrado sempre maggiore dello stesso. Come in altre città fortificate d'Italia (vedi Verona), gli spazi aperti relativi alle architetture militari monumentali in disuso risultano spesso prossimi alla decadenza e divengono sede dei loschi traffici che la città rifiuta altrove. 


\section{GLI OBIETTIVI}

Alla luce delle considerazioni appena delineate, il progetto di recupero della rocca di Ravenna viene visto come opportunità per la riqualificazione del centro storico e dell'intera città, dato che i sistemi di spazi aperti attorno al monumento vengono ridisegnati per lo svago e la sosta e vanno a sopperire alla penuria di spazi aperti pubblici e a giardino.

La configurazione dei nuovi spazi aperti della rocca, è vista quindi come occasione unica di luogo di aggregazione, di strutture polivalenti di uso flessibile caratteristiche per quest'area e che si vuole rappresentino momenti simbolici della vita umana, del rapporto dell'uomo con l'ambiente e con la propria città.

Per il progetto appare fondamentale la rivitalizzazione del monumento mantenendo salde le scelte gestionali già attuate. Dunque, riprogettazione del teatro, con particolare attenzione ai materiali impiegati i quali, secondo gli intenti, non dovrebbero prevaricare formalmente e visivamente la preesistenza ma integrarsi ad essa con leggerezza, senza mimetizzare le intenzioni progettuali, rendendole in ogni caso palesi e perfettamente leggibili anche dall'esterno. Il progetto nasce dalla necessità di superare gli squilibri urbani che si sono andati approfondendo fra la città e la rocca e per il bisogno di colmare le carenze, dal punto di vista qualitativo, dei servizi collettivi, ridefinendo e ricucendo, attraverso un'architettura pianificatrice, lo strappo col resto del tessuto urbano.

\section{LA METODOLOGIA}

Il progetto accoglie integralmente la destinazione a teatro della rocca Brancaleo$n e$, frutto di un'esperienza ventennale che risulta essere incoraggiata dal successo crescente e dall'elevato apporto culturale che dona alla vita sociale della città.

Il progetto è fondamentalmente una ricontestualizzazione dell'esistente, non avulsa dal reale, ma inserita in esso; denuncia la propria presenza che, compenetrata con quella del monumento, dovrebbe dare origine ad un nuovo uso del luogo.

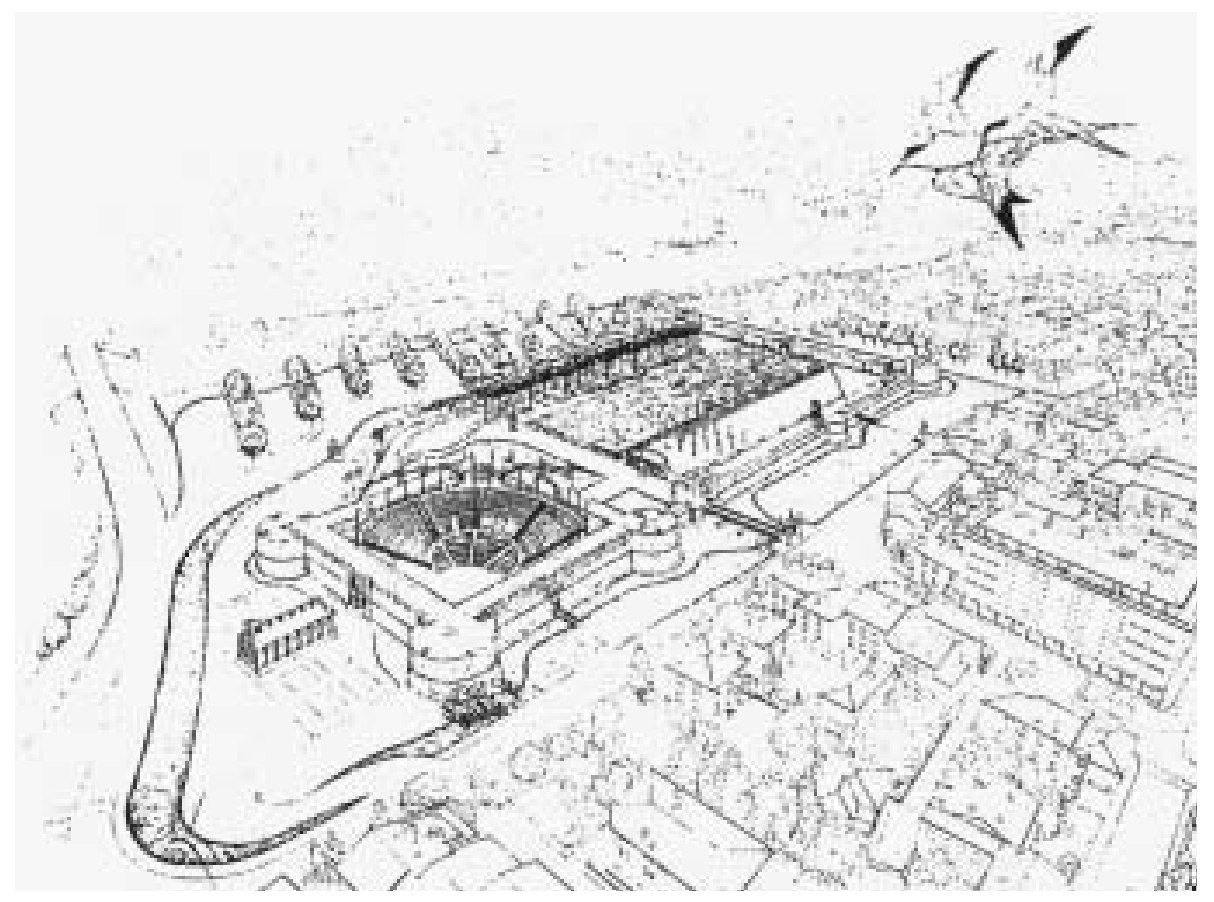

Figura 2 - Disegno prospettico del progetto. 
Il progetto non si esaurisce con la riqualificazione della zona della rocca, ma una parte altrettanto importante spetta alla fase progettuale relativa ai suoi spazi aperti.

Qui il verde viene modellato sia nelle forme tradizionali, tipiche di questa regione, sia in forme nuove, moderne. $\grave{E}$ in questa logica che si deve leggere il frammento di territorio boscoso apparentemente disposto in maniera irregolare, $\mathrm{ma}$ in realtà pianificato secondo un criterio modulare che s'ispira alle pinete impiantate dai Romani sulla costa adriatica per limitare l'erosione del mare o, viceversa, la ricerca sulle essenze del sottobosco e l'introduzione del Birdgardening. Quest'ultimo rappresenta, da un lato una nuova sensibilità nei confronti della natura, dall'altro sottolinea il carattere di continuità con l'antica concezione del giardino: luogo di relazioni armoniose, in cui i colori dei fiori e dei frutti, il canto degli uccelli, le forme bizzarre delle farfalle e degli insetti si mescolano contribuendo a definirlo come "Luogo di Delizia".

Il bosco, dunque, da realizzarsi con Pinus pinea, vuole essere un richiamo al territorio che circonda la città, a quelle pinete che nel corso dei secoli hanno rappresentato una fonte inesauribile di vita e sostentamento per gli abitanti del luogo. Se il bosco e la pineta coi loro simboli possono apparire un frammento spontaneo, irregolare e generico, il successivo passaggio compositivo è di natura squisitamente paradigmatica: si tratta di ricreare col verde un'immagine che rappresenta l'unicum storico e antiquario di Ravenna.

La scelta è caduta - non a caso - sul viso più seducente e famoso dell'arte musiva ravennate, il viso dell'Imperatrice Teodora, conservato nello spazio mistico e profondo di silenzi della Basilica di S. Vitale.

La trasposizione di parte del suddetto frammento su scala urbana rappresenta, in nuce, un programma di valorizzazione del monumento e un punto di riferimento per l'auspicata rinascita e lo sviluppo culturale della città. La realizzazione si basa su uno studio particolareggiato delle essenze erbacee perenni e l'immagine-manifesto di Teodora/Ravenna risulta un vasto "mosaico" di specie floreali, di profumi e colori mutevoli secondo il variare delle stagioni. Una continua metamorfosi nello spazio e nel tempo.

Il leit-motiv portante dell'intero intervento è quello di riuscire ad imprimere una dinamica a tutto il progetto, un progetto in continua evoluzione percepibile soprattutto nelle mutazioni cromatiche del giardino fiorito o nella maturazione dei materiali, intesa in senso organico, presente negli elementi della piazza, della pietra e del legno.

Il monumento sul quale il progetto è intervenuto per rivitalizzarlo necessitava di un contesto che lo rendesse indipendente e tuttavia profondamente integrato in tutte le trasformazioni proposte. La configurazione del nuovo spazio del teatro disegnato dal progetto si pone come una sorta di proiezione della rocca verso l'esterno. La fortificazione e gli spazi aperti che la circondano diventano un unico elemento ben definito e stabilmente riconoscibile a differenza di quanto accade attualmente, quando l'uso solo stagionale impedisce che questo manufatto rivesta l'importanza di scala urbana che gli compete.

Questo spazio urbano, una volta realizzato, non apparirà come un episodio meramente aggregato agli altri spazi verdi a disposizione di Ravenna, ma sarà un versatile e flessibile polo di sviluppo, con una sua precisa identità, connettendosi di nuovo al tessuto cittadino dal quale per tanto tempo è stato emarginato.

\section{GLI ESITI}

Nonostante il progetto rappresenti ancora solo il risultato di una tesi di laurea, si ritiene che gli obiettivi e la metodologia adoperati, possano essere di un 
certo interesse e quindi viene fatto rientrare a pieno titolo nel tema della valorizzazione degli spazi aperti monumentali.

La tesi di laurea (Brandoli-Baldisserri) è stata discussa il 15 luglio 1991 ed è stata coordinata e seguita dalla prof. Renata Rippa in qualità di relatrice, titolare della cattedra di "Arte dei Giardini e Architettura del Paesaggio" presso il Dipartimento di Progettazione dell'Architettura del Politecnico di Milano.

Autori del progetto: Davide Brandoli, Stefano Baldisserri

Titolo del progetto: La rocca, il teatro, il giardino: un progetto per Ravenna

\section{RIFERIMENTI BIBLIOGRAFICI E ICONOGRAFICI}

I materiali utilizzati per la redazione di questa scheda, sono stati tratti da: BRANDOLI DAVIDE e BALDisserRi STEFAnO, La rocca, il teatro, il giardino: un progetto per Ravenna, "Paesaggio urbano", sett./ott. 1993. 



\title{
LUOGHI PER L'ARTE
}

\author{
Emanuela Morelli
}

"Nel giardino e nel parco si colloca forse il maggior tentativo di riconciliazione progettuale fra uomo e natura".

Con la creazione degli spazi espositivi all'aperto si può osservare che la nascita di un'opera d'arte è legata strettamente ad un preciso luogo.

Larte, in quanto linguaggio e comunicazione $e^{2}$, divenendo cerniera tra lo spazio sociale e culturale della società contemporanea ed il mondo naturale, ha in questo contesto l'arduo compito di ristabilire il difficile e contrastante rapporto che l'uomo ha da sempre cercato di instaurare con la natura.

Gli spazi aperti d'arte, come quelli di seguito presentati, differiscono dai comuni musei o dalle gallerie all'aperto in quanto le opere, non essendo collocate in un ambiente che svolge ruolo passivo di cornice e sfondo, attivano uno scambio sinergico con il paesaggio circostante costituendo un percorso iniziatico di rivelazioni e scoperta: "Il parco, la campagna, la natura sono siti nei quali l'arte si integra con profonda interazione di energie, da cui scaturisce quasi un percorso iniziatico, e dunque un coinvolgimento diverso da quello che offre un museo"3.

L'opera d'arte diviene allora "monumento"4 collocato entro uno spazio collettivo e di fruizione pubblica. Il visitatore segue un percorso, costretto, stabilito o dato dal proprio intuito, scandito da una alternanza di luoghi segreti, intimi, pubblici. Attraverso questo movimento l'individuo può instaurare, tramite l'opera stessa, un processo evocatore originale delle caratteristiche intrinseche dell'ambiente. Con il suo rapporto dialettico, in contrapposizione o di coerenza formale, l'opera intende quindi celebrare il luogo.

Il gesto artistico stimola così un dialogo tra il paesaggio e lo spazio sociale, talvolta contraddittorio, ma sempre vitale, che esplica una nuova visione ed un nuovo modo di osservare il luogo.

Il sito, principio ispiratore, è il motivo di esistere dell'opera d'arte, la quale "diviene un filtro magico attraverso cui leggere il paesaggio stesso, levarne i significati nascosti"

\footnotetext{
1 Francesco Gurrieri, Art spaces a Celle, "Professione Architetto", periodico degli architetti toscani, 6, novembre-dicembre 1996, pag. 17.

2 "Larte è linguaggio, è comunicazione, non è un medicinale curativo. L'arte non è fatta per risolvere problemi sociali, ma per esprimerli. Non è compito suo risolverli; l'arte è linguaggio, è comunicazione, è la forma più comunicativa del pensiero umano, soprattutto di quello cittadino. E ancora una volta non si tratta di centralità o di periferia culturale: è un fatto che riguarda la nostra totalità, il nostro essere urbano che ha diritto di esprimersi pienamente." (PIERRE RESTANY in Dibattito sull'arte ambientale, http://www.aevo.com/townart/indice.html).

${ }^{3}$ Anna Mazzanti, Il territorio dell'arte, in Gianni Pettena, Patrizia Pietrogrande e MariaChiara Pozzana (a cura di), Giardini, Parchi e Paesaggio. L'avventura delle idee in Toscana dall'Ottocento a oggi, Le Lettere, Firenze 1998, pag. 165.

${ }^{4}$ Monumento, dal latino monumentum, derivato da monere, ricordare, è la testimonianza viva del passato (Dizionario Enciclopedico di Architettura ed Urbanistica).

${ }^{5}$ Renato Trippa, Giardini di oggi in Italia in Alberta CazZani (a cura di), Architettura del verde. L'esperienza paesaggistica italiana, BE-MA editrice, Milano 1994, pag. 17.
} 
L'artista studia attentamente l'ambiente, la sua storia, la morfologia, la composizione delle specie vegetali, i colori e il gioco di luce ed ombra, il clima, le sensazioni che induce.

Il sito diviene dunque il punto di partenza per la riproposizione dell'opera, che genera di conseguenza spazio qualificato e pregiato.

L'obiettivo dell'arte è di conseguenza quello di trasformarsi in strumento di conoscenza del mondo naturale e delle antiche origini dell'uomo ${ }^{6}$, il mezzo per avvicinarsi alla Natura ${ }^{7}$, un'occasione per viverla e rivelarla, creando così un momento e un'opportunità di crescita individuale e collettiva.

Grazie ad essa, mediatrice ed evocatrice delle culture antenate con principi sostanzialmente animistici, scaturisce difatti un dialogo arte-natura-verità che rafforza le specificità del contesto paesistico.

Il paesaggio quindi non è inteso come rappresentazione scenografica e cornice, ma elemento stesso fondativo dell'opera, tramite appunto la sua reinterpretazione.

Questa stessa crea luoghi che fungono da simbolico tramite fra le istituzioni umane e le mutevoli concezioni della Natura.

Grazie sia a studiosi quali Isa Belli Barsali e Alessandro Tagliolini, con la conseguente identificazione del valore storico e monumentale del giardino, sia alle arti visive, con il loro carattere sperimentale, il ricercare la verità con atteggiamenti provocatori e guardare la "Natura del XX secolo, tecnologica, industriale, pubblicitaria, urbana" ", "sorgente di espressività e di materiali nuovi per l'espressione artistica", si è potuto riconquistare una concezione contemporanea del giardino e del paesaggio.

Già nelle epoche passate le tecniche, l'ingegno, l'arte, avevano contribuito a realizzare opere simboliche e forme indagatrici rappresentanti l'incessante ricerca dell'uomo verso la verità e la conoscenza della Natura, portatrice di mistero, inquietudine e passioni, generatrice al tempo stesso di vita e di morte.

Nell'antico e vasto parco di Pratolino ${ }^{10}$, per citare uno dei più significativi giardini della seconda metà del XVI secolo, fu proprio la tecnologia corrente del periodo a mettersi a servizio dell'arte come mezzo di decodificazione delle leggi della natura. Automi, sculture giganti come l'Appennino ${ }^{11}$, collocati entro una elaborata struttura vegetazionale, creavano stupore, nuove suggestioni e interpretazioni fantastiche del luogo. "I significati delle allegorie seminate nel parco sembrano profilare un itinerario catartico, una sorta di iniziazione ai misteri del mondo naturale"12.

Il più recente parco di Pinocchio a Collodi ${ }^{13}$ evidenzia invece le complesse relazioni tra scultura, paesaggio e architettura. Il percorso da intraprendere è

${ }^{6}$ Vedi la scheda di Enrica Dall'Ara, riferita all'Hortus conclusus a Benevento, in cui le sculture di Mimmo Paladino rievocano i Sanniti, antiche popolazioni della città.

${ }^{7}$ Vedi la scheda di Emanuela Morelli, riferita al giardino Hic terminus haeret, di Daniel Spoerri.

${ }^{8}$ Pierre Restany in Giulio Carlo Argan, L'arte moderna 1770/970, Sansoni editore, Firenze I edizione 1970, II edizione 1971, pag. 657.

${ }^{9}$ PIERRE Restany in Dibattito sull'arte ambientale, http://www.aevo.com/townart/indice.html.

${ }^{10}$ Ubicato nell'attuale comune di Vaglia in prossimità di Firenze, i lavori cominciarono nel 1568. La bellezza e la spettacolarità del parco di Pratolino nacque grazie all'interazione di due grandi personaggi: Francesco I dei Medici, proprietario della vasta tenuta, figura scientifica, tecnologica e al tempo stesso inquieta, e Bernardo Buontalenti, artista e "ingegnere dei fiumi", il cui estro nasceva dall'interpretazione del capriccio e dell'artificio. Il parco era suddiviso in due parti: a nord della villa chiamato Degli Antichi ed a sud detto Dei Moderni. Nel 1823, sotto la famiglia Demidoff, fu trasformato da Joseph Frietsch in parco paesaggistico.

${ }^{11}$ La statua dell'Appennino, definita "la meraviglia delle meraviglie di Pratolino", fu eseguita nel 1580 dal Giambologna. Nel suo aspetto pietrificato convergono lineamenti umani naturali. Le sue dimensioni sono tali da poterla percorrere al suo interno, tra grotte e stanze.

${ }^{12}$ Alessandro Tagliolini, Storia del giardino italiano, La Casa Usher, Firenze 1988, pag. 167.

${ }^{13}$ Parco di Pinocchio e Paese dei balocchi, Collodi, provincia di Pistoia, luogo già famoso grazie alla vicina Villa Garzoni (vedi a tal proposito la scheda di Antonella Valentini, riferita al restauro di Villa Garzoni). Nel 1956 sono nate le prime sculture nel giardino ideato dagli architetti Baldi e De Luigi. Tra gli autori principali dello spazio architettonico e vegetale si menzionano Pietro Porcinai, Marco Zanuso, Pietro Consagra e Giovanni Michelucci. 
definito dal tema della fiaba, costituito da una successione di stanze isolate che costituiscono gli episodi, separate e protette da un interessante apparato vegetazionale. Come in un giardino romantico tale successione, ricca di rivelazioni allegoriche, induce sorpresa, fantasia e gioco.

Il paesaggio è definito qualitativamente da una direzione principale e generatrice: fin dagli albori della storia, l'uomo ha sempre agito in funzione di essa.

La nascita e il divenire quindi di un'opera d'arte ambientale non può prescindere dalla sua collocazione nel luogo di appartenenza. Il processo di riqualificazione di tale spazio avviene difatti orientando il gesto artistico in relazione alla direttrice generatrice ${ }^{14}$. Il risultato che ne scaturisce, così come nel giardino e a scala più vasta nel paesaggio, è dinamico e la crescita e il mutamento dello spazio nel tempo diventano le variabili secondo cui il progetto si evolve senza mai raggiungere la sua stessa compiutezza.

Lopera incompiuta è quasi sempre presente in queste realizzazioni di artepaesaggio proprio "come la vita di ogni uomo nella totalità della storia e nell'eternità della natura. Per questo l'arte (...) ha il compito di fissare l'effimera figura del vivente oltre il passaggio del tempo"15.

Il paesaggio diviene quindi un'opera aperta, archivio vivente, laboratorio di ricerca della verità nel costante, talvolta coerente e talvolta difficile, rapporto tra uomo e natura.

Abbiamo così esempi, come il parco di Villa Celle a Pistoia ${ }^{16}$ e il Giardino di Daniel Spoerri a Seggiano, che diventano un simposio di idee tra artificiale e naturale, in cui varietà e complessità instaurano il rapporto dialettico vivace e dinamico con il luogo, senza alterare l'antica struttura di fondo.

Il Parco di Villa Celle, realizzato grazie agli interventi di molti artisti, ed il Giardino dei Tarocchi a Garavicchio ${ }^{17}$, opera monografica di Niki de Saint Phalle, sono diventati nel tempo, gli esempi più significativi di una condizione culturale che si sta sempre più affermando in Italia.

A fianco degli esempi di spazi d'arte "visitati" nelle schede seguenti, ve ne sono molti altri in Italia ugualmente di notevole interesse, tra cui si menzionano:

- Fiumara d'arte, Castel di Tusa, provincia di Messina, in Sicilia. Il percorso che individua le varie opere artistiche si svolge lungo il tracciato del fiume Halesus. La prima realizzazione risale al 1986. Il progetto generale è di Antonio Presti in collaborazione con artisti quali, Consagra, Di Palma, Nagasawa, Schiavocampo.

- Campo del Sole a Tuoro, sulle rive del Lago Trasimeno. Lintervento progettua$\mathrm{le}^{18}$ si riferisce alla zona di Campo del Sole e ad una vasta zona naturalistica.

"Il progetto parte da una analisi e da considerazioni che prevedono la localizzazione di funzioni compatibili con il contesto ambientale, cioè l'utilizzazione dell'ambiente naturale, le attività sportive, la ricettività dell'area e il rapporto con il lago"19. Qui ventisette artisti sono stati chiamati a erigere un proprio totem, prevalentemente realizzato con pietra serena grigia locale, dentro una radura a forma di cerchio. L'effetto che ne consegue è la trasformazione di tale spazio in un luogo sacro. "A parte la sua iconografia di riferimento solare archetipa, l'insieme tende costituire un luogo di riflessione, di medita-

\footnotetext{
${ }^{14}$ Vedi saggio introduttivo di BRUNO CORÀ, l'esperienza di arte ambientale, "Quaderno n. 4, Arte ambientata Arte Ambientale", periodico a cura del Centro per l'Arte Contemporanea Luigi Pecci - Prato, 2001.

${ }^{15}$ Massimo Venturi Ferriolo, L'artista delle metamorfosi, "Architettura del Paesaggio", 6, monografico dedicato ad Alessandro Tagliolini, Alinea editrice, Firenze giugno 2001, pag. 3.

${ }^{16}$ Vedi la scheda di Anna Lambertini, riferita a Villa Celle.

${ }^{17}$ Vedi la scheda di Emanuela Morelli, riferita al Giardino dei Tarocchi.

${ }^{18}$ Progetto redatto dagli architetti Cristiano Toraldo di Francia e Andrea Noveri, con la consulenza per la parte ambientale e paesaggistica dello studio Natura Progetto. L'intervento è stato iniziato nel 1985.

${ }^{19}$ Ines RomitTi, Campo del Sole, colonne sul lago Trasimeno, "Professione Architetto", 6, Alinea Editrice, Firenze 1996.
} 
zione, di memoria, di incontro e di familiarità. (...) In questo senso la tavola-desco centrale, che supporta un simbolo solare, si offre come il termine domestico di un percorso, quasi iniziatico, come il luogo di incontro e appunto del dialogo" 20 .

- MACAM (Museo d'Arte Contemporanea all'aperto di Maglione), Maglione Canadese, Torino.

- Gli abitanti del piccolo borgo urbano della provincia di Torino hanno messo a disposizione di settanta artisti i muri delle proprie abitazioni, come supporto per la realizzazione delle opere d'arte.

- Cretto di Alberto Burri, comune di Ghibellina, in Sicilia.

Progettato nel 1981 in ricordo del terribile terremoto del 1968 che distrusse interamente la città vecchia. L'opera è un enorme lenzuolo di cemento bianco "crettato", che ricopre le rovine dell'antico centro urbano. "Quest'opera esprime tutta la capacità del «monumento» di rappresentare oggi il grande racconto storico alla cui frammentazione pure stiamo ormai assistendo; rappresenta la complessità dei nostri giorni produttori di labirinti dove ogni punto è insieme punto di arrivo e di partenza." 21

- Arte Sella - Sella di Borgo Valsugana, in Trentino. E una biennale di arte contemporanea, nata nel 1986. Le opere, che devono esprimere il rapporto con la natura basato sul rispetto, traendo da essa ispirazione e stimolo, sono ottenute in genere con sassi, foglie, rami e tronchi, mentre più raramente sono utilizzati oggetti materiali o colori artificiali.

Non è un caso comunque che gran parte degli interventi presentati in questa sezione si trovino nel centro Italia e in particolare nella Toscana. Questa regione è difatti caratterizzata da un paesaggio fortemente espressivo della sua storia culturale, in cui valli intensamente coltivate si alternano con aree ad alto valore naturalistico. La storia della sua trasformazione, legata anche a gesti comuni e quotidiani, è ancora viva e funzionante nel tessuto del paesaggio: essa si può ancora leggere nei terrazzamenti, nelle opere di ingegneria e regimazione idraulica, nell'artigianato attivo specie nei piccoli paesi, nonché nel rapporto prolifico che questa civiltà ha sempre avuto nei confronti dell'arte.

Questi paesaggi, specie dell'area meridionale della Toscana, tra la Maremma e la provincia di Siena, richiamano, oggi come nel passato, molti artisti stranieri, i quali non si pongono come semplici visitatori, ma eleggono a propri luoghi di residenza soprattutto i piccoli centri urbani, un po' isolati, dove è ancora forte l'integrazione tra nuove esigenze di vita e tradizioni locali.

A fronte di questa situazione l'amministrazione della Regione Toscana, con il proposito di incentivare e valorizzare queste risorse di arte contemporanea, ha redatto in questi ultimi anni, il progetto regionale "promozione del sistema di arte moderna e contemporanea in Toscana - Sentieri nell'arte" di cui fanno parte, oltre il Parco di Villa Celle, il Giardino dei Tarocchi e il giardino Hic terminus haeret:

- il Giardino dei Suoni di Paul Fuchs, in località Boccheggiano, Grosseto;

- il Giardino di sculture - gli Ominidi - di Kurt Metzler, nella sua proprietà, a Jesa, Siena;

${ }^{20}$ Intervista a ENRICO CRISPOLTI, Le colonne di Campo del sole, "Arredo Urbano", 46, dedicato a "costruire l'immaginario - l'architettura del monumento in Italia e nel mondo: evoluzione e nuove tendenze", IN/ASA, novembre-dicembre 1991, pag. 97.

${ }^{21}$ Aldo Maria Di Chio, La forza dell'espressione. Progettare monumenti a Gibellina, "Arredo Urbano", 46, dedicato a "costruire l'immaginario - l'architettura del monumento in Italia e nel mondo: evoluzione e nuove tendenze", IN/ASA, novembre-dicembre 1991, pag. 66.

${ }^{22}$ La rassegna si svolge all'interno dei Giardini delle Rose e nel percorso lungo le mura. Nel 1991, la XXI edizione è stata dedicata all'opera di Alessandro Tagliolini (1931-2000), scultore-paesaggista come egli stesso amava definirsi, personaggio significativo nel panorama paesaggistico italiano e internazio- 
- Forme nel verde a S. Quirico d'Orcia, Siena ${ }^{22}$;

- I progetti di travertino, a Rapolano Terme, Siena;

- Affinità Elettive intervento di cinque artisti a San Gimignano, Siena;

- Artisti nel centro storico a Peccioli, Pisa. Un percorso contemporaneo nel centro storico di Peccioli;

- Dopopaesaggio a Certaldo, Firenze. "Dove l'uomo può confrontarsi con la natura e creare esperienze individuali"23;

- Tuscia Electa, itinerari contemporanei attraverso alcune cittadine del Chianti fiorentino e senese.

Quest'ultimo progetto, nato nel 1996, è diventato un importante appuntamento annuale per la realtà toscana, dove installazioni effimere di arte contemporanea sono appositamente studiate nel luogo che le accoglierà. L'intento è quello di fornire un ulteriore segno, espressivo dell'attuale società, nella stratificazione storico-culturale del paesaggio, sperimentando la possibilità di instaurare una continuità nel rapporto dialettico tra il mondo passato e quello presente.

Alcune di queste "sperimentazioni" sono state pienamente riconosciute dalla collettività e sono diventate segni permanenti.

nale. È stato autore di molti testi importanti riguardanti la storia del giardino, e progettista di interventi di restauro, quali la Certosa di San Lorenzo di Padula e Villa Rufolo a Ravello (complessi storico-monumentali situati entrambi nella regione Campania), e di parchi e giardini urbani e privati (Il parco delle terme di Sciacca): sono opere di architettura del paesaggio dove natura ed artificio "incessantemente" dialogano tra loro.

${ }^{23}$ Anna Mazzanti, 'Dopopaesaggio' Castello di Santa Maria del Fiore, in Gianni Pettena, Patrizia Pietrogrande e Mariachiara Pozzana (a cura di), Giardini, Parchi e Paesaggio. L'avventura delle idee in Toscana dall'Ottocento a oggi, Le Lettere, Firenze 1998, pag. 173. 



\title{
"SpaZI D'ARTE a Celle: \\ L'INVENZIONE DI UN COLLEZIONISTA APPASSIONATO"
}

\author{
Anna Lambertini
}

A Santomato, nel paesaggio agrario collinare toscano alle porte di Pistoia, un parco creato intorno alla metà dell'Ottocento è divenuto per volere del suo attuale proprietario, Giuliano Gori, un importante museo all'aperto d'arte ambientale contemporanea.

"Il desiderio e la curiosità di verificare il comportamento dell' artista contemporaneo di fronte a una committenza che gli offra l'opportunità di realizzare un progetto, in cui lo spazio venga usato come parte integrante della propria opera, e non più come semplice contenitore, rappresenta la molla che ci ha convinti a riprendere l'interrotta tradizione delle installazioni nel parco"1. Ecco come il suo "inventore" spiega la nascita degli Spazi d'Arte nel parco di Celle, la cui innegabile peculiarità consiste nel configurarsi come un paesaggio storico che accoglie in sé molte idee contemporanee di paesaggio, attraverso la definizione di luoghi qualificati dall'intervento dell'artista.

Celle merita attenzione non solo per la qualità ed eccezionalità degli esiti spaziali raggiunti, ma anche perché in essa pare concretizzarsi in forma paradigmatica la definizione di paesaggio

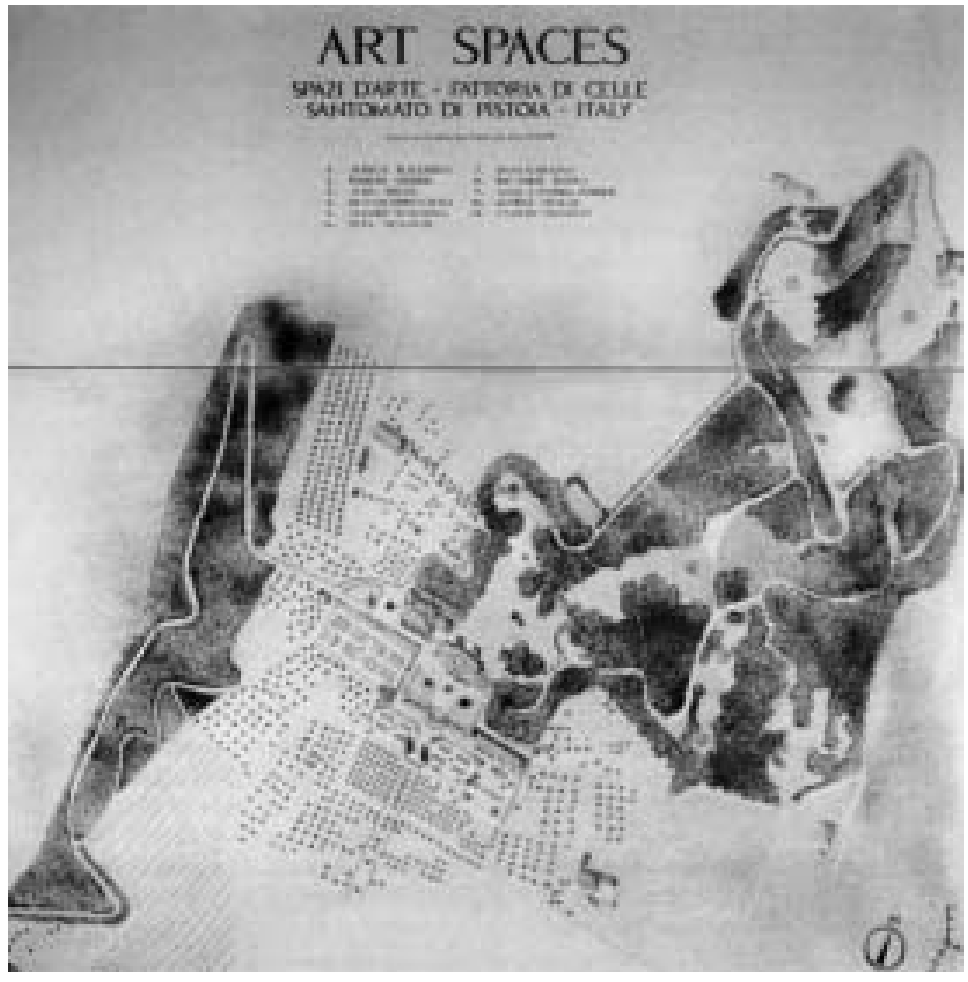

Figura 1 - Planimetria di Art Spaces redatta dallo Studio Arch. Francesco Gurrieri nel 1983. come opera aperta, come archivio vivente del rapporto uomo/natura, e ancora di paesaggio come palinsesto, unico testo scritto e riscritto nel corso del tempo ${ }^{2}$.

\section{BREVI NOTE STORICHE SU CELLE}

Comprendere a fondo il valore dell'esperienza condotta a Celle non può prescindere dal considerarne il contesto territoriale e paesaggistico facendo riferimento alla sua evoluzione nel tempo.

\footnotetext{
* Si ringraziano per il prezioso contributo: Marco Cei, che ha reso disponibile il suo materiale documentario su Celle e Miranda Mac Phail, che ha fornito informazioni aggiornate sui recenti sviluppi della collezione.

${ }^{1}$ Giuliano Gori in Pierre Restany (a cura di), Arte Ambientale, Allemandi Editore, Torino 1994, pag. 9.

${ }^{2}$ Cfr. Lionella Scazzosi, Paesaggio, Paysage, Paisaje, Landscape, Landschaft, Landschap, Krajobraz... politiche e culture del paesaggio in Europa e negli Stati Uniti: una lettura trasversale in Politiche e culture del paesaggio, a cura di Lionella Scazzosi, Gangemi Editore, Roma 1999, pagg. 17-36.
} 


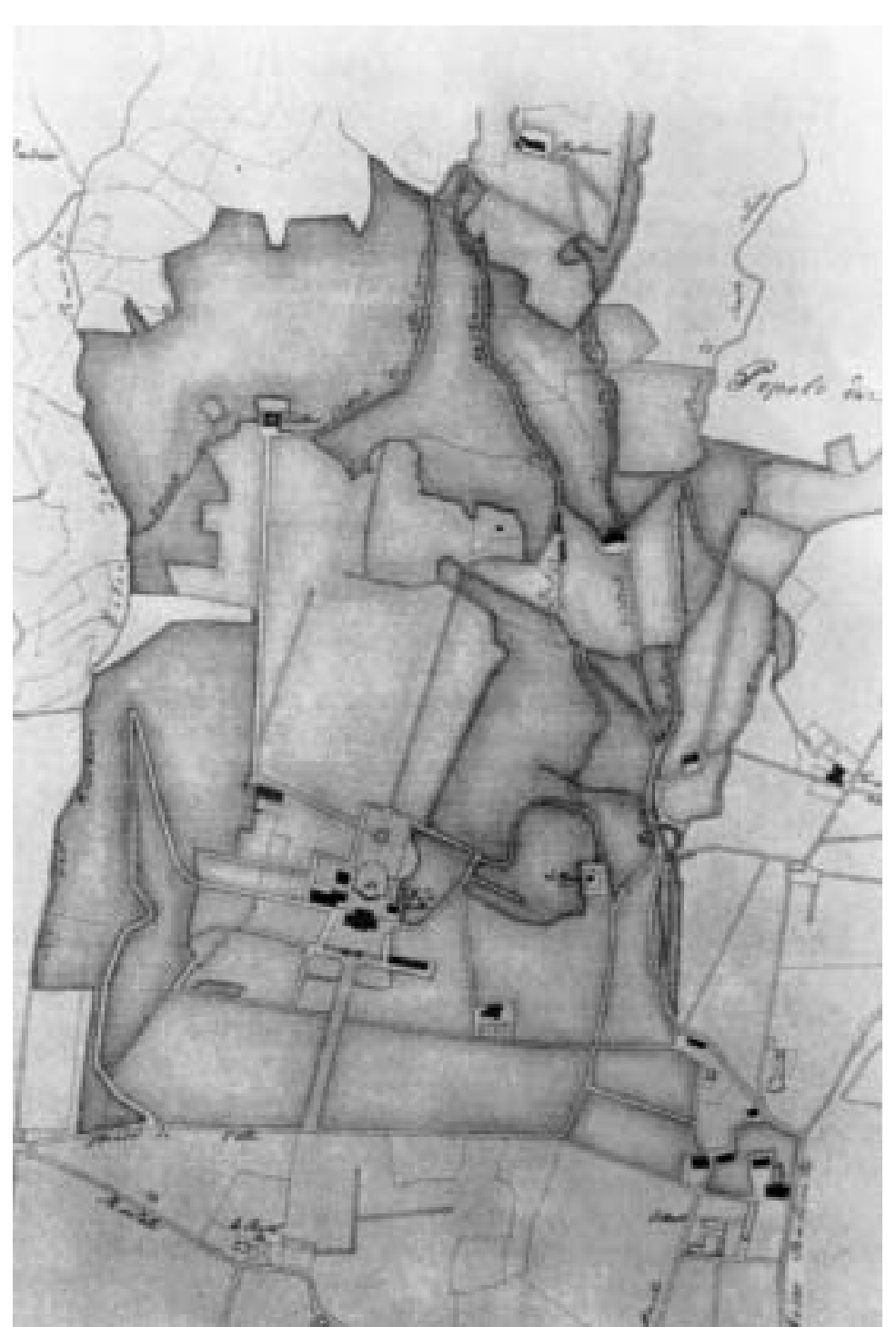

Figura 2 - Individuazione della proprietà di Celle in una vecchia mappa catastale.
Le fonti storiche ci permettono di far risalire l'origine delle prime fabbriche di Celle intorno al Mille, epoca in cui la zona costituisce territorio di frontiera tra i domini di diverse casate e le città di Pistoia e Firenze.

Il toponimo, abbastanza diffuso e comune a molti casali e piccoli nuclei insediativi della Toscana $^{3}$, inizia a comparire in documenti e carte dalla metà del XIII secolo a indicare un luogo individuato da alcuni corsi d'acqua ${ }^{4}$. A quel tempo la zona era dominata dalla famiglia pistoiese dei Cancellieri.

Nella metà del XV secolo la tenuta di Celle risulta di proprietà di un'altra potente famiglia della zona: i Pazzaglia, mentre nel XVI secolo l'impianto originale di semplice struttura fortificata viene modificato per creare un edificio più articolato e complesso. Un secolo dopo Celle è citata tra i possessi della famiglia Fabroni.

È a partire dalla metà del XVII secolo che, per volere di Ignazio Fabroni, si pone mano ad un progetto di trasformazione dell'edificio principale della fattoria in villa padronale, cui viene conferita una veste architettonica degna del gusto dell'epoca e di rappresentare il prestigio dei proprietari, come testimonia un documento del 1663 in cui si cita "casa detta il Casone, con tinaia, orto e gelseto, circondato di mura serve per villa e habitatione del padrone in detto Comune (S. Mato)"

Significative modifiche sono apportate anche all'esterno della villa dove vengono creati giardini terrazzati all' italiana in cui "si piantarono bussoli" e "si posero gli Alcipressi"

Il programma di miglioramento e ammodernamento della tenuta sarà proseguito da un altro Fabroni, il più illustre nella storia della famiglia, Carlo Agostino (1651-1727), che fu cardinale fiorentino sotto il papa Clemente XI. È grazie a lui che saranno condotte numerose sistemazioni agrarie (destinate soprattutto alla coltivazione di olivo, vite e grano), realizzate nuove piantagioni (tra cui anche una di gelsi presumibilmente per l'allevamento del baco da seta), costruite nuove fabbriche all'interno della tenuta, come la piccola cappella a pianta centrale di gusto neoclassico edificata sul retro della villa.

La sequenza degli spazi esterni, giardini-parco-bosco, sarà organizzata in applicazione degli schemi compositivi geometrico-formali dello stile barocco e strutturata da un asse centrale che assumerà la villa come principale quinta di riferimento.

${ }^{3}$ Come riferisce il Repetti, il toponimo Celle fa riferimento o alla presenza di una cappellina (sacellum, cella, sacrarium) o ad una grotta naturale o artefatta oppure al nome del suo primo fondatore Cello. E. RePETTI, Dizionario geografico, fisico, storico della Toscana, Firenze 1833.

${ }^{4}$ Cfr. Marco Cei , "Il parco di Celle a Pistoia", Edifir, Firenze 1994.

${ }^{5}$ In MARCo Cei, op. cit., 1994.

${ }^{6}$ Preziose informazioni e descrizioni di Celle e di scene di vita quotidiana dei Fabroni ci sono fornite da un minuzioso diario di ricordi di Ignazio Fabroni "Ricordi di villeggiatura, di caccia e d'altro..." 1665-1690, citato in MARCO CeI “Il parco di Celle a Pistoia”, Edifir 1994, pagg. 12-16. 
L'impianto barocco del parco resta pressoché inalterato fino all'inizio del secolo XIX. Solo una concessione viene fatta alla moda dell'epoca: in risposta alla dilagante mania ottocentesca per le chinoiseries, nei giardini sul retro della villa viene costruita una voliera in mattoni e ferro a pianta ottagonale e cuspide a pennacchio, disegnata dal poeta Bartolomeo Sestini ${ }^{7}$ nel 1812.

Grazie alle descrizioni riportate in un Catasto del 1823 realizzato per Leopoldo II, è possibile desumere notizie utili a capire l'assetto agrario e forestale della fattoria.

Le parti boscate, in particolare, presentavano un'alta diversificazione d'uso: querceta (formata per lo più da Quercus robur e Quercus pubescens), ceduo di castagni per ottenere palerie assortite, ragnaie per l'uccellagione, albereta con pioppi, stipeto (sottobosco di saggina per scope) e noccioleta (per la raccolta dei frutti) ${ }^{8}$.

Qualche anno dopo, sotto la gestione del conte di origine piemontese Damiano Caselli, ben altra sarà l'influenza esercitata sull'assetto e l'estetica del parco dal prevalere del gusto del tempo: in applicazione dei dettami dell'arte dei giardini inglesi, venuta ormai di gran moda anche in Italia grazie alla diffusione di vari trattati di interpreti e cultori dell'allora 'via moderna al giardino

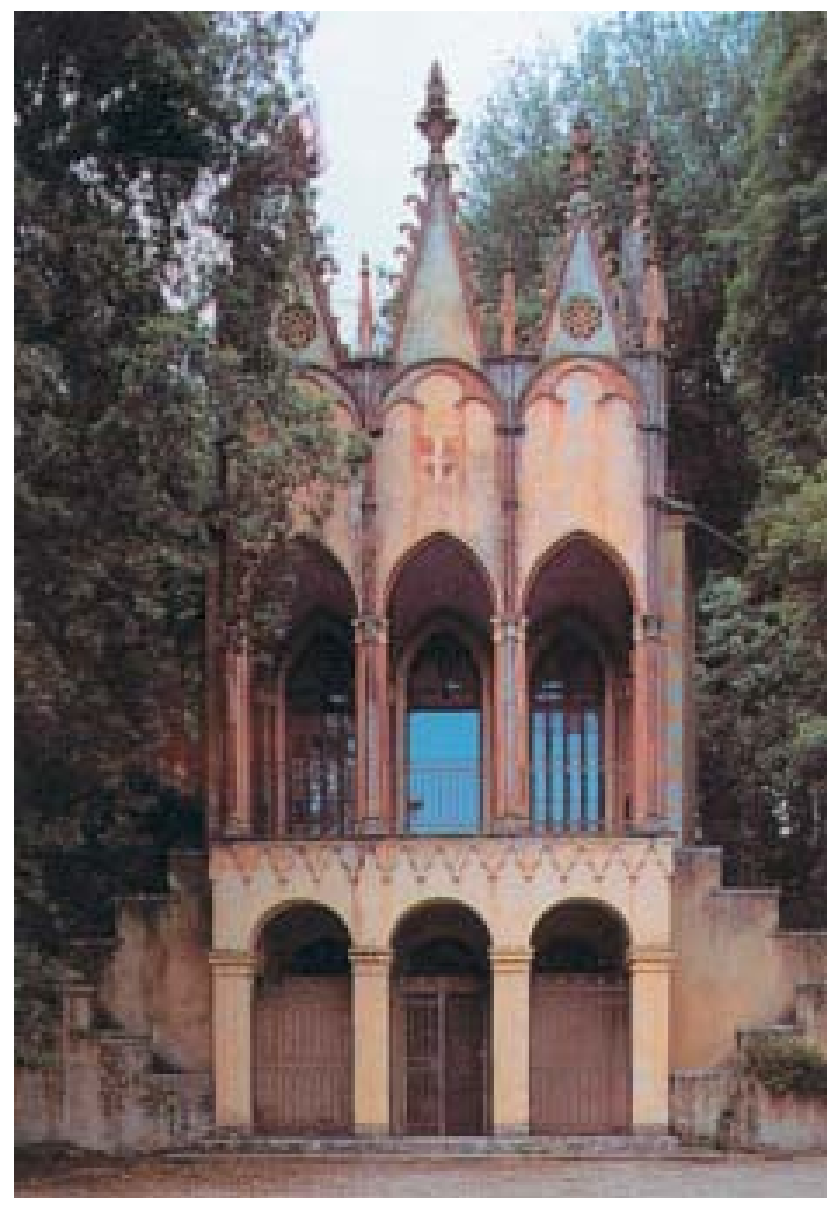

Figura 3 - La Casina Neogotica.
Figura 4 - All'interno: Dani Karavan, Cerimonia del tè, 1999.

${ }^{7}$ Bartolomeo Sestini studiò "ornato e prospettiva" all'Accademia delle Arti di Firenze, dedicandosi anche allla poesia ed allo studio dei classici, fu autore di varie opere letterarie tra cui "Amori Campestri" (1814), gli "Idilli" (1816). Cfr. Francesco Gurrieri, “Art Spaces a Celle", in "Professione Architetto", 6/1992, Alinea, Firenze 1992, pag. 19.

${ }^{8}$ In Marco CeI, op. cit., 1994, pag. 34. 
paesaggistico' ${ }^{\prime 9}$, sarà attuato un radicale intervento di trasformazione degli spazi esterni della Fattoria.

L'esito dell'intervento sarà tale da portarci oggi a riconoscere che "la villa di Celle a Santomato, per organicità e stato di conservazione, è una delle più importanti dell'intera Toscana. L'insieme di architettura, scultura, parco giardino, fontane ed opere d'arte, costituisce un episodio significativo e riassuntivo della cultura sette-ottocentesca del Granducato" ${ }^{10}$.

\section{Sul parco ottocentesco}

Un'epigrafe posta nel parco, "nell'ombilico della scogliera", oltre a indicare la paternità in Giovanni Gambini di parte del progetto ottocentesco fissandone esattamente il periodo di costruzione, palesa nell'affermazione conclusiva lo spirito dell'intervento:

"Negli anni 44 e 45 del secolo XIX / costruivasi questa scogliera sopra / muro grosso in pianta B. a22 - alto 27 / Gio. Gambini di Pistoia richiesto a ornare questa parte del bosco, compiuta l'isola / e i dintorni del lago, la immaginò, la diresse / e senza gli ordigni usati dall'arte, la / condusse conforme natura si piace / mostrarsi più originale e severa".

Giovanni Gambini, architetto e pittore paesaggista, compie la sua opera di modellamento della natura e di creazione di un nuovo paesaggio in adesione all'estetica del pittoresco ${ }^{11}$ : il parco può essere letto come il risultato dell'applicazione di un esercizio di stile, di quello stile, più precisamente, educato alla "natura liberamente rappresentata attraverso l'artificio delle forme nascoste"12.

Un esercizio di stile, certo, ma con tutto il repertorio di espedienti formali e compositivi recitato e condotto con sensibilità e sapienza.

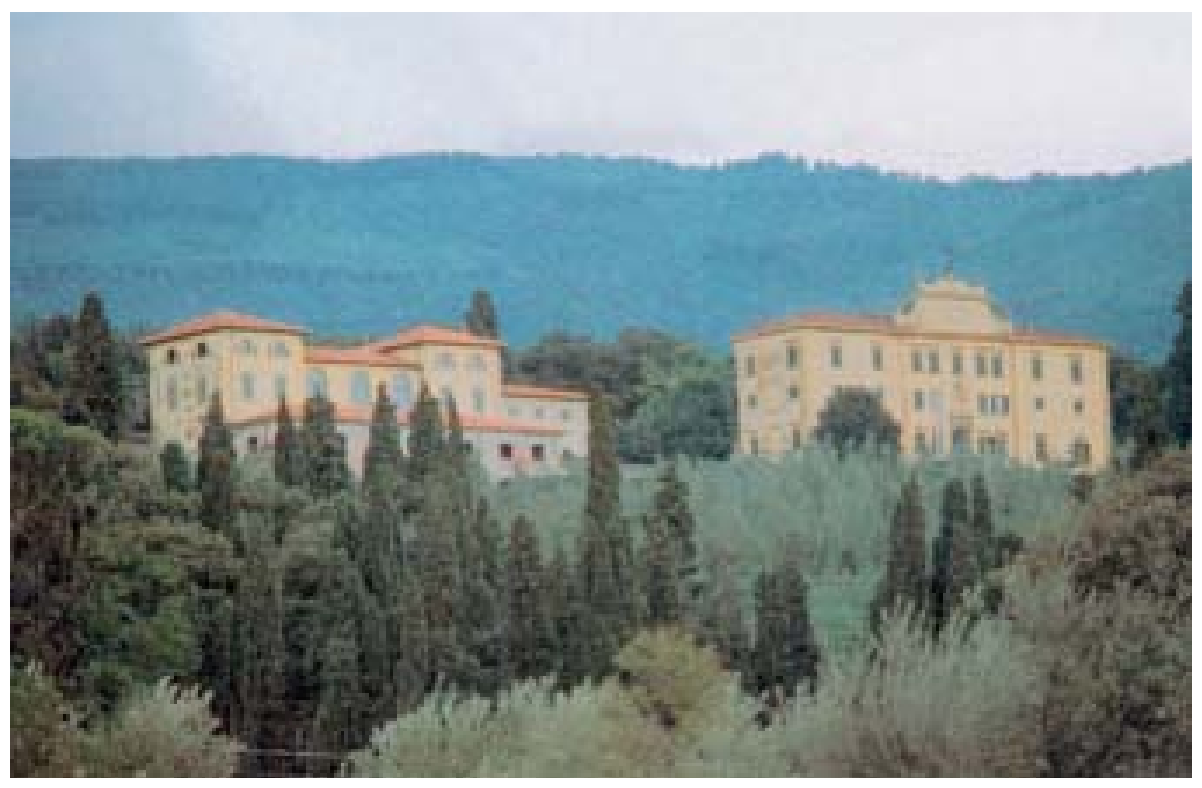

Figura 5 - Il complesso Villa, Fattoria e Limonaia.

${ }^{9}$ In Italia la dilagante moda del giardino informale sarà influenzata soprattutto dalle seguenti opere editoriali: IPPOLITO PINDEMONTE, Dissertazione su i giardini inglesi e sul merito in ciò dell'talia, Padova 1792; Luigi Mabil, Teoria dell'arte de giardini, Remondini, Bassano 1801; ErCole Silva, Dell'arte de' giardini inglesi, Genio Tipografico, Milano 1801.

${ }^{10}$ Francesco Gurrieri, Nuovi spazi d'arte contemporanea a Celle in Arte Ambientale, PIerre Restany (a cura di), Allemandi editore, Torino 1994, pag. 20.

${ }^{11}$ Sul tema cfr. Massimo Venturi Ferriolo, Giardino e paesaggio dei romantici, Guerini e Associati, Milano 1998, e RafFaele Milani Il pittoresco. L'evoluzione del Gusto tra classico e romantico, Universale Laterza, Bari 1996.

${ }^{12}$ Massimo Venturi Ferriolo, op. cit., 1998. 
È soprattutto con l'occhio del pittore che possiamo immaginarci il Gambini intento alla realizzazione del parco e, rubando un'immagine già evocata da Walpole per elogiare le capacità espressive di William Kent, occupato a "piantar quadri".

I caratteri naturali del luogo vengono, per così dire, raffinati e corretti: la morfologia variabile del terreno e le altimetrie sono sfruttate abilmente per introdurre nuovi elementi di attrazione visiva e punti di vista privilegiati, il corso del torrente Brana che attraversa il parco modificato per creare un ampio lago, le masse boscate ridisegnate. I percorsi sinuosi che si aprono all'improvviso su una veduta, il lago con l'isoletta, l'orrido con la cascata, l'impianto vegetazionale e tutto il corredo di fabriques, sono elementi di una costruzione paesaggistica impostata su una successione di scene pittoriche e di valori, dove è annullata quella possibilità di un'unica prospettiva dominante che aveva invece guidato la redazione del parco settecentesco.

Lattitudine del Gambini a comporre il paesaggio avvalendosi della sua sensibilità pittorica si mostra chiaramente nell'impostazione della complessa scena del lago plasmato in forme naturali, inserito nel "seno di una chiusa valletta, che è a mezzo del parco" e su cui "sorge una bella isoletta, giacente per metà su grotte praticabili "13, dove viene riproposto da vari punti di vista ma con uguale evidenza lo spettacolo della 'bellezza vivente', della bella disposizione della natura.

"Sebbene il parco del Gambini sia certamente fatto di terra, rocce, acqua e piante, è una natura artificiale costruita in conformità con l'estetica del pittoresco allora dominante, che richiedeva grotte, cascate e ruderi, nonchè un paesaggio cangiante segnato da zone pastorali, oscuri boschetti, ponticelli rustici, placidi laghetti con precisi luoghi d'ormeggio, nascondigli segreti e speciali punti d'osservazione che consentono di inquadrare la natura in modo da ricordare le tele di Lorrain, Poussin e Salvator Rosa" ${ }^{14}$.

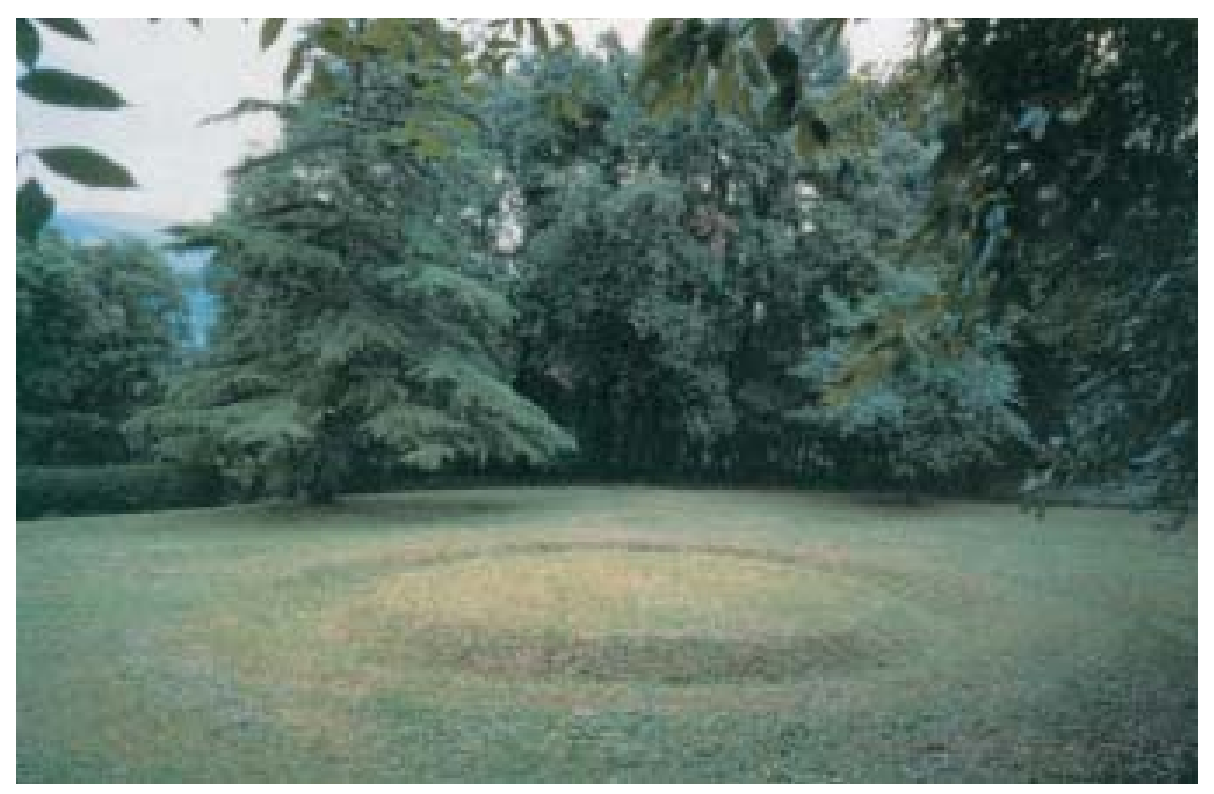

Figura 6 - Richard Long, Cerchio d'erba, 1985.

Il tempietto in stile neoclassico, esostilo e a pianta circolare, collocato sull'isoletta, ad esempio, è facilmente leggibile come una variazione su un tema pittorico e di composizione dell'arte dei giardini ormai ampiamente rappresentato e descritto.

\footnotetext{
${ }^{13}$ Giuseppe Tigri, Pistoia e il suo territorio, Pescia e i suoi dintorni, Pistoia 1854, da MARCo CeI, op. cit., 1994, pag. 35 .

${ }^{14}$ Robert Hobbs, Ambientazione alla fattoria di Celle in Pierre Restany (a cura di), Arte Ambientale, Allemandi editore, Torino 1994, pag. 38.
} 
Opera d'arte in sé, il parco del Gambini possiede un impianto narrativo fondato sull'articolazione di visioni della natura e di architetture nella natura con cui si rimanda ad altri luoghi ed altre epoche. Anche a Celle, "il giardino diventa enciclopedia: percorrendolo si sfogliano diversi capitoli del libro del mondo" 15 .

L'immaginazione viene stimolata soprattutto attraverso l'uso di uno speciale vocabolario di oggetti e scene emblematiche messo a punto per evocare figure letterarie e situazioni favolose (l'orrido con la cascata, la Casina neo-gotica), anche esotiche (come nel caso dell'inserimento del Monumento Sepolcrale Egiziano, in cui "elementi assirici si fondono con elementi egiziani e con alcune elaborazioni proprie del primo eclettismo"16).

Il gusto per il pittoresco si afferma per toccare qui, ancor più che la dimensione romantica, quella romanzesca ${ }^{17}$; la distinzione di fondo tra le due viene individuata da Venturi Ferriolo nel ruolo nascosto o evidente del mediatore del desiderio che sta tra soggetto e oggetto: "sono romantiche le opere che riflettono la presenza del mediatore senza mai svelarla, e romanzesche quelle che invece la svelano"18.

Nella cifra del romanzesco si collocano anche alcuni progetti di fabriques non relizzate che avrebbero dovuto essere inserite nel programma decorativo immaginato dal Gambini. È il caso di un edificio neoclassico di cui si conservano i disegni, di un anfiteatro e di "un Castello, con Rocche e Torri" da costruirsi "in luogo elevato in vista, e di fronte al lago" e "che ben chiuderebbe la scena di questo" di cui si fa menzione in un Ricordo manoscritto preparato dal Gambini per una guida dell'epoca sul territorio pistoiese ${ }^{19}$.

In particolare, l'inserimento di una struttura fortificata nel parco è un tema al contempo architettonico e letterario, intimamente connesso alla fortunata stagione del racconto epico-cavalleresco e del Gothic Revival, inaugurata già quasi un secolo prima con "Il Castello di Otranto" di Horace Walpole.

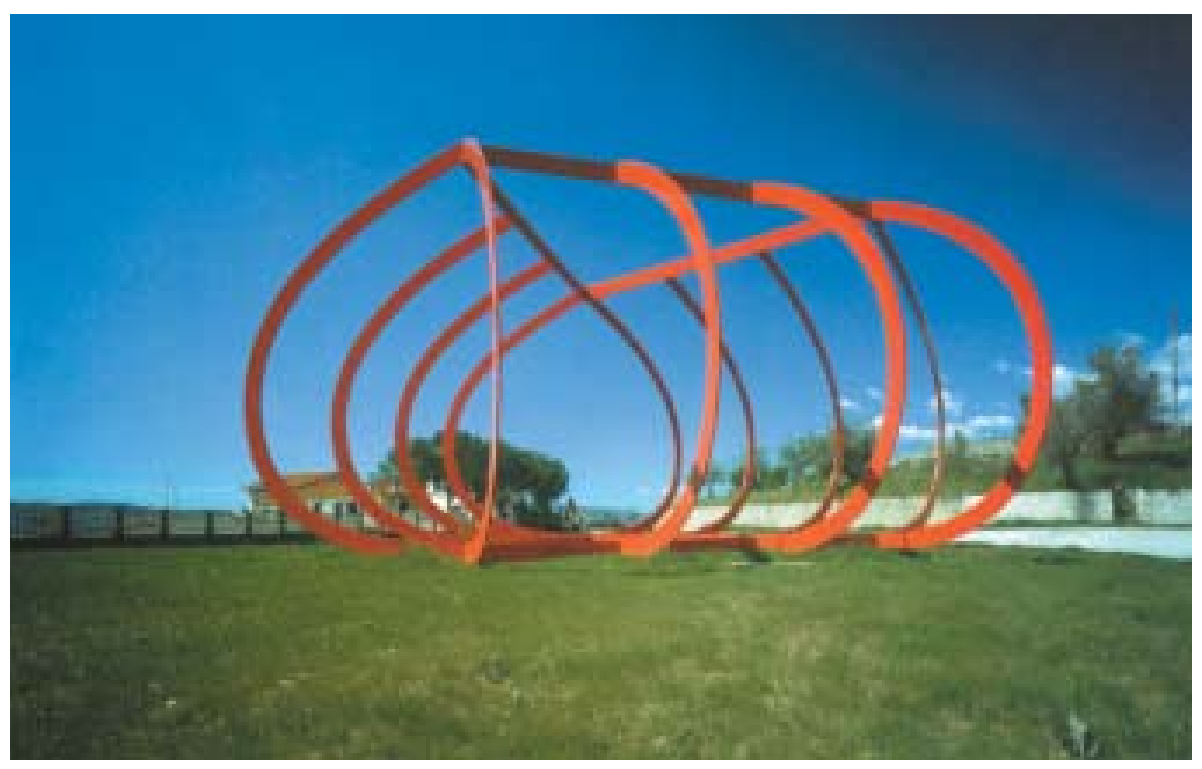

Figura 7 - Alberto Burri, Grande Ferro Celle, 1986.

${ }^{15}$ Monique Mosser, "Le architetture paradossali ovvero piccolo trattato sulle "fabriques" in MONIQUE Mosser e GeOrge Teyssot (a cura di), Architettura dei giardini di occidente, Electa, Milano 1999, pag. 259.

${ }^{16}$ MARCo CeI, op. cit., 1994, pag. 22.

17 "Il romanzesco che stimola sorpresa e meraviglia e il <<magico >> che si apparenta al sublime concorrono a quella concordia discors che è l'anima del nuovo giardino. Entrambi contribuiscono ora al nuovo stile degli impianti paesaggistici, che vengono erroneamente addebitati ad un presupposto stile romantico, già ampiamente contestato da Siegmar Gerndt, prima, e da Rosario Assunto, dopo". MASSIMO VeNTURI FERRIOLO, op. cit., 1998, pag. 71.

${ }^{18}$ Massimo Venturi Ferriolo, op. cit., 1998, pagg. 69-70.

${ }^{19}$ In MARCO CEI, op. cit., 1994, pag. 23 e pag. 36. 


\section{Dal parco ottocentesco all'origine degli "Spazi d'Arte"}

Negli anni Sessanta dell'Ottocento la tenuta, dopo alcuni passaggi di proprietà, venne acquistata dal conte Ferdinando Gatteschi, la cui moglie era dama d'onore della regina Margherita di Savoia. Fino alla fine del secolo, Celle si qualifica così come il teatro elegante delle sfarzose scene di vita dell'alta società legata al neonato Regno d'Italia.

All'inizio del Novecento, in seguito ad una bancarotta finanziaria, il Gatteschi è costretto a vendere villa, parco, fattoria e poderi ad un ricco borghese, Giuseppe Matteini, che dà avvio ad una serie di interventi di restauro dei vari fabbricati e di ampliamento del parco con la piantagione di un nuovo bosco.

La proprietà passò poi al bibliofilo ed umanista Tammaro De Marinis, che trasformò Celle in un vivace centro culturale frequentato da scrittori, artisti, e personalità varie, tra cui Ugo Ojetti, Paul Valéry, Bernard Berenson. Durante la seconda Guerra Mondiale a Celle si installò un comando militare tedesco e fu in quel periodo che il complesso subì i maggiori danni, alla cui riparazione il proprietario si dedicò appena terminato il conflitto bellico.

Alla morte del De Marinis, nel 1969, la tenuta fu di nuova messa in vendita e quindi acquistata dal collezionista Giuliano Gori, che l'anno successivo vi si trasferì con la famiglia e la sua collezione di opere d'arte.

Da questo momento, comincia a prendere forma il progetto di realizzare a Celle un vero e proprio polo artistico con valenza espositiva dedicato all'arte contemporanea, con cui poter colmare un vuoto del panorama culturale toscano di quegli anni.

\section{GLI OBIETTIVI: IL COLLEZIONISTA E LA SUA IDEA}

Non è possibile mettere a fuoco l'obiettivo di base che ha guidato la realizzazione di Spazi d'Arte, senza introdurre qualche riferimento biografico sul loro "inventore": pochi altri progetti, infatti, appaiono come questo plasmati in forma così marcata sul carattere e la personalità del loro ideatore. Celle costituisce la figura nel tappeto ${ }^{20}$ di un disegno di vita personale condotto nel segno dell'arte.

Il collezionista di origine pratese Giuliano Gori è un personaggio singolare: appassionato cultore d'arte, fin da giovane viaggia per tutto il mondo per vedere, conoscere, acquistare opere che a partire dagli anni Sessanta dello scorso secolo in poi andranno a costituire il corpus di una delle più significative raccolte private europee di arte moderna e contemporanea.

Dopo un primo interesse per la produzione delle avanguardie artistiche dei primi decenni del Novecento, Gori, anche affascinato dai contenuti delle Biennali di Venezia degli anni Sessanta e Settanta, sposta la sua attenzione sui movimenti artistici di quel periodo e avvia nuove esplorazioni negli ambiti dell'arte povera e dell'astrattismo.

L'acquisto di Celle, a cui è connessa oltre alla possibilità di poter disporre di ampi e numerosi spazi in cui sistemare le opere, quella di restituire al complesso storico nuova vita, costituisce il passaggio fondamentale che condurrà ai nuovi orientamenti dell'attività di composizione della raccolta d'arte.

Il primo nucleo della collezione trova spazio all'interno degli edifici della proprietà: nella villa dove la famiglia risiede, in una parte del fienile e nella fattoria, mentre qualche grande scultura moderna (opere di Cascella, Pomodoro, Marini) viene collocata all'esterno.

\footnotetext{
${ }^{20}$ Con riferimento al tema letterario e di critica introdotto con il romanzo omonimo di HenRY JAMES. "La figura nel tappeto, il disegno che a prima vista l'occhio non coglie ma che si rivela poi essere ciò che dà unità e senso all'opera, tenendone insieme tutte le parti, è un dispositivo di grande ricchezza." da BenedetTA Bini, "La menzogna nel tappeto" introduzione alla edizione Sellerio del 2002 de La figura nel tappeto, di HENRY JAMES (1896), pag. 17.
} 
L'incontro con il critico d'arte Amnon Barzel nel 1976, presente alla Biennale di Venezia di quell'anno come commissario del padiglione israeliano, nonché la visita alla rassegna d'arte "Documenta" di Kassel del 1977, in cui è posto in primo piano il ruolo della land art nell'ambito degli orientamenti dell'arte contemporanea, risultano episodi decisivi nel conferire allo sviluppo della collezione un nuovo carattere.

Amnon Barzel aveva realizzato qualche anno prima in Israele un museo all'aperto di cinquanta sculture, in un lembo di terra sulle rive del Giordano. Giuliano Gori prende in considerazione l'idea di creare a Celle qualcosa di simile: un percorso espositivo dove le opere siano parte integrante del paesaggio.

L'idea è affascinante e merita di essere messa in pratica: nel giro di pochi mesi alcuni artisti vengono convocati e viene creata una commissione consultiva composta da esperti ${ }^{21}$.

Alla commissione viene affidato anche il compito di definire un codice di comportamento a cui attenersi per regolare gli interventi nel parco senza danneggiarne gli elementi naturali.

E così, all'insegna della massima di Carlo Belli 'I diritti dell'arte iniziano dove terminano quelli della natura", viene dato avvio al lavoro dei primi artisti che porterà nel 1982 all'inaugurazione ufficiale del nucleo iniziale degli Spazi d'Arte22.

\section{LA METODOLOGIA: CELLE OPERA APERTA}

La particolarità e raffinatezza dell'idea che sottende al progetto di collezione d'arte ambientale di Celle ed il carattere di programma in continuo aggiornamento, rimandano, più che ad un'impostazione metodologica, alla poetica di uno speciale modus operandi, sviluppato nel tempo da Giuliano Gori in superamento di una più tradizionale concezione di raccolta d'arte.

"I concetti su cui si basano gli Spazi d'Arte sono la creazione di opere d'arte non-nomadi, in contrapposizione all'ottica del museo visto quale contenitore d'arte pre-esistente. Nel parco di Celle, il sito dell'opera diviene lo studio dell'artista e gli elementi di natura diventano i suoi materiali, i suoi contenuti e il punto di partenza" (Amnon Barzel, 1992).

L'agire in situ dell'artista determina il senso dell'opera, che "nasce dunque appositamente per un luogo preciso, vive nel tempo con esso e diviene indispensabile per la sua lettura" 23 .

Gli artisti, che Giuliano Gori individua seguendo una sua intuizione personale $\mathrm{o}$ in base all'interesse che il loro lavoro ha suscitato in lui, vengono invitati a trascorrere un soggiorno a Celle. In questo modo hanno la possibilità di scegliere e studiare lo spazio per la loro installazione, che può essere realizzata all'interno dei locali del complesso architettonico o all'aperto, e in seguito possono cominciare a lavorare.

Questa prassi, consente di incorporare nel processo di elaborazione dell'opera il valore di quel "tempo psicologico di ricezione delle cose" ${ }^{24}$ legato ad una fruizione sinestetica dello spazio che dovrà essere trasformato. Racconta, a proposito

\footnotetext{
${ }^{21}$ Faranno parte della commissione: Amnon Barzel, Renato Barilli (critico d'arte), Francesco Gurrieri (architetto), Knud Jensen (fondatore del Museo Louisiana in Danimarca), Manfred Schneckenurger (direttore per gli anni 1977 e 1987 di Documenta a Kassel).

22 All'inaugurazione degli Spazi d'Arte, il $1^{\circ}$ giugno 1982, le prime nove installazioni all'aperto erano state realizzate dai seguenti artisti: Alice Aycock, Dani Karavan, Robert Morris, Dennis Oppenheim, Anne e Patrick Poirier, Ulrich Ruckriem, Mauro Staccioli, George Trakas, Richard Serra. All'interno dei locali della Fattoria, restaurati ad hoc, trovarono invece collocazione sei nuove opere.

${ }^{23}$ Stefania Gori, "La collezione d'arte ambientale alla Fattoria di Celle", in Arte Ambientata Arte Ambientale, Centro per l'arte contemporanea Luigi Pecci, Quaderno 4, Gli Ori, Prato 2001.

${ }^{24}$ Raffaele Milani, L'arte del paesaggio, Il Mulino, Bologna 2001, pag. 74.
} 
di ciò, il Gori che: "all'inizio gli artisti arrivavano e pensavano di avere capito tutto in base alla prima impressione, invece questo parco va preso a piccole dosi, perché ci vuole tanto tempo prima di presentare il progetto" 25 .

Se il legame stretto tra installazione e luogo suggerisce, attraverso l'azione compiuta, un nuovo valore dello spazio, il rapporto che si viene a creare tra artista e collezionista è fondamentale per comprendere il senso di tutta l'operazione culturale della collezione di arte ambientale. L'artista, infatti, ha modo di sviluppare una speciale intesa prima che con il luogo, con Giuliano Gori e la sua famiglia, da cui viene ospitato durante il periodo di ideazione e realizzazione dell'opera. In questo modo, Gori ha la possibilità di seguire tutto il processo del fare artistico e di porsi come interlocutore attivo fin dalla fase di scelta del sito in cui collocare l'opera. Fa notare Pierre Restany che " $\mathrm{i}$ lavori installati all'esterno della Fattoria di Celle sono frutto di un'osmosi intellettuale e sensibile tra l'artista ed il collezionista" (Pierre Restany, 1992).

Un'osmosi creata attraverso l'elaborazione di un non sempre facile, se pur fecondo, scambio dialettico tra collezionista e artista, come evidenzia l'artista Beverly Pepper: "...il mio rapporto con Giuliano Gori, come è successo anche con alcuni artisti di Celle, è stato molto vivace e "battagliato", perché lui desidera avere un controllo, come una specie di imprinting sulle opere, ottenendo sì un grande rispetto per i luoghi, ma forse anche opere più limitate dagli artisti”26.

La vocazione di Giuliano Gori a trasformarsi da collezionista-acquirente di opere già immesse sul mercato dell'arte a collezionista-promotore e partecipante attivo alle opere studiate in un luogo per quel luogo e non commerciabili, costituisce la chiave di comprensione del filo conduttore della collezione di Spazi d'Arte, costruita, in analisi finale, prevalentemente intorno al gusto personale del suo proprietario.

Attualmente, dopo la costituzione del primo nucleo di nove installazioni all'aperto per cui Giuliano Gori si avvalse del lavoro curatoriale di Amnon Barzel, è il collezionista stesso, affermando la sua autonomia culturale da critici e orientamenti del mercato dell'arte, a curare in prima persona il programma Spazi d'Arte, avvalendosi della collaborazione della dott.ssa Miranda Mac Phail.

\section{GLI ESITI: UN PAESAGGIO PER L'ARTE CONTEMPORANEA}

Nella sua configurazione attuale la tenuta di Celle si estende per venticinque ettari recintati di superficie collinare che comprendono oltre al parco ottocentesco, caratterizzato da una rigogliosa copertura boschiva in cui è dominante la formazione del querceto ${ }^{27}$, terreni agricoli in declivio coltivati a vite ed olivo.
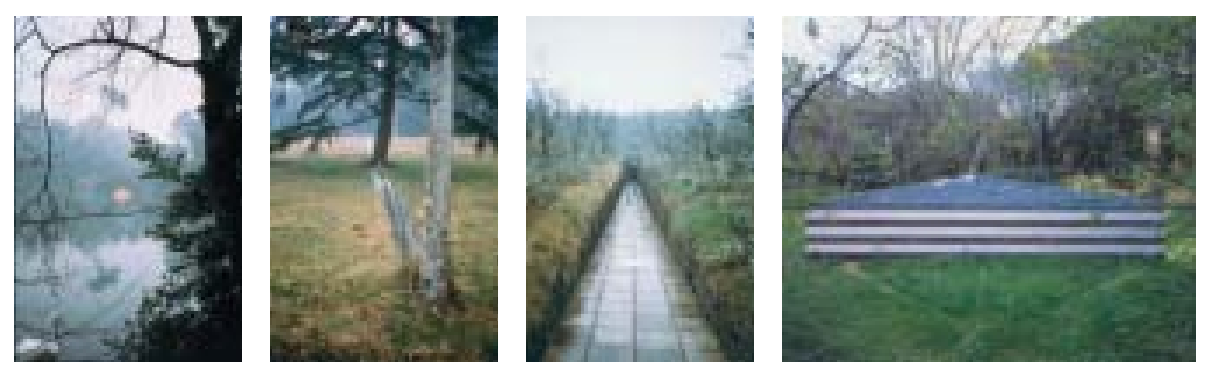

Figure 8, 9, 10,11 - Alcuni Spazi d'Arte: lavori di Marta Pan, Dani Karavan, Bukichi Inoue, Robert Morris.

25 Antonella Massa, I Parchi Museo di scultura contemporanea, Loggia dei Lanzi, Firenze 1995, pag. 37.

${ }^{26}$ MARCo CeI, op. cit., 1994.

27 "La copertura vegetale è costituita prevalentemente da cedui di querce caducifoglie, cui si consocia il 
In questo paesaggio storico, risultato di un'attività produttiva che continua ad essere esercitata attraverso i secoli e di un'attività estetica, sono distribuite le installazioni di arte ambientale, trentasette fino ad oggi, espressione di una rinnovata alleanza tra opera e ambiente.

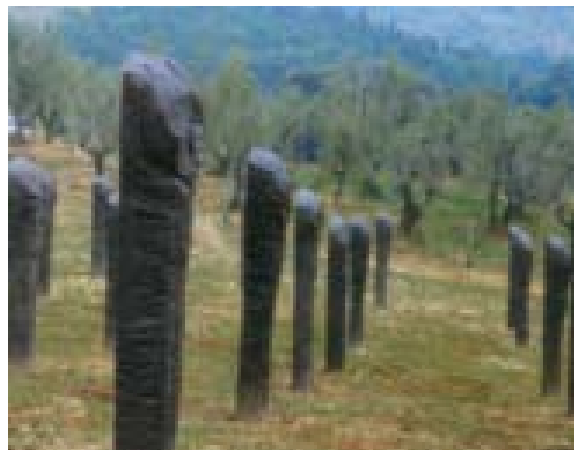

Figura 12 - Magdalena Abakanowicz, Catarsis, 1985.

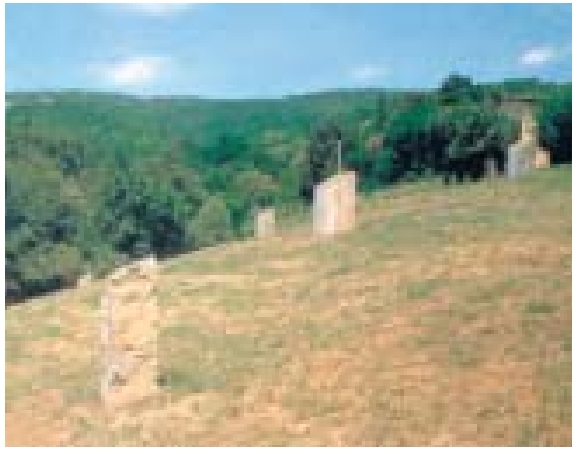

Figura 13 - Richard Serra, Open Field Vertical Elevations, 1982.

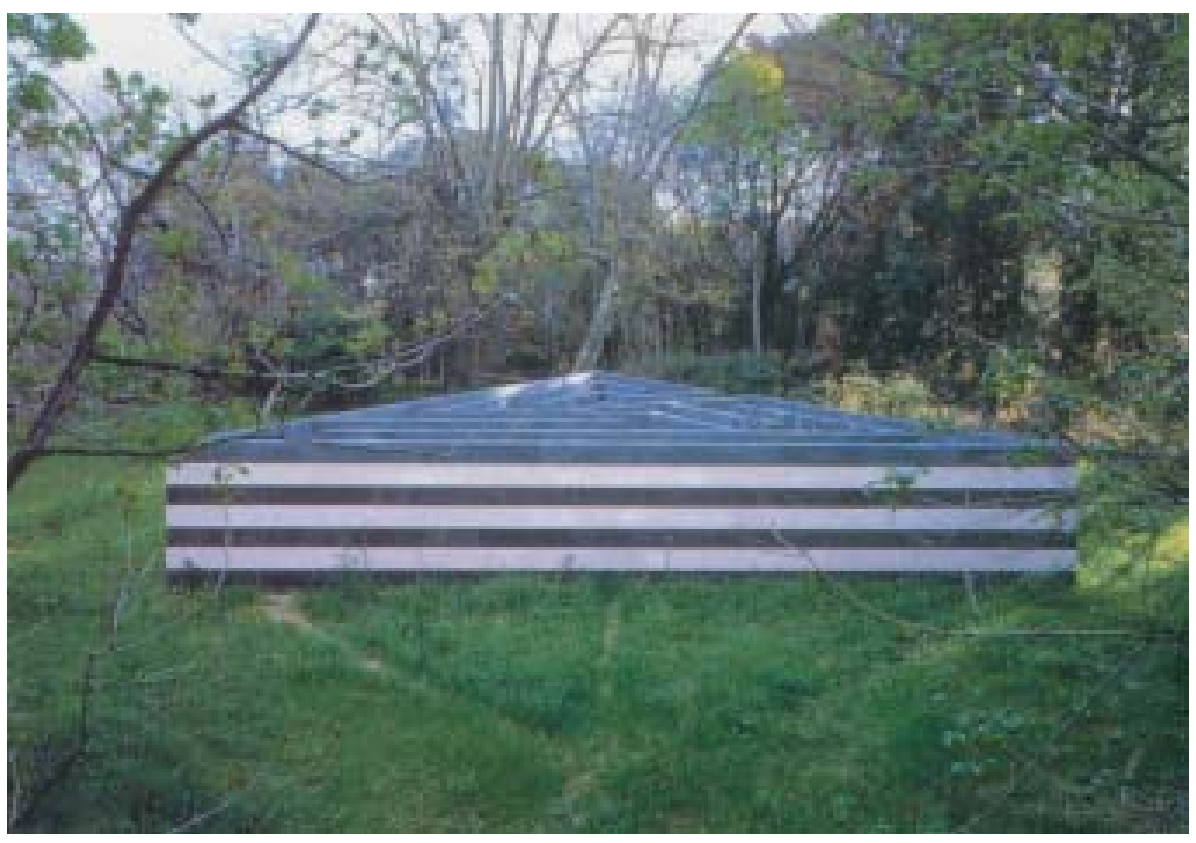

Figura 14 - Robert Morris, Labirinto, 1982.

Il percorso espositivo, che come già accennato si sviluppa anche dentro gli edifici, all'esterno si snoda prevalentemente attraverso il parco ottocentesco, ma invade anche l'area agricola dove alcuni artisti hanno preferito lavorare. Come entrando in una magica wunderkammer dai confini dilatati, ricca di mirabilia e curiosa, a Celle si è coinvolti in uno speciale viaggio di conoscenza.

È forse quella del viaggio, anche inteso come "strumento di esplorazione estetica" ${ }^{28}$, la metafora più avvincente ed immediata per comprendere l'esperienza di

pino marittimo e il cipresso. Alla formazione dominante del querceto si consociano numerose altre specie tipiche di questo piano vegetazionale (acero campestre, carpino, orniello, ginepro, castagno, pruno, corbezzolo), oppure introdotte (robinia, cedri, etc.) (...). Man mano che si guadagna quota, si nota che la latifoglia in genere prende il sopravvento sulla conifera, tanto che nella parte più alta, che coincide con la foresta dell'Acquerino, domina la faggeta pura”. Silvia MARTELli in MARCO CEI, op. cit., 1994, pag. 28.

28 "Il viaggio, qualunque sia la destinazione, reale (...) o immaginaria (...), è stato uno strumento di esplorazione estetica, per una ricerca dell'autentico e dell'inviolato, in opposizione all'immagine sfigurata della terra”, in RAFFAELE MiLANI, L'arte del paesaggio, Il Mulino, Bologna 2001, pag. 61. 


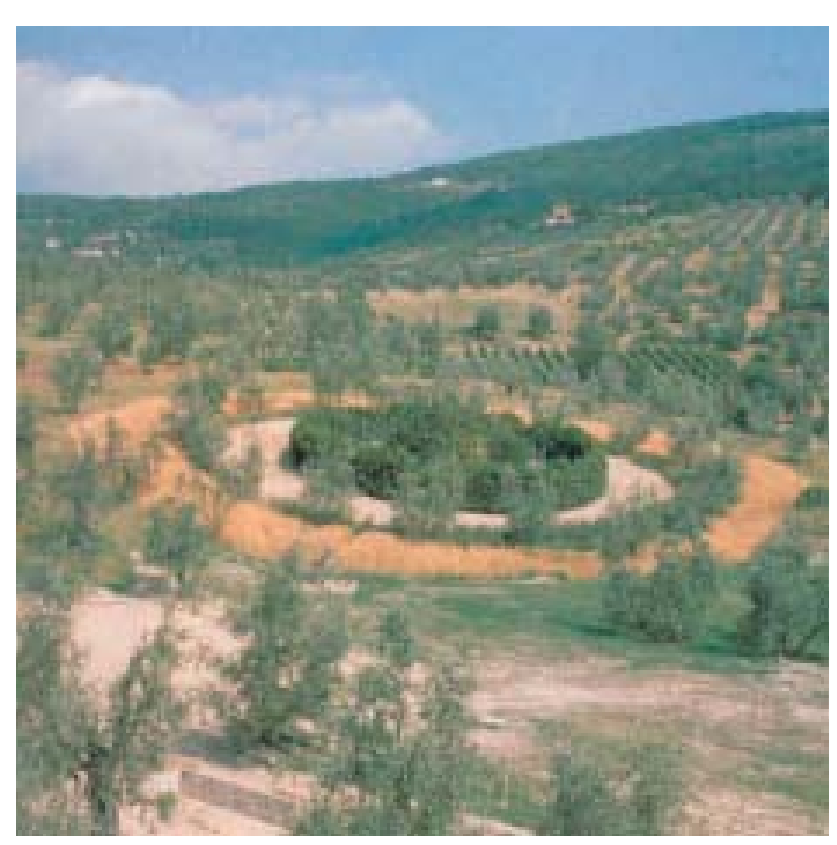

Figura 15 - Alan Sonfist, Cerchi nel tempo, 1985.

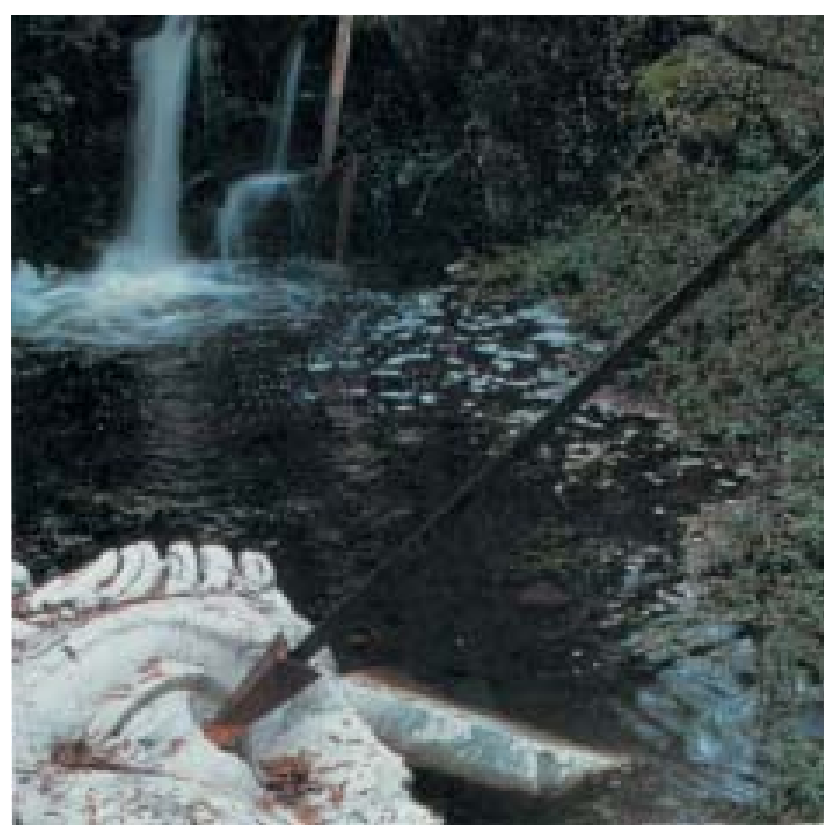

Figura 16 - Anne \& Patrick Poirier, La morte di Efialte, 1982.

Celle e per cogliere la suggestione di questo paesaggio contenitore di molte idee di paesaggio.

Il tema del viaggio reca in sé i motivi dello straniamento, del movimento nel tempo e nei luoghi e dell'orientamento, gli stessi a cui ci riconduce la lettura delle singole opere e dello sviluppo della collezione nel suo insieme. La partenza è annunciata fuori del cancello d'accesso principale alla tenuta, quindi aldilà dei suoi limiti fisici, da una grande scultura metallica verniciata di un rosso squillante. Si tratta de "Il Grande Ferro Celle" di Alberto Burri (1986), segnale visivo e di orientamento della collezione, divenuto anche simbolo degli Spazi d'Arte.

Una volta entrati all'interno della proprietà, dopo aver salito un tortuoso viale alberato ed essere arrivati in prossimità del complesso architettonico costituito da Fattoria, Villa, Limonaia e Fienile, gli effetti di un'improvvisa quanto attesa accelerazione degli stimoli della percezione saranno evidenti: "L'impressione sarà cumulativa, una soglia sarà varcata, la quantità diventerà qualità, penetreremo in un nuovo territorio. In nessun altro luogo come a Celle assisteremo a un dialogo così vario e intenso col genius loci”29.

\section{Le installazioni di arte ambientale}

A Celle sono state prodotte molte 'specie di spazi'.

Alcune delle opere realizzate fanno chiaramente riferimento alla tradizione dell'Arte dei giardini, riproponendo tòpoi e figure che da sempre hanno popolato parchi e giardini.

È il caso del Labirinto (1984-93) di Robert Morris, una costruzione triangolare sviluppata su un piano inclinato, che nell'uso dei materiali recupera la tradizione architettonica delle facciate delle chiese toscane, ma nel suo potenziale simbolico appare molto vicina alla Casa storta di Bomarzo. Uno spazio dai contorni duri e taglienti, spezzettato al suo interno per offrire un percorso obbligato stretto tra mura alte due metri e fasciate di bianco/verde.

"Il Labirinto non solo impedisce di vedere aldilà dei suoi spazi cogenti, ma disorienta il visitatore costringendolo a procedere in salita e in discesa

\footnotetext{
${ }^{29}$ KNUd W. Jensen, Il genius loci a Celle, in Arte Ambientale, Allemandi editore, Torino 1994, pag. 63.
} 
lungo i sentieri inclinati. (...) L'opera di Morris rappresenta il suo monumento al dubbio" 30 .

Anche il lavoro dell'artista americana Beverly Pepper, famosa per le sue anfisculture (opere che esprimono una sintesi di anfiteatro e scultura), si colloca in un rapporto di continuità con la tradizione dei giardini. Spazio Teatro Celle, presentato come un omaggio a Pietro Porcinai, è al contempo un'opera di land art, che modella e plasma una depressione naturale del terreno, e un luogo teatrale che, in speciali occasioni, quando Celle si anima con manifestazioni culturali di vario tipo, è in grado di accogliere trecentocinquanta spettatori.

Si tratta in questo caso di una committenza esplicita: la collocazione dell'opera tiene conto oltre che della vocazione naturale del luogo anche della volontà di Giuliano Gori di dare corpo ad un'idea concepita dal Gambini per il parco ottocentesco e che prevedeva appunto la costruzione di un anfiteatro al di sotto della Casina neogotica. Nel lavoro della Pepper lo spazio scenico si raggomitola in fondo al pendio, restando protetto da una collinetta inerbita di cui è stato asportato uno spicchio.

Iperuranio (1996) di Idetoshi Nagasawa evoca almeno due figure del giardino storico italiano, il gioco d'acqua e l'Hortus conclusus, e crea un riferimento alla cultura zen del paesaggio. L'opera è costituita da una costruzione rettangolare in marmo chiaro delle Apuane e si presenta come un recinto duro "dove quello che viene distribuito è proprio un bene prezioso: l'acqua. L'acqua disegna due cascate sotto le quali il passeggero è invitato a introdursi per accedere a questo recinto..."31.

Altri interventi invece paiono irrompere nelle scene disegnate dal progetto pittoresco per offrirne inedite letture. La Scultura flottante (1990) di Marta Pan, si muove liberamente sospinta dal vento nelle acque del grande lago in cui immette il vigore del suo arancione vibrante. "Assertivamente artificiale, quest'opera cerca di incantare piuttosto che confrontarsi direttamente, di affascinare piuttosto che provocare" 32 .

Joseph Kosuth in Modus operandi Celle ha utilizzato una lastra di vetro a doppio spessore, collocata in prossimità del tempietto costruito sull'isoletta, come elemento di frammentazione fisica dello spazio: giungendo dalla scala che dall'approdo delle barche conduce alla sommità dell' isola, risulta impossibile avvicinarsi alla fabrique. Sulla lastra di vetro, un'iscrizione che riporta un'affermazione di Nietzche pare volere fornire ulteriori spunti di riflessione "sulla grande illusione che è alla base del parco"33.

Il confronto con il genius loci ha dato origine, come si è detto, ad una pluralità e complessità di interpretazioni: ha spinto alcuni artisti ad indirizzare il loro lavoro utilizzando chiari riferimenti al mondo mitologico (Poirier, Spagnulo) o all'arte e la letteratura italiana antica (Ian Hamilton Finlay, Alice Aycock) o ancora ad accentuare i toni di contrasto nella dialettica artificiale/naturale (Dani Karavan, Sol Lewitt, Dennis Oppenheim, Mauro Staccioli, George Trakas).

Di alcune opere è possibile cogliere la connotazione mimetica, di altre l'emergente monumentalità. Di volta in volta, lo spazio viene scavato e ricavato dentro lunghi corridoi (Bukichi Inoue), suddiviso da linee decise ad unire in nuove relazioni gli elementi preesistenti ed a sottolineare inequivocabili assialità (Dani Karavan), delicatamente modellato (Richard Long) o insistentemente puntualizzato con l'introduzione di forme scolpite e ripetute seguendo una modularità più o meno serrata (Magdalena Abakanowicz, Richard Serra).

\footnotetext{
${ }^{30}$ Robert Hobbs, Ambientazione alla Fattoria di Celle, in Arte Ambientale, Collezione Gori - Fattoria di Celle, Firenze 1993, da MARCO CEI, op. cit., 1994, pag. 85.

31 Bruno CorÀ, L'esperienza dell'arte ambientale, in Arte Ambientata Arte Ambientale, Centro per l'arte contemporanea Luigi Pecci, Quaderno 4, Gli Ori, Prato 2001, pag. 8.

32 Robert HobBs, op. cit., 1994, pag. 41.

33 Robert HobBs, op. cit., 1994, pag. 41.
} 
Alcuni artisti hanno preferito lavorare fuori dai limiti del parco ottocentesco, scegliendo come referenti il paesaggio agrario e i segni della campagna toscana: Alan Sonfist, per esempio, che con Cerchi del tempo ha realizzato un'opera di forte valenza evocativa di memorie mitiche o mitologiche. Intorno ad una minuta 'foresta primordiale' l'artista ha costruito una serie di cerchi concentrici, utilizzando materiale vegetale vivente, sculture in bronzo come metafora di un impianto arboreo, pietre. Il concetto di circolarità, nelle intenzioni dell'artista, viene ribadito dal fluire dei ritmi stagionali delle attività agricole che modellano il paesaggio da cui l'opera è ospitata.

Ad indicare la complementarietà tra spazi espositivi interni e installazioni all'aperto, alcuni lavori sono scomposti in più parti, poste sia nel parco che negli edifici della Villa e della Fattoria. È il caso delle opere Enfiteusi I e II di Enrico Castellani e Cerchio di erba e Anello Verde di Prato di Richard Long. "Sebbene in questo modo tali elementi sembrano stabilire legami tra i diversi spazi, in realtà pongono in evidenza un senso di alienazione oltre che di disorientamento"34.

\section{CONCLUSIONI}

Oltre ad essere laboratorio artistico permanente e collezione, Celle è anche un polo culturale ed un centro espositivo di rilevanza internazionale, in cui vengono allestite periodicamente mostre temporanee. Due case coloniche della tenuta sono state di recente restaurate proprio per fornire locali da utilizzare a questo scopo: Casa Peppe e Cascina Terrarossa.

Nel giugno 2002 Celle, festeggiando i suoi vent'anni di attività per l'arte contemporanea, con la mostra intitolata "Magnete" 35 ha costituito la quarta tappa della rassegna d'arte in toscana "Continuità" ${ }^{6}$, con circa centocinquanta artisti rappresentati attraverso l'esposizione di opere di pittura, scultura, film e video.

Grazie all'idea di Giuliano Gori e attraverso gli interventi degli artisti contemporanei chiamati a confrontarsi con lo spirito del luogo, il gusto del Pittoresco che aveva guidato la redazione del parco ottocentesco pare oggi, ad un secolo e mezzo di distanza, continuare a svilupparsi per evolversi nella direzione del Romantico più puro, da intendersi nella connotazione che ci offre Venturi Ferriolo "...romantico è diventato ogni giardino al di là del proprio stile, oltre il giardinaggio artistico, come nel caso di Goethe, modello universale dei Romantici.

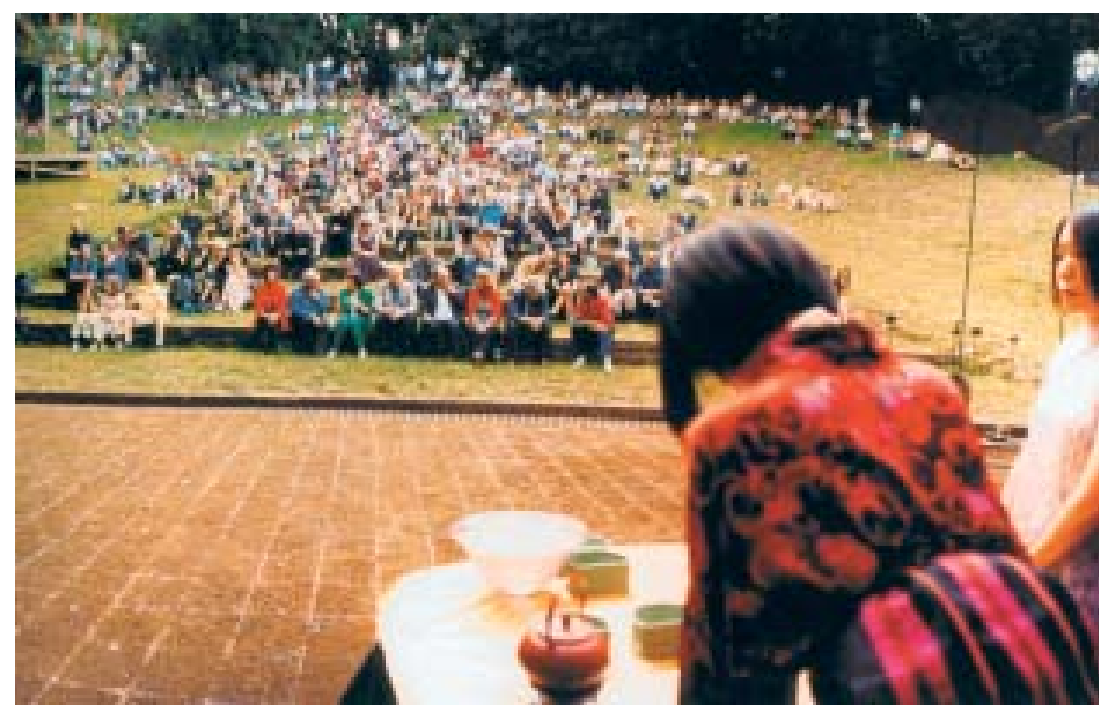

Figura 17 - Spazio teatro Celle: cerimonia del tè. Allestimento estate 1999.

\footnotetext{
${ }^{34}$ Robert HobBs, op. cit., 1994, pag. 49.

${ }^{35}$ La mostra "Magnete. Presenze artistiche straniere in Toscana nella seconda metà del XX secolo", aperta dal 3 giugno al 30 settembre 2002, è stata curata da Angela Vettese.

36 "Continuità. Arte in Toscana 1945-2000", programma di mostre proposto dallo SMAC (Sistema Metropolitano d'Arte Contemporanea), in quattro sedi diverse: Palazzo Strozzi, Firenze: "Arte in Toscana 1945-1967"; Palazzo Fabroni Arti Visive Contemporanee, Pistoia: "Arte in Toscana 1968 1989"; Centro per l'Arte Contemporanea L. Pecci, Prato "Arte in Toscana 1990-2000 e collezionismo del contemporaneo in Toscana"; Fattoria di Celle, Santomato: "Magnete. Presenze artistiche straniere in Toscana nella seconda metà del XX secolo".

${ }^{37}$ Massimo Venturi Ferriolo, op. cit., 1998, pag. 168.
} 
Possiamo parlare di desiderio profuso in un giardino o in un progetto, nel suo significato pregnante di ideazione, idea, proposito più o meno definito riguardo a qualcosa che si ha intenzione di fare o d'intraprendere come fatto soggettivo, «spirituale» di rivelazione del luogo. Romantico non è progetto di maniera, è quindi desiderio e non stile..." ${ }^{37}$. A Celle si è solidificata l'immaginazione di un collezionista appassionato.

\section{RIFERIMENTI BIBLIOGRAFICI}

Arte ambientata Arte ambientale, Quaderno 4, periodico a cura del Centro per l'arte contemporanea Luigi Pecci, Gli Ori, Prato 2001.

Cei Marco, Il Parco di Celle a Pistoia. Araba fenice del giardino, Edifir, Firenze 1994.

FAGONE VITTORIO, Arte nella natura: una nuova alleanza tra opera e ambiente, in OMODEO LAURA SALE (a cura di), Architectura \& natura, Mazzotta editore, Milano 1994.

Gori STEFAniA, La collezione d'arte ambientale alla Fattoria di Celle, in Arte Ambientata Arte Ambientale, Centro per l'arte contemporanea Luigi Pecci, Quaderno 4, Gli Ori, 2001, Prato, pagg. 28-30.

Guccione Biagio, Villa di Celle in Parchi e Giardini contemporanei. Cenni sullo specifico paesaggistico, Alinea, Firenze 2001, pagg. 100-102.

Gurrieri Francesco, Art Spaces a Celle, "Professione Architetto", 6, Alinea 1992, pagg. 17-25.

Massa Antonella, Spazi d'arte alla fattoria di Celle in I Parchi Museo di scultura contemporanea, Loggia dei Lanzi, Firenze 1995.

Mazzanti Anna, Spazi d'arte alla fattoria di Celle in Pettena Gianni, Pietrogrande Patrizia e Pozzana Mariachiara (a cura di), Giardini parchi paesaggi. L'avventura delle idee in Toscana dall'Ottocento a oggi, Le Lettere, Sesto Fiorentino 1998.

Palazzoli Daniela (a cura di), Il secondo Paradiso, Fabbri Editori, Milano 1992.

Pettena Gianni, Pietrogrande Patrizia e Pozzana Mariachiara (a cura di), Giardini parchi paesaggi. L'avventura delle idee in Toscana dall'Ottocento a oggi, Le Lettere, Sesto Fiorentino 1998.

Restany Pierre (a cura di), Arte Ambientale, Allemandi editore, Torino 1994.

Vettese Angela, Land art dal sapore pittoresco, "Il Sole 24 Ore", Domenica 24 Agosto, n. 224, pag. 27.

Siti web:

$<$ www.sculpture.org/documents/parks/p\&g/gori/gori.htm> $<$ www.po-net.prato.it/artestoria/contrmp/eng/celle.htm>

\section{RIFERIMENTI ICONOGRAFICI}

Figure 1, 2: rielaborazione di Anna Lambertini da CeI Marco, Il Parco di Celle a Pistoia. Araba fenice del giardino, Edifir, Firenze 1994, pag. 26 e pag. 21.

Figure 3, 4, 17: rielaborazione di Anna Lambertini di fotografie Carlo Fei, pubblicate in BRUNO CorÀ (a cura di), Dani Karavan, Gli Ori - Maschietto \& Musolino, Pontedera 1999, pagg.160, 165, 167.

Figura 5: rielaborazione di Anna Lambertini da CEI MARCO, op. cit., 1994, tavole a colori fuori numerazione di pagina.

Figure 6, 15, 16: rielaborazione di Anna Lambertini da PALAzzoli Daniela (a cura di), Il secondo Paradiso, Fabbri Editori, Milano 1992, pagg. 214, 215.

Figure 7, 12, 13, 14: rielaborazione di Anna Lambertini da Arte ambientata Arte ambientale, Quaderno 4, periodico a cura del Centro per l'arte contemporanea Luigi Pecci, Gli Ori, Prato 2001, pagg. 29, 8, 30 .

Figure 8, 9,10,11: fotografie di Anna Lambertini. 
SCHEDA DI PROGETTO

Nome:

Tipo di intervento:

Committente:

Luogo:

Piano curatoriale

della collezione d'arte ambientale:

Iter del progetto:
Superficie:

Spazi d'Arte a Celle

realizzazione di un parco museo d'arte ambientale all'aperto come parte di un polo espositivo d'arte moderna e contemporanea

privato (Sig. Giuliano Gori)

Celle, Santomato, Pistoia. Toscana

1981-1982: Giuliano Gori con la consulenza artistica di Amnon Barzel Commissione consultiva: Renato Barilli, Francesco Gurrieri, Knud Jensen, Manfred Scheneckenburger

Opere dal 1982 ad oggi: Giuliano Gori con la consulenza artistica di Miranda Mac Phail

25 ettari recintati

1982. Costituzione del primo nucleo della collezione esterna d'arte ambientale con esecuzione di 9 installazioni e apertura ufficiale al pubblico;

1982-1992. Gli Spazi d'Arte all'esterno comprendono 30 opere. Si amplia anche la collezione interna a cui vengono dedicati l'edificio principale della fattoria, parte del fienile e spazi all'interno della villa;

1992-2002. Sviluppo ulteriore della collezione, 37 opere all'aperto. Restauro delle case coloniche Casa Peppe e Cascina Terrarossa per essere destinate ai fini museali, con carattere espositivo temporaneo

Artisti presenti nella collezione interna (elenco non esaustivo):

In Villa: Balla, Boccioni, Braque, Calder, Chia, Magritte, Wharol, Picasso, Segal, Stella; All'ultimo piano della Villa sono ospitate opere commissionate nel 1982 di: Michelangelo Pistoletto, Luciano Fabro, Giulio Paolini, Gilberto Zorio, Giuseppe Penone, Nicola De Maria, Aldo Spoldi, Gianni Ruffi, Mimmo Paladino;

In Fattoria (restaurata nel 1985): Roberto Barni, Luciano Ori, Nunzio, Pietro Coletta, Giuseppe Chiari, Robert Morris, Andrei Roiter, Magdalena Abakanovicz;

Nel Fienile: Sol LeWitt, Richard Long, Emilo Vedova, Luigi Mainolfi, Robert Morris

Elenco degli artisti e delle opere di arte ambientale all'aperto (aggiornato al maggio 2002)

1. Alberto Burri, Grande ferro Celle, 1986, acciaio verniciato, (h. cm 525)

2. Stephen Cox, Mago, 1993, pietra

3. Roberto Barni, Servi muti, 1988, bronzo (h. cm 170)

4. Fabrizio Corneli, Meridiana, 1997, bronzo, pietra

5. Jean-Michel Folon, L'albero dai frutti d'oro, 2002, bronzo

6. Ulrich Ruckriem, Senza titolo, 1982, pietra serena (h. cm 240)

7. Robert Morris, Labirinto, 1982, serpentino, trani, cemento, (h. cm 200)

8. Alice Aycock, Le reti di Solomone, 1982, acciaio, cemento

9. Robert Morris \& Claudio Parmigiani, Melancholia II, marmo, bambù, 2002

10. Dennis Oppenheim, Formula Compound (A Combustion Chamber) (An Exorcism), 1982, acciaio

11. Dani Karavan, Cerimonia del Tè, 1999, specchi, tè verde, porcellana

12. Beverly Pepper, Spazio teatro Celle - Omaggio a Pietro Porcinai, 1992, ghisa, terra, tufo

13. Mauro Staccioli, Scultura Celle, 1982, cemento (h. cm 800)

14. Max Neuhaus, Sound Installation, 1983, frequenza audio

15. Bukichi Inoue, Il mio buco nel cielo, 1985-89, legno, pietra, cemento, vetro, acqua

16. Ian Hamilton Finlay, Il bosco Virgiliano, 1985, bronzo

15. Jaume Plensa, Gemelli, 1998, vetro

16. Alan Sonfist, Cerchi del Tempo, 1985, bronzo, vegetale, galestro (diametro m 50)

17. Frank Breidenbruch \& A.R. Penck, Centro spirituale, 1995-97, marmo, acciaio inox

18. Aiko Miyawaki, Utsurohi, 1996, acciaio inox

19. Giuseppe Spagnulo, Daphne, 1987-88, ulivo, acciaio, bronzo, (h. cm 570) 
20. Dani Karavan, Linea I-II-III + IV, 1982, cemento bianco

21. Michel Gerard, Cellsmic, 1992, bronzo, alluminio, vetro

22. Richard Serra, Open Field Vertical Elevations, 1982, Colombino di Firenzuola

23. Robert Morris, I caduti ed i salvati, 2000, resina

24. Hidetoshi Nagasawa, Iperuranio, 1996, marmo, acqua

25. Marta Pan, Scultura flottante Celle, 1990, alluminio verniciato

26. Joseph Kosuth, Modus Operandi Celle, 1987, vetro (h. cm 200)

27. Olavi Lanu, Le tre pietre, 1985, resina

28. Anne \& Patrick Poirier, La morte di Efialte, 1982, bronzo, marmo

29. George Trakas, Il sentiero dell'amore, 1982, legno, acciaio, terracotta

30. Sol LeWitt, Cubo senza cubo,1986-88, cemento, m. 5 x 5

31. Susana Solano, Acotacion, 1990, corten

32. Richard Long, Cerchio di erba, 1985, erba

33. Fabrizio Corneli, Grande estruso, 1987-88, ferro zincato

34. Magdalena Abakanowicz, Katarsis, 1985, bronzo

35. Fausto Melotti, Tema e variazioni II, 1981, acciaio inox, (h. cm 600)

36. Enrico Castellani, Enfiteusi II, 1987, acciaio corten e inox

37. Menashe Kadishman, Luce del mattino (pecore e pecore), 1993-94, granito, ferro

Elenco degli artisti di cui sono state allestite installazioni o mostre temporanee:

Stefano Arienti, Hossein Golba, Robert Morris, Luciano Ori, Claudio Parmeggiani, Beverly Pepper, Jaume Pensa, Costas Tsoclis 


\title{
Il GIardino del Tarocchi di NIKI de SaInt Phalle A GARAVICCHIO, CAPALBIO
}

\author{
Emanuela Morelli
}

\section{IL CONTESTO}

Percorrendo l'Aurelia, in prossimità del confine tra la Toscana ed il Lazio, improvvisamente appaiono un gruppo di statue giganti e scintillanti, poste su una delle prime colline coltivate prevalentemente ad olivo e che discendono verso la pianura e al mar Tirreno, che svettano tra la macchia mediterranea ed il paesaggio brullo tipico della Maremma. Mentre ci si avvicina la composizione sparisce dalla nostra vista come se fosse stata un'apparizione e, attraversando i campi di olivi, ciò che ci troviamo davanti è solo un severo muro realizzato in tufo, tipica pietra locale, con un grande foro circolare e centrale che funge da porta di ingresso. Oltrepassata questa linea di demarcazione e abbandonato quindi simbolicamente ogni aspetto della vita quotidiana, veniamo avvolti dal mondo fantastico del Giardino dei Tarocchi di Niki de Saint Phalle.

Nata a Parigi (Neully-sur-Seine) nel 1930, da padre francese e madre americana, Niki de Saint Phalle ha realizzato molte opere famose, tra cui insieme a Jean Tinguely, la Fontana Stravinsky nel 1983, nella piazzetta a lato del Centre Pompidou di Parigi ${ }^{1}$.

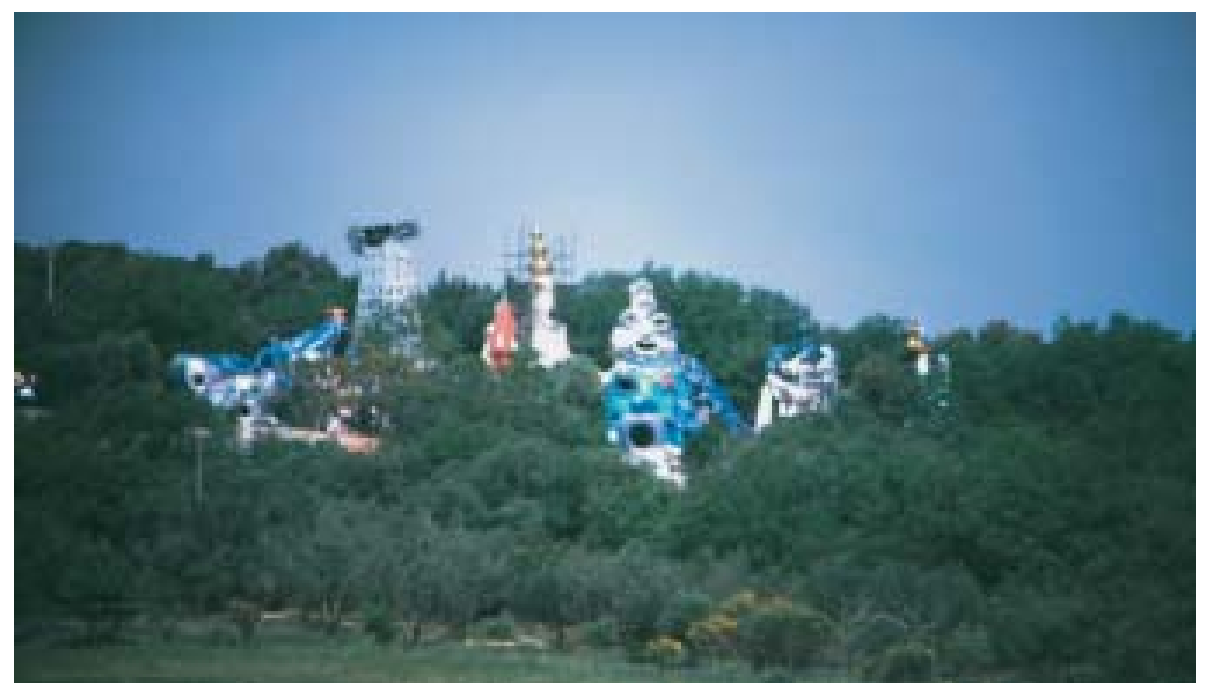

Figura 1 - Vista dalla via Aurelia del complesso delle statue del Giardino dei Tarocchi.

\footnotetext{
${ }^{1}$ Negli anni Cinquanta, mentre lavora come mannequin e cover girl per riviste di moda francesi ed americane, inizia i suoi primi esperimenti e studi sulla pittura. Durante il 1952 viaggia con suo marito attraverso la Francia, la Spagna e l'Italia, e rimane profondamente impressionata dalle cattedrali, definendole simboli di un'idea collettiva ed imponenti opere architettoniche realizzate grazie alla forza di molte persone. Nel 1954 torna in Spagna e scopre Antoni Gaudí, in particolare il Parco Guell, realizzato tra il 1900 ed il 1920 con l'uso di diversi materiali. Da questa esperienza nasce in lei l'esigenza di realizzare un giorno il proprio giardino, luogo di gioia per bambini e per adulti, in cui si possa vivere insieme arte e natura. Dopo una grave crisi depressiva, torna a Parigi ed incontra Jean Tinguely, con cui percorrerà professionalmente e sentimentalmente gran parte della sua vita.
} 
L'effetto contrastante generato tra l'imponente complesso delle sculture del Giardino dei Tarocchi e il carattere brullo del paesaggio maremmano aveva suscitato inizialmente un impatto negativo sugli abitanti del luogo. Questi difatti sulle prime reagirono in modo estremo, cercando di impedire la realizzazione dell'opera stessa. A causa di questo problema il giardino subì frequenti interruzioni e rallentamenti. Ma per Niki de Saint Phalle non si trattava di nascondere o mitigare l'atto umano, in questo caso artistico, con l'ambiente circostante, ma di integrare l'uno all'altro, impostando un vivace rapporto dialettico con il paesaggio.

Le sculture quindi dovevano generare una sorta di contrasto "amichevole" tra le forme dell'arte e della natura: "scegliendo di rivestirle di un mosaico di specchi nei quali la natura si riflette e si infrange in mille punti di vista entrando e invadendo le forme scultoree, in un eccitante processo, come lo chiamò Tinguely, di vis-a visification"2.

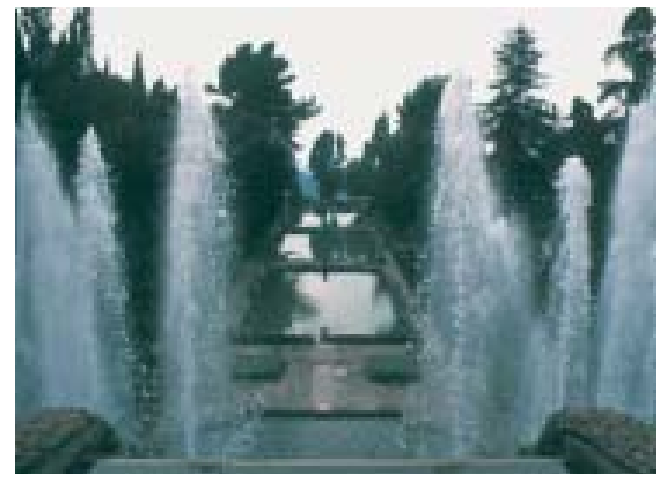

Figura 2 - Il Giardino di Villa d'Este a Tivoli.

Grazie alla tenacità dell'artista il Giardino dei Tarocchi è divenuto nel tempo uno degli esempi più significativi, apprezzati e famosi, in cui arte, natura e cultura dialogano in maniera attiva. Questo riconoscimento è avvenuto in seguito anche nella stessa popolazione locale, per la quale adesso l'opera rappresenta, anziché ostilità, motivo di orgoglio e di identità.

Nella realizzazione del giardino di Niki de Saint Phalle sono evidenti soluzioni estetiche e simboliche che rimandano alla storia del giardino italiano. Grande influenza, per sua stessa ammissione, hanno avuto i giochi sfarzosi d'acqua del giardino di Villa d'Este a Tivoli $^{3}$ e l'atmosfera misteriosa che avvolge le giganti sculture dei mostri del Sacro Bosco di Bomarzo ${ }^{4}$, entrambi distanti pochi chilometri da Garavicchio.

In questi anni, in cui la sua arte diviene una terapia per liberarsi dai "propri demoni" e per ricucire le "fratture con il mondo degli uomini" (PierRe Restany, in AnNa MAZZAnTi (a cura di), Niki de Saint Phalle - Il Giardino dei Tarocchi, edizioni Charta, Milano 1997, pag. 30) entra a far parte della corrente dei Noveau réalisme (unica figura femminile), realizza i famosi Tiri, opere pittoriche nate dall'esplosione di palloncini riempiti di colore causata dagli spari di una carabina, e la rappresentazione delle sue prime figure (grottesche) femminili, dalle cui ferite fuoriescono vari oggetti. Sono gli anni in cui, grazie alla forza distruttrice delle sue opere, si svincola dalla realtà quotidiana mettendo a fuoco la propria immaginazione.

Tra il 1965 ed il 1966, recuperata la propria personalità e femminilità come fonte generatrice, ispirata anche dalla gravidanza della sua amica Clarissa Rivers, comincia ad elaborare la Nanas, una figura gigante femminile, assimilabile alla "dea-madre" dotata di grandi forme rotondeggianti e colorate, (la Hon-en Katedral del 1966, per il Museo di Stoccolma, in posizione di partoriente, percorribile al suo interno, lunga ventotto metri e alta sette, e Le paradise fantastique, per il Padiglione Francese all'Expo del 1967 a Montréal, dove avviene il salto dall'atto progettuale plastico della scultura all'atto plastico ambientale).

Seguono una serie di realizzazioni importanti, quasi tutte in collaborazione con Jean Tinguely, tra cui: La testa del Ciclope (monumento nazionale) a Milly-La Fôret (Francia) nel 1969, Il Golem, un mostro di grandi dimensioni con tre lingue che diventano scivoli per i bambini nel parco-giochi Rabinovich di Gerusalemme nel 1972, il Dragone per bambini in un giardino privato a Knokke (Belgio) nel 1973, la Fontana Stravinsky per la piazza del Centre Pompidou, a Parigi, nel 1983, la fontana di Chateau-Chinon, paese di origine del presidente francese Mitterand nel 1988, il Giardino dei Tarocchi a Garavicchio (Italia) dal 1980 al 1997.

Nel mese di maggio del 2002, Niki de Saint Phalle muore in California, dopo una lunga malattia che le ha colpito i polmoni, "cosciente di aver lasciato un segno nella storia dell'arte dell'ultimo secolo, ma non soltanto di quello" (Gianluigi Denega, La scomparsa della de Saint Phalle, "La Repubblica", 23 maggio 2002).

2 Anna Mazzanti, Il Giardino dei Tarocchi, in Gianni Pettena, Patrizia Pietrogrande e Mariachiara Pozzana (a cura di), Giardini, Parchi, Paesaggi. L'avventura delle idee in Toscana dall'Ottocento a oggi, Le Lettere, Firenze 1998.

${ }^{3}$ Il giardino di Villa d'Este è stato realizzato da Pirro Ligorio nel 1550, nella "Valle Gaudente" del fiume Aniene. È uno dei giardini più spettacolari del Rinascimento, per i suoi innumerevoli ed esuberanti giochi d'acqua. La chiave allegorica "è il mezzo di lettura di tutto il percorso che il Ligorio disegna nel verde soggiogando il visitatore" (AlESSANDro TAGLIOlini, Storia del giardino italiano, La Casa Usher, Firenze 1988, pagg. 196-197), in cui si contrappongono i temi della virtù e del vizio.

${ }^{4}$ Per il Sacro Bosco di Bomarzo si rimanda alla nota n. 4 della scheda sul Giardino Hic Terminus Haeret di Daniel Spoerri. 
Sono opere storiche che hanno affascinato ed ispirato l'artista, la quale ha rielaborato e riproposto le stesse soluzioni estetiche e simboliche nel proprio giardino. I riferimenti al Giardino del conte Vicino Orsini non si esplicano solo nelle proporzioni giganti delle sculture realizzate in pietra tufacea ed un tempo dipinte e nell'effetto sorpresa che le stesse producono creando un ambiente magico e stregato che può essere in alcuni momenti di smarrimento e di instabilità, ma anche nelle scritte che durante il percorso accompagnano e guidano il visitatore, come pure nel tentativo di creare un luogo che fuoriesca dall'ambito culturale urbano.

In entrambe le opere difatti vi è il tentativo di fuggire dalla città e la sua negazione come istituzione: Niki cerca un luogo lontano per la realizzazione del suo giardino, possibilmente in Africa o in America Latina, "l'Orsini ricerca nel luogo, attraverso i miti e le leggende, l'etica della propria esistenza nel clima rurale $(. .$.$) in questa sua fuga il legame con la cultura è determinato dai libri che$ parlano di paesi sconosciuti, dell'ambiente esotico, che alimentano la sua evasione della realtà o meglio comprovano con la realtà le sue visioni fantastiche" 5 . Salta quindi la rappresentazione convenzionale degli oggetti e i riferimenti tra una rappresentazione e l'altra: gli esseri viventi sono più grandi degli edifici, e questi ultimi, come La casa inclinata del Bosco di Bomarzo, hanno equilibrio precario o si spezzano come nella Torre di Babele di Niki de Saint Phalle.

\section{GLI OBIETTIVI}

"Nel 1955 andai a Barcellona e vidi per la prima volta il meraviglioso Parco Guell di Gaudí. Capii che mi ero imbattuta nel mio maestro e nel mio destino. Tremavo in tutto il corpo. Sapevo che anche io, un giorno, avrei costruito il mio Giardino della Gioia. Un piccolo angolo di Paradiso. Un luogo di incontro tra l'uomo e la natura. Ventiquattro anni dopo mi sarei imbarcata nella più grande opera della mia vita: il Giardino dei Tarocchi. (...) Come in tutte le fiabe, lungo il cammino alla ricerca del tesoro mi sono imbattuta in draghi, streghe, maghi e nell'Angelo della temperanza" ${ }^{6}$.

Durante un periodo di convalescenza in Svizzera, nel 1974, Niki de Saint Phalle, ritrova Marella Caracciolo Agnelli, sua amica e fotografa già negli anni Cinquanta a New York, quando era mannequin. A lei confida di aver fatto proprio in quei giorni un sogno in cui costruiva grandi sculture per un giardino, legate al tema dei Tarocchi, e di sentire l'esigenza di realizzarlo. Nasce quindi la volontà di realizzare un giardino dedicato ai 22 Arcani Maggiori delle carte dei Tarocchi (sono ventidue difatti le sculture che lo compongono), simboli universali che si trovano con disegni simili tra loro in tutte le parti del mondo, dalla Francia, all'Egitto, all'India: "Se la vita è un gioco di carte, noi siamo nati senza conoscere il nostro ruolo, e tuttavia dobbiamo giocare la nostra mano. Durante i secoli l'uomo ha amato giocare con i Tarocchi. Poeti, filosofi, alchimisti, artisti si sono votati alla scoperta dei loro significati" .

"I tarocchi sono solo un gioco o indicano una filosofia di vita? (...) Questi simboli delle carte sono qui per insegnarci qualcosa in più

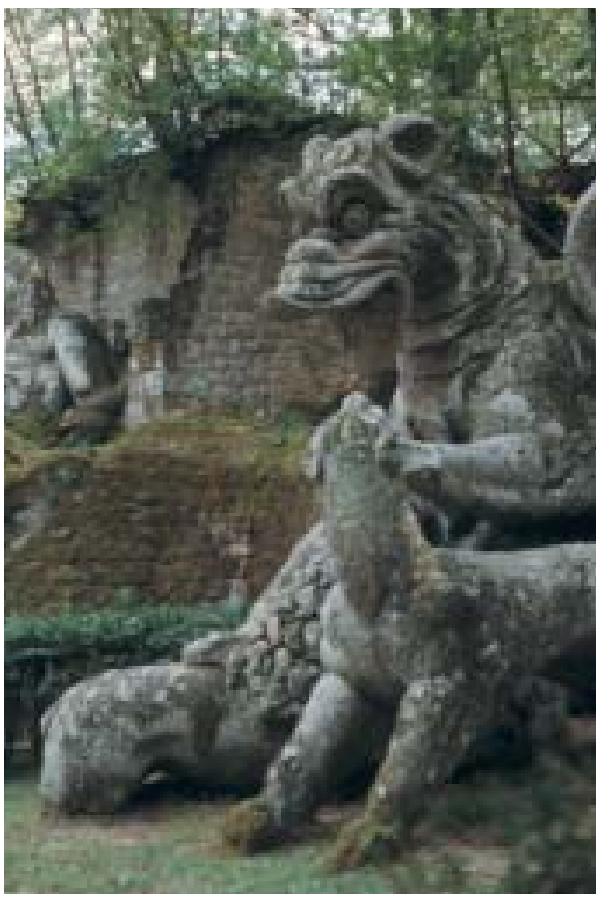

Figura 3 - Il Drago del Sacro Bosco di Bomarzo.

\footnotetext{
${ }^{5}$ Alessandro Tagliolini, Storia del giardino italiano, La Casa Usher, Firenze 1988, pag. 209.

${ }^{6}$ Niki de Saint Phalle, Il Giardino dei Tarocchi, Edizione Benteli, Berna 1997.

${ }^{7}$ Niki de Saint Phalle in Franco Zagari, Architettura del giardino contemporaneo, Mondadori, Milano 1988, pag. 52.
} 
riguardo al nostro avventuroso e meraviglioso viaggio che è questa vita" ${ }^{8}$. Inizialmente, come il conte Vicino Orsini, l'artista aveva immaginato tale giardino in un luogo distante dalle aree fortemente urbanizzate e industrializzate in quanto doveva presentare ancora molti caratteri di natura incontaminata e selvatica, oltre ad appartenere a popoli in cui le tradizioni e gli antichi usi artigianali erano ancora vivi. Aveva quindi idealmente pensato a posti come l'Africa e l'America Latina, che oltre a garantire quiete e concentrazione, ispiravano atmosfere magiche. Ma Carlo e Nicola Caracciolo, fratelli di Marella, le offrono nel frattempo, per la costruzione del giardino, una porzione di terreno, più precisamente una ex-cava, della loro proprietà toscana in Maremma, terra dove un tempo avevano vissuto gli Etruschi, selvatica e al tempo stesso ricca di storia e di tradizioni. Niki rimane entusiasta del luogo e accetta di edificare lì l'opera più importante della sua vita come nel 1962 l'oroscopo le aveva annunciato'.

\section{LA METODOLOGIA}

Il giardino viene costruito sui dislivelli originati dalla precedente cava, in un'area collinare caratterizzata dal paesaggio di olivi, sughere e di macchia mediterranea.

Tra il 1979 ed il 1980 viene effettuata una prima "pulitura del terreno" e realizzato il primo modello delle sculture che andranno a formare il Giardino di Garavicchio, mentre nel decennio successivo, tra il 1980 ed 1990, la scultrice impiegherà gran parte del suo tempo per la realizzazione dell'opera, facendo esperimenti e inventando soluzioni ai vari problemi, sia di ordine economico che tecnico, in cui si imbatterà.

L'organizzazione del giardino ci ricorda come impianto una cittadella, ed il luogo baricentrico della composizione si individua nella piazza centrale ubicata nell'anfiteatro creato dalla ex-cava, dove si trova il complesso Mago, Sacerdotessa,

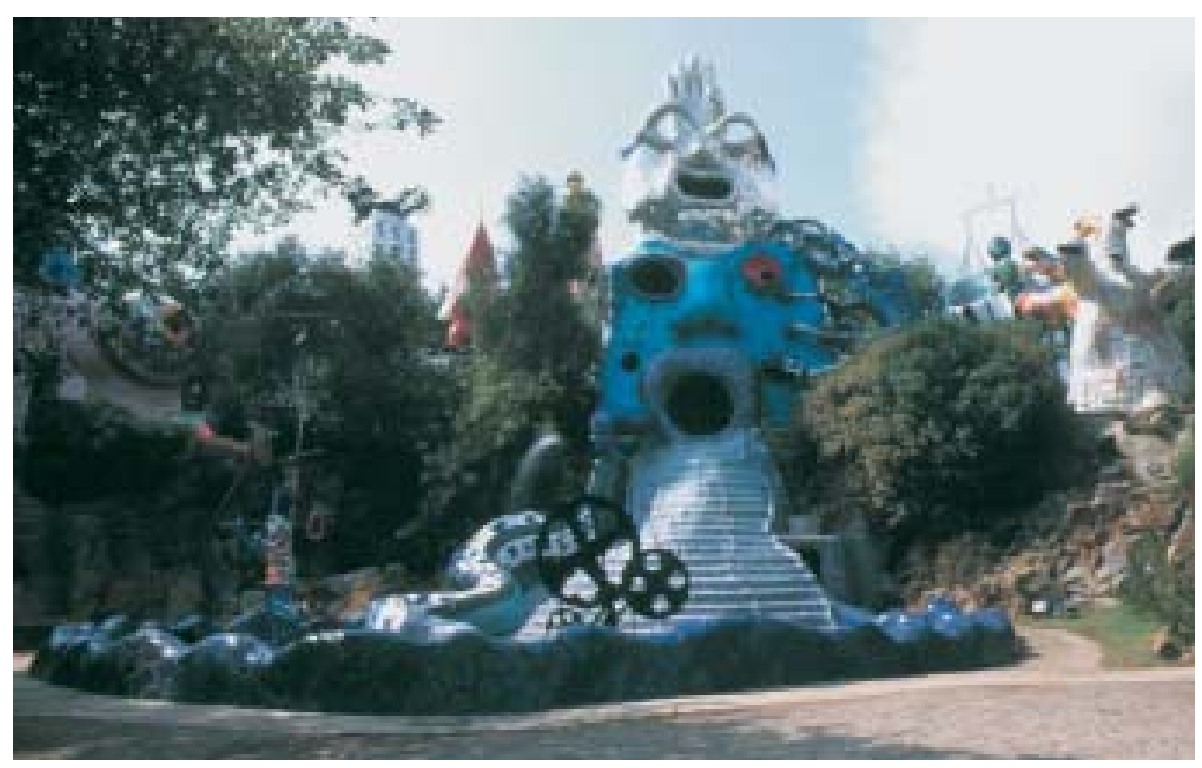

Figura 4 - Il nucleo centrale del giardino composto dalle sculture Il Mago-La Sacerdotessa attorno alla vasca d'acqua ed al centro la Ruota della Fortuna.

\footnotetext{
${ }^{8}$ Niki de Saint Phalle in Anna Mazzanti (a cura di), Niki de Saint Phalle, edizioni Charta, Milano 1990, Catalogo.

9 "Nel 1962 il mio oroscopo già aveva annunciato che avrei realizzato qui in Italia l'opera della mia vita”, Niki de Saint Phalle in Anna MazZanti (a cura di), op. cit, Milano 1990.
} 
Ruota della Fortuna disposto attorno ad una vasca d'acqua, e a cui la Sfinge-Imperatrice sembra fare la guardia.

All'interno di quest'ultima scultura, che ha paragonato alla "grande madre", Niki de Saint Phalle ha vissuto durante la realizzazione del giardino, con la camera da letto disposta in un seno, e la cucina nell'altro. Posizionata sull'orlo dell'anfiteatro roccioso, questa enorme ed imponente figura femminile si affaccia nella piazza centrale con la vista proiettata verso la pianura ed il mare, e sembra quasi assumere il ruolo di guardiano e custode del luogo.

Da questo nucleo poi partono una serie di vialetti e di piccole piazzette. Gli ambienti sono delimitati dalle pareti colorate e luminose delle sculture, pari ad edifici, e dalla presenza di alberi, per lo più olivi e querce come nel Castello, alternando luoghi "pubblici" a quelli più intimi.

All'interno del giardino non sono volutamente previste visite guidate per rispettare sia l'aspetto esoterico, sia la libertà delle sensazioni e delle percezioni che il luogo suscita in ogni visitatore. Difatti non esiste una sequenza 'ufficiale' da percorrere e le sculture sono disposte istintivamente nell'ambiente, senza nessuna logica predeterminata, così come la successione delle carte nel gioco dei Tarocchi. Ognuno qui è libero di dirigersi su vialetti e scale, a seconda del proprio volere, intuito e istinto, disegnando un percorso dato dalla propria immaginazione.

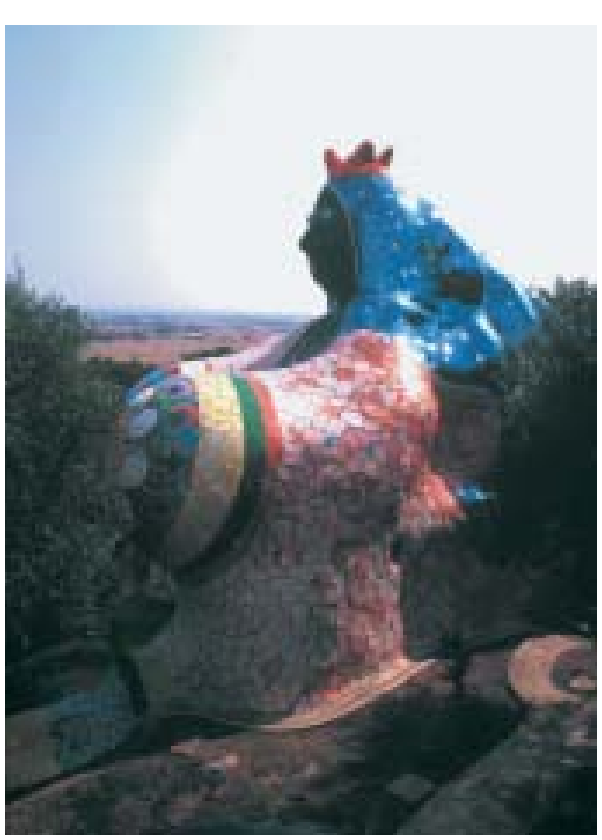

Figura 5 - la Sfinge e sullo sfondo il paesaggio della costa maremmana.

Le sculture sono realizzate con un'armatura di tondini di metallo lavorato a mano e saldato, su cui è stato spruzzato del cemento, rivestito poi, come una pelle, da una miriade di tessere colorate in ceramica, poliestere, vetro e specchi.

Alcune delle opere sono visitabili al suo interno, così come la già citata Imperatrice dove Niki ha appunto vissuto durante gli anni di costruzione del giardino, la Giustizia, la testa del Mago, la Cappella.

Gli Skinny, riferibili ad un più tardo periodo dell'evoluzione dell'artista, sono sculture filiformi, che assumono l'aspetto della linea di definizione della figura della carta: sono figure ariose in cui nella loro trasparenza si può osservare la natura che le avvolge.

Nella fase di cantiere del progetto ed in particolare durante la costruzione delle grandi sculture, la vegetazione presente è stata salvaguardata e protetta. In molti casi le piante sono state coperte da teli bianchi durante la costruzione delle grandi sculture, in modo tale che gli spruzzi di cemento non ricadessero su di esse. Altri cespugli di piante aromatiche, tipiche della macchia mediterranea quali il timo, il lentisco, il rosmarino e la lavanda, sono stati piantati nel giardino per aumentare le sensazioni olfattive. A queste poi si associano esperienze sonore, che vanno dal fruscio del vento tra le foglie, al suono delle cicale nei mesi estivi, al 'ruscellare' dell'acqua, ai cigolii dei congegni macchinosi e ferrosi di Jean Tinguely. La composizione organica del giardino di conseguenza influisce su tutto l'apparato sensoriale del visitatore, avvolgendolo ed inondandolo di magia e di sensazioni.

Niki de Saint Phalle è stata in realtà molto attenta al rapporto che la sua opera instaurava di volta in volta, con il paesaggio che la ospitava. Nel caso di alcune figure giganti, come il Mago e la Torre di Babele, per la loro troppo imponenza, durante la fase di realizzazione, sono state ridotte le dimensioni previste nel modellino affinché si potesse trovare una più armoniosa integrazione con l'ambiente circostante. Sono proprio le sculture più emergenti nel paesaggio (la mano del Mago e la Torre di Babele) ad essere rivestite di specchi, oltre che per il loro valore simbolico, per mitigare l'eccessiva esuberanza delle loro dimensioni, affinché avvenisse una sorta di smaterializzazione della forma.

Se confrontiamo il diverso uso della vegetazione tra il Sacro Bosco di Bomarzo e il Giardino di Garavicchio, possiamo osservare che in Bomarzo la 


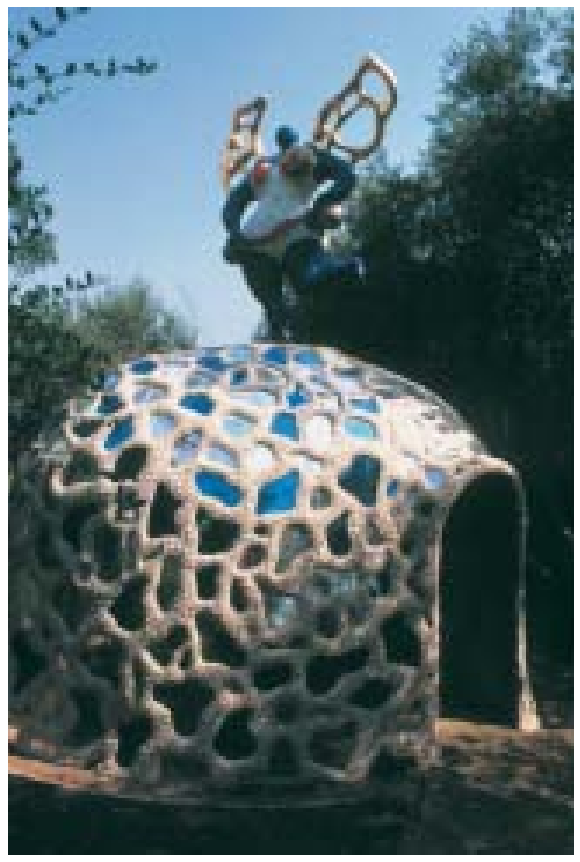

Figura 6 - La Cappella sormontata da un angelo.

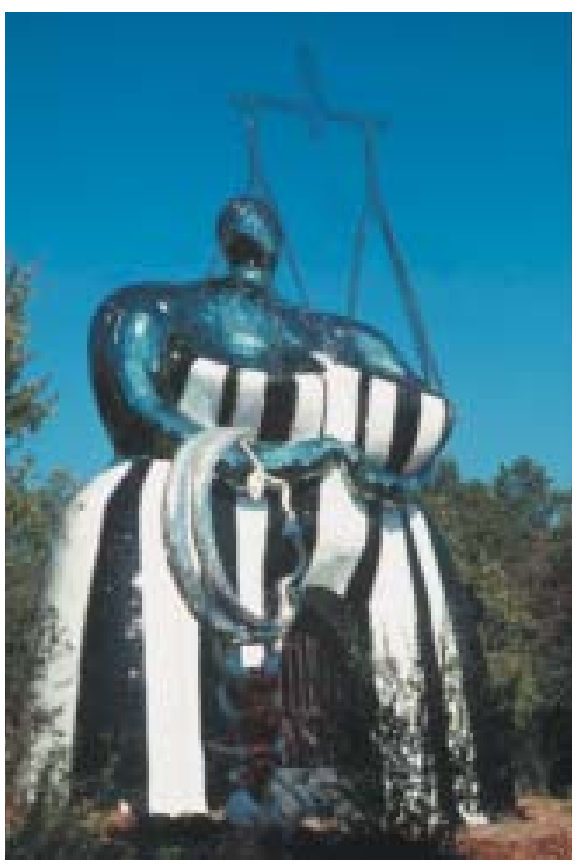

Figura 7 - La Giustizia.

natura, e sopratutto il bosco decisamente più ombroso ed umido, induce un maggior senso di mistero e di inquietudine con riferimenti tipici del periodo manierista, in cui si perde la "visione antropocentrica, rassicurante e razionale, che aveva dominato il periodo rinascimentale" ${ }^{10}$. Nel Giardino dei Tarocchi la vegetazione prende invece una gioiosa vitalità che si esprime sui caratteri della flora mediterranea profumata e disordinata.

Ma il parco di riferimento primigenio (da cui è partita la prima idea costruttrice) è il parco Guell a Barcellona di Gaudí. In questo parco giocano molti fattori che ricorrono poi nel giardino di Garavicchio: l'esuberanza dei colori come espressione artistica e simbolica, l'abolizione della linea retta come elemento di espressione e l'uso di forme curve e arcuate, più capaci di rendere libera l'espressione artistica e di maggior imprevedibilità: un meccanismo di energie, sensazioni, magia, tra simboli, memorie primitive e gioco.

Nel 1991 muore improvvisamente Jean Tinguely. Durante la costruzione del Museo a lui dedicato a Basilea, Niki de Saint Phalle conosce l'architetto Mario Botta con cui instaura un rapporto di amicizia e di collaborazione (lavoreranno assieme per la realizzazione dell'Arca di Noè del 1996, un'altra installazione monumentale per i bambini a Gerusalemme). Tra il 1996 ed il 1997 Niki chiede all'architetto di costruire un ingresso per il suo giardino a Garavicchio.

Botta progetta una lunga linea di demarcazione, più propriamente un muro in pietra locale, il tufo, con un'unica apertura centrale circolare, che funge da confine tra il mondo esterno ed il mondo interno.

È un filtro, luogo di transito, per la decantazione e la purificazione del visitatore dalle inquietudini quotidiane. Oltrepassato questo limite egli può addentrarsi nel luogo magico ed onirico del giardino.

L'entrata, essenziale nel disegno, che riporta il colore del paesaggio locale, doveva essere espressamente in contrasto con il lavoro della scultrice, ricco dal punto di vista cromatico, nella varietà dei materiali utilizzati, dotato di grandi forme tondeggianti: "Mario ha concepito una struttura molto maschile: un

${ }^{10}$ Antonella Massa, Itinerari italiani - I parchi museo di scultura contemporanei, Loggia de' Lanzi, 1995, pag. 74. 


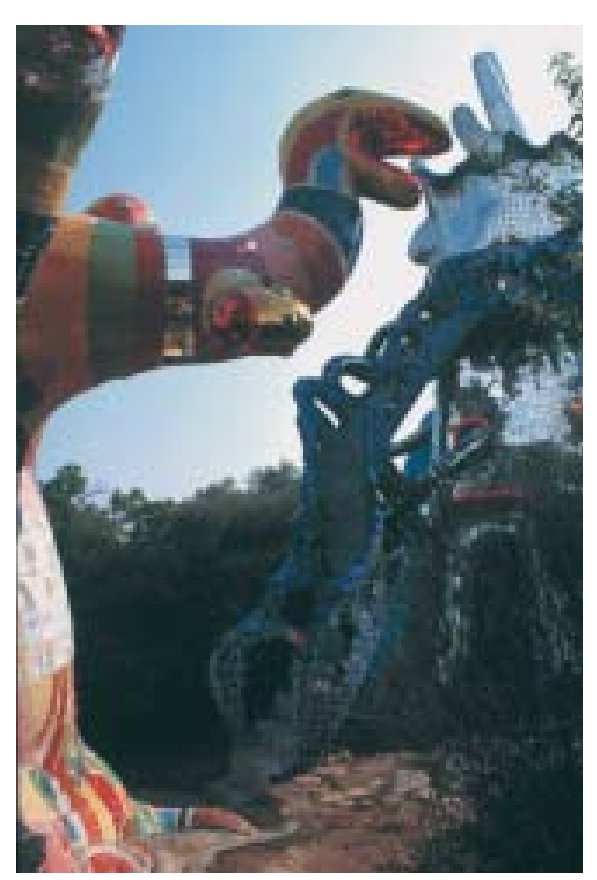

Figura 8 - Particolare del rivestimento delle sculture.

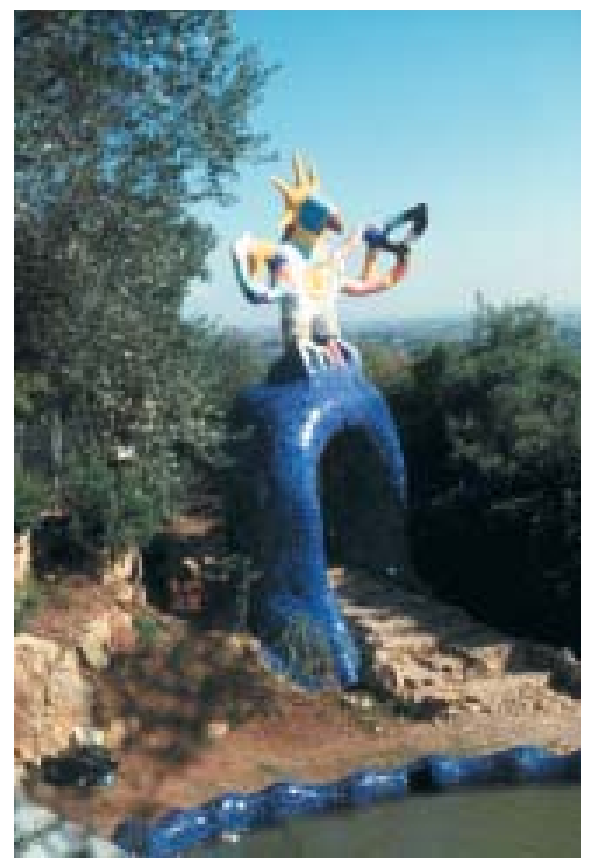

Figura 9 - Prove di colore sul Sole.

muro di pietra locale simile ad una fortezza che marca chiaramente la separazione tra il mondo esterno e quello interno. Il muro simboleggia per me una protezione, come il drago che nelle fiabe protegge il tesoro" ${ }^{11}$.

Ogni colore all'interno del giardino è stato appositamente studiato in relazione alla figura della scultura e del luogo che doveva creare. Esso ha un preciso riferimento simbolico: il blu all'interno ed all'esterno della Papessa indica il colore spirituale, il blu elettrico delle nanas rappresenta il colore della chiaroveggenza e dell'estasi, il verde è utilizzato come colore della forza e della vitalità primigenia, il rosso come colore della procreazione, il giallo, come l'oro che riveste la Morte, diviene il colore dell'intelligenza e della spiritualità (interpretazione positiva della morte come senso della rinascita).

\section{GLI ESITI}

Il Giardino di Garavicchio si può reputare un'opera totale ed organica.

Grazie alla presenza invadente della natura esso sembra sempre in movimento, anche quando i meccanismi ferrosi di Tinguely sono fermi.

Cambiando i punti di vista, durante il cammino, esso ci offre sempre una visione e un'emozione diversa, che rimanda anche al fascino magico del mondo etrusco con cui Niki dialoga idealmente e fisicamente. Rare però sono le visioni dall'interno verso l'esterno. Il paesaggio si esprime principalmente al suo interno, nella sua ricca e tipica vegetazione ed il contatto con i campi coltivati del contesto agricolo circostante è, in realtà ben delimitato. Solo in alcuni punti, per lo più giungendo sulla sommità di alcune sculture, è possibile notare la pianura del litorale costiero, organizzato su un'ampia maglia agricola.

Dopo diciassette anni di duro lavoro nel 1997 il giardino apre ufficialmente al pubblico: "Oggi il giardino è quasi finito. $\mathrm{O}$ forse no? No, il lavoro al giardino

\footnotetext{
${ }^{11}$ Niki de Saint Phalle, op. cit., Berna 1997, pag. 68.
} 
continuerà in una forma $\mathrm{o}$ in un'altra fin quando vivrò. La mia opera finirà il giorno in cui morirò e comincerò la mia nuova vita" 12 .

Il giardino, strettamente collegato alla sua genitrice, è soprattutto il frutto di una liberazione interiore. Nella libertà dei materiali, nell'indagare ed osservare continuamente, Niki ricerca se stessa: l'esigenza di una continua metamorfosi, di un infinito movimento come crescita spirituale, come rinnovamento. Esso diviene così lo strumento con cui si individua la soluzione per poter placare i propri demoni nascosti.

L'elaborazione e la costruzione dell'intero progetto sono state per la scultrice una grande esperienza spirituale, a cui ha dedicato, anche nei momenti di malattia (come l'artrite e l'intossicazione dal poliestere che utilizzava per ricoprire le sue sculture) tutte le sue forze.

Le tappe del processo si possono leggere anche sulle figure simboliche, sui colori e nelle scritte delle piastrelle che compongono come un mosaico l'intero rivestimento del luogo, e che ci fanno da guida e da interprete della sua figura, lungo l'intero percorso.

La natura femminile di Niki de Saint Phalle è percepibile in ogni angolo del giardino ma, al tempo stesso, si sente quanto essa sia fortemente legata all'aspetto maschile del metallo e della saldatura, peculiarità del suo compagno. Jean Tinguely, che era stato nel suo passato tra i fondatori della corrente del Nouveau réalisme, ha difatti qui realizzato le prime strutture in acciaio delle figure della Sfinge-Imperatrice, della Papessa-Sacerdotessa e del Mago, e ha portato a termine da solo il Papa, che era la sua figura preferita. Anche se alcune opere sono state realizzate da altri collaboratori che hanno acquisito le sue tecniche, egli ha discusso e collaborato con Niki alla realizzazione dell'intero complesso.

In particolare la Ruota della fortuna, il lampo che decapita la torre di Babele, l'Ingiustizia dentro la Giustizia, il Carro dell'Imperatrice e il Mondo, portano indubbiamente la sua impronta artistica.

Il giardino, oltre ad esternare le grandi personalità dei due artisti e la contrapposizione tra maschile e femminile, nasce da un lavoro collettivo di persone che hanno contribuito ad arricchirlo con il loro talento e la loro professionalità (alcuni artisti e specialisti nelle varie tecniche della saldatura, delle ceramiche e nel taglio degli specchi, ma altri semplici operai o contadini locali, invasi dall'entusiasmo dell'artista).

Niki chiama così il giardino la sua "Cattedrale", nome che non solo esterna l'imponenza dell'opera, ma anche la forza collettiva del cantiere che lo ha realizzato. Simile ad una bottega neo-medioevale di artisti ed artigiani, ognuno era libero di proporre e sperimentare soluzioni diverse ai vari casi. Al tempo stesso, come in una società matriarcale, come lei stessa diceva, ogni decisione finale veniva presa dall'artista, quasi come a sottolineare e definire la propria esistenza attraverso la realizzazione dell'opera.

Vengono brevemente descritte alcune delle più significative opere tra le ventidue sculture.

\section{La Papessa, Il Mago, la Ruota della Fortuna.}

Il complesso è ubicato all'interno dell' anfiteatro della ex-cava. È un luogo che è stato attentamente studiato durante la sua realizzazione, visto che doveva fare i conti anche con la morfologia del terreno. La verde vegetazione invade i contorni delle sculture e fa risaltare la luminosità dei colori delle sculture in cui predominano gli specchi del Mago e il colore azzurro del contorno della vasca e della Papessa, mentre lo spazio ristretto dell'anfiteatro amplifica la percezione del gigantesco gruppo scultoreo.

\footnotetext{
${ }^{12}$ Niki de Saint Phalle in Anna Mazzanti (a cura di), op. cit., Milano 1990, Catalogo.
} 


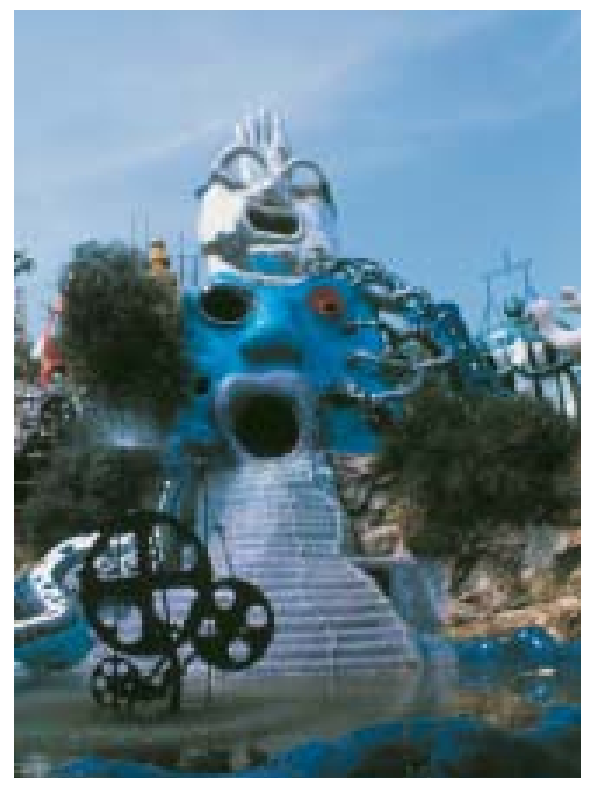

Figura 10 - Il Mago e la Papessa.

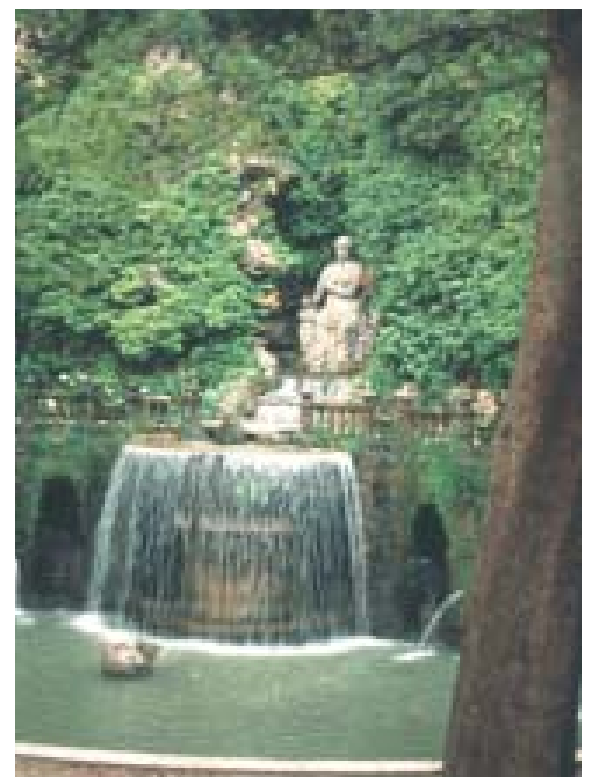

Figura 11 - La Fontana dell'Ovato, Villa d'Este.

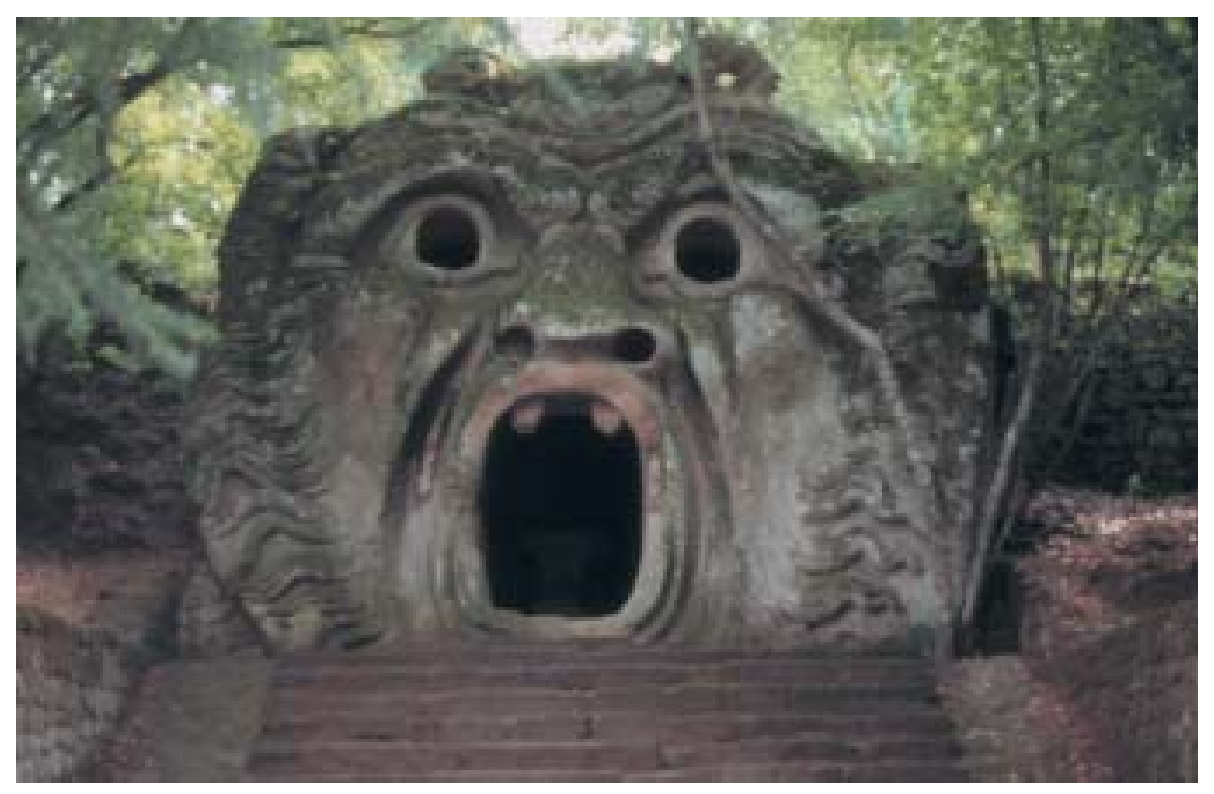

Figura 12 - L’Orco del Sacro Bosco di Bomarzo.

Il Mago con la mano alzata sopra la testa, ricoperto di specchi, è la "carta di Dio". Simbolo della forza del mondo, della luce, dell'energia pura e del gioco, risiede al di sopra della Grande Sacerdotessa, che esprime il potere femminile dell'intuizione e il potenziale dell'irrazionale inconscio.

Questa scultura, rivestita di colore azzurro, con occhi spalancati e una grande bocca aperta, da dove esce l'acqua che scorre poi sopra i gradini è certamente un omaggio sia alla scultura L'Orco del giardino di Bomarzo, che l'artista aveva visitato durante un suo viaggio nel 1962, sia alle sculture rigogliose di acqua presenti nel Giardino di Villa d'Este. L'acqua che scende fa poi muovere la Ruota della Fortuna realizzata da Jean Tinguely, simbolo della vita: "ciò che sale inevitabilmente dovrà scendere"13. 


\section{Il Castello dell'Imperatore}

L'Imperatore è il simbolo del potere maschile inteso sia negativamente che positivamente. Rappresenta il controllo e la conquista, ed è caratterizzato dall'organizzazione e dall'aggressività, portatore di scienza, medicine e guerra.

Il Castello è l'opera più completa dal punto di vista architettonico, una cittadella in cui è forte l'eredità di Gaudí come maestro spirituale: formata da torri, da un camminamento di ronda e da ventidue colonne che sorreggono un loggiato minuziosamente rivestite di tessere colorate. Al centro vi un cortile, e la vegetazione si incunea in ogni luogo.

Gli alberi di olivi presenti sono essi stessi divenuti elementi integranti della scultura e definiscono lo spazio all'interno della composizione.

Nella vasca al centro del cortile del Castello ci sono quattro figure femminili sdraiate, anch'esse colorate, spruzzanti acqua dai loro seni. Ė segno di gioiosa fertilità che ricorda le sculture con i getti d'acqua di Villa d'Este.

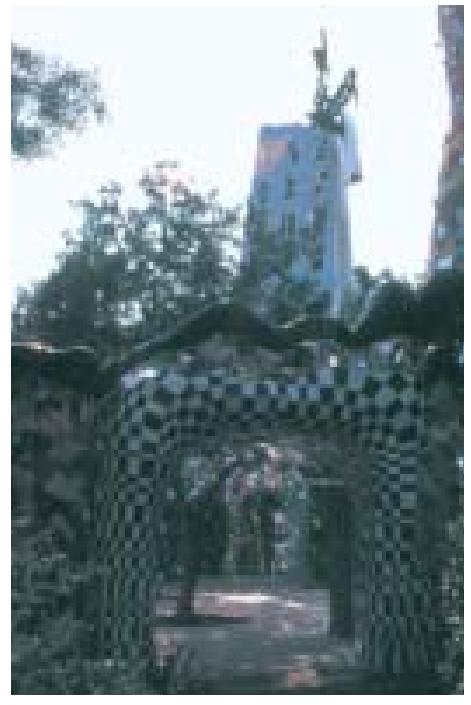

Figura 13 - L'entrata del Castello.

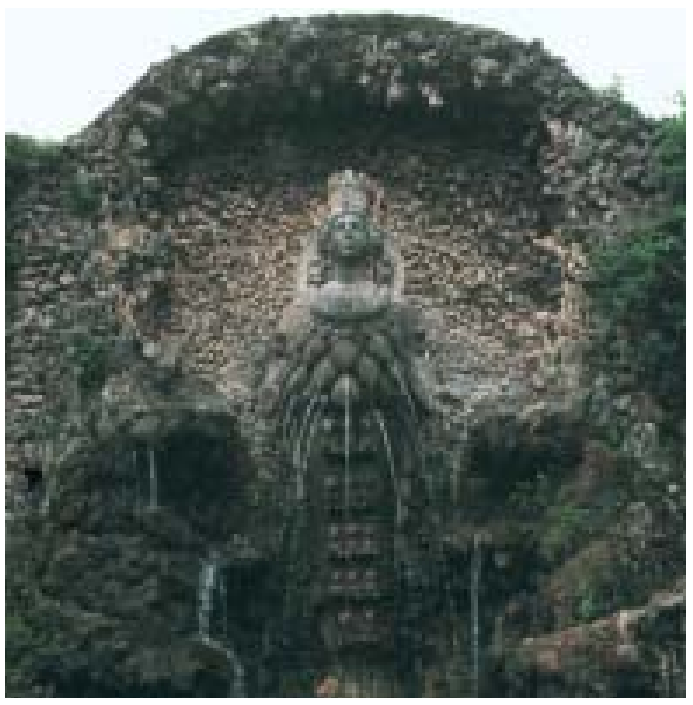

Figura 14 - Fontana di Villa d'Este.

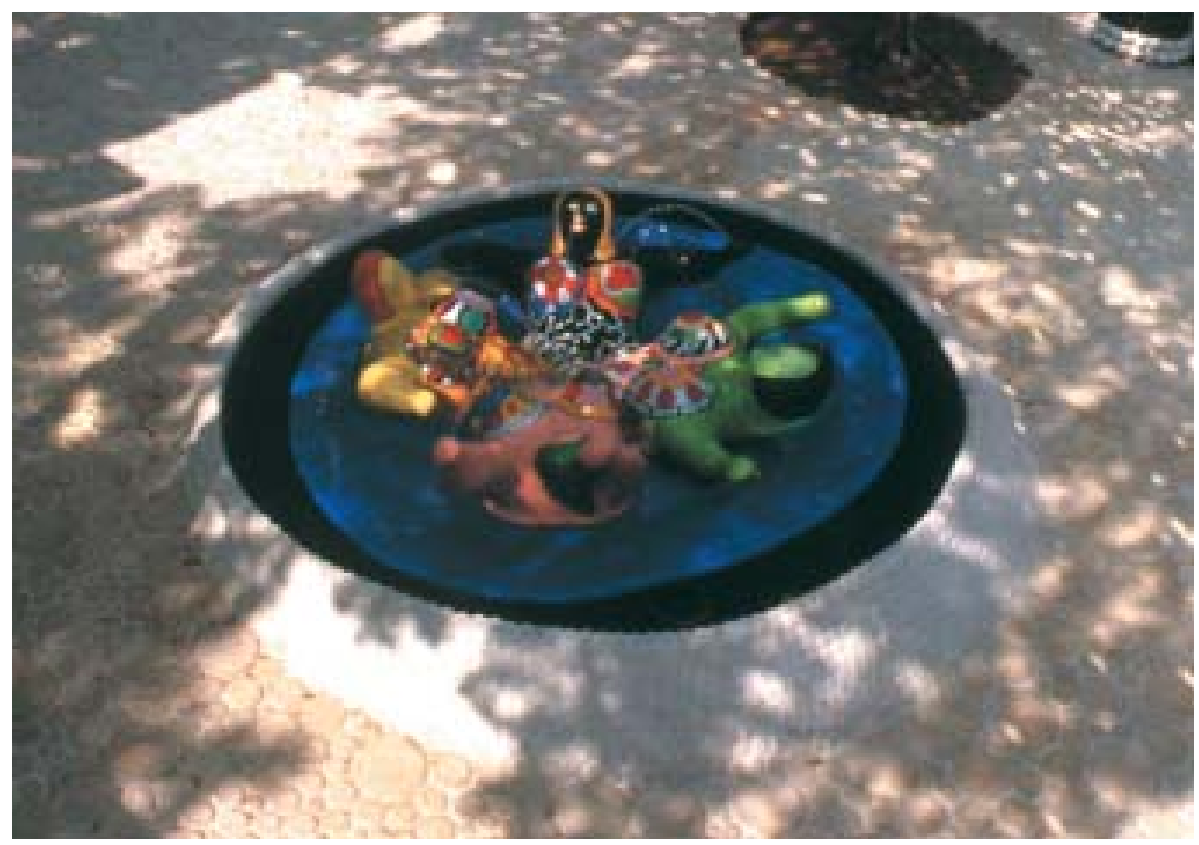

Figura 15 - La Fontana situata al centro del cortile del Castello: composta da quattro figure femminili sdraiate, spruzzano acqua dai loro seni. 
Sull'intera composizione svetta la Torre di Babele, completamente ricoperta di specchi, decapitata da un fulmine realizzato da Jean Tinguely, simbolo delle costruzioni fisiche e mentali: "Bisogna rompere le mura della mente in modo da poter guardare oltre" 14 .

\section{RIFERIMENTI BIBLIOGRAFICI}

Centro per l’Arte Contemporanea del Luigi Pecci (a cura di), Quaderno n. 4, Arte ambientata Arte Ambientale, Prato 2001.

DE Saint Phalle Niki, Il Giardino dei Tarocchi, Edizioni Benteli, Berna, 1997.

Massa Antonella, Itinerari italiani, I parchi Museo di scultura contemporanea, Loggia de' Lanzi, 1995.

Mazzanti Anna (a cura di), Niki de Saint Phalle - il Giardino dei Tarocchi, Edizioni Charta, Milano 1997.

Pettena Gianni, Pietrogrande Patrizia e Pozzana Mariachiara (a cura di), Giardini, Parchi, Paesaggi. L'avventura delle idee in Toscana dall'Ottocento a oggi, Le Lettere, Firenze 1998.

Schulz-Hoffmann Carla (Herausgegeben), Niki de Saint Phalle, Prestel-Verlag, Bonn 1987.

ZAGARI FranCO, Larchitettura del giardino contemporaneo, A. Mondadori Editore, Milano, 1998.

\section{RIFERIMENTI ICONOGRAFICI}

Le fotografie sono di Emanuela Morelli.

\section{SCHEDA DI PROGETTO}

Tipo di intervento:

Località:

Proprietà:

Progettista:

Anno di realizzazione:

Superficie complessiva:

Specie arboree impiegate: giardino realizzato da artista contemporanea

Garavicchio, comune di Capalbio, provincia di Grosseto

appartenuta in precedenza ai fratelli Caracciolo, è attualmente proprietà della Fondazione "Il Giardino dei Tarocchi” di cui presidente è Niki de Saint Phalle

Niki de Saint Phalle con la collaborazione di Jean Tinguely

$1979-1997$

circa 2-3 ettari

vegetazione autoctona tipica della macchia mediterranea: sono presenti prevalentemente olivi, sughere e arbusti aromatici 



\title{
IL GIARDINO HIC TERMINUS HAERET DI DANIEL SPOERRI A SEGGIANO
}

\author{
Emanuela Morelli
}

\section{IL CONTESTO}

Daniel Spoerri, di origini romene e con ampia formazione culturale, ha percorso una vita complessa e ricca di eventi, in cui ha praticato danza, mimo, ha lavorato nel teatro ed è stato poeta, scrittore e scultore ${ }^{1}$.

Nel 1992 si stabilisce tra Arcidosso e Seggiano, due piccoli paesi della Maremma alle pendici del Monte Amiata, attratto dalla possibilità di comprare, secondo le fonti di alcuni suoi conoscenti, una "costruzione instabile" che ricorda la casa inclinata del Sacro Bosco di Bomarzo. L'edificio risulta però essere una anonima costruzione recente in cattive condizioni strutturali, ma durante il suo soggiorno amiatino rimane così affascinato dal paesaggio da acquistare la fattoria "il Giardino" poco distante dal paese di Seggiano. La proprietà risulta completamente abbandonata da anni e la vegetazione spontanea ha già cominciato a riconquistare le vecchie aree coltivate.

Nonostante l'aspetto selvaggio (o forse proprio per questo), Spoerri appena la visita, ha l'impressione che sia il luogo adatto per inserire le proprie sculture e poter realizzare il giardino che paradossalmente diviene quello che lui definisce il suo più grande impegno della vita.

Daniel Spoerri chiama il proprio giardino Hic terminus haeret: la frase è quella che Didone grida nel proprio dolore di amante abbandonata prima di suicidarsi e davanti all'inevitabile destino stabilito dagli Dei in cui Enea deve partire dall'isola della regina Fenicia per tornare a Roma ${ }^{2}$. Per lo scultore significa "qui aderiscono i confini": terminus non ha solo il significato di fine, ma si riferisce a Giove, il dio

\footnotetext{
${ }^{1}$ Nato a Galati, cittadina portuale della Romania sul Danubio, nel 1930, si trasferisce in Svizzera a causa delle persecuzioni naziste che videro la morte di suo padre, di origini ebraiche, nel 1941 in un campo di concentramento. Vivendo principalmente di borse di studio, diviene primo ballerino dell'Opera di Berna e frequenta corsi di mimo. Si accosta, sempre in quel periodo, al teatro dell'assurdo, per poi divenire aiuto regista al teatro di Darmstadt. Nel 1959 costituisce con altri artisti, tra cui Arp, Cristo, Duchamp, Niki de Saint Phalle e Tinguely, le edizioni MAT, Multiplication d'Art Trasformable, ovvero una forma di espressione liberatoria in cui l'arte "si muove o che si lascia muovere". Gli anni Sessanta sono caratterizzati dalla realizzazione delle opere che in seguito lo renderanno famoso, i Tableauxpièges (Quadri trappola). Esse rappresentano tavole apparecchiate dadaisticamente con oggetti comuni e poi affisse alle pareti come quadri.

Spoerri è anche una delle personalità emergenti dalla corrente artistica dei Noveau réalism, discendente diretta dell'avanguardia dadaista, in cui si riscopre il ruolo estetico dell'oggetto non più osservato dal punto di vista quotidiano e dove l'arte viene utilizzata come strumento di scoperta, esperienza ed interazione.

Nel 1962 aderisce al gruppo internazionale dei Fluxus, tra i cui fondatori troviamo Marcel Duchamp, che ha come obiettivo l'eliminazione delle barriere tra chi crea e compone e chi invece ascolta, in poche parole instaurare un rapporto tra l'artista e chi osserva, tra cantante e pubblico.

Alla fine degli anni Sessanta anni apre dei locali dove il cibo viene associato all'esposizioni di arte e in cui le gallerie d'arte sono assimilabili a dei ristoranti (Eat Art).

Durante la sua vita è vissuto in molte parti del mondo: in Svizzera, con la madre ed i fratelli, dove conosce Jean Tinguely (con lui compie un lungo viaggio in Europa), a Parigi e dopo aver soggiornato a New York con altri artisti, risiede solitario su l'isola greca Simi per circa un anno e mezzo, per poi spostarsi nuovamente a Dusseldorf, nel sud della Francia, ed infine a Seggiano, dove ha costruito il suo giardino divenuto poi ufficialmente Fondazione il 25 luglio del 1996.
} 
della luce e dell'agricoltura degli Etruschi, vecchi antenati di questo luogo, che lo chiamavano anche Tinia, il guardianocustode dei confini e del passaggio inteso anche in senso temporale; haeret prende invece il significato di "aderire a qualcosa", di spazio in cui due cose si uniscono, si fondono, e diventano una cosa sola, come il maschile ed il femminile, il giorno e la notte, la quiete ed il moto, la vita e la morte, temi fondamentali della struttura portante del giardino.

Così come i giardini antichi divenivano elementi cardine all' interno della struttura del paesaggio antropico, anche in questo caso il giardino di Spoerri, nel pieno rispetto dell'ambiente che lo accoglie, diviene un altro tassello fondamentale, un segno nel contesto paesistico, in cui si può osservare la continuità della storia della dialettica tra uomo e natura. Qui il paesaggio non viene vissuto solo nel suo aspetto scenografico, ma nel suo significato più complesso, costituito da elementi stori-

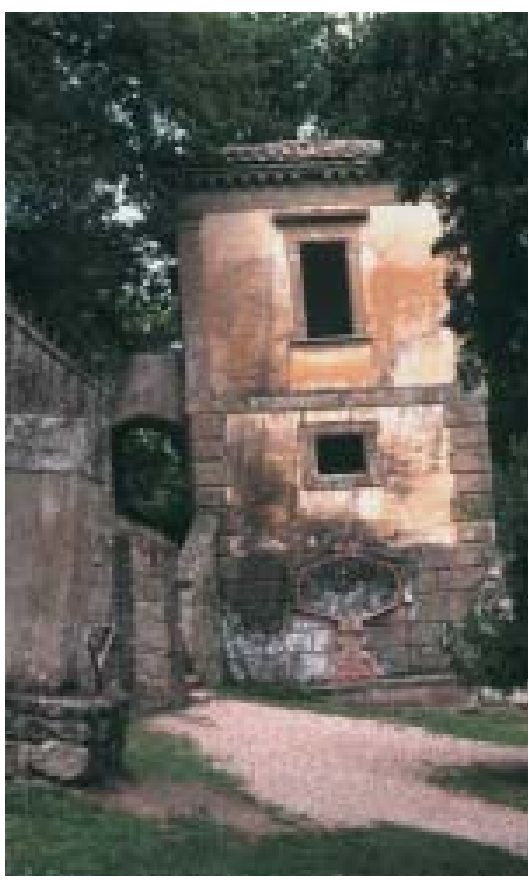

Figura 1 - La casa inclinata del Sacro Bosco di Bomarzo. co-culturali, naturali e estetico-percettivi.

Daniel Spoerri, che già nel suo passato era stato affascinato da alcuni giardini storici, tra cui il già citato Sacro Bosco di Bomarzo ${ }^{3}$, nei primi anni Ottanta approfondisce i temi riguardanti la storia e l'evoluzione del giardino come opera d'arte grazie alla sua attività di docente presso l'Accademia di Belle Arti di Monaco di Baviera e di Vienna.

Proprio il corso viennese è dedicato al mito di Polifilio, che sembra aver ispirato la realizzazione del Sacro Bosco di Bomarzo ${ }^{4}$, il parco di mostri realizzati in pietra nel Cinquecento, non molto lontano dal paese di Seggiano.

Nel giardino di Spoerri la fonte ispiratrice proveniente da Bomarzo non si esplica tanto nella realizzazione estetica e formale delle sculture, ma piuttosto nel suo carattere di mistero e nella sua filosofia: la lotta tra desiderio e realtà, tra l'immaginazione e la verità, "la natura intesa come teatro interagente dell'azione, un misto di realtà e di evocazioni attraverso i rumori, gli odori e l'insieme è l'universo che ci sopravvive, che si tenta di ridurre, di tagliare e dominare (...) dove la vita si congiunge alla morte"

${ }^{2}$ Libro IV dell'Eneide di Virgilio, verso 614.

${ }^{3}$ Daniel Spoerri visita il Sacro Bosco di Bomarzo nel 1964, durante uno dei suoi primi viaggi in Italia.

${ }^{4}$ L'Hypnerotomachia Poliphili è un racconto illustrato, scritto dal frate domenicano veneziano Francesco Colonna a metà del Quattrocento ed edito nel 1499. Racconta il sogno d'amore di Polifilio che intraprende un avventuroso viaggio per rivedere la sua amante Ponia. La storia si svolge sull'isola Citera che ha forma perfetta e circolare, su cui vivono Venere e Amore. Il racconto è arricchito da numerose illustrazioni, gran parte delle quali forniscono dettagli sulla realizzazione perfetta del giardino attraverso la ricerca del bello.

A questo libro sembra che si sia ispirato il conte Vicino Orsini (anche per la parentela che univa gli Orsini ai Colonna) per la realizzazione del Sacro Bosco di Bomarzo nel 1552, una località presso Viterbo. Il parco che "manifesta la volontà di eludere una progettazione del giardino in termini convenzionali per esprimere una creazione del proprio mondo interiore" (AlESSANDRO TAGLIOLINI, Storia del giardino italiano, La Casa Usher, Firenze 1988, pagg. 206-207) è dedicato alla giovane moglie precocemente scomparsa, in cui forse il conte, tramite un percorso tra grandi sculture di mostri e architetture in pietra, voleva rievocarla in un luogo di meraviglie e di mistero.

5 Anna Mazzanti in Anna Mazzanti (a cura di), Il Giardino di Daniel Spoerri, Maschietto \& Mugolino editore, Siena 1998, pag. 46. 


\section{GLI OBIETTIVI}

La visione che Daniel Spoerri ha della natura è tutt'altro che idilliaca: per molti anni, fino a quando vive nelle grandi metropoli come Parigi, è per lui inesistente ed appartiene ad un mondo che non lo riguarda. Di essa, come lui stesso dice, ha solo ricordi negativi di quando era ragazzo ed era costretto a contenere l'esuberanza delle siepi del giardino di sua zia in Svizzera attraverso la potatura.

Egli ha quindi il sospetto che l'uomo in realtà tema la natura e realizzi giardini per domarla e poter viverla serenamente, così come i "primitivi" usavano fare danze, indossare maschere e compiere rituali vari, nel tentativo di esorcizzarla: "la cultura è l'allontanamento della natura. In tal modo noi contrapponiamo ad essa qualcosa, nel tentativo di esorcizzarla"6.

$\mathrm{Ma}$ al tempo stesso non si può evitarla del tutto, essa comunque continua ad essere un'attrazione per l'uomo, anche perché in lei riconosce "la più diretta procreatrice eterna, simbolo dell'incessante divenire"7.

Spoerri davanti alla vastità e alla potenza della natura quindi dichiara che "cercando di collocare con discrezione nel paesaggio questi Segni, figure contro il dolore ed esercizi per combattere la paura (come Jean Tinguely li ha definiti in un testo su una mia esposizione del 1985) si inizia a riflettere su essa, si inizia forse a comprenderla, ci si avvicina prudentemente ad essa per verificare se le si resiste. (...). Nelle mie opere io libero le mie inquietudini e vi rinasco"8.

Così l'artista comincia ad inserire con molta esitazione le sue sculture, i bronzi, nella natura quasi più con il tentativo di nasconderle piuttosto che mostrarle.

Prendendo via via sempre più confidenza con il processo di dialettica tra arte e paesaggio, egli si pone l'obiettivo di "proiettare in questa natura non solo il bello ma anche il terribile, il mesto l'attraente e il ripugnante, a rammentarci che anche noi siamo natura con tutto il bello e tutto lo spaventevole. È solo la presenza della morte nella pienezza vitale a fare la veramente la vita; c'est la vie..."

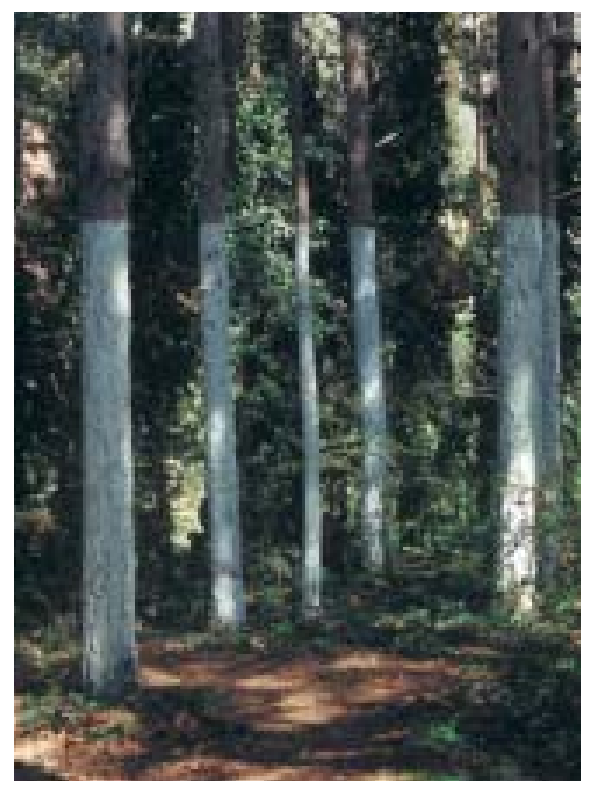

Figura 2 - Il Bosco di Platone, Karl Gerstner.

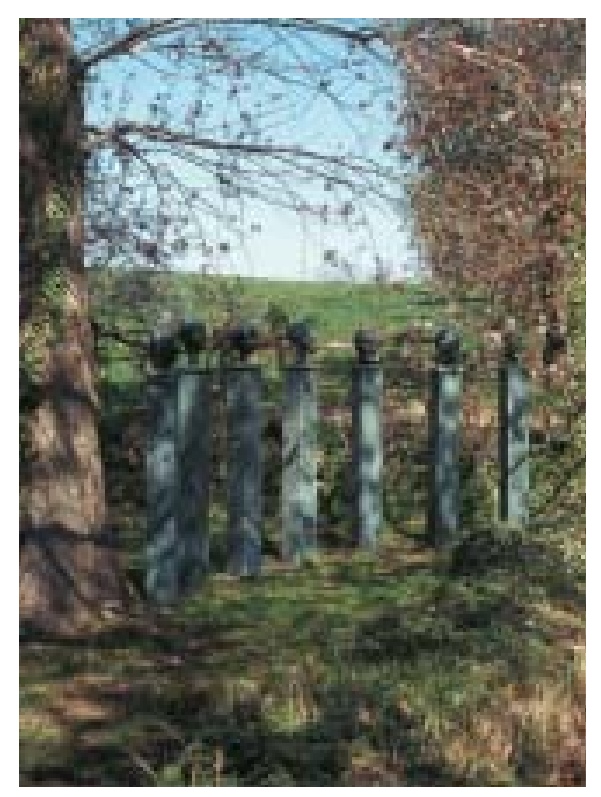

Figura 3 - Alcune debolezze umane, Eva Aeppli.

\footnotetext{
${ }^{6}$ Daniel Spoerri in Anna MazZanti (a cura di), op. cit., 1998, pag. 7.

${ }^{7}$ ANNA MAZZANTI in ANNA MAZZANTI (a cura di), op. cit., 1998, pag. 46.

${ }^{8}$ Daniel Spoerri in AnNa Mazzanti (a cura di), op. cit., 1998, pag. 7.

${ }^{9}$ Daniel Spoerri in Anna MazZanti (a cura di), Il Giardino di Daniel Spoerri - Ultime Installazioni, Maschietto\&Mugolino, Siena 2000, pag. 3.
} 


\section{LA METODOLOGIA}

Il giardino era parte di una proprietà agraria più vasta appartenuta alla famiglia degli Ugurgeri, presente in questo luogo sicuramente già dal Trecento, periodo a cui risalgono i primi documenti scritti sulla proprietà.

Grande sedici ettari, il giardino è situato sul Monte Amiata, una montagna di origine vulcanica, nella fascia vegetazionale immediatamente sotto i 700 metri di altitudine caratterizzata dalla tipica vegetazione a roverella.

Quando Spoerri ne diviene proprietario, questo si trovava in forte stato di abbandono: la natura andava già ad riappropriarsi delle parti coltivate con le sue specie pioniere, gli olivi non erano stati più potati e le macchie di bosco avevano il sottobosco ormai impenetrabile e infestato da rampicanti.

L'artista ha quindi ritenuto indispensabile, come prima operazione, un recupero dell'intero luogo, degli edifici colonici e dell'intero sistema degli spazi aperti, in cui si dovevano ristabilire confini e limiti delle varie zone, controllando le invasioni della vegetazione. Nella proprietà si è così ricostruito un rapporto lirico ed un equilibrio tra i pieni e i vuoti che si possono ricollegare, nel loro movimento e nelle pause, ai ritmi che lo scultore ha appreso durante il suo passato di ballerino e di mimo.

Nella tenuta si trovano tracce delle coltivazioni tipiche del luogo, olivo, vite e castagno, sistemate su terrazzamenti ottenuti grazie lo "spietramento", visto che il terreno presenta anche zone con elevate pendenze ed è costituito da argille e calcari marnosi, con elevata ricchezza di scheletro. Gran parte delle aree coltivate sono state recuperate attraverso la potatura ed evitando trattamenti chimici: il bosco di castagno è stato ripulito dalla rinnovazione naturale, mentre gli olivi, la cui cultivar è l'"olivastra Seggianese" (tipica di questa zona), sono stati potati per ottenere la forma tipica dell'olivo a "vaso cespugliato".

Purtroppo oggi le coltivazioni a vigneto sono quasi del tutto scomparse, solo in alcune aree, specie in quelle non ancora interessate dall'intervento di recupero, si trovano alcune piante di viti ormai inselvatichite. È previsto comunque il reinserimento di tale coltivazione in una piccola zona del giardino.

In prossimità degli edifici sono presenti dei frutteti, soprattutto ciliegi, susini e fichi, che però non sono in buone condizioni per la loro anzianità e per l'at-

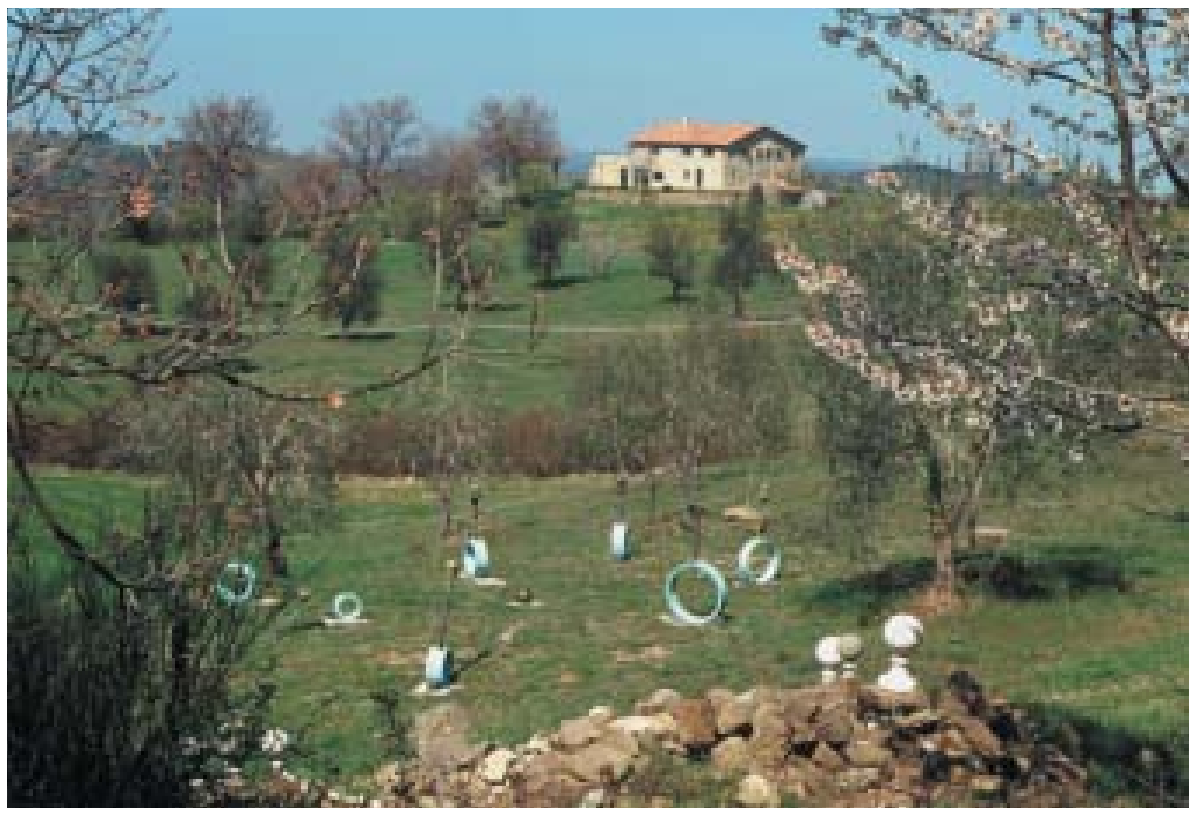

Figura 4 - I giocolieri di Daniel Spoerri, disposti nel vasto prato centrale del giardino. 


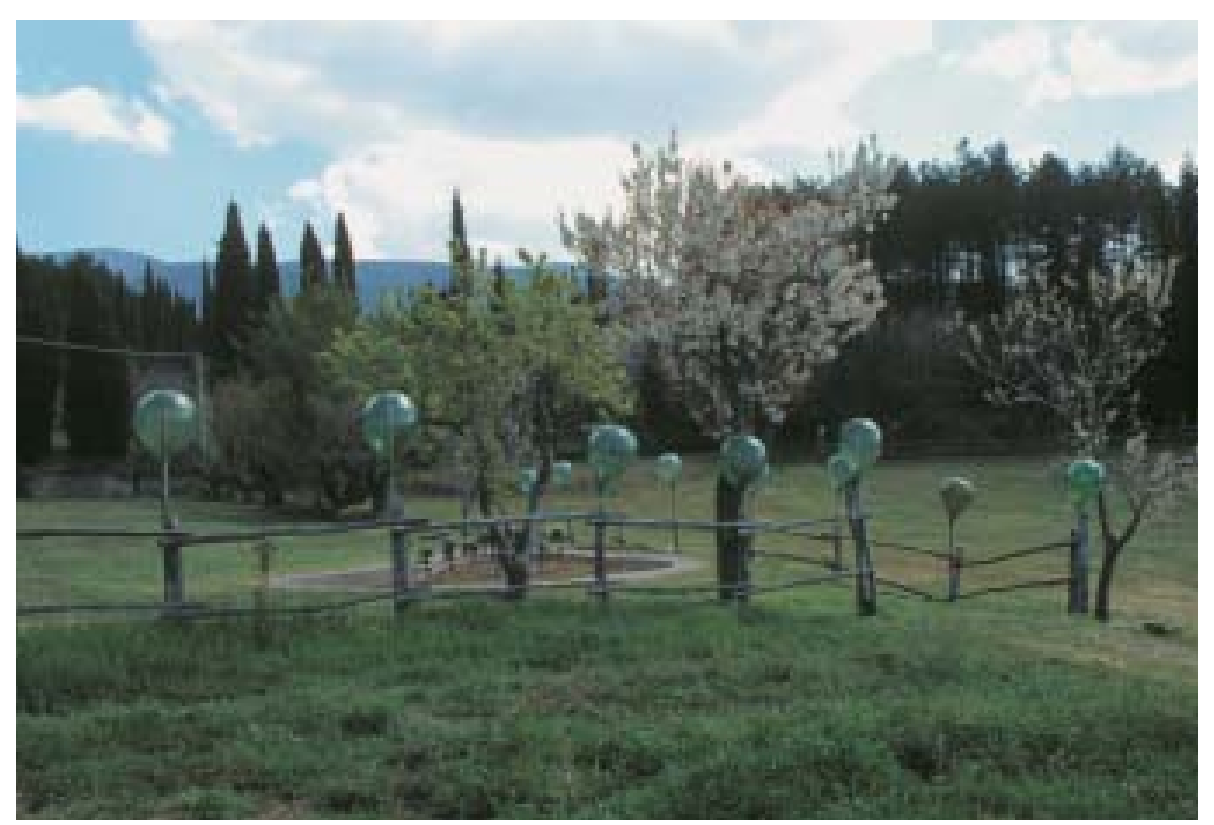

Figura 5 - Il Frutteto in prossimità degli edifici colonici; si notano inoltre Le damigiane bevute e i Guerrieri della notte.

tacco di malattie. Anche in questo caso il proprietario ha previsto il recupero dell'area attraverso la reintroduzione degli alberi da frutto attivando una ricerca che individui le antiche varietà presenti e cercando di ripristinare l'impianto agricolo complessivo della vecchia proprietà.

Per la visita del giardino, così come per il vicino Giardino dei Tarocchi di Niki de Saint Phalle, non esiste un percorso ideale, ma piuttosto un percorso funzionale, che senza interruzioni ritorna al punto di partenza e che scopre via via la presenza delle sculture, talvolta nascoste, inducendo sensazioni di sorpresa.

$\grave{E}$ una specie di percorso iniziatico e rivelatorio, in cui ci viene chiesto di affrontare "le prove e le sorprese della vita" ${ }^{10}$.

Daniel Spoerri diviene quindi autore e regista della propria opera. Il giardino è composto da oltre una settantina di sculture, gran parte realizzate da lui ma anche da altri artisti, circa una ventina, quasi tutti di area svizzero-tedesca e di età diverse: sono quelli a lui più cari e compagni di vita, come Eva Aeppli e Jean Tinguely.

Qui gli artisti sono stati lasciati liberi nel loro intervento ma, l'imprevisto progettuale che ne scaturisce deve rientrare in un disegno unitario di dialogo con proposizioni sostanzialmente animistiche. In questo caso l'opera artistica viene realizzata nel sito, gli artefici ne devono studiare la morfologia, la fisionomia dei caratteri vegetali e i suoi contenuti culturali, in modo tale da interagire in modo organico con l'ambiente circostante.

Larte diviene quindi uno strumento di conoscenza e di avvicinamento alla natura, creando un'occasione per vivere assieme sia il mondo naturale e lo spazio sociale e culturale dell'uomo.

Lo stesso Daniel Spoerri afferma che quando era "atterrato" in queste "terre campagnole", non conosceva assolutamente nulla della natura, nessun nome di pianta e nessun suo comportamento. Eppure adesso le cose sono cambiate: "Come mai poi tutto questo è cambiato, come mai adesso so cosa sono gli ulivi e come si potano, come mai a poco a poco ho finito per osservare le piante con 
lo stesso interesse con cui osservo l'arte nei musei, come mai io trovi così avvincente la messa in scena di un'opera nel paesaggio e spesso abbia modificato o spostato un'installazione perché non mi pareva in sintonia con il luogo (...) non lo so con precisione e non voglio neppure saperlo"11.

Oltre alle tavolette in nero che indicano il nome, l'autore e l'anno di realizzazione dell'opera, durante il tragitto si individuano anche tavolette, questa volta in bianco, che indicano le specie botaniche presenti (alle originarie quaranta specie, Daniel Spoerri ha previsto l'inserimento di nuove, tutte autoctone e di cui nessuna conifera dei recenti rimboschimenti vicini). Il cartellino del percorso botanico porta il nome della pianta in latino, italiano, dialettale e tedesco, nonché una breve descrizione riguardo all'interesse ecologico e produttivo della tradizione locale, oltre a segnalazioni simboliche o letterarie.

Se ciò può sembrare una esigenza di appropriazione, di conoscenza e di necessità per instaurare un rapporto dialettico con il luogo, conseguenza inevitabile è la nascita del senso di rispetto e di umiltà in rapporto al paesaggio.

Nonostante il progetto di recupero, il giardino continua a mantenere volutamente un certo aspetto selvatico per rispettare quel carattere di segretezza e di mistero che il luogo deve avere: si lascia quindi che il tempo ristabilisca un rapporto più armonioso tra i passaggi instaurati fra l'opera dell'uomo e i luoghi della natura, come per esempio il far crescere l'erba tra le fessure dei gradini di tufo, o il creare passaggi intermedi di vegetazione arbustiva ed erbacea tra le zone a prato e quelle a bosco.

Il percorso nasce da un angolo della casa di Spoerri e, attraversando il prato e risalendo nel bosco, compie un cerchio da cui parte un sentiero che segue i margini del giardino: è una sorta di sentiero più breve e pratico per toccare tutti i punti del giardino. Qui però il visitatore non si sente tenuto a seguirlo: egli può attraversare i prati come meglio gli si addice. Seguendo il proprio senso di orientamento, in modo da creare un individuale percorso dato dalle proprie relazioni intime che instaura e che gli suggerisce il luogo, attiva così anche un processo mnemonico e di riflessione che rafforza il ricordo della visita.

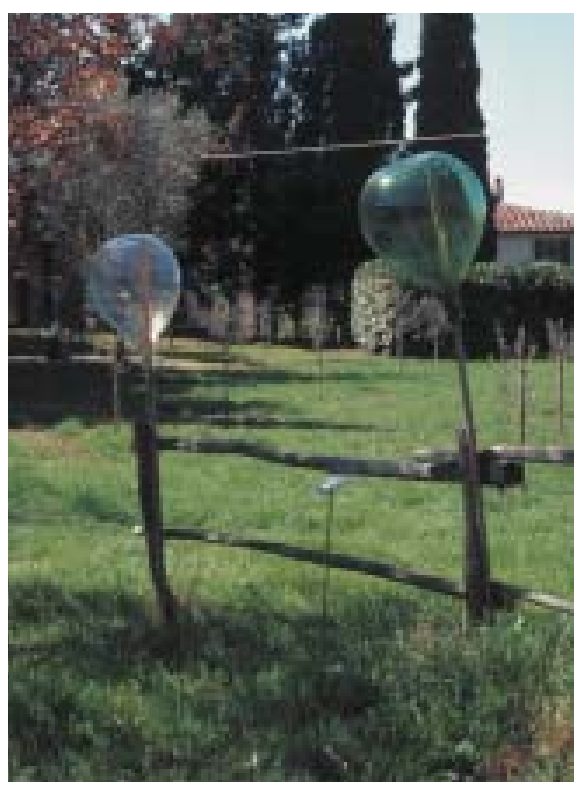

Figura 6 - Le damigiane bevute, Daniel Spoerri.

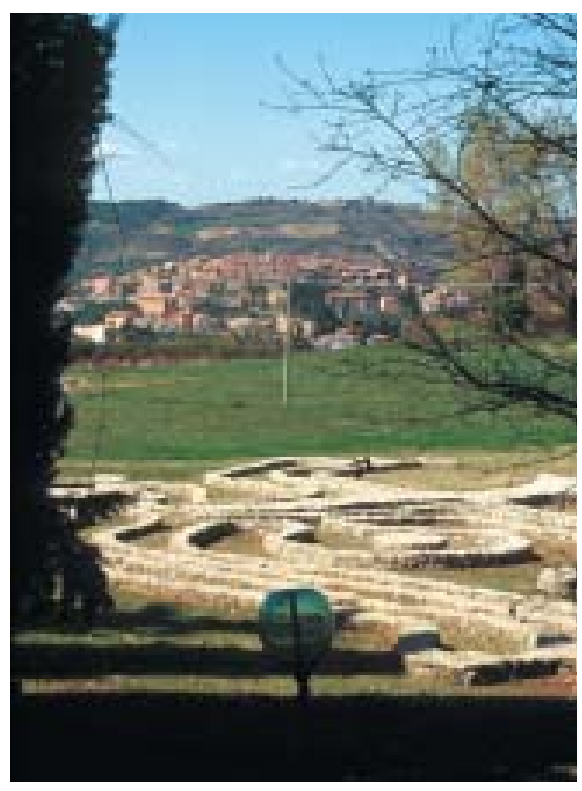

Figura 7 - Particolare del Labirinto di Daniel Spoerri, e sullo sfondo il paese di Seggiano.

\footnotetext{
${ }^{11}$ Daniel Spoerri in Anna Mazzanti (a cura di), op. cit., Siena 2000, pag. 3.
} 
Le opere sono inserite nel giardino in modo tale che esse non prevalgono sulla natura che le ospita, evitando quindi posizioni monumentali ed egocentriche, ma anzi suscitando sorpresa e imprevisto.

E un modo di collocare gli oggetti nei parchi, che rimanda alla filosofia del giardino romantico.

Il contesto non diviene una cornice teatrale, ma piuttosto le opere scultoree si nascondono in esso senza mostrarsi al meglio, riprendendo e sottolineando i segni dell'ambiente che li circonda o talvolta contrapponendosi ad essi, ma sempre in rapporto dialettico.

I rumori percepiti dalle foglie o dall'acqua provenienti dalle due sorgenti esistenti, $i$ vari dislivelli del terreno, i pieni ed i vuoti delle radure e delle macchie boscate, concorrono insieme alle sculture a infondere percezioni e sensazioni, che rimandano ad archetipi e a luoghi magici, relazionandosi sia con la natura, sia con l'orizzonte paesistico.

Le opere nel giardino acquistano una nuova espressione rispetto ad una loro eventuale collocazione in un museo. Le patine date dagli agenti atmosferici, dalla terra e dall'acqua rivestono le superfici, facendogli perdere la lucentezza del metallo: le sculture diventano "macchiate" e si mescolano alle gradazioni dei toni di colore dell'ambiente, mitigando la loro "aggressività" e facendole sembrare, come lui stesso dice, "meno cattive".

All'esterno e all'interno degli edifici della proprietà si trovano vari utensili ed oggetti della tradizione agricola locale disposti in maniera particolare. È una visione liberatoria dove gli oggetti vengono rappre-

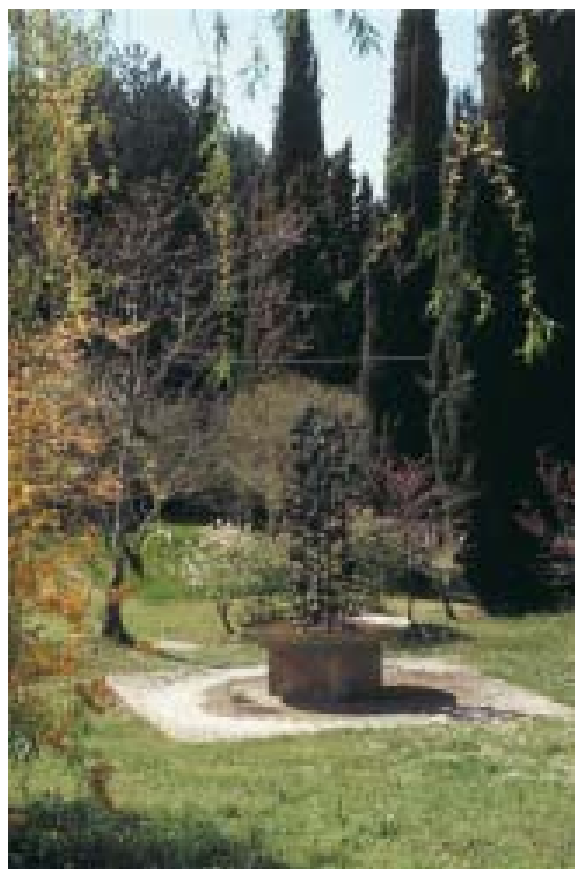

Figura 8 - Il gocciolatoio di tritacarne, Daniel Spoerri. sentati con soluzioni visive insolite che possono diventare espressione d'arte (come le damigiane bevute, capovolte su pali che formano una recinzione e $\mathrm{i}$ guanti da operaio disposti sull'asta degli Unicorni), in cui si scopre una vita nascosta dell'oggetto, diversa dal suo uso quotidiano. Questo modo di osservare ed interpretare l'oggetto discende dal passato di Spoerri come componente della corrente artistica Nouveau réalisme ${ }^{12}$.

Il giardino diviene una sorta di enunciati in cui rappresentare ciò che è essenziale. Muovendosi al suo interno ci si imbatte nelle forze che lo fanno vivere ed in quelle che, se non dominate, lo potrebbero distruggere: la natura da una parte selvaggia ma al tempo stesso controllata, così come lo specchio di acqua, piatto ed immobile rappresenta il movimento delle foglie al vento: "... Solo in qualcosa di natura profondamente diversa e contraria, la natura e l'animo tragico delle cose si riflettono... E se volete riprendere un mare un tempesta, o gli orrori di una guerra, state calmi"13.

"E con ciò saremo di nuovo all'inizio, all'uomo e al sentimento di paura o di insufficienza, di nudità, fuori dal niente, per contrapporre al niente qualcosa che è niente $\mathrm{e}$ che tornerà al niente" 14 .

\section{GLI ESITI DEL PROGETTO}

Il giardino è ancora in continua evoluzione. Nascono nuove installazioni e alcune di quelle "più anziane" subiscono mutamenti o spostamenti per perfezionare l'inserimento nell'ambiente.

\footnotetext{
12 "Questi nuovi realisti considerano il mondo come un quadro, la grande opera di cui si appropriano certi frammenti dotati di significato universale. Ci mostrano il reale negli aspetti diversi della sua totalità espressiva...(Pierre Restany). (...) L'evento estetico deve prodursi nel contesto della fenomenologia del mondo moderno, illuminarne certi aspetti significativi”. GIULIO CARLO ARGAN, L'arte moderna 1770/970, Sansoni editore, Firenze, II edizione 1971, pag. 657.

${ }^{13}$ Anna Maria Ortese in AnNa Mazzanti (a cura di), op. cit., Siena 1998, pag. 20.

${ }^{14}$ Daniel Spoeri in Anna MazZanti (a cura di), op. cit., Siena 1998, pag. 9.
} 


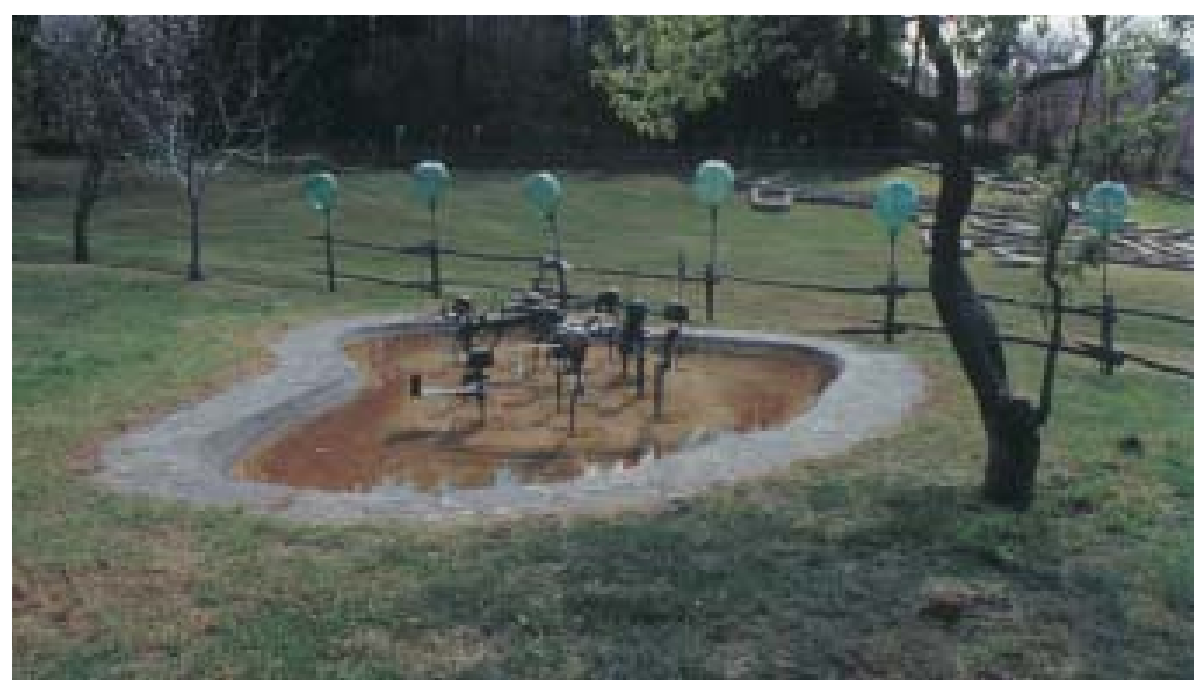

Figura 9 - Guerrieri della notte, Daniel Spoerri. Tredici elementi di bronzo del 1982 ora collocati in una vasca d'acqua. "Ne deriva una tensione dialettica tra il segno di forza e di moltiplicazione e la natura, che d'altronde in questa zona avvallata e verde di prati, che si apre in vasti orizzonti, afferma indiscutibile la sua supremazia”.

Visto l'enorme quantità di sculture presenti nel giardino, (circa una settantina), vengono di seguito segnalate quelle definite più significative e che esaltano maggiormente il proprio rapporto con il contesto paesistico.

\section{Il recinto degli Unicorni / Ombelico del mondo / Omphalos (1991)}

Autore Daniel Spoerri

Il complesso delle sculture, formanti un recinto, è realizzato sulla sommità di una collina a nord della proprietà.

Fonti storiche e leggende ipotizzano che originariamente fosse il punto in cui era nato il centro urbano di Seggiano. Accertato comunque che anticamente era un luogo di culto (è presente anche una piccola chiesetta dove sono sepolti gli antichi proprietari), è anche il luogo più panoramico del giardino, da cui si gode una splendida vista che si apre verso la vallata, sul paese di Seggiano e sui profili del Monte Amiata.

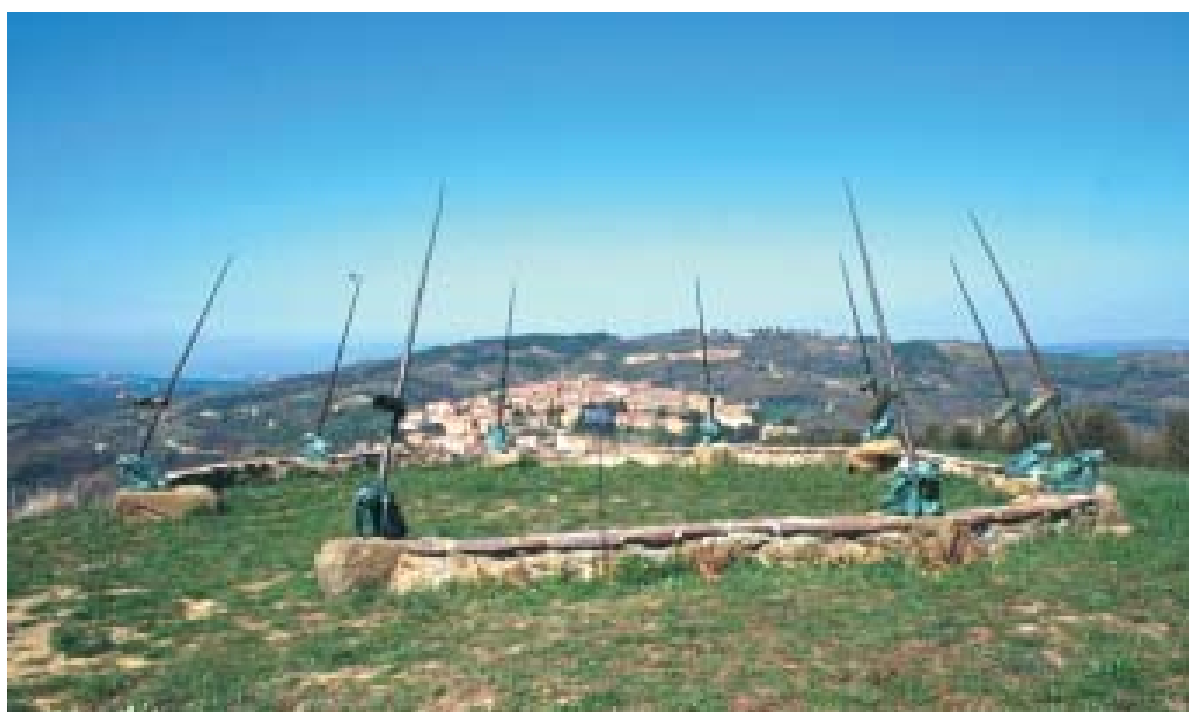

Figura 10 - Gli Unicorni/Ombelico del mondo, Daniel Spoerri. Sullo sfondo il paese di Seggiano, anch'esso disposto sulla sommità di una collina. 


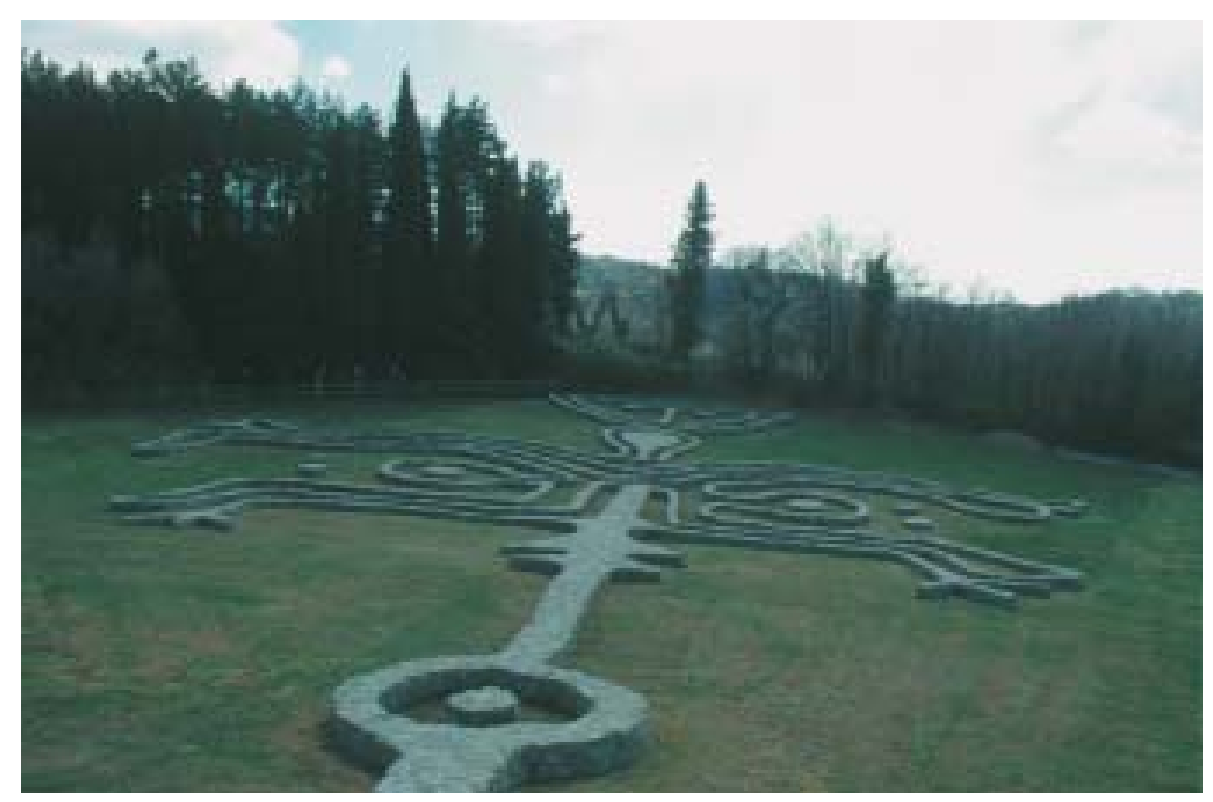

Figura 11 - Veduta panoramica del sentiero murato labirintiforme nel pratone sottostante gli edifici colonici, delimitato a sinistra da un filare di conifere mentre a destra la vista si apre sulla valle del paese di Seggiano.

Qui la dialettica tra opera d'arte e paesaggio esalta maggiormente il genius loci.

Non ci sono dubbi infatti che l'opera sia stata realizzata appositamente per questo luogo.

Su di un basso muretto in pietra a forma circolare, sono disposti ad intervalli regolari nove crani in bronzo di animali, da cui partono lunghi corni, che come lance in mano a cavalieri invisibili, convergono in un punto ideale del cielo.

Tra paesaggio ed opera si instaura un rapporto reciproco di esaltazione, e che motiva l'esistenza dell'opera solo in questo preciso luogo.

La sensazione che scaturisce è di essere in presenza di un luogo fantastico dove si incontrano antiche forze magiche.

Sentiero murato labirintiforme (1996-98)

Autore Daniel Spoerri

L'opera viene realizzata nell'ampio pratone sottostante gli edifici, contornato da una folta vegetazione arborea da una parte, mentre l'altro lato si riconduce, risalendo il terreno, alla collina degli Unicorni.

Anche qui troviamo un basso muretto in pietra (alto circa $50 \mathrm{~cm}$ ) che ricalca un petrogrifo precolomboniano. La linea che compone il disegno si curva, compie cerchi, per poi tornare nuovamente indietro e ripercorrere parallelamente il tracciato di un'altra, poi se ne distacca, ricerca, ricurva e ritorna indietro.

La realizzazione evoca il tema del labirinto, tanto caro fin dai tempi remoti alla storia del giardino ed ivi collocato da sempre, ma che qui perde il suo senso di inquietudine e di smarrimento per l'alta visibilità del percorso.

È il tema del percorso della vita, della contrapposizione e della continua attrazione che invece guida l'intero andamento del segno: il contrasto tra il mondo femminile e quello maschile, tra la vita e la morte, temi dominanti dell'intero giardino. $\mathrm{Al}$ momento che ci allontaniamo da esso, in un punto di vista che lo abbraccia tutto, ne comprendiamo il disegno: "l'atto creativo fra Cosmo e Natura, origine di ogni forma di vita"15. 
I giocolieri (1985)

Autore Daniel Spoerri

È un complesso di sculture che rappresentano ognuna una particolare figura umana (il poeta, il prete, il perseguitato, il maschio, la donna nell'atto di grattarsi, il cavaliere, l'ufficiale delle SS). Questi giocolieri sono disposti intorno ad una palla, rappresentazione simbolica del mondo.

L'intera figurazione del "gioco", il tentativo appunto di appropriarsi della palla, è collocata tra l'erba del grande prato centrale della proprietà.

Il corpo di ogni scultura è costituito da un cerchio, vuoto al suo interno: ne risulta un elemento leggero e dinamico che sembra inseguire la pendenza del terreno collinare, mentre il colore patinato del bronzo giunge ad una perfetta armonia ed integrazione con le tonalità dell'ambiente circostante.

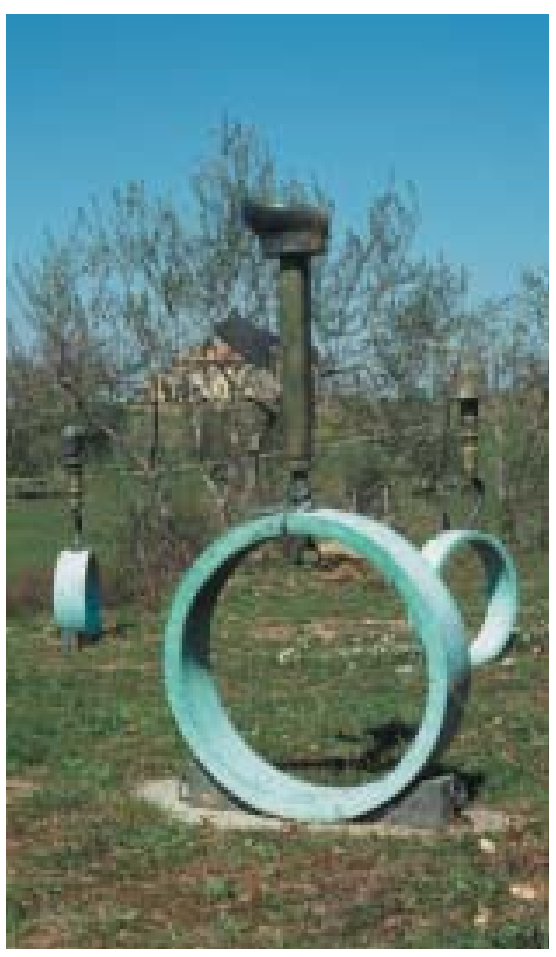

Figura 12 - Particolare de I giocolieri: L’ufficiale delle SS.

\section{RIFERIMENTI BIBLIOGRAFICI}

Argan GiUlio Carlo, L'arte moderna 1770/970, Sansoni editore, Firenze I edizione 1970, II edizione 1971.

Feliziani Manuela (a cura di) Il Giardino di Daniel Spoerri - HIC TERMINUS HAERET, Sito web, URP provincia di Grosseto, 1998.

MaZZANTI ANna (a cura di), Il giardino di Daniel Spoerri, Maschietto\&Musolino, Siena 1998.

MazzANTI ANNA (a cura di), Il Giardino di Daniel Spoerri - Ultime Installazioni, Maschietto\&Mugolino, Siena 2000.

MaZZANTI ANNA, Daniel Spoerri e la Natura. Storia di un rapporto artistico conflittuale attraverso il Giardino del Monta Amiata, "Architettura del Paesaggio", 7, Alinea editrice Novembre 2001, pagg. 58-60.

TAGLIOLINI AlESSANDRO, Storia del giardino italiano, La Casa Usher, Firenze 1988.

ZoppI MARIELLA, Storia del giardino europeo, editori Laterza, Bari 1995.

\section{RIFERIMENTI ICONOGRAFICI}

Le fotografie sono di Emanuela Morelli, la nota scritta della Figura 9 è tratta da: MAZZANTI ANNA (a cura di), Il giardino di Daniel Spoerri, Maschietto \& Musolino, Siena 1998, pag. 57. 
SCHEDA DI PROGETTO

Tipo di intervento:

Località:

Proprietà:

Progettista e collaboratori:

Anno di realizzazione:

Superficie complessiva:

Specie arboree impiegate: giardino realizzato da artista contemporaneo

Fattoria Il Giardino, comune di Seggiano, provincia di Grosseto

Daniel Spoerri

Daniel Spoerri è autore e regista della propria opera. Il giardino è comunque composto da oltre una settantina di sculture, gran parte realizzate da lui ma anche da altri artisti, circa una ventina, quasi tutti di area svizzero-tedesca e di età diverse, che sono quelli a lui più cari e compagni di vita, come Topor, Eva Aeppli e Jean Tinguely.

Il percorso botanico è curato dall'architetto paesaggista Irma Beniamino

La realizzazione è cominciata con l'acquisto nel 1991 della proprietà da parte di Daniel Spoerri: Il 25 luglio del 1996 è diventata Fondazione (Hic Terminus Haeret) ma il giardino è in realtà ancora in continua evoluzione

La superficie della Fondazione e di circa 16 ettari. In realtà il giardino era parte di una proprietà agricola più vasta degli Ugurgeri, una famiglia senese, presente qui da prima del 1300

Sono stati effettuati delle operazioni di recupero sulla vegetazione autoctona e presente e di alcune aree coltivate, in specie gli oliveti ed i frutteti. Siamo ad una quota di $650 \mathrm{~m}$. sul livello del mare, e la vegetazione presente è caratterizzata, dalla roverella, Quercus pubescens, castagno, Castanea sativa, coltivazioni di olivi, Olea europea 'Olivastra Seggianese' e macchia tipica boschiva, tra cui la rara specie endemica italiana, crespolina, Santolina etrusca, che cresce spontaneamente solo in Toscana, Umbria e Lazio. 



\title{
L'HORTUS CONCLUSUS A BENEVENTO
}

\author{
Enrica Dall'Ara
}

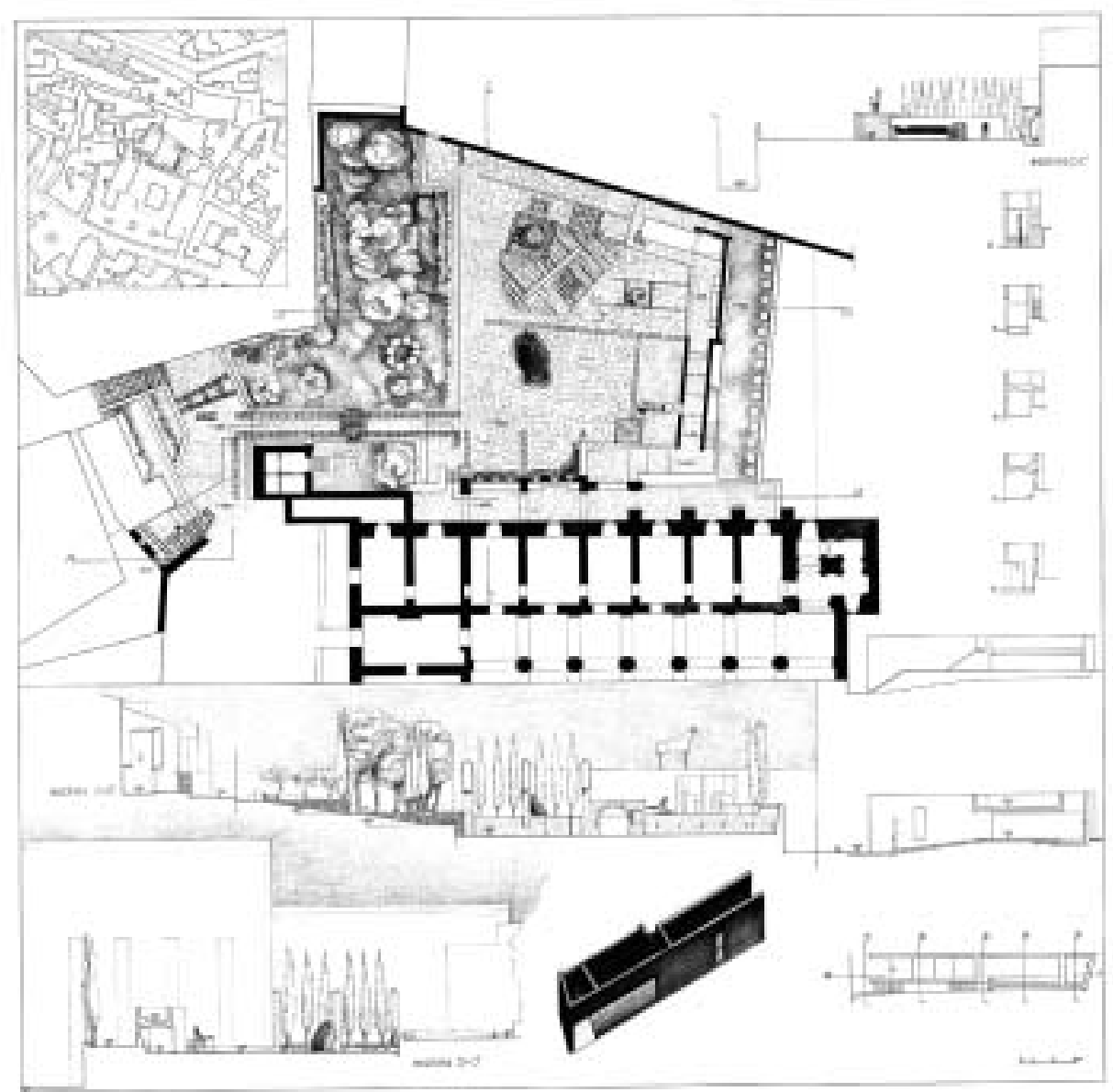

Figura 1 - Elaborati progettuali: planimetria, prospetti e sezioni, con assonometria della galleria.

\section{IL CONTESTO}

All'artista Mimmo Paladino era stato chiesto di realizzare un gruppo scultoreo per la propria città, Benevento. Egli appartiene, con Sandro Chia, Francesco Clemente, Enzo Cucchi, Nicola De Maria, alla corrente, riconducibile al movimento Post-Modern, che il critico Bonito Oliva definisce sulla rivista "Flash Art" Transavanguardia, nel novembre del 1979, significando un riscontrato allontanamento dal darwinismo linguistico delle avanguardie del secondo dopoguerra nell'assunzione di un "atteggiamento nomade di reversibilità di tutti i linguaggi del passato" ${ }^{1}$. A questa labilità del discorso, guidato da un "pensiero debole" quale proposto, in Italia, dai filosofi Gianni Vattimo e Pier Aldo Rovatti, si affianca un 
orientamento verso soluzioni neo-espressioniste, a causa di una razionalità non cartesiana, che cede spazio alla tensione, alla fisicità, alla "primarietà"

L'occasione per l'esposizione delle opere di Mimmo Paladino a Benevento è offerta dal restauro più ampio, di cui è curatore l'architetto Palmieri, legato al recupero funzionale dell'ex-convento di San Domenico utilizzato precedentemente come sede del Tribunale e attualmente sede amministrativa della nuova Università di Benevento.

\section{L'OBIETTIVO}

L'artista invita l'architetto Roberto Serino alla realizzazione, per ideazione congiunta, di un luogo il cui "tema architettonico è quello della ridefinizione dei "margini" entro i quali mettere in scena la rappresentazione dell'arte": lo spazio che nel Seicento era un orto pensile diviene hortus conclusus.

\section{LA METODOLOGIA}

La formazione del temenos ${ }^{3}$ - all'interno è uno spazio ieratico in cui le sculture dell'artista sono sia genitrici sia sacerdotesse - è perseguita, su un lato, mediante la costruzione di una doppia parete che costituisce una galleria, continuazione di un vecchio passaggio di accesso, recuperato.

Il recinto è immaginato come spazio percorribile: dalla prospettiva che apre sul giardino le figure vengono anticipate ma subito negate per l'itinerario che devia. La galleria ha funzione di limbo. Intende realizzare un luogo di distacco che consenta di lasciare la contingenza della città. Attraverso di essa l'orto stesso si allontana e non è possibile percepire nessun traguardo oltre il blu assorbente della parete di fronte, da cui si riversa acqua come da una fonte sacra.

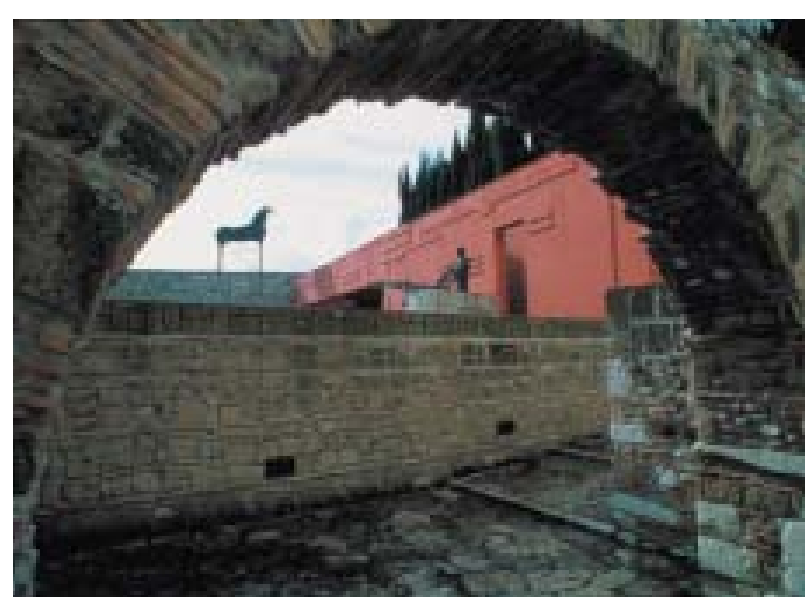

Figura 2 - prospettiva che anticipa le figure del giardino.
Una finestra fa riapparire, transitando, l'interno, senza consentire l'accesso.

La scultura che si oppone al passaggio assume il compito di allungare il periodo della percorrenza, avvisando che gli eventi successivi avranno un carattere di alterità: questo fornisce giustificazione all'estraneazione temporale.

Ulteriormente è possibile, tramite le scale che conducono ad un giardino degli odori, indugiare per altri spazi.

Lideazione sembra muovere attorno alla volontà di creare un'anti-camera necessaria alla scoperta delle opere di Paladino che si riveleranno, per le forme sintetiche, quasi archetipiche e slegate dal contesto. Allo stesso tempo saranno evocatrici di una civiltà antica - quella dei Sanniti che fondarono Benevento - animate da una fantasia mitologica, lungo un tracciato di continuità culturale ${ }^{4}$.

2 Il concetto di primarietà è ripreso da Flavio Caroli, I Mostri di una domenica tedesca, in La giovane pittura in Germania, catalogo, Galleria d'Arte Moderna, Bologna, 1982, e da FlaVIO CAROLI, Il Magico primario, in Magico primario, Fabbri, Milano 1982, cit. in LEA VERGINE, L'arte in trincea - Lessico delle tendenze artistiche 1960-1990, Skira, Milano 1996, pagg. 246-247.

${ }^{3}$ Il termine viene riferito ad un altro progetto di Roberto Serino - la chiesa, non realizzata, per Gibellina - in Benedetta Gravagnuolo, Architetture scolpite con la luce: opere di Roberto Serino, "Controspazio", 6, Gangemi Editore, Roma 1994, pagg. 34-46.

4 "Dal Neo-espressionismo la Transavangurdia prende il concetto di immagine ridotta ai suoi elementi espressivi fondamentali, realizzati con tratti violenti e colori accesi. Ma contemporaneamente se ne distacca per una visione meno drammatica ma più solare. In particolare per quel gusto tutto mediterraneo di un'affabulazione sensuale che si ritrova soprattutto in Paladino. (...) Nella Transavanguardia troviamo infatti il ricorso alla memoria e alla citazione, con la scelta optata verso la tradizione delle 


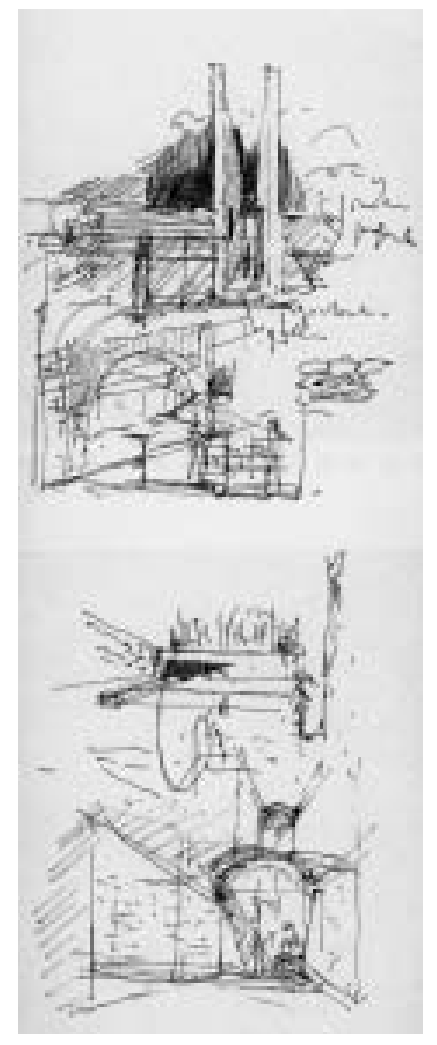

Figura 3 - Schizzi per la galleria.

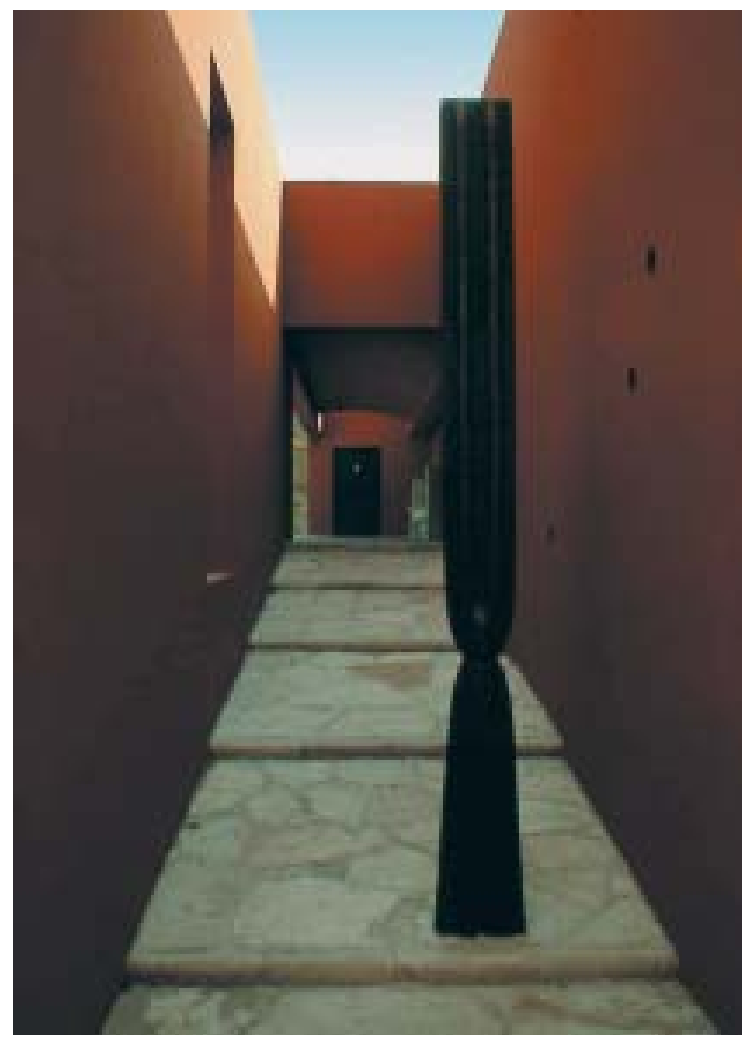

Figura 4 - La galleria.

\section{GLI ESITI}

Dopo il preludio della galleria l'accesso avviene improvviso, lateralmente, mediante un portale ampio, che immette nella piazza dei "Rimandi". È una superficie di pietra ad opus incertum, dal sapore antichissimo, con una sensazione quasi tattile, rugosa, variabile nella tessitura, mentre lo sguardo gira a indagarla nella sua interezza. Su questa superficie poggiano, ruotando con movenze lentissime - in realtà è l'osservatore a spostarsi nell'esplorazione - un disco al centro, una conchiglia, una campana, una testa equina, un lungo teschio di bucranio ${ }^{5}$. Il rapporto con un ambiente consueto è sensibile solo nel giardino, progettato da Palmieri, sul quale sono presenti i tetti di altri edifici, ed in qualche modo richiamato da un ombrello rovesciato a terra ai piedi di un albero che spacca, esile, la crosta di pietra.

Il cavallo rivolto oltre il confine dell'orto, a vedetta, proietta verso cose assenti all'interno, stabilendo un potere sulla città invisibile. Unitamente ai colori primari del rosso e del blu cobalto contro lo sfondo di cipressi, la sua maschera d'oro e la sagoma nera ritagliata definiscono un'iconografia bidimensionale e statica, quasi fosse una raffigurazione arcaica di sovranità. La statua oggettiva la poetica di Paladino: “(...) Ogni

Avanguardie storiche. Memoria e citazione che rimangono però legati esclusivamente allo stile. I riferimenti poetici, specie in Paladino sono ben altri. Essi riguardano la riscoperta della memoria "profonda", quella dove, con spirito quasi metafisico, le forme restano fisse in istanti senza tempo. Devitalizzati dalle tensioni contingenti, per mostrare l'essenza delle cose, le forme stesse si riducono a quel nocciolo duro che più duramente e lentamente il tempo riesce a scalfire (...). (Nell'opera di Paladino) ricorrono immagini che rimandano ad un universo arcano e primitivo, dove le forme sono tradotte in segni eleganti e semplificati." Da Mimmo Paladino, in www. Francescomorante.it/pag_3/318a.

${ }^{5}$ Le statue dell' Hortus Conclusus non hanno un titolo assegnato dall'autore coerentemente alla volontà di identificare l'intero spazio come opera unica. I nomi utilizzati hanno solo un valore descrittivo-identificativo. 


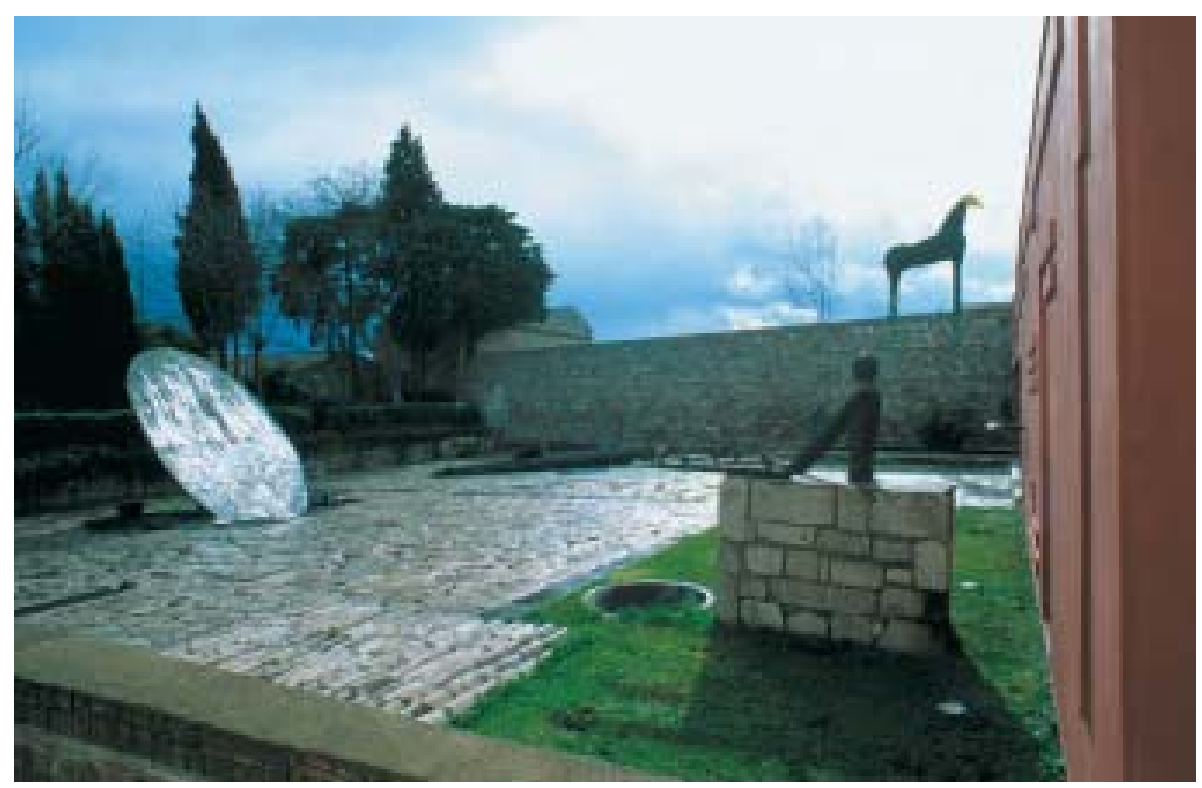

Figura 5 - Interno dell'Hortus conclusus.

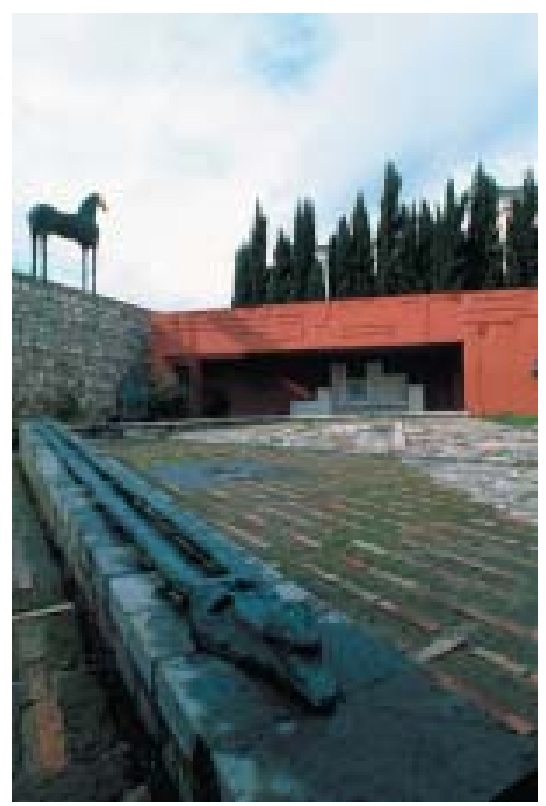

Figura 6 - Interno dell' Hortus conclusus.

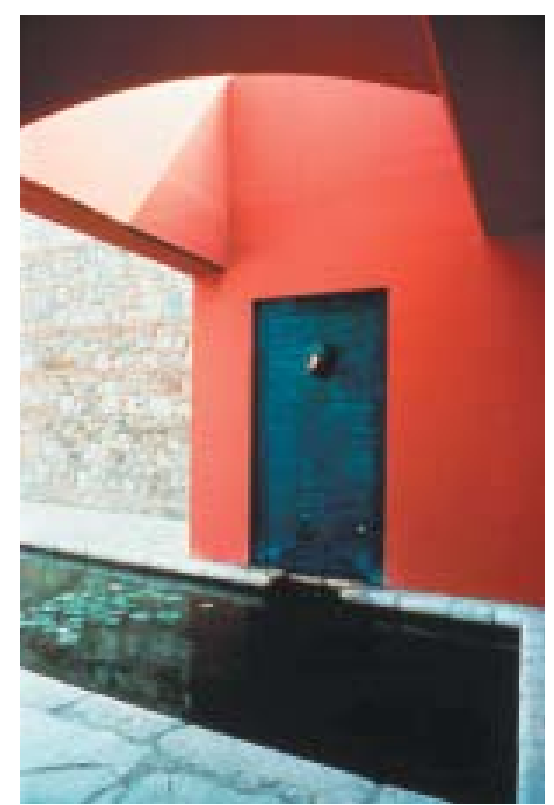

Figura 7 - Acqua dal blu cobalto.

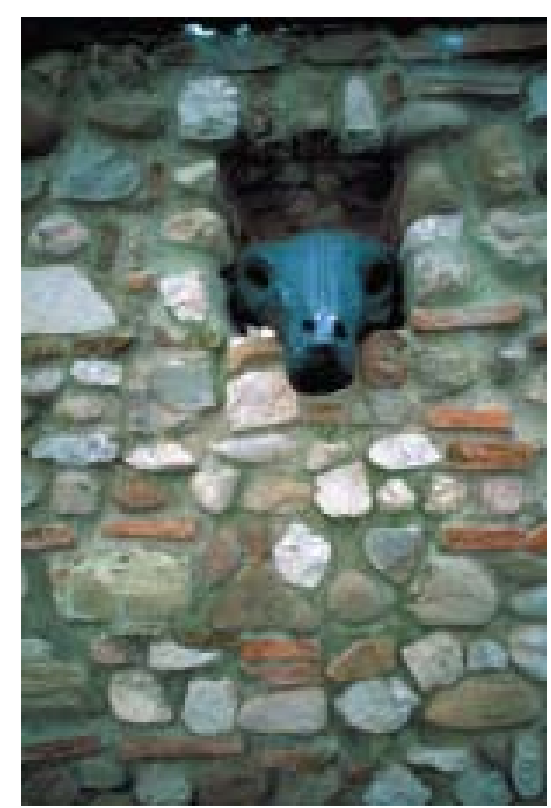

Figura 8 - Figura nell'Hortus conclusus.

mio lavoro è sempre e totalmente in equilibrio, nel senso che il movimento non è mai dichiarato (...). Io mi considero un artista sospeso ad un filo: posso cadere da un momento all'altro, ma non voglio cadere da nessuna delle due parti"'.

Osservandole nei rimandi coreografici, le sculture di Paladino potrebbero raccontarsi qualcosa come le fanciulle de "Il Marinaio"7 di Fernando Pessoa.

Anche lo spazio dell' Hortus sembra volere ospitare un "dramma statico in un quadro"8 conferendo un significato intenso al suo essere conclusus.

Ogni elemento attribuisce fissità.

${ }^{6}$ Da Intervista con Michele Bonuomo, in Terrae motus, catalogo, Electa, Napoli 1984, cit. in LEA VERGINE, L'arte in trincea - Lessico delle tendenze artistiche 1960-1990, Skira, Milano 1996, pag. 250.

${ }^{7}$ FERnANDo Pessoa, Il marinaio, Einaudi, Torino 1988.

${ }^{8}$ Così Ferdinando Pessoa definisce Il marinaio. Il titolo originale dell'opera è O Marinheiro. Drama estatico em um quadro. 


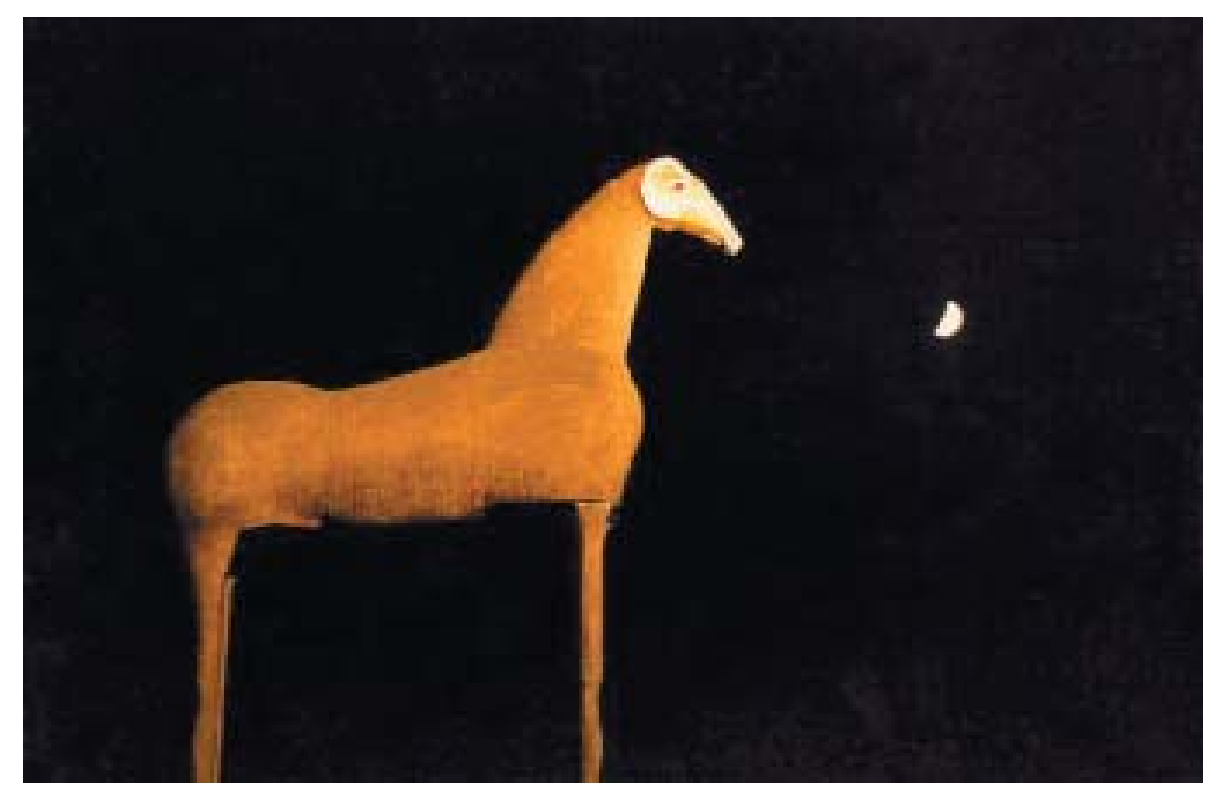

Figura 9 - Il cavallo.

Seppure l'acqua sgorga ovunque non c'è trasformazione, in quanto la terra immediatamente la inghiotte, scivolata sul disco. Dal muro blu della galleria si stende, piatta, in un rettangolo fermo su cui si duplica il profilo immobile del cavallo. Una figura stabilizza i gomiti di braccia troppo lunghe su un piano di pietra, per lasciarsi cadere acqua dalle mani, senza un gesto, mentre nell'erba si dispongono colonne in serie orizzontale, impossibilitate al crollo. Semplicemente l'acqua itera l'esistenza di queste divinità.

Se sia fisica oppure onirica non è chiaro e si creano le componenti di cui sono costituiti i miti: realtà energiche conosciute mediante una fantasia primitiva.

Oppure è il sogno che si materializza?

"PRIMA VEGLIATRICE: L'ora non è mai suonata.

SECONDA VEGLIATRICE: Non potevamo sentirla, non ci sono orologi qui vicino. Tra poco dovrebbe essere giorno.

TERZA VEGLIATRICE:. No, l'orizzonte è buio.

PRIMA VEGLIATRICE: Non desiderate, sorelle mie, che ci intratteniamo raccontando quel che siamo state? È bello ed è sempre falso.

SECONDA VEGLIATRICE: No, non ne parliamo. E poi, siamo state qualcosa?

PRIMA VEGLIATRICE: Forse. Non lo so. Ma ad ogni modo è sempre bello parlare del passato. Le ore sono colate e noi abbiamo serbato il silenzio. Io, da parte mia, sono rimasta a guardare la fiamma di quella candela. A volte trema, a volte diventa più gialla, a volte impallidisce. Non so perché succeda questo. Ma forse che noi sappiamo, sorelle mie, perché le cose succedono?" ${ }^{10}$.

\footnotetext{
${ }^{9}$ Il particolare rapporto fra realtà inconscia e realtà materica proprio della Transavanguardia è delineato in maniera lucidissima da Giulio Carlo Argan: "La Transavanguardia rivendica la parte del corpo anche come veicolo di energie dell'inconscio. Le stesse immagini conservano una pesantezza fisica, rimangono impastate con la materia del colore. Non trascorrono con la labilità propria delle immagini della televisione o della pubblicità, ristagnano e costringono a certi tempi di osservazione. La natura di quelle immagini è ambigua: da un lato sono appesantite dalla materia pittorica, dall'altra inconsistenti come nomi, quasi si volesse provare visivamente il nesso lacaniano di inconscio e linguaggio. Il ristagno è come una ripetizione e con la ripetizione l'immagine si altera e corrompe, diventa abituale, la si riconosce senza neppure guardarla, ma proprio perciò finisce per assumere un senso vagamente mitico. Infine ci si accorge che ha una sua vitalità soltanto fisiologica, ma tenace (...).", in Achille Bonito Oliva (a cura di), Avanguardia Transavanguardia, Electa, Milano 1982, cit. in LeA VERGINE, L'arte in trincea - Lessico delle tendenze artistiche 1960-1990, Skira, Milano 1996, pag. 243.

${ }^{10}$ Da Fernando Pessoa, Il marinaio, Einaudi, Torino 1988, pag. 5.
} 


\section{RIFERIMENTI BIBLIOGRAFICI}

Bonito Oliva Achille (a cura di), Avanguardia Transavanguardia, Electa, Milano 1982.

Cappiello Vito, Architettura contemporanea in Campania, “D’A”, 13, pag. 34 e pagg. 36-38.

Croiset Pierre Alain (a cura di), "Casabella”, numero monografico Il disegno degli spazi aperti, 597-598, Milano 1993, pag. 44.

De Fusco Renato, La Campania - Architettura ed Urbanistica del Novecento, in G. Pugliese Carrattelli (a cura di), Storia e civiltà della Campania, L'Ottocento e il Novecento, Electa, Napoli 1995, pag. 121 e pagg. 146-147.

Gravagnuolo BenedetTa, Architetture scolpite con la luce, "Controspazio", 6, Gangemi Editore, Roma 1994, pagg. 34-36.

Gravagnuolo Benedetta, Hortus Infinitus, "Architettura e Arte”, 3, 1998, pagg. 58-61.

Hortus Conclusus. Almanacco dell'Architettura Italiana, Electa, Milano 1993, pagg. 62-63.

LiaO Alice, Hortus Conclusus, in "www. Lightforum.com/magazine", 2000-2001.

NeOnato Francesca, Un recinto sacro, "Acer”, 6, Il Verde Editoriale, Milano 2001, pagg. 32-35.

Pansera Anty e Vitta Maurizio, Guida all'Arte contemporanea, Marietti, Casale Monferrato 1986.

VERGINE LEA, L'arte in trincea - Lessico delle tendenze artistiche 1960-1990, Skira, Milano 1996.

Siti web:

Hortus Conclusus, in <www.Comune.benevento.it/Turismo/ Hortus $>$.

Hortus Conclusus, in <www.Francescomorante.it/pag_3/318ab $>$.

Mimmo Paladino, in <www.Francescomorante.it/pag_3/318a $>$.

Post modern, in <www.Francescomorante.it/pag_3/318>.

\section{RIFERIMENTI ICONOGRAFICI}

Figure 1-8: per gentile concessione di Roberto Serino.

Figura 9: foto Le Pera, da Hortus Conclusus, www.Comune.benevento.it/Turismo/ Hortus.

Figura 10: per gentile concessione di Roberto Serino.

\section{SCHEDA DI PROGETTO}

Tipo di intervento:

Committente:

Progetto e direzione lavori:

Progetto illuminazione:

Impresa di costruzione:

Superficie:

Tempi: recupero di un orto pensile e progettazione di un giardino pubblico - spazio espositivo

Amministrazione Comunale di Benevento

Mimmo Paladino

Arch. Roberto Serino

con Pasquale Palmieri

Filippo Cannata - Filippo Cannata \& Co.

Donato Callisto

Vivai Zaini di Durazzano (BN)

$2.200 \mathrm{mq}$

Progettazione 1991

Realizzazione1992 
IL RECUPERO AMBIENTALE DI UN ATTIVITÀ ESTRATTIVA

\title{
DISMESSA DA LUOGO DI PRODUZIONE MATERIALE A LUOGO \\ DI PRODUZIONE CULTURALE: CAVA BORELLA A VAGLI
}

\author{
Sabrina Tozzini
}

\section{IL CONTESTO}

Situata nel comune di Vagli di Sotto (provincia di Lucca) ed all'interno del Parco Naturale delle Apuane, la cava Borella appartiene al sistema ambientale della Val d'Arnetola, una formazione valliva minore dell'alta Garfagnana, formata da un torrente affluente dell'Edron, costituendo una realtà particolare, soprattutto grazie alla propria morfologia di valle parallela alla principale, quella del Serchio. Racchiusa tra la linea di cresta principale delle Apuane ed una seconda catena di contrafforti ed esposta a nord, ha scoraggiato nei secoli la sua messa a coltura. Facendo parte del demanio comune degli abitanti di Vagli, è stata principalmente governata a pascolo e ceduo, compatibilmente al regime di Uso Civico, che impone la rinnovabilità della risorsa, ma anche, secondo le testimonianze archivistiche, al "cavar pietre" per l'utilizzazione diretta dei cavatori, attività che la legislazione Estense, sotto il cui controllo si trovava il Comune di Vagli, consentiva anche nei terreni demaniali.

La sua posizione naturalmente isolata, il suo uso parsimonioso per la necessità di lasciare il capitale ambientale anche alle generazioni future, hanno permesso la conservazione dei caratteri originari del luogo, almeno fino all'apertura delle cave alla metà del Novecento, e comunque, dato il carattere tutto sommato poco intensivo che esse hanno assunto e pur con le discrepanze relative alla loro presenza, anche fino ai nostri giorni. Infatti si tratta sì di un'area a forte vocazione marmifera, ma ben diversa da quella più a nord, controllata dalla Provincia di Massa e Carrara, dove l'industria del marmo ha ormai assunto proporzioni gigantesche anche rispetto alla risorsa stessa.

L'ambiente geomorfologico è di tipo Carsico e vi si possono ammirare abissi ed inghiottitoi, mentre la vegetazione appartiene alla serie del faggio, che ora si addensa in formazioni boschive frammiste di conifere ed ora si distribuisce in esemplari sparsi sui pascoli, aprendosi completamente sui calcari nudi ed i vaccineti delle vette.

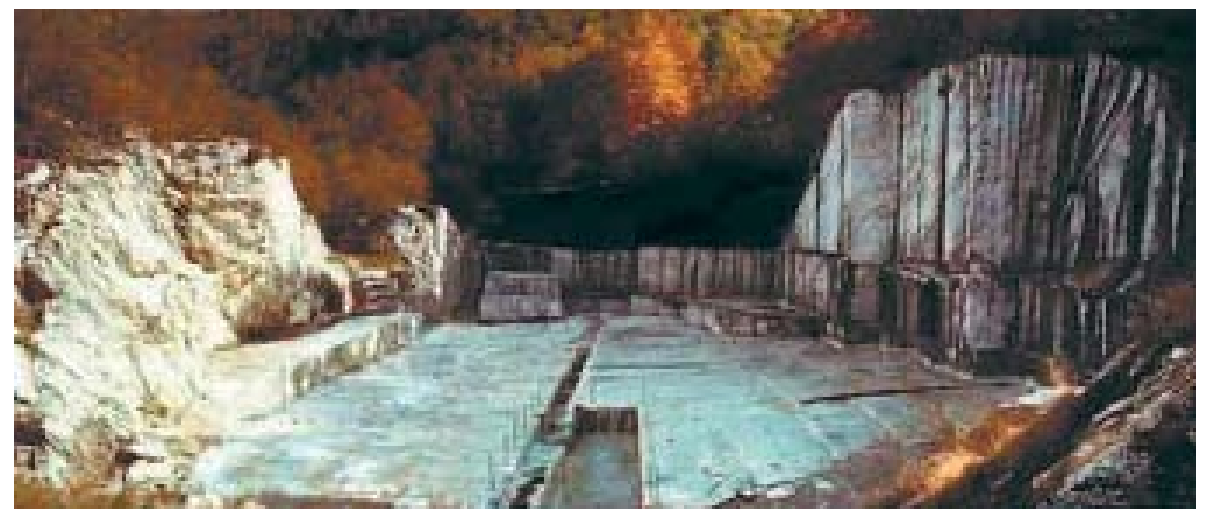

Figura 1 - Visuale della Cava Borella. 
Numerose ed interessanti le presenze storiche, tra percorsi luoghi di culto ed insediamenti pastorali, ma anche testimonianze della tecnologia degli antichi cavatori e delle prime industrie marmifere, che un recente progetto vorrebbe raccogliere e illustrare in una cava vicina.

\section{GLI OBIETTIVI}

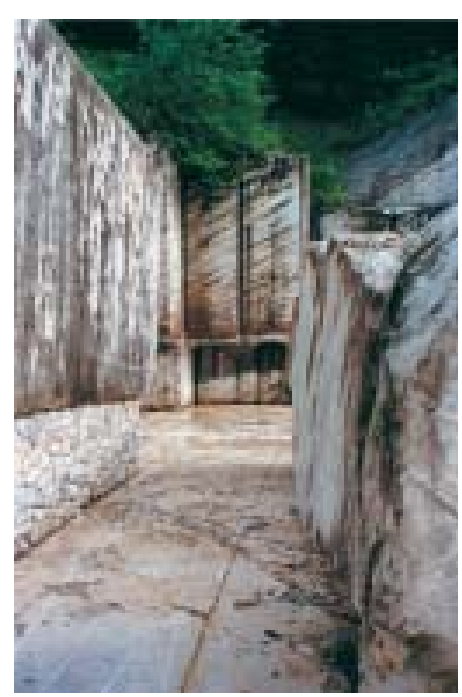

Figura 2 - L'ingresso alla cava.

Il progetto è stato promosso per la parte artistica da Maurizio Guidi e Andrea Tesseri, creatori del progetto EVOCAVA, l'associazione culturale che attualmente si occupa della gestione del luogo, e per la parte di progettazione ambientale dall'architetto Pietro Luigi Biagioni. Finalità dell'intervento ${ }^{1}$ è il recupero ambientale della cava e la minimizzazione dell'impatto della stessa nell'ambiente circostante, attraverso la rimozione del materiale di scarto, i così detti "ravaneti", che raccolgono il pietrisco avanzato dalle lavorazioni, accantonato sul piano di lavorazione della cava oppure gettato dalle pendici dei monti, a creare le tipiche "slavine" bianche visibili ovunque sulle Apuane. Parallelamente agli obiettivi naturalistici è comunque fortemente presente l'istanza di rinnovamento della memoria legata al marmo, ancora così sentita dalle popolazioni locali, per configurarsi non come un corpo estraneo, come brutale sfruttamento di risorse, ma come oggetto della memoria, di un lungo rapporto tra la montagna e la sua gente. Rapporto in evoluzione, che trasforma cultura materiale in cultura artistica e propone nuove interpretazioni del paesaggio, nuove possibili soluzioni per la convivenza aree tutelate e cultura antropica.

\section{LA METODOLOGIA}

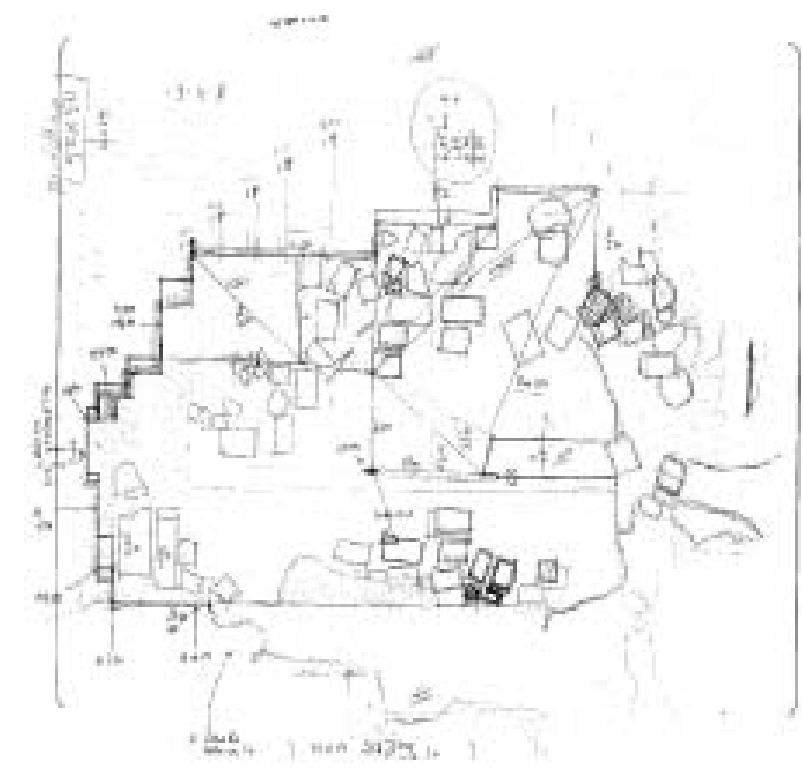

Figura 3 - Rilievo della situazione preesistente.
Completamente improntato al rispetto del sito, i progettisti si sono imposti il metodo del mantenimento di quanto preesistente, limitando gli interventi allo stretto necessario, soprattutto in relazione alla accessibilità del luogo e alla sicurezza dei visitatori. Una "scultura trovata": il titolo della pubblicazione di presentazione suggerisce la chiave di lettura per l'intera opera. La cava consiste in un'apertura di metri 50 per 30 , con una profondità massima di metri 20, ed è stata aperta dentro una collinetta che la contiene completamente. Il tipo di coltivazione che vi si è attuato ha conservato un piano di calpestio orizzontale, a cui si accede da uno stretto ingresso simile ad un canyon scavato nel marmo, che permette di apprezzare ancora di più lo spazio plastico e surreale dell'interno, dove le pareti verticali che la delimitano conservano ancora le tracce delle diverse tecniche di estrazione, più antiche in alto e più recenti in basso. Sono stati mantenuti e valorizzati i vecchi sentieri d'accesso alla cava, posti a quote più alte e quindi non più utilizzati, ma punti di vista di grande effetto verso l'interno.

Sono stati rimossi dal pavimento interno blocchi semilavorati che ostacolavano la visuale dello spazio, ed è stato operato un disgaggio delle parti pericolanti. Particolare attenzione è stata posta alla pulizia e alla messa

${ }^{1}$ Il progetto ha partecipato al Premio Mediterraneo del Paesaggio, promosso dalla Regione Toscana con la Junta de Andalucía e la Région Languedoc-Roussillon, nel 2000. 
in sicurezza della corona della cava, il margine in cui si ricongiungono marmo e vegetazione, la necessaria recinzione è stata ricavata infoltendo la faggeta di bordo e utilizzando rami di faggio intrecciati per maggior protezione almeno fino alla crescita delle nuove piantine messe a dimora. Su una parte del fronte, che invece restava libero dalla vegetazione, si è preferito utilizzare la recinzione metallica comunemente usata dai cavatori per mezzo di ancoraggi d'acciaio inseriti nella roccia e cavi metallici.

L'illuminazione della cava non è di tipo stabile, ma segue piuttosto le esigenze sceniche degli eventi da rappresentare, mentre l'approvvigionamento elettrico viene garantito dalla presenza dell'originale cabina ENEL, restaurata. Si è reso necessario anche un ripristino della viabilità, che conduce $\mathrm{i}$ visitatori a piedi dal paese di Vagli di Sotto alla Val d'Arnetola.

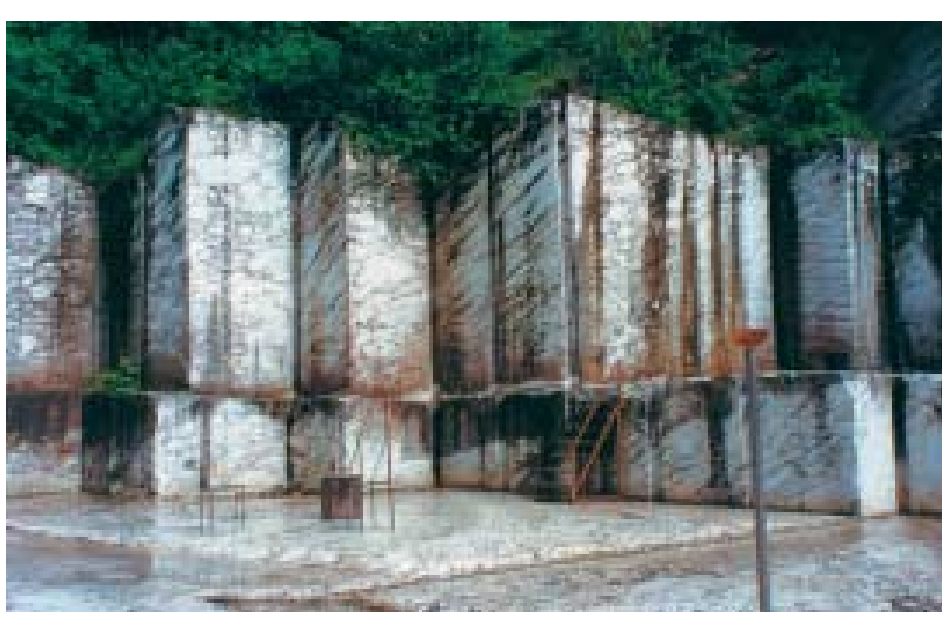

Figura 4 - Veduta della cava all'interno.

\section{GLI ESITI}

Pur trattandosi di un intervento "leggero", di una rilettura di una testimonianza storica più che di un progetto in senso stretto, l'esperienza ha aperto un interessante campo di riflessione sul tema naturalità e presenze antropiche. In particolar modo il caso è interessante per localizzazione interna al Parco Naturale delle Alpi Apuane e, pur essendo nato da un'iniziativa esterna, si colloca perfettamente nell'ambito della gestione strategica del parco stesso. Notevole il successo di pubblico che ha ottenuto, nonostante l'iniziale titubanza dell'ammi-nistrazione comunale di Vagli, che conoscendo l'asperità del proprio territorio e la non facile accessibilità del luogo, temeva che si costruisse la proverbiale "cattedrale nel deserto"2.

"Dal settembre 1998 lo spazio della cava ha ospitato attività culturali che si articolano in due settori, uno di produzione artistica e l'altro orientato alla didattica. Ancora prima che i lavori fossero completamente conclusi sono stati allestiti nella cava tre spettacoli teatrali e una mostra di sculture sul tema del marmo"3.

Tale successo è dato sicuramente dalla forza evocativa che la cava stessa, nelle sue particolarità morfologiche di realtà suggestiva ed insieme raccolta, racchiude in sé, ma anche nella capacità dei progettisti di esplicitare la coscienza di un valore culturale endogeno, profondamente condiviso dalle popolazioni locali, ma anche dai visitatori esterni, che trovano qui un senso di riconciliazione tra uomo e natura. Si tratta infatti di un progetto paesaggistico che è anche un'operazione di archeologia industriale, valorizzazione di una memoria che travalica simbolicamente i limiti del locale, ed entra a far parte del patrimonio culturale comune della nostra società post-industriale.

\footnotetext{
${ }^{2}$ Dichiara il Sindaco Agostino Matteo Landi “...pensare addirittura di poter trasformare una zona bellissima ma anche boscosa ed impervia, posta a 1200 metri di quota, in un teatro mi sembrava davvero troppo. Quali artisti avrebbero accettato di impegnarsi nell'impresa? E chi sarebbe andato fin lassù per assistere ad uno spettacolo? Eppure, nonostante tutto, i due appassionati e competenti amici di Castelnuovo riuscirono a convincere l'Amministrazione Comunale. E fu un bene! L'idea EVOCAVA si è andata sempre più affermando. E sempre più numerosi gruppi di Pellegrini della Montagna" hanno risalito la valle di Arnetola per assistere al miracolo di un ritrovato accordo tra arte e natura, cultura e tradizione". Cit. in Associazione Evocava, Cava Borella. Una scultura trovata, Tipolito 2000, Lucca 1999.

${ }^{3}$ Junta de Andalucía, Région Languedoc-Roussillon, Regione Toscana, Premio Mediterraneo del paesaggio PMP 2000, Artes Gráficas, Sevilla 2001.
} 


\section{RIFERIMENTI BIBLIOGRAFICI}

Associazione Evocava, Cava Borella. Una scultura trovata, Tipolito 2000, Lucca 1999. Junta de Andalucía, Région Languedoc-Roussillon, Regione Toscana, Premio Mediterraneo del paesaggio PMP 2000, Artes Gráficas, Sevilla 2001.

Siti web: <www.evocava.it>

\section{RIFERIMENTI ICONOGRAFICI}

Figura 1: Associazione culturale EVOCAVA, sito Internet www.evocava.it Figure 2, 4: fotografie di Antonella Valentini.

Figura 3: Associazione Evocava, Cava Borella. Una scultura trovata Tipolito 2000, Lucca 1999, pag. 8.

\section{SCHEDA DI PROGETTO}

Progetto:

Luogo:

Committente:

Tipologia di recupero:

Superficie:

Progettisti:

Anno di progettazione:

Anno di realizzazione:

Costo intervento: recupero ambientale di una cava di marmo dismessa e suo utilizzo per attività culturali

Val d'Arnetola, comune di Vagli di Sotto, provincia di Lucca

Associazione Culturale Evocava

spazio teatrale ed espositivo

$1.500 \mathrm{mq}$

Progettazione Ambientale Dott. Arch. Pietro Luigi Biagioni Creazione progetto artistico Maurizio Guidi e Andrea Tessieri

1996

1999

$€ 140.000,00$ 


\title{
PAESAGGI STORICI
}

\author{
Antonella Valentini
}

Tra la fine degli anni Sessanta ed i primi anni Ottanta, in Italia avviene la maturazione dell'apparato concettuale che sta alla base della teoria sul restauro dei giardini e della stessa definizione di giardino storico ${ }^{1}$. Principalmente si ha il riconoscimento della storicità di un giardino e la sua identificazione come monumento e bene culturale. Questo processo si realizza autonomamente ed in ritardo rispetto agli studi che hanno condotto a mettere a punto la teoria sul restauro delle opere architettoniche; lo dimostra il fatto che gli inizi del XX secolo sono caratterizzati da ripristini e rifacimenti in stile, come avveniva sui monumenti già dalla metà del secolo precedente. Il passaggio dal concetto generico di "giardino italiano" a quello di "giardino storico" si ha nel secondo dopoguerra ma se ne acquisisce coscienza solo negli anni Settanta, quando l'IFLA (International Federation of Landscape Architects) crea una sezione sui giardini storici e, in accordo con l'ICOMOS (International Council on Monuments and Sites), organizza nel 1971 a Fontainebleau un primo convegno sulla loro tutela. Nel 1981, a seguito dei lavori della VI assemblea generale dell'ICOMOS, nasce la Carta dei Giardini Storici², detta anche Carta di Firenze, che stila la prima definizione di giardino storico accettata internazionalmente: una "composizione architettonica e vegetale che, dal punto di vista della storia e dell'arte, presenta un interesse pubblico. Come tale è considerato come un monumento"3 e dunque, al pari dei monumenti architettonici, deve essere salvaguardato. In Italia un giardino può essere classificato di interesse storico-artistico, diventando quindi oggetto di tutela (e di notifica), secondo due leggi che risalgono al 1939, riunificate recentemente in un unico decreto legislativo ${ }^{4}$. La legge 1089/1939 prevede che siano considerate di interesse storico-artistico "le cose" di almeno cinquanta anni di età e questo garantisce che vi rientrino a buon diritto anche giardini realizzati agli inizi del XX secolo ed in particolare negli anni Trenta, periodo che possiamo considerare l'ultima stagione d'oro per l'arte dei giardini italiana ${ }^{5}$.

Un aspetto importante sottolineato dalla Carta di Firenze è il ruolo fondamentale rivestito all'interno di un giardino storico dalla componente vegetale, che è una

\footnotetext{
${ }^{1}$ Per ripercorrere le tappe di questo percorso, si veda LiONELLA SCAZZOSI, Il giardino opera aperta: la conservazione delle architetture vegetali, Alinea, Firenze 1993, oppure MARIACHIARA POZZANA, Giardini storici. Principi e tecniche della conservazione, Alinea, Firenze 1996.

${ }^{2}$ La Carta dei Giardini Storici, redatta a Firenze il 21 maggio 1981 dal Comitato Internazionale dei Giardini Storici (Comité International des Jardins et Sites Historiques), di cui era presidente Réné Pechêre, è stata registrata il 15 dicembre 1982 dall'ICOMOS. La Carta è suddivisa in quattro sezioni: (a) definizioni e obiettivi, (b) manutenzione, conservazione, restauro e ripristino, (c) utilizzazione, (d) protezione legale e amministrativa.

${ }^{3}$ Carta dei Giardini Storici, 1981, art. 1.

${ }^{4}$ Legge 1089/1939 Tutela delle cose dinteresse artistico e storico e Legge 1497/1939 Protezione delle bellezze naturali. Queste due leggi sono state abrogate con il D.L. 490/1999, che in realtà ne ripropone quasi integralmente i testi.

${ }^{5}$ Nel 1931 a Firenze è allestita una mostra dal titolo "Il giardino italiano", che raccoglie famosi esempi di giardini italiani e che coincide con l'ultima occasione di interesse per questo tema nel nostro paese. All'interno di questa esperienza si parla anche di restauro ed è evidente un atteggiamento che tende al ripristino ed alla ricostruzione di giardini ormai scomparsi utilizzando disegni ed iconografia antica.
} 
componente dinamica, non statica, deteriorabile e rinnovabile ${ }^{6}$. Questa affermazione implica che la vegetazione, in quanto soggetta a modificazioni ed alterazioni, debba essere gestita, curata e quindi anche restaurata, in modo che possa essere mantenuto integro il valore complessivo del bene. Per tenere sotto controllo questo dinamismo la catalogazione della componente vegetale di un giardino storico diventa un momento indispensabile e si realizza attraverso un inventario degli elementi vegetali, che è presupposto conoscitivo e strumento metodologico mediante cui attivare la gestione e la conservazione del nostro patrimonio culturale ${ }^{7}$.

La caratteristica peculiare delle architetture vegetali, il loro essere in continua trasformazione, implica che in mancanza di idonei e periodici interventi si possa innescare in breve termine un processo di degrado. La manutenzione di un giardino è infatti essenziale per la riuscita di un progetto di restauro, il quale non si esaurisce nel giro dei pochi mesi in cui sono effettuati gli interventi, ma dura nel tempo e si costruisce lentamente, assecondando il ritmo naturale della crescita degli elementi vegetali, e può vanificarsi in breve tempo se le cure colturali non vengono eseguite con regolarità e con tecniche adeguate. Un giardino in cui è effettuata una corretta manutenzione ordinaria riduce la necessità di onerosi interventi straordinari, anche se l'equilibrio di un sistema così complesso è condizionato da numerosi fattori esterni ed imprevedibili come i cambiamenti di proprietà, la diminuzione di interesse, la riduzione delle risorse economiche, la presenza di tecnici non specializzati, l'impossibilità di utilizzare mezzi meccanici per le lavorazioni anche più semplici. Il calendario dei lavori di manutenzione, di cui ogni progetto di restauro correttamente redatto si avvale, è lo strumento che garantisce alla proprietà di eseguire gli interventi manutentivi, ma non essendo previsto quale parte integrante del progetto di restauro (non costituisce cioè un elaborato "ufficiale" consegnato alle Sovrintendenze per l'approvazione), viene purtroppo spesso disatteso, come si è verificato nel caso del restauro del giardino di Villa Garzoni a Collodi.

La manutenzione ordinaria non è il solo elemento in grado di sostenere la buona riuscita di un restauro; un altro aspetto da tener presente deriva dal fatto che il giardino storico è un monumento nato per un uso elitario in contesti culturali e sociali completamente diversi da quelli contemporanei; un'opera d'arte "vivente" che più difficilmente delle architetture si presta al riuso. Aprire al pubblico queste strutture così delicate esponendole ad un notevole flusso di visitatori può comportare problemi di conservazione del bene, se non si considera il giardino storico come un vero e proprio museo all'aperto, regolamentandone gli usi, i tempi e le modalità per la visita. È solo negli ultimi anni che una tale politica ha superato l'ostilità della popolazione, che vedeva in questo modo limitare la godibilità del bene, non accorgendosi della necessità di trovare nuovi modi di fruizione per garantire la tutela del patrimonio. Ne è un esempio significativo l'esperienza del giardino di Boboli a Firenze ${ }^{8}$.

${ }^{6}$ L'articolo 2 della Carta dei Giardini Storici recita: "il giardino storico è una composizione di architettura il cui materiale è principalmente vegetale, dunque vivente e come tale deteriorabile e rinnovabile. Il suo aspetto risulta così da un perpetuo equilibrio, nell'andamento ciclico delle stagioni, fra lo sviluppo e il deperimento della natura e la volontà d'arte e d'artificio che tende a conservarne perennemente lo stato".

7 L'esigenza di mettere a punto una strategia comune per la catalogazione del patrimonio vegetale dei giardini storici nasce dalla fine degli anni Settanta, a partire dal convegno di S. Quirico d'Orcia nel 1978. Sono state proposte, da enti diversi, differenti tipi di scheda di analisi - che assieme alla cartografia costituisce l'inventario del patrimonio vegetale - ma la più utilizzata è quella messa a punto dall'ICCD (Istituto Centrale per il Catalogo e la Documentazione) che risulta costituita da una scheda PG, mirata all'acquisizione di informazioni catastali, storiche, fisionomiche, ed un inserto $P G / B$ per la raccolta dei principali dati ambientali, vegetazionali, floristici.

${ }^{8}$ In realtà non doveva essere vinta solo l'ostilità della popolazione, ma la tendenza della cultura urbanistica a considerare anche il verde storico ai fini del soddisfacimento degli standard di verde pubblico come quantità minima di superficie prevista per abitante; atteggiamento che è stato solo in parte superato dalla pianificazione comunale degli ultimi anni. 
Il gruppo italiano del comitato ICOMOS-IFLA ha approvato un documento nel quale si afferma che il giardino storico è "un insieme polimaterico, progettato dall'uomo", la cui natura storica, appunto, si esplicita nel fatto di essere un'opera d'arte che appartiene al passato, ma che deve vivere nel futuro e che, benché "rinnovabile biologicamente, non è riproducibile nella sua complessità polimaterica" ${ }^{\prime 10}$. Sebbene la doppia natura del giardino storico - monumento architettonico e monumento vegetale e dunque in continua metamorfosi - abbia costituito l'ostacolo principale per individuare, all'interno della generale disciplina sul restauro, uno specifico ambito teorico e tecnico relativo alla conservazione dell'opera d'arte "giardino", i recenti sviluppi della materia ${ }^{11}$, hanno contribuito a fugare ogni dubbio circa la necessità di tale operazione, sottolineando che si può pensare al giardino "come a una composizione musicale, che ha una struttura fissa definita nello spartito, ma che viene ripetuta all'infinito e con possibili varianti, rimanendo sempre la stessa opera composta originariamente. Ovviamente il paragone è solo concettuale, essendo il giardino opera d'arte ben diversa, ma l'idea dell'artista, sviluppata nel primo progetto, può essere ripetuta molte volte nel cambiare delle stagioni e nella rotazione della vegetazione, conservando la sua identità iniziale"12. Uno dei principali problemi metodologici, infatti, è la necessità di adeguare l'intervento di restauro del giardino storico alla sua stessa natura di monumento in continua evoluzione, come ben evidenziano le parole di Giovanni Carbonara: "in quanto vivente la sua conservazione ed il suo restauro non potranno prescindere dalle leggi biologiche e genetiche del mondo vegetale; in quanto opera d'arte, da quelle della più generale riflessione sulla tutela dei beni culturali" ${ }^{13}$. È stato spesso evidenziato dagli studiosi della materia ${ }^{14}$, come la conservazione dei giardini, anche se ambito disciplinare giovane e dotato di una propria specificità, sia da riferire a quello ormai codificato della conservazione delle opere d'arte, e sia da definire nella sua unità metodologica ${ }^{15}$.

La Carta di Firenze definisce alcuni principi fondamentali attraverso cui condurre le operazioni di manutenzione e di restauro per la salvaguardia dei giardini storici, per i quali "si può eventualmente raccomandare il ripristino"16, ed in particolare per "...le parti del giardino più prossime a un edificio, al fine di metterne in risalto l'intima connessione" 17 anche se, nei casi in cui "...un giardino è

\footnotetext{
${ }^{9}$ Proposta per una carta del restauro dei giardini storici. Documento approvato all'unanimità dai partecipanti alla tavola rotonda organizzata dall'Accademia delle Arti e del Disegno, Firenze, 12 settembre 1981.

${ }^{10}$ Mariachiara Pozzana, op. cit., 1996, pag. 20.

${ }^{11}$ L'utilizzo delle tecniche archeologiche per il restauro dei giardini storici, che si sono sviluppate a partire dagli anni Ottanta, consente di studiare il giardino come documento di se stesso, al di là della deperibilità dei suoi elementi costitutivi. Il restauro dei Giardini Segreti di Villa Borghese a Roma è avvenuto basandosi su una importante campagna di indagini archeologiche.

${ }^{12}$ Mariachiara PozZana, op. cit., 1996, pag. 22.

${ }^{13}$ Giovanni Carbonara, Problemi di restauro dei giardini storico artistici, in Vincenzo Cazzato (a cura di), Tutela dei giardini storici bilanci e prospettive, Ministero per i Beni Culturali e Ambientali Ufficio Studi, Roma 1898, pag. 184.

${ }^{14}$ Ad esempio da MARCo DEZZI BARDESCHI, Quei giardini fuggitivi come gli anni..., in LIONELLA SCAZZOSI e MAURIZIO Boriani (a cura di), Il giardino e il tempo. Conservazione e manutenzione delle architetture vegetali, Guerini e Associati, Milano 1992, pag. 12.

${ }^{15}$ La teoria di una unità metodologica degli interventi di conservazione è stata impostata da Cesare Brandi agli inizi degli anni Sessanta, ma per quanto riguarda i giardini deve essere ancora chiaramente enunciata. Resta ancora valida, a quarant'anni di distanza, la definizione di Brandi per il quale il restauro "costituisce il momento metodologico del riconoscimento dell'opera d'arte, nella sua consistenza fisica e nella sua duplice polarità estetica e storica, in vista della sua trasmissione al futuro", come pure valgono e possono essere applicati anche all'ambito dei giardini storici, i due principi del restauro da lui postulati e cioè "si restaura solo la materia dell'opera d'arte" e "il restauro deve mirare al ristabilimento della unità potenziale dell'opera d'arte, purché ciò sia possibile senza commettere un falso artistico o un falso storico, e senza cancellare ogni traccia del passaggio dell'opera d'arte nel tempo" (CESARE BRANDI, Teoria del restauro, Einaudi, Torino 1977, I ed. 1963, pagg. 6-8).

${ }^{16}$ Carta dei Giardini Storici, 1981, art. 9.

${ }^{17}$ Carta dei Giardini Storici, 1981, art. 15.
} 
totalmente scomparso o si possiedono solo degli elementi congetturali sui suoi stati successivi, non si potrà allora intraprendere un ripristino valido dell'idea del giardino storico"18. Questo riferimento all'idea del ripristino suscita in molti studiosi ed architetti italiani una forte reazione in nome del principio della irripetibilità della risorsa, primo fra tutti Marco Dezzi Bardeschi e Isa Belli Barsali, la quale, in un suo celebre articolo apparso nel $1983^{19}$, mette in evidenza come ogni giardino sia sempre stato oggetto nel tempo di trasformazioni che costituiscono la testimonianza di una particolare fase della sua storia, ognuna delle quali deve essere conservata. Il principio della conservazione di ogni stratificazione storica è comunque richiamato anche nella Carta di Firenze, costituendo un criterio fondamentale ampiamente condiviso dagli operatori e sul quale si basa ogni intervento di restauro ${ }^{20}$, come testimoniano gli esempi riportati dei restauri dei giardini di Villa Garzoni, di Boboli e di Villa Borghese. Quest'ultima rappresenta un importante esempio di ripristino, basato su numerose indagini conoscitive (archeologiche, d'archivio, della trattatistica, dell'iconografia e cartografia), mirato all'evocazione del giardino storico settecentesco.

In questa sezione sono presentati alcuni casi studio considerati significativi nel panorama italiano: tre riguardano il restauro di parchi e giardini storici, il quarto, il Piano per il Parco dell'Appia Antica, si interessa della conservazione di un paesaggio caratterizzato da valenze storico-archeologiche oltre che naturalistiche e paesistiche.

Il giardino di Villa Borghese e quello di Boboli si distinguono per la loro importanza nella storia dell'arte italiana dei giardini, per la loro magnificenza (legato ai Principi Borghese il primo, alla Famiglia Medici il secondo), e per il loro essere inseriti in contesti fortemente "qualificati" come quelli di due città d'arte, Roma e Firenze, con le quali convivono in rapporto dialettico. Due giardini simbolizzazione del potere, affermazione dell'autorità di principi e nobili che, durante i secoli XVI e XVIII, trovava proprio nei giardini la sua massima espressione. Anche Villa Garzoni è espressione della potenza e degli sforzi economici dei suoi proprietari e creatori; essa rappresenta infatti uno dei molti giardini di ville signorili sorte nel paesaggio rurale a presidio del territorio, in questo caso la campagna toscana e precisamente l'ambito collinare tra Lucca e Pistoia. Diverso è il caso dell'area solcata dalla via Appia Antica che si distingue per la dimensione del territorio interessato (circa 3.500 ettari) e per la presenza di monumenti architettonici e resti archeologici (tombe, acquedotti, torri medioevali, casali rinascimentali, catacombe, eccetera) inseriti nel contesto agricolo e naturalistico tipico dell'agro romano.

Un filo conduttore accomuna questi luoghi, indipendentemente dalla dimensione e dalle loro peculiarità: l'essere paesaggi storici da salvaguardare attraverso accurati progetti di restauro ed attente opere di manutenzione e gestione. Un dato emerge dall'osservazione dei progetti presentati: la necessità di impostare il lavoro sulla base della pluridisciplinarità, cioè la cooperazione ed

${ }^{18}$ Carta dei Giardini Storici, 1981, art. 17.

${ }^{19}$ ISA Belli Barsali, I giardini non si sbucciano, "Italia Nostra", 221, 1983, pagg. 32-36.

${ }^{20}$ Nell'art. 15 della Carta dei Giardini Storici si legge: "L'intervento di restauro deve rispettare l'evoluzione del giardino in questione. Come principio non si potrà privilegiare un'epoca a spese di un'altra a meno che il degrado o il deperimento di alcune parti possano eccezionalmente essere l'occasione per un ripristino fondato su vestigia o su documenti ricusabili." L'idea del ripristino è invece ancora ampiamente dibattuta ed in Italia prevale la tendenza al suo rifiuto. In Europa esistono esempi importanti di ripristino, come il giardino olandese di Het Loo che è stato interamente ricostruito, tra il 1977 e il 1984, sulla base di una ricca documentazione (documenti d'archivio, iconografia, trattatistica) ed attraverso scavi archeologici. La ricostruzione ha suscitato varie critiche in quanto si è andati a sostituire la sistemazione ottocentesca ormai storicizzata con il giardino barocco originale, interessando anche statue e fontane (ricostruite ex novo con materiali moderni) che un efficiente gruppo di giardinieri preposti alla manutenzione si adopera perché siano mantenute costantemente lucide, sottraendo al giardino quella patina di storicità che si formerebbe naturalmente in un giardino storico autentico. 
integrazione di diverse professionalità (architetto, geologo, fitopatologo, agronomo, storico dell'arte, solo per citarne alcuni) che è elemento peculiare di un progetto di restauro, costituendone garanzia di qualità.

In Italia non esiste ancora una sistematizzazione dello strumento preposto alla conservazione del giardino storico, il cosiddetto "piano generale della conservazione" ${ }^{21}$ sul modello inglese o tedesco, benché sia stata tentata una compilazione di norme ed elaborati che ne fanno parte ${ }^{22}$, pur nel riconoscimento dell'estrema variabilità della casistica legata ai caratteri specifici di ogni singolo progetto. Il Master Plan di Boboli è significativo di questa necessità di costruire una visione unitaria e coordinata di tutti gli aspetti e problematiche che contraddistinguono il giardino storico, rappresentando lo strumento di pianificazione degli interventi progettuali previsti.

${ }^{21}$ Con questa definizione si intende "uno strumento che deve fornire le linee generali che costituiscono una guida per l'intervento sul giardino storico, ai diversi livelli e scale del lavoro. Sarà perciò comprensivo di tutti gli interventi necessari su vegetazione e opere d'arte, e riunirà anche indicazioni che possono essere sviluppate in piani di settore cioè di gestione e manutenzione." (MARIACHIARA POZZANA, op. cit., 1996, pag. 159). I modelli di riferimento sono il Master Plan inglese e il Parkpflegewerk tedesco.

${ }^{22}$ Cfr. Mariachiara PozZana, op. cit., 1996, pagg. 159-167. 



\title{
IL RESTAURO del GIARDINO DI VILLA GARZONI A COLlOd*
}

\author{
Antonella Valentini
}

\section{IL CONTESTO}

Il restauro del patrimonio vegetale del giardino di Villa Garzoni a Collodi nel comune di Pescia, in provincia di Pistoia, è stato realizzato agli inizi degli anni Novanta. Tale progetto ha ottenuto un premio dalla Comunità Europea nel 1993, anno dedicato al restauro dei giardini storici ${ }^{1}$.

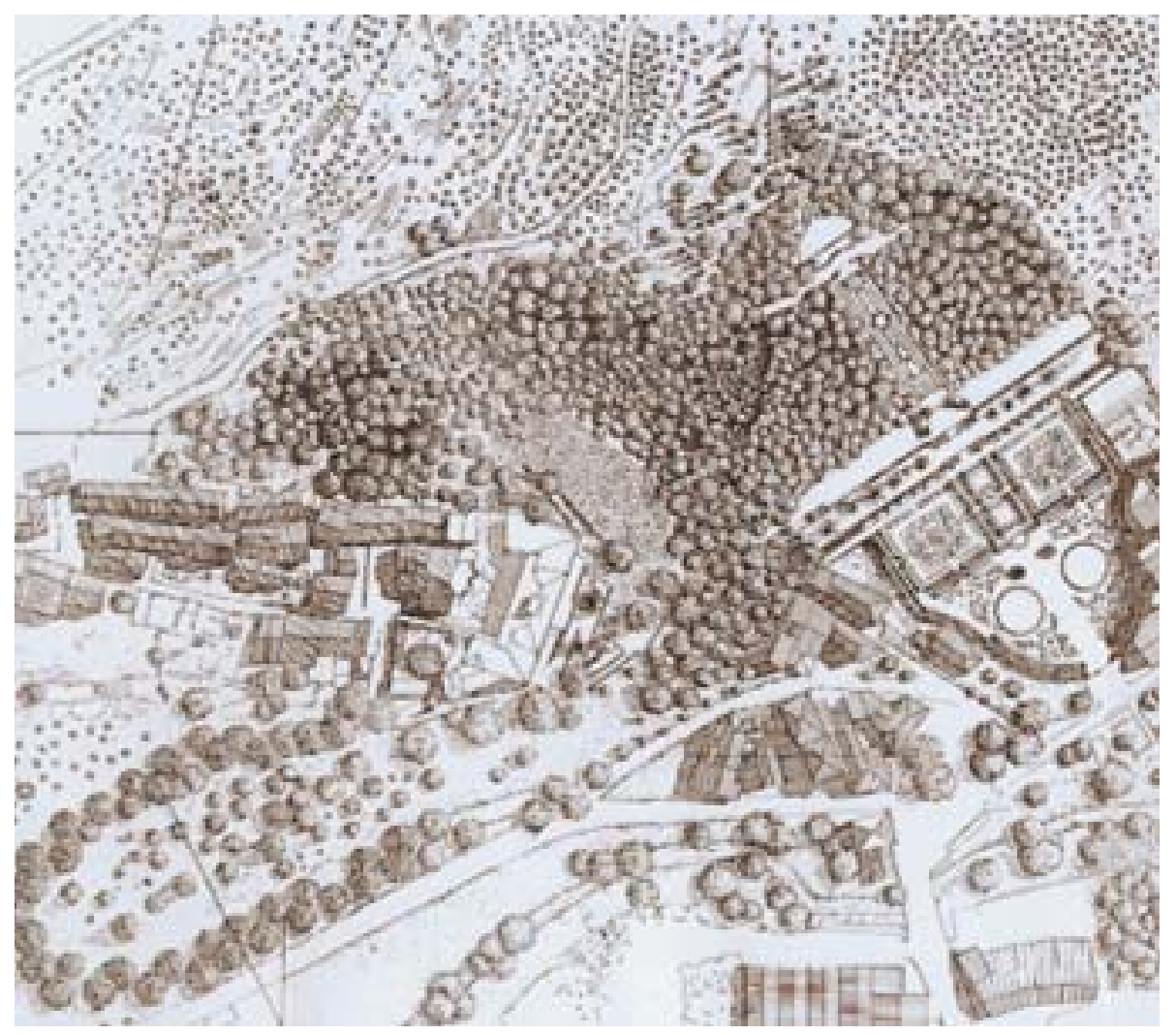

Figura 1 - Planimetria della villa e giardino Garzoni a Collodi.

* Si ringrazia.la Dott.ssa Silvia Martelli per il suo prezioso contributo e la disponibilità dimostrata fornendo documentazione, riferimenti bibliografici e consigli.

${ }^{1}$ Il progetto di restauro della vegetazione è stato redatto dalla Dott. For. Silvia Martelli nel 1992-93 ed i lavori sono stati eseguiti nel 1994. Il restauro della parte architettonica del giardino (percorsi, statuaria) e della villa, affidata allo studio Gurrieri-De Vita, è in corso di progettazione.

Il complesso di Villa Garzoni è stato recentemente acquistato da Enrico Preziosi, titolare dell'omonimo marchio di giocattoli, con l'intenzione di farne un luogo di riferimento internazionale per eventi culturali dedicati all'infanzia, dove possono svolgersi mostre, convegni, spettacoli. L'operazione commerciale-culturale a vari livelli - dalla campagna promozionale di una linea di giochi "Pinocchio" alla promozione di un parco tematico dedicato al burattino di legno in un'area vicina al parco storico - coinvolge direttamente la villa ed il giardino. Per il loro restauro sembra che il Ministero dei Beni Culturali si sia impegnato a finanziare il $40 \%$ degli interventi previsti (costo presunto 50 milioni di euro, di cui 12 per il giardino). Nel 2001 è stato firmato l'Accordo di Programma tra la Regione Toscana, i Comuni di Capannori e Pescia, le Province di 
Il giardino Garzoni è un esempio di giardino scenografico barocco. Nato nel Seicento come grande teatro all'aperto, visivamente autonomo rispetto alla villa, che si trova a lato e non sull'asse della composizione, ha un ingresso indipendente direttamente dalla strada pubblica. Si configura come una scena teatrale di forte impatto visivo, non preceduta da alcuna sistemazione intermedia che la faccia scoprire gradualmente e progettata per essere abbracciata dalla vista con un unico sguardo. Il giardino si adatta alla natura del luogo, sviluppandosi in altezza su un ripido pendio e creando una spettacolare composizione (qui, come all'Isola Bella sul Lago Maggiore o in altri giardini settecenteschi, si conferma il principio barocco della spettacolarità), la cui verticalità è accentuata se vista dal basso, mentre dall'alto, per un particolare effetto illusionistico, le varie terrazze si ricompongono a formare un unico dolce declivio. L'influenza francese, che dalla seconda metà del Seicento in Toscana ed in particolare nella lucchesia è molto sentita $^{2}$, a Villa Garzoni si lega alla tradizione italiana dei terrazzamenti. Il giardino si configura come uno spazio scenico (la sua stessa forma, a campana rovesciata, ricorda quella di un teatro) definito da viali di spalliere di alloro e cipresso, con le quinte ottenute dal Bosco di Lecci, la platea costituita dal prato nel piano corrispondente alla campana rovesciata, il proscenio rappresentato dai terrazzamenti con le grotte, le fontane, le statue. Questo apparato "tendeva alla messa in scena di un viatico di salvazione, enfatizzato dalla ripida salita, culminante col Romitorio, circoscritta da una corona di cipressi che solennizzava il carattere mistico del luogo" ${ }^{\prime 3}$. Un considerevole numero di statue, con soggetti comici, epici, favolistici e mitologici accentua questo carattere teatrale ${ }^{4}$.

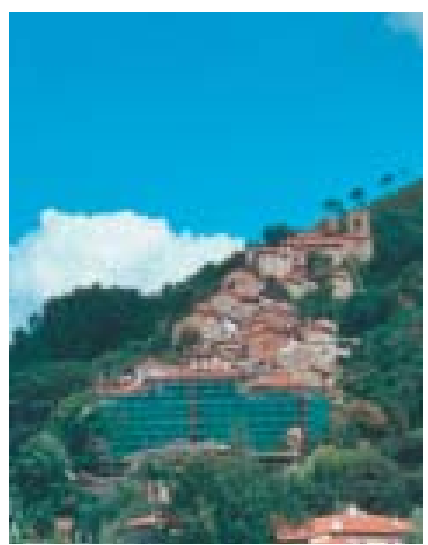

Figura 2 - La villa (in restauro) con Collodi alle sue spalle.

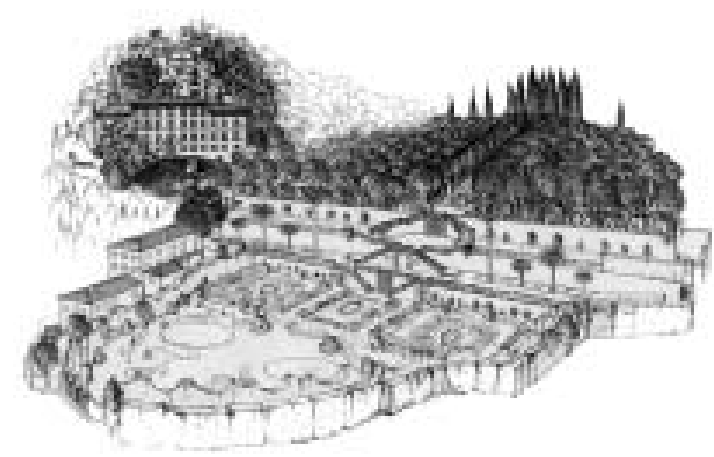

Figura 3 - Vista assonometrica del giardino con la villa e, sul retro, il paese di Collodi.

Lucca e Pistoia, la Fondazione Nazionale "Carlo Collodi” per la realizzazione del "Parco degli amici europei di Pinocchio a Collodi”. Il master plan del parco tematico (di sette ettari di superficie) è stato affidato agli architetti Luigi e Sabina Snozzi e Gustavo Groisman, mentre un "bosco delle meraviglie" è stato progettato dal paesaggista tedesco Stefan Tischer e vari padiglioni ispirati alle diverse favole sono stati ideati da architetti di fama internazionale tra cui Alvaro Siza, Zaha Hadid, Tobia Scarpa.

${ }^{2}$ Nel XVII secolo lo Stato di Lucca vive un momento di particolare splendore e nella sua campagna fioriscono molte ville con i loro giardini che, elementi di transizione tra l'abitazione ed il paesaggio nel quale si inseriscono, sono caratterizzati da imponenti effetti scenografici. Ne sono esempi la Villa Torrigiani a Camigliano, ma soprattutto Villa Pecci Blunt a Marlia.

3 Vincenzo Cazzato e Maria Adriana Giusti, Teatri di Verzura in Italia in Vincenzo Cazzato, Marcello Fagiolo e Maria Adriana Giusti, Teatri di Verzura. La scena del giardino dal Barocco al Novecento, Edifir, Firenze 1993, pag. 103. M. Adriana Giusti rileva come questa idea della sacra rappresentazione che si svela nell'ascesa espiatoria, accomuni il progetto per Villa Garzoni a quello di Carlo Fontana per Villa Chigi di Cetinale in provincia di Siena (1670 ca.), evidenziando un collegamento, ancora tutto da esplorare, tra Roma e l'ambito lucchese.

${ }^{4}$ Il programma iconografico che ha guidato la collocazione e la scelta delle varie statue non è stato ancora completamente decifrato. È stato sottolineato il rapporto del giardino Garzoni con Pratolino e Boboli per la presenza di alcuni elementi (la statua del cinghiale e quella del contadino con il barile) e per la compresenza di mitologia greca e romana con motivi rustici (cfr. FILIPPO PIZZONI, Il giardino arte e storia, Leonardo Arte-Elemond, Venezia 1999). 


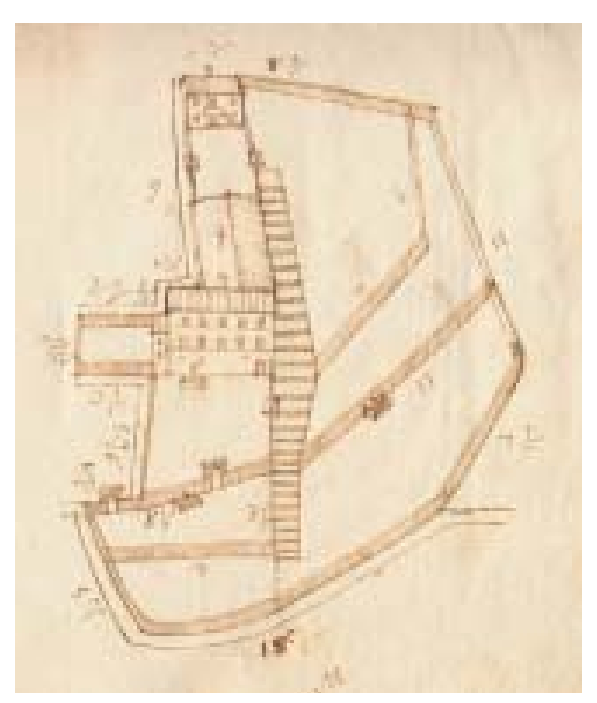

Figura 4 - Planimetria del Martilogio del 1550.

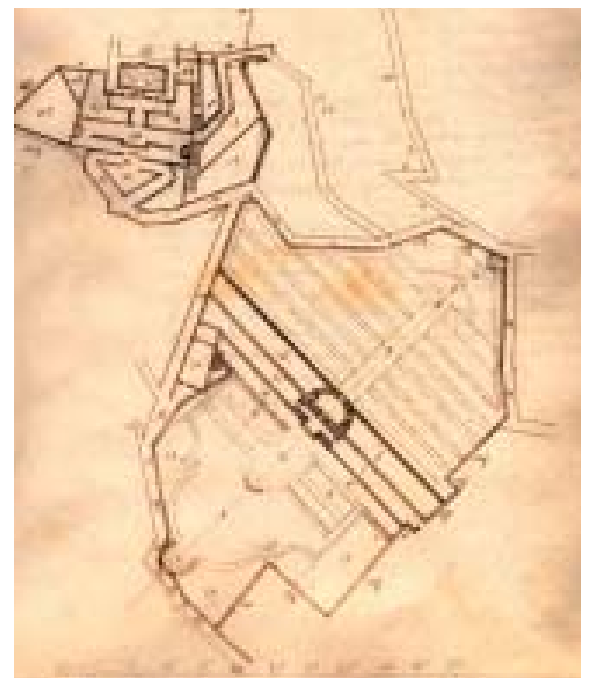

Figura 5 - Planimetria del Terrilogio del 1680.

La trasformazione del giardino durante il corso del Settecento avviene su registri iconografici e simbolici che, attraverso il potenziamento idraulico, la statuaria e la realizzazione del Teatrino di Verzura, confermano il progetto scenico iniziato nel secolo precedente. Ma è proprio nel Settecento che si assiste ad un vero e proprio cambiamento di scena, dal sacro al profano. Si perde la dimensione di sacralità, ma si conserva la ricchezza degli effetti scenografici in particolar modo dedicati al "movimento", rappresentato dalla statua della Fama "che vive di moto, ed acquista forza correndo" 5 collocata nel bacino in sommità, ed alla "metamorfosi", raffigurata dalle statue di Apollo e Dafne e dal mascherone anamorfico in rocce e tufo che si scorge nel piano inclinato della catena d'acqua. Nel giardino, infatti, si trova l'unico esempio di cui siamo a conoscenza di mascherone anamorfico ${ }^{6}$, purtroppo ora in pessimo stato di conservazione. $\grave{E}$ una sapiente illusione ottica in quanto avvicinandosi l'immagine si dissolve: è possibile scorgerla solo dallo spazio semicircolare davanti all'attuale cancello di ingresso, anche se in realtà il punto di vista privilegiato si trova sulla collina prospiciente. "Il punto di vista esterno elimina (...) il limite della chiusa estendendo il giardino nel paesaggio. Non è da escludere che l'anamorfosi sia stata un raffinato tentativo di ricucire otticamente la cesura tra villa e giardino imposta dalla particolare orografia del terreno. Il punto di stazione è infatti anche in asse con la villa, a meno di qualche grado, e nell'assoluta frontalità delle due immagini la villa e il giardino - che appaiono come dipinte su un unico quadro, sembra così essere stato recuperato anche l'aspetto più significativo del giardino anamorfico di Kircher, ossia l'indissolubile unità visiva tra villa, giardino e paesaggio"7.

La storia della famiglia Garzoni è strettamente legata alle vicende del paese di Collodi, castello fortificato già in epoca romana ed alle vicende delle lotte tra guelfi e ghibellini per il possesso della Valdinievole ${ }^{8}$. Il complesso di Villa Garzoni era inserito in un territorio, quello della campagna lucchese, caratterizzato

\footnotetext{
${ }^{5}$ Eneide, IV, 174-183 in MARIA AdRIANA GIUSTI, Teatri di vegetazione: flora, pomona e la verzura in Vincenzo Cazzato, Marcello Fagiolo e Maria Adriana Giusti, op. cit., 1993, pag. 67.

${ }^{6}$ Cfr. FILIPPO CAMEROTA, Il giardino anamorfico: sviluppi di unïdea cartesiana in MARIA ADRIANA GIUSTI, Alessandro TAgliolini, Il giardino delle muse. Arti e artifici nel barocco europeo, Edifir, Firenze 1995.

${ }^{7}$ Filippo CAMEROTA, op. cit., 1995, pag. 266.

${ }^{8} \mathrm{La}$ famiglia Garzoni fu esiliata a Lucca quando la zona cadde in mano fiorentina nel $1328 \mathrm{con}$ la morte di Castruccio Castracani, signore ghibellino che aveva occupato Pistoia fino alle porte di Firenze. I Garzoni tentarono in ogni modo di cacciare dal territorio pistoiese i guelfi fiorentini e reimpadronirsi dei loro possedimenti partecipando attivamente alle lotte tra lucchesi e fiorentini fino alla definitiva restituzione di Collodi a Lucca nel 1442.
} 
fino alla metà del XX secolo dalla compresenza di attività agricole e selvicolturali e segnato sia da forme di sistemazione agraria, come ciglioni e terrazzamenti per sfruttarne i pendii collinari ed opere per la regimazione delle acque, sia da ampi boschi destinati allo svago ed alla caccia dei signori terrieri del Seicento e Settecento. In questa maglia agricolo forestale emergeva la villa, con il borgo di Collodi alle sue spalle.

La villa, risultato di un progetto di ristrutturazione iniziato verso la metà del XVII secolo, è un imponente edificio di forma regolare con la facciata nobile rivolta a valle, verso sud, posto in posizione elevata rispetto al paesaggio circostante, sul quale si apre con ampie vedute?

Dalla metà del XX secolo il contesto paesaggistico cambia profondamente ed il complesso risulta in parte compromesso da nuove urbanizzazioni ed attività industriali e turistiche, che ne mutano soprattutto il rapporto visuale e dialettico con il paese ${ }^{10}$. La tessitura agraria tradizionale invece sopravvive nei rilievi collinari, conservando ancora intatte le prospettive verso monte. Un altro importante parco, inaugurato nel 1972, dialoga visivamente con il parco storico: il Giardino di Pinocchio, opera di Pietro Porcinai, in cui il paesaggista crea lo scenario per ambientare i vari episodi della fiaba di Carlo Lorenzini lungo un percorso punteggiato dalle sculture di Pietro Consagra e le trovate costruttive di Marco Zanuso.

\section{LA STORIA DEL GIARDINO}

Di dimensioni pari a circa 200 metri di profondità per 130 di larghezza, circondato da muri, il giardino non ha nessun rapporto assiale con la villa, alla quale è collegato mediante due rampe a gradoni. Si sviluppa su tre ripiani terrazzati preceduti da uno spazio pianeggiante caratterizzato dalla presenza di un Parterre de broderie attorno a due vasche circolari, di gusto prettamente francese. Salendo

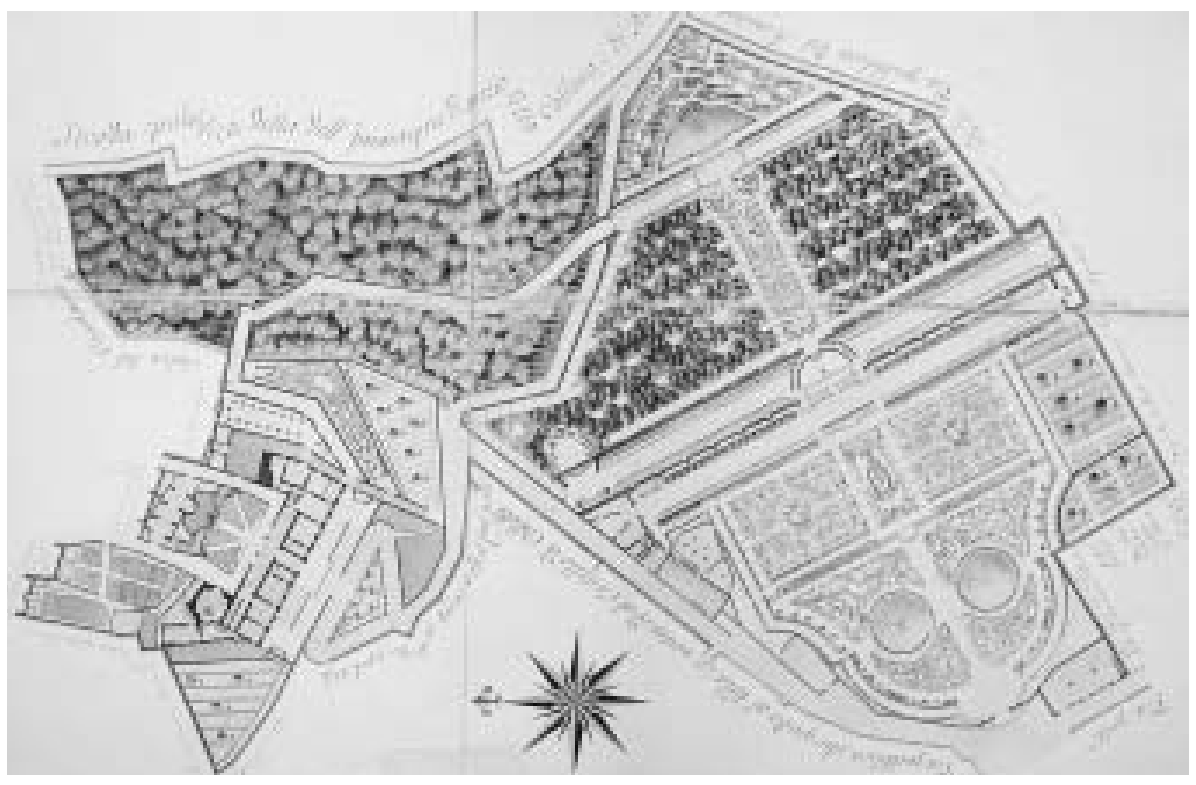

Figura 6 - Planimetria del Terrilogio del 1797.

\footnotetext{
${ }^{9}$ Probabilmente il proprietario Romano Garzoni quando scelse la posizione della villa come residenza della propria casata, in un ameno paesaggio circondato da boschi per la caccia, ben conosceva il trattato di Giovanni di Vincenzo Saminiati Dell'edificar delle case e palazzi in villa e dell'ordinar dei giardini ed orti. Manoscritto in 5 fascicoli datato 1580-1590, Archivio di Stato di Lucca, Dono Paganini n. 3. Il trattato è riportato in ISA Belli BARSALI, La Villa a Lucca dal XV al XX secolo, Appendice a cura di Mario Barsali, De Luca Ed., Roma 1964.
} 


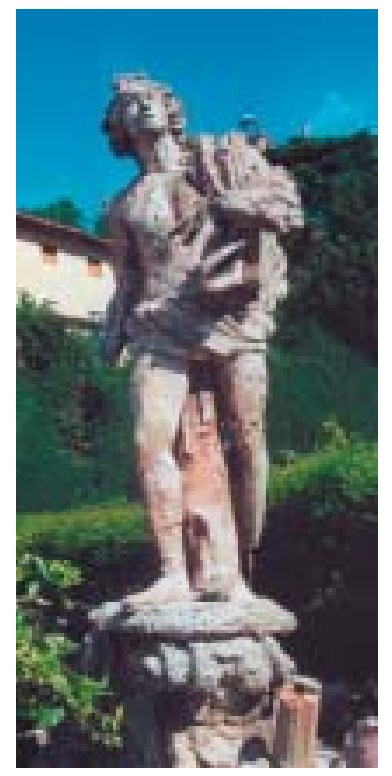

Figura 7 - La statua di Apollo.

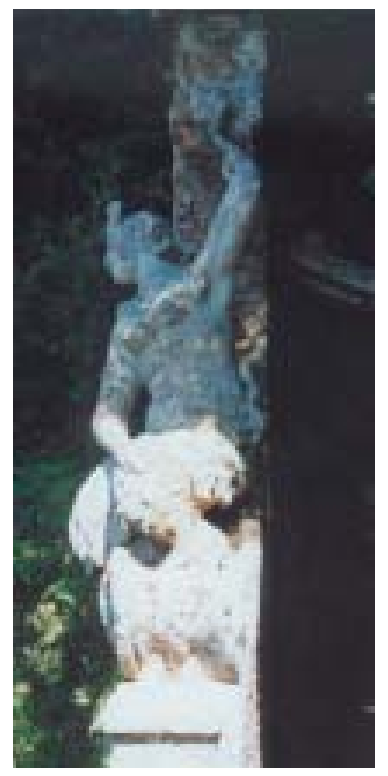

Figura 8 - Uno dei due Satiri.

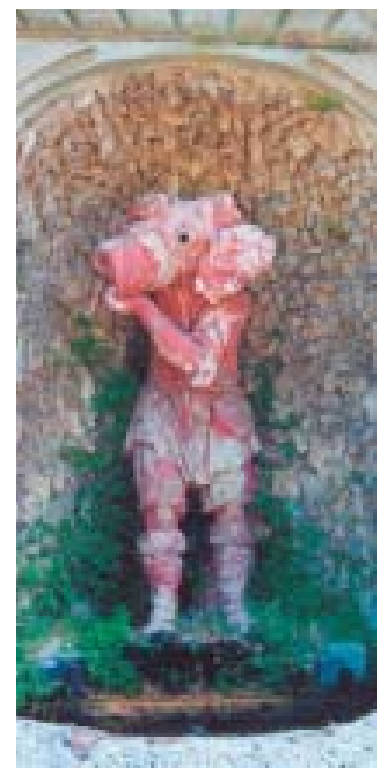

Figura 9 - La statua del Villano.

una piccola scala in mosaico di ciottoli si arriva ad un secondo Parterre à l'anglaise, posto su un piano leggermente inclinato, costituito da tre campi di cui i due laterali riportano al centro lo stemma di Famiglia e quello centrale la cifra. Una scala monumentale a doppia rampa porta al primo terrazzamento detto il Viale delle Palme, collegato, mediante una seconda scala a doppia rampa sotto la quale si trova un ninfeo del VII secolo, al secondo piano terrazzato denominato Viale di Pomona (a destra) e Viale degli Imperatori (a sinistra), alla fine del quale si trova il Teatro di Verzura. Una terza scala curvilinea a doppia rampa conduce al terzo ripiano dove si trova il Bosco di Lecci, tagliato longitudinalmente da una catena d'acqua che parte da una vasca in tufo dove primeggia la statua della Fama. Da qui partono, verso destra, il Viale del Turco e, verso sinistra, il Viale delle Camelie che mette in comunicazione il giardino con la villa per mezzo di un ponte in muratura, la cui zona sottostante è occupata dal Labirinto. Si prosegue poi nel Viale dei Poveri, dal quale si raggiunge, per mezzo di una ripida scalinata, il Boschetto di Bambì. Da qui, attraversando un altro ponte, si giunge al Viale del Gomito e al Viale del Porcellino che ci riconduce al Teatro di Verzura ed al Parterre. Il giardino è punteggiato di statue facenti parte di un programma iconografico che comprendeva diversi temi e generi: Flora, Diana, i Fauni, Bacco e Cerere, Apollo e Dafne nel parterre d'ingresso, ed ancora, il Villano che versa acqua dal barile, le muse della Tragedia e della Commedia, il Cinghiale, le Stagioni, i Satiri.

Della prima fase cinquecentesca del giardino si possiede solo una breve descrizione ed un disegno sommario contenuti in un Martilogio redatto dal perito agrimensore Agostino Pergola nel 1550: “...Una chiusa con casa, ovvero palazzo (...) con orto et giardini con bellissime fontane et più muraglie sopra di se posta in castello di Collodi"11. Il giardino è dunque costituito da un'area di

${ }^{10}$ È stato utilizzato, ad esempio, come sede di un monumento ai caduti lo spazio circolare posto di fronte al cancello di ingresso della villa che in origine faceva parte del giardino (probabilmente formato da una quinta di cipressi speculare al boschetto dei cipressi della parte più alta), ma soprattutto costituiva il luogo preferenziale per osservare il mascherone anamorfico. Questo spazio è stato recentemente liberato da funzioni improprie (baracchino per souvenirs, cassonetti per l'immondizia, parcheggi), restituendogli l'antica funzione di punto privilegiato per godere della prospettiva del giardino.

11 Martilogio dei Beni dello Spettabile Romano Garzoni, 1550, Archivio di Stato di Lucca (ASL), Fondo Garzoni, filza n. 27 in Silvia MARTElli, Il giardino Garzoni a Collodi: documenti per la storia e proposte per la conservazione, "Arte dei Giardini”, 2, 1993, pag. 13. 
piccole dimensioni accanto al palazzo, ornata da fontane e distribuita su tre livelli di terreno, posti a monte dell'attuale Viale dei Poveri (dove ora, nel terrazzo intermedio, si trova il Labirinto). Una seconda zona al di là del torrente che scorre a fianco della villa, descritta come "...bellissima chiusa parte olivata et parte vignata con molti frutti con acqua per il mezzo, con molte selvaggine..." 12 è un luogo produttivo; qui si svilupperà il giardino nel XVII secolo.

La prima fonte che ci descrive in dettaglio la sistemazione secentesca del complesso è l'opera di un poeta, Francesco Sbarra, Le Pompe di Collodi del $1652^{13}$. Una pianta del 1680, a corredo del Terrilogio Duccini ${ }^{14}$, ritrae questo primo progetto, in cui si può osservare come l'impianto del giardino sia rimasto sostanzialmente immutato fino ai nostri giorni nei suoi elementi costitutivi: un grande piazzale d'ingresso in prato circondato da siepi di cipresso (in luogo dell'attuale Parterre de broderie) il quale, sebbene abbia un carattere scenografico, conserva un aspetto rustico-produttivo essendo coltivato a fieno; una zona più elevata con siepi di mirto che delimitano spazi geometrici (dove oggi esiste il Parterre à l'anglaise); i terrazzamenti con spalliere di agrumi, nicchie per statue, grotte per scherzi d'acqua e fontane; il Labirinto; il Bosco di Lecci "che giace in poggio tosato sopra egualmente, diviso in molti viali con le sponde di lauri silvestri, e racchiuso da spalliere di cipressi tessuti...” e sulla sommità “... una cappellina dipinta con una stanza contigua detta il Romitorio con un boschetto di cipressi viali con spalliere di cipressi, e di lauri, dentro li quali sono ripartimenti di finestre con molti globi sopra repartiti con bella mostra"15. Il piccolo giardino cinquecentesco a lato della villa è descritto come "giardino di fiori".

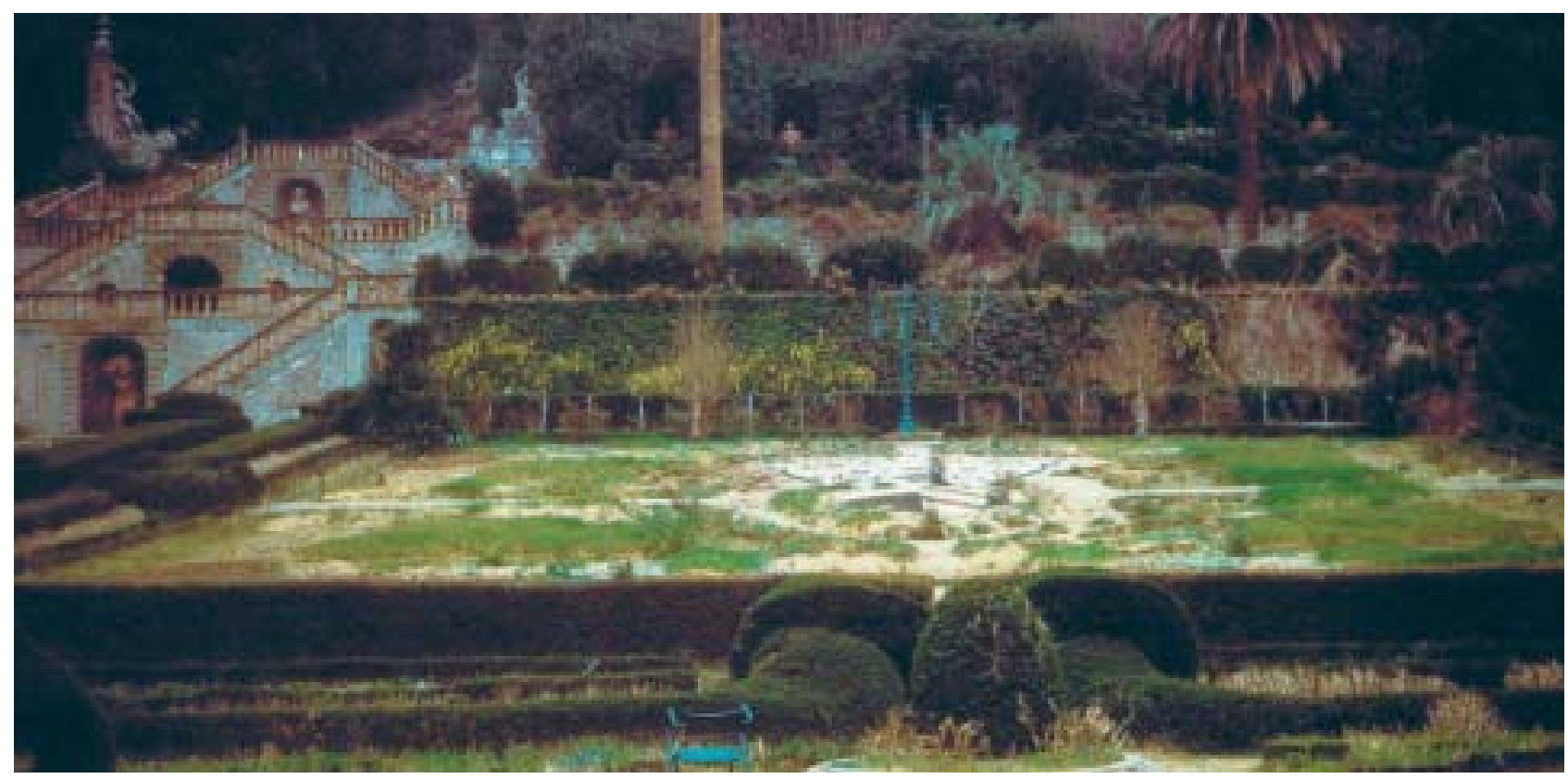

Figura 10 - Veduta dei parterres prima dell'intervento di restauro (1993).

${ }^{12}$ Martilogio, op. cit., 1550, in ARIANNA BECHINI, Evoluzione storica del giardino Garzoni a Collodi attraverso lo studio della struttura idrica, "Bollettino degli Ingegneri" 8-9, 2001, pag. 12.

${ }^{13}$ Francesco Sbarra, Le Pompe di Collodi Delitiosissima Villa del Signor Cavalier Romano Garzoni, Lucca 1652. Da quel momento il giardino, la cui realizazione è attribuita al proprietario Romano Garzoni, diventa famoso e visitato da personaggi illustri come Anna de' Medici e Ferdinando d'Austria, che qui soggiornarono nel 1662. Una delle meraviglie del giardino decantate nel poema era l'ars topiaria: "in mille guise si contorce e si stende / il bel cipresso hora scherzante, hor grave, / Hor esprime una Torre, hor una Nave, / Hor di fera, Hor d'augel sembianze prende".

${ }_{14}$ Terrilogio, come il precedente Martilogio, sono sinonimi in area lucchese di cabreo (elenco o catasto dei beni feudali in genere).

${ }^{15}$ Terrilogio Duccini, 1680, ASL, Fondo Garzoni, filza n.29 in Silvia MarTelli, op. cit., 1993, pag. 13. 
L'intervento settecentesco, attribuito all'architetto Ottaviano Diodati ${ }^{16}$ che affianca Romano Garzoni, pronipote del primo creatore del giardino, non altera la struttura scenografica generale. Anzi, questa viene amplificata con la realizzazione della catena d'acqua con la statua della Fama, del Teatro di Verzura, della Fabbrica dei Bagnetti, dove i signori si immergevano al suono degli orchestrali e che va a sostituire il Romitorio, dei parterres con lo stemma e la cifra della Famiglia, del parterre de broderie in bosso e pietre colorate e delle due vasche circolari all'ingresso in sostituzione del prato "a guisa di theatro" secentesco. Questo nuovo assetto del giardino è rappresentato in molte composizioni celebrative, in dipinti ed in planimetrie di corredo ai Terrilogi, che descrivono il giardino “...ornato con prospettive architettoniche Statue, Grotteschi con scherzi d'acqua, Vasche con Acque salenti, Scale con Balaustrate, Viali con siepi vive di Cipressi e Lauri, Parterre, Boschetti di Cedri, Vasi di Agrumi ed altro che tutto insieme presenta all'occhio un nobilissimo Prospetto..." 17 .

Nell'Ottocento e nel Novecento il giardino non subisce trasformazioni sostanziali nell'impianto generale, ma l'apertura al pubblico ${ }^{18}$ provoca una modifica del carattere stesso del giardino: dalla sobrietà cromatica originale si passa ad una forte presenza di ricche fioriture colorate causata da introduzioni casuali ed improprie dal punto di vista botanico. Anche i vari passaggi di proprietà hanno contribuito al lento degrado, che ha subito un arresto solo agli inizi degli anni Novanta, quando i penultimi proprietari hanno iniziato i lavori di restauro della vegetazione.

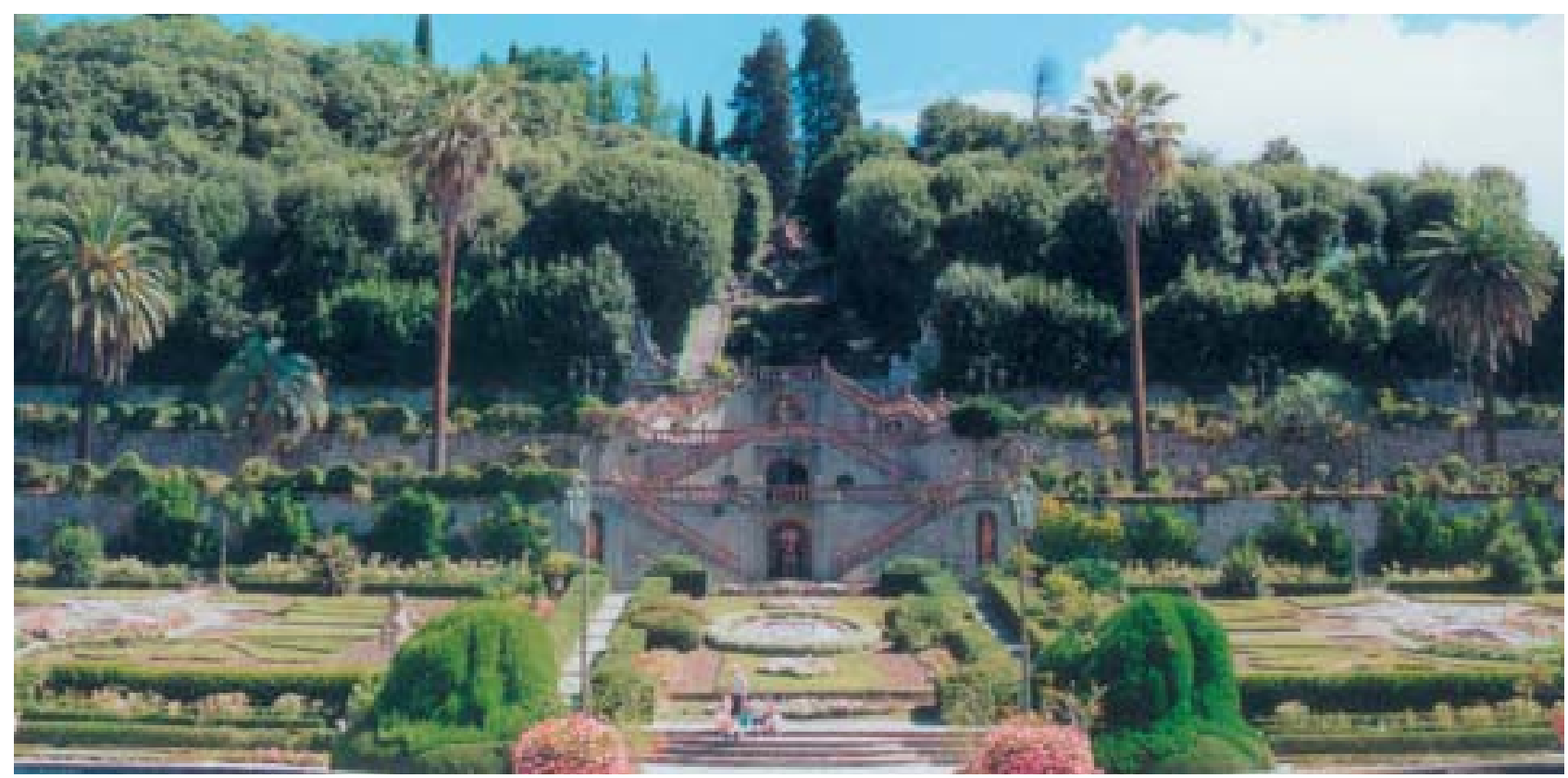

Figura 11 - Veduta dei parterres restaurati (2002).

\footnotetext{
${ }^{16}$ Ottaviano Diodati ha lavorato anche alla villa ed alla Palazzina dell'Orologio, collocata all'interno del cortile e progettata da Filippo Juvarra intorno al 1730 ed ha legato il suo nome all'edizione lucchese dell'Encyclopédie ou dictionnaire raisonné des sciences des arts et des métiers (che curò negli anni 17581771). Questo fatto avvalora la tesi del legame dell'ambiente lucchese con la cultura francese. La fama del giardino Garzoni divenne tale che Carlo VII di Napoli si rivolse a Diodati per un progetto (mai realizzato) per il parco di Caserta ed il re di Polonia Stanislao Poniatowski richiese un disegno del giardino, che fu eseguito da Francesco Cecchi nel 1793 (in questo acquerello è chiaramente visibile il mascherone anamorfico della catena d'acqua).

${ }^{17}$ Terrilogio dei Beni Stabili, 1797, ASL, Fondo Garzoni, filza n. 152 in Silvia Martelli, op. cit., 1993, pag. 17.
} 


\section{GLI OBIETTIVI DEL PROGETTO DI RESTAURO}

La fase analitica del progetto ha operato su livelli complementari e distinti. $\mathrm{Da}$ un lato il giardino è stato indagato attraverso una serie di analisi ambientali che hanno messo in evidenza i principali dati climatici, pedologici, idrografici, geologici e paesaggistici; dall'altro ne è stata ricostruita l'evoluzione storica, per comprendere la struttura stessa del giardino e valutare le scelte progettuali effettuate nel corso del tempo. Questa ricostruzione storica è stata effettuata anche attraverso lo studio della trattatistica italiana e straniera che ha permesso di approfondire la conoscenza di importanti elementi costitutivi. Per l'interpretazione dei parterres, ad esempio, è stato fatto riferimento ai testi di $\mathrm{Mollet}^{19}$ e di Dezallier D’Argenville ${ }^{20}$.

Infine, è stato "fotografato" lo stato attuale del giardino attraverso un rilievo vegetazionale (elaborato in scala 1:250) associato ad una descrizione tecnica dello stato della vegetazione, rilevando patologie presenti, fallanze, pratiche manutentive scorrette (tipo potature sbagliate o mancanti), assenza di controllo delle piante infestanti e concimazioni errate. Al momento del restauro, infatti, il giardino versava in un cattivo stato di conservazione; in alcune sue parti era stato abbandonato, mentre in altre, in particolare nei parterres, erano state effettuate scelte storicamente scorrette dal punto di vista botanico.

Il progetto di restauro aveva come obiettivo principale "la conservazione critica di tutte le fasi storiche che nel corso del tempo si sono sovrapposte ed intrecciate" 21 e a questo scopo è stata condotta una accurata indagine storica, attraverso la comparazione dei quattro periodi principali del giardino (corrispondenti al Seicento, Settecento, Ottocento e Novecento).

In molte parti, in particolare lungo i viali nei quali il giardino è articolato, gli interventi previsti sono stati indirizzati alla conservazione e alla valorizzazione degli elementi esistenti, ottenute soprattutto attraverso un miglioramento gestionale ed una accurata programmazione delle operazioni da effettuare sul patrimonio vegetale (potature, risarcimenti delle fallanze, eccetera). Inoltre, è stata ritenuta indispensabile la sostituzione delle specie vegetali introdotte in modo improprio durante il corso dei secoli, come ad esempio per la siepe in pittosporo (Pittosporum tobira) nel Viale del Porcellino sostituita con una siepe in bosso (Buxus sempervirens) o la messa a dimora lungo i muri che sostengono le terrazze delle originali spalliere di agrumi al posto di specie come lillà, edera e gelsomino (Syringa vulgaris, Hedera helix, Jasminum officinale). Un trattamento diverso è stato riservato a quegli elementi che, pur introdotti "impropriamente", hanno oramai un valore storico in quanto rappresentano il manifestarsi del gusto e dello stile di un epoca, come il filare di palme (Washingtonia filifera) del Viale delle Palme ed il Boschetto di Bambù (Plyllostachys sp.), entrambi piantati con molta probabilità agli inizi del XX secolo.

Il progetto forniva inoltre puntuali indicazioni per una serie di interventi quali: le modalità di piantagione per ripristinare il disegno delle siepi di bosso dei parterres in parte mancanti; le potature delle siepi alte di bosso che circondano il piazzale d'ingresso (nel giardino secentesco questa siepe era in cipresso, di cui rimane solo qualche esemplare, sostituita da bosso e tasso nel Novecento); la sostituzione delle specie improprie (thuje, erba della Pampas, lagerstroemie, coto-

\footnotetext{
${ }^{18}$ Non si conosce la data esatta in cui il giardino è stato aperto al pubblico, tuttavia l'indicazione dei giorni di visita in una guida inglese ai giardini italiani del 1912 ne testimonia l'apertura già agli inizi del XX secolo. (Cfr. AuBrey LE BlOnd, The old gardens of Italy. How to visit them, John Lane, the Bodley Head, London 1912).

${ }^{19}$ ANDRÈ MOLLET, Le jardin de plasir, Stockholm 1651.

${ }^{20}$ Antoine Joseph Dezailler D’Argenville, La théorie et la Pratique du Jardinage, Mariette, Paris 1709 (I ed.).

${ }^{21}$ Silvia Martelli, Relazione del Progetto di Restauro, dattiloscritto, 1993.
} 


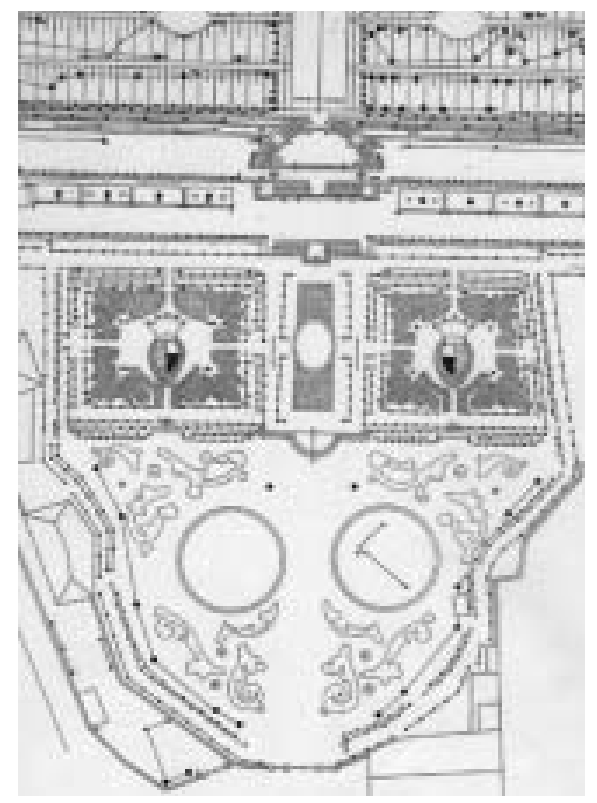

Figura 12 - Particolare della tavola di rilievo vegetazionale.

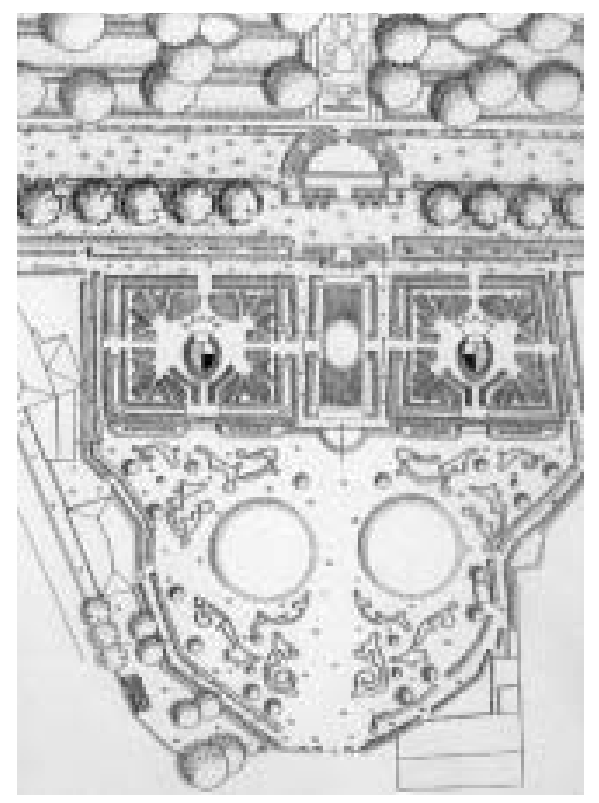

Fig. 13 - Particolare della tavola di progetto.

neaster, eccetera); la sistemazione delle piante in vaso, poste sui piedistalli decorati lungo la siepe di confine, esclusivamente di agrumi (specificamente limoni, eliminando le fioriture, tipo bouganville).

\section{LA METODOLOGIA}

Il progetto di restauro è stato impostato "scomponendo" il giardino nei diversi elementi compositivi che lo costituiscono (il Parterre, il Teatro, il Labirinto, il Viale delle Palme, eccetera), di ciascuno dei quali è stata ricostruita la storia in modo che emergessero chiaramente l'evoluzione e la stratificazione, allo scopo di effettuare le migliori scelte progettuali senza per questo perdere di vista la composizione generale. Questi elementi, quattordici in tutto, sono diventate "tipologie" di intervento e di ognuna è stato elaborato un progetto specifico.

Un importante tema progettuale è quello relativo al disegno dei parterres, presenti nel piano d'ingresso e nel retrostante piano inclinato. Nel Seicento l'area di ingresso era costituita, come abbiamo visto, da un grande prato mentre nel pendio adiacente erano presenti lunghe siepi di mirto; nel Settecento sono realizzati i parterres de broderie nel primo piano e nel secondo piano inclinato, delimitati da siepi di bosso, specie vegetale che a Collodi, come in molti altri giardini italiani, è andata a sostituire il mirto probabilmente per motivi legati alla

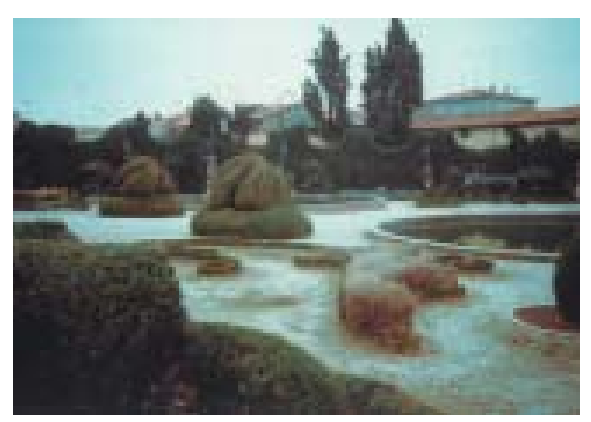

Figura 14 - Il Parterre de broderie prima del restauro.

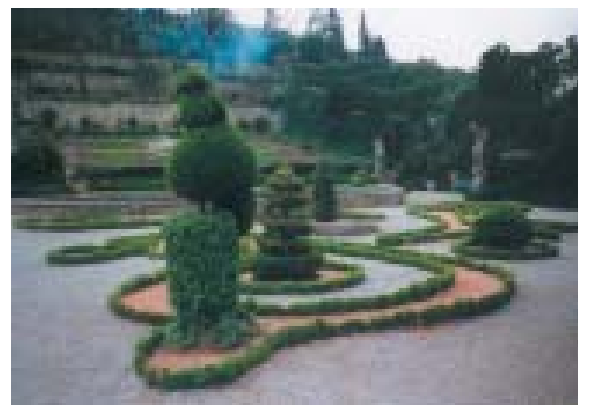

Figura 15 - Il Parterre de broderie dopo il restauro. 
sua maggiore resistenza alle avversità climatiche. Nell'Ottocento il disegno dei parterres appare profondamente trasformato, sia quelli d'ingresso modificati in "...quattro campi fioriti ad uso di giardino", che quelli sul pendio sostituiti da quattro aree rettangolari descritte come "...terreno vestito di fiori ad uso di giardino"22. Al momento dell'intervento di restauro, invece, il disegno settecentesco risultava ristabilito, anche se semplificato e modificato con fioriture stagionali all'interno delle siepi.

Il progetto ha scelto di restituire l'antico splendore del disegno originale settecentesco ai parterres attorno alle vasche nell'area d'ingresso, con l'eliminazione delle fioriture stagionali e la loro sostituzione con sabbie colorate che, sia i documenti settecenteschi sul giardino Garzoni sia la trattatistica francese prima ricordata, concordano nel descrivere all'interno di siepi di bosso ${ }^{23}$.

La scelta invece compiuta per i parterres sul piano inclinato è stata determinata sia da motivazioni tecniche che da esigenze gestionali. La presenza di un parterre a ricamo su una superficie inclinata è di difficile gestione per il dilavamento che si verifica a causa della pendenza, senza una continua manutenzione e senza un frequente apporto di nuovo materiale, operazioni che venivano costantemente eseguite in epoca settecentesca ma che ora appaiono inattuabili. La sostituzione delle fioriture stagionali con sabbia colorata, quindi, sarebbe stata impraticabile. Il progetto ha mantenuto allora il disegno settecentesco, sostituendolo con un parterre à l'anglaise, in prato, circondato da piattabande fiorite, la cui scelta delle specie vegetali è stata effettuata sulla base delle descrizioni dei manuali e trattati citati e delle descrizioni storiche del giardino ${ }^{24}$.

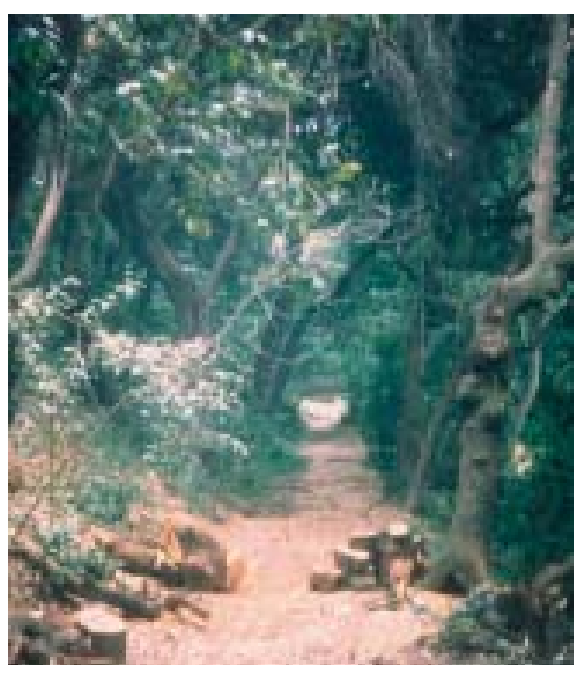

Figura 16 - Il Bosco di Lecci prima del restauro.

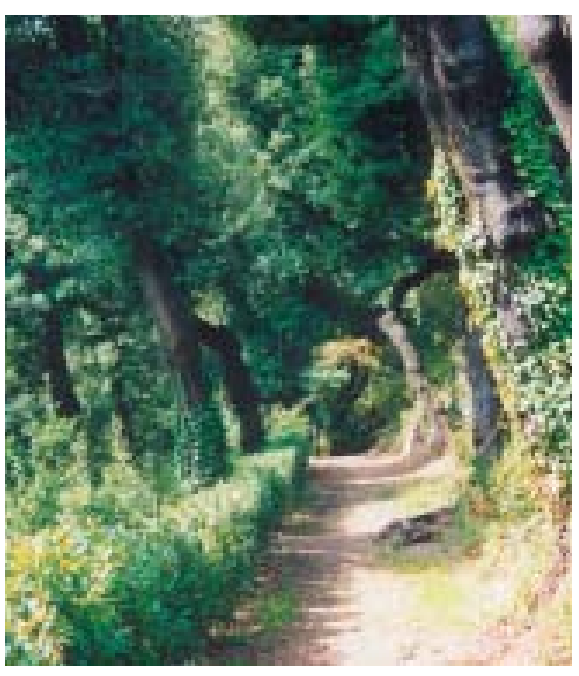

Figura 17 - Il Bosco di Lecci dopo il restauro.

${ }^{22}$ Catasto Nuovo, Planimetria, 1836, ASL, busta n. 464 e Catasto Nuovo, Giornale di campagna, 1836, ASL, busta n. 399, in SiLVIA MARTELLI, Il giardino Garzoni a Collodi: documenti per la storia e proposte per la conservazione, "Arte dei Giardini", 2, 1993, pag. 19.

${ }^{23} \mathrm{Il}$ parterre de broderie è composto da arabeschi che assomigliano a ricami del primo Seicento. Il primo parterre barocco a ricamo si deve a Claude Mollet ed era delimitato da una bordura di bosso, anche se si diffondono bordure di fiori orlate da bosso, in particolare sotto la spinta innovatrice di André Le Notre. Il fondo era costituito da materiali colorati: "I parterre a ricamo sono così chiamati, poiché il bosso con il quale sono formati è disposto a disegni che imitano il ricamo; il fondo del terreno è cosparso di sabbie colorate per meglio staccare il fogliame del bosso..." (Antoine Joseph DeZAiller D’ArgenVille, op. cit., in Silvia Martelli, op. cit., 1993, pag. 25).

Che i parterres del giardino Garzoni fossero così realizzati si rileva da una descrizione settecentesca, la quale recita: "...più parterri, parte di verdi, con urnette simili, e cassette di fiori, e parte a secci, che sono di diverse qualità di pietre di vari colori..." (Descrizione senza data, ASL, Fondo Garzoni, filza n.55, in Silvia MarTelli, op. cit., 1993, pag. 24).

${ }^{24}$ Una descrizione del parterre à l'anglaise la troviamo ancora in D'Argenville: "I parterre all'inglese sono più semplici e constano di grandi tappeti verdi, a prato, di un sol pezzo o a pochi scomparti; possono 
Un secondo tema progettuale è il restauro del Bosco di Lecci, che si è conservato perfettamente immutato nella sua struttura dal Seicento ad oggi, a parte la creazione, nel secolo successivo, della catena d'acqua in luogo del semplice viale centrale di separazione. Nella planimetria secentesca il bosco è suddiviso in vialetti paralleli fiancheggiati da alte siepi di alloro (Laurus nobilis) ed è costituito da lecci (Quercus ilex) potati nella parte superiore in forma geometrica, che costituiscono un nucleo denso, circondato da una quinta di cipressi (Cupressus sempervirens). Al momento dell'intervento di restauro questa forma geometrica e compatta era resa meno evidente dall'eccessiva crescita degli alberi e dalla nascita spontanea di altre specie vegetali non sempreverdi, la cortina di cipressi era scomparsa e le siepi di alloro si erano diradate e sfrangiate. Lo sviluppo esagerato della chioma aveva reso instabili le piante, il cui apparato radicale non era altrettanto sviluppato in quanto per secoli i lecci erano stati potati per contenerne la crescita. Lo squilibrio aveva provocato il disassamento di alcuni esemplari, fino ad arrivare addirittura al crollo e a cedimenti delle scarpate che collegano i vialetti. Il progetto, dunque, è stato rivolto al recupero della forma originaria della composizione, per ottenere un bosco con individui non troppo sviluppati e di altezza controllata. Questo è stato ottenuto attraverso il taglio fitosanitario, eliminando le piante morte ma rimaste in piedi ed i seccumi degli alberi e della siepe ed il taglio di alcuni esemplari, consentendo così anche la crescita di giovani piante che in futuro avrebbero sostituto quelle attuali e favorendo lo sviluppo del sottobosco (ciclamini, edera). La ricostituzione delle siepi di alloro nella loro altezza originaria di tre metri ha contribuito al ripristino dell'immagine originale di questo luogo.

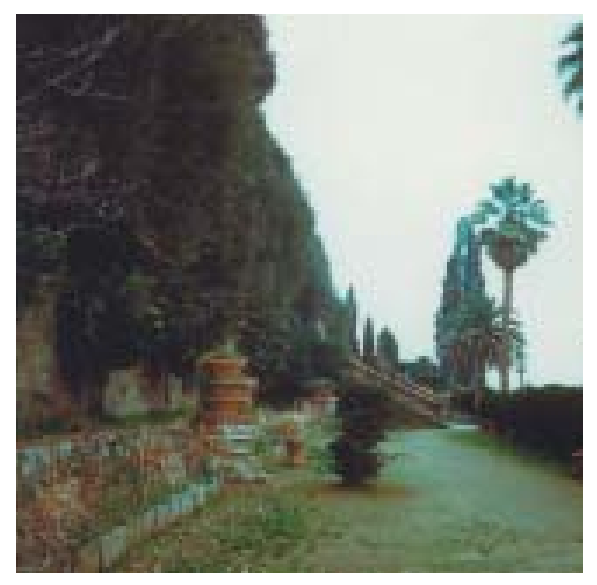

Figura 18 - Il Viale degli Imperatori prima del restauro.

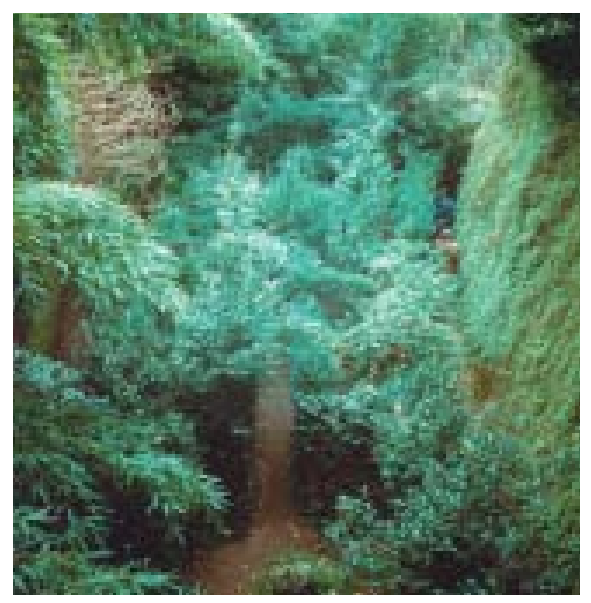

Figura 20 - Il Labirinto prima del restauro.

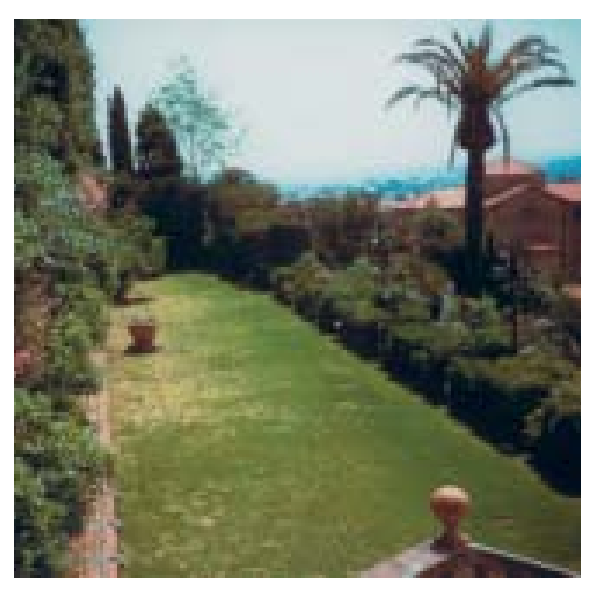

Figura 19 - Il Viale degli Imperatori dopo il restauro.

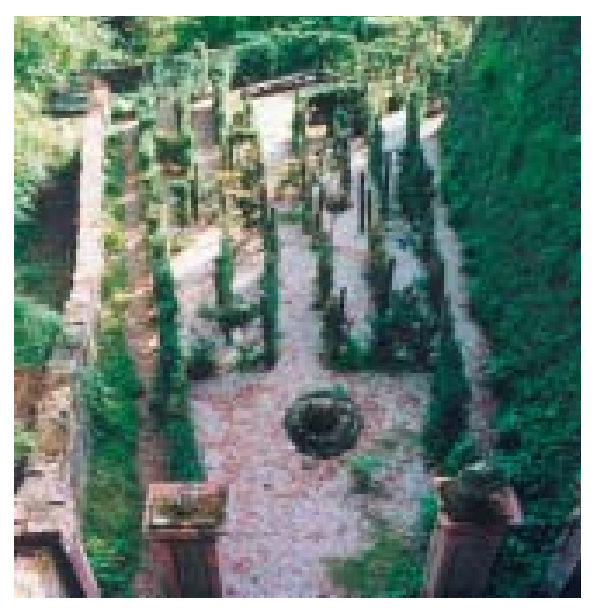

Figura 21 - Il Labirinto dopo il restauro. 


\section{GLI ESITI}

Il restauro del giardino Garzoni aveva previsto un calendario di lavori di manutenzione da effettuarsi in ogni singola stagione e per ogni tipologia di intervento individuata; tuttavia la manutenzione costante e regolare è stata eseguita con cura solo per alcuni anni, limitandosi poi agli interventi più urgenti, come spesso succede in molti giardini storici. In particolare è stata concentrata al parterre d'ingresso, mentre altre zone più marginali sono state trascurate, provocando un regredire della situazione alle condizioni precedenti il restauro ed annullando i risultati conseguiti.

Due architetture vegetali, il Labirinto secentesco ed il Teatro di Verzura settecentesco, si presentavano al momento del progetto di restauro fortemente degradate, anche se, essendo costituite prevalentemente da bosso, specie vegetale a crescita lenta, l'alterazione si era manifestata lentamente e poteva essere recuperata con facilità mediante un efficace programma manutentivo che prevedesse accurate potature ed adeguati risarcimenti. Il restauro del Labirinto è stato compiuto nel corso dei lavori di manutenzione del giardino, contemporaneamente al restauro di due statue poste nel parterre d'ingresso e delle arcate intorno al bacino d'acqua della statua della Fama ${ }^{25}$. Il Teatrino di Verzura non è invece mai stato restaurato.

Il progetto del 1993 indicava i vari interventi necessari per ripristinare la composizione originale ed individuava soprattutto le cure indispensabili per una corretta manutenzione e gestione suggerendo, ad esempio, un maggior controllo ed educazione dei visitatori, la cui presenza ha un impatto considerevole su strutture delicate come il Teatro od il Labirinto. Per questo può destare preoccupazione l'intenzione di Enrico Preziosi il quale, a proposito del parco tematico dedicato a Pinocchio che vorrebbe realizzare nelle colline davanti al parco storico di cui Villa Garzoni costituirebbe il fulcro, vuole attirare in questo luogo un milione di visitatori l'anno. L'imprenditore sottolinea la necessità di realizzare parcheggi, alberghi ed infrastrutture. Senza entrare nel merito del progetto del parco divertimenti e di questo tipo di operazione (che comunque ha lo scopo di valorizzare un'area economicamente depressa), ciò che preoccupa è la possibilità che una proposta come questa possa stravolgere, come è già accaduto in passato con interventi di ben minore impatto, quella indissolubile unità visiva tra villa, giardino e paesaggio che era stata uno degli obiettivi dei suoi creatori.

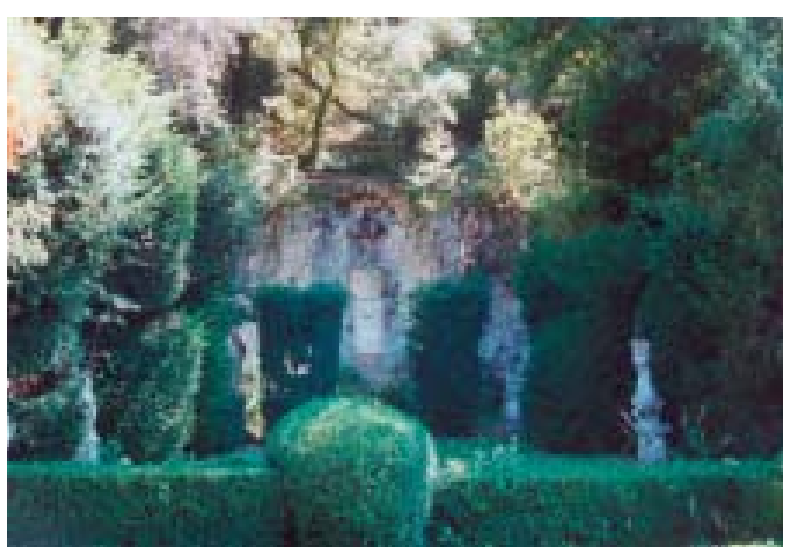

Figura 22 - Il Teatro di Verzura.

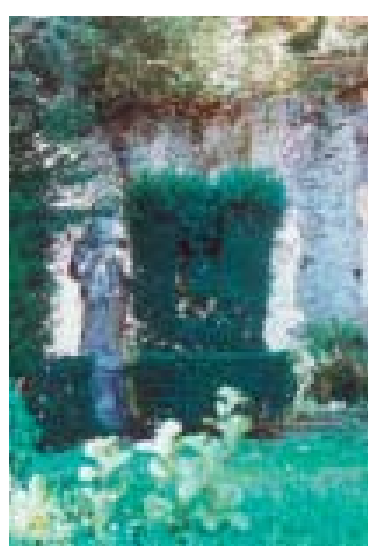

Figura 23 - Una delle statue del Teatro.

essere contornati da piattabande di fiori (...) chiusi da filari di bosso; essi devono presentarsi come una superficie colorata ben livellata e perciò vanno distinti per la loro altezza in alti, medi o nani. I più usati sono quelli di altezza media: tulipani, narcisi, giacinti (...) I fiori alti non si adattano ai parterre...per piattabande molto basse si usano fiori nani: viola, ciclamino, margherita, primola, zafferano" (ANTOINE Joseph Dezailler D’Argenville, op. cit., in Silvia Martelli, op. cit., 1993, pag. 25).

${ }^{25} \mathrm{Nel} 1997$ è stato redatto il rilievo del labirinto ed un accurato progetto esecutivo per ripristinarne il disegno originale, eseguito nello stesso anno. 


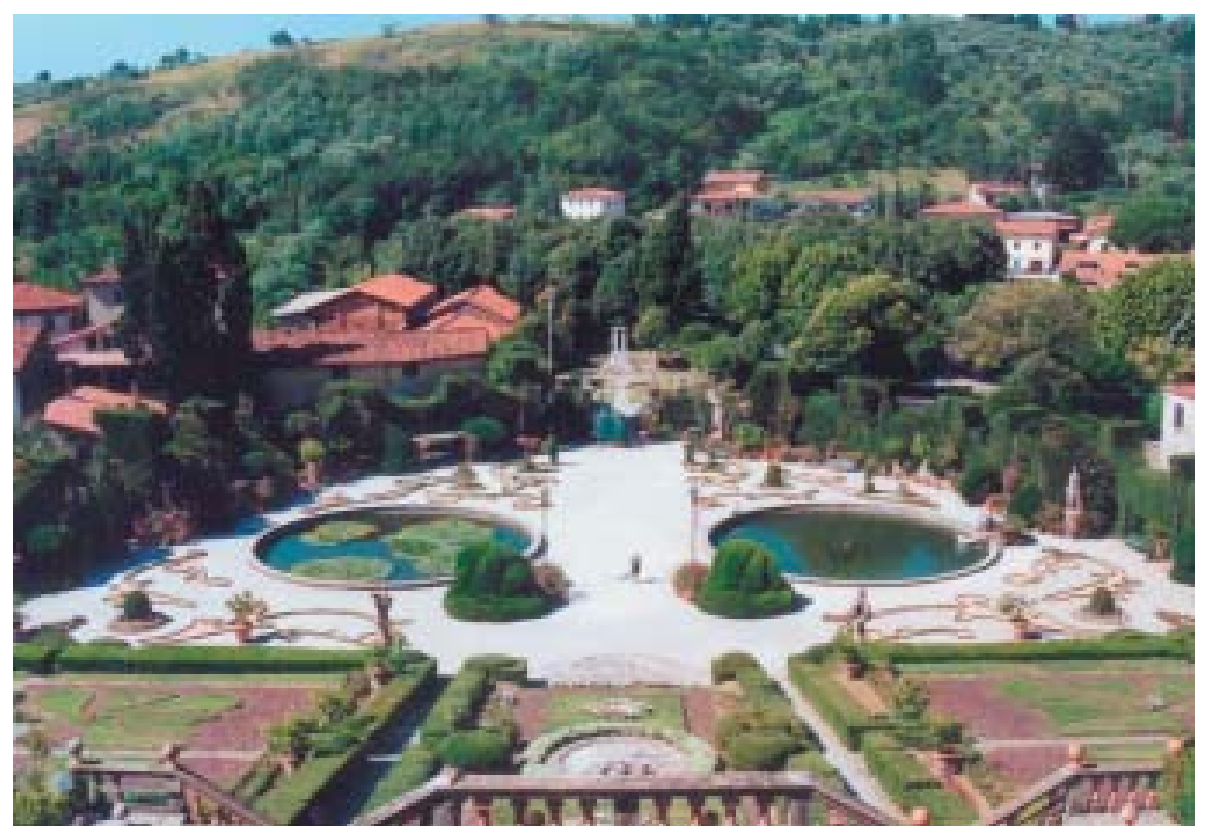

Figura 24 - Veduta dalla seconda rampa della scalinata del parterre d'ingresso e del parterre nel piano inclinato.

\section{RIFERIMENTI BIBLIOGRAFICI}

BECHINI ARIANNA, Evoluzione storica del giardino Garzoni a Collodi attraverso lo studio della struttura idrica, "Bollettino degli Ingegneri", 8-9, 2001, pagg. 11-19.

Belli Barsali IsA, La Villa a Lucca dal XV al XX secolo, De Luca Ed., Roma 1964.

Caliterna Piero, Cellie Giulia e De Vita Maurizio, Il giardino Garzoni a Collodi, "Recuperare l'edilizia”, 2, anno II, marzo 1998.

CAZZANi AlberTa (a cura di), Architettura del verde. L'esperienza paesaggistica italiana, Be-Ma, Milano 1994.

Cazzato Vincenzo, Fagiolo Marcello e Giusti Maria Adriana, Teatri di Verzura. La scena del giardino dal Barocco al Novecento, Edifir, Firenze 1993.

Fariello FranCESCO, Architettura dei giardini, Edizioni dell'Ateneo, Roma 1985.

Giusti MaRia AdRIANA e TAgliolini Alessandro, Il giardino delle muse. Arti e artifici nel barocco europeo, Edifir, Firenze 1995.

LE BLOND AUBREY, The old gardens of Italy. How to visit them, John Lane, the Bodley Head, London 1912.

Maniglio Calcagno AnNalisa (a cura di), Giardini e parchi lucchesi nella storia del paesaggio d'Italia, Pacini Fazi, Lucca 1992.

MARTELli SiLVIA, Il Giardino Garzoni a Collodi. Documenti per la storia e proposte per la conservazione, "Arte dei giardini", 2, 1993, pagg. 13-28.

Martelli Silvia, Giardino Garzoni a Collodi. Guida alla visita, Alinea, Firenze 2002.

PIZZONI FILIPPO, Il giardino arte e storia, Leonardo Arte, Venezia 1999.

PONTE Alessandra, "Le pompe di Collodi" il giardino di villa Garzoni, in MOSSER MONIQUe e TeYsSOT GEORGE, L'architettura dei giardini d'Occidente dal Rinascimento al Novecento, Electa, Milano 1990, pagg. 177-180.

PozZANa MARIa ChIARA, I giardini di Firenze e della Toscana. Guida completa, Giunti, Firenze 2001.

Regione Toscana, Giardini di Toscana, Edifir, Firenze 2001.

TAgliolini Alessandro, Storia del giardino italiano, La casa Usher, Firenze 1988.

\section{RIFERIMENTI ICONOGRAFICI}

Figure 1, 3: disegno di Roberta Palloni.

Figure 4- 6: Archivio di Stato di Lucca Fondo Garzoni, filze n. 27 c. 1, n. 29 c. 20 , n. 152 c. 4. Autorizzazione n. prot. 5168-V/9 del 6 settembre 2002, su concessione del Ministero per i Beni Culturali e Ambientali.

Figure 2, 7, 8, 9, 11, 22, 23 e 24: fotografie di Antonella Valentini. 
Figure 10 e 14-21: fotografie di Silvia Martelli.

Figure 12, 13: particolari delle tavole di progetto di restauro, per gentile concessione di Silvia Martelli.

\section{SCHEDA DI PROGETTO}

Progetto:

Luogo:

Proprietà:

Tipologia di giardino:

Superficie:

Progettista e Direttore Lavori:

Anno di progettazione:

Anno di realizzazione:

Imprese esecutrici:

Costo intervento di restauro:

Costo manutenzione:

Impresa di manutenzione: restauro del patrimonio vegetale del giardino di Villa Garzoni

Collodi, Pescia, in provincia di Pistoia

privata, Dilezza s.r.l.

giardino storico aperto al pubblico

3 ettari

Dott. For. Silvia Martelli, Firenze

$1992-1993$

1994 restauro dei parterres, bosco dei lecci, viali

1997 lavori di manutenzione ordinaria del giardino (la cui supervisione generale è affidata alla Dott. S. Martelli):

progettazione esecutiva e restauro del Labirinto

Altri interventi affidati agli Arch.tti M. De Vita e P. Caliterna, Firenze:

- restauro di due statue nel parterre de broderie

- restauro degli archi posti attorno alla vasca della Fama

non è mai stato eseguito il restauro per il boschetto dei cipressi, il Teatro di Verzura ed il Boschetto del Bambù (di quest'ultimo nel 1993 è stata effettuata solo una ripulitura del canneto)

Atlante Soc. Cop., Vernio, Prato

(potatura e sistemazione dei boschetti, sagomatura delle siepi dell'area dei parterres)

Pinochi, Montecatini Terme, Pistoia

(sistemazione broderies e del canneto)

Ditta Fratelli del Ministro, Pescia, Pistoia

(svuotatura delle vasche)

Giardinieri della Villa Garzoni

(sistemazione broderies e delle spalliere di agrumi)

300 milioni di lire (Finanziamento Comunità Europea)

i proventi degli incassi di ingresso al giardino sono devoluti alla manutenzione annua (circa mezzo miliardo di lire, pari a $€ 250.000,00$ )

giardinieri della Villa Garzoni (n. 5 negli anni 1993-1997) 


\title{
Il Master Plan del Giardino di Boboll a Firenze*
}

\author{
Anna Lambertini
}

\section{IL CONTESTO}

Il Giardino di Boboli, forse il più noto tra i giardini medicei, si trova nella fascia di contatto tra due sistemi spaziali ben definiti: quello urbano, dello spazio murato, dei quartieri di Santo Spirito e San Niccolò, e quello collinare, dello spazio aperto diffuso, delle sistemazioni ottocentesche del Poggi e del paesaggio agrario storico.

Nato dall'unione dell'antico 'Orto dei Pitti', posto alle spalle del Palazzo omonimo ${ }^{1}$ acquistato nel 1550 da Eleonora di Toledo moglie di Cosimo I de' Medici, con una porzione della contigua area agricola collinare ${ }^{2}$, fin dalle sue origini si configura come trait d'union tra paesaggio costruito e paesaggio coltivato.

La scelta di Cosimo I di fare dell'imponente complesso architettonico preesistente una nuova reggia per la corte ducale appare dettata proprio dalla disponibilità di ampi spazi liberi alle spalle dell'edificio, adatti ad essere trasformati in un nuovo magnifico giardino, grazie al quale il Palazzo avrebbe potuto competere per sfarzo e ricchezza con le altre dimore regali europee.

La zona risultava inoltre ben presidiata ${ }^{3}$ : compresa tra i bastioni cinquecenteschi d'Oltrarno, appena costruiti come linea difensiva durante la guerra contro Siena, e le mura trecentesche rinforzate da poderosi baluardi, appariva proprio come quella "porta posteriore (della città) fortificata da ogni lato in modo eccezionale indicata nel De Re Aedificatoria come perfetta figura del palazzo del tiranno" ${ }^{\text {. }}$

La felice posizione della collina di Boboli, dominante su Firenze, che diviene così al contempo oggetto di contemplazione e di controllo, è l'ideale per attuare quella strategia di appropriazione della città, inscenata dal principe per l'affermazione del suo potere politico, non solo in forma simbolica o visiva. "Trasformando in giardino la collina di Boboli, Cosimo privatizza e sequestra una parte assai estesa della città e fa di essa un bene esclusivo e geloso da tra-

*Si ringraziano per l'indispensabile contributo Loris Stefanini, che ha fornito una copia del Master Plan, e Giorgio Galletti.

${ }^{1}$ Palazzo Pitti fu fatto costruire a partire dal 1451 da Luca di Bonaccorso Pitti, che aveva acquistato i terreni per la sua edificazione da una potente famiglia fiorentina, i Rossi. Nel 1454 Pitti acquistò poi anche un podere contiguo dai frati di Monteoliveto.

2 "Il vecchio Orto de' Pitti fu acquistato da Eleonora nel febbraio del 1550 e misurava centoquarantasei staiora, era delimitato dal «murus seu el bastione di Sua Ex.tia», dalle «moenia flor.ina via mediante» e dai beni «monnalium S. Felicitatis». A questo nucleo portante si aggiunge nel maggio del '50 il podere di Bogoli, estorto alle monache di Santa Felicita con la promessa di un conguaglio in «beni e denari» che ancora nel 1568 risulta lontana dall'essere soddisfatta. L'area misurava settantaquattro staiora." In ALESSANDRO RINALDI, «Quattro pitaffi senza lettere»: I primi anni del giardino di Boboli e lo 'spartimento' del Tribolo in Boboli 90 - Atti del Convegno Internazionale, Voll. 1 e 2, Edifir, Firenze 1991, pagg. 19-30.

${ }^{3}$ La sicurezza del luogo, dal punto di vista difensivo, era stata comprovata durante l'assedio del 1530, quando Boboli si trovò a fronteggiare lo schieramento delle truppe imperiali poste sul colle di Giramonte e a rispondere con efficacia al fuoco delle loro artiglierie.

${ }_{4}^{4}$ AlesSANDRO RINALDI, Giardini e metamorfosi urbana a Firenze tra Medioevo e Rinascimento, pagg. 15-30 in Daniela Cinti, Giardini \& Giardini. Il verde storico nel centro di Firenze, Electa, Milano 1998, pag. 24. 


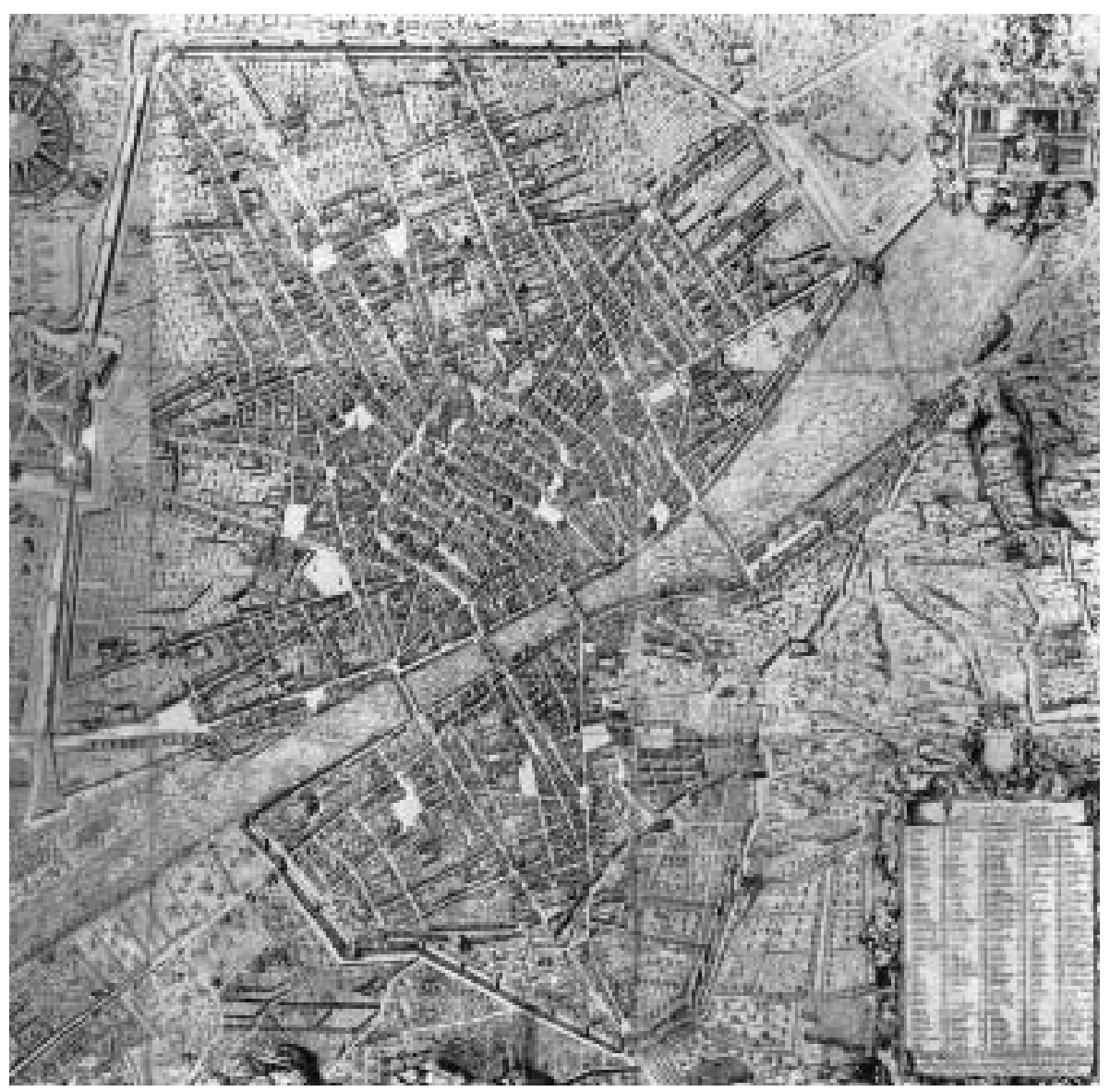

Figura 1 - Stefano Buonsignori "Novae pulcherrimae civitatis Florentiae topographiae accuratissime delineata" (1584).

sportare, come una preda di guerra, nel proprio corredo patrimoniale, in mezzo agli altri possedimenti territoriali"'5.

In quello straordinario ritratto storico della forma urbis fiorentina cinquecentesca, fornito dal monaco olivetano Stefano Buonsignori e datato 1584, che è la Nova pulcherrimae civitas Florentiae topografia accuratissime delineata, Palazzo Pitti ed il Giardino di Boboli appaiono incorporati nei limiti sud-orientali della città fortificata dal cui centro prendono distacco. Il collegamento fisico tra la dimora del principe ed il cuore urbano viene garantito attraverso un percorso aereo privilegiato e protetto: il Corridoio Vasariano. "Come un filo d'Arianna esso permette al principe di districarsi nel labirinto della città e della storia e ritrovare l'approdo al retroterra sicuro e protetto della natura e del giardino"6. Agganciato al bordo del perimetro urbano fortificato il complesso "palatino" di Pitti, con la sua relazione vantaggiosa tra palazzo e giardino, domina così la città e si proietta al contempo alla conquista del territorio aldilà delle mura.

\section{LA STORIA DEL GIARDINO. IL PROGETTO CINQUECENTESCO}

Niccolò Pericoli, detto il Tribolo, che già aveva realizzato per i Medici il giardino della Villa di Castello, fu incaricato del progetto di sistemazione degli spazi esterni della nuova dimora ducale.

\footnotetext{
${ }^{5}$ AlesSANDRO RinAldi, op. cit., 1998, pag. 24.

${ }^{6}$ AlesSANDRO Rinaldi, op. cit., 1998, pag. 25.
} 
Per il disegno del giardino fu utilizzato uno schema compositivo tradizionale, basato sull'accentuazione di una visuale prospettica dominante, in asse col palazzo, e su un'articolazione spaziale fortemente geometrica che tuttavia tendeva a valorizzare i caratteri del preesistente paesaggio agrario e a sfruttare la morfologia naturale del terreno.

Scrive il Vasari che il Tribolo fece "tutto lo spartimento del monte in quel modo che gli sta, accomodando tutte le cose con bel giudizio ai luoghi loro", così da soddisfare i desideri di Cosimo che "voleva ornare quel luogo con giardini, boschi e fontane e vivai ed altre cose simili"7.

La vasta conca esistente alle spalle del palazzo, formata in seguito all'escavazione della pietra forte che era servita come materiale da costruzione, fu utilizzata per realizzare un ampio anfiteatro naturale, incorniciato da terrazzamenti con boschetti di piante in prevalenza decidue.

È proprio questo l'elemento ritenuto più originale nell'impianto cinquecentesco: la riproposizione di un tema architettonico che utilizza la forma ad ippodromo, cioè uno spazio rettangolare chiuso da un'esedra, in chiave naturale, con la cavea (denominata nei documenti cinquecenteschi semplicemente "Il Prato") definita da una struttura verde, non murata. Il Palazzo, affacciandosi su questo spazio scenico, ne diviene al contempo muto e imponente spettatore e quinta architettonica conclusiva.

Già nella sua prima redazione Boboli assume dunque la valenza di "luogo teatrale" per eccellenza: è lo spazio delle rappresentazioni simboliche inscenate attraverso il raffinato programma iconologico attuato nel giardino, è l'ambito privilegiato destinato ad offrire una cornice adeguata ai rituali della vita di corte, è architettura teatrale reale utilizzata per l'allestimento di spettacoli, feste e cerimonie ${ }^{8}$.

Il Tribolo morì appena un anno dopo l'assegnazione dell'incarico, e i lavori furono proseguiti dal genero Davide Fortini, che si attenne comunque al progetto originario. Nel 1561 gli subentrò nella direzione dei lavori Bartolomeo Ammannati, e, a tutt'oggi, lo stato di conoscenza delle fonti storiche non permette di stabilire con esattezza il quadro delle attribuzioni tra lui ed il Tribolo nell'ideazione del piano progettuale del giardino.

La configurazione assunta dal giardino di Boboli alla fine del Cinquecento, dopo l'intervento dell'Ammannati, ci viene descritta con minuzia pittorica in una delle celeberrime lunette dipinte da Giusto Utens nel 1599, in cui è leggibile la stretta connessione tra palazzo e giardino.

L'organizzazione spaziale appare regolata da un rigoroso ordo geometricus, che, come fa notare il Galletti, ripropone gli schemi colturali illustrati nel famoso trattato agrario trecentesco di Piero de'Crescenzi 'Liber ruralium commodorum'. "Ne' verzieri ciascuna sorte d'arbori in suo ordine si dee porre, non mescolata con altra, ad accrescimento di piacere e vaghezza (...), di gran diletto è avere ne' propri luoghi abbondanza di buoni arbori, e di diverse generazioni"’.

Le zone a nord furono sistemate secondo compartimenti quadrangolari e piantate con olivi, alberi da frutto e vigne, un piccolo giardino segreto fu dedicato alla coltivazione dei frutti nani, in cima alla collina fu creato un giardino dei semplici, mentre una grande ragnaia detta 'della banda di Santa Felicita" fu piantata nella zona est. Ecco riprodotto con raffinata cura il paesaggio della collina coltivata.

Il paesaggio dei boschi venne introdotto per definire e incorniciare la scena centrale su cui si affacciava il retro del palazzo: sempre raggruppati in spartimenti

\footnotetext{
${ }^{7}$ In Alessandro Tagliolini, Storia del Giardino Italiano, La Casa Husher, Firenze 1994, pag. 154.

${ }^{8}$ L'11 maggio 1589, per esempio, in occasione delle nozze tra Ferdinando e Cristina di Lorena furono allestiti un Torneo alla Sbarra ed una spettacolare Naumachia nel Cortile, trasformato per l'occasione dall'estro del Buontalenti in un ingegnoso spazio teatrale dotato di complesse strutture sceniche e di un raffinato apparato coreografico.

'In Giorgio Galletti, Giardino di Boboli Master Plan, Ministero per i Beni e le Attività Culturali, Soprintendenza delle Province di Firenze, Prato, Pistoia, Firenze 2000, pag. 13.
} 
ordinati, vennnero messi a dimora 12 cerri (Quercus cerris), 12 faggi (Fagus sp.), 12 aceri (Acer sp.), 12 tigli (Tilia sp.), 12 platani (Platanus sp.), 12 castagni (Castanea sativa), 12 noci (Juglans regia), 12 cornioli (Cornus sanguinea), e ancora, 6 tamerici (Tamaryx gallica), 6 scotani (Cotynus sp.), 2 "secomori" (forse Fycus sycomorus), 12 frassini (Fraxinus sp.), $12 \mathrm{olmi}$ (Ulmus sp.). A questa sequenza di boschetti decidui, che dovevano formare una sorta di tappeto vegetale cangiante caratterizzato da una vivace varietà cromatica e dalla contrapposizione dei diversi effetti di tessitura vegetale prodotti dalle chiome degli alberi, faceva da corona terminale una piantata di sempreverdi, cipressi (Cupressus sempervirens) e abeti (Abies sp.).

Nella ricchezza dell'impianto vegetale e nella tendenza a volere riproporre l'infinita varietà della natura, si riflette la competenza nel campo delle scienze naturali di Cosimo, che va ricordato anche come il committente degli Orti Botanici di Pisa (1543) e di Firenze (1545). La presenza di un giardino dei semplici coltivato sopra il bastione del Cavaliere, oltre a rappresentare un altro elemento di innovazione rispetto alla tradizione umanistica dell'arte dei giardini, conferma il particolare interesse del duca per lo studio del mondo vegetale e la botanica ${ }^{10}$ dimostrato anche con l'esercizio di pratiche di giardinaggio. Annota nel 1754 il naturalista toscano Giovanni Targioni Tozzetti, a proposito dell'abilità di Cosimo I come 'giardiniere': "Sua grande intelligenza di tenere i frutti nani e di fare le cerchiate a mezza botte, (e il) divertimento che si prendeva in potare ed innestare i frutti con le sue proprie mani"11.

Nell'articolato programma decorativo del giardino si riscontra la curiosità per lo studio e la classificazione degli elementi del mondo minerale, che si concretizza in particolare nella realizzazione delle prime grotte artificiali (la Grotticina di Madama e la Grotta Madama). "Le Grotte di Boboli ricostruiscono infatti uno spaccato delle interiora naturae in cui si generano e 'maturano' pietre e fossili. Nella studiata semioscurità degli anfratti artificiali brilla un campionario dei tesori con i quali la Madre Natura, come illustra il ciclo pittorico dello studiolo di Francesco I, alimenta l'industria mineraria toscana e rifornisce generosamente le collezioni medicee"12.

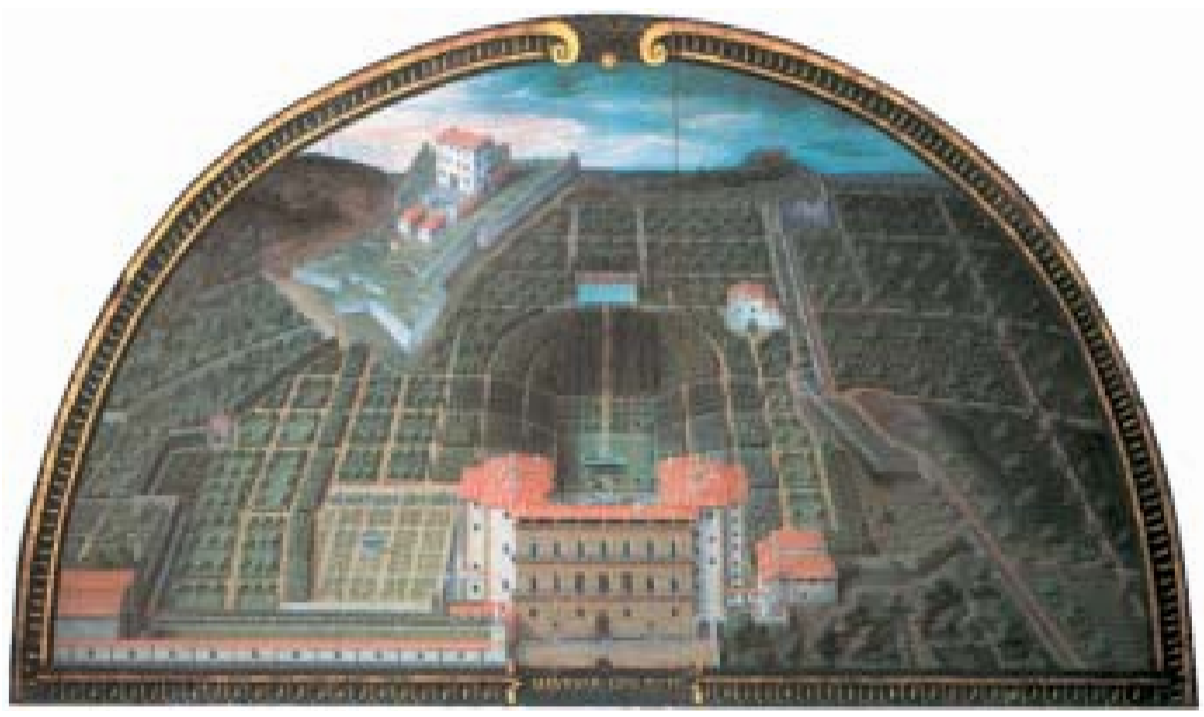

Figura 2 - Giusto di Utens, lunetta raffigurante Palazzo Pitti, (1599-1629). Firenze, Museo Storico Topografico.

\footnotetext{
${ }^{10}$ Cfr. Giorgio Galletti, op. cit., 2000, pag. 14.

${ }^{11}$ In Pietro Roccasecca, Un giardino in area urbana, in Boboli 90 - Atti del Convegno Internazionale, Vol. 2, Edifir, Firenze 1999, pagg. 577-585.

${ }^{12}$ AlESSANDRO RINALDI, Giardini e metamorfosi urbana a Firenze tra Medioevo e Rinascimento di DANIELA CINTI in Giardini \& Giardini. Il verde storico nel centro di Firenze, Electa, Milano 1998, pagg. 15-30.
} 
I lavori di piantagione e sistemazione del terreno procedettero di pari passo con la realizzazione di un complesso sistema di drenaggio, per contrastare il dilavamento superficiale dei terreni in pendenza, e di quello idraulico, finalizzato all'alimentazione delle conserve d'acqua (indispensabili per le coltivazioni) e delle fontane, figure sceniche e simboliche di valore centrale nella lettura dello spettacolo della terza natura allestito nel giardino cinquecentesco.

L'esperienza nel campo dell'ingegneria idraulica, derivata dai precedenti lavori di costruzione del giardino di Castello, permise di condurre con efficacia l'opera di captazione della sorgente della Ginevra, che scaturiva naturalmente nella soprastante collina di Arcetri, e di convogliarne le acque in un grande bacino posto aldisopra dell'Anfiteatro. Da qui attraverso un condotto si poté alimentare la Fontana dell'Oceano, realizzata dal Giambologna tra il 1574 ed il 1576, inserita inizialmente al centro del Prato come da disegno del Tribolo. Un altro condotto andava poi ad alimentare una seconda conserva d'acqua, costruita in prossimità della parte terminale del corridoio vasariano (di collegamento tra Palazzo Pitti e Palazzo Vecchio), in corrispondenza del vivaio iniziato dal Vasari nel 1563 e nucleo primitivo della Grotta Grande ${ }^{13}$.

Gli interventi di regimazione delle acque, oltre a testimoniare una prodigiosa abilità nell'uso delle tecniche di ingegneria idraulica, ci riconducono al tema della cultura manierista del giardino come microcosmo dominato dall'intelligenza e dal sapere del principe, luogo eletto in cui la natura può essere domata e piegata al volere dell'uomo.

"Il ciclo completo di formazione e trasformazione delle acque è oggetto di una rappresentazione plastica nella fontana di Giunone realizzata da Bartolomeo Ammannati, destinata inizialmente al Salone dei Cinquecento e poi trasferita a Boboli sulla terrazza del quarto lato del cortile. Nella grotta sottostante il principe celebra se stesso come legislatore della natura e come costruttore del palazzo e del giardino: sotto le spoglie bibliche di Mosé spezza la rupe di pietra forte con cui è costruita la fabbrica e che nella versione formalizzata dei conci rustici riveste il cortile, facendone scaturire la sorgente che mette idealmente in moto il ciclo delle fontane. Ma la figura mitologica che meglio interpreta l'aspirazione di Cosimo al ruolo di supremo reggitore delle acque è quella di Nettuno. L'immagine del dio può essere proposta nella versione folgorante e armata di Stoldo Lorenzi oppure in quella del colosso pacificatore che sovrasta attualmente l'Isola ma che era stata inizialmente pensata per il centro del teatro di verzura alle spalle di Palazzo Pitti"14.

A Bartolomeo Ammannati si deve il completamento del Cortile Grande del Palazzo: due nuove ali, ortogonali al corpo quattrocentesco, furono allineate alle fasce esterne dell'Anfiteatro a sottolineare la coesione spaziale e formale tra giardino e palazzo, ma anche a suggellare la continuità tra due ambienti scenici, il cortile e l'anfiteatro.

Nel 1574, Cosimo I abdica in favore del figlio Francesco che lo sostituisce alla reggenza di quello che è diventato il Granducato di Toscana.

Si può ritenere che cinque anni più tardi, quando in occasione delle nozze tra il Granduca e Bianca Cappello venne allestito un torneo nel Cortile, il primo nucleo del giardino fosse stato completato ${ }^{15}$. Intanto, alla direzione dei lavori era stato affiancato all'Ammannati Bernardo Buontalenti, a cui si deve il progetto della Grotta Grande, eseguita tra il 1583 ed il 1587, e in cui fu incorporato il preesistente vivaio vasariano.

\footnotetext{
${ }^{13}$ Cfr. Giorgio Galletti, op. cit., 2000, pag. 5.

${ }^{14}$ Alessandro Rinaldi, op. cit., 1998, pag. 26.

15 Giorgio Galletti, op. cit. 2000, pagg. 30-31.
} 


\section{IL SEICENTO}

Nel Seicento il giardino attraversa la sua seconda importante fase di costruzione.

Morto Francesco I nel 1587, morto anche il suo successore, il fratello Ferdinando, nel 1609 diviene Granduca Cosimo II, figlio di quest'ultimo.

È grazie a lui che, a partire dal 1612, il giardino verrà ampliato aldilà delle mura trecentesche, verso l'attuale Porta Romana. Incaricato di seguire il progetto fu Giulio Parigi, cui si affiancò nella direzione lavori per le opere di ingegneria Gherardo Mechini.

La nuova area venne organizzata lungo un ampio Viale, detto dei Cipressi, secondo un asse trasversale rispetto a quello principale esistente che sembra come "scoccato" dai boschetti superiori del giardino cinquecentesco verso ovest per tagliare simmetricamente i terreni di nuova acquisizione.

Nella sua redazione secentesca, Boboli sarà modellato per favorire l'allestimento delle scenografie barocche: l'attivazione del dispositivo di un nuovo asse dominante permetterà di innescare una sequenza di rapporti visuali e prospettici tra le varie 'stanze' del giardino. "In ogni direzione si volga lo sguardo, si percepisce la perfezione della costruzione geometrica, ancora secondo la concezione visiva rinascimentale, ma con una dilatazione più ampia che sfocia in una teatralità pienamente barocca"16.

Gli interventi di trasformazione spaziale sono rivestiti di nuove connotazioni simboliche attraverso l'adozione di schemi costruttivi (ragnaie e labirinti) che, anche se già in uso nel giardino rinascimentale, diventano per il giardino barocco elementi di forte caratterizzazione e vengono a Boboli riproposti in ripetizione quasi ossessiva.

Al di sopra del nuovo viale vennero piantati in successione tre grandi labirinti: il primo per cui i lavori iniziarono nel 1613, detto poi Labirinto Vecchio, era il più grande e di forma ovale. A questo fecero seguito gli altri, uno circolare, piantato nel 1622 ed il seguente, a pianta ottagonale, nel 1638. Un quarto labirinto ottagonale era disposto simmetricamente aldisotto del Viale. Al termine della sequenza dei labirinti un percorso coperto da un'architettura vegetale formata dall'allineamento di due filari di leccio con i rami intrecciati, detto la Cerchiata Grande, affiancato da altri due passaggi coperti detti le Cerchiate Piccole, tagliava perpendicolarmente il Viale dei Cipressi incrociandolo a metà percorso.

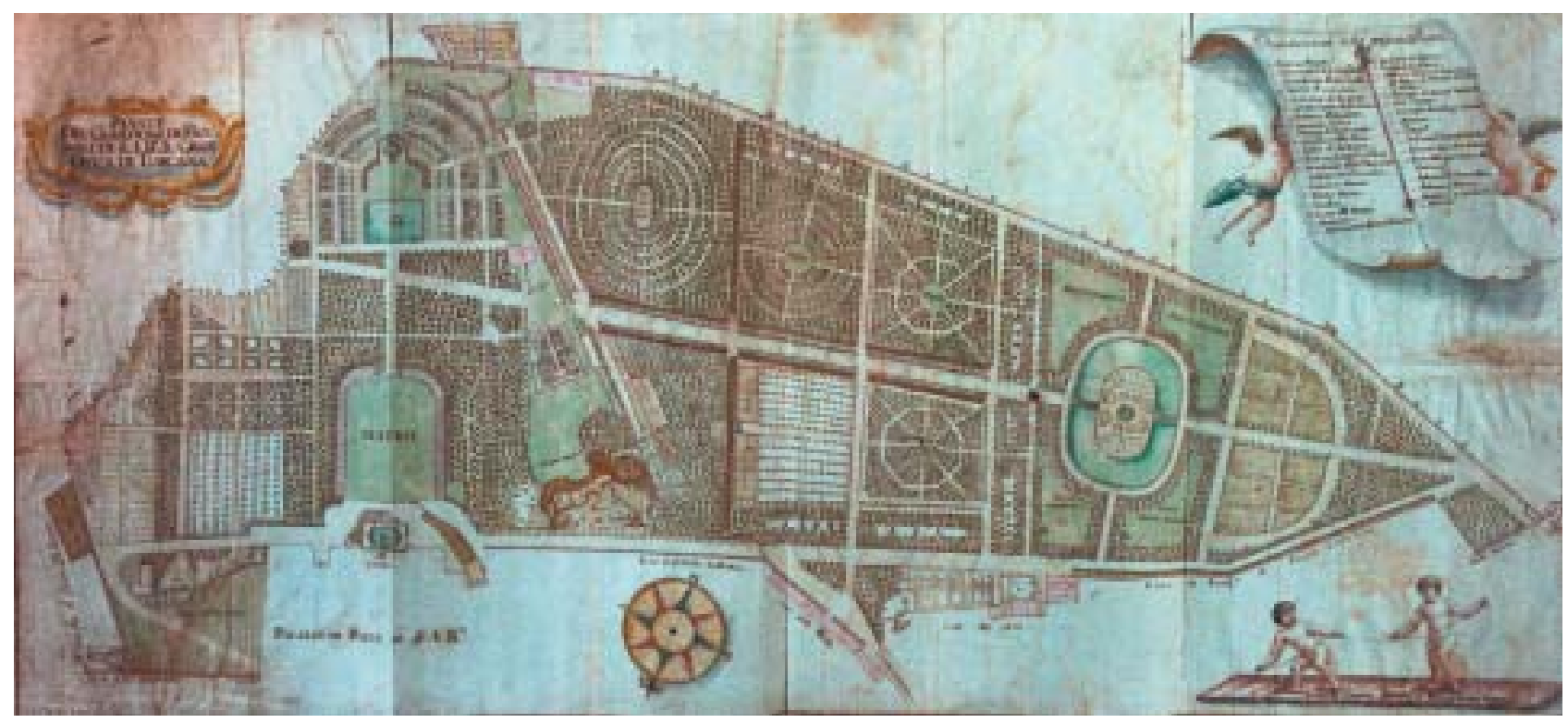

Figura 3 - Michele Gori, "Pianta del Giardino di Boboli di S.A.R. il Granduca di Toscana”, (1709). Firenze BNCF, Nuove accessioni, cartella 7.159. 
Alla sommità nord del Viale furono create due terrazze destinate alla coltivazione degli agrumi a spalliera. Proseguendo, il Viale arrivava alla zona dell'Anfiteatro, per cui dal 1630 al 1634 si condussero i lavori che portarono alla definizione delle gradinate in muratura; al di sopra dell'Anfiteatro venne inoltre realizzato, a delimitazione della vasca rettangolare cinquecentesca (la conserva d'acqua in cui erano state portata le acque della Sorgente Ginevra), un grande invaso formato da una serie di terrazzamenti degradanti, quasi un secondo anfiteatro, chiamato il Bacino del Forcone per la collocazione al centro della vasca di una statua in bronzo del Nettuno con tridente, opera di Stoldo Lorenzi.

Risulta dalle planimetrie e dalle fonti storiche che i terrazzamenti erano decorati con preziose broderies fiorite, poste a coronamento di un gigantesco stemma mediceo disegnato con siepi di bosso e visibile dal Palazzo. All'estremità sud del Viale venne realizzato invece un dispositivo spaziale di sicuro effetto scenografico, il bacino dell'Isola, un ampio spazio di forma ellittica, circondato da cipressi (Cupressus sempervirens). Al centro, fu inserita una grande vasca con un' isola sistemata come giardino di fiori in vaso compartimentato, contenente in origine una statua di Venere circondata da amorini, e accessibile attraverso due ponti lineari realizzati lungo la direttrice del Viale. Nel 1637, la statuaria originale venne smantellata su indicazione di Alfonso Parigi detto il Giovane, che fece inserire la Fontana dell'Oceano del Giambologna, precedentemente collocata al centro del Prato cinquecentesco.

Pur mantenendo la continuità con i temi compositivi svolti nel disegno cinquecentesco, la concezione spaziale del progetto del Parigi attua quella strategia di teatralizzazione dell'impianto del giardino tipica del gusto barocco e la svolge con successo: uscendo dal recinto protettivo originario, il giardino si sviluppa per assumere un aspetto più grandioso e per moltiplicare i suoi luoghi scenici.

L'uso dello schema geometrico a compartimenti mantenuto nella composizione secentesca ben si presta alla realizzazione delle ragnaie, la cui tecnica di costruzione venne applicata ad ampie parti di Boboli, "divenendo un vero modulo costruttivo vegetale. La maglia dei vialetti delimitava insulae di verzura dalla forma di lunghe strisce, entro le quali era distribuita con regolarità geometrica la piantagione, dando luogo ad innumerevoli corridoi dalla prospettiva allargatissima" ${ }^{17}$.

Vennero costruite la grande ragnaia dell'Isola, che ne circondava il bacino, e quella della Pace, sotto le mura trecentesche, affiancata da una catena d'acqua, la cosiddetta Fontana dei Mostaccini.

\section{IL SETTECENTO}

Nel 1737, dopo la morte dell'ultimo rappresentante della dinastia medicea, il Granducato di Toscana passa agli Asburgo-Lorena ed ha inizio una nuova stagione politica e culturale. Boboli attraversa un primo periodo di abbandono e incuria, per poi ritrovare nuova vita a partire dal 1765 con l'insediamento di Pietro Leopoldo a Firenze. Furono avviati vari interventi di risistemazione del giardino, il cui disegno planimetrico generale restò sostanzialmente invariato, anche se sottoposto ad una formalizzazione più accentuata dell'ordine geometrico attraverso l'uso di spalliere di sempreverdi. Vennero introdotte alcune innovazioni: la creazione di un Jardin potager dotato di serre calde per la coltivazione di piante esotiche; la costruzione di una limonaia e di una Kaffehaus di gusto viennese disegnate da Zanobi del Rosso; un giardino botanico contiguo a Boboli fu annesso al Gabinetto di Fisica e Storia Naturale istituito nel 1771; il profilo del Bacino del Forcone venne rimodulato in chiave rococò.

\footnotetext{
${ }^{17}$ Giorgio Galletti, op. cit., 2000, pag. 15.
} 


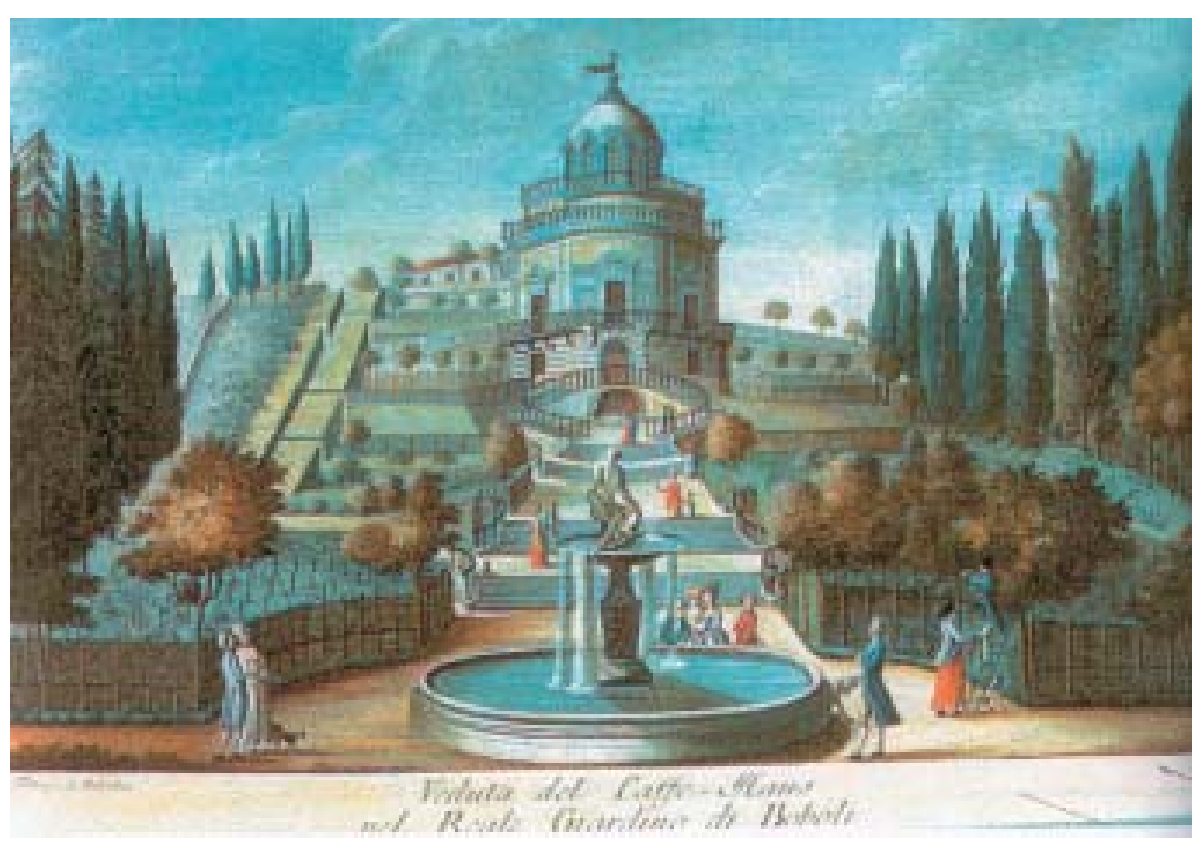

Figura 4 - Aniello Lamberti, Veduta del Kaffeehaus (1790 ca.).

Boboli si arricchì di nuovi elementi ornamentali e della statuaria della collezione di Ferdinando I che da Villa Medici a Roma venne trasferita a Firenze. Della collezione faceva parte anche l'Obelisco Egizio che sarà collocato nel 1790 al centro dell'Anfiteatro.

Allo stesso anno risale una serie di undici incisioni di Aniello Lamberti raffiguranti il Giardino di Boboli dopo gli interventi ed i restauri lorenesi. Nelle tavole gli spazi del giardino appaiono delimitati da alte siepi formali di sempreverdi; questo particolare fa presumere che è quello lorenese il periodo in cui la costruzione di alte spalliere di siepe mista fu estesa anche ai viali non destinati all'uccellagione, affiancati invece nel Seicento da siepi in forma libera ${ }^{18}$

\section{L'OTTOCENTO}

Nel 1799 le truppe napoleoniche invadono Firenze ed i Lorena lasciano la città; due anni dopo, con l'istituzione del Regno di Etruria, la Toscana passa sotto Lodovico di Borbone, per poi essere assegnato nel 1807, con il Trattato di Fontainebleau, all'impero francese. Questi vari passaggi di proprietà, cui è collegata la mancanza di cura e manutenzione del giardino, fanno sì che Boboli si affacci al nuovo secolo in uno stato di evidente abbandono. Tuttavia, ancora all'inizio dell'Ottocento, Boboli mantiene pressochè inalterato il disegno cinque-secentesco.

Quando Elena Baciocchi, sorella di Napoleone, nominata Granduchessa di Toscana giunge a Firenze nel 1808, esplicita subito il proposito di attuare una grande trasformazione dell'ormai superato modello geometrico-formale del giardino in chiave paesaggistica. È la moda del tempo, e anche una grandiosa opera storica come Boboli non ne può rimanere immune. I lavori di sistemazione del giardino vengono affidati a Giuseppe Cacialli, che aveva assunto l'incarico di "Architetto dei Regi Palazzi e Possessioni". Vengono avviati alcuni interventi di ristrutturazione, che prevedono anche il ripristino dei giochi d'acqua della Grotta Grande. Per aprire un nuovo viale alberato con platani (Platanus occidentalis) viene distrutto il primo corridoio della grande Ragnaia dell'Isola.

${ }^{18}$ Giorgio Galletti, op. cit., 2000, pagg. 10-11. 


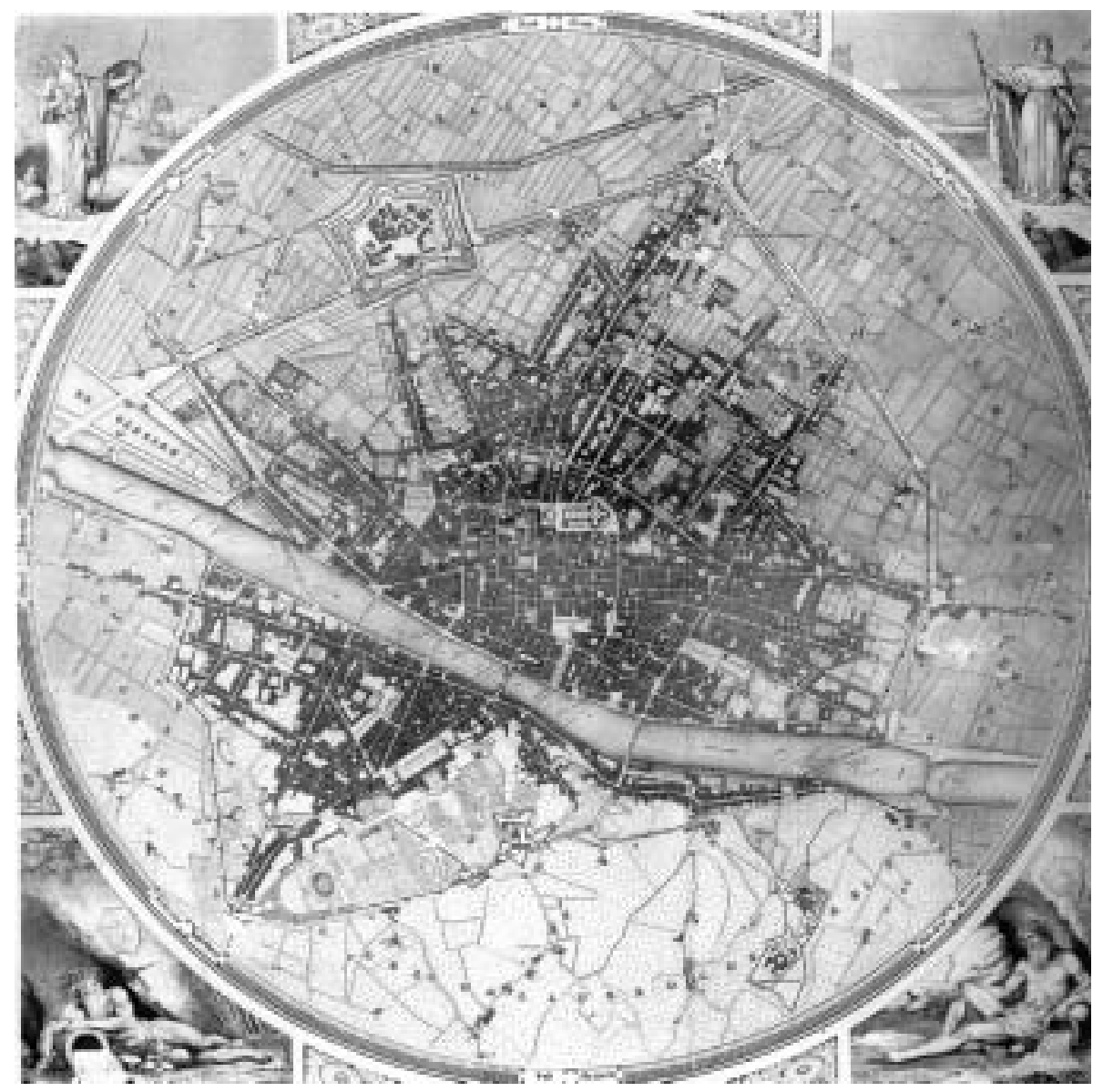

Figura 5 - Federico Fantozzi, "Pianta geometrica di Firenze sulla proporzione di 1 a 4500" (1843).

Nel 1814, con la caduta dell'Impero Napoleonico il Granducato ritorna ai Lorena. Ferdinando III decide di restaurare Boboli restituendolo "alla primiera sua forma, secondo il disegno del Tribolo e del Buontalenti"19 e affida il compito all'architetto Pasquale Poccianti che da immediatamente corso a nuovi progetti, tra cui la costruzione di due tiepidari e di una Grotta presso l'ingresso di Annalena.

Ė nel 1834, dopo la morte di Ferdinando III, che Boboli subisce l'intervento di trasformazione più pesante e distruttivo con la costruzione di un sinuoso viale carrozzabile di collegamento tra la sommità del Viale dei Cipressi e la zona dell'Isola. I tre grandi labirinti secenteschi vengono cancellati, e il tentativo di realizzare con la vegetazione arborea laterale al Viale dei Cipressi amene quinte naturali fallisce: $\mathrm{i}$ boschetti di leccio, per mancanza di manutenzione, finirono in breve tempo per trasformarsi in disordinata boscaglia destinata alla produzione di legna. I piani originari della zona dei Labirinti furono inoltre maldestramente alterati a causa dei significativi riporti di terra necessari alla definizione del nuovo tracciato.

Il tentativo ottocentesco di annullare il formalismo geometrico dell'impianto originario, rompendo la sequenza compartimentata del giardino con una forte linea a serpentina, appare dunque quanto mai goffo e scorretto anche dal punto di vista paesaggistico: imbriglia la successione degli spazi secenteschi ordinati secondo una griglia ortogonale senza riuscire a svincolarsi dalle antiche direttrici rettilinee.

Un secondo intervento ottocentesco di sostanziale modifica dell'impianto precedente è costituito dalla costruzione nel 1852 di un Giardino Botanico ad opera di Filippo Parlatore, botanico palermitano, che trasformò l'area del geo-

${ }^{19}$ F. Inghirami 1835, in Giorgio GalletTi, op. cit., 2000, pag. 45. 
metrico Jardin potager, posto su un terrazzamento sostenuto da un alto muro lungo una ragnaia, secondo i canoni del giardino all'inglese. Furono disegnati percorsi e aiuole dal profilo sinuoso, con leggeri movimenti di terra furono create dolci ondulazioni. Il nuovo Giardino Botanico, denominato "Superiore" per distinguerlo da quello leopoldino, venne organizzato in funzione di una distribuzione sistematica delle piante per provenienza geografica. Il Tepidario Grande fu riadattato per permettere la coltivazione delle numerose specie di piante tropicali introdotte e la vasca ovest, detta delle Ninfee, fu trasformata in un bacino ornamentale con l'inserimento di una roccaglia di gusto pittoresco. Un Aquarium, risalente probabilmente al 1874, di forma circolare e suddiviso in quarantotto celle, del tutto simile a quello realizzato dallo stesso Parlatore per l'Orto Botanico di Palermo, trovò posto nella fascia est per ospitare la sezione di raccolta scientifica delle piante acquatiche ${ }^{20}$.

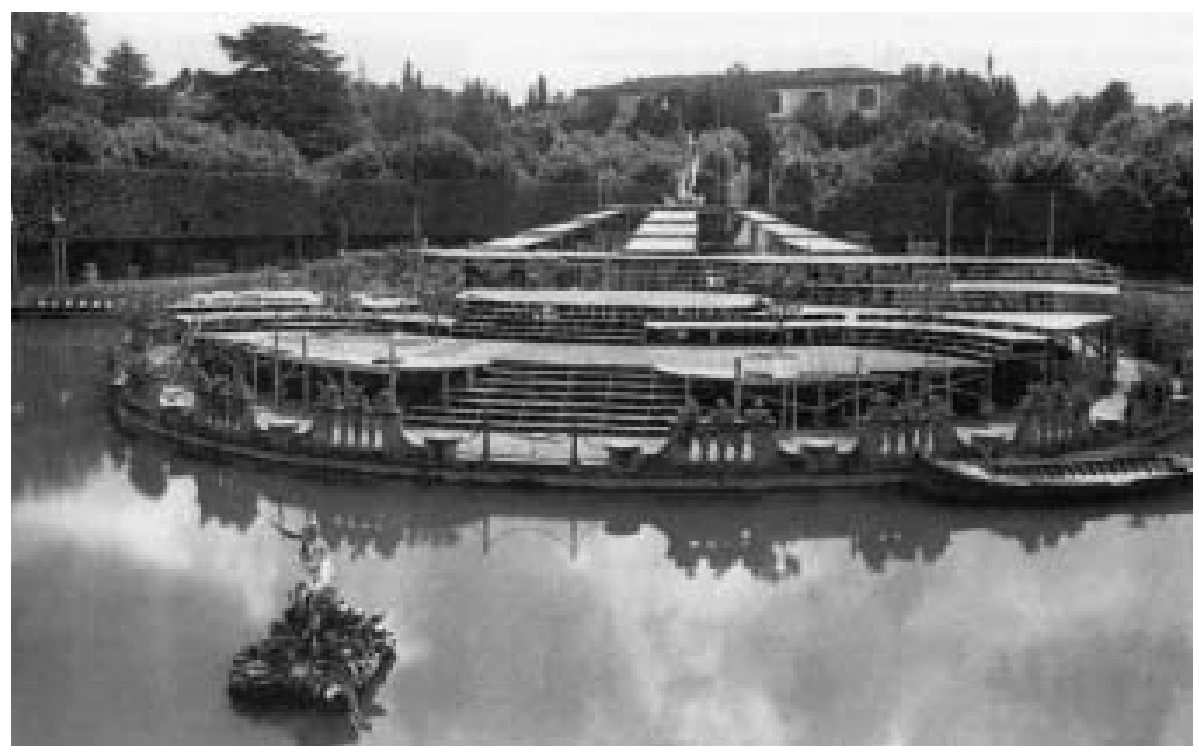

Figura 6 - L'Isola di Boboli allestita per lo spettacolo teatrale La Tempesta, regia di Giorgio Strehler (1953).

\section{DAL NOVECENTO AD OGGI}

Quello del Parlatore può essere considerato come l'ultimo della sequenza storica degli interventi significativi di trasformazione di Boboli, che ha attraversato tutto il Novecento senza subire ulteriori modifiche della sua configurazione spaziale.

Nel 1905 Palazzo Pitti ed il giardino divengono di proprietà demaniale, e durante tutto il corso dello scorso secolo il complesso fu oggetto di vari restauri, ora volti a recuperare un edificio, ora una parte di giardino, ora elementi della statuaria.

La vocazione di Boboli a luogo teatrale resta confermata dal numero sostanzioso di spettacoli all'aperto che a partire dal 1933 vi sono allestiti ${ }^{21}$.

Nel 1966 Boboli viene aperto al pubblico: l'accesso al giardino è offerto in forma libera e incondizionata. L'apertura gratuita lascia per molti anni questi spazi ad un uso pubblico incontrollato e spesso poco rispettoso. Anno dopo anno

${ }^{20}$ Cfr. Giorgio Galletti, op. cit., 2000, pagg. 167-169.

${ }^{21}$ Si rimanda per approfondimenti sull'argomento a: ALESSANDRO SARDELLI, "La città e il giardino: uso pubblico di Boboli all'inizio del Novecento" ed a MARIA AlBERTI “Teatro all'aperto - Teatro di stato: il Sogno di Reinhardt a Boboli (1933) come antefatto ai temi dibattuti dal Convegno Volta (Roma 1934)", in Boboli 90 - Atti del Convegno, Vol. 1, Edifir, Firenze 1999, pagg. 358-381. 
a Boboli si sono andati accentuando gli aspetti del degrado legati ad una fruizione troppo 'disinvolta': compattazione dei suoli, vandalismo verso statue ed edifici, sporcizia. A questi, vanno sommati i fenomeni di degrado ambientale dovuti al crescente inquinamento atmosferico e al progressivo processo di dilavamento dei terreni in pendenza ormai quasi del tutto privi della rete di drenaggio, così come gli effetti di una manutenzione del patrimonio vegetazionale poco accorta. Tra questi ultimi, il metodo di potatura utilizzato per il contenimento dell'architettura arborea, la capitozzatura ${ }^{22}$, eseguita con tagli drastici di motosega, ha finito per causare oltre al degrado del paesaggio vegetale anche la propagazione di fitopatologie e marciumi nelle piante. La numerosa popolazione di lecci (Quercus ilex) ne è risultata particolarmente danneggiata.

All'inizio degli anni Ottanta, periodo in cui il dibattito sui problemi del restauro e della conservazione dei parchi e giardini storici diviene più acceso, l'allora Direttore dell'Unità Organica Funzionale Ville, Parchi e Giardini, Arch. Massimo de Vico Fallani, riuscì ad imprimere un decisivo impulso alla rivalutazione dei giardini medicei in consegna alla Soprintendenza per i Beni Architettonici ed Ambientali delle Province di Firenze, Prato e Pistoia. Grazie alla sua competenza e al suo lavoro furono realizzati i primi dettagliati rilievi floristici di Boboli "moderna" e avviate indagini storiche.

In occasione del convegno nazionale Boboli '90, tenuto nel 1989 a Firenze per fare il punto sullo stato della conoscenza del giardino, fu possibile portare all'attenzione degli organi ministeriali italiani la necessità di intervenire per impedire l'ormai devastante avanzamento del degrado, accentuato dalla forte affluenza giornaliera di pubblico giunta a contare circa quattro milioni di visitatori all'anno. I partecipanti al convegno furono concordi sulla necessità di sottoporre il giardino di Boboli alle stesse misure di gestione della frequentazione pubblica adottate per il sistema museale nazionale e quindi all'applicazione di una tassa di

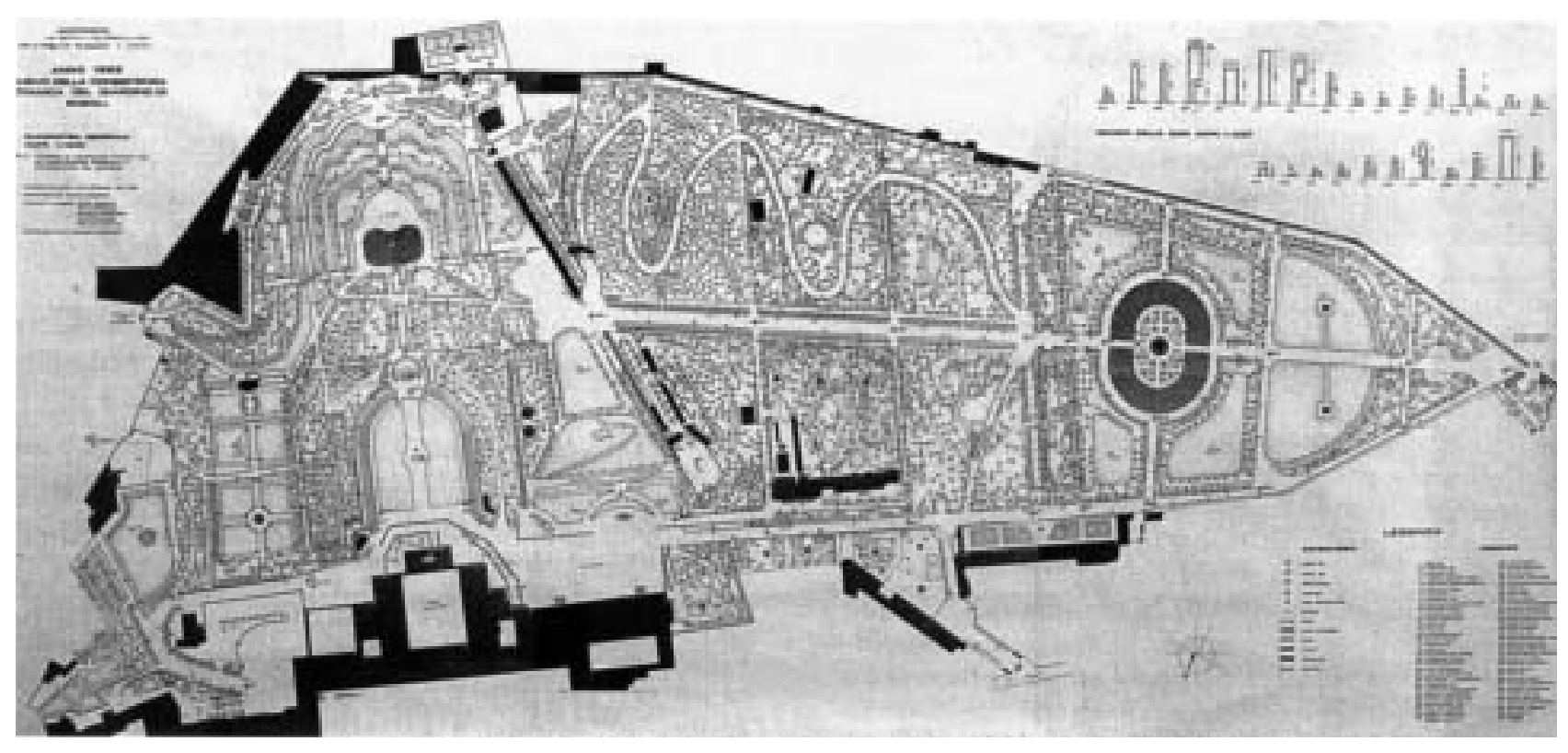

Figura 7 - Rilievo floristico del Giardino di Boboli fatto realizzare da Massimo Vico Fallani nel 1982.

${ }^{22}$ La capitozzatura è una tecnica di coltivazione applicabile soltanto ad alcune specie arboree, e consiste nel ceduare la pianta ad alcuni metri da terra (circa 3-4 metri) ed aspettare l'emissione di nuovi polloni da parte delle gemme dormienti. A Boboli, per contenere i costi di manutenzione, questi interventi sono stati praticati per molto tempo seguendo intervalli di 10-15 anni; applicando turni così lunghi, il taglio veniva fatto su rami che avevano avuto modo di raggiungere notevoli dimensioni, operazione che portava la pianta ad indebolirsi sempre più. 
ingresso, che fu introdotta nel 1993. Questa disposizione, che suscitò anche molte polemiche, permise di porre un limite alla tendenza da parte di residenti e turisti a considerare Boboli alla stregua di un qualsiasi parco o giardino di quartiere e non piuttosto come un prezioso e fragile complesso di carattere museale da rispettare e proteggere. Il numero dei visitatori all'anno è attualmente di circa un milione, cifra che porta Boboli ad essere il secondo museo fiorentino più frequentato.

\section{Il Master Plan: GLI OBietTivi}

La necessità di creare un piano organico di restauro per il Giardino di Boboli in grado di individuare e definire un programma coerente di interventi volti al recupero, alla conservazione, alla gestione ed alla manutenzione del ricco e prezioso sistema museale, appariva da tempo evidente in particolar modo in considerazione del suo duplice aspetto di bene storico-culturale e di parco pubblico, unica area verde usufruibile nella zona d'oltrarno fiorentino. Se durante i lavori del convegno Boboli '90 era emersa la preoccupazione per il crescente avanzamento del degrado di molte delle opere architettoniche e di arredo, non era risultato di certo incoraggiante neanche il quadro delle conoscenze sullo stato dell'impianto vegetale e le sue condizioni fitosanitarie ${ }^{23}$.

La considerazione che gli interventi di restauro e conservazione del giardino e delle sue architetture non potevano più essere affidati alla estemporaneità delle decisioni quotidiane e alla semplice gestione delle attività di manutenzione ordinaria, fece maturare l'idea di elaborare un documento programmatico di linee guida.

Tra il 1998 ed il 1999, l'allora responsabile dell'Unità Organica Funzionale Ville, Parchi e Giardini, Arch. Giorgio Galletti, usufruendo di una borsa di ricerca dell'istituto statunitense "Studies in Landscape Architecture" di Dumbarton Oaks, ebbe modo di lavorare alla redazione del Master Plan di Boboli. Per la sua stesura, il Galletti si è avvalso dell'importante apparato documentario precedentemente costituito, di un repertorio significativo di studi e informazioni già raccolte in vari ambiti di ricerca, dell'indispensabile collaborazione di colleghi, studiosi, tecnici e dei giardinieri di Boboli, oltre che di una esperienza ormai decennale di attività gestionale diretta e degli esiti di meticolose ricerche d'archivio condotte in prima persona.

Il Master Plan contiene "un complesso ordine di operazioni preliminari al progetto esecutivo, che hanno lo scopo di costruire una visione unitaria di tutte le problematiche inerenti il sito oggetto dell'intervento. La conoscenza storica, i dati d'archivio, l'analisi visuale dello stato di fatto, i rilievi, l'interpretazione dello spazio, l'analisi del degrado, gli usi passati e attuali, l'ottimizzazione della gestione, i metodi di coltivazione si assommano nella individuazione di una strategia che garantisca il massimo dello standard qualitativo nel restauro e nella manutenzione future." 24 Il Master Plan di Boboli si presenta quindi come uno strumento finalizzato alla pianificazione della progettazione.

Considerata la natura e la storia del bene oggetto di attenzione progettuale, il tema d'interesse centrale è apparso pertanto fin dall'inizio quello di individuare accanto alle linee direttrici per gli interventi di restauro, alcuni indirizzi di 'pianificazione' della manutenzione.

${ }^{23}$ Si legga al riguardo: Romano Gellini, Alberto Giuntoli, PaOlo Grossoni e Silvia Schiff, $I l$ Giardino di Boboli: aspetti vegetazionali, condizioni fitosanitarie e metodologie di intervento, in Boboli 90..., op. cit., 1999, pagg. 195-211.

${ }^{24}$ Giorgio GalletTI, op. cit., 2000, pag. 1. 
L'autore dichiara esplicitamente che "il Master Plan non intende risolvere tutti i problemi, ma li pone, li analizza, li schematizza in tavole riassuntive di facile consultazione. Le soluzioni future potranno essere diverse, molteplici, maggiormente approfondite con progetti particolareggiati. Nuovi studi storici e nuovi documenti d'archivio potranno ulteriormente contribuire a colmare le molte incertezze e i rischi di errori. Questo documento è il frutto di un'esperienza vissuta giorno per giorno in giardino, in archivio, in biblioteca, che non potrà dettare legge assoluta, ma potrà essere uno strumento utile a chi dovrà operare in futuro in questo luogo eccezionale della storia" 25 .
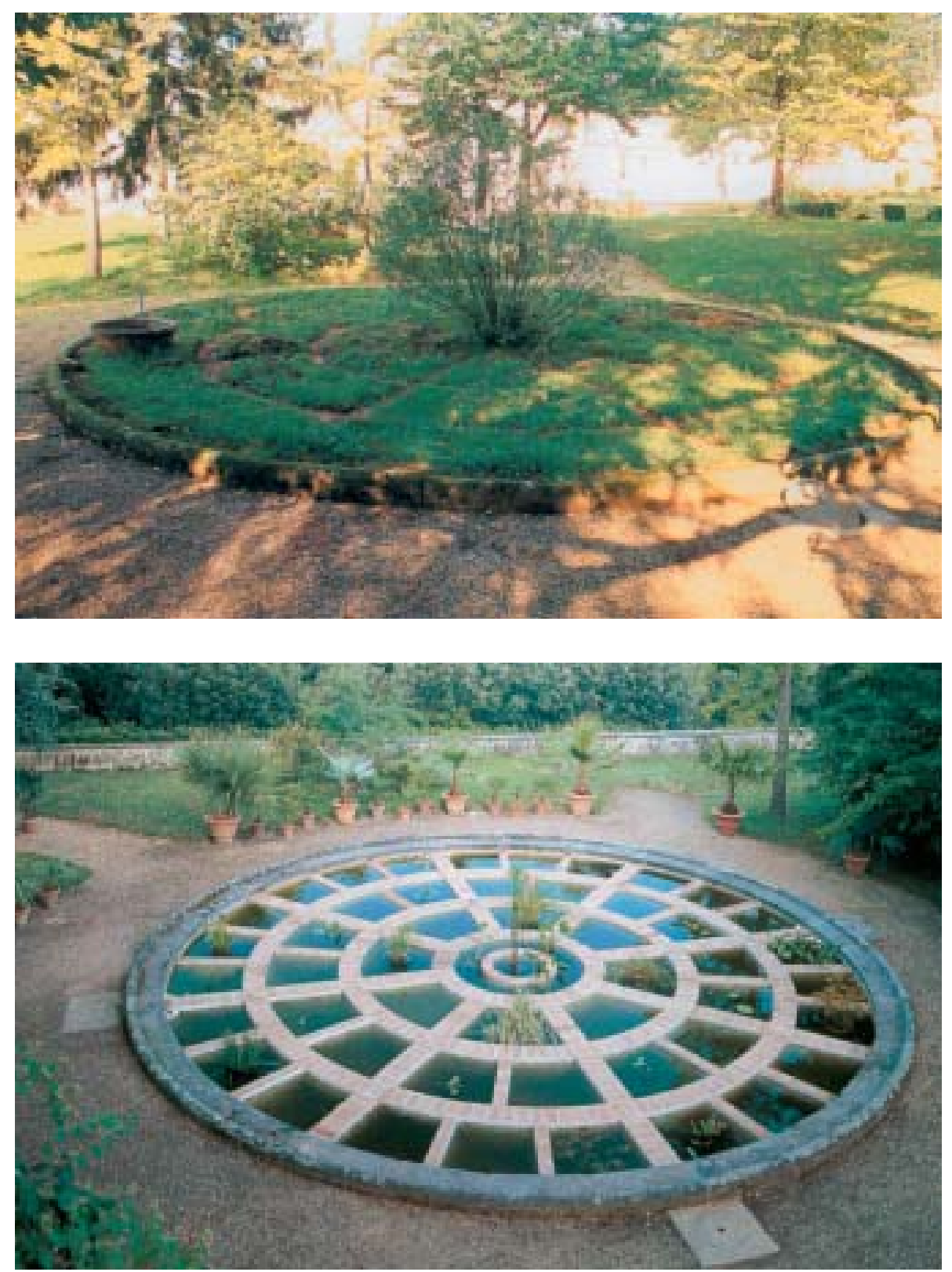

Figure 8-9 - L'Aquarium del Giardino Superiore della Botanica, prima e dopo gli interventi di restauro effettuati dal 1994 al 1996. 
Il documento elaborato si compone di:

- Premessa, in cui sono sinteticamente definiti obiettivi e contenuti del Piano;

- Riassunto storico, con cui si ripercorre a grandi linee l'evoluzione del giardino dalle sue origini fino ad oggi;

- Lettura critica - interpretativa del paesaggio di Boboli e della sua influenza nella storia dei giardini, in cui le trasformazioni storiche sono analizzate così da poter mettere in evidenza le fasi di formazione della struttura del paesaggio che oggi vediamo;

- Tavola cronologica, che ricostruisce in forma puntuale un percorso nel tempo segnalando tutte le notizie, gli interventi e riferimenti storici documentati utili per conoscere Boboli, dal 1451 al 1996;

- Raccolta e analisi critico-descrittiva di tutte le planimetrie storiche del giardino conosciute fino ad oggi, partendo da un brano della Pianta di Firenze di Stefano Buonsignori del 1584 e concludendo con la "Pianta geometrica di Firenze" eseguita da Federico Fantozzi nel 1843;

- Sezione dedicata alla definizione degli interventi di restauro proposti per il giardino, che viene scomposto in sedici zone omogenee. È questa la parte più consistente del documento;

- Note sugli interventi di manutenzione ordinaria;

- Preventivo di massima, con cui viene conferito un carattere di maggiore operatività, al Master Plan, attraverso una stima dei costi complessivi degli interventi ipotizzati in forma puntuale, fondamentale per la valutazione delle risorse finanziarie reali da attivare.

Il piano progettuale è rivolto al restauro delle strutture architettoniche e dell'impianto vegetazionale, mentre per i problemi ed i temi del restauro delle singole opere della statuaria presenti nel giardino rimanda alla redazione da parte di uno storico dell'arte di ulteriori studi e progetti specifici.

\section{METOdOLOGIA: BobOLI TRA CONSERVAZIONE, RESTAURO E MANUTENZIONE}

La metodologia di lavoro che ha condotto alla redazione del Master Plan è impostata sulla concezione di Boboli come di un unicum paesaggistico, intendendo con questo che "le sculture come le architetture, dall'Oceano del Giambologna, dallo Stanzone degli Agrumi, alla Kaffehaus, alla Grotta del Buontalenti sono complementari alla vegetazione ed insieme ad essa costituiscono un paesaggio storico di eccezionale rilevanza. La lettura di questo paesaggio, per cui è stato necessario lo studio delle fonti, dei trattati ed il filtro dell'esperienza quotidiana, ha gettato le basi per una futura strategia di restauro" 26 .

Leggere Boboli come un testo unitario polimaterico, del resto, appare l'unica operazione possibile per affrontare il difficile problema del suo restauro, osservazione valida per il restauro di ogni giardino e parco storico. La natura intrinseca del bene 'giardino storico', come è noto, implica l'attivazione di una pluralità di competenze professionali e tecniche e la necessità di raccogliere un sapere multidisciplinare che può provenire solo attraverso il contributo di figure diverse anche in tempi differenti.

Il percorso che ha portato all'individuazione delle linee guida per gli interventi di restauro e di conservazione si è mosso dalla fase preliminare di conoscenza del giardino $^{27}$, studiato, letto, osservato e interpretato sia attraverso accurate e ripetute

${ }^{26}$ Giorgio Galletti, Giardino di Boboli Master Plan, Ministero per i Beni e le Attività Culturali, Sop. Firenze, Prato, Pistoia, Firenze 2000.

27 "La documentazione storica rappresenta il primo gradino della conoscenza di un giardino storico. La ricerca si conduce, come per i beni architettonici, su fonti letterarie e documentarie edite ed inedite, su cartografia, materiale iconografico e fotografico, fonti che in generale costituiscono la base per la conoscenza storica del giardino e si qualificano come un insieme ricco e vario...", MARIACHIARA POZZANA, Giardini storici. Principi e tecniche della conservazione, Alinea editrice, Firenze 1996, pag. 104. 
ricerche di archivio e di fonti documentarie storiche sia come oggetto in sé, 'documento di sé stesso' su cui sono stati condotti, attraverso gli anni, rilievi strumentali diretti (floristici, pedologici, architettonici, eccetera) e analisi visuali.

Nel suo lavoro il Galletti ha integrato la documentazione già raccolta e gli studi realizzati anche da altri, "confezionando" un prodotto scientifico che tiene conto quindi di un corpus molto nutrito di indagini conoscitive ed ha il merito di averle sapute organizzare ed elaborare in forma critica.

Lo studio della cartografia storica urbana e di progetto, ricca soprattutto nella sua produzione settecentesca, ha permesso di conoscere la configurazione del giardino nelle sue varie fasi. In particolare la scoperta, effettuata dallo stesso Galletti durante le indagini d'archivio, della "Pianta del Giardino di Boboli di S.A.R. il Granduca di Toscana", elaborata da Michele Gori nel 1709, è risultata di grande utilità per la conoscenza di Boboli dopo gli interventi secenteschi di ampliamento e trasformazione in chiave barocca del giardino. Questo prezioso documento iconografico, caratterizzato da una notevole precisione e dalla descrizione minuziosa di molti particolari ancora sconosciuti, ha fornito riferimenti utili in particolare per l'interpretazione critica sulla cui base sono state mosse le proposte di restauro per la zona dell'Isola e dei Labirinti.

Pur partendo da una lettura unitaria del giardino, in considerazione della complessità della sua struttura e della sua storia, il Master Plan per rendere più agevole ed efficace la definizione dei vari interventi di restauro, individua all'interno del giardino sedici aree caratteristiche, "ciascuna valutata come entità a sé stante".

Per quanto riguarda alcune zone, questa operazione è stata agevolata dalla natura del giardino "squisitamente all'italiana" e quindi di per sé organizzato geometricamente e rigorosamente compartimentato.

Altre zone invece sono state circoscritte in applicazione di valutazioni di tipo meramente operativo. Ė quest' ultimo il caso delle aree del Viale dei Cipressi, dei Labirinti e dell'Isola, che di fatto dal punto di vista paesaggistico e storico non sarebbero scindibili l'una dalle altre perché frutto di un unico progetto originario e di un'unica sequenza spaziale, ma che sono state suddivise in tre unità distinte in riferimento alla differente natura dei problemi di restauro ad esse connessi.

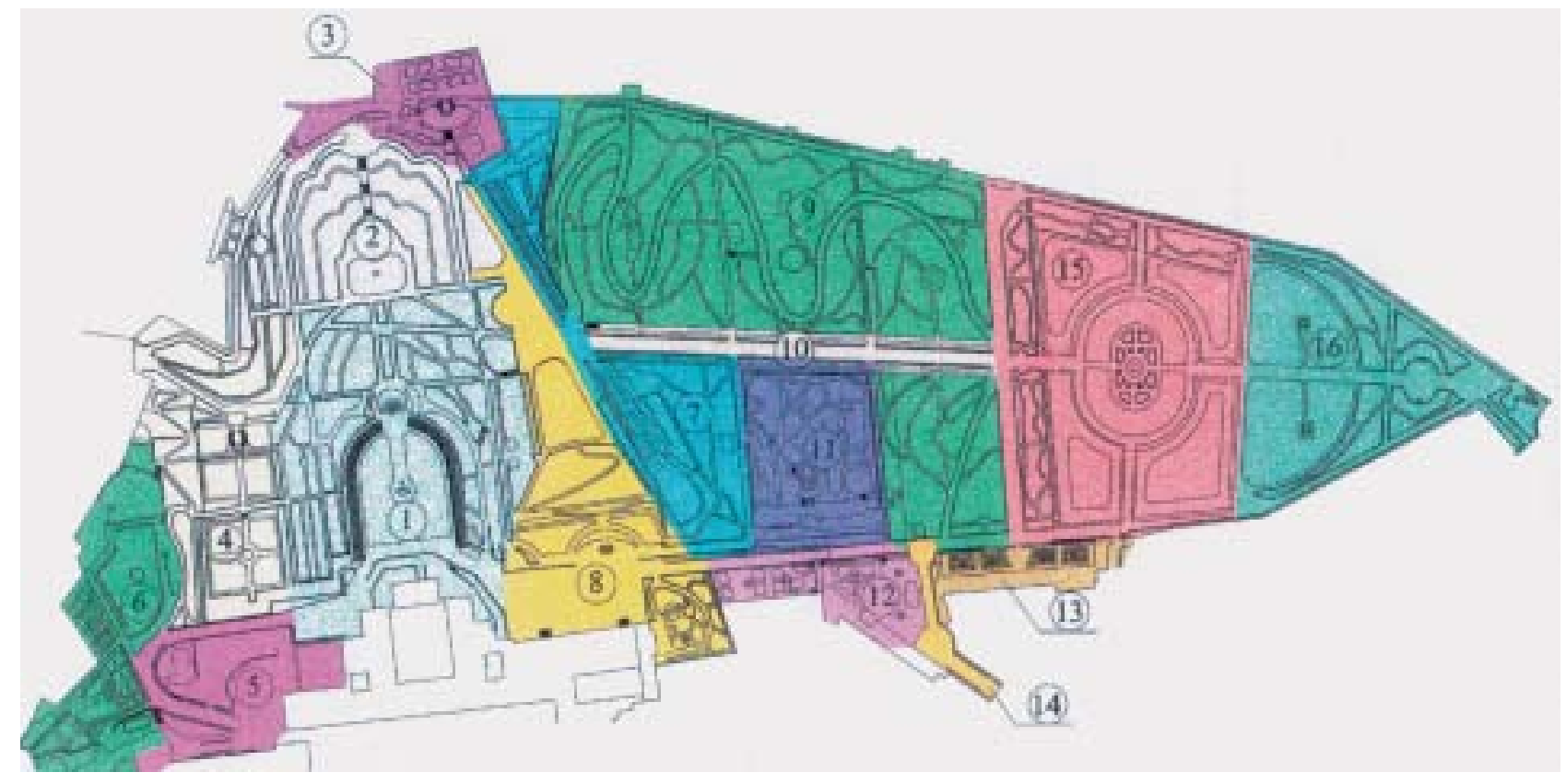

Figura 10 - Planimetria generale del Master Plan, con individuazione delle 16 aree caratteristiche. 
L'individuazione delle aree caratteristiche ha permesso inoltre di ipotizzare interventi unitari, finalizzati all'attivazione di un più efficace e meno dispersivo programma di lavori.

Le sedici aree omogenee individuate, così come è possibile desumere dalla planimetria generale di riferimento, sono le seguenti:

1. L'Anfiteatro, con Anfiteatro, Viale Obliquo, Viale di Cerere, Rampe del Forcone, Ghiacciaie, Giardino delle Camelie, Giardino del Carciofo.

2. Il Bacino del Forcone, con Emiciclo, Vasca di Nettuno, Statua della Dovizia.

3. Il Giardino del Cavaliere, con Fontana delle Scimmie, Giardino, Piazzola d'ingresso, Vasche delle Trote e del Cavaliere.

4. Kaffehaus, con Kaffehaus e Giardino.

5. Il Piazzale di Bacco e La Grotta Grande, con Piazzale di Bacco, Cortile Nonfinito, Viale dei Prigionieri, Grotta Grande.

6. Area di Madama, con Grotta e giardino di Madama, Giardino di Giove, Prato del Menabuoni.

7. Giardini della Lavacapo e della Sughera.

8. Meridiana, Prato dei Castagni, Conte di Torino

9. I Labirinti, con Labirinto Superiore, Labirinto Intermedio, Labirinto Inferiore, Labirinto Ovest, Viale Carrozzabile Est, Cerchiata Grande, Viale dei Mostaccini, Ragnaia di Giove.

10. Il Viale dei Cipressi, con in più le Cerchiate Piccole.

11. Il Giardino Botanico Superiore, con Tiepidario Grande, Serra delle Medimilla, Vasca delle Ninfee, Aquarium, Ingresso, Serra per la moltiplicazione, Serra degli Ananas, Tepidario Piccolo, Serra delle orchidee, Vasche, Aiuole.

12. Il Giardino Botanico Inferiore, Terrazzamento superiore ed inferiore.

13. La Limonaia.

14. Ingresso di Annalena.

15. Il Bacino dell'Isola, con l'Isola, le Ragnaie dell'Isola, Viale della Stella, Viale degli Obelischi, Prati dell'Isola, Viale dei Platani.

16. Il Prato delle Colonne.

Per ogni area è stata elaborata una scheda sintetica, contenente tutti i dati ritenuti essenziali ai fini della elaborazione delle linee guida di intervento e organizzati così da delineare: profilo storico, descrizione delle condizioni attuali, proposte di restauro.
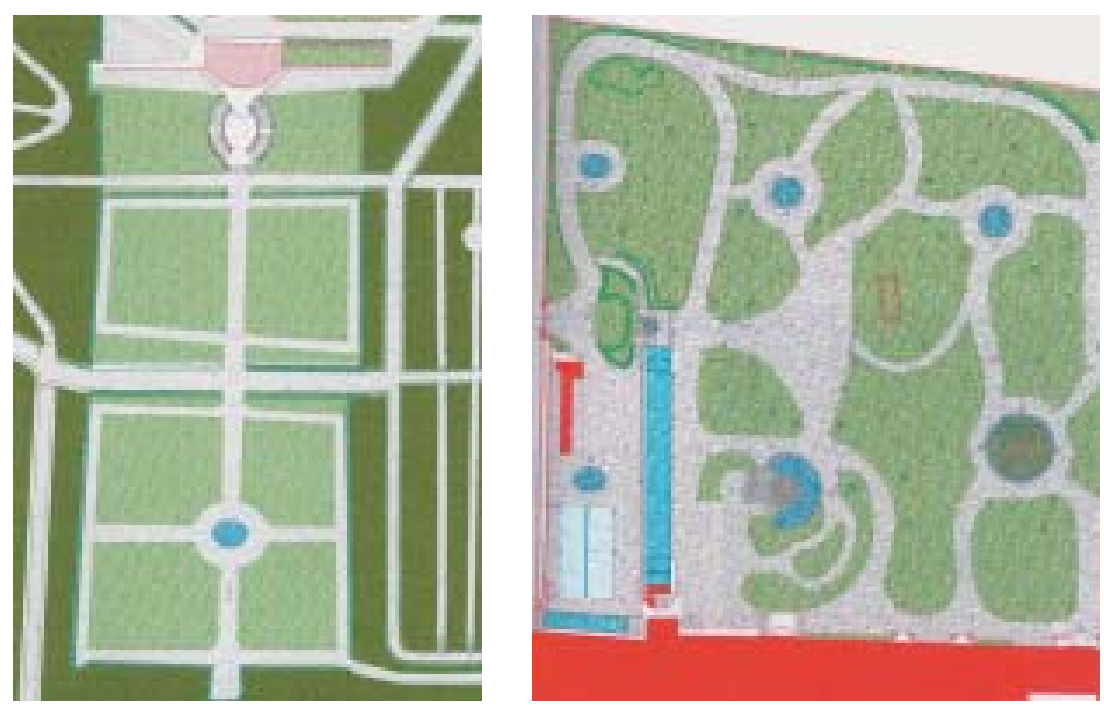

Figura 11 - Planimetrie di rilievo di tre delle sedici aree caratteristiche. In ordine: la 1, Anfiteatro; la 4, Kaffehaus; la 11, Giardino della Botanica Superiore. 
In riferimento alla varietà dei temi progettuali incontrati, alle differenti condizioni di conservazione delle varie parti del giardino e al grado di definizione delle indicazioni desumibili dal quadro della conoscenza storica, il Master Plan propone interventi che fanno riferimento ad approcci diversi della teoria del restauro del giardino storico: ora si indica una scelta di tipo puramente conservativo, ora la ricostruzione filologica di un brano storico cancellato dal tempo o dall'incuria, ora si punta alla riqualificazione paesistica attraverso il 'ripristino' o l'introduzione di elementi allusivi o evocativi di precedenti sistemazioni storiche.

Come spiega l'autore," questa variabilità metodologica non sembri un'incoerenza o il frutto di un eclettismo di comodo. Essa va intesa invece nel senso di un'attitudine aperta, non arroccata a posizioni dogmatiche e acritiche, ma flessibile alle situazioni e alle esigenze particolari”28.

Forse, il Master Plan di Boboli ha invece il difetto di presentarsi senza una sezione generale dedicata allo stato attuale del giardino interpretato nel suo insieme, e senza uno schema riassuntivo dei temi di indagine, degli aspetti del degrado e dei problemi di restauro, utile per focalizzare in un quadro unitario le tematiche affrontate. Sarebbe stato utile, inoltre, fornire una sorta di abaco di particolari delle soluzioni tipo tecniche-operative, quasi un libretto delle istruzioni, per la realizzazione degli interventi di manutenzione, in cui tradurre anche l'esperienza già acquisita 'sul campo'.

\section{GLI ESITI: GLI INTERVENTI REALIZZATI}

Nell'ultimo decennio il volto di Boboli ha riacquistato splendore: la maggiore attenzione posta nelle pratiche di manutenzione delle sue architetture vegetali, l'introduzione di un nuovo metodo di potatura della vegetazione arborea, la reintroduzione dell'orticultura ornamentale nei giardini dell'Isola, del Cavaliere e dalla Limonaia, così come la riduzione del carico annuo di visitatori, sono la dimostrazione che una nuova sensibilità e un atteggiamento culturale più responsabile hanno potuto e saputo guidare le scelte gestionali. La possibilità di innalzare lo standard della qualità delle opere di manutenzione ordinaria ha rappresentato il primo obbiettivo da raggiungere per porre le premesse alla redazione del Master Plan.

"Manutenzione ed uso comportano necessariamente anche interventi, siano essi di conservazione e/o di adeguamento dell'esistente, siano essi di nuova realizzazione: è proprio la qualità di questi interventi (e, naturalmente, la loro quantità e distribuzione) che risulta decisiva per la salvaguardia del paesaggio storico" 29 . L'attuazione di un piano generale di restauro e conservazione per Boboli è strettamente correlata alla possibilità di disporre delle necessarie risorse finanziarie, che, secondo un calcolo previsionale di massima presentato nel Master Plan, erano quantificabili al 1999 e solo per gli interventi su architetture e struttura verde, in 10,800 milioni di euro circa.

La dotazione finanziaria annuale ordinaria disponibile per la manutenzione è di circa quarantacinque mila euro, sufficienti appena per attuare gli interventi sulla struttura vegetale (taglio delle siepi, sfalcio dei prati, potature).

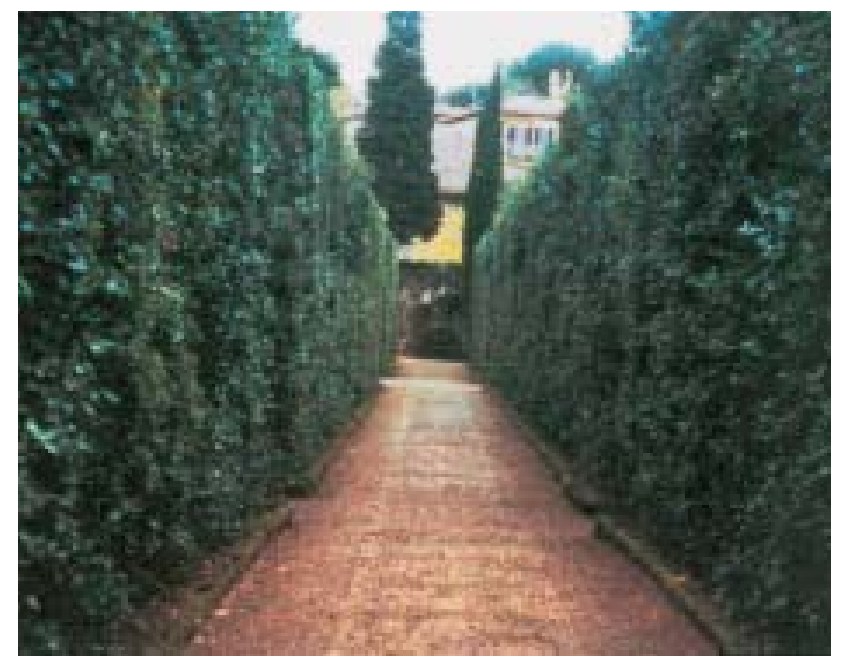

Figura 12 - La Ragnaia di Giove dopo il restauro.

\footnotetext{
${ }^{28}$ Giorgio Galletti, op. cit., 2000, pag. 2.

${ }^{29}$ MAURIZIO BORIANI, Il paesaggio come "paesaggio costruito". Manutenzione, conservazione ed uso di un patrimonio storico, "A-Letheia", 7, Alinea, Firenze 1996, pagg. 7-8.
} 
Qualche anno fa il Governo italiano ha destinato a Boboli circa quattro milioni di euro, in applicazione della L. 662 del 23/12/1996, sulla attribuzione dei proventi ottenuti dai giochi del lotto. L'arrivo di queste risorse, di cui al momento è stata assegnata solo una parte, ha permesso la conclusione di alcuni cantieri di restauro precedentemente avviati e l'apertura di altri, attualmente in corso, svolti seguendo le indicazioni del Master Plan.

Sono in corso:

- Intervento nel settore del Labirinto Superiore e completamento del recupero del Prato della Danza o Fontana degli Uccellini, avviato nel 1994. Il progetto è finalizzato alla ricostruzione delle quinte di delimitazione della stanza centrale del Labirinto, unico elemento spaziale rimasto della configurazione rappresentata nella pianta del Gori. Un doppio sistema di siepi sempreverdi monospecie (una interna più bassa in Buxus sempervirens per richiamare il muretto secentesco e l'altra, sul limite esterno lungo lo sviluppo delle fondazioni della recinzione secentesca, in Viburnum tinus) andrà a delineare il perimetro del Prato. La zona sarà inoltre dotata di impianto di irrigazione e di alcuni semplici arredi in marmo per favorire la sosta, verrà ripristinato il sistema dei drenaggi superficiali e ultimato il restauro della fontana centrale recuperata durante i lavori precedenti.

- Ripulitura della zona della Fontana dei Mostaccini, con messa a dimora di una nuova spalliera di lecci (Quercus ilex) e restauro dei mascheroni.

- Restauro della Fontana del Carciofo.

- Restauro della parte sud dell'Anfiteatro, con illuminazione delle edicole.

- Restauro dello Stanzonaccio, in cui saranno collocate le statue originali del giardino.

- Sistemazione della zona pavimentata antistante il pronao della Grotta del Buontalenti, con recupero del piano del lastricato cinquecentesco, scoperto nel 1998, sottostante la quota attuale, con riqualificazionne del Piazzale di Bacco.

Sono stati recentemente conclusi:

- il lavoro di piantagione di una vigna, che ha comportato lo smantellamento di un boschetto fortemente degradato di Quercus ilex, e la ricostruzione di un frutteto di frutti nani con varietà usate nel Settecento nel Giardino della Kaffehaus, in riproposizione dell'immagine settecentesca rappresentata in uno degli undici dipinti di Aniello Limberti del 1790.

- Recupero del sistema delle Ragnaie a lato dell'Anfiteatro, con un restauro iniziato nel 1989. La ricostruzione di questa rigorosa architettura vegetale, composta da alte siepi potate di Laurus nobilis, Quercus ilex, Viburnum tinus, è stata effettuata con attenzione filologica, secondo uno schema di piantagione desunto dal trattato storico della fine del Cinquecento di Giovanni Antonio Popoleschi. Le Ragnaie sono ben visibili ma non accessibili al pubblico.

- Restauro della scala a tenaglia di accesso al Giardino del Cavaliere, mediante sostituzione di gradini e intervento conservativo della balaustra neogotica della terrazza circolare allo sbarco della scala e quello del tetto della limonaia, per cui partiranno presto i lavori di recupero della facciata.

L'elenco non è comprensivo di tutti i cantieri, ma permette di valutare la consistenza degli interventi in atto, volti al recupero, alla conservazione ed alla manutenzione di un paesaggio storico 'ad altissima definizione'.

Boboli è stato recuperato anche nella sua dimensione teatrale e di luogo d'arte e per l'arte: il restauro della "Stanza verde" del Labirinto Superiore ha riconsegnato alla città un raffinato spazio scenico, in cui vengono organizzati nella stagione estiva concerti di musica classica. Nel 1999, inoltre, il giardino ha ospita- 
to l'esposizione di scultura contemporanea all'aperto "Miti ed Eroi", con opere dell'artista rumeno Igor Mitoray. Da qualche anno sono state collocate lungo i percorsi alcune fontanine in ghisa disegnate dall'artista Hossein Golba.

La redazione del Master Plan di Boboli e la sua applicazione, si collocano nel panorama culturale italiano del restauro dei giardini e parchi storici come una tappa importante di un percorso ancora tutto da costruire e che ha un carattere di forte sperimentazione: se il giardino può essere considerato come "un'opera profondamente immersa nel tempo e nello spazio" 30 , allora occorrerà attendere qualche anno, prendere un po' di distacco, per poter valutare fino in fondo i primi risultati del processo attuale di rifigurazione di un così complesso e fragile sistema.

\section{RIFERIMENTI BIBLIOGRAFICI}

Alberti Maria, “Teatro all'aperto - Teatro di stato: il Sogno di Reinhardt a Boboli (1933) come antefatto ai temi dibattuti dal Convegno Volta (Roma 1934)", in Boboli 90 - Atti del Convegno, Vol. 1, Edifir, Firenze 1999, pagg. 371-381.

Boboli '90 - Atti del Convegno Internazionale, Voll. 1 e 2, Edifir, Firenze 1991.

Boboli - Atti delle conferenze tenute nell'ambito dell'occasione didattica "Il giardino come laboratorio di sperimentazione", Comune di Firenze, Firenze 1994.

Basso Francesca, Celli Chiara e Rossi Cristina, I Labirinti di Boboli: Ipotesi di riqualificazione, in Sperimentazioni. Restauro di Giardini e Parchi Storici, Edizioni Regione Toscana, Firenze 1996, pagg. 49-64.

Bencivenni Mario e de Vico Fallani Massimo, Giardini pubblici a Firenze dall'Ottocento a oggi, Edifir, Firenze 1998.

Centro D, Il Giardino Botanico di Boboli, Centro D, Firenze 1996.

Cinti Daniela, Giardini \& Giardini. Il verde storico nel centro di Firenze, Electa, Milano 1998.

FAgiolo MarCello, "Un continuum spazio-temporale", in Arte dei Giardini. Storia e Restauro, n. 1/1991, Alinea, Firenze 1991.

Galletti Giongio, Paoletti, Cacialli, e Poccianti, architetti del Regio Giardino di Boboli in Boboli 90 - Atti del Convegno Internazionale, Vol. 2, Edifir, Firenze 1991, pagg. 505-524.

GALLETTI GIORGIO, Linsula vegetale come struttura morfologica del Giardino di Boboli, in BORIANI Maurizio e SCAzzosi Lionella (a cura di), Il giardino e il tempo, Milano 1992.

Galletti Giorgio, Un aspetto del restauro di Boboli: il problema dei Labirinti, in MACERA Mirella (a cura di), I giardini del Principe, Atti del Convegno internazionale "Parchi e Giardini Storici, Parchi Letterari”, Torino 1994, pagg. 143-153.

Galletti Giongio, La gestione dei giardini di proprietà statale a Firenze, in Sperimentazioni. Restauro di Giardini e Parchi Storici, Edizioni Regione Toscana, Firenze 1996, pagg. 11-15.

Galletti Giorgio, Giardino di Boboli Master Plan - Paesaggio e Architettura, Ministero per i Beni e le Attività Culturali, Soprintendenza per i Beni Ambientali e Architettonici per le province di Firenze, Prato e Pistoia, Firenze 2000.

Gellini Romano, Giuntoli Alberto, Grossoni Paolo e Schiff Silvia, Il Giardino di Boboli: aspetti vegetazionali, condizioni fitosanitarie e metodologie di intervento, in Boboli 90..., op. cit. 1991, pagg. 195-211.

GURRIERI FRANCESCO, Il giardino storico dall'empirismo al metodo: un itinerario difficile, in CAZZATO Vincenzo (a cura di), Tutela dei giardini storici. Bilanci e prospettive, Roma 1999, pagg. 152-158.

Mastrorocco Mila, Le mutazioni di Proteo. I giardini medicei del Cinquecento, Sansoni Editore, Firenze 1981.

Pozzana Mariachiara, Selvatici, Labirinti, Ragnaie a Boboli in Boboli '90 - Atti del Convegno Internazionale, Vol. 2, Edifir, Firenze 1991, pagg. 485-491.

RINALDI ALESSANDRO, Giardini e metamorfosi urbana a Firenze tra Medioevo e Rinascimento, in CINTI DANIELA, Giardini \& Giardini. Il verde storico nel centro di Firenze, Electa, Milano 1998, pagg. 15-30.

RocCASECCA PIETRO, Un giardino in area urbana, in Boboli '90 - Atti del Convegno Internazionale, Vol. 2, Edifir, Firenze 1991, pagg. 577-585.

SARDELLI AlesSANDRO, La città e il giardino: uso pubblico di Boboli allinizio del Novecento in Boboli 90 - Atti del Convegno, Vol. 1, Edifir, Firenze 1991, pagg. 358-370.

Tagliolini Alessandro, Storia del Giardino Italiano, La Casa Husher, Firenze 1994.

\footnotetext{
${ }^{30}$ Marcello Fagiolo, "Un continuum spazio-temporale", in Arte dei Giardini. Storia e Restauro, n. 1/1991, Alinea, Firenze 1991, pag. 16.
} 


\section{RIFERIMENTI ICONOGRAFICI}

Figure 1, 5: rielaborazione di Anna Lambertini da CinTI Daniela, Giardini \& Giardini. Il verde storico nel centro di Firenze, Electa, Milano 1998, pagg. 79, 83.

Figure 2-4, 6, 10-12: rielaborazione di Anna Lambertini da GALLETTI GIORGIO, Giardino di Boboli Master Plan - Paesaggio e Architettura, Ministero per i Beni e le Attività Culturali, Soprintendenza per i Beni Ambientali e Architettonici per le Province di Firenze, Prato e Pistoia, Firenze 2000, pagg. 50, 52, 98, 11, 63, 65, 97, 197, 7.

Figura 7: rielaborazione di Anna Lambertini da BenCiVEnNi Mario e DE Vico Fallani Massimo, Giardini pubblici a Firenze dall'ottocento a oggi, Edifir, Firenze 1998, pag. 259.

Figure 8, 9: rielaborazione di Anna Lambertini da Lolli GHetTi MARIO (et al.), Il Giardino Botanico di Boboli, Centro D, Firenze 1996, pagg. 5-6.

\section{SCHEDA DI PROGETTO}

Progetto:

Luogo:

Proprietà:

Tipologia di giardino:

Numero visitatori all'anno:

Superficie:

Redazione del progetto:

Collaborazione al progetto:

Consulenza:

Contributi fondamentali:

Rilievi e assistenza grafica:

Anno di presentazione ufficiale del Master Plan:
Master Plan del Giardino di Boboli

Boboli, Firenze, Toscana

pubblica demaniale, sotto il controllo della Soprintendenza per i Beni Ambientali e Architettonici delle Province di Firenze, Prato e Pistoia

giardino storico mediceo, aperto al pubblico con pagamento di tassa di ingresso

circa un milione

30 ettari

Arch. Giorgio Galletti, Direttore Unità Organica Funzionale Ville, Parchi e Giardini, della Soprintendenza per i Beni ambientali ed Architettonici per le province di Firenze, Prato e Pistoia Firenze, fino all'anno 2001

Geom. Loris Stefanini, Direttore operativo Giardino di Boboli

Elisabeth Banks Associates, Londra

Luigi Zangheri, Detlef Heikamp, Silvia Mascalchi, Riccardo Spinelli per le ricerche storiche. Emanuele Bonicolini, Riccardo Panichi, Ivo Matteuzzi, Paolo Basetti, Paolo Ravegnani per la collaborazione

Studio Stefano Brandi - Geoarte S.T.A., Firenze

2000 


\title{
IL RIPRISTINO dEI GIARDINI SEGRETI DI VILLA BORGHESE
}

\author{
Anna Lambertini
}

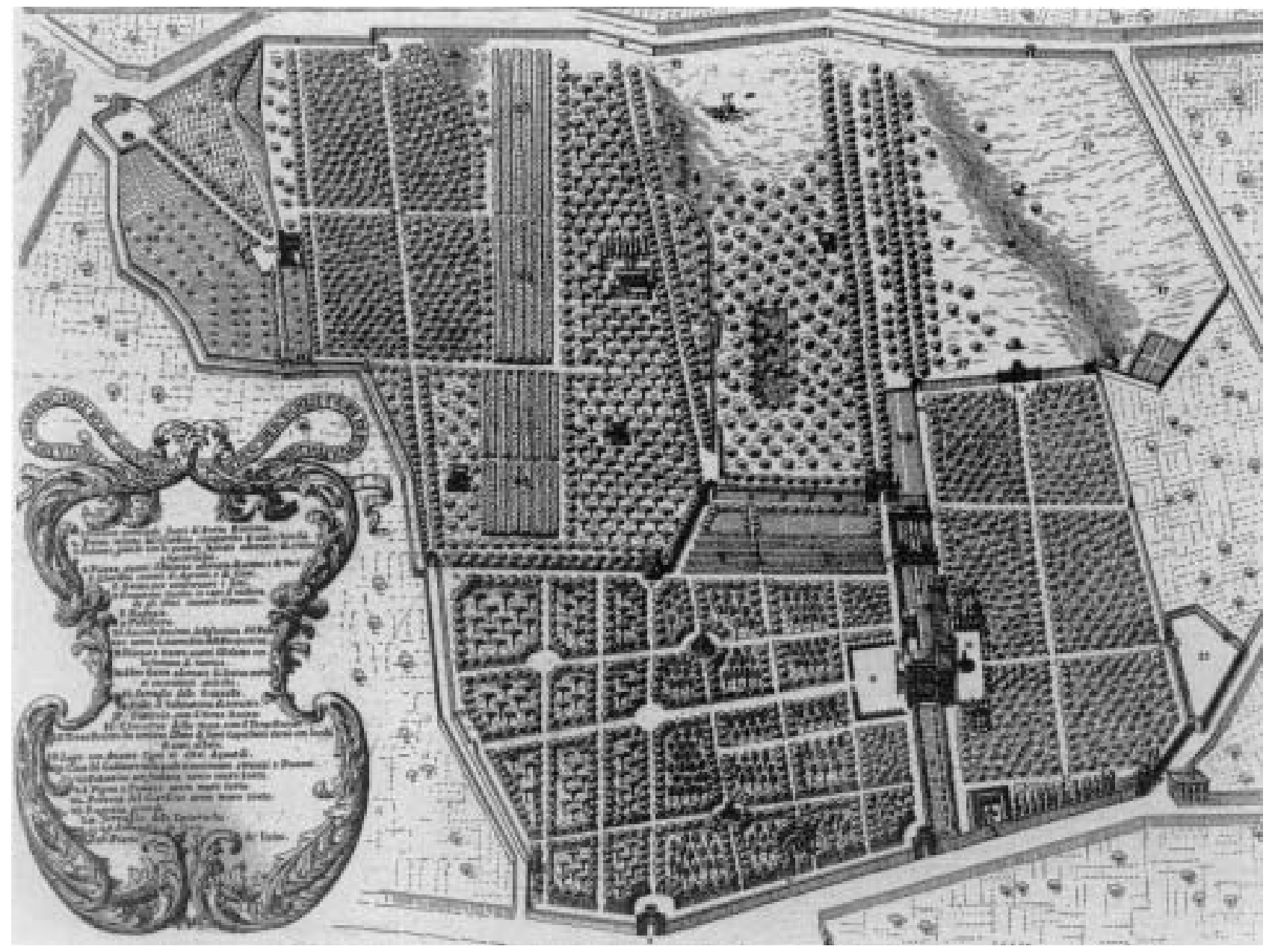

Figura 1 - Pianta prospettica di Simone Felice Delino pubblicata dal Falda in "Li giardini di Roma nel 1667".

\section{Il CONTESTO: IL PARCO DEI MUSEI}

Raccontare la storia del Parco di Villa Borghese è come raccontare la storia di una parte di Roma.

Nata all'inizio del Seicento fuori dalla città, oltre le Mura Aureliane, "fra le vigne” della campagna, Villa Borghese si presenta oggi come un'ampia area verde inglobata all'interno di un tessuto urbano denso, circondata dalle costruzioni che nel tempo hanno progressivamente eroso il sistema degli spazi aperti del paesaggio agrario storico. Definita recentemente Parco dei Musei, contiene al suo interno la Galleria Borghese e il Museo Canonica ed ha in adiacenza la Galleria d'Arte Moderna ed il Museo Etrusco di Villa Giulia. Il Parco dei Musei, che si estende per 
ottanta ettari, si configura nella Roma contemporanea come un ricco sistema museale diffuso a scala urbana, in cui il carattere espositivo è attribuibile non solo ai singoli elementi contenuti, ma al complesso nel suo insieme. L'articolata composizione di spazi aperti, differenziati per tipologia, storia e tipo di fruizione, oltre a fungere da connettivo per architetture di pregio e monumenti, costituisce quasi un catalogo di modelli paesistici prodotti nel tempo. Giardini geometrici, giardini all'inglese, il bioparco (trasformazione dell'ex giardino zoologico inaugurato nel 1911), porzioni di paesaggio naturale, formano un insieme di alto valore, oltre che storico, ambientale e naturalistico che è stato recentemente oggetto di un poderoso programma di interventi di riqualificazione e valorizzazione.

I Giardini Segreti, allineati in composta sequenza sui lati minori del Casino nobile, sono inseriti in questo contesto paesistico, ritenuto tra i più famosi dell'area urbana romana. Oggi, recuperati allo stato di abbandono in cui sono rimasti per decenni, si presentano come preziosi microcosmi vegetali dedicati al gusto della moda orticola secentesca.

\section{La Storia di Villa Borghese. Dal giardino privato al parco pubblico}

"Quasi cento passi fuori Porta Pinciana, è situata verso Oriente la Villa Borghese la quale, parte in pianura, parte in valle, parte in piacevoli salite, mostra nell'ampiezza, e varietà del sito, nella maestà delle fabbriche, nella copia delle acque, e nelle opere di Pittura, e Scoltura, di non havere che invidiare a gli anti-

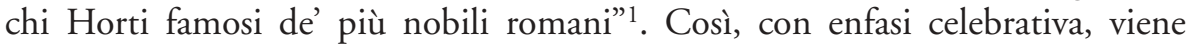
descritta dal Manilli nel 1650 la nuova dimora appena realizzata dai principi Borghese. Lopera fu commissionata da Scipione Caffarelli, nipote di Paolo V Borghese salito al soglio papale nel 1605, che assumendo il nome della nobile casata decise di affermarne il prestigio con la creazione di una residenza sontuosa. La donazione da parte di un nobile romano ai Borghese di una vigna posta fuori la cinta muraria, in un luogo ideale per la bellezza del paesaggio e per la vicinanza alla città, rappresentò l'occasione per la scelta del sito. Si costitù quindi il primo nucleo di una grandiosa proprietà, che raggiunse $\mathrm{i}$ circa cento ettari di estensione in seguito a successive acquisizioni, proseguite fino al 1620.

I progetti di trasformazione cominciarono nel 1608 e vennero affidati a Flaminio Ponzio ed al suo allievo Giovanni Vasanzio; a loro successe, nel 1621, Girolamo Rainaldi. La sistemazione dei giardini fu seguita dallo stesso Rainaldi assieme a Domenico Savino di Montepulciano, mentre le opere di ingegneria idraulica furono condotte dall'esperto Giovanni Fontana. È ancora il Manilli a descriverci il risultato dei lavori e la configurazione secentesca degli spazi esterni della villa, che si estendeva "per lo spazio di tre miglia di giro, dalla banda suddetta di Porta Pinciana, fin'a Muro torto, vicino alla porta Flaminia: et è tutto quest'ambito cinto, non solo di mura all'intorno, d'altezza, le più basse, di venti palmi d'architetto; ma nel di dentro contiene ancora molti recinti minori, che distinguendo il luogo in più parti, lo rendono vago alla vista"2.

Emerge da questa descrizione storica la particolarità della villa di essere suddivisa in tre parti distinte, delimitate da recinzioni murarie con porte di accesso e definite semplicemente "recinti".

Nel primo recinto era inserito, lateralmente rispetto al fronte del palazzo, il portale d'ingresso principale, che si apriva su un viale carrozzabile posto a pochi

${ }^{1}$ JaCopo Manilli, Villa Borghese fuori di Porta Pinciana, Roma 1650, in Beata DI GAdDO, L'Architettura di Villa Borghese, "Groma Quaderni", 5, Università degli Studi di Roma "La Sapienza", D.A.C.C., Roma 1997, pag. 15.

2 In Alessandro Tagliolini, I giardini di Roma, Newton Compton Editori, Roma 1980, pagg. 217- 218, della Seconda Edizione. 
passi fuori dalla Porta Pinciana. Questa parte era chiamata Giardino Boschereccio perché comprendeva un ampio settore alberato con pini, cipressi ed olmi (Pinus pinea, Cupressus sempervirens e Ulmus sp.), strutturato in forma geometrica.

I boschetti, così disegnati e costituiti in prevalenza da sempreverdi, dovevano formare uno spettacolare effetto di contrasto cromatico con la facciata chiara e fastosamente decorata del palazzo, che si affacciava su un ampio piazzale rettangolare, leggibile come un vuoto architettonico strategico nella sequenza dei 'pieni' arborati. Per sottolineare l'assialità dei percorsi e definire con più precisione le geometrie degli spazi furono messi a dimora filari alberati monospecifici con siepi continue, formati da cipressi (Cupressus sempervirens) o olmi (Ulmus sp.) nei viali più larghi e da lecci (Quercus ilex) in quelli più stretti. Coprivano alla vista vari tratti della cinta muraria soprattutto melangoli e melograni (Citrus aurantium e Punica granatum), nei punti più soleggiati, e lecci (Quercus ilex) e siepi di alloro (Laurus nobilis), in quelli più ombreggiati.

Il secondo recinto, che proteggeva il giardino privato del principe, era detto Giardino della Prospettiva: volto verso il prospetto posteriore del palazzo presentava un articolato corredo ornamentale di statue antiche e altorilievi e riproponeva l'idea di giardino come antiquarium. In questo recinto, a cui non si poteva accedere con le carrozze, si trovavano le scuderie e la conigliera. Un ampio bosco, costituito da una piantagione di oltre seicento lecci (Quercus ilex) si stendeva dietro il palazzo occupando tutta la zona fino ai limiti della cinta muraria.

Nella fascia di contatto tra il secondo ed il terzo recinto, collocati ai fianchi del casino del Nobile erano disposti i "Giardini secreti di Agrumi e fiori", preziosi spazi dedicati a coltivazioni ornamentali che si svolgevano come raffinati tappeti verdi ricamati davanti alla palazzina della Meridiana e all'Uccelliera, disegnate dal Rainaldi.

Il terzo recinto, infine, il Parco, costituiva la parte più estesa e veniva identificato con la pars rustica della villa, i cui limiti sfumavano percettivamente nel paesaggio agrario circostante. Fu questa l'ultima parte della villa ad essere sistematae ad essere mantenuta per quasi tutti i due secoli successivi a coltura agricola e a parco naturale con le sistemazioni caratteristiche delle 'vigne' romane: l'hortus olitorius, i boschetti, le ragnaie. In una vallata dai morbidi contorni, venne realizzato un bacinorettangolare in cui trovarano dimora varie specie di uccelli acquatici e che fu posto al centro di una piantagione regolare di quarantadue platani (Platanus sp.), in parte ancora esistente.

Nel volume di incisioni di giardini romani pubblicato da Gian Battista Falda nel 1667, viene presentata la "Pianta prospettica" di Villa Borghese di Simone Felice Delino in cui ci viene mostrata l'organizzazione spaziale del complesso trentaquattro anni dopo la conclusione dei lavori.

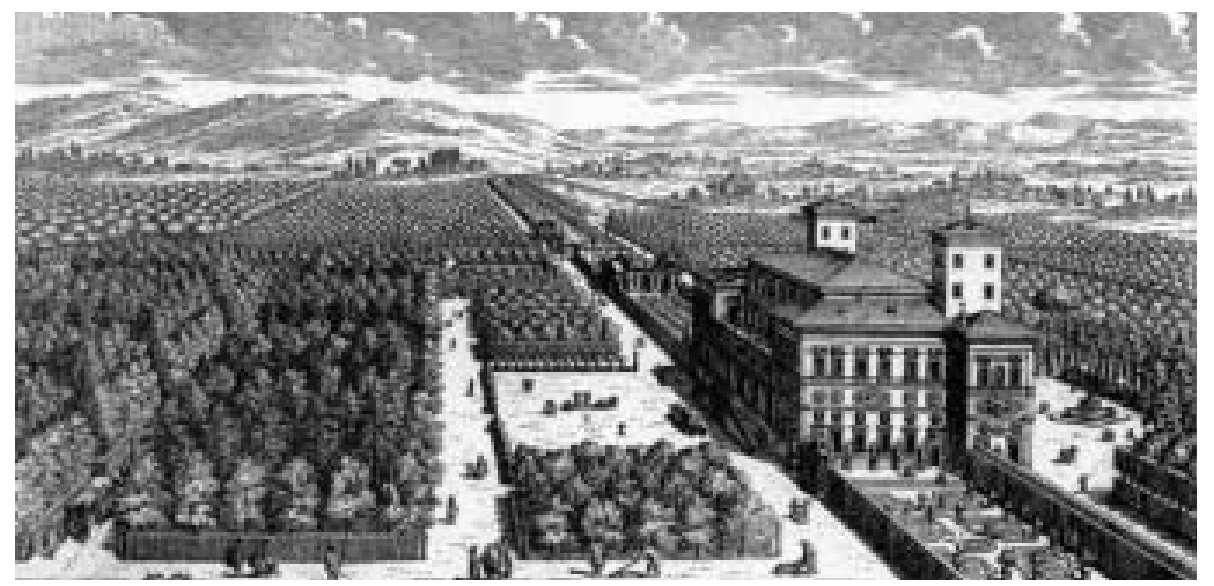

Figura 2 - Veduta prospettica di Villa Borghese, Simone Felice Delino, 1670. 
Il modello di riferimento che aveva guidato la redazione del progetto era ancora quello già ampiamente sviluppato in epoca rinascimentale: la villa, in posizione dominante, doveva dettare le regole di composizione generali impartendo con il suo corpo di fabbrica le assialità principali, impostate su una griglia ortogonale. Ma poiché il terreno, per la massima parte caratterizzato da una certa variabilità altimetrica, presentava qualità poco adatte all'applicazione di uno schema troppo rigido destinato ad essere attuato con successo solo su un terreno pianeggiante, l'impianto generale, pur mantenendo il formalismo geometrico, finirà per essere adattato alla morfologia naturale. Limpostazione della rete dei viali, che definisce una partitura di spazi quadrangolari di dimensioni e inclinazione variabili, perde la rigorosa ortogonalità, ma restano rispettati i valori formali di base improntati alla creazione di importanti assi prospettici da e verso la Villa. La chiusura continua lungo il perimetro della proprietà ad opera del recinto murario, favorì la creazione di una serie di episodi architettonici e ornamentali utilizzati come importanti fondali per risolvere in forma scenografica la conclusione dei viali. La mancata assialità rispetto al palazzo del portale di ingresso principale, viene recuperata attraverso la forte accentuazione di questo elemento architettonico $^{3}$. La creazione di un'esedra di verzura, orientata verso l'interno del recinto, è utilizzata come dispositivo spaziale per introdurre il percorso da seguire fino al centro della scena dominata dal palazzo, un'architettura compatta sormontata da due torri, posta nella parte più elevata del primo recinto.

Nonostante la successione di recinti, già alla sua origine, la Villa venne aperta nella sua "pars urbana" al pubblico: una targa posta all'ingresso invitava i cittadini ad entrare per godere delle delizie dei giardini.

La configurazione dei recinti rimase pressoché inalterata fino alla fine del Settecento, quando, per volere di Marcantonio Borghese, si diede avvio ad importanti interventi di trasformazione dell'assetto secentesco. Sollecitato dai cambiamenti culturali introdotti dall'Illuminismo, attraverso cui si erano andati a trasformare anche a Roma i valori della tradizione figurativa dell'architettura e dell'arte dei giardini, e in considerazione delle mutate relazioni spaziali tra villa e città indotte dall'espansione urbana di un secolo di storia, il principe si decise ad imprimere un'immagine aggiornata alla sua dimora.

La lettura ed il confronto di due planimetrie storiche, una del 1776 ed una del 1809, riferite all'assetto di Villa Borghese prima e dopo i lavori settecenteschi, permettono di cogliere, anche solo attraverso l'analisi del diverso lessico del giardino utilizzato, la differenza tra due modi di concepire la villa e la simbologia di esibizione del potere. Nella planimetria del 1809 scompaiono le scritte in latino utilizzate, in quella precedente, per individuare tòpoi colti del giardino ideati in continuità con la tradizione classica (come hortus olitorius, licitarium, nemus) e compaiono invece termini come museo, ippodromo, cappella, ad indicare la "volontà di una maggiore partecipazione alla vita collettiva nella Villa"

"Anche la vegetazione arborea, pazientemente ed uniformemente disegnata nella prima, subisce, nella seconda versione, una interpretazione molto più differenziata: il bosco di lecci dietro al casino diventa una perfetta trama geometrica di piccoli punti mentre, sempre un bosco di lecci, ma nel Giardino del Lago, diventa un intricato intreccio fra vialetti tortuosi. La forzatura di interpretazione grafica è però molto efficace perché esalta i valori formali delle due parti del giardino: quello seicentesco, il cui rigido impianto simboleggia il trionfo della concezione assolutistica del potere, e la parte dei nuovi lavori, dove la libertà e la tolleranza creano un paesaggio aderente ai nuovi canoni estetici" ${ }^{5}$.

${ }^{3}$ Cfr. BeATA DI Gaddo, L'architettura di Villa Borghese, "Groma Quaderni”, 5, Università degli Studi di Roma "La Sapienza”, D.A.A.C., Roma 1997, pag. 38.

${ }^{4}$ BEATA DI GADDO, op. cit., 1997, pag. 103.

${ }^{5}$ Ibidem. 
I lavori di modifica, che interessarono soprattutto la struttura del terzo recinto, furono condotti dal 1776 al 1793 dai due architetti romani Antonio e Mario Asprucci, padre e figlio.

Nell'Ottocento la superficie del parco venne ampliata dal successore di Marcantonio Borghese, il figlio Camillo, che dopo un primo periodo di disinteresse per la proprietà, confermato anche dalla vendita ai francesi della preziosa collezione di opere d'arte conservata nella Villa, decise di dedicarsi invece alla sua valorizzazione.

Il progetto di sistemazione delle nuove parti acquisite fu affidato nel 1822 a Luigi Canina, architetto di origine piemontese residente a Roma che qualche anno dopo conquistò fama europea come archeologo.

Il progetto del Canina riuscì a rendere unitario col resto del giardino il nuovo spazio dalla forma molto irregolare, caratterizzato da un terreno fortemente scosceso e separato dal resto della proprietà da una strada pubblica, risolvendo brillantemente tutti i problemi di connessione fisica e formale. Per garantire la continuità dei percorsi dentro il parco furono costruiti due ponti che, superandola in quota, ovviavano alla cesura fisica costituita dalla strada pubblica.

Nel 1849 durante l'assedio di Roma da parte delle truppe francesi, parco e villa subirono gravi danni dovuti ai bombardamenti: molti arredi furono distrutti, così come alcuni degli edifici accessori.

Dopo l'Unità d'Italia la Villa venne contesa ai Borghese dallo Stato italiano che ne rivendicava la vocazione a parco pubblico, in virtù della targa apposta nel Seicento all'ingresso del primo recinto. Dopo varie battaglie legali, anche per evitare che villa e parco potessero essere irrimediabilmente compromesse da impietose speculazioni edilizie, nel 1901 si arrivò al loro acquisto da parte dello Stato, che due anni dopo ne fece cessione al Comune di Roma. Nel 1908, per permettere la continuità spaziale tra due importanti sistemi di aree pubbliche, furono realizzati un ponte di superamento della Via del Muro Torto ed un ampio viale di collegamento tra il Piazzale del Pincio e Villa Borghese.

Dopo il periodo di desolante abbandono in cui il complesso era rimasto dalla metà dell'Ottocento, l'Amministrazione comunale diede avvio ad alcuni interventi di riqualificazione di Villa Borghese. Vennero attuati lavori di risistemazione dei giardini segreti e dei piazzali antistanti il Palazzo, trasformati secondo una libera reinterpretazione dei modelli del giardino storico formale, così come dettavano i principi del revival del 'giardino all'italiana'. "Era stata così cancellata la storia e l'evoluzione dei siti e contemporaneamente appiattita, secondo un'interpretazione univoca, l'immagine dei giardini"6.

Anche il nuovo assetto novecentesco non era destinato a durare: durante la II Guerra Mondiale i giardini vennero trasformati in orti di guerra e destinati alla coltivazione di cavoli e patate.

Da allora, a parte sporadici e casuali interventi di manutenzione, giardini e parco sono rimasti in condizioni di degrado fino al 1998, anno in cui è stato possibile predisporre i vari lavori di ripristino.

\section{GIARDINI SEGRETI: DALLA STORIA AL RESTAURO ${ }^{7}$}

Il Giardino segreto, derivazione diretta dello spazio medievale dell'hortus conclusus, rappresenta un carattere ricorrente nella composizione dei giardini delle ville rinascimentali e barocche; raccolto entro alte mura protettive, segnava il

\footnotetext{
${ }^{6}$ Alberta Campitelli, I Giardini Segreti di Villa Borghese: dalla storia al restauro in Maria AdRIANA Giusti (a cura di), I Tempi della Natura. Restauro e restauri di giardini storici., Edifir, Firenze 1999, pag. 183.

${ }^{7}$ Cfr. Alberta Campitelli op. cit., 1999, pagg. 177-193.
} 
passaggio dallo spazio chiuso dell'abitazione del principe a quello aperto del parco. Questo carattere riservato e protetto lo rendeva il luogo ideale per la coltivazione di specie botaniche rare e preziose, destinate al diletto del suo proprietario e di pochi fortunati suoi intimi. La presenza della cinta muraria offriva un ottimo supporto per le piante di agrumi da coltivare a spalliera.

Nel Seicento, in risposta alla dilagante "tulipomania", furono creati in tutta Europa giardini segreti per l'esposizione delle varie bulbacee divenute oggetto di culto e scambio per collezionisti e appassionati, tra cui si ricordano a Roma anche i Borghese.

I Giardini Segreti di Villa Borghese riproponevano in forma codificata una tipologia secentesca ampiamente affermata, sul cui assetto e composizione la trattatistica dell'epoca si dilungava in precise descrizioni.

Alla fine del Seicento a Villa Borghese erano presenti tre giardini segreti, allineati lungo i prospetti minori del Casino nobile (oggi Museo Borghese): il Primo Giardino, Giardino de melangoli verso mezzogiorno; il Secondo Giardino tra Casino e Uccelliera, il Giardino segreto de fiori a tramontana; il Terzo Giardino tra Uccelliera e Meridiana, Giardino dé fiori o della fioritura

Leggendo la descrizione del Manilli, veniamo a sapere che all'epoca del suo scritto, nel 1650, solo due giardini erano dedicati alla coltivazione ornamentale. "Da due Stanze degli Appartamenti terreni, s'esce in due Giardini segreti: il primo de' quali, verso Mezzogiorno, detto il Giardino de' Melangoli, lungo 455 e largo 88 palmi, termina con la strada maestra. I due muri lunghi, dalle bande, son coperti d'agrumi diversi. Da capo venne abbellito da una parte della Facciata di fianco del Palazzo e all'altro capo, al muro della via maestra, da una facciata d'opere di scoltura. Vien composto questo Giardino di 144 alberi di Melangoli, divisi in 24 fila. Nel principio, vicino al Palazzo, si vedon ne' muri due fontane di marmo simili, con due teste di Drago, che sbucando da picciole caverne, versan l'acqua in due conchiglie. (....) Il Giardino all'altro lato del Palazzo, volto a Tramontana, è lungo 400 palmi, e dell'istessa larghezza, che l'altro. Hà similmente i due muri lunghi coperti di più forti d'agrumi, con due fontane picciole, simili in tutto à quelle dell'altro Giardino. Vien diviso in dieci compartimenti, spartiti pel lungo in due ordini, e cinti di spalliere basse di mortella. In ogni compartimento son piantati sei alberi di melangoli: e in terra in varii quadretti più fiori di Tulipani, d'Anemoni, di Giunchiglie, di Giacinti, e d'altri fiori i più rari che si trovino. (....) S'entra per quest'andito nel Gallinaro, contiguo all'Uccelliera, che è un Cortile largo come i Giardini, e lungo 297 palmi, con una fontana vicina à quattro stanze, che servon per i Polli, e con otto alberi di Gelsi, piantati quattro per banda"

La sistemazione del terzo giardino risale al 1680 e fu fatta contestualmente alla costruzione della palazzina della Meridiana.

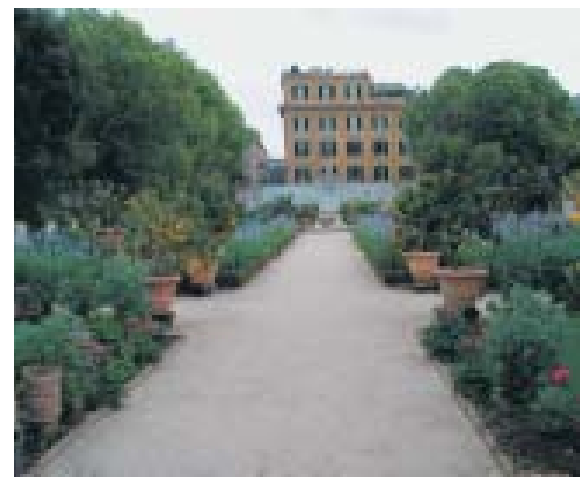

Figura 3 - Il Giardino Vecchio.

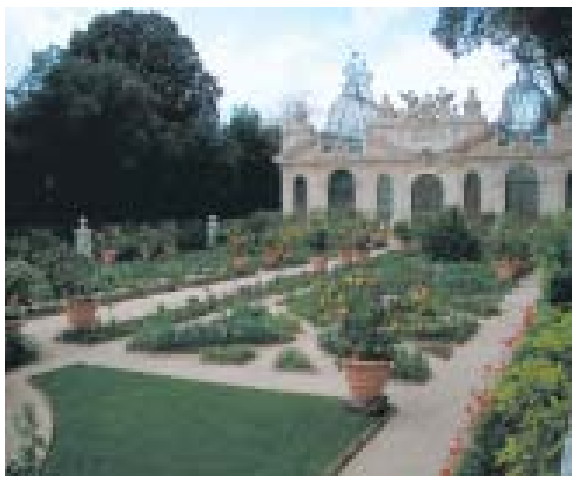

Figura 4 - Il Giardino dell'Uccelliera.

${ }^{8}$ JaCopo Manilli, op. cit., Roma 1650, in BeATA Di GADDO, op.cit., Roma 1997, pag. 73. 
Concepiti come delle vere e proprie stanze all'aperto, i giardini vennero inseriti dunque in una lunga fascia di terreno serrata entro due alte cinte murarie, innestate ai lati del Palazzo. Giardini ed edifici formavano un unicum architettonico: l'inserimento dell'Uccelliera e della palazzina della Meridiana determinò una partitura dal ritmo regolarmente cadenzato che, partendo dal limite orientale, proponeva un'alternanza di vuoti e di pieni, con una pausa più lunga in corrispondenza del Palazzo.

Ai tre giardini secenteschi ne seguirà poi un quarto, posto a conclusione della sequenza sul lato occidentale: il Giardino di Coltivazione, realizzato negli anni Venti del Novecento in luogo dell'antica Serra delle Tartarughe riportata nella cartografia settecentesca.

\section{Gli ObietTIVI E LA METODOLOGIA DEL PROGETTO: RESTAURo o 'RIPRISTINO'?}

Lo stanziamento dei finanziamenti straordinari per il Giubileo 2000 ha consentito a Roma, come in altre città d'arte italiane, la realizzazione di importanti interventi di riqualificazione urbana e di recupero di significativi ambiti di interesse storico-monumentale. Tra questi si colloca anche il restauro dei Giardini di Villa Borghese, a cui sono stati destinati novecentotrenta mila euro.

I giardini, che si trovavano in uno stato di avanzato degrado anche in seguito alla cantierizzazione dei lavori di restauro del Museo Borghese smantellati nel 1997, sono stati oggetto di numerose indagini conoscitive. La fase indispensabile della raccolta dei dati è stata condotta con grande cura da un gruppo di lavoro interdisciplinare costituito da architetti, storici dell'arte dei giardini e dell'architettura, botanici.

La notevole mole di informazioni prodotta, attraverso lo studio della cartografia storica, le ricerche di archivio, lo studio della trattatistica e dell'iconografia botanica storica, ha permesso di ripercorrere le varie fasi di realizzazione dei giardini e la loro evoluzione nel tempo dovuta ai cambiamenti della moda e del gusto nell'orticoltura ornamentale, ma non ha reso possibile la definizione dell'originario assetto secentesco e delle successive trasformazioni per tutti i tre Giardini Segreti.

Nel 1998, sono stati pertanto condotte indagini archeologiche applicando una metodologia ampiamente sperimentata ${ }^{9}$ in ambito europeo, non distruttiva. I risultati degli scavi stratigrafici effettuati sono stati analizzati in relazione ai dati raccolti.

Questa operazione ha permesso di completare e rendere più sicuro il quadro conoscitivo, rendendo certi alcuni elementi o aggiungendone di nuovi.

Per esempio nel caso del Giardino Vecchio, i saggi archeologici hanno confermato la tesi dell'esistenza di un giardino pre-borghesiano nel nucleo originario della proprietà, costituito dalla Vigna donata ai Borghese nel 1608. Il ritrovamento delle tracce di una cisterna, di una probabile neviera e di un muro di cinta testimoniano un uso non solo utilitario, ma anche di decoro dell'area campestre poi trasformata in dimora principesca, come dimostrano anche la presenza di piante di agrumi.

Altre strutture emerse negli scavi hanno poi fornito nuovi elementi relativi agli interventi commissionati da Scipione Borghese, come il ritrovamento del sistema di fontane in marmo bigio africano, addossato ai muri del primo recinto, citato nelle descrizioni storiche del Manilli, e come ancora il rinvenimento di due filari di supporti di malta utilizzati per alloggiare i pali di legno che servivano a sostenere i pergolati di agrumi lungo il muro di cinta. I due filari

\footnotetext{
${ }^{9}$ Sui metodi di indagine archeologica applicati ai giardini cfr. LUIGI ZANGHERI, L'archeologia per il restauro dei giardini storici europei, "Quasar", 19, 1998, pagg. 5-10.
} 
sono riferiti a due epoche diverse di impianto e testimoniano la continuità d'uso nel tempo della spalliera di agrumi come elemento caratteristico dei giardini segreti, nel Seicento e nel Settecento.

Nel terzo Giardino, o Giardino della Meridiana, la campagna di scavi, con il ritrovamento della struttura delle fondazioni delle serre ottocentesche di cui viene fatta menzione in una descrizione del 1815 , ha confermato il cambio di uso che il giardino ebbe, passando, nel corso di circa due secoli, da giardino per l'esposizione di fiori pregiati a giardino per la coltivazione delle piante esotiche.

La constatazione di una successione di trasformazioni profonde avvenute nel tempo ha reso improponibile per i progettisti la definizione di un assetto storico prevalente sugli altri e "soprattutto, per nessuna epoca è stato possibile ricostruire con una certa attendibilità il disegno delle aiuole ed il tipo di fioriture impiegate"10.

Infatti, per quanto riguarda la ricostruzione della composizione botanica dei giardini, in alcuni casi sono stati individuati elenchi abbastanza dettagliati di fioriture, ma, confrontando descrizioni e documentazioni cartografiche anche di epoche tra loro vicine, i dati sono risultati discordanti.

"Questa situazione, non inattesa in quanto il giardino è per sua natura manufatto mutevole più di qualunque altro, ha indotto un acceso dibattito sul tipo di intervento da effettuare per ripristinare dei giardini in quelle che si presentavano ormai come aree incolte senza alcuna traccia di disegno di aiuole" ${ }^{11}$.

Alla fine, l'impossibilità di condurre un intervento di restauro filologicamente corretto, ha spinto i progettisti verso il 'ripristino' di un modello, optando per un'evocazione del giardino storico secentesco, attraverso l'adozione di un impianto formale e di un repertorio botanico desunti dalla trattatistica dell'epoca. Particolare cura è stata posta anche nella scelta dei materiali e degli elementi di arredo: per esempio la cordonatura di delimitazione delle aiuole è stata realizzata con mattoncini in cotto fabbricati appositamente.

\section{GLI ESITI: LA RICOSTRUZIONE DI UN MODELLO STORICO}

Dallo stato di totale abbandono in cui erano stati lasciati dal secondo dopoguerra, i Giardini Segreti sono stati ricondotti a nuova vita: il risultato dell'accurato lavoro di indagine storica e di conoscenza ha portato infine alla ricostruzione di un modello storico e alla scelta di riproporre il gusto e il disegno di un ambiente secentesco. I giardini, impostati lungo un unico asse centrale di simmetria, sono tornati a formare con l'architettura degli edifici un'unità spaziale e architettonica, in riproposizione di una sequenza di notevole effetto scenografico e decorativo.

Il Giardino Vecchio (in origine Giardino de' Melangoli), proprio perché il più antico, è stato riproposto in forma mista come esempio di un "Giardino di fiori e di agrumi". Per il suo disegno e la sua composizione, il gruppo di progetto si é in parte ispirato alla tradizione coronaria, in voga tra la metà e la fine del Cinquecento, e in parte a brani della iconografia storica del giardino proposta dalle vedute di Simone Felice Delino, contenute nella raccolta di incisioni di Giovanni Battista Falda (c. 1678-1683). L'impianto planimetrico è stato suggerito dalla partitura plastica del tratto di cinta muraria che costituisce la quinta conclusiva del giardino: nicchie, riquadri e inserti scultorei hanno determinato l'impostazione e le dimensioni della griglia dei percorsi, "secondo i principi di compartimentazione architettonica adottati già dai primi del Cinquecento anche nella realizzazione dei giardini”"12.

${ }^{10}$ Alberta CAmpitelli, I Giardini Segreti di Villa Borghese: dalla storia al restauro, in Maria Adriana GiUsti (a cura di), I Tempi della Natura. Restauro e restauri di giardini storici. Edifir, Firenze 1999, pag. 193.

${ }^{11}$ AlberTa CAmpitelli, op. cit., 1999, pag. 193.

${ }^{12}$ Dipartimento Politiche Ambientali e Agricole, Comune di Roma, Il giardino Vecchio in I Giardini Segreti di Villa Borghese, (Brochure con schede), Fratelli Palombi Editori, Roma 1999. 
Nelle aiuole, una ricca gamma varietale di piante con prevalenza di arbustive aromatiche ed erbacee, ripropone le fioriture dell'hortus coronarius $^{13}$, il "giardino delle ghirlande" di tradizione medievale recuperata ed enfatizzata nell'Umanesimo e nel Rinascimento.

Il tema degli agrumi viene svolto con la presentazione di una collezione di agrumi in vaso posti su piedistalli di varie altezze (secondo un modello visibile ancora oggi nel Giardino Buonaccorsi a Potenza Picena), e con la piantagione di melangoli (Citrus aurantium) e limoni (Citrus limon) allevati a spalliera lungo il muro settentrionale. Al centro della composizione generale, assume il valore di fulcro di distribuzione dell'elemento vitale del giardino la Fontana settecentesca con va-

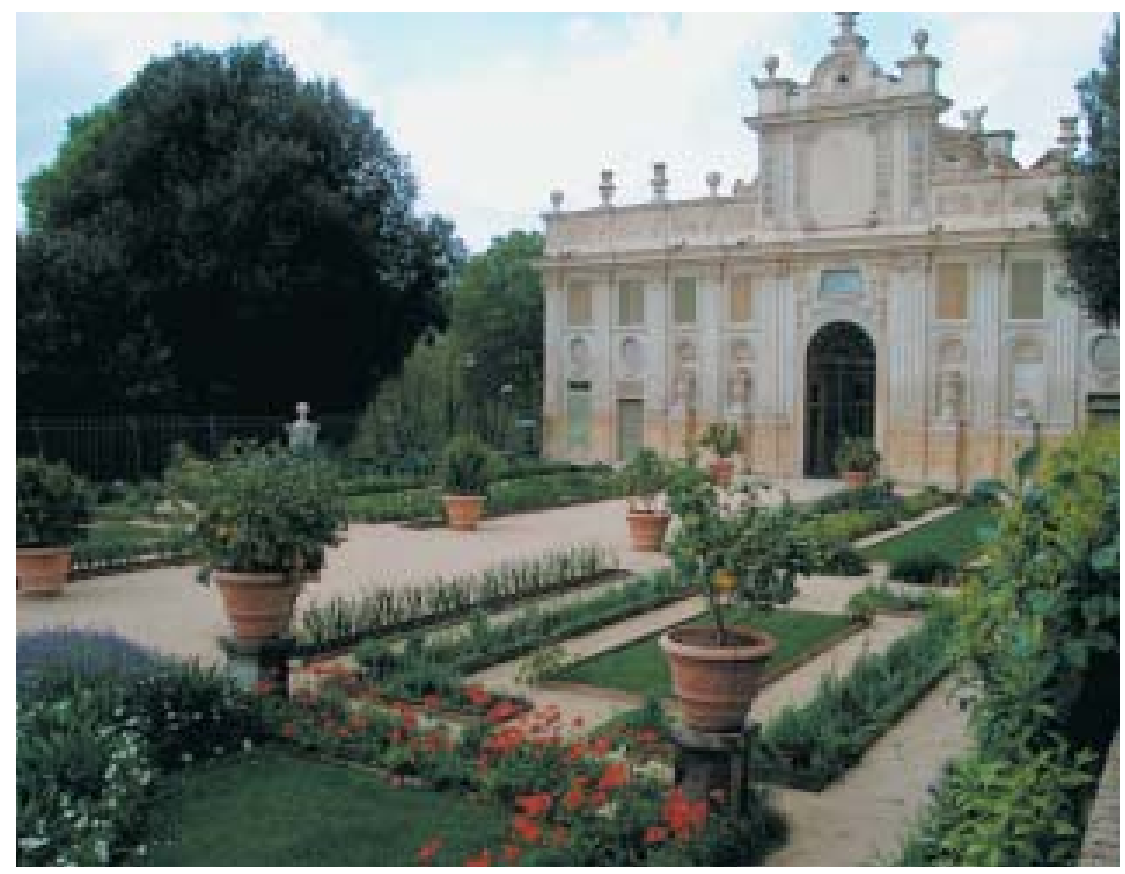

Figura 5 - Il Giardino della Meridiana. sca quadrilobata, documentata nella planimetria del 1776 , in cui l'acqua scaturisce dal becco di un'aquila in travertino (simbolo dei Borghese) posta su una roccaglia.

Il Giardino dell'Uccelliera ripropone il modello di un giardino di fiori della prima metà del Seicento desunto dallo studio delle fonti pubblicate e manoscritte a quell'epoca. Hanno in particolare guidato le scelte formali e di composizione botanica le indicazioni contenute negli scritti di Tobia Aldini e Giovan Battista Ferrari ${ }^{14}$, e la documentazione archivistica sui giardini di fiori in area romana di Francesco Caetani (1625) e di Antonio Barberini (circa 1645). Suddiviso in "spartimenti di fiori" che formano ricami geometrici finemente lavorati, risultato dell'attento studio delle associazioni botaniche, il giardino presenta una ricca collezione di bulbose e rizomatose, a conferma della sua antica vocazione a "giardino di cipolle"15.

"La connotazione seicentesca di questo giardino è resa dall'uso enfatizzato di anemoni (Anemone sp.), la rizomatosa da fiore più importante in area romana, da dove veniva esportata in Italia ed in Europa. (...) Il (Tulipa) è volutamente rappresentato sottotono, nella forma di Tulipa sylvestris e di una collezione di tulipani botanici sottostanti i vasi di gelsomini e posti in prossimità del Casino dell'Uccelliera. Questo perché la moda del tulipano si afferma in Italia con un ritardo di alcuni decenni rispetto ai Paesi Bassi”16.

$\mathrm{Al}$ centro, anche qui è collocata la Fontana settecentesca originale, che, in analogia con quella del Giardino Vecchio, è formata da una vasca quadrilobata

\footnotetext{
${ }_{13}$ Nell'hortus coronarius venivano coltivate le piante destinate alla composizione di ghirlande, festoni e collane vegetali, a cui veniva riconosciuto un valore magico, terapeutico e simbolico.

${ }^{14} \mathrm{Ci}$ si riferisce più precisamente a: TOBIA AlDINI, "Exactissima Descriptio Rariorum Quarandum Plantarum, que continentur Romae in Horto Farnesiano", Roma 1625 e Giovan BATTISTA FerRARI "Flora overo cultura dei fiori", Pierantonio Facciotti, Roma 1638.

${ }^{15}$ Definizione riportata nella documentazione storica ad indicare la presenza di una pregiata collezione di bulbose, famosa tra i cultori e oggetto di scambi tra i proprietari dei giardini più preziosi. "E noto che il cardinale Borghese si faceva inviare bulbi da ogni dove e, tra gli altri, il cardinale Guido Bentivoglio, nunzio apostolico a Bruxelles, con grande sollecitudine si affrettava a spedirgli le varietà più rare e pregiate che riusciva e reperire nei Paesi Bassi”. AlberTa CAMPITElli, op. cit., 1999, pag. 188.

${ }^{16}$ Dipartimento Politiche Ambientali e Agricole, Comune di Roma, Il giardino dell'Uccelliera in I Giardini Segreti di Villa Borghese, (Brochure con schede), Fratelli Palombi Editori, Roma 1999.
} 
animata da una scultura zoomorfa in travertino, questa volta un drago alato, altro simbolo della casata dei Borghese, dalla cui bocca esce lo zampillo d'acqua.

Il Giardino della Meridiana costituisce, sia in termini spaziali sia in termini di interpretazione di un modello legato ad un preciso periodo storico, la prosecuzione progettuale dei primi due. Qui l'intenzione è stata quella di evocare la cultura orticola in voga alla fine del Seicento, sempre attraverso l'uso di piante, erbacee perenni ed annuali e bulbose da fiore, introdotte in quel periodo. Tra tutte le bulbose trionfa il tulipano (Tulipa sp.), che appare anche raffigurato nelle decorazioni a stucco della facciata est della palazzina della Meridiana. Il legame con i temi ornamentali della palazzina è rafforzato anche dall'inserimento nel piano delle aiuole di un motivo centrale stellare, una "rosa dei venti", a cui viene mutato l'impianto botanico nelle diverse stagioni e che presenta così una duplice rappresentazione, estiva ed invernale.

Il Giardino di Coltivazione è stato pensato come luogo deputato, appunto, alla coltivazione, propagazione, conservazione e sperimentazione delle specie botaniche impiegate negli altri giardini. Per il suo disegno, il gruppo di progetto si è basato su elementi formali desunti da documenti tardo cinquecenteschi ed esempi reali ancora esistenti. Anche in questo caso lo spazio viene suddiviso secondo uno schema rigorosamente geometrico e simmetrico, il cui ritmo viene impartito dai contrafforti presenti lungo la cinta muraria. L'assetto planimetrico assume una forma ad ippodromo, in riproposizione di un motivo ricorrente nei giardini secenteschi romani.

Contestualmente al recupero dei Giardini Segreti, è stato attuato anche un intervento di ricostruzione del giardino formale realizzato nei primi decenni del Novecento nel piazzale retrostante il Palazzo ed oggi chiamato Giardino del Museo. Il disegno novecentesco, con elaborate broderies delimitate da bordure geometriche di bosso (Buxus sempervirens), è stato riproposto, a testimonianza del revival del giardino geometrico e formale che a partire dal primo decennio del Novecento imperversò in affermazione del modello 'all'italiana'.

Il fervore editoriale degli ultimi vent'anni sulla teoria e la pratica del restauro dei giardini storici, testimonia l'importanza e lo sviluppo di un dibattito culturale su un tema che in Italia comincia ad avere solo oggi significativi esempi di applicazione operativa. Il recupero dei Giardini Segreti è uno di questi e si colloca con ragione nel quadro nazionale di illustrazione degli orientamenti in atto. La necessità di restituire la originale unitarietà al complesso Palazzo-Giardini-Palazzine di Villa Borghese, così come le valutazioni sullo stato di conservazione dei singoli

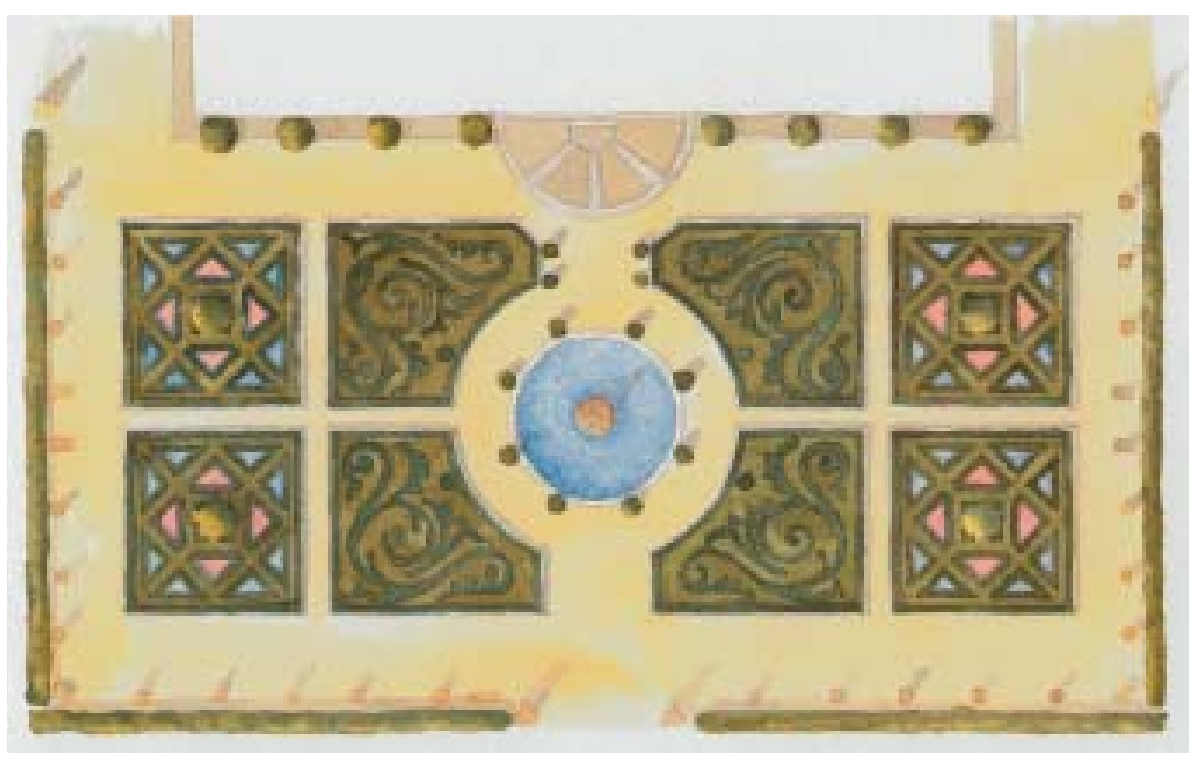

Figura 6 - Il Giardino del Museo, disegno acquarellato del progetto di restauro. 
giardini ridotti ormai a semplici aiuole inerbite, hanno portato sul tavolo delle scelte progettuali il difficile problema dell'indirizzo metodologico da seguire.

Nel far prevalere "l'evocazione di forme e linguaggi seicenteschi" ${ }^{17}$, si è preferito attuare un'opera di ricostruzione critico-creativa delle preesistenze, che, grazie al sapiente uso del cospicuo apparato documentario, ha dato origine ad una successione di modelli storici di giardino. La declinazione del tema "giardino segreto" appare svolta per suggerire una riflessione sull'idea di processualità storica intimamente connessa al concetto di giardino come luogo mutevole, sensibile al passaggio del tempo e delle mode. Nell'impossibilità di eseguire una restituzione rigorosamente filologica, è stato posto l'accento sul valore espositivo dei giardini; ne sono prova soprattutto l'accurata ricerca progettuale condotta sugli impianti botanici e l'attenzione posta in fase di costruzione e piantagione degli spartimenti fioriti, concepiti come piccoli microcosmi in trasformazione al passaggio delle stagioni.

Lintervento così attuato non si limita ad evocare un'immagine di giardino storico, ma sollecita un preciso comportamento da parte del visitatore che "sembra avvicinarsi a quello di un collezionista, comportamento che impone una vista ravvicinata del singolo fiore, da esaminare guardando in giù, a testa bassa, piuttosto che ammirando la complessità della composizione"18.

\section{RIFERIMENTI BIBLIOGRAFICI}

CAmpitelli Alberta, I Giardini Segreti di Villa Borghese: dalla storia al restauro in GIUSTI MARIA ADriana (a cura di), I Tempi della Natura. Restauro e restauri di giardini storici, Edifir, Firenze 1999, pagg. 177-196.

Di GADDO BEATA, L'Architettura di Villa Borghese, "Groma Quaderni”, 5, Università degli Studi di Roma "La Sapienza”, D.A.C.C., Roma 1997.

Di Giovine Mirella (a cura di), Guida al Verde di Roma, Comune di Roma, Lozzi \& Rossi Editori, Roma 2000.

Dipartimento Politiche Ambientali e Agricole, Comune di Roma, I Giardini Segreti di Villa Borghese, (Brochure con schede), Fratelli Palombi Editori, Roma 1999.

Giusti Maria Adriana e Tagliolini Alessandro (a cura di), Il giardino delle Muse. Arti e artifici nel barocco europeo, Edifir, Firenze 1995.

Roma dalla storia alla natura, "Gli Speciali di Folia", inserto allegato ad "Acer", 6, 2000, Il Verde editoriale, Milano 2000.

Pozzana Mariachiara, Giardini storici. Principi e tecniche della conservazione, Alinea editrice, Firenze 1996.

Segre AdA, La metamorfosi e il giardino italiano nel Seicento, in GiUsti Maria Adriana e TAGLIOLINI AlESSANDro (a cura di), Il giardino delle Muse. Arti e artifici nel barocco europeo, Edifir, Firenze 1995, pagg. 97-122.

Tongiorgi Tomasi Lucia, Tulipomania. Addenda, in Giusti Maria Adriana e Tagliolini AlesSANDRO (a cura di), Il giardino delle Muse. Arti e artifici nel barocco europeo, Edifir, Firenze 1995, pagg. 79-97.

Tagliolini Alessandro, I giardini di Roma, Newton Compton editori, Roma 1980, pagg. 217218, della Seconda Edizione.

Tagliolini Alessandro, Storia del giardino italiano, La Casa Husher, Firenze 1994.

\section{RIFERIMENTI ICONOGRAFICI}

Figure 1, 2: rielaborazione di Anna Lambertini da DI GADDO BEATA, L'Architettura di Villa Borghese, "Groma Quaderni”, 5, Università degli Studi di Roma "La Sapienza", D.A.C.C., Roma 1997, pag. 26 e pag. 102.

Figure 3, 4, 5: fotografie di Alessandra Cazzola.

Figura 6: rielaborazione di Anna Lambertini da Dipartimento Politiche Ambientali e Agricole, Comune di Roma, I Giardini Segreti di Villa Borghese, (Brochure con schede), Fratelli Palombi Editori, Roma 1999.

${ }^{17}$ Maria Adriana Giusti (a cura di), I Tempi della Natura. Restauro e restauri di giardini storici. Edifir, Firenze 1999, pag. 12.

${ }^{18}$ Mariachiara Pozzana, Giardini storici. Principi e tecniche della conservazione, Alinea editrice, Firenze 1996. 
SCHEDA DI PROGETTO

Nome:

Luogo:

Proprietà:

Tipologia di giardini:

Superficie:

Gruppo di Progetto:

Gruppo di lavoro:

Studi archeologici:

Anno di realizzazione:

Tempi di realizzazione:

Impresa esecutrice:

Fornitori piante:

Costi di realizzazione: ripristino dei Giardini Segreti di Villa Borghese

Roma, Lazio

pubblica, Comune di Roma

giardini storici, aperti al pubblico un giorno alla settimana con visita guidata

$9100 \mathrm{mq}$

Arch. Mirella di Giovine (Dipartimento Politiche Ambientali ed Agricole, Comune di Roma)

Dott.ssa Alberta Campitelli

Dott.ssa Ada Segre (consulenza botanica)

Arch. Aniello Ancona, Arch. Santo Ceravolo, Gianni Letizia, Geom. Carlo Marinucci, Tecnico agrario Stefano Carletti, Geom. Franco Spirito

condotti nel 1997 da Cooperativa Parsifal, Dott.ssa Anne Allimant

2000

180 giorni

Sarappalti SpA - Caldani Piante

Agrumi: Az. Agr. Oscar Tintori

Arbusti e Aromatiche: F.lli Margheriti - Torsanlorenzo e Chiusi

Bulbi e rizomatose: Vivaio Floriana di J. Sheibal; Present Garden di P. Goemans

Bulbose antiche particolari: Villa La Petraia, Firenze

Erbacee perenni e aromatiche: Priola di P.L. Priola; Susigarden G. Geott e S.Lukas

Iris e piante acquatiche: Guido degli Innocenti, Firenze

Rose Antiche: Il Mondo delle Rose di Duquennoy

Sementi annuali: Chiltern Seed, Bortree Stile; Eric Schweizer Semens

Tappeti erbosi: Bindi Pratopronto

Vasi Cotto Impruneta: Terracotte Enzo Zago

930 mila euro circa (Finanziamenti per il Piano del grande Giubileo 2000) 


\title{
IL PARCO DELL'APPIA ANTICA
}

\author{
Alessandra Cazzola
}

\section{IL CONTESTO}

Il territorio adiacente la via Appia Antica è delimitato a nord dalla cinta delle Mura Aureliane di Roma, a ovest dalla via Ardeatina e dalla ferrovia Roma-Napoli, a est dalla via Tuscolana e dalla via Appia Nuova fino a Frattocchie, mentre a sud lambisce l'abitato di S. Maria delle Mole e il fosso ai margini dell'area archeologica di Tellene.

La superficie è di circa 3.500 ettari, in cui sono compresi: la valle della Caffarella (250 ha), il Parco archeologico della via Latina, il Parco degli Acquedotti (240 ha), un tratto della via Appia Antica all'interno dei comuni di Ciampino e Marino.

Anche se è evidente che la componente naturale del paesaggio del Parco dell'Appia è stata profondamente plasmata nel corso dei millenni dall'opera dell'uomo, alla base della caratterizzazione di questo territorio vi è comunque l'evoluzione geologica e geomorfologica dell'area. È stata l'attività del Vulcano Laziale, specie nella fase in cui ha prodotto colate imponenti della lunghezza di diversi chilometri che coinvolsero direttamente la zona dell'Appia (come la cosiddetta colata di Capo Bove), a modellare gli aspetti caratteristici del paesaggio "originario" della zona, creando quella "piattaforma" dall'andamento pianeggiante, più rilevata rispetto alle pianure paludose circostanti, su cui fu poi tracciata la via Appia.

All'attività vulcanica si è aggiunta l'azione degli agenti atmosferici, in particolare delle acque superficiali, che hanno inciso in misura diversa terreni a differente grado di erodibilità, contribuendo all'aspetto tipicamente ondulato del terreno e

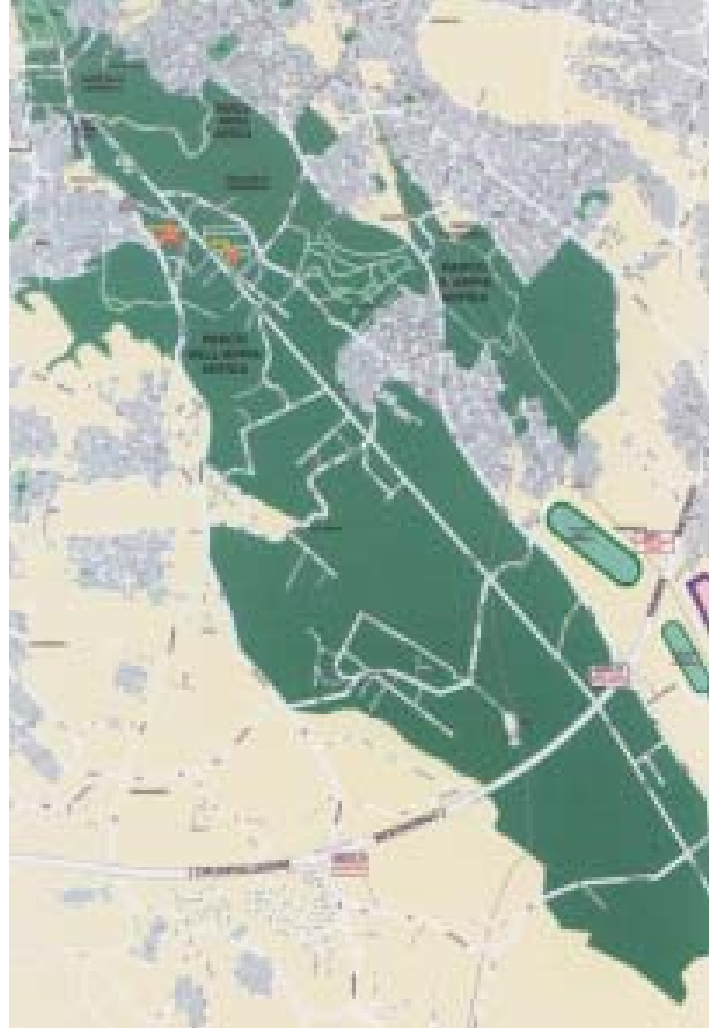

Figura 1 - La localizzazione del parco dell'Appia rispetto al centro di Roma. creando in alcuni tratti una rete di numerosi e profondi solchi dovuti all'erosione delle acque superficiali, separati da strette lingue pianeggianti di terreno.

Il territorio, inserito nel contesto naturalistico tipico dell'agro romano, presenta una prevalente utilizzazione agricola, con grandi estensioni di colture agrarie e di pascoli nella parte centrale; nelle aree più vicine ai centri abitati si hanno appezzamenti medio-piccoli di colture a seminativo, serre, e un gran numero di orti abusivi, che frammentano e degradano il paesaggio. La capacità produttiva tende comunque a regredire a causa dell'intenso intervento antropico. Laddove l'impatto umano lo consente, resistono boschetti di latifoglie miste con residui di querce, aceri, sambuchi, eccetera, relitti delle precedenti formazioni forestali, e la vegetazione ripariale e palustre lungo i corsi d'acqua e nelle zone umide, con esemplari di pioppi e salici.

La principale valenza del Parco è sicuramente quella storico-archeologica, costituita dall'insieme di tombe, sepolcri, acquedotti e ville che costeggiano la via Appia Antica, dalle torri medievali e dai casali sorti dal Rinascimento fino al 
XIX secolo. Ma all'interno del Parco sono presenti anche complessi differenti, ad iniziare dal circo di Massenzio per finire col sistema sotterraneo costituito dalle catacombe di Pretestato, Domitilla, S. Sebastiano, S. Callisto e dalle Catacombe Ebraiche. I monumenti della valle della Caffarella ed il comprensorio della via Latina (di cui fanno parte il Parco archeologico della via Latina, il sistema degli acquedotti e la villa dei Sette Bassi) completano il quadro.

La pianificazione che ha interessato il Parco dell'Appia Antica è stata molto lunga e articolata e successivamente vengono sintetizzati $i$ due momenti più significativi: il Piano di Assetto proposto da Italia Nostra nel 1976 - e mai realizzato - e il Piano di Assetto predisposto dall'Ente Parco tra il 1998 e il 2002 (anno nel quale è stato adottato).

\section{Il Piano di assetto dell'area del Parco dell'Appia di Italia Nostra (1976)}

Nel lavoro di Italia Nostra, coordinato dalla Prof.ssa Vittoria Calzolari e culminato nel volume curato dal Prof. Massimo Olivieri (Piano per il parco dell'Appia Antica, Italia Nostra - sezione di Roma, Roma 1984), per la prima volta, dopo almeno vent’anni di dibattiti e polemiche, veniva presentata una proposta di assetto complessivo, che rispettava le caratteristiche storico-archeologiche e naturalistico-paesistiche territoriali del parco, e una gran quantità di dati riuniti in singoli blocchi informativi e analisi, che hanno rappresentato la base fondamentale per la proposta stessa.

\section{GLI OBIETTIVI}

Il primo obiettivo del progetto è stato quello di individuare un metodo attraverso il quale fare sì che i successivi progetti e interventi fossero coerenti all'idea complessiva del piano di assetto e alle finalità proposte in esso, piuttosto che definire un modello unico di assetto fisico che nelle sue diverse fasi di attuazione avrebbe potuto subire delle modifiche. Il Piano ha quindi organizzato e disegnato un sistema di relazioni costruite sulle potenzialità ambientali del luogo, sulle situazioni e sulle esigenze di tipo più urbanistico, sulle esigenze e sugli effetti delle modalità con cui vengono usati determinati spazi e sugli esiti formali che questo riuso comporta.

Il raggiungimento di questo obiettivo si è quindi fondato sulla realizzazione di quattro linee-guida:

1. la ricomposizione del sistema delle aree archeologiche e delle strutture delle altre epoche, al fine di non creare una sorta di frattura tra le aree archeologiche e il resto del Parco (sia attraverso l'uso di barriere fisiche, sia con altre sistemazioni vegetali), ma al contrario di evidenziare il legame tra tessuti storici e paesaggio rendendo leggibili le diverse stratificazioni (compresa l'«archeologia sotterranea» delle cave e cunicoli di epoca romana e più tarda);

2. la ricostruzione dell'unità formale del Parco alla grande scala, mettendo in evidenza quelli che sono i caratteri geomorfologici fondamentali ai quali corrispondono diverse possibili colture. Ad esempio: il rimboschimento dei pendii e la creazione di ampie zone a bosco compatto, il recupero del sistema delle acque partendo dall'Almone, la riprogettazione dei margini del Parco dove maggiori sono le esigenze di aprirlo verso la città e di curare l'affaccio di quest'ultima su di esso;

3. l'utilizzazione degli edifici esistenti nel Parco, che è sicuramente più facile per quelli che sono considerati di qualità storico-architettonica (forti, casali, complessi di vario genere), non lo è altrettanto per le circa duecentoquaranta ville, regolari e abusive, presenti entro il perimetro del Parco e per le quali il Piano ipotizzava la demolizione nel caso di edifici "inconciliabili" col contesto e la graduale acquisizione da parte di Enti Pubblici dei rimanenti; 

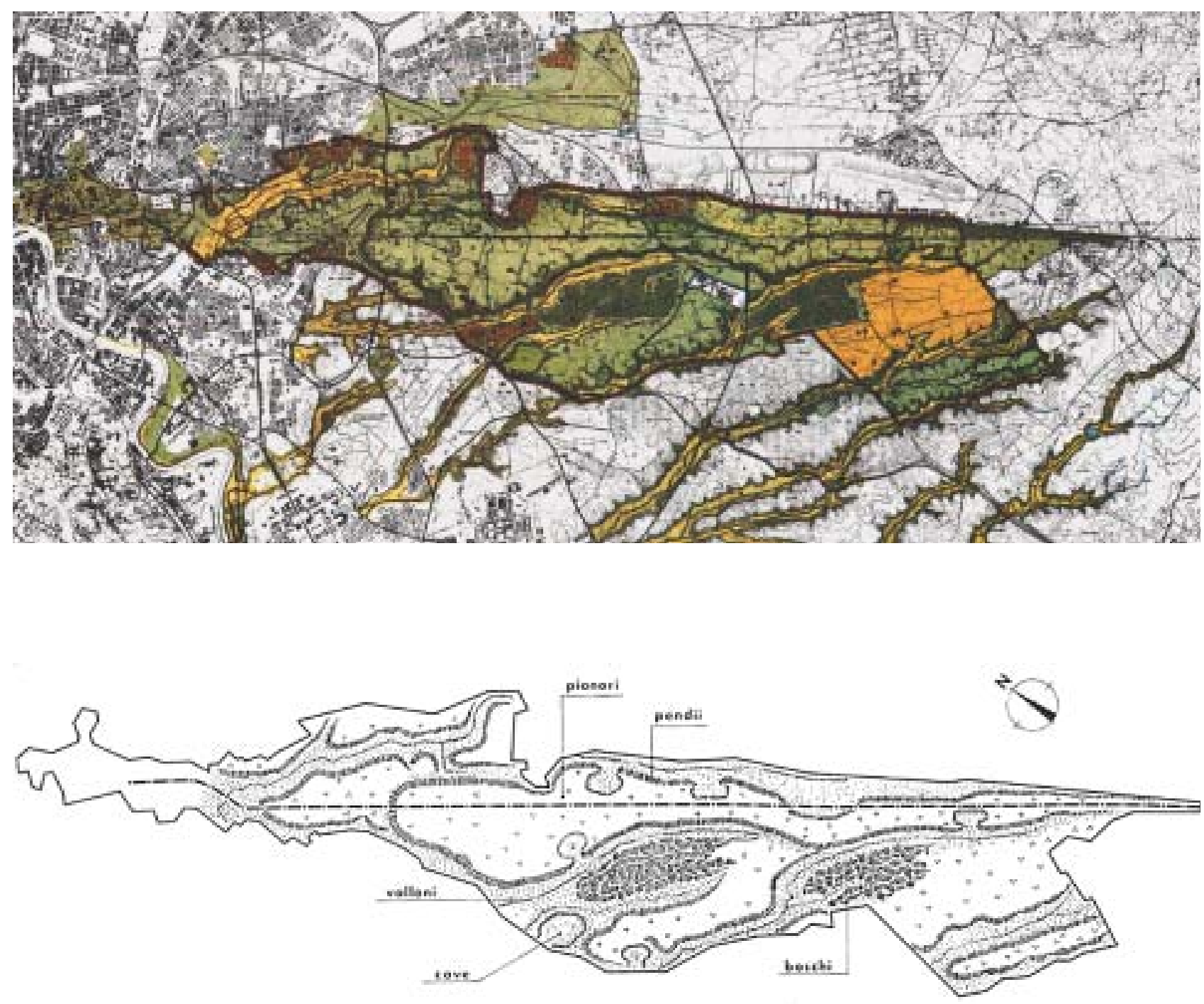

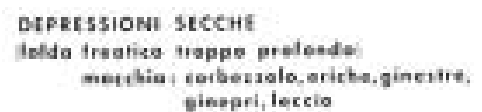

metchia i corbezasolo, oricto

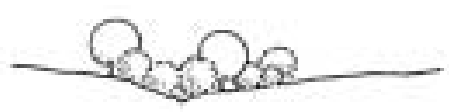

PIANOEI

inceseggiers to masuhin nelln erponiest dai pesdii pine demastite, roverella

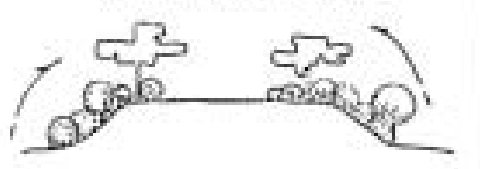

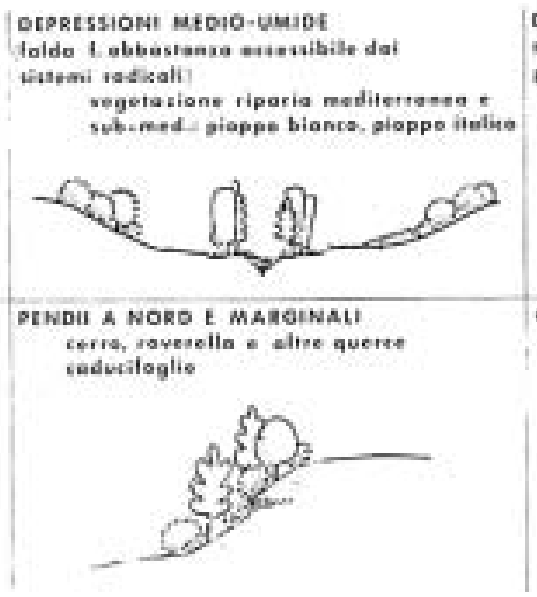

DEPRESSIONI CON ACOUA AFFORANTE

iendenta a riprodurte espetti del passaggia pentins:

cargino, farnie, pioppe b. teliei gieppiglasane arientole

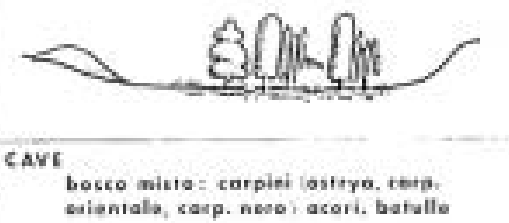

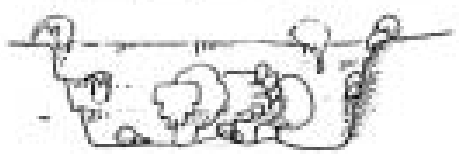

Figure 2 - (sopra) e 3 (sotto).

La tavola di piano con la quale si visualizza il possibile assetto finale del territorio a grande scala (sopra) viene letta in relazione con: le sezioni attraverso il quale viene indicato il rapporto che si prevede di ricreare tra la vegetazione, il suolo, il sistema delle acque, l'esposizione dei versanti e le pendenze (sotto); gli schemi che mettono in correlazione i caratteri ambientali con i tipi e le densità d'uso compatibili; le tavole urbanistiche che valutano la domanda di aree verdi da parte dei quartieri circostanti e l'accessibilità da questi ultimi al parco; gli schemi che offrono la "griglia - tipo" che la Pubblica Amministrazione dovrebbe utilizzare al fine di coordinare e controllare gli obiettivi, i costi e i benefici. 
4. il mantenimento delle attività produttive esistenti (in particolare l'attività dell'agricoltura), con, da una parte, la conservazione dell'uso agricolo laddove esista una struttura fondiaria abbastanza congrua o dove l'uso produttivo sia conciliabile con le finalità del Parco, e, dall'altra parte, la previsione di altre forme di agricoltura specializzata (vivai, colture legnose, orti) o di pascoli nelle zone a Parco per le quali è prevista la riorganizzazione.

Il secondo obiettivo della proposta di assetto è stato quello di ricercare una metodologia di programmazione che coordinasse i problemi di gestione tecnica con quelli di tipo istituzionale e legislativo.

È stato quindi previsto di:

- subordinare i vari possibili finanziamenti a precisi piani di attuazione e di gestione;

- assicurare la partecipazione da parte di rappresentanze degli organismi di base coinvolti in ogni fase progettuale;

- provvedere ad una collaborazione di alto livello scientifico con esperti italiani e stranieri;

- prevedere un'attuazione del Piano "diluita" in 10-15 anni attraverso l'applicazione di programmi biennali.

La proposta di assetto comprendeva anche uno schema di proposta di legge per il finanziamento, la realizzazione e la gestione del Parco. Nello schema veniva proposto un contributo di 15 miliardi, da erogare in un lasso di tempo di 5 anni, a favore del Comune di Roma, da finalizzare all'esproprio delle aree e alla costituzione di un" "azienda - consorzio" tra il Comune di Roma e la Regione Lazio per la realizzazione e gestione del Parco. A questo fine veniva previsto un contributo straordinario di 30 miliardi, distribuito su dieci annualità, insieme alla predisposizione di programmi attuativi biennali dai quali sarebbero dovute venire le indicazioni circa gli edifici da demolire perché incompatibili con le finalità del Parco.

\section{LA METODOLOGIA}

Lo studio è stato condotto applicando il criterio dell'interdisciplinarietà, con lo scopo di ottenere un'immagine del territorio del parco che fosse il prodotto di un'ampia complessità di relazioni tra i diversi elementi o processi propri di ciascuna disciplina (idrogeologia, pedologia, botanica, agronomia, archeologia, storia, urbanistica, eccetera). Dalle diverse informazioni ottenute sono scaturite tutta la serie di decisioni riguardo a ciò di cui era assolutamente necessario tener conto (ossia quelle che oggi verrebbero chiamate «invarianti») e tutti quei suggerimenti utili riguardo ai comportamenti che si sarebbero dovuti tenere sia in termini generali, sia per ciò che riguarda determinati luoghi e/o determinate azioni.

Un secondo criterio, ritenuto basilare nella conduzione dello studio, è stato quello di proiettare il piano in una dimensione territoriale di grande scala, considerando quindi un sistema molto più ampio del quale fanno parte i Colli Albani, le aree comprese fra questi ultimi, il Parco e il fiume Tevere, e quelle tra i Colli Albani e il mare. Il Parco dell'Appia Antica è stato in questo modo considerato da un lato come diretta zona di passaggio e di integrazione tra le aree archeologiche del centro storico della città e i Colli Albani con il loro - allora solo previsto parco regionale, dall'altro lato come zona fulcro dello stesso sistema.

I criteri adottati per studiare l'area di interesse non si sono, quindi, limitati ad esaminare i vari elementi esclusivamente all'interno dei suoi stretti confini, bensì ad analizzare a fondo anche le aree limitrofe, allo scopo di individuare i rapporti del Parco con la città costruita e di specificare in proposte di maggior dettaglio quanto previsto ed indicato nelle studio a carattere sovracomunale. Ė stato quindi 
proposto che le aree contermini, in base alla caratteristiche di ciascuna, diventassero o zone da includere nel perimetro del Parco, oppure zone filtro, o ancora aree di penetrazione del verde nel costruito, o elementi di connessione con altri ambiti aventi interesse naturalistico, storico, archeologico, paesistico.

Un terzo criterio, decisivo ai fini della proposta, è stato quello della tutela rivolta non solo ai beni archeologici, che rappresentano sicuramente gli elementi di maggiore notorietà del Parco, ma anche alla natura, al paesaggio e alle preesistenze storiche, come i casali. La proposta del piano è orientata al raggiungimento di una "tutela attiva" attraverso la quale ottenere:

- la valorizzazione e la contestualizzazione dei resti archeologici rispetto ad un territorio il cui paesaggio è caratterizzato (e lo è stato soprattutto storicamente) dai rapporti che intercorrono tra tali resti e altri elementi di diversa natura;

- la protezione e la valorizzazione degli ambiti naturalistici e dei complessi vegetazionali;

- la realizzazione di coperture boschive laddove sussistono maggiori problemi di degrado e il mantenimento di ampie aree a pascolo e a colture;

- la riutilizzazione e, laddove necessario, il recupero dei casali ai fini propri del Parco.

Il disegno complessivo che ne risulta è fortemente caratterizzato dalla contemporanea presenza di elementi "naturali" (le zone alberate e i prati, i cigli della colata lavica, i corsi d'acqua, eccetera), che sottolineano la struttura fisica del territorio e che, pur essendo in rapporto di organicità con gli altri elementi, hanno una funzione egemone e strutturante, e di elementi più "antropici" quali i complessi monumentali, la struttura rettilinea della via Appia, i forti e i casali, le aree attrezzate, eccetera.

\section{GLI ESITI}

Il progetto prevedeva la realizzazione del Piano di assetto secondo due fasi di programmazione e attuazione che si sarebbero dovute svolgere in un periodo di dieci anni. Nello specifico:

1 a fase - 6 mesi / 1 anno

Questa fase sarebbe servita ad aggiornare le conoscenze, ad evitare che determinate situazioni - le più a rischio - si deteriorassero ulteriormente, a migliorare e a cominciare ad utilizzare alcune parti del Parco e, infine, a creare tutte le premesse (studi, accordi, finanziamenti, eccetera) per poter iniziare la operazioni che riguardavano poi le fasi successive.

$2^{\text {a }}$ fase - 10/15 anni

La seconda fase sarebbe stata articolata in programmi biennali o modulata secondo i Programmi Pluriennali di attuazione del Comune di Roma e, per il primo biennio, avrebbe compreso la costituzione di un organismo addetto alla programmazione e all'attuazione del Piano del Parco e la ridefinizione e l'approvazione del Progetto definitivo con le indicazioni delle linee strutturali e i criteri di sistemazione del Parco dell'Appia Antica, gli ambiti per i quali sviluppare i progetti attuativi separati, le priorità nelle acquisizione dei terreni e degli edifici e la loro distribuzione nell'arco dei 10-15 anni e, infine, le convenzioni attraverso le quali fruire tutto ciò che per un tempo più o meno lungo resterà di proprietà privata.

\section{Il Piano di Assetto Dell’Ente Parco (1998-2002)}

Nel 1997 l'Ente Parco dell'Appia Antica ha avviato i lavori per predisporre il proprio Piano di Assetto, con la scelta di fondo di redigere il Piano con le 
strutture ed il personale degli Uffici stessi dell'Ente. Nel luglio del 2002 il Piano è stato adottato dal Consiglio Direttivo dell'Ente Parco. L'obiettivo principale, sin dalle fasi iniziali, è stato quello di costruire un Piano di Assetto che fosse contemporaneamente uno strumento di programmazione e pianificazione territoriale e uno strumento per la gestione del Parco. Per fare ciò la fase iniziale dei lavori è stata tutta tesa a predisporre un cospicuo bagaglio di conoscenze, predisponendo un sistema informativo (GIS) che rappresentasse nello stesso tempo sia un contenitore entro il quale riversare tutte le informazioni provenienti dalle diverse ricerche fatte fino ad oggi sull'argomento, sia un patrimonio di informazioni che fosse continuamente aggiornabile e consultabile da cittadini, ricercatori, progettisti, eccetera.

\section{GLI OBIETTIVI}

Il Piano, che al momento è in Regione per l'approvazione definitiva, ha come obiettivi principali:

1. ricostruire l'intera unità territoriale e paesaggistica del Parco e creare le condizioni ottimali per uno sviluppo sostenibile;

2. adeguare le politiche della mobilità cittadina sul territorio, ponendo particolare attenzione a quelle che sono le strade di maggior scorrimento (via Appia Pignatelli, via di Tor Carbone, via Ardeatina) e individuando alcune soluzioni di medio periodo in comune accordo con quelle che dovrebbero essere le indicazioni del Nuovo Piano Regolatore del Comune di Roma;

3. ampliare i confini del Parco, in maniera tale da comprendere e riconnettere tra loro aree con un alto valore paesaggistico;

4. eliminare i detrattori ambientali rappresentati soprattutto da insediamenti commerciali che attualmente occupano spazi di notevole interesse (paesaggistico, archeologico, eccetera), attraverso l'attuazione di un processo di "delocalizzazione delle attività produttive", che prevede la risistemazione delle stesse in aree esterne al Parco, e la riconversione, con usi compatibili, dei manufatti storici, nonché la demolizione dei manufatti più recenti e di scarso valore;

5. ripristinare l'attività agricola, intendendola come l'elemento-matrice del recupero paesaggistico, in ampie zone del Parco: attraverso le previsioni del Piano di Assetto verrà istituita una sorta di «Banca del terreno» attraverso la quale l'Ente Parco si farà mediatore tra i proprietari di lotti di medie-piccole dimensioni (intorno ai 5.000 metri quadrati), che non hanno alcuna possibilità di utilizzare i loro terreni, e agricoltori e allevatori che, al contrario, possono impiegare quegli stessi appezzamenti per le loro attività pagando un affitto ai proprietari;

6. incentivare i processi di rinaturalizzazione attualmente in corso in aree del
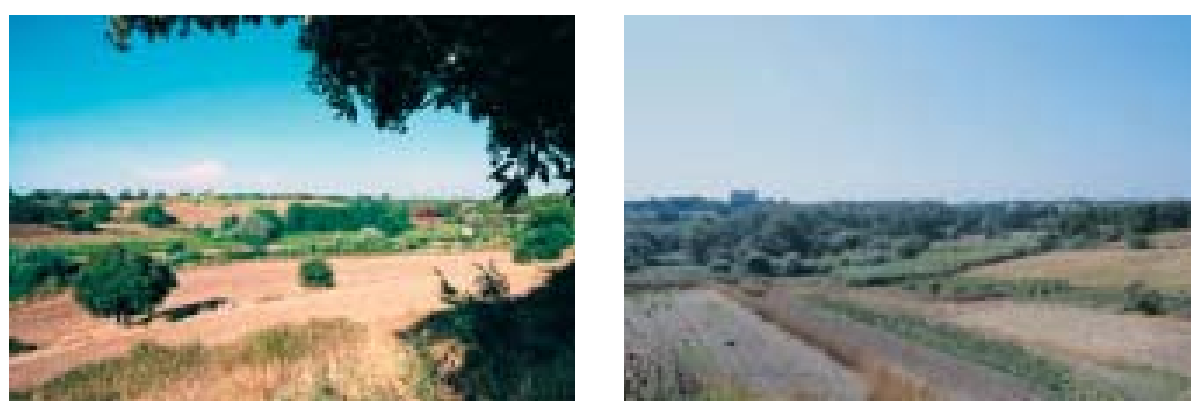

Figure 4, 5. - Alcuni esempi di aree agricole entro l'area del Parco dell'Appia Antica. 
Parco importanti, come ad esempio le ex cave e le zone a ridosso dei corsi d'acqua principali (l'Almone e il fosso di Tor Carbone);

7. consolidare gli elementi ritenuti strutturanti il paesaggio, attraverso il ripristino dell'agricoltura che si farebbe promotrice di tutta una serie di interventi di reintegrazione di siepi, corsi d'acqua, boschetti, eccetera;

8. contribuire al bilancio ecologico dell'intera città di Roma e dei comuni limitrofi, rappresentando lo snodo centrale dei vari corridoi ecologici che vanno dai Castelli Romani al parco di Veio, da Decima Malafede alla zona del litorale e della pineta di Castel Porziano;

9. costruire un rapporto fitto di scambio continuo con i cittadini, attraverso una politica di fruizione controllata che individui alcune aree marginali (non particolarmente interessanti) dove localizzare gli spazi di massima fruizione; da questi viene poi fatto partire il sistema di sentieri che si sviluppa poi nel Parco e il ricco sistema di "Centri visita" finalizzati principalmente alla didattica ambientale per le scuole e i cittadini;

10. realizzare le condizioni ottimali affinché la ricerca archeologica possa essere pianificata e realizzata senza grossi impedimenti di tipo urbanistico.

\section{LA METODOLOGIA}

La struttura del Piano di Assetto viene delineata attraverso 24 tavole di sintesi, in scala 1:10.000, e una serie di dossier/elenchi ad esse collegati.

La parte analitica del Piano è formata dalle seguenti cartografie:

- inquadramento territoriale del sistema dei parchi e delle altre aree protette intorno alla città di Roma;

- sistema dei confini amministrativi e dello stato di diritto (vincoli L.1089/39, L.1497/39, L.431/85, eccetera);

- mosaico degli strumenti urbanistici vigenti nei comuni entro i quali ricade il Parco;

- sistema viario e della mobilità;

- sistema ambientale con l'individuazione dei caratteri fisici, dei complessi litologici e dei caratteri podologici, del bacino idrografico e del sistema idrografico di superficie, della fisionomica della vegetazione;

- cartografie storiche con l'evoluzione dell'insediamento dalla protostoria fino al XX secolo.

La parte progettuale del Piano è invece costituita da:

- carte prescrittive, nelle quali viene indicata la zonizzazione con le zone A, B e C, così come richiesta dalla legge nazionale sui parchi (L.394/91);

- carte gestionali, che mostrano, oltre alla cosiddetta gestione ambientale e paesistica, le modalità della fruizione e il sistema della mobilità e della circolazione interna al Parco;

- carte progettuali, con l'individuazione dell'ampliamento dei limiti del Parco e con l'inquadramento dei progetti attuativi (processi di rinaturalizzazione, interventi sul sistema delle acque, eccetera).

I dossier che accompagnano le diverse cartografie rappresentano delle schedature di informazioni su diversi argomenti, tra i quali è opportuno ricordare:

- il sistema della circolazione;

- i detrattori principali del paesaggio;

- le aree degli insediamenti commerciali da delocalizzare;

- la struttura delle proprietà.

RIFERIMENTI BIBLIOGRAFICI 
Per le vicende storiche:

Benevolo Leonardo, Roma da ieri a domani, Laterza, Roma-Bari 1971.

Benevolo Leonardo Roma dal 1870 al 1990, Laterza, Roma-Bari 1992.

BORTOLOTTI LANDO, Roma fuori le mura, Laterza, Roma-Bari 1988.

Cederna Givlio (a cura di), Antonio Cederna: storia moderna dell'Appia Antica, Legambiente e Italia Nostra, Roma 1997.

Comune Di Roma, Roma capitale 1870-1911, Catalogo della mostra «Architettura e Urbanistica. Uso e trasformazione della città storica», Marsilio, Venezia 1984.

Frutaz Amato Pietro, Le carte del Lazio, Staderini, Roma 1972.

Frutaz Amato Pietro, Le piante di Roma, Tip. L. Salomone, Roma 1962.

InSOlera ITAlo, Roma moderna, Einaudi, Torino 1971.

Morandi MaUrIZIO, L'Appia Antica dal piano del '31 ad oggi, "Urbanistica”, 46-47, INU edizioni, Roma 1966, pag. 58.

Olivieri Massimo, Appia Antica: cronologia di un parco mancato, in PAOlo BerdinI (a cura di) "La città senza piano: le trasformazioni urbanistiche di Roma negli anni Ottanta», INU sezione Lazio, Roma 1992, pagg. 87-112.

Quarra Alessandro, L'Appia nella recente urbanistica romana, "Capitolium", 3, F.lli Palombi, Roma 1969, pag. 78.

RAVAGLIOLI ARMANDO, Il parco dell'Appia: sconfitta di una generazione?, "Roma/Rome", 1, Roma 1990, pag. 65.

Per la descrizione dei luoghi:

Alvisi GIOvanni, Nuove individuazioni di preesistenze archeologiche, "Urbanistica", 46-47, INU edizioni, Roma 1966, pag. 26.

Arnoldus-Huyzendueld Antonia, Il Paesaggio geologico ed i geotopi della Campagna Romana, "Quaderni dell'Ambiente", 5, Comune di Roma - ENEA, Fratelli Palombi, Roma 1997.

CABIANCA VINCENZO e QUILICI LORENZO, I beni culturali archeologici del territorio romano, "Urbanistica", 54-55, INU edizioni, Roma 1969, pagg. 82-108.

Campitelli Alberta, La Campagna romana dall'antichità al Medioevo: il territorio della X Circoscrizione, Comune di Roma X Circoscrizione, Roma 1999.

CANina Luigi, La prima parte della via Appia dalla porta Capena a Boville, Roma 1853.

Cignini Bruno, Massari Giuseppe e Pignatti SANDro, L'ecosistema Roma: ambiente e territorio, Fratelli Palombi, Roma 1995.

CoArelli Filippo, Guida Archeologica di Roma, Arnoldo Mondadori, Venezia 1974.

CoArelli Filippo, Dintorni di Roma, Arnoldo Mondadori, Roma-Bari 1981.

Istituto Tecnico per Geometri Giovanni Boaga, Il Parco degli Acquedotti, Legambiente Lazio, Roma 1997.

Lanciani Rodolfo, Rovine e scavi di Roma antica (titolo originale: The ruins \& excavations of ancient Rome), London 1897, Quasar, Roma 1985, pagg. 73-83 e pagg. 343-349.

QUILICI LORENZO, Il patrimonio archeologico della valle della Caffarella, "Urbanistica", 46-47, INU edizioni, Roma 1966, pag. 69.

Quilici Lorenzo, La via Appia da Roma a Bovillae, Bulzoni, Roma 1977.

Quilici Lorenzo, La via Latina da Roma a Castel Savelli, Bulzoni, Roma 1978.

QUilici Lorenzo, Via Appia: da porta Capena ai Colli Albani, Fratelli Palombi, Roma 1989.

QUILICI Lorenzo, Via Appia: dalla pianura pontina a Brindisi, Fratelli Palombi, Roma 1989.

Staccioli Romolo A, Le vie Appia e Latina, Tascabili Economici Newton, Roma 1996.

StacCioli Romolo A, Gli acquedotti di Roma antica, "Roma ieri oggi domani", anno IV, F.lli Palombi, Roma 1991, pag. 81.

Staccioli Romolo A, Gli acquedotti di Roma antica, Tascabili Economici Newton, Roma 1996.

Tomassetti Giuseppe, La Campagna Romana Antica, Medievale e Moderna, vol. IV: via Latina, Leo S. Olschki, Firenze 1976.

Per la pianificazione del parco:

FIORI NICA, Il parco degli Acquedotti, "Roma ieri oggi domani”, 70, anno VII, F.lli Palombi, Roma 1994, pag. 42.

Insolera ItAlo e Perego Francesco (a cura di), Archeologia e città: storia moderna dei Fori di Roma, Laterza, Roma-Bari 1983.

Olivieri Massimo (a cura di), Piano per il parco dell'Appia Antica, Italia Nostra - sezione di Roma, Roma 1984.

Per i personaggi:

Cederna Giulio (a cura di), Antonio Cederna: storia moderna dell'Appia Antica, Legambiente e Italia Nostra, Roma 1997.

Ministero per i Beni e le Attività Culturali, Centro di documentazione Antonio 
Cederna, Beni culturali, urbanistica e paesaggio nell'opera di Antonio Cederna: 1921-1996, (CD rom a cura dell'Ente), Roma 1999.

Siti web:

<www.parcoappiaantica.org>

$<$ www.parks.it/parco.appia.antica/par.html>

$<$ www.romacivica.net/tarcaf/caffapp/infnatst.htm>

$<$ www.romacivica.net/tarcaf/parcoaa.htm>

\section{RIFERIMENTI ICONOGRAFICI}

Figura 1: Di GIOvine Mirella (a cura di), Guida al verde di Roma: alla scoperta dei parchi naturali, delle ville storiche e dei giardini pubblici, Lozzi \& Rossi, Roma 2000, pag. 114.

Figure 2, 3: Olivieri MAssimo (a cura di), Piano per il parco dell'Appia Antica, Italia Nostra sezione di Roma, Roma 1984, tav. XXI, pag. 167 e tav. XXII, pag. 169.

Figura 4: www.parcoappiaantica.org, foto a cura dell'Ente Parco.

Figura 5: Comune di Roma, Nuovo Piano Regolatore Generale - Guida alla progettazione negli ambiti di paesaggio, Roma 2000.

\section{SCHEDA DI PROGETTO}

Progetto:

Luogo:

Superficie:

Progettisti:

Anno di progettazione:

SCHEDA DI PROGETTO

Progetto:

Luogo:

Committente:

Superficie:

Progettisti:

Anno di progettazione:

Stato di attuazione:
Piano di Assetto dell'area del Parco dell'Appia

Comune di Roma, Comune di Ciampino, Comune di Marino (Provincia di Roma)

3.500 ha circa

Italia Nostra (coordinamento Prof.ssa Vittoria Calzolari)

1976
Piano di Assetto del Parco dell'Appia

Comune di Roma, Comune di Ciampino, Comune di Marino (Provincia di Roma)

Ente Parco dell'Appia Antica

3.500 ettari circa

Ente Parco dell'Appia Antica

$1997-2002$

il Piano di Assetto è stato adottato dal Consiglio Direttivo dell'Ente Parco nel luglio 2002. 



\title{
IDEE E PROGETTI
}

\author{
Claudia Cassatella
}

In un momento di grande fermento culturale, quale sta attraversando la ricerca sul paesaggio in Italia, è normale che siano molto più numerose le proposte ed i progetti, rispetto alle realizzazioni. Intendiamo qui presentare tre casi dei quali non è ancora possibile mostrare le realizzazioni compiute (benché alcune siano già avviate), ma che non sarebbero potute mancare in questa pubblicazione, poiché rappresentano elaborazioni significative su alcuni temi centrali nella ricerca italiana: il paesaggio agrario, in particolare quello storico, ed il paesaggio fluviale. Di comune hanno anche il fatto - frequente nel contesto italiano - di essere progetti di valorizzazione di risorse naturali e culturali esistenti.

Il "Programma di Paesaggio Chianti" (inserito nel Piano Territoriale di Coordinamento della Provincia di Firenze) getta le basi per interventi di recupero delle aree di paesaggio storico mezzadrile, per una superficie di circa 23.000 ettari.

Nella campagna pavese è in progetto una via verde pedonale e ciclabile (19 chilometri il percorso principale), lungo un'antica via d'acqua, per il collegamento tra la Certosa, la città di Pavia e il fiume Ticino, che interessa 3.500 ettari di aree agricole.

Il progetto programma "Torino città d'acque" sta realizzando il collegamento continuo dei 74 chilometri di sponde dei quattro fiumi torinesi, aggiungendo ai 420 ettari dei parchi esistenti 830 ettari di nuove aree verdi.

\section{Il paradigma della reticolarità}

In concreto questi progetti trattano di sistemazioni idrauliche, sistemazioni spondali, corridoi ecologici, percorsi e manufatti storici. Sono progetti di connessioni: connessioni fisiche (percorsi), ecologiche (corridoi per la flora e la fauna), ma anche (e, forse, soprattutto) "sintattiche"; sono cioè progetti che restituiscono leggibilità a quei legami tra luoghi, oggetti ed usi che ci rendono il senso del paesaggio, le relazioni tra le sistemazioni dei versanti, tra le strade di crinale e i poderi, tra i fiumi, la collina e la città.

Questi progetti possono essere ricondotti al paradigma della reticolarità che pervade le scienze fisiche come quelle umane, e in particolare, per quanto ci riguarda, l'ecologia, la geografia, la semiotica (l'approccio strutturalista alla lettura del paesaggio $)^{1}$. Essi mettono in rete non solo gli elementi appartenenti allo stesso livello di analisi (ad esempio le risorse fisiche connesse in reti ambientali, i segni della storia in testi paesistici) ed allo stesso settore d'intervento, ma anche i diversi livelli tra di loro, cercando di valorizzare congiuntamente le risorse naturali e culturali. Prendiamo ad esempio il concetto di greenway, o corridoio verde, che è presente sia nel caso di Pavia sia in quello di Torino: "sistemi di territori

\footnotetext{
${ }^{1}$ Cfr., a proposito del paradigma della reticolarità nella pianificazione paesistica, ROBERTO GAMBINO, Progetti per l'ambiente, Franco Angeli, Milano 1996, e più in generale Claudia Cassatella, Iperpaesaggi, Testo \& Immagine, Torino 2001.
} 
lineari tra loro connessi, protetti e sviluppati in modo da ottenere benefici di tipo ecologico, ricreativo, storico-culturale"2.

Lo sfruttamento di questa trans-settorialità e trans-scalarità può consentire anche di ottimizzare le risorse dell'intervento. Nei casi esaminati le connessioni hanno una funzionalità a scala locale (ad esempio il collegamento tra luoghi urbani o tra centri vicini), ma sono pensate per contribuire al funzionamento della scala territoriale: ad esempio nel caso piemontese, la connessione tra i fiumi si allaccia ad una rete di percorsi collinari e si integra in un vasto disegno della Regione Piemonte, denominato Corona Verde, che valorizza la continuità ecologica tra la montagna e le colline del Monferrato attraverso il difficile ostacolo costituito dall'area metropolitana torinese.

\section{I fiumi, i canali, il paesaggio agrario storico}

Fiumi, canali artificiali, sistemazioni dei versanti rivestono un'importanza cruciale nel contesto della delicatissima situazione idrogeologica italiana.

Il Po è il maggior fiume italiano, uno dei maggiori in Europa. La Regione Piemonte ha avviato un Progetto Po fin dai primi anni Ottanta, periodo in cui venivano elaborate proposte di recupero ambientale anche per altri grandi fiumi come il Reno e la Loira. Il tema del paesaggio fluviale fu sviluppato in quegli anni soprattutto con studi di matrice ecologica ${ }^{3}$. La legge 18 maggio 1989 n. 183 per la tutela del suolo ${ }^{4}$ ha stabilito che le misure di tutela ambientale facciano riferimento ai bacini idrografici, tramite appositi "Piani di Bacino": questi sono piani che integrano i diversi aspetti settoriali ed i diversi livelli di pianificazione.

Oltre al fitto reticolo idrografico, l'Italia possiede una minutissima rete di canali artificiali, in particolare nella Pianura Padana, che dà luogo a paesaggi unici (soprattutto nel vercellese e novarese, nel ferrarese, nella pianura veneta). È un sistema assai delicato, che soffre per l'abbandono della manutenzione continua di un tempo, talvolta per la cementificazione dei canali, quando non per il loro intombamento. Alcune volte i canali restano, inglobati nella città o nella metropoli, unici corridoi cui si può affidare una seppur ardua continuità ecologica tra l'urbano e la campagna; nei casi più fortunati, oltre alle sponde, si dispone di una fascia sufficiente per la fruizione ciclo-pedonale. In molte città italiane quindi, il sistema del verde ricalca il sistema delle acque (secondo quel modello di reticolarità di si è accennato).

Il caso di Pavia è un'esemplare applicazione in cui una pista ciclabile segue una roggia e collega più centri tramite un percorso studiato per offrire il godimento dei brani più belli del paesaggio agrario.

Gli stessi problemi di manutenzione e conservazione della rete irrigua si trovano, ampliando lo sguardo, per gli altri elementi del paesaggio agrario tradizionale, come ad esempio nei muretti di contenimento dei terrazzamenti: molti versanti montani e collinari, rimodellati dall'uomo, soffrono oggi per l'abbandono, creando anche situazioni di rischio idrogeologico. Il celebre paesaggio delle Cinque Terre è uno di questi luoghi, il Chianti un altro - paesaggi simbolo di quell'integrazione tra uomo e ambiente, di quella continuità tra passato e presente, per cui i paesaggi italiani sono noti in tutto il mondo. L'abbandono delle colture tradizionali pone seri problemi alla conservazione di questi paesaggi, poiché, nonostante ci si renda conto che il paesaggio agrario non può essere sottratto alle dinamiche produttive e sociali della società rurale per "museificarlo", nondimeno la sua conservazione sembra irrinunciabile, in quanto esso reca i simboli dell'identità storica del paese. La soluzione viene indicata nel concetto di sostenibilità.

\footnotetext{
${ }^{2}$ Definizione dell'Associazione Italiana Greenways, cit. da Michele Ercolini, in questo volume.

${ }^{3}$ Cfr. ad esempio Pompeo FabBri, Il Paesaggio fluviale, Guerini Studio, Milano 1986.

${ }^{4}$ La definizione di "suolo" contenuta nel primo articolo della legge è molto ampia: "il territorio, il suolo, il sottosuolo, gli abitati e le opere infrastrutturali”.
} 
Il tema è avvertito in tutto il vecchio continente e, infatti, il Consiglio d'Europa ha promosso diversi studi sulla sostenibilità del paesaggio agrario ed incentivi per il sostegno di politiche agricole che abbiano tra gli obiettivi anche la conservazione del paesaggio tradizionale (ad esempio la salvaguardia delle siepi). Essa sfugge alle possibilità d'intervento diretto di tipo pubblico ed è affidata invece alla manutenzione continua da parte dei proprietari. Poiché molte tradizioni stanno andando perdute, si moltiplicano in Europa le "guide per gli interventi" o i "manuali per i proprietari" (ad esempio in Inghilterra, redatte dalla Countryside Commission, o in Francia, ad opera delle varie municipalità).

Anche il programma per il Chianti elabora una "guida", corredata da indicazioni economiche, inserendosi perfettamente nella linea di ricerca sulla sostenibilità socio-economica delle politiche paesistiche e sulla partecipazione della popolazione alle stesse.

I tre esempi presentati non possono certo esaurire queste tematiche, delle quali abbiamo accennato l'importanza. Tuttavia ci sembrano sufficientemente indicativi dell'attenzione e dell'approccio integrato alla valorizzazione congiunta delle risorse del territorio, tramite l'individuazione di reti ambientali, reti fruitive, reti di beni storico-culturali, che nel complesso costituiscono la complessità del progetto di paesaggio. 



\title{
LA SCOPERTA DELLA PRESENZA DEI FIUMI IN CITÀ: TORINO CITTÀ D'ACQUE*
}

\author{
Claudia Cassatella
}

\section{IL CONTESTO}

Le Corbusier vide in Torino una delle migliori posizioni naturali al mondo. La città giace ai piedi delle Alpi, sulle rive del Fiume Po, il maggiore fiume italiano che qui riceve quattro affluenti (Dora Riparia, Stura, Sangone, Dora Baltea). "Il panorama di Torino si presenta, a chi lo osservi da uno dei tanti belvedere della vicina collina, estesissimo e tutto in giro cerchiato dall'immensa chiostra alpina. La città giace per la maggior parte sulla riva sinistra del Po; le sue vie sono quasi tutte rettifili perpendicolari o paralleli al fiume": così la guida del Touring Club Italiano nel 1930, che apre l'illustrazione della città con quattro immagini dei "Murazzi", gli argini ottocenteschi, un tempo ricovero delle barche ed oggi luogo di vita notturna.

I fiumi di Torino hanno avuto un ruolo difensivo, produttivo e di svago fino agli anni Cinquanta del secolo scorso, quando sono stati costretti dall'espansione urbana a scorrere tra argini, fabbriche e case, scomparendo alla vista. Ciò ha causato fenomeni di degrado ambientale, ma anche l'indebolimento del ruolo simbolico dei fiumi nell'immaginario collettivo e nella vita della città. Ad esempio, fino al secondo dopoguerra esistevano persino stabilimenti balneari sulle rive dei fiumi, mentre oggi i punti in cui ci si può avvicinare all'acqua sono pochi. Con il motto "Riqualificare le sponde, riconquistare i fiumi" la Città di Torino ha inteso avviare un progetto-programma per la realizzazione di un sistema di parchi fluviali che crei connessioni ecologiche e fruitive tra Po, Dora Riparia, Stura e Sangone.

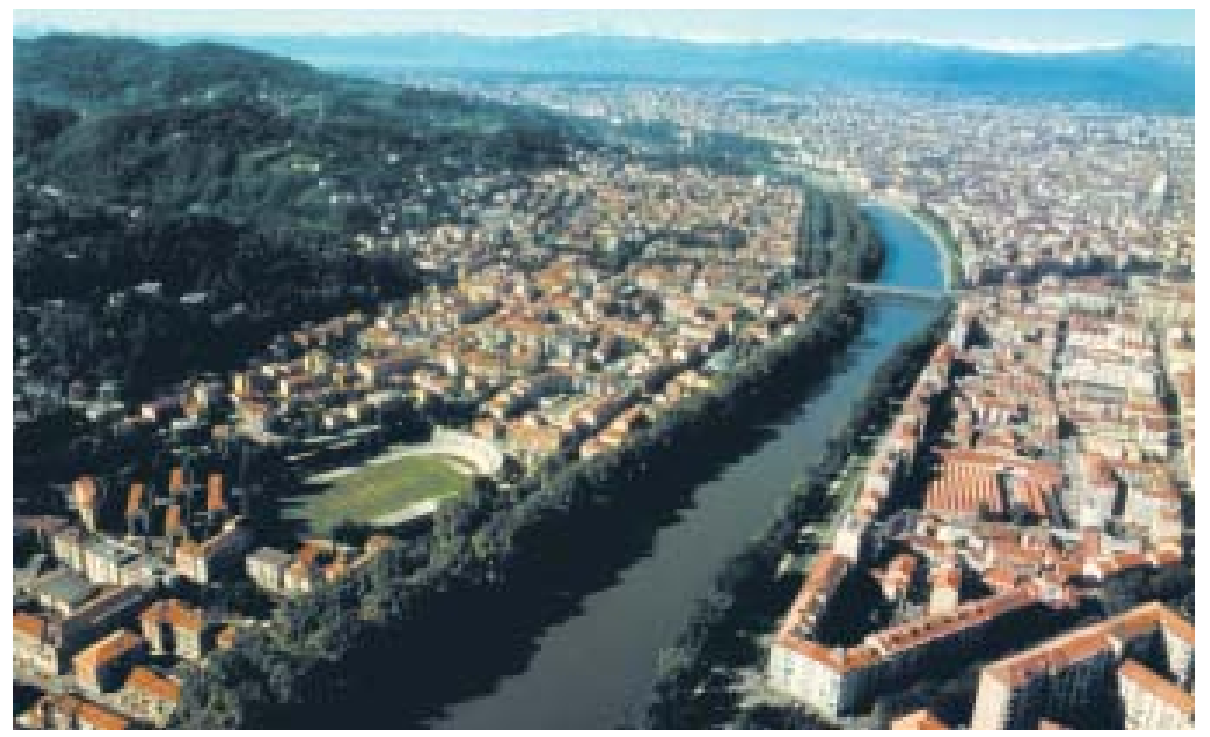

Figura 1 - Il Po nel tratto torinese, sullo sfondo l'arco alpino.

* Ringraziamenti: l'autrice desidera ringraziare il Settore Verde Pubblico della Città di Torino per la disponibilità e cortesia usata. 


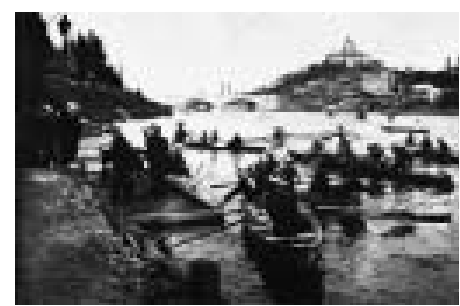

Figura 2 - Un gruppo di canottieri sul Po nell'Ottocento.

\section{Storia dei fiumi e della città}

"Il Po, il maggior fiume d'Italia (632 chilometri dalle sorgenti del Monviso alla foce in Adriatico) era il fiume della pesca e delle feste, via di comunicazione delle valli alpine con il capoluogo. Il Sangone fu a lungo il fiume del riposo e degli orti e venne a lungo utilizzato come luogo di svago. Praticamente fino alle soglie del secolo scorso. La Dora era il fiume della produzione: l'acqua, grande e quasi unica, forza motrice prima del vapore e del gasolio. La Stura restò a lungo "fuori le mura", luogo ancora naturale, ma per molti secoli troppo lontano dalle fortificazioni che cingevano la città per avere un uso concreto" 1 .

Il Po è sempre stato oggetto di navigazione a fini ricreativi: sull'acqua si svolgevano feste regali e popolari già nel XVII secolo. Sulle sue sponde fu costruito il Castello del Valentino (XVI sec.) come dimora di piacere, poi affiancato dall'Orto Botanico (1729), sull'altra sponda fu aperto lo Zoo (dal 1955 al 1993). Il Parco del Valentino ospitò varie esposizioni nazionali ed internazionali tra Ottocento e Novecento arricchendosi di padiglioni eclettici. Nell'Ottocento nacquero circoli remieri che vantano un'attività ininterrotta fino ad oggi. Il fiume era navigabile e, dal 1926 al 1930, un idrovolante partiva dal Valentino per Milano, mentre oggi si effettua solo un breve tratto di navigazione turistica. Le celebrazioni per il centenario dell'Unità d'Italia (1961) portarono nuove sistemazioni del lungofiume ed episodi di architettura moderna, quali gli edifici per esposizioni progettati da Pier Luigi Nervi.

La Dora invece è stata fin dal Settecento il luogo di insediamento delle attività manifatturiere, fuori dalla cinta urbana, fino a venir canalizzata a metà del secolo scorso e persino coperta in un tratto che è tornato alla luce solo un anno fa, grazie ad un programma di trasformazione di un'area industriale dismessa.

Il Sangone è un torrente che ha sempre rifornito la città di acqua proveniente dalle valli alpine: non a caso proprio qui fu costruito il primo acquedotto cittadino nel 1859. Nei pressi sorgeva un'altra delle dimore sabaude, il Castello di Miraflores (XVI sec.). Fino al 1960, quando è stato raggiunto dall'urbanizzazione, il fiume è stato la meta di gite fuori porta (vi sorgevano veri e propri "Lidi") ed il luogo degli orti urbani (recentemente oggetto di un programma di riordino).

Il torrente Stura, infine, ha conservato anch'esso il carattere di "fuori porta" e presenta tratti di una certa naturalità, seppur inselvatichita. Costituisce un corridoio ecologico fondamentale verso il Parco della Mandria (sede di un'altra dimora sabauda) e le Valli di Lanzo, ed è particolarmente ricco di avifauna (la garzaia dell'Isolone di Bertolla è l'unico caso europeo, con Amsterdam, di garzaia in contesto urbano).

\section{La varietà di situazioni ambientali e fruitive}

Lo stato di fatto presenta dunque una grande varietà di situazioni e valenze storiche, naturalistiche, paesaggistiche, fruitive. Sono presenti tratti canalizzati e

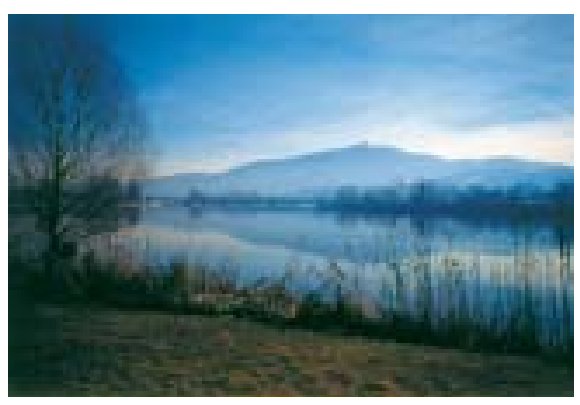

Figura 3 - L'area della Confluenza tra Po e Stura.

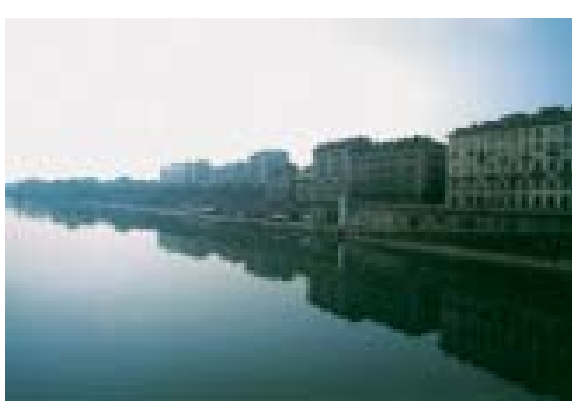

Figura 4 - I Murazzi del Po ne costituiscono il tratto più urbano.

${ }^{1}$ Gianni Boscolo, I fiumi, in Città di Torino, Torino Città d'acque, 2001, cd-rom. 


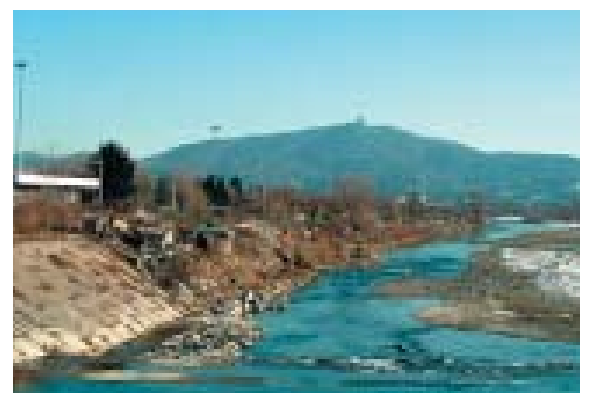

Figura 5 - Un tratto di sponda di Stura occupato da orti abusivi e in attesa di riqualificazione.

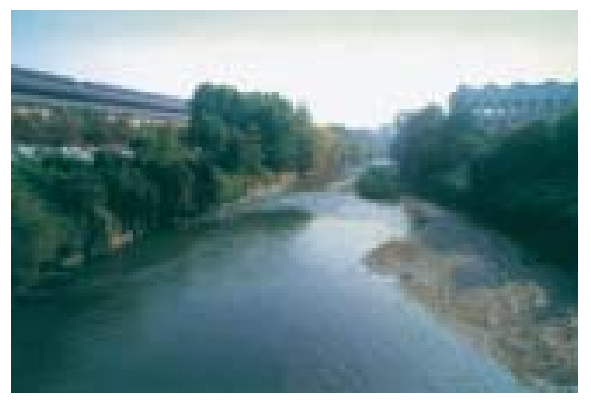

Figura 6 - Un tratto di Dora stretto tra le fabbriche; la dismissione di questi stabilimenti consentirà il recupero ambientale delle sponde e la creazione di un parco.

tratti naturaliformi (ad esempio i Laghetti Falchera). Si riscontrano aree degradate, occupate abusivamente da orti urbani e campi nomadi, da discariche e persino da attività produttive, tanto che uno dei maggiori impegni finanziari del progetto è l'esproprio di queste aree, che talvolta necessitano di bonifiche chimico-fisiche (ad esempio l'area Basse di Stura); lungo il torrente Stura si estende inoltre l'area della discarica di rifiuti urbani torinese. Sulle sponde dei fiumi si trovano le dimore extraurbane dei Savoia (che costituiscono la cosiddetta "Corona di delizie"), episodi urbani come il sistema Piazza Vittorio Veneto - Chiesa della Gran Madre di Dio, ponti ottocenteschi in muratura e in ferro, lungofiume edificati come i Murazzi (che costituiscono un polo di grande vivacità urbana), parchi storici come il Valentino, grandi parchi attrezzati di epoca moderna.

Non mancano le possibilità di fruizione: navigazione turistica, canottaggio, piste ciclabili, aree gioco; ma esistono anche aree naturalistiche in cui la fruizione è scoraggiata. Uno degli slogan iniziali del progetto Città d'acque proponeva di recuperare persino la balneabilità del fiume (il nuovo impianto di depurazione è già in opera). Ma la fruizione è avvenuta finora "per punti" piuttosto che per reti, legata anche ad una percezione frammentaria della presenza dei fiumi in città: i cittadini conoscono il tratto del Po in prossimità del centro storico, ma difficilmente nelle loro mappe mentali sanno collegarlo agli altri fiumi. Soprattutto manca, per lunghi tratti, la possibilità concreta di percorrere le sponde.

\section{GLI OBIETTIVI}

\section{Il sistema del verde: il contesto territoriale e la progettualità in corso}

L'attenzione per i fiumi come sistema nacque già negli anni Settanta, concretizzandosi nel "Rapporto preliminare di studi sul sistema del verde" (1983) finalizzato alla revisione del PRG, che introduceva, insieme alla proposta di un "Anello Verde"2 collinare, quella di un "Verde Azzurro" fluviale. Gabriele Bovo, coordinatore del progetto per il Settore Verde Pubblico Nuove Opere, illustra la nascita e l'inquadramento urbanistico di Città d'acque: "Nel dicembre 1993 la Giunta comunale $(. .$.$) deliberò l'attuazione di tale progetto-programma che si poneva$ come obiettivo la pianificazione e la realizzazione di interventi di riqualificazione e risanamento ambientale delle fasce verdi spondali dei fiumi cittadini per la realizzazione di un unico sistema continuo di parchi fluviali (esteso su $37 \mathrm{~km}$ di alveo e quindi $74 \mathrm{~km}$ di sponde) collegati da reti di percorsi pedonali, ciclabili, naturalistici e didattici, in cui fossero evidenziate e tutelate per ogni corso d'acqua le

\footnotetext{
${ }^{2}$ L'Anello Verde costituisce una rete di 30 chilometri di percorsi nel parco della collina torinese (che si stende tra Po e Monferrato), anch'esso in fase di realizzazione e collegato al lungo Po.
} 


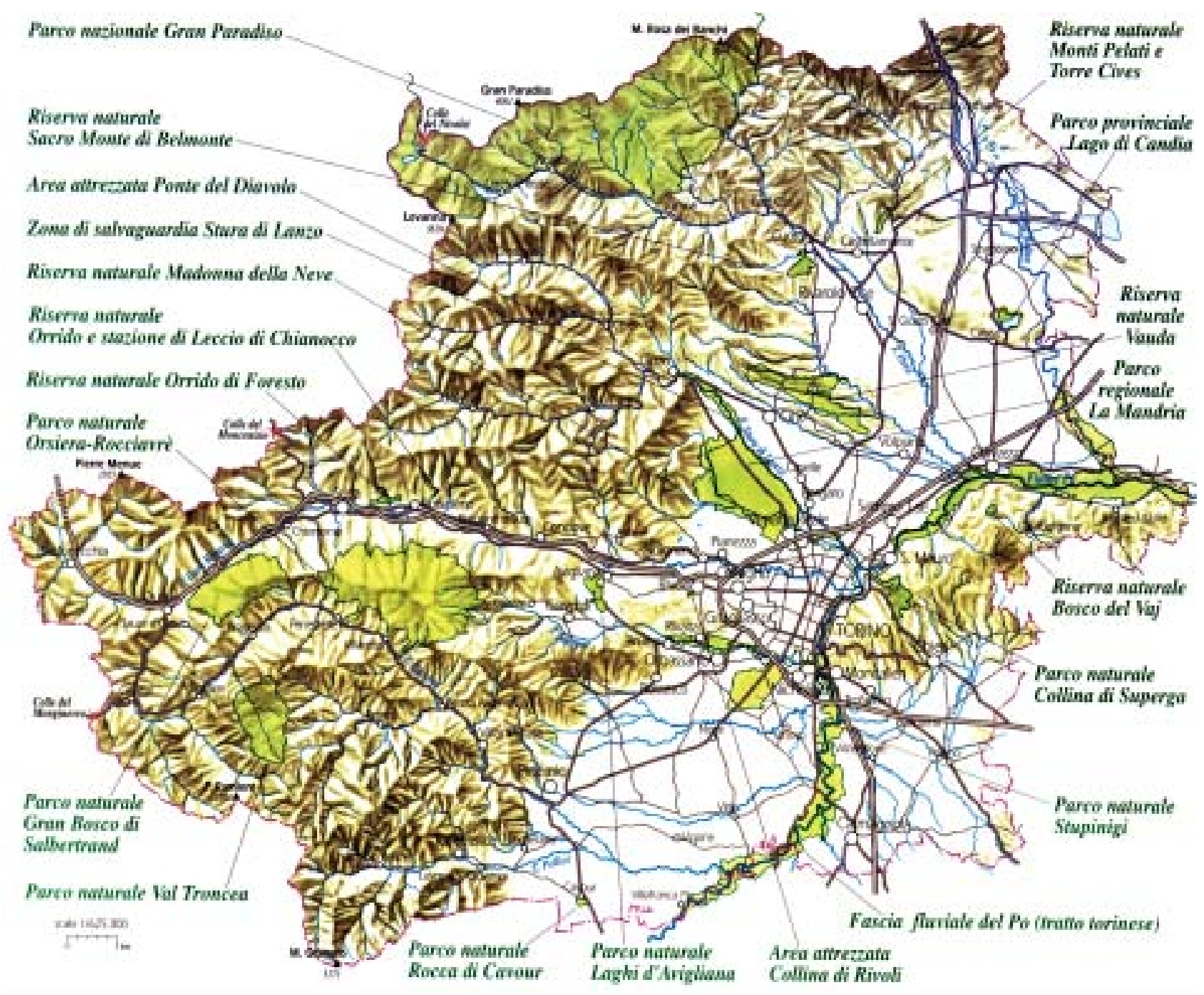

Figura 7 - I corsi d'acqua e le aree protette della provincia di Torino.

rispettive peculiarità naturalistiche e faunistiche, le emergenze architettoniche, i caratteri percettivi e descrittivi della loro storia, intervenendo su aree inaccessibili o altamente degradate o più semplicemente da riqualificare. (...) Nello stesso periodo un ulteriore elemento propulsivo venne dall'inserimento del progetto nel Programma Regionale denominato "Corona Verde" varato con l'obiettivo di completare e collegare in modo organico il sistema delle aree protette regionali esistenti intorno al capoluogo piemontese che comprendono antiche proprietà sabaude (La Mandria, Stupinigi, Vallere, Superga), parchi ed aree attrezzate (Ponte del Diavolo, Laghi di Avigliana, Collina di Rivoli), riserve naturali e zone di salvaguardia (Vauda, Bosco del Vaj, Madonna della neve-Monte Lera, Stura di Lanzo).

Il sistema verde di Torino Città d'acque avrà quindi in tal senso lo scopo di costituire una fascia di transizione fra i parchi cittadini dell'area urbana centrale e l'ambiente meno antropizzato e progressivamente naturaliforme dei parchi estensivi collinari e fluviali periferici (Sangone e Stura), sino ai parchi regionali della fascia periurbana che a loro volta costituiranno la corona verde di collegamento verso il contesto agricolo e forestale delle valli pedemontane.

L'obiettivo finale, sarà quindi la realizzazione di una omogenea e continua struttura verde di connessione fra gli ambiti delle valli alpine a nord e del sistema collinare dell'alto Monferrato a sud, comprendente sottosistemi di collegamento e fruizione antropica come ciclopiste, percorsi ginnici, percorsi didattici, strut- 

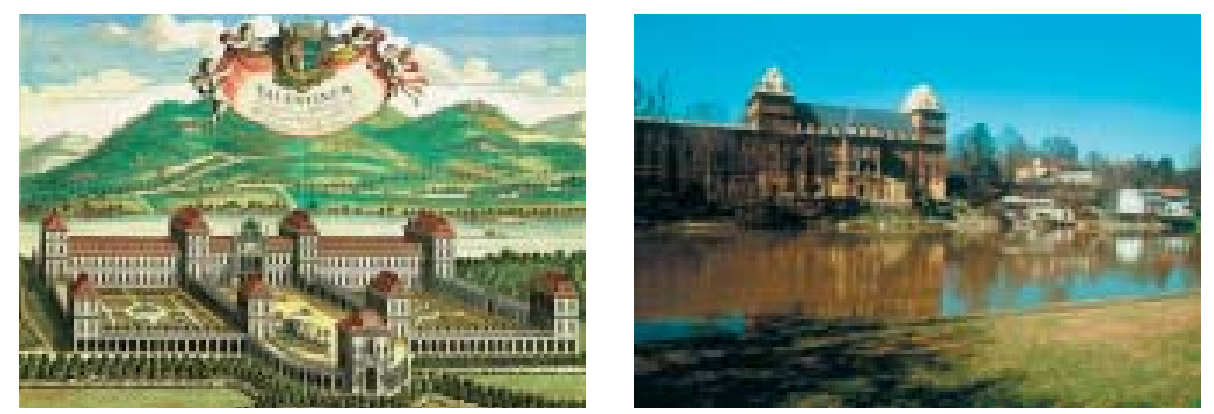

Figure 8-9 - Il Castello del Valentino, una delle residenze reali di casa Savoia, nel progetto di Castellamonte pubblicato nel Theatrum sabaudiae (Blaeu, Amsterdam, 1682), e in una veduta attuale.

ture di ricezione turistica e vaste aree di tutela naturalistica e faunistica collegate con un sistema di corridoi ecologici"'.

I progetti "Corona di delizie", "Anello Verde" e "Torino Città d'acque" trovano dunque una sintesi ed integrazione nel progetto regionale di "Corona Verde"4: questa integrazione, che si esplica in connessioni ecologiche e funzionali, rappresenta un'interessante caso di integrazione tra livelli di progettualità a scale diverse ed a livelli amministrativi differenti.

"Città d'acque" persegue dunque obiettivi a due livelli, quello urbano e quello territoriale.

\section{LA METODOLOGIA}

Gli studi di supporto (in particolare la modellizzazione ecologica ed il Piano Faunistico dei Fiumi Torinesi) hanno mostrato la potenzialità di costituzione di corridoi ecologici con riferimento al contesto metropolitano ed addirittura regionale. Altri studi che interessano l'area sono: il Piano d'Area dell'Ente Parco del Po tratto Torinese, il PRG Città di Torino, il Progetto stralcio della fascia fluviale del Po dell'Autorità di Bacino, il Progetto Speciale Periferie.

Nel 1998 il Settore Verde Pubblico ha enucleato un Settore Nuove Opere per dare attuazione al progetto. Nel 1999 gli architetti Maurizio Cilli e Maurizio Zucca hanno redatto un Masterplan che costituisce lo sfondo di riferimento per tutti gli interventi, individuando ambiti caratterizzati da diverse valenze ecologiche, paesaggistiche, funzionali, di cui sono presi in considerazione, ad esempio, tipologia di area verde, classificazione P.R.G., uso, tipo di sponda e di alveo, attività sportivo-ricreative, attrezzature per la navigazione, visuali, edifici caratterizzanti, accessibilità automobilistica.

I principali tipi di intervento riguardano la bonifica delle fasce spondali, l'incremento dei bacini di esondazione ed il consolidamento degli argini ${ }^{5}$, l'acquisizione delle aree di interesse, il potenziamento della navigazione turistica.

La progettazione di ciascun ambito ha seguito la procedura ordinaria per l'affidamento e l'esecuzione, perciò sono moltissimi i progettisti, i consulenti e di

\footnotetext{
${ }^{3}$ Gabriele Bovo, Inquadramento urbanistico, in Città di Torino, Torino Città d'acque, 2001, cd-rom.

${ }^{4}$ Il progetto Corona Verde ripensa ed evoca concettualmente l'organizzazione del territorio frutto del volere dei duchi e dei re sabaudi tra Seicento e primo Settecento: un insieme di residenze a corona della città capitale che l'architetto Amedeo di Castellamonte definì negli anni settanta del Seicento "Corona di Delitie", intendendo con tale metafora l'idea di un sistema di maisons de plaisance funzionalmente connesse attraverso un' articolata rete stradale e una vasta estensione di giardini, rotte di caccia e territori destinati alla produzione agricola. Di questo articolato sistema oggi permangono la reggia di Venaria Reale e La Mandria, la Villa della Regina, il Castello del Valentino e quello di Moncalieri, la Palazzina di Caccia di Stupinigi, il Castello di Rivoli.

${ }^{5}$ Negli ultimi anni alcuni eventi alluvionali hanno colpito gravemente la città (l'ultimo, drammatico, nel 1999), mostrando la fragilità della situazione idrogeologica a monte, ma anche la necessità di maggiore cura degli alvei nel tratto cittadino.
} 


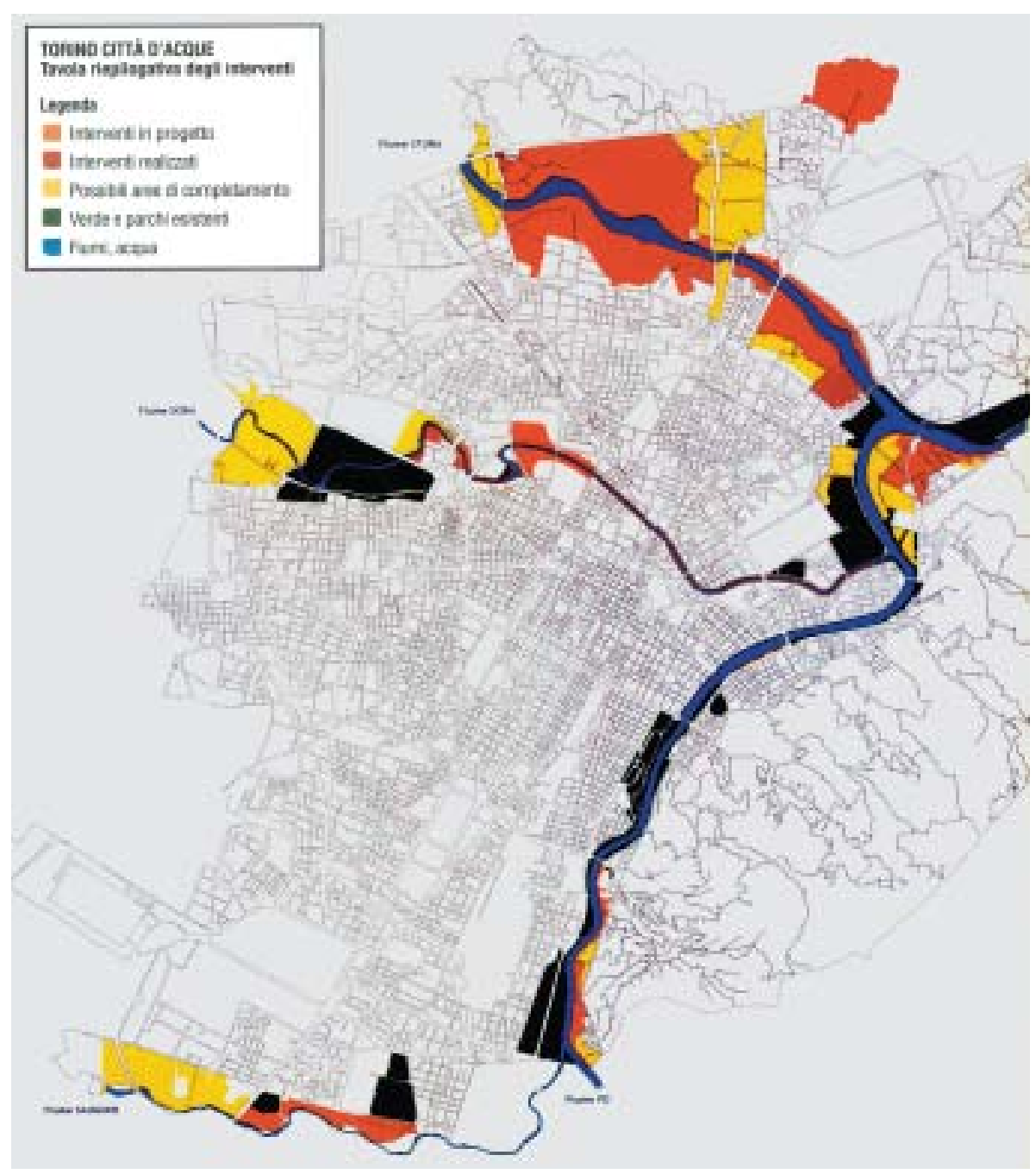

Figura 10 - Città di Torino, schema riassuntivo del progetto Città d'acque.

conseguenza gli approcci progettuali. In generale valgono gli indirizzi dettati dalla Città: utilizzo di tecniche di ingegneria naturalistica, gestione naturalistica della flora e della fauna, preferenza per materiali naturali come il legname, gli stabilizzanti stradali senza bitumi, le piante autoctone.

Aspetti particolari riguardano il coinvolgimento di associazioni naturalistiche nella gestione delle aree (sull'esempio della Pro Natura Torino per i sentieri collinari), la sinergia con altri progetti speciali della città, quali il Progetto Periferie, ed altre attività volte nella direzione di incentivare la partecipazione della cittadinanza.

La città di Torino si estende per 1.300 ettari e possiede 16.000 .000 metri quadrati di aree verdi pubbliche, raggiungendo uno standard di $17 \mathrm{mq} / \mathrm{abitante}$ (grandemente incrementato dagli anni Settanta, quando raggiungeva appena i $3,6 \mathrm{mq}$ per abitante). Con questo progetto acquisirà nuove aree verdi per 8.300.000 metri quadrati, che si aggiungeranno ai 4.200 .000 metri quadrati di aree esistenti lungo i 74 chilometri di sponde.

\section{GLI ESITI}

\section{Il futuro: unidentità di città fluviale?}

Le realizzazioni sono in corso. I recenti eventi alluvionali hanno imposto di dare priorità agli interventi sulle sponde e di fare cambiamenti anche importanti 
ai progetti che, a causa della legge italiana che regola le opere pubbliche (L. 109/1994), hanno dovuto talvolta ripercorrere l'iter approvativo. Il risanamento ambientale di molte aree è in corso, dalla bonifica delle acque e dei terreni, agli interventi sul verde. Sono già stati realizzati alcuni chilometri di nuovi percorsi ciclabili ed il ripristino di alcuni approdi (ricorrendo alla riprogettazione in stile per gli attracchi storici); al restauro dei ponti storici si aggiungerà un nuovo attraversamento pedonale; ovunque è stato arricchito l'arredo urbano con modelli tipici della Città. Se questi interventi soffrono di una certa standardizzazione dal punto di vista architettonico (proprio per la ripetitività di certi elementi), cui forse sopperisce la caratterizzazione dei siti (sia naturale sia urbana), è indubbio che anche i pochi interventi giunti a compimento hanno consentito l'accesso dei cittadini alle sponde, modificando il rapporto della città con i propri fiumi - e si è in dubbio se parlare di una "riscoperta" o di una vera e propria "invenzione" di Torino come "città fluviale". La presenza fisica di ben quattro fiumi non è sufficiente, infatti, a definirla tale, finché manchi la consapevolezza collettiva di questa risorsa. Il Po è forse per Torino ciò che è il Tevere per Roma, l'Arno per Firenze. Forse, ma gli altri fiumi? La Città si sta impegnando molto nella comunicazione degli obiettivi di questo progetto, che è vasto e ricco di interrelazioni con altri territori ed altri agenti. La sensibilità ambientale sta crescendo anche grazie alle immagini paesistiche che, finalmente, i fiumi riescono ad offrire.

\section{RIFERIMENTI BIBLIOGRAFICI}

Atlante dei parchi, suppl. n. 1 a "Piemonte Parchi", 6, Regione Piemonte, Torino 2001.

Bovo Gabriele (a cura di), Un fiume di verde, "Acer", 3, Il Verde Editoriale, Milano 2000, pagg. 78-84.

CitTÀ di Torino, Assessorato Per l'Ambiente, Il sistema del verde urbano. Prime proposte d'intervento lungo le sponde del Po e nella collina di Torino, doc. 1, 1981.

CitTÀ Di TORINO, Torino Città d'acque, cd-rom, 2001.

Corona Verde. Torino Città d'acque, "Folia speciale", suppl. al n. 6 di "Acer", Il Verde Editoriale, Milano 2001.

PARCHI VERSO IL 2000, suppl. n.1 a "Piemonte Parchi”, 2(78), Regione Piemonte, Torino 1998.

ToRINo PIEMONTE. Parchi e giardini, suppl. n.1 a "Piemonte Parchi”, 7, Regione Piemonte, Torino 2000.

Touring Club Italiano, Piemonte. Attraverso l'Italia, vol. I, T.C.I., Milano 1930.

Siti web: <www.comune.torino.it/ambiente/verde/index.htm>

\section{RIFERIMENTI ICONOGRAFICI}

Figura 1: per gentile concessione della Città di Torino, Settore Turismo.

Figura 2: Il volto di Torino 1880-1915, La Stampa, Torino 1987, pag. 137; per gentile concessione dell'Archivio Luciana Gawronska Frassati, Torino.

Figure 3, 5, 11: Foto Ezio Demagistris; per gentile concessione dell'autore.

Figure 4, 6: Foto Maurizio Stabio; per gentile concessione della Città di Torino, Settore Verde Pubblico Nuove Opere.

Figura 7: Atlante dei parchi, suppl. n. 1 a "Piemonte Parchi”, 6, Regione Piemonte, Torino 2001, pag. 20; per gentile concessione della Regione Piemonte, Settore Pianificazione Aree Protette.

Figura 8: Torino, Il Castello del Valentino (I, 28) Incisione anonima su disegno (1668) di Giovanni Tommaso Borgonio, Theatrum Sabaudiae, Blaeu, Amsterdam, 1682; per gentile concessione dell'Archivio Storico della Città di Torino (è vietata la riproduzione o duplicazione con qualsiasi mezzo).

Figura 9: Foto Gianni Boscolo; per gentile concessione del Centro di Documentazione e Ricerca sulle Aree Protette, Regione Piemonte, Settore Pianificazione Aree Protette.

Figura 10: per gentile concessione della Città di Torino, Settore Verde Pubblico Nuove Opere.

Figura 12: fotografia di Claudia Cassatella. 
SCHEDA DI PROGETTO

Progetto:

Tipo di intervento:

Luogo:

Committente:

Progettisti:

Iter del progetto:

Dati dimensionali:

Costi di realizzazione:

Ambiti di progetto:

Elementi vegetali utilizzati:

Materiali per consolidamenti:

Materiali per pavimentazioni:

Arredo urbano: progetto programma "Torino Città d'acque"

creazione parco fluviale e riqualificazione aree spondali e parchi esistenti

Torino

Città di Torino

Settore Verde Pubblico Nuove Opere (responsabile procedimento: Dott. Oscar Peano, coordinamento ed esecuzione: Dott. Gabriele Bovo)

Masterplan degli architetti Maurizio Cilli e Maurizio Zucca (1999)

progetti particolareggiati di autori vari

1983, rapporto preliminare di studi sul sistema del verde (1994 approvazione revisione PRG)

1993, delibera comunale del progetto programma di sistema continuo di parchi fluviali

1999, masterplan

dal 1998 in corso, progetti particolareggiati e realizzazioni grado di progettazione/realizzazione variabile a seconda degli ambiti

lunghezza sponde $74 \mathrm{~km}$

superficie alvei fluviali $3.300 .000 \mathrm{mq}$

superficie aree verdi esistenti $4.200 .000 \mathrm{mq}$

nuove aree verdi in progetto $8.300 .000 \mathrm{mq}$

aree verdi a progetto terminato $15.800 .000 \mathrm{mq}$

totale verde pubblico comunale esistente $16.000 .000 \mathrm{mq}, 17 \mathrm{mq} / \mathrm{ab}$

stima sommaria degli investimenti ad oggi, 64 miliardi di lire (€ 33.258.000), in previsione altri 70 miliardi di lire

fiume Po (Parco del Meisino, Zona Fioccardo, Attracchi fluviali, Sponda Murazzi Colletta, Parco dello Zoo); fiume Dora Riparia (Parco fluviale di via Calabria, Sponde tra i corsi Umbria e Potenza, Parco della Spina 3); torrente Stura di Lanzo (Parco Stura, Parco dell'Arrivore, Parco della Stura Nord e Sud, Laghetti Falchera); torrente Sangone (Parco Sangone)

arbusti ed alberi autoctoni, in particolare specie ripariali e planiziali

scogliere a masi ciclodici vincolati rinverdite con talee di salice, palificate in legno a parete singola rinverdite con talee di salice e nocciolo, biostuoie antierosione, coperture diffuse in talee di salice, canalette in legname e pietrame

vari, in particolare stabilizzati non bituminosi per i percorsi ciclabili

panche, cestini, pali di illuminazione e fontane modello Città di Torino (anche modelli storici), ed altri; giochi bimbi, attrezzature sportive, WC, casette e tettoie per orti urbani 


\title{
LA GREENWAY DELLA BATTAGLIA DI PAVIA
}

\author{
Michele Ercolini
}

\section{IL CONTESTO}

"Il termine greenway deriva dall'unione di greenbelt e parkway ed è dunque legato al concetto di collegamento tra le aree verdi, assumendo un significato leggermente diverso negli Stati Uniti, dove è nato negli anni Sessanta e dove assume il ruolo prevalente di area verde lineare, ed in Europa dove richiama principalmente l'idea di percorso ${ }^{1 "}$ ".

Nate principalmente per garantire il collegamento e la continuità delle società vegetali ed animali, le greenways vengono oggi considerate dei veri e propri "sistemi di territori lineari tra loro connessi, protetti e sviluppati in modo da ottenere benefici di tipo ecologico, ricreativo, storico-culturale ${ }^{2 "}$, capaci di realizzare una rete viaria alle diverse scale, locale, regionale, nazionale, europea e di perseguire molteplici finalità, sfruttando le caratteristiche dei territori ed adattandosi alla morfologia dei luoghi.

Le greenways dunque, "corridoi multiscopo di sistemi ecologici, culturali, storico-ricreativi, turistici ${ }^{1}$ ", si prefiggono, in primis, l'obiettivo di evitare l'imposizione di zone verdi "artificiali", puntando altresì alla tutela, al recupero e soprattutto alla rivalorizzazione delle risorse ambientali, naturali e paesistiche esistenti: fiumi, sentieri, zone collinari, aree montuose, eccetera.

Il progetto in esame si sviluppa nelle quattro principali realtà territoriali pavesi:

- la realtà agricola, con campi di mais, riso, soia, divisi da una rete di canali e rogge di origine storica;

- la realtà "urbana-agricola", segnata dalla presenza di terreni incolti e da una forte e spesso "disordinata" e "disorientata" urbanizzazione;

- la realtà urbana consolidata, rappresentata da Pavia, città ricca di acqua, storia, spazi verdi;

- la realtà fluviale del Ticino e degli altri corsi d'acqua, con i loro meandri, le zone umide, i manufatti di interesse idrico-storico, eccetera.

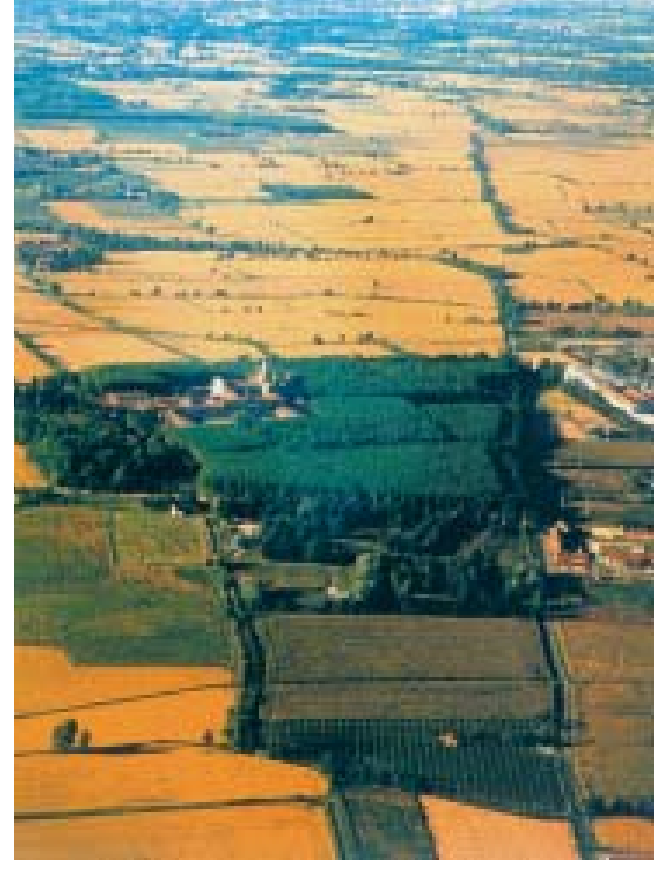

Figura 1 - Veduta aerea della Certosa.

Ma il contesto entro cui deve essere inquadrato l'intervento è certamente di carattere storico-culturale; infatti, il progetto in questione vuole ripercorrere ma soprattutto reinterpretare un evento risalente al 1525 tra i più significativi della storia europea: la Battaglia di Pavia. 
Questo conflitto, che vide confrontarsi da una parte l'imperatore Re di Spagna, Carlo V d'Asburgo, e dall'altra il Re di Francia, Francesco I, pose fine alle ostilità politiche e militari tra questi due paesi, stabilendo con la vittoria di Carlo V, la supremazia della Spagna in Italia. Non a caso, l'area dell'itinerario principale coincide proprio con i luoghi più significativi della battaglia, ossia la zona del Parco urbano della Roggia Vernavola e quella del Parco di Gian Galeazzo Visconti, inserendosi all'interno di un paesaggio storico-agrario regolare, armonioso, in cui risulta ancora ben evidente l'impronta risalente al periodo romano. Ritroviamo infatti, l'assoluta regolarità della divisione dei campi, la perfetta ortogonalità delle centuriazioni e, ancora, la rete viaria dell'epoca, rappresentata dalla strada di origine romana che collegava Mediolanum a Ticinum.

Non si può però dimenticare che la greenway, nei suoi 19 chilometri di percorso, deve anche "confrontarsi" con la realtà ben differente della campagna immediatamente esterna ai confini della città, caratterizzata da un paesaggio rurale-urbano monotono, rigido ed uniforme.

\section{GLI OBIETTIVI}

L'obiettivo principale dell'intero progetto è rappresentato essenzialmente dalla volontà di garantire, oltre alla piena accessibilità al percorso, un certo livello di continuità, sia per motivi di flusso antropico che ecologico. Un obiettivo però non facile da raggiungere a causa dell'elevato grado di frammentazione del territorio pavese, conseguente alle infrastrutture stradali, alle strozzature dovute all'edificato ed a veri e propri sbarramenti causati da orti urbani ed occupazioni abusive.

Nel progettare il parco lineare si è cercato quindi di trovare "delle soluzioni di compromesso, aggirando aree impossibili, attraversando e riattraversando la Roggia, restringendo il sentiero al minimo (fino a 1,5 metri di larghezza), proponendo ponti, sottopassaggi, sovrappassi, attraversamenti con semafori, eccetera ${ }^{2 "}$.

Tra le altre molteplici finalità, ricordiamo:

- la volontà di saldare le due porzioni di territorio del Parco Urbano della Vernavola, prossimamente interessate dalla frattura causata dalla costruzione della tangenziale Nord;

- valorizzare l'intero sistema idrico-storico delle rogge e dei canali, dei navigli e dei fontanili;

- garantire, in particolare, la protezione delle sponde delle rogge e arricchirne la vegetazione oggi impoverita da pratiche colturali intensive;

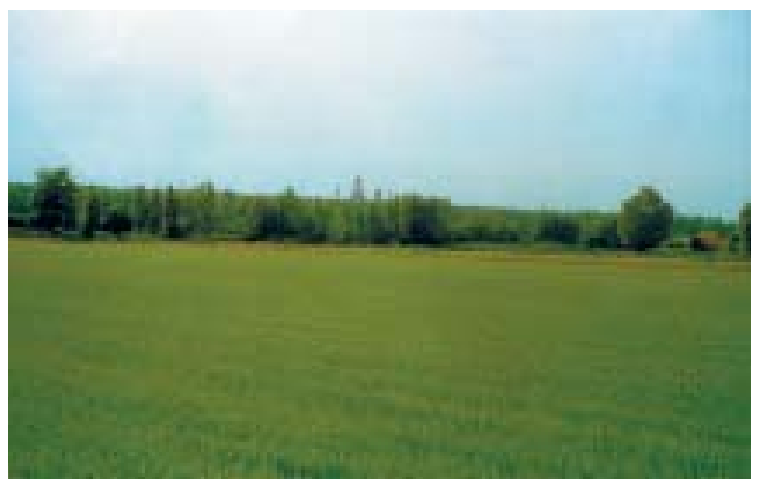

Figura 2 - La città di Pavia vista dalla Greenway.

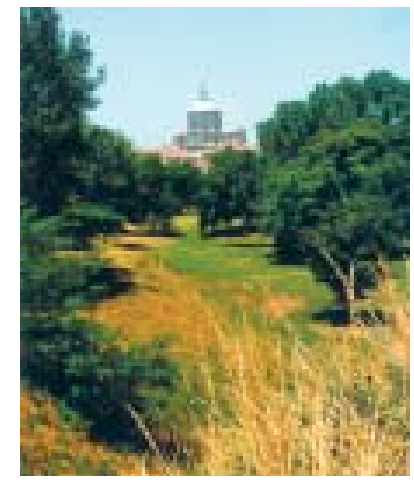

Figura 3 - La Certosa dalla Greenway.

\footnotetext{
${ }^{2}$ Definizione dell'Associazione Italiana Greenways.
} 
- riorganizzare il paesaggio agricolo, attraverso infrastrutture lineari e di sosta che colleghino con una rete accessibile ai fruitori (residenti, turisti), gli elementi di elevato pregio ambientale-storico-culturale con le realtà agricolerurali, anche ai fini di una fruizione agrituristica;

- garantire una migliore fruibilità del sistema di ciclomobilità, migliorando l'integrazione tra le vie ciclabili e le aree verdi;

- avviare un'azione di tutela e riqualificazione degli ambiti di rilevanza ecologica, tentando di diversificare il più possibile la struttura e la composizione della vegetazione in relazione alle peculiarità di ogni sito (biotopi, ecotopi, zone di transizione, eccetera);

- attuare una ricostruzione ambientale per evidenziare l'evoluzione storica dell'uso del suolo;

- garantire una valorizzazione delle risorse naturalistiche esistenti, attraverso il mantenimento del patrimonio vegetazionale delle aree private e l'impianto di siepi, macchie di bosco, filari;

- ricostruire la vegetazione potenziale (Carpino Quercetum roboris, Carpinion e Alno Union) sfruttando una vasta varietà di specie arbustive ed arboree;

- ricostruire la vegetazione spondale utilizzando un'elevata densità di impianto;

- dare vita infine, ad una serie di iniziative e servizi per attrarre i consistenti flussi turistici che arrivano alla Certosa, nella città di Pavia.

\section{LA METODOLOGIA}

Il percorso metodologico della greenway di Pavia si fonda principalmente sullo studio (analisi-sintesi-valutazione) delle risorse naturali-storiche e paesistiche del sito, attuato attraverso "il sistema di sovrapposizione dei dati e valutazione basata su criteri oggettivi tematici ${ }^{3}$." Sovrapponendo le singole mappe tematiche si è così arrivati a carte intermedie (mappa storica, naturalistica, idrico-agricola, eccetera), dalla cui interpretazione e valutazione sono scaturite le scelte progettuali.

La fase di analisi si è concentrata in particolar modo sullo studio delle risorse storico-paesistiche, cercando di valutare fino a che punto gli aspetti attuali del territorio pavese siano ricollegabili agli scenari dell'epoca cinquecentesca. Si sono studiati per tanto tutti i dati, i documenti, le carte riguardanti le coltivazioni, i confini, la rete viaria, gli aspetti vegetazionali, i manufatti storici, i percorsi d'acqua, relativi al periodo della Battaglia, per sottolineare eventuali aspetti di continuità o rilevanti modifiche.

Le carte realizzate per questa fase analitica hanno descritto l'evoluzione storica del territorio individuando tre momenti: il periodo precedente alla Battaglia, con la città circondata dalle antiche mura; il periodo del Settecento e dell'Ottocento, in cui si evidenzia soprattutto la trasformazione del paesaggio agricolo ed urbano, le zone di espansione e le tipologie delle colture; la situazione attuale, con l'individuazione dei siti di interesse storico, la città, il paesaggio, i collegamenti e il potenziale d'uso dell'area.

Si è inoltre valutato il sistema dei corsi d'acqua e delle zone umide rapportandolo agli aspetti vegetazionali dell'uso del suolo. A ciò ha fatto seguito la redazione di una carta delle risorse idriche con indicati: i vari tipi di corsi d'acqua esistenti, quelli naturali (come il Ticino, il Vernavola) e quelli artificiali (Roggia Grande, Colombara); i punti di interesse, come i manufatti idraulici di rilevanza storica e/o tecnologica, i fontanili; gli elementi detrattori, come le strozzature, le emissioni inquinanti, eccetera.

${ }^{3}$ Daniela Zandonella Necca e Katherina Ziman Scudo, La Greenway della Battaglia di Pavia dalla Certosa al Ticino, "Ambiente Costruito", 1, 2000, pag. 16. 
Tra le risorse analizzate ritroviamo ovviamente anche quelle naturali: per gli aspetti geologici si sono raccolte informazioni sui suoli e sottosuoli, finalizzate alla ricostruzione del processo di formazione del paesaggio e alla tutela delle aree di significante interesse fisiografico.

Per il sistema idrico superficiale si sono presi in considerazione i principali corsi d'acqua: la Roggia Vernavola, la Bareggia, la Sartirara, il Loggione, la Barona, il Cavo Boromeo, il Cavo Kewenkuller, la Grande, il Cavo Sessino, il Cavo Stampa, il Cavone, la Carona e la Barcheggiana. La componente idricosuperficiale è stata inoltre valutata sia rapportandola, come visto in precedenza, ai vari tipi d'uso del suolo, sia alle possibilità di movimento delle specie animali e dell'uomo; infine, sono state prese in considerazione anche le rilevanti valenze storiche legate agli aspetti tecnologici del sistema idrico.

Si è passati poi allo studio della componente vegetazionale con particolare riferimento al sistema delle ripe e alla circolazione delle specie, confrontando la realtà attuale con quella dell'epoca, allo scopo di ricavarne indicazioni e suggerimenti utili per una loro rivalorizzazione.

Per ciò che concerne invece gli aspetti relativi alla fauna, sono state individuate le specie animali di maggior rilevanza, al fine di ricavare elementi utili per la loro conservazione, tutela e per garantirne un buon livello di continuità nei collegamenti.

Nella metodologia progettuale dunque, emerge in maniera piuttosto evidente come "l'attenzione interpretativa dello svolgimento della Battaglia, abbia inteso precisare quanto gli aspetti naturali influirono sul suo corso. Se ne sono così ricavati suggerimenti per possibili interventi, inclusi i collegamenti fra siti particolarmente rilevanti, che permettano di meglio ripercorrere i luoghi della Battaglia a scopo sia didattico che turistico-ricreativo" ${ }^{4}$.

\section{GLI ESITI}

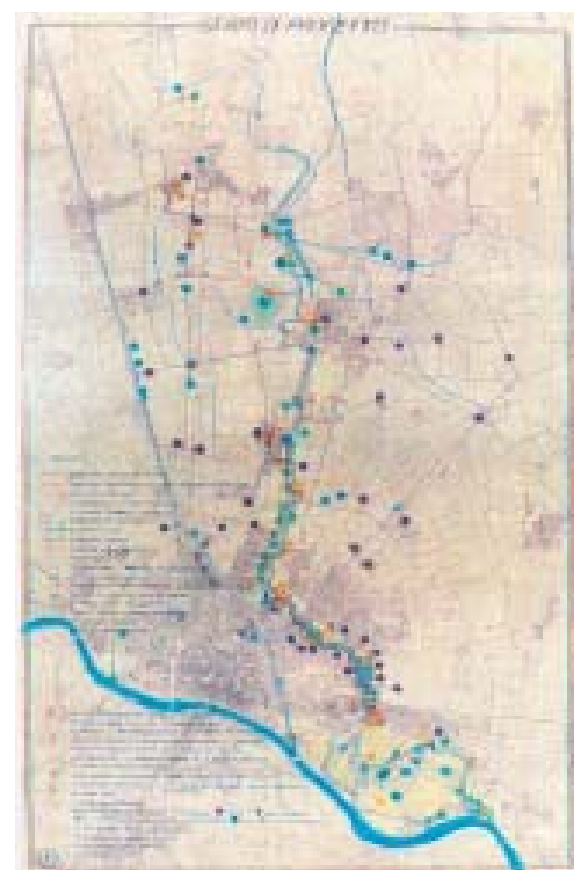

Figura 5 - Planimetria progetto definitivo.
Il progetto si è proposto di rivalutare questa porzione di territorio pavese partendo da esperienze simili di recupero del paesaggio culturale, sviluppatesi negli Stati Uniti (paese in cui sono nate le greenways).

Si è così arrivati a definire un percorso progettuale in grado di gestire la complessità delle principali componenti/risorse territoriali presenti nell'area: risorse naturali, culturali, agricole, paesistiche, eccetera. L'intervento nello specifico prevede, come ampiamente descritto in apertura, la realizzazione di un itinerario verde pedonale e ciclabile "interpretativo" degli avvenimenti storici (la Battaglia di Pavia), caratterizzanti il contesto esaminato.

Il sentiero principale segue quasi per intero il corso della Roggia Vernavola; questa scelta non è però casuale in quanto la Roggia già rappresenta un vero e proprio "sistema lineare-naturale" di collegamento tra la zona agricola, la città e il fiume Ticino, raggruppando tra l'altro al suo interno, le principali risorse ambientali-storiche del comprensorio.

Il percorso, che si snoda per una lunghezza pari a 19 chilometri in tratti di ampiezza variabile (dai 3 a 4 metri in ambito urbano fino ai 30-40 nelle aree "naturali"), realizza una vera e propria rete "di percorsi articolati gerarchicamente, individuando una maglia fondamentale ed una serie di alternative, anelli e circuiti complementari stretta- 
mente connessi, per una fruizione più capillare e tematica del territorio (le vie d'acqua e gli antichi manufatti, le vie romane, i luoghi del Parco Visconteo, le cascine, i mulini, ecc)"5. Si è cercato in particolar modo di sfruttare itinerari già esistenti, puntando al loro riconoscimento, recupero e/o adeguamento, evitando il più possibile la creazione di nuovi percorsi.

Si possono così individuare oltre a quello principale, tre tipologie di itinerari tematici: quello "naturalistico", che si muove all'interno delle aree di più alto valore idrico, botanico e faunistico; l'itinerario "storico" che crea una rete di collegamento tra i monumenti più significativi; e "l'itinerario della Battaglia", che traccia una rete di collegamento tra gli elementi di valore storico-culturale inerenti il conflitto del 1525, ripercorrendo per scopi turistico-didattico-ricreativi i luoghi più significativi della Battaglia.

Il tutto risulta sempre e comunque finalizzato a garantire una conoscenza più dettagliata e approfondita delle diverse risorse esaminate.

La greenway può essere poi ulteriormente suddivisa in quattro differenti unità geografiche. Il tratto della Valle del Ticino, che parte dalla confluenza tra il fiume Ticino e la Roggia Vernavola, per concludersi a S. Lazzaro; il tratto della Bassa Valle della Vernavola, compreso tra Viale Cremona e Via Vigentina; il tratto dell'Alta Valle Vernavola, tra la Torretta e il confine amministrativo di S. Genesio ed Uniti; infine, il tratto definito "Piano agricolo" che ha inizio dal confine amministrativo di S. Genesio ed Uniti per concludersi alla Certosa di Pavia.

Attualmente è in via di approvazione il progetto definitivo ed esecutivo relativo al "Tratto C" dell'Alta Valle Vernavola. La scelta di questo itinerario e non di altri è dovuta sia all'ormai imminente costruzione della Tangenziale Nord, che necessita di un collegamento e di un'opera di mitigazione ambientale, sia alla già accertata disponibilità delle aree interessate dall'intervento.

La greenway di Pavia dunque, può essere considerata un vero e proprio "filo conduttore verde, che lega la città ai campi e alla natura, unendo i vari sistemi e offrendo occasioni di riassetto generale" 8 .

Sotto questo profilo, la realizzazione della via verde pavese rappresenta certamente qualcosa di "inedito" e di qualità superiore ad ogni altra fino ad oggi progettata in Italia.

Non a caso, si è parlato sempre di "itinerario" e non di mera "pista ciclabile", volendo così sottolineare un tipo d'intervento assai più ricco e complesso di una semplice strada per ciclisti. L'itinerario è stato qui progettato per risultare un percorso a valenza storico-culturale e paesistica collegante fra loro gli elementi più qualificanti, per una fruizione organica del contesto in cui esso è inserito. Un percorso che ci introduce, ci guida alla "scoperta" del paesaggio, dei luoghi storici e monumentali, delle ricchezze culturali del territorio pavese.

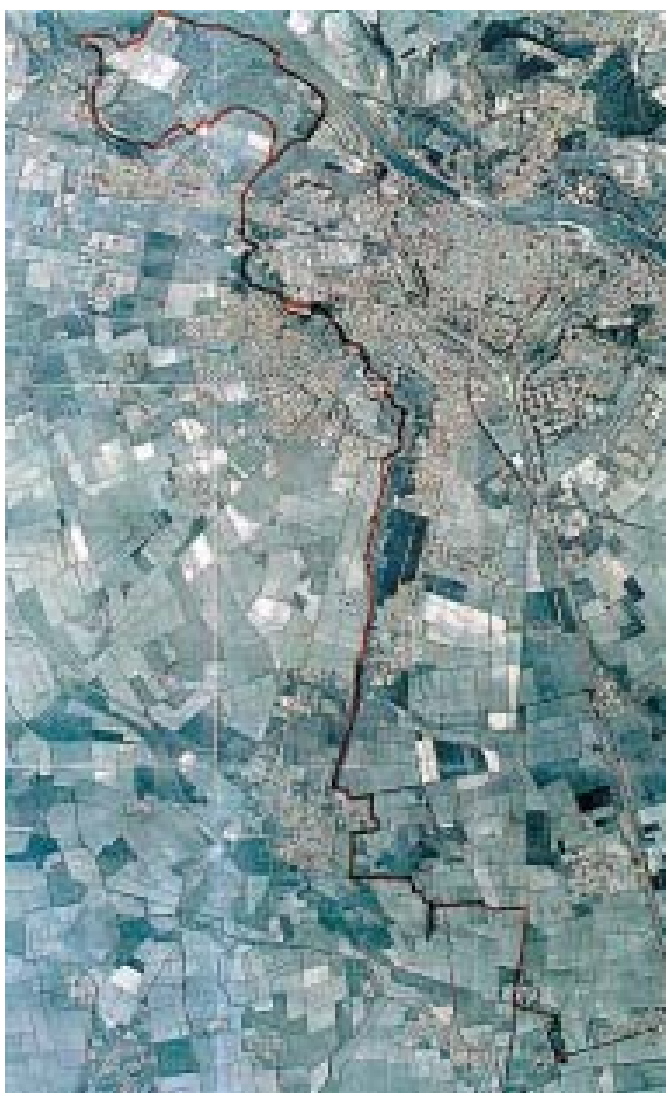

Figura 6 - La Greenway riportata su una foto aerea del 1994.

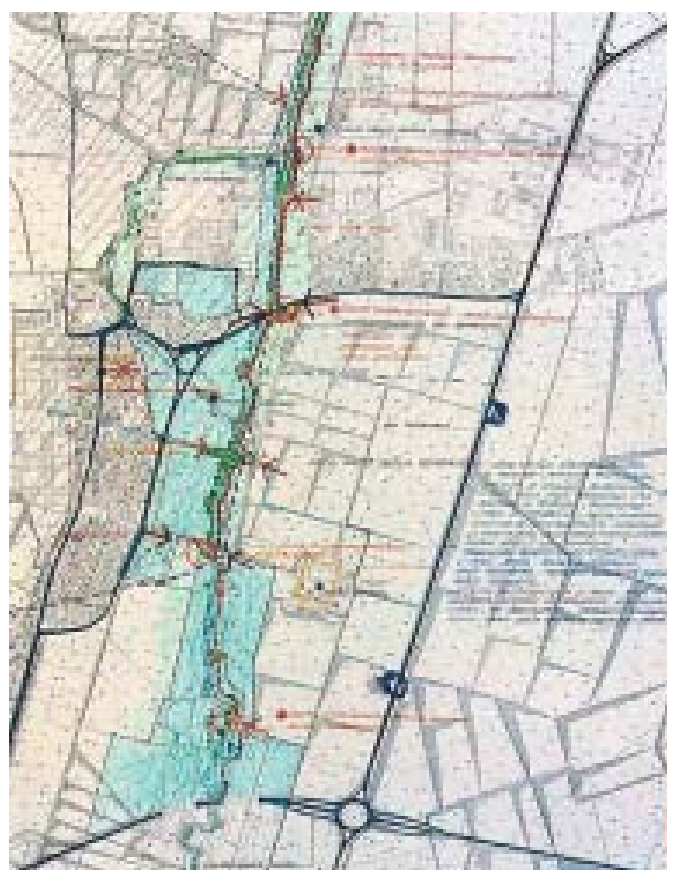

Figura 7 - Planimetria progetto definitivo: tratto C.

\footnotetext{
${ }^{5}$ Clare Littlewood, La Greenway della Battaglia di Pavia, progetto di una via verde pedonale e ciclabile tra la Certosa di Pavia e il fiume Ticino, "Architettura del Paesaggio", 5, 2000, pag. 39.

${ }^{6}$ Daniela Zandonella Necca e Katherina Ziman Scudo, op. cit., 2000, pag. 21.

${ }^{7}$ Daniela Zandonella Necca e Katherina Ziman Scudo, op. cit., 2000, pag. 19.

${ }^{8}$ Clare LitTlewood, op. cit., 2000, pag. 39.
} 


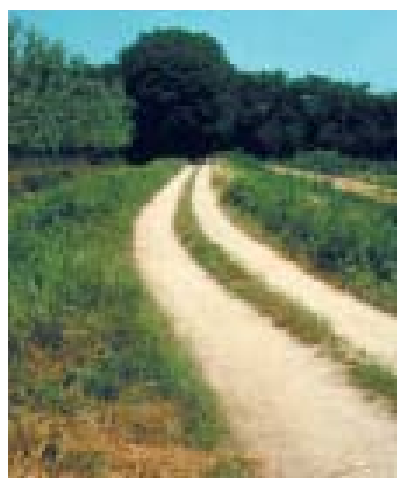

Figura 8 - Tratto dell'itinerario naturalistico.

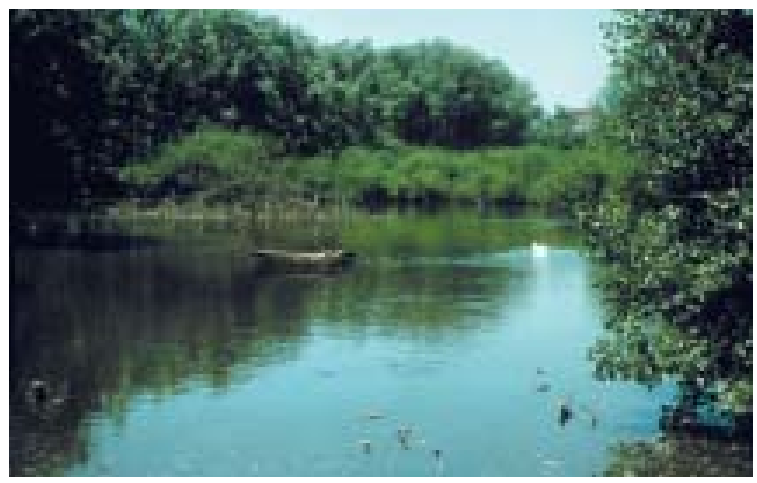

Figura 9 - Zona umida del Parco della Vernavola.

Concludiamo ricordando un interessante progetto, promosso dall'Università di Pavia e attualmente allo studio, riguardante l'estensione della greenway all'interno di un contesto territoriale più ampio; è prevista cioè, la realizzazione di una "corsia verde" non più limitata però al comprensorio pavese ma che consente di collegare la città di Milano a Pavia, Pavia a Voghera e Voghera a Varzì. Questo porterebbe tra l'altro, al significativo recupero della viabilità storica della strada romana in direzione mare e al ripristino delle fasce fluviali, a tutt'oggi in gran parte occupate e coltivate abusivamente.

\section{RIFERIMENTI BIBLIOGRAFICI}

ANGrilli Massimo, Greenways, "Urbanistica”, 113, 1999, pagg. 92-97.

Littlewood Clare, La Greenway della Battaglia di Pavia, progetto di una via verde pedonale e ciclabile tra la Certosa di Pavia e il fiume Ticino, "Architettura del Paesaggio", 5, 2000, pagg. $38-45$.

TURner TOM, Greenways: theory and history, Conferenza Facoltà di Agraria - Università di Milano, 17 maggio 2001.

Zandonella Necca Daniela e Ziman Scudo Katherina, La Greenway della Battaglia di Pavia dalla Certosa al Ticino, "Ambiente Costruito", 1, 2000, pagg. 16-26.

Siti web: <www.comune.pv.it>

\section{RIFERIMENTI ICONOGRAFICI}

Figure 1-7: Clare Littlewood, La Greenway della Battaglia di Pavia, progetto di una via verde pedonale e ciclabile tra la Certosa di Pavia e il fiume Ticino, "Architettura del Paesaggio", 5, 2000, pagg. 38-45.

Figure 8, 9: immagini gentilmente fornite dalla Dott. Arch. Katherina Ziman Scudo. 
Progetto:

Luogo:

Committente:

Superficie:

Progettisti: realizzazione di una "via verde", pedonale e ciclabile, per il collegamento tra la Certosa, la città di Pavia e il fiume Ticino. La lunghezza del percorso principale è pari a $19 \mathrm{~km}$

la zona oggetto dell'intervento interessa, insieme a quello di Pavia, quattro comuni limitrofi: Borgarello, Certosa, Giussago, S. Genesio ed Uniti. L'area è delimitata, a Nord dalla Roggia Roggione, dalla torre del Mangano e dalla Certosa, a Sud dal fiume Ticino e dalla città di Pavia, ad Est dalla strada di Lardirago e ad Ovest dalla statale dei Giovi e dal naviglio pavese

Comune di Pavia

3.500 ettari comprendenti:

Campi coltivati (riso, mais, soia) $\quad 2.880$ ha $(80 \%)$

Pioppeti

Vegetazione naturale

105 ha $(3 \%)$

Incolti

105 ha $(3 \%)$

Aree urbanizzate

335 ha $(11 \%)$

Arch. Paolo Agliardi, Arch. Daniela Zandonella Necca, Arch. Katherina Ziman Scudo (progetto esecutivo)

Gruppo di lavoro (progetto preliminare):

- Donata Vicini - Musei civici di Pavia

- Pierluigi Tozzi - Dipartimento di Geografia Storica del Mondo Antico, Università degli Studi di Pavia

- Francesco Sartori - Dipartimento di Ecologia del Territorio e degli Ambienti Terrestri, Università degli Studi di Pavia

- Giuseppe Marchetti - Dipartimento di Ecologia del Territorio e degli Ambienti Terrestri, Università degli Studi di Pavia

- Riccardo Groppali - Dipartimento di Ecologia del Territorio e degli Ambienti Terrestri, Università degli Studi di Pavia

- Marco Calandra - Associazione Amici della Battaglia

- Luigi Casali - Associazione Amici della Battaglia

Strutture previste:

4 centri interpretativi, 4 centri di servizio, 6 punti di ristoro

Anno di progettazione:

1998 



\title{
IL "PROGRAMMA DI PAESAGGIO CHIANTI" Nel PIANo TerRITORIALE DI CoORdinamento DELLA PROVINCIA DI FIRENZE
}

\author{
Alessandra Cazzola
}

\section{IL CONTESTO}

Il progetto presentato riguarda il paesaggio del Chianti limitatamente alla sola provincia di Firenze ${ }^{1}$, in quanto elaborazione sperimentale di uno dei cosiddetti Programmi di paesaggio previsti dal Piano Territoriale di Coordinamento della provincia di Firenze.

L'area interessata dal progetto ha un'estensione di circa 230 chilometri quadrati e grosso modo coincide con la Val di Greve e il versante di destra della Pesa, ove le innovazioni delle pratiche agricole stanno provocando profonde trasformazioni del paesaggio, andando a disperdere l'immagine storica propria di questa porzione di territorio toscano ed incidendo sugli assetti idrogeologici ed ambientali.

La delimitazione effettuata dipende prevalentemente da considerazioni di tipo geo-morfologico e dal fatto che solo nei versanti indicati è possibile rintracciare alcuni tratti significativi del paesaggio mezzadrile; non sono state quindi prese in considerazione questioni attinenti alla denominazione del vino "Chianti", in quanto queste obbediscono a motivazioni di altro genere.

Il Chianti fiorentino fino a trenta-quaranta anni fa presentava le tipiche caratteristiche del paesaggio mezzadrile delle colline toscane per larga parte inalterate. Luogo di castelli, pievi, fattorie, la struttura del paesaggio si è consolidata sul sistema di crinale, già tracciate dalla viabilità etrusca e attraversate da taluni percorsi sussidiari romani. La villa o il castello, sedi dell'azienda mezzadrile, si disponevano all'incrocio fra il crinale principale e quello secondario; dai crinali si dipanavano i terreni articolati in poderi, rappresentando ciascuno una sorta di microcosmo di specie naturali e di coltivi. La chiusura dell'intero sistema avveniva nel fondo valle, lungo il corso d'acqua, dove il più delle volte veniva sistemato il mulino e altre attrezzature annesse.

In riferimento anche a quelli che sono i principali obiettivi del progetto, è soprattutto il limite sud, dato dal confine provinciale, a presentare le maggiori incongruenze, perché vengono artificialmente tagliate zone appartenenti alla provincia di Siena, del tutto simili da un punto di vista morfologico ed agronomico. È comunque difficile, dal punto di vista operativo, immaginare politiche di tutela ambientale e di incentivo agli imprenditori agricoli che si fermino ai confini fra le province di Siena e Firenze, anche se gli aspetti metodologici del progetto non vengono messi in discussione dai limiti attuali

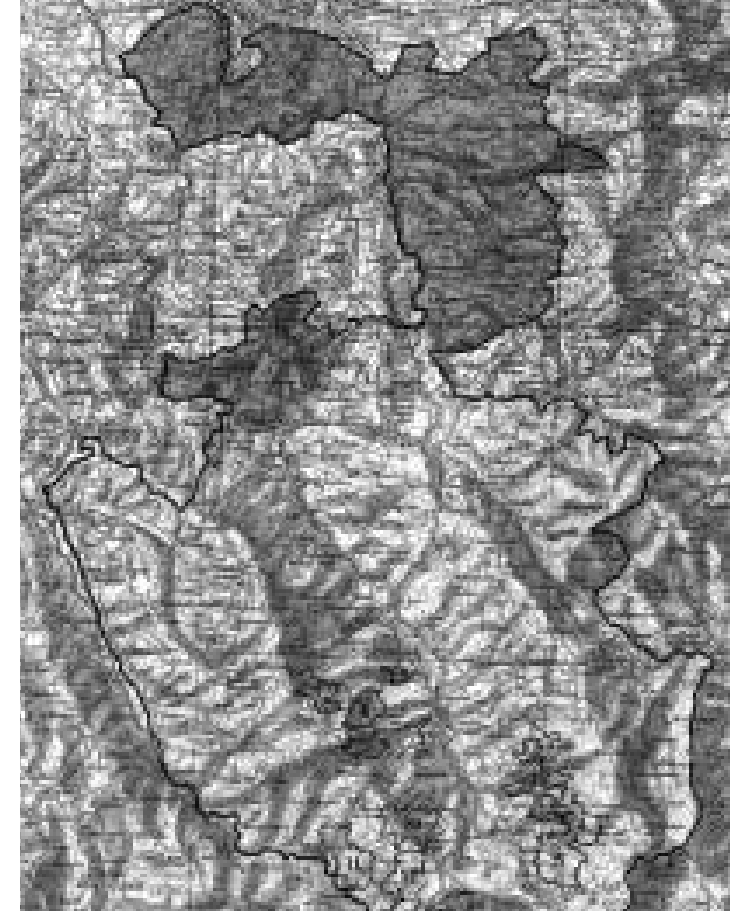

Figura 1 - L'area del progetto di tutela del paesaggio chiantigiano.

${ }^{1}$ I comuni chiantigiani sono: Barberino Val d'Elsa, Greve in Chianti, San Casciano in Val di Pesa, Tavarnelle in Val di Pesa nella provincia di Firenze e i comuni di Castellina in Chianti, Castelnuovo Berardenga, Gaiole in Chianti, Radda in Chianti nella provincia di Siena. 


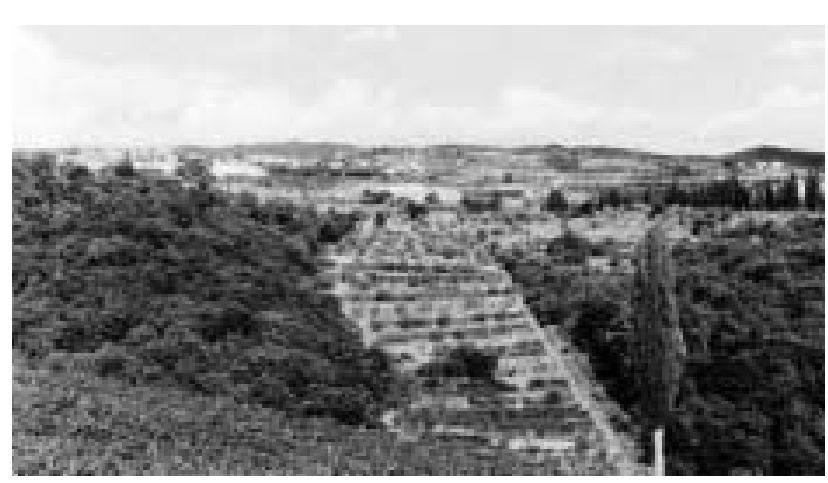

Figura 2. - La fotografia mostra un esempio relativamente conservato di sistemazioni tradizionali.

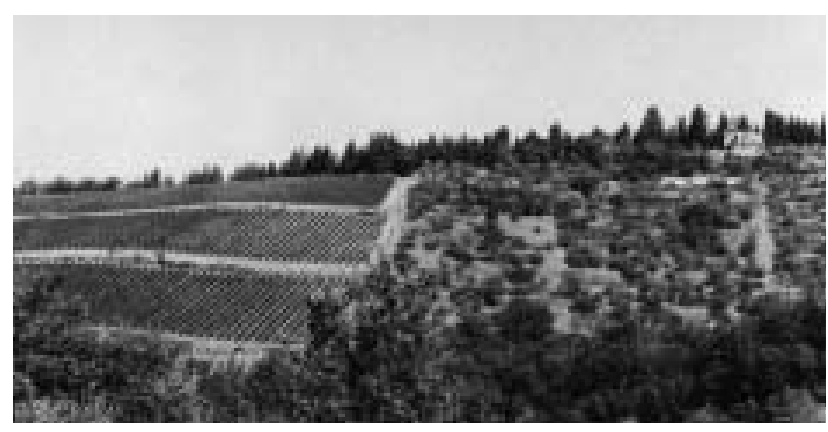

Figura 3. - In questo caso, invece, le colture e le sistemazioni tradizionali sono state in parte sostituite da un vigneto specializzato con impianti a "rittochino" interrotti da muri realizzati con mezzi meccanici. del Programma di Paesaggio, poiché il campione di aree di paesaggio mezzadrile individuato è rappresentativo di unità paesaggistiche sufficientemente variegate. Diventa dunque fondamentale considerare il progetto di tutela del paesaggio del Chianti fiorentino all'interno di una strategia unitaria e integrata delle politiche urbanistiche, ambientali e paesaggistiche dei comuni del Chianti, dove i confini amministrativi (comunali e provinciali) possono al massimo definire le articolazioni di questa strategia.

La realtà sociale chiantigiana, inoltre, è particolarmente composita e come tale al suo interno presenta conflitti la cui mediazione risulta essere particolarmente difficile. Da una parte, infatti, vi sono i produttori agricoli che vogliono avere un'ampia libertà nell'adattare il paesaggio alla tecnologia imposta e richiesta dal mercato; dall'altra troviamo gli imprenditori i cui prodotti sono promossi e valorizzati proprio dai contenuti storici del paesaggio (solo in alcuni casi le due figure coincidono, dando così origine ad interessanti, seppure episodiche, forme di tutela individuale); da un'altra ancora, infine, c'è la cosiddetta società civile, ovvero i residenti che non solo godono del "bel paesaggio" ma che intendono anche preservare i valori immobiliari delle loro proprietà, e le varie associazioni politico-culturali preoccupate della possibile scomparsa di preziose testimonianze storiche.

\section{GLI OBIETTIVI}

L'impostazione teorica del progetto nasce dalla constatazione che le politiche di tutela del paesaggio difficilmente possono rappresentare politiche di conservazione integrale. Nel Programma di paesaggio, quindi, l'approccio è stato di "tipo strutturale", ovvero il paesaggio storico chiantigiano è stato studiato come una struttura articolata in tre livelli fondamentali, nella quale i livelli superiori condizionano ma non determinano quelli inferiori.

Tre sono i fondamentali livelli strutturali del paesaggio collinare chiantigiano individuati:

- il primo livello è costituito dalla struttura profonda del territorio, ovvero il sistema territoriale fondativo che, essendo dotato di resistenza, permane nel tempo e che, nel caso del Chianti, è caratterizzato da un sistema insediativo definito da una viabilità matrice di base e dall'edificazione ad essa collegata (borghi, castelli, ville, chiese, eccetera);

- la struttura del secondo livello, cioè la struttura portante del paesaggio mezzadrile, è quella condizionata dal sistema insediativo storico, disposto lungo i crinali principali o secondari, e dal reticolo idrografico;

- il terzo ed ultimo livello strutturale, quello delle coltivazioni tradizionali, si può dire che è oggi praticamente scomparso, in quanto la sua morfologia, definita dagli usi, dalle tecniche agrarie e dalle modalità di coltivazione, non ha resistito alle dinamiche più o meno recenti di trasformazione. Quello che ancora sopravvive, anche se per limitate estensioni, è il suo "scheletro", ossia il sistema di contenimento dei terreni e il sistema di controllo delle acque superficiali che modellava buona parte delle pendici collinari. 
La strategia di base del progetto è dunque basata sulla possibilità di modificare alcune regole di uno specifico livello strutturale del tipico paesaggio mezzadrile, mantenendo però le relazioni fra le principali componenti e il senso d'ordine sostanziale. A seconda del maggiore o minore valore di testimonianza storica e del grado di conservazione tuttora esistente, è stato proposto di conservare uno o più "livelli strutturali", consentendo però di modificare, a partire da un certo livello, con certe modalità e a certe condizioni, l'organizzazione territoriale o alcuni suoi elementi.

Gli obiettivi fondamentali del programma sono stati quelli di:

- definire i caratteri della struttura profonda del territorio, tutelandoli e rafforzandoli;

- individuare tutte quelle porzioni di territorio ove è ancora possibile riconoscere il paesaggio mezzadrile e la sua organizzazione, confermandola in alcune sue regole consolidate e razionali (la localizzazione dei manufatti, la viabilità poderale, la disposizione delle aree con copertura boschiva, le sistemazioni agrarie tradizionali compatibili con certi livelli e qualità di produzione, eccetera);

- fornire indirizzi di recupero e tutela, insieme ad una valutazione dei costi degli interventi necessari, consentendo quindi una serie di trasformazioni a scala più piccola, secondo nuove regole che si accordino alla struttura complessiva individuata in precedenza.

\section{LA METODOLOGIA}

Il primo livello di analisi è consistito nell'individuare, descrivere e rappresentare la struttura profonda del territorio, cercando di riconoscere i suoi caratteri distintivi, ossia:

- la strutturalità, intesa come ruolo di base che caratterizza e condiziona tutti i sistemi e gli elementi che definiscono la morfologia del paesaggio;

- la connettività, cioè il fatto che i suoi elementi siano fra loro legati dal punto di vista morfologico e funzionale;

- la profondità storica, intesa come continuità formale (e quindi coerenza morfologica) rispetto all'evolversi delle forme di organizzazione sociale ed economica;

- la contestualità, cioè la permanenza di legami fisici e funzionali nel contesto territoriale;

- la resistenza alle trasformazioni territoriali, che la rende ancora riconoscibile dal punto di vista morfologico.

La struttura profonda è stata definita nei suoi caratteri fisici secondo l'individuazione di tre sistemi fondamentali fra loro connessi, matrici a loro volta della morfologia generale del paesaggio:

- il sistema insediativo storico;

- il reticolo idrografico principale, in quanto espressione dei caratteri litologici e dell'evoluzione dei processi della dinamica morfologica;

- $i$ boschi e le foreste, che attualmente si presentano come un insieme di aree con copertura boschiva di varia natura e con diversi regimi di conduzione e che, se collegate fra loro e opportunamente gestite, potrebbero contribuire a formare un ecosistema complesso, biologicamente funzionale.

Successivamente, il secondo livello di analisi ha riguardato l'individuazione e la descrizione delle aree di paesaggio mezzadrile. Nella ricerca sono stati assunti tre parametri concorrenti per l'individuazione di tali aree: il primo, ha interessato le loro dimensioni che, anche se non sono state definite a priori, sono state scelte in 

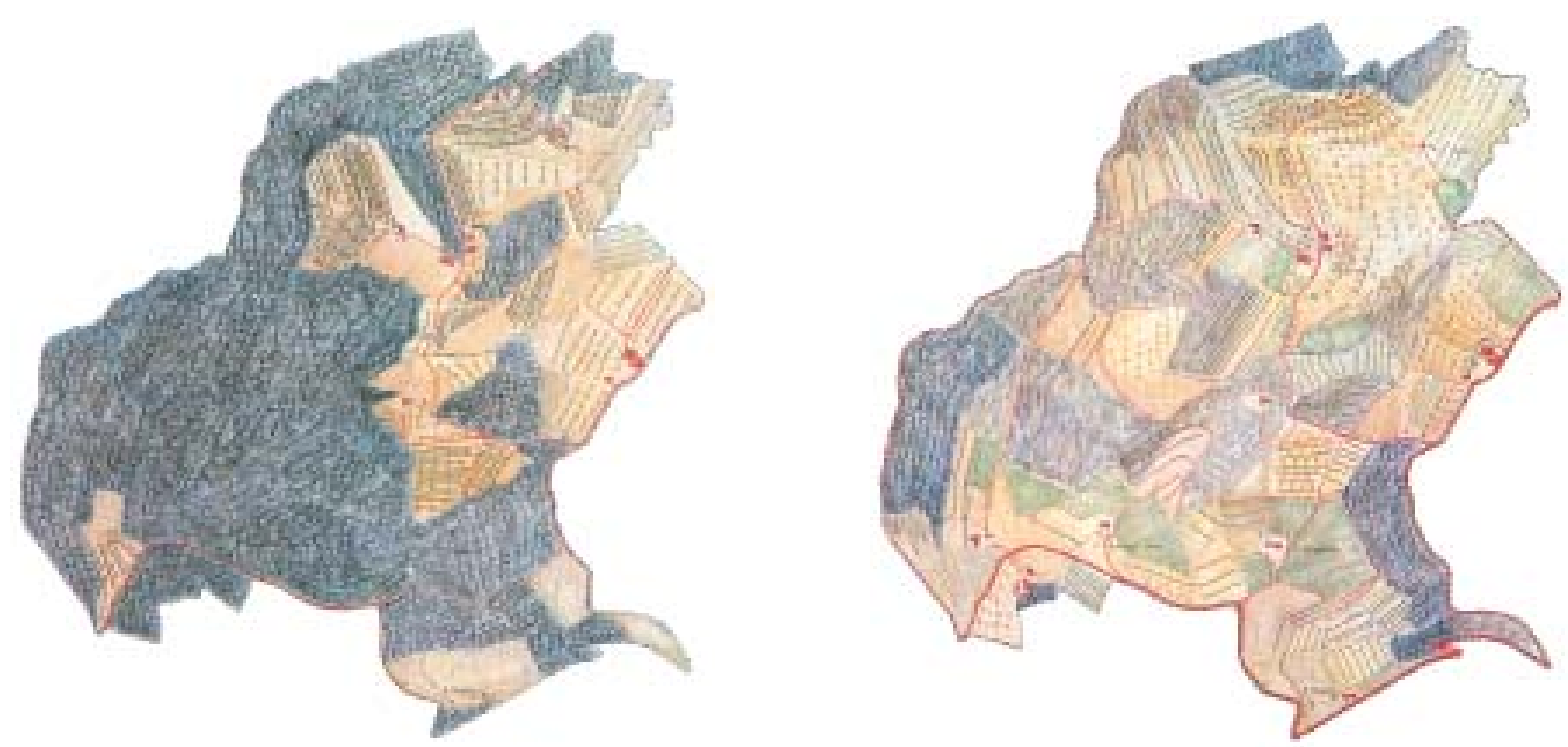

Figure 4, 5 - L'area di paesaggio mezzadrile di San Cresci: a sinistra la ricostruzione dello stato di fatto nel 1825 in base al Catasto Generale Toscano; a destra la ricostruzione dello stato di fatto nel 1989-1998 in base alla Carta Tecnica Regionale e ad una serie di sopralluoghi (nelle tavole sono rappresentati gli elementi fondamentali presenti alla data della documentazione utilizzata: le strade vicinali e poderali, l'edilizia rurale con le aree di pertinenza, i terrazzamenti, i ciglioni, le lunette, gli oliveti, i vigneti maritati all'olivo e quelli in coltura specializzata, i seminativi, i boschi e il reticolo idrografico principale e secondario).

modo tale da consentire interventi paesaggistici, ambientali e agronomici di un certo respiro (empiricamente, tralasciando piccole zone che possono essere considerate solo dei frammenti, l'ampiezza minima è quindi risultata di circa 40 ettari).

Il secondo parametro ha studiato gli elementi che definiscono la "storicità" dell'area. Esclusa la permanenza di assetti di coltura promiscua - quasi ovunque scomparsi o, dove presenti, con un valore del tutto residuale - l'attenzione è stata incentrata sulle infrastrutture e sulle sistemazioni agrarie di supporto e, precisamente sul quel complesso di opere che da una parte modellavano e contenevano i terreni e dall'altra ne assicuravano la stabilità idrogeologica. È stata perciò assunta come decisiva, per l'individuazione delle aree di paesaggio mezzadrile, la presenza di una rete di viabilità vicinale e poderale e di elementi come muri a secco, ciglioni, dreni tradizionali, tali da funzionare ancora come un sistema agronomico e idraulico, in uno stato di conservazione sufficiente per consentirne il ripristino o una limitata e controllata trasformazione.

Il terzo parametro, infine, si è interessato della frequenza delle sistemazioni agrarie, che non devono presentarsi come gruppi isolati, ma, al contrario, è necessario che definiscano una diffusa organizzazione paesaggistica.

In definitiva, un'area di paesaggio mezzadrile deve essere abbastanza estesa tanto da permettere politiche coordinate e integrate di intervento e da presentare opere di sistemazione agraria sufficientemente dense, relazionate fra loro, e in uno stato di degrado tale da non impedirne una parziale o totale conservazione.

Il terzo livello di analisi, infine, ha coinciso con il problema più complesso della ricerca: mettere a punto un metodo speditivo ma allo stesso tempo attendibile, per valutare le operazioni di ripristino e di trasformazione di ciascuna area di paesaggio mezzadrile e i relativi costi.

In primo luogo, è stata individuata un'area campione in cui fossero presenti le diverse tipologie di sistemazioni agrarie; in secondo luogo, è stata compiuta un'analisi approfondita di ciascuna tipologia rispetto alle tecniche costruttive impiegate, alle modalità di manutenzione, restauro o ripristino, alle possibilità di evoluzione diacronica della tipologia stessa, ai materiali da usare, ai costi, ecce- 
tera. A questo punto, ogni tipologia è stata articolata a seconda di un parametro di degrado o di dissesto e dell'intervento proposto ${ }^{2}$.

Sono stati così individuati undici interventi-tipo, ciascuno definito dall'incrocio fra la tipologia base (muro o acquidoccio), e l'intervento, conseguente o possibile, riferito al suo stato di degrado/dissesto.

Per ogni intervento-tipo è stata preparata una scheda identificativa con la descrizione sintetica delle operazioni di intervento e il calcolo dei costi, sulla base di una definizione analitica delle opere necessarie e dei loro prezzi in economia. Al momento di effettuare la ricerca sul campo è dunque possibile stabilire in modo speditivo la tipologia dell'opera di sistemazione agraria, il tipo di intervento e il costo relativo.

\section{GLI ESITI}

Il primo livello paesaggistico, la struttura profonda del territorio, è stato descritto e rappresentato a partire dal sistema insediativo storico definito secondo criteri di coerenza morfologica e non sulla base di un'astratta periodizzazione. La rappresentazione cartografica è stata quindi disegnata con tecniche che assicurano un'immediata ed evidente leggibilità della forma del territorio ed è stata studiata una sorta di normativa impostata su misure di tutela proposte come indirizzi per gli strumenti urbanistici comunali ed espresse nella forma di criteri o regole funzionali, morfologiche e tipologiche da rispettare, in relazione a:

- la viabilità matrice;

- gli elementi puntuali disposti lungo la viabilità matrice;

- le aree di pertinenza della viabilità matrice;

- i centri abitati esistenti per le parti morfologicamente incoerenti;

- i nuovi nuclei residenziali;

- la protezione visiva dei crinali;

- le relazioni fra crinali e fondovalle.

Il Programma di paesaggio ha poi fornito anche alcuni indirizzi e criteri per il miglioramento e la gestione delle aree boschive, nonché la stima dei costi unitari degli interventi proposti.

Per quanto riguarda il secondo livello strutturale, il paesaggio mezzadrile, i risultati della ricerca confermano le preoccupazioni e la fondatezza degli obiettivi che fin dall'inizio hanno guidato il progetto.

In tutta l'area presa in esame, che come accennato misura circa 23.000 ettari, sono ancora individuabili 1300 ettari di paesaggio mezzadrile, suddivisi in tredici aree di dimensioni variabili da 40 a 160 ettari, pari a meno del 6\% della superficie complessiva.

È quindi possibile "consegnare" alle future generazioni soltanto alcuni residui di paesaggio storico - costruito fra il XIII secolo e il primo scorcio del Novecento, con momenti salienti nel Rinascimento e nell'Ottocento - testimoni della cultura e del lavoro di una formazione sociale che ha improntato come nessun’altra di sé il territorio.

Per ogni area, o per gruppi di aree, sono stati formulati indirizzi e criteri articolati nei seguenti punti:

- gestione e miglioramento delle colture arboree della vite e dell'olivo (individuando per gli oliveti gli interventi tipo e i relativi costi unitari) e delle aree

${ }^{2}$ Per fare un esempio, i muri a secco sono stati divisi nelle tipologie: "muro di contenimento della viabilità", "muro di ciglione", "muro di sottoscarpa"; successivamente, ognuna di queste tipologie è stata ulteriormente distinta in ragione degli interventi necessari per assicurarne un corretto funzionamento ("manutenzione ordinaria", "rifacimento parziale", "rifacimento a seguito di fenomeni di spanciamento", "demolizione e ricostruzione", eccetera). 

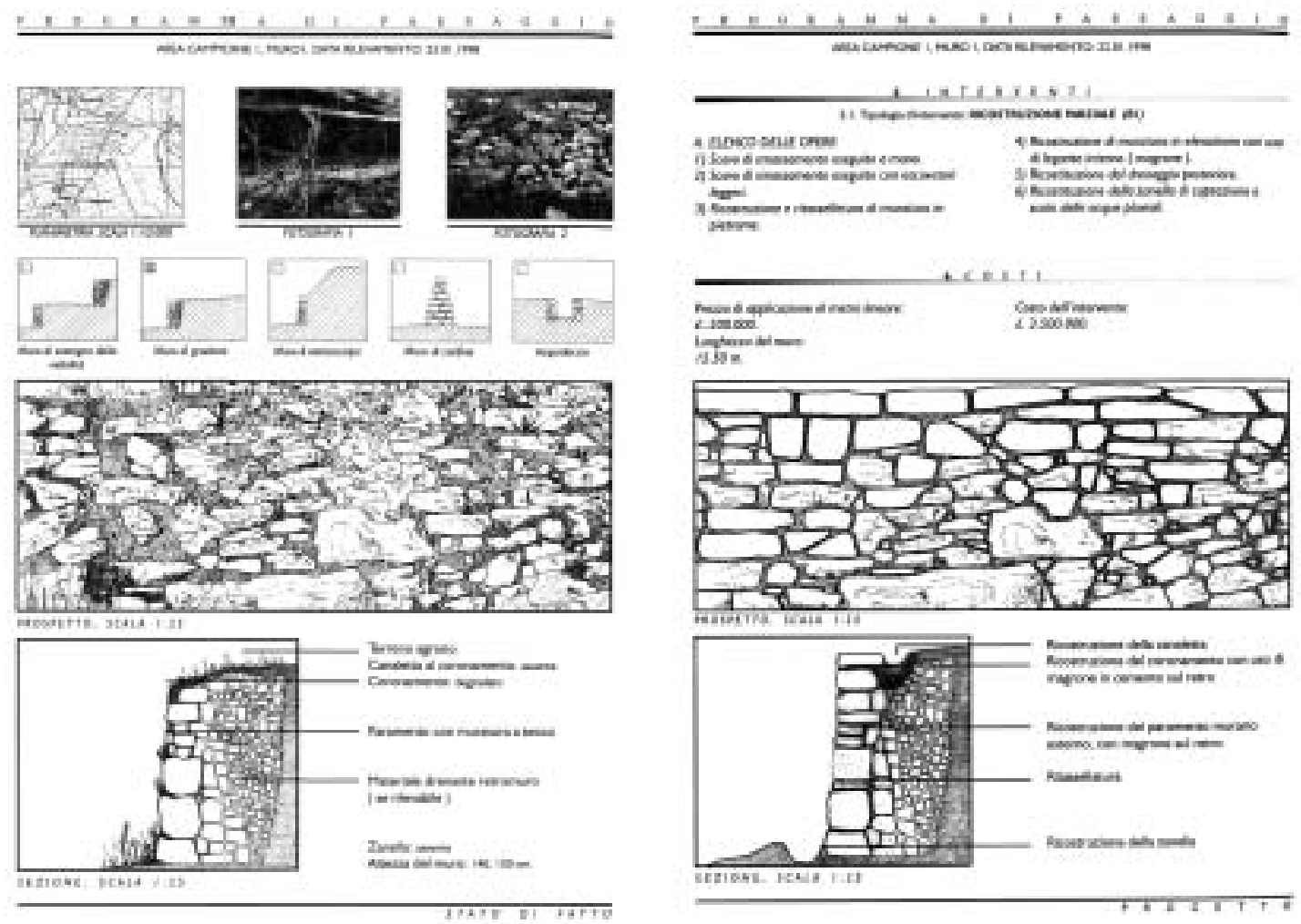

Figure 6, 7 - Schede tipo del "Manuale": a sinistra la scheda con il rilievo della sistemazione di un tipo di muretto a secco; a destra la scheda con gli interventi consigliati per il recupero e i relativi costi previsti.

con copertura boschiva (definendo le operazioni tipo a seconda delle caratteristiche forestali e i relativi costi unitari);

- gestione e miglioramento del reticolo idrografico superficiale con la stima dei costi;

- stima dei costi necessari per la manutenzione e il ripristino delle sistemazioni idraulico-agrarie tradizionali.

Per il terzo ed ultimo livello strutturale, costituito dalle sistemazioni idrauliche tradizionali (muri a secco, ciglioni, acquidocci), l'idea guida è stata quella di preparare un "Manuale" che da una parte consentisse di calcolare i costi di ripristino del paesaggio agrario nelle diverse aree e, dall'altra, potesse essere immediatamente utilizzato dagli operatori agricoli per eseguire in modo corretto gli interventi opportuni. Questo "Manuale" è costituito da:

- introduzione esplicativa;

- abaco delle tipologie esistenti e degli interventi proposti;

- scheda di rilievo esemplificativa con le relative istruzioni;

- schede di intervento articolate in una parte descrittiva dello stato attuale e una parte esplicativa degli interventi, con i prezzi di applicazione al metro lineare;

- norme di attuazione con la descrizione delle opere-tipo e dei prezzi medi.

La tutela ed il recupero del paesaggio storico chiantigiano non può, però, essere pensato senza una partecipazione attiva dei diversi soggetti interessati: le amministrazioni pubbliche, gli imprenditori agricoli, la popolazione rurale. Gli strumenti da utilizzare devono quindi necessariamente essere diversi e coordinati tra loro: servono, in primo luogo, progetti ed, in secondo luogo, finanziamenti da erogare nella veste di incentivi alla produzione e direttamente finalizzati al 
ripristino delle sistemazioni idraulico-agrarie; in terzo luogo occorre una qualche forma di gestione che sia in grado di coordinare le politiche con i progetti e faciliti l'accesso da parte degli imprenditori ai finanziamenti.

Laspetto più interessante del Programma di paesaggio Chianti è rappresentato proprio da questo suo essere innanzi tutto uno strumento per la programmazione paesistico-territoriale. Il progetto si è dunque fermato al primo punto, offrendo indirizzi, criteri, progetti, stime dei costi, alla comunità chiantigiana e a tutta la società toscana, rappresentando la base di partenza e di conoscenza per poter affrontare gli altri due punti, non limitandosi esclusivamente a dettare regole e ad imporre vincoli per la tutela delle sistemazioni idraulico-agrarie di tipo tradizionale.

Allo stato attuale, il progetto costituisce un lavoro in progress che va discusso, integrato e migliorato, in modo tale che si possa consapevolmente decidere se la tutela del paesaggio chiantigiano è sostenibile anche da un punto di vista sociale ed economico.

\section{RIFERIMENTI BIBLIOGRAFICI}

Per il "Programma di Paesaggio":

Alfaioli Alessandro (et al.), La tutela del paesaggio delle colline: il Piano Guida della Provincia di Firenze, supplemento a "Paesaggio Urbano", 5, Maggioli, Rimini 1998.

BALDESCHI PAOlO (a cura di), Il Chianti fiorentino: un progetto per la tutela del paesaggio, Laterza, Roma-Bari 2000.

BALDESCHI PAOLO, Un progetto per la tutela del paesaggio storico chiantigiano metodologia e risultati, contributo al Seminario del Dottorato di ricerca in Progettazione Paesistica «Paradigmi di lettura per il paesaggio agrario: componenti, relazioni, scenari», Firenze 7 maggio 2001.

Per il Piano Territoriale di Coordinamento della Provincia di Firenze:

BAldesChi PAOlO, Il piano territoriale di coordinamento della provincia di Firenze, in "Paesaggio Urbano", 3, Maggioli, Rimini 1996, pagg. 8-9.

Conti Riccardo e Ulivieri Luigi (a cura di), Piano Territoriale di Coordinamento Provinciale, Franco Angeli, Milano 2000.

Cusmano Mario Guido (a cura di), Città e insediamenti. Dalle prospettive dell'area vasta alla costruzione dello statuto dei luoghi, Franco Angeli, Milano 2002.

Piano Territoriale di Coordinamento Provinciale: Progetto preliminare, (a cura della provincia di Firenze - Settore Pianificazione Territoriale), Firenze 1996.

Ulivieri Luigi (a cura di), Lineamenti del piano territoriale di coordinamento della Provincia di Firenze, Provincia di Firenze, Firenze 1995.

ULIVIERI LUIGI (a cura di), PTCP: conoscere per progettare: voci, frammenti, esperienze nel "cantiere" del Piano, "Ad Arnum. Quaderni dell'Assessorato Urbanistica e Assetto del Territorio della Provincia di Firenze", 2, Firenze 1994.

Ulivieri LUIGI, La struttura del piano, "Paesaggio Urbano", 3, Maggioli, Rimini 1996, pagg. 20-21.

Valutazioni sull'assetto territoriale della provincia di Firenze: interpretazioni e indicazioni di metodo e operative, "Ad Arnum. Quaderni dell'Assessorato Urbanistica e Assetto del Territorio della Provincia di Firenze", 0, Firenze 1990.

Verso il piano territoriale di coordinamento (a cura della prov. di Firenze), Firenze 1992.

Siti web: <www.provincia.firenze.it>

\section{RIFERIMENTI ICONOGRAFICI}

Le immagini sono tratte da: BALDESCHI PAOLO (a cura di), Il Chianti fiorentino: un progetto per la tutela del paesaggio, Laterza, Roma-Bari 2000, pagg. X, VI, VI, 60, 61, 174, 175. 
SCHEDA DI PROGETTO

Progetto:

Luogo:

Committente:

Superficie:

Progettisti:

Anno di progettazione:

Stato di attuazione: intervento di recupero delle aree di paesaggio storico mezzadrile

comuni di Barberino Val d'Elsa, Greve in Chianti, San Casciano in Val di Pesa, Tavarnelle in Val di Pesa (provincia di Firenze)

Provincia di Firenze

$230 \mathrm{kmq}$ circa

Paolo Baldeschi (coordinatore responsabile)

Guia Agostini (ingegneria ambientale)

Angiolo Caselli (sistemazioni idraulico-agrarie tradizionali)

David Fantini (analisi e rappresentazione del territorio)

Alessandro Fonseca (agronomia)

Carlo Alberto Garzonio (geologia)

Paolo Giustiniani (ingegneria idraulica)

Gianluca Guiducci (agronomia)

Luciano Lucani (scienze forestali)

Stefano Monni (ingegneria idraulica)

Rino Vardaro (architettura e rappresentazione del territorio)

1998

realizzata la prima fase della definizione degli ambiti e delle metodologie di intervento 

Finito di stampare in Firenze presso la tipografia editrice Polistampa Giugno 2004 